Air Sampling Associates, Inc.

Client Sample ID: HARDIN STACK RUN 2 HNO3/H2O2

TOTAL Metals

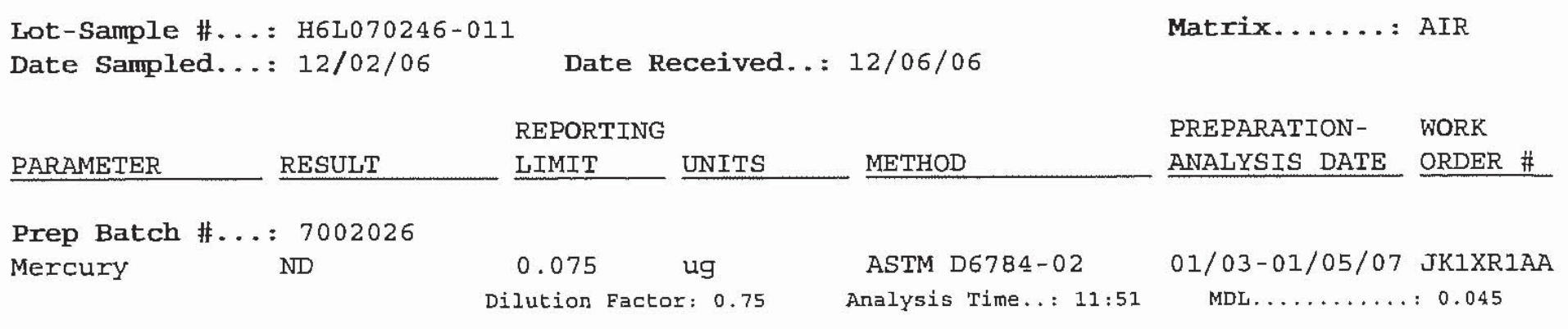


Air Sampling Associates, Inc.

Client Sample ID: HARDIN STACK RON 2 KMNO4/H2SO4

TOTAL Metals

Lot-Sample \#...: H6L070246-012

Date Sampled...: 12/02/06

Date Received. .: 12/06/06

REPORTING

LIMIT

UNITS

METHOD

Prep Batch \#...: 7002028

Mercury
12.1
ASTM D6784-02

Analysis Time..: 11:55
Matrix....... AIR

PREPARATION- WORK ANALYSIS DATE ORDER \#

01/04/07 JK1XT1AA

MDL $. \ldots \ldots \ldots: 0.28$ 
Air Sampling Associates, Inc.

Client Sample ID: FARDIN STACK RUN 3 FH

TOTAL Metals

Lot-Sample \#...: H6L070246-015

Date Sampled...: 12/02/06

PARAMETER

RESULT

Prep Batch \#...: 7002025

Mercury $0.0090 \mathrm{~B}$
Date Received..: 12/06/06

REPORTING LIMIT UNITS 0.010 ug
Dilution Factor: 0.1
Matrix...... AIR

PREPARATION- WORK ANALYSIS DATE ORDER \# ASTM D6784-02 01/02-01/05/07 JK1X51AA Analygis Time..: $11: 27$

\section{NOTE (S) :}

B Estimated result. Result is less than RL. 
Air Sampling Associates, Inc.

Client Sample ID: HARDIN STACK RUN 3 KCL

TOTAL Metals

Irot-Sample 壮... : H6L0702\$6-016

Date Sampled...: 12/02/06

PARAMETER

Prep Batch \#...: 7002028

Mercury
$0.080 \mathrm{~B}$

RESULT

0.12

Dilution Factor: 1.25

LIMIT

UNITS
Date Received..: 12/06/06

REPORTING METHOD

ASTM D6784-02

Analysis Time..: 11:11
Matrix...... AIR

PREPARATION- WORK ANALYSIS DATE ORDER \#

01/04/07 JKבX81AA MDL......... 0.075

$\operatorname{NOTE}(\mathrm{S})$ :

B Estimated result. Result is less than RL. 
Air Sampling Associates, Inc.

Client Sample ID: HARDIN STACK RUN 3 HNO3/H2O2

TOTAL Metals

Irot-Sample \#...: H6L070246-017

Date Sampled...: 12/02/06

PARAMETER

Prep Batch \#...: 7002026

Mercury

0.064
Date Received..: 12/06/06

REPORTING LIMIT UNITS

$0.063 \quad$ ug
Dilution Factor: 0.63 METHOD
Matrix....... AIR

PREPARATION - WORK ANALYSIS DATE ORDER \#

ASTM D6784-02

Analysis Time..: 11:53
01/03-01/05/07 JK10A7AA MDL.......... 0.038 
Air Sampling Associates, Inc.

Client Sample ID: HARDIN STACK RON 3 KMNO4/H2SO4

TOTAL Metals

Lot-Sample \#... : H6L070246-018

Date Sampled...: 12/02/06

PARAMETER

Prep Batch \#...: 7002028

Mercury
6.4

Date Received. .: 12/06/06

REPORTING LIMIT

UNITS
METHOD

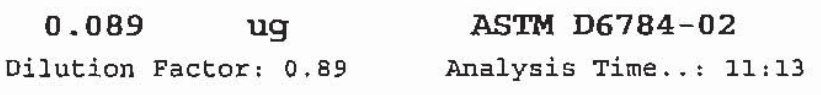

Matrix.....: AIR

PREPARATION- WORK ANALYSIS DATE ORDER \#

01/04/07 JK10E1AA MDL......... 0.053 
Air Sampling Associates, Inc.

Client Sample ID: HARDIN SDA INLET DUCT RUN I FH TOTAL Metals

Lot-Sample \#... : H6L070246-021

Date Sampled...: 12/01/06

PARAMETER

Prep Batch \#...: 7002025

Mercury
0.61

Date Received. .: 12/06/06

REPORTING LIMIT UNITS METHOD ASTM D6784-02 Analysis Time..: 11:29

Matrix...... AIR

PREPARATION- WORK ANALYSIS DATE ORDER \#

01/02-01/05/07 JK1051AA MDL.......... 0.0060 
Air Sampling Associates, Inc.

Client Sample ID: HARDIN SDA INLBT DUCT RUN I KCL

TOTAL Metals

Lot-Sample \#. . : : H6L070246-022

Date Sampled...: 12/01/06

PARAMETER

Prep Batch \#...: 7002028

Mercury
1.6
Date Received. .: 12/06/06

REPORTING

LIMIT

UNITS

ug

Dilution Factor: 1.11
Matrix...... AIR

PREPARATION- WORK ANALYSIS DATE ORDER \#

01/04/07 JK1061AA 
Air Sampling Associates, Inc.

Client Sample ID: HARDIN SDA DNLET DUCT RUN 1 HNO3/H2O2

TOTAL Metals

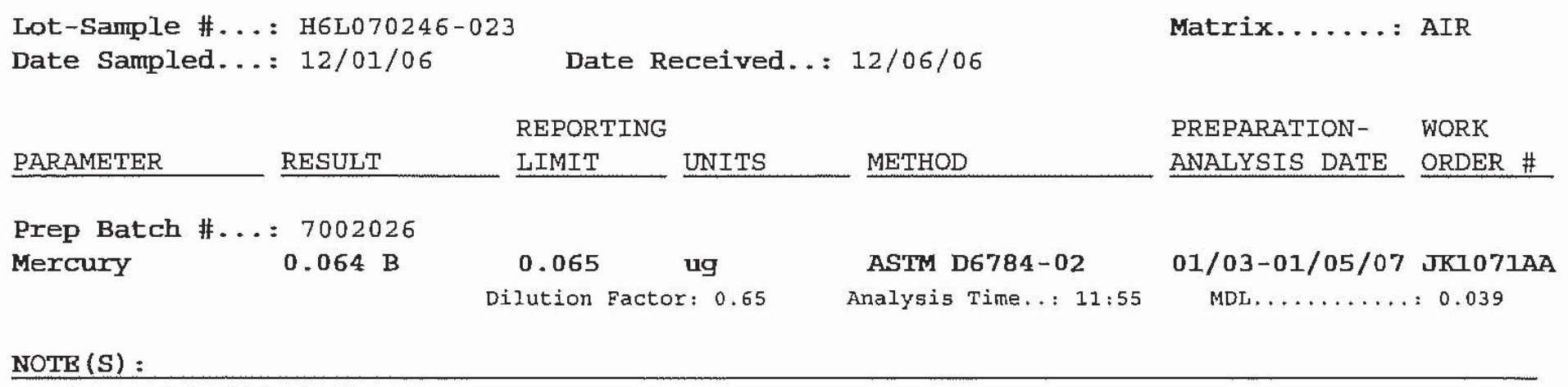

B Estimated result, Result is less than RL. 
Air Sampling Associates, Inc.

Client Sample ID: HARDIN SDA INLET DUCT RUN 1 KMNO4/H2SO4

TOTAL Metals

Lot-Sample \#...: H6L070246-024

Date Sampled...: 12/01/06

PARAMETER

Prep Batch \#...: 7002028

Mercury
3.6
Date Received. .: 12/06/06

REPORTING LIMIT UNITS

ug Dilution Factor: 0.89
Matrix......: AIR

PREPARATION- - WORK ANALYSIS DATE ORDER \#

01/04/07 JK11A1AA

MDL........ 0.053 
Air Sampling Associates, Inc.

Client Sanple ID: HARDIN SDA INLET DUCT RUN 2 FH TOTAL Metals

Lot-Sample \#...: H6Iد070246-027

Date Sampled. . : 12/02/06

PARAMETER

Prep Batch \#...: 7002025

Mercury
1.5
Date Received. .: 12/06/06

REPORTING LIMIT

UNITS

ug

Dilution Factor: 0.5
Matrix.....: AIR

PREPARATION- WORK ANALYSIS DATE ORDER \#

ASTM D6784-02

Analysis Time..: $12: 08$
01/02-01/05/07 JK1IFIAA MDL......... 0.030 
Air Sampling Associates, Inc.

Client Sample ID: HARDIN SDA INLET DUCT RUN 2 KCL TOTAL Metals

Lot-Sample \#. . : H6L070246-028

Date Sampled...: 12/02/06

PARAMETER

RESULT

Prep Batch \#...: 7002028

Mercury
1.7
Date Received..: 12/06/06

REPORTING LIMIT UNITS $\begin{array}{ll}0.12 & \text { ug } \\ \text { Dilution } & \text { Factor: } 1.15\end{array}$
Matrix..... : AIR

PREPARATION- WORK ANALYSIS DATE ORDER \#

ASTM D6784-02 01/04/07 JK11K1AA

Analysis Time..: 11:19 
Air Sampling Associates, Inc.

Client Sample ID: HARDIN SDA INLET DUCT RUN 2 HNO3/H2O2

TOTAL Metals

Lot-Sample \#...: H6L070246-029

Date Sampled...: 12/02/06

PARAMETER

Prep Batch \#...: 7002026

Mercury
0.10
Date Received..: 12/06/06

REPORTING IIMIT UNITS

ug Dilution Factor: 0.63
Matrix...... AIR

PREPARATION- WORK ANALYSIS DATE ORDER \# ASTM D6784-02 01/03-01/05/07 JK11LIAA Analysis Time. ; 11:57 
Air Sampling Associates, Inc.

Client Sample ID: HARDIN SDA INLET DUCT RUN 2 KMNO4/H2SO4

TOTAL Metals

Lot-Sample \#. . : H6L070246-030

Date Sampled...: 12/02/06

PARAMETER

Prep Batch \#...: 7002028

Mercury
4.6
Date Received..: 12/06/06

REPORTING LIMIT UNITS

ug Dilution Factor: 0.92 METHOD
Matrix...... AIR

PREPARATION- WORK ANALYSIS DATE ORDER \#

ASTM D6784-02

Analysis Time..: 11:25
01/04/07 JKZ1Q1AA MDL......... 0.055 
Air Sampling Associates, Inc.

Client Sample ID: HARDIN SDA INLET DUCT RUN 3 FH

TOTAL Metals

Irot-Sample 非... H6L070246-033

Date Sampled...: 12/02/06

PARAMETER

Pxep Batch \#...: 7002025

Mercury

$$
0.64
$$

Date Received..: 12/06/06

REPORTING LIMIT

0.010 ug

Dilution Factor: 0.1
Matrix...... AIR

PREPARATION- WORK ANALYSIS DATE ORDER \#

ASTM D6784-02
Analysis Time..: 11:33

01/02-01/05/07 JK1121AA MDL......... 0.0060 
Air Sampling Associates, Inc.

Client Sample ID: HARDIN SDA TNLET DUCT RUN 3 KCL

TOTAL Metals

Lrot-Sample \#. . . : H6L070246-034

Date Sampled...: 12/02/06

PARAMETER

Prep Batch \#...: 7002028

Mercury
3.3
Date Received..: 12/06/06

REPORTING LIMIT UNITS METHOD

ASTM D6784-02

Analysis Time..: 11:27
Matrix....... AIR

DREPARATION- WORK ANALYSIS DATE ORDER \#

01/04/07 JK1131AA MDL........ 0.049 
Air Sampling Associates, Inc.

Client Sample ID: HARDIN SDA INLET DUCT RUN 3 HNO3/H2O2

TOTAL Metals

Lot-Sample \#... : H6L070246-035

Date Sampled...: 12/02/06

Date Received..: 12/06/06

REPORTING

LIMIT

RESULT

Prep Batch \#...: 7002026

Mercury
$0.058 \mathrm{~B}$
0.068 ug

Dilution Factor: 0.68

UNITS
METHOD

ASTM D6784-02

Analysis Time..: 12:03
Matrix...... AIR

PREPARATION- WORK ANALYSIS DATE ORDER \#

01/03-01/05/07 JK1141AA

MDL......... 0.041

NOTE (S) :

B Estimated result. Result is less than RL. 
Air Sampling Associates, Inc.

Client Sample ID: HARDIN SDA INLET DUCT RUN 3 KMNO4/H2SO4

TOTAL Metals

Lot-Sample \# . . : H6L070246-036

Date Sampled...: 12/02/06

Date Received. .: 12/06/06

REPORTING

LIMIT

RESULT

Prep Batch \#...: 7002028

Mercury
0.79
0.11 ug

Dilution Factor: 1.11
Matrix......: AIR

PREPARATION- WORK ANALYSIS DATE ORDER \#

ASTM D6784-02

Analysis Time..: $11: 29$
01/04/07 JK1171AA MOL......... 0.067 
Air Sampling Associates, Inc.

\section{Client Sample ID: HARDIN SDA INLET+STACK RB 0. IN HNO3}

\section{TOTAL Metals}

Lot-Sample \#...: H6L070246-037

Date Sampled...: 12/01/06

PARAMETER

Prep Batch \#...: 7002025

Mercury
ND
Date Received. .: 12/06/06

REPORTING LIMIT UNITS

$0.010 \quad$ ug
Dilution Factor: 0.1
Matrix...... : AIR

PREPARATION - WORK METHOD
ASTM D6784-02 01/02-01/05/07 JK12A1AA Analysis Time..: 11:39 
Air Sampling Associates, Inc.

Client Sanple ID: HARDIN SDA INLET+STACK RB KCL TOTAL Metals

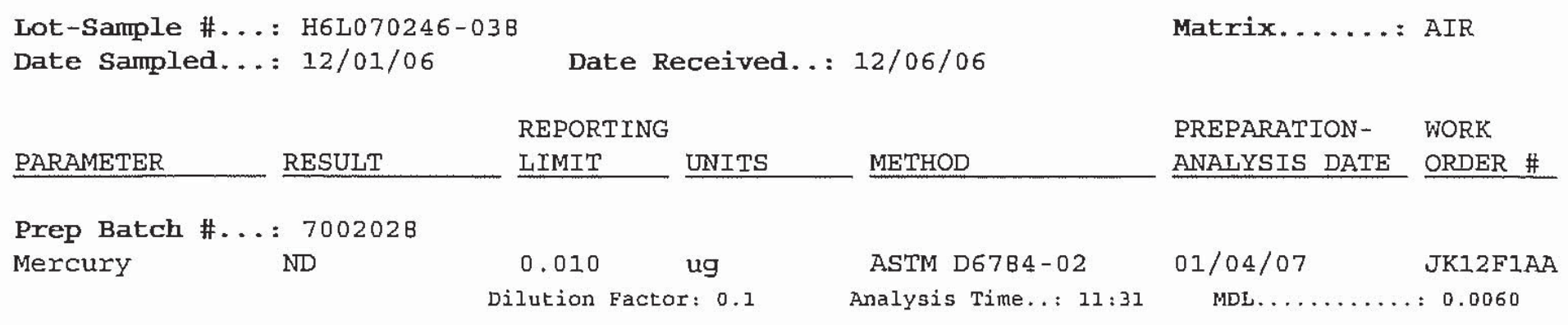


Air Sampling Associates, Inc.

Client Sample ID: HARDIN SDA INLET+STACK RB HNO3/H2O2

TOTAL Metals

Irot-Sample \#... : H6L070246-039

Date Sampled...: 12/01/06

PARAMETER

Prep Batch \#...: 7002026

Mercury

0.034
Date Received. .: 12/06/06

REPORTING

LIMIT

UNITS

0.024 ug
ilution Eactor: 0.24
Matrix...... AIR

PREPARATION- WORK ANALYSIS DATE ORDER \#

ASTM D6784-02 01/03-01/05/07 JK12G1AA Analysis Time..: 12:06 
Air Sampling Associates, Inc.

Client Sample ID: HARDIN SDA INLET+STACK RB KMNO4/H2SO4/HYD

TOTAL Metals

Lot-Sample \#...: H6L070246-040

Date Sampled...: 12/01/06

PARAMETER

Prep Batch 模...: 7002028

Mercury
ND
Date Received..: 12/06/06

REPORTING LIMIT UNITS 0.011 ug
ilution Factor: 0.11
Matrix....... AIR

PREPARATION- WORK ANALYSIS DATE ORDER \#

01/04/07 JK12H1AA MDL.........: 0.0066 


\section{METHOD BLANK RRPORT}

TOTAL Metals

Client Lot \#...: H6L070246

Matrix....... A AIR

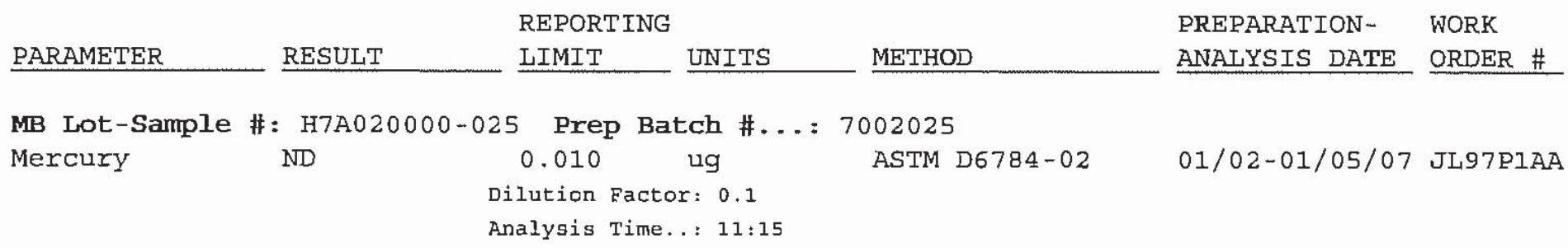

MB Lot-Sample \#: H7A020000-026 Prep Batch \#...: 7002026

$\begin{array}{ccccc}\text { Mercury ND } & 0.025 & \text { ug } & \text { ASTM D6784-02 } & \text { 01/03-01/05/07 JL97R1AA } \\ \text { Dilution Factor: } 0.25 & \\ \text { Analysis Time, : 11:41 } & \end{array}$

MB Lot-Sample \#: H7A020000-028 Prep Batch \#...: 7002028

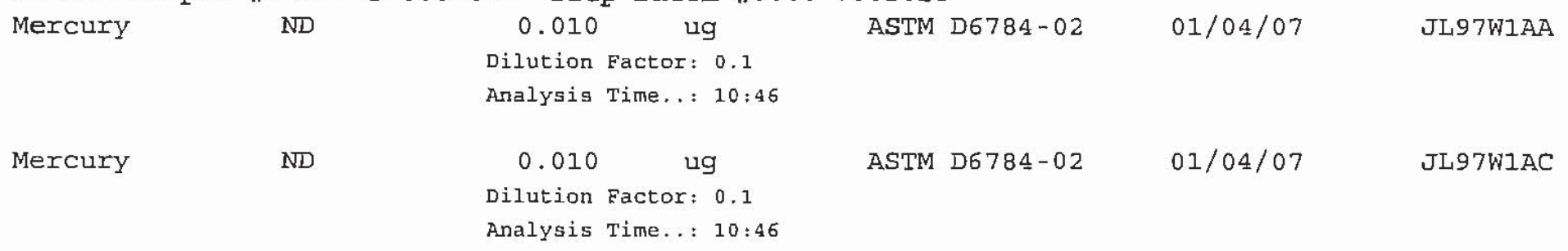

NOTE (S) :

Calculations are performed before rounding to avoid round-off errors in calsulated results. 


\section{LABORA'TORY CONTROL SAMPLE EVALUATION REPORT}

\section{TOTAL Metals}

Lot-Sample \#. . : : H6L070246

PARAMETER

Mercury
PERCENT RECOVERY

RECOVERY LIMITS

$97 \quad(80-120)$

$(80-120) 2.7 \quad(0-20)$

RPD
Matrix........ AIR

PREPARATION - PREP-

METHOD

ASTM D6784-02

ASTM D6784-02

Analysis Time... : $11 ; 17$

NOTE $(\mathrm{S}):$

Calculations are perforned before rounding to avoid round-off errors in calculated results. 


\section{LAABORATORY CONTROI SAMPLE DATA REPORT}

TOTAL Metals

Lot-Sample \#. . : H6L070246

Matrix........ AIR

\begin{tabular}{|c|c|c|c|c|c|c|c|c|}
\hline PARAMETER & $\begin{array}{l}\text { SPIKE } \\
\text { AMOUNT } \\
\end{array}$ & $\begin{array}{l}\text { MEASURED } \\
\text { AMOUNT } \\
\end{array}$ & UNITS & $\begin{array}{l}\text { PERCNT } \\
\text { RECVRY }\end{array}$ & RPD & METHOD & $\begin{array}{l}\text { PREPARATION- } \\
\text { ANALYSIS DATE }\end{array}$ & $\begin{array}{l}\text { PREP } \\
\text { BATCH \# }\end{array}$ \\
\hline \multirow[t]{2}{*}{ Mercury } & 0.500 & 0.487 & ug & 97 & & ASTM D6784-02 & $01 / 02-01 / 05 / 07$ & 7002025 \\
\hline & 0.500 & 0.474 & ug & 95 & 2.7 & ASTM D6784-02 & $01 / 02-01 / 05 / 07$ & 7002025 \\
\hline
\end{tabular}

NOTE (S) :

Calculations are performed before rounding to avoid round-off errors in calculated results. 
LABORATORY CONTROI SAMPLE EVALUATION REPORT

\section{TOTAL Metals}

Client Lot \#...: H6L070246

Matrix.......: AIR

\begin{tabular}{|c|c|c|c|c|c|}
\hline PARAMETER & $\begin{array}{l}\text { PERCENT } \\
\text { RECOVERY } \\
\end{array}$ & $\begin{array}{l}\text { RECOVERY } \\
\text { LIMITS } \\
\end{array}$ & METHOD & $\begin{array}{l}\text { PREPARATION- } \\
\text { ANALYSIS DATE }\end{array}$ & WORK ORDER \# \\
\hline IcS Lot-Sample\#: & $\mathrm{H} 7 \mathrm{AO} 2000 \mathrm{C}$ & Prep B & $\operatorname{cch} \# \ldots=70020$ & & \\
\hline \multirow[t]{2}{*}{ Mercury } & 80 & $(80-120)$ & ASTM D67B4-02 & $01 / 03-01 / 05 / 07$ & JL97R1AC \\
\hline & & \multicolumn{2}{|c|}{ Dilution Factor: 0.25} & Time.. : 11:44 & \\
\hline ICS Lot-Sample\#: & $\mathrm{H} 7 \mathrm{AO} 2000$ & Prep B & tch \#...: 7002 & & \\
\hline \multirow{2}{*}{ Mercury } & 97 & $(80-120)$ & ASTM D6784-02 & $01 / 04 / 07$ & JL97W1AD \\
\hline & & \multicolumn{2}{|c|}{ Dilution Factor: 0.1} & Time. : $10: 4 \mathrm{~B}$ & \\
\hline Mercury & 97 & $(80-120)$ & ASTM D6784-02 & $01 / 04 / 07$ & JL97W1AE \\
\hline
\end{tabular}

NOTE (S) :

Calculations are performed before rounding to avoid round-off errors in calculated results. 


\section{LABORATORY CONTROL SAMPLE DATA REPORT}

\section{TOTAL Metals}

Client Lot \#...: H6L070246

PARAMETER

SPIKE MEASURED AMOUNT AMOUNT
PERCNT RECVRY METHOD
Matrix........ AIR

PREPARATION- WORK ANALYSIS DATE ORDER \#

LCS Lot-Sample\#: H7A020000-026 Prep Batch \#...: 7002026

Mercury

1.251 .00

80

ASTM D6784-02

01/03-01/05/07 JL97R1AC

Dilution Factor: 0.25

LCS Lot-Sample\#: H7A020000-028 Prep Batch \#...: 7002028

Mercury

0.500

0.483

ug Dilution Factor: 0.1

Mercury

0.500

0.483
ASTM D6784-02

Analysis Time..: $10: 48$

ASTM D6784-02

Analysis Time..: 10:48
$01 / 04 / 07$

$01 / 04 / 07$
97

Dilution Factor: 0.1

\section{NOTE (S) :}

Calculations are performed before rounding to avoid round-off errors in calculated results. 


\section{MATRIX SPIKE SAMPLE EVALUATION RBPORT}

TOTAL Metals

Client Lot \#...: H6L070246

Date Sampled...: 12/01/06
Date Received. . : 12/06/06

DERCENT RECOVERY

PARAMETER RECOVERY LIMITS RPD LIMITS METHOD
Matrix.......: AIR

PREPARATION - WORK ANALYSIS DATE ORDER \#

01/03-01/05/07 JK1WF1AC 01/03-01/05/07 JKIWFIAD

MS Lot-Sample \#: H6L070246-005 Prep Batch \#...: 7002026

Mercury 88 $(80-120)$ A.STM D6784-02

$(80-120) 2.8 \quad(0-20)$ A.STM D6784-02 Dilution Factor: 0.6

Analysis Time..: $11: 47$

$\operatorname{NOTE}(S)$ :

Calculations are performed before rounding to avoid round-off errors in calculated results. 
MATRIX SPIKE SAMPLE DATA REPORT

TOTAL Metals

Client Lot \#...: H6L070246

Date Sampled...: 12/01/06
Date Received. .: 12/06/06

PERCNT RECVRY RPD METHOD
Matrix........ AIR

PREPARATION - WORK ANALYSIS DATE ORDER \#

PARAMETER AMOUNT AMT

MEASRD AMOUNT UNITS

MS Lot-Sample \#: H6L070246-005 Prep Batch \#...: 7002026

Mercury

$$
\begin{aligned}
& 0.041 \quad 0.600 \quad 0.569 \text { ug } 88 \text { ASTM D67B4-02 01/03-01/05/07 JK1WF1AC } \\
& \begin{array}{llllllll}
0.041 & 0.600 & 0.586 & \text { ug } & 91 & 2.8 & \text { ASTM D67B4-02 01/03-01/05/07 JK1WF1AD }
\end{array} \\
& \text { Dilution Factor: } 0.6 \\
& \text { Analysis Time..: } 11: 47
\end{aligned}
$$

NOTB (S) :

Calculations are performed before rounding to aveid round-off errors in calculated results. 
MATRIX SPIKE SAMPLE EVALUATION REPORT

TOTAL Metals

Client Lot \#...: H6L070246

Date Sampled...: 12/01/06
Date Received..: 12/06/06

\begin{tabular}{llll} 
PERCENT & RECOVERY & RPD & \\
RECOVERY & LIMITS & RPD & LIMITS METHOD \\
\hline
\end{tabular}

Matrix....... AIR

PREPARATION - WORK ANALYSIS DATE ORDER \#

01/04/07 JKIWE1AC 01/04/07 JKIWEIAD

MS Lot-Sample \#: H6L070246-004 Prep Batch \#.. : 7002028

Mercury Dilution Factor: 1.32

Analysis Time..: 10:53

$\operatorname{NOTB}(S)$ :

Calculations are performed before rounding to avoid round-off errors in calculated results. 
MATRIX SPIKR SAMELE DATA RBPORT

TOTAL Metals

Client Lot \#...: H6L070246

Date Sampled...: 12/01/06
Matrix.......: AIR

Date Received..: 12/06/06

PERCNT

PREPARATION-

WORK

SAMPLE SPIKE MEASRD RECVRY RPD METHOD

\section{ANALYSIS DATE}

MS Lot-Sample \#: H6L070246-004 Prep Batch \#...: 7002028

Mercury

\begin{tabular}{|c|c|c|c|c|c|c|c|c|c|}
\hline \multirow{2}{*}{$\begin{array}{l}0.11 \\
0.11\end{array}$} & 1.32 & 1.41 & ug & 99 & & ASTM & D6784-02 & $01 / 04 / 07$ & JKIWEIAC \\
\hline & 1.32 & 1.48 & ug & 104 & 4.6 & ASTM & D6784-02 & $01 / 04 / 07$ & JKIWEIAD \\
\hline \multicolumn{9}{|c|}{ Dilution Factor: 1.32} & \\
\hline & & & ${ }^{\circ}$ & $0: 53$ & & & & & \\
\hline
\end{tabular}

NO'TB (S) :

Calculations are performed before rounding to avoid round-off errors in calculated results. 
MATRIX SPIKE SAMPLE EVALUATION REPORT

TOTAL Metals

Client Lot \#...: H6L070246

Date Sampled...: 12/01/06
Date Received..: 12/06/06

\begin{tabular}{|c|c|c|c|c|}
\hline PERCENT & RECOVERY & & RPD & \\
\hline RECOVERY & LIMITS & RPD & LIMITS & METHOD \\
\hline
\end{tabular}

Matrix........ AIR

PREPARATION - WORK ANALYSIS DATE ORDER \#

01/04/07 JKIWGIAC 01/04/07 JKIWGIAD

\author{
NC, MSB $(80-120)$ \\ NC, MSB $(80-120)$ \\ Dilution Eactor: 0.78 \\ Analysis Time. . : 11:02 \\ ASTM D6784-02 \\ ASTM D6784-02
}

\section{$\operatorname{NOTB}(S)$ :}

Calculations are performed before rounding to avoid round-off errors in calculated results.

NC The recovery and/or RPD were not calculated.

MSIB The recovery and RPD were not calculated because the sumpic amount was greater than four times the spike amount. 
MATRIX SPIKB SAMPLE DATA REPORT

TOTAI, Metals

PREPARATION- WORK ANALYSIS DATE ORDER \#
Client Lot \#...: H6L070246

Date Sampled...: 12/01/06
Matrix.......: AIR

Date Received. .: 12/06/06 RECVRY RPD METHOD
PARAMETER AMOUNT AMT
RAR

AMOUNT UNITS
MS Lot-Sample \#: H6L070246-006 Prep Batch \#...: 7002028

Mercury

$\begin{array}{lccc}6.8 & 0.780 & 7.88 & \text { ug } \\ 6.8 & 0.780 & 8.11 & \text { Qualifiers: NC, MSB } \\ & & \text { Qualifiers: NC, MSB } \\ & & \text { Dilution Factor: } 0.78 \\ & \text { Analysis Time. : } 11: 02\end{array}$

$\begin{array}{lll}\text { ASTM D6784-02 } & 01 / 04 / 07 & \text { JKIWGIAC } \\ \text { ASTM D6784-02 } & 01 / 04 / 07 & \text { JKIWG1AD }\end{array}$

\section{$\operatorname{NOTB}(\mathrm{S})$ :}

Calculations are performed before rounding to avoid round-off errors in calculated results.

NC The recovery and/or RPD were not calculated.

MSB The reeovery and RPD were not calcuinted because the sample amount was greater than four times the spike amount. 
STL Knoxville

Mercury Data Reporting Form

Post Digestion Spike

Units: $u g / L(p p b)$

Instrument ID: Leeman HydraAA $\mathrm{Hg}$

Data File Name: H010507. PRN

\begin{tabular}{|c|c|c|c|c|}
\hline Element & $\begin{array}{c}\text { PDS } \\
\text { JK1WAA }\end{array}$ & $\begin{array}{c}\text { Original Sample } \\
\text { JK1WA }\end{array}$ & Spike Added & $\begin{array}{c}\text { Percent } \\
\text { Recovery }\end{array}$ \\
\hline $\mathrm{Hg}$ & 1.36 & 0.34 & 1.0 & 102 \\
\hline
\end{tabular}


Sample Data Summary 
Air Sampling Associates, Inc.

client Sample I: HARDIN STACK RUN 1 FILTER A117

General Chemistry

Lot-Sample \#...: H6L070246-001

Date Sampled...: 12/01/06
Hork Order \#. . . : JK1V2

Date Received... 12/06/06
Matrix.......: AIR

PREPARATION- PREP ANALYSIS DATE BATCH \#
PARAMETER

RESULT

Particulates (total) 387
RL

0.50

mg

Dilution Factor: 1
METHOD

CFR60A 5 
Air Sampling Associates, Inc.

client sample ID: HARDIN STACK RON 1 ACBTONE

General Chemistry

Lot-Sarmle \#...: H6L070246-002 Hork Order \#...: JKIV7

Date Sampled...: 12/01/06
Date Received..: 12/06/06
Matrix........ AIR

PREPARATION- PREP ANALYSIS DATE BATCH \# $12 / 12-12 / 14 / 06 \quad 6346334$
PARAMETER

Particulates (total) 16.3
RI $\quad$ UNITS

$0.50 \mathrm{mg}$

Dilution Factor: 1
METHOD

CFR60A 5 
Air Sampling Associates, Inc.

client Sample ID: HARDIN STACK RUN 2 FILTKR A118

General Chemistry

Lot-Sample \#...: H6L,070246-007

Date Sampled...: 12/02/06
Work Order \#...: JK1W8

Date Received. .: 12/06/06
Matrix........ AIR

PREPARATION- PREP ANALYSIS DATE BATCH \# $12 / 12-12 / 13 / 06 \quad \overline{6346334}$
PARAMETER

Particulates (total) 383
$\mathrm{RL}$ 0.50

Ing

Dilution Factor: 1
METHOD

CFR6 0A 5 MDL ........ 0.50 
Air Sampling Associates, Inc.

client sample ID: HARDIN STACK RUN 2 ACETONE

General Chemistry

Lot-Sample \#...: H6L070246-008 work Order \#...: JK1XA

Date Sampled... 12/02/06
Date Received..: 12/06/06
Matrix....... AIR

PREPARATION- PREP ANALYSIS DATE BATCH \#

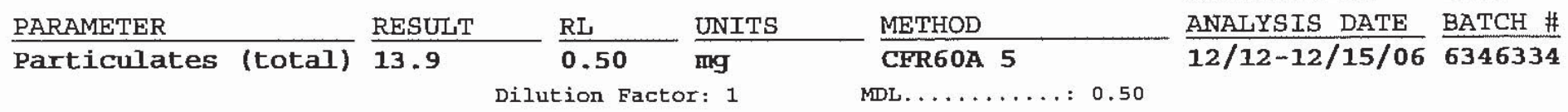


Air Sampling Associates, Inc.

Client Sample ID: HARDIN STACK RUN 3 FILTKR A119

General Chemistry

Lot-Sample \#...: H6L070246-013 Work Order \#...: JK1X1

Date Sampled...: 12/02/06
Date Received..: 12/06/06
Matrix........ AIR

PREPARATION- PREP ANALYSIS DATE BATCH \#
PARAMETER

RESULT

Particulates (total) $\mathbf{3 9 2}$
RI 0.50

Dilution Factor: 1
METHOD

CFR60A 5 12/12-12/14/06 6346334 
Air Sampling Associates, Inc.

client Sample ID: HARDIN STACK RUN 3 ACETONE

General Chemistry

Lot-Sample \#...: H6L070246-014 Work Order \#...: JK1X4

Date Sampled...: 12/02/06

\begin{abstract}
Date Received...: 12/06/06
\end{abstract}
Matrix......: AIR

PREPARATION- PREP ANALYSIS DATE BATCH \#

$\frac{\text { PARAMETER }}{\text { Particulates (total) }} \frac{\text { RESULT }}{\mathbf{1 2 . 1}} \frac{\text { RL }}{\mathbf{0 . 5 0}} \frac{\text { UNITS }}{\mathbf{m g}}$ Dilution Factor: $1 \quad \frac{\text { METHOD }}{\text { CFR60A } \mathbf{5}}$


Air Sampling Associates, Inc.

Client Sample ID: HARDIN SDA TNLFT DUCT RUN 1 FILTER (THIMBLE 53)

General Chemistry

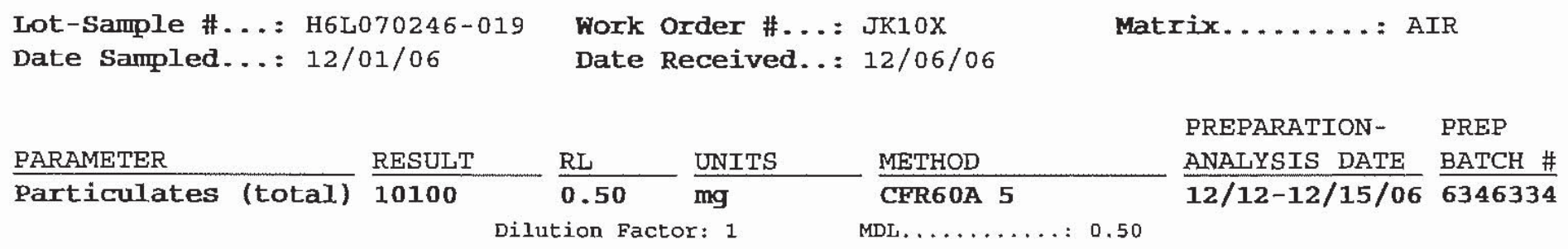


Air Sampling Associates, Inc.

client Sample ID: HARDTN SDA INLET DUCT RUN 1 ACBTONE

General Chemistry

Lot-Sample \#...: H6L070246-020

Date sampled...: 12/01/06
Work Order \#...: JKI01

Date Received..: 12/06/06
Matrix........ AIR

PREPARATION- PREP ANALYSIS DATE BATCH \#
PARAMETER

RESULT

Particulates (total) $\mathbf{3 2 5 0}$
RL 0.50

Dilution Factor: 1
METHOD

CFR60A 5 
Air Sampling Associates, Inc.

Client Sample ID: HARDIN SDA INLET DUCT RUN 2 FILTER (THIMBLE 54)

General Chemistry

Lot-Sample \#. . : H6L070246-025 Work Order \#...: JK11C

Date Sampled...: 12/02/06
Date Received. .: 12/06/06
Matrix........ AIR

PREPARATION - PREP ANALYSIS DATE BATCH \#
PARAMETER

Particulates (total) 12700
$\underline{R L}$

0.50
UNITS

mig

Dilution Factor: 1
METHOD

CFR60A 5 MDL . ........ 0.50 
Air Sampling Associates, Inc.

client Sample ID: HARDIN SDA INLET DUCT RON 2 ACETONE

General Chemistry

Iot-Sample \#...: H6L070246-026 Work Order \#...: JK11D

Date Sampled...: 12/02/06
Date Received. : $12 / 06 / 06$
Matrix......... AIR

PREPARATION - PREP ANALYSIS DATE BATCH \#

$\frac{\text { PARAMETER }}{\text { Particulates (total) }} \frac{\text { RESULT }}{2930} \frac{\text { RL }}{\mathbf{0 . 5 0}} \frac{\text { UNITS }}{\mathrm{mg}}$ Dilution Factor: $1 \quad \frac{\text { METHOD }}{\text { CFR60A } 5} \frac{\text { ANALYSIS DATE }}{12 / 12-12 / 14 / 06} \frac{\text { BATCH \# }}{6346334}$


Air Sampling Associates, Inc.

client Sample ID: HARDIN SDA INLET DUCT RUN 3 FILTER (THIMBLE 55)

General Chenistry

Lot-Sample \#...: H6L070246-031 Fork Order \#...: JK11X

Date sampled...: 12/02/06

\begin{abstract}
Date Received. .: 12/06/06
\end{abstract}
Matrix........ AIR

PREPARATION- PREP ANALYSIS DATE BATCH \#

$\frac{\text { PARAMETER }}{\text { Particulates (total) }} \frac{\text { RESULT }}{12500} \frac{\text { RL }}{0.50} \frac{\text { UNITS }}{\mathrm{mg}} \frac{\text { METHOD }}{\text { CFR60A 5 }} \frac{\text { ANALYSIS DATE }}{12 / 12-12 / 15 / 06} \frac{\text { BATCH \# }}{6346334}$


Aix Sampling Associates, Inc.

client Sample ID: HARDIN SDA INLFT DUCT RUN 3 ACETONE

Genexal Chemistry

Lot-Sample \#...: H6L070246-032 work Order \#...: JK111

Date Sampled...: 12/02/06

\author{
Date Received. .: 12/06/06
}

Matrix........ AIR

PREPARATION- $\quad$ PREP ANALYSIS DATE BATCH \#

\begin{tabular}{|c|c|c|c|c|c|c|c|}
\hline PARAMETER & & RESULT & $\underline{\mathrm{RL}}$ & UNITS & METHOD & ANALYSIS DATE & $\mathrm{BATCH} \#$ \\
\hline Particulates & (total) & 730 & 0.50 & $\mathrm{mg}$ & CFR60A 5 & $12 / 12-12 / 14 / 06$ & 6346334 \\
\hline
\end{tabular}


Sample Receipt Documentation 


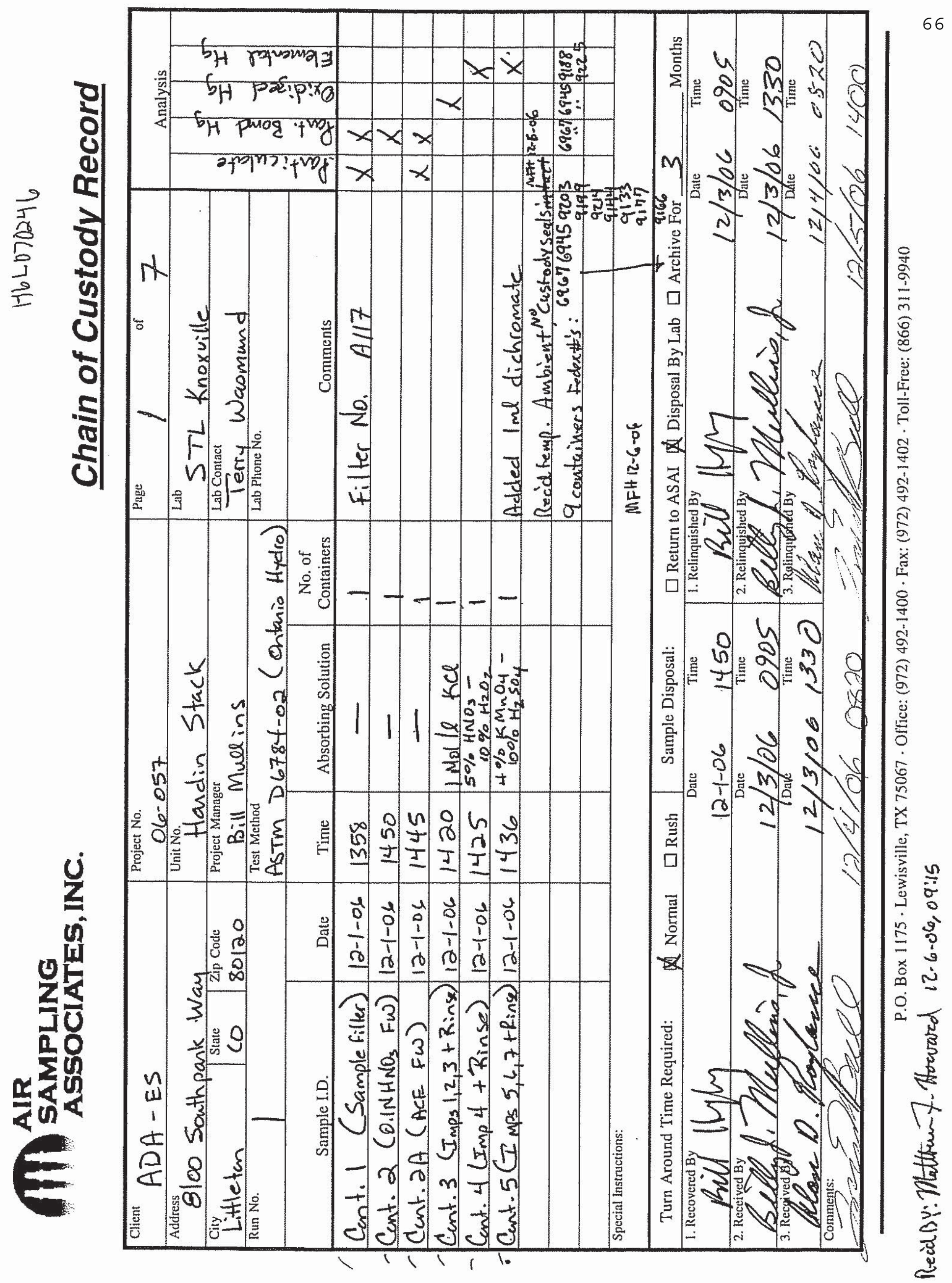




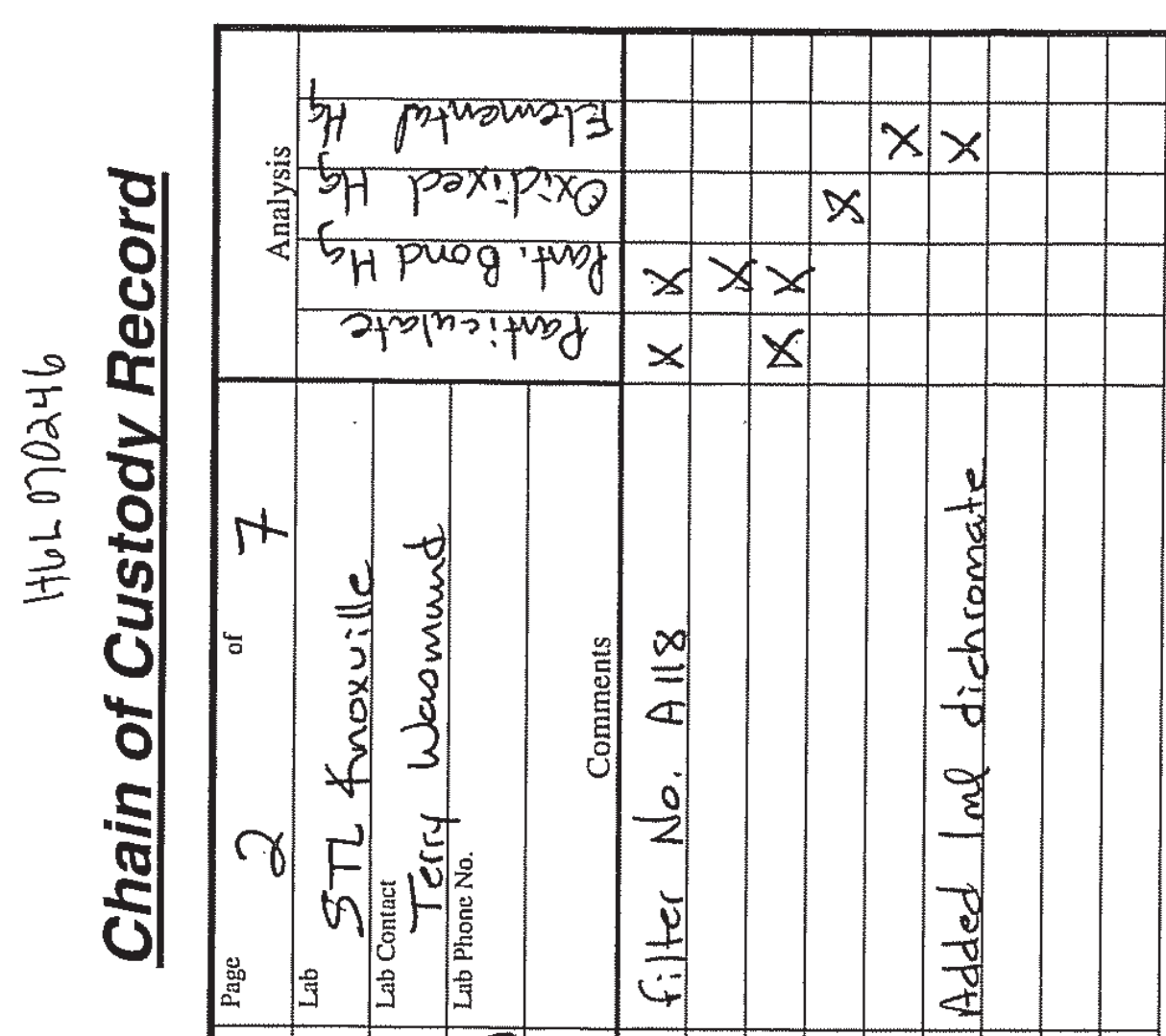




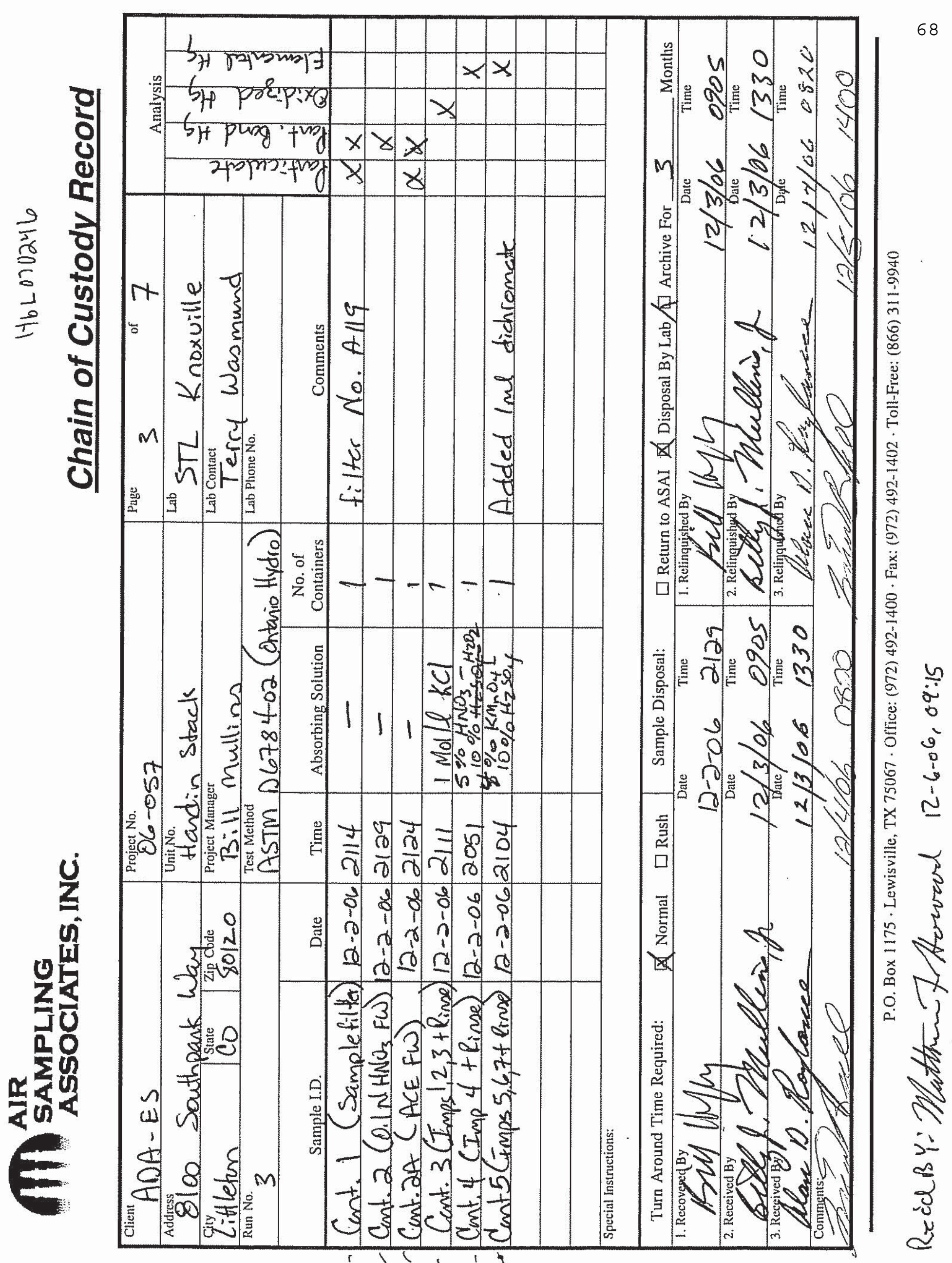




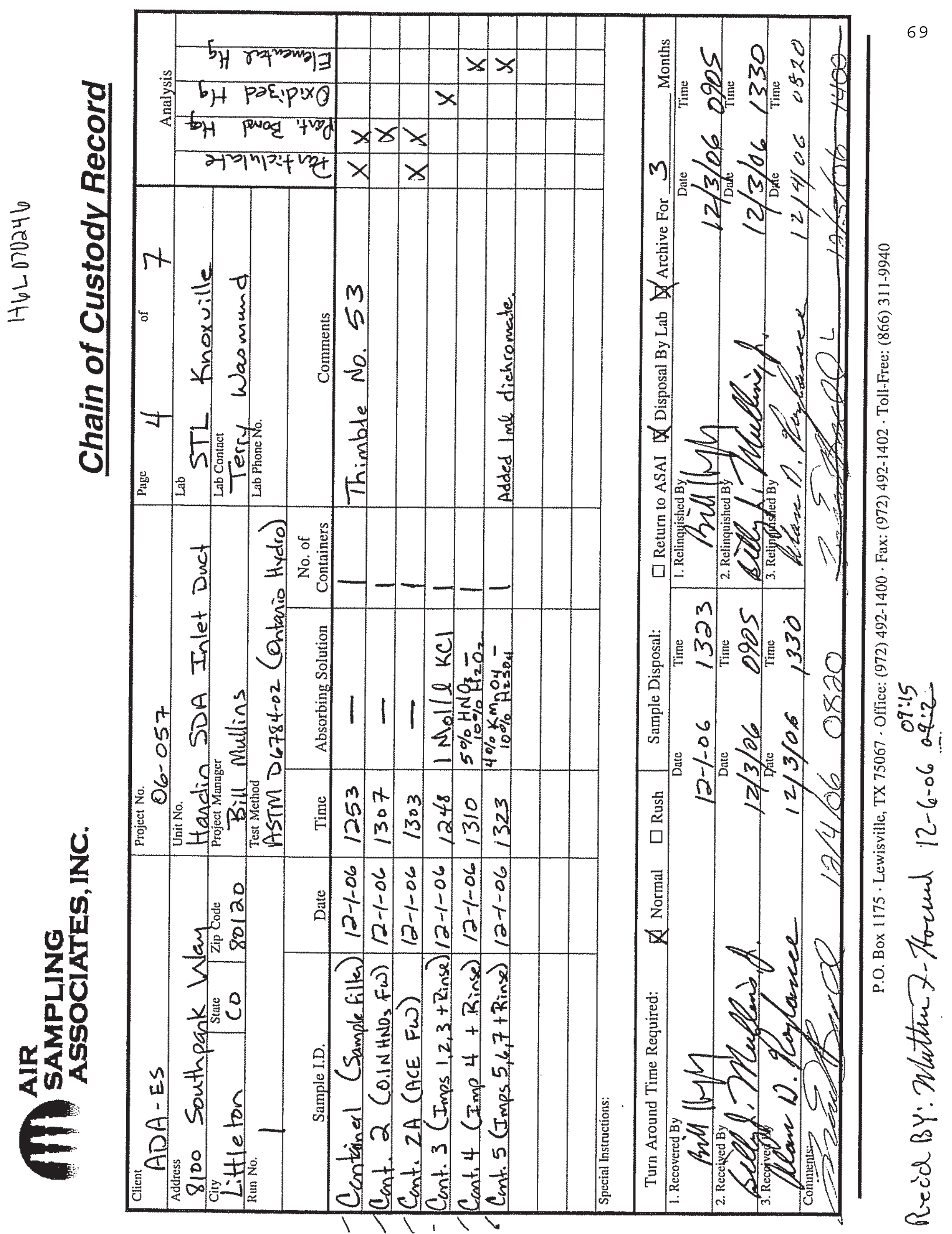




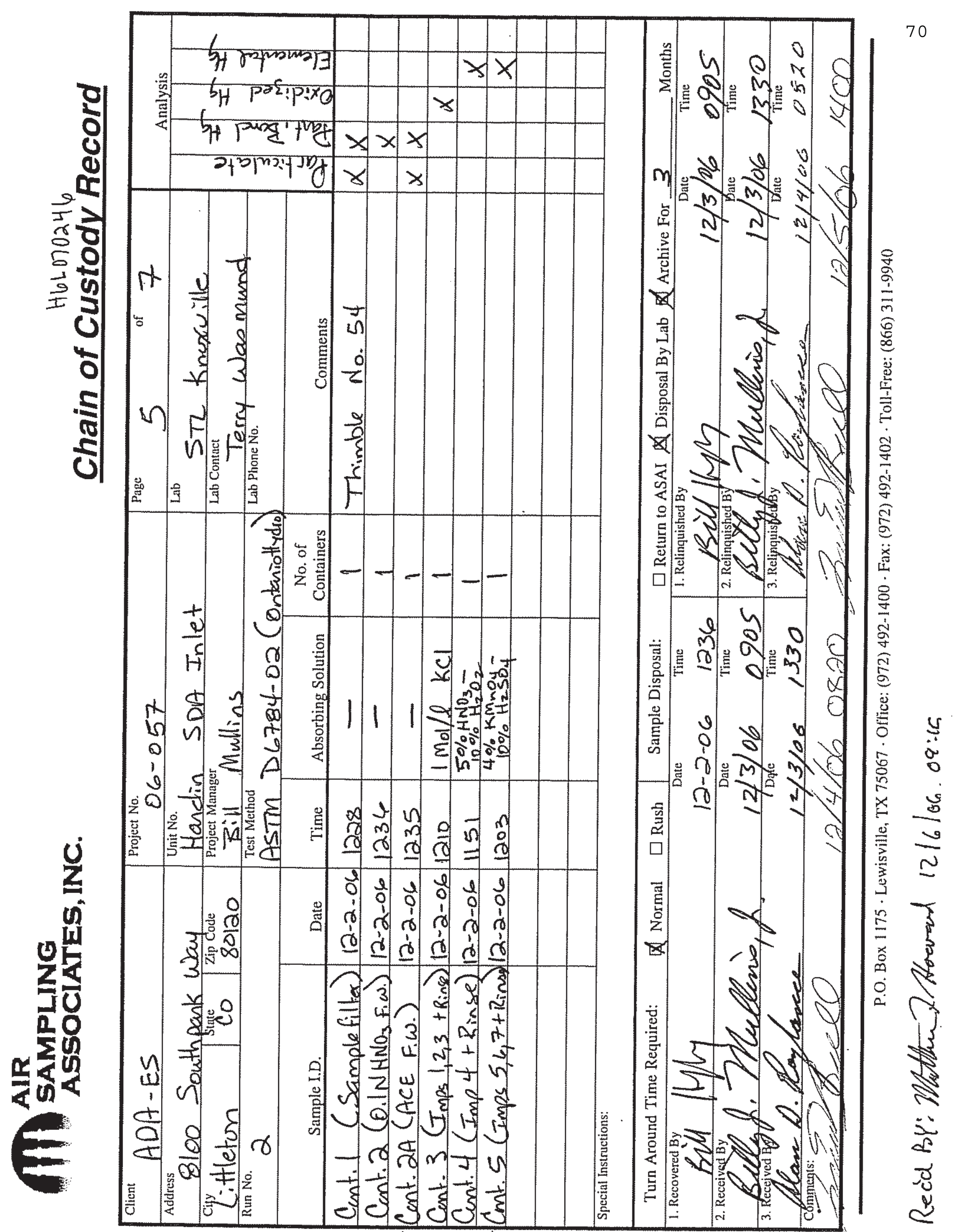




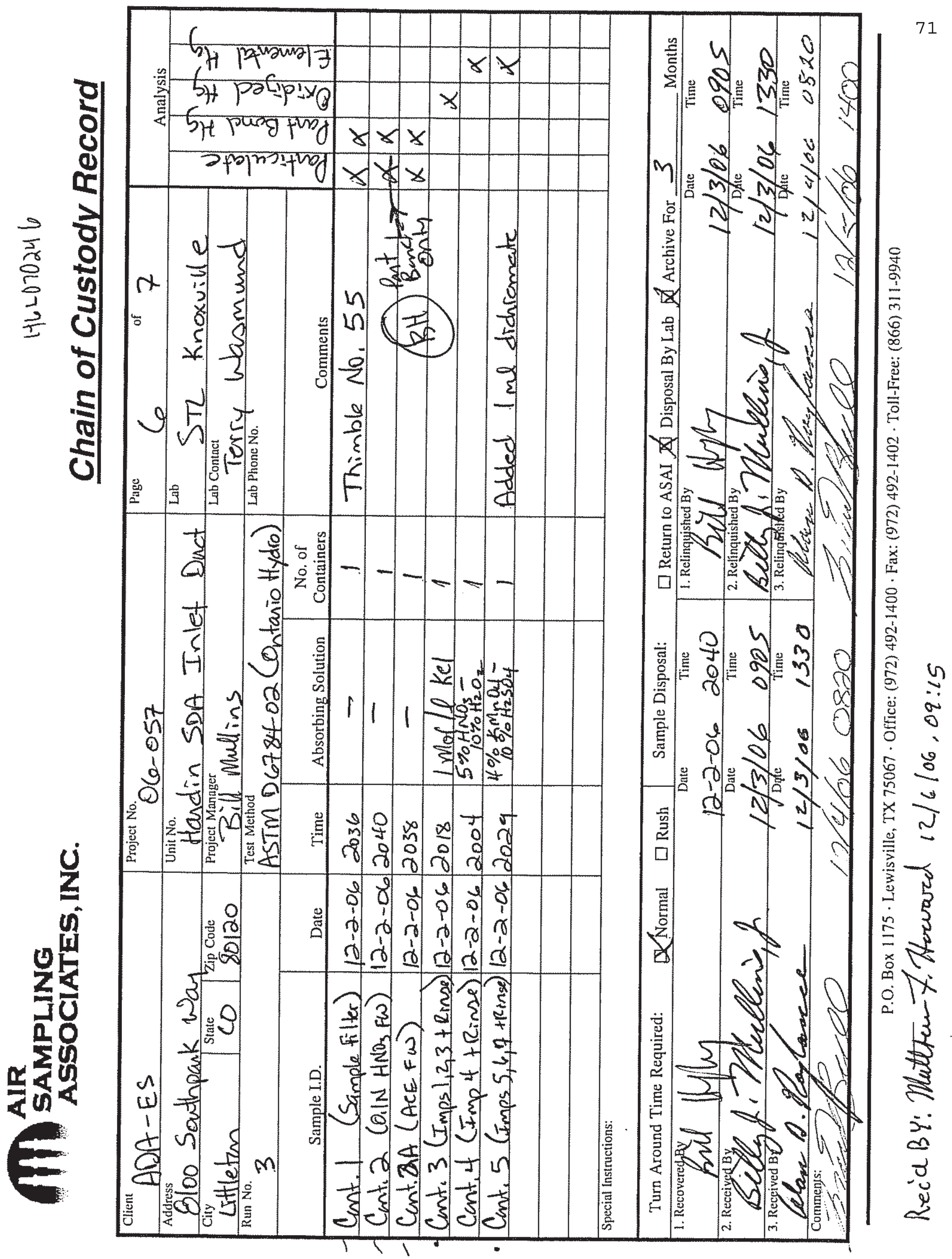




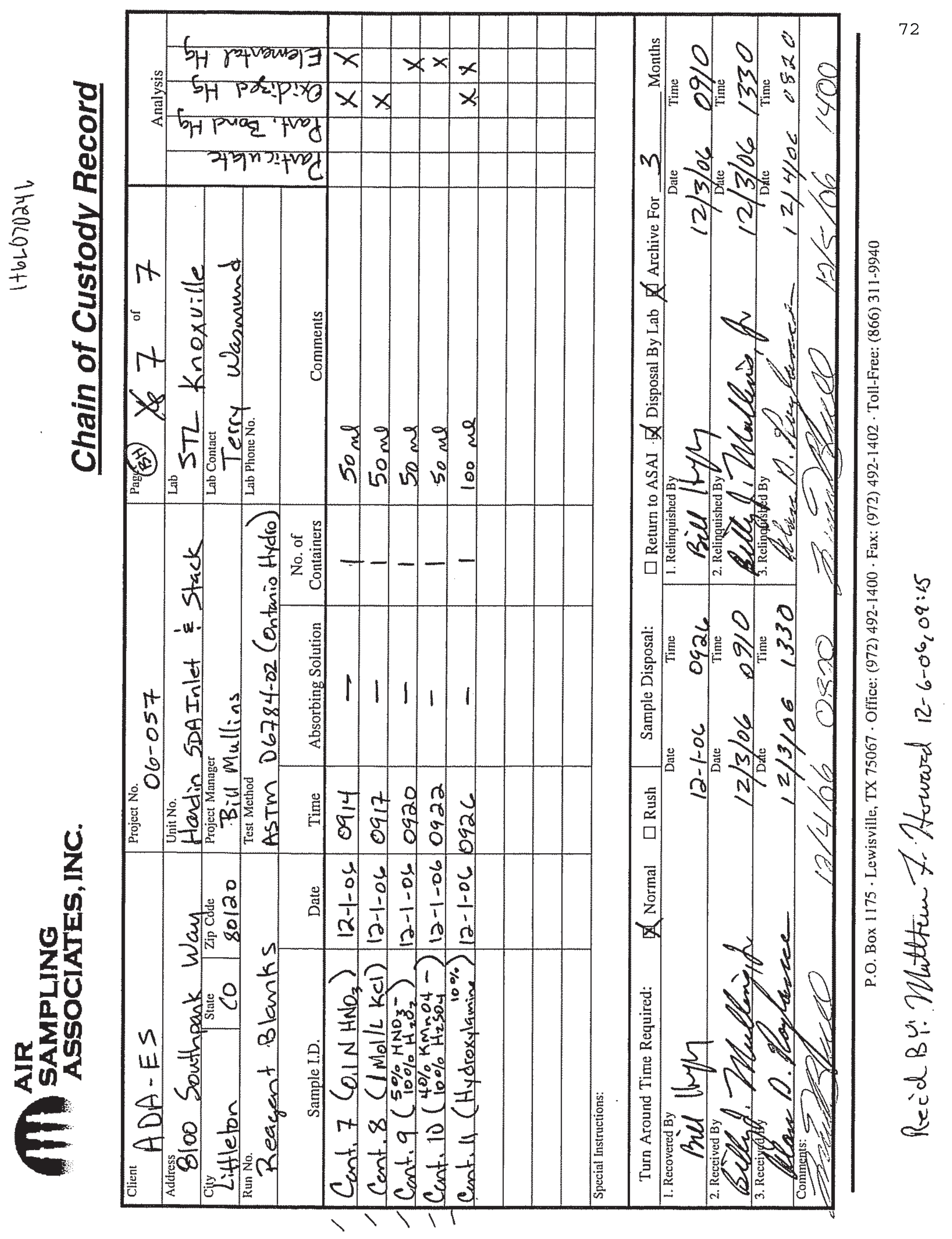




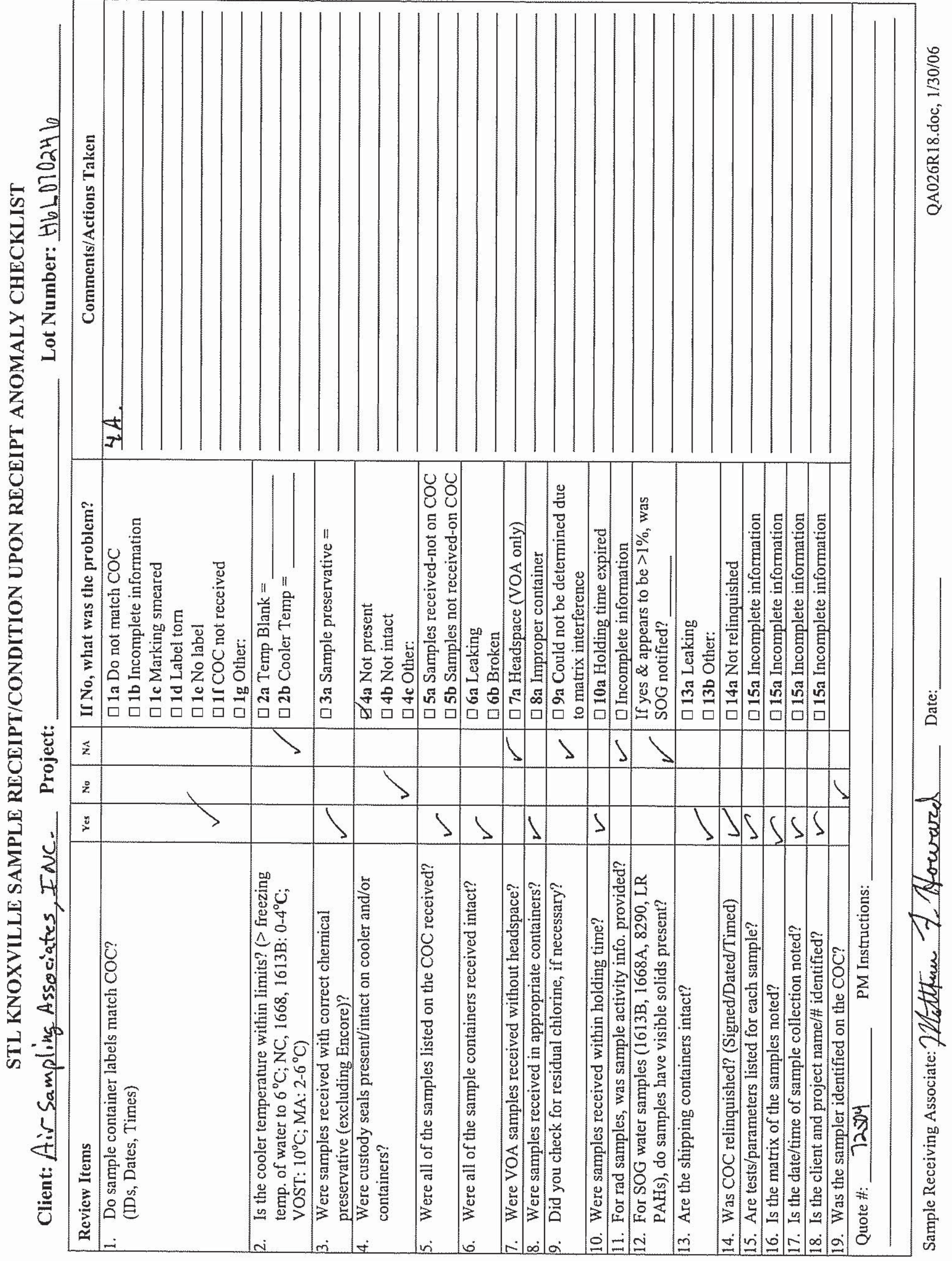


Metals 
Sample Results 
Air Sampling Associates, Inc.

client Sample ID: HARDIN STACK RUN 1 FH

TOTAI Metals

Lot-Sample \#...: H6L070246-003

Date Sampled...: 12/01/06

PARAMETER

Prep Batch \#...: 7002025

Mercury
Date Received. . : 12/06/06

REPORTING

LIMIT

UNITS

$0.010 \quad$ ug
Dilution Factor: 0.1
Matrix...... AIR

PREPARATION- WORK ANALYSIS DATE ORDER \#

ASTM D6784-02 01/02-01/05/07 JKבWA1AA Analysis Time..: $11: 21$ 
Air Sampling Associates, Inc.

Client Sample ID: HARDIN STACK RUN 1 KCL

\section{TOTAI Metals}

Lot-Sample \#. . : H6L070246-004

Date Sampled...: 12/01/06

PARAMETER

RESULT

Prep Batch \#...: 7002028

Mercury

$0.11 \mathrm{~B}$

0.13

ug

Dilution Factor: 1.32

REPORTING

UNITS

METHOD

ASTM D6784-02
Analysis Time. : : 10:51

Matrix...... : AIR

PREPARATION- WORK ANALYSIS DATE ORDER \#

01/04/07 JK1WEIAA

MDL......... 0.079

NOTE $(S)$ :

B Estimated result. Result is less than RL. 
Air Sampling Associates, Inc.

Client Sample ID: HARDIN STACK RUN 1 HNO3/H2O2

TOTAL Metals

Lot-Sample \#...: H6L070246-005

Date Sampled...: 12/01/06

PARAMETER

Prep Batch \#...: 7002026

Mercury $\quad 0.041 \mathrm{~B}$
0.060 ug
Dilution Factor: 0.6
Date Received. : 12/06/06

REPORTING

LIMIT

UNITS METHOD

Matrix..... : AIR

PREPARATION- WORK ANALYSIS DATE ORDER \#

01/03-01/05/07 JK1WFIAA

Analysis Time..: 11:46

$\operatorname{NOTE}(S):$

B Estimated result. Result is less than RL. 
Air Sampling Associates, Inc.

Client Sample ID: HARDIN STACK RUN I KMNO4/H2SO4

TOTAL Metals

Lot-Sample \#...: H6L070246-006

Date Sampled...: 12/01/06

PARAMETER

RESULT

Prep Batch \#...: 7002028

Mercury

6.8
Date Received. . : 12/06/06

REPORTING

LIMIT

0.078 ug

Dilution Factor: 0.78
Matrix.....: AIR

PREPARATION- WORK ANALYSIS DATE ORDER \#

$\begin{array}{ccc}\text { ASTM D6784-02 } & 01 / 04 / 07 & \text { JKIWGLAA } \\ \text { Analysis Time..: 11:00 } & \text { MDL . . . . }\end{array}$


Air Sampling Associates, Inc.

Client Sample ID: HARDIN STACK RUN 2 FH

TOTAL Metals

Lot-Sample \#...: H6L070246-009

Date Sampled...: 12/02/06

PARAMETER

RESULT

Prep Batch \#...: 7002025

Mercury
ND
Date Received. . : 12/06/06

REPORTING

LIMIT

UNITS

METHOD

ASTM D6784-02
Analysis Time..: 11:25
Matrix....... AIR

PREPARATION- WORK ANALYSIS DATE ORDER \#

01/02-01/05/07 JKIXDIAA MDi.......... 0.0060 
Air Sampling Associates, Inc.

Client Sample ID: HARDIN STACK RUN 2 KCL

TOTAL Metals

Lot-Sample \#...: H6L070246-010

Date Sampled...: 12/02/06

PARAMETER

RESULT

Prep Batch \#...: 7002028

Mercury
ND
Date Received. .: 12/06/06

REPORTING

LIMIT

UNITS

METHOD

ASTM D6784-02

Analysis Time..: 11:06
Matrix....... AIR

PREPARATION- WORK ANALYSIS DATE ORDER \#

01/04/07 JKIXELAA MDL......... 0.008 
Air Sanpling Associates, Inc.

Client Sample ID: HARDIN STACK RUN 2 HNO3/H2O2

TOTAL Metals

Lrot-Sanple \#...: H6L070246-011

Date Sampled...: 12/02/06

PARAMETER

Prep Batch \#...: 7002026

Mercury

RESULT
7002026
ND

Date Received. .: 12/06/06

REPORTING

LIMIT

UNITS METHOD

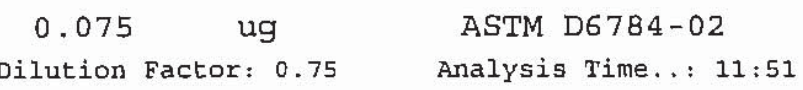

Matrix...... A AIR

PREPARATION - WORK ANALYSIS DATE ORDER \#

01/03-01/05/07 JK1XR1AA MDL......... 0.045 
Air Sampling Associates, Inc.

client Sample ID: HARDIN STACK RUN 2 KMNO4/H2SO4

TOTAL Metals

Lot-Sample \#...: H6L070246-012

Date Sampled...: 12/02/06

Date Received..: 12/06/06

REPORTING LIMIT

RESULT

Prep Batch \#...: 7002028

Mercury

\begin{abstract}
12.1
\end{abstract}

$\begin{array}{ccc}0.46 & \text { ug } & \text { ASTM D6784-02 } \\ \text { Dilution Factor: } 4.6 & \text { Analysis Time. : 11:55 }\end{array}$

Matrix......: AIR

PREPARATION- WORK ANALYSIS DATE ORDER \#

01/04/07 JKIXTIAA 
Air Sampling Associates, Inc.

Client Sample ID: HARDIN STACK RUN 3 FH

TOTAL Metals

Lot-Sample \#. . : H6L070246-015

Date Sampled...: 12/02/06

PARAMETER

Prep Batch \#...: 7002025

Mercury $0.0090 \mathrm{~B}$
Date Received. . : 12/06/06

REPORTING LIMIT

0.010

ug

Dilution Factor: 0.1
Matrix....... AIR

PREPARATION - WORK ANALYSIS DATE ORDER \#

\section{ASTM D6784-02}

Analysis Time..: $11: 27$
01/02-01/05/07 JK1X51AA MDL.......... 0.0060

NOTE $(S)$ :

B Estimated result. Result is less than RL. 
Air Sampling Associates, Inc.

Client Sample ID: HARDIN STACK RUN 3 KCL

TOTAL Metals

Lot-Sample \#... : H6L070246-016

Date Sampled...: 12/02/06

Date Received..: 12/06/06

REPORTING

LIMIT

PARAMETER

RESULT

Prep Batch \#...: 7002028

Mercury $\quad 0.080 \mathrm{~B}$

0.12 ug

Dilution Factor: 1.25

NOTR (S) :
Matrix......: AIR

PREPARATION- WORK ANALYSIS DATE ORDER \#

01/04/07 JK1X81AA MDL......... : 0.075

B Estimated result. Result is less than RL. 
Air Sampling Associates, Inc.

Client Sample ID: HARDIN STACK RON 3 HNO3/H2O2

TOTAL Metals

Lot-Sample \#...: H6L070246-017

Date Sampled...: 12/02/06

PARAMETER

Prep Batch \#...: 7002026

Mercury

0.064
Date Received. .: 12/06/06

REPORTING

LIMIT

UNITS

ug

0.063

Dilution Factor: 0.63 METHOD
Matrix...... : AIR

PREPARATION-

WORK

ASTM D6784-02

Analysis Time..: 11:53
01/03-01/05/07 JK10A1AA

MDL......... : 0.038 
Air Sampling Associates, Inc.

Client Sample ID: HARDIN STACK RUN 3 KMNO4/H2SO4

TOTAL Metals

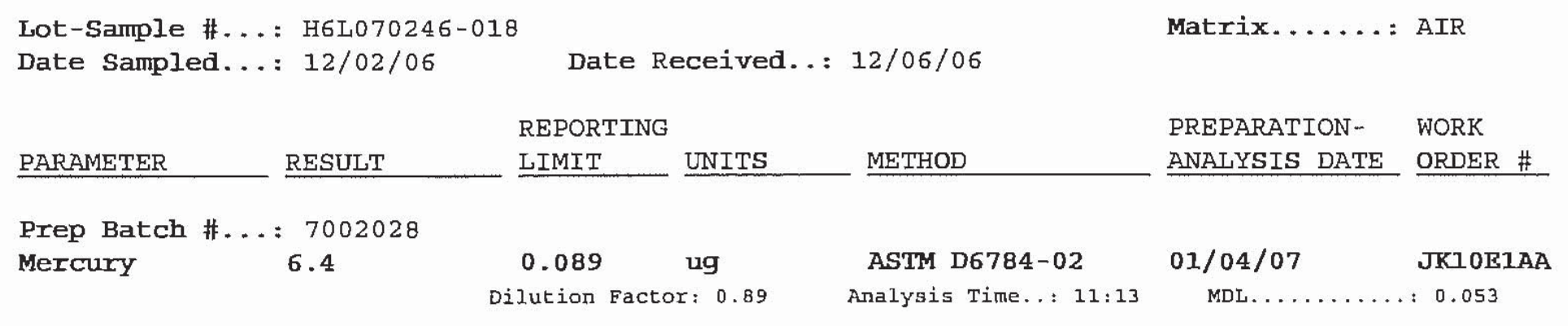


Air Sampling Associates, Inc.

client Sample ID: HARDIN SDA INLET DUCT RUN 1 FH

TOTAL Metals

Lot-Sample \#. . : : H6L070246-021

Date Sampled...: 12/01/06

PARAMETER

Prep Batch \#...: 7002025

Mercury

\begin{abstract}
0.61
\end{abstract}
Date Received..: 12/06/06

REPORTING LIMIT UNITS

$0.010 \quad \mathrm{ug}$
ilution Factor: 0.1 METHOD
Matrix......: AIR

PREPARATION- WORK ANALYSIS DATE ORDER \#

ASTM D6784-02

Analysis Time..: 11:29
01/02-01/05/07 JK1051AA MDL.......... 0.0060 
Air Sampling Associates, Inc.

Client Sample ID: HARDIN SDA INLET DUCT RUN 1 KCL

TOTAL Metals

Lot-Sample \#. . : H6L070246-022

Date Sampled...: 12/01/06

PARAMETER

Prep Batch \#...: 7002028

Mercury
1.6
Date Received..: 12/06/06

REPORTING LIMIT

UNITS

ug

Dilution Factor: 1.11
Matrix....... AIR

PREPARATION- WORK ANALYSIS DATE ORDER \#

$\begin{array}{ccc}\text { ASTM D6784-02 } & 01 / 04 / 07 & \text { JK1061AA } \\ \text { Analysis Time. : } 11: 15 & \text { MDL.......... } & 0.067\end{array}$


Air Sampling Associates, Inc.

client Sample ID: HARDIN SDA INIGT DUCT RUN 1 HNO3/H2O2

TOTAL Metals

Lot-Sample \#... : H6L070246-023

Date Sampled...: 12/01/06

PARAMETER

Prep Batch \#...: 7002026

Mercury $0.064 \mathrm{~B}$
Date Received..: 12/06/06

REPORTING

LIMIT

UNITS

METHOD

\section{ASTM D6784-02}

Analysis Time... 11:55
Matrix...... AIR

PREPARATION - WORK ANALYSIS DATE ORDER \#

01/03-01/05/07 JK1071AA MDL......... 0.039

NOTE (S) :

B Estimated result, Result is less than RL. 
Air Sampling Associates, Inc.

Client Sample ID: HARDTN SDA INLET DUCT RUN 1 KMNO4/H2SO4

\section{TOTAL Metals}

Lot-Sample \#...: H6L070246-024

Date Sampled...: 12/01/06

Date Received..: 12/06/06

REPORTING

LIMIT

RESULT

Prep Batch \#...: 7002028

Mercury
3.6 ug

Dilution Factor: 0.89
Matrix......: AIR

PREPARATION- WORK ANALYSIS DATE ORDER \#

01/04/07 JK11A1AA

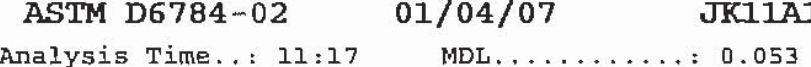


Air Sampling Associates, Inc.

Client Sample ID: HARDIN SDA INLET DUCT RUN 2 FH TOTAL Metals

Lot-Sample \#...: H6L070246-027

Date Sampled...: 12/02/06

PARAMETER

Prep Batch \#...: 7002025

Mercury
1.5
Date Received..: 12/06/06

REPORTING

LIMIT UNITS

ug

Dilution Factor: 0.5 METHOD
Matrix......: AIR

PREPARATION- WORK ANALYSIS DATE ORDER \#

ASTM D6784-02

Analysis Time..: 12:0日
01/02-01/05/07 JK11FIAA MDL.......... 0.030 
Air Sampling Associates, Inc.

Client Sample ID: HARDIN SDA INLET DUCT RUN 2 KCL

TOTAL Metals

Lot-Sample \#...: H6L070246-028

Date Sampled...: 12/02/06
Date Received..: 12/06/06

REPORTING

IIMIT

UNITS

$\mathrm{ug}$

0.12

Dilution Factor: 1.15
1.7
Prep Ba
Mercury

RESULT
Matrix...... AIR

PREPARATION- WORK ANALYSIS DATE ORDER \#

ASTM D6784-02 01/04/07 JK11KבAA

MDL........ 0.069 
Air Sampling Associates, Inc.

Client Sample ID: HARDIN SDA INIET DUCT RUN 2 HNO3/H2O2

TOTAL Metals

Lot-Sample \#. . : H6L070246-029

Date Sampled...: 12/02/06

PARAMETER

Prep Batch \#. . : 7002026

Mercury

$$
0.10
$$

Date Received..: 12/06/06

REPORTING LIMIT UNITS

ug Dilution Factor: 0.63
Matrix......: AIR

PREPARATION - WORK ANALYSIS DATE ORDER \#

01/03-01/05/07 JK11L1AA MDL......... 0.038 
Air Sampling Associates, Inc.

Client Sample ID: HARDIN SDA INLET DUCT RUN 2 KMNO4/H2SO4

TOTAL Metals

Lrot-Sample \#...: H6L070246-030

Date Sampled...: 12/02/06

PARAMETER

Prep Batch \#...: 7002028

Mercury
4.6
Date Received..: 12/06/06

REPORTING

LIMIT

UNITS

0.092 ug
Dilution Factor: 0.92
Matrix..... : AIR

PREPARATION- WORK ANALYSIS DATE ORDER \#

$\begin{array}{ccc}\text { ASTM D6784-02 } & 01 / 04 / 07 & \text { JK11Q1AA } \\ \text { Analysis Time..: 11:25 } & \text { MDL......... } 0.055\end{array}$


Air Sampling Associates, Inc.

Client Sample ID: HARDIN SDA INLET DUCT RUN 3 FH

TOTAL Metals

Irot-Sample \#...: H6L070246-033

Date Sampled...: 12/02/06

PARAMETER

Prep Batch \#...: 7002025

Mercury
0.64

Date Received..: 12/06/06

REPORTING

LIMIT

UNITS METHOD

ASTM D6784-02

Analysis Time..: $11: 33$

Matrix...... AIR

PREPARATION- WORK ANALYSIS DATE ORDER \#

01/02-01/05/07 JK1121AA MDL......... : 0.0060 
Air Sampling Associates, Inc.

Client Sample ID: HARDIN SDA INLET DUCT RUN 3 KCL

TOTAL Metals

Lot-Sample \#. . : : H6L070246-034

Date Sampled...: 12/02/06

Date Received. . : 12/06/06

REPORTING

LIMIT

UNITS

Prep Batch \#...: 7002028

Mercury

3.3

0.081 ug

Dilution Factor: 0.81
METHOD

Matrix......: AIR

PREPARATION - WORK ANALYSIS DATE ORDER \#

ASTM D6784-02

Analysis Time... 11:27
01/04/07 JK1131AA

MDL. ........ 0.049 
Air Sampling Associates, Inc.

client Sample ID: HARDTN SDA TNLET DUCT RUN 3 HNO3/H2O2

Total Metals

Lot-Sample \#...: H6L070246-035

Date Sampled...: 12/02/06

Date Received. . : 12/06/06

REPORTING

LIMIT

UNITS

METHOD

Prep Batch 将...: 7002026

Mercury

$0.058 \mathrm{~B}$
0.068 ug

Dilution Factor: 0.68
ASTM D6784-02

Analysis Time..: 12:03
Matrix....... AIR

PREPARATION - WORK ANALYSIS DATE ORDER \#

01/03-01/05/07 JK1141AA

MDE........ 0.041

NOTE (S) :

B Estimated result. Result is less than RL. 
Air Sampling Associates, Inc.

Client Sample ID: HARDIN SDA INLET DUCT RON 3 KMNO4/H2SO4

TOTAL Metals

Lot-Sample \#. . : H6L070246-036

Date Sampled...: 12/02/06

PARAMETER

Prep Batch \#...: 7002028

Mercury

0.79
Date Received. .: 12/06/06

REPORTING LIMIT

UNITS

ug

Dilution Factor: 1.11
Matrix......: AIR

PREPARATION- WORK ANALYSIS DATE ORDER \#

ASTM D6784-02 01/04/07

MDL . . . . . . : 0.067 
Air Sampling Associates, Inc.

Client Sample ID: HARDIN SDA INLET+STACK RB 0. IN HNO3

TOTAL Metals

Lot-Sample \#... : H6L070246-037

Date Sampled...: 12/01/06

Date Received..: $12 / 06 / 06$

REPORTING

LIMIT

UNITS

Prep Batch \#...: 7002025

Mercury

ND

0.010 ug

Dilution Factor: 0.1
METHOD

Matrix...... AIR

PREPARATION- WORK ANALYSIS DATE ORDER \#

ASTM D6784-02 01/02-01/05/07 JK12A1AA


Air Sampling Associates, Inc.

Client Sample ID: HARDIN SDA INLET+STACK RB KCL

TOTAL Metals

Lot-Sample \#. . : H6L070246-038

Date Sampled...: 12/01/06

PARAMETER

Prep Batch \#...: 7002028

Mercury
ND
Date Received. : 12/06/06

REPORTING

LIMIT

UNITS

METHOD

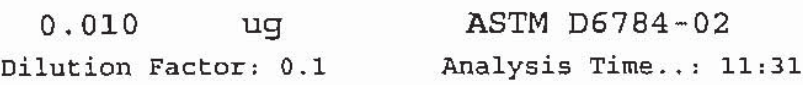

Matrix...... AIR

PREPARATION- WORK ANALYSIS DATE ORDER \#

01/04/07 JK12F1AA MDL.......... 0.0060 
Air Sampling Associates, Inc.

Client Sample ID: HARDIN SDA INLITT+STACK RB HNO3/H2O2

TOTAL Metals

Lot-Sample \#. . : H6L070246-039

Date Sampled...: 12/01/06

PARAMETER

RESULT

Prep Batch \#...: 7002026

Mercury
0.034

Date Received..: 12/06/06

REPORTING

LIMIT

UNITS

METHOD

ASTM D6784-02

Analysis Time... 12:06

Matrix....... AIR

PREPARATION - WORK ANALYSIS DATE ORDER \#

01/03-01/05/07 JK12G1AA MDL........ 0.014 
Air Sampling Associates, Inc.

Client Sample ID: HARDIN SDA INLET+STACK RB KMNO4/H2SO4/HYD

TOTAL Metals

Lot-Sample \#...: H6L070246-040

Date Sampled...: 12/01/06

PARAMETER

Prep Batch \#...: 7002028

Mercury
$\mathrm{ND}$
Date Received..: 12/06/06

REPORTING

IIMIT

UNITS METHOD

0.011 ug A5TM D6784-02

Dilution Factor: 0.11
Analysis Time..: 11:33
Matrix.....: AIR

PREPARATION - WORK ANALYSIS DATE ORDER \#

01/04/07 JK12H1AA MDL......... : 0.0066 


\section{QC Summary}




\section{METHOD BLANK REPORT}

TOTAL Metals

Client Lot \#...: H6L070246

Matrix....... AIR

PARAMETER

RESULT

MB Lot-Sample \#: H7A020000-025

Mercury
ND

$$
0.010
$$

Dilution Factor: 0.1

Analysis Time..: 11:15
PREPARATION - WORK ANALYSIS DATE ORDER \#

01/02-01/05/07 JL97P1AA

ANALYSIS DATE ORDER \#

MB Lot-Sample \#: H7A020000-026 Prep Batch \#. . : 7002026

Mercury

ND

0.025

ug

ASTM D6784-02

01/03-01/05/07 JL97R1AA

Dilution Factor: 0.25

Analysis Time..; 21:41

MB Lot-Sample \#: H7A020000-028 Prep Batch \#...: 7002028

Mercury

ND

0.010

ug

Dilution Factor: 0.1

Analysis Time..; 10:46

ASTM D6\\84-02

$01 / 04 / 07$

JL97W1AA

Mercury

ND

0.010

ug

Dilution Factor: 0.1

Analysis Time..: 10:46

NOTE (S) :

Calculations are performed before rounding to avaid round-off errors in calculated results. 


\section{LABORATORY CONTROL SAMPLE EVALUATION REPORT}

\section{TOTAL Metals}

Lot-Sample \#. . : H6L070246

\begin{tabular}{|c|c|c|c|}
\hline \multirow{3}{*}{$\frac{\text { DARAMETER }}{\text { Mercury }}$} & PERCENT & RECOVERY & RPD \\
\hline & RECOVERY & LIMITS & RPD LIMITS \\
\hline & 97 & $(80-120)$ & \\
\hline & 95 & $(80-120)$ & $(0-20)$ \\
\hline
\end{tabular}

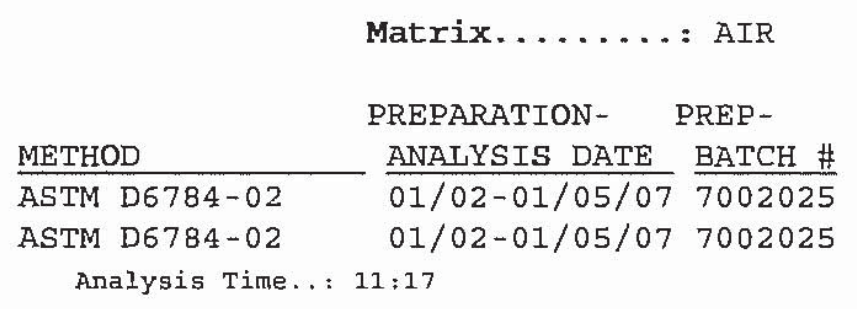

Matrix...... A AIR

PREPARATION- PREPANALYSIS DATE BATCH \# $01 / 02-01 / 05 / 077002025$

$: 17$

\section{NOTE $(S)$ :}

Calculations are performed beforc rounding to avoid round-off errors in calculated results. 


\section{LABORATORY CONTROL SAMPLE DATA REPORT}

\section{TOTAL Metals}

Lot-Sample \#. . : H6L070246

Matrix........ AIR

\begin{tabular}{|c|c|c|}
\hline & SPIKE & MEASURE \\
\hline PARAMETER & AMOUNT & AMOUNT \\
\hline \multirow[t]{2}{*}{ Mercury } & 0.500 & 0.487 \\
\hline & 0.500 & 0.474 \\
\hline
\end{tabular}

$\begin{array}{ll}\text { DNITS } & \text { PERCNT } \\ \text { Ug } & \frac{\text { RECVRY }}{97} \\ \text { ug } & 95\end{array}$

Dilution Factor: 0.1

\begin{tabular}{|c|c|c|}
\hline \multicolumn{3}{|c|}{ Matrix........ . AIR } \\
\hline METHOD & $\begin{array}{l}\text { PREPARATION- } \\
\text { ANALYSIS DATE }\end{array}$ & $\begin{array}{l}\text { PREP } \\
\text { BATCH \# }\end{array}$ \\
\hline ASTM D6784-02 & $01 / 02-01 / 05 / 07$ & 7002025 \\
\hline ASTM D6784-02 & $01 / 02-01 / 05 / 07$ & 7002025 \\
\hline
\end{tabular}

NOTE (S) :

Calculations are performed before rounding to avoid round-off errors in calculated results. 


\section{LABORATORY CONTROL SAMPLE EVALUATION REPOR'T}

\section{TOTAL Metals}

Client Lot \#...: H6L070246

Matrix........ AIR

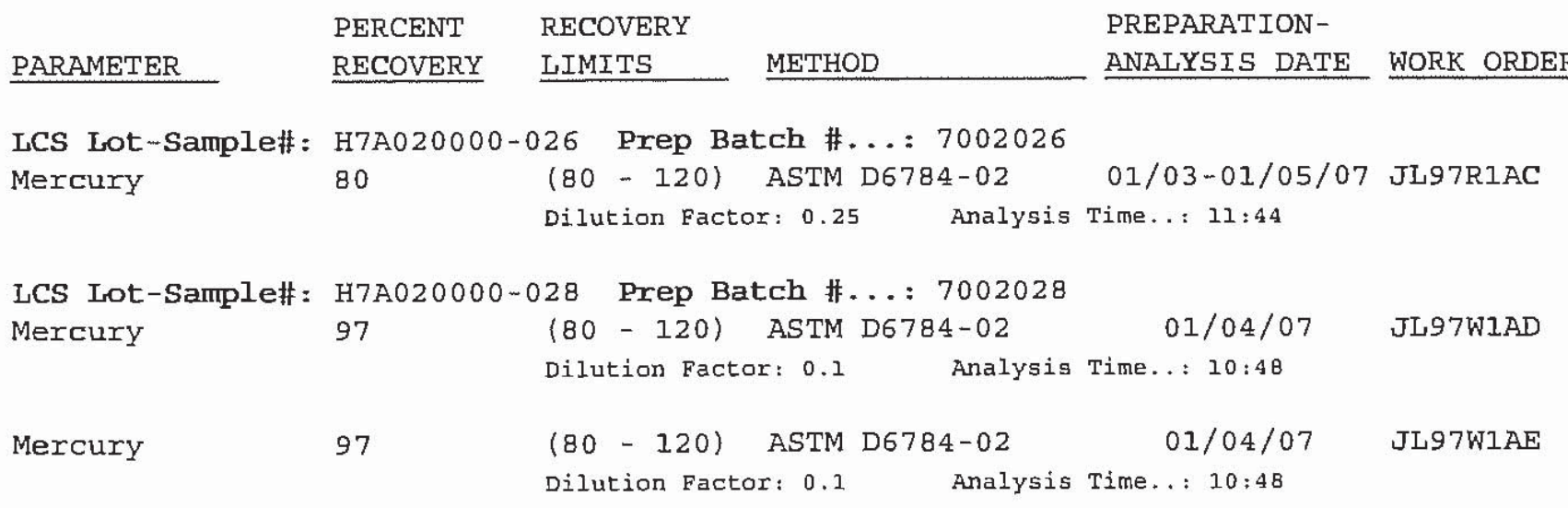

NOTE (S) :

Calculations are performed before rounding to avoid round-off errors in calculated results. 


\section{LABORATORY CONIROL SAMPLE DATA REPORT}

TOTAL Metals

Client Irot \#...: H6L070246

Matrix....... A AIR

PERCNT

PREPARATION - WORK

PARAMETER AMOUNT AMOUNT

UNITS
RECVRY METHOD ANALYSIS DATE ORDER \#

LCS Lot-Sample\#: H7A020000-026 Prep Batch \#...: 7002026

Mercury

1. 25

1.00

ug

80

ASTM D6784-02

01/03-01/05/07 JL97RIAC

Dilution Factor: 0.25

Analysis Time..: 11:44

LCS Lot-Sample\#: H7A020000-028 Prep Batch \#...: 7002028

Mercury

0.500

0.483

ug

97

ASTM D6784-02

$01 / 04 / 07$

JL97WIAD

Mercury

0.500

0.483

Dilution Factor: 0.1

Analysis Time..: 10:48

ug
Dilution Factor: 0.1

ASTM D6784-02

$01 / 04 / 07$

JL97WIAE

NOTR $(S)$ :

Calculations are performed before rounding to avoid round-off ertors in calculated results. 
MATRIX SPIKE SAMPLB EVALUATION RRPORT

TOTAL Metals

Client Lot \#...: H6L070246

Date sampled...: 12/01/06
Matrix........ AIR

Date Received..: 12/06/06

\begin{tabular}{|c|c|c|c|c|}
\hline PERCENT & RECOVERY & & RPD & \\
\hline RECOVERY & LIMITS & RPD & LIMITS & METHOD \\
\hline
\end{tabular}

MS Lot-Sample \#: H6L070246-005 Prep Batch \#...: 7002026

88

$(80-120)$

ASTM D6784-02

$(80-120) 2.8 \quad(0-20)$ ASTM D6784-02 Dilution Factor: 0.6

Analysis Time. . : $11: 47$
PREPARATION - WORK ANALYSIS DATE ORDER \#

01/03-01/05/07 JKIWFIAC 01/03-01/05/07 JKIWFIAD

NOTE (S) :

Calculations are performed before rounding to avoid round-off errors in calculated results. 
MATRIX SPIKE SAMPLE DATA REPORT

TOTAL Metals

Client Iot \#...: H6L070246

Date Sampled...: 12/01/06
Matrix........ AIR

Date Received. . : 12/06/06

PREPARATION-

WORK

SAMPLE SPIKE

MEASRD

RECVRY RPD METHOD

ORDER \#

MS Iot-Sanmle \#: H6L070246-005 Prep Batch \#...: 7002026 Mercury

$$
\begin{array}{ccccccc}
0.041 & 0.600 & 0.569 & \text { ug } & B 8 & & \text { ASTM D6784-02 01/03-01/05/07 JK1WF1AC } \\
0.041 & 0.600 & 0.586 \text { ug } & 91 & 2.8 & \text { ASTM D67B4-02 01/03-01/05/07 JK1WF1AD } \\
\text { Dilution Factor: } 0.6 & \\
\text { Analysis Time. : } 11: 47 &
\end{array}
$$

NOTE (S) :

Calculations are performed before rounding to avoid round-off errors in calculated results. 
MATRIX SPIKE SAMPLE EVALUATION REPORT

TOTAL Metals

Client Lot \#...: H6L070246

Date Sampled...: 12/01/06
Date Received..: 12/06/06

\begin{tabular}{llll} 
PERCENT & RECOVERY & RPD & \\
RECOVERY & LIMITS & RPD & LIMITS METHOD \\
\hline
\end{tabular}

MS Lot-Sample \#: H6L070246-004 Prep Batch \#... : 7002028

Mercury 99

104

\author{
$(80-120) \quad$ ASTM D6784-02 \\ $(80-120) 4.6 \quad(0-20)$ ASTM D6784-02 \\ Dilution Factor: 1.32 \\ Analysis Time..: 10:53
}

Matrix........ AIR

PREPARATION- WORK ANALYSIS DATE ORDER \#

01/04/07 JK1WE1AC

01/04/07 JKIWEIAD

NOTE (S) :

Calculations are performed before rounding to avoid round-off errors in calculated results. 
MATRIX SPIKE SAMPIE DATA REPORT

TOTAL Metals

Client Lot \#...: H6L070246

Date Sampled...: 12/01/06
Date Received..: 12/06/06

$$
\text { SAMPLE SPIKE }
$$

MEASRD

PARAMETER AMOUNT AMT
PERCNT
RECVRY RPD METHOD
Matrix....... AIR

PREPARATION - WORK ANALYSIS DATE ORDER \#

MS Lot-Sample \#: H6I070246-004 Prep Batch \#...: 7002028

Mercury

\begin{tabular}{|c|c|c|c|c|c|c|c|c|c|}
\hline 0.11 & 1.32 & 1.41 & ug & 99 & & ASTM & D6784-02 & $01 / 04 / 07$ & $J K \perp W E \perp A C$ \\
\hline 0.11 & 1.32 & 1.48 & ug & 104 & 4.6 & ASTM & D6784-02 & $01 / 04 / 07$ & JKIWEIAD \\
\hline & & & & & & & & & \\
\hline
\end{tabular}

NOTE (S) :

Calculations are performed before rounding to avoid round-off errors in calculated results. 
MATRIX SPIKR SAMPLE EVALUATION REPORT

TOTAL Metals

client Irot \#...: H6L070246

Date Sampled...: 12/01/06
Matrix....... AIR

Date Received..: 12/06/06

RPD

RPD LIMITS METHOD
PREPARATION- WORK ANALYSIS DATE ORDER \#

01/04/07 JKIWGIAC 01/04/07 JKIWGIAD

MS Lot-Sample \#: H6L070246-006 Prep Batch \#...: 7002028

Mercury

$$
\begin{array}{rrrr}
\text { NC, MSB } & (80-120) & \text { ASTM D6784-02 } \\
\text { NC, MSB } & (80-120) & (0-20) & \text { ASTM D6784-02 } \\
& \text { Dilution Factor: } 0.78 \\
& \text { Analysis Time. : } 11: 02
\end{array}
$$

NOTB $(S)$ :

Calculations are perfornied before rounding to avoid round-off errors in calculazed results. NC The recovery and/or RPD were not calculated.

MSB The recovery and RPD were not calculated because the sample amount was greater than four times the spike amount. 
MATRIX SPIKE SAMPLE DATA REPORT

TOTAL Metals

client Lot \#...: H6L070246

Date Sampled...: 12/01/06
Date Received..: 12/06/06 RECVRY RPD METHOD
Matrix....... A AIR

PREPARATION- WORK ANALYSIS DATE ORDER \#

PARAMETER AMOUNT AMT

AMOUNT UNITS

MS Lot-Sample \#: H6L070246-006 Prep Batch \#... 7002028 Mercury

$\begin{array}{cccc}6.8 & 0.780 & 7.88 & \text { ug } \\ 6.8 & 0.780 & 8.11 & \text { Qualifiers: NC,MSB } \\ & & \text { Qualifiers: NC,MSB } \\ & \text { Dilution Factor: } 0.78 \\ & \text { Analysis Time. : } 11: 02\end{array}$

ASTM D6784-02

$01 / 04 / 07$

JKIWGIAC

ASTM D6784-02

$01 / 04 / 07$

JKIWGIAD

Analysis Time. : 11:02

\section{NOTE $(S):$}

Calculations are performed before rounding to avoid round-off errors in colculated results.

NC The recovery and/or RPD were not calculated.

MSB The recovery and RPD were not calculated because the sample amount was grenter than four times uhe spike amount. 


\section{STL Knoxville}

\section{Mercury Data Reporting Form}

Post Digestion Spike

Units: $u g / L(p p b)$

Instrument ID: Leeman HydraAA Hg

Data File Name: H010507. PRN

\begin{tabular}{|c|c|c|c|c|}
\hline Element & $\begin{array}{c}\text { PDS } \\
\text { JK1WAA }\end{array}$ & $\begin{array}{c}\text { Original Sample } \\
\text { JKIWA }\end{array}$ & Spike Added & $\begin{array}{c}\text { Percent } \\
\text { Recovery }\end{array}$ \\
\hline $\mathrm{Hg}$ & 1.36 & 0.34 & 1.0 & 102 \\
\hline
\end{tabular}


Quality Control Results

Mercury 


\section{STL Knoxville}

Mercury Data Reporting Form

Initial Calibration Verification

Units: $u g / L(p p b)$

Instrument ID: Leeman HydraAA Hg An

Data File Name: M010407.PRN

\begin{tabular}{|c|c|c|c|c|c|c|c|c|c|c|c|c|c|}
\hline \multirow[b]{2}{*}{ Elem } & \multirow[b]{2}{*}{$\begin{array}{l}\text { True } \\
\text { Conc }\end{array}$} & \multicolumn{2}{|c|}{$\begin{array}{c}\text { Ck2icv } \\
1 / 4 / 2007 \\
9: 50 \mathrm{AM}\end{array}$} & \multirow[b]{2}{*}{ Found } & \multirow[b]{2}{*}{$\begin{array}{c}\% \\
\operatorname{Rec}\end{array}$} & \multirow[b]{2}{*}{ Found } & \multirow[b]{2}{*}{$\begin{array}{c}\% \\
\operatorname{Rec}\end{array}$} & \multirow[b]{2}{*}{ Found } & \multirow[b]{2}{*}{$\begin{array}{c}\% \\
\operatorname{Rec}\end{array}$} & \multirow[b]{2}{*}{ Found } & \multirow[b]{2}{*}{$\begin{array}{l}\% \\
\operatorname{Rec}\end{array}$} & \multirow[b]{2}{*}{ Found } & \multirow[b]{2}{*}{$\begin{array}{l}\% \\
\operatorname{Rec}\end{array}$} \\
\hline & & Found & $\begin{array}{c}\% \\
\operatorname{Rec} \\
\end{array}$ & & & & & & & & & & \\
\hline $\mathrm{Hg}$ & 2.5 & 2.43 & 97.2 & & & & & & & & & & \\
\hline
\end{tabular}


STL Knoxville

Mercury Data Reporting Form

Continuing Calibration Verification

Units: $u g / L(p p b)$

Instrument ID: Leeman HydraAA $\mathrm{Hg}$ An

Data File Name: M010407.PRN

\begin{tabular}{|c|c|c|c|c|c|c|c|c|c|c|c|c|c|}
\hline \multirow[b]{2}{*}{ Elem } & \multirow[b]{2}{*}{$\begin{array}{l}\text { True } \\
\text { Conc }\end{array}$} & \multicolumn{2}{|c|}{$\begin{array}{c}\mathrm{Ck} 3 \mathrm{ccv} \\
1 / 4 / 2007 \\
9: 56 \mathrm{AM} \\
\end{array}$} & \multicolumn{2}{|c|}{$\begin{array}{c}\mathrm{Ck} 3 \mathrm{ccv} \\
1 / 4 / 2007 \\
10: 14 \mathrm{AM}\end{array}$} & \multicolumn{2}{|c|}{$\begin{array}{c}\mathrm{Ck} 3 \mathrm{ccV} \\
1 / 4 / 2007 \\
10: 33 \mathrm{AM}\end{array}$} & \multicolumn{2}{|c|}{$\begin{array}{c}\text { Ck3ccv } \\
1 / 4 / 2007 \\
10: 56 \mathrm{AM} \\
\end{array}$} & \multicolumn{2}{|c|}{$\begin{array}{c}\text { Ck3ccv } \\
1 / 4 / 2007 \\
11: 21 \mathrm{AM}\end{array}$} & \multicolumn{2}{|c|}{$\begin{array}{c}\text { Ck3ccv } \\
1 / 4 / 2007 \\
11: 35 \mathrm{AM}\end{array}$} \\
\hline & & Found & $\begin{array}{c}\% \\
\operatorname{Rec}\end{array}$ & Found & $\begin{array}{c}\% \\
\operatorname{Rec}\end{array}$ & Found & $\begin{array}{c}\% \\
\text { Rec }\end{array}$ & Found & $\begin{array}{c}\% \\
\operatorname{Rec}\end{array}$ & Found & $\begin{array}{l}\% \\
\operatorname{Rec}\end{array}$ & Found & $\begin{array}{c}\% \\
\operatorname{Rec}\end{array}$ \\
\hline $\mathrm{Hg}$ & 5.0 & 4.92 & 98.4 & 4.94 & 98.8 & 4.94 & 98.8 & 4.95 & 99.0 & 4.91 & 98.2 & 4.89 & 97.8 \\
\hline
\end{tabular}




\section{STL Knoxville}

Mercury Data Reporting Form

Continuing Calibration Verification
Units: $u g / L(p p b)$

Instrument ID: Leeman HydraAA Hg An

Data File Name: M010407.PRN

\begin{tabular}{|c|c|c|c|c|c|c|c|c|c|c|c|c|c|}
\hline \multirow[b]{2}{*}{ Elem } & \multirow[b]{2}{*}{$\begin{array}{l}\text { True } \\
\text { Conc }\end{array}$} & \multicolumn{2}{|c|}{$\begin{array}{c}\text { Ck3ccv } \\
1 / 4 / 2007 \\
11: 57 \mathrm{AM}\end{array}$} & & & & & & & & & & \\
\hline & & Found & $\begin{array}{l}\% \\
\operatorname{Rec}\end{array}$ & Found & $\begin{array}{l}\% \\
\text { Rec }\end{array}$ & Found & $\begin{array}{l}\% \\
\operatorname{Rec}\end{array}$ & Found & $\begin{array}{l}\% \\
\text { Rec }\end{array}$ & Found & $\begin{array}{l}\% \\
\operatorname{Rec}\end{array}$ & Found & $\begin{array}{l}\% \\
\text { Rec }\end{array}$ \\
\hline $\mathrm{Hg}$ & 5.0 & 4.87 & 97.4 & & & & & & & & & & \\
\hline
\end{tabular}




\section{STL Knoxville}

Mercury Data Reporting Form

Contract Required Detection Limit Standard(s)
Units: $u g / L(p p b)$

Instrument ID: Leeman HydraAA Hg An

Data File Name: M010407.PRN

\begin{tabular}{|c|c|c|c|c|c|c|c|c|c|c|c|c|c|}
\hline \multirow[b]{2}{*}{ Elem } & \multirow[b]{2}{*}{$\begin{array}{l}\text { True } \\
\text { Conc }\end{array}$} & \multicolumn{2}{|c|}{$\begin{array}{c}\text { CRA } \\
1 / 4 / 2007 \\
9: 54 \mathrm{AM}\end{array}$} & \multirow[b]{2}{*}{ Found } & \multirow[b]{2}{*}{$\begin{array}{l}\% \\
\text { Rec }\end{array}$} & \multirow[b]{2}{*}{ Found } & \multirow[b]{2}{*}{$\begin{array}{l}\% \\
\text { Rec }\end{array}$} & \multirow[b]{2}{*}{ Found } & \multirow[b]{2}{*}{$\begin{array}{l}\% \\
\operatorname{Rec}\end{array}$} & \multirow[b]{2}{*}{ Found } & \multirow[b]{2}{*}{$\begin{array}{c}\% \\
\operatorname{Rec}\end{array}$} & \multirow[b]{2}{*}{ Found } & \multirow[b]{2}{*}{$\begin{array}{c}\% \\
\text { Rec }\end{array}$} \\
\hline & & Found & $\begin{array}{c}\% \\
\operatorname{Rec}\end{array}$ & & & & & & & & & & \\
\hline $\mathrm{Hg}$ & 0.2 & 0.18 & 88.0 & & & & & & & & & & \\
\hline
\end{tabular}




\section{STL Knoxville}

\section{Mercury Data Reporting Form}

Initial Calibration Blank(s)

Units: $u g / L(p p b)$

Instrument ID: Leeman HydraAA Hg An

Data File Name: M010407.PRN

\begin{tabular}{|c|c|c|c|c|c|c|c|c|c|c|c|c|c|}
\hline \multirow[b]{2}{*}{ Elem } & \multirow{2}{*}{$\begin{array}{c}\text { Reporting } \\
\text { Limit }\end{array}$} & \multicolumn{2}{|c|}{$\begin{array}{c}\text { ICB } \\
1 / 4 / 2007 \\
9: 52 \mathrm{AM}\end{array}$} & \multirow[b]{2}{*}{ Found } & \multirow[b]{2}{*}{ Flag } & \multirow[b]{2}{*}{ Found } & \multirow[b]{2}{*}{ Flag } & \multirow[b]{2}{*}{ Found } & \multirow[b]{2}{*}{ Flag } & \multirow[b]{2}{*}{ Found } & \multirow[b]{2}{*}{ Flag } & \multirow[b]{2}{*}{ Found } & \multirow[b]{2}{*}{ Flag } \\
\hline & & Found & Flag & & & & & & & & & & \\
\hline $\mathrm{Hg}$ & 0.1 & 0.06 & $\mathrm{U}$ & & & & & & & & & & \\
\hline
\end{tabular}




\section{STL Knoxville}

\section{Mercury Data Reporting Form}

Continuing Calibration Blank(s)
Units: $u g / L(p p b)$

Instrument ID: Leeman HydraAA Hg Ana

Data File Name: M010407.PRN

\begin{tabular}{|c|c|c|c|c|c|c|c|c|c|c|c|c|c|}
\hline \multirow[b]{2}{*}{ Elem } & \multirow{2}{*}{$\begin{array}{c}\text { Reporting } \\
\text { Limil }\end{array}$} & \multicolumn{2}{|c|}{$\begin{array}{l}\text { Cklccb } \\
1 / 4 / 20017 \\
9: 58 \mathrm{AM}\end{array}$} & \multicolumn{2}{|c|}{$\begin{array}{c}\text { Cklccb } \\
1 / 4 / 2007 \\
10: 16 \mathrm{AM}\end{array}$} & \multicolumn{2}{|c|}{$\begin{array}{c}\text { Ckiccb } \\
1 / 4 / 2007 \\
10: 35 \mathrm{AM}\end{array}$} & \multicolumn{2}{|c|}{$\begin{array}{c}\text { Cklccb } \\
1 / 1 / 2007 \\
10: 58 \mathrm{AM}\end{array}$} & \multicolumn{2}{|c|}{$\begin{array}{c}\text { Cklccb } \\
1 / 1 / 2007 \\
11: 23 \text { AM }\end{array}$} & \multicolumn{2}{|c|}{$\begin{array}{c}\text { Ck1ccb } \\
1 / 4 / 2001 \\
11: 37 \text { AM }\end{array}$} \\
\hline & & Found & Flag & Found & Flag & Found & Flag & Found & Flag & Found & Flag & Found & Flag \\
\hline $\mathrm{Hg}$ & 0.1 & 0.06 & U & -0.07 & B & 0.06 & U & 0.06 & U & 0.06 & U & 0.06 & U \\
\hline
\end{tabular}




\section{STL Knoxville}

Mercury Data Reporting Form

Continuing Calibration Blank(s)
Units: $u g / L(p p b)$

Instrument ID: Leeman HydraAA Hg An

Data File Name: M010407.PRN

\begin{tabular}{|c|c|c|c|c|c|c|c|c|}
\hline & & \multicolumn{2}{c|}{$\begin{array}{c}\text { Cklccb } \\
1 / 4 / 2007 \\
11: 59 \mathrm{AM}\end{array}$} & & & & & \\
\cline { 3 - 10 } Elem & $\begin{array}{c}\text { Reporting } \\
\text { Limit }\end{array}$ & \begin{tabular}{c} 
Found $\quad$ Flag \\
\hline $\mathrm{Hg}$
\end{tabular} & Found Flag & Found Flag & Found Flag & Found Flag & Found Flag \\
\hline
\end{tabular}




\section{STL Knoxville}

Mercury Data Reporting Form

Instrument Detection Limits

IDL Completion Date:12/14/2006
Units: $u g / L(p p b)$

Instrument ID: Leeman HydraAA Hg An

Data File Name: M010407.PRN

\begin{tabular}{|c|c|c|c|}
\hline Element & $\begin{array}{c}\text { Wavelength } \\
(\mathbf{n m})\end{array}$ & $\begin{array}{c}\text { Reporting } \\
\text { Limit }\end{array}$ & IDL \\
\hline $\mathrm{Hg}$ & 253.70 & 0.1 & 0.06 \\
\hline
\end{tabular}




\section{STL Knoxville}

\section{Sample ID Nomenclature}

The sample ID consists of 5 alpha-numeric characters followed by a suffix in the 6 th position that designates the sample type:

\begin{tabular}{cll} 
Suffix & & \multicolumn{1}{c}{ Sample Type: } \\
\cline { 1 - 1 } B & & Method Blank \\
C & & Laboratory Control Sample \\
L & & Laboratory Control Sample Duplicate \\
S & & Matrix Spike \\
D & & Matrix Spike Duplicate \\
X & & Sample Duplicate \\
P & Serial Dilution \\
A & Post Digestion Spike \\
Z\# & Dilution; \# = Dilution Factor
\end{tabular}

Mercury Report Generator Version 2.0 


\section{STL Knoxville}

Mercury Data Reporting Form

Initial Calibration Verification

Units: ug/L (ppb)

Instrument ID: Leeman HydraAA Hg An

Data File Name: M010507.PRN

\begin{tabular}{|c|c|c|c|c|c|c|c|c|c|c|c|c|c|}
\hline \multirow[b]{2}{*}{ Elem } & \multirow[b]{2}{*}{$\begin{array}{l}\text { True } \\
\text { Conc }\end{array}$} & \multicolumn{2}{|c|}{$\begin{array}{c}\text { Ck2icv } \\
\text { 1/5/2007 } \\
10: 05 \text { AM }\end{array}$} & & & & & & & & & & \\
\hline & & Found & $\begin{array}{c}\% \\
\operatorname{Rec}\end{array}$ & Found & $\begin{array}{l}\% \\
\operatorname{Rec}\end{array}$ & Found & $\begin{array}{c}\% \\
\operatorname{Rec}\end{array}$ & Found & $\begin{array}{l}\% \\
\operatorname{Rec}\end{array}$ & Found & $\begin{array}{l}\% \% \\
\operatorname{Rec}\end{array}$ & Found & $\begin{array}{l}\% \\
\operatorname{Rec}\end{array}$ \\
\hline $\mathrm{Hg}$ & 2.5 & 2.48 & 99.2 & & & & & & & & & & \\
\hline
\end{tabular}




\section{STL Knoxville}

\section{Mercury Data Reporting Form}

Continuing Calibration Verification
Units: $u g / L(p p b)$

Instrument ID: Leeman HydraAA Hg An

Data File Name: M010507.PRN

\begin{tabular}{|c|c|c|c|c|c|c|c|c|c|c|c|c|c|}
\hline \multirow[b]{2}{*}{ Elem } & \multirow[b]{2}{*}{$\begin{array}{l}\text { True } \\
\text { Conc }\end{array}$} & \multicolumn{2}{|c|}{$\begin{array}{c}\text { Ck3ccv } \\
1 / 5 / 2007 \\
10: 11 \mathrm{AM}\end{array}$} & \multicolumn{2}{|c|}{$\begin{array}{c}\text { Ck3ccv } \\
1 / 5 / 2007 \\
10: 30 \mathrm{AM}\end{array}$} & \multicolumn{2}{|c|}{$\begin{array}{c}\text { Ck3ccv } \\
1 / 5 / 2007 \\
10: 48 \mathrm{AM}\end{array}$} & \multicolumn{2}{|c|}{$\begin{array}{c}\text { Ck3ccv } \\
1 / 5 / 2007 \\
11: 11 \mathrm{AM}\end{array}$} & \multicolumn{2}{|c|}{$\begin{array}{c}\mathrm{Ck} 3 \mathrm{ccv} \\
1 / 5 / 2007 \\
11: 35 \mathrm{AM}\end{array}$} & \multicolumn{2}{|c|}{$\begin{array}{c}\text { Ck3ccv } \\
1 / 5 / 2007 \\
11: 59 \mathrm{AM}\end{array}$} \\
\hline & & Found & $\begin{array}{c}\% \\
\operatorname{Rec}\end{array}$ & Found & $\begin{array}{c}\% \\
\operatorname{Rec}\end{array}$ & Found & $\begin{array}{c}\% \\
\operatorname{Rec}\end{array}$ & Found & $\begin{array}{c}\% \\
\operatorname{Rec}\end{array}$ & Found & $\begin{array}{c}\% \\
\operatorname{Rec}\end{array}$ & Found & $\begin{array}{c}\% \\
\operatorname{Rec}\end{array}$ \\
\hline $\mathrm{Hg}$ & 5.0 & 4.83 & 96.6 & 4.80 & 96.0 & 4.86 & 97.2 & 4.84 & 96.8 & 4.83 & 96.6 & 4.90 & 98.0 \\
\hline
\end{tabular}




\section{STL Knoxville}

\section{Mercury Data Reporting Form}

\section{Continuing Calibration Verification}

Units: $\mathrm{ug} / \mathrm{L}(\mathrm{ppb})$

Instrument ID: Leeman HydraAA Hg An

Data File Name: M010507.PRN

\begin{tabular}{|c|c|c|c|c|c|c|c|c|c|c|c|c|c|}
\hline \multirow[b]{2}{*}{ Elem } & \multirow[b]{2}{*}{$\begin{array}{l}\text { True } \\
\text { Conc }\end{array}$} & \multicolumn{2}{|c|}{$\begin{array}{c}\text { Ck3ccv } \\
1 / 5 / 2007 \\
12: 10 \mathrm{PM}\end{array}$} & \multirow[b]{2}{*}{ Found } & \multirow[b]{2}{*}{$\begin{array}{c}\% \\
\operatorname{Rec}\end{array}$} & \multirow[b]{2}{*}{ Found } & \multirow[b]{2}{*}{$\begin{array}{c}\% \\
\operatorname{Rec}\end{array}$} & \multirow[b]{2}{*}{ Found } & \multirow[b]{2}{*}{$\begin{array}{l}\% \\
\operatorname{Rec}\end{array}$} & \multirow[b]{2}{*}{ Found } & \multirow[b]{2}{*}{$\begin{array}{c}\% \\
\operatorname{Rec}\end{array}$} & \multirow[b]{2}{*}{ Found } & \multirow[b]{2}{*}{$\begin{array}{l}\% \\
\operatorname{Rec}\end{array}$} \\
\hline & & Found & $\begin{array}{l}\% \\
\operatorname{Rec}\end{array}$ & & & & & & & & & & \\
\hline $\mathrm{Hg}$ & 5.0 & 4.88 & 97.6 & & & & & & & & & & \\
\hline
\end{tabular}




\section{STL Knoxville}

Mercury Data Reporting Form

Contract Required Detection Limit Standard(s)
Units: $u g / L(p p b)$

Instrument ID: Leeman HydraAA Hg An

Data File Name: M010507.PRN

\begin{tabular}{|c|c|c|c|c|c|c|c|c|c|c|c|c|c|}
\hline \multirow[b]{2}{*}{ Elem } & \multirow[b]{2}{*}{$\begin{array}{l}\text { True } \\
\text { Conc }\end{array}$} & \multicolumn{2}{|c|}{$\begin{array}{c}\text { CRA } \\
1 / 5 / 2007 \\
10: 09 \mathrm{AM}\end{array}$} & \multirow[b]{2}{*}{ Found } & \multirow[b]{2}{*}{$\begin{array}{l}\% \\
\operatorname{Rec} \\
\end{array}$} & \multirow[b]{2}{*}{ Found } & \multirow[b]{2}{*}{$\begin{array}{l}\% \\
\operatorname{Rec}\end{array}$} & \multirow[b]{2}{*}{ Found } & \multirow[b]{2}{*}{$\begin{array}{l}\% \\
\operatorname{Rec}\end{array}$} & \multirow[b]{2}{*}{ Found } & \multirow[b]{2}{*}{$\begin{array}{l}\% \\
\operatorname{Rec}\end{array}$} & \multirow[b]{2}{*}{ Found } & \multirow[b]{2}{*}{$\begin{array}{c}\% \\
\operatorname{Rec}\end{array}$} \\
\hline & & Found & $\begin{array}{c}\% \\
\operatorname{Rec}\end{array}$ & & & & & & & & & & \\
\hline $\mathrm{Hg}$ & 0.2 & 0.19 & 95.0 & & & & & & & & & & \\
\hline
\end{tabular}




\section{STL Knoxville}

\section{Mercury Data Reporting Form}

Initial Calibration Blank(s)

Units: $u g / L(p p b)$

Instrument ID: Leeman HydraAA Hg An

Data File Name: M010507.PRN

\begin{tabular}{|c|c|c|c|c|c|c|c|c|c|c|c|c|c|}
\hline \multirow[b]{2}{*}{ Elem } & \multirow{2}{*}{$\begin{array}{l}\text { Reporting } \\
\text { Limit }\end{array}$} & \multicolumn{2}{|c|}{$\begin{array}{c}\text { ICB } \\
1 / 5 / 2007 \\
10: 07 \mathrm{AM}\end{array}$} & \multirow[b]{2}{*}{ Found } & \multirow[b]{2}{*}{ Flag } & \multirow[b]{2}{*}{ Found } & \multirow[b]{2}{*}{ Flag } & \multirow[b]{2}{*}{ Found } & \multirow[b]{2}{*}{ Flag } & \multirow[b]{2}{*}{ Found } & \multirow[b]{2}{*}{ Flag } & \multirow[b]{2}{*}{ Found } & \multirow[b]{2}{*}{ Flag } \\
\hline & & Found & Flag & & & & & & & & & & \\
\hline $\mathrm{Hg}$ & 0.1 & 0.06 & $\mathrm{U}$ & & & & & & & & & & \\
\hline
\end{tabular}




\section{STL Knoxville}

\section{Mercury Data Reporting Form}

Continuing Calibration Blank(s)
Units: $u g / L(p p b)$

Instrument ID: Leeman HydraAA Hg Ana

Data File Name: M010507.PRN

\begin{tabular}{|c|c|c|c|c|c|c|c|c|c|c|c|c|c|}
\hline \multirow[b]{2}{*}{ Elcm } & \multirow{2}{*}{$\begin{array}{c}\text { Reporting } \\
\text { Limil }\end{array}$} & \multicolumn{2}{|c|}{$\begin{array}{c}\text { Cklccb } \\
1 / 5 / 2007 \\
10: 1+\mathrm{AM}\end{array}$} & \multicolumn{2}{|c|}{$\begin{array}{c}\text { Cklccb } \\
1 / 5 / 2007 \\
10: 32 \mathrm{AM}\end{array}$} & \multicolumn{2}{|c|}{$\begin{array}{c}\text { Cklccb } \\
1 / 5 / 2007 \\
10: 50 \mathrm{AM}\end{array}$} & \multicolumn{2}{|c|}{$\begin{array}{c}\text { Cklccb } \\
1 / 5 / 2007 \\
11: 13 \text { AM }\end{array}$} & \multicolumn{2}{|c|}{$\begin{array}{c}\text { Cklccb } \\
1 / 5 / 2007 \\
11: 37 \text { AM }\end{array}$} & \multicolumn{2}{|c|}{$\begin{array}{c}\text { Cklccb } \\
1 / 5 / 2007 \\
12: 01 \mathrm{PM}\end{array}$} \\
\hline & & Found & Flag & Found & Flag & Found & Flag & Found & Flag & Found & Flag & Found & Flang \\
\hline $\mathrm{Hg}$ & 0.1 & 0.06 & U & 0.06 & U & 0.06 & $\mathrm{U}$ & 0.06 & U & 0.06 & $\mathrm{U}$ & 0.06 & U \\
\hline
\end{tabular}




\section{STL Knoxville}

Mercury Data Reporting Form

Continuing Calibration Blank(s)
Units: ug/L (ppb)

Instrument ID: Leeman HydraAA $\mathrm{Hg} \mathrm{An}$

Data File Name: M010507.PRN

\begin{tabular}{|c|c|c|c|c|c|c|c|c|c|c|c|c|c|}
\hline \multirow[b]{2}{*}{ Elem } & \multirow[b]{2}{*}{$\begin{array}{l}\text { Reporting } \\
\text { Limit }\end{array}$} & \multicolumn{2}{|c|}{$\begin{array}{c}\text { Cklccb } \\
1 / 5 / 2007 \\
12: 12 \text { PM }\end{array}$} & \multirow[b]{2}{*}{ Found } & \multirow[b]{2}{*}{ Flag } & \multirow[b]{2}{*}{ Found } & \multirow[b]{2}{*}{ Flag } & \multirow[b]{2}{*}{ Found } & \multirow[b]{2}{*}{ Flag } & \multirow[b]{2}{*}{ Found } & \multirow[b]{2}{*}{ Flag } & \multirow[b]{2}{*}{ Found } & \multirow[b]{2}{*}{ Flag } \\
\hline & & Found & Flag & & & & & & & & & & \\
\hline $\mathrm{Hg}$ & 0.1 & 0.06 & $\mathrm{U}$ & & & & & & & & & & \\
\hline
\end{tabular}




\section{STL Knoxville}

\section{Mercury Data Reporting Form}

Post Digestion Spike

Units: $u g / L(p p b)$

Instrument ID: Leeman HydraAA Hg

Data File Name: H010507. PRN

\begin{tabular}{|c|c|c|c|c|}
\hline Element & $\begin{array}{c}\text { PDS } \\
\text { JK1WAA }\end{array}$ & $\begin{array}{c}\text { Original Sample } \\
\text { JK1WA }\end{array}$ & Spike Added & $\begin{array}{c}\text { Percent } \\
\text { Recovery }\end{array}$ \\
\hline $\mathrm{Hg}$ & 1.36 & 0.34 & 1.0 & 102 \\
\hline
\end{tabular}


STL Knoxville

Mercury Data Reporting Form

Instrument Detection Limits

IDL Completion Date:12/14/2006
Units: $u g / L(p p b)$

Instrument ID: Leeman HydraAA Hg An

Data File Name: M010507.PRN

\begin{tabular}{|c|c|c|c|}
\hline Element & $\begin{array}{c}\text { Wavelength } \\
(\mathbf{n m})\end{array}$ & $\begin{array}{c}\text { Reporting } \\
\text { Limit }\end{array}$ & IDL \\
\hline $\mathrm{Hg}$ & 253.70 & 0.1 & 0.06 \\
\hline
\end{tabular}




\section{STL Knoxville}

\section{Sample ID Nomenclature}

The sample ID consists of 5 alpha-numeric characters followed by a suffix in the 6 th position that designates the sample type:

\begin{tabular}{cll} 
Suffix & & \multicolumn{1}{c}{ Sample Type: } \\
\cline { 1 - 1 } B & & Method Blank \\
C & & Laboratory Control Sample \\
L & & Laboratory Control Sample Duplicate \\
S & & Matrix Spike \\
D & Matrix Spike Duplicate \\
X & Sample Duplicate \\
P & Serial Dilution \\
A & Post Digestion Spike \\
Z\# & Dilution; \# = Dilution Factor
\end{tabular}

Mercury Report Generator Version 2.0 
Raw Data Mercury 


\section{STL Knoxville \\ Mercury Analysis Cover Sheet}

\begin{tabular}{|r|l|l|l|}
\hline Analyst & D(a) & Instrument & Leeman Hydra AA Mercury Analyzer \\
\hline Run Date & $01 / 04 / 07$ & Chart Name & M010407 \\
\hline
\end{tabular}

\begin{tabular}{||l|l|c|l||}
\hline \multicolumn{2}{|c|}{ Daily Maintenance } & \multicolumn{3}{c|}{ As Needed Maintenance } \\
\hline$\square$ & Check tubing. & $\checkmark$ & Adjust/change Hg lamp. \\
\hline \multirow{2}{*}{$\begin{array}{l}\text { Check maintenance schedule } \\
\text { flags. }\end{array}$} & $\square$ & Clean or replace optical cell. \\
\cline { 3 - 5 } & & $\square$ & Lubricate pump and autosampler arm. \\
\cline { 3 - 5 } & & $\square$ & Change the drying tube. \\
\hline
\end{tabular}


Instrument Upload

Run Log - Page 1 :

Started Thu Jan 4 13:14:22 2007 by WILBURND

Data File: UPL\$KNX_DATA_ROOT: $<$ LHG $>$ M010407.PRN; 1

\begin{tabular}{|c|c|c|c|c|c|c|c|}
\hline \# & WorkOrder & Dilution & Date & Time & Batch & Lot & Instrume \\
\hline- & 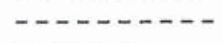 & $\cdots$ & 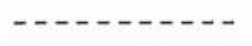 & $-\ldots-1$ & & & \\
\hline 1 & CK2ICV & 1.00 & 04 -JAN-2007 & $09: 50: 37$ & & & HG \\
\hline 2 & ICB & 1.00 & 04 -JAN-2007 & $09: 52: 22$ & & & HG \\
\hline 3 & CRA & 1.00 & $04-J A N-2007$ & $09: 54: 18$ & & & HG \\
\hline 4 & CK3 CCV & 1.00 & 04 -JAN-2007 & $09: 56: 14$ & & & HG \\
\hline 5 & $\mathrm{CK} \perp \mathrm{CCB}$ & 1.00 & 04 -JAN-2007 & $09: 58: 21$ & & & HG \\
\hline 6 & MDL1 & 1.00 & $04-J A N-2007$ & $10: 00: 08$ & & & HG \\
\hline 7 & MDL2 & 1.00 & $04-J A N-2007$ & $10: 01: 55$ & & & HG \\
\hline 8 & MDL3 & 1.00 & 04 -JAN-2007 & $10: 04: 02$ & & & HG \\
\hline 9 & MDL4 & 1.00 & $04-J A N-2007$ & $10: 05: 54$ & & & HG \\
\hline 10 & MDL5 & 1.00 & $04-J A N-2007$ & $10: 07: 50$ & & & HG \\
\hline 11 & MDL6 & 1.00 & 04 -JAN-2007 & $10: 10: 19$ & & & HG \\
\hline 12 & MDL7 & 1.00 & 04 -JAN-2007 & $10: 12: 08$ & & & HG \\
\hline 13 & $\mathrm{CK} 3 \mathrm{CCV}$ & 1.00 & $04-J A N-2007$ & $10: 14: 05$ & & & HG \\
\hline 14 & CKICCB & 1.00 & 04 -JAN-2007 & $10: 16: 05$ & & & HG \\
\hline 15 & MDL1 & 1.00 & $04-J A N-2007$ & $10: 17: 54$ & & & HG \\
\hline 16 & MDL2 & 1.00 & $04-J A N-2007$ & $10: 20: 26$ & & & HG \\
\hline 17 & MDL3 & 1.00 & .04 -JAN-2007 & $10: 22: 52$ & & & HG \\
\hline 18 & MDL4 & 1.00 & $04-J A N-2007$ & $10: 24: 42$ & & & $\mathrm{HG}$ \\
\hline 19 & MDL5 & 1.00 & $04-J A N-2007$ & $10: 26: 30$ & & & HG \\
\hline 20 & MDL6 & 1.00 & $04-J A N-2007$ & $10: 28: 21$ & & & HG \\
\hline 21 & MDL7 & 1.00 & $04-J A N-2007$ & $10: 30: 47$ & & & HG \\
\hline 22 & CK3CCV & 1.00 & $04-J A N-2007$ & $10: 33: 26$ & & & HG \\
\hline 23 & CK1CCB & 1.00 & $04-J A N-2007$ & $10: 35: 34$ & & & HG \\
\hline 24 & JL99EBT & 1.00 & $04-J A N-2007$ & $10: 37: 24$ & 7003044 & H7A020000 & HG \\
\hline 25 & JMCENCT & 1.00 & $04-J A N-2007$ & $10: 39: 10$ & 7003044 & H7A0 30000 & HG \\
\hline 26 & JL57ET & 1.00 & $04-J A N-2007$ & $10: 41: 08$ & 7003044 & H6L270125 & HG \\
\hline 27 & JL57EST & 1.00 & $04-J A N-2007$ & $10: 42: 56$ & 7003044 & H6L270125 & HG \\
\hline 28 & JL57EDT & 1.00 & $04-J A N-2007$ & $10: 44: 48$ & 7003044 & H6L 270125 & HG \\
\hline 29 & JL97WB & 0.10 & 04 -JAN-2007 & $10: 46: 39$ & 7002028 & $\mathrm{H} 7 \mathrm{~A} 020000$ & HG \\
\hline 30 & JL97WC & 0.10 & $04-J A N-2007$ & $10: 48: 41$ & 7002028 & $\mathrm{H} 7 \mathrm{AO} 20000$ & HG \\
\hline 31 & JK1WE & 1.32 & $04-J A N-2007$ & $10: 51: 03$ & 7002028 & H6L070246 & HG \\
\hline 32 & JKIWES & 1.32 & 04 -JAN-2007 & $10: 53: 00$ & 7002028 & H6L070246 & HG \\
\hline 33 & JKIWED & 1.32 & $04-J A N-2007$ & $10: 54: 57$ & 7002028 & H6L070246 & $\mathrm{HG}$ \\
\hline 34 & $\mathrm{CK} 3 \mathrm{CCV}$ & 1.00 & $04-J A N-2007$ & $10: 56: 46$ & . & & HG \\
\hline 35 & $\mathrm{CK} \perp \mathrm{CCB}$ & 1.00 & $04-J A N-2007$ & $10: 58: 37$ & & & HG \\
\hline 36 & JKIWG & 0.78 & $04-J A N-2007$ & $11: 00: 24$ & 7002028 & H6L070246 & HG \\
\hline 37 & JK1WGS & 0.78 & $04-J A N-2007$ & $11: 02: 44$ & 7002028 & H6L070246 & HG \\
\hline 38 & JK1WGD & 0.78 & $04-J A N-2007$ & $11: 04: 36$ & 7002028 & H6L070246 & HG \\
\hline 39 & JK1XE & 1.47 & $04-J A N-2007$ & $11: 06: 48$ & 7002028 & H6L070246 & HG \\
\hline 40 & JK1XT & 0.92 & $04-J A N-2007$ & $11: 08: 41$ & 7002028 & H6L070246 & HG \\
\hline 41 & JK1X8 & 1.25 & $04-J A N-2007$ & $11: 11: 18$ & 7002028 & H6L070246 & HG \\
\hline 42 & JK10E & 0.89 & $04-J A N-2007$ & $11: 13: 26$ & 7002028 & H6L 070246 & HG \\
\hline 43 & JK106 & 1.11 & $04-J A N-2007$ & $11: 15: 34$ & 7002028 & H6L 070246 & HG \\
\hline 44 & JK11A & 0.89 & 04 -JAN-2007 & $11: 17: 34$ & 7002028 & H6L070246 & HG \\
\hline
\end{tabular}


Instrument Upload

Run Log - Page 2 :

Started Thu Jan 4 13:14:22 2007 by WILBURND

Data File: UPL\$KNX_DATA_ROOT: $<$ LHG $>$ M010407.PRN; 1

Run Log - Page 2 :

-

\begin{tabular}{|c|c|c|c|c|c|c|c|}
\hline \# & WorkOrder & Dilution & Date & Time & Batch & Lot & Instrument \\
\hline- & 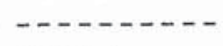 & 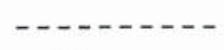 & - & 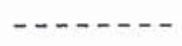 & $-\cdots$ & - - - & $\cdots$ \\
\hline 45 & JK11K & 1.15 & $04-J A N-2007$ & $11: 19: 24$ & 7002028 & H6L 070246 & HG \\
\hline 46 & $\mathrm{CK} 3 \mathrm{CCV}$ & 1.00 & $04-J A N-2007$ & $11: 21: 12$ & & & HG \\
\hline 47 & $\mathrm{CK} \perp \mathrm{CCB}$ & 1.00 & $04-J A N-2007$ & $11: 23: 19$ & & & HG \\
\hline 48 & JK11Q & 0.92 & $04-J A N-2007$ & $11: 25: 18$ & 7002028 & H6L0 70246 & HG \\
\hline 49 & JKI13 & 0.81 & $04-J A N-2007$ & $11: 27: 55$ & 7002028 & H6L 070246 & HG \\
\hline 50 & JK117 & 1.11 & $04-J A N-2007$ & $11: 29: 43$ & 7002028 & H6L 070246 & HG \\
\hline 51 & $\mathrm{JK} 12 \mathrm{~F}$ & 0.10 & $04-J A N-2007$ & $11: 31: 40$ & 7002028 & H6L070246 & HG \\
\hline 52 & JK12H & 0.11 & $04-J A N-2007$ & $11: 33: 30$ & 7002028 & H6L070246 & $\mathrm{HG}$ \\
\hline 53 & $\mathrm{CK} 3 \mathrm{CCV}$ & 1.00 & $04-J A N-2007$ & $11: 35: 19$ & & & HG \\
\hline 54 & $\mathrm{CK} \perp \mathrm{CCB}$ & 1.00 & $04-J A N-2007$ & $11: 37: 17$ & & & HG \\
\hline 55 & JK1XTZ5 & 4.60 & $04-J A N-2007$ & $11: 55: 32$ & 7002028 & H6L070246 & HG \\
\hline 56 & $\mathrm{CK} 3 \mathrm{CCV}$ & 1.00 & $04-J A N-2007$ & $11: 57: 24$ & & & HG \\
\hline 57 & $\mathrm{CK} \perp \mathrm{CCB}$ & 1.00 & $04-J A N-2007$ & $11: 59: 21$ & & & HG \\
\hline
\end{tabular}


STL Knoxville

Method Information

Method Name: 7pt.std/SW-846 7470A/7471A/0.1 RL

Instrument Calibrated Every ? Hours: $\quad 24$

Correlation Coefficient $>/=: \quad 0.995$

CRA Required?: Yes

Duplicate RPD: 20
Element: Hg Chart Number: m010407.prn

Number of Calibration Standards: $\quad 6$

Run ICV Immediately After Calibration?: Yes

Number of Samples Between CCVs: $\quad 10$

Calculate Duplicates by CLP or SW-846?: SW-846 Protocol

$\begin{array}{rr}\text { CRA Recovery Range: } & 30.0 \\ \text { ICV Recovery Range: } & 10.5 \\ \text { CCV Recovery Range: } & 20.5 \\ \text { LCS Recovery Range: } & 10.5 \\ \text { MS/MSD Recovery Range: } & 15.5\end{array}$

Letter to Signify Blanks: B

Letter to Signify LCS's: C

Letter to Signify LCSD's: L

Letter to Signify Dilutions: $\quad Z$

Letter to Signify Duplicates: $\mathrm{X}$

Letter to Signify MS's: $\quad$ S

Letter to Signify MSD's: D

Letters to Signify MSA's: MSA+

Number of Additions: $\quad 3$
CRA Conc. (ug/L): $\quad 0.2$

ICV Conc. (ug/L): $\quad 2.5$

CCV Conc. (ug/L): 5

LCS Spike Level (ug/L): 5

MS Spike Level (ug/L): 1

\section{Calibration Standard Concentrations (ug/L)}

Standard 1 Conc: 0.1

Standard 5 Conc: 5
Standard 2 Conc: 0.2

Standard 6 Conc: 10
Standard 3 Conc: 0.5

Standard 7 Conc: 0
Standard 4 Conc: 1

Standard 8 Conc: 0

ICB/CCB Check Level (ug/L): $0.059 \quad$ Prep Blank Check Level (ug/L): $0.049 \quad$ Reporting Limit (ug/L): 0.1 
STL Knoxville Mercury Data Review Checklist

Method: 7pt.std/SW-846 7470A/7471A/0.1 RL

\begin{tabular}{|c|c|c|c|c|c|c|c|}
\hline Analyst: & \multicolumn{3}{|c|}{ DKW } & Instrument: & \multicolumn{2}{|c|}{ Leeman Hydra AA Mercury Analyzer } \\
\hline Run Date: & Jan-04-07 & Calibration Number: & 1 & Chart Name: & m010407.prn & Element: & Hg \\
\hline
\end{tabular}

\begin{tabular}{|c|c|c|c|c|}
\hline A. Calibration/Instrument Run QC & N/A & Yes & No & Comments \\
\hline 1. Instrument calibrated every 24 hours? & & $\mathrm{x}$ & & Calibration was 2 hours and 25 minutes long \\
\hline $\begin{array}{l}\text { 2. Instrument calibrated using a minimum of a blank and } 5 \\
\text { standards? }\end{array}$ & & $x$ & & \\
\hline $\begin{array}{l}\text { 3. Do standard absorbances increase as standard } \\
\text { concentrations increase? }\end{array}$ & & $x$ & & \\
\hline 4. Instrument calibrated with standards at the SOP levels? & & $x$ & & \\
\hline 5. Correlation coefficient greater than or equal to 0.995 ? & & $x$ & & Correlation coefficient was 0.9999 \\
\hline 6. ICV analyzed directly after calibration? & & $\mathrm{x}$ & & \\
\hline 7. ICV within specified control limits? & & $x$ & & + \\
\hline 8. ICB analyzed immediately after ICV? & & $x$ & & \\
\hline 9. ICB within acceptable range? & & $x$ & & \\
\hline 10. CRA analyzed at the beginning of the run? & & $x$ & & \\
\hline 11. CRA results within acceptable range? & & $x$ & & \\
\hline 12. All CCV's within specified control limits? & & $x$ & & \\
\hline 13. All CCB's within acceptable range? & & $x$ & & \\
\hline 14. All CCB's analyzed immediately after CCV's? & & $x$ & & \\
\hline
\end{tabular}

B. Client Sample and QC Sample Results
1.Were all sample results within the linear range of the instrument?
2. Were there any samples with results more negative than Report Limit?
3.Were samples analyzed within the holding time?

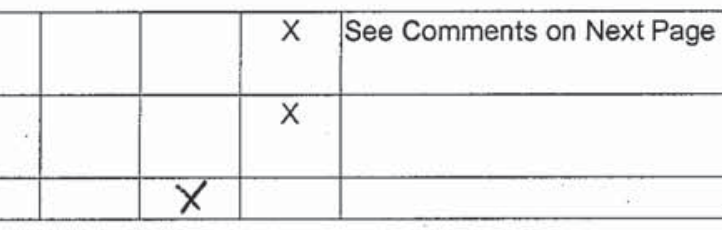

\section{Preparation/Matrix QC}

1.Was one method blank prepared for each batch?

2.Were all method blanks less than the specified level?

3.Was an LCS prepared for each batch?

4.Were all LCS recoveries within QC limits?

5. Were matrix spikes, sample duplicates and/or post-

digestion spikes run at required frequency?

6. Were all matrix spike recoveries within $Q C$ limits?

7.Were post-digestion spike recoveries within $\mathrm{QC}$ limits?

8. Were duplicate RPDs within QC limits?

D. Other

1. Current IDL data on file?

2. Current MDL data on file?

3. Are all nonconformances documented appropriately?

4.Were all project specific instructions followed?

Question

Number: Problem(s) Found:

B1 Sample JK1WGS at 1/4/2007 11:02:44 AM exceeded linear range and an acceptable dilution was NOT found. MSB, NC N

B1 Sample JK1WGD at 1/4/2007 11:04:36 AM exceeded linear range and an acceptable dilution was NOT found. S RIDANALI $2 E$

C6 Matrix spike sample JL57EST at 1/4/2007 10:42:56 AM had 98.6 recovery.

C6 Matrix spike sample JL57EDT at $1 / 4 / 2007$ 10:44:48 AM had $500 \%$ recovery.

C6 Matrix spike sample JK1WGS at 1/4/2007 11:02:44 AM had 132\% recovery. MSB,NC

C6 Matrix spike sample JK1WGD at 1/4/2007 11:04:36 AM had 162\% recovery. $\begin{aligned} & \text { DID NOT } \\ & \text { REANALYZE }\end{aligned}$ 
STL Knoxville Mercury Data Review Checklist
Analyst: DKW
Date: $1 / 4 / 07$

Analyst Comments: 


\section{STL KNOXVILLE}

\section{INTERMEDIATE STANDARD - VERIFICATION}

Stock Std Name: ICP 080

Element: $\mathrm{Hg}$

Stock Source: Ultra

Stock Std Lot \#: F Foo 424

Stock Std Expiration Date: $8 / 31 / 08$

Stock Conc.: $1000 \mathrm{ppm}$

Acid Lot: $\quad$ B 44032
Stock Vol $(\mathrm{ml}) / 100 \mathrm{ml}: 1.0 \mathrm{ml}$

Date Prepared: $1 / 2 / 07$

Expiration Date: $2 / 2 / 07$

QC Check: $140 / 0307$

Initials: KND

Matrix: $2 \% \mathrm{HNO}_{3}$

\section{WORKING STANDARD -- VERIFICATION}

$1 \mathrm{ml}$ Intermediate Standard $/ 100 \mathrm{ml} \mathbf{0 . 1 5} \%$ Concentrated Nitric Acid

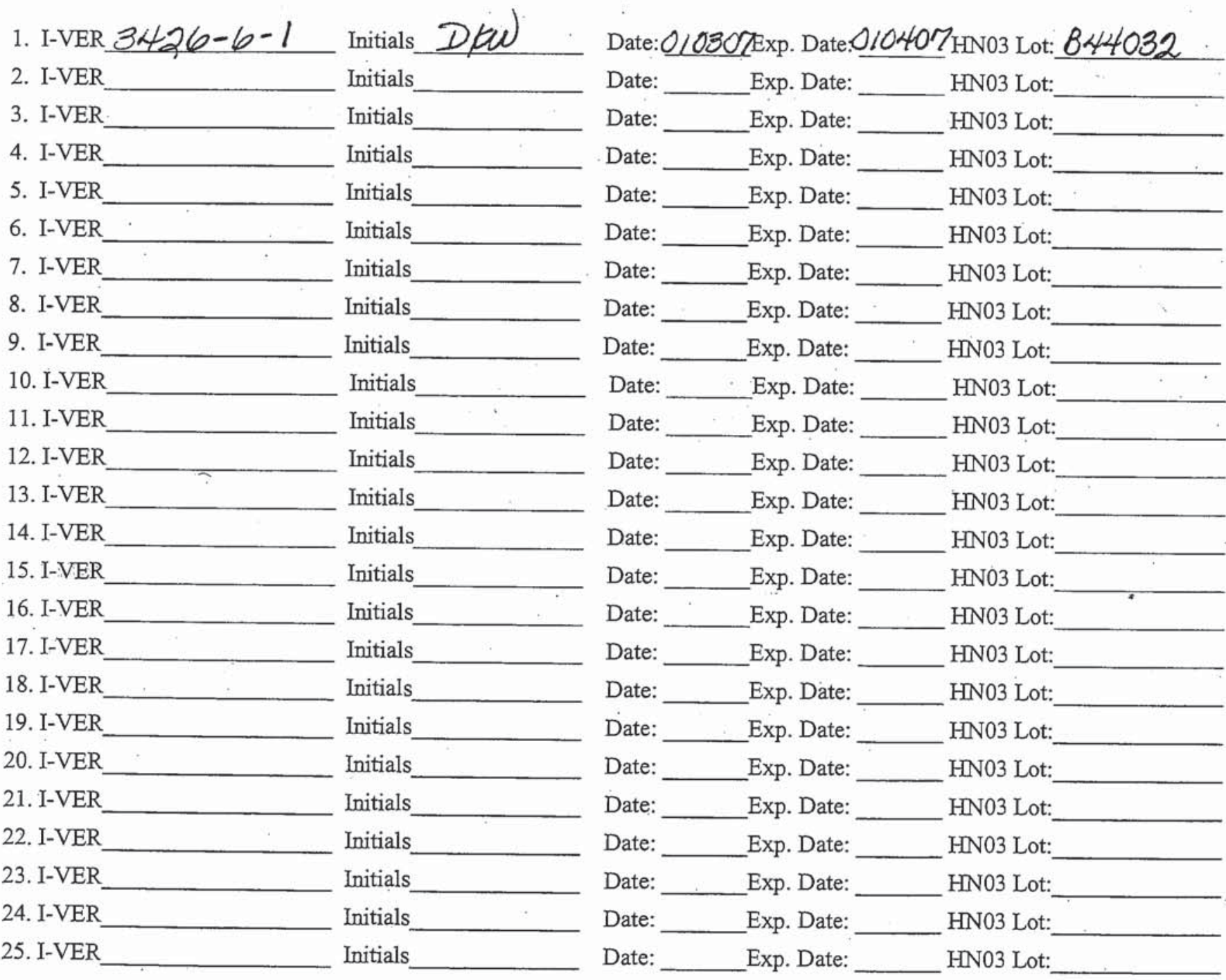




\section{STL KNOXVILLE}

\section{INTERMEDIATE STANDARD --VERIFICATION}

Stock Std Name: ICP 080

Element: $\mathrm{Hg}$

Stock Source: Ultra

Stock Std Lot \#: F $\quad$ oo 424

Stock Std Expiration Date: $8 / 31 / 08$

Stock Conc.: 1000 ppm

Acid Lot: $B 44032$
Stock Vol $(\mathrm{ml}) / 100 \mathrm{ml}: 1.0 \mathrm{ml}$

Date Prepared: $1 / 2 / 07$

Expiration Date: $2 / 2 / 07$

QC Check: 4010307

Initials: $\quad \mathrm{KND}$

Matrix: $2 \% \mathrm{HNO}_{3}$

\section{WORKING STANDARD - VERIFICATION}

$1 \mathrm{ml}$ Intermediate Standard/100 ml $0.15 \%$ Concentrated Nitric Acid

\begin{tabular}{|c|c|}
\hline 1. I-VER $3426-6-1$ & Initials D $D$ W \\
\hline 2. I-VER & Initials \\
\hline 3. I-VER & Initials \\
\hline 4. I-VER & Initials \\
\hline 5. I-VER & Initials \\
\hline 6. I-VER & Initials \\
\hline 7. I-VER & Initials \\
\hline 8. I-VER_ & Initials \\
\hline 9. I-VER_ & Initials_ \\
\hline 10. I-VER & Initials \\
\hline 11. I-VER & Initials \\
\hline 12. I-VER & Initials_ \\
\hline 13. I-VER & Initials \\
\hline 14. I-VER & Initials \\
\hline 15. I-VER & Initials \\
\hline 16. I-VER & Initials \\
\hline 17. I-VER & Initials \\
\hline 18. I-VER & Initials \\
\hline 19. I-VER_ & Initials \\
\hline 20. I-VER & Initials \\
\hline 21. I-VER_ & Initials \\
\hline 22. I-VER_ & Initials \\
\hline 23. I-VER & Initials \\
\hline 24. I-VER & Initials \\
\hline 25. I-VER & Initials \\
\hline
\end{tabular}

Date:010307Exp. Date:010407 HN03 Lot: 844032

Date:__Exp. Date:

Date:__Exp. Date:

HNO3 Lot:

Date:

Exp. Date:

HNO3 Lot:

Date:

Exp. Date:

HNO3 Lot:

Date:

Exp. Date:

HNO3 Lot:

Date:

Exp. Date:

Date:

Exp. Date:

HNO3 Lot:

Date:

Exp. Date:

Date:

Exp. Date:

HNO3 Lot:

Date:

Exp. Date:

HN03 Lot:

Date:

Exp. Date:

HN03 Lot:

Date:

Exp. Date:

HN03 Lot:

Date:

Exp. Date:

HN03 Lot:

Date:

Exp. Date:

Date:

Exp. Date:

HN03 Lot:

Date:

Exp. Date:

Date:

Exp. Date:

HNO3 Lot:

Date:

Exp. Date:

Date:

Exp. Date:

Date:

Exp. Date:

HN03 Lot:

Date:

Exp. Date:

Date:

Exp. Date:

Date:

Exp. Date:

Date:

Exp. Date:

HN03 Lot:

HN03 Lot:

HN03 Lot:

HN03 Lot:

HNO3 Lot:

HN03 Lot:

HNO3 Lot:

HN03 Lot:

HN03 Lot:

HNO3 Lot:

HN03 Lot: 
Protocol: 7ptcurve

\section{We. lin.".}
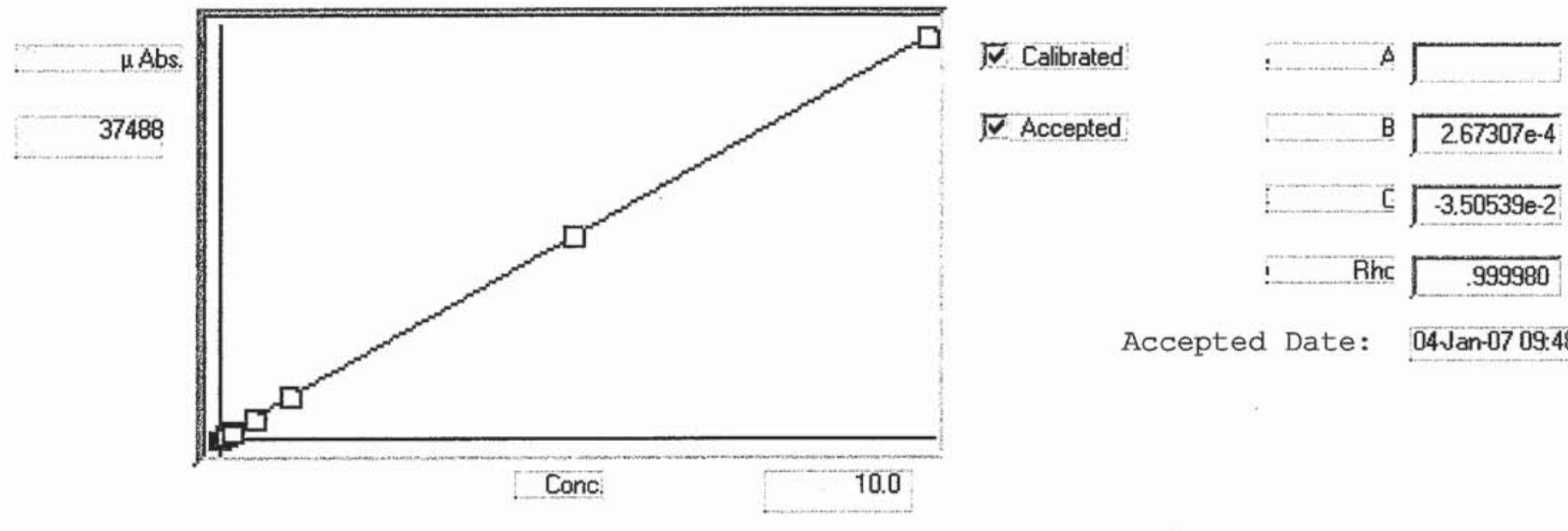

Accepted Date: 04Jan-0709:48

\begin{tabular}{|c|c|c|c|c|c|c|c|c|c|}
\hline S & Conc. & Calc. & Dev. & Mean & SD or $\% R S D$ & $\operatorname{Rep} 1$ & Rep 2 & $\operatorname{Rep} 3$ & Rep 4 \\
\hline 01 & .00000 & -.022 & -.022 & 50 & 0 & 50 & & & \\
\hline 02 & .10000 & .103 & .003 & 517 & $0 \%$ & 517 & & & \\
\hline 03 & .20000 & .166 & -.034 & 753 & $0 \%$ & 752 & & & \\
\hline 04 & .50000 & .513 & .013 & 2053 & $0 \%$ & 2052 & & & \\
\hline 05 & 1.0000 & 1.03 & .032 & 3992 & $0 \%$ & 3992 & & & \\
\hline 06 & 5.0000 & 5.02 & .021 & 18917 & $0 \%$ & 18916 & & & \\
\hline 07 & 10.000 & 9.99 & -.014 & 37489 & $0 \%$ & 37488 & & & \\
\hline 08 & & & & & & & & & \\
\hline 09 & & & & & & & & & \\
\hline 10 & & & & & & & & & \\
\hline
\end{tabular}


STL - Knoxville

$09: 34: 3604$ Jan 2007
Folder: $\quad$ M010307

Protocol: 7ptcurve

Line Conc. Units SD/RSD $\quad 1 \quad 3 \quad 2 \quad 3 \quad 4 \quad 5$

** Standard: 1 Rep: 1

Seq: 0

09:34:36 04 Jan 07 HG

$\mathrm{Hg} \quad .000 \quad \mathrm{ppb} \quad 50$

** Standard: 2 Rep: 1

Seq: $109: 36: 3504$ Jan 07 HG

Hg $\quad .100 \quad \mathrm{ppb} \quad 517$

$\star \star \star$ Standard: 3 Rep: 1

Seq: $209: 38: 4204$ Jan 07 HG

$\begin{array}{llll}\mathrm{Hg} & .200 \quad \mathrm{ppb} & 752\end{array}$

$\star \star \star$ Standard: 4 Rep: 1

Seq: $3 \quad 09: 40: 3904$ Jan $07 \quad$ HG

$\mathrm{Hg} \quad .500 \quad \mathrm{ppb} \quad 2052$

*** Standard: 5 Rep: $1 \quad$ Seq: $4 \quad 09: 42: 5004$ Jan 07 HG

$\mathrm{Hg} \quad 1.00 \quad \mathrm{ppb} \quad 3992$

*** Standard: 6 Rep: $1 \quad$ Seq: $5 \quad 09: 44: 4804$ Jan 07 HG

Hg $\quad 5.00 \quad$ ppb $\quad 18916$

*** Standard: 7 Rep: $1 \quad$ Seq: $6 \quad 09: 47: 1104$ Jan 07 HG

Hg $\quad 10.0 \quad \mathrm{ppb} \quad 37488$

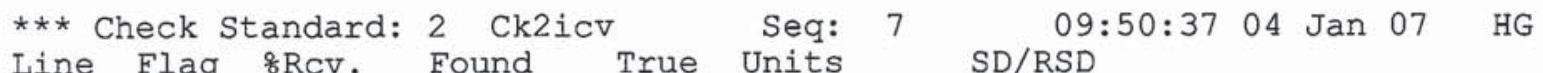
$\begin{array}{lrrrrrr}\text { Line Flag } & \text { \&Rcv. } & \text { Found } & \text { True } & \text { Units } & \text { SD/RSD } \\ \text { Hg } & 97.1 & 2.43 & 2.50 & \mathrm{ppb} & .000 & \%\end{array}$

*** Sample ID: ICB Seq: $8 \quad$ 09:52:22 04 Jan 07 HG $\mathrm{Hg} \quad-.046 \quad \mathrm{ppb} \quad .000 \%-.046$

*** Sample ID: CRA CRA Seq: $9 \quad$ 09:54:18 04 Jan 07 HG

$\begin{array}{lllll}\mathrm{Hg} & .176 \quad \mathrm{ppb} & .000 & \circ & .176\end{array}$

*** Check Standard: $3 \mathrm{Ck3ccv}$ Seq: $10 \quad 09: 56: 1404$ Jan 07 HG

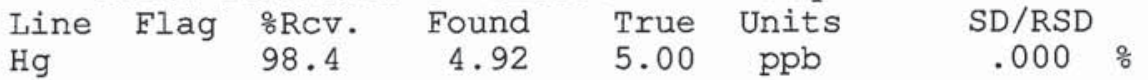

*** Check Standard: 1 Cklccb Seq: 11 09:58:21 04 Jan 07 HG

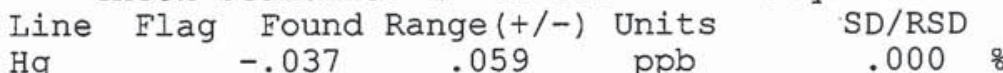

*** Sample ID: MDL1 Seq: $12 \quad$ 10:00:08 04 Jan 07 HG Hg $\quad .086 \quad \mathrm{ppb} \quad .000$ \% $\quad .086$

*** Sample ID: MDL2 Seq: $13 \quad$ 10:01:55 04 Jan 07 HG

$\mathrm{Hg} \quad .062 \quad \mathrm{ppb} \quad .000 \quad \% \quad .062$

*** Sample ID: MDL3 Seq: $14 \quad$ 10:04:02 04 Jan 07 HG

Hg $\quad .106 \quad$ ppb $\quad .000 \quad \% \quad .106$

*** Sample ID: MDL4 Seq: $15 \quad$ 10:05:54 04 Jan 07 HG

$\mathrm{Hg} \quad .063 \quad \mathrm{ppb} \quad .000 \quad .063$ 
STL - Knoxville

10:07:50 04 Jan 2007

Line Conc. Units

*** Sample ID: MDL5

SD/RSD

Page

2148

12

3

Seq: 16

10:07:50 04 Jan 07

5
Hg $\quad .072 \quad \mathrm{ppb}$

$\star \star \star$ Sample ID: MDL 6

$\mathrm{Hg} \quad .079 \quad \mathrm{ppb}$

** Sample ID: MDL7 $\begin{array}{llllll}\mathrm{Hg} & .081 & \mathrm{ppb} & .000 & \% & .081\end{array}$

$\star \star \star$ Check Standard: $3 \mathrm{Ck} 3 \mathrm{ccv}$
Line Flag \%Rcv. Found Tru

$\mathrm{Hg}$

$\star \star \star$
Line Flag Standard: $1 \mathrm{Ck} 1 \mathrm{ccb}$

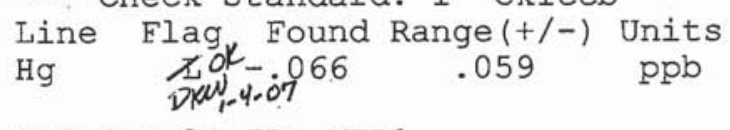

*** Sample ID: MDL1

$\mathrm{Hg} \quad .063 \quad \mathrm{ppb}$

$\star \star \star$ Sample ID: MDL2 KCL $0.1 \mathrm{ppb}$

$.000 \% \quad .072$

Seq: 17 10:10:19 04 Jan 07 HG

$.000 \% \quad .079$

Seq: $18 \quad 10: 12: 0804 \mathrm{Jan} 07$ HG

Hg $\quad .079 \quad \mathrm{ppb}$

** Sample ID: MDL3 $.000 \% \quad .063$

Seq: 19 Units

ppb

(9)
10:14:05 04 Jan 07 HG SD/RSD
Seq: $20 \quad 10: 16: 0504$ Jan 07 HG $.000 \%$

Seq: $21 \quad 10: 17: 5404$ Jan 07 HG $\begin{array}{lllllll}\mathrm{Hg} & .088 & \mathrm{ppb} & .000 & 8 & .088\end{array}$

$\star * \star$ Sample ID: MDL4

Seq: $24 \quad 10: 24: 4204$ Jan 07 HG $\begin{array}{llllll}\mathrm{Hg} & .083 & \mathrm{ppb} & .000 & \% & .083\end{array}$

*** Sample ID: MDL5

Seq: $25 \quad 10: 26: 3004$ Jan 07 HG

$\mathrm{Hg} \quad .088 \quad \mathrm{ppb}$

*** Sample ID: MDL6

$.000 \% \quad .088$

$\begin{array}{llllll}\mathrm{Hg} & .077 & \mathrm{ppb} & .000 & \% & .077\end{array}$

*** Sample ID: MDL7

Seq: $27 \quad 10: 30: 4704$ Jan 07 HG

$\begin{array}{lllll}\mathrm{Hg} & .099 \quad \mathrm{ppb} & .000 \quad \% & .099\end{array}$

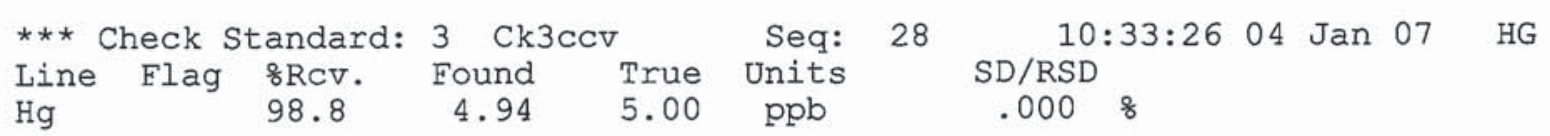

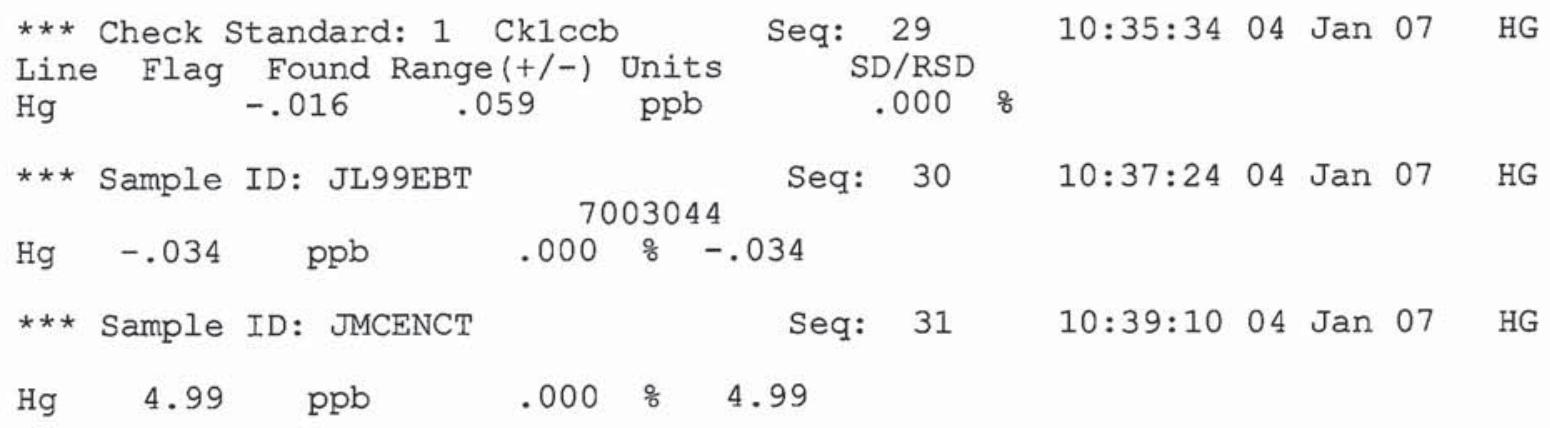


STL - Knoxville

10:41:08 04 Jan 2007

Line Conc. Units

$\mathrm{SD} / \mathrm{RSD}$

Protocol: 7ptcurve

Pace

*** Sample ID: JL57ET

Seq: 32

10:41:08 04 Jan 07 HG

$\begin{array}{lllllllll}\mathrm{Hg} & -.033 & \mathrm{ppb} & .000 & 8 & -.033\end{array}$

*** Sample ID: JL57EST

Seq: $33 \quad 10: 42: 5604$ Jan 07 HG

$\mathrm{Hg}$

$$
\begin{array}{lllll}
4.93 \quad \mathrm{ppb} & .000 \quad 8 & 4.93
\end{array}
$$

*** Sample ID: JL57EDT

Seq: $34 \quad 10: 44: 48$ 04 Jan 07 HG

$\begin{array}{lllllllll}H g & 5.00 & \mathrm{ppb} & .000 & \% & 5.00\end{array}$

*** Sample ID: JL97WB

$\mathrm{Hg} \quad-.060 \quad \mathrm{ppb}$

7002028 Seq: 35

10:46:39 04 Jan 07 HG

*** Sample ID: JL97WC

Seq: $36 \quad 10: 48: 4104$ Jan 07 HG

$\begin{array}{lllllll}H g & 4.83 & \mathrm{ppb} & .000 & \% & 4.83\end{array}$

*** Sample ID: JK1WE

Seq: 37 10:51:03 04 Jan 07 HG

$\begin{array}{lllllll}\mathrm{Hg} & .084 & \mathrm{ppb} & .000 & \% & .084\end{array}$

*** Sample ID: JK1WES

Seq: 38 10:53:00 04 Jan 07 HG

$\begin{array}{llllll}\mathrm{Hg} & 1.07 & \mathrm{ppb} & .000 & \circ & 1.07\end{array}$

*** Sample ID: JK1WED

Seq: $39 \quad 10: 54: 57$ 04 Jan 07 HG

$\begin{array}{llllll}\text { Hg } & 1.12 & \mathrm{ppb} & .000 & \% & 1.12\end{array}$

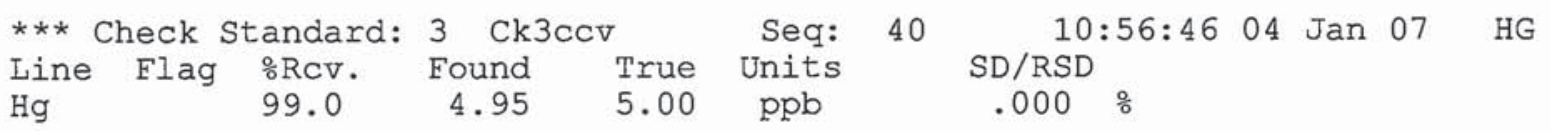

$\begin{array}{llllll}\mathrm{Hg} & 99.0 & 4.95 & 5.00 & \mathrm{ppb} & .000\end{array}$

$\begin{array}{llllll}* * * \text { Check Standard: } 1 \text { Cklccb } & \text { Seq: } 41 & 40: 58: 3704 \text { Jan } 07 & \text { HG } \\ \text { Line Flag Found Range }(+/-) & \text { Units } & \text { SD/RSD } & & \end{array}$ $\begin{array}{llllll}\mathrm{Hg} & -.053 & .059 & \mathrm{ppb} & .000\end{array}$

*** Sample ID: JK1WG Seq: $42 \quad$ 11:00:24 04 Jan 07 HG

$\begin{array}{llllll}\mathrm{Hg} & 8.78 \quad \mathrm{ppb} & .000 & \% & 8.78\end{array}$

*** Sample ID: JK1WGS Seq: 43 1l:02:44 04 Jan 07 HG

$\begin{array}{llllll}H g & 10.1 & \mathrm{ppb} & .000 & \% & 10.1\end{array}$

*** Sample ID: JK1WGD Seq: $44 \quad 11: 04: 3604$ Jan 07 HG

$\begin{array}{llllll}\mathrm{Hg} & 10.4 & \mathrm{ppb} & .000 & \text { o } & 10.4\end{array}$

*** Sample ID: JK1XE Seq: $45 \quad 11: 06: 48$ 04 Jan 07 HG

$\mathrm{Hg} \quad-.014 \quad \mathrm{ppb} \quad .000 \%-.014$

*** Sample ID: JK1XT Seq: $46 \quad$ 11:08:41 04 Jan 07 HG

$\begin{array}{lllllll}\mathrm{Hg} & 12.5 \quad \mathrm{ppb} & .000 & \circ & 12.5\end{array}$

*** Sample ID: JK1X8 Seq: $47 \quad$ 11:11:18 04 Jan 07 HG

$\begin{array}{llllll}\text { Hg } & .064 & \mathrm{ppb} & .000 & \% & .064\end{array}$ 
STL - Knoxville

$11: 13: 2604$ Jan 2007
Folder: M010407

Protocol: 7ptcurve

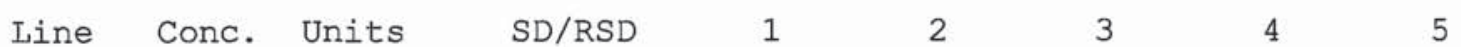

*** Sample ID: JK10E

Seq: 48 11:13:26 04 Jan 07 HG

$\begin{array}{llllll}\text { Hg } & 7.18 & \mathrm{ppb} & .000 & \text { \% } & 7.18\end{array}$

** Sample ID: JK106

Seq: $49 \quad 11: 15: 3404$ Jan 07 HG

$\begin{array}{llllll}\text { Hg } & 1.43 & \mathrm{ppb} & .000 & 8 & 1.43\end{array}$

** Sample ID: JK11A

Seq: $50 \quad 11: 17: 3404$ Jan 07 HG

$\begin{array}{lllll}\mathrm{Hg} & 3.99 & \mathrm{ppb} & .000 \quad \% & 3.99\end{array}$

*** Sample ID: JK11K

Seq: $51 \quad 11: 19: 2404$ Jan 07 HG

Hg $\quad 1.46 \quad \mathrm{ppb} \quad .000 \% 1.46$

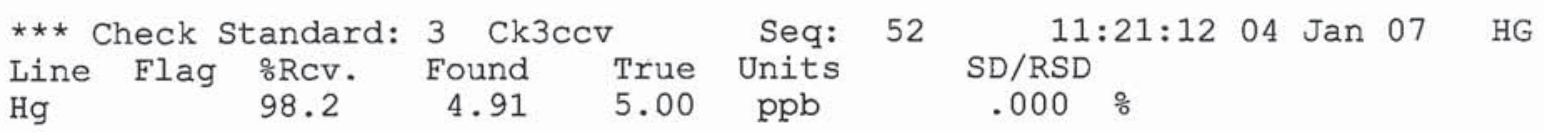

*** Check Standard: 1 Cklccb
Line Flag Found Range $(+/-)$ Units $\begin{array}{llll}\mathrm{Hg} & -.021 & .059 & \mathrm{ppb}\end{array}$

$\star \star *$ Sample ID: JK11Q

Seq: $54 \quad 11: 25: 1804$ Jan $07 \quad$ HG

$\begin{array}{lllll}H g & 5.00 \quad \mathrm{ppb} & .000 \quad \% \quad 5.00\end{array}$

*** Sample ID: JK113 Seq: $55 \quad$ 11:27:55 04 Jan 07 HG

Hg $\quad 4.03 \quad \mathrm{ppb} \quad .000 \quad 8 \quad 4.03$

*** Sample ID: JKl17 Seq: $56 \quad$ 11:29:43 04 Jan 07 HG $\mathrm{Hg} \quad .708 \quad \mathrm{ppb} \quad .000 \% \quad .708$

** Sample ID: JK12F

Seq: $57 \quad 11: 31: 4004$ Jan 07 HG

$\mathrm{Hg} \quad-.034 \quad \mathrm{ppb} \quad .000 \quad \% \quad-.034$

*** Sample ID: JK12H

Seq: $58 \quad 11: 33: 3004$ Jan 07 HG

$\mathrm{Hg} \quad-.015 \quad \mathrm{ppb} \quad .000 \quad \% \quad-.015$

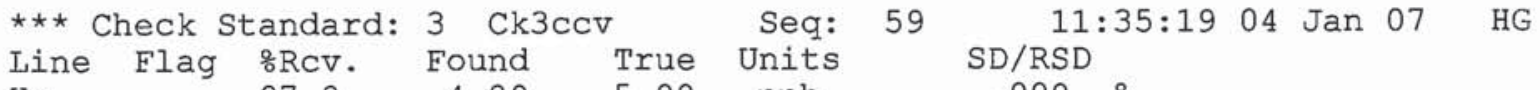
$\begin{array}{llllll}\mathrm{Hg} & 97.8 & 4.89 & 5.00 & \mathrm{ppb} & .000\end{array}$

$\star \star \star$ Check Standard: 1 Cklccb
Line Flag Found Range $(+/-)$ Units $\mathrm{Hg} \quad-.028 \quad .059 \quad \mathrm{ppb}$ Seq: $\quad 60 \quad 11: 37: 1704$ Jan $07 \quad$ HG $\mathrm{SD} / \mathrm{RSD}$ .000 \&

*** Sample ID: JK1XTZ5

Seq: $61 \quad 11: 55: 3204$ Jan 07 HG

$\begin{array}{llllll}H g & 2.62 & \mathrm{ppb} & .000 & \% & 2.62\end{array}$

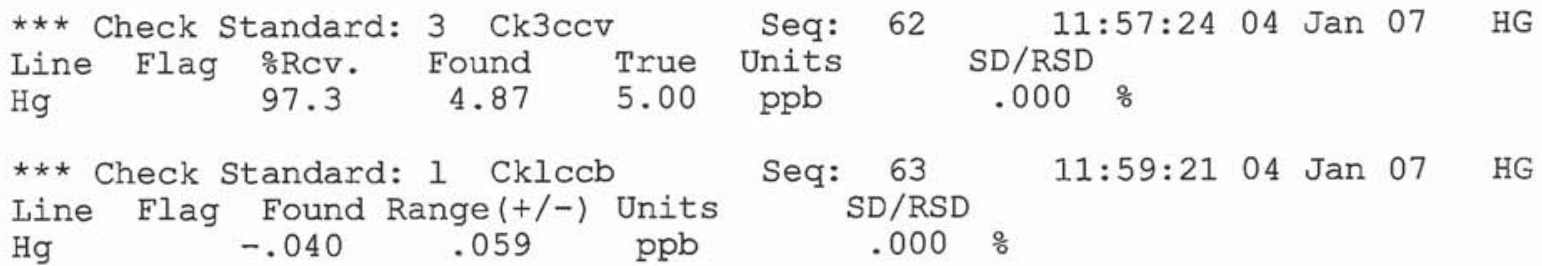




\section{STL Knoxville}

Mercury Analysis Cover Sheet

Scanned

\begin{tabular}{|r|c|c|c|}
\hline Analyst & $D(\omega)$ & Instrument & Leeman Hydra AA Mercury Analyzer \\
\hline Run Date & $1 / 5 / 07$ & Chart Name & M0/0507 \\
\hline
\end{tabular}

\begin{tabular}{||l|l|c|l||}
\hline \multicolumn{2}{|c|}{ Daily Maintenance } & \multicolumn{2}{c|}{ As Needed Maintenance } \\
\hline \multirow{2}{*}{\begin{tabular}{l} 
Clags. \\
\cline { 3 - 4 }
\end{tabular}} & $\begin{array}{l}\text { Check tubing. } \\
\text { flack maintenance schedule }\end{array}$ & $\square$ & Clean or replace optical cell. \\
\hline & & $\square$ & Lubricate pump and autosampler arm. \\
\cline { 3 - 4 } & & $\square$ & Change the drying tube. \\
\hline
\end{tabular}


Instrument Upload

Started Fri Jan 5. 11:40:01 2007 by WILBURND

Run Log - Page 1 :

Data File: UPL\$KNX_DATA_ROOT: $<$ LHG $>$ M010507.PRN; 1

\begin{tabular}{|c|c|c|c|c|c|c|c|}
\hline \# & WorkOrder & Dilution & Date & Time & Batch & Lot & Instrument \\
\hline & $----\cdots-$ & 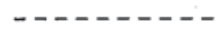 & $-\cdots-1-1-1$ & $-\cdots-\cdots$ & --- & & \\
\hline 1 & CK2ICV & 1.00 & $05-J A N-2007$ & $10: 05: 28$ & & & HG \\
\hline 2 & ICB & 1.00 & $05-J A N-2007$ & $10: 07: 24$ & & & HG \\
\hline 3 & CRA & 1.00 & $05-J A N-2007$ & $10: 09: 20$ & & & HG \\
\hline 4 & $\mathrm{CK} 3 \mathrm{CCV}$ & 1.00 & $05-J A N-2007$ & $10: 11: 09$ & & & HG \\
\hline 5 & $\mathrm{CK} 1 \mathrm{CCB}$ & 1.00 & $05-J A N-2007$ & $10: 14: 08$ & & & HG \\
\hline 6 & MDL1 & 1.00 & $05-J A N-2007$ & $10: 15: 56$ & & . & HG \\
\hline 7 & MDL2 & 1.00 & $05-J A N-2007$ & $10: 18: 14$ & & & HG \\
\hline 8 & MDL3 & 1.00 & $05-J A N-2007$ & $10: 20: 05$ & & & HG \\
\hline 9 & MDL4 & 1.00 & 05 -JAN-2007 & $10: 21: 52$ & & & HG \\
\hline 10 & MDL5 & 1.00 & $05-J A N-2007$ & $10: 23: 50$ & & & HG \\
\hline 11 & MDL6 & 1.00 & $05-J A N-2007$ & $10: 25: 46$ & & & HG \\
\hline 12 & MDL7 & 1.00 & $05-J A N-2007$ & $10: 27: 54$ & & & HG \\
\hline 13 & $\mathrm{CK} 3 \mathrm{CCV}$ & 1.00 & $05-J A N-2007$ & $10: 30: 21$ & & & HG \\
\hline 14 & $\mathrm{CK} \perp \mathrm{CCB}$ & 1.00 & $05-J A N-2007$ & $10: 32: 18$ & & & HG \\
\hline 15 & MDL1 & 1.00 & $05-J A N-2007$ & $10: 34: 09$ & & & HG \\
\hline 16 & MDL2 & 1.00 & $05-J A N-2007$ & $10: 36: 01$ & & & HG \\
\hline 17 & MDL3 & 1.00 & $05-J A N-2007$ & $10: 37: 53$ & & & HG \\
\hline 18 & MDL4 & 1.00 & $05-J A N-2007$ & $10: 40: 01$ & & & HG \\
\hline 19 & MDL5 & 1.00 & $05-J A N-2007$ & $10: 41: 58$ & & & HG \\
\hline 20 & MDL6 & 1.00 & $05-J A N-2007$ & $10: 43: 56$ & & & HG \\
\hline 21 & MDL7 & 1.00 & $05-J A N-2007$ & $10: 46: 04$ & & & HG \\
\hline 22 & $\mathrm{CK} 3 \mathrm{CCV}$ & 1.00 & $05-J A N-2007$ & $10: 48: 21$ & & & HG \\
\hline 23 & CK1CCB & 1.00 & $05-J A N-2007$ & $10: 50: 10$ & & & HG \\
\hline 24 & JL971B & 0.10 & $05-J A N-2007$ & $10: 52: 11$ & 7002029 & $\mathrm{H} 7 \mathrm{~A} 020000$ & HG \\
\hline 25 & JL971C & 0.10 & $05-J A N-2007$ & $10: 54: 39$ & 7002029 & $\mathrm{H} 7 \mathrm{~A} 020000$ & HG \\
\hline 26 & JL971L & 0.10 & $05-J A N-2007$ & $10: 56: 37$ & 7002029 & $\mathrm{H} 7 \mathrm{~A} 020000$ & HG \\
\hline 27 & JLHWK & 0.20 & $05-J A N-2007$ & $10: 58: 24$ & 7002029 & H6L140394 & HG \\
\hline 28 & JLHWKA & 0.20 & $05-J A N-2007$ & $11: 00: 17$ & 7002029 & H6L140394 & HG \\
\hline 29 & JLHXD & 0.20 & 05-JAN-2007 & $11: 02: 10$ & 7002029 & H6L140394 & HG \\
\hline 30 & JLHXF & 0.20 & $05-J A N-2007$ & $11: 03: 58$ & 7002029 & H6L140394 & HG \\
\hline 31 & JLHXH & 0.10 & $05-J A N-2007$ & $11: 05: 48$ & 7002029 & H6L140394 & HG \\
\hline 32 & JLHXK & 0.10 & $05-J A N-2007$ & $11: 07: 43$ & 7002029 & H6L140394 & HG \\
\hline 33 & JLHXM & 0.10 & $05-J A N-2007$ & $11: 09: 45$ & 7002029 & H6L140394 & HG \\
\hline 34 & $\mathrm{CK} 3 \mathrm{CCV}$ & 1.00 & $05-J A N-2007$ & $11: 11: 32$ & & & HG \\
\hline 35 & $\mathrm{CK} 1 \mathrm{CCB}$ & 1.00 & $05-J A N-2007$ & $11: 13: 21$ & & & HG \\
\hline 36 & JL97PB & 0.10 & $05-J A N-2007$ & $11: 15: 29$ & 7002025 & $\mathrm{H} 7 \mathrm{~A} 020000$ & HG \\
\hline 37 & JL97PC & 0.10 & $05-J A N-2007$ & $11: 17: 27$ & 7002025 & $\mathrm{H} 7 \mathrm{~A} 020000$ & HG \\
\hline 38 & JL97PL & 0.10 & $05-J A N-2007$ & $11: 19: 38$ & 7002025 & $\mathrm{H} 7 \mathrm{~A} 020000$ & HG \\
\hline 39 & JKIWA & 0.10 & $05-J A N-2007$ & $11: 21: 27$ & 7002025 & H6L070246 & HG \\
\hline 40 & JKIWAA & 0.10 & $05-J A N-2007$ & $11: 23: 14$ & 7002025 & H6L070246 & HG \\
\hline 41 & JK1XD & 0.10 & $05-J A N-2007$ & $11: 25: 13$ & 7002025 & H6L070246 & HG \\
\hline 42 & JK1X5 & 0.10 & $05-J A N-2007$ & $11: 27: 13$ & 7002025 & H6L070246 & HG \\
\hline 43 & JK105 & 0.10 & $05-J A N-2007$ & $11: 29: 00$ & 7002025 & H6L070246 & HG \\
\hline 44 & JKI1F & 0.50 & $05-J A N-2007$ & $11: 31: 17$ & 7002025 & H6L070246 & HG \\
\hline
\end{tabular}




$\begin{array}{ll}\text { Instrument Upload } & \text { Run Log - Page } 2: \\ : & \text { Started Fri Jan } 5 \text { 11:40:01 } 2007 \text { by WILBURND } \\ : & \text { Data File: UPL\$KNX_DATA_ROOT:<LHG>M010507.PRN; } 1\end{array}$

\begin{tabular}{|c|c|c|c|c|c|c|c|}
\hline$\#$ & WorkOrder & Dilution & Date & Time & Batch & Lot & Instrument \\
\hline--- & ------- & -------- & --------- & $-\cdots-\cdots$ & $-\ldots-\cdots$ &.------- & $-\cdots$ \\
\hline 45 & JK112 & 0.10 & $05-J A N-2007$ & $11: 33: 35$ & 7002025 & H6L070246 & HG \\
\hline 46 & $\mathrm{CK} 3 \mathrm{CCV}$ & 1.00 & $05-J A N-2007$ & $11: 35: 37$ & & & HG \\
\hline 47 & CK1CCB & 1.00 & $05-J A N-2007$ & $11: 37: 34$ & & & HG \\
\hline 48 & JK12A & 0.10 & $05-J A N-2007$ & $11: 39: 43$ & 7002025 & H6L070246 & HG \\
\hline 49 & JL97RB & 0.25 & $05-J A N-2007$ & $11: 41: 55$ & 7002026 & $\mathrm{H} 7 \mathrm{~A} 020000$ & HG \\
\hline 50 & JL97RC & 0.25 & $05-J A N-2007$ & $11: 44: 14$ & 7002026 & $\mathrm{H} 7 \mathrm{~A} 020000$ & HG \\
\hline 51 & JK1WF & 0.60 & $05-J A N-2007$ & $11: 46: 04$ & 7002026 & H6L070246 & HG \\
\hline 52 & JK1WFS & 0.60 & $05-J A N-2007$ & $11: 47: 54$ & 7002026 & H6L070246 & HG \\
\hline 53 & JKIWFD & 0.60 & $05-J A N-2007$ & $11: 49: 46$ & 7002026 & H6L070246 & HG \\
\hline 54 & JKIXR & 0.75 & $05-J A N-2007$ & $11: 51: 57$ & 7002026 & H6L070246 & HG \\
\hline 55 & JK10A & 0.63 & $05-J A N-2007$ & $11: 53: 48$ & 7002026 & H6L070246 & HG \\
\hline 56 & JK107 & 0.65 & $05-J A N-2007$ & $11: 55: 35$ & 7002026 & H6L070246 & HG \\
\hline 57 & JK11L & 0.63 & $05-J A N-2007$ & $11: 57: 33$ & 7002026 & H6L070246 & HG \\
\hline 58 & $\mathrm{CK} 3 \mathrm{CCV}$ & 1.00 & $05-J A N-2007$ & $11: 59: 24$ & & & HG \\
\hline 59 & $\mathrm{CK} 1 \mathrm{CCB}$ & 1.00 & $05-J A N-2007$ & $12: 01: 12$ & & & HG \\
\hline 60 & JK114 & 0.68 & $05-\mathrm{JAN}-2007$ & $12: 03: 28$ & 7002026 & H6L070246 & HG \\
\hline 61 & JK12G & 0.24 & $05-J A N-2007$ & $12: 06: 15$ & 7002026 & H6L070246 & HG \\
\hline 62 & JK11FZ5 & 0.50 & $05-J A N-2007$ & $12: 08: 04$ & 7002025 & H6L070246 & HG \\
\hline 63 & $\mathrm{CK} 3 \mathrm{CCV}$ & 1.00 & $05-J A N-2007$ & $12: 10: 04$ & & & HG . \\
\hline 64 & $\mathrm{CK} \perp \mathrm{CCB}$ & 1.00 & $05-J A N-2007$ & $12: 12: 32$ & & & HG \\
\hline
\end{tabular}




\section{STL Knoxville}

\section{Method Information}

Method Name: Ontario Hydro

Instrument Calibrated Every ? Hours: 24

Correlation Coefficient $>/=\mathbf{0} \quad 0.995$

CRA Required?: Yes

Duplicate RPD: 20
Element: $\mathrm{Hg}$

Chart Number: m010507.prn

Number of Calibration Standards: 6

Run ICV Immediately After Calibration?: Yes

Number of Samples Between CCVs: 10

Calculate Duplicates by CLP or SW-846?: SW-846 Protocol

\begin{abstract}
CRA Recovery Range: $\quad 30.0$
ICV Recovery Range: ' 10.5

CCV Recovery Range: $\quad 20.5$

LCS Recovery Range: $\quad 20.5$

MS/MSD Recovery Range: $\quad 20.5$
\end{abstract}

CRA Conc. (ug/L): $\quad 0.2$

ICV Conc. (ug/L): $\quad 2.5$

CCV Conc. (ug/L): 5

LCS Spike Level (ug/L): 5

MS Spike Level (ug/L): $\quad 1$

$\begin{array}{rcrcr}\text { Letter to Signify Blanks: } & \text { B } & \text { Position to Signify Blanks: } & 6 \\ \text { Letter to Signify LCS's: } & \text { C } & \text { Position to Signify LCS's: } & 6 \\ \text { Letter to Signify LCSD's: } & \text { L } & \text { Position to Signify LCSD's: } & 6 \\ \text { Letter to Signify Dilutions: } & \text { Z } & \text { Position to Signify Dilutions: } & 6 \\ \text { Letter to Signify Duplicates: } & \text { X } & \text { Position to Signify Duplicates: } & 6 \\ \text { Letter to Signify MS's: } & \text { S } & \text { Position to Signify MS's: } & 6 \\ \text { Letter to Signify MSD's: } & \text { D } & \text { Position to Signify MSD's: } & 6 \\ \text { Letters to Signify MSA's: } & \text { MSA+ } & \text { Position to Signify MSA's: } & 6 \\ \text { Number of Additions: } & 3 & & \end{array}$

Calibration Standard Concentrations (ug/L)

\begin{tabular}{|c|c|c|c|}
\hline Standard 1 Conc: 0.1 & Standard 2 Conc: 0.2 & Standard 3 Conc: 0.5 & Standard 4 Conc: 1 \\
\hline Standard 5 Conc: 5 & Standard 6 Conc: 10 & Standard 7 Conc: 0 & Standard 8 Conc: 0 \\
\hline
\end{tabular}

ICB/CCB Check Level (ug/L): $0.099 \quad$ Prep Blank Check Level (ug/L): $0.099 \quad$ Reporting Limit (ug/L): 0.1

IDL (ug/L): $0.06 \quad$ Date of IDL: $12 / 14 / 2006$ 


\section{STL Knoxville Mercury Data Review Checklist}

Method: Ontario Hydro

\begin{tabular}{|c|c|c|c|c|c|c|c|}
\hline Analyst: & \multicolumn{2}{|c|}{ DKW } & Instrument: & \multicolumn{2}{c|}{ Leeman Hydra AA Mercury Analyzer } \\
\hline Run Date: & Jan-05-07 & Calibration Number: & 1 & Chart Name: & m010507.prn & Element: & $\mathrm{Hg}$ \\
\hline
\end{tabular}

\section{A. Calibration/Instrument Run QC}

1. Instrument calibrated every 24 hours?

2. Instrument calibrated using a minimum of a blank and 5 standards?

3. Do standard absorbances increase as standard concentrations increase?

4. Instrument calibrated with standards at the SOP levels?

5. Correlation coefficient greater than or equal to 0.995 ?

6. ICV analyzed directly after calibration?

7. ICV within specified control limits?

8. ICB analyzed immediately after ICV?

9. ICB within acceptable range?

10. CRA analyzed at the beginning of the run?

11. CRA results within acceptable range?

12. All CCV's within specified control limits?

13. All CCB's within acceptable range?

14. All CCB's analyzed immediately after CCV's?

\section{B. Client Sample and QC Sample Results}

1.Were all sample results within the linear range of the instrument?

2.Were there any samples with results more negative than Report Limit?

3.Were samples analyzed within the holding time?

\begin{tabular}{|c|c|c|c|l|} 
& N/A & Yes & No & Comments \\
\hline 5 & & $X$ & & Calibration was 2 hours and 22 minutes long \\
\hline & & $X$ & & \\
& & $X$ & & \\
\hline$?$ & & $X$ & & \\
\hline & & $X$ & & Correlation coefficient was 0.9996 \\
\hline & & $X$ & & \\
\hline & & $X$ & & \\
\hline & $X$ & & \\
\hline & & $X$ & & \\
\hline & & $X$ & & \\
\hline & $X$ & & \\
\hline & & $X$ & & \\
\hline
\end{tabular}

\section{Preparation/Matrix QC}

1. Was one method blank prepared for each batch?

2.Were all method blanks less than the specified level?

3.Was an LCS prepared for each batch?

4. Were all LCS recoveries within $Q C$ limits?

5. Were matrix spikes, sample duplicates and/or postdigestion spikes run at required frequency?

6. Were all matrix spike recoveries within $\mathrm{QC}$ limits?

7.Were post-digestion spike recoveries within $Q C$ limits?

8.Were duplicate RPDs within QC limits?

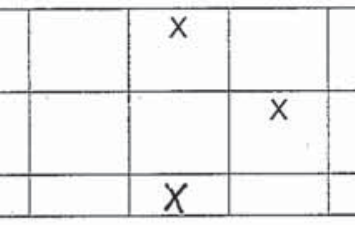

D. Other

1. Current IDL data on file?

2. Current MDL data on file?

3. Are all nonconformances documented appropriately?

4. Were all project specific instructions followed?

Analyst: DXW

Date: $01 / 05 / 07$

\section{Analyst Comments:}




\section{STL KNOXVILLE}

\section{INTERMEDLATE STANDARD - CALIBRATION}

Stock Std Name: PLHG4-2Y

Element: Hg

Stock Source: Spex

Stock Std Lot \#: $12-43 \mathrm{HG}$

Stock Std Expiration Date: $4 / 30 / 07$

Stock Conc.: $1000 \mathrm{ppm}$

$\mathrm{HNO}_{3}$ Lot B44032
Stock Vol (ml)/100ml: $1 \mathrm{ml}$

Date Prepared: $1 / 2 / 07$

Expiration Date: 2 2/2/0

QC Check: M010307

Initials: $K N D$

Matrix: $2 \% \mathrm{HNO}_{3}$

\section{WORKING STANDARD - CALIBRATION}

One ml Intermediate Standard $/ 100 \mathrm{ml} 0.15 \%$ Concentrated Nitric Acid

1. I-CAL $3425-6-1$

2. I-CAL $3425-6-2$

3. I-CAL $3425-6-3$

4. .I-CAL

5. .I-CAL

6. .I-CAL

7. .I-CAL

8. .I-CAL

9. I- $\mathrm{CAL}$

10..I-CAL

11..I-CAL

12..I-CAL

13. I-CAL

14. I-CAL

15. I-CAL

16. I-CAL

17. I-CAL

18. .I-CAL

19. .I-CAL

20. .I-CAL

21. I-CAL

22. I-CAL

23. I-CAL

24. .I-CAL

25. I-CAL

\begin{tabular}{l} 
Initials KND \\
Initials KND \\
Initials DKW \\
Initials \\
Initials \\
Initials \\
Initials \\
Initials \\
Initials \\
Initials \\
Initials \\
Initials \\
Initials \\
Initials \\
Initials \\
Initials \\
Initials \\
Initials \\
Initials \\
Initials \\
Initials \\
Initials \\
Initials \\
Initials \\
Initials \\
\hline
\end{tabular}

Date: $1 / 2 / 07$

Exp. Date: $1 / 3 / 07$ Date: $1 / 3 / 07$ Exp. Date: $1 / 4 / 07$ HN03 Lot: B 34032 Date: 010407 Exp. Date: 010507 HN03 Lot: B44032 Date: Exp. Date: Date:_Exp. Date: HN03 Lot: B4Y032

Date: Exp. Date: HN03 Lot:

Date: Exp. Date: HNO3 Lot:

Date: Exp. Date:

Date: Exp. Date: Date: Exp. Date:

Date: Exp. Date: HN03 Lot: Date: Exp. Date:

Date: Exp. Date: HNO3 Lot: Date: Exp. Date: HNO3 Lot: Date: Exp. Date: HN03 Lot:

Date: Exp. Date: HNO3 Lot: Date: Exp. Date: HNO3 Lot:

Date: Exp. Date:

Date: Exp. Date: HNO3 Lot: HNO3 Lot: Date: Exp. Date: HNO3 Lot: Date: Date: Exp. Date: HN03 Lot: HNO3 Lot: HNO3 Lot: HNO3 Lot: HN03 Lot: HN03 Lot: HNO3 Lot: Date: Exp. Date: HNO3 Lot: Date: Exp. Date: HNO3 Lot: Date: Exp. Date: HNO3 Lot: HNO3 Lot: 


\section{STL KNOXVILLE}

\section{INTERMEDIATE STANDARD - VERIFICATION}

Stock Std Name: ICP 080

Element: $\mathrm{Hg}$

Stock Source: Ultra

Stock Std Lot \#: F $\quad F 0424$

Stock Std Expiration Date: $8 / 31 / 08$

Stock Conc.: $1000 \mathrm{ppm}$

Acid Lot: B44032
Stock Vol (ml) $/ 100 \mathrm{ml}: 1.0 \mathrm{ml}$

Date Prepared: $1 / 2 / 0>$

Expiration Date: $2 / 2 / 0>$

QC Check: $\mathrm{M010307}$

Initials: $\quad K N D$

Matrix: $2 \% \mathrm{HNO}_{3}$

\section{WORKING STANDARD - VERIFICATION}

$1 \mathrm{ml}$ Intermediate Standard $/ 100 \mathrm{ml} \mathbf{0 . 1 5} \%$ Concentrated Nitric Acid

1. I-VER $3426-6-1$

2. I-VER $3424-4-2$

3. I-VER

4. I-VER

5. I-VER

6. I-VER

7. I-VER

8. I-VER

9. I-VER

10. I-VER

11. I-VER

12. I-VER

13. I-VER

14. I-VER

15. I-VER

16. I-VER

17. I-VER

18. I-VER

19. I-VER

20. I-VER

21. I-VER

22. I-VER

23. I-VER

24. I-VER

25. I-VER

\begin{tabular}{l} 
Initials D PW \\
Initials D WW \\
Initials \\
Initials \\
Initials \\
Initials \\
Initials \\
Initials \\
Initials \\
Initials \\
Initials \\
Initials \\
Initials \\
Initials \\
Initials \\
Initials \\
Initials \\
Initials \\
Initials \\
Initials \\
Initials \\
Initials \\
Initials \\
Initials \\
Initials \\
\hline
\end{tabular}

Date:010307Exp. Date.010407HN03 Lot: 844032 Date: 010407Exp. Date:010507HN03 Lot: $B 44032$

Date:__Exp. Date:

Date: __ Exp. Date: HN03 Lot:

Date: ___Exp. Date: HN03 Lot:

Date:___Exp. Date HN03 Lot:

Date: Exp. Date: HNO3 Lot:

Date: Exp. Date: HNO3 Lot:

Date: Exp. Date: HN03 Lot:

Date: Exp. Date: HNO3 Lot:

Date: __ Exp. Date: HN03 Lot: Date: Exp. Date: Date:___ Exp. Date: HN03 Lot: Date: ___ Exp. Date: Date:___Exp. Date: Date: __ Exp. Date: HNO3 Lot: Date: __ Exp. Date: Date:___ Exp. Date: Date: __ Exp. Date: Date: ____ Exp. Date: HNO3 Lot: Date: __ Exp. Date: Date:___Exp. Date: HN03 Lot: Date: ___Exp. Date: Date: ___Exp. Date: HNO3 Lot: Date: Exp. Date: HN03 Lot: HN03 Lot: HN03 Lot: HNO3 Lot: HNO3 Lot: HN03 Lot: HN03 Lot: HN03 Lot: HNO3 Lot: HN03 Lot: 
Protocol: 7ptcurve

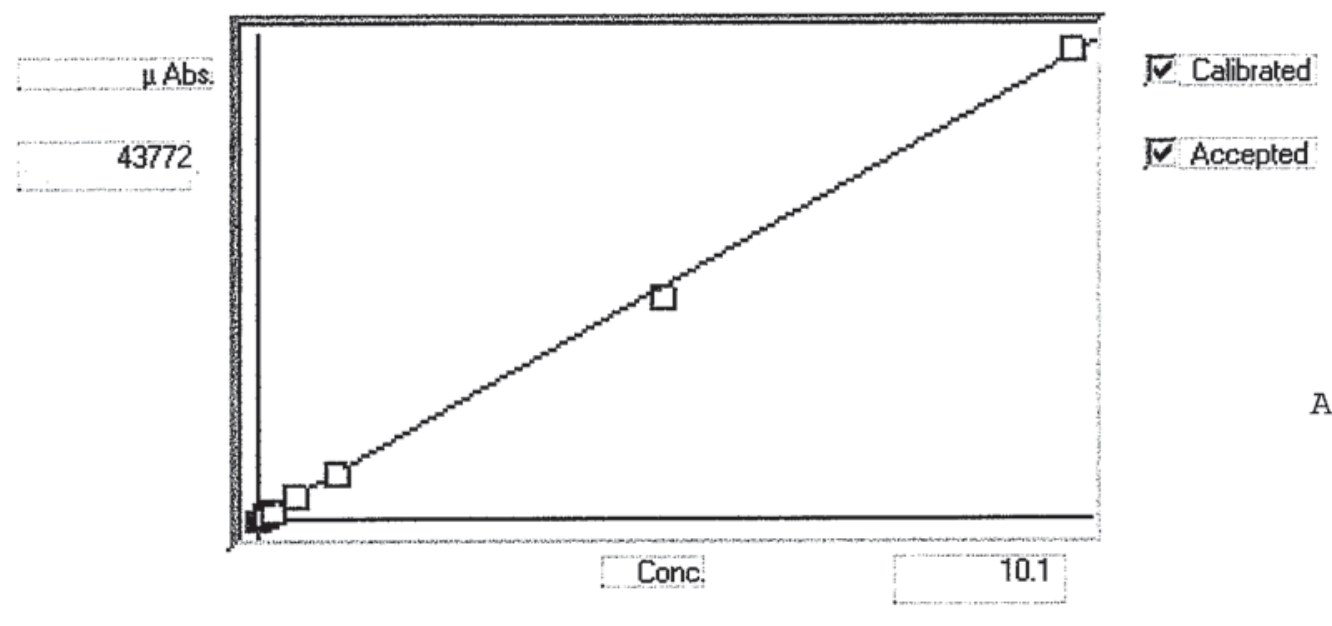

\section{Wt lin."IV}

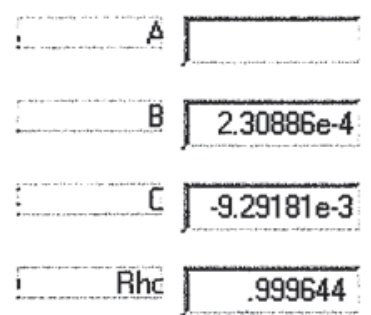

Accepted Date: 05Jan-07 10:02

$\mathrm{s}$
0
0
0
0
0
0
0

\begin{tabular}{|c|c|c|c|c|c|c|c|c|c|}
\hline Conc. & Calc. & Dev. & Mean & SD or \%RSD & Rep 1 & Rep 2 & Rep 3 & $\operatorname{Rep} 4$ & Rep 5 \\
\hline .00000 & .014 & .014 & 103 & 0 & 103 & & & & \\
\hline .10000 & .122 & .022 & 568 & $0 \%$ & 567 & & & & \\
\hline .20000 & .205 & .005 & 926 & $0 \%$ & 926 & & & & \\
\hline .50000 & .545 & .045 & 2400 & $0 \%$ & 2400 & & & & \\
\hline 1.0000 & 1.04 & .036 & 4528 & $0 \%$ & 4528 & & & & \\
\hline 5.0000 & 4.78 & -.219 & 20750 & $0 \%$ & 20749 & & & & \\
\hline 10.000 & 10.1 & .097 & 43772 & $0 \%$ & 43772 & & & & \\
\hline
\end{tabular}


Line Conc. Units

1

2

3

4

5

Standard: 1 Rep: 1

Seq: 0

09:50:53 05 Jan 07

HG

$\begin{array}{llll}\mathrm{Hg} & .000 \quad \mathrm{ppb} & 103\end{array}$

*** Standard: 2 Rep: 1

Seq : 1

09:52:41 05 Jan 07 HG

$\begin{array}{llll}\mathrm{Hg} & .100 \quad \mathrm{ppb} & 567\end{array}$

*** Standard: 3 Rep: 1

Seq: 2 09:54:30 05 Jan 07 HG

Hg $\quad .200 \quad$ ppb $\quad 926$

*** Standard: 4 Rep: 1

Seq: 3 09:56:18 05 Jan 07 HG

Hg $\quad .500 \quad \mathrm{ppb} \quad 2400$

*** Standard: 5 Rep: 1

Seq: $4 \quad 09: 58: 17$ 05 Jan 07 HG

$\begin{array}{llll}\text { Hg } & 1.00 \quad \mathrm{ppb} & 4528\end{array}$

*** Standard: 6 Rep: 1

Seq: 5 10:00:05 05 Jan 07 HG

Hg $\quad 5.00 \quad \mathrm{ppb} \quad 20749$

*** Standard: 7 Rep: 1

Seq: $6 \quad 10: 01: 52$ 05 Jan 07 HG

Hg $\quad 10.0 \quad \mathrm{ppb} \quad 43772$

*** Check Standard: 2 Ck2icv Seq: 7 10:05:28 05 Jan 07 HG Line Flag \&Rcv. Found

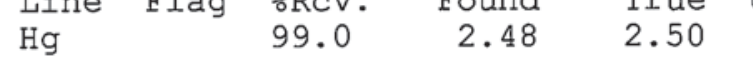
Units $\mathrm{SD} / \mathrm{RSD}$

$\mathrm{ppb} \quad .000 \%$

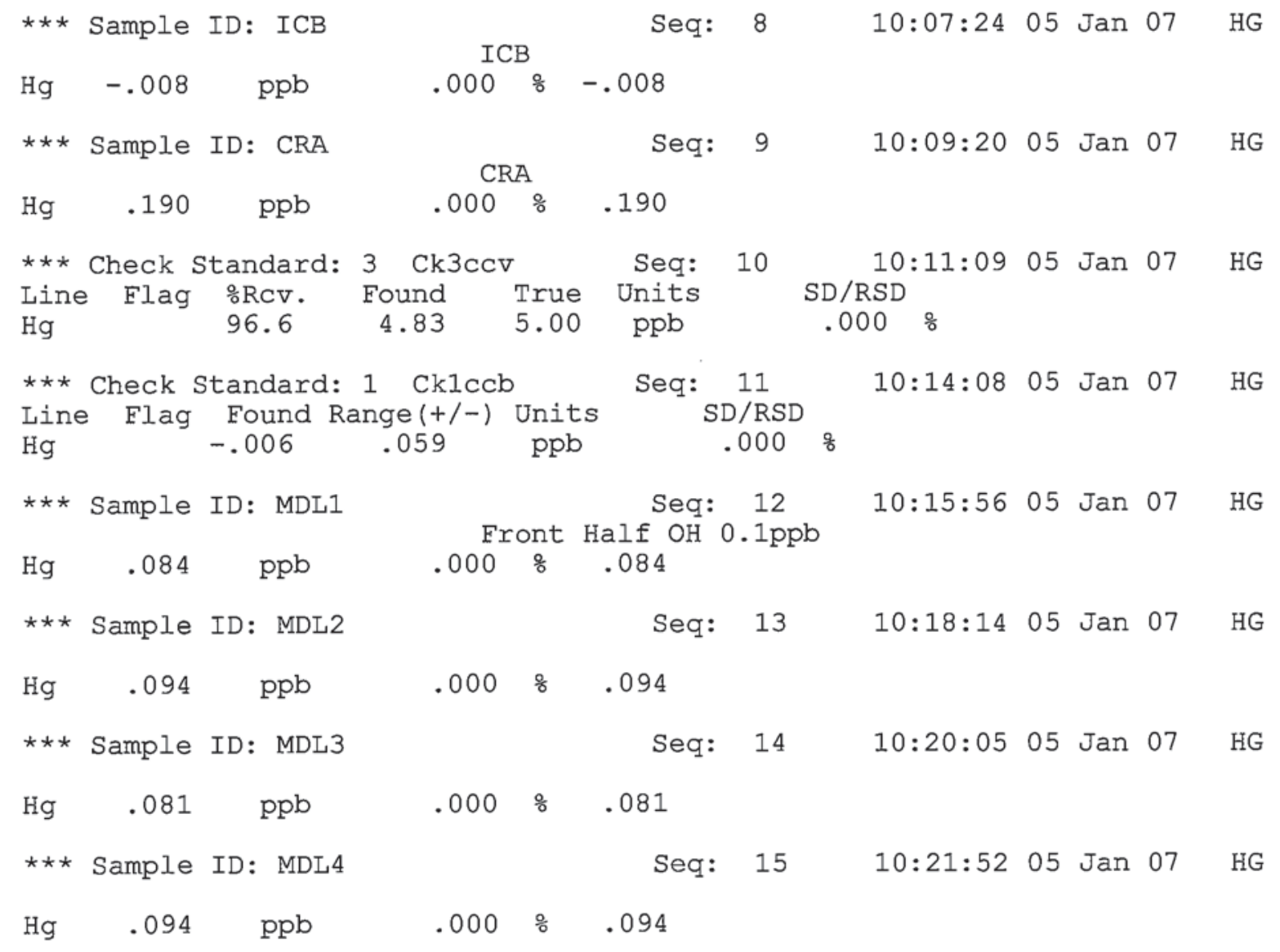


STL - Knoxville

$10: 23: 50 \quad 05$ Jan 2007

Line Conc. Units

SD/RSD

Folder: M010507

Protocol: 7ptcurve

*** Sample ID: MDL5

Seq: 16

$10: 23: 50 \quad 05$ Jan 07 HG

$\begin{array}{llllll}\mathrm{Hg} & .091 & \mathrm{ppb} & .000 & 8 & .091\end{array}$

*** Sample ID: MDL 6

Seq: 17 10:25:46 05 Jan 07 HG

$\mathrm{Hg} \quad .100 \quad \mathrm{ppb} \quad .000 \quad \& \quad .100$

** Sample ID: MDL7

Seq: $18 \quad 10: 27: 5405$ Jan 07 HG

Hg $\quad .129 \quad \mathrm{ppb} \quad .000 \quad .129$

*** Check Standard: $3 \mathrm{Ck3ccv}$ Seq: 19 10:30:21 05 Jan 07 HG $\begin{array}{lrrrcc}\text { Line Flag } & \% \text { RCv. } & \text { Found } & \text { True } & \text { Units } & \text { SD/RSD } \\ \text { Hg } & 96.0 & 4.80 & 5.00 & \text { ppb } & .000 \%\end{array}$

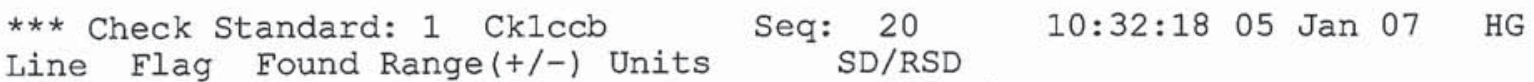

Hg $\quad-.012 \quad .059 \quad \mathrm{ppb} \quad .000 \%$

*** Sample ID: MDLI

Seq: 21 10:34:09 05 Jan 07 HG

$\mathrm{Hg} \quad .041 \quad \mathrm{ppb}$ Back Half $\mathrm{OH} 0.1 \mathrm{ppb}$

*** Sample ID: MDL2

Seq : 22

10:36:01 05 Jan 07 HG

$\begin{array}{llllll}\mathrm{Hg} & .062 & \mathrm{ppb} & .000 & \% & .062\end{array}$

*** Sample ID: MDL3

Seq: 23 10:37:53 05 Jan 07 HG

$\begin{array}{llllll}\mathrm{Hg} & .044 & \mathrm{ppb} & .000 & \% & .044\end{array}$

*** Sample ID: MDL4

Seq: 24 10:40:01 05 Jan 07 HG

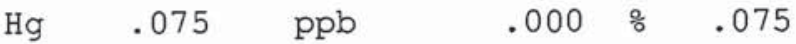

$\star \star \star$ Sample ID: MDL5

Seq: 25 10:41:58 05 Jan 07 HG

$\begin{array}{lllll}\mathrm{Hg} & .048 \quad \mathrm{ppb} & .000 & \% & .048\end{array}$

$\star \star *$ Sample ID: MDL6

Seq: 26 10:43:56 05 Jan 07 HG

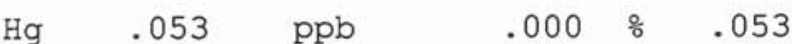

*** Sample ID: MDL7

Seq: 27 10:46:04 05 Jan 07 HG

$\begin{array}{llllll}\mathrm{Hg} & .080 & \mathrm{ppb} & .000 & 8 & .080\end{array}$

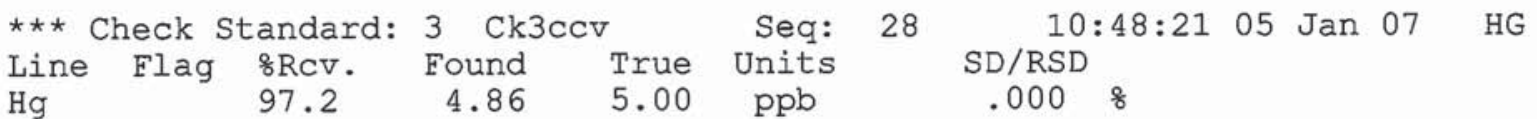

*** Check Standard: $1 \mathrm{Cklccb}$

Line Flag Found Range(+/-) Units

Hg $\quad .011 \quad .059 \quad \mathrm{ppb}$

Seq: $29 \quad 10: 50: 1005$ Jan 07 HG $\mathrm{SD} / \mathrm{RSD}$ $.000 \%$

*** Sample ID: JL971B

Seq: $30 \quad 10: 52: 1105$ Jan 07 HG

Hg $-.017 \mathrm{ppb} \quad .000 \%$ \%

** Sample ID: JL971C Seq: $31 \quad$ 10:54:39 05 Jan 07 HG

$\begin{array}{lllll}\mathrm{Hg} & 4.91 & \mathrm{ppb} & .000 \quad \% \quad 4.91\end{array}$ 
STL - Knoxville

$10: 56: 3705$ Jan 2007

Line Conc. Units

Sample ID: JL971L

1

Seq: $32 \quad 10: 56: 3705$ Jan 07 HG

$\begin{array}{llllll}H g & 5.15 & \mathrm{ppb} & .000 & \% & 5.15\end{array}$

*** Sample ID: JLHWK Seq: $33 \quad$ 10:58:24 05 Jan 07 HG

$\mathrm{Hg}-.013 \mathrm{ppb} \quad .000 \%-.013$ DIGESTED 10 PPb STD.

*** Sample ID: JLHWKA 9ML SAMple +'ML Seq: 34 11:00:17 05 Jan 07 HG

Hg $\quad 1.04 \quad \mathrm{ppb} \quad .000 \quad \% \quad 1.04$

$\star \star \star$ Sample ID: JLHXD

Seq: $35 \quad 11: 02: 1005 \mathrm{Jan} 07$ HG

$\begin{array}{llllll}H g & .003 & \mathrm{ppb} & .000 & \% & .003\end{array}$

Sample ID: JLHXE

Seq: $36 \quad 11: 03: 5805$ Jan 07 HG

$\begin{array}{llllll}H g & .016 & \mathrm{ppb} & .000 & \circ & .016\end{array}$

*** Sample ID: JLHXH

Seq: $\quad 37$

11:05:48 05 Jan 07 HG

$\begin{array}{llllll}\mathrm{Hg} & .006 & \mathrm{ppb} & .000 \quad \% & .006\end{array}$

*** Sample ID: JLHXK

Seq: 38 11:07:43 05 Jan 07 HG

$\begin{array}{llllll}\mathrm{Hg} & .052 & \mathrm{ppb} & .000 & \% & 052\end{array}$

*** Sample ID: JLHXM

Seq: 39 11:09:45 $05 \mathrm{Jan} 07$ HG

Hg $\quad .003 \quad \mathrm{ppb} \quad .000 \quad \& \quad .003$

*** Check Standard: $3 \mathrm{Ck3ccv}$ Seq: 40 11:11:32 05 Jan 07 HG Line Flag :RCV. Found True Units SD/RSD

$\begin{array}{llllll}\mathrm{Hg} & 96.9 & 4.84 & 5.00 & \mathrm{ppb} & .000\end{array}$

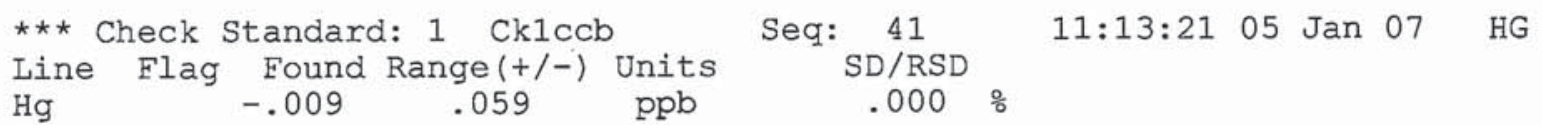

*** Sample ID: JL97PB Seq: $42 \quad$ 11:15:29 05 Jan 07 HG

Hg $\quad .006 \quad \mathrm{ppb} \quad .000 \%$ H6L070246 EH

*** Sample ID: JL97PC Seq: $43 \quad$ 11:17:27 05 Jan 07 HG

$\begin{array}{lllll}H g & 4.87 \quad \mathrm{ppb} & .000 \quad 8 \quad 4.87\end{array}$

*** Sample ID: JL97PL Seq: $44 \quad$ 11:19:38 05 Jan 07 HG

Hg $\quad 4.74 \quad \mathrm{ppb} \quad .000 \quad \% \quad 4.74$

*** Sample ID: JK1WA Seq: $45 \quad$ 11:21:27 05 Jan 07 HG

Hg $.378 \quad \mathrm{ppb} \quad .000 \% .378$ DIESTED 10 Ppb STD

*** Sample ID: JK1WAA 9 mL SAmpLE + 1 ML DIESTED 10 Peq: $46 \quad$ 11:23:14 05 Jan 07 HG

Hg $\quad 1.36 \quad \mathrm{ppb} \quad .000 \quad \% \quad 1.36$

*** Sample ID: JK1XD Seq: $47 \quad$ 11:25:13 05 Jan 07 HG

$\mathrm{Hg} \quad .044 \quad \mathrm{ppb} \quad .000 \quad \% \quad .044$ 
STL - Knoxville

$11: 27: 1305$ Jan 2007

Line Conc. Units

Sample ID: JK1X5

$\mathrm{SD} / \mathrm{RSD}$

Folder: M010507

Protocol: 7ptcurve

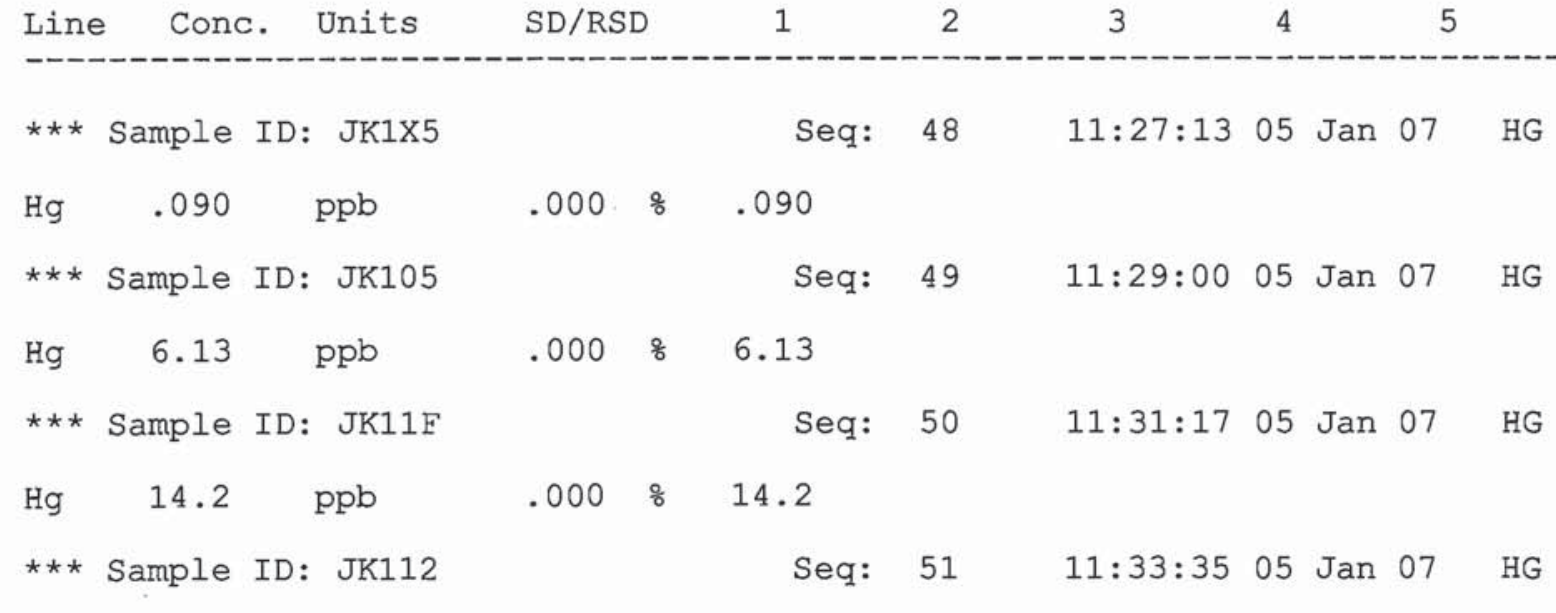

Hg $\quad 6.43 \quad \mathrm{ppb} \quad .000 \quad \% \quad 6.43$

$\begin{array}{llllllll}* \star * \text { Check Standard: } 3 \mathrm{Ck} 3 \mathrm{ccv} & \text { Seq: } 52 & 11: 35: 3705 \text { Jan } 07 \text { HG } \\ \text { Line Flag \%Rcv. Found True Units } & \text { SD/RSD }\end{array}$ $\begin{array}{lrrrcc}\text { Line Flag } & \% \text { Rcv. } & \text { Found } & \text { True } & \text { Units } & \text { SD/RSD } \\ \mathrm{Hg} & 96.6 & 4.83 & 5.00 & \mathrm{ppb} & .000 \%\end{array}$

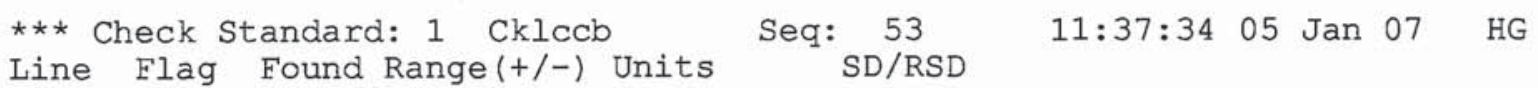

$\begin{array}{lllll}\mathrm{Hg} & -.026 & .059 & \mathrm{ppb} & .000 \%\end{array}$

*** Sample ID: JK12A Seq: $54 \quad$ 11:39:43 05 Jan 07 HG

$\begin{array}{lllll}\mathrm{Hg} & .012 \quad \mathrm{ppb} & .000 \quad \% \quad 012\end{array}$

*** Sample ID: JL97RB Seq: $55 \quad$ 11:41:55 05 Jan 07 HG

$\begin{array}{llll}\mathrm{Hg} & .008 \quad \mathrm{ppb} & .000 \% & .008\end{array}$

*** Sample ID: JL97RC Seq: $56 \quad$ 11:44:14 05 Jan 07 HG

$\mathrm{Hg} \quad 4.00 \quad \mathrm{ppb} \quad .000 \% \quad 4.00$

*** Sample ID: JK1WF Seq: $57 \quad$ 11:46:04 05 Jan 07 HG

Hg $\quad .069 \quad \mathrm{ppb} \quad .000 \% \quad .069$

** Sample ID: JK1WFS Seq: $58 \quad$ 11:47:54 05 Jan 07 HG

$\begin{array}{lllll}\mathrm{Hg} & .949 \quad \mathrm{ppb} & .000 \quad \% & .949\end{array}$

*** Sample ID: JK1WFD Seq: $59 \quad$ 11:49:46 05 Jan 07 HG

$\mathrm{Hg} \quad .976 \quad \mathrm{ppb} \quad .000 \quad \% \quad .976$

*** Sample ID: JK1XR Seq: $60 \quad$ 11:51:57 05 Jan 07 HG

$\begin{array}{lllll}\mathrm{Hg} & .058 \quad \mathrm{ppb} & .000 \quad \% \quad 058\end{array}$

** Sample ID: JK10A Seq: $61 \quad$ 11:53:48 05 Jan 07 HG

$\begin{array}{lllll}\mathrm{Hg} & .102 \quad \mathrm{ppb} & .000 \quad 8 & .102\end{array}$

*** Sample ID: JK107 Seq: $62 \quad$ 11:55:35 05 Jan 07 HG

$\begin{array}{lllll}\mathrm{Hg} & .099 \quad \mathrm{ppb} & .000 & \% & .099\end{array}$

*** Sample ID: JK11L Seq: $63 \quad$ 11:57:33 05 Jan 07 HG

Hg $\quad .160 \quad \mathrm{ppb} \quad .000 \% \quad .160$ 


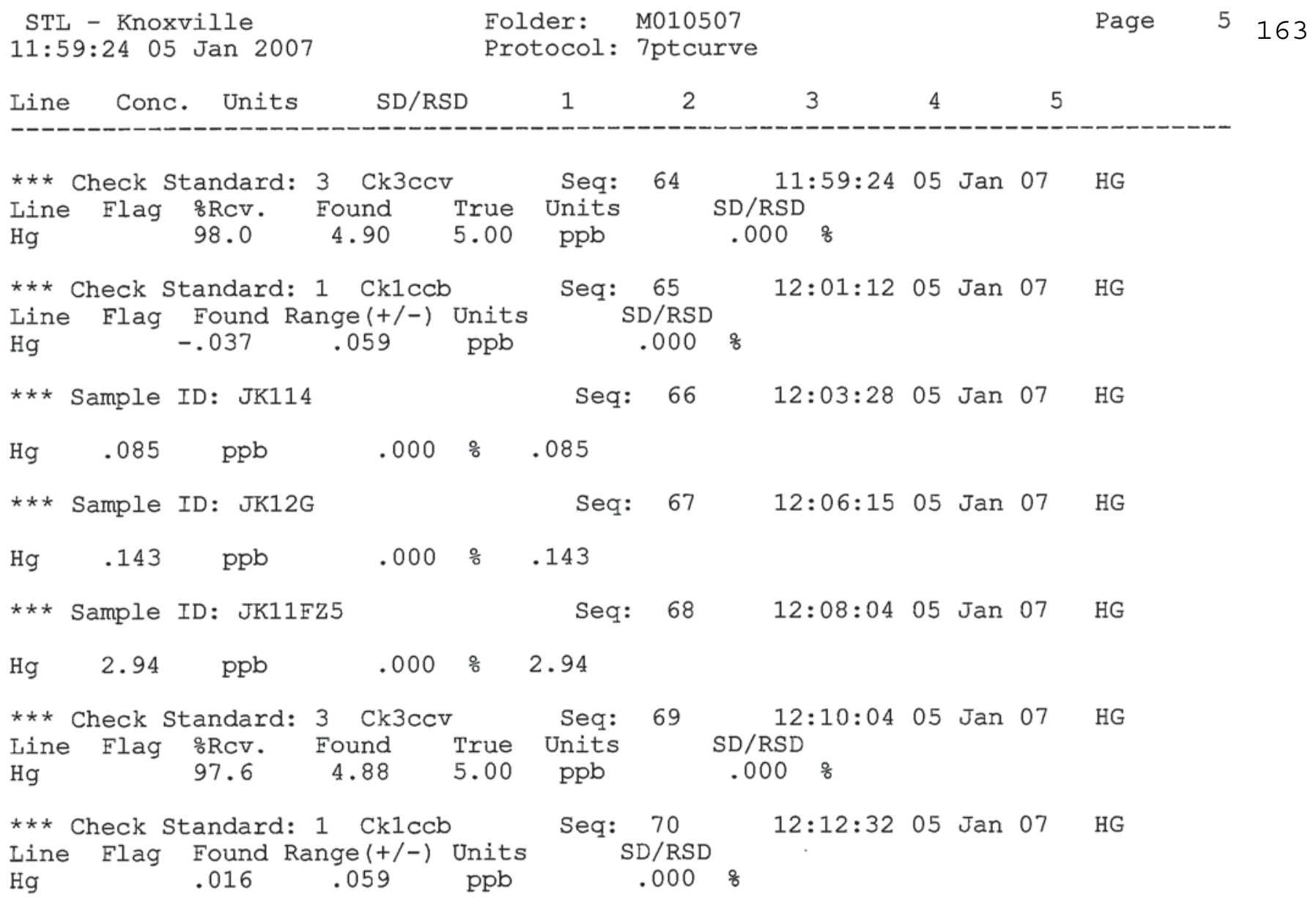

Page

5163

11:59:24 05 Jan 2007

Protocol: 7ptcurve

Line Conc. Units SD/RSD

2

3

5

$* * *$ Check Standard: $3 \mathrm{Ck} 3 \mathrm{ccv}$

Line Flag \%Rcv. Found

$\mathrm{Hg}$

Seq: 64 $4.90 \quad 5.00$

Units ppr

11:59:24 05 Jan 07 HG

$\mathrm{SD} / \mathrm{RSD}$

.000

*** Check Standard: $1 \mathrm{Ck} 1 \mathrm{ccb}$

Line Flag Found Range(+/-) Units $\mathrm{Hg}$

Seq: $65 \quad$ 12:01:12 05 Jan 07 HG $-.037 \quad .059 \quad \mathrm{ppb}$

$\mathrm{SD} / \mathrm{RSD}$

$.000 \%$

*** Sample ID: JK114

Seq: $66 \quad 12: 03: 2805$ Jan 07 HG

$\begin{array}{llllll}\mathrm{Hg} & .085 & \mathrm{ppb} & .000 & \% & .085\end{array}$

*** Sample ID: JK12G

Seq: $67 \quad 12: 06: 1505$ Jan 07 HG

$\begin{array}{llllll}\mathrm{Hg} & .143 & \mathrm{ppb} & .000 & \% & .143\end{array}$

*** Sample ID: JK11FZ5

Seq: 68 12:08:04 05 Jan 07 HG

$\begin{array}{llllll}\mathrm{Hg} & 2.94 & \mathrm{ppb} & .000 & \% & 2.94\end{array}$

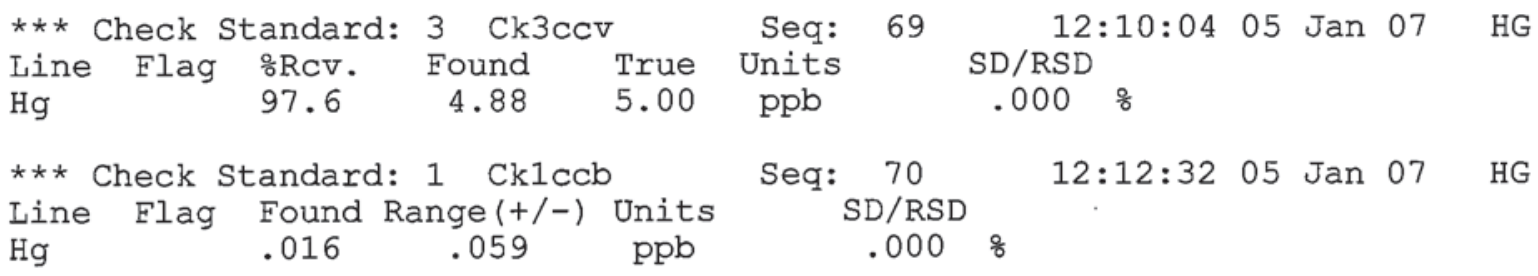


Miscellaneous Data 
जo

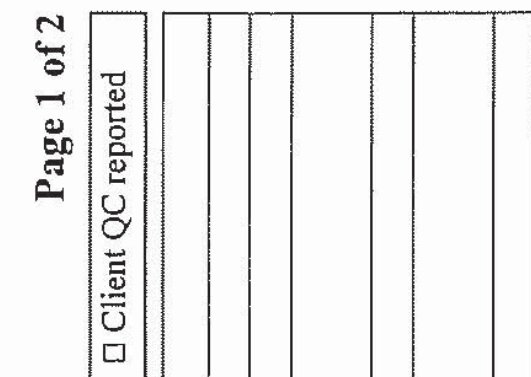

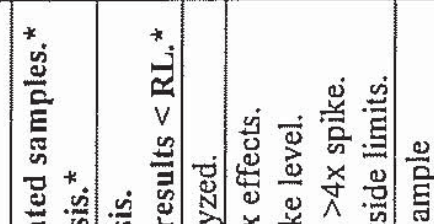

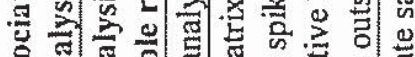

总

ㄷㄴ은

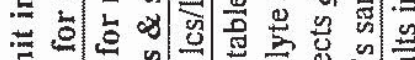

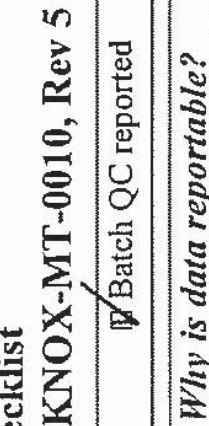

E의

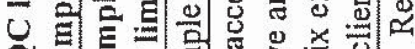

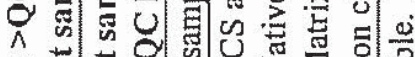

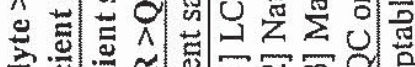

군

들 웝

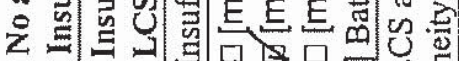

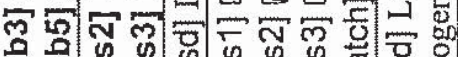

E है

밈ㅁㅁㅁㅁㅁㅁㅁㅇㅛ

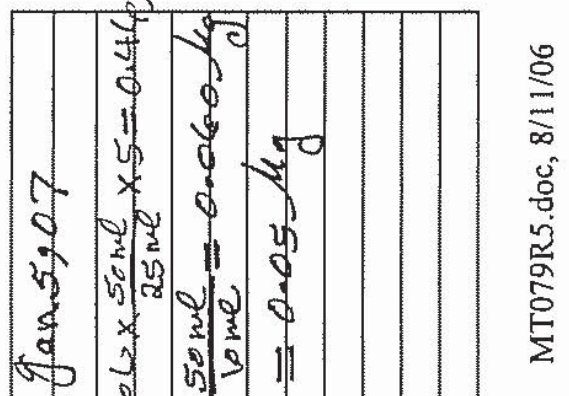

U马

б。

焉

$>>>>>>>$

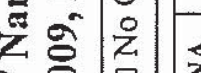

$\varangle$

跑

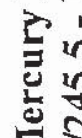

-

$>$

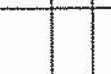

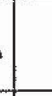$$
>>
$$

$>>>$

$+5$

$\therefore$ के को

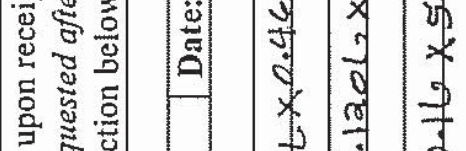

क्ष

资言密

牙

E哥吕

ㅁㅁ맘 


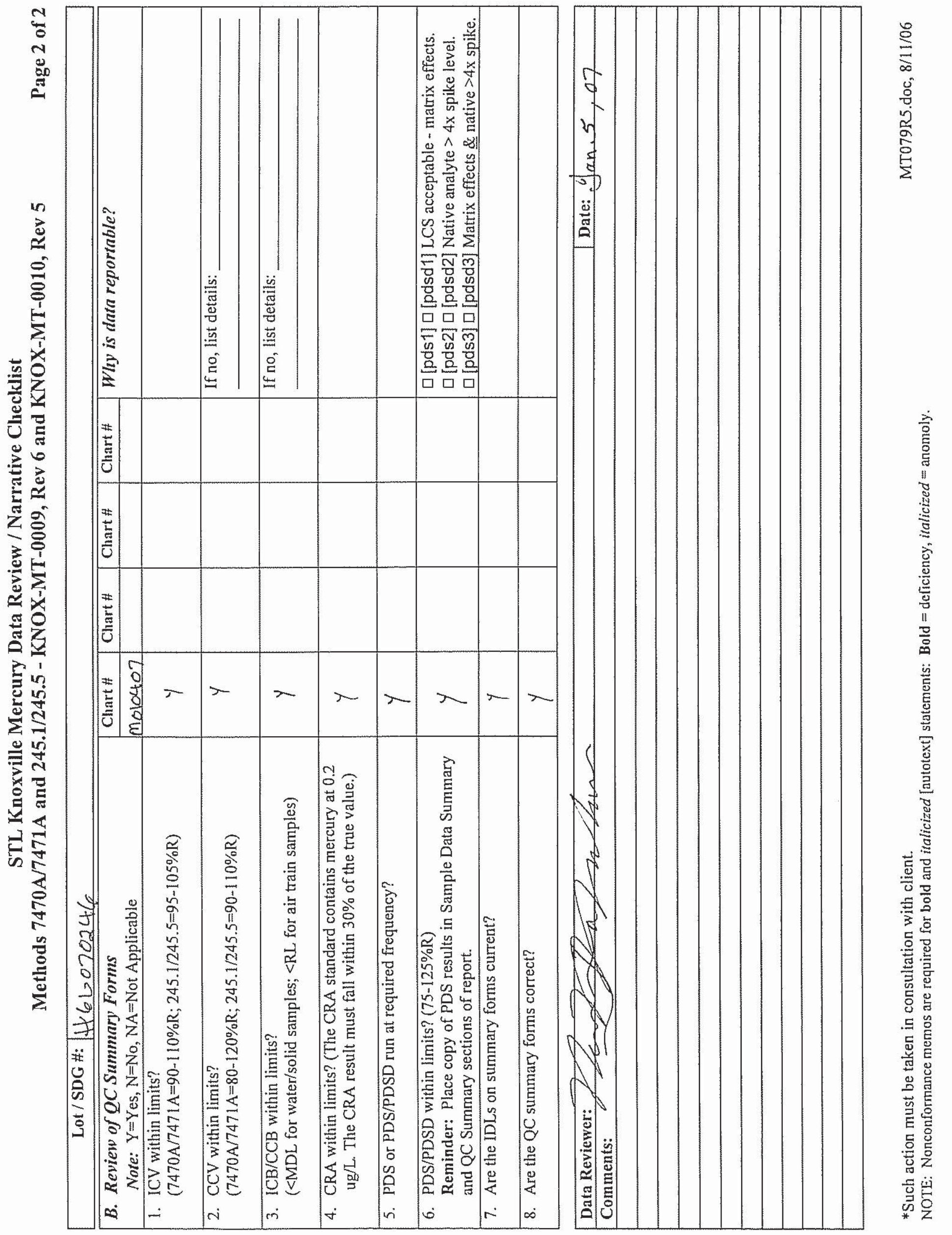




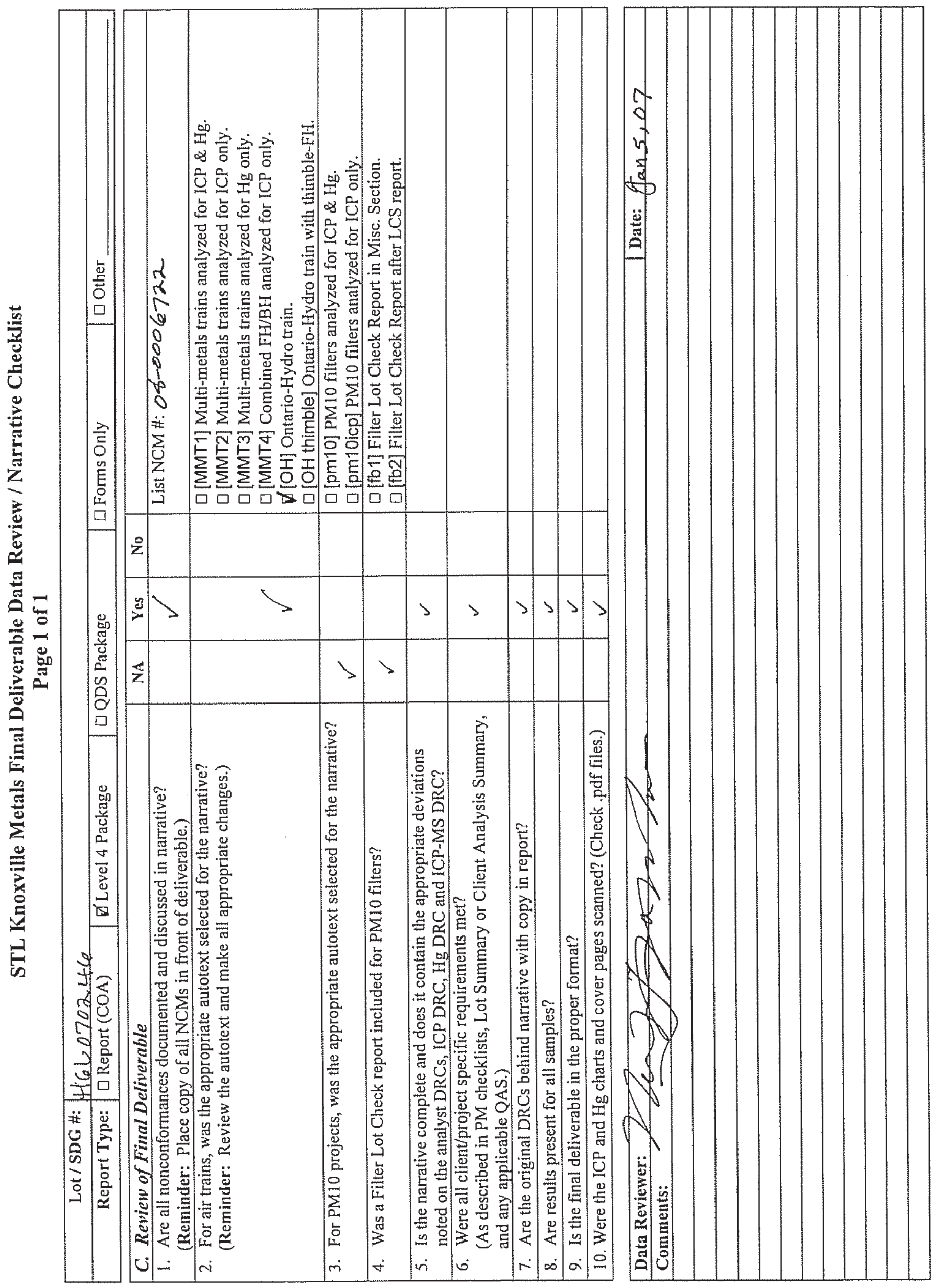


STL Knoxville

Analyst: $K N D / D K N$

$\square$ Hg in PM10 filters: KNOX-IP-0003, Rev. 4

口 MMT train: KNOX-MT-0006, Rev. 9

\section{Mercury Air Prep Spilking Form}

Date: $01 / 04 / 07$
Batch: 7002025

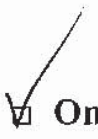

Bath A Temp: 950

Bath B Temp: $940 \mathrm{C}$

(Acceptance range $90^{\circ}-95^{\circ} \mathrm{C}$ )

\begin{tabular}{|c|c|c|c|c|c|c|}
\hline \multirow{2}{*}{ Standard Type } & \multirow{2}{*}{ Standard ID } & \multicolumn{2}{|c|}{ Intermediate Std. } & \multirow{2}{*}{$\begin{array}{l}\text { Volume } \\
\text { Used }(\mathrm{ml})\end{array}$} & \multirow{2}{*}{$\begin{array}{c}\text { Final } \\
\text { Volume } \\
\text { (ml) }\end{array}$} & \multirow{2}{*}{$\begin{array}{c}\text { Final } \\
\text { Concentration } \\
\text { (ugg/L) }\end{array}$} \\
\hline & & Source & Conc.(ug/L) & & & \\
\hline Calibration Std 1 & N/A & & N/A & N/A & 50 & Blank \\
\hline Calibration Std 2 & $1-\mathrm{CAL}-3425-6-3$ & SPEX & 100 & 0.05 & 50 & 0.1 \\
\hline Calibration Std 3 & I-CAL- & & 100 & 0.1 & 50 & 0.2 \\
\hline Calibration Std 4 & I-CAL- & & 100 & 0.25 & 50 & 0.5 \\
\hline Calibration Std 5 & I-CAL- & & 100 & 0.5 & 50 & 1.0 \\
\hline Calibration Std 6 & I-CAL- & & 100 & 2.5 & 50 & 5.0 \\
\hline Calibration Std 7 & I-CAL & - & 100 & 5.0 & 50 & 10.0 \\
\hline
\end{tabular}

\begin{tabular}{|c|c|c|c|c|c|c|}
\hline \multirow{2}{*}{$\begin{array}{c}\text { Standard } \\
\text { Type } \\
\end{array}$} & \multirow{2}{*}{ Standard ID } & \multicolumn{2}{|c|}{ Intermediote Std. } & \multirow{2}{*}{$\begin{array}{c}\text { Amount } \\
\text { Used (ml) }\end{array}$} & \multirow{2}{*}{$\begin{array}{c}\text { Final } \\
\text { Volume } \\
(\mathrm{ml})\end{array}$} & \multirow{2}{*}{$\begin{array}{l}\text { Final Conc. } \\
(\mathrm{ug} / \mathrm{L})\end{array}$} \\
\hline & & Source & Conc.(ug/L) & & & \\
\hline ICV & I-VER-3426-6-2 & ULTRH & 100 & 1.25 & 50 & 2.5 \\
\hline $\mathrm{CCV}$ & 1-CAL $3425-6-3$ & PEX & 100 & 2.5 & 50 & 5.0 \\
\hline CRA & I-CAL- & 1 & 100 & 0.1 & 50 & 0.2 \\
\hline
\end{tabular}

\begin{tabular}{|c|c|c|c|c|c|c|c|}
\hline \multirow{2}{*}{ Standard Type } & \multirow{2}{*}{ Standard ID } & \multicolumn{2}{|c|}{ Intermediate Std. } & \multirow{2}{*}{$\begin{array}{c}\text { Amount Used } \\
@ \text { ICP } \\
\text { prep/final } \\
\text { volume }\end{array}$} & \multirow{2}{*}{$\begin{array}{l}\text { ICP Sample } \\
\text { Volume } \\
\text { used @ Hg } \\
\text { prep (ml) }\end{array}$} & \multirow{2}{*}{$\begin{array}{c}\text { Final } \\
\text { Conc. @ } \\
\text { Hg prep } \\
\text { (ug/L) } \\
\end{array}$} & \multirow{2}{*}{$\begin{array}{c}\text { Final } \\
\text { Amount } \\
\text { (ug) }\end{array}$} \\
\hline & & Source & Conc.(ug/L) & & & & \\
\hline Air (PM10) LCS & I-CAL- & & 10,000 & $0.250 \mathrm{ml} / 50 \mathrm{ml}$ & 5.0 & 5.0 & 11.25 \\
\hline Air (PM10) MS & I-CAL- & & 10,000 & $0.050 \mathrm{ml} / 50 \mathrm{ml}$ & 5.0 & 1.0 & 2.25 \\
\hline
\end{tabular}

\begin{tabular}{|c|c|c|c|}
\hline \multicolumn{4}{|c|}{ REAGENTS } \\
\hline Conc. $\mathrm{H}_{2} \mathrm{SO}_{4}$ Lot $\mathrm{H}$ : & $c 15045$ & $5 \% \mathrm{KMnO}_{4}$ ID \#: & $B-3122-12-C$ \\
\hline Conc. $\mathrm{HNO}_{3}$ Lot \#: & $B 44032$ & $5 \% \mathrm{~K}_{2} \mathrm{~S}_{2} \mathrm{O}_{8}$ ID H: & $D-2638-17-E$ \\
\hline Conc. HCL Lot & C42A23 & $\mathrm{SnCl}_{2}$ Solution ID \#: & $A-3382-20-B$ \\
\hline $12 \% \mathrm{NaCl}^{-N_{2}} \mathrm{OH} \mathrm{HCL}$ ID H: & $c-3267-3-E$ & & \\
\hline
\end{tabular}




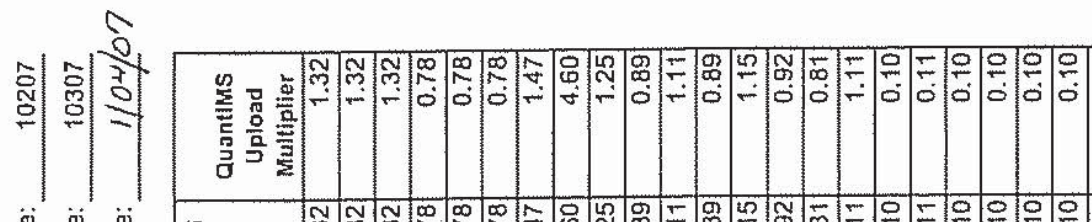

产产兽

翣品

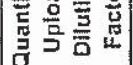

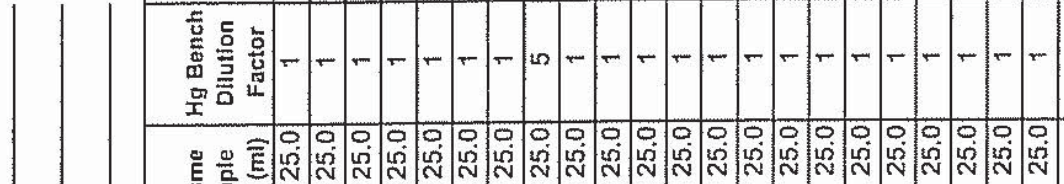

总竞

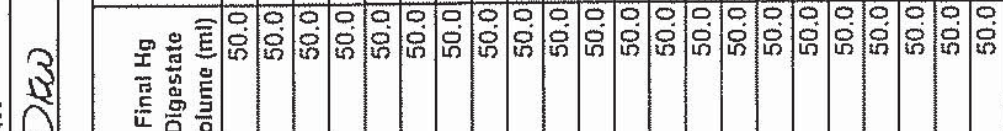

号穷 莖总言

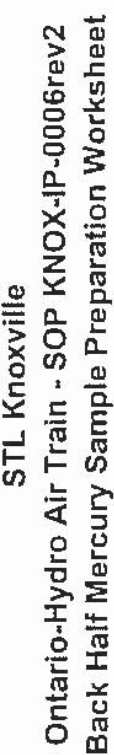

荠齐

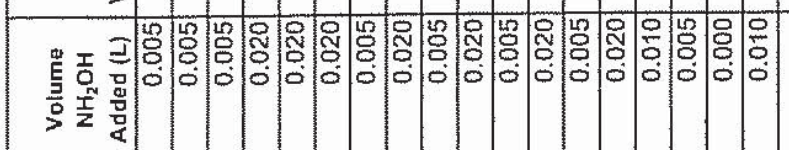

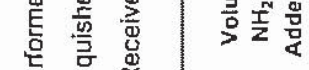

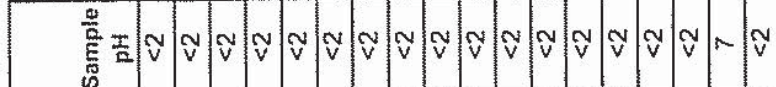

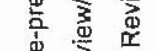

व.

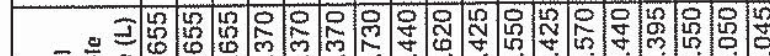

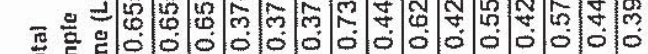

品

(a) 000000000

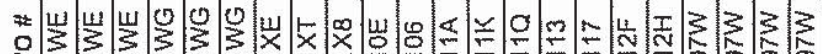

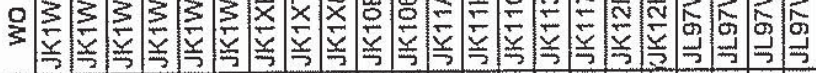

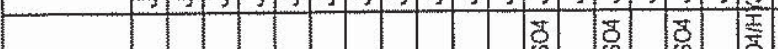

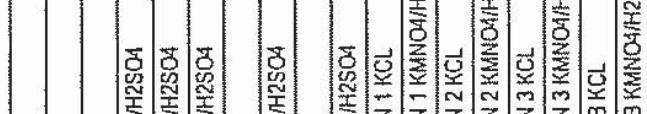

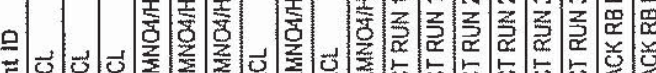

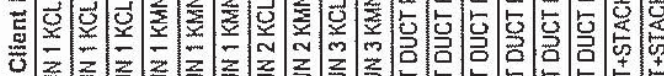

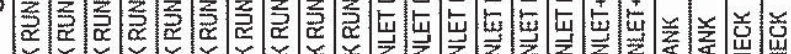

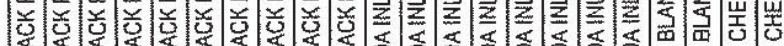

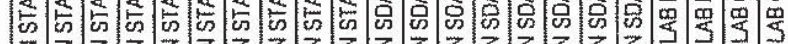

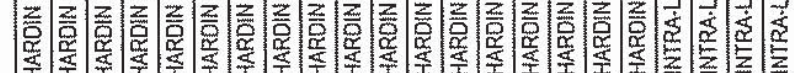

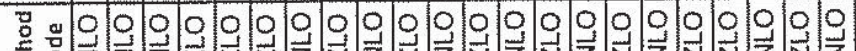

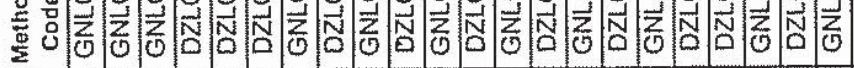

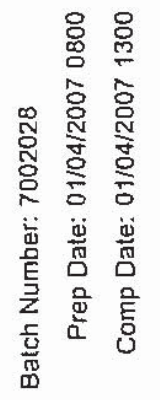

$\infty \infty 0$

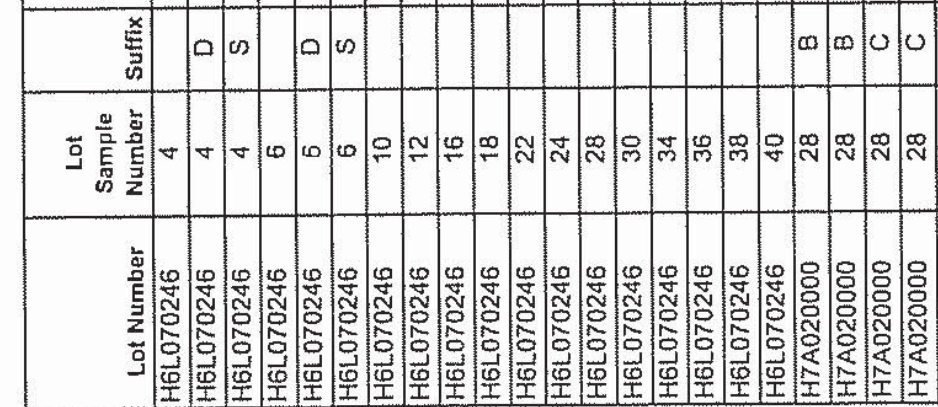

$\vec{E} \vec{E}$

㖞 ํํㅇ

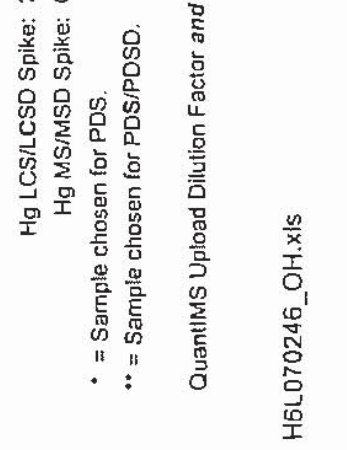


STL Knoxville

Mercury Air Prep Spiking Form

Analyst: KND/DKW Date: $01 / 03 / 07 \quad$ Batch: 2002028

Bath A Temp: $93^{\circ} \mathrm{C}$

Bath B Temp: $940 \mathrm{C}$

(Acceptance range $90^{\circ}-95^{\circ} \mathrm{C}$ )

$\square$ Hg in PM10 filters: KNOX-IP-0003, Rev. 4

$\checkmark$ Ontario Hydro: KNOX-IP-0006, Rev. 2

口 MMT train: KNOX-MT-0006, Rev. 9

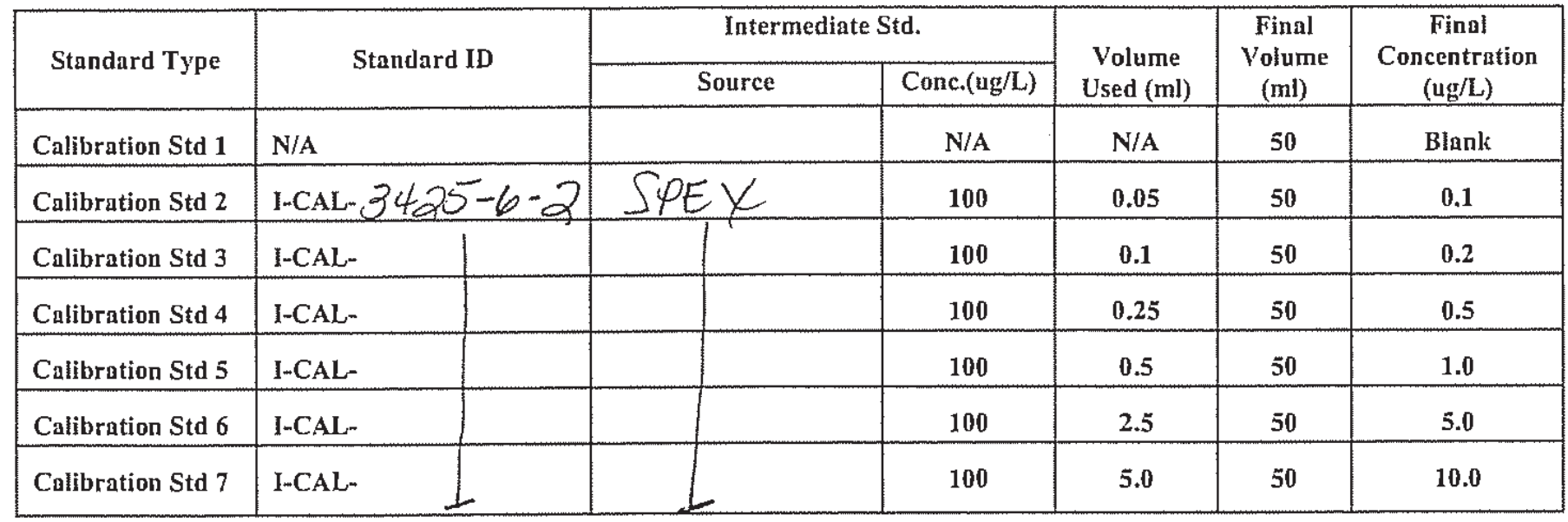

\begin{tabular}{|c|c|c|c|c|c|c|}
\hline \multirow{2}{*}{$\begin{array}{c}\text { Standard } \\
\text { Type } \\
\end{array}$} & \multirow{2}{*}{ Standard ID } & \multicolumn{2}{|c|}{ Intermediate Std. } & \multirow{2}{*}{$\begin{array}{c}\text { Amount } \\
\text { Used (ml) } \\
\end{array}$} & \multirow{2}{*}{$\begin{array}{c}\text { Final } \\
\text { Volume } \\
(\mathrm{ml})\end{array}$} & \multirow{2}{*}{$\begin{array}{c}\text { Final Conc. } \\
(\mathrm{ug} / \mathrm{L})\end{array}$} \\
\hline & & Source & Conc.(ug/L) & & & \\
\hline $\mathrm{ICV}$ & I-VER- $3426-6-1$ & KLTPA & 100 & 1.25 & 50 & 2.5 \\
\hline $\mathrm{CCV}$ & I-CAL-3425-10-2 & SPEX & 100 & 2.5 & 50 & 5.0 \\
\hline CRA & I-CAL- & $L$ & 100 & 0.1 & 50 & 0.2 \\
\hline
\end{tabular}

\begin{tabular}{|l|l|c|c|c|c|c|c|}
\hline \multirow{2}{*}{ Standard Type } & \multirow{2}{*}{ Standard ID } & \multicolumn{2}{|c|}{ Intermediate Std. } & $\begin{array}{c}\text { Amount Used } \\
\text { @ ICP } \\
\text { prep/final } \\
\text { volume }\end{array}$ & $\begin{array}{c}\text { ICP Sample } \\
\text { Volume } \\
\text { used @ Hg } \\
\text { prep (m) }\end{array}$ & $\begin{array}{c}\text { Final } \\
\text { Conc. @ } \\
\text { Hg prep } \\
\text { (ug/L) }\end{array}$ & $\begin{array}{c}\text { Final } \\
\text { Amount } \\
\text { (ug) }\end{array}$ \\
\cline { 2 - 8 } & Source & Conc.(ug/L) & 11.25 \\
\hline Air (PM10) LCS & I-CAL- & & 10,000 & $0.250 \mathrm{ml} / 50 \mathrm{ml}$ & 5.0 & 5.0 & 11.0 \\
\hline Air (PM10) MS & I-CAL- & & 10,000 & $0.050 \mathrm{ml} / 50 \mathrm{ml}$ & 5.0 & 1.0 & 2.25 \\
\hline
\end{tabular}

\begin{tabular}{|c|c|c|c|}
\hline \multicolumn{4}{|c|}{ REAGENTS } \\
\hline Conc. $\mathrm{H}_{2} \mathrm{SO}_{4}$ Lot \#: & 015045 & $5 \% \mathrm{KMnO}_{4}$ ID \#: & $B-3122-12-C$ \\
\hline Conc. $\mathrm{HNO}_{3}$ Lot \#: & 344032 & $5 \% \mathrm{~K}_{2} \mathrm{~S}_{2} \mathrm{O}_{8}$ ID \#: & $D-21038-17-E$ \\
\hline Conc. HCL Lot \#: & 00001 & $\mathrm{SnCl}_{2}$ Solution ID \#: & $A-3382 \div 20-A$ \\
\hline $12 \% \mathrm{NaClNH}_{2} \mathrm{OH} \cdot \mathrm{HCL}$ ID \#: & $C-3267-3-E$ & & \\
\hline
\end{tabular}




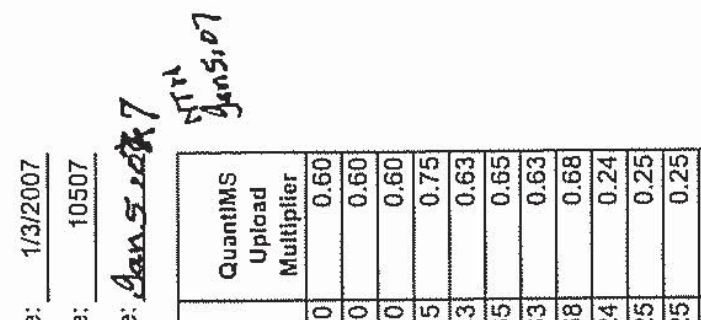

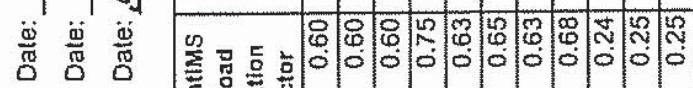

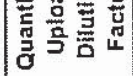

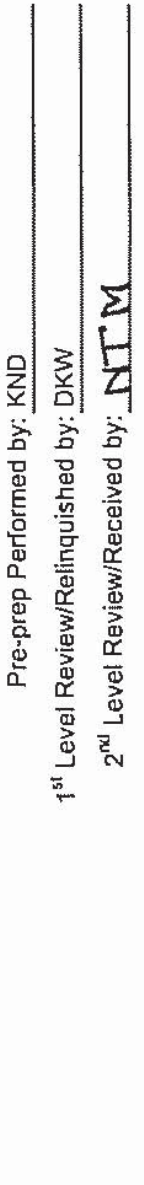

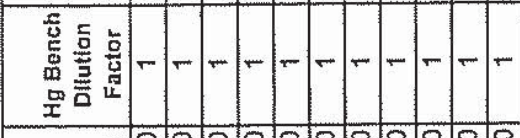

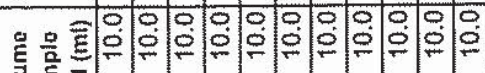

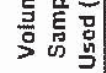

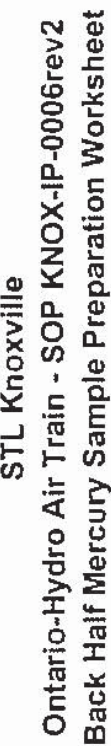

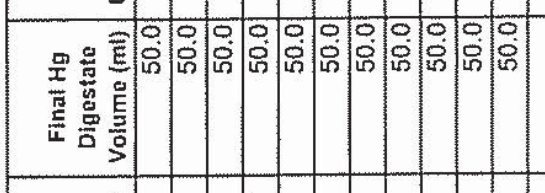

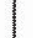

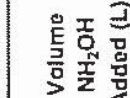

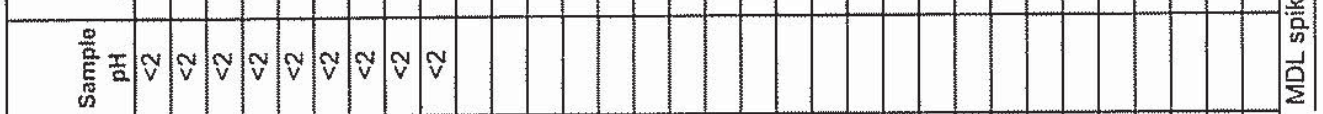

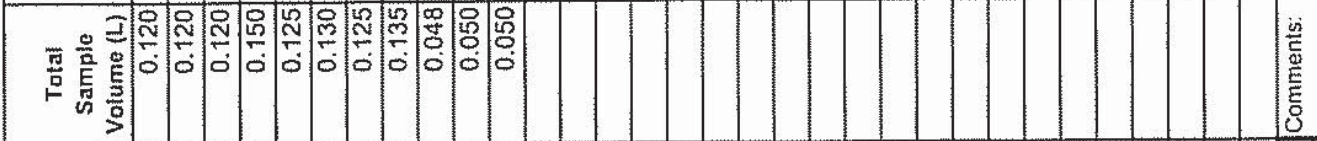
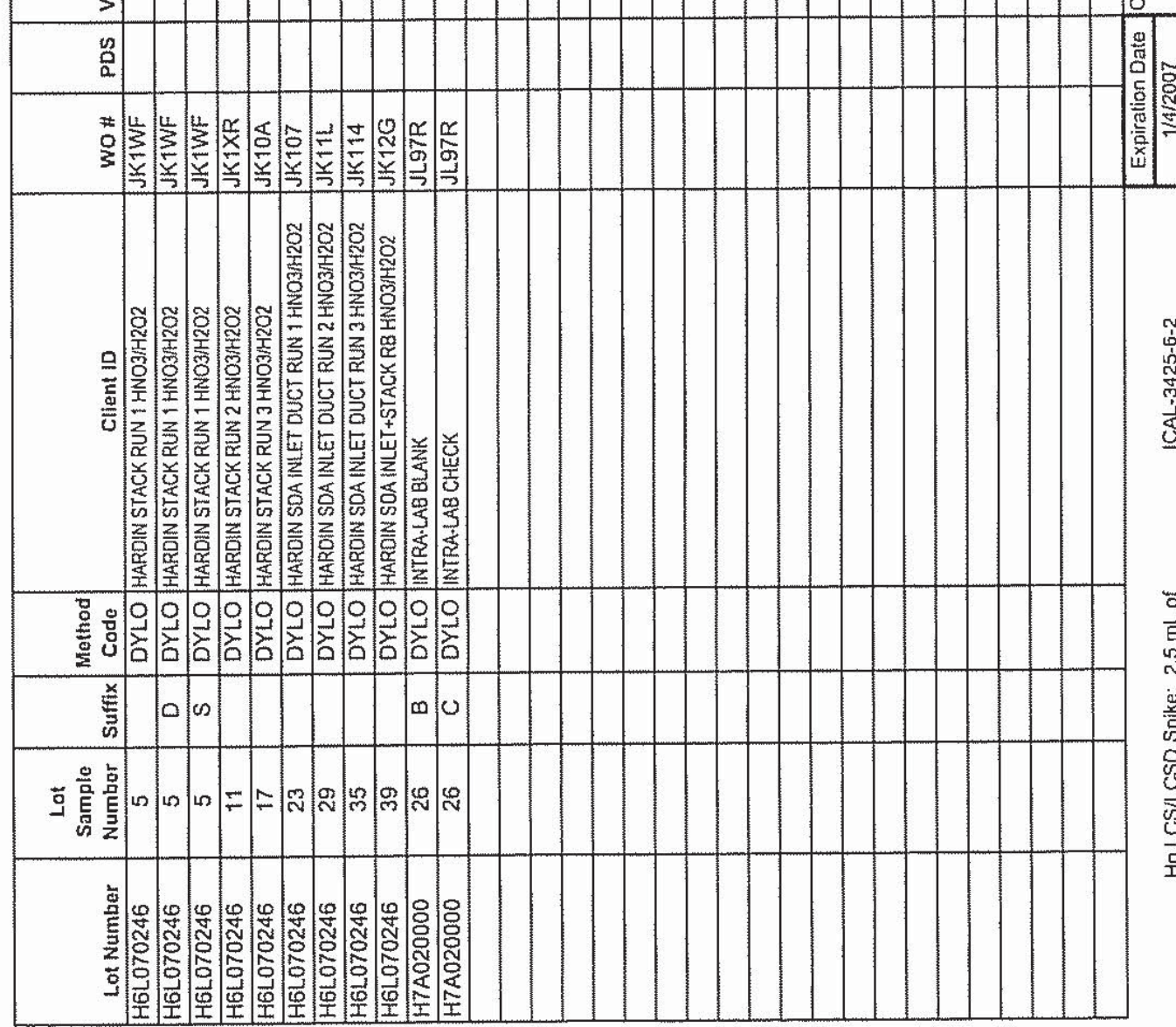

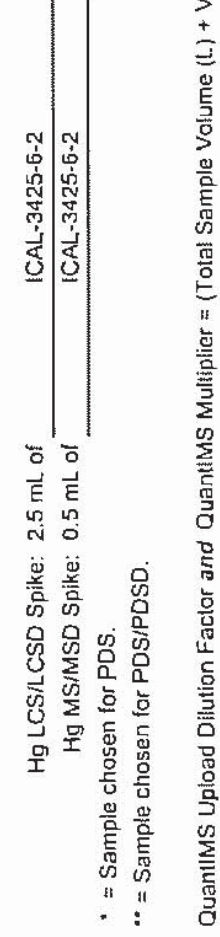


STL Knoxville

Analyst: KND/DKW Date: $01 / 04 / 07$

Spiking Form

Batch:

Bath A Temp: $93^{\circ}$

Bath B Temp: $940 \mathrm{C}$

(Acceptance range $90^{\circ}-95^{\circ} \mathrm{C}$ )

口 Hg in PM10 filters: KNOX-IP-0003, Rev. 4

D Ontario Hydro: KNOX-IP-0006, Rev. 2

口 MMT train: KNOX-MT-0006, Rev. 9

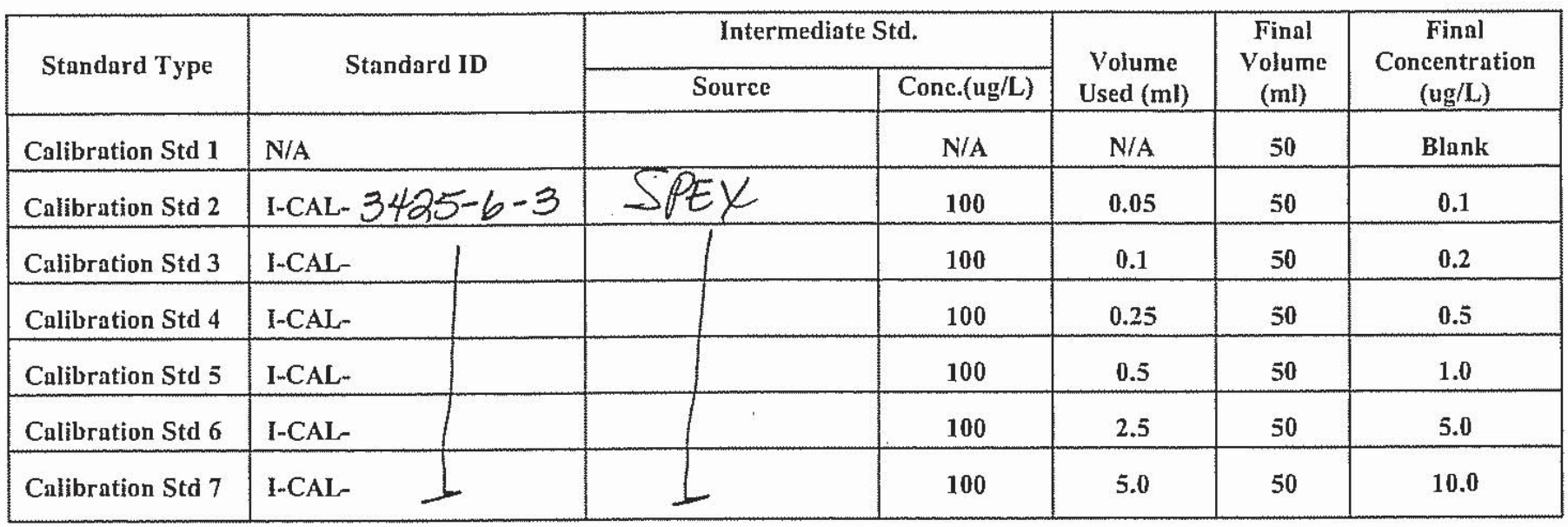

\begin{tabular}{|c|c|c|c|c|c|c|}
\hline \multirow{2}{*}{$\begin{array}{c}\text { Standard } \\
\text { Type }\end{array}$} & \multirow{2}{*}{ Standard ID } & \multicolumn{2}{|c|}{ Intermediate Std, } & \multirow{2}{*}{$\begin{array}{c}\text { Amount } \\
\text { Used (ml) }\end{array}$} & \multirow{2}{*}{$\begin{array}{l}\text { Final } \\
\text { Volume } \\
\text { (ml) }\end{array}$} & \multirow{2}{*}{$\begin{array}{l}\text { Final Conc. } \\
(u g / L)\end{array}$} \\
\hline & & Source & Conc.(ug/L) & & & \\
\hline ICV & IVER- $342 b-6-2$ & ULTRA & 100 & 1.25 & 50 & 2.5 \\
\hline $\mathrm{CCV}$ & I-CAL $-3425-6-3$ & EX & 100 & 2.5 & 50 & 5.0 \\
\hline CRA & I-CAL- & $\perp$ & 100 & 0.1 & 50 & 0.2 \\
\hline
\end{tabular}

\begin{tabular}{|c|c|c|c|c|c|c|c|}
\hline \multirow{2}{*}{ Standard Type } & \multirow{2}{*}{ Standard ID } & \multicolumn{2}{|c|}{ Intermediate Std. } & \multirow{2}{*}{$\begin{array}{l}\text { Amount Used } \\
\text { @ ICP } \\
\text { prep//inal } \\
\text { volume } \\
\end{array}$} & \multirow{2}{*}{$\begin{array}{l}\text { ICP Snmple } \\
\text { Volume } \\
\text { used @ Hg } \\
\text { prep (ml) }\end{array}$} & \multirow{2}{*}{$\begin{array}{l}\text { Final } \\
\text { Conc. @ } \\
\text { Hg prep } \\
\text { (ug/L) } \\
\end{array}$} & \multirow{2}{*}{$\begin{array}{l}\text { Final } \\
\text { Amount } \\
\text { (ug) }\end{array}$} \\
\hline & & Source & Conc.(ug/L) & & & & \\
\hline Air (PM10) LCS & I-CAL- & & 10,000 & $0.250 \mathrm{~mL} / 50 \mathrm{ml}$ & 5.0 & 5.0 & 11.25 \\
\hline Air (PM10) MS & I-CAL & & 10,000 & $0.050 \mathrm{ml} / 50 \mathrm{ml}$ & 5.0 & 1.0 & 2.25 \\
\hline
\end{tabular}

\begin{tabular}{|c|c|c|c|}
\hline \multicolumn{4}{|c|}{ REAGENTS } \\
\hline Conc. $\mathrm{H}_{2} \mathrm{SO}_{4}$ Lot & C.5045 & $5 \% \mathrm{KMnO}_{4}$ ID H: & $B-3122-12-C$ \\
\hline Conc. $\mathrm{HNO}_{3}$ Lot \#: & 844032 & $5 \% \mathrm{~K}_{2} \mathrm{~S}_{2} \mathrm{O}_{8}$ ID \#: & $D-2638-17-E$ \\
\hline Conc. HCL Lot \#: & $1+2422$ & $\mathrm{SnCl}_{2}$ Solution ID H: & $A-3382-20-B$ \\
\hline $12 \% \mathrm{NaClNH}_{2} \mathrm{OH} \cdot \mathrm{HCL}$ ID \#: & $c-3267-3-E$ & & \\
\hline
\end{tabular}




\section{Wet Chemistry}


Sample Summary 
Air Sampling Associates, Inc.

Client Sample ID: HARDTN STACK RUN 1 FILTKR Al17

General Chemistry

Lot-Sample \#...: H6L070246-001

Date Sampled...: 12/01/06
Work Order \#... JK1V2

Date Received..: 12/06/06
Matrix........ AIR

PREPARATION - PREP ANALYSIS DATE BATCH \#

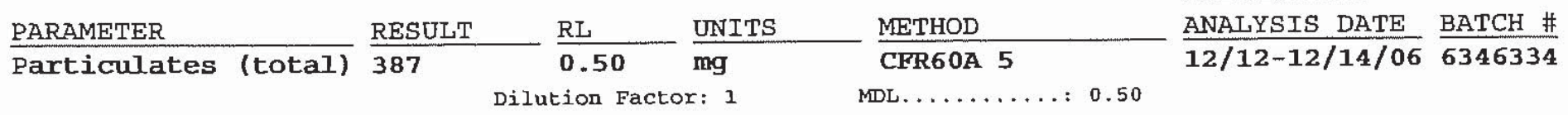


Air Sampling Associates, Inc.

Client Sample ID: HARDIN STACK RUN 1 ACETONB

General Chemistry

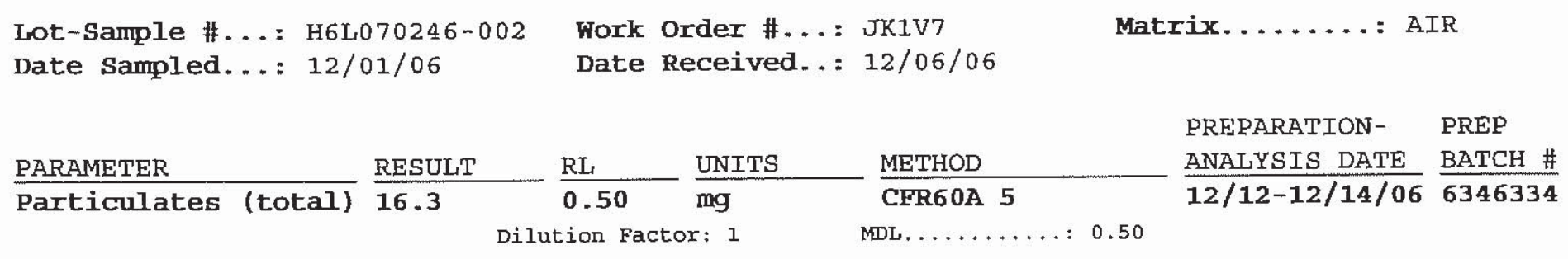


Air Sampling Associates, Inc.

Client Sample ID: HARDIN STACK RUN 2 FILTKR A118

General Chemistry

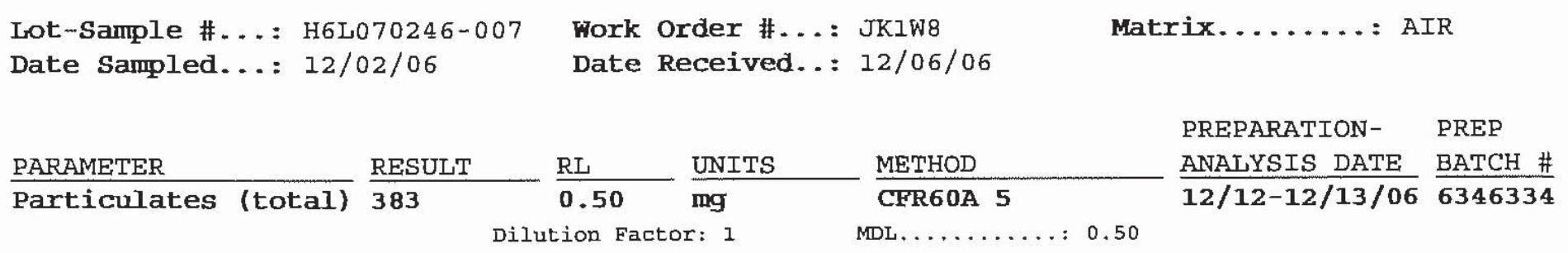


Air Sampling Associates, Inc.

client Sample ID: HARDIN STACK RUN 2 ACETONE

\section{General Chemistry}

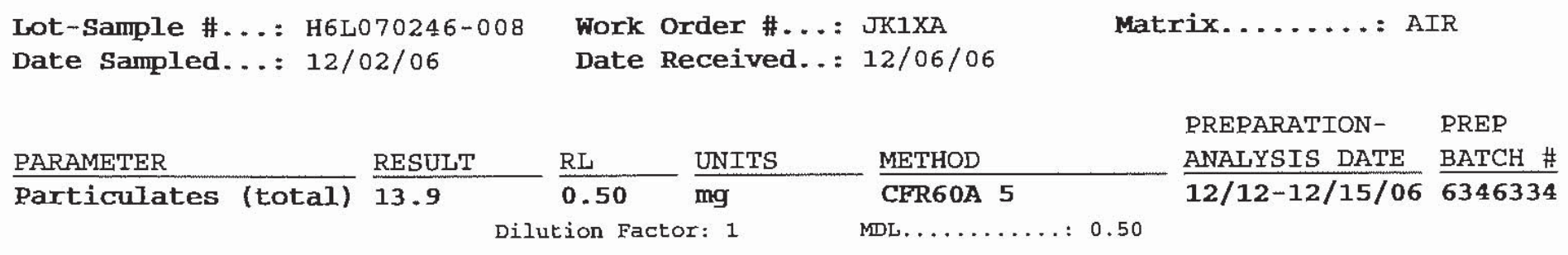


Air Sampling Associates, Inc.

\section{client Sample ID: HARDIN STACK RUN 3 FILTER A719}

\section{General Chemistry}

Lot-Sample \#. . : H6L070246-013

Date Sampled...: 12/02/06
Hork Order \#...: JK1X1

Date Received..: 12/06/06
Matrix.......: AIR

PREPARATION- $\quad$ PREP ANALYSIS DATE BATCH \#

$\frac{\text { PARAMETER }}{\text { Particulates (total) }} \frac{\text { RESULT }}{392} \frac{\text { RL }}{\mathbf{0 . 5 0}} \frac{\text { UNITS }}{\mathrm{mg}} \frac{\text { METHOD }}{\text { CFR60A 5 }} \frac{\text { ANALYSIS DATE }}{\mathbf{1 2 / 1 2 - 1 2 / 1 4 / 0 6}} \frac{\text { BATCH \# }}{\mathbf{6 3 4 6 3 3 4}}$


Air Sampling Associates, Inc.

Client Sample ID: HARDIN STACK RUN 3 ACETONE

General Chemistry

Lot-Sample \#...: H6L070246-014 Fork Order \#...: JK1X4

Date Sampled...: 12/02/06
Date Received. . : 12/06/06
Matrix.......: AIR

PREPARATION- PREP ANALYSIS DATE BATCH \#
PARAMETER RESULT

Particulates (total) 12.1
UNITS

mg Dilution Factor: 1
METHOD

CFR60A 5 
Air Sampling Associates, Inc.

client Sample ID: HARDIN SDA TNLET DUCT RON 1 FILTKR (THTMBLE 53)

General Chemistry

Lot-Sample \#...: H6L070246-019 Fork Order \#...: JK10X

Date Sampled...: 12/01/06

\begin{abstract}
Date Received. . : 12/06/06
\end{abstract}
Matrix......... AIR

PREPARATION- PREP $\frac{\text { ANALYSIS DATE }}{12 / 12-12 / 15 / 06} \frac{\text { BATCH \# }}{6346334}$ 
Air Sampling Associates, Inc.

\section{client Sample ID: HARDIN SDA INLEP DUCT RUN 1 ACETONE}

\section{General Chemistry}

Lot-Sample \#... : H6L070246-020

Date Sampled...: 12/01/06
Work Order \#...: JK101

Date Received. .: 12/06/06
Matrix........ AIR

PREPARATION- PREP

ANALYSIS DATE BATCH \#
PARAMETER

RESULT

Particulates (total) $\mathbf{3 2 5 0}$
RI, mg Dilution Factor: 1
METHOD

CFR60A 5 MDL . . . . . . . 0.50 
Air Sampling Associates, Inc.

client Sample ID: HARDIN SDA INLET DUCT RUN 2 FILTTR (THIMBLB 54)

General Chemistry

Lot-Sample \#...: H6L070246-025 Work order \#...: JK11C

Date Sampled... : 12/02/06

\begin{abstract}
Date Received. .: 12/06/06
\end{abstract}
Matrix........ AIR

PREPARATION- PREP ANALYSIS DATE BATCH \# $12 / 12-12 / 15 / 06 \quad \overline{6346334}$
PARAMETER

Particulates (total) 12700
RL $\quad$ UNITS

$0.50 \mathrm{mg}$

Dilution Factor: 1
METHOD

CFR6 0A 5

MDL......... 0.50 
Air Sampling Associates, Inc.

client Sample ID: HARDIN SDA INLET DUCT RUN 2 ACETONB

General Chemistry

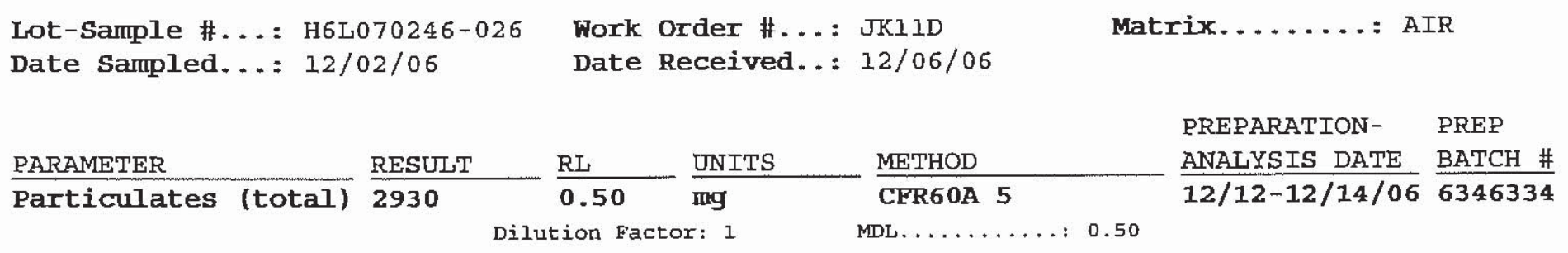


Air Sampling Associates, Inc.

client sample ID: HARDIN SDA INLET DUCT RUN 3 FILTER (THTMBIE 55)

General Chemistry

Lot-Sample \#...: H6L070246-031 work Order \#...: JK11X

Date Sampled...: 12/02/06

\author{
Date Received... 12/06/06
}

Matrix.......: AIR

PREPARATION- PREP

ANALYSIS DATE BATCH \#

$\frac{\text { PARAMETER }}{\text { Particulates (total) }} \frac{\text { RESULT }}{\mathbf{1 2 5 0 0}} \frac{\text { RL }}{\mathbf{0 . 5 0}} \frac{\text { UNITS }}{\mathrm{mg}} \frac{\text { METHOD }}{\text { Dilution Factor: } 1} \quad \frac{\text { ANALYSIS DATE }}{\mathbf{1 2 / 1 2 - 1 2 / 1 5 / 0 6}} \frac{\text { BATCH \# }}{6346334}$


Air Sampling Associates, Inc.

Client Sample ID: HARDIN SDA INI.ET DOCT RUN 3 ACETONE

General Chemistry

Lot-Sample \#...: H6L070246-032 Work Order \#...: JK111

Date sampled... : 12/02/06

\begin{abstract}
Date Received..: 12/06/06
\end{abstract}
Matrix........ AIR

PREPARATION- PREP ANALYSIS DATE BATCH \#

$\frac{\text { PARAMETER }}{\text { Particulates (total) }} \frac{\text { RESULT }}{730}-\frac{\text { RL }}{\mathbf{0 . 5 0}} \frac{\text { UNITS }}{\mathrm{mg}} \frac{\text { METHOD }}{\text { CFR60A } 5} \frac{\text { ANALYSIS DATE }}{12 / 12-12 / 14 / 06} \frac{\text { BATCH \# }}{6346334}$


Raw Data 


\section{Total Particulates}




\section{STL Knoxville Particulate Matter Determination Data Review/Narrative Checklist Method: SW-846 Method 0050 by SOP KNOX-WC-0006, Rev. 2 \\ Page 1 of 1}

\begin{tabular}{|r|c|}
\hline Batch Number: & 6346334 \\
\hline Lot Number(s): & H6 6070246 \\
\hline
\end{tabular}

\begin{tabular}{|c|c|c|c|c|c|}
\hline $\begin{array}{l}\text { Review Items } \\
\text { A. Calibration }\end{array}$ & N/A & $\mathbf{Y}$ & $\mathbf{N}$ & If No, why is data reportable? & $\sqrt{1}$ \\
\hline $\begin{array}{l}\text { 1. Was the balance calibration checked with } 5,50 \& 100 \\
\text { gram weights prior to use? }\end{array}$ & & $\checkmark$ & & & \\
\hline $\begin{array}{l}\text { 2. Was the balance checked with } 100 \text { gram weight after } \\
\text { every } 10 \text { samples? }\end{array}$ & & $\sqrt{ }$ & & & \\
\hline 3. Was the balance tared before each weighing? & & $\checkmark$ & & & $\sqrt{v}$ \\
\hline 4. Was the balance ID documented? & & $\sqrt{ }$ & & & \\
\hline \multicolumn{6}{|l|}{ B. Client Sample and QC Sample Results } \\
\hline \multicolumn{6}{|l|}{$\begin{array}{l}\text { 1. Were all special project requirements met? (Were PM } \\
\text { Checklists, Lot Summary, and any applicable QAS } \\
\text { reviewed?) }\end{array}$} \\
\hline 2. Were sample IDs verified? & & $\sqrt{ }$ & & · & \\
\hline 3. Were all transcriptions checked? & & $\checkmark$ & & & \\
\hline 4. Calculations checked for enor? & & $\checkmark$ & & & \\
\hline $\begin{array}{l}\text { 5. Final report acceptable? (Results correct, units correct, } \\
\text { deviations noted in narrative, and analysis dates } \\
\text { correct.) }\end{array}$ & & $\sqrt{ }$ & & & \\
\hline \multicolumn{6}{|l|}{ C. Preparation/Matrix QC } \\
\hline $\begin{array}{l}\text { 1. Were all sample weighings performed in duplicate (at } \\
\text { least)? }\end{array}$ & & $\checkmark$ & & & \\
\hline 2. Was the average of the last two weighings used? & & $\sqrt{ }$ & & & $\sqrt{1}$ \\
\hline \multicolumn{6}{|l|}{ D. Other } \\
\hline 1. Are all nonconformances documented appropriately? & $\sqrt{ }$ & & & NCM No.: & $\sqrt{A}$ \\
\hline
\end{tabular}

\begin{tabular}{|c|c|}
\hline \begin{tabular}{l|l} 
Analyst: Ju thumphrey & Date: $12 / 15 / 06$ \\
\end{tabular} & 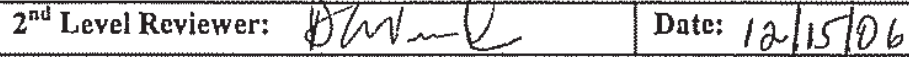 \\
\hline Comments: & Comments: \\
\hline * samples were fly ash which are hydroscopic & Tolats data with \\
\hline Large samplef ${ }^{j \mu H}$ weights kept weighed results & sisiniss in \\
\hline within $1 \%$ & namative because rannot \\
\hline & thent ale desits requend \\
\hline 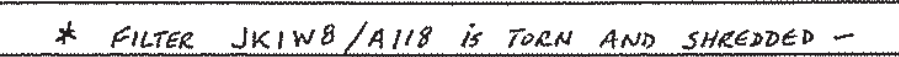 & frim (Xuant IMS \\
\hline SOME SMALL PIECES WERE LOST IN HANDLING & \\
\hline FILTER, REPORTED FIRST WEIGHING RESULT & \\
\hline * NO TARE WEIGHTS PROVIDED FOR FILTERS - & \\
\hline REPGRTED RESULTS AS WEIGHED & \\
\hline
\end{tabular}



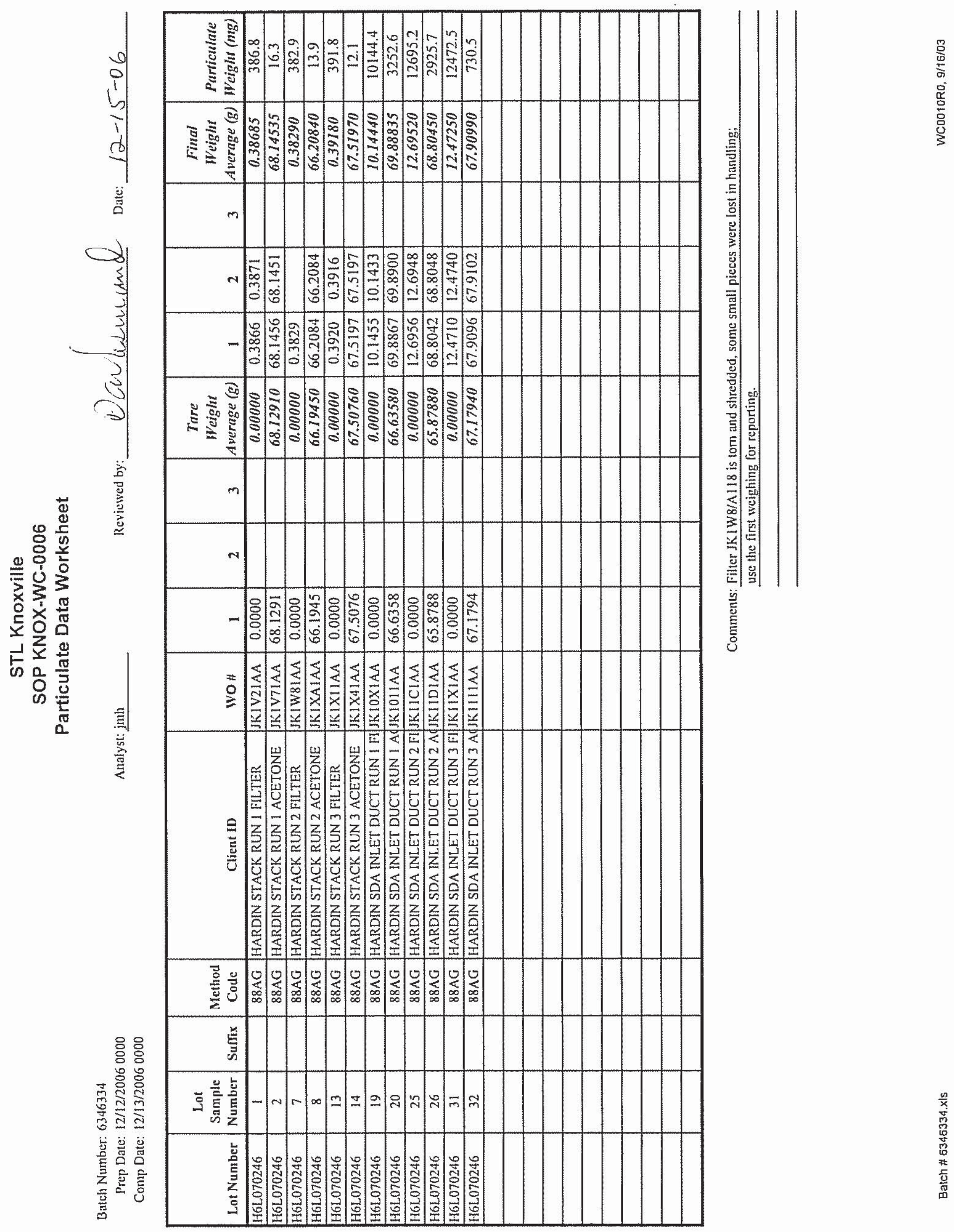


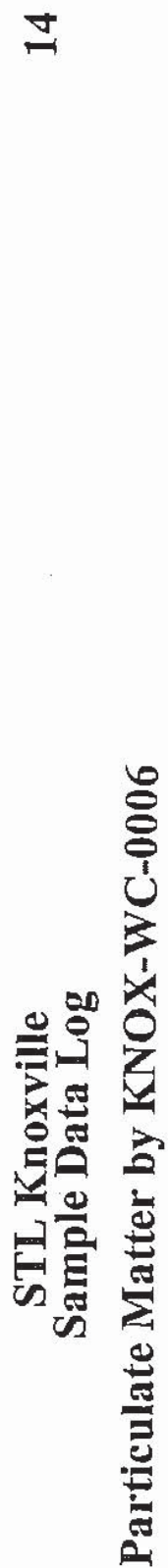

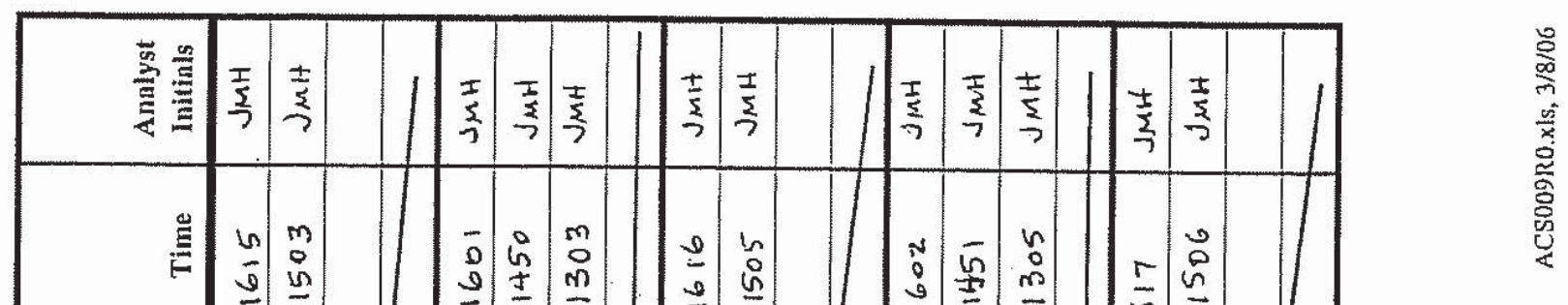

\begin{tabular}{|c|c|c|c|c|c|c|c|c|c|c|c|}
\hline & 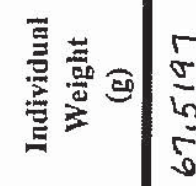 & $\mid \begin{array}{c}5 \\
6 \\
5 \\
0\end{array}$ & \multicolumn{2}{|c|}{\begin{tabular}{l|l}
0 & 10 \\
0 & 4 \\
$g$ & $\exists$ \\
0 & \\
0 &
\end{tabular}} & 焉 & $\left|\begin{array}{l}0 \\
0 \\
0 \\
0 \\
0 \\
0\end{array}\right|$ & $\begin{array}{c}0 \\
0 \\
0 \\
\infty \\
0 \\
0\end{array}$ & \multicolumn{2}{|c|}{ 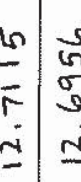 } & 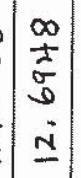 & 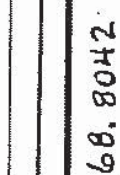 \\
\hline
\end{tabular}

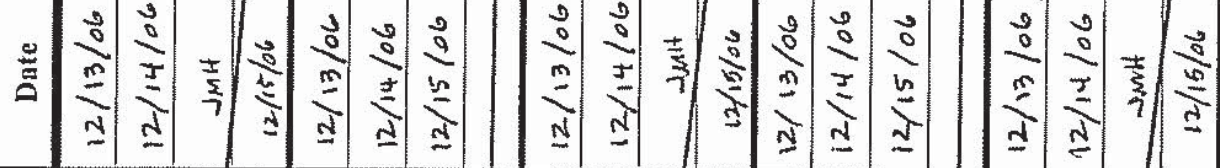

$20 x-y$

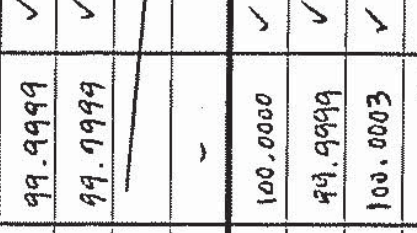

$>>$

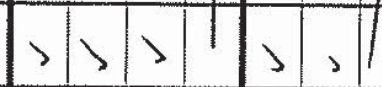

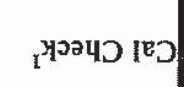

\begin{tabular}{l|l}
$\sigma$ & $\sigma$ \\
$\sigma$ & $\sigma$ \\
$\sigma$ & $\sigma$
\end{tabular}

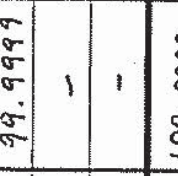

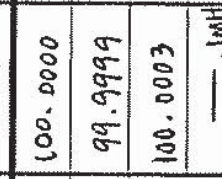

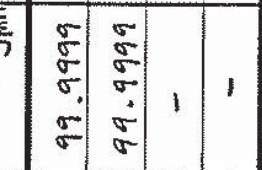



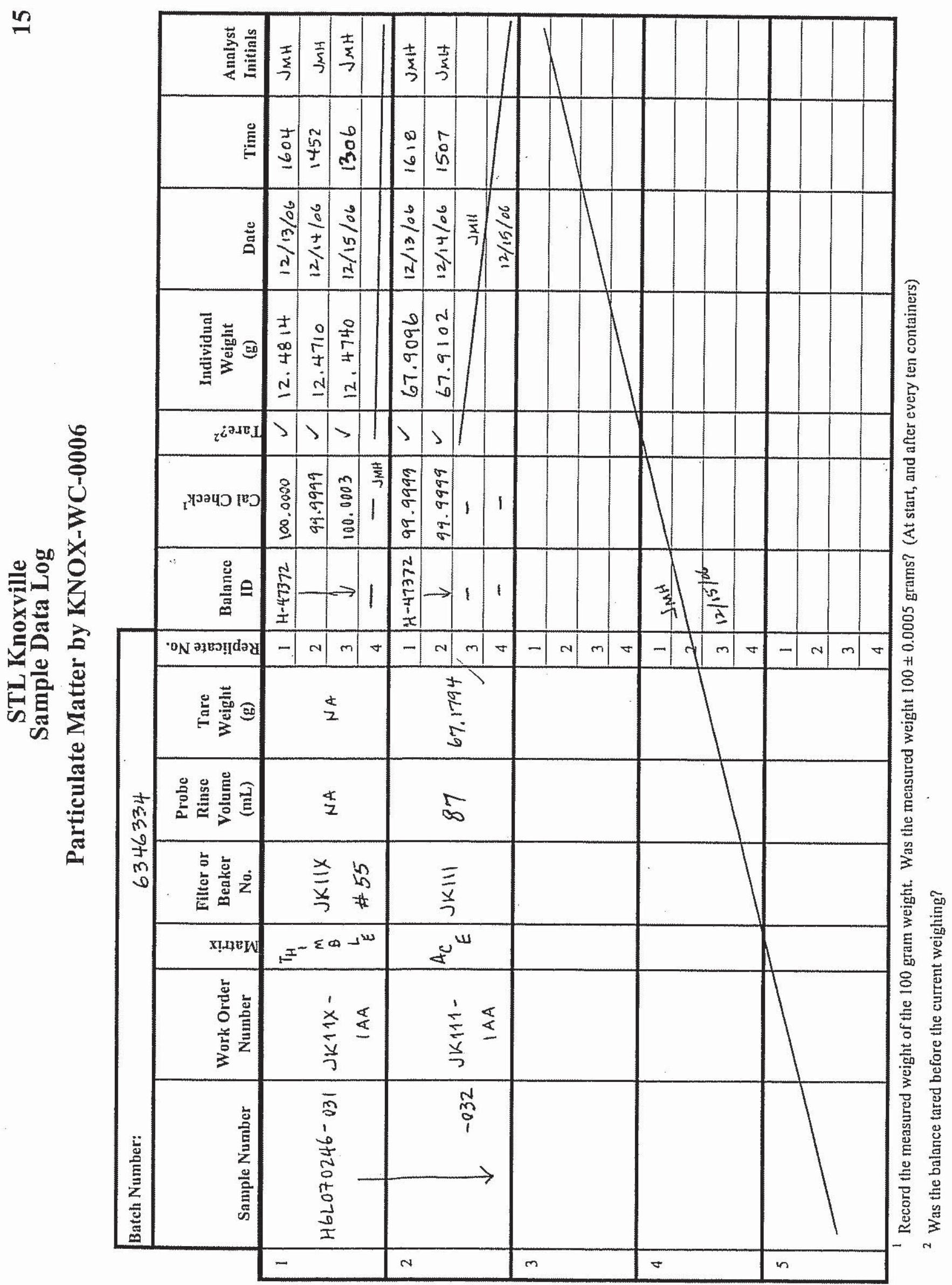
STL Knoxville

Tare Weight Log

Particulate Matter by KNOX-WC-0006

\begin{tabular}{|c|c|c|c|c|c|c|c|c|c|c|c|}
\hline & $\begin{array}{c}\text { Filter or } \\
\text { Beaker } \\
\text { No. }\end{array}$ & 莣 & 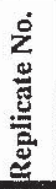 & Balance ID & 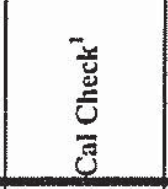 & है. & $\begin{array}{l}\text { Individual } \\
\text { Weight (g) } \\
\end{array}$ & Date & Time & $\begin{array}{c}\text { Analyst } \\
\text { Initials } \\
\end{array}$ & $\begin{array}{c}\text { Tare } \\
\text { Weight } \\
\text { (g) }\end{array}$ \\
\hline \multirow[t]{4}{*}{1} & \multirow{4}{*}{$\rho k^{18 \sigma}$} & \multirow{4}{*}{$F$} & 1 & 14.47372 & 100.0000 & $\checkmark$ & 33.4243 & $12-12-06$ & $10: 15$ & $\angle D W$ & \multirow{4}{*}{33.4244} \\
\hline & & & 2 & $\downarrow$ & $\downarrow$ & $V$ & 33.4245 & $\downarrow$ & $15: 30$ & $k$ & \\
\hline & & & 3 & & & & & & & & \\
\hline & & & 4 & & & & tow & & & & \\
\hline \multirow[t]{4}{*}{2} & \multirow{4}{*}{$p k^{13^{30}}$} & \multirow{4}{*}{6} & 1 & H- 47372 & 100,0000 & $\checkmark$ & 46.1156 & $12-12-06$ & $10: 16$ & $\angle D \omega$ & \multirow[b]{4}{*}{46.1157} \\
\hline & & & 2 & $j$ & $\downarrow$ & $\checkmark$ & 46,1158 & $k$ & $15: 31$ & $\downarrow$ & \\
\hline & & & 3 & & & & & & & & \\
\hline & & & 4 & & & & $\mathrm{hOW}$ & & & & \\
\hline \multirow[t]{4}{*}{3} & \multirow{4}{*}{ pq1831 } & \multirow{4}{*}{ F } & 1 & $4-47372$ & 100,0000 & $\checkmark$ & 32.1098 & $23-103-06$ & $10: 17$ & $\angle \Delta \omega$ & \multirow[b]{4}{*}{32.1100} \\
\hline & & & 2 & $\psi$ & $\downarrow$ & $\checkmark$ & 32,1102 & $\sqrt{k}$ & $15: 32$ & $\downarrow$ & \\
\hline & & & 3 & & & & & & & & \\
\hline & & & 4 & & & 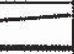 & 100 & & & & \\
\hline \multirow[t]{4}{*}{4} & \multirow{4}{*}{$P K^{18^{3}}$} & \multirow{4}{*}{ F } & 1 & 4.47372 & 100.0000 & 2 & 35.2943 & $12-12-06$ & $10: 18$ & $\angle D A O$ & \multirow{4}{*}{35.2945} \\
\hline & & & 2 & $k$ & $\downarrow$ & $\checkmark$ & 35.2947 & $j$ & $15: 33$ & $k$ & \\
\hline & & & 3 & & & & & & & & \\
\hline & & & 4 & & & & 1.000 & & & & \\
\hline \multirow[t]{4}{*}{5} & \multirow{4}{*}{ JKIV7 } & \multirow{4}{*}{$A_{C_{E}}$} & 1 & $\mathrm{H}-41372$ & 100.0003 & $\checkmark$ & 68.1291 & $12-12-06$ & 1229 & $J_{M H}$ & \multirow{4}{*}{68.1291} \\
\hline & & & 2 & & & & y,ant. & & & & \\
\hline & & & 3 & & & & & & & & \\
\hline & & & 4 & & & & $12-12-06$ & & & $=$ & \\
\hline 6 & & & 1 & $\mathrm{H}-47372$ & 100.0000 & $\checkmark$ & 66.1945 & $12-12-06$ & 1230 & $J_{M H}$ & \\
\hline & $I K 1 \times A$ & ${ }^{A} C_{G}$ & 2 & & & & junt & & & & 1945 \\
\hline & & & 3 & & & & & 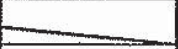 & & & 66.1740 \\
\hline & & & 4 & & & & $12-12.06$ & & & $=$ & \\
\hline 7 & & & 1 & $\mathrm{H}-47372$ & 100.0000 & $\checkmark$ & 67.5076 & $12-12.06$ & 1231 & JMH & \\
\hline & JK1X4 & $A_{C_{c}}$ & 2 & & & & & & & & $10>-7 h$ \\
\hline & & & 3 & & & & & Juth & & & 67.5076 \\
\hline & & & 4 & & & & & $12-12-a$ & & $=$ & \\
\hline 8 & & & 1 & $\mathrm{H}-47372$ & 100.0800 & $\checkmark$ & 66.6358 & $12-12.06$ & 1232 & JMH & \\
\hline & & $l_{c}$ & 2 & & & & & & & & 16358 \\
\hline & $3 \times 101$ & & 3 & & & & & JMH & & & 166.6300 \\
\hline & & & 4 & & & & & $12-12.06$ & & $=$ & \\
\hline 9 & & & 1 & $H-47372$ & 100.0000 & $\checkmark$ & 65,8788 & $12-12-06$ & 1233 & $J M H$ & \\
\hline & $T \times 110$ & $A_{C_{E}}$ & 2 & & & & & & & & 168788 \\
\hline & JRाis & & 3 & & & & & Jilli & & & 65.8180 \\
\hline & & & 4 & & & & & $12-12-06$ & & $=$ & \\
\hline 10 & & & 1 & $\mathrm{H}-47372$ & 100.0000 & $\checkmark$ & 67.1794 & $12-12-06$ & 1234 & JMH & \\
\hline & KK 111 & ${ }^{A} c_{E}$ & 2 & $=$ & $=$ & & & & & & 671794 \\
\hline & djorate & & 3 & & & & & JMIF & & & $61.17 \mathrm{~T}$ \\
\hline & & & 4 & & & & & $12-12-86$ & & 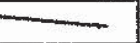 & \\
\hline
\end{tabular}




\section{Sample Receipt Documentation}




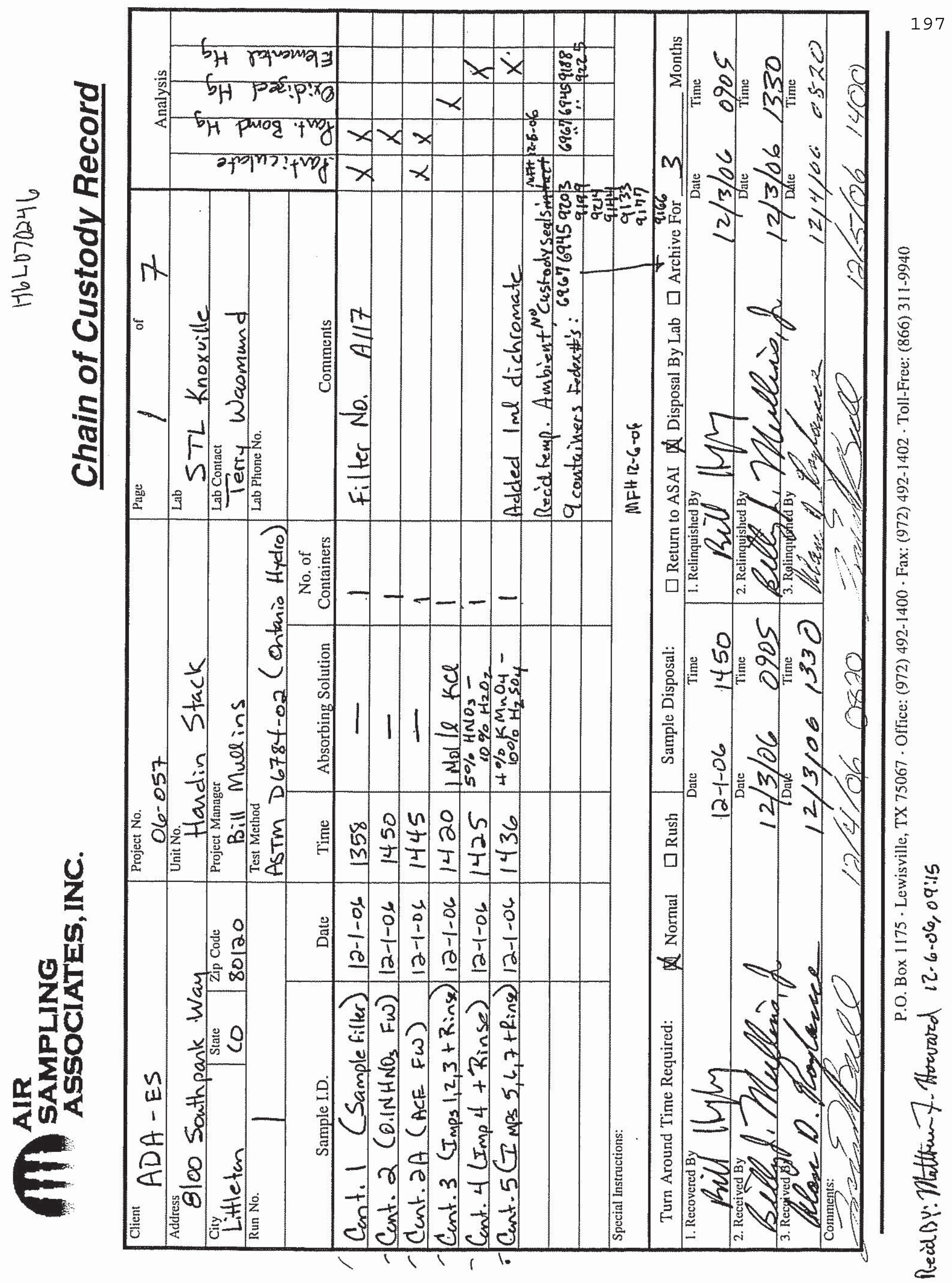




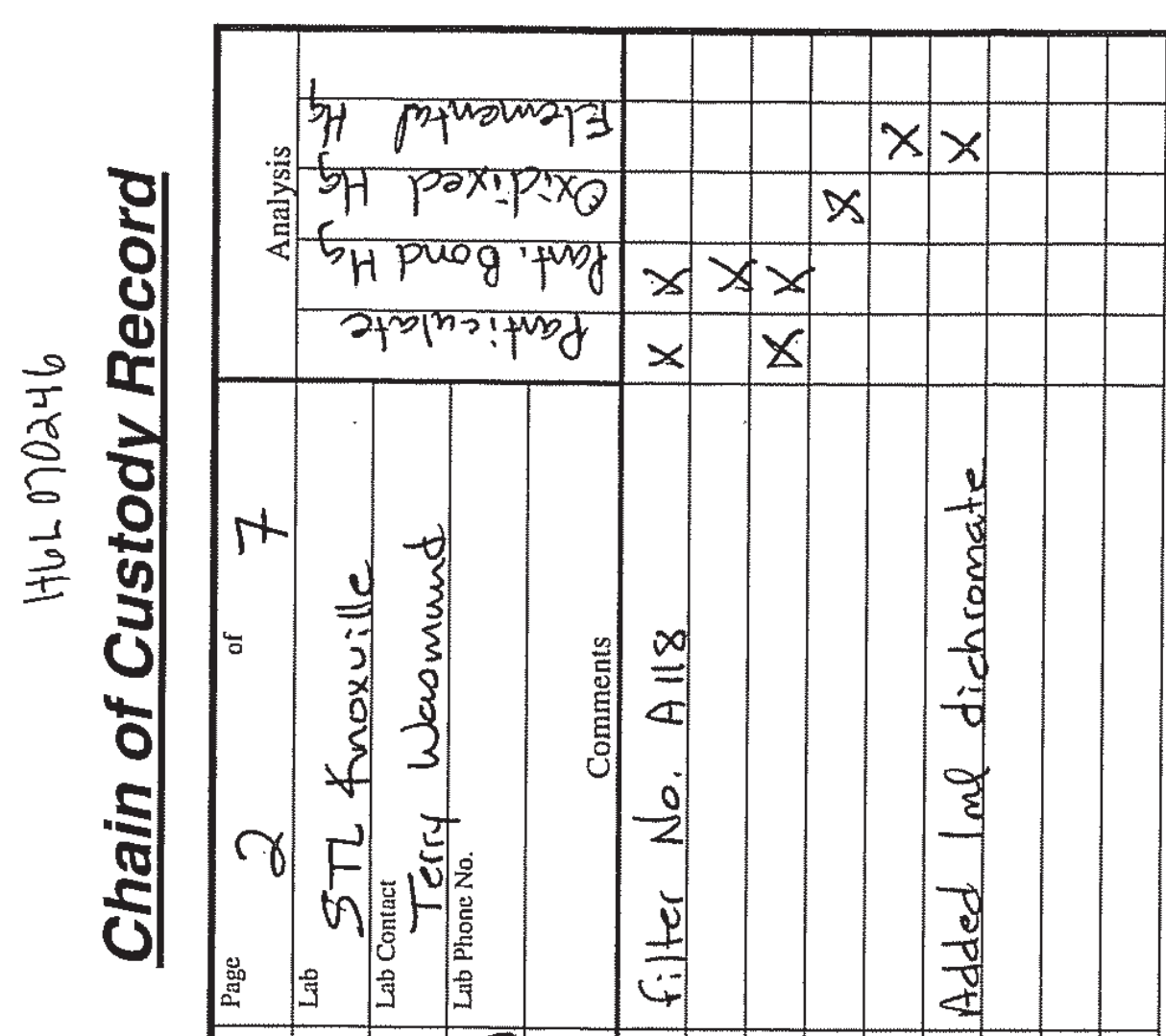




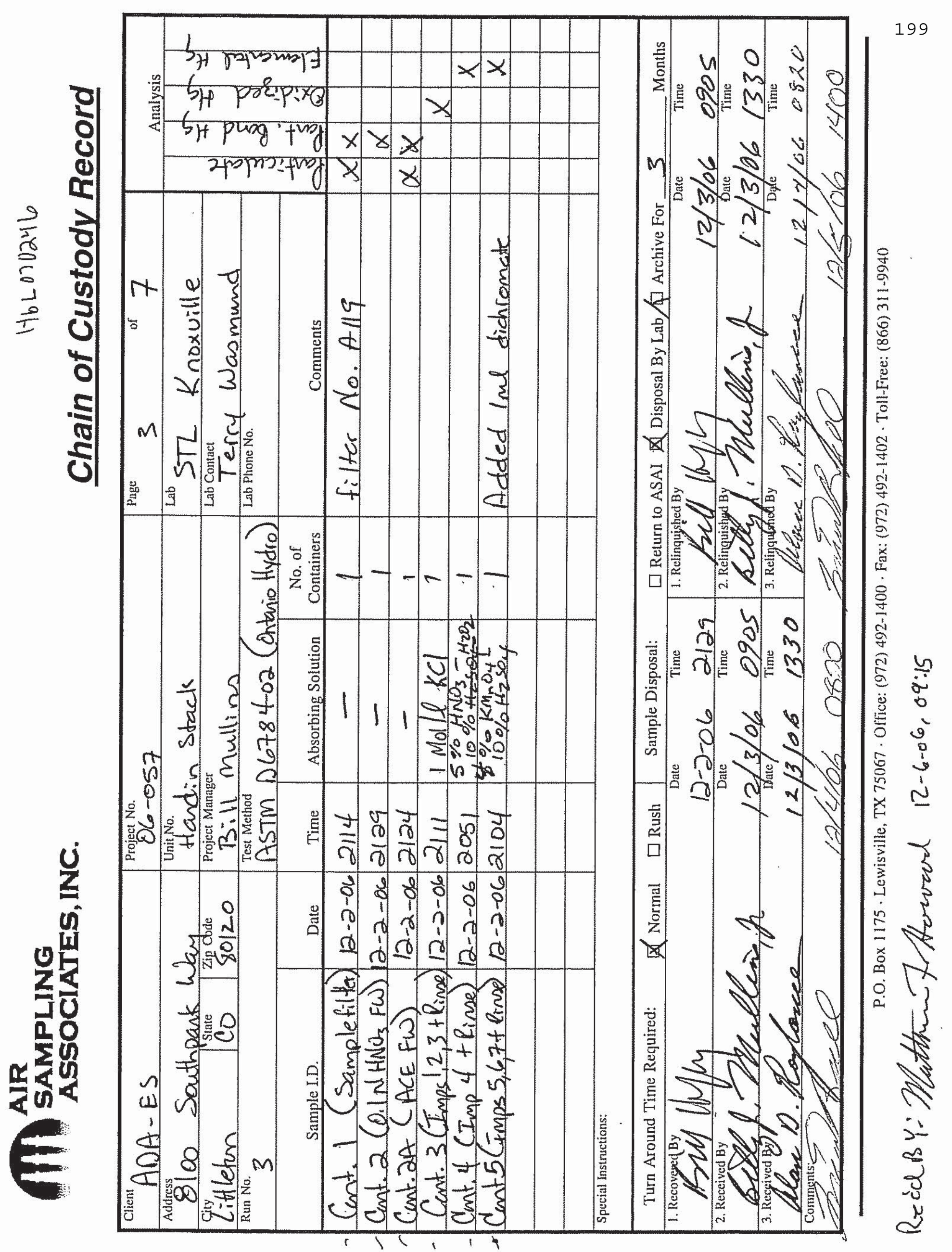




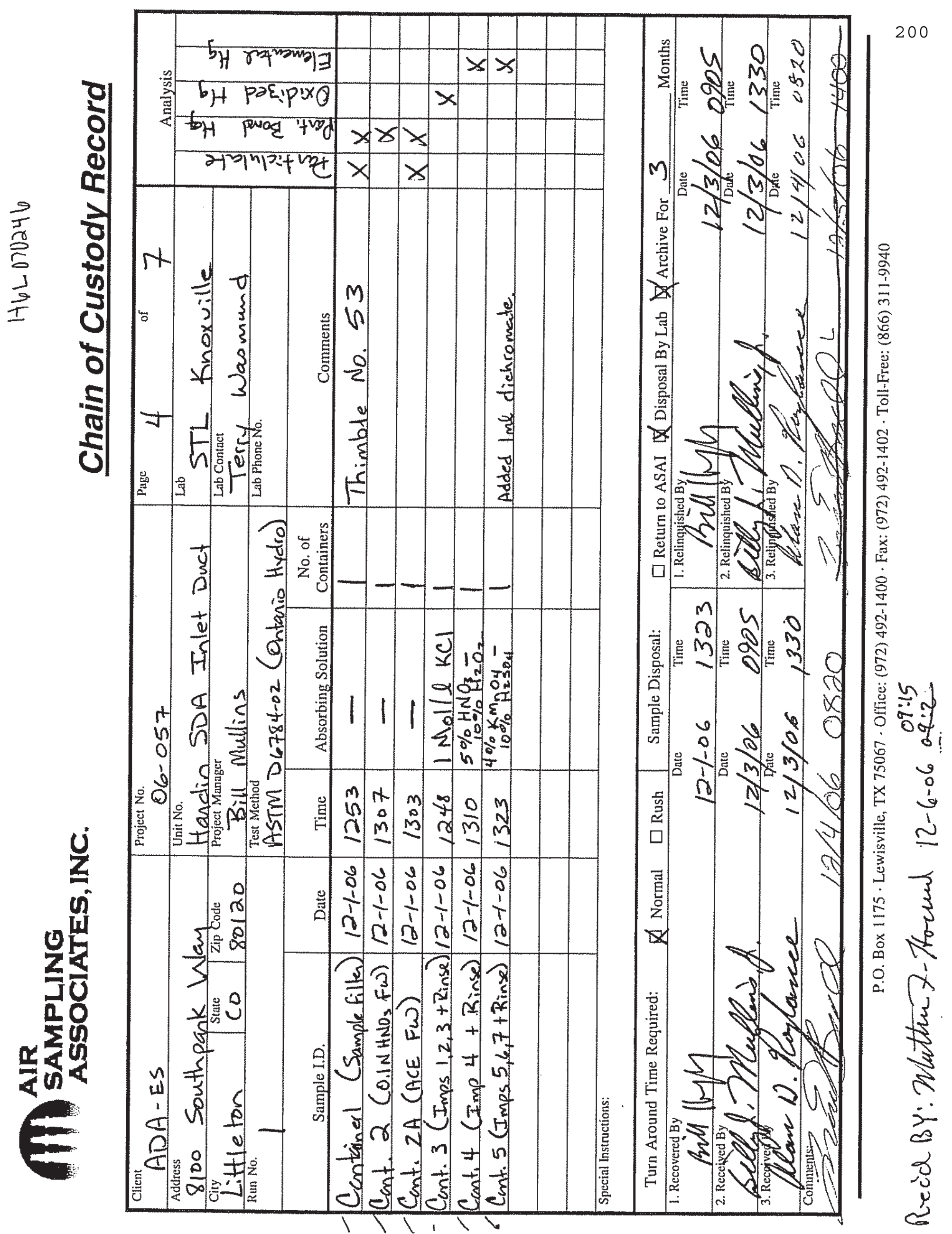




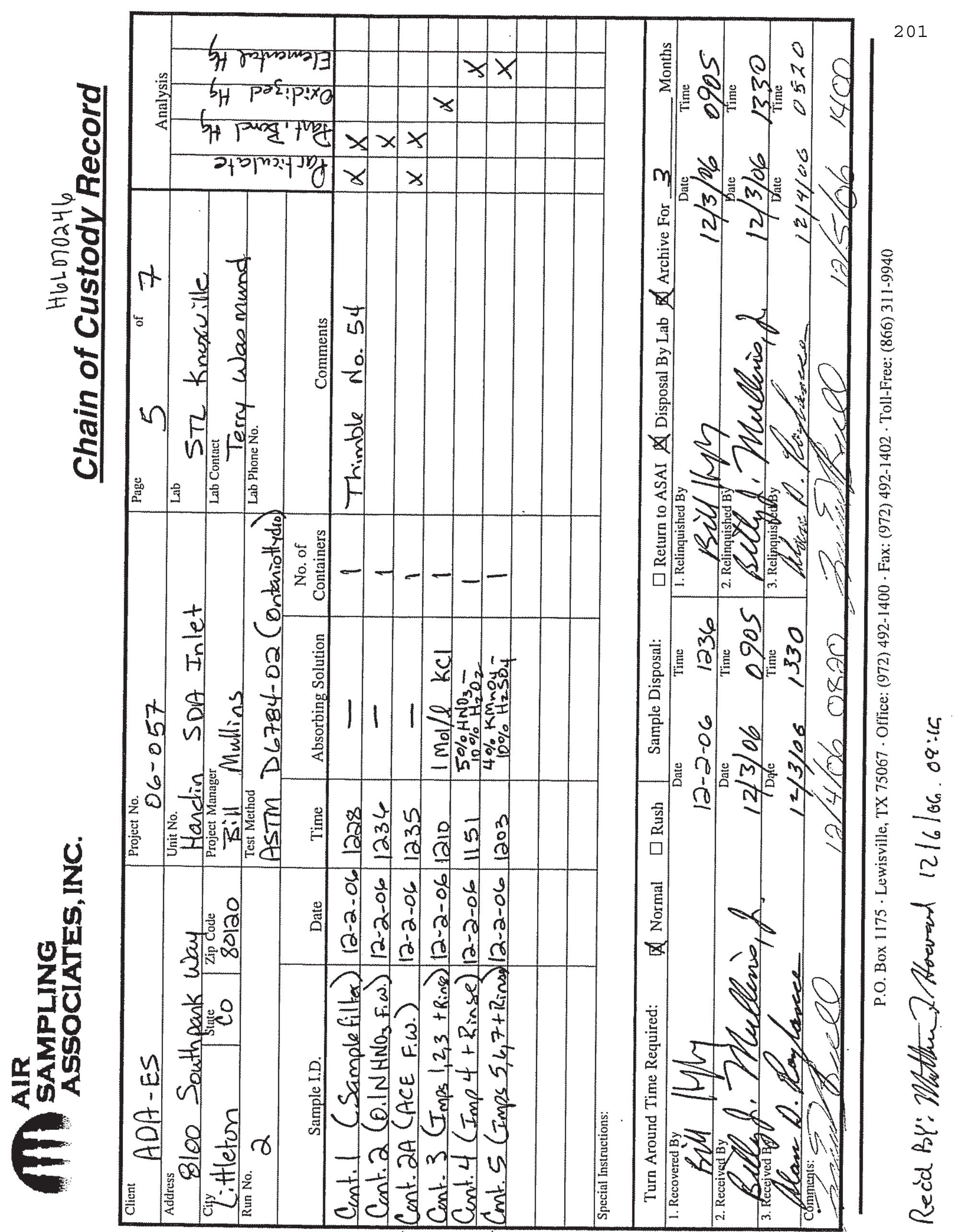




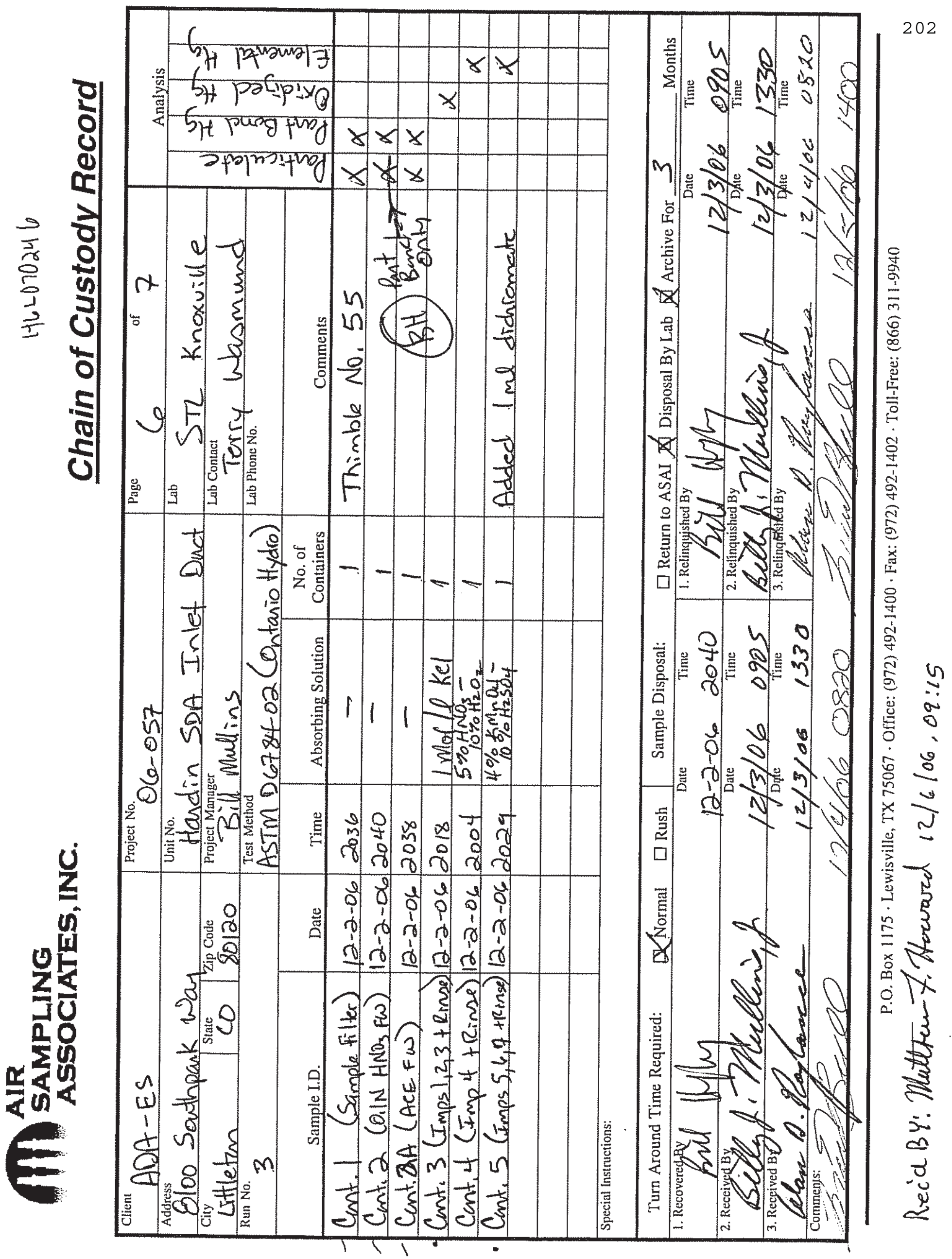




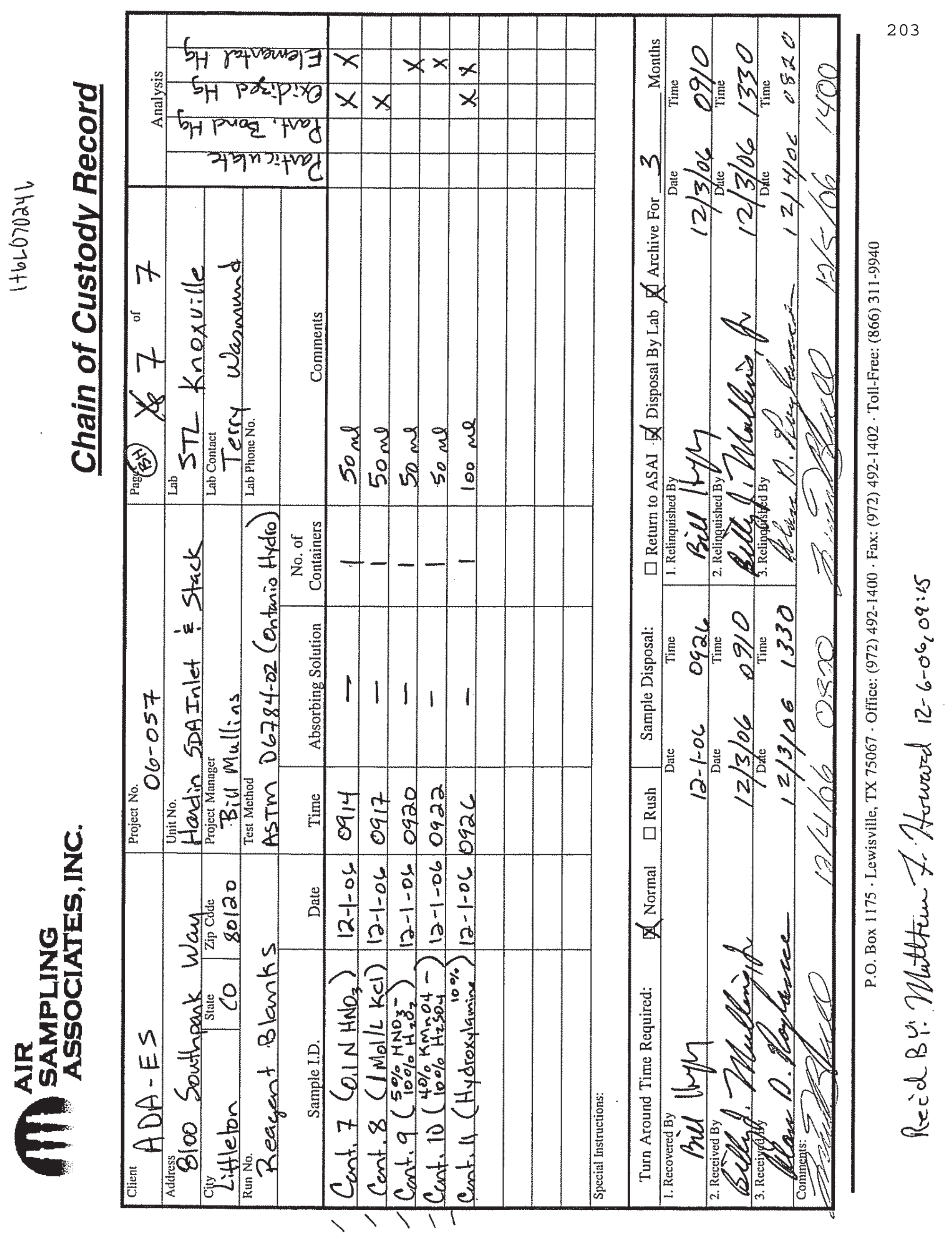




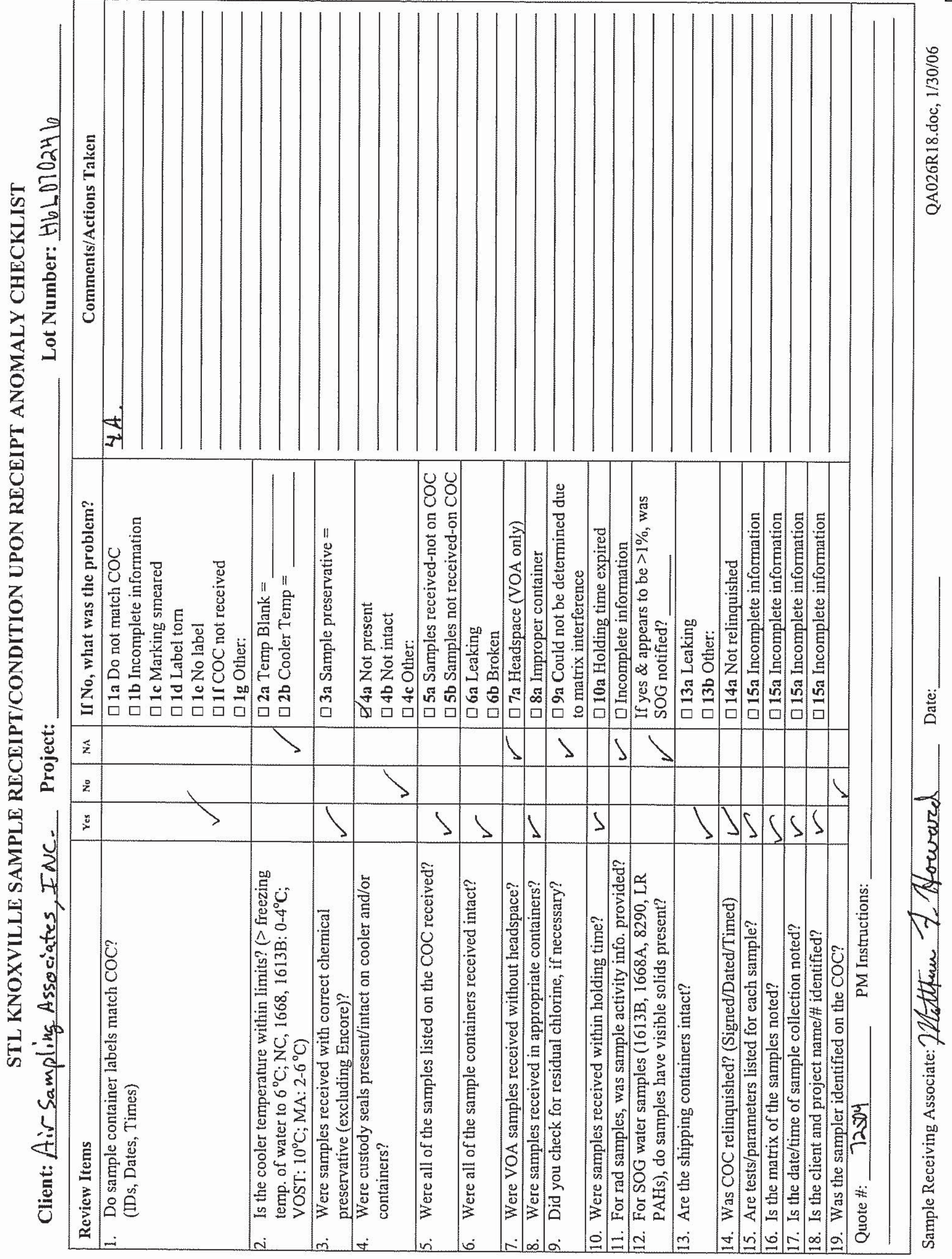


H6L140394 Analytical Report............................................. 1

Sample Receipt Documentation ....................................... 26

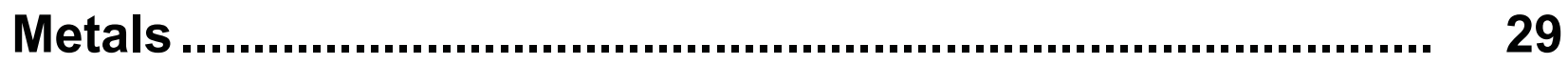

Sample Results ...................................................... 30

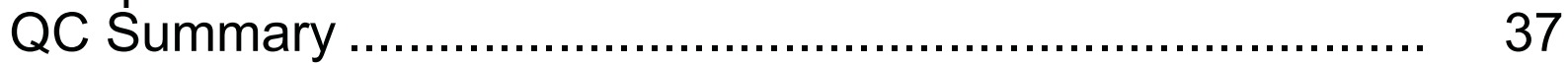

Quality Control Results Mercury .................................. 42

Raw Data Mercury …................................................ 53

Miscellaneous Data................................................... 67

Wet Chemistry .............................................................. 73

Sample Summary ................................................... 74

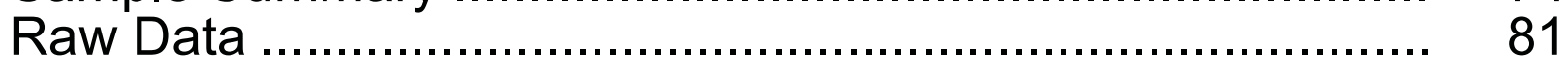

Sample Receipt Documentation ......................................... 87

Total Number of Pages ...................................................... $\quad 89$ 


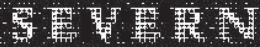 \\ STL Knoxville \\ 5815 Middlebrook Pike \\ Knoxville, TN 37921 \\ Tel: 8652913000 Fax: 8655844315 \\ www.stl-inc.com}

\section{ANALYTICAL REPORT}

PROJECT NO. 06-057

Hardin, Montana

Lot \#: H6L140394

\section{Bill Hefley}

Air Sampling Associates, Inc.

407-2B Holford's Prairie Road

Lewisville, TX 75056

SEVERN TRENT LAABORATORIES, INC.

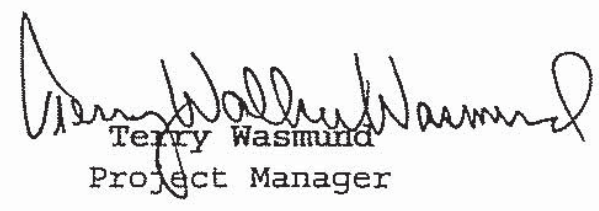

January 8, 2007 


\section{QC DATA ASSOCIATION SUMMARY}

H6L140394

Sample Preparation and Analysis Control Numbers

\begin{tabular}{|c|c|c|c|c|c|}
\hline SAMPLE\# & MATRIX & $\begin{array}{l}\text { ANALYTICAL } \\
\text { METHOD }\end{array}$ & $\begin{array}{l}\text { LEACH } \\
\text { BATCH \# }\end{array}$ & $\begin{array}{l}\text { PREP } \\
\text { BATCH \# }\end{array}$ & MS RUN\# \\
\hline \multirow[t]{2}{*}{001} & AIR & CFR60A 5 & & 6354260 & \\
\hline & AIR & ASTM D6784-02 & & 7002029 & \\
\hline \multirow[t]{2}{*}{002} & AIR & CFR60A 5 & & 6354260 & \\
\hline & AIR & ASTM D6784-02 & & 7002029 & \\
\hline \multirow[t]{2}{*}{003} & AIR & CFR60A 5 & & 6354260 & \\
\hline & AIR & ASTM D6784-02 & & 7002029 & \\
\hline \multirow[t]{2}{*}{004} & AIR & CFR60A 5 & & 6354260 & \\
\hline & AIR & ASTM D6784-02 & & 7002029 & \\
\hline \multirow[t]{2}{*}{005} & AIR & CFR60A 5 & & 6354260 & \\
\hline & AIR & ASTM D6784-02 & & 7002029 & \\
\hline \multirow[t]{2}{*}{006} & AIR & CFR60A 5 & & 6354260 & \\
\hline & AIR & ASTM D6784-02 & & 7002029 & \\
\hline
\end{tabular}




\section{ANALYTICAL METHODS SUMMARY}

H6L140394

$\begin{array}{ll}\text { PARAMETER } & \begin{array}{l}\text { ANALYTICAL } \\ \text { METHOD }\end{array} \\ \begin{array}{l}\text { Mercury (Ontario Hydro) } \\ \text { Particulate Emissions }\end{array} & \begin{array}{l}\text { ASTM D6784-02 } \\ \text { CFR60A } 5\end{array} \\ \text { References: } & \\ \text { ASTM Annual Book Of ASTM Standards. } & \\ \text { CFR60A "Test Methods", 40 CFR, Part 60, Appendix A, July 1, } 1993 .\end{array}$




\section{SAMPLE SUMMARY}

H6L140394

WO \# SAMPLE\# CLIENT SAMPLE ID

JLHWK 001 HARDIN SDA DUCT+STACK RB FILTER 66

$\begin{array}{llll}\text { JLHXD } & 002 & \text { HARDIN SDA DUCT+STACK RB FILTER } 67\end{array}$

JLHXF 003 HARDIN SDA DUCT+STACK RB FILTER 68

JLHXH 004 HARDIN SDA DUCT+STACK RB FILTER A120

JLHXK 005 HARDIN SDA DUCT+STACK RB FILTER A121

JLHXM 006 HARDIN SDA DUCT+STACK RB FILTER A122
SAMPLED SAMP

DATE

$12 / 11 / 0616: 00$

$12 / 11 / 06 \quad 16: 00$

$12 / 11 / 0616: 00$

$12 / 11 / 0616: 00$

$12 / 11 / 0616: 00$

$12 / 11 / 0616: 00$

NOTB (S) :

- The analytical results of the samples listed above are presented on the following pages.

- All calculations are performed before rounding to avoid round-off errors in calculated results.

- Results noted as "ND" were not detected at or above the stated limit.

- This repart must not be reproduced, exeept in fuil, without the written approval of the laboratory.

- Results for the following paramesers are never reported on a dry weight basis: color, corrosivity, density, nashpoint, ignitability, Jayers, odor,

paint fitter test, pH, porosity pressure, reactivity, redox potential, specific gravity, spot tests, solids, solubility, temperature, viscosity, and weight. 


\section{PROJECT NARRATIVE H6L140394}

The results reported herein are applicable to the samples submitted for analysis only.

This report shall not be reproduced except in full, without the written approval of the laboratory.

\section{The original chain of custody documentation is included with this report.}

\section{Sample Receipt}

Custody seals were not present upon sample receipt at STL Knoxville.

The chain of custody documentation did not list the sample IDs for the filter samples. For $\log$ in purposes the sample ID will be taken from the filter itself.

\section{Quality Control and Data Interpretation}

Unless otherwise noted, all holding times and QC criteria were met and the test results shown in this report meet all applicable NELAC requirements.

\section{Metals}

These stack gas samples were prepared and analyzed using STL Knoxville standard operating procedure KNOX-IP-0006 which is based on ASTM Method D6784-02, "Standard Test Method for Elemental, Oxidized, Particle-Bound and Total Mercury in Flue Gas Generated from Coal-Fired Stationary Sources (Ontario Hydro Method)" with modifications from EPA Method 29. SW-846 Method 7470A, as incorporated in STL Knoxville standard operating procedure KNOX-MT-0009, was used to perform the final instrument analysis.

Acid digestion was performed on the front half particulate filter fractions using $\mathrm{HNO}_{3}$, $\mathrm{HCl}$ and $\mathrm{HF}$. After digestion, the $\mathrm{HF}$ was sequestered using $\mathrm{H}_{3} \mathrm{BO}_{3}$ followed by another heating cycle. These digestates were adjusted to final volume and a portion was digested for CVAA analysis in order to determine the particle-bound mercury. Results were calculated using the following equation:

STL Knoxville maintains the following certifieations, approvals and accreditations: Arkansas DEQ Cert. \#05-043-0, Calirornia DHS ELAP Cert. \#2423, Colorado DPHE, Connecticut DPH Cert. \#PH-0223, Florida DOH Cert. \#E87177,

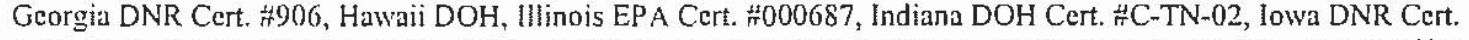
\#375, Kansas DHE Cert. 萿E-10349, Kentucky DEP Lab ID 490101 , Louisiana DEQ Cert. \#03079, Louisiana DOHH Cert. 莎LA030024, Maryland DHMH Cert. \#277, Massachusets DEP Cert. \#M-TN009, Michigan DEQ Lab ID \#9933, New Jersey DEP Cert. \#TN(001, New York DOH Lab \#10781, North Carolina DPH Lab ID DEHNR Cert. \#64, Ohio EPA VAP Cert. \#CL0059, Oklahoma DEQ ID \#9415, Pennsylvania DEP Cert. \#68-00576, South Carolina DHEC Lab ID \#84001001, Tennessee DOH Lab ID \#02014, Utah DOH Cert. \# QUAN3, Virginia DGS Lab ID $\# 00165$, Washington DOE Lab $\# \mathrm{Cl} 20$, West Virginia DEP Cert. $\# 345$, Wisconsin DNR Lab ID $\# 998044300$, Naval Faeilitics Engineering Service Center and USDA Soil Pemit $\stackrel{m}{\pi} \mathrm{S}-46424$. This list of approvals is subject to change and does not imply that laboratory certification is available for all parameters reported in this environmental sample data report. 


\section{PROJECT NARRATIVE H6L140394}

$H g, u g=(H g, u g / L) *($ Micr. Digestate Volume, $L) *\left(\frac{\text { Final Volume Hg Digestate }(m L)}{\text { Volume Micr. Digestate Used }(m L)}\right) *$ Bench Dilution

Thimbles were received instead of filters for three of the front half samples. Each thimble was divided in two because the digestion vessels are not large enough for the entire thimble. Acid digestion was performed on each half of the front half particulate thimbles using $\mathrm{HNO}_{3}, \mathrm{HCl}$ and $\mathrm{HF}$. Heating was done by microwave. For each of the two digestates, the $\mathrm{HF}$ was sequestered using $\mathrm{H}_{3} \mathrm{BO}_{3}$ followed by another microwave cycle, and the final volume for each was adjusted to $100 \mathrm{~mL}$. These digestates were combined (total volume of $200 \mathrm{~mL}$ ) and a portion was digested for CVAA analysis in order to determine the particle-bound mercury. Results were calculated using the following equation:

$H g, u g=(H g, u g / L) *($ Micr. Digestate Volume, L $) *\left(\frac{\text { Final Volume Hg Digestate }(m L)}{\text { Volume Micr. Digestate Used }(m L)}\right) *$ Bench Dilution

Please note that the dilution factor reported on the sample result form for the front half samples is actually a combination of preparation factors and bench dilution factors.

\section{Wet Chemistry}

The measurement of the mass of particulate matter trapped by the particulate filter and probe rinse derived from an M-5 sampling train was performed using SOP number KNOX-WC-0006 (based on EPA Methods 0050 and 5). Microfiber filters, Petri dishes, and $150 \mathrm{~mL}$ beakers are carefully inspected and tare weighed to constant weight. After sample collection, the filters are dried, and then carefully weighed to constant weight to determine the mass of particulate matter trapped on the filters. The acetone probe rinse solution is evaporated to dryness, and then weighed to constant weight to determine the total particulate mass collected in the rinse. The total particulate mass collected by an M5 train is the sum of the particulate filter and the acetone probe rinse residue weights.

Filter thimbles and disks that were not prepared by STL Knoxville were submitted for analysis. The reported weight is the gross weight, which includes the total weight of the

STL Knoxville maintains the following certifications, approvals and accreditations: Arkansas DEQ Cert. \#05-043-0, California DHS ELAP Cert. \#2423, Colorado DPHE, Connecticut DPH Cert. HPH-0223, Florida DOH Cert. \#E87177, Georgia DNR Cert. \#906, Hawaii DOH, Illinois EPA Cert. ${ }^{\circ} 000687$, Indiana DOH Cert. \#C-TN-02, lowa DNR Cert. \#375, Kansas DHE Cert. \#E-10349, Kentucky DEP Lab ID Cert. 蒜LA030024, Maryland DHMH Cert. \#277, Massachusetts DEP Cert. New Jersey DEP Cert. \#TN001, New York DOH Lab \#10781, North Carolina DPH Lab ID \#21705, North Carolina DEHNR Cert. $\# 64$, Ohio EPA VAP Cert. \#CL0059, Oklahoma DEQ ID \#9415, Pennsylvania DEP Cert. \#68-00576, South Carolina DHEC Lab ID \#84001001, Tennessee DOH Lab ID $\# 02014$, Utah DOH Cert. \# QUAN3, Virginia DGS

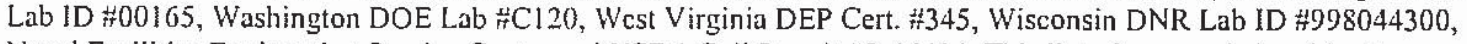
Naval Facilities Enginecring Service Center and USDA Soil Permit ${ }^{H S}$-46424. This list of approvals is subject to change and docs not inply that laboratory certification is available for all parameters reported in this environmental sample data report. 


\section{PROJECT NARRATIVE \\ H6L 140394}

filter and the entrained particulate material. Unfortunately, the laboratory information system will not report sufficient digits to obtain a precise net particulate weight when the tare weight is subtracted from the gross weight. The actual raw data is tabulated below for the user's convenience.

\begin{tabular}{|l|l|l|c|}
\hline $\begin{array}{c}\text { Lot-Sample } \\
\text { Number } \\
\text { Work Order } \\
\text { Number }\end{array}$ & \multicolumn{1}{|c|}{ Client ID } & $\begin{array}{c}\text { Gross } \\
\text { Weight } \\
\text { Average } \\
\text { (mg) }\end{array}$ \\
\hline H6L140394-001 & JLHWK1AC & HARDIN SDA DUCT+STACK RB FILTER 66 & 2855.6 \\
\hline H6L140394-002 & JLHXD1AC & HARDIN SDA DUCT+STACK RB FILTER 67 & 2934.5 \\
\hline H6L140394-003 & JLHXF1AC & HARDIN SDA DUCT+STACK RB FILTER 68 & 2510.3 \\
\hline H6L140394-004 & JLHXH1AC & $\begin{array}{l}\text { HARDIN SDA DUCT+STACK RB FILTER } \\
\text { A120 }\end{array}$ & 360.0 \\
\hline H6L140394-005 & JLHXK1AC & $\begin{array}{l}\text { HARDIN SDA DUCT+STACK RB FILTER } \\
\text { A121 }\end{array}$ & 359.8 \\
\hline H6L140394-006 & JLHXM1AC & $\begin{array}{l}\text { HARDIN SDA DUCT+STACK RB FILTER } \\
\text { A122 }\end{array}$ & 359.4 \\
\hline
\end{tabular}

STL Knoxville maintains the following certifications, approvals and acereditations: Arkansas DEQ Cert. California DHS ELAP Cert. \$2423, Colorado DPHE, Connecticut DPH Cert. \#PH-0223, Florida DOH Cert. \#E87177,

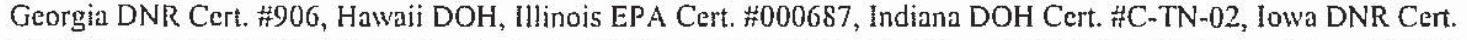
$\# 375$, Kansas DHE Cert. \#E-10349, Kentucky DEP Lab ID \#90101, Louisiana DEQ Cert. \#03079, Louisiana DOHH Cert. 呍030024, Maryland DHMH Cert. \#277, Massachusetts DEP Cert. \#M-TN009, Michigan DEQ Lab ID \#9933, New Jersey DEP Cert. \#TN001, New York DOH Lab \#10781, North Carolina DPH Lab ID \#21 705, North Carolina DEHNR Cert. \#64, Ohio EPA VAP Cert. ${ }_{7}$ CL0059, Oklahoma DEQ ID \#9415, Pennsylvania DEP Cert. \#68-00576, South Carolina DHEC Lab ID \#S4001001, Tennessec DOH Lab ID \#02014, Utah DOH Cert. \# QUAN3, Virginia DGS Lab ID \#00165, Washington DOE Lab \#C120, West Virginia DEP Cert. \#345, Wisconsin DNR Lab ID Naval Facilities Engineering Service Center and USDA Soil Permit \#S-46424. This list of approvals is subject to change and does not imply that laboratory certification is available for all parameters reported in this environmental sample data report. 
Sample Data Summary 
Air Sampling Associates, Inc.

Client Sample ID: BARDIN SDA DUCT+STACK RB FILTER 66

TOTAL Metals

Lot-Sample \#. . : H6L140394-001

Date Sampled...: 12/11/06

Date Received. .: 12/12/06

REPORTING

LIMIT

UNITS

0.020 ug
Dilution Factor: 0.2
ND
RESULT

Prep Batch 拉: 7002029

Mercury
Matrix.... . : AIR

PREPARATION - WORK ANALYSIS DATE ORDER \# 
Air Sampling Associates, Inc.

Client Sample ID: HARDIN SDA DUCT+STACK RB FILTER 67

TOTAL Metals

Lot-Sample \#. . . : H6L140394-002

Date Sampled...: 12/11/06

PARAMETER

RESULT

Prep Batch \#. . : 7002029

Mercury

ND
Date Received. .: 12/12/06

REPORTING LIMIT UNITS METHOD

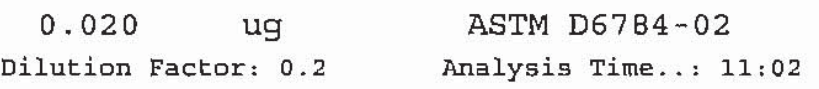

Matrix...... AIR

PREPARATION - WORK ANALYSIS DATE ORDER \#

01/02-01/05/07 JLHXD1AA MDL. ........ 0.012 
Air Sampling Associates, Inc.

Client Sample ID: HARDIN SDA DUCT+STACK RB FILTER 68

TOTAL Metals

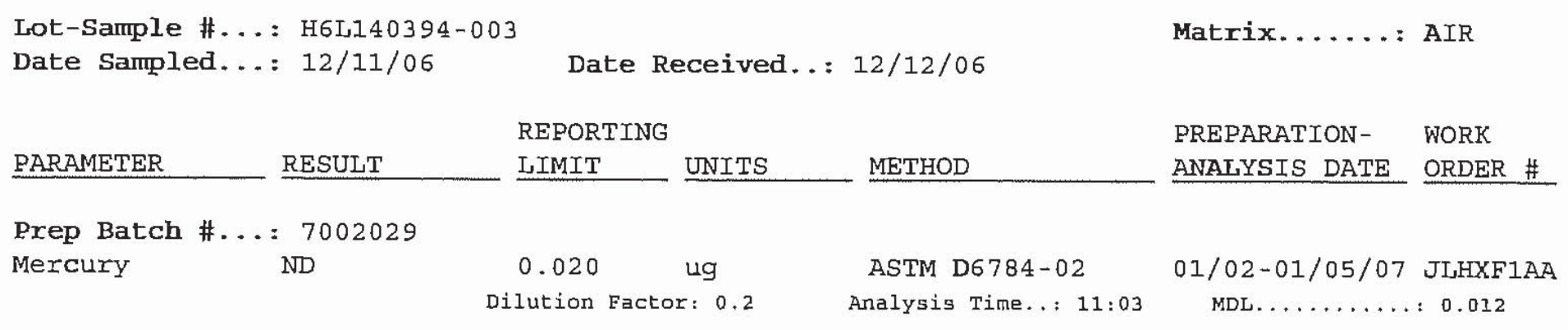


Air Sampling Associates, Inc.

Client Sample ID: HARDIN SDA DUCT+STACK RB FILTER AZ20

TOTAL Metals

Lot-Sample \#...: H6L140394-004

Date Sampled. . : 12/11/06

PARAMETER

Prep Batch \#...: 7002029

Mercury
ND
Date Received. .: 12/12/06

REPORTING

LIMIT

UNITS

0.010 ug
Dilution Factor: 0.1
Matrix..... AIR

PREPARATION- WORK ANALYSIS DATE ORDER \#

ASTM D6784-02 01/02-01/05/07 JLHXH1AA Analysis Time..: 11:05 
Air Sampling Associates, Inc.

Client Sample ID: HARDIN SDA DUCT+STACK RB FILTER A121

TOTAL Metals

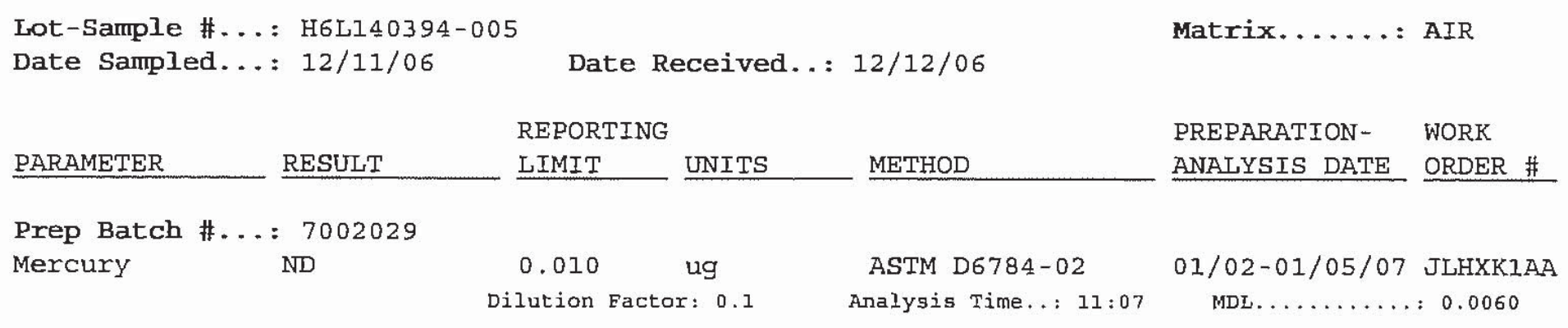


Air Sampling Associates, Inc.

Client Sample ID: HARDIN SDA DUCT+STACK RB FILTKR Al22.

TOIAL Metals

Irot-Sample \#...: H6L140394-006

Date Sampled...: 12/11/06

Date Received. .: 12/12/06

REPORTING

LIMIT

RESULT

Prep Batch \#...: 7002029

Mercury
ND

0.010 ug

Dilution Factor: 0.1
Matrix...... AIR

PREPARATION- WORK ANALYSIS DATE ORDER \#

01/02-01/05/07 JLHXM1AA MDL......... 0.0060 
METHOD BLANK REPORT

TOTAL Metals

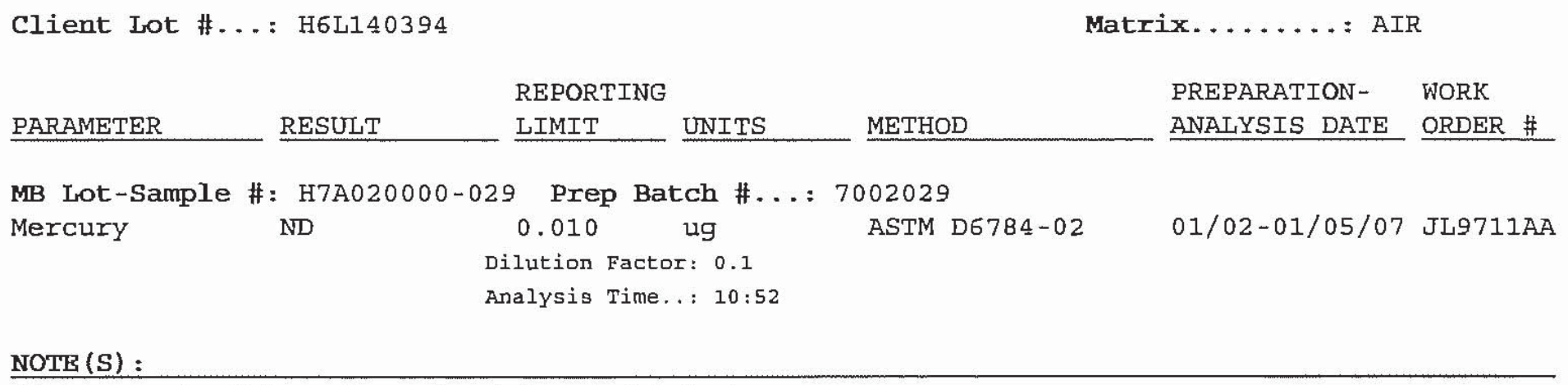

Calculations are performed before rounding to avoid round-off errors in calculated results. 


\section{LABORATORY CONTROL SAMPLE EVALUATION RBPORT}

TOTAL Metals

Lot-Sample \#. . : : H6L140394

PARAMETER

Mercury

$$
\text { PERCENT RECOVERY }
$$

RECOVERY LIMITS

$98 \quad(80-120)$

103

$$
(80-120) 4.8 \quad(0-20)
$$

Dilution Factor: 0.1
Matrix........ A AIR

PREPARATION- PREPANALYSIS DATE BATCH \#

$01 / 02-01 / 05 / 077002029$

$01 / 02-01 / 05 / 07 \quad 7002029$

ASTM D6784-02

Analysis Time... 10:54

NOTE (S) :

Calculations are performed before rounding to avoid round-off errors in calculated results. 


\section{LABORATORY CONTROL SAMPLE DATA REPORT}

TOTAL Metals

Lot-Sample \#. . . : H6L140394

\begin{tabular}{|c|c|c|c|c|}
\hline & SPIKE & MEASURED & & PERCNT \\
\hline PARAMETER & AMOUNT & AMOUNT & UNITS & RECVRY \\
\hline \multirow[t]{2}{*}{ Mercury } & 0.500 & 0.491 & $\mathrm{ug}$ & 98 \\
\hline & 0.500 & 0.515 & ug & 103 \\
\hline
\end{tabular}

Matrix........ AIR

PREPARATION- PREP ANALYSIS DATE BATCH \# 01/02-01/05/07 7002029 01/02-01/05/07 7002029 ASTM D6784-02

4.8 A5TM D67B4-02 Analysis Time..: 10:54

NOTB (S) :

Calculations are performed before rounding to avoid round-off errors in calculated results. 


\section{STL Knoxville}

Mercury Data Reporting Form

Post Digestion Spike

Units: $u g / L(p p b)$

Instrument ID: Leeman HydraAA $\mathrm{Hg}$

Data File Name: H010507. PRN

\begin{tabular}{|c|c|c|c|c|}
\hline Element & $\begin{array}{c}\text { PDS } \\
\text { JLHWKA }\end{array}$ & $\begin{array}{c}\text { Original Sample } \\
\text { JLHWK }\end{array}$ & Spike Added & $\begin{array}{c}\text { Percent } \\
\text { Recovery }\end{array}$ \\
\hline $\mathrm{Hg}$ & 1.04 & $\mathrm{ND}$ & 1.0 & 104 \\
\hline
\end{tabular}


Sample Data Summary 
Air Sampling Associates, Inc.

client Sample ID: HARDIN SDA DUCT+STACK RB FILTER 66

General Chemistry

Lot-Sample \#...: H6L140394-001 Work Order \#...: JLHWK

Date Sampled...: 12/11/06
Date Received..: 12/12/06
Matrix........ AIR

PREPARATION- PREP ANALYSIS DATE BATCH \#
PARAMETER

Particulates (total) $\mathbf{2 8 6 0}$
RESULT
RL 0.50 UNITS mg Dilution Factor: 1
METHOD

CFR60A 5 MDL . . . . . . : 0.50 
Air Sampling Associates, Inc.

Client Sample ID: HARDIN SDA DUCT+STACK RB FILTKR 67

General Chenistry

Lot-Sample \#...: H6L140394-002 Work Order \#...: JLHXD

Date Sampled...: 12/11/06
Date Received..: 12/12/06
Matrix........: AIR

PREPARATION- PREP ANALYSIS DATE BATCH \#
PARAMETER

RESULT

2930
RL UNITS

0.50 mg

Dilution Factor: 1
METHOD

CFR60A 5 $12 / 19-12 / 20 / 066354260$ 
Air Sampling Associates, Inc.

Client Sample ID: HARDIN SDA DUCT+STACK RB FILTER 68

General Chemistry

Jot-Sample \#...: H6L140394-003 Fork Order \#...: JLHXF

Date Sampled...: $12 / 11 / 06$
Date Received..: 12/12/06
Matrix....... AIR

PREPARATION- PREP ANALYSIS DATE BATCH \#
PARAMETER

RESULT

Particulates (total) 2510

RL

0.50

Dilution Factor:
UNITS

mg

METHOD

CFR60A 5 
Air Sampling Associates, Inc.

Client Sample ID: HARDIN SDA DUCT+STACK RB FILTKR A120

\section{General Chemistry}

Lot-Sample \#...: H6L140394-004

Date Sampled...: 12/11/06 work Order \#...: Л ЛHXH

Date Received. : : 12/12/06
Matrix........ AIR

PREPARATION- PREP ANALYSIS DATE BATCH \#
PARAMETER

Particulates (total) 360
RESULT

RL 0.50

Dilution Factor: 1
METHOD CFR6 0A 5 
Air Sampling Associates, Inc.

Client Sample ID: HARDIN SDA DUCT+STACK RB PILTER A121

General Chemistry

Lot-Sample \#...: H6L140394-005 work Order \#...: JLHXK

Date Sampled...: 12/11/06
Date Received... 12/12/06
Matrix....... AIR

PREPARATION- PREP ANALYSIS DATE BATCH \#
PARAMETER

Particulates (total) $\mathbf{3 6 0}$
RESULT

RL UNITS

$0.50 \mathrm{mg}$

Dilution Factor: 1
METHOD

CFR60A 5

MDL. ......... 0.50 
Air Sampling Associates, Inc.

client Sample ID: HARDIN SDA DUCT+STACK RB FILTKR A122

General Chemistry

Lot-Sample \#. . : H6L140394-006

Date Sampled...: 12/11/06
Work Order \#...: JLHXM

Date Received. .: 12/12/06
Matrix.......: AIR

PREPARATION - PREP ANALYSIS DATE BATCH \# 12/19-12/20/06 $\overline{6354260}$
PARAMETER

RESULT

Particulates (total) 359
RL_ــــــINS

$0.50 \mathrm{mg}$

Dilution Factor: 1
METHOD

CFR60A 5 
Sample Receipt Documentation 


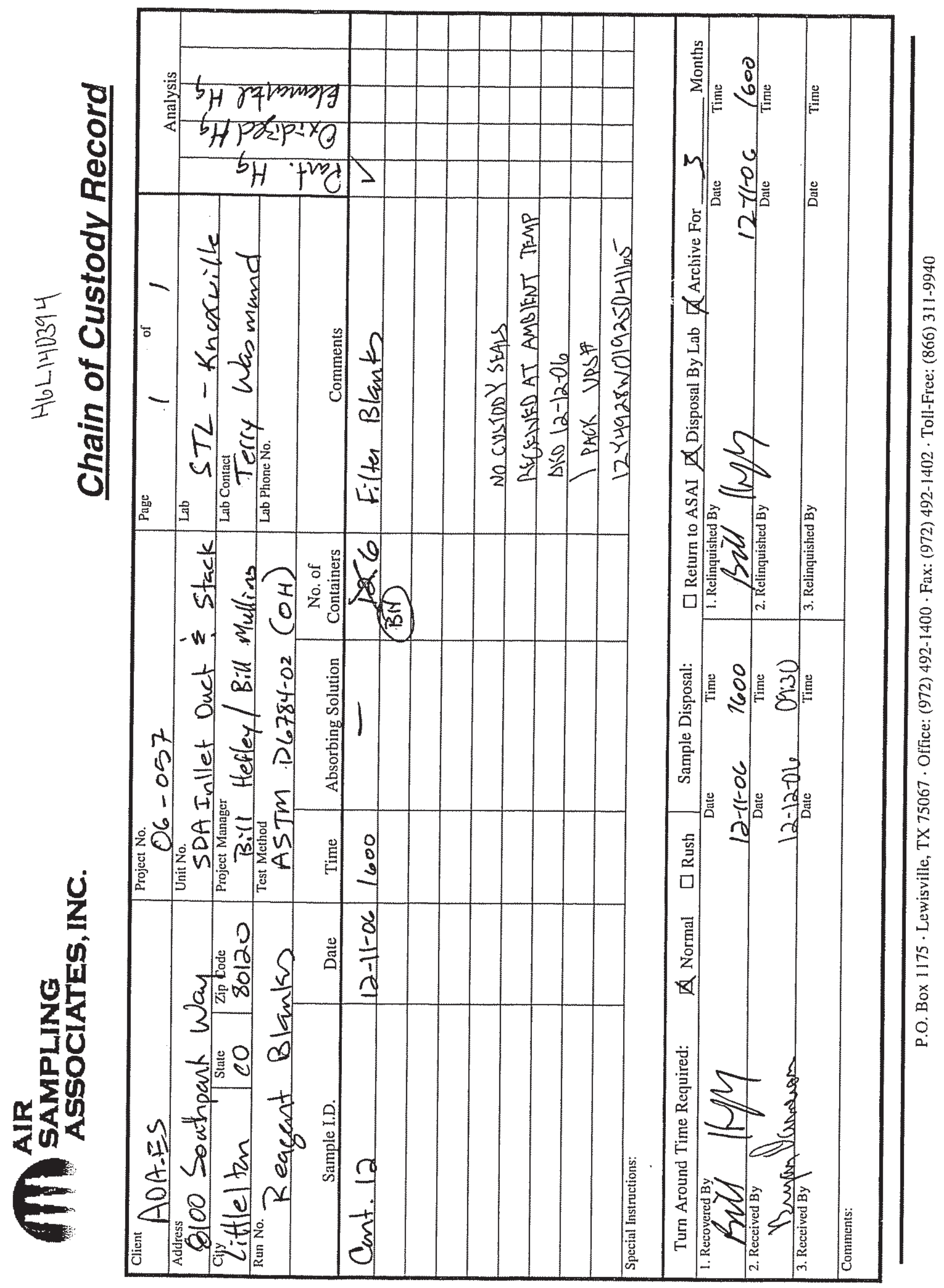




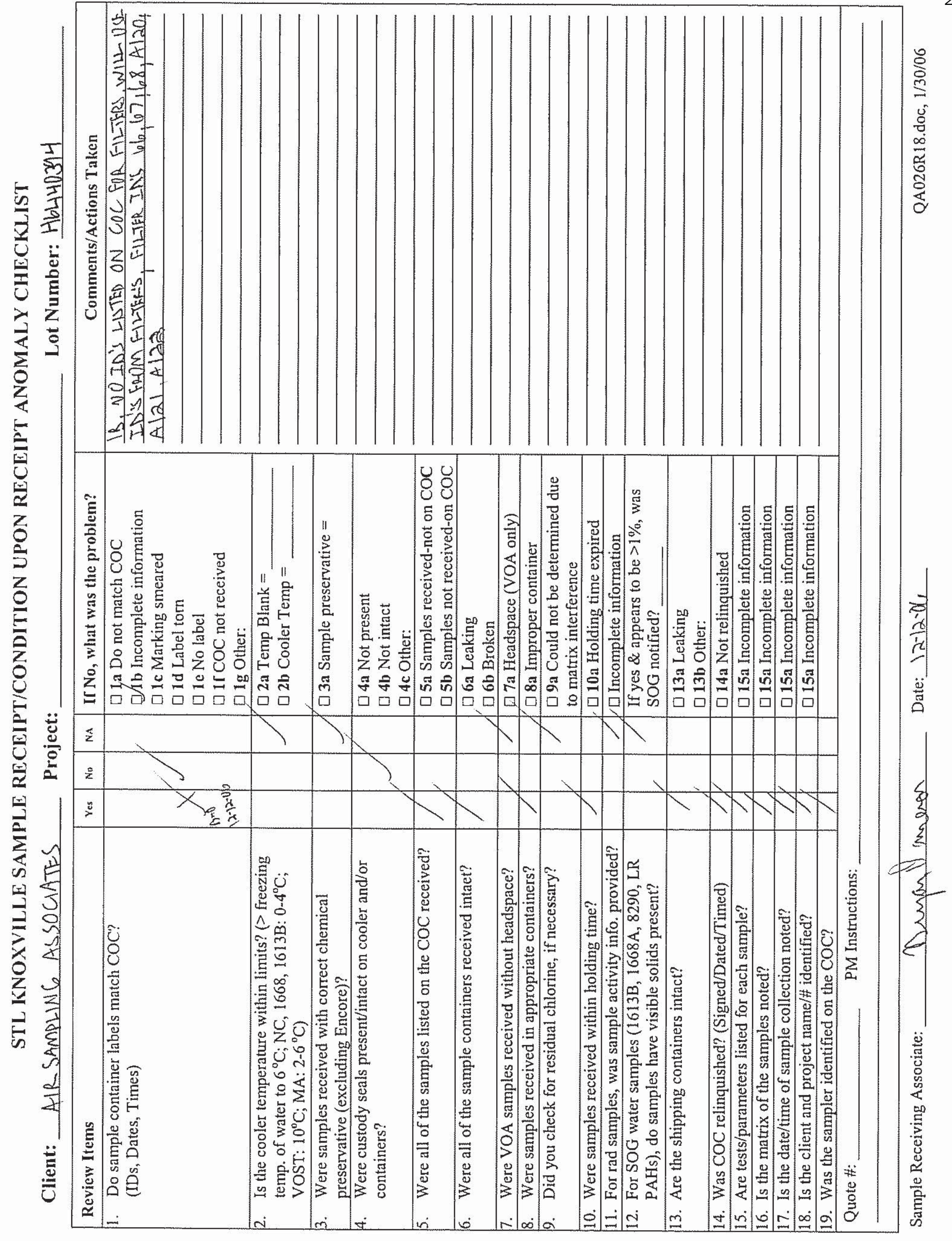


Metals 
Sample Results 
Air Sampling Associates, Inc.

Client Sample ID: HARDIN SDA DUCT+STACK RB FILTBR 66

TOTAL Metals

Lot-Sample \#...: H6L140394-001

Date Sampled...: 12/11/06

PARAMETER

Prep Batch \#...: 7002029

Mercury
$\mathrm{ND}$
Date Received. .: 12/12/06

REPORTING

LIMIT

UNITS

0.020 ug
Dilution Factor: 0.2
Matrix...... AIR

PREPARATION- WORK ANALYSIS DATE ORDER \#

ASTM D6784-02 01/02-01/05/07 JLHWKIAA

Analysis Time..: 10:58 
Air Sampling Associates, Inc.

Client Sample ID: HARDIN SDA DUCT+STACK RB FILTKR 67

\section{TOTAL Metals}

Lot-Sample \#...: H6L140394-002

Date Sampled...: 12/11/06

Date Received. .: 12/12/06

REPORTING

LIMIT

UNITS

METHOD

Prep Batch \#...: 7002029

Mercury

\begin{abstract}
ND
\end{abstract}

\author{
0.020 \\ ug \\ Dilution Factor: 0.2
}

Matrix...... AIR

PREPARATION - WORK ANALYSIS DATE ORDER \#

01/02-01/05/07 JLHXD1AA

MDL......... : 0.012 
Air Sampling Associates, Inc.

Client Sample ID: HARDIN SDA DUCT+STACK RB FILTER 68

TOTAL Metals

Lot--Sample \#...: H6L140394-003

Date Sampled...: 12/11/06

Date Received..: 12/12/06

REPORTING LIMIT

RESULT

Prep Batch \#...: 7002029

Mercury
$\mathrm{ND}$
$0.020 \quad \mathrm{ug}$

Dilution Factor: 0.2
Matrix...... A AIR

PREPARATION - WORK ANALYSIS DATE ORDER \#

01/02-01/05/07 JLHXF1AA MDL......... 0.012 
Air Sampling Associates, Inc.

Client Sample ID: HARDIN SDA DUCT+STACK RB FILTER A120

\section{TOTAL Metals}

Lot-Sample \#...: H6LI40394-004

Date Sampled...: 12/11/06

Date Received. .: 12/12/06

REPORTING

LIMIT

RESULT

Prep Batch \#...: 7002029

Mercury
$\mathrm{ND}$

$0.010 \mathrm{ug}$

Dilution Factor: 0.1
Matrix...... : AIR

PREPARATION- WORK ANALYSIS DATE ORDER \# METHOD

ASTM D6784-02

01/02-01/05/07 JLHXH1AA Analysis Time..: 11:05 
Air Sampling Associates, Inc.

Client Sample ID: HARDIN SDA DUCT+STACK RB PILTER Al21

TOTAL Metals

Lot-Sample \#...: H6L140394-005

Date Sampled...: 12/11/06

PARAMETER

Prep Batch \#...: 7002029

Mercury
$\mathrm{ND}$
Date Received..: 12/12/06

REPORTING LIMIT UNITS $0.010 \quad \mathrm{ug}$
Dilution Factor: 0.1
Matrix....... AIR

PREPARATION - WORK ANALYSIS DATE ORDER \# ASTM D6784-02 01/02-01/05/07 JLHXK1AA Analysis Time..: 11:07 
Air Sampling Associates, Inc.

Client Sample ID: HARDIN SDA DUCT+STACK RB FILTKR A122

TOTAL Metals

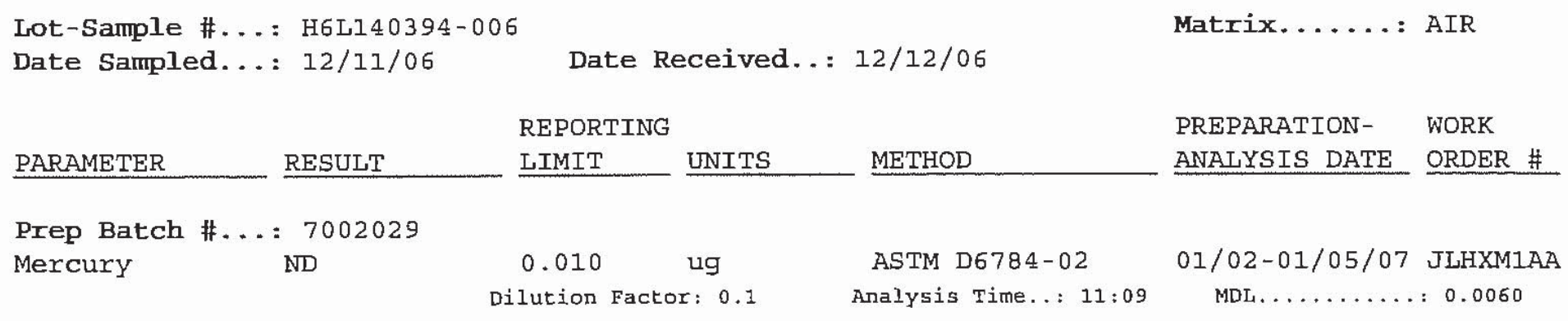


QC Summary 
METHOD BLANK REPORT

TOTAL Metals

Client Lot \#...: H6L140394

\begin{tabular}{lll} 
& Matrix......... AIR \\
REPORTING & PREPARATION- & WORK \\
LIMIT & METTS & MNALYSIS DATE ORDER \# \\
\hline
\end{tabular}

MB Lot-Sample \#: H7A020000-029 Prep Batch \#. . : 7002029

Mercury

ND

0.010

ug

ASTM D6784-02

01/02-01/05/07 JL9711AA

Dilution Factor: 0.1

Analysis Time..: 10:52

NOTE (S) :

Calculations are performed before rounding to avoid round-off errors in calculated results. 


\section{LABORATORY CONTROL SAMPLE EVALUATION RBPORT}

\section{TOTAL Metals}

Lot-Sample \#. . : H6L140394

\begin{tabular}{|c|c|c|c|c|}
\hline \multirow{3}{*}{$\frac{\text { PARAMETER }}{\text { Mercury }}$} & \multirow{2}{*}{$\begin{array}{l}\text { PERCENT } \\
\text { RECOVERY } \\
\end{array}$} & RECOVERY & \multicolumn{2}{|c|}{ RPD } \\
\hline & & LIMITS & RPD & LIMITS \\
\hline & 98 & $(80-120)$ & & \\
\hline & 103 & $(80-120)$ & 4.8 & $(0-20)$ \\
\hline
\end{tabular}

\begin{tabular}{|c|c|}
\hline & Matrix....... A AIR \\
\hline METHOD & $\begin{array}{l}\text { PREPARATION- } \\
\text { ANALYSIS DATE } \\
\text { AATCH \# }\end{array}$ \\
\hline ASTM D6784-02 & $01 / 02-01 / 05 / 07 \overline{7002029}$ \\
\hline ASTM D $6784-02$ & $01 / 02-01 / 05 / 077002029$ \\
\hline
\end{tabular}

Matrix....... AIR

PREPARATION- PREPANALYSIS DATE BATCH \# $01 / 02 \sim 01 / 05 / 077002029$

NOTE (S) :

Calculations are performed before rounding to avoid round-off errors in calculated results. 


\section{LABORATORY CONTROL SAMPLE DATA RBPORT}

\section{TOTAL Metals}

Lot-Sample \#. . .: H6L140394

Matrix....... : AIR

\begin{tabular}{|c|c|c|c|c|c|c|c|c|}
\hline $\mathrm{AB}$ & $\begin{array}{l}\text { SPIKE } \\
\text { AMOUNT }\end{array}$ & $\begin{array}{l}\text { MEASURED } \\
\text { AMOUNT }\end{array}$ & UNITS & $\begin{array}{l}\text { PERCNT } \\
\text { RECVRY }\end{array}$ & RPD & METHOD & $\begin{array}{l}\text { PREPARATION- } \\
\text { ANALYSIS DATE }\end{array}$ & $\begin{array}{l}\text { PREP } \\
\text { BATCH \# }\end{array}$ \\
\hline \multirow[t]{2}{*}{ ercur } & 0.500 & 0.491 & $\mathrm{ug}$ & 98 & & ASTM D6784-02 & $\overline{01 / 02-01 / 05 / 07}$ & 7002029 \\
\hline & 0.500 & 0.515 & ug & 103 & 4.8 & ASTM D6784-02 & $01 / 02-01 / 05 / 07$ & 7002029 \\
\hline
\end{tabular}

NOTT $(S)$ :

Calculations are performed before rounding to avoid round-off errors in calculated results. 


\section{STL Knoxville}

\section{Mercury Data Reporting Form}

Post Digestion Spike

Units: $\mathrm{ug} / \mathrm{L}(\mathrm{ppb})$

Instrument ID: Leeman HydraAA Hg

Data File Name: H010507. PRN

\begin{tabular}{|c|c|c|c|c|}
\hline Element & $\begin{array}{c}\text { PDS } \\
\text { JLHWKA }\end{array}$ & $\begin{array}{c}\text { Original Sample } \\
\text { JLHWK }\end{array}$ & Spike Added & $\begin{array}{c}\text { Percent } \\
\text { Recovery }\end{array}$ \\
\hline $\mathrm{Hg}$ & 1.04 & $\mathrm{ND}$ & 1.0 & 104 \\
\hline
\end{tabular}


Quality Control Results Mercury 


\section{STL Knoxville}

Mercury Data Reporting Form

Initial Calibration Verification

Units: ug/L (ppb)

Instrument ID: Leeman HydraAA Hg An

Data File Name: M010507.PRN

\begin{tabular}{|c|c|c|c|c|c|c|c|c|c|c|c|c|c|}
\hline \multirow[b]{2}{*}{ Elem } & \multirow[b]{2}{*}{$\begin{array}{l}\text { True } \\
\text { Conc }\end{array}$} & \multicolumn{2}{|c|}{$\begin{array}{c}\text { Ck2icy } \\
1 / 5 / 2007 \\
10: 05 \mathrm{AM}\end{array}$} & \multirow[b]{2}{*}{ Found } & \multirow[b]{2}{*}{$\begin{array}{c}\% \\
\operatorname{Rec}\end{array}$} & \multirow[b]{2}{*}{ Found } & \multirow[b]{2}{*}{$\begin{array}{l}\% \\
\operatorname{Rec}\end{array}$} & \multirow[b]{2}{*}{ Found } & \multirow[b]{2}{*}{$\begin{array}{l}\% \\
\mathrm{Rec}\end{array}$} & \multirow[b]{2}{*}{ Found } & \multirow[b]{2}{*}{$\begin{array}{c}\% \\
\operatorname{Rec}\end{array}$} & \multirow[b]{2}{*}{ Found } & \multirow[b]{2}{*}{$\begin{array}{l}\% \\
\operatorname{Rec}\end{array}$} \\
\hline & & Found & $\begin{array}{l}\% \\
\operatorname{Rec}\end{array}$ & & & & & & & & & & \\
\hline $\mathrm{Hg}$ & 2.5 & 2.48 & 99.2 & & & & & & & & & & \\
\hline
\end{tabular}




\section{STL Knoxville}

\section{Mercury Data Reporting Form}

Continuing Calibration Verification

Units: $u g / L(p p b)$

Instrument ID: Leeman HydraAA Hg An

Data File Name: M010507.PRN

\begin{tabular}{|c|c|c|c|c|c|c|c|c|c|c|c|c|c|}
\hline \multirow[b]{2}{*}{ Elem } & \multirow[b]{2}{*}{$\begin{array}{l}\text { True } \\
\text { Conc } \\
\end{array}$} & \multicolumn{2}{|c|}{$\begin{array}{c}\text { Ck3ccv } \\
1 / 5 / 2007 \\
10: 11 \mathrm{AM}\end{array}$} & \multicolumn{2}{|c|}{$\begin{array}{c}\mathrm{Ck} 3 \mathrm{ccV} \\
1 / 5 / 2007 \\
10: 30 \mathrm{AM} \\
\end{array}$} & \multicolumn{2}{|c|}{$\begin{array}{c}\mathrm{Ck} 3 \mathrm{ccv} \\
1 / 5 / 2007 \\
10: 48 \mathrm{AM}\end{array}$} & \multicolumn{2}{|c|}{$\begin{array}{c}\text { Ck3ccv } \\
1 / 5 / 2007 \\
11: 11 \mathrm{AM}\end{array}$} & \multicolumn{2}{|c|}{$\begin{array}{c}\text { Ck3ccv } \\
1 / 5 / 2007 \\
11: 35 \mathrm{AM}\end{array}$} & \multicolumn{2}{|c|}{$\begin{array}{c}\text { Ck3ccv } \\
1 / 5 / 2007 \\
11: 59 \mathrm{AM}\end{array}$} \\
\hline & & Found & $\begin{array}{c}\% \\
\operatorname{Rec}\end{array}$ & Found & $\begin{array}{c}\% \\
\operatorname{Rec}\end{array}$ & Found & $\begin{array}{c}\% \\
\operatorname{Rec}\end{array}$ & Found & $\begin{array}{l}\% \\
\operatorname{Rec}\end{array}$ & Found & $\begin{array}{c}\% \\
\operatorname{Rec}\end{array}$ & Found & $\begin{array}{c}\% \\
\operatorname{Rec}\end{array}$ \\
\hline $\mathrm{Hg}$ & 5.0 & 4.83 & 96.6 & 4.80 & 96.0 & 4.86 & 97.2 & 4.84 & 96.8 & 4.83 & 96.6 & 4.90 & 98.0 \\
\hline
\end{tabular}




\section{STL Knoxville}

\section{Mercury Data Reporting Form}

Continuing Calibration Verification
Units: ug/L (ppb)

Instrument ID: Leeman HydraAA Hg An

Data File Name: M010507.PRN

\begin{tabular}{|c|c|c|c|c|c|c|c|c|c|c|c|c|c|}
\hline \multirow[b]{2}{*}{ Elem } & \multirow[b]{2}{*}{$\begin{array}{l}\text { True } \\
\text { Conc }\end{array}$} & \multicolumn{2}{|c|}{$\begin{array}{c}\text { Ck3ccv } \\
1 / 5 / 2007 \\
12: 10 \mathrm{PM}\end{array}$} & \multirow[b]{2}{*}{ Found } & \multirow[b]{2}{*}{$\begin{array}{l}\% \\
\operatorname{Rec}\end{array}$} & \multirow[b]{2}{*}{ Found } & \multirow[b]{2}{*}{$\begin{array}{l}\% \\
\operatorname{Rec}\end{array}$} & \multirow[b]{2}{*}{ Found } & \multirow[b]{2}{*}{$\begin{array}{c}\% \\
\operatorname{Rec}\end{array}$} & \multirow[b]{2}{*}{ Found } & \multirow[b]{2}{*}{$\begin{array}{l}\% \\
\operatorname{Rec}\end{array}$} & \multirow[b]{2}{*}{ Found } & \multirow[b]{2}{*}{$\begin{array}{c}\% \\
\operatorname{Rec}\end{array}$} \\
\hline & & Found & $\begin{array}{c}\% \\
\operatorname{Rec}\end{array}$ & & & & & & & & & & \\
\hline $\mathrm{Hg}$ & 5.0 & 4.88 & 97.6 & & & & & & & & & & \\
\hline
\end{tabular}




\section{STL Knoxville}

Mercury Data Reporting Form

Contract Required Detection Limit Standard(s) Units: ug/L (

Instrument ID: Leeman HydraAA Hg An

Data File Name: M010507.PRN

\begin{tabular}{|c|c|c|c|c|c|c|c|c|c|c|c|c|c|}
\hline \multirow[b]{2}{*}{ Elem } & \multirow[b]{2}{*}{$\begin{array}{l}\text { True } \\
\text { Conc }\end{array}$} & \multicolumn{2}{|c|}{$\begin{array}{c}\text { CRA } \\
1 / 5 / 2007 \\
10: 09 \text { AM }\end{array}$} & \multirow[b]{2}{*}{ Found } & \multirow[b]{2}{*}{$\begin{array}{l}\% \\
\operatorname{Rec}\end{array}$} & \multirow[b]{2}{*}{ Found } & \multirow[b]{2}{*}{$\begin{array}{l}\% \\
\operatorname{Rec}\end{array}$} & \multirow[b]{2}{*}{ Found } & \multirow[b]{2}{*}{$\begin{array}{l}\% \\
\operatorname{Rec}\end{array}$} & \multirow[b]{2}{*}{ Found } & \multirow[b]{2}{*}{$\begin{array}{l}\% \\
\operatorname{Rec}\end{array}$} & \multirow[b]{2}{*}{ Found } & \multirow[b]{2}{*}{$\begin{array}{l}\% \\
\operatorname{Rec}\end{array}$} \\
\hline & & Found & $\begin{array}{l}\% \\
\operatorname{Rec}\end{array}$ & & & & & & & & & & \\
\hline $\mathrm{Hg}$ & 0.2 & 0.19 & 95.0 & & & & & & & & & & \\
\hline
\end{tabular}




\section{STL Knoxville}

\section{Mercury Data Reporting Form}

Initial Calibration Blank(s)

Units: ug/L (ppb)

Instrument ID: Leeman HydraAA Hg An

Data File Name: M010507.PRN

\begin{tabular}{|c|c|c|c|c|c|c|c|c|c|c|c|c|c|}
\hline \multirow[b]{2}{*}{ Elem } & \multirow{2}{*}{$\begin{array}{c}\text { Reporting } \\
\text { Limit }\end{array}$} & \multicolumn{2}{|c|}{$\begin{array}{c}\mathrm{ICB} \\
1 / 5 / 2007 \\
10: 07 \mathrm{AM}\end{array}$} & \multirow[b]{2}{*}{ Found } & \multirow[b]{2}{*}{ Flag } & \multirow[b]{2}{*}{ Found } & \multirow[b]{2}{*}{ Flag } & \multirow[b]{2}{*}{ Found } & \multirow[b]{2}{*}{ Flag } & \multirow{2}{*}{ Found } & \multirow[b]{2}{*}{ Flag } & \multirow[b]{2}{*}{ Found } & \multirow[b]{2}{*}{ Flag } \\
\hline & & Found & Flag & & & & & & & & & & \\
\hline $\mathrm{Hg}$ & 0.1 & 0.06 & U & & & & & & & & & & \\
\hline
\end{tabular}


STL Knoxville

Mercury Data Reporting Form

Continuing Calibration Blank(s)

Units: $u g / L(p p b)$

Instrument ID: Leeman HydraAA Hg Ana

Data File Name: M010507.PRN

\begin{tabular}{|c|c|c|c|c|c|c|c|c|c|c|c|c|c|}
\hline \multirow[b]{2}{*}{ Elen } & \multirow{2}{*}{$\begin{array}{c}\text { Reporting } \\
\text { Limit }\end{array}$} & \multicolumn{2}{|c|}{$\begin{array}{c}\text { Cklccb } \\
1 / 5 / 2007 \\
10: 1+\mathrm{AM}\end{array}$} & \multicolumn{2}{|c|}{$\begin{array}{c}\text { CkI ccb } \\
1 / 5 / 2007 \\
10: 32 \mathrm{AM}\end{array}$} & \multicolumn{2}{|c|}{$\begin{array}{c}\text { Cklccb } \\
1 / 5 / 2007 \\
10: 50 \mathrm{AM}\end{array}$} & \multicolumn{2}{|c|}{$\begin{array}{c}\text { Cklccb } \\
1 / 5 / 2007 \\
11: 13 \mathrm{AM}\end{array}$} & \multicolumn{2}{|c|}{$\begin{array}{c}\mathrm{Cklccb} \\
1 / 5 / 2007 \\
11: 37 \mathrm{AM}\end{array}$} & \multicolumn{2}{|c|}{$\begin{array}{c}\text { Cklccb } \\
1 / 5 / 2007 \\
12: 01 \mathrm{PM}\end{array}$} \\
\hline & & Found & Flag & Found & Flag & Found & Flag & Found & Flag & Found & Flag & Found & Flag \\
\hline $\mathrm{Hg}$ & 0.1 & 0.06 & U & 0.06 & U & 0.06 & $\mathrm{U}$ & 0.06 & $\mathrm{U}$ & 0.06 & $\mathrm{U}$ & 0.06 & U \\
\hline
\end{tabular}




\section{STL Knoxville}

Mercury Data Reporting Form

Continuing Calibration Blank(s)
Units: $u g / L(p p b)$

Instrument ID: Leeman HydraAA Hg An

Data File Name: M010507.PRN

\begin{tabular}{|c|c|c|c|c|c|c|c|c|c|c|c|c|c|}
\hline \multirow[b]{2}{*}{ Elem } & \multirow[b]{2}{*}{$\begin{array}{l}\text { Reporting } \\
\text { Limit }\end{array}$} & \multicolumn{2}{|c|}{$\begin{array}{c}\text { Cklccb } \\
1 / 5 / 2007 \\
12: 12 \text { PM }\end{array}$} & & & & & & & & & & \\
\hline & & Found & Flag & Found & Flag & Found & Flag & Found & Flag & Found & Flag & Found & Flag \\
\hline $\mathrm{Hg}$ & 0.1 & 0.06 & U & & & & & & & & & & \\
\hline
\end{tabular}




\section{STL Knoxville}

\section{Mercury Data Reporting Form}

Post Digestion Spike

Units: $u g / L(p p b)$

Instrument ID: Leeman HydraAA Hg

Data File Name: H010507. PRN

\begin{tabular}{|c|c|c|c|c|}
\hline Element & $\begin{array}{c}\text { PDS } \\
\text { JLHWKA }\end{array}$ & $\begin{array}{c}\text { Original Sample } \\
\text { JLHWK }\end{array}$ & Spike Added & $\begin{array}{c}\text { Percent } \\
\text { Recovery }\end{array}$ \\
\hline $\mathrm{Hg}$ & 1.04 & $\mathrm{ND}$ & 1.0 & 104 \\
\hline
\end{tabular}




\section{STL Knoxville}

\section{Mercury Data Reporting Form}

Instrument Detection Limits

IDL Completion Date:12/14/2006
Units: ug/L (ppb)

Instrument ID: Leeman HydraAA Hg An

Data File Name: M010507.PRN

\begin{tabular}{|c|c|c|c|}
\hline Element & $\begin{array}{c}\text { Wavelength } \\
(\mathrm{nm})\end{array}$ & $\begin{array}{c}\text { Reporting } \\
\text { Limit }\end{array}$ & IDL \\
\hline $\mathrm{Hg}$ & 253.70 & 0.1 & 0.06 \\
\hline
\end{tabular}




\section{STL Knoxville}

\section{Sample ID Nomenclature}

The sample ID consists of 5 alpha-numeric characters followed by a suffix in the 6 th position that designates the sample type:

\begin{tabular}{cll} 
Suffix & & \multicolumn{1}{c}{ Sample Type: } \\
\cline { 1 - 1 } B & & Method Blank \\
C & & Laboratory Control Sample \\
L & & Laboratory Control Sample Duplicate \\
S & & Matrix Spike \\
D & Matrix Spike Duplicate \\
X & Sample Duplicate \\
P & Serial Dilution \\
A & Post Digestion Spike \\
Z\# & Dilution; \#= Dilution Factor
\end{tabular}


Raw Data Mercury 


\section{STL Knoxville}

Mercury Analysis Cover Sheet

Scanned

\begin{tabular}{|r|c|c|c|}
\hline Analyst & $D(\omega)$ & Instrument & Leeman Hydra AA Mercury Analyzer \\
\hline Run Date & $1 / 5 / 07$ & Chart Name & M0/0507 \\
\hline
\end{tabular}

\begin{tabular}{|c|c|c|c|}
\hline \multicolumn{2}{|r|}{ Daily Maintenance } & \multicolumn{2}{|r|}{ As Needed Maintenance } \\
\hline D & Check tubing. & $\square$ & Adjust/change Hg lamp. \\
\hline \multirow[t]{3}{*}{ a } & \multirow[t]{3}{*}{$\begin{array}{l}\text { Check maintenance schedule } \\
\text { flags. }\end{array}$} & $\square$ & Clean or replace optical cell. \\
\hline & & $\square$ & Lubricate pump and autosampler arm. \\
\hline & & $\square$ & Change the drying tube. \\
\hline
\end{tabular}


Instrument Upload

Started Fri Jan 5. 11:40:01 2007 by WILBURND

Run Log - Page 1 :

Data File: UPL\$KNX_DATA_ROOT: $<$ LHG $>$ M010507.PRN; 1

\begin{tabular}{|c|c|c|c|c|c|c|c|}
\hline \# & WorkOrder & Dilution & Date & Time & Batch & Lot & Instrument \\
\hline & $----\cdots-$ & 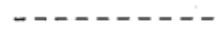 & $-\cdots-1-1-1$ & $-\cdots-\cdots$ & --- & & \\
\hline 1 & CK2ICV & 1.00 & $05-J A N-2007$ & $10: 05: 28$ & & & HG \\
\hline 2 & ICB & 1.00 & $05-J A N-2007$ & $10: 07: 24$ & & & HG \\
\hline 3 & CRA & 1.00 & $05-J A N-2007$ & $10: 09: 20$ & & & HG \\
\hline 4 & $\mathrm{CK} 3 \mathrm{CCV}$ & 1.00 & $05-J A N-2007$ & $10: 11: 09$ & & & HG \\
\hline 5 & $\mathrm{CK} 1 \mathrm{CCB}$ & 1.00 & $05-J A N-2007$ & $10: 14: 08$ & & & HG \\
\hline 6 & MDL1 & 1.00 & $05-J A N-2007$ & $10: 15: 56$ & & . & HG \\
\hline 7 & MDL2 & 1.00 & $05-J A N-2007$ & $10: 18: 14$ & & & HG \\
\hline 8 & MDL3 & 1.00 & $05-J A N-2007$ & $10: 20: 05$ & & & HG \\
\hline 9 & MDL4 & 1.00 & 05 -JAN-2007 & $10: 21: 52$ & & & HG \\
\hline 10 & MDL5 & 1.00 & $05-J A N-2007$ & $10: 23: 50$ & & & HG \\
\hline 11 & MDL6 & 1.00 & $05-J A N-2007$ & $10: 25: 46$ & & & HG \\
\hline 12 & MDL7 & 1.00 & $05-J A N-2007$ & $10: 27: 54$ & & & HG \\
\hline 13 & $\mathrm{CK} 3 \mathrm{CCV}$ & 1.00 & $05-J A N-2007$ & $10: 30: 21$ & & & HG \\
\hline 14 & $\mathrm{CK} \perp \mathrm{CCB}$ & 1.00 & $05-J A N-2007$ & $10: 32: 18$ & & & HG \\
\hline 15 & MDL1 & 1.00 & $05-J A N-2007$ & $10: 34: 09$ & & & HG \\
\hline 16 & MDL2 & 1.00 & $05-J A N-2007$ & $10: 36: 01$ & & & HG \\
\hline 17 & MDL3 & 1.00 & $05-J A N-2007$ & $10: 37: 53$ & & & HG \\
\hline 18 & MDL4 & 1.00 & $05-J A N-2007$ & $10: 40: 01$ & & & HG \\
\hline 19 & MDL5 & 1.00 & $05-J A N-2007$ & $10: 41: 58$ & & & HG \\
\hline 20 & MDL6 & 1.00 & $05-J A N-2007$ & $10: 43: 56$ & & & HG \\
\hline 21 & MDL7 & 1.00 & $05-J A N-2007$ & $10: 46: 04$ & & & HG \\
\hline 22 & $\mathrm{CK} 3 \mathrm{CCV}$ & 1.00 & $05-J A N-2007$ & $10: 48: 21$ & & & HG \\
\hline 23 & CK1CCB & 1.00 & $05-J A N-2007$ & $10: 50: 10$ & & & HG \\
\hline 24 & JL971B & 0.10 & $05-J A N-2007$ & $10: 52: 11$ & 7002029 & $\mathrm{H} 7 \mathrm{~A} 020000$ & HG \\
\hline 25 & JL971C & 0.10 & $05-J A N-2007$ & $10: 54: 39$ & 7002029 & $\mathrm{H} 7 \mathrm{~A} 020000$ & HG \\
\hline 26 & JL971L & 0.10 & $05-J A N-2007$ & $10: 56: 37$ & 7002029 & $\mathrm{H} 7 \mathrm{~A} 020000$ & HG \\
\hline 27 & JLHWK & 0.20 & $05-J A N-2007$ & $10: 58: 24$ & 7002029 & H6L140394 & HG \\
\hline 28 & JLHWKA & 0.20 & $05-J A N-2007$ & $11: 00: 17$ & 7002029 & H6L140394 & HG \\
\hline 29 & JLHXD & 0.20 & 05-JAN-2007 & $11: 02: 10$ & 7002029 & H6L140394 & HG \\
\hline 30 & JLHXF & 0.20 & $05-J A N-2007$ & $11: 03: 58$ & 7002029 & H6L140394 & HG \\
\hline 31 & JLHXH & 0.10 & $05-J A N-2007$ & $11: 05: 48$ & 7002029 & H6L140394 & HG \\
\hline 32 & JLHXK & 0.10 & $05-J A N-2007$ & $11: 07: 43$ & 7002029 & H6L140394 & HG \\
\hline 33 & JLHXM & 0.10 & $05-J A N-2007$ & $11: 09: 45$ & 7002029 & H6L140394 & HG \\
\hline 34 & $\mathrm{CK} 3 \mathrm{CCV}$ & 1.00 & $05-J A N-2007$ & $11: 11: 32$ & & & HG \\
\hline 35 & $\mathrm{CK} 1 \mathrm{CCB}$ & 1.00 & $05-J A N-2007$ & $11: 13: 21$ & & & HG \\
\hline 36 & JL97PB & 0.10 & $05-J A N-2007$ & $11: 15: 29$ & 7002025 & $\mathrm{H} 7 \mathrm{~A} 020000$ & HG \\
\hline 37 & JL97PC & 0.10 & $05-J A N-2007$ & $11: 17: 27$ & 7002025 & $\mathrm{H} 7 \mathrm{~A} 020000$ & HG \\
\hline 38 & JL97PL & 0.10 & $05-J A N-2007$ & $11: 19: 38$ & 7002025 & $\mathrm{H} 7 \mathrm{~A} 020000$ & HG \\
\hline 39 & JKIWA & 0.10 & $05-J A N-2007$ & $11: 21: 27$ & 7002025 & H6L070246 & HG \\
\hline 40 & JKIWAA & 0.10 & $05-J A N-2007$ & $11: 23: 14$ & 7002025 & H6L070246 & HG \\
\hline 41 & JK1XD & 0.10 & $05-J A N-2007$ & $11: 25: 13$ & 7002025 & H6L070246 & HG \\
\hline 42 & JK1X5 & 0.10 & $05-J A N-2007$ & $11: 27: 13$ & 7002025 & H6L070246 & HG \\
\hline 43 & JK105 & 0.10 & $05-J A N-2007$ & $11: 29: 00$ & 7002025 & H6L070246 & HG \\
\hline 44 & JKI1F & 0.50 & $05-J A N-2007$ & $11: 31: 17$ & 7002025 & H6L070246 & HG \\
\hline
\end{tabular}




$\begin{array}{ll}\text { Instrument Upload } & \text { Run Log - Page } 2: \\ : & \text { Started Fri Jan } 5 \text { 11:40:01 } 2007 \text { by WILBURND } \\ : & \text { Data File: UPL\$KNX_DATA_ROOT:<LHG>M010507.PRN; } 1\end{array}$

\begin{tabular}{|c|c|c|c|c|c|c|c|}
\hline \# & WorkOrder & Dilution & Date & Time & Batch & Lot & Erume \\
\hline . & 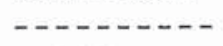 & $\ldots$ & - - & $\ldots$ & $-\ldots$ & 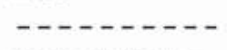 & \\
\hline 45 & JK112 & 0.10 & $05-J A N-2007$ & $11: 33: 35$ & 7002025 & H6L070246 & HG \\
\hline 46 & $\mathrm{CK} 3 \mathrm{CCV}$ & 1.00 & $05-J A N-2007$ & $11: 35: 37$ & & & HG \\
\hline 47 & CK1CCB & 1.00 & $05-J A N-2007$ & $11: 37: 34$ & & & HG \\
\hline 48 & JK12A & 0.10 & $05-J A N-2007$ & $11: 39: 43$ & 7002025 & H6L070246 & HG \\
\hline 49 & JL97RB & 0.25 & $05-J A N-2007$ & $11: 41: 55$ & 7002026 & $\mathrm{H} 7 \mathrm{~A} 020000$ & HG \\
\hline 50 & JL97RC & 0.25 & $05-J A N-2007$ & $11: 44: 14$ & 7002026 & $\mathrm{H} 7 \mathrm{~A} 020000$ & HG \\
\hline 51 & JKIWF & 0.60 & 05 -JAN-2007 & $11: 46: 04$ & 7002026 & H6L070246 & HG \\
\hline 52 & JK1WFS & 0.60 & $05-J A N-2007$ & $11: 47: 54$ & 7002026 & H6L070246 & HG \\
\hline 53 & JKIWFD & 0.60 & $05-J A N-2007$ & $11: 49: 46$ & 7002026 & H6L070246 & HG \\
\hline 54 & JKIXR & 0.75 & 05 -JAN-2007 & $11: 51: 57$ & 7002026 & H6L 070246 & HG \\
\hline 55 & JK10A & 0.63 & $05-J A N-2007$ & $11: 53: 48$ & 7002026 & H6L070246 & HG \\
\hline 56 & JK107 & 0.65 & $05-J A N-2007$ & $11: 55: 35$ & 7002026 & H6L070246 & HG \\
\hline 57 & JK11L & 0.63 & $05-J A N-2007$ & $11: 57: 33$ & 7002026 & H6L070246 & HG \\
\hline 58 & $\mathrm{CK} 3 \mathrm{CCV}$ & 1.00 & $05-J A N-2007$ & $11: 59: 24$ & & & HG \\
\hline 59 & $\mathrm{CK} \perp \mathrm{CCB}$ & 1.00 & 05-JAN-2007 & $12: 01: 12$ & & & HG \\
\hline 60 & JK114 & 0.68 & $05-J A N-2007$ & $12: 03: 28$ & 7002026 & H6L070246 & HG \\
\hline 61 & JK12G & 0.24 & $05-J A N-2007$ & $12: 06: 15$ & 7002026 & H6L070246 & HG \\
\hline 62 & JK11FZ5 & 0.50 & $05-J A N-2007$ & $12: 08: 04$ & 7002025 & H6L070246 & HG \\
\hline 63 & $\mathrm{CK} 3 \mathrm{CCV}$ & 1.00 & $05-J A N-2007$ & $12: 10: 04$ & & & HG \\
\hline 64 & CK1CCB & 1.00 & $05-J A N-2007$ & $12: 12: 32$ & & & HG \\
\hline
\end{tabular}




\section{STL Knoxville}

\section{Method Information}

Method Name: Ontario Hydro

Instrument Calibrated Every ? Hours: 24

Correlation Coefficient $>/=: \quad 0.995$

CRA Required?: Yes

Duplicate RPD: 20
Element: $\mathrm{Hg}$

Chart Number: m010507.prn

Number of Calibration Standards: 6

Run ICV Immediately After Calibration?: Yes

Number of Samples Between CCVs: 10

Calculate Duplicates by CLP or SW-846?: SW-846 Protocol

$\begin{array}{rr}\text { CRA Recovery Range: } & 30.0 \\ \text { ICV Recovery Range: } & 10.5 \\ \text { CCV Recovery Range: } & 20.5 \\ \text { LCS Recovery Range: } & 20.5 \\ \text { MS/MSD Recovery Range: } & 20.5\end{array}$

Letter to Signify Blanks: B

Letter to Signify LCS's: C

Letter to Signify LCSD's: L

Letter to Signify Dilutions: $\quad Z$

Letter to Signify Duplicates: $\mathrm{X}$

Letter to Signify MS's: S

Letter to Signify MSD's: D

Letters to Signify MSA's: MSA+

Number of Additions: $\quad 3$
CRA Conc. (ug/L): $\quad 0.2$

ICV Conc. (ug/L): $\quad 2.5$

CCV Conc. (ug/L): 5

LCS Spike Level (ug/L): 5

MS Spike Level (ug/L): $\quad 1$

Calibration Standard Concentrations (ug/L)

Standard 1 Conc: 0.1

Standard 5 Conc: 5

ICB/CCB Check Level (ug/L): 0.099
Prep Blank Check Level (ug/L): $0.099 \quad$ Reporting Limit (ug/L): 0.1

IDL (ug/L): $0.06 \quad$ Date of IDL: $12 / 14 / 2006$ 
STL Knoxville Mercury Data Review Checklist

Method: Ontario Hydro

\begin{tabular}{|c|c|l|c|c|c|c|c|}
\hline Analyst: & \multicolumn{3}{|c|}{ DKW } & Instrument: & \multicolumn{2}{|c|}{ Leeman Hydra AA Mercury Analyzer } \\
\hline Run Date: & Jan-05-07 & Calibration Number: & 1 & Chart Name: & m010507.prn & Element: & Hg \\
\hline
\end{tabular}

\section{A. Calibration/Instrument Run QC}

1. Instrument calibrated every 24 hours?

2. Instrument calibrated using a minimum of a blank and 5 standards?

3. Do standard absorbances increase as standard concentrations increase?

4. Instrument calibrated with standards at the SOP levels?

5. Correlation coefficient greater than or equal to 0.995 ?

6. ICV analyzed directly after calibration?

7. ICV within specified control limits?

8. ICB analyzed immediately after ICV?

9. ICB within acceptable range?

10. CRA analyzed at the beginning of the run?

11. CRA results within acceptable range?

12. All CCV's within specified control limits?

13. All CCB's within acceptable range?

14. All CCB's analyzed immediately after CCV's?

B. Client Sample and QC Sample Results

1.Were all sample results within the linear range of the instrument?

2.Were there any samples with results more negative than Report Limit?

3.Were samples analyzed within the holding time?

\begin{tabular}{|c|c|c|c|l|} 
& N/A & Yes & No & Comments \\
\hline 5 & & $X$ & & Calibration was 2 hours and 22 minutes long \\
\hline & & $X$ & & \\
& & $X$ & & \\
\hline$?$ & & $X$ & & \\
\hline & & $X$ & & Correlation coefficient was 0.9996 \\
\hline & & $X$ & & \\
\hline & & $X$ & & \\
\hline & $X$ & & \\
\hline & & $X$ & & \\
\hline & & $X$ & & \\
\hline & $X$ & & \\
\hline & & $X$ & & \\
\hline
\end{tabular}

C. Preparation/Matrix QC

1.Was one method blank prepared for each batch?

2. Were all method blanks less than the specified level?

3.Was an LCS prepared for each batch?

4. Were all LCS recoveries within $Q C$ limits?

5. Were matrix spikes, sample duplicates and/or postdigestion spikes run at required frequency?

6. Were all matrix spike recoveries within $\mathrm{QC}$ limits?

7.Were post-digestion spike recoveries within $Q C$ limits?

8. Were duplicate RPDs within QC limits?

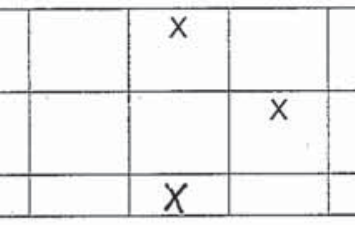

D. Other

1. Current IDL data on file?

2. Current MDL data on file?

3. Are all nonconformances documented appropriately?

4. Were all project specific instructions followed?

Analyst: DXW

Date: $01 / 05 / 07$

Analyst Comments: 


\section{STL KNOXVILLE}

\section{INTERMEDIATE STANDARD - CALIBRATION}

Stock Std Name: PLHG4-2Y

\section{Element: $\mathrm{Hg}$}

Stock Source: Spex

Stock Std Lot \#: $12-43 \mathrm{HG}$

Stock Std Expiration Date: $4 / 30 / 07$

Stock Conc.: $1000 \mathrm{ppm}$

$\mathrm{HNO}_{3}$ Lot $\mathrm{B} 44032$
Stock Vol (ml)/100ml: $1 \mathrm{ml}$

Date Prepared: $1 / 2 / 07$

Expiration Date: 2 2/2/0

QC Check: M010307

Initials: $K N D$

Matrix: $2 \% \mathrm{HNO}_{3}$

\section{WORKING STANDARD - CALIBRATION}

One ml Intermediate Standard $/ 100 \mathrm{ml} 0.15 \%$ Concentrated Nitric Acid

1. I-CAL $3425-6-1$

2. I-CAL $3425-6-2$

3. I-CAL $3425-6-3$

4. .I-CAL

5. .I-CAL

6. .I-CAL

7. .I-CAL

8. .I-CAL

9. I- $\mathrm{CAL}$

10..I-CAL

11..I-CAL

12..I-CAL

13. I-CAL

14. I-CAL

15. I-CAL

16. I-CAL

17. I-CAL

18. .I-CAL

19. .I-CAL

20. I-CAL

21. I-CAL

22. I-CAL

23. I-CAL

24. I-CAL

25. I-CAL
Initials /CND

Initials KND

Initials DFW

Initials

Initials

Initials

Initials

Initials

Initials

Initials

Initials

Initials

Initials

Initials

Initials

Initials

Initials

Initials

Initials

Initials

Initials

Initials

Initials

Initials

Initials
Date: $1 / 2 / 07$ Exp. Date: $1 / 3 / 07$ Date: $1 / 3 / 07$ Exp. Date: $1 / 4 / 07$ Date: 010407 Exp. Date: 010507

Date:

Date:

Exp. Date:

Date:

Exp. Date:

Exp. Date:

Date:

Exp. Date:

Date:

Date:

Date:

Date:

Date:

Date:

Date:

Date:

Date:

Date:

Date:

Date:

Date:

Date:

Date:

Date:

Date:

Date:
Exp. Date:

Exp. Date:

Exp. Date:

Exp. Date:

Exp. Date:

Exp. Date:

Exp. Date:

Exp. Date:

Exp. Date:

Exp. Date:

Exp. Date:

Exp. Date:

Exp. Date:

Exp. Date:

Exp. Date:

Exp. Date:

Exp. Date:

Exp. Date:
HN03 Lot: 344032 HN03 Lot: B44032 HN03 Lot: B4Y032 HNO3 Lot: HN03 Lot: HNO3 Lot: HNO3 Lot: HNO3 Lot: HNO3 Lot: HNO3 Lot: HNO3 Lot: HNO3 Lot: HNO3 Lot: HN03 Lot: HN03 Lot: HN03 Lot: HNO3 Lot: HNO3 Lot: HNO3 Lot: HN03 Lot: HN03 Lot: HNO3 Lot: HNO3 Lot: HN03 Lot: HN03 Lot: 


\section{STL KNOXVILLE}

\section{INTERMEDIATE STANDARD - VERIFICATION}

Stock Std Name: ICP 080

Element: $\mathrm{Hg}$

Stock Source: Ultra

Stock Std Lot \#: F $\quad F 0424$

Stock Std Expiration Date: $8 / 31 / 08$

Stock Conc.: $1000 \mathrm{ppm}$

Acid Lot: B44032
Stock Vol (ml) $/ 100 \mathrm{ml}: 1.0 \mathrm{ml}$

Date Prepared: $1 / 2 / 0>$

Expiration Date: $2 / 2 / 0>$

QC Check: $\mathrm{M010307}$

Initials: $\quad K N D$

Matrix: $2 \% \mathrm{HNO}_{3}$

\section{WORKING STANDARD - VERIFICATION}

$1 \mathrm{ml}$ Intermediate Standard/100 ml $0.15 \%$ Concentrated Nitric Acid

1. I-VER $3426-6-1$

2. I-VER $3424-4-2$

3. I-VER

4. I-VER

5. I-VER

6. I-VER

7. I-VER

8. I-VER

9. I-VER

10. I-VER

11. I-VER

12. I-VER

13. I-VER

14. I-VER

15. I-VER

16. I-VER

17. I-VER

18. I-VER

19. I-VER

20. I-VER

21. I-VER

22. I-VER

23. I-VER

24. I-VER

25. I-VER

\begin{tabular}{l} 
Initials D PW \\
Initials D WW \\
Initials \\
Initials \\
Initials \\
Initials \\
Initials \\
Initials \\
Initials \\
Initials \\
Initials \\
Initials \\
Initials \\
Initials \\
Initials \\
Initials \\
Initials \\
Initials \\
Initials \\
Initials \\
Initials \\
Initials \\
Initials \\
Initials \\
Initials \\
\hline
\end{tabular}

Date:010307Exp. Date.010407HN03 Lot: 844032 Date: 010407Exp. Date:010507HN03 Lot: $B 44032$

Date:__Exp. Date:

Date: __ Exp. Date: HN03 Lot:

Date: ___Exp. Date: HN03 Lot:

Date:___Exp. Date HN03 Lot:

Date: Exp. Date: HNO3 Lot:

Date: Exp. Date: HNO3 Lot:

Date: Exp. Date: HN03 Lot:

Date: Exp. Date: HNO3 Lot:

Date: __ Exp. Date: HN03 Lot: Date: Exp. Date: Date:___ Exp. Date: HN03 Lot: Date: ___ Exp. Date: Date:___Exp. Date: Date: Exp. Date: HNO3 Lot: Date: __ Exp. Date: Date: ___ Exp. Date: Date: __ Exp. Date: Date:___Exp. Date: HNO3 Lot: Date: __ Exp. Date: Date:___Exp. Date: HN03 Lot: Date: ___Exp. Date: Date: ___ Exp. Date: HNO3 Lot: Date: Exp. Date: HNO3 Lot: HN03 Lot: HN03 Lot: HNO3 Lot: HNO3 Lot: HN03 Lot: HN03 Lot: HN03 Lot: HNO3 Lot: HN03 Lot: 
Protocol: 7ptcurve

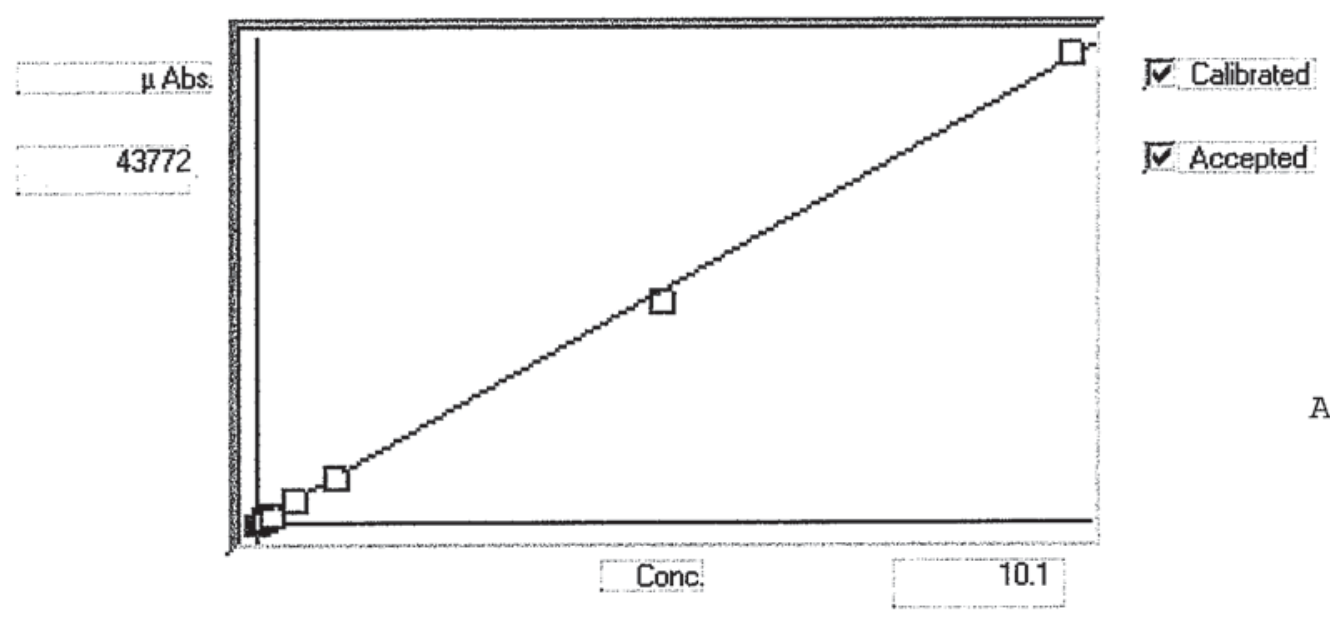

\section{Wt lin."I}

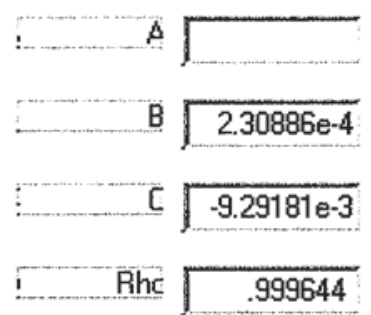

Accepted Date: 05Jan-07 10:02

$\mathrm{s}$
0
0
0
0
0
0
0

\begin{tabular}{|c|c|c|c|c|c|c|c|c|c|}
\hline Conc. & Calc. & Dev. & Mean & SD or \%RSD & Rep 1 & Rep 2 & Rep 3 & $\operatorname{Rep} 4$ & Rep 5 \\
\hline .00000 & .014 & .014 & 103 & 0 & 103 & & & & \\
\hline .10000 & .122 & .022 & 568 & $0 \%$ & 567 & & & & \\
\hline .20000 & .205 & .005 & 926 & $0 \%$ & 926 & & & & \\
\hline .50000 & .545 & .045 & 2400 & $0 \%$ & 2400 & & & & \\
\hline 1.0000 & 1.04 & .036 & 4528 & $0 \%$ & 4528 & & & & \\
\hline 5.0000 & 4.78 & -.219 & 20750 & $0 \%$ & 20749 & & & & \\
\hline 10.000 & 10.1 & .097 & 43772 & $0 \%$ & 43772 & & & & \\
\hline
\end{tabular}


STL - Knoxville

09:50:53 05 Jan 2007
Folder: M010407A

Protocol: 7ptcurve

Line Conc. Units SD/RSD

1

2

3

4

5

Standard: 1 Rep: 1

Seq : 0

09:50:53 05 Jan 07

HG

$\begin{array}{llll}\mathrm{Hg} & .000 \quad \mathrm{ppb} & 103\end{array}$

*** Standard: 2 Rep: 1

Seq: 1

09:52:41 05 Jan 07 HG

$\begin{array}{llll}\mathrm{Hg} & .100 \quad \mathrm{ppb} & 567\end{array}$

*** Standard: 3 Rep: 1

Seq : 2

09:54:30 05 Jan 07 HG

Hg $\quad .200 \quad$ ppb $\quad 926$

*** Standard: 4 Rep: 1

Seq: 3

09:56:18 05 Jan $07 \quad$ HG

Hg $\quad .500 \quad \mathrm{ppb} \quad 2400$

*** Standard: 5 Rep: 1

Seq : 4

09:58:17 05 Jan 07 HG

$\begin{array}{lll}\mathrm{Hg} & 1.00 \quad \mathrm{ppb} & 4528\end{array}$

*** Standard: 6 Rep: 1

Seq : 5

10:00:05 05 Jan 07 HG

$\mathrm{Hg} \quad 5.00 \quad \mathrm{ppb} \quad 20749$

*** Standard: 7 Rep: 1

Seq: $6 \quad 10: 01: 52$ 05 Jan 07 HG

Hg $\quad 10.0 \quad \mathrm{ppb} \quad 43772$

$\begin{array}{llllll}* * * \text { Check Standard: } & 2 \text { Ck2icv } & \text { Seq: } & \text { 10:05:28 } 05 \text { Jan } 07 & \text { HG } \\ \text { Line Flag oRcv. Found True Units } & \text { SD/RSD }\end{array}$

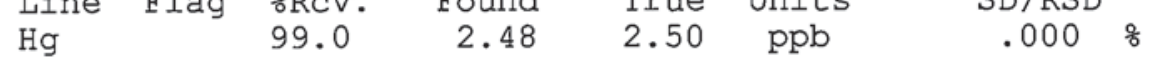

*** Sample ID: ICB Seq: $8 \quad 10: 07: 2405$ Jan 07 HG

$\mathrm{Hg} \quad-.008 \quad \mathrm{ppb} \quad .000 \%-.008$

*** Sample ID: CRA Seq: $9 \quad 10: 09: 2005$ Jan 07 HG

Hg $\quad .190 \quad \mathrm{ppb} \quad .000 \%$

$* * *$ Check Standard: $3 \mathrm{Ck3ccv}$ Seq: $10 \quad 10: 11: 0905$ Jan 07 HG Line Flag \%Rcv. Found True Units SD/RSD

$\begin{array}{lllllll}\text { Hg } & 96.6 & 4.83 & 5.00 & \text { ppb } & .000 & \%\end{array}$

$* * *$ Check Standard: 1 Cklccb
Line Flag Found Range (+/-) Units $\mathrm{Hg} \quad-.006 \quad .059 \quad \mathrm{ppb} \quad .000 \quad \%$

*** Sample ID: MDL1 Seq: 12 10:15:56 05 Jan 07 HG Hg $\quad .084$ ppb $\quad .000 \%$ Front Half OH $0.1 \mathrm{ppb}$

*** Sample ID: MDL2 Seq: $13 \quad$ 10:18:14 05 Jan 07 HG $\begin{array}{lllll}\mathrm{Hg} & .094 \quad \mathrm{ppb} & .000 \quad \% \quad & .094\end{array}$

*** Sample ID: MDL3 Seq: $14 \quad$ 10:20:05 05 Jan 07 HG $\begin{array}{lllll}\mathrm{Hg} & .081 & \mathrm{ppb} & .000 \quad \% \quad .081\end{array}$

*** Sample ID: MDL4 Seq: $15 \quad$ 10:21:52 05 Jan 07 HG

Hg $\quad .094 \quad$ ppb $\quad .000 \quad \% \quad .094$ 
STL - Knoxville

$10: 23: 50 \quad 05$ Jan 2007

Line

Sample ID: MDL5

SD/RSD

Folder: M010507

Protocol: 7ptcurve

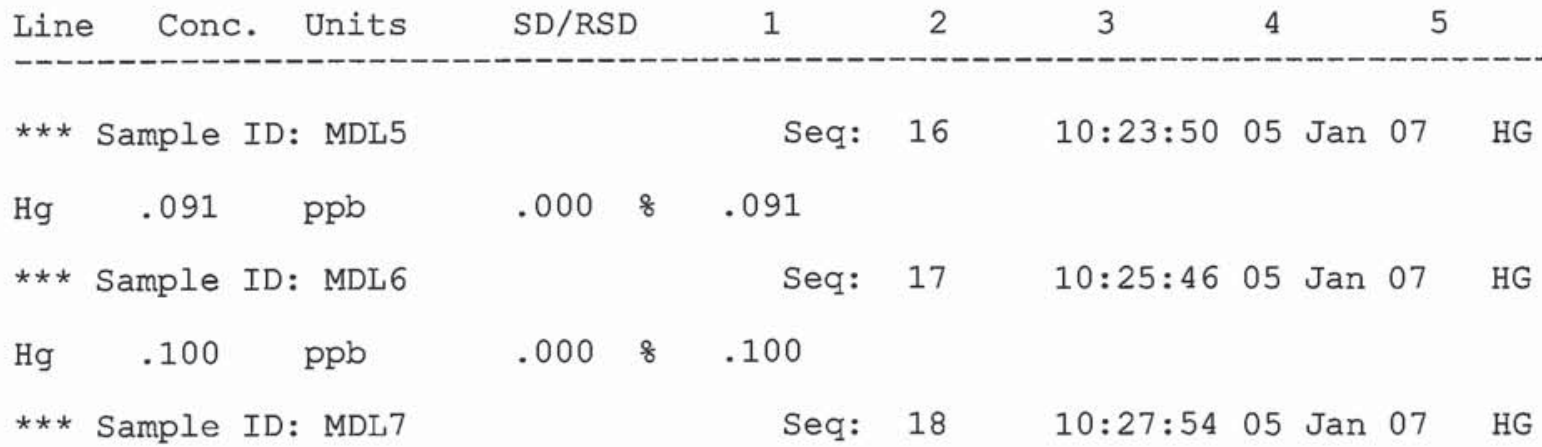

$\begin{array}{llllll}H g & .129 & \mathrm{ppb} & .000 & \% & .129\end{array}$

*** Check Standard: $3 \mathrm{Ck3ccv}$ Seq: 19 10:30:21 05 Jan 07 HG

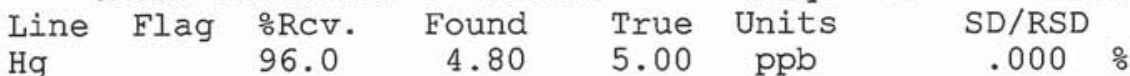
$\begin{array}{llllll}* * * \text { Check Standard: } 1 \text { Cklccb } & \text { Seq: } 20 & 10: 32: 1805 \text { Jan } 07 \text { HG } \\ \text { Line Flag Found Range }(+/-) \text { Units } & \text { SD/RSD } & & \end{array}$

$\begin{array}{lllll}\mathrm{Hg} & -.012 & .059 & \mathrm{ppb} & .000\end{array}$

$\star \star \star$ Sample ID: MDLI

Seq: 21 10:34:09 05 Jan 07 HG

$\mathrm{Hg} \quad .041 \quad \mathrm{ppb}$ Back Half $\mathrm{OH} 0.1 \mathrm{ppb}$

$\star * *$ Sample ID: MDL2

Seq: 22 10:36:01 $05 \mathrm{Jan} 07$ HG

$\begin{array}{llllll}\mathrm{Hg} & .062 & \mathrm{ppb} & .000 & \text { \% } & .062\end{array}$

*** Sample ID: MDL3

Seq: $23 \quad 10: 37: 5305$ Jan 07 HG

$\begin{array}{llllll}\mathrm{Hg} & .044 & \mathrm{ppb} & .000 & \% & .044\end{array}$

*** Sample ID: MDL4

Seq: 24 10:40:01 05 Jan 07 HG

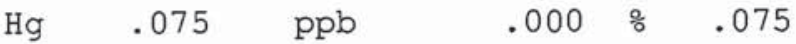

** Sample ID: MDL5

Seq: 25 10:41:58 05 Jan 07 HG

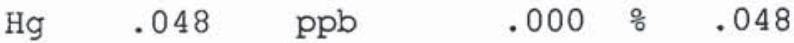

$\star \star \star$ Sample ID: MDL6

Seq: $26 \quad 10: 43: 5605$ Jan 07 HG

$\begin{array}{llllll}\mathrm{Hg} & .053 & \mathrm{ppb} & .000 & \circ & .053\end{array}$

** Sample ID: MDL7

Seq: $27 \quad 10: 46: 0405$ Jan 07 HG

$\begin{array}{llllll}\mathrm{Hg} & .080 & \mathrm{ppb} & .000 & 8 & .080\end{array}$

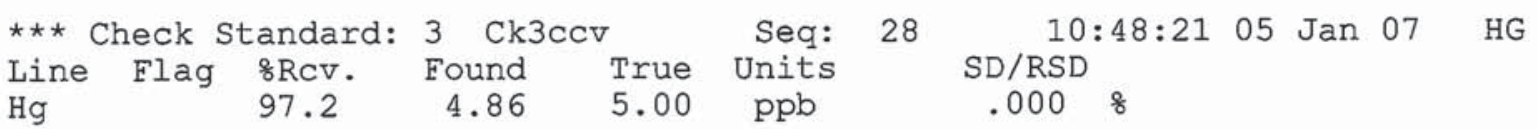

*** Check Standard: $1 \mathrm{Cklccb}$

Line Flag Found Range(+/-) Units

Hg $\quad .011 \quad .059 \quad \mathrm{ppb}$

Seq: $29 \quad 10: 50: 1005$ Jan 07 HG $\mathrm{SD} / \mathrm{RSD}$ $.000 \%$

*** Sample ID: JL971B

Seq: $30 \quad 10: 52: 1105 \mathrm{Jan} 07$ HG

Hg -.017 ppb $\quad .000 \%$ \% -.017

** Sample ID: JL971C Seq: $31 \quad$ 10:54:39 05 Jan 07 HG

$\begin{array}{lllll}\mathrm{Hg} & 4.91 \quad \mathrm{ppb} & .000 \quad \% \quad 4.91\end{array}$ 
STL - Knoxville

$10: 56: 3705$ Jan 2007

Line Conc. Units

\section{*** Sample ID: JL971L}

3

Seq: 32

10:56:37 05 Jan 07

HG

$\begin{array}{lllll}H g & 5.15 & \mathrm{ppb} & .000 \quad \% & 5.15\end{array}$

$\star \star \star$ Sample ID: JLHWK

Seq : 33

10:58:24 05 Jan 07 HG

$\mathrm{Hg}-.013 \mathrm{ppb} \quad .000 \%-.013$ DIGESTED 10 PPb STD.

*** Sample ID: JLHWKA 9ML SAMple +'IML DIGEST 34 11:00:17 05 Jan 07 HG

Hg $\quad 1.04 \quad \mathrm{ppb} \quad .000 \quad \% \quad 1.04$

$\star \star \star$ Sample ID: JLHXD

Seq: $35 \quad 11: 02: 1005$ Jan $07 \quad$ HG

$\mathrm{Hg} \quad .003 \quad \mathrm{ppb} \quad .000 \% \quad .003$

Sample ID: JLHXE

Seq: $36 \quad 11: 03: 5805 \mathrm{Jan} 07$ HG

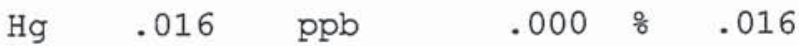

*** Sample ID: JLHXH

Seq : 37

11:05:48 05 Jan 07 HG

$\begin{array}{llllll}\mathrm{Hg} & .006 & \mathrm{ppb} & .000 & 8 & .006\end{array}$

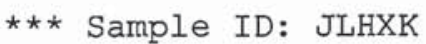

Seq: $38 \quad 11: 07: 4305$ Jan 07 HG

$\begin{array}{llllll}\mathrm{Hg} & .052 & \mathrm{ppb} & .000 & 8 & .052\end{array}$

*** Sample ID: JLHXM

Seq: 39 11:09:45 05 Jan 07 HG

$\mathrm{Hg} \quad .003 \quad \mathrm{ppb} \quad .000 \quad 8 \quad .003$

** Check Standard: $3 \mathrm{Ck3ccv}$ Seq: 40 11:11:32 05 Jan 07 HG Line Flag \&RCV. Found True Units SD/RSD

$\begin{array}{llllll}\mathrm{Hg} & 96.9 & 4.84 & 5.00 & \mathrm{ppb} & .000\end{array}$

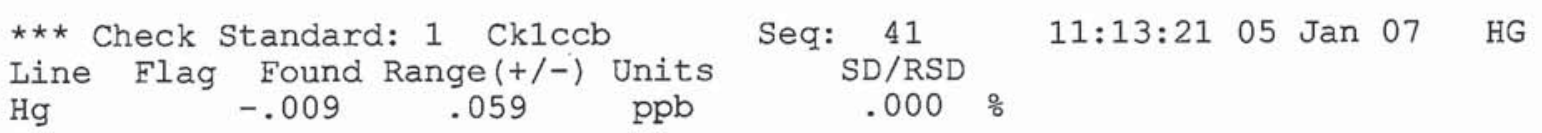

*** Sample ID: JL97PB Seq: $42 \quad$ 11:15:29 05 Jan 07 HG

Hg $\quad .006 \quad$ ppb $\quad .000 \%$ \% .006

*** Sample ID: JL97PC Seq: $43 \quad$ 11:17:27 05 Jan 07 HG

$\begin{array}{lllll}\mathrm{Hg} & 4.87 & \mathrm{ppb} & .000 \quad 8 \quad 4.87\end{array}$

*** Sample ID: JL97PL Seq: $44 \quad$ 11:19:38 05 Jan 07 HG

Hg $\quad 4.74 \quad \mathrm{ppb} \quad .000 \quad \% \quad 4.74$

*** Sample ID: JK1WA Seq: $45 \quad$ 11:21:27 05 Jan 07 HG

Hg $.378 \quad \mathrm{ppb} \quad .000 \% .378$ DIESTED 10 PPb STD

*** Sample ID: JK1WAA 9 mL SAMpLE + 1 ML DIESTED 10 PPb $46 \quad 11: 23: 1405$ Jan 07 HG

Hg $\quad 1.36 \quad \mathrm{ppb} \quad .000 \quad 1.36$

*** Sample ID: JK1XD Seq: $47 \quad$ 11:25:13 05 Jan 07 HG

Hg $\quad .044 \quad \mathrm{ppb} \quad .000 \quad \% \quad .044$ 
STL - Knoxville

$11: 27: 1305$ Jan 2007

Line Conc. Units

Sample ID: JK1X5

$\mathrm{SD} / \mathrm{RSD}$

Folder: M010507

Protocol: 7ptcurve

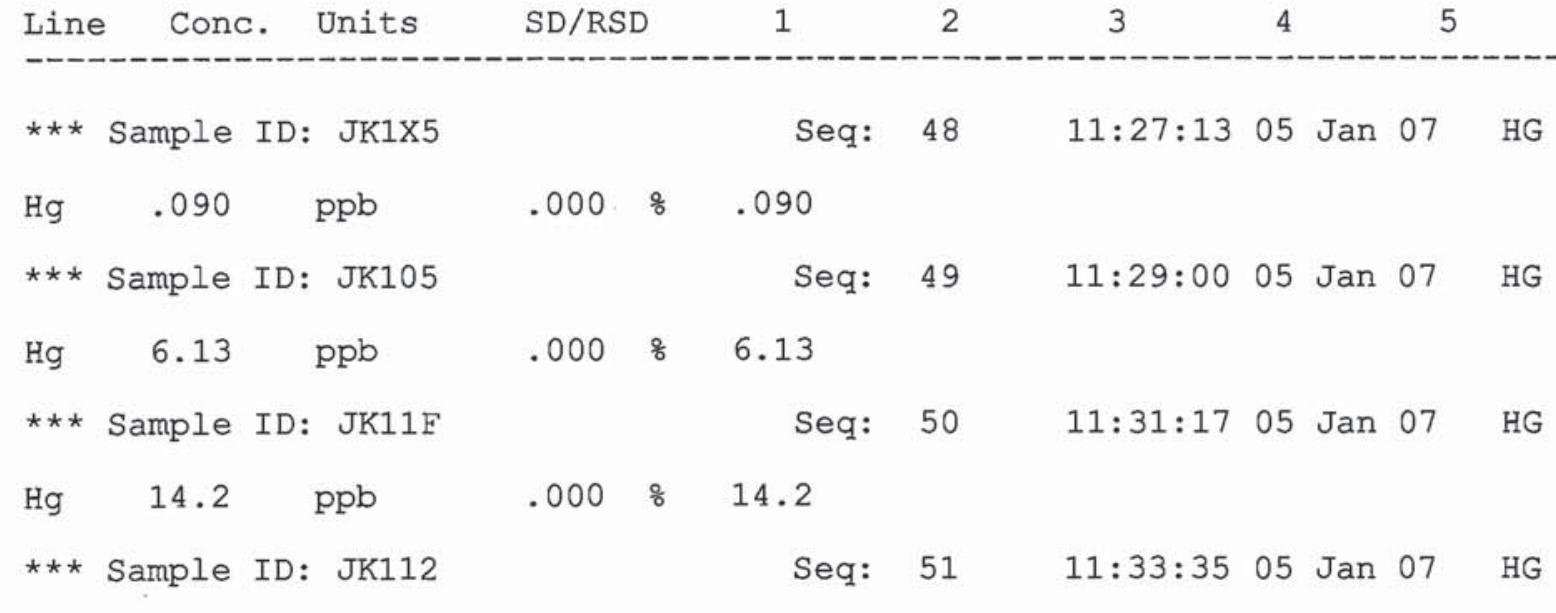

Hg $\quad 6.43 \quad \mathrm{ppb} \quad .000 \quad \% \quad 6.43$

$\begin{array}{llllllll}* \star * \text { Check Standard: } 3 \mathrm{Ck} 3 \mathrm{ccv} & \text { Seq: } 52 & 11: 35: 3705 \text { Jan } 07 \text { HG } \\ \text { Line Flag \%Rcv. Found True Units } & \text { SD/RSD }\end{array}$ $\begin{array}{lrrrcc}\text { Line Flag } & \% \text { Rcv. } & \text { Found } & \text { True } & \text { Units } & \text { SD/RSD } \\ \mathrm{Hg} & 96.6 & 4.83 & 5.00 & \mathrm{ppb} & .000 \%\end{array}$

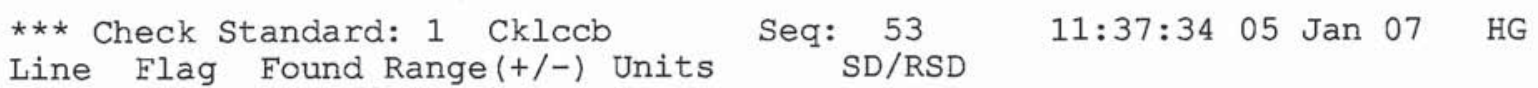

$\begin{array}{lllll}\mathrm{Hg} & -.026 & .059 & \mathrm{ppb} & .000 \%\end{array}$

*** Sample ID: JK12A Seq: $54 \quad$ 11:39:43 05 Jan 07 HG

$\begin{array}{lllll}\mathrm{Hg} & .012 \quad \mathrm{ppb} & .000 \quad 8 & .012\end{array}$

*** Sample ID: JL97RB Seq: $55 \quad$ 11:41:55 05 Jan 07 HG

$\begin{array}{llll}\mathrm{Hg} & .008 \quad \mathrm{ppb} & .000 \% & .008\end{array}$

*** Sample ID: JL97RC Seq: $56 \quad$ 11:44:14 05 Jan 07 HG

$\mathrm{Hg} \quad 4.00 \quad \mathrm{ppb} \quad .000 \% \quad 4.00$

*** Sample ID: JK1WF Seq: $57 \quad$ 11:46:04 05 Jan 07 HG

Hg $\quad .069 \quad \mathrm{ppb} \quad .000 \% \quad .069$

*** Sample ID: JK1WFS Seq: $58 \quad$ 11:47:54 05 Jan 07 HG

$\begin{array}{lllll}\mathrm{Hg} & .949 \quad \mathrm{ppb} & .000 \quad \% & .949\end{array}$

*** Sample ID: JK1WFD Seq: $59 \quad$ 11:49:46 05 Jan 07 HG

$\mathrm{Hg} \quad .976 \quad \mathrm{ppb} \quad .000 \quad \% \quad .976$

*** Sample ID: JK1XR Seq: $60 \quad$ 11:51:57 05 Jan 07 HG

$\begin{array}{lllll}\mathrm{Hg} & .058 \quad \mathrm{ppb} & .000 \quad \% \quad 058\end{array}$

** Sample ID: JK10A Seq: $61 \quad$ 11:53:48 05 Jan 07 HG

$\begin{array}{lllll}\mathrm{Hg} & .102 \quad \mathrm{ppb} & .000 \quad 8 & .102\end{array}$

*** Sample ID: JK107 Seq: $62 \quad$ 11:55:35 05 Jan 07 HG

$\mathrm{Hg} \quad .099 \quad \mathrm{ppb} \quad .000 \quad \% \quad .099$

*** Sample ID: JK11L Seq: $63 \quad$ 11:57:33 05 Jan 07 HG

Hg $\quad .160 \quad \mathrm{ppb} \quad .000 \% \quad .160$ 


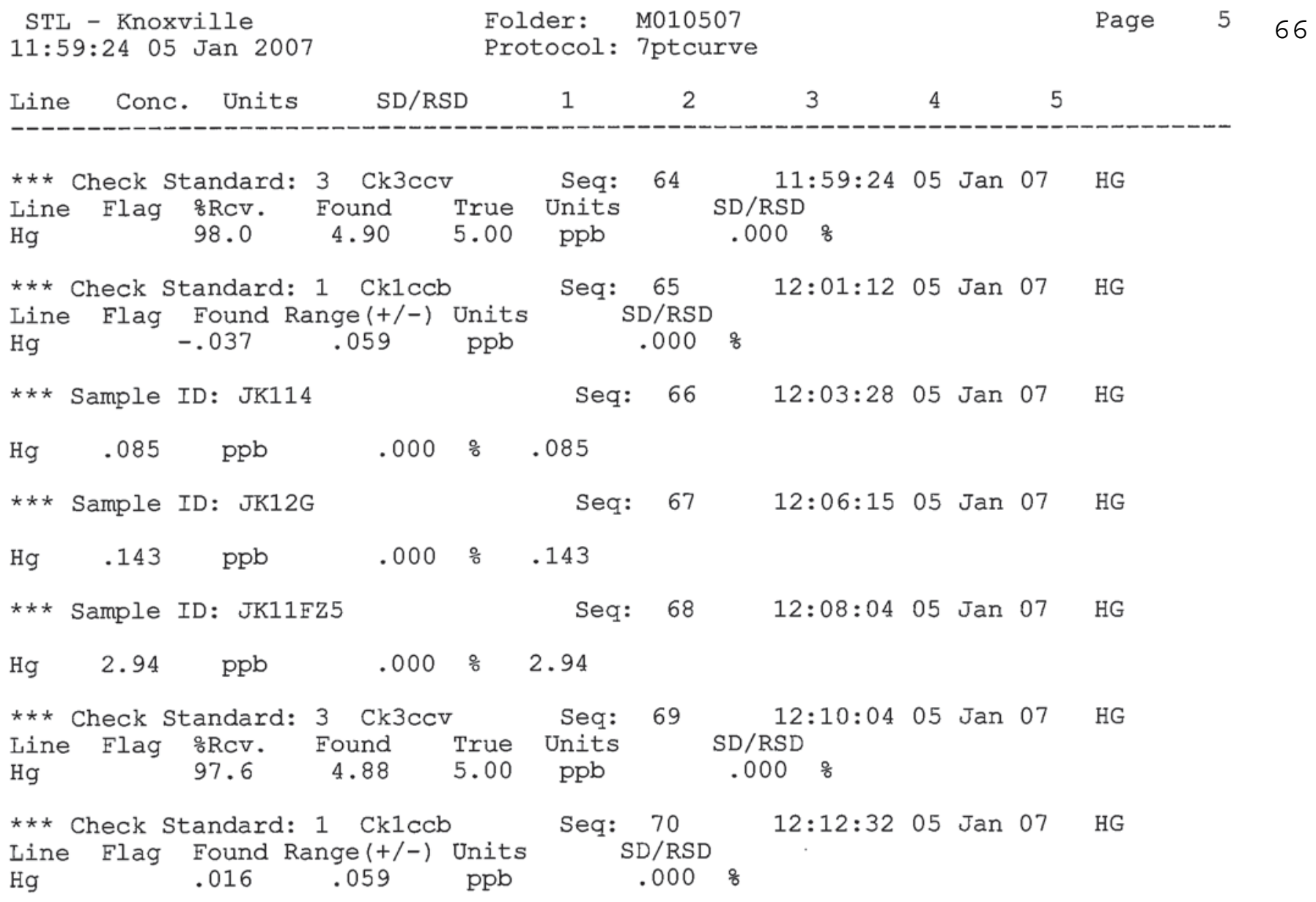

Page

5

66

11:59:24 05 Jan 2007

Protocol: 7ptcurve

Line Conc. Units SD/RSD

2

3

5

$* * *$ Check Standard: $3 \mathrm{Ck} 3 \mathrm{ccv}$

Line Flag prev. Found

$\mathrm{Hg}$

Seq: 64 $4.90 \quad 5.00$

Units ppr

11:59:24 05 Jan 07 HG

$\mathrm{SD} / \mathrm{RSD}$

.000

*** Check Standard: $1 \mathrm{Ck} 1 \mathrm{ccb}$

Line Flag Found Range(+/-) Units $\mathrm{Hg}$

Seq: 65 12:01:12 05 Jan 07 HG $-.037 \quad .059 \quad \mathrm{ppb}$

$\mathrm{SD} / \mathrm{RSD}$

$.000 \%$

*** Sample ID: JK114

Seq: $66 \quad 12: 03: 2805$ Jan 07 HG

$\begin{array}{llllll}\mathrm{Hg} & .085 & \mathrm{ppb} & .000 & \% & .085\end{array}$

*** Sample ID: JK12G

Seq: $67 \quad 12: 06: 1505$ Jan 07 HG

$\begin{array}{llllll}\mathrm{Hg} & .143 & \mathrm{ppb} & .000 & \% & .143\end{array}$

*** Sample ID: JK11FZ5

Seq: 68 12:08:04 05 Jan 07 HG

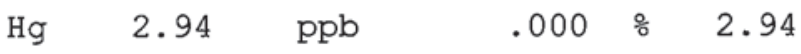

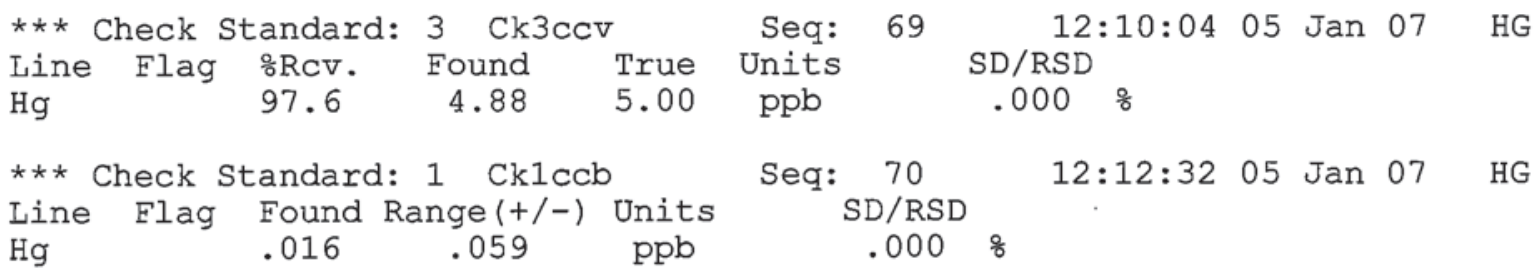


Miscellaneous Data 


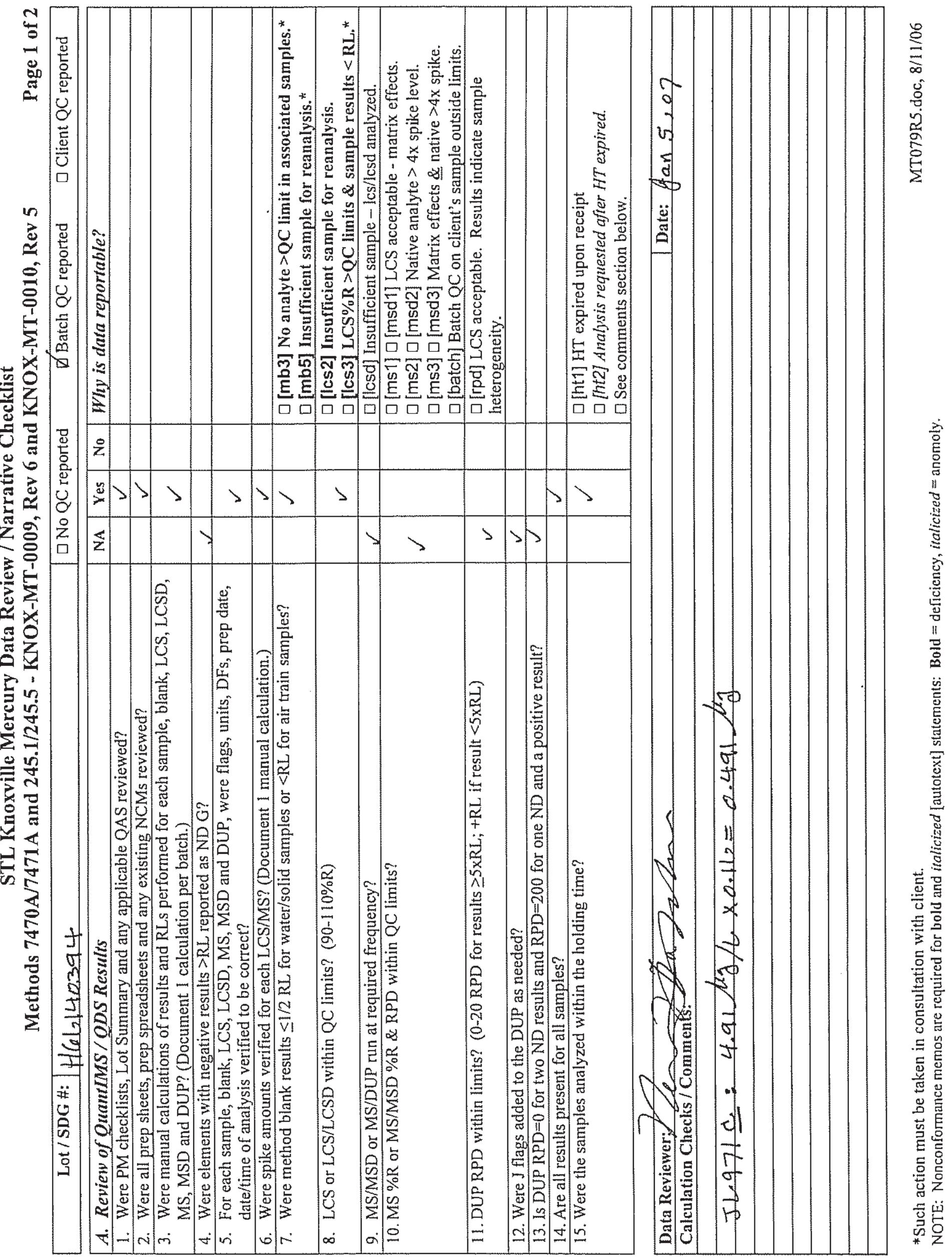




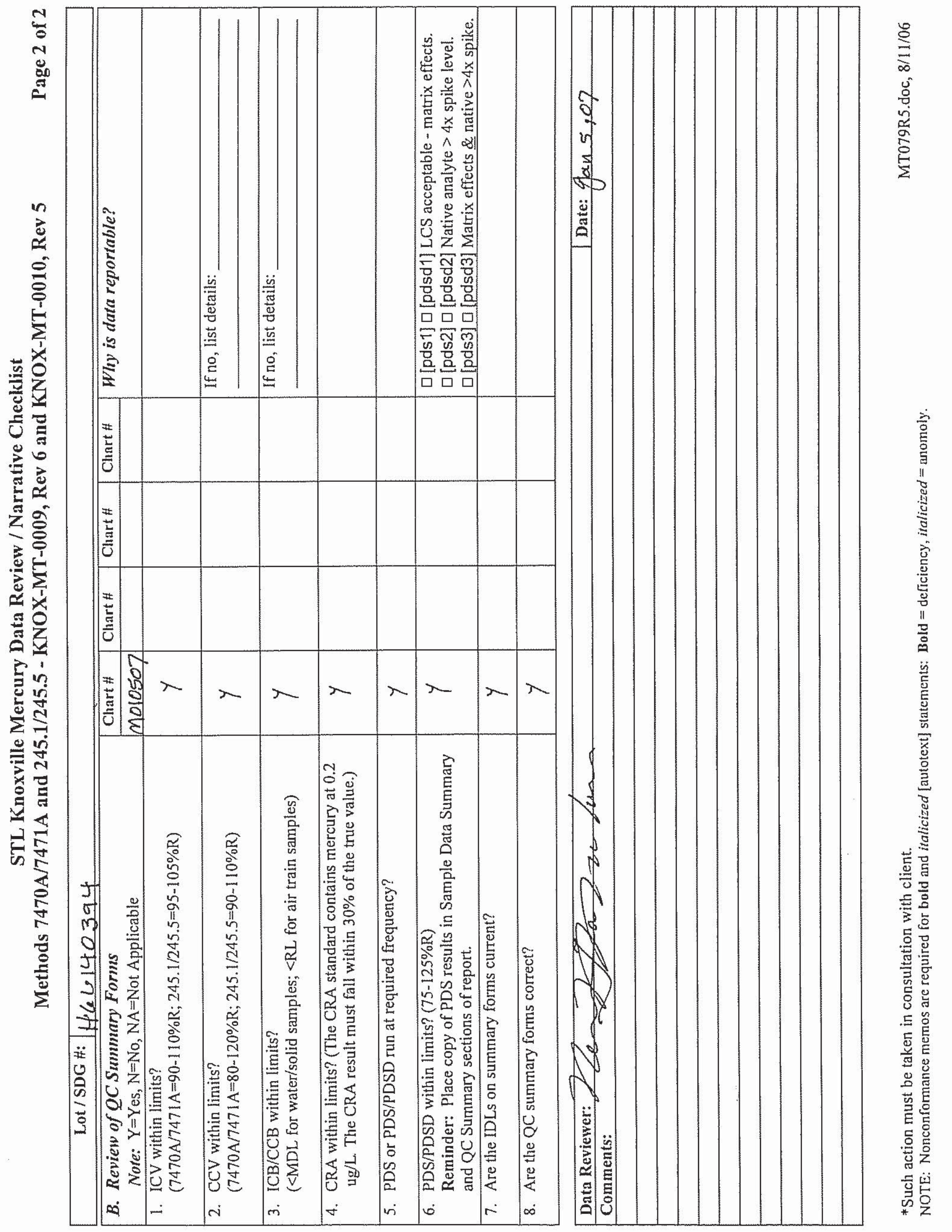




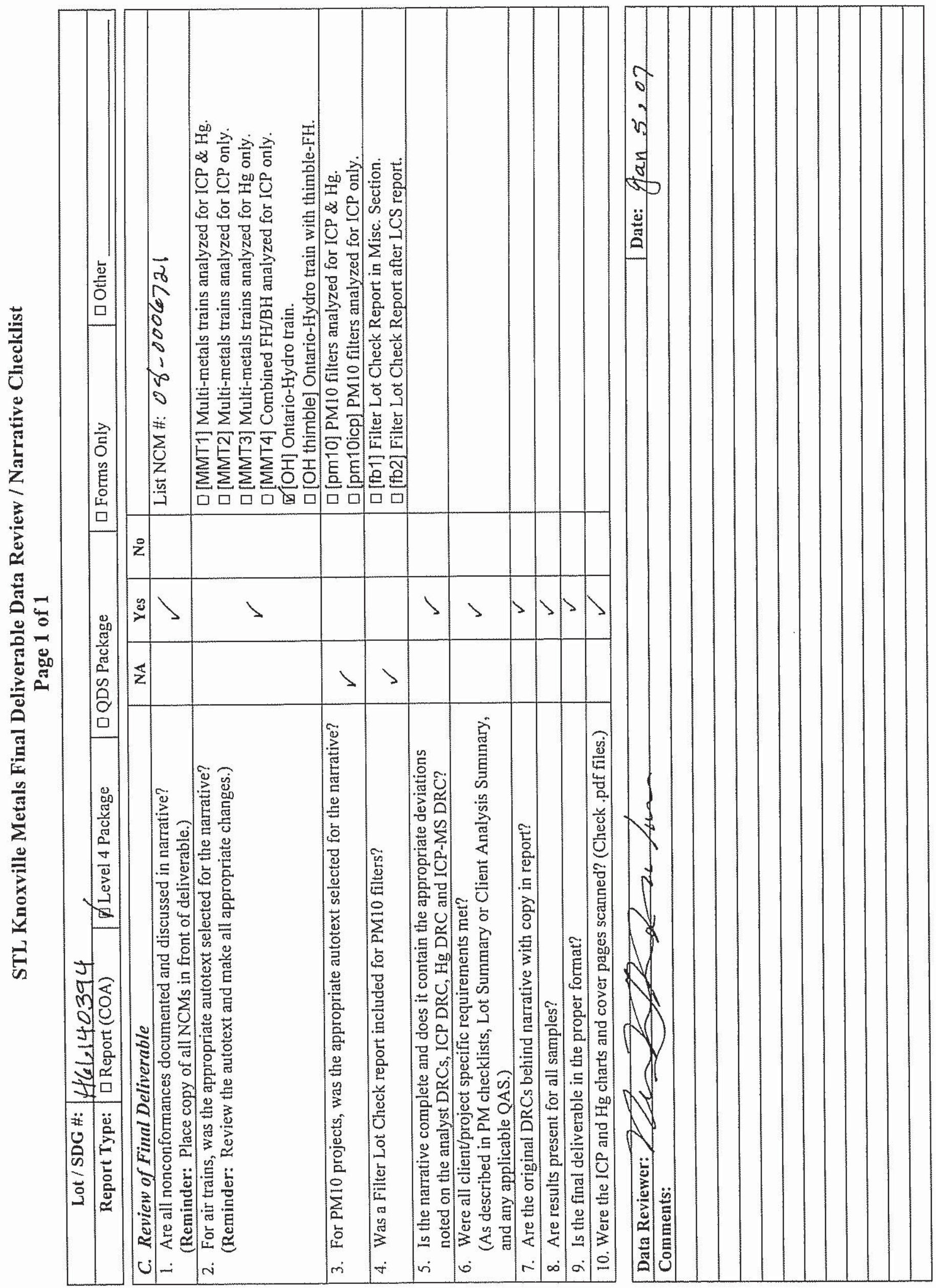




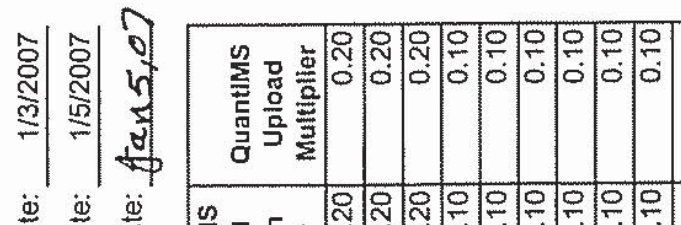

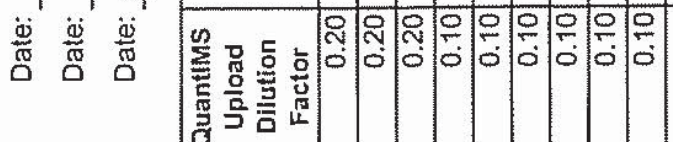

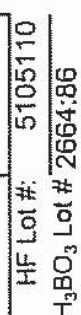

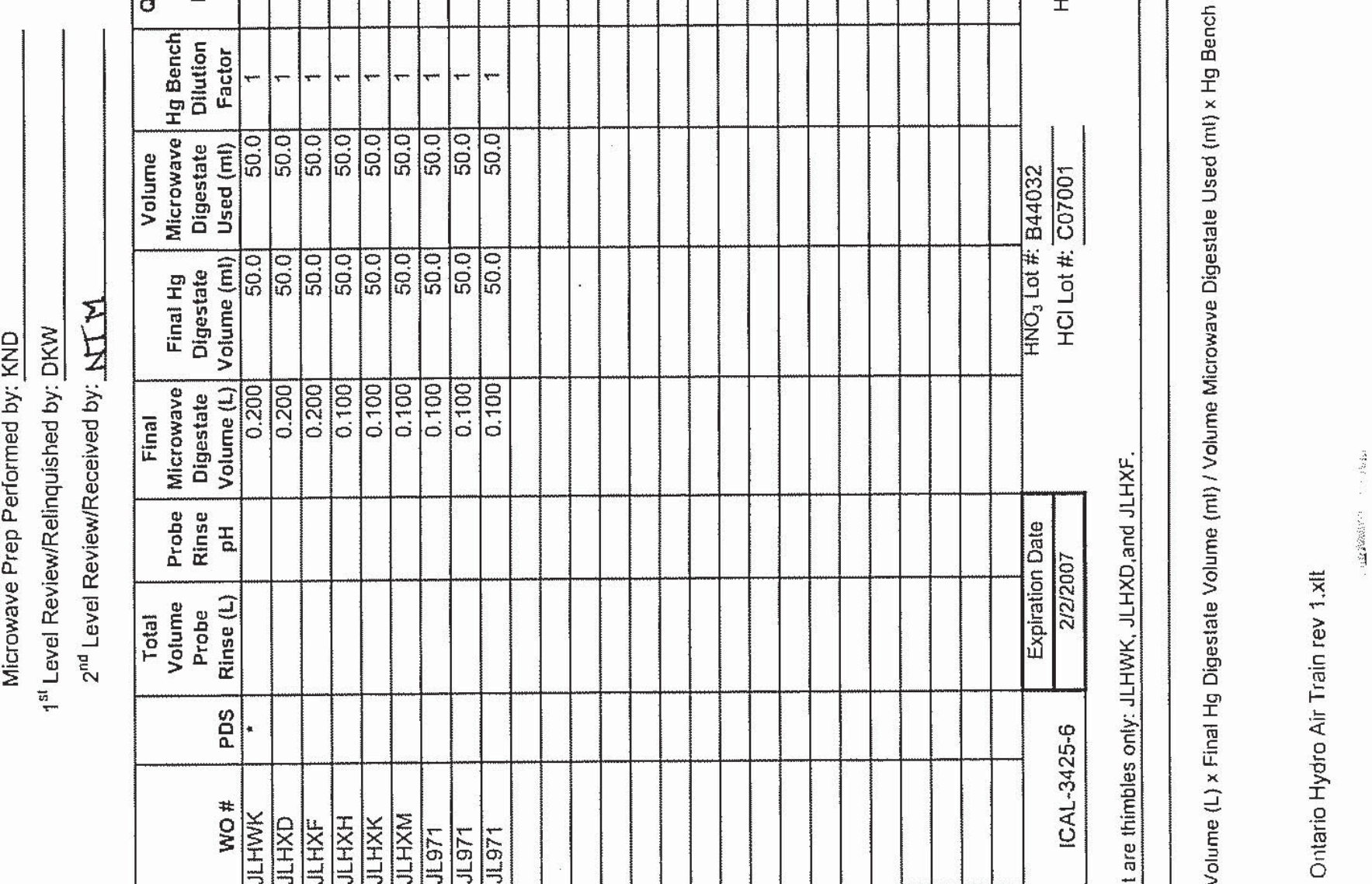

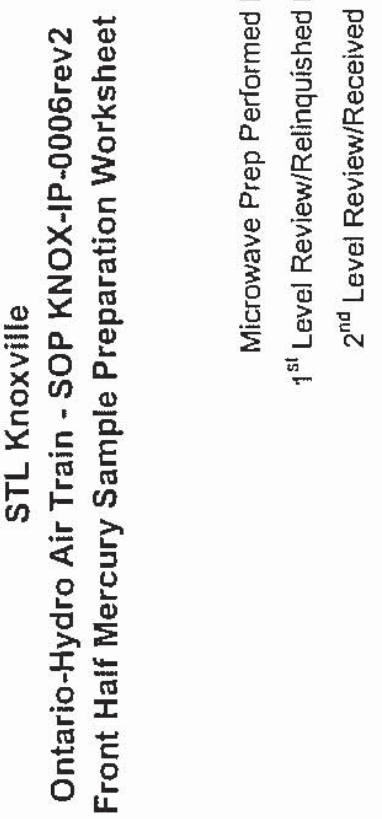

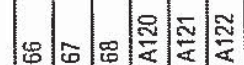

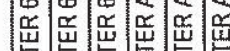

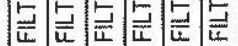

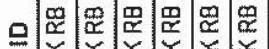

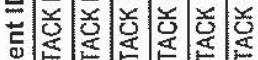

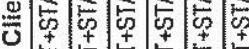

o

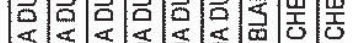

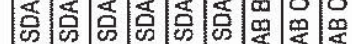

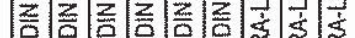

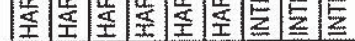

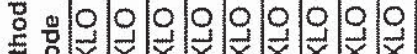

恶

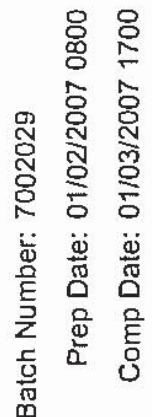

$\infty 0$.

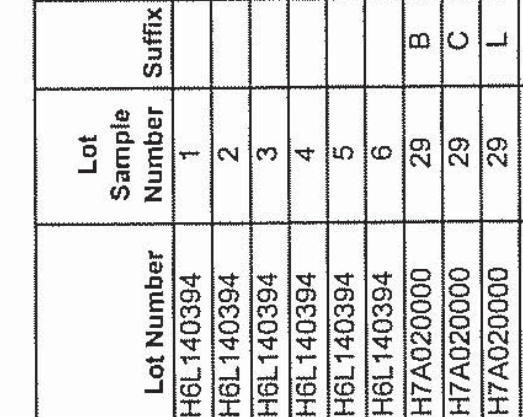

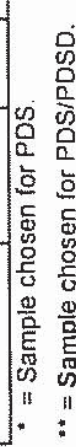


STL Knoxville

Mercury Air Prep Spiking Form

Mercury Air Prep Spiking Form
$01 / 04 / 07 \quad$ Batch: 7002029

Hg in PM10 filters: KNOX-IP-0003, Rev, 4

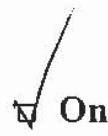

MMT train: KNOX-MT-0006, Rev. 9

Bath A Temp: $93^{\circ}$

Bath B Temp: 240 C

(Acceptance range $90^{\circ}-95^{\circ} \mathrm{C}$ )

\begin{tabular}{|c|c|c|c|c|c|c|}
\hline \multirow{2}{*}{ Standard Type } & \multirow{2}{*}{ Standard ID } & \multicolumn{2}{|c|}{ Intermediate Std. } & \multirow{2}{*}{$\begin{array}{c}\text { Volume } \\
\text { Used (ml) }\end{array}$} & \multirow{2}{*}{$\begin{array}{c}\text { Final } \\
\text { Volume } \\
(\mathrm{ml})\end{array}$} & \multirow{2}{*}{$\begin{array}{c}\text { Final } \\
\begin{array}{c}\text { Concentration } \\
\text { (ug/L) }\end{array} \\
\end{array}$} \\
\hline & & Source & Conc. $(u g / L)$ & & & \\
\hline Colibration Std 1 & N/A & & N/A & N/A & 50 & Blank \\
\hline Calibration Std 2 & I-CAL- $3425-6-3$ & SPEX & 100 & 0.05 & 50 & 0.1 \\
\hline Calibration Std 3 & I-CAL- & & 100 & 0.1 & 50 & 0.2 \\
\hline Calibration Std 4 & I-CAL- & & 100 & 0.25 & 50 & 0.5 \\
\hline Calibration Std 5 & I.CAL- & 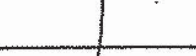 & 100 & 0.5 & 50 & 1.0 \\
\hline Calibration Std 6 & I-CAL- & . & 100 & 2.5 & 50 & 5.0 \\
\hline Calibration Std 7 & I-CAL- $\perp$ & L & 100 & 5.0 & 50 & 10.0 \\
\hline
\end{tabular}

\begin{tabular}{|c|c|c|c|c|c|c|}
\hline \multirow{2}{*}{$\begin{array}{c}\text { Standard } \\
\text { Type } \\
\end{array}$} & \multirow{2}{*}{ Standard ID } & \multicolumn{2}{|c|}{ Intermediate Std. } & \multirow{2}{*}{$\begin{array}{l}\text { Amount } \\
\text { Used (ml) }\end{array}$} & \multirow{2}{*}{$\begin{array}{c}\text { Final } \\
\text { Volume } \\
\text { (ml) }\end{array}$} & \multirow{2}{*}{$\begin{array}{l}\text { Final Conc } \\
\text { (ug/L) }\end{array}$} \\
\hline & & Source & Conc.(ug/L) & & & \\
\hline ICV & I-VER- $342 b-6-2$ & ULTPA & 100 & 1.25 & 50 & 2.5 \\
\hline $\mathrm{CCV}$ & I-CAL $3425-6-3$ & PEX & 100 & 2.5 & 50 & 5.0 \\
\hline CRA & I-CAL- & 1 & 100 & 0.1 & 50 & 0.2 \\
\hline
\end{tabular}

\begin{tabular}{|c|c|c|c|c|c|c|c|}
\hline \multirow{2}{*}{ Standard Type } & \multirow{2}{*}{ Standard ID } & \multicolumn{2}{|c|}{ Intermediate Std. } & \multirow{2}{*}{$\begin{array}{l}\text { Amount Used } \\
\text { @ ICP } \\
\text { prep/final } \\
\text { volume }\end{array}$} & \multirow{2}{*}{$\begin{array}{c}\text { ICP Sample } \\
\text { Volume } \\
\text { used @ } \mathrm{Hg} \\
\text { prep }(\mathrm{ml})\end{array}$} & \multirow{2}{*}{$\begin{array}{c}\text { Final } \\
\text { Conc. @ } \\
\text { Hg prep } \\
\text { (ug/L) } \\
\end{array}$} & \multirow{2}{*}{$\begin{array}{l}\text { Final } \\
\text { Amount } \\
\text { (ug) }\end{array}$} \\
\hline & & Source & Conc.(ug/L) & & & & \\
\hline Air (PM10) LCS & I-CAL- & & 10,000 & $0.250 \mathrm{ml} / 50 \mathrm{ml}$ & 5.0 & 5.0 & 11.25 \\
\hline Air (PM10) MS & I-CAL- & & 10,000 & $0.050 \mathrm{ml} / 50 \mathrm{ml}$ & 5.0 & 1.0 & 2.25 \\
\hline
\end{tabular}

\begin{tabular}{|c|c|c|c|}
\hline \multicolumn{4}{|c|}{ REAGENTS } \\
\hline Conc. $\mathrm{H}_{2} \mathrm{SO}_{4}$ Lot & $c 15045$ & $5 \% \mathrm{KMnO}_{4} \mathrm{XD}_{\mathrm{H}}:$ & $B-3122-12-C$ \\
\hline Conc. $\mathrm{HNO}_{3}$ Lot \#: & 844032 & $5 \% \mathrm{~K}_{2} \mathrm{~S}_{2} \mathrm{O}_{8}$ ID & $D-2638-17-E$ \\
\hline Conc. HCL Lot H: & $1+2422$ & $\mathrm{SnCl}_{2}$ Solution ID \#: & $A-3382-20-B$ \\
\hline $12 \% \mathrm{NaClNH}_{2} \mathrm{OH} \cdot \mathrm{HCL}$ ID \#: & $c-3267-3-E$ & & \\
\hline
\end{tabular}


Wet Chemistry 
Sample Summary 
Air Sampling Associates, Inc.

Client Sample ID: HARDIN SDA DUCT+STACK RB PILTER 66

General Chenistry

Lot-Sample \#...: H6L140394-001 Work Order \#...: JLHWK

Date Sampled. . . : 12/11/06
Date Received. .: 12/12/06
Matrix....... AIR

PREPARATION- PREP

ANALYSIS DATE BATCH \#
PARAMETER

Particulates (total) 2860

RL

0.50 mig Dilution Factor: 1
METHOD

CFR60A 5 MDL......... 0.50 
Air Sampling Associates, Inc.

Client Sample ID: HARDIN SDA DUCT+STACK RB FILTKR 67

General Chemistry

Iot-Sample \#...: H6L140394-002

Date Sampled... : 12/11/06
Work Order \#...: ЛLHXD

Date Received. .: 12/12/06
Matrix........ AIR

PREPARATION- $\quad$ PREP

ANALYSIS DATE BATCH \#

PARAMETER

Particulates (total) 2930
RESULT

RL

$0.50 \mathrm{mg}$ Dilution Factor: 1

METHOD

CFR60A 5

MDL.......... 0.50 
Air Sampling Associates, Inc.

client sample ID: HARDIN SDA DUCT+STACK RB FILTKR 68

General Chemistry

Lot-Sample \#...: H6L140394-003 work Order \#...: JLHXF

Date Sampled...: 12/11/06
Date Received. . : 12/12/06
Matrix.......: AIR

PREPARATION- $\quad$ PREP ANALYSIS DATE BATCH \#
PARAMETER

Particulates (total) 2510
RESULT

$\mathrm{RL}$ 0.50 Dilution Factor:

METHOD

CFR60A 5 $\operatorname{MDL} . \ldots \ldots \ldots \ldots: 0.50$ 
Air Sampling Associates, Inc.

Client Sample ID: HARDIN SDA DUCT+STACK RB FILTER A120

General Chemistry

Lot-Sample \#...: H6L140394-004 work Order \#...: JLHXH

Date sampled...: 12/11/06
Date Received..: 12/12/06
Matrix.......... AIR

PREPARATION- PREP

ANALYSIS DATE BATCH \#

$\frac{\text { PARAMETER }}{\text { Particulates (total) }} \frac{\text { RESULT }}{360} \frac{\text { RL }}{0.50} \frac{\text { UNITS }}{\mathrm{mg}} \frac{\text { METHOD }}{\text { Dilution Factor: } 1} \quad \frac{\text { ANALYSIS DATE }}{\text { 12/19-12/20/06 }} \frac{\text { BATCH \# }}{6354260}$


Air Sampling Associates, Inc.

Client Sample ID: HARDIN SDA DUCT+STACK RB FILTER A121

General Chemistry

Lot-Sample \#...: H6L140394-005 work Order \#...: ЛLHXK

Date sampled...: 12/11/06

\begin{abstract}
Date Received. . : 12/12/06
\end{abstract}
Matrix....... AIR

PREPARATION- PREP ANALYSIS DATE BATCH \#
PARAMETER

Particulates (total) 360
RESULT

$\mathrm{RL}$

0.50 $\mathrm{mg}$
METHOD CFR60A 5 $12 / 19-12 / 20 / 06 \quad \overline{6354260}$ 
Air Sampling Associates, Inc.

Client Sample ID: HARDTN SDA DUCT+STACK RB FILTER A122

General Chemistry

Lot-Sample \#. . : : H6L140394-006

Date Sampled... : 12/11/06
Work Order \#...: JLHXM

Date Received. . : 12/12/06
Matrix....... A AIR

PREPARATION- PREP ANALYSIS DATE BATCH \#

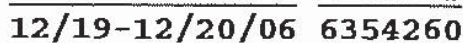

PARAMETER

Particulates (total) 359
RESULT

$\underline{R L}$ 0.50

Dilution Factor: 1

UNITS

METHOD
CFR60A 5
MDL.........: 0.50


Raw Data 
Total Particulates 


\section{STL Knoxville Particulate Matter Determination Data Review/Narrative Checklist Method: SW-846 Method 0050 by SOP KNOX-WC-0006, Rev. 2 Page 1 of 1}

\begin{tabular}{|r|r|}
\hline Batch Number: & 6354260 \\
\hline Lot Number(s): & 462140394 \\
\hline
\end{tabular}

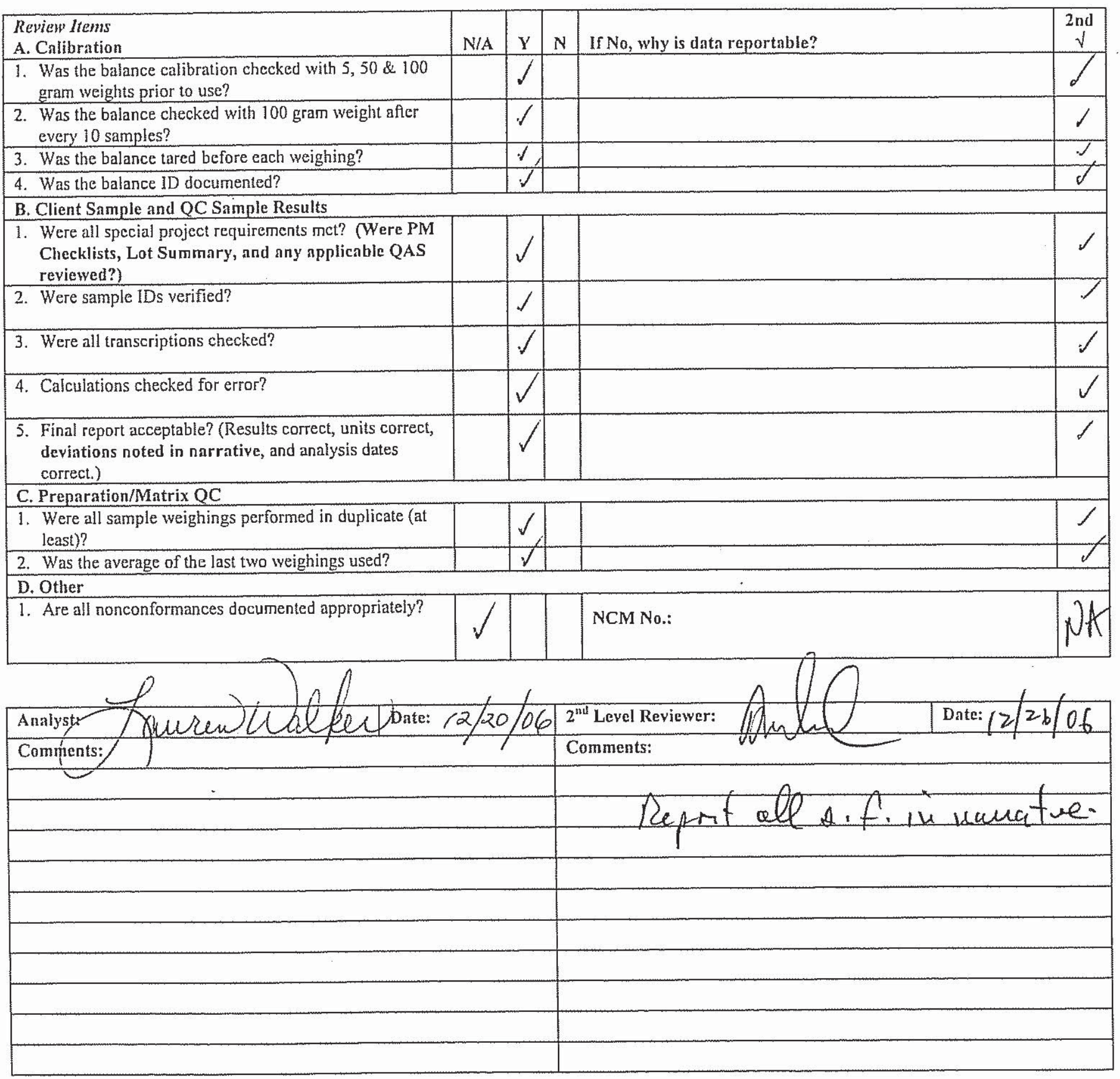




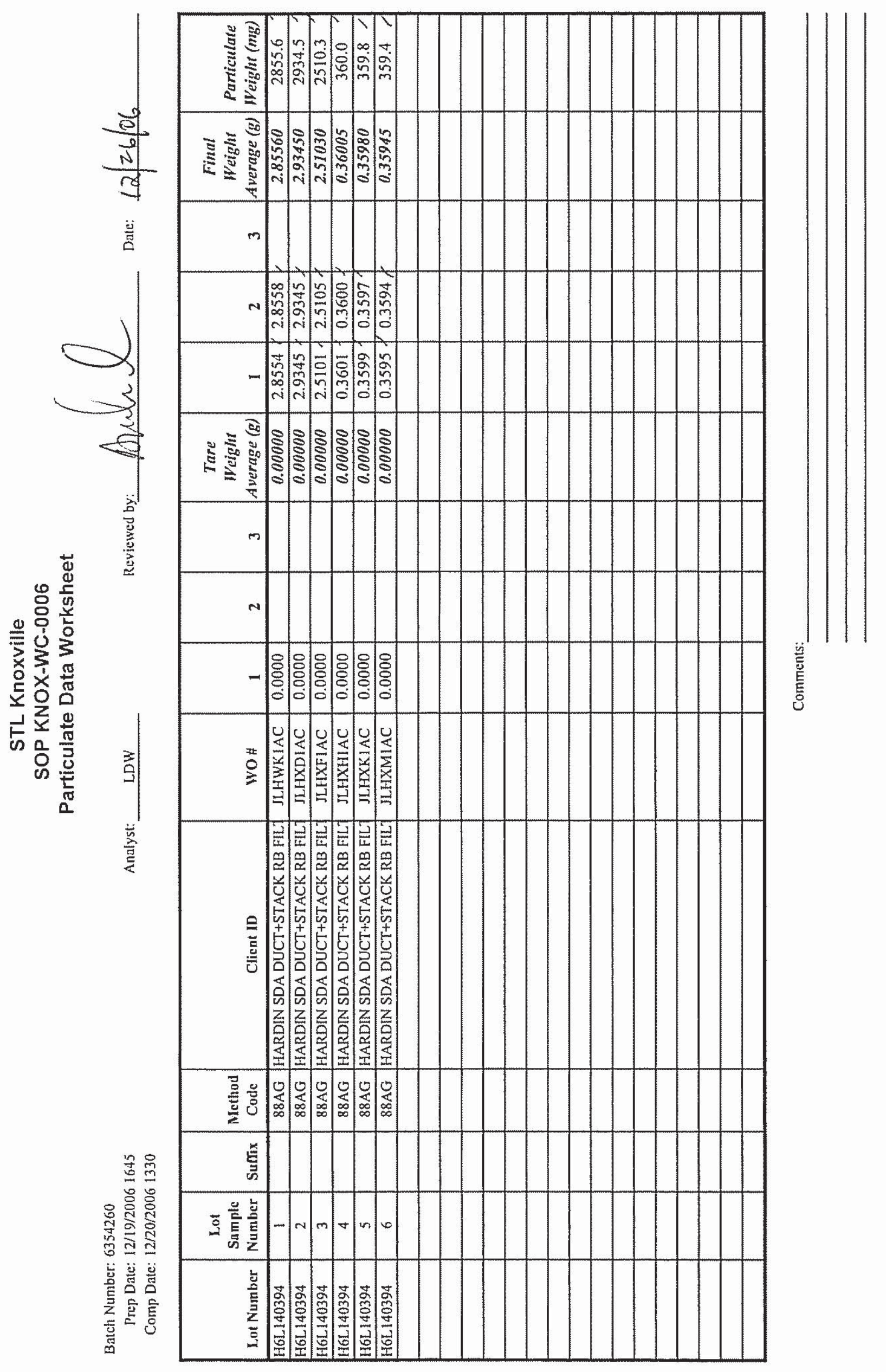




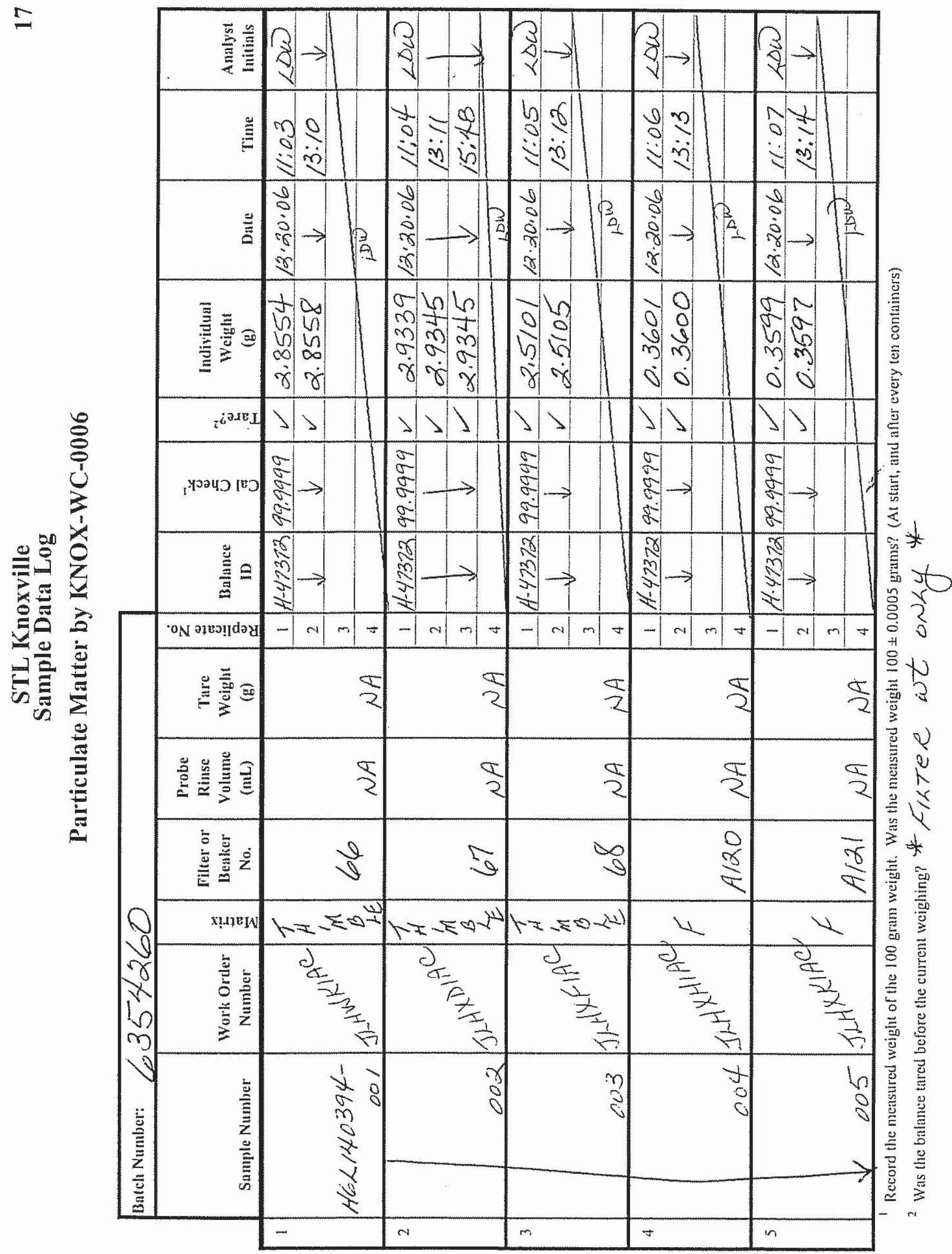




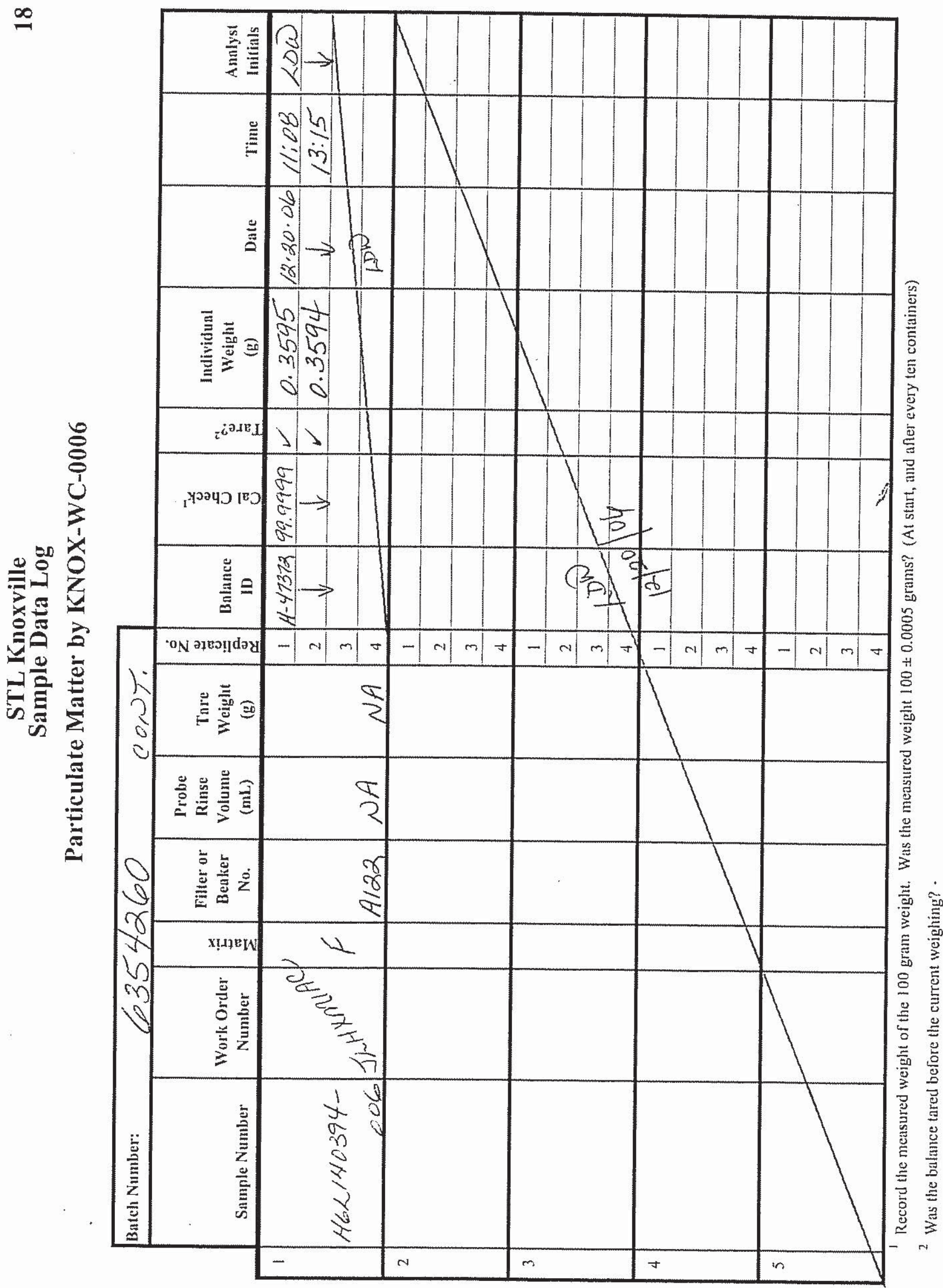


Sample Receipt Documentation 


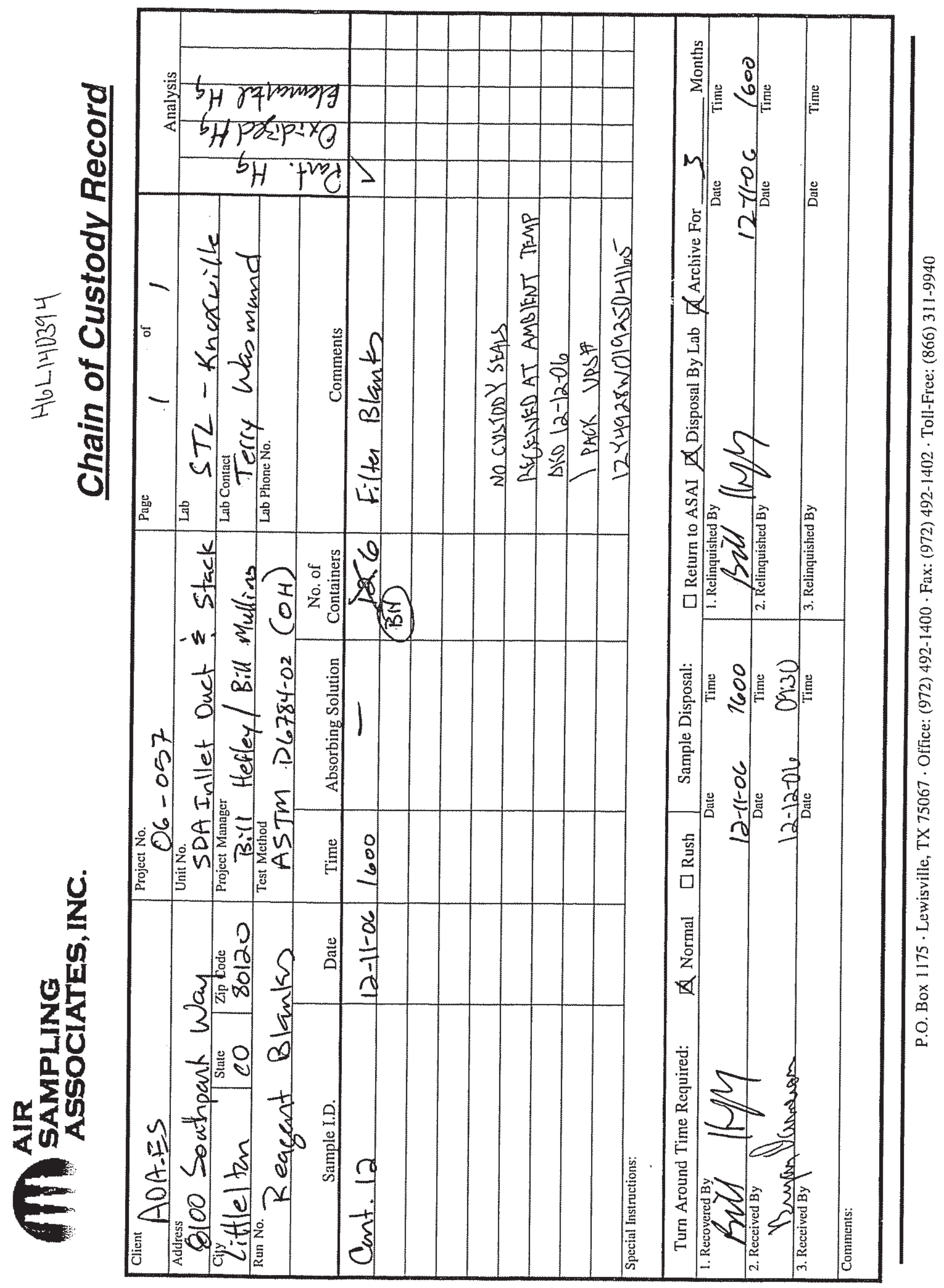




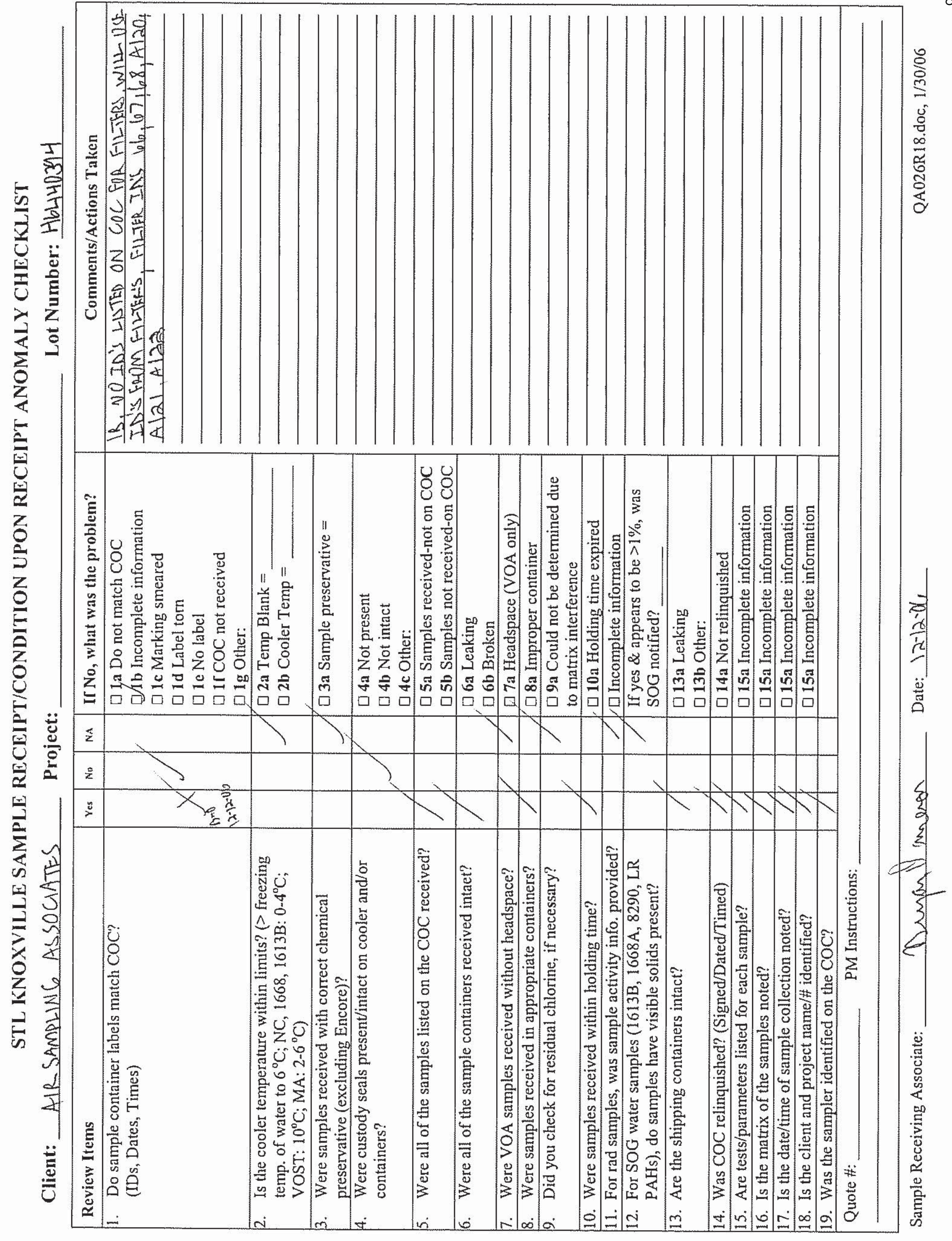


Appendix I:

NCASI Method 8A (Sulfur Trioxide) Analytical Data 


\section{SULFUR TRIOXIDE EMISSION DATA}

Job No.: $\quad$ 06-057

Job Name: $\underline{A D A-E S}$
Location: $\underline{\text { Hardin, MT }}$

Unit ID: SDA Inlet Duct

\begin{tabular}{|c|c|c|c|c|}
\hline Run No. & 1 & 2 & 3 & \\
\hline Date & $11 / 30 / 06$ & $11 / 30 / 06$ & $12 / 01 / 06$ & \\
\hline Time & $1323-1423$ & $1648-1748$ & $1401-1501$ & \\
\hline$\underline{N}$ - normality of $\mathrm{Ba}\left(\mathrm{ClO}_{4}\right)_{2}$ & 0.0099 & 0.0099 & 0.0099 & \\
\hline$M L_{1}-m l$ in impinger & 113 & 131 & 81 & \\
\hline $\mathrm{ML}_{\mathrm{A}}-\mathrm{ml}$ in aliquot & 1.0 & 1.0 & 1.0 & \\
\hline \multirow{3}{*}{$M L_{B}-\mathrm{ml}$ of $\mathrm{Ba}\left(\mathrm{ClO}_{4}\right)_{2}$ to titrate } & 0.2 & 0.2 & 0.1 & \\
\hline & 0.3 & 0.2 & 0.2 & \\
\hline & 0.2 & 0.1 & 0.2 & \\
\hline Avg. $\mathrm{ML}_{\mathrm{B}}-\mathrm{ml}$ of $\mathrm{Ba}\left(\mathrm{ClO}_{4}\right)_{2}$ to titrate & 0.2 & 0.2 & 0.2 & \\
\hline $\mathrm{ML}_{\mathrm{BB}}-\mathrm{ml}$ of $\mathrm{Ba}\left(\mathrm{ClO}_{4}\right)_{2}$ to titrate blank & 0.05 & 0.05 & 0.05 & \\
\hline$T_{m}$ - avg. gs meter temp. $\left({ }^{\circ} \mathrm{F}\right)$ & 70 & 74 & 72 & \\
\hline 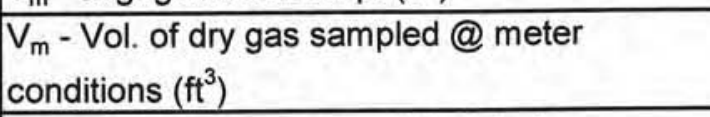 & 21.571 & 22.079 & 21.825 & \\
\hline$P_{b}$ - barometric pressure ("Hg Abs.) & 26.94 & 26.96 & 27.14 & \\
\hline $\begin{array}{l}\text { Q } \mathrm{s} \text { - stack gas Vol dry @ standard conditions } \\
\left(\mathrm{SCFM}^{*}\right)\end{array}$ & NA & NA & NA & \\
\hline $\mathrm{C}_{1}-\mathrm{SO}_{3}$ in impinger (mgs) & 6.71 & 7.78 & 4.81 & \\
\hline $\mathrm{ppm} \mathrm{SO}_{3}$ & 3.69 & 4.20 & 2.60 & \\
\hline $\mathrm{C}_{\mathrm{SO}_{3}}$ - emission rate of $\mathrm{SO}_{3}$ (lbs/day) & NA & $\mathrm{NA}$ & NA & \\
\hline $\mathrm{C}_{\mathrm{s}}$ - emission rate of $\mathrm{S}$ (Ibs/day) & $\mathrm{NA}$ & NA & NA & \\
\hline
\end{tabular}

* 29.92" Hg, $68^{\circ} \mathrm{F}\left(760 \mathrm{~mm} \mathrm{Hg}, 20^{\circ} \mathrm{C}\right)$

$C_{1}=\frac{M L_{1} \times\left(M_{B} \times M L_{B B}\right) \times \underline{N} \times 40}{M L_{A}}$

$C_{\text {sO3 }}=p p m S_{3} \times Q_{s} \times 299.1 \times 10^{-6}$

$\mathrm{ppmSO}_{3}=\frac{0.6021 \times \mathrm{C}_{1} \times\left(\mathrm{T}_{\mathrm{M}}+460\right)}{\mathrm{V}_{\mathrm{m}} \times \mathrm{P}_{\mathrm{b}}}$

$\mathrm{C}_{\mathrm{s}}=\frac{\mathrm{C}_{\mathrm{SO} 3}}{2.5}$ 
Appendix J:

Chain of Custodies 


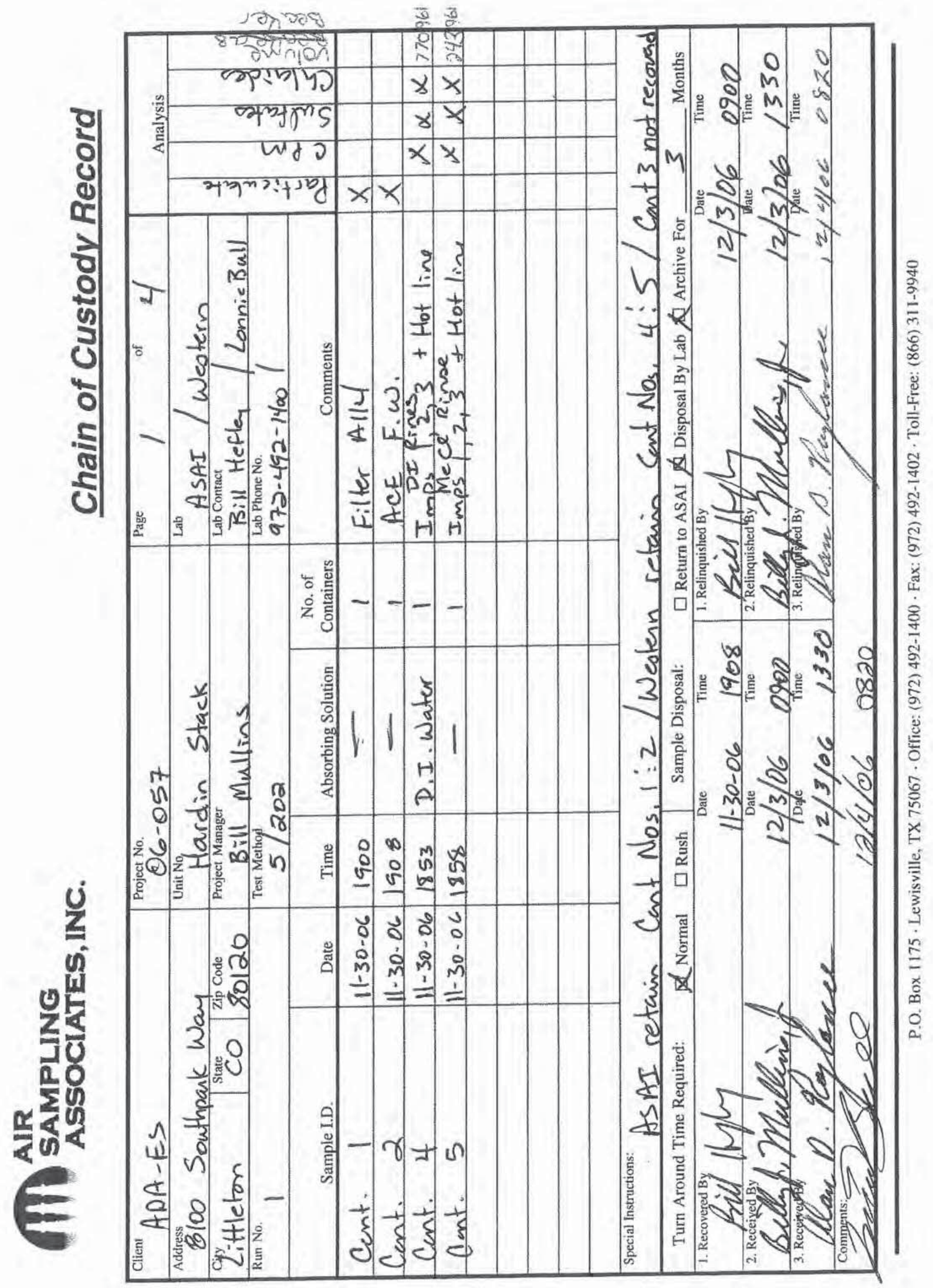




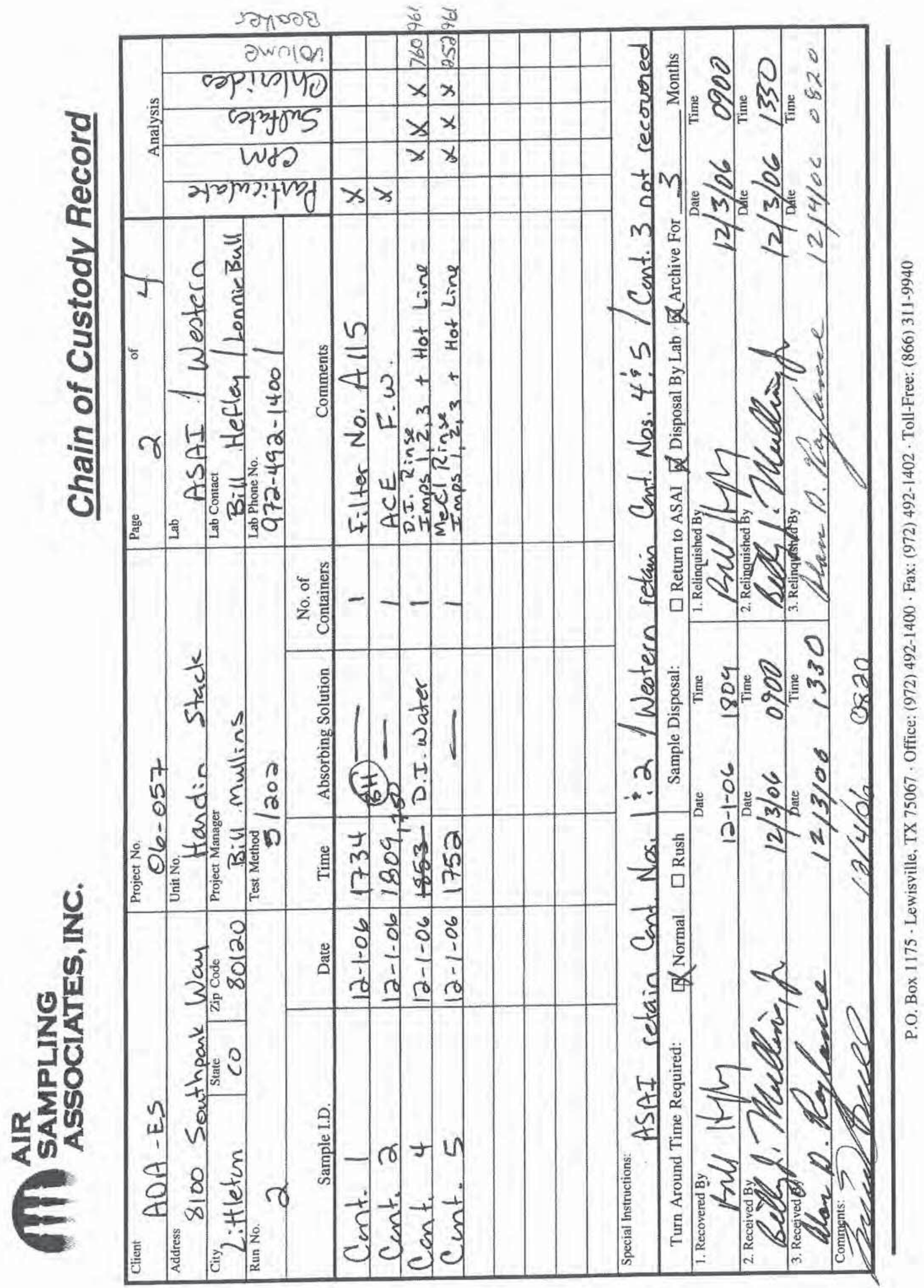




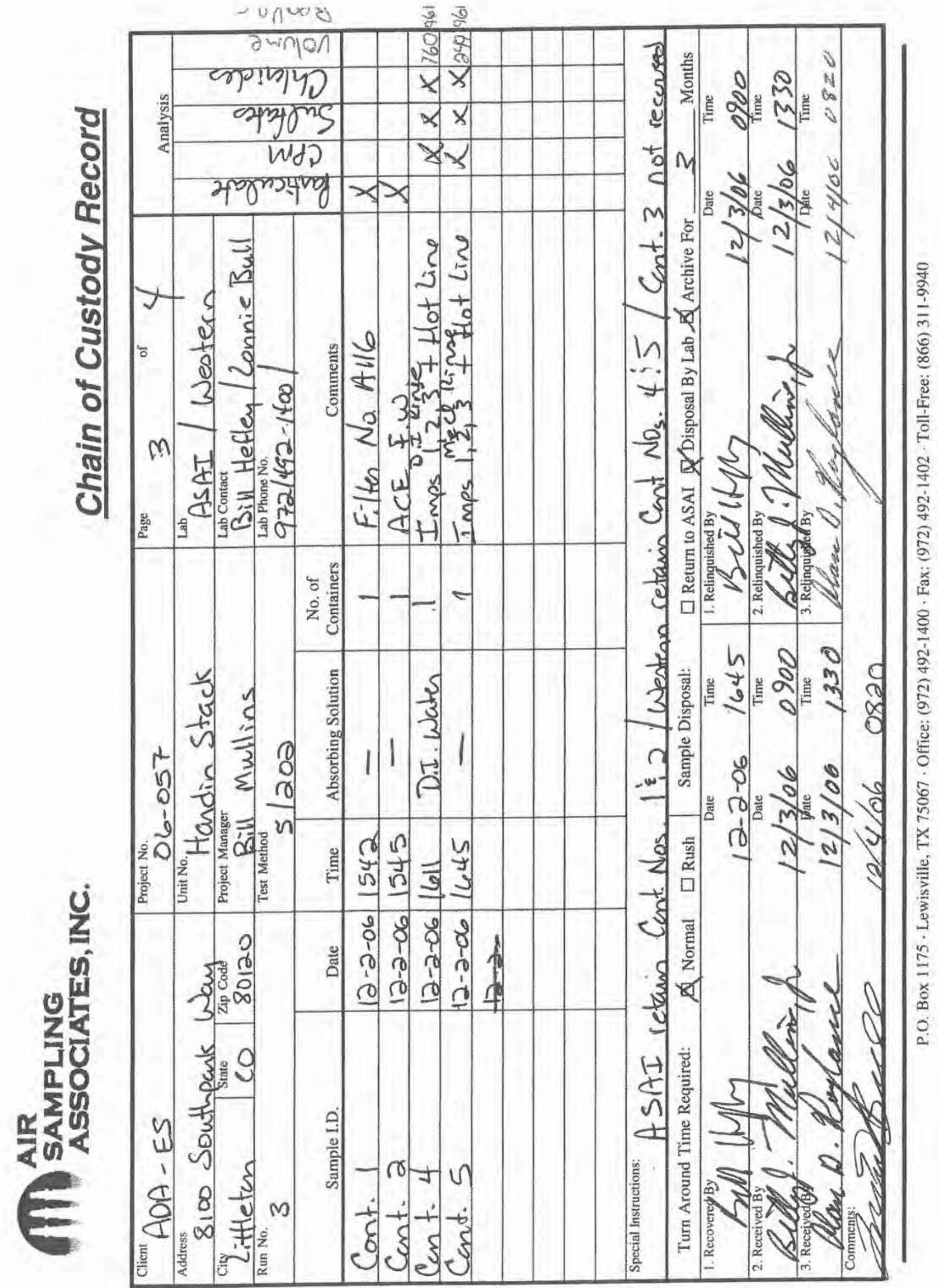




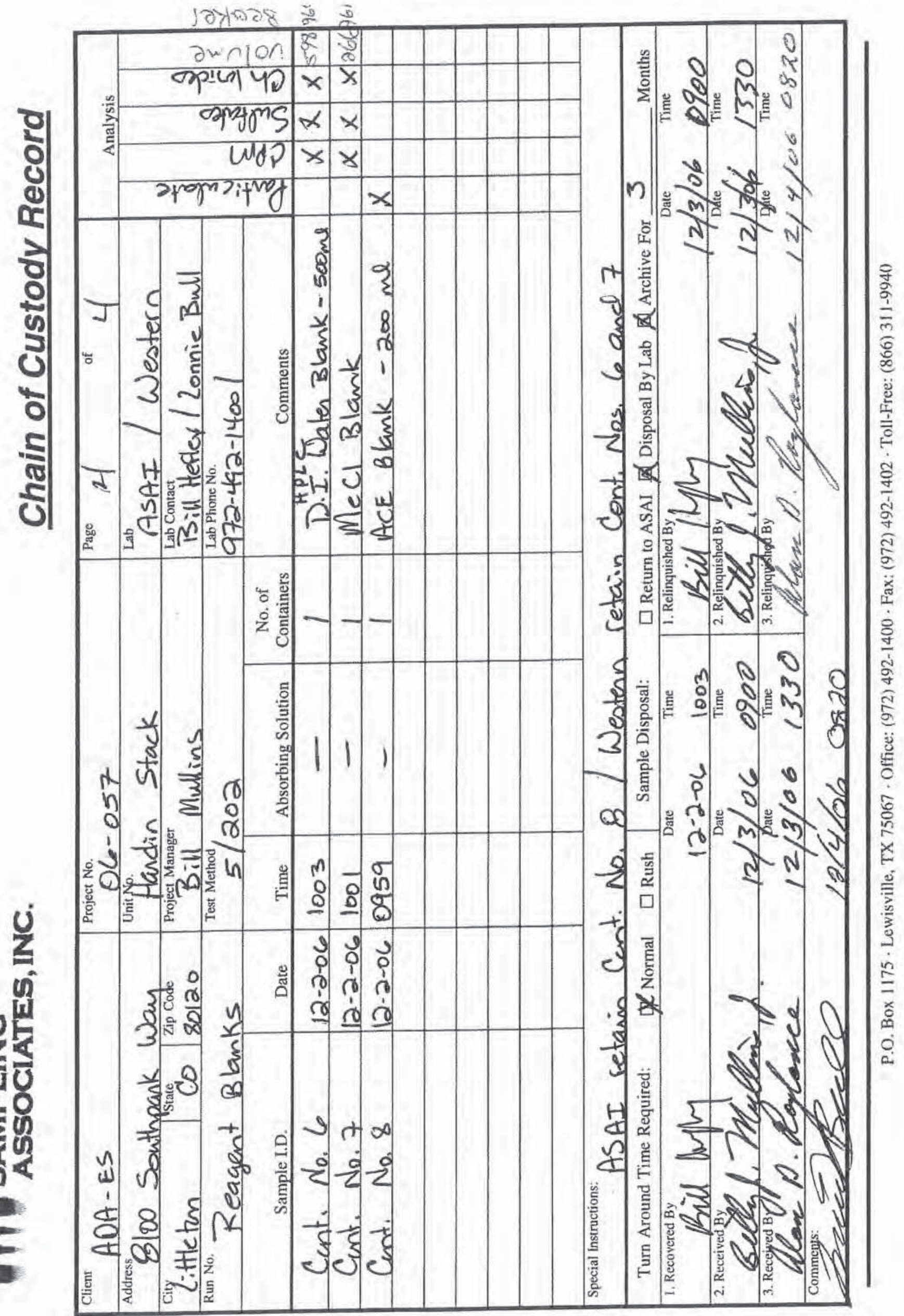




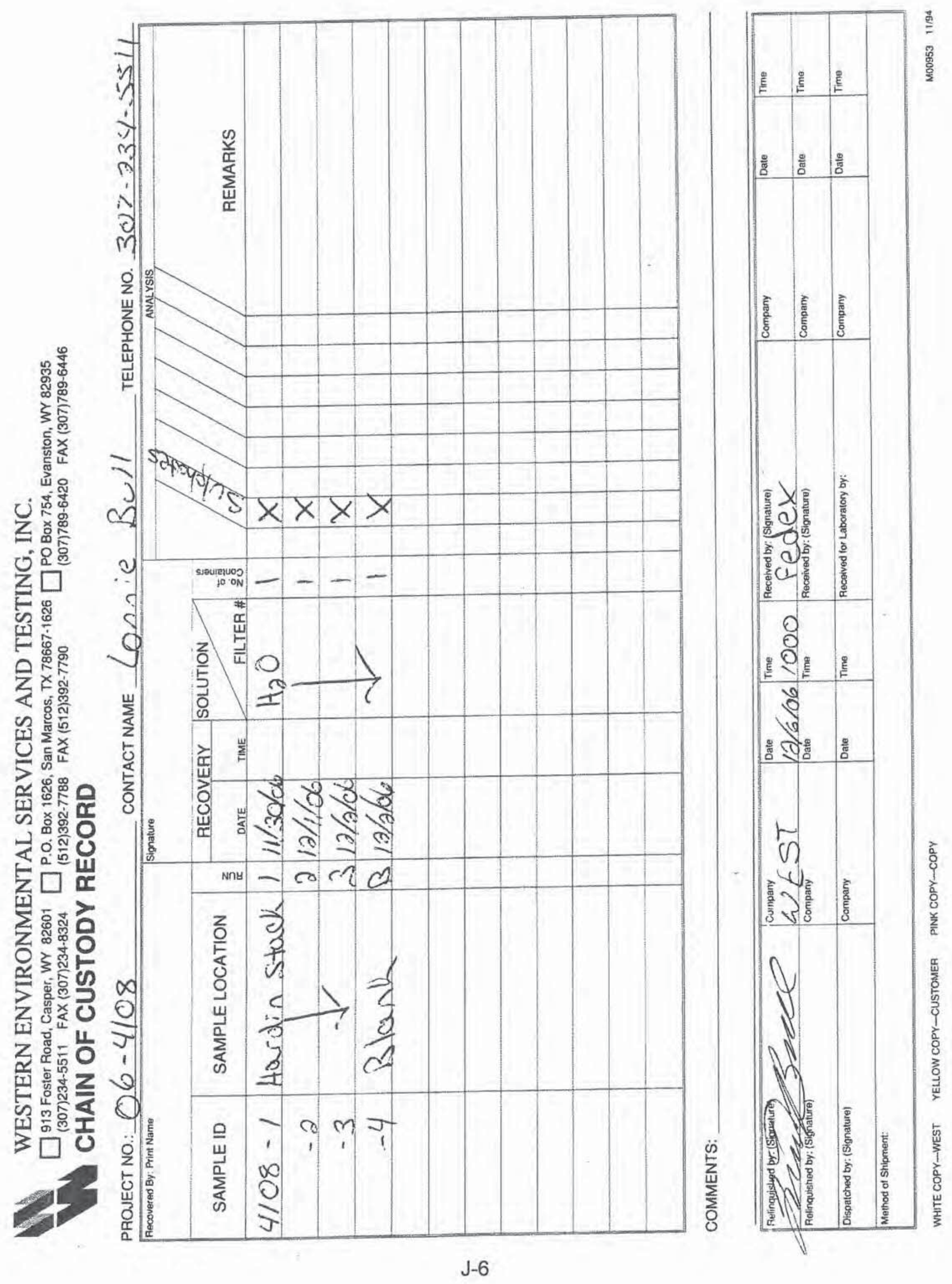



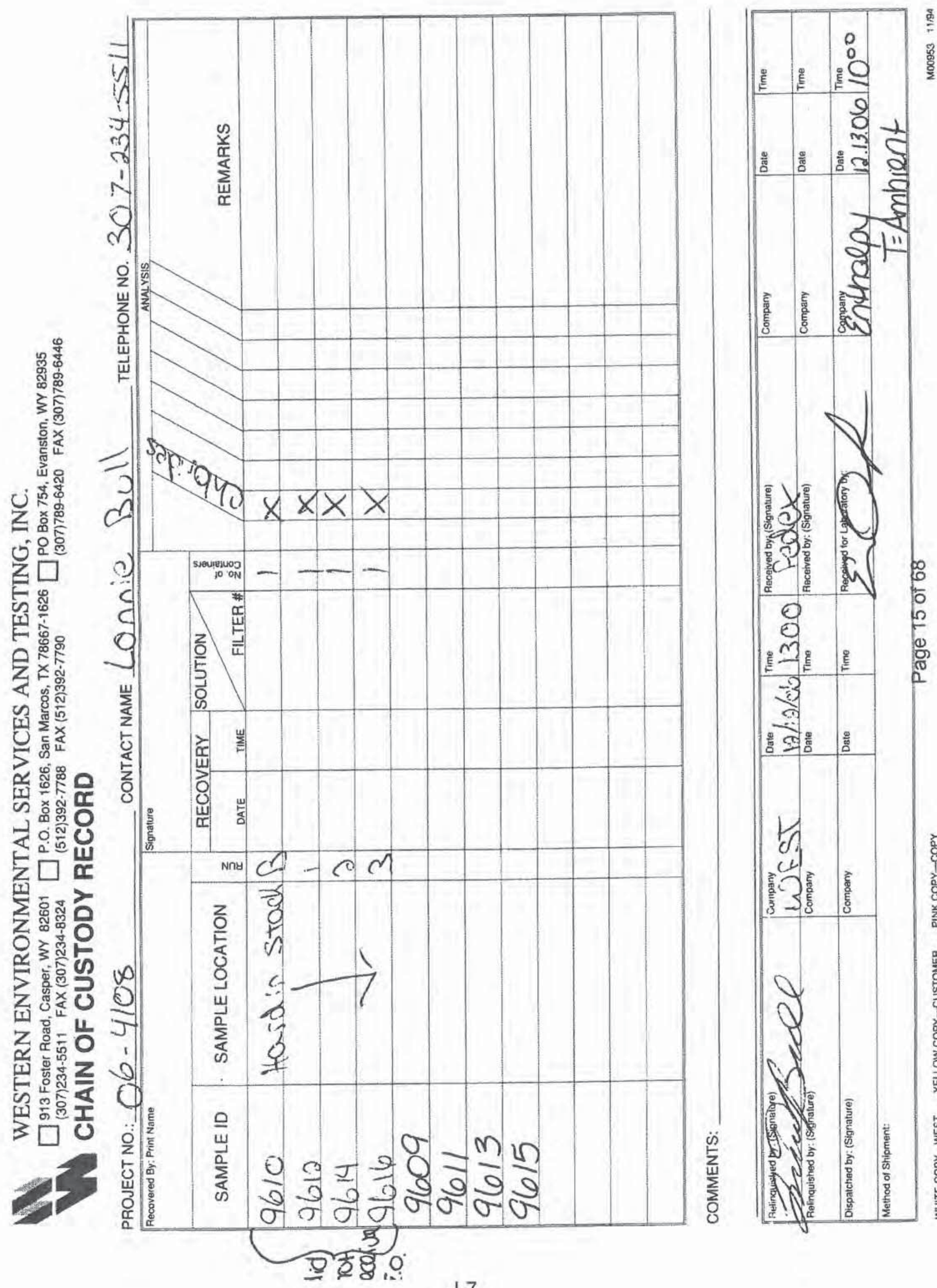


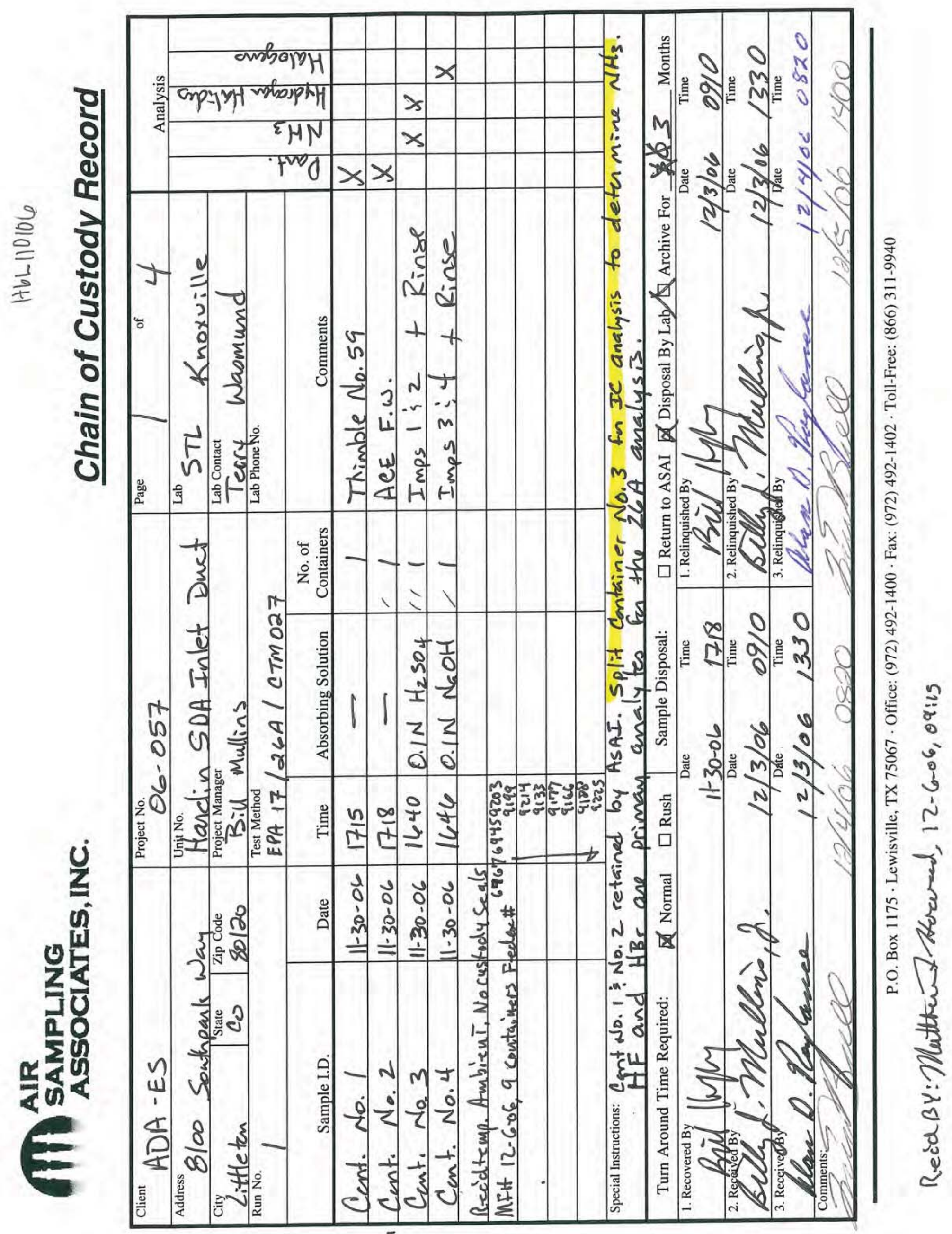




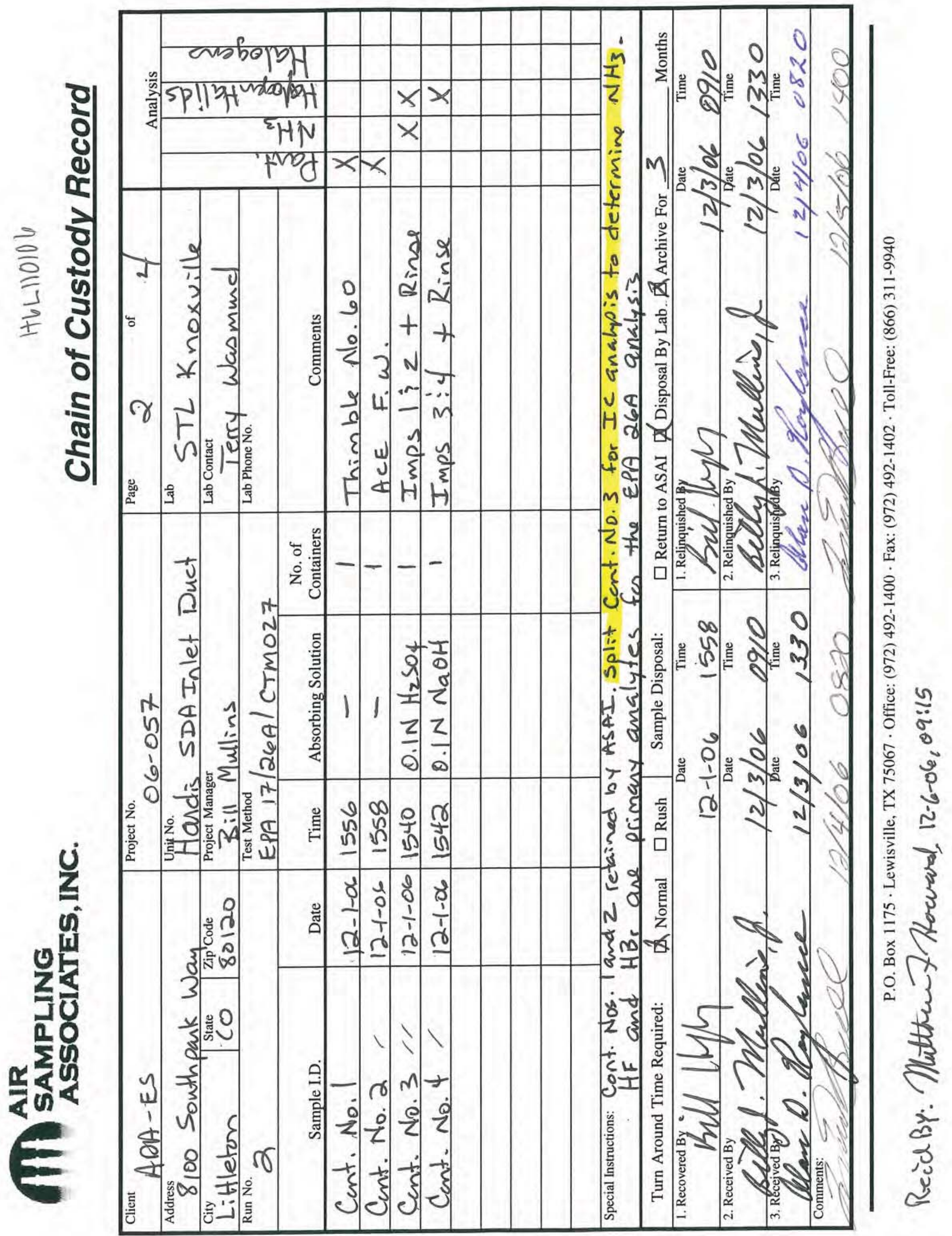




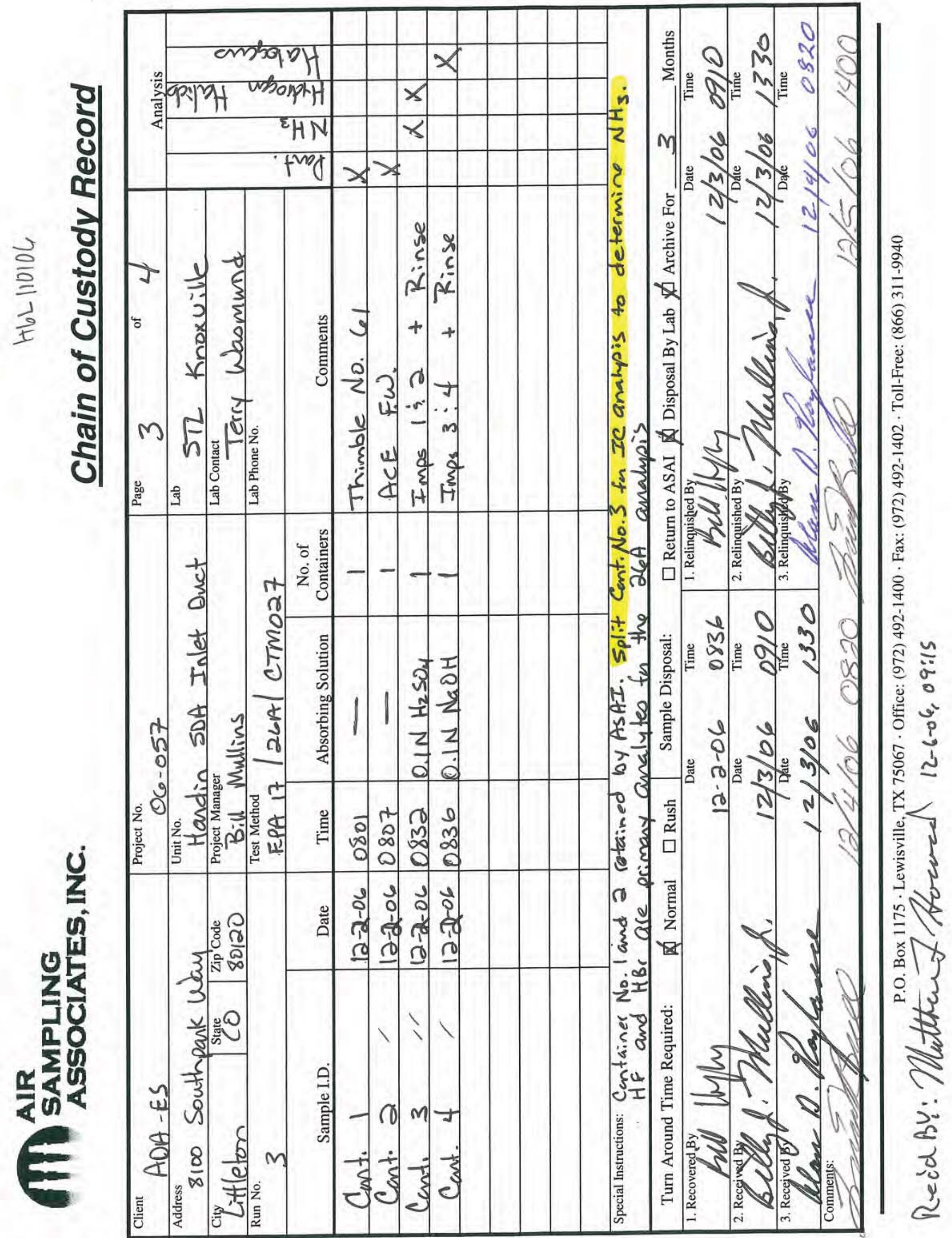




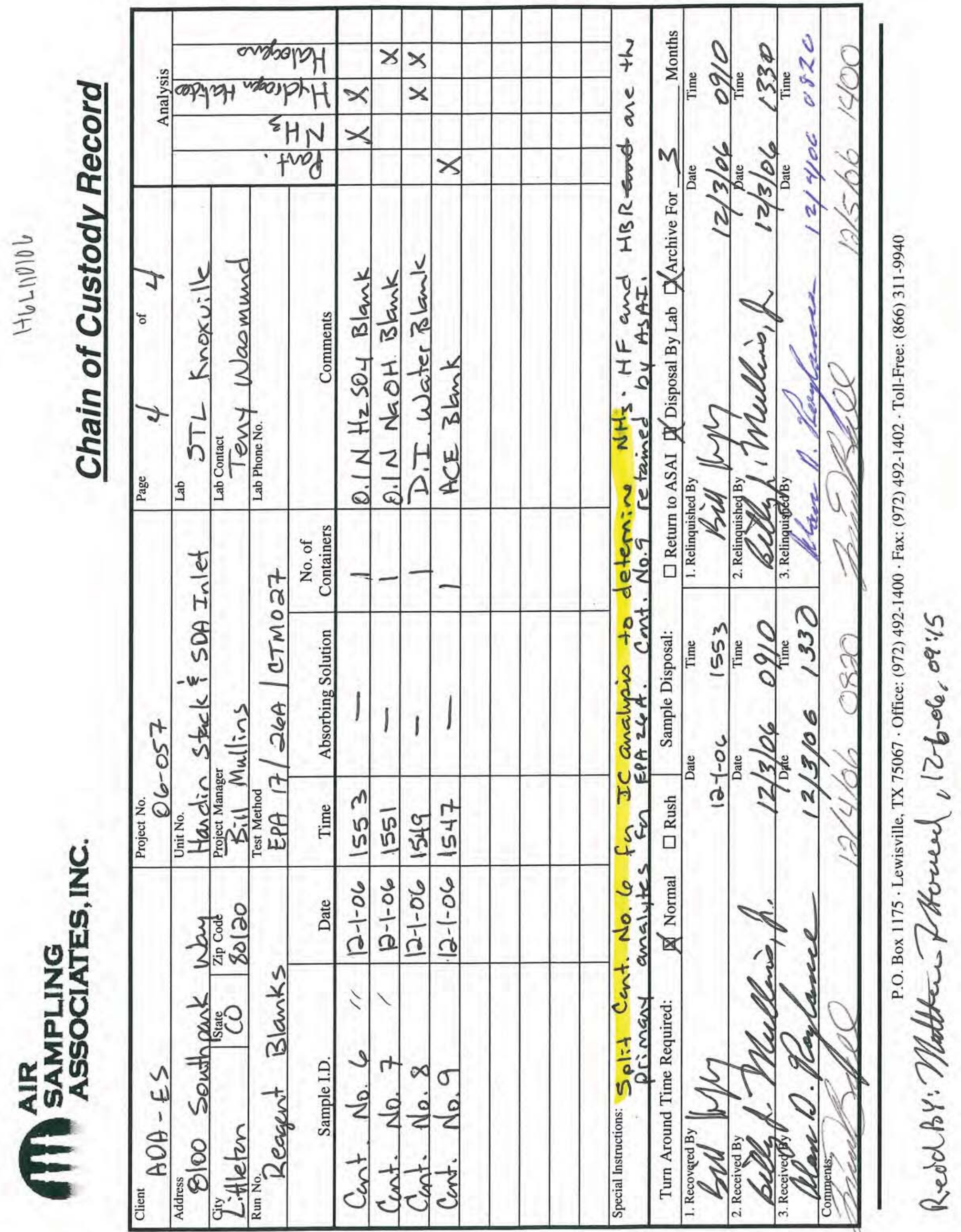




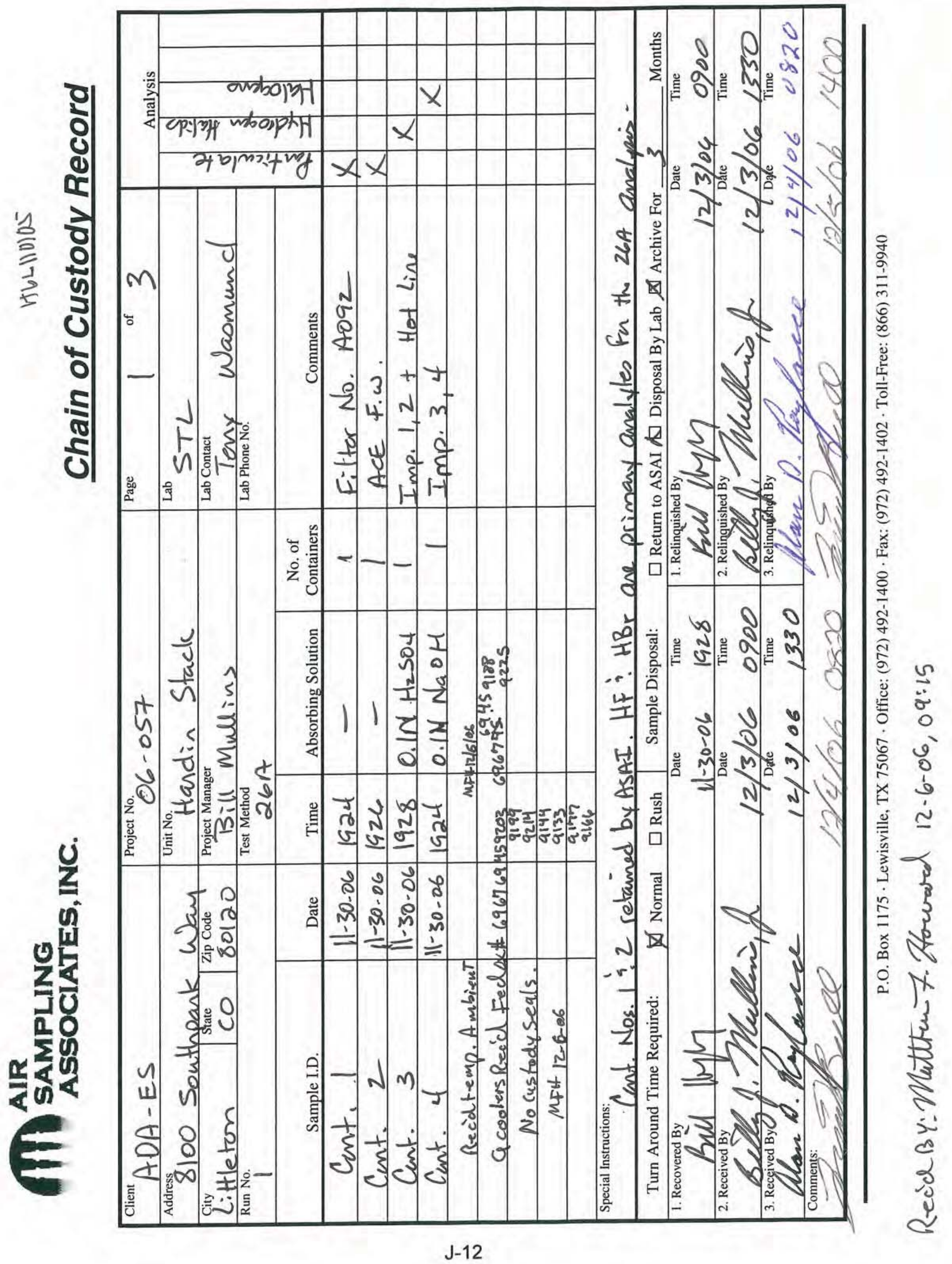




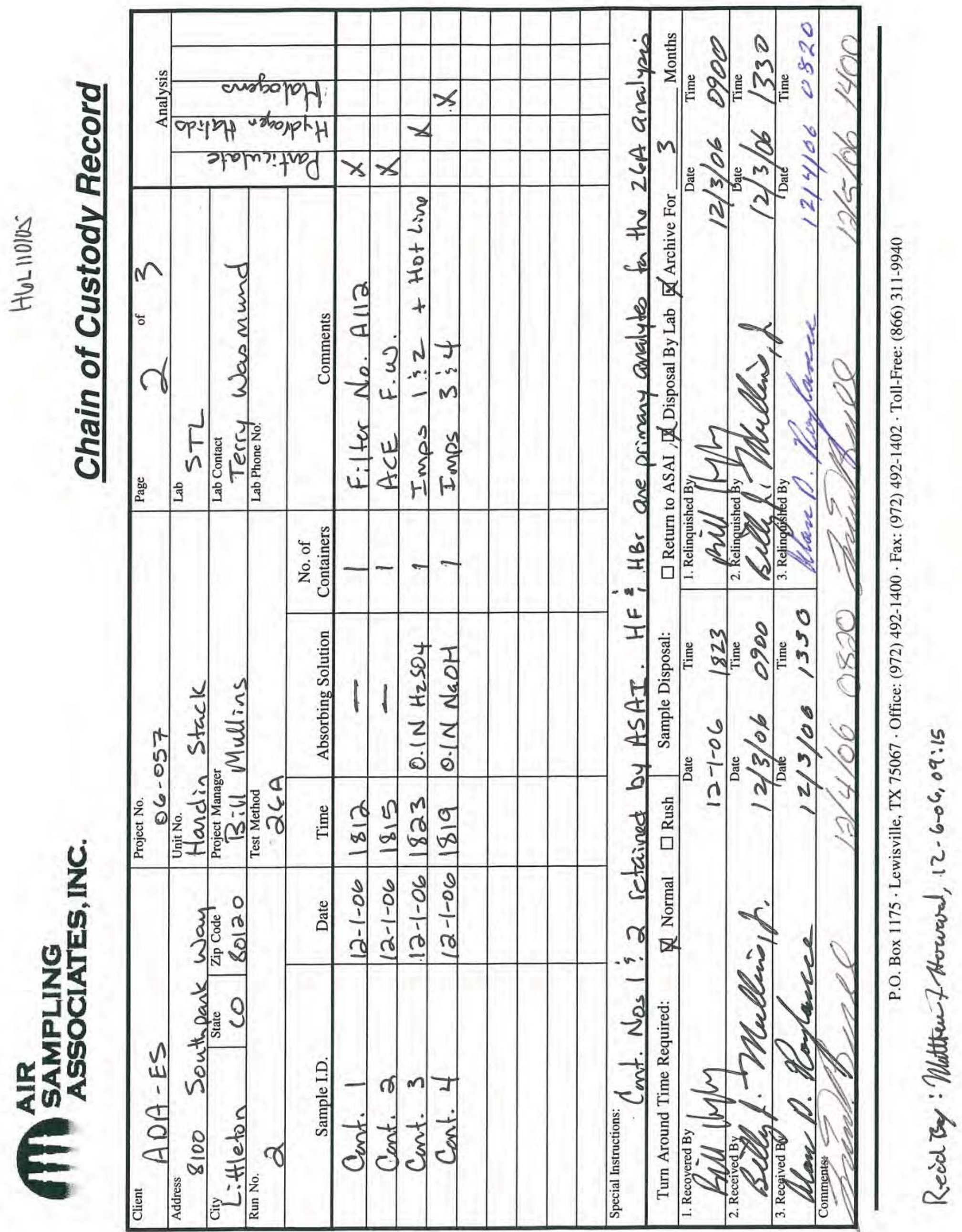




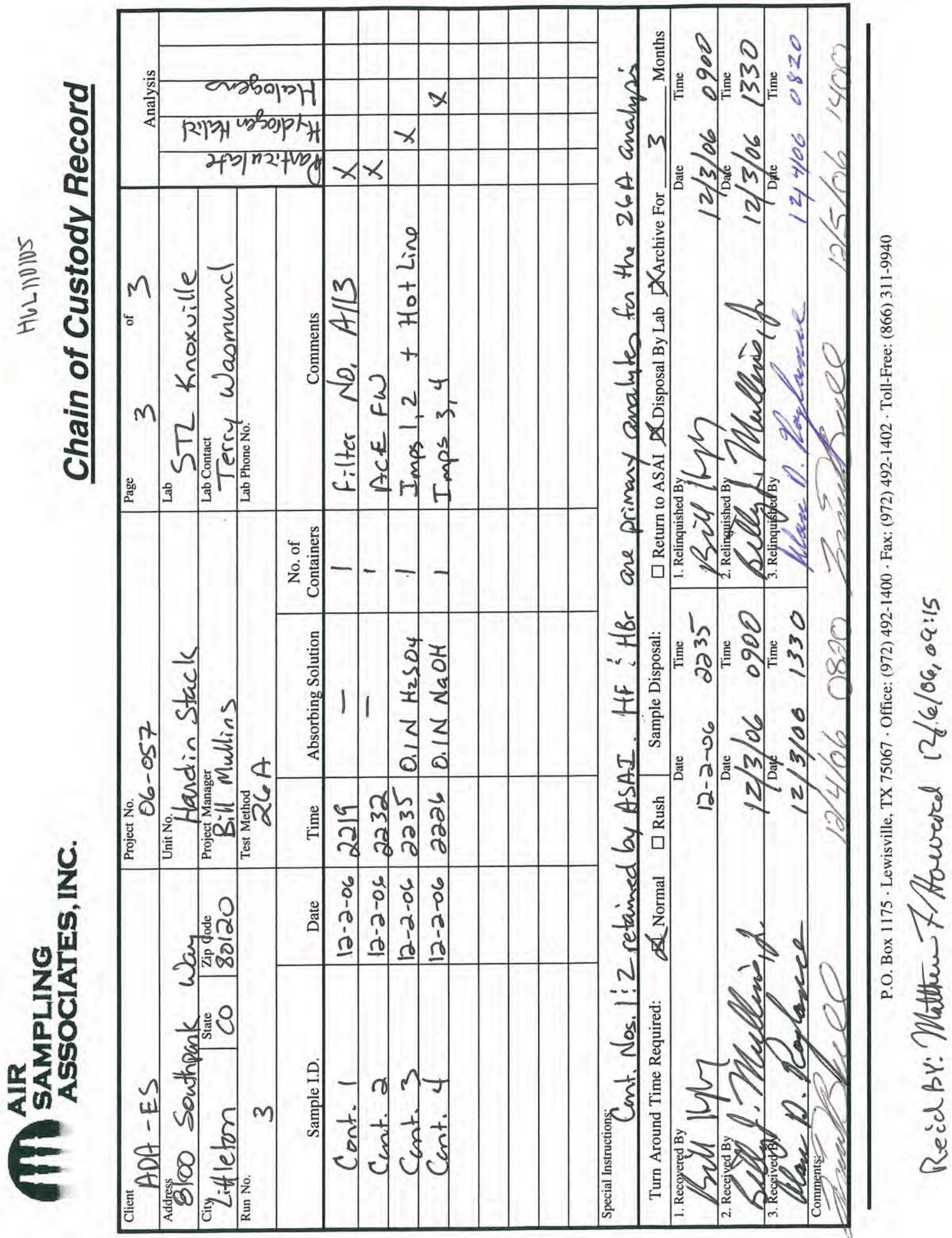




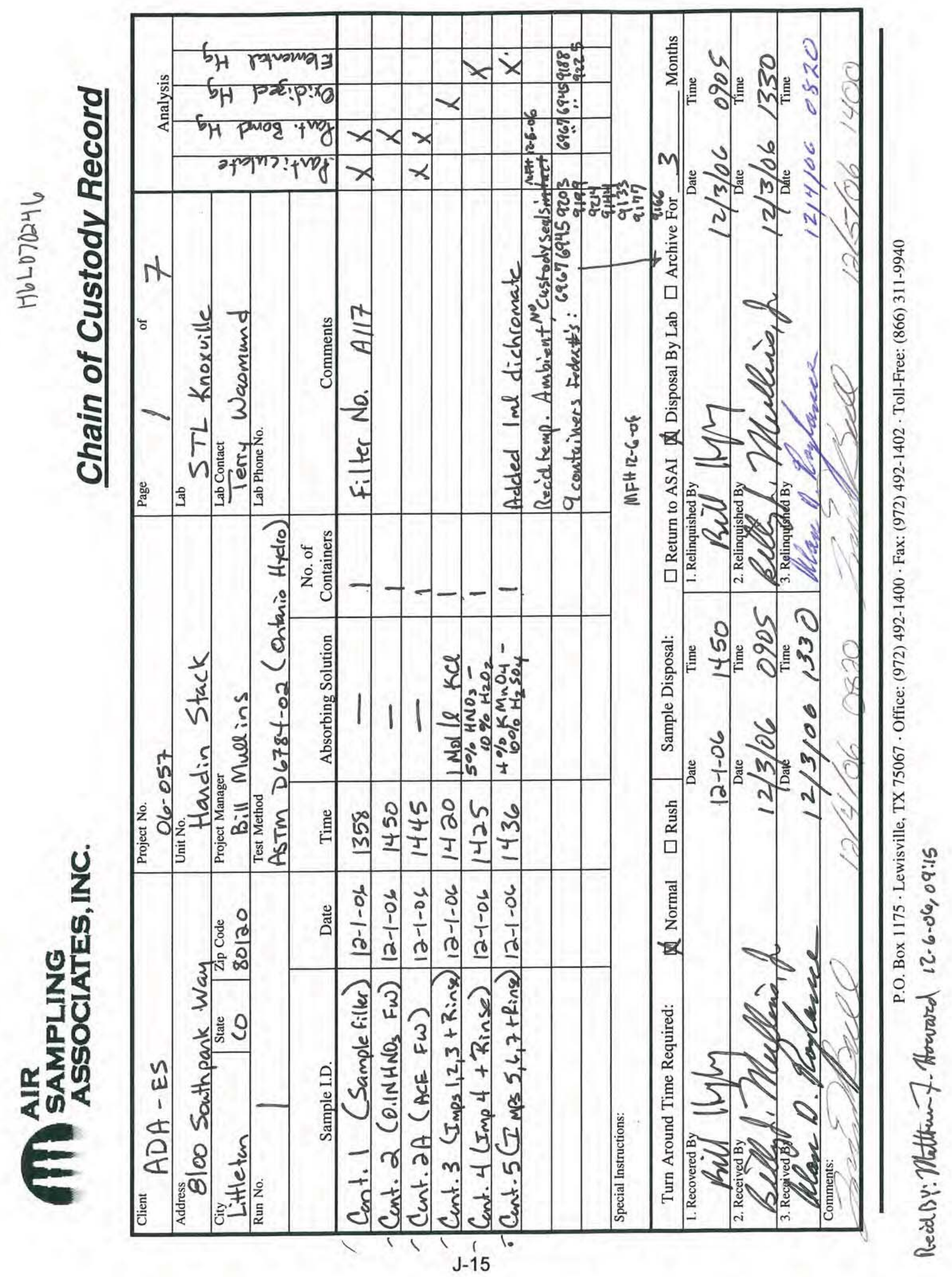




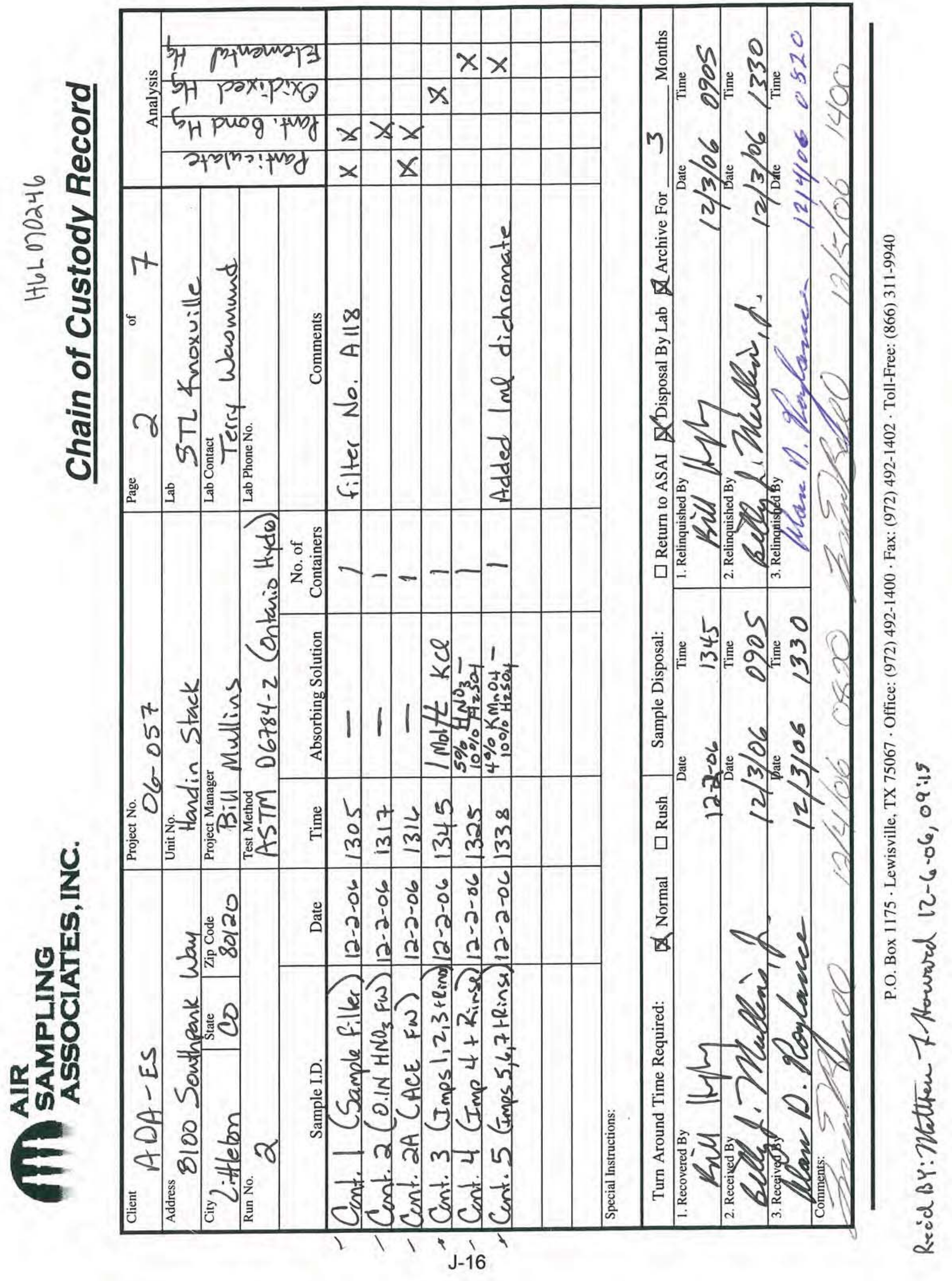




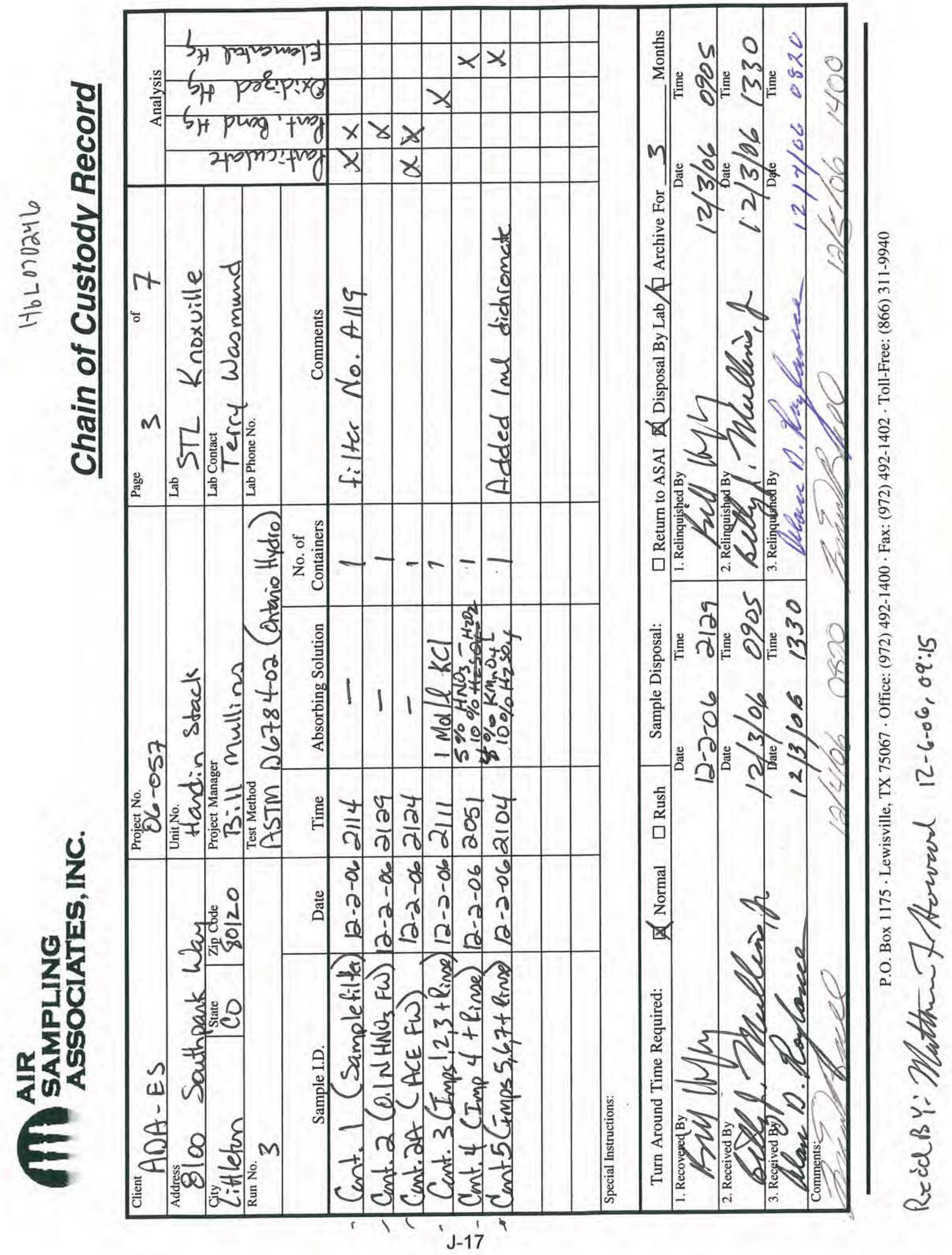




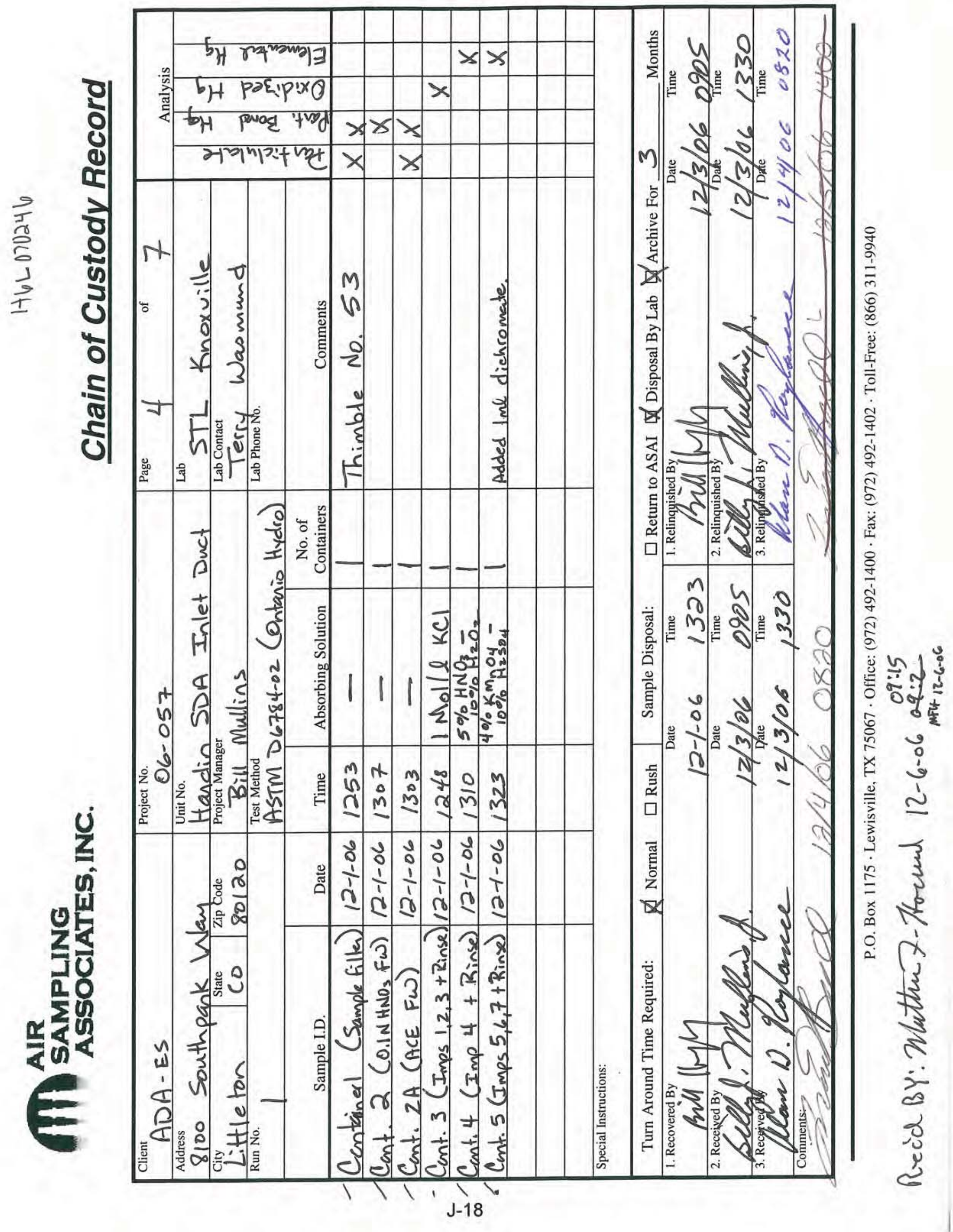




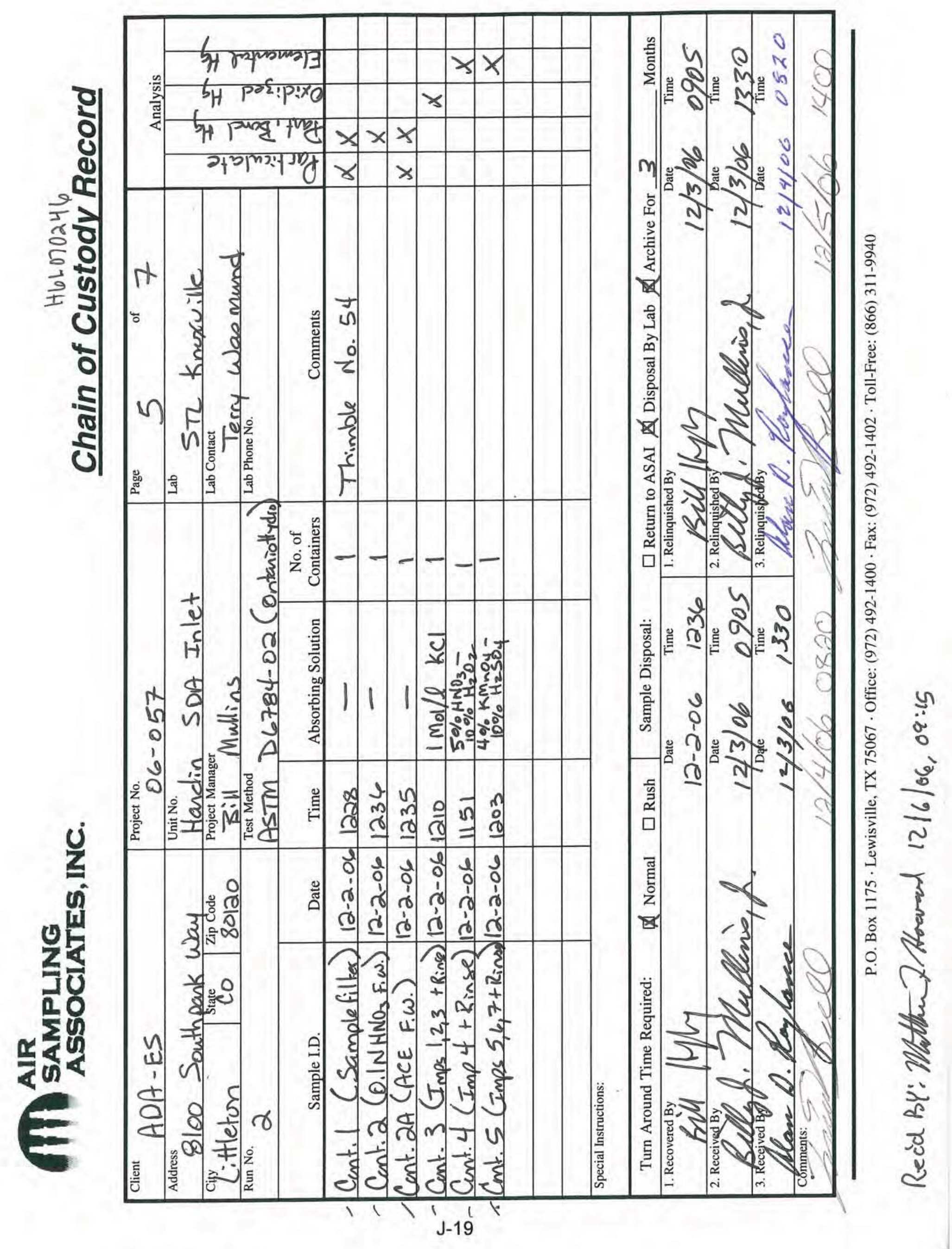




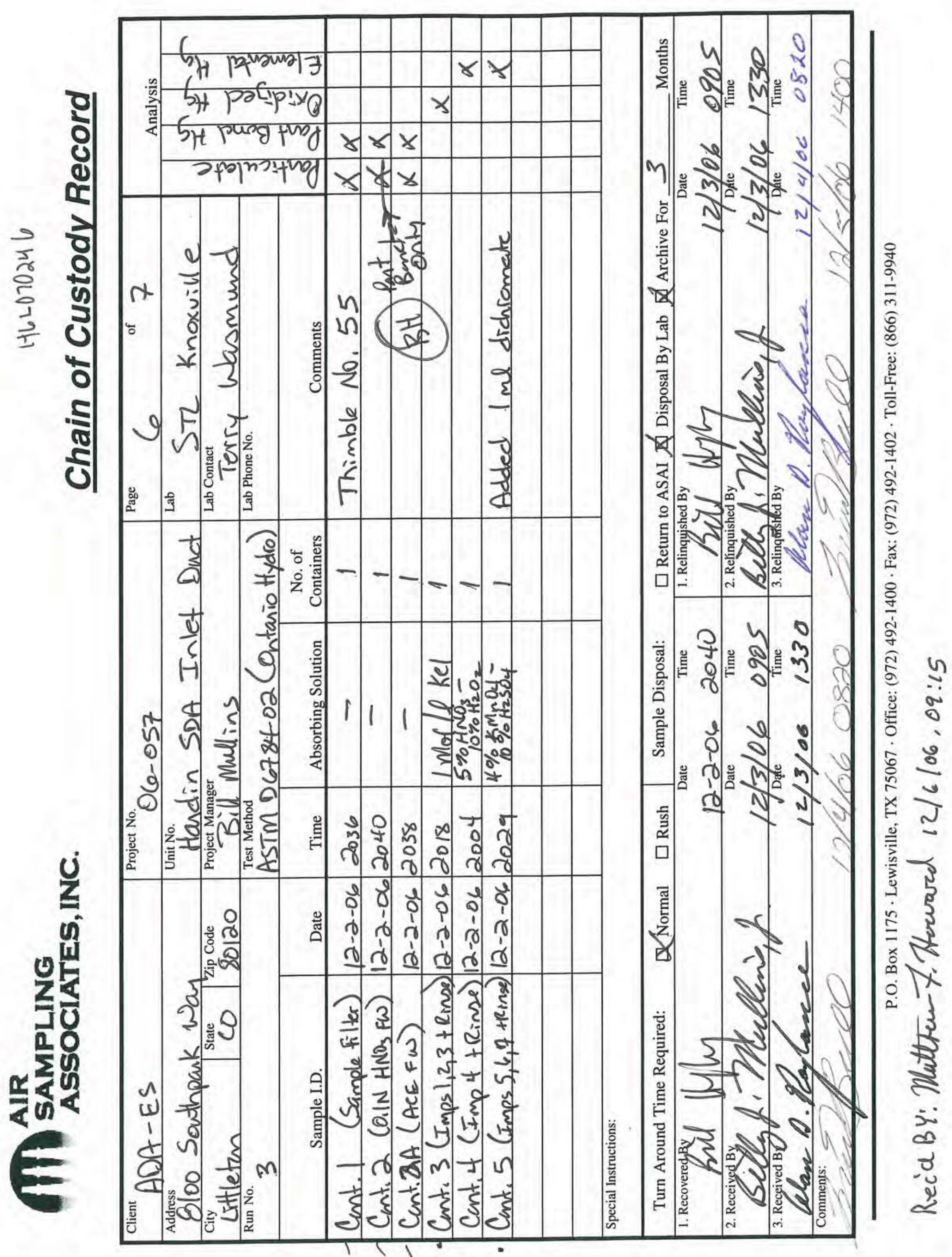




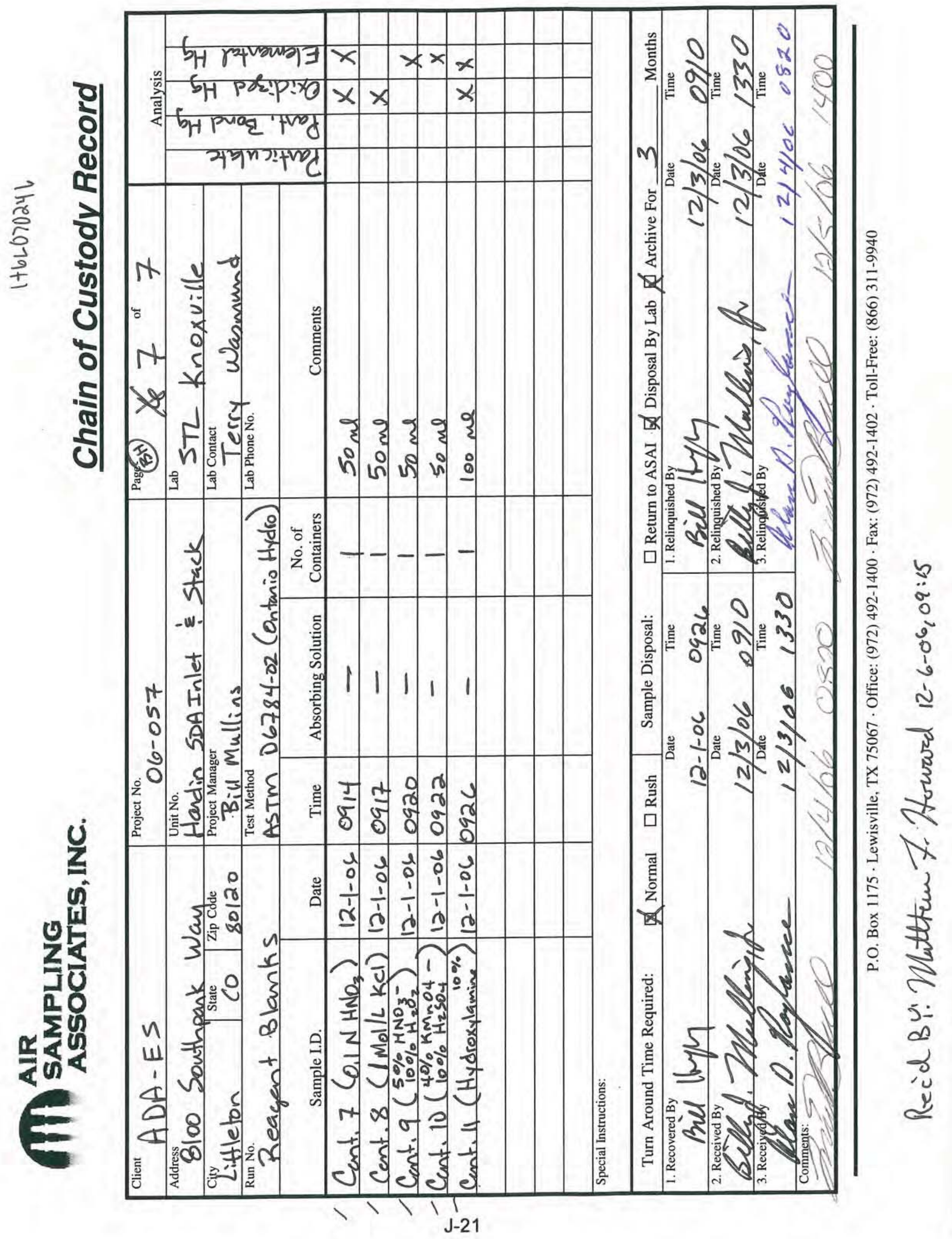




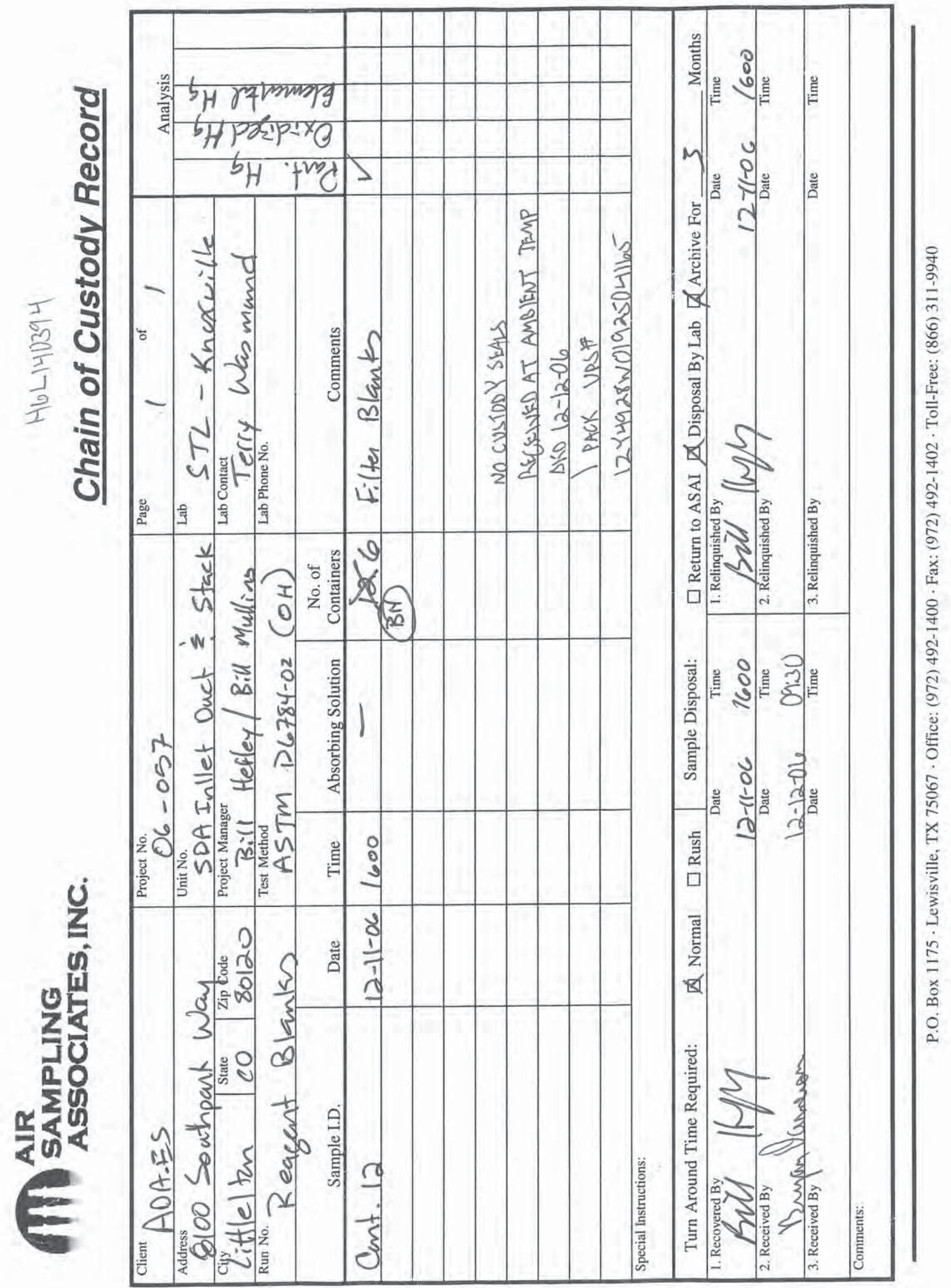




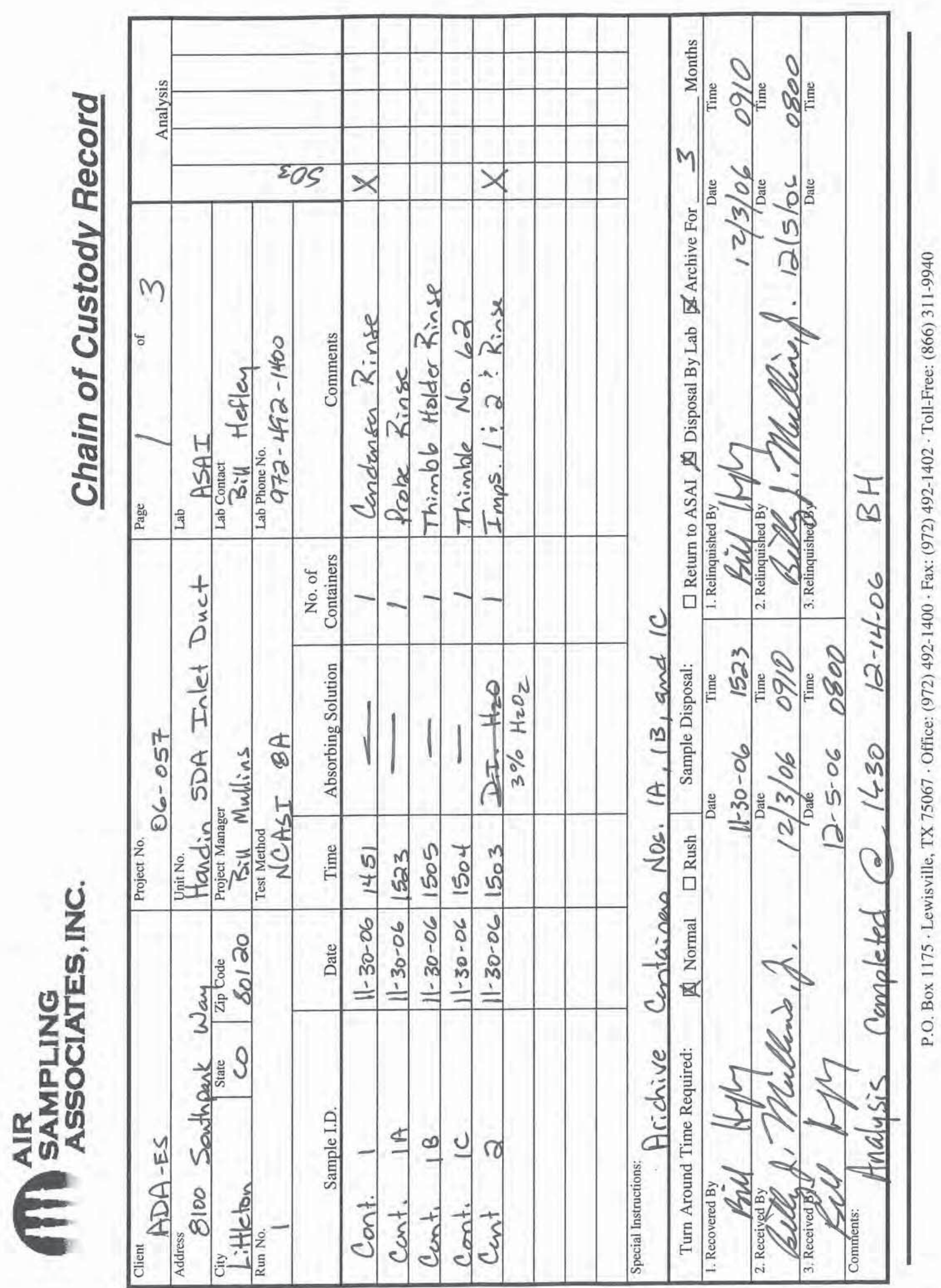




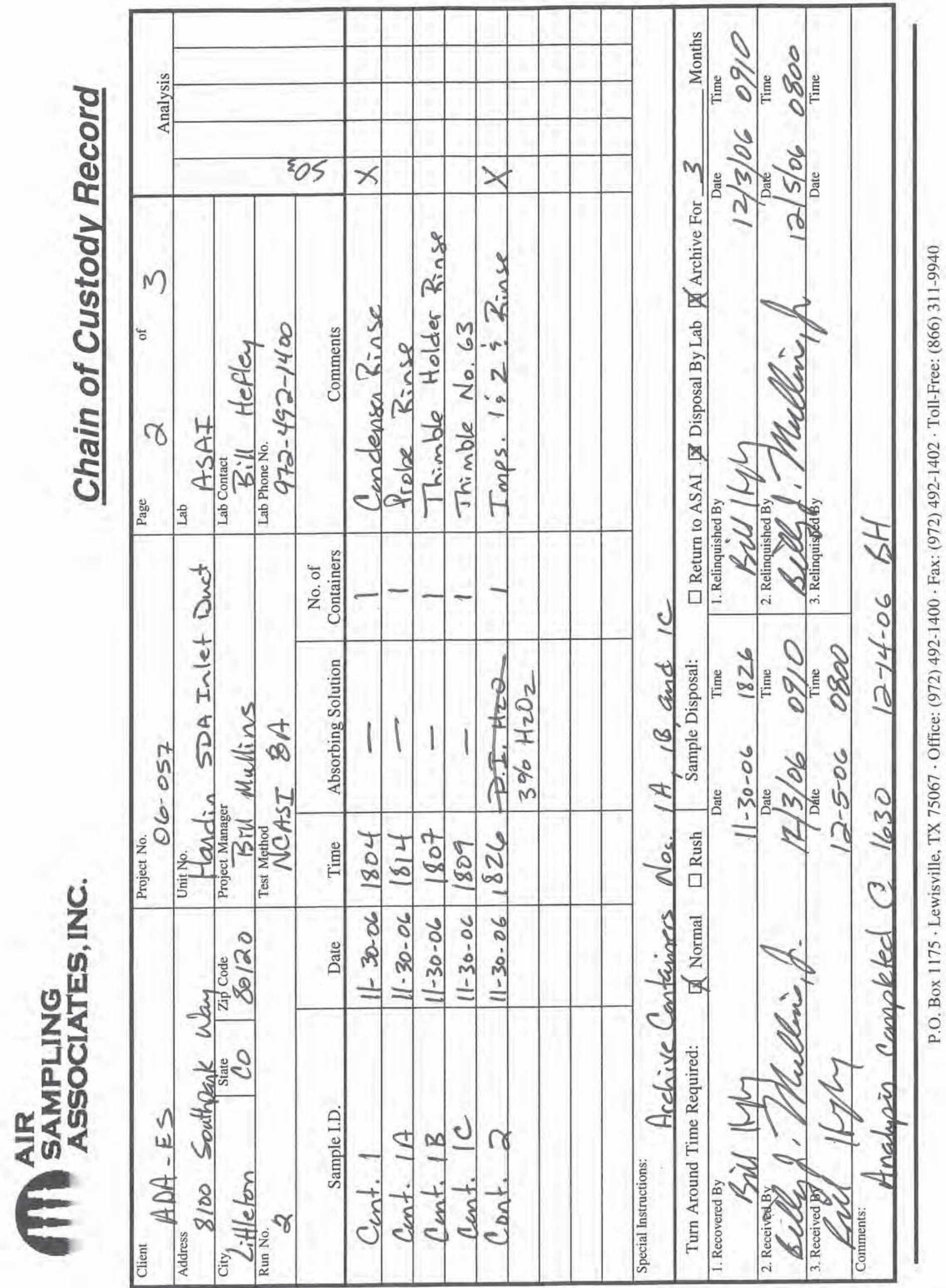




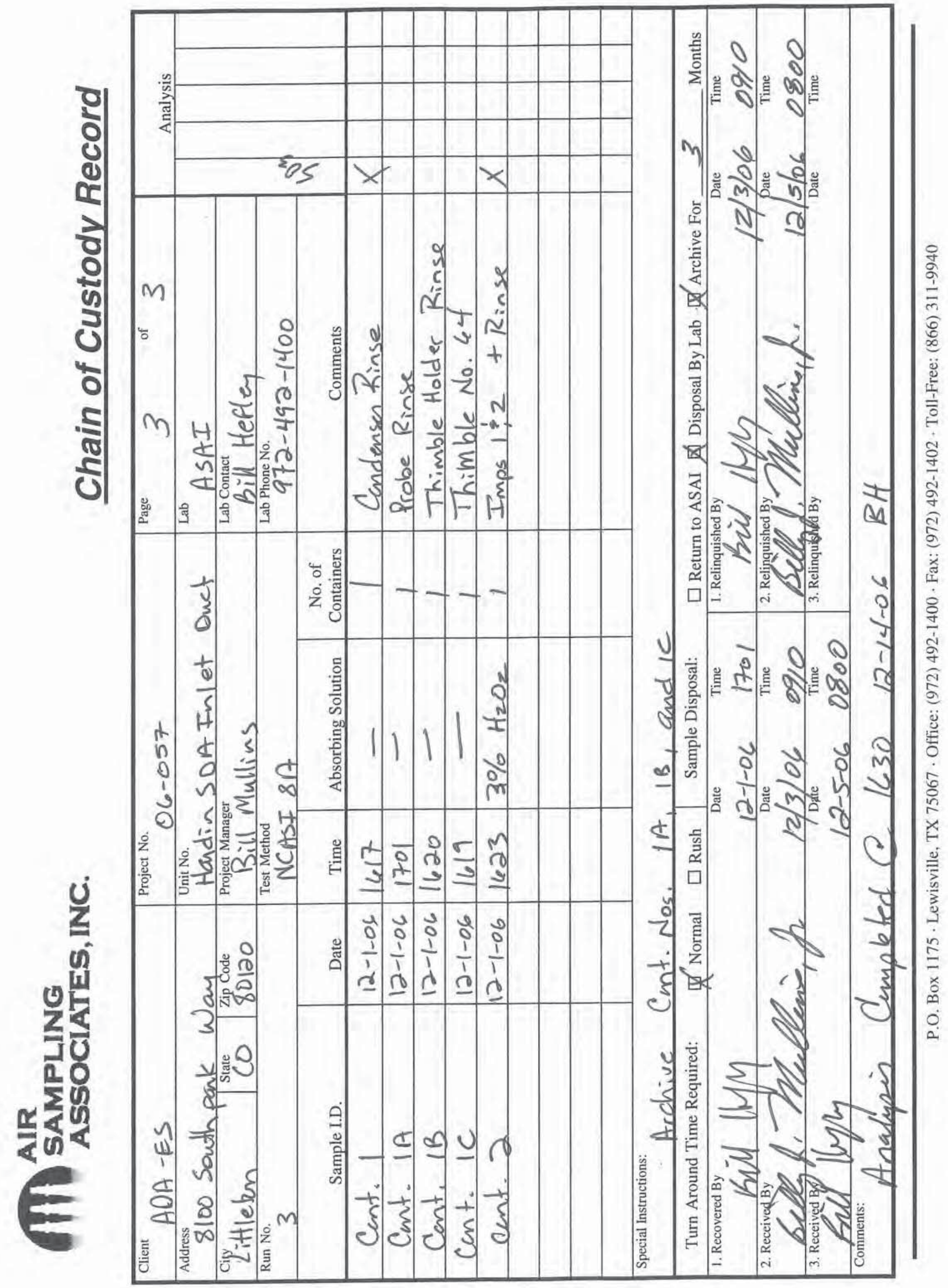


Appendix K:

Resumes of Test Personnel 
BILLY J. MULLINS, JR.; President

Education

Post Graduate Study Environmental Engineering at Southern Methodist University; Dallas, Texas 1970.

M.S. 1969, New York University; New York, New York, in Civil Engineering (Air Resources).

B.S. 1968, Texas Tech University; Lubbock, Texas, in Civil Engineering (Water Resources). Studies in Engineering at the U.S. Naval Academy; Annapolis, Maryland, 1963-1964

Professional Attended Short Course on Air Pollution Engineering at the University

Training of Texas at Austin, February 1970.

$\underline{\text { Courses }}$

Attended four-week management course presented by the American Management Association, 1976.

Certification Registered Professional Engineer

Certified Visible Emissions Evaluator

Licensed Private Pilot (Multi-Engine-Land, Instrument)

Diplomat in the American Academy of Environmental Engineers

Inductee into the Stack Sampling Hall of Fame

Certified as Qualified Environmental Professional (QEP)

Professional Air \& Waste Management Association - Past Chairman, Past Vice Memberships Chairman, and Past Board of Directors of North Texas Chapter and Southwest Section; Past Chairman, Consultants Committee; Past Chairman, Source Measurement Committee

Source Evaluations Society - Past President, Past Board of Directors

\section{American Management Association}


MULLINS (p. 2)

Publications Authored "Real World Experience with USEPA's New Sampling and Analytical Methods for Conducting Risk Burn," May 1998.

Co-authored "Sulfur Compound Emissions of the Petroleum Production Industry," December 1974.

Co-authored "Field Procedure for Stabilizing Hydrogen Sulfide Samples to be Analyzed Using Modified Methylene Blue Technique," presented at the Conference on Ambient Air Quality Measurements, Austin, Texas, March 1975.

Co-authored "Atmospheric Emissions Survey of the Sour Gas Industry," October 1975.

Co-authored "Technique for Insuring the Validity of Samples for High Concentrations of Sulfur Dioxide Using the EPA Method 5 Sampling Train," presented at the Third National Conference on Energy and the Environment, College Corner, Ohio, September 1975.

Teaching Experience
Conducted training seminars on sampling methods periodically since 1974 to present.

Conducted a one-day seminar on Part 75 Testing over ten times in 1993 and 1994.

Served as a lecturer in the Environmental Protection Agency's (EPA) training course number 450, "Source Sampling for Particulate Pollutants," for two years from January 1974 to October 1975 and March, 1992.

Conducted a two-day training course entitled "technical Assistance in Source Sampling" at lowa State University, Ames, lowa, for the Environmental Protection Agency (EPA), October 1974.

Conducted Environmental Protection Agency's (EPA) training course number 450, "Source Sampling for Particulate Pollutants," at Research Triangle Park, North Carolina, September 1975. 
MULLINS (p. 3)

Teaching

Experience

$\underline{\text { (Cont'd) }}$
Technical

Experience
Conducted a two-day short course entitled "Performing and Observing Source Sampling," Dallas, Texas, July 1976, May 1977, October 1977, November 1987 and November 1988; Lake Charles, Louisiana, May 1977; Casper Wyoming, May 1977; Point Comfort, Texas, November 1992.

Served as a lecturer in the Environmental Protection Agency's twoday seminar entitled "Asphalt Industry Environmental Solutions," presented in Dallas, Texas, March 21-22, 1979.

Conducted a two-day short course entitled "Performing and Observing Source Sampling," Phoenix, Arizona, August, 1990, for the State of Arizona, Department of Environmental Quality; Lincoln, Nebraska, March 1980, for the State of Nebraska, Air Quality Control Division.

Directed and performed stack sampling on over 2000 sources of which over 500 were sampled simultaneously using more than one sampling train at several points in the flue gas stream; 1972-present.

Directed and performed over 200 short-term ambient air studies using mobile sampling vans and various ambient air sampling equipment; 1972-present.

Designed, directed and operated over 20 permanent ambient air networks of various size and duration for a variety of parameters; 1972-present.

Designed surface and underground drainage systems for residential subdivisions, public works projects, and shopping centers; 19691972.

Designed several residential subdivisions including lot layout, street design, drainage design, and utility design; 1969-1972. 
MULLINS (p. 4)

Research

Projects

Related

$\underline{\text { Projects }}$
Supervised and conducted a study made by the Hawaiian Sugar Planters' Association to characterize the emissions for several bagasse-fired boilers, April-May 1976.

Supervised and conducted a study made by the Rio Grande Valley Sugar Growers, Inc. to determine the area affected by the burning of sugarcane fields prior to harvesting, November 1974-April 1975.

Supervised and conducted a study by a lightweight aggregate manufacturer to develop a material balance around the process through sampling and analysis of several parameters, November 1973.

Conducted a study in New York City to attempt to develop a correlation in the ambient air between carbon dioxide and sulfur dioxide to provide a tool for predicting air pollution predicting air pollution episodes, January-May 1969.

Served as Chairman of the Engineering Foundation Conference on Stack Sampling and Source Evaluation in Destin, Florida, 2002, and Santa Barbara, California, 1985.

Served as Co-Chairman of the Engineering Foundation Conference on Stack Sampling and Source Evaluation in Destin, Florida, 2001.

Served as Session Chairman at the Engineering Foundation Conference on Stack Sampling and Source Evaluation in Hershey, Pennsylvania, 1984; San Diego, California, 1993; and in Palm Coast, Florida, 1994. 
BILLY L. HEFLEY; Associate

Education

Professional

Training

$\underline{\text { Certification }}$

Professional

Memberships

Technical

Experience
B.S. 1992, East Central University; Ada, Oklahoma, in Environmental Science with a concentration in Environmental Management.

Attended 40-hour Occupational and Environmental Training Program on Hazardous Materials (CFR 1910.120) Dallas, Texas, April 1993.

Also attended an 8-hour refresher course January 1994, February 1995, January 1996, and May 2004.

Attended 8-hour Safe Hazardous Materials Transportation Training Program (HM-126F and HM-181) Dallas, Texas, October 1994.

Attended Bill Mullins' Performing and Observing Source Sampling Short Course; Dallas, Texas, January 1995.

Certified Visible Emissions Evaluator

\section{Air \& Waste Management Association Source Evaluations Society}

Participated in the sampling of over 750 sources, including several of which were sampled simultaneously using more than one sampling train. Thoroughly trained in all EPA testing procedures, 1992present.

Participated in an auditing program for a permanent eight-station sulfur dioxide ambient air network in East Texas, 1992-1993.

Participated in a semi-monthly ambient air monitoring survey for organic compounds at a petrochemical facility located in South Texas, 1992-1993.

Participated in EPA's Information Collection Request for Mercury conducting more than 46 simultaneous tests for Speciated Mercury using the "Standard Test Method for Elemental, Oxidized, ParticleBound, and Total Mercury in Flue Gas Generated from Coal-Fired Stationary Sources (Ontario Hydro Method)." September 1999-May 2000. 
$\operatorname{HEFLEY~(p.~2)~}$

Experienced in the analysis of commercial calibration gas cylinders for $\mathrm{NO}_{\mathrm{x}}, \mathrm{SO}_{2}, \mathrm{CO}_{2}$, and $\mathrm{O}_{2}$.

Experience with calibration techniques for all field testing equipment.

Thoroughly trained in the operation and routine maintenance of the following:

Anarad Model AR50-C Carbon Dioxide Analyzer

Anarad Model AR880 Oxides of Nitrogen Analyzer

Anarad Model AR23 Oxygen Analyzer

Anarad Model AR30C2 Sulfur Dioxide Analyzer

California Analytical Model 300-HFID Total Hydrocarbon Analyzer

Servomex Model 1440 Carbon Dioxide Analyzer

Servomex Model 1440 Oxygen Analyzer

Teledyne Model 326 Oxygen Analyzer

Thermo Environmental Model 10AR \& 10S Oxides of Nitrogen

Analyzer

Thermo Electron Model 46C Oxides of Nitrogen Analyzer

Thermo Electron Model 48C Oxides of Nitrogen Analyzer

Western Research Model 721A Sulfur Dioxide Analyzer 
GARY B. GOLDMAN; Associate

Education

$\underline{\text { Certification }}$

Professional

Memberships

Technical

Experience
B.S. 1993, University of Texas at Arlington; Arlington, Texas, in Geology.

Certified Visible Emissions Evaluator

\section{Source Evaluation Society}

Senior Emissions Evaluator with the Texas Commission on Environmental Quality, responsible for oversight of all source testing activities within the State of Texas, Region 4, which encompasses 19 counties in the North Texas region, 1999-2005.

Participated in the sampling of over 750 sources, including several of which were sampled simultaneously utilizing more than one sampling train, 1994-1999.

Thoroughly trained in all EPA testing procedures specified in Title 40 of the Code of Federal Regulations, Part 60.

Thoroughly trained in Test Methods for Evaluating Solid Waste, Physical/Chemical Methods, 0010 Through 0100 Series.

Participated in EPA's 3-D probe study. May - August 1997.

Experienced in the analysis of commercial calibration gas cylinders for $\mathrm{NO}_{x}, \mathrm{SO}_{2}, \mathrm{CO}_{2}$, and $\mathrm{O}_{2}$.

Experienced with calibration techniques for all field testing equipment.

Thoroughly trained in the operation and routine maintenance of the following:

Anarad Model AR50-C Carbon Dioxide Analyzer

Anarad Model AR880 Oxides of Nitrogen Analyzer

Anarad Model AR23 Oxygen Analyzer

Anarad Model AR30C2 Sulfur Dioxide Analyzer 
GOLDMAN (p. 2)

California Analytical Model 300-HFID Total Hydrocarbon Analyzer

Servomex Model 1440 Carbon Dioxide Analyzer

Servomex Model 1440 Oxygen Analyzer

Teledyne Model 326 Oxygen Analyzer

Thermo Environmental Model 10AR/S Oxides of Nitrogen Analyzer

Thermo Electron Model 46C Oxides of Nitrogen Analyzer

Thermo Electron Model 48C Oxides of Nitrogen Analyzer

Western Research Model 721A Sulfur Dioxide Analyzer

Professional Attended 40-hour Occupational and Environmental Training Program

Training on Hazardous Materials (CFR 1910.120) Dallas, Texas, April 1993.

Attended HAZWOPER 8-hour refresher course, 1994-2005.

Attended the following Environmental Protection Agency (EPA) Courses:

Course 345, Emission Capture and Gas Handling System Inspection

Course 380, Fugitive Source Inspection

Course 400, Introduction to Hazardous Air Pollutants

Course 413, Control of Particulate Emissions

Course 415, Control of Gaseous Emissions

Course 418, Control of Nitrogen Oxide Emissions

Course 427, Combustion Evaluation

Course 444, Air Pollution Field Enforcement

Course 445, Inspection of Particle Control Devices

Course 446, Inspection Safety Procedures

Course 450, Source Sampling for Particulate Pollutants

Course 452, Principles and Practice of Air Pollution Control

Course 455, Inspection of Gas Control Devices and Selected Industries

Course 474, Continuous Emission Monitoring

Course 482, Sources and Control of Volatile Organic Air

Pollutants

Attended the following California Air Resources Board (CARB) Courses

Course 221, Continuous Emission Monitoring

Course 233, Solvent Cleaning: Degreasing Operations

Course 242, Hot Mix Asphalt Facilities

Course 245, Cement Plants

Course 270, Incinerators 
PATRICK SELAKOVICH; Associate

Education

Professional

Training

Professional

Memberships

Technical

Experience
B.S.B.A. 1992, University of Arkansas; Fayetteville, Arkansas, in General Business.

Attended 24-hour Occupational and Environmental Training Program on Hazardous Materials (CFR 1910.120) Dallas, Texas, April 1997.

Attended 40-hour Occupational and Environmental Training Program on Hazardous Materials (CFR 1910.120) \& 'Train the Trainer', Gettysburg, Pennsylvania, July 1998.

Also attended an 8-hour refresher course January 2000, January 2001, and May 2004.

Attended OSHA General Industry Safety and Health Training, May 1999.

\section{Society For Human Resource Management}

Participated in the sampling of over 150 sources, including several of which were sampled simultaneously using more than one sampling train. Thoroughly trained in all EPA testing procedures, 1996present.

Participated in EPA's 3-D probe study. May - August 1997.

Experience with calibration techniques for all field testing equipment.

Thoroughly trained in the operation and routine maintenance of the following:

California Analytical Model 300-HFID Total Hydrocarbon Analyzer Servomex Model 1440 Carbon Dioxide Analyzer

Servomex Model 1440 Oxygen Analyzer

Thermo Electron Model 46C Oxides of Nitrogen Analyzer Thermo Electron Model 48C Oxides of Nitrogen Analyzer Western Research Model 721A Sulfur Dioxide Analyzer 
SCOT JACKSON; Associate

Education

Professional

Training

Certification

Technical

Experience
B.S.B.A. May 1978, Mountain View Jr. College, in General Business.

Purchasing Supervisor for METCO Environmental, Inc. in charge of inventory and supplies. January 1995 - April 2005.

Attended 40-hour Occupational and Environmental Training Program on Hazardous Materials (CFR 1910.120), Dallas, Texas, May 2000.

Attended Fed-Ex Hazardous Goods Shipping Training, June 2004.

\section{Certified Visible Emissions Evaluator}

Participated in the sampling of over 100 sources, including several of which were sampled simultaneously using more than one sampling train. Thoroughly trained in all EPA testing procedures, 1995present.

Experience with calibration techniques for all field testing equipment.

Thoroughly trained in the operation and routine maintenance of the following:

California Analytical Model 300-HFID Total Hydrocarbon Analyzer Servomex Model 1440 Carbon Dioxide Analyzer Servomex Model 1440 Oxygen Analyzer

Thermo Electron Model 42C Oxides of Nitrogen Analyzer Thermo Electron Model 48C Oxides of Nitrogen Analyzer Western Research Model 721A Sulfur Dioxide Analyzer 


\author{
STACK SAMPLING REPORT \\ FOR \\ MERCURY SPECIATION AND HYDROGEN HALIDE \& HALOGEN \\ TESTING \\ ON THE \\ ROCKY MOUNTAIN POWER \\ HARDIN POWER PLANT \\ SDA INLET DUCT AND STACK \\ HARDIN, MONTANA \\ PROJECT NO. 07-024
}

APRIL 2007

PREPARED FOR:

ADA-ES, INC.

8100 SOUTHPARK WAY

LITTLETON, CO 80120

PREPARED BY:

AIR SAMPLING ASSOCIATES, INC.

P.O. BOX 1175

LEWISVILLE, TEXAS 75067

(Total Number of pages including cover: 529 ) 


\section{TABLE OF CONTENTS}

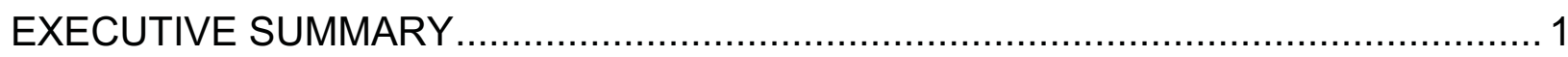

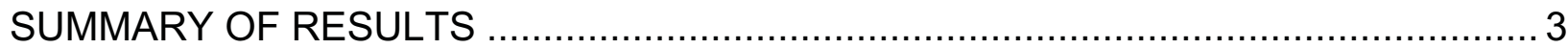

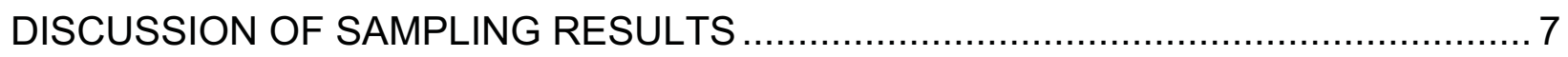

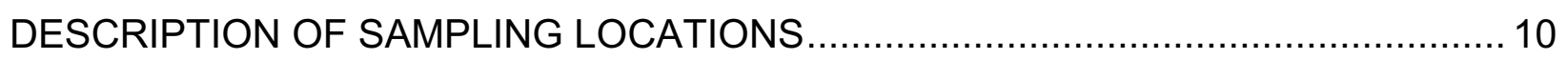

SAMPLING AND ANALYTICAL PROCEDURES …............................................. 13

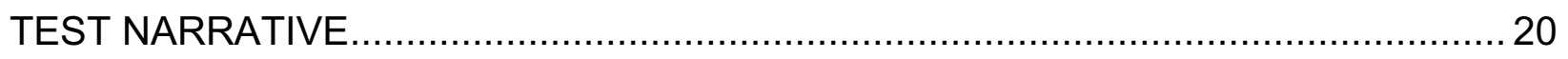

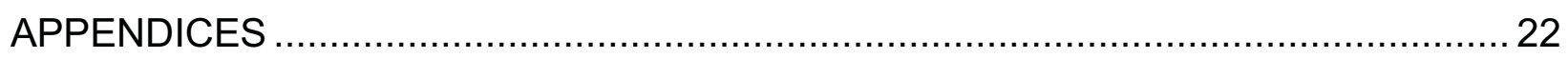

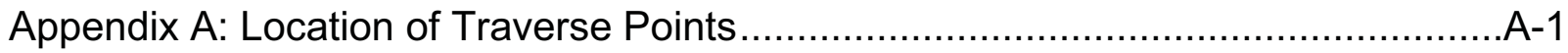

Appendix B: Nomenclature and Equations for Calculation of Source Emissions .......B-1

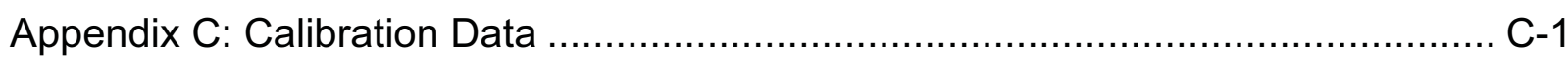

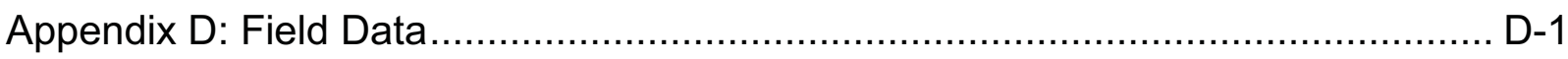

Appendix E: EPA Test Methods 5 and 26A (Particulate Matter and

Hydrogen Halides \& Halogens) Analytical Data - Stack.......................E-1

Appendix F: EPA Test Methods 5, 17 (Particulate Matter), and ASTM D6784-02

(Ontario Hydro) Mercury Speciation Test Method Analytical Data .........F-1

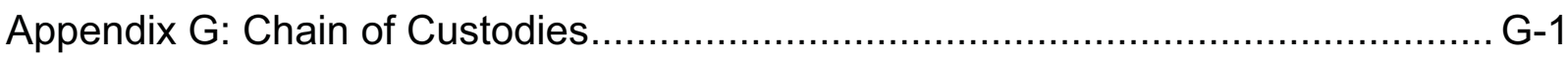

Appendix H: Resumes of Test Personnel ..................................................... H-1 


\section{TABLES}

Table 1: Summary of Sampling Results

Table 2: SDA Inlet Duct Particulate Matter and Speciated Mercury

Summary of Sampling Results. 4

Table 3: Stack Particulate Matter and Hydrogen Halides \& Halogens

Summary of Sampling Results 5

Table 4: Stack Particulate Matter and Speciated Mercury

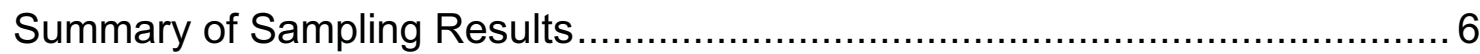

Table 5: EPA Test Method 26A Condenser/Absorbing System ................................ 14

Table 6: ASTM D6784-02 (Ontario Hydro) Speciated Mercury Test Method

Condenser/Absorbing System ............................................................... 17

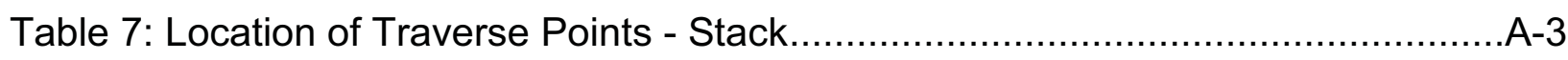

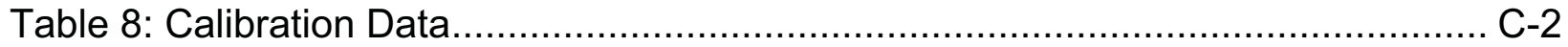

\section{FIGURES}

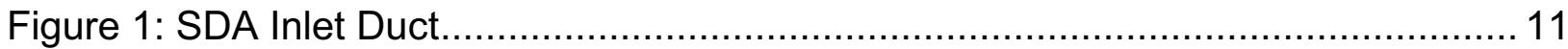

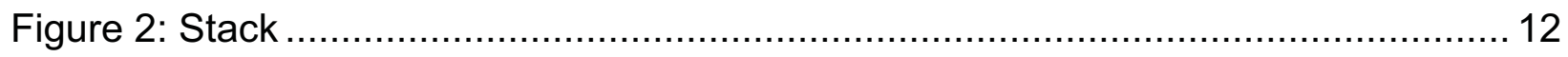

Figure 3: EPA Test Methods 5 and 26A Stack Sampling Train ................................ 15

Figure 4: ASTM D6784-02 (Ontario Hydro) Mercury Speciation Test Method

Sampling Train - SDA Inlet Duct......................................................... 18

Figure 5: ASTM D6784-02 (Ontario Hydro) Mercury Speciation Test Method

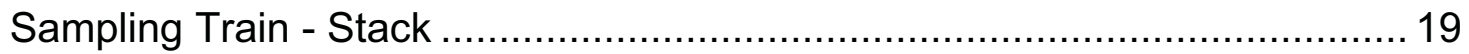

Figure 6: Location of Traverse Points - SDA Inlet Duct ........................................

Figure 7: Location of Traverse Points - Stack ................................................... 


\section{EXECUTIVE SUMMARY}

Air Sampling Associates, Inc. of Lewisville, Texas conducted stack testing at the Rocky Mountain Power, Hardin Power Plant, located in Hardin, Montana. The purpose of the stack testing was to determine the speciated mercury emissions at the SDA Inlet Duct and to determine the speciated mercury emissions and the hydrogen halide and halogen emissions at the Stack. The testing was conducted on April 24 through April 27, 2007.

The sampling team consisted of Mr. Bill Mullins, Mr. Bill Hefley, Mr. Patrick Selakovich, Mr. Scot Jackson, and Mr. Dane Odde. Mr. Mullins was the test team leader.

Mr. Jerry Amrhein of ADA-ES was the project manager.

The procedures set forth in Title 40 of the Code of Federal Regulations, Part 60 (40CFR60), Appendix A, Test Methods 1, 2, 3, 4, 5, 17, 26A, and ASTM D6784-02 (Ontario Hydro) Mercury Speciation Method were followed during the testing.

Triplicate samples for particulate matter collected at the Stack according to EPA Test Method 5, in conjunction with EPA Test Method 26A, indicated an average of 18.34 pounds per hour, based on the 'front half' analysis of the sampling train.

Triplicate samples for speciated mercury collected at the SDA Inlet Duct according to the ASTM D6784-02 (Ontario Hydro) Mercury Speciation Method indicated that the average total mercury was equal to $2.944 \mathrm{lbs} / 10^{12} \mathrm{Btu}$. The average total mercury was $22.99 \%$ particulate mercury, $13.73 \%$ oxidized mercury, and $63.28 \%$ elemental mercury. Samples for particulate matter collected using the optional test procedures in the ASTM D6784-02 (Ontario Hydro) Mercury Speciation Method indicated an average of 9184.01 pounds per hour, based on the analysis of the 'front half' of the sampling trains. 
Triplicate samples for hydrogen halides \& halogens collected at the Stack indicated an average concentration of hydrogen bromide and an average concentration of bromine that were below the detectable limit of the sampling procedure. The sampling results indicated an average concentration of hydrogen chloride that was below the detectable limit of the sampling procedure and an average concentration of chlorine that was below the detectable limit of the sampling procedure. The sampling results indicated an average concentration of hydrogen fluoride and an average concentration of fluoride that were below the detectable limit of the sampling procedure. Samples for particulate matter collected using the optional test procedures in EPA Test Method 26A indicated an average of 18.34 pounds per hour, based on the analysis of the 'front half' of the sampling trains.

Triplicate samples for speciated mercury collected at the Stack according to the ASTM D6784-02 (Ontario Hydro) Mercury Speciation Method indicated that the average total mercury was equal to $3.926 \mathrm{lbs} / 10^{12} \mathrm{Btu}$. The average total mercury was $79.66 \%$ elemental mercury. Samples for particulate matter collected using the optional test procedures in the ASTM D6784-02 (Ontario Hydro) Mercury Speciation Method indicated an average of 28.29 pounds per hour, based on the analysis of the 'front half' of the sampling trains. 


\section{SUMMARY OF RESULTS}

Table 1: Summary of Sampling Results

\begin{tabular}{|l|c|c|}
\hline $\begin{array}{l}\text { Sampling Trains } \\
\text { - Parameters }\end{array}$ & $\begin{array}{c}\text { SDA Inlet Duct } \\
\text { Average }\end{array}$ & $\begin{array}{c}\text { Stack } \\
\text { Average }\end{array}$ \\
\hline EPA 5 / 26A & -- & 14.74 \\
- Moisture (\%) & -- & 295,494 \\
- Flow Rate (DSCFM) & -- & 18.34 \\
- Particulate Matter (Ibs/hr - front & -- & $<0.779$ \\
- half) & & \\
\hline EPlorine (mg/dscm) & & 15.21 \\
- Moisture (\%) & & 310,113 \\
- Flow Rate (DSCFM) & 10.85 & \\
- Particulate Matter (Ibs/hr- front & 305,317 & 28.29 \\
$\quad$ half) & $9,184.01$ & 3.926 \\
\hline
\end{tabular}




\section{(11) SAMPLING \\ ASSOCIATES, INC.}

\section{SUMMARY OF RESULTS}

Table 2: SDA Inlet Duct Particulate Matter and Speciated Mercury

Summary of Sampling Results

\begin{tabular}{|c|c|c|c|c|}
\hline Run No. & 1 & 2 & 3 & Average \\
\hline Test Date & 04/26/07 & $04 / 26 / 07$ & 04/26/07 & ----- \\
\hline Test Time & $1020-1302$ & $1445-1657$ & 1810-2012 & ----- \\
\hline Flow Rate - DSCFM* & 295,561 & 309,321 & 311,069 & 305,317 \\
\hline Stack Temp. - 'F & 320 & 324 & 325 & 323 \\
\hline $\mathrm{O}_{2}$ - \% Vol. (dry) & 6.5 & 6.6 & 6.6 & 6.6 \\
\hline $\mathrm{CO}_{2}-\% \mathrm{Vol}$. (dry) & 12.8 & 12.7 & 12.7 & 12.7 \\
\hline Percent Excess Air & 43.7 & 44.6 & 44.6 & 44.3 \\
\hline Moisture Content - \% & 11.13 & 10.85 & 10.56 & 10.85 \\
\hline Percent Isokinetic & 96.2 & 96.2 & 96.1 & 96.2 \\
\hline Sample Volume - DSCM & 1.392 & 1.456 & 1.433 & 1.427 \\
\hline $\begin{array}{l}\text { Particulate Matter } \\
\text { - gr/dscf (Front Half) } \\
\text { - Ibs/hr (Front Half) }\end{array}$ & $\begin{array}{c}4.0495 \\
10,257.30\end{array}$ & $\begin{array}{c}3.1221 \\
8,276.37\end{array}$ & $\begin{array}{c}3.3829 \\
9,018.37\end{array}$ & $\begin{array}{c}3.518 \\
9,184.01\end{array}$ \\
\hline $\begin{array}{l}\text { Particle Bound Mercury } \\
-\mu g \\
-\mu g / d s c m \\
-I b s / 10^{12} B_{t u}^{* *}\end{array}$ & $\begin{array}{c}1.3000 \\
0.9339 \\
0.828\end{array}$ & $\begin{array}{c}0.9700 \\
0.6662 \\
0.595\end{array}$ & $\begin{array}{c}0.9800 \\
0.6839 \\
0.610\end{array}$ & $\begin{array}{l}1.0833 \\
0.7613 \\
0.678\end{array}$ \\
\hline $\begin{array}{l}\text { Oxidized Mercury } \\
-\mu g \\
-\mu g / d s c m \\
-I b s / 10^{12} B t u^{* *}\end{array}$ & $\begin{array}{c}0.6500 \\
0.4670 \\
0.414\end{array}$ & $\begin{array}{c}0.5700 \\
0.3915 \\
0.349\end{array}$ & $\begin{array}{c}0.7200 \\
0.5024 \\
0.449\end{array}$ & $\begin{array}{c}0.6467 \\
0.4536 \\
0.404\end{array}$ \\
\hline $\begin{array}{l}\text { Elemental Mercury } \\
-\mu g \\
-\mu g / d s c m \\
-l b s / 10^{12} B_{t u}^{* *}\end{array}$ & $\begin{array}{c}2.7300 \\
1.9612 \\
1.738\end{array}$ & $\begin{array}{c}3.2600 \\
2.2390 \\
1.999\end{array}$ & $\begin{array}{c}2.9700 \\
2.0726 \\
1.850\end{array}$ & $\begin{array}{c}2.9867 \\
2.0909 \\
1.862\end{array}$ \\
\hline $\begin{array}{l}\text { Total Mercury } \\
-\mu g \\
-\mu g / d s c m \\
-I b s / 10^{12} B_{t u}^{* *}\end{array}$ & $\begin{array}{c}4.6800 \\
3.3621 \\
2.980 \\
\end{array}$ & $\begin{array}{c}4.8000 \\
3.2967 \\
2.943 \\
\end{array}$ & $\begin{array}{c}4.6700 \\
3.2589 \\
2.909 \\
\end{array}$ & $\begin{array}{c}4.7167 \\
3.3059 \\
2.944 \\
\end{array}$ \\
\hline
\end{tabular}




\section{SAMPLING \\ ASSOCIATES, INC.}

\section{SUMMARY OF RESULTS}

Table 3: Stack Particulate Matter and Hydrogen Halides \& Halogens

Summary of Sampling Results

\begin{tabular}{|c|c|c|c|c|}
\hline Run No. & 1 & 2 & 3 & Average \\
\hline Test Date & $04 / 27 / 07$ & $04 / 27 / 07$ & $04 / 27 / 07$ & ----- \\
\hline Test Time & 0900-1017 & $1110-1227$ & 1407-1523 & ----- \\
\hline Flow Rate - DSCFM* & 306,890 & 279,393 & 300,200 & 295,494 \\
\hline Stack Temp. - ${ }^{\circ} \mathrm{F}$ & 223 & 220 & 228 & 224 \\
\hline $\mathrm{O}_{2}$ - \% Vol. (dry) & 6.6 & 6.7 & 6.7 & 6.7 \\
\hline $\mathrm{CO}_{2}-\% \mathrm{Vol}$. (dry) & 12.7 & 12.6 & 12.6 & 12.6 \\
\hline Percent Excess Air & 44.6 & 45.6 & 45.6 & 45.3 \\
\hline Moisture Content - \% & 15.16 & 14.63 & 14.43 & 14.74 \\
\hline Percent Isokinetic & 101.0 & 98.6 & 99.0 & 99.5 \\
\hline Sample Volume - DSCM & 1.252 & 1.113 & 1.200 & 1.188 \\
\hline $\begin{array}{l}\text { Particulate Matter } \\
\text { - gr/dscf (Front Half) } \\
\text { - Ibs/hr (Front Half) }\end{array}$ & $\begin{array}{c}0.0086 \\
22.53\end{array}$ & $\begin{array}{c}0.0056 \\
13.41\end{array}$ & $\begin{array}{c}0.0074 \\
19.07\end{array}$ & $\begin{array}{c}0.0072 \\
18.34\end{array}$ \\
\hline $\begin{array}{l}\text { Hydrogen Bromide } \\
\text { - mg } \\
-m g / d s c m\end{array}$ & $\begin{array}{l}<0.496 \\
<0.396\end{array}$ & $\begin{array}{l}<0.425 \\
<0.382\end{array}$ & $\begin{array}{l}<0.481 \\
<0.401\end{array}$ & $\begin{array}{l}<0.467 \\
<0.393\end{array}$ \\
\hline $\begin{array}{l}\text { Bromine } \\
\quad-m g \\
-m g / d s c m\end{array}$ & $\begin{array}{l}<0.275 \\
<0.220\end{array}$ & $\begin{array}{l}<0.250 \\
<0.225\end{array}$ & $\begin{array}{l}<0.290 \\
<0.242\end{array}$ & $\begin{array}{l}<0.272 \\
<0.229\end{array}$ \\
\hline $\begin{array}{l}\text { Hydrogen Chloride } \\
-m g \\
-m g / d s c m\end{array}$ & $\begin{array}{l}<1.010 \\
<0.807\end{array}$ & $\begin{array}{l}<0.864 \\
<0.776\end{array}$ & $\begin{array}{l}<0.977 \\
<0.814\end{array}$ & $\begin{array}{l}<0.950 \\
<0.799\end{array}$ \\
\hline $\begin{array}{l}\text { Chlorine } \\
\quad-m g \\
-m g / d s c m\end{array}$ & $\begin{array}{l}<0.550 \\
<0.439\end{array}$ & $\begin{array}{l}<0.500 \\
<0.449\end{array}$ & $\begin{array}{l}1.740 \\
1.450\end{array}$ & $\begin{array}{l}<0.930 \\
<0.779\end{array}$ \\
\hline $\begin{array}{l}\text { Hydrogen Fluoride } \\
\text { - mg } \\
-m g / d s c m\end{array}$ & $\begin{array}{l}<0.516 \\
<0.412\end{array}$ & $\begin{array}{l}<0.442 \\
<0.397\end{array}$ & $\begin{array}{l}<0.500 \\
<0.417\end{array}$ & $\begin{array}{l}<0.486 \\
<0.409\end{array}$ \\
\hline $\begin{array}{l}\text { Fluorine } \\
\quad-m g \\
-m g / d s c m \\
\end{array}$ & $\begin{array}{l}<0.290 \\
<0.232 \\
\end{array}$ & $\begin{array}{l}<0.263 \\
<0.236\end{array}$ & $\begin{array}{l}<0.305 \\
<0.254\end{array}$ & $\begin{array}{l}<0.286 \\
<0.241\end{array}$ \\
\hline
\end{tabular}

* 29.92" $\mathrm{Hg}, 68^{\circ} \mathrm{F}\left(760 \mathrm{~mm} \mathrm{Hg}, 20^{\circ} \mathrm{C}\right)$ 


\section{SAMPLING}

\section{SUMMARY OF RESULTS}

Table 4: Stack Particulate Matter and Speciated Mercury Summary of Sampling Results

\begin{tabular}{|c|c|c|c|c|}
\hline Run No. & 1 & 2 & 3 & Average \\
\hline Test Date & $04 / 26 / 07$ & $04 / 26 / 07$ & $04 / 26 / 07$ & $\begin{array}{ll}---- \\
--\end{array}$ \\
\hline Test Time & $1020-1305$ & $1445-1650$ & $1810-2015$ & ----- \\
\hline Flow Rate - DSCFM* & 304,308 & 310,931 & 315,099 & 310,113 \\
\hline Stack Temp. - ${ }^{\circ} \mathrm{F}$ & 214 & 215 & 216 & 215 \\
\hline $\mathrm{O}_{2}-\%$ Vol. (dry) & 6.8 & 6.9 & 6.8 & 6.8 \\
\hline $\mathrm{CO}_{2}-\% \mathrm{Vol}$. (dry) & 12.7 & 12.6 & 12.7 & 12.7 \\
\hline Percent Excess Air & 46.8 & 47.8 & 46.8 & 47.1 \\
\hline Moisture Content - \% & 15.19 & 15.38 & 15.06 & 15.21 \\
\hline Percent Isokinetic & 98.5 & 97.4 & 98.0 & 98.0 \\
\hline Sample Volume - DSCM & 2.018 & 2.040 & 2.079 & 2.046 \\
\hline $\begin{array}{l}\text { Particulate Matter } \\
\text { - gr/dscf (Front Half) } \\
\text { - Ibs/hr (Front Half) }\end{array}$ & $\begin{array}{c}0.0094 \\
24.57\end{array}$ & $\begin{array}{c}0.0071 \\
18.85\end{array}$ & $\begin{array}{c}0.0154 \\
41.46\end{array}$ & $\begin{array}{c}0.0106 \\
28.29\end{array}$ \\
\hline $\begin{array}{l}\text { Particle Bound Mercury } \\
\text { - } \mu \mathrm{g} \\
\text { - } \mu \mathrm{g} / \mathrm{dscm} \\
-\mathrm{lbs} / 10^{12} \mathrm{Btu}^{* *}\end{array}$ & $\begin{array}{c}0.0110 \\
0.0055 \\
0.005\end{array}$ & $\begin{array}{c}0.0580 \\
0.0284 \\
0.026\end{array}$ & $\begin{array}{c}0.0290 \\
0.0139 \\
0.013\end{array}$ & $\begin{array}{c}0.0327 \\
0.0159 \\
0.014\end{array}$ \\
\hline $\begin{array}{l}\text { Oxidized Mercury } \\
-\mu g \\
-\mu g / d s c m \\
-~ I b s / 10^{12} B t u^{\star *}\end{array}$ & $\begin{array}{c}1.8000 \\
0.8920 \\
0.807\end{array}$ & $\begin{array}{l}1.800 \\
0.8824 \\
0.804\end{array}$ & $\begin{array}{c}1.7000 \\
0.8177 \\
0.740\end{array}$ & $\begin{array}{c}1.7667 \\
0.8640 \\
0.784\end{array}$ \\
\hline $\begin{array}{l}\text { Elemental Mercury } \\
\text { - } \mu g \\
\text { - } \mu \mathrm{g} / \mathrm{dscm} \\
-\operatorname{lbs} / 10^{12} \text { Btu }^{* *}\end{array}$ & $\begin{array}{c}6.8500 \\
3.3944 \\
3.072\end{array}$ & $\begin{array}{c}7.4900 \\
3.6716 \\
3.347\end{array}$ & $\begin{array}{c}6.8100 \\
3.2756 \\
2.965\end{array}$ & $\begin{array}{c}7.0500 \\
3.4472 \\
3.128\end{array}$ \\
\hline $\begin{array}{l}\text { Total Mercury } \\
\text { - } \mu g \\
-\mu g / d s c m \\
-~ l b s / 10^{12} B t u^{* *}\end{array}$ & $\begin{array}{c}8.6610 \\
4.2919 \\
3.884\end{array}$ & $\begin{array}{c}9.3480 \\
4.5824 \\
4.177\end{array}$ & $\begin{array}{c}8.539 \\
4.1072 \\
3.718\end{array}$ & $\begin{array}{c}8.8493 \\
4.3272 \\
3.926\end{array}$ \\
\hline
\end{tabular}




\title{
DISCUSSION OF SAMPLING RESULTS
}

\author{
SDA Inlet Duct
}

\section{Particulate Matter and Speciated Mercury}

The three tests for particulate matter and speciated mercury taken at the SDA Inlet Duct appeared to be an accurate representation of the actual emissions during the tests. All leak checks performed on the sampling train and the pitot tubes indicated no leaks before or after each test. The indicative parameters calculated from the field data were in reasonable agreement. The measured moisture contents for the three runs were within $2.6 \%$ of the mean value. The measured flow rates (DSCFM) for the tests were within $3.2 \%$ of the mean value. The rates of sampling for the three tests were within the specified limits (90 to 110 percent isokinetic). The greatest deviation from 100\% isokinetic was $3.9 \%$.

The concentrations (gr/dscf - Front Half) of particulate matter for the three tests showed a range of -11.26 percent to +15.10 percent variation from the mean value of 3.518 $\mathrm{gr} / \mathrm{dscf}$ - front half.

The calculated emissions (lbs $/ 10^{12} \mathrm{Btu}$ ) of total mercury for the three tests showed a range of $-1.19 \%$ to $+1.22 \%$ deviation from the mean value of $2.944 \mathrm{lbs} / 10^{12} \mathrm{Btu}$. 


\section{Stack}

\section{Particulate Matter and Hydrogen Halides \& Halogens}

The three tests for particulate matter and hydrogen halides \& halogens taken at the Stack appeared to be an accurate representation of the actual emissions during the tests. All leak checks performed on the reference method sampling train and pitot tubes showed no leaks before or after testing. The indicative parameters of the tests were in close agreement. The measured moisture contents $(\% \mathrm{M})$ were within $2.8 \%$ of the mean value. The measured flow rates (DSCFM) were within $5.4 \%$ of the mean value. The rates of sampling for the tests were within the specified limits ( $90 \%$ to $110 \%$ isokinetic). The greatest deviation from $100 \%$ isokinetic was $1.4 \%$.

The concentrations (gr/dscf - Front Half) of particulate matter for the three tests showed a range of -22.2 percent to +19.4 percent deviation from the mean value of 0.0072 $\mathrm{gr} / \mathrm{dscf}$ - front half. The large variation is due the lower emissions during the second test when a coal mill was stopped.

The concentrations $(\mathrm{mg} / \mathrm{dscm})$ of hydrogen bromide and bromine for the three tests were below the minimum reporting limit of the reference method.

The concentrations $(\mathrm{mg} / \mathrm{dscm})$ of hydrogen chloride and chlorine for the three tests were below the minimum reporting limit of the reference method. A small amount of chlorine was detected during the third test that was approximately three times the minimum reporting limit of the reference method.

The concentrations $(\mathrm{mg} / \mathrm{dscm}$ ) of hydrogen fluoride and fluorine for the three tests were below the minimum reporting limit of the reference method. 


\section{Particulate Matter and Speciated Mercury}

The three tests for particulate matter and speciated mercury on the Stack appeared to be an accurate representation of the actual emissions during the tests. All leak checks performed on the sampling train and the pitot tubes indicated no leaks before or after each test. The indicative parameters calculated from the field data were in reasonable agreement. The measured moisture contents for the three runs were within $1.1 \%$ of the mean value. The measured flow rates (DSCFM) for the tests were within $1.9 \%$ of the mean value. The rates of sampling for the three tests were within the specified limits (90 to 110 percent isokinetic). The greatest deviation from $100 \%$ isokinetic was $2.6 \%$.

The concentrations ( $\mathrm{gr} / \mathrm{dscf}$ - Front Half) of particulate matter for the three tests showed a range of -33.2 percent to +44.8 percent variation from the mean value of 0.0106 $\mathrm{gr} / \mathrm{dscf}-$ front half. The large variation is due to significantly higher emissions during the third test.

The calculated emissions ( $\mathrm{lbs} / 10^{12} \mathrm{Btu}$ ) of total mercury for the three tests showed a range of $-5.31 \%$ to $+6.38 \%$ deviation from the mean value of $3.926 \mathrm{lbs} / 10^{12} \mathrm{Btu}$. 


\section{DESCRIPTION OF SAMPLING LOCATIONS}

The sampling ports on the SDA Inlet Duct are approximately 36 feet $91 / 2$ inches above the ground. The sampling ports are located 13 feet 4 inches (1.12 equivalent duct diameters) downstream from a bend in the duct and 56 feet 3 inches (4.75 equivalent duct diameters) upstream from a bend in the duct.

The sampling ports on the Stack are approximately 123 feet $11 / 2$ inches above the ground. The sampling ports are located 33 feet 1 1/2 inches (3.64 stack diameters) downstream from a constriction in the stack and $>18$ feet $23 / 8$ inches ( $>2.00$ stack diameters) upstream from the outlet to the stack. 


\section{SAMPLING LOCATION}

Figure 1: SDA Inlet Duct

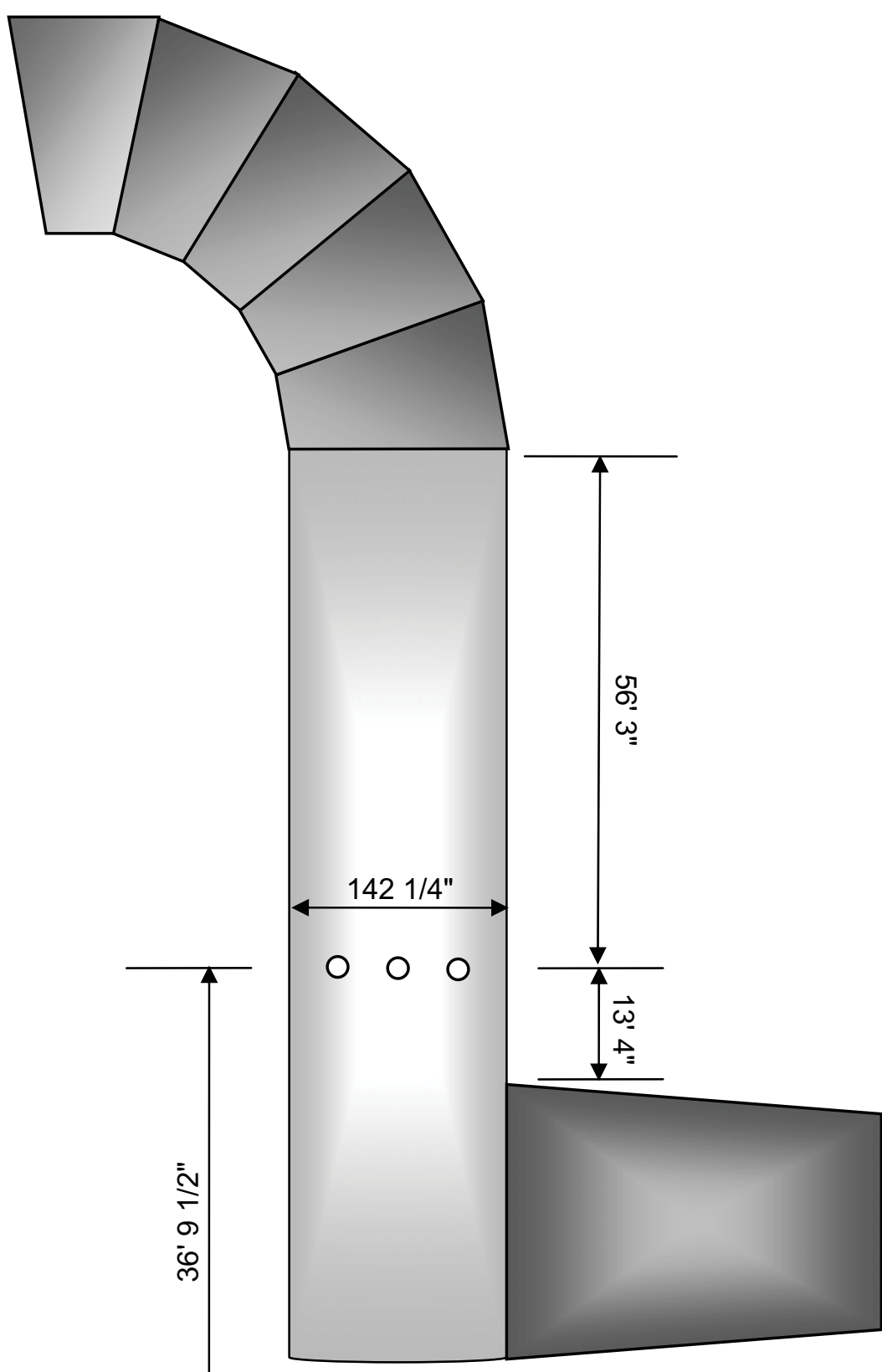




\section{SAMPLING LOCATION}

Figure 2: Stack

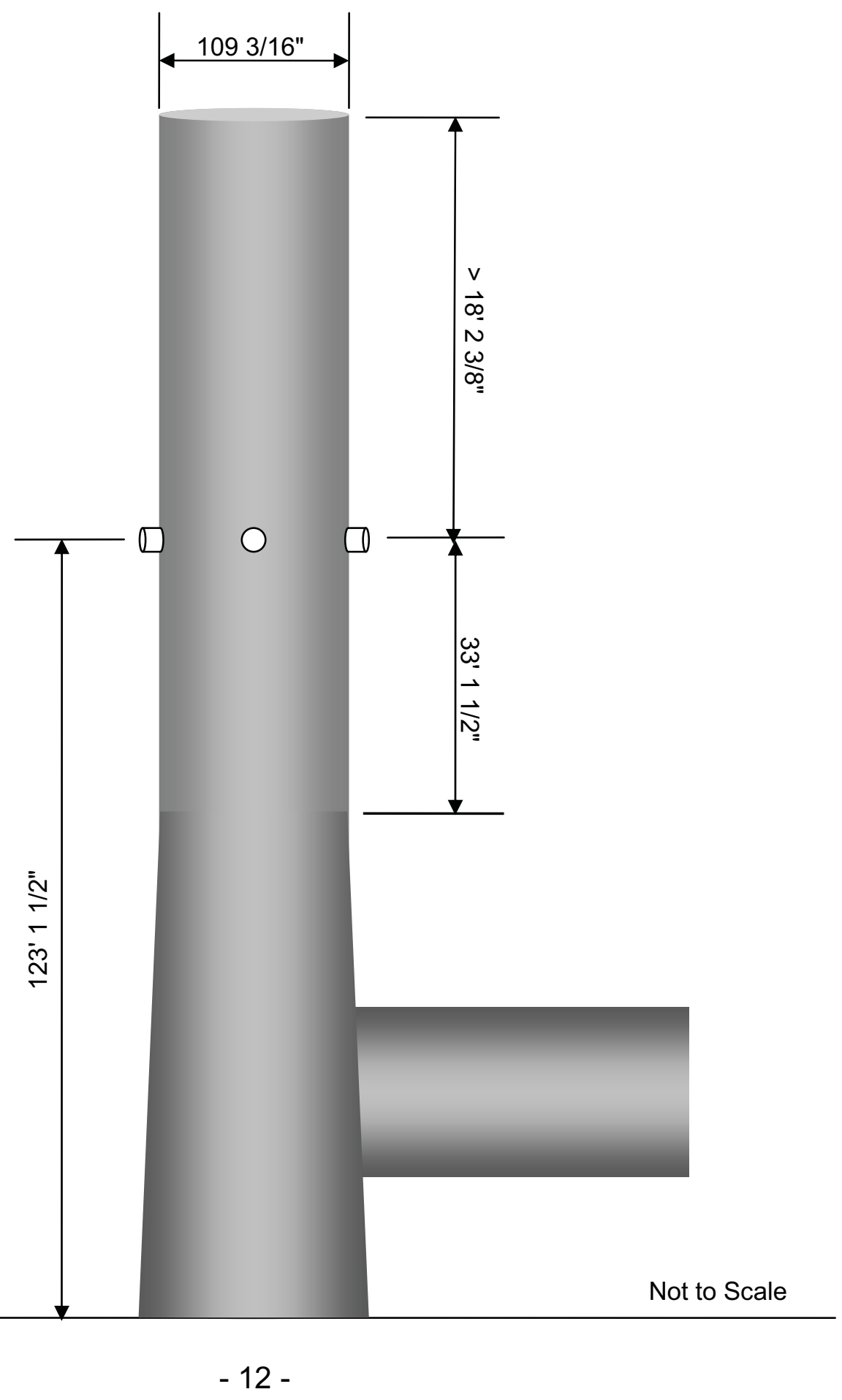




\section{SAMPLING AND ANALYTICAL PROCEDURES}

The procedures set forth in 40CFR60, Appendix A, Test Methods 1, 2, 3, 4, 5, 17, 26A, and ASTM D6784-02 (Ontario Hydro) Mercury Speciation Test Method were followed during testing.

Four Traverse points were sampled from three ports on the SDA Inlet Duct for a total of twelve traverse points. All traverse points were checked for cyclonic flow and the average angle was equal to 2.4 degrees. The pitot tube lines were checked for leaks before and after each test under a vacuum and a pressure. The lines were also checked for clearance and the manometer was zeroed before each test.

Twelve traverse points were sampled from two ports on the Stack for a total of twentyfour traverse points. All traverse points were checked for cyclonic flow and the average angle was equal to 6.0 degrees. The pitot tube lines were checked for leaks before and after each test under a vacuum and a pressure. The lines were also checked for clearance and the manometer was zeroed before each test.

\section{Particulate Matter and Hydrogen Halides \& Halogens}

Particulate matter and hydrogen halides \& halogens samples were taken at the Stack sampling location according to EPA Test Methods 1, 2, 3, 4, 5, and 26A. For each run at the Stack, samples of three minute duration were taken at each of twenty-four traverse points for a total test time of 72 minutes. Data was recorded at three minute intervals.

The sampling train was leak checked at the end of the sampling probe at 15" of mercury vacuum before each test, and again at the conclusion of each test at the highest 


\section{II}

vacuum recorded during sampling. This was done to predetermine the possibility of a diluted sample.

The 'front half' of the sampling train at the Stack contained the following components:

Glass nozzle

Heated glass lined probe @ $248^{\circ} \mathrm{F} \pm 25^{\circ} \mathrm{F}$

Heated fiberglass filter @ $248^{\circ} \mathrm{F} \pm 25^{\circ} \mathrm{F}$

Heated Teflon line @ $248^{\circ} \mathrm{F} \pm 25^{\circ} \mathrm{F}$

The 'back half' of the sampling train at the Stack sampling location contained the following components:

Table 5: EPA Test Method 26A Condenser/Absorbing System

\begin{tabular}{|c|c|c|}
\hline Impinger No. & Impinger Type & Absorbing Solution \\
\hline 1 & Greenburg-Smith & $100 \mathrm{ml} 0.1 \mathrm{~N} \mathrm{H}_{2} \mathrm{SO}_{4}$ \\
\hline 2 & Greenburg-Smith & $100 \mathrm{ml} 0.1 \mathrm{~N} \mathrm{H}_{2} \mathrm{SO}_{4}$ \\
\hline 3 & Modified & $100 \mathrm{ml} 0.1 \mathrm{~N} \mathrm{NaOH}$ \\
\hline 4 & Modified & $100 \mathrm{ml} 0.1 \mathrm{~N} \mathrm{NaOH}$ \\
\hline 5 & Modified & 〜200 g silica gel \\
\hline
\end{tabular}

Integrated Orsat samples were collected during each test. The samples were analyzed according to EPA Method 3 to determine the stack gas molecular weight. 


\section{AIR

Figure 3: EPA Test Methods 5 and 26A Stack Sampling Train

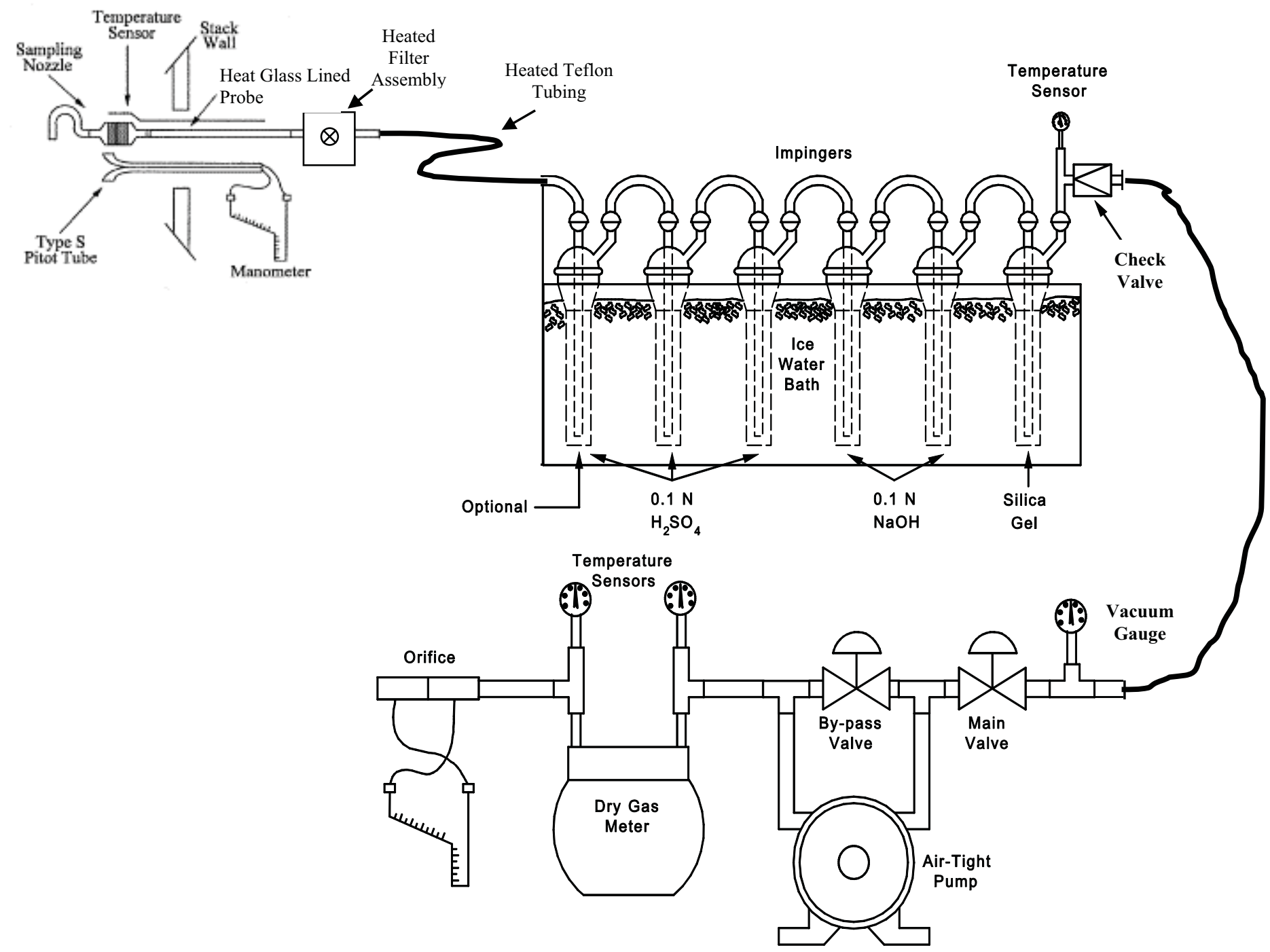




\section{Particulate Matter and Speciated Mercury}

Speciated mercury and particulate matter samples were taken at the SDA Inlet Duct and at the Stack sampling locations according to EPA Test Methods 1, 2, 3, 4, 5, and 17; and ASTM D6784-02 (Ontario Hydro) Mercury Speciation Test Method. For each run at the SDA Inlet Duct, samples of ten minute duration were taken at each of twelve traverse points for a total test time of 120 minutes. Data was recorded at five minute intervals. For each run at the Stack, samples of five minute duration were taken at each of twenty-four traverse points for a total test time of 120 minutes. Data was recorded at five minute intervals.

The sampling trains were leak checked at the end of the sampling probes at 15 " of mercury vacuum before each test, and again at the conclusion of each test at the highest vacuum recorded during sampling. This was done to predetermine the possibility of a diluted sample.

The 'front half' of the sampling train at the SDA Inlet Duct contained the following components:

Glass nozzle

Fiberglass Thimble

Heated glass lined probe @ $248^{\circ} \mathrm{F} \pm 25^{\circ} \mathrm{F}$

Heated glass back-up filter @ $248^{\circ} \mathrm{F} \pm 25^{\circ} \mathrm{F}$

The 'front half' of the sampling train at the Stack contained the following components:

Glass nozzle

Heated glass lined probe @ $248^{\circ} \mathrm{F} \pm 25^{\circ} \mathrm{F}$

Heated fiberglass filter @ $248^{\circ} \mathrm{F} \pm 25^{\circ} \mathrm{F}$

Heated Teflon line @ $248^{\circ} \mathrm{F} \pm 25^{\circ} \mathrm{F}$ 
The 'back half' of the sampling trains, contained the following components:

Table 6: ASTM D6784-02 (Ontario Hydro) Speciated Mercury Test Method Condenser/Absorbing System

\begin{tabular}{|c|c|c|}
\hline Impinger No. & Impinger Type & Absorbing Solution \\
\hline 1 & Modified & $100 \mathrm{ml} 1 \mathrm{~N} \mathrm{KCl}$ \\
\hline 2 & Modified & $100 \mathrm{ml} 1 \mathrm{~N} \mathrm{KCl}$ \\
\hline 3 & Greenburg-Smith & $100 \mathrm{ml} 1 \mathrm{~N} \mathrm{KCl}$ \\
\hline 4 & Modified & $100 \mathrm{ml} 5 \% \mathrm{v} / \mathrm{v} \mathrm{HNO}_{3}, 10 \% \mathrm{v} / \mathrm{v} \mathrm{H}_{2} \mathrm{O}_{2}$ \\
\hline 5 & Modified & $100 \mathrm{ml} 4 \% \mathrm{w} / \mathrm{v} \mathrm{H}_{2} \mathrm{SO}_{4}, 10 \% \mathrm{v} / \mathrm{v} \mathrm{KMn}_{4}$ \\
\hline 6 & Modified & $100 \mathrm{ml} 4 \% \mathrm{w} / \mathrm{v} \mathrm{H}_{2} \mathrm{SO}_{4}, 10 \% \mathrm{v} / \mathrm{v} \mathrm{KMnO}_{4}$ \\
\hline 7 & Greenburg-Smith & $100 \mathrm{ml} 4 \% \mathrm{w} / \mathrm{v} \mathrm{H}_{2} \mathrm{SO}_{4}, 10 \% \mathrm{v} / \mathrm{v} \mathrm{KMn}_{4}$ \\
\hline 8 & Modified & $\sim 200 \mathrm{~g}$ silica gel \\
\hline
\end{tabular}

Integrated Orsat samples were collected at each sampling location during each test. The samples were analyzed according to EPA Method 3 to determine the stack gas molecular weight. 
Figure 4: ASTM D6784-02 (Ontario Hydro) Mercury Speciation Test Method Sampling Train - SDA Inlet Duct

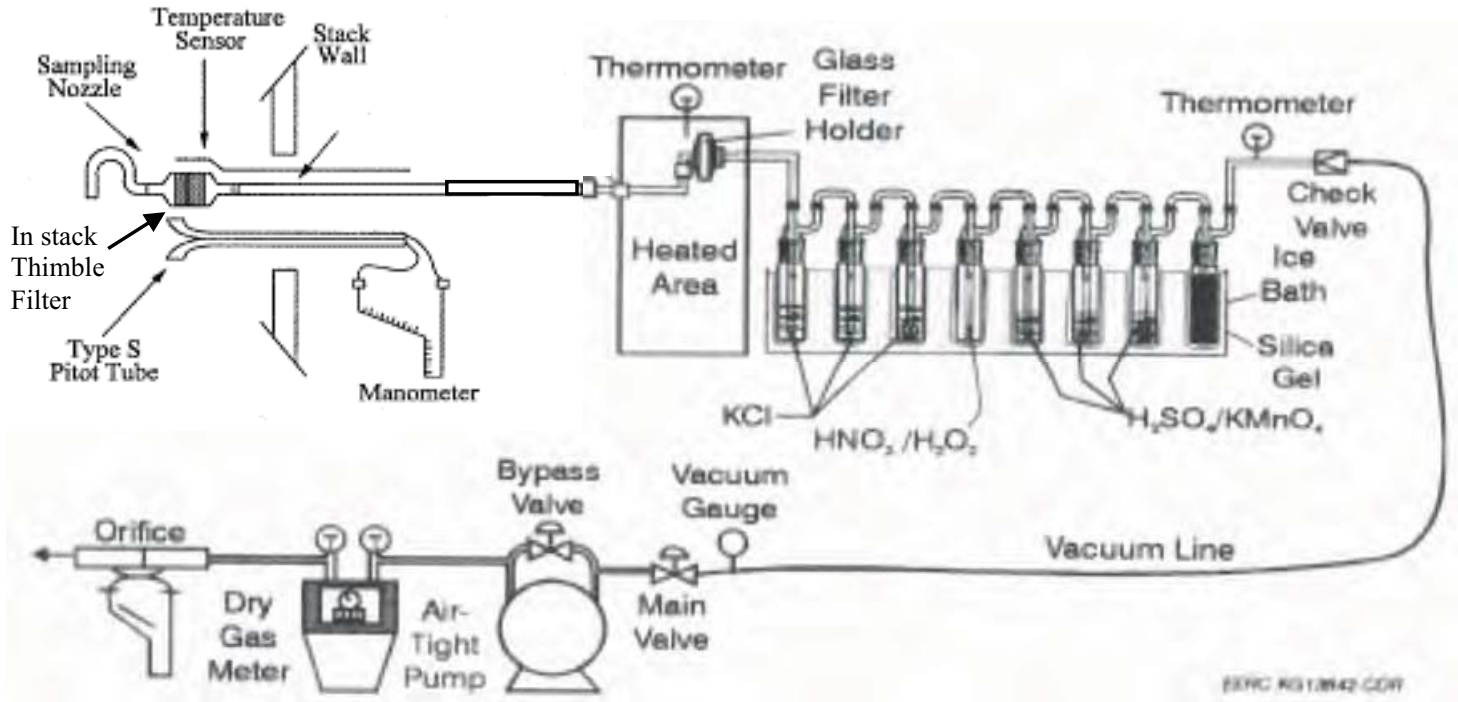


Figure 5: ASTM D6784-02 (Ontario Hydro) Mercury Speciation Test Method Sampling Train - Stack

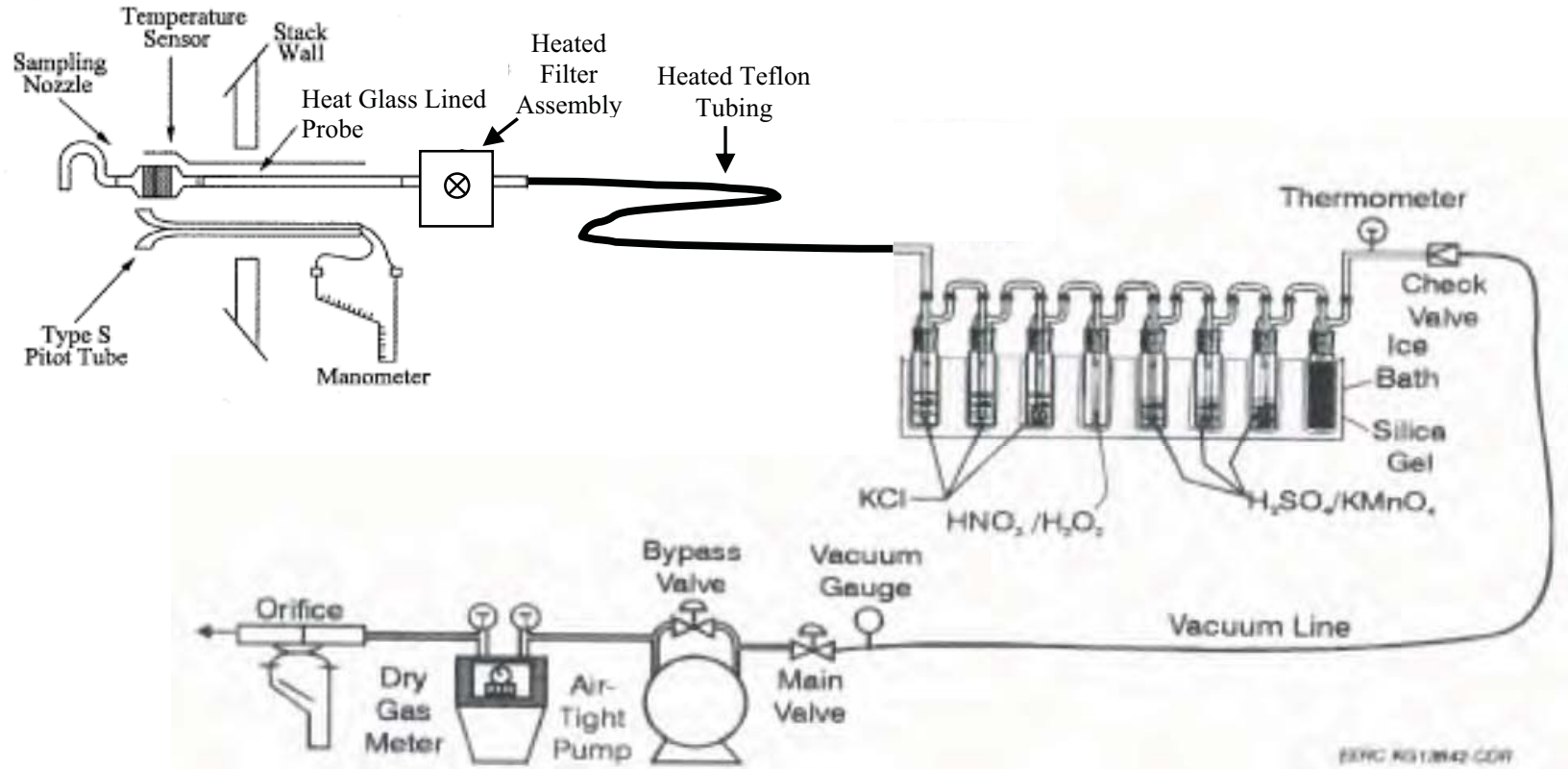




\section{TEST NARRATIVE}

Personnel from Air Sampling Associates, Inc. arrived at the Rocky Mountain Power, Hardin Power Plant, located in Hardin, Montana at 8:00 a.m. on Tuesday, April 24, 2007. The sampling trailer was placed into position and electrical power was established. The sampling equipment was moved onto the SDA Inlet Duct and the Stack before securing the equipment for the night at 5:00 p.m.

On Wednesday, April 25, 2007, personnel returned to the plant at 8:00 a.m. More sampling equipment was moved onto the SDA Inlet Duct and the sample recovery area was established. Personnel prepared for the next day's testing before departing the plant at 11:30 a.m.

On Thursday, April 26, 2007, personnel returned to the plant at 6:45 a.m. The sampling equipment was prepared for testing and the first simultaneous test on the Stack and on the SDA Inlet Duct for mercury speciation began at 10:20 a.m. The test continued until 11:41 a.m. when the test was stopped due to plant operational problems. The first test resumed at 12:20 p.m. and continued until its completion at 1:05 p.m. The second simultaneous test for speciated mercury was taken from 2:45 p.m. until 4:57 p.m. The third simultaneous test for speciated mercury was taken from 6:10 p.m. until 8:15 p.m. The samples were recovered and the sampling equipment was moved off of the SDA Inlet Duct before personnel departed the plant at 11:00 p.m.

On Friday, April 27, 2007, personnel returned to the plant at 7:00 a.m. The sampling equipment was prepared for testing and the first test on the Stack using EPA Method 26A began at 9:00 a.m. Testing continued until the completion of the third test at 3:23 p.m. 


\section{III}

The sampling equipment was moved off of the Stack and loaded into the sampling trailer. The samples were taken to Air Sampling Associates, Inc.'s office in Lewisville, Texas for analysis and shipment to the subcontracted laboratories.

Operations at the Rocky Mountain Power, Hardin Power Plant, SDA Inlet Duct and Stack, located in Hardin, Montana, were completed at 5:30 p.m. on Friday, April 27, 2007. 


\section{APPENDICES}

Appendix A: Location of Traverse Points

Appendix B: Nomenclature and Equations for Calculation of Source Emissions

Appendix C: Calibration Data

Appendix D: Field Data

Appendix E: EPA Test Methods 5 and 26A (Particulate Matter and Hydrogen Halides \& Halogens) Analytical Data - Stack

Appendix F: EPA Test Methods 5, 17 (Particulate Matter), and ASTM D6784-02 (Ontario Hydro) Mercury Speciation Test Method Analytical Data

Appendix G: Chain of Custodies

Appendix H: Resumes of Test Personnel 
Appendix A:

Location of Traverse Points 


\section{Appendix A}

\section{Location of Traverse Points SDA Inlet Duct}

The sampling ports are located 13 feet 4 inches (1.12 equivalent duct diameters) downstream from a bend in the bend in the duct and 56 feet 3 inches (4.75 equivalent duct diameters) upstream from a bend in the duct. The locations of the traverse points were calculated as follows:

Figure 6: Location of Traverse Points - SDA Inlet Duct

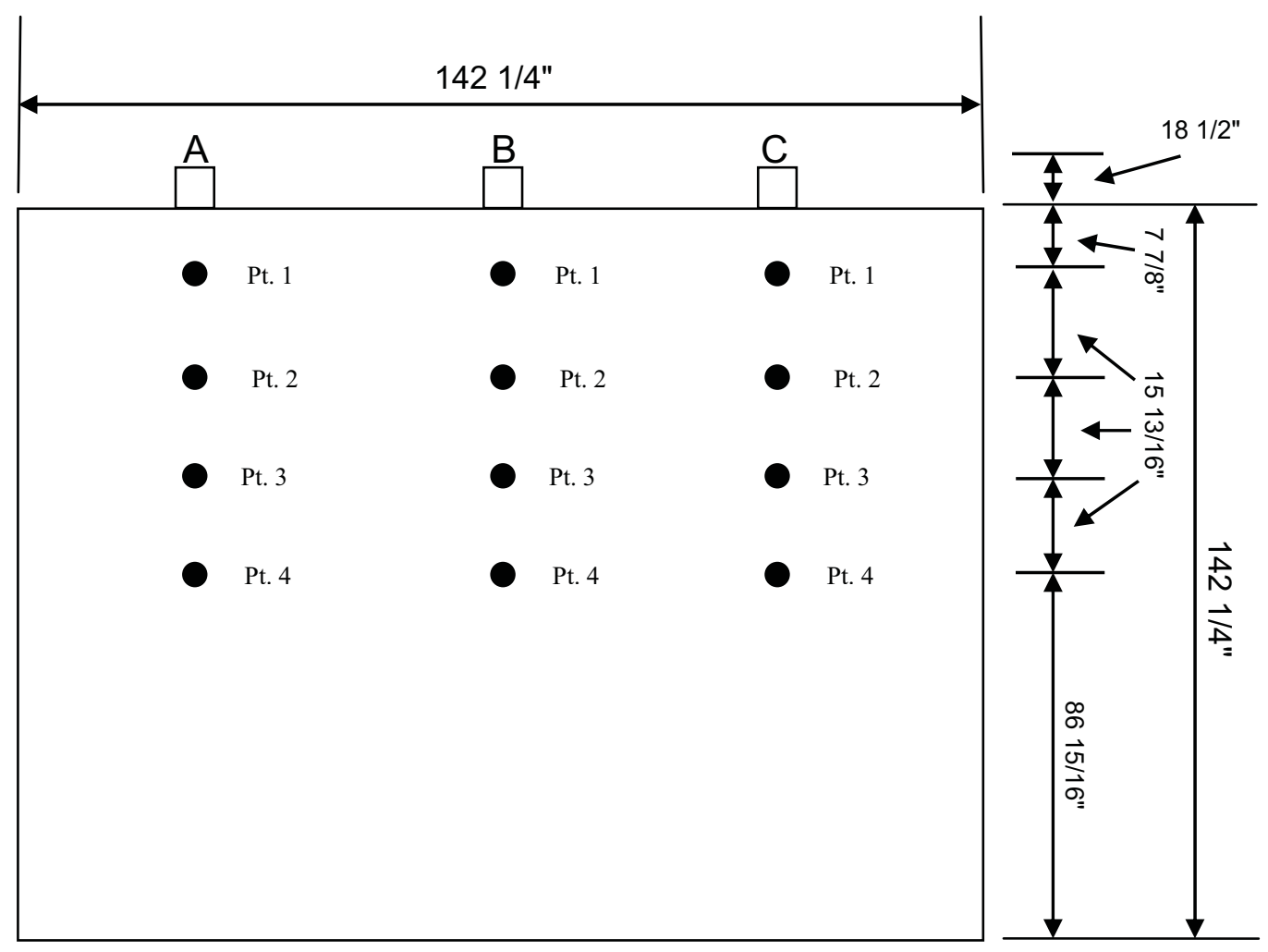

Note: Physical limitations prevented the complete traversing of the SDA Inlet Duct. 


\section{Appendix A}

\section{Location of Traverse Points Stack}

The sampling ports are located 33 feet 1 1/2 inches (3.64 stack diameters) downstream from a constriction in the stack and $>18$ feet $23 / 8$ inches ( $>2.00$ stack diameters) upstream from the outlet to the stack. The locations of the traverse points were calculated as follows:

Table 7: Location of Traverse Points - Stack

\begin{tabular}{|c|c|c|}
\hline \multicolumn{3}{|c|}{ Port \& Wall Thickness $=151 / 8$ inches } \\
\hline Insid & ameter $=109$ & \\
\hline $\begin{array}{c}\text { Point } \\
\text { Number }\end{array}$ & $\begin{array}{c}\text { Percent of } \\
\text { Stack Diameter }\end{array}$ & $\begin{array}{l}\text { Distance } \\
\text { from Wall }\end{array}$ \\
\hline 1 & 2.1 & $25 / 16 "$ \\
\hline 2 & 6.7 & $75 / 16 "$ \\
\hline 3 & 11.8 & $127 / 8 "$ \\
\hline 4 & 17.7 & $195 / 16 "$ \\
\hline 5 & 25.0 & $275 / 16 "$ \\
\hline 6 & 35.6 & $387 / 8 "$ \\
\hline 7 & 64.4 & $705 / 16 "$ \\
\hline 8 & 75.0 & $817 / 8 "$ \\
\hline 9 & 82.3 & $897 / 8 "$ \\
\hline 10 & 88.2 & $965 / 16 "$ \\
\hline 11 & 93.3 & $1017 / 8 "$ \\
\hline 12 & 97.9 & $1067 / 8 "$ \\
\hline
\end{tabular}




\section{Appendix A}

Figure 7: Location of Traverse Points - Stack

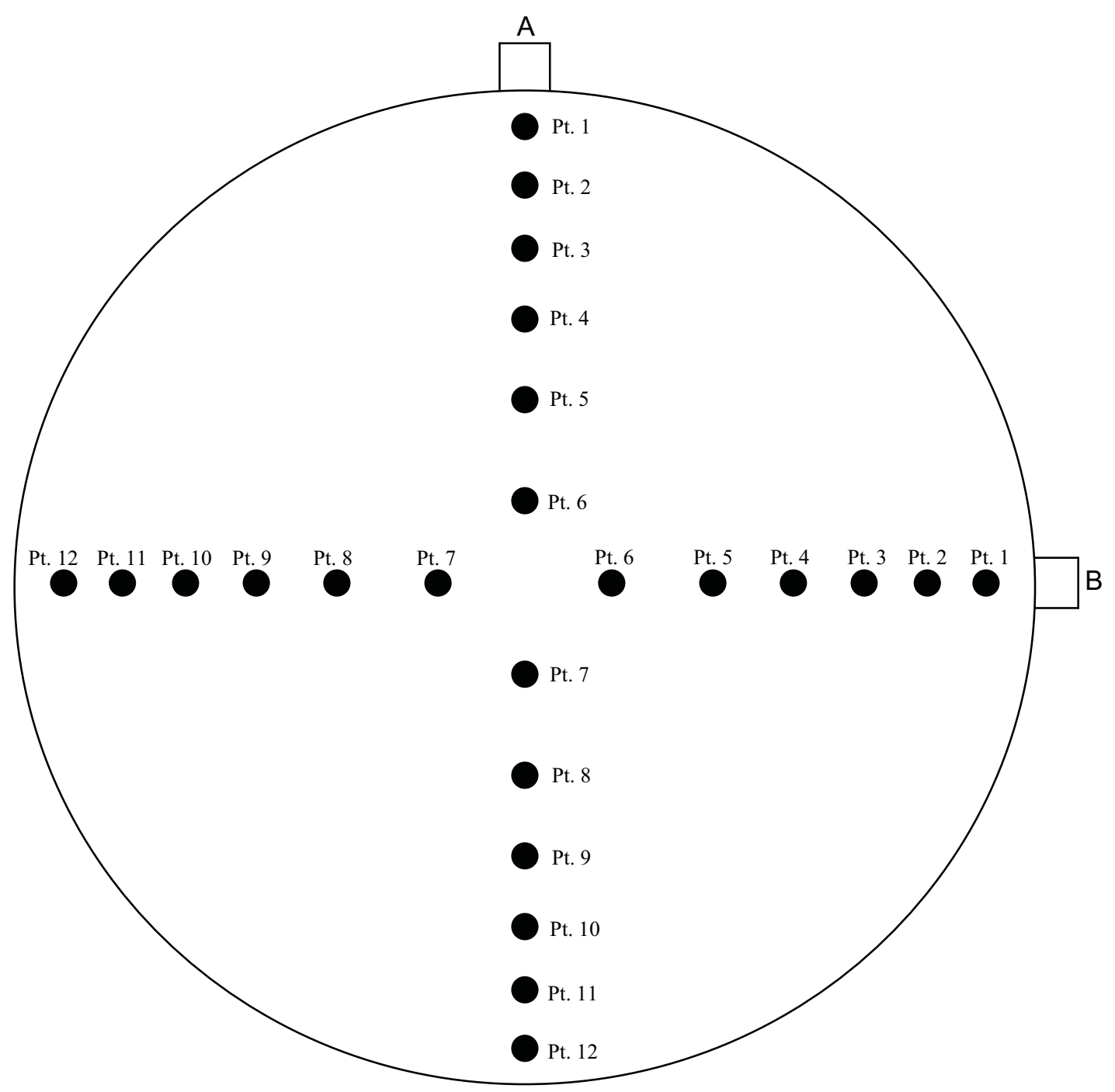

Not to scale. 
Appendix B:

Nomenclature and Equations for Calculation of Source Emissions 


\section{Nomenclature For Flow Rate and Moisture Calculations}

\begin{tabular}{|c|c|c|c|}
\hline Symbol & $\begin{array}{l}\text { English } \\
\text { Units }\end{array}$ & $\begin{array}{l}\text { Metric } \\
\text { Units }\end{array}$ & Description \\
\hline$A_{s}$ & in. $^{2}$ & $\mathrm{~m}^{2}$ & Stack Area \\
\hline $\mathrm{C}_{\mathrm{an}}$ & $g r / d s c f^{*}$ & $\mathrm{~g} / \mathrm{dscm}^{*}$ & $\begin{array}{l}\text { Particulate - probe, cyclone, } \\
\text { and filter }\end{array}$ \\
\hline $\mathrm{C}_{\mathrm{ao}}$ & $\mathrm{gr} / \mathrm{dscf}{ }^{*}$ & $\mathrm{~g} / \mathrm{dscm}^{*}$ & Particulate -total \\
\hline $\mathrm{C}_{\mathrm{at}}$ & $\begin{array}{c}\mathrm{gr} / \mathrm{CF} @ \\
\text { stack conditions }\end{array}$ & $\mathrm{g} / \mathrm{m} 3$ & $\begin{array}{l}\text { Particulate - probe, cyclone, } \\
\text { and filter }\end{array}$ \\
\hline $\mathrm{C}_{\mathrm{au}}$ & $\begin{array}{c}\text { gr/CF @ } \\
\text { stack conditions }\end{array}$ & $\mathrm{g} / \mathrm{m} 3$ & Particulate - total \\
\hline $\mathrm{C}_{\mathrm{aw}}$ & $\mathrm{lbs} / \mathrm{hr}$ & $\mathrm{kg} / \mathrm{hr}$ & $\begin{array}{l}\text { Particulate - probe, cyclone, } \\
\text { and filter }\end{array}$ \\
\hline $\mathrm{C}_{\mathrm{ax}}$ & $\mathrm{lbs} / \mathrm{hr}$ & $\mathrm{kg} / \mathrm{hr}$ & Particulate - total \\
\hline $\mathrm{C}_{\mathrm{p}}$ & & & Pitot Tube Calibration Factor \\
\hline$D_{n}$ & in. & $\mathrm{m}$ & Sampling Nozzle Diameter \\
\hline$\% \mathrm{EA}$ & & & $\begin{array}{l}\text { Percent Excess Air at } \\
\text { Sampling Point }\end{array}$ \\
\hline g & $32.2 \mathrm{ft} / \mathrm{sec}^{2}$ & & Acceleration of gravity \\
\hline$\% l$ & & & Percent Isokinetic \\
\hline$\% \mathrm{M}$ & & & $\begin{array}{l}\text { Percent Moisture in the Stack } \\
\text { Gas by Volume }\end{array}$ \\
\hline$M_{d}$ & & & Mole Fraction of Dry Gas \\
\hline$m_{f}$ & $\mathrm{mg}$ & $\mathrm{mg}$ & $\begin{array}{l}\text { Particulate - probe, cyclone, } \\
\text { and filter }\end{array}$ \\
\hline $\mathrm{mt}$ & $\mathrm{mg}$ & $\mathrm{mg}$ & Particulate -total \\
\hline$M_{\text {water }}$ & $18 \mathrm{lb} / \mathrm{lb}-\mathrm{mole}$ & & Molecular Weight of Water \\
\hline MW & $\mathrm{lb} / \mathrm{lb}$-mole & g/g-mole & $\begin{array}{l}\text { Molecular Weight of Stack } \\
\text { Gas }\end{array}$ \\
\hline $\mathrm{MW}_{\text {air }}$ & $28.84 \mathrm{lb} / \mathrm{lb}-\mathrm{mole}$ & & Molecular Weight of Air \\
\hline $\mathrm{MW}_{\mathrm{d}}$ & Ib/lb-mole & g/g-mole & $\begin{array}{l}\text { Molecular Weight of Dry Stack } \\
\text { Gas }\end{array}$ \\
\hline
\end{tabular}


Symbol

$P_{b}$

$P_{m}$

Ps

$\Delta \mathrm{P}$

$\mathrm{P}_{\text {std }}$

$\mathrm{Q}_{a}$

$Q_{s}$

R

$T_{m}$

$T_{t}$

$\mathrm{T}_{\mathrm{s}}$

$\mathrm{T}_{\text {std }}$

$V_{m}$

$V \mathrm{~m}_{\text {std }}$

$\mathrm{V}_{\mathrm{s}}$

$\mathrm{V}_{\mathrm{w}}$

$V w_{\text {gas }}$
English

Units

"Hg Absolute

$" \mathrm{H}_{2} \mathrm{O}$

"Hg Absolute

$" \mathrm{H}_{2} \mathrm{O}$

29.92" $\mathrm{Hg}$

ACFM

DSCFM $^{*}$

21.83" Hg-

$\mathrm{ft}^{3} / \mathrm{lb}$-mole ${ }^{\circ} \mathrm{R}$

${ }^{\circ} \mathrm{F}$

$\min$

${ }^{\circ} \mathrm{F}$

$528^{\circ} \mathrm{R}$

$\mathrm{ft}^{3}$

dscf* $^{*}$

fpm

$\mathrm{ml}$

$\operatorname{scf}^{*}$
Metric

$\underline{\text { Units }}$

$\mathrm{mm} \mathrm{Hg}$

$\mathrm{mm} \mathrm{H}_{2} \mathrm{O}$

$\mathrm{mm} \mathrm{Hg}$

$\mathrm{mm} \mathrm{H}_{2} \mathrm{O}$

$760 \mathrm{~mm} \mathrm{Hg}$

$\mathrm{m}^{3} / \mathrm{hr}$

$\mathrm{dscm} / \mathrm{hr} r^{*}$

${ }^{\circ} \mathrm{C}$

$\min$

${ }^{\circ} \mathrm{C}$

$293^{\circ} \mathrm{K}$

$\mathrm{m}^{3}$

$\mathrm{dscm}^{*}$

$\mathrm{m} / \mathrm{sec}$

$\mathrm{ml}$

$\mathrm{scm}^{*}$
Description

Barometric Pressure

Orifice Pressure drop

Stack Pressure

Velocity Head of Stack Gas

Standard Barometric Pressure

Stack Gas Volume at Actual Stack Conditions

Stack Gas Volume at 29.92"

$\mathrm{Hg}, 528^{\circ} \mathrm{R}$, dry

Universal Gas Constant

Average Gas Meter

Temperature

Net Time of Test

Stack Temperature

Standard Temperature

Volume of Dry Gas Sampled

@ Meter Conditions

Volume of Dry Gas Sampled @ Standard Conditions

Stack Velocity @ Stack Conditions

Total Water Collected in Impingers and Silica Gel

Volume of Water Vapor Collected @ Standard Conditions 
Symbol

$\rho_{\text {air }}$

$\rho_{\text {water }}$

$\rho_{\text {man }}$
English

Units

$0.0748 \mathrm{lbs} / \mathrm{ft}^{3}$

$1 \mathrm{~g} / \mathrm{ml}$

$62.32 \mathrm{lbs} / \mathrm{ft}^{3}$
Metric

$\underline{\text { Units }}$

Description

Density of Air

Density of Water

Density of Manometer Oil

(Inches of Water)

Standard Conditions: $29.92 " \mathrm{Hg}, 68^{\circ} \mathrm{F}\left(760 \mathrm{~mm} \mathrm{Hg}, 2{ }^{\circ} \mathrm{C}\right)$ 


\section{EXAMPLE CALCULATIONS}

1. Volume of dry gas sampled at standard conditions. *

$$
\begin{aligned}
& V_{m s t d}=V_{m}\left(\frac{T_{s t d}}{T_{m}+460}\right)\left[\frac{P_{b}+\frac{P_{m}}{13.6}}{P_{s t d}}\right] \\
& V_{m s t d}=17.65 V_{m}\left[\frac{P_{b}+\frac{P_{m}}{13.6}}{T_{m}+460}\right]=d s c f \\
& V_{m s d}=d s c f \times 0.028317=d s c m
\end{aligned}
$$

2. Volume of water vapor collected at standard conditions. *

$$
\begin{aligned}
& V_{w_{g a s}}=\frac{\left(V_{w}-g m s \mathrm{SO}_{2}-g m s H_{2} S\right) \rho_{\text {water }} R T_{\text {std }}}{P_{\text {std }} M_{\text {water }} 453.6} \\
& V_{w_{g a s}}=0.0472\left(V_{w}-g m s \mathrm{SO}_{2}-g m s H_{2} S\right)=s c f \\
& V_{w_{g a s}}=s c f \times 0.028317=s c m
\end{aligned}
$$

3. Percent moisture in stack gas.

$$
\% M=\frac{V_{w_{g a s}}}{V_{m_{s t d}}+V_{w_{g a s}}} \times 100=\%
$$


4. Mole fraction of dry gas.

$$
M_{d}=\frac{100-\% M}{100}
$$

5. Average molecular weight of dry stack gas.

$$
\begin{aligned}
M W_{d}=\left[\% \mathrm{CO}_{2} \times \frac{44}{100}\right]+\left[\% \mathrm{O}_{2} \times \frac{32}{100}\right]+\left[\% N_{2} \times \frac{28}{100}\right]+\left[\% \mathrm{CO} \times \frac{28}{100}\right] & =\mathrm{lb} / \mathrm{lb}-\text { mole } \\
& =\mathrm{g} / \mathrm{g}-\mathrm{mole}
\end{aligned}
$$

6. Molecular weight of stack gas.

$$
M W=M W_{d} \times M_{d}+18\left(1-M_{d}\right)=\frac{l b}{l b-m o l e}=g / g-\text { mole }
$$

7. Percent excess air at sampling point.

$$
\% E A=\frac{100\left[\% \mathrm{O}_{2}-(0.5 \% \mathrm{CO})\right]}{0.265\left(\% \mathrm{~N}_{2}\right)-\left[\% \mathrm{O}_{2}-(0.5 \% \mathrm{CO})\right]}
$$

8. Stack Pressure.

$$
P_{s}=P_{b}+\frac{\text { Stack Pressure " } \mathrm{H}_{2} \mathrm{O}}{13.6}=" \mathrm{Hg} \text { Absolute }
$$

$$
P_{s}=" H g \text { Abs. } \times 25.4=m m H g
$$

9. Stack velocity at stack conditions.

$$
\begin{aligned}
& V_{s}=C_{p} 60\left[\frac{2 g \times \rho_{\text {man }} \times P_{\text {std }} \times M W_{\text {air }} \times\left(T_{s}+460\right) \times \Delta P}{12 \times \rho_{\text {air }} \times P_{s} \times M W \times T_{\text {std }}}\right]^{1 / 2} \\
& V_{s}=5,123.8 C_{p}\left[\frac{\left(T_{s}+460\right)}{P_{s} \times M W}\right]^{1 / 2} \sqrt{\Delta P} \text { average }=\mathrm{fpm} \\
& V_{s}=f p m \times 0.00508=\mathrm{m} / \mathrm{sec}
\end{aligned}
$$


10. Dry stack gas volume at standard conditions. *

$$
\begin{aligned}
& Q_{s}=\frac{1}{144} V_{s} \times A_{s} \times M_{d} \times \frac{T_{s t d}}{T_{s}+460} \times \frac{P_{s}}{P_{s t d}} \\
& Q_{s}=\frac{0.123 V_{s} \times A_{s} \times M_{d} \times P_{s}}{T_{s}+460}=D S C F M
\end{aligned}
$$

$Q_{s}=D S C F M \times 1.6990=d s \mathrm{~cm} / \mathrm{hr}$

11. Actual stack gas volume at stack conditions.

$$
\begin{aligned}
& Q_{a}=\frac{V_{s} \times A_{s}}{144}=A C F M \\
& Q_{a}=A C F M \times 1.6990=\mathrm{m}^{3} / \mathrm{hr}
\end{aligned}
$$

12. Percent Isokinetic

$$
\begin{aligned}
& \% \mathrm{l}=\frac{V_{\text {mstd }} \times\left(T_{s}+460\right) \times P_{s t d} \times 100 \times 144 i n^{2} / \mathrm{ft}^{2}}{M_{d} \times T_{s t d} \times P_{s} \times T_{t} \times V_{s}\left(\frac{\Pi \times D_{n}{ }^{2}}{4}\right)} \\
& \% \mathrm{I}=\frac{1039 \times V_{\text {sstd }} \times\left(T_{s}+460\right)}{M_{d} \times P_{s} \times T_{t} \times V_{s} \times D_{n}{ }^{2}}
\end{aligned}
$$

* 29.92" Hg, $68^{\circ} \mathrm{F}\left(760 \mathrm{~mm} \mathrm{Hg}, 20^{\circ} \mathrm{C}\right)$ 
13. Particulate - Probe, cyclone, and filter.

$$
\begin{aligned}
& C_{a n}=\frac{m_{f}}{V_{m_{s t d}}} \times \frac{1 \mathrm{gr}}{64.8 \mathrm{mg}} \\
& C_{a n}=0.0154 \times \frac{\mathrm{mf}}{V_{\text {mstd }}} \mathrm{gr} / \mathrm{dscf} \text { * } \\
& C_{\text {an }}=\mathrm{gr} / \mathrm{dscf} \times 2.290=\mathrm{g} / \mathrm{dscm} \text { * }
\end{aligned}
$$

14. Particulate total.

$$
\begin{aligned}
& \mathrm{C}_{\mathrm{ao}}=0.0154 \times \frac{\mathrm{m}_{\mathrm{t}}}{\mathrm{V}_{\mathrm{mstd}}}=g r / d s c f \text { * } \\
& \mathrm{C}_{\mathrm{ao}}=\mathrm{gr} / \mathrm{dscf} \times 2.290=\mathrm{g} / \mathrm{dscm} \text { * }
\end{aligned}
$$

15. Particulate - probe, cyclone, and filter at stack conditions.

$$
\begin{aligned}
& C_{a t}=C_{a n} \times \frac{P_{s}}{P_{\text {std }}} \times \frac{\left(T_{s t d}\right)}{\left(T_{s}+460\right)} \times M_{d} \\
& C_{a t}=\frac{17.65 \times C_{a n} \times P s \times M d}{T x+460}=g r / C F \\
& C_{a t}=g r / C F \times 2.290=g / m^{3}
\end{aligned}
$$

16. Particulate - total, at stack conditions.

$$
\begin{aligned}
& C_{a u}=\frac{17.65 \times C_{a o} \times P_{s} \times M_{d}}{T_{s}+460}=g r / C F \\
& C_{a u}=g r / C F \times 2.290=g / m^{3}
\end{aligned}
$$




\section{AII

17. Particulate - probe, cyclone, and filter.

$$
\begin{aligned}
& C_{a w}=C_{a n} \times Q_{s} \times \frac{60 \mathrm{~min} .}{1 \mathrm{hr}} \times \frac{1 \mathrm{lb}}{7,000 \mathrm{gr}} \\
& C_{a w}=0.00857 \times C_{a n} \times Q_{s}=\mathrm{lbs} / \mathrm{hr} \\
& C_{a w}=\mathrm{lbs} / \mathrm{hr} \times 0.4536=\mathrm{kg} / \mathrm{hr}
\end{aligned}
$$

18. Particulate - total.

$$
\begin{aligned}
& C_{a x}=0.00857 \times C_{a o} \times Q_{s}=l b s / h r \\
& C_{a x}=l b s / h r \times 0.4536=\mathrm{kg} / \mathrm{hr}
\end{aligned}
$$

19. Mercury $-\mu \mathrm{g} / \mathrm{dscm}$

$$
\mu \mathrm{g} / \mathrm{dscm}=\mu \mathrm{g} \div\left(\mathrm{V}_{\mathrm{mstd}} \times 0.028317 \mathrm{~m}^{3} / \mathrm{ft}^{3}\right)
$$

*29.92" Hg, $68^{\circ} \mathrm{F}\left(760 \mathrm{~mm} \mathrm{Hg}, 20^{\circ} \mathrm{C}\right)$ 


\title{
11.
}

\author{
Summary of Results \\ Mercury Speciation Results
}

Rocky Mountain Power - Hardin Power Plant

SDA Inlet Duct

\begin{tabular}{|c|c|c|c|c|}
\hline $\begin{array}{l}\text { Run No. } \\
\text { Test Date: } \\
\text { Test Time: }\end{array}$ & $\begin{array}{c}1 \\
4 / 26 / 2007 \\
1020-1302\end{array}$ & $\begin{array}{c}2 \\
4 / 26 / 2007 \\
1445-1657\end{array}$ & $\begin{array}{c}3 \\
4 / 26 / 2007 \\
1810-2012\end{array}$ & Average \\
\hline \multicolumn{5}{|c|}{ Volume of Dry Gas Sampled @ Standard Conditions } \\
\hline dscm & 1.392 & 1.456 & 1.433 & 1.427 \\
\hline dscf & 49.156 & 51.407 & 50.596 & 50.386 \\
\hline \multicolumn{5}{|l|}{ Oxygen } \\
\hline$\%$ volume & 6.5 & 6.6 & 6.6 & 6.6 \\
\hline \multicolumn{5}{|l|}{ Particulate Mercury } \\
\hline$\mu g$ & 1.3000 & 0.9700 & 0.9800 & 1.0833 \\
\hline$\mu \mathrm{g} / \mathrm{dscf}$ & 0.0264 & 0.0189 & 0.0194 & 0.0216 \\
\hline$\mu \mathrm{g} / \mathrm{dscm}$ & 0.9339 & 0.6662 & 0.6839 & 0.7613 \\
\hline $\mathrm{lbs} / 10^{12} \mathrm{Btu}$ & 0.828 & 0.595 & 0.610 & 0.678 \\
\hline$\%$ of total $\mathrm{Hg}$ & $27.78 \%$ & $20.21 \%$ & $20.99 \%$ & $22.99 \%$ \\
\hline \multicolumn{5}{|l|}{ Oxidized Mercury } \\
\hline$\mu g$ & 0.6500 & 0.5700 & 0.7200 & 0.6467 \\
\hline$\mu g / d s c f$ & 0.0132 & 0.0111 & 0.0142 & 0.0128 \\
\hline$\mu \mathrm{g} / \mathrm{dscm}$ & 0.4670 & 0.3915 & 0.5024 & 0.4536 \\
\hline $\mathrm{lbs} / 10^{12} \mathrm{Btu}$ & 0.414 & 0.349 & 0.449 & 0.404 \\
\hline$\%$ of total $\mathrm{Hg}$ & $13.89 \%$ & $11.88 \%$ & $15.42 \%$ & $13.73 \%$ \\
\hline \multicolumn{5}{|l|}{ Elemental Mercury } \\
\hline$\mu \mathrm{g}$ & 2.7300 & 3.2600 & 2.9700 & 2.9867 \\
\hline$\mu \mathrm{g} / \mathrm{dscf}$ & 0.0555 & 0.0634 & 0.0587 & 0.0592 \\
\hline$\mu \mathrm{g} / \mathrm{dscm}$ & 1.9612 & 2.2390 & 2.0726 & 2.0909 \\
\hline $\mathrm{lbs} / 10^{12} \mathrm{Btu}$ & 1.738 & 1.999 & 1.850 & 1.862 \\
\hline$\%$ of total $\mathrm{Hg}$ & $58.33 \%$ & $67.92 \%$ & $63.60 \%$ & $63.28 \%$ \\
\hline \multicolumn{5}{|l|}{ Total Mercury } \\
\hline$\mu \mathrm{g}$ & 4.6800 & 4.8000 & 4.6700 & 4.7167 \\
\hline$\mu \mathrm{g} / \mathrm{dscf}$ & 0.0952 & 0.0934 & 0.0923 & 0.0936 \\
\hline$\mu \mathrm{g} / \mathrm{dscm}$ & 3.3621 & 3.2967 & 3.2589 & 3.3059 \\
\hline $\mathrm{lbs} / 10^{12} \mathrm{Btu}$ & 2.980 & 2.943 & 2.909 & 2.944 \\
\hline Oxygen based F factor & 9,780 & 9,780 & 9,780 & 9,780 \\
\hline
\end{tabular}




\title{
11.
}

\author{
Summary of Results \\ Mercury Speciation Results
}

Rocky Mountain Power - Hardin Power Plant

Stack

\begin{tabular}{|c|c|c|c|c|}
\hline $\begin{array}{l}\text { Run No. } \\
\text { Test Date: } \\
\text { Test Time: }\end{array}$ & $\begin{array}{c}1 \\
4 / 26 / 2007 \\
1020-1305\end{array}$ & $\begin{array}{c}2 \\
4 / 26 / 2007 \\
1445-1650\end{array}$ & $\begin{array}{c}3 \\
4 / 26 / 2007 \\
1810-2015\end{array}$ & Average \\
\hline \multicolumn{5}{|c|}{ Volume of Dry Gas Sampled @ Standard Conditions } \\
\hline dscm & 2.018 & 2.04 & 2.079 & 2.046 \\
\hline dscf & 71.281 & 72.049 & 73.417 & 72.249 \\
\hline \multicolumn{5}{|l|}{ Oxygen } \\
\hline$\%$ volume & 6.8 & 6.9 & 6.8 & 6.8 \\
\hline \multicolumn{5}{|l|}{ Particulate Mercury } \\
\hline$\mu g$ & 0.0110 & 0.0580 & 0.0290 & 0.0327 \\
\hline$\mu \mathrm{g} / \mathrm{dscf}$ & 0.0002 & 0.0008 & 0.0004 & 0.0005 \\
\hline$\mu \mathrm{g} / \mathrm{dscm}$ & 0.0055 & 0.0284 & 0.0139 & 0.0159 \\
\hline $\mathrm{lbs} / 10^{12} \mathrm{Btu}$ & 0.005 & 0.026 & 0.013 & 0.014 \\
\hline$\%$ of total $\mathrm{Hg}$ & $0.13 \%$ & $0.62 \%$ & $0.34 \%$ & $0.36 \%$ \\
\hline \multicolumn{5}{|l|}{ Oxidized Mercury } \\
\hline$\mu g$ & 1.8000 & 1.8000 & 1.7000 & 1.7667 \\
\hline$\mu g / d s c f$ & 0.0253 & 0.0250 & 0.0232 & 0.0245 \\
\hline$\mu \mathrm{g} / \mathrm{dscm}$ & 0.8920 & 0.8824 & 0.8177 & 0.8640 \\
\hline $\mathrm{lbs} / 10^{12} \mathrm{Btu}$ & 0.807 & 0.804 & 0.740 & 0.784 \\
\hline$\%$ of total $\mathrm{Hg}$ & $20.78 \%$ & $19.26 \%$ & $19.91 \%$ & $19.98 \%$ \\
\hline \multicolumn{5}{|l|}{ Elemental Mercury } \\
\hline$\mu \mathrm{g}$ & 6.8500 & 7.4900 & 6.8100 & 7.0500 \\
\hline$\mu \mathrm{g} / \mathrm{dscf}$ & 0.0961 & 0.1040 & 0.0928 & 0.0976 \\
\hline$\mu \mathrm{g} / \mathrm{dscm}$ & 3.3944 & 3.6716 & 3.2756 & 3.4472 \\
\hline $\mathrm{lbs} / 10^{12} \mathrm{Btu}$ & 3.072 & 3.347 & 2.965 & 3.128 \\
\hline$\%$ of total $\mathrm{Hg}$ & $79.09 \%$ & $80.12 \%$ & $79.75 \%$ & $79.66 \%$ \\
\hline \multicolumn{5}{|l|}{ Total Mercury } \\
\hline$\mu \mathrm{g}$ & 8.6610 & 9.3480 & 8.5390 & 8.8493 \\
\hline$\mu \mathrm{g} / \mathrm{dscf}$ & 0.1215 & 0.1297 & 0.1163 & 0.1225 \\
\hline$\mu \mathrm{g} / \mathrm{dscm}$ & 4.2919 & 4.5824 & 4.1073 & 4.3272 \\
\hline $\mathrm{lbs} / 10^{12} \mathrm{Btu}$ & 3.884 & 4.177 & 3.718 & 3.926 \\
\hline Oxygen based F factor & 9,780 & 9,780 & 9,780 & 9,780 \\
\hline
\end{tabular}


SOURCE EMISSION SURVEY

JOB NUMBER: 07-024

JOB NAME: ADA-ES

LOCATION: Hardin, Montana

UNIT TESTED: SDA Inlet Ontario Hydro

SOURCE EMISSION CALCULATIONS

\begin{tabular}{|c|c|c|c|c|c|}
\hline & & & & NUMBER & \\
\hline SYMBOL & DESCRIPTION & UNITS & 1 & 2 & 3 \\
\hline DATE & & & $04 / 26 / 07$ & $04 / 26 / 07$ & $04 / 26 / 07$ \\
\hline BEGIN TIME & & & 1020 & 1445 & 1810 \\
\hline END TIME & & & 1302 & 1657 & 2012 \\
\hline$P(b)$ & BAROMETRIC PRESSURE & $\begin{array}{l}\text { "Hg Abs. } \\
(\mathrm{mm} \mathrm{Hg})\end{array}$ & $\begin{array}{r}26.96 \\
(685.00) \\
\end{array}$ & $\begin{array}{r}26.93 \\
(684.00) \\
\end{array}$ & $\begin{array}{r}26.93 \\
(684.00) \\
\end{array}$ \\
\hline$P(m)$ & ORIFICE PRESSURE DROP & $\begin{array}{l}\text { " } \mathrm{H} 2 \mathrm{O} \\
(\mathrm{mm} \mathrm{H} 2 \mathrm{O})\end{array}$ & $\begin{array}{r}0.577 \\
(14.700) \\
\end{array}$ & $\begin{array}{r}0.631 \\
(16.000) \\
\end{array}$ & $\begin{array}{r}0.626 \\
(15.900) \\
\end{array}$ \\
\hline & DGM CALIBRATION FACTOR & & 1.004 & 1.004 & 1.004 \\
\hline$V(m)$ & $\begin{array}{l}\text { VOLUME DRY GAS SAMPLED } \\
@ \text { METER CONDITIONS } \\
\text { LEAK CHECK VOLUME }\end{array}$ & $\begin{array}{l}\text { ft. }{ }^{\wedge} \\
\left(m^{\wedge} 3\right) \\
\text { ft. }{ }^{\wedge} 3\end{array}$ & $\begin{array}{c}55.077 \\
(1.560) \\
0.000 \\
\end{array}$ & $\begin{array}{c}58.194 \\
(1.648) \\
0.406 \\
\end{array}$ & $\begin{array}{r}58.127 \\
(1.646) \\
0.000 \\
\end{array}$ \\
\hline$T(m)$ & $\begin{array}{l}\text { AVERAGE GAS METER } \\
\text { TEMPERATURE }\end{array}$ & $\begin{array}{l}\text { DEG.F } \\
\text { (DEG.C) }\end{array}$ & $\begin{array}{c}74 \\
(23) \\
\end{array}$ & $\begin{array}{c}79 \\
(26) \\
\end{array}$ & $\begin{array}{c}87 \\
(31) \\
\end{array}$ \\
\hline$V(m[s t d])^{*}$ & $\begin{array}{l}\text { VOLUME DRY GAS SAMPLED } \\
\text { @ STANDARD CONDITIONS* }\end{array}$ & $\begin{array}{l}\text { DSCF } \\
\text { (DSCM) }\end{array}$ & $\begin{array}{l}49.156 \\
(1.392) \\
\end{array}$ & $\begin{array}{l}51.407 \\
(1.456) \\
\end{array}$ & $\begin{array}{l}50.596 \\
(1.433) \\
\end{array}$ \\
\hline$V(w)$ & $\begin{array}{l}\text { TOTAL WATER COLLECTED, } \\
\text { IMPINGERS \& SILICA GEL }\end{array}$ & $\mathrm{ml}$ & 130.4 & 132.5 & 126.5 \\
\hline $\mathrm{V}($ w[gas $])$ & $\begin{array}{l}\text { VOLUME WATER VAPOR } \\
\text { COLLECTED @ STANDARD } \\
\text { CONDITIONS* }\end{array}$ & $\begin{array}{l}\text { SCF } \\
\text { (SCM) }\end{array}$ & $\begin{array}{c}6.155 \\
(0.174)\end{array}$ & $\begin{array}{c}6.254 \\
(0.177)\end{array}$ & $\begin{array}{c}5.971 \\
(0.169)\end{array}$ \\
\hline$\% M$ & $\begin{array}{l}\text { MOISTURE IN STACK GAS } \\
\text { BY VOLUME }\end{array}$ & $\%$ & 11.13 & 10.85 & 10.56 \\
\hline $\mathrm{Md}$ & MOL FRACTION OF DRY GAS & & 0.8887 & 0.8915 & 0.8944 \\
\hline $\mathrm{Tt}$ & NET TIME OF TEST & MINUTES & 120 & 120 & 120 \\
\hline
\end{tabular}

* 68 Deg.F, 29.92 "Hg (20 Deg.C, 760 mm Hg) 


\section{SOURCE EMISSION CALCULATIONS}

JOB NUMBER: 07-024

JOB NAME: ADA-ES

LOCATION: Hardin, Montana

UNIT TESTED: SDA Inlet Ontario Hydro

\begin{tabular}{|c|c|c|c|c|c|}
\hline \multicolumn{3}{|c|}{40} & \multicolumn{3}{|c|}{ RUN NUMBER } \\
\hline SYMBOL & DESCRIPTION & UNITS & 1 & \begin{tabular}{l|l}
2 \\
\end{tabular} & 3 \\
\hline $\mathrm{CO} 2$ & & $\%$ & 12.8 & 12.7 & 12.7 \\
\hline $\mathrm{O} 2$ & & $\%$ & 6.5 & 6.6 & 6.6 \\
\hline $\mathrm{CO}$ & & $\%$ & 0.0 & 0.0 & 0.0 \\
\hline N2 & & $\%$ & 80.7 & 80.7 & 80.7 \\
\hline$\%$ EA & $\begin{array}{l}\text { EXCESS AIR @ SAMPLING } \\
\text { POINT }\end{array}$ & $\%$ & 43.7 & 44.6 & 44.6 \\
\hline MWd & $\begin{array}{l}\text { MOLECULAR WEIGHT OF } \\
\text { DRY STACK GAS }\end{array}$ & $\begin{array}{l}\text { LB/LB-MOLE } \\
\text { (g/g-MOLE) }\end{array}$ & $\begin{array}{r}30.31 \\
(30.31) \\
\end{array}$ & $\begin{array}{r}30.30 \\
(30.30)\end{array}$ & $\begin{array}{r}30.30 \\
(30.30) \\
\end{array}$ \\
\hline MW & $\begin{array}{l}\text { MOLECULAR WEIGHT OF } \\
\text { STACK GAS }\end{array}$ & $\begin{array}{l}\text { LB/LB-MOLE } \\
\text { (g/g-MOLE) }\end{array}$ & $\begin{array}{c}28.94 \\
(28.94) \\
\end{array}$ & $\begin{array}{r}28.96 \\
(28.96) \\
\end{array}$ & $\begin{array}{c}29.00 \\
(29.00) \\
\end{array}$ \\
\hline $\mathrm{Cp}$ & PITOT TUBE CALIBRATION & & 0.824 & 0.822 & 0.824 \\
\hline DELTAP & $\begin{array}{l}\text { VELOCITY HEAD OF STACK } \\
\text { GAS }\end{array}$ & $\begin{array}{l}\text { "H2O } \\
(\mathrm{mm} H 20)\end{array}$ & $\begin{array}{r}0.906 \\
(23.000)\end{array}$ & $\begin{array}{r}0.995 \\
(25.300)\end{array}$ & $\begin{array}{r}0.986 \\
(25.000) \\
\end{array}$ \\
\hline DELTAP $P^{\wedge}(1 / 2)$ & & "H2O & 0.939 & 0.987 & 0.984 \\
\hline Ts & STACK TEMPERATURE & $\begin{array}{l}\text { DEG. F } \\
\text { (DEG. C) }\end{array}$ & $\begin{array}{c}320 \\
(160) \\
\end{array}$ & $\begin{array}{c}324 \\
(162) \\
\end{array}$ & $\begin{array}{r}325 \\
(163) \\
\end{array}$ \\
\hline Ps & STACK PRESSURE & $\begin{array}{l}\text { "Hg Abs. } \\
(\mathrm{mm} \mathrm{Hg}) \\
\text { "H2O }\end{array}$ & $\begin{array}{r}25.64 \\
(651.00) \\
-18.00 \\
\end{array}$ & $\begin{array}{r}25.53 \\
(648.00) \\
-19.00 \\
\end{array}$ & $\begin{array}{r}25.75 \\
(654.00) \\
-16.00 \\
\end{array}$ \\
\hline Vs & $\begin{array}{l}\text { STACK VELOCITY @ STACK } \\
\text { CONDITIONS }\end{array}$ & $\begin{array}{l}\text { FPM } \\
(\mathrm{m} / \mathrm{SEC} .)\end{array}$ & $\begin{array}{r}4,065 \\
(21) \\
\end{array}$ & $\begin{array}{r}4,281 \\
(22) \\
\end{array}$ & $\begin{array}{r}4,260 \\
(22) \\
\end{array}$ \\
\hline As & STACK AREA & $\begin{array}{l}\text { (SQ.INCHES) } \\
\text { (SQ.METERS) }\end{array}$ & $\begin{array}{r}20,235 \\
(13) \\
\end{array}$ & $\begin{array}{r}20,235 \\
(13) \\
\end{array}$ & $\begin{array}{r}20,235 \\
(13) \\
\end{array}$ \\
\hline Qs & $\begin{array}{l}\text { DRY STACK GAS VOLUME @ } \\
\text { STANDARD CONDITIONS* }\end{array}$ & $\begin{array}{l}\text { DSCFM } \\
\text { (DSCM/HR) }\end{array}$ & $\begin{array}{c}295,561 \\
(502,158)\end{array}$ & $\begin{array}{r}309,321 \\
(525,536)\end{array}$ & $\begin{array}{c}311,069 \\
(528,506)\end{array}$ \\
\hline $\mathrm{Qa}$ & $\begin{array}{l}\text { ACTUAL STACK GAS VOLUME } \\
\text { @ STACK CONDITIONS }\end{array}$ & $\begin{array}{l}\text { ACFM } \\
\left(m^{\wedge} 3 / H R\right)\end{array}$ & $\begin{array}{r}571,169 \\
(970,416) \\
\end{array}$ & $\begin{array}{r}601,527 \\
(1,021,994) \\
\end{array}$ & $\begin{array}{r}598,553 \\
(1,016,942) \\
\end{array}$ \\
\hline Dn & SAMPLING NOZZLE DIAM. & $\begin{array}{l}\text { IN. } \\
(\mathrm{m})\end{array}$ & $\begin{array}{r}0.193 \\
(0.005)\end{array}$ & $\begin{array}{r}0.193 \\
(0.005) \\
\end{array}$ & $\begin{array}{c}0.191 \\
(0.005) \\
\end{array}$ \\
\hline$\% \mathrm{I}$ & PERCENT ISOKINETIC & $\%$ & 96.2 & 96.2 & 96.1 \\
\hline
\end{tabular}

*68 Deg.F, 29.92 "Hg (20 Deg.C, 760 mm Hg) 


\section{SOURCE EMISSION CALCULATIONS}

JOB NUMBER: 07-024

JOB NAME: ADA-ES

LOCATION: Hardin, Montana

UNIT TESTED: SDA Inlet Ontario Hydro

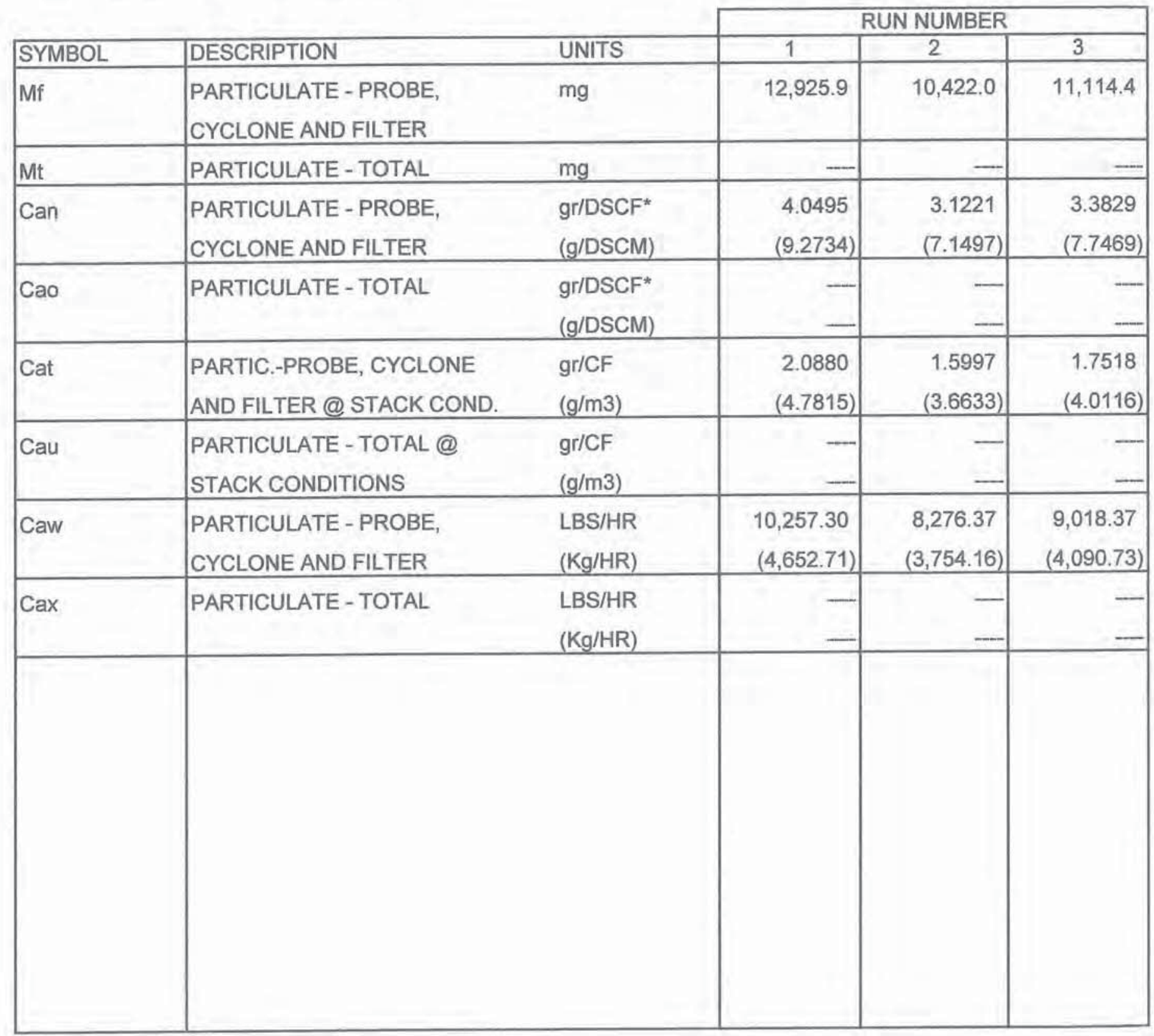

*68 Deg.F, 29.92 "Hg (20 Deg.C, 760 mm Hg) 
SOURCE EMISSION SURVEY

JOB NUMBER: 07-024

JOB NAME: ADA-ES

LOCATION: Hardin, Montana

UNIT TESTED: Stack EPA Method 26A

SOURCE EMISSION CALCULATIONS

\begin{tabular}{|c|c|c|c|c|c|}
\hline & & & & NUMBER & \\
\hline SYMBOL & DESCRIPTION & UNITS & 1 & 2 & 3 \\
\hline DATE & & & $04 / 27 / 07$ & $04 / 27 / 07$ & $04 / 27 / 07$ \\
\hline BEGIN TIME & & & 0900 & 1110 & 1407 \\
\hline END TIME & & & 1017 & 1227 & 1523 \\
\hline$P(b)$ & BAROMETRIC PRESSURE & $\begin{array}{l}\text { "Hg Abs. } \\
(\mathrm{mm} \mathrm{Hg})\end{array}$ & $\begin{array}{r}27.05 \\
(687.00) \\
\end{array}$ & $\begin{array}{r}27.05 \\
(687.00) \\
\end{array}$ & $\begin{array}{r}26.99 \\
(686.00) \\
\end{array}$ \\
\hline$P(m)$ & ORIFICE PRESSURE DROP & $\begin{array}{l}\text { " } \mathrm{H} 2 \mathrm{O} \\
(\mathrm{mm} \mathrm{H} 2 \mathrm{O})\end{array}$ & $\begin{array}{r}1.313 \\
(33.400) \\
\end{array}$ & $\begin{array}{r}1.086 \\
(27.600) \\
\end{array}$ & $\begin{array}{r}1.252 \\
(31.800) \\
\end{array}$ \\
\hline & DGM CALIBRATION FACTOR & & 0.998 & 0.998 & 0.998 \\
\hline$V(m)$ & $\begin{array}{l}\text { VOLUME DRY GAS SAMPLED } \\
\text { @ METER CONDITIONS } \\
\text { LEAK CHECK VOLUME }\end{array}$ & $\begin{array}{l}\text { ft. } 3 \\
\left(\mathrm{~m}^{\wedge} 3\right) \\
\text { ft. }{ }^{\wedge} 3\end{array}$ & $\begin{array}{c}48.359 \\
(1.369) \\
0.000 \\
\end{array}$ & $\begin{array}{c}44.009 \\
(1.246) \\
0.000 \\
\end{array}$ & $\begin{array}{c}47.712 \\
(1.351) \\
0.000 \\
\end{array}$ \\
\hline$T(m)$ & $\begin{array}{l}\text { AVERAGE GAS METER } \\
\text { TEMPERATURE }\end{array}$ & $\begin{array}{l}\text { DEG.F } \\
\text { (DEG.C) }\end{array}$ & $\begin{array}{r}64 \\
(18) \\
\end{array}$ & $\begin{array}{c}76 \\
(24) \\
\end{array}$ & $\begin{array}{r}78 \\
(26) \\
\end{array}$ \\
\hline$V(m[s t d])^{*}$ & $\begin{array}{l}\text { VOLUME DRY GAS SAMPLED } \\
\text { @ STANDARD CONDITIONS* }\end{array}$ & $\begin{array}{l}\text { DSCF } \\
\text { (DSCM) }\end{array}$ & $\begin{array}{l}44.219 \\
(1.252) \\
\end{array}$ & $\begin{array}{l}39.316 \\
(1.113) \\
\end{array}$ & $\begin{array}{l}42.391 \\
(1.200) \\
\end{array}$ \\
\hline$V(w)$ & $\begin{array}{l}\text { TOTAL WATER COLLECTED, } \\
\text { IMPINGERS \& SILICA GEL }\end{array}$ & $\mathrm{ml}$ & 167.4 & 142.7 & 151.4 \\
\hline$V(w[$ gas $])$ & $\begin{array}{l}\text { VOLUME WATER VAPOR } \\
\text { COLLECTED @ STANDARD } \\
\text { CONDITIONS* }\end{array}$ & $\begin{array}{l}\text { SCF } \\
\text { (SCM) }\end{array}$ & $\begin{array}{c}7.901 \\
(0.224)\end{array}$ & $\begin{array}{c}6.735 \\
(0.191)\end{array}$ & $\begin{array}{l}7.146 \\
(0.202)\end{array}$ \\
\hline$\% \mathrm{M}$ & $\begin{array}{l}\text { MOISTURE IN STACK GAS } \\
\text { BY VOLUME }\end{array}$ & $\%$ & 15.16 & 14.63 & 14.43 \\
\hline Md & MOL FRACTION OF DRY GAS & & 0.8484 & 0.8537 & 0.8557 \\
\hline $\mathrm{Tt}$ & NET TIME OF TEST & MINUTES & 72 & 72 & 72 \\
\hline
\end{tabular}

*68 Deg.F, 29.92 "Hg (20 Deg.C, 760 mm Hg) 


\section{SOURCE EMISSION CALCULATIONS}

JOB NUMBER: 07-024

JOB NAME: ADA-ES

LOCATION: Hardin, Montana

UNIT TESTED: Stack EPA Method 26A

\begin{tabular}{|c|c|c|c|c|c|}
\hline \multirow{2}{*}{ SYMBOL } & \multirow[b]{2}{*}{ DESCRIPTION } & \multirow[b]{2}{*}{ UNITS } & \multicolumn{3}{|c|}{ RUN NUMBER } \\
\hline & & & 1 & 2 & 3 \\
\hline $\mathrm{CO} 2$ & & $\%$ & 12.7 & 12.6 & 12.6 \\
\hline $\mathrm{O} 2$ & & $\%$ & 6.6 & 6.7 & 6.7 \\
\hline $\mathrm{CO}$ & & $\%$ & 0.0 & 0.0 & 0.0 \\
\hline N2 & & $\%$ & 80.7 & 80.7 & 80.7 \\
\hline$\% E A$ & $\begin{array}{l}\text { EXCESS AIR @ SAMPLING } \\
\text { POINT }\end{array}$ & $\%$ & 44.6 & 45.6 & 45.6 \\
\hline MWd & $\begin{array}{l}\text { MOLECULAR WEIGHT OF } \\
\text { DRY STACK GAS }\end{array}$ & $\begin{array}{l}\text { LB/LB-MOLE } \\
\text { (g/g-MOLE) }\end{array}$ & $\begin{array}{r}30.30 \\
(30.30) \\
\end{array}$ & $\begin{array}{c}30.28 \\
(30.28) \\
\end{array}$ & $\begin{array}{r}30.28 \\
(30.28) \\
\end{array}$ \\
\hline MW & $\begin{array}{l}\text { MOLECULAR WEIGHT OF } \\
\text { STACK GAS }\end{array}$ & $\begin{array}{l}\text { LB/LB-MOLE } \\
\text { (g/g-MOLE) }\end{array}$ & $\begin{array}{r}28.43 \\
(28.43) \\
\end{array}$ & $\begin{array}{r}28.49 \\
(28.49) \\
\end{array}$ & $\begin{array}{r}28.51 \\
(28.51) \\
\end{array}$ \\
\hline $\mathrm{Cp}$ & PITOT TUBE CALIBRATION & & 0.826 & 0.824 & 0.824 \\
\hline DELTAP & $\begin{array}{l}\text { VELOCITY HEAD OF STACK } \\
\text { GAS }\end{array}$ & $\begin{array}{l}\text { "H2O } \\
(\mathrm{mm} \text { H2O) }\end{array}$ & $\begin{array}{r}3.983 \\
(101.200) \\
\end{array}$ & $\begin{array}{r}3.304 \\
(83.900) \\
\end{array}$ & $\begin{array}{r}3.810 \\
(96.800) \\
\end{array}$ \\
\hline DELTA $P \wedge(1 / 2)$ & & "H2O & 1.994 & 1.805 & 1.949 \\
\hline Ts & STACK TEMPERATURE & $\begin{array}{l}\text { DEG. F } \\
\text { (DEG. C) }\end{array}$ & $\begin{array}{r}223 \\
(106) \\
\end{array}$ & $\begin{array}{c}220 \\
(104) \\
\end{array}$ & $\begin{array}{c}228 \\
(109) \\
\end{array}$ \\
\hline Ps & STACK PRESSURE & $\begin{array}{l}\text { "Hg Abs. } \\
\text { (mm Hg) } \\
\text { "H2O }\end{array}$ & $\begin{array}{r}26.90 \\
(683.00) \\
-2.05 \\
\end{array}$ & $\begin{array}{r}26.94 \\
(684.00) \\
-1.45 \\
\end{array}$ & $\begin{array}{c}26.88 \\
(683.00) \\
-1.50 \\
\end{array}$ \\
\hline Vs & $\begin{array}{l}\text { STACK VELOCITY @ STACK } \\
\text { CONDITIONS }\end{array}$ & $\begin{array}{l}\text { FPM } \\
\text { (m/SEC.) }\end{array}$ & $\begin{array}{r}7,975 \\
(41) \\
\end{array}$ & $\begin{array}{r}7,173 \\
(36) \\
\end{array}$ & $\begin{array}{r}7,797 \\
(40) \\
\end{array}$ \\
\hline As & STACK AREA & $\begin{array}{l}\text { (SQ.INCHES) } \\
\text { (SQ.METERS) }\end{array}$ & $\begin{array}{r}9,363 \\
(6) \\
\end{array}$ & $\begin{array}{r}9,363 \\
(6) \\
\end{array}$ & $\begin{array}{r}9,363 \\
(6) \\
\end{array}$ \\
\hline Qs & $\begin{array}{l}\text { DRY STACK GAS VOLUME @ } \\
\text { STANDARD CONDITIONS* }\end{array}$ & $\begin{array}{l}\text { DSCFM } \\
\text { (DSCM/HR) }\end{array}$ & $\begin{array}{c}306,890 \\
(521,406)\end{array}$ & $\begin{array}{c}279,393 \\
(474,689)\end{array}$ & $\begin{array}{r}300,200 \\
(510,040)\end{array}$ \\
\hline Qa & $\begin{array}{l}\text { ACTUAL STACK GAS VOLUME } \\
\text { @ STACK CONDITIONS }\end{array}$ & $\begin{array}{l}\text { ACFM } \\
\left(m^{\wedge} 3 / \mathrm{HR}\right)\end{array}$ & $\begin{array}{r}518,556 \\
(881,027) \\
\end{array}$ & $\begin{array}{r}466,400 \\
(792,414) \\
\end{array}$ & $\begin{array}{r}506,950 \\
(861,308) \\
\end{array}$ \\
\hline$\overline{D n}$ & SAMPLING NOZZLE DIAM. & $\begin{array}{l}\text { IN. } \\
(\mathrm{m})\end{array}$ & $\begin{array}{r}0.154 \\
(0.004) \\
\end{array}$ & $\begin{array}{r}0.154 \\
(0.004) \\
\end{array}$ & $\begin{array}{c}0.154 \\
(0.004) \\
\end{array}$ \\
\hline$\% 1$ & PERCENT ISOKINETIC & $\%$ & 101.0 & 98.6 & 99.0 \\
\hline
\end{tabular}

*68 Deg.F, 29.92 "Hg (20 Deg.C, 760 mm Hg) 


\section{SOURCE EMISSION CALCULATIONS}

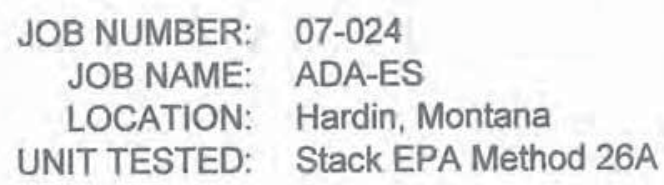

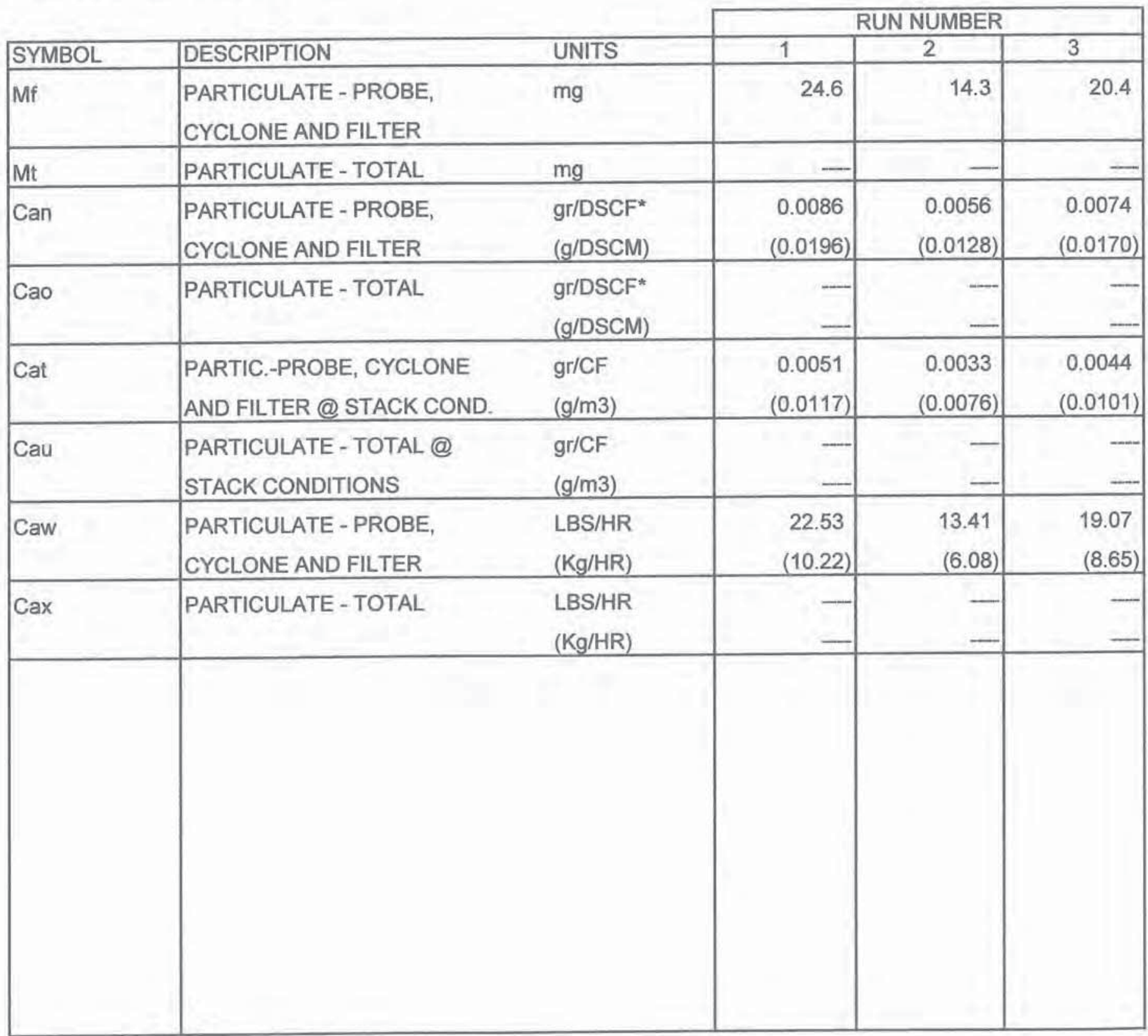

* 68 Deg.F, 29.92 "Hg (20 Deg.C, 760 mm Hg) 
SOURCE EMISSION SURVEY

JOB NUMBER: 07-024

JOB NAME: ADA-ES

LOCATION: Hardin, Montana

UNIT TESTED: Stack Ontario-Hydro

SOURCE EMISSION CALCULATIONS

\begin{tabular}{|c|c|c|c|c|c|}
\hline \multirow{2}{*}{ SYMBOL } & \multirow[b]{2}{*}{ DESCRIPTION } & \multirow[b]{2}{*}{ UNITS } & \multicolumn{3}{|c|}{ RUN NUMBER } \\
\hline & & & 1 & 2 & 3 \\
\hline DATE & & & $04 / 26 / 07$ & $04 / 26 / 07$ & $04 / 26 / 07$ \\
\hline BEGIN TIME & & & 1020 & 1445 & 1810 \\
\hline END TIME & & & 1305 & 1650 & 2015 \\
\hline$P(b)$ & BAROMETRIC PRESSURE & $\begin{array}{l}\text { "Hg Abs. } \\
(\mathrm{mm} \mathrm{Hg})\end{array}$ & $\begin{array}{r}26.96 \\
(685.00) \\
\end{array}$ & $\begin{array}{r}26.93 \\
(684.00) \\
\end{array}$ & $\begin{array}{r}26.93 \\
(684.00) \\
\end{array}$ \\
\hline$P(m)$ & ORIFICE PRESSURE DROP & $\begin{array}{l}\text { " } \mathrm{H} 2 \mathrm{O} \\
(\mathrm{mm} \mathrm{H} 2 \mathrm{O})\end{array}$ & $\begin{array}{r}1.286 \\
(32.700) \\
\end{array}$ & $\begin{array}{r}1.343 \\
(34.100) \\
\end{array}$ & $\begin{array}{r}1.369 \\
(34.800) \\
\end{array}$ \\
\hline & DGM CALIBRATION FACTOR & & 0.998 & 0.998 & 0.998 \\
\hline$V(m)$ & $\begin{array}{l}\text { VOLUME DRY GAS SAMPLED } \\
\text { @ METER CONDITIONS } \\
\text { LEAK CHECK VOLUME }\end{array}$ & $\begin{array}{l}\text { ft.^3 } \\
\left(m^{\wedge} 3\right) \\
\text { ft.^3 }\end{array}$ & $\begin{array}{c}79.415 \\
(2.249) \\
0.000 \\
\end{array}$ & $\begin{array}{c}81.555 \\
(2.309) \\
0.000 \\
\end{array}$ & $\begin{array}{c}83.098 \\
(2.353) \\
0.000 \\
\end{array}$ \\
\hline$T(m)$ & $\begin{array}{l}\text { AVERAGE GAS METER } \\
\text { TEMPERATURE }\end{array}$ & $\begin{array}{l}\text { DEG.F } \\
\text { (DEG.C) }\end{array}$ & $\begin{array}{r}72 \\
(22) \\
\end{array}$ & $\begin{array}{r}80 \\
(27) \\
\end{array}$ & $\begin{array}{r}80 \\
(27) \\
\end{array}$ \\
\hline$V(m[s t d])^{*}$ & $\begin{array}{l}\text { VOLUME DRY GAS SAMPLED } \\
\text { @ STANDARD CONDITIONS* }\end{array}$ & $\begin{array}{l}\text { DSCF } \\
\text { (DSCM) }\end{array}$ & $\begin{array}{l}71.281 \\
(2.018) \\
\end{array}$ & $\begin{array}{l}72.049 \\
(2.040) \\
\end{array}$ & $\begin{array}{l}73.417 \\
(2.079) \\
\end{array}$ \\
\hline$V(w)$ & $\begin{array}{l}\text { TOTAL WATER COLLECTED, } \\
\text { IMIPINGERS \& SILICA GEL }\end{array}$ & $\mathrm{ml}$ & 270.5 & 277.4 & 275.8 \\
\hline$V($ w[gas] $)$ & $\begin{array}{l}\text { VOLUME WATER VAPOR } \\
\text { COLLECTED @ STANDARD } \\
\text { CONDITIONS* }\end{array}$ & $\begin{array}{l}\text { SCF } \\
\text { (SCM) }\end{array}$ & $\begin{array}{l}12.768 \\
(0.362)\end{array}$ & $\begin{array}{l}13.093 \\
(0.371)\end{array}$ & $\begin{array}{l}13.018 \\
(0.369)\end{array}$ \\
\hline$\% \mathrm{M}$ & $\begin{array}{l}\text { MOISTURE IN STACK GAS } \\
\text { BY VOLUME }\end{array}$ & $\%$ & 15.19 & 15.38 & 15.06 \\
\hline Md & MOL FRACTION OF DRY GAS & & 0.8481 & 0.8462 & 0.8494 \\
\hline $\mathrm{Tt}$ & NET TIME OF TEST & MIINUTES & 120 & 120 & 120 \\
\hline
\end{tabular}

*68 Deg.F, 29.92 "Hg (20 Deg.C, $760 \mathrm{~mm} \mathrm{Hg}$ ) 


\section{SOURCE EMISSION CALCULATIONS}

JOB NUMBER: $\quad$ 07-024

JOB NAME: ADA-ES

LOCATION: Hardin, Montana

UNIT TESTED: Stack Ontario-Hydro

\begin{tabular}{|c|c|c|c|c|c|}
\hline \multirow{2}{*}{ SYMBOL } & \multirow[b]{2}{*}{ DESCRIPTION } & \multirow[b]{2}{*}{ UNITS } & \multicolumn{3}{|c|}{ RUN NUMBER } \\
\hline & & & 1 & 2 & 3 \\
\hline $\mathrm{CO} 2$ & & $\%$ & 12.7 & 12.6 & 12.7 \\
\hline $\mathrm{O} 2$ & & $\%$ & 6.8 & 6.9 & 6.8 \\
\hline $\mathrm{CO}$ & & $\%$ & 0.0 & 0.0 & 0.0 \\
\hline N2 & & $\%$ & 80.5 & 80.5 & 80.5 \\
\hline$\% \mathrm{EA}$ & $\begin{array}{l}\text { EXCESS AIR @ SAMPLING } \\
\text { POINT }\end{array}$ & $\%$ & 46.8 & 47.8 & 46.8 \\
\hline MWd & $\begin{array}{l}\text { MOLECULAR WEIGHT OF } \\
\text { DRY STACK GAS }\end{array}$ & $\begin{array}{l}\text { LB/LB-MOLE } \\
\text { (g/g-MOLE) }\end{array}$ & $\begin{array}{r}30.30 \\
(30.30)\end{array}$ & $\begin{array}{c}30.29 \\
(30.29) \\
\end{array}$ & $\begin{array}{r}30.30 \\
(30.30) \\
\end{array}$ \\
\hline MW & $\begin{array}{l}\text { MOLECULAR WEIGHT OF } \\
\text { STACK GAS }\end{array}$ & $\begin{array}{l}\text { LB/LB-MOLE } \\
\text { (g/g-MOLE) }\end{array}$ & $\begin{array}{c}28.44 \\
(28.44) \\
\end{array}$ & $\begin{array}{c}28.40 \\
(28.40) \\
\end{array}$ & $\begin{array}{r}28.45 \\
(28.45) \\
\end{array}$ \\
\hline $\mathrm{Cp}$ & PITOT TUBE CALIBRATION & & 0.826 & 0.824 & 0.826 \\
\hline DELTAP & $\begin{array}{l}\text { VELOCITY HEAD OF STACK } \\
\text { GAS }\end{array}$ & $\begin{array}{l}\text { "H2O } \\
(\mathrm{mm} \mathrm{H} 2 \mathrm{O}) \\
\end{array}$ & $\begin{array}{r}3.888 \\
(98.800) \\
\end{array}$ & $\begin{array}{r}4.098 \\
(104.100) \\
\end{array}$ & $\begin{array}{r}4.158 \\
(105.600) \\
\end{array}$ \\
\hline DELTAP $\wedge(1 / 2)$ & & "H2O & 1.967 & 2.022 & 2.038 \\
\hline Ts & STACK TEMPERATURE & $\begin{array}{l}\text { DEG. F } \\
\text { (DEG. C) }\end{array}$ & $\begin{array}{c}214 \\
(101) \\
\end{array}$ & $\begin{array}{c}215 \\
(102) \\
\end{array}$ & $\begin{array}{r}216 \\
(102) \\
\end{array}$ \\
\hline Ps & STACK PRESSURE & $\begin{array}{l}\text { "Hg Abs. } \\
(\mathrm{mm} \mathrm{Hg}) \\
\text { "H2O }\end{array}$ & $\begin{array}{r}26.85 \\
(682.00) \\
-1.45 \\
\end{array}$ & $\begin{array}{r}26.78 \\
(680.00) \\
-2.05 \\
\end{array}$ & $\begin{array}{r}26.82 \\
(681.00) \\
-1.50 \\
\end{array}$ \\
\hline Vs & $\begin{array}{l}\text { STACK VELOCITY @ STACK } \\
\text { CONDITIONS }\end{array}$ & $\begin{array}{l}\text { FPM } \\
\text { (m/SEC.) }\end{array}$ & $\begin{array}{r}7,821 \\
(40) \\
\end{array}$ & $\begin{array}{r}8,042 \\
(41) \\
\end{array}$ & $\begin{array}{r}8,119 \\
(41) \\
\end{array}$ \\
\hline As & STACK AREA & $\begin{array}{l}\text { (SQ.INCHES) } \\
\text { (SQ.METERS) }\end{array}$ & $\begin{array}{r}9,363 \\
(6) \\
\end{array}$ & $\begin{array}{r}9,363 \\
(6) \\
\end{array}$ & $\begin{array}{r}9,363 \\
(6) \\
\end{array}$ \\
\hline Qs & $\begin{array}{l}\text { DRY STACK GAS VOLUME @ } \\
\text { STANDARD CONDITIONS* }\end{array}$ & $\begin{array}{l}\text { DSCFM } \\
\text { (DSCM/HR) }\end{array}$ & $\begin{array}{c}304,308 \\
(517,019)\end{array}$ & $\begin{array}{c}310,931 \\
(528,272)\end{array}$ & $\begin{array}{r}315,099 \\
(535,353)\end{array}$ \\
\hline $\mathrm{Qa}$ & $\begin{array}{l}\text { ACTUAL STACK GAS VOLUME } \\
@ \text { STACK CONDITIONS }\end{array}$ & $\begin{array}{l}\text { ACFM } \\
\left(m^{\wedge} 3 / H R\right) \\
\end{array}$ & $\begin{array}{r}508,536 \\
(864,003) \\
\end{array}$ & $\begin{array}{r}522,926 \\
(888,451) \\
\end{array}$ & $\begin{array}{r}527,876 \\
(896,861) \\
\end{array}$ \\
\hline$D n$ & SAMPLING NOZZLE DIAM. & $\begin{array}{l}\text { IN. } \\
(\mathrm{m})\end{array}$ & $\begin{array}{r}0.154 \\
(0.004) \\
\end{array}$ & $\begin{array}{c}0.154 \\
(0.004) \\
\end{array}$ & $\begin{array}{r}0.154 \\
(0.004) \\
\end{array}$ \\
\hline$\% 1$ & PERCENT ISOKINETIC & $\%$ & 98.5 & 97.4 & 98.0 \\
\hline
\end{tabular}

*68 Deg.F, 29.92 "Hg (20 Deg.C, 760 mm Hg) 


\section{SOURCE EMISSION CALCULATIONS}

JOB NUMBER: 07-024

JOB NAME: ADA-ES

LOCATION: Hardin, Montana

UNIT TESTED: Stack Ontario-Hydro

\begin{tabular}{|c|c|c|c|c|c|}
\hline & & & & NUMBER & \\
\hline SYMBOL & DESCRIPTION & UNITS & 1 & 2 & 3 \\
\hline Mf & PARTICULATE - PROBE, & $\mathrm{mg}$ & 43.6 & 33.1 & 73.2 \\
\hline & CYCLONE AND FILTER & & & & \\
\hline Mit & PARTICULATE - TOTAL & $\mathrm{mg}$ & - & - & - \\
\hline Can & PARTICULATE - PROBE, & gr/DSCF* & 0.0094 & 0.0071 & 0.0154 \\
\hline & CYCLONE AND FILTER & (g/DSCM) & $(0.0216)$ & $(0.0162)$ & $(0.0352)$ \\
\hline Cao & PARTICULATE - TOTAL & gr/DSCF* & - & - & - \\
\hline & & (g/DSCM) & - & - & $=$ \\
\hline Cat & PARTIC.-PROBE, CYCLONE & $\mathrm{gr} / \mathrm{CF}$ & 0.0056 & 0.0042 & 0.0091 \\
\hline & AND FILTER @ STACK COND. & $(\mathrm{g} / \mathrm{m} 3)$ & $(0.0128)$ & $(0.0096)$ & $(0.0208)$ \\
\hline Cau & PARTICULATE - TOTAL @ & $\mathrm{gr} / \mathrm{CF}$ & - & - & - \\
\hline & STACK CONDITIONS & $(\mathrm{g} / \mathrm{m} 3)$ & - & - & - \\
\hline Caw & PARTICULATE - PROBE, & LBS/HR & 24.57 & 18.85 & 41.46 \\
\hline & CYCLONE AND FILTER & $(\mathrm{Kg} / \mathrm{HR})$ & $(11.14)$ & $(8.55)$ & $(18.81)$ \\
\hline Cax & PARTICULATE - TOTAL & LBS/HR & - & 一 & 一 \\
\hline & & $(\mathrm{Kg} / \mathrm{HR})$ & - & - & - \\
\hline & & & & & \\
\hline & & & & & \\
\hline & & & & & \\
\hline & & & & & \\
\hline & & & & & \\
\hline & & & & & \\
\hline & & & & & \\
\hline
\end{tabular}

* 68 Deg.F, 29.92 "Hg (20 Deg.C, 760 mm Hg) 
Appendix C:

Calibration Data 


\section{Appendix C}

\section{Table 8: Calibration Data}

Pre-Test Calibrations:

\section{Equipment}

Dry Gas Meter 1-1

Digital Temperature Indicator 1-1

Dry Gas Meter 1-1 Orifice

Dry Gas Meter 1-2

Digital Temperature Indicator 1-2

Dry Gas Meter 1-2 Orifice

Pitot Tube 2-1

Pitot Tube 2-2

Pitot Tube 2-3

Pitot Tube 2-4

Nozzle 5-1

Nozzle 6-2

Nozzle 7-2

\section{Calibration Factor $\quad \underline{\text { Calibration Date }}$}

0.998

04/03/07

$04 / 03 / 07$

04/03/07

1.004

04/03/07

04/03/07

04/03/07

0.826

03/29/07

0.824

04/03/07

0.824

01/02/07

0.822

04/02/07

0.154

04/04/07

0.191

04/04/07

0.193

04/04/07 
Post-Test Calibrations:

\section{Equipment}

Dry Gas Meter 1-1

Digital Temperature Indicator 1-1

Dry Gas Meter 1-2

Digital Temperature Indicator 1-2

Pitot Tube 2-1

Pitot Tube 2-2

Pitot Tube 2-3

Pitot Tube 2-4

Nozzle 5-1

Nozzle 6-2
Calibration Factor

0.985

0.991

0.822

0.823

0.823

0.824

0.154

0.191

\section{Calibration Date}

05/18/07

$05 / 18 / 07$

05/18/07

05/18/07

05/21/07

05/21/07

$05 / 21 / 07$

05/21/07

05/18/07

05/18/07 
Calibration Data

Dry Gas Meter Calibration

\begin{tabular}{rc} 
Meter Console No. & ASAI 1-1 \\
\cline { 2 - 2 } Date Calibration Performed: & $04 / 03 / 07$ \\
\end{tabular}

$\Delta \mathrm{H}$ Setting

\begin{tabular}{c}
$\left(\right.$ " $\left.\mathrm{H}_{2} \mathrm{O}\right)$ \\
\hline 0.50 \\
1.00 \\
1.50 \\
2.00 \\
3.00 \\
4.00 \\
Average
\end{tabular}

\begin{tabular}{c}
$\mathrm{C}_{\mathrm{DG}}$ \\
\hline 1.002 \\
\hline 1.000 \\
\hline 1.002 \\
\hline 1.000 \\
\hline 0.992 \\
\hline 0.991 \\
\hline 0.998 \\
\hline
\end{tabular}

Variation +: $0.40 \%$ Variation -: $-0.70 \%$

Certified by: Scot Jachsen 04/03/07

Calibrator (Signature / Date) Bill Hefley 04/03/07 QA Officer (Signature / Date) 
Calibration Data

Dry Gas Meter Calibration

Meter Console No.

ASAI 1-1

Date Calibration Performed:

04/03/07

$\begin{array}{rlrl}\text { Run } \frac{1}{\mathrm{~Pb}} & =29.61 \\ \text { Wet Test Meter } \mathrm{C}_{\mathrm{f}}=1.020 & \mathrm{Hg}\end{array}$

Wet Test Meter No. ASAI-0

Dry Gas Meter Meter

Meter

Temperature

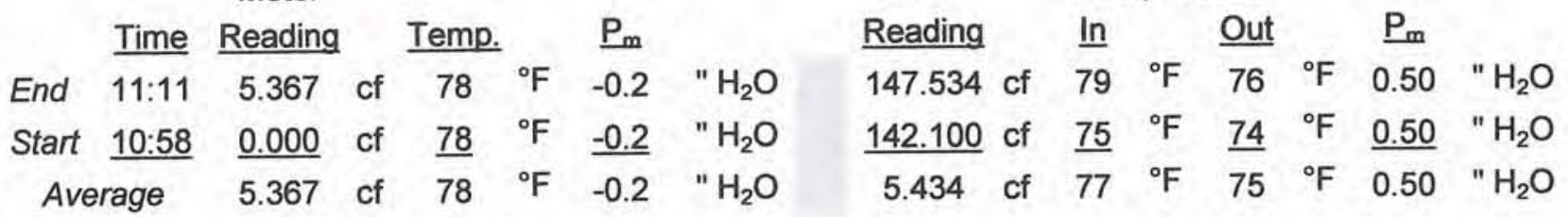

Wet Test Meter $V_{m s d}=17.65 \times V_{m}\left[\frac{P_{b}+\frac{P_{m}}{13.6}}{\left(T_{m}+460\right)}\right] x C_{f}=\underline{5.315} \mathrm{dcsf}$
Dry Gas Meter $V_{m s d}=17.65 \times \mathrm{Vm}\left[\frac{P_{b}+\frac{P_{m}}{13.6}}{\left(T_{m}+460\right)}\right]=\underline{5.305} \mathrm{dcsf}$ Calibration Factor $\left(C_{D G}\right)=\frac{\text { Wet Test Meter } V_{\text {mstd }}}{\text { Dry Gas Meter } V_{\text {mst }}}=\underline{1.002}$ 


\author{
Calibration Data \\ Dry Gas Meter Calibration
}

Meter Console No.

ASAI 1-1

Date Calibration Performed:

04/03/07

$\begin{aligned} \text { Run } \frac{1}{\mathrm{~Pb}} & =29.61 \mathrm{Hg} \\ \text { Wet Test Meter } \mathrm{C}_{\mathrm{f}}=1.020 \text { Console Pump Vacuum } & =-5.0^{\mathrm{H}} \mathrm{Hg}\end{aligned}$

Wet Test Meter No. ASAl-O

Dry Gas Meter Meter Meter Temperature

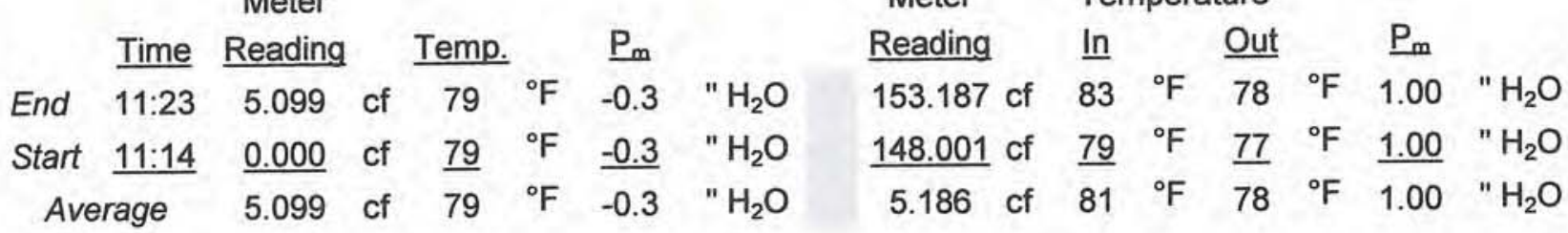

Wet Test Meter $V_{\text {msd }}=17.65 \times V_{m}\left[\frac{P_{b}+\frac{P_{m}}{13.6}}{\left(T_{m}+460\right)}\right] x C_{f}=\underline{5.039} \mathrm{dcsf}$ Dry Gas Meter $V_{m s d}=17.65 \times \mathrm{Vm}\left[\frac{P_{b}+\frac{P_{m}}{13.6}}{\left(T_{m}+460\right)}\right]=\underline{5.039} \mathrm{dcsf}$ Calibration Factor $(C D G)=\frac{\text { Wet Test Meter } V_{\text {std }}}{\text { Dry Gas Meter } V_{\text {std }}}=1.000$ 


\author{
Calibration Data \\ Dry Gas Meter Calibration
}

Meter Console No.

ASAI 1-1

Date Calibration Performed:

04/03/07

Run 1 at $1.5^{\prime \prime} \Delta \mathrm{H}$

Wet Test Meter $C_{f}=1.020$

$\mathrm{Pb}=29.61 " \mathrm{Hg}$

Console Pump Vacuum $=-5.0 \mathrm{Hg}$

Wet Test Meter No. ASAl-0 Meter

Meter

Dry Gas Meter

\begin{tabular}{|c|c|c|c|c|c|c|c|c|c|c|c|c|c|c|}
\hline \multirow[b]{3}{*}{ End } & \multicolumn{3}{|c|}{ Meter } & \multirow[b]{2}{*}{ Temp. } & \multirow{2}{*}{\multicolumn{2}{|c|}{$\underline{P_{m}}$}} & & \multirow{2}{*}{$\begin{array}{c}\text { Meter } \\
\text { Reading }\end{array}$} & \multicolumn{3}{|c|}{ Temperature } & \multirow{2}{*}{\multicolumn{2}{|c|}{$\underline{P_{m}}$}} & \multirow[b]{3}{*}{$" \mathrm{H}_{2} \mathrm{O}$} \\
\hline & Time & Reading & & & & & & & In & & Out & & & \\
\hline & $11: 41$ & 10.248 & cf & 79 & ${ }^{\circ} \mathrm{F}$ & -0.5 & " $\mathrm{H}_{2} \mathrm{O}$ & $164.136 \mathrm{cf}$ & 87 & ${ }^{\circ} \mathrm{F}$ & 80 & ${ }^{\circ} \mathrm{F}$ & 1.50 & \\
\hline Start & 11:26 & $\underline{0.000}$ & cf & $\underline{79}$ & ${ }^{\circ} \mathrm{F}$ & $\underline{-0.5}$ & $" \mathrm{H}_{2} \mathrm{O}$ & 153.704 cf & $\underline{81}$ & ${ }^{\circ} \mathrm{F}$ & $\underline{78}$ & ${ }^{\circ} \mathrm{F}$ & $\underline{1.50}$ & $" \mathrm{H}_{2} \mathrm{O}$ \\
\hline Ave & rage & 10.248 & cf & 79 & ${ }^{\circ} \mathrm{F}$ & -0.5 & " $\mathrm{H}_{2} \mathrm{O}$ & $10.432 \mathrm{cf}$ & 84 & ${ }^{\circ} \mathrm{F}$ & 79 & ${ }^{\circ} \mathrm{F}$ & 1.50 & $" \mathrm{H}_{2} \mathrm{O}$ \\
\hline
\end{tabular}

Wet Test Meter $V_{m s d}=17.65 \times V_{m}\left[\frac{P_{b}+\frac{P_{m}}{13.6}}{\left(T_{m}+460\right)}\right] \times C_{f}=\underline{10.123} \mathrm{dcsf}$
Dry Gas Meter Vmsd $=17.65 \times \mathrm{Vm}\left[\frac{P_{b}+\frac{P_{m}}{13.6}}{\left(T_{m}+460\right)}\right]=\underline{10.106} \mathrm{dcsf}$ Calibration Factor $(C D G)=\frac{\text { Wet Test Meter } V_{m_{s t d}}}{D r y \text { Gas Meter } V_{m_{s t d}}}=1.002$ 


\author{
Calibration Data \\ Dry Gas Meter Calibration
}

Meter Console No.

Date Calibration Performed:

ASAI 1-1

04/03/07

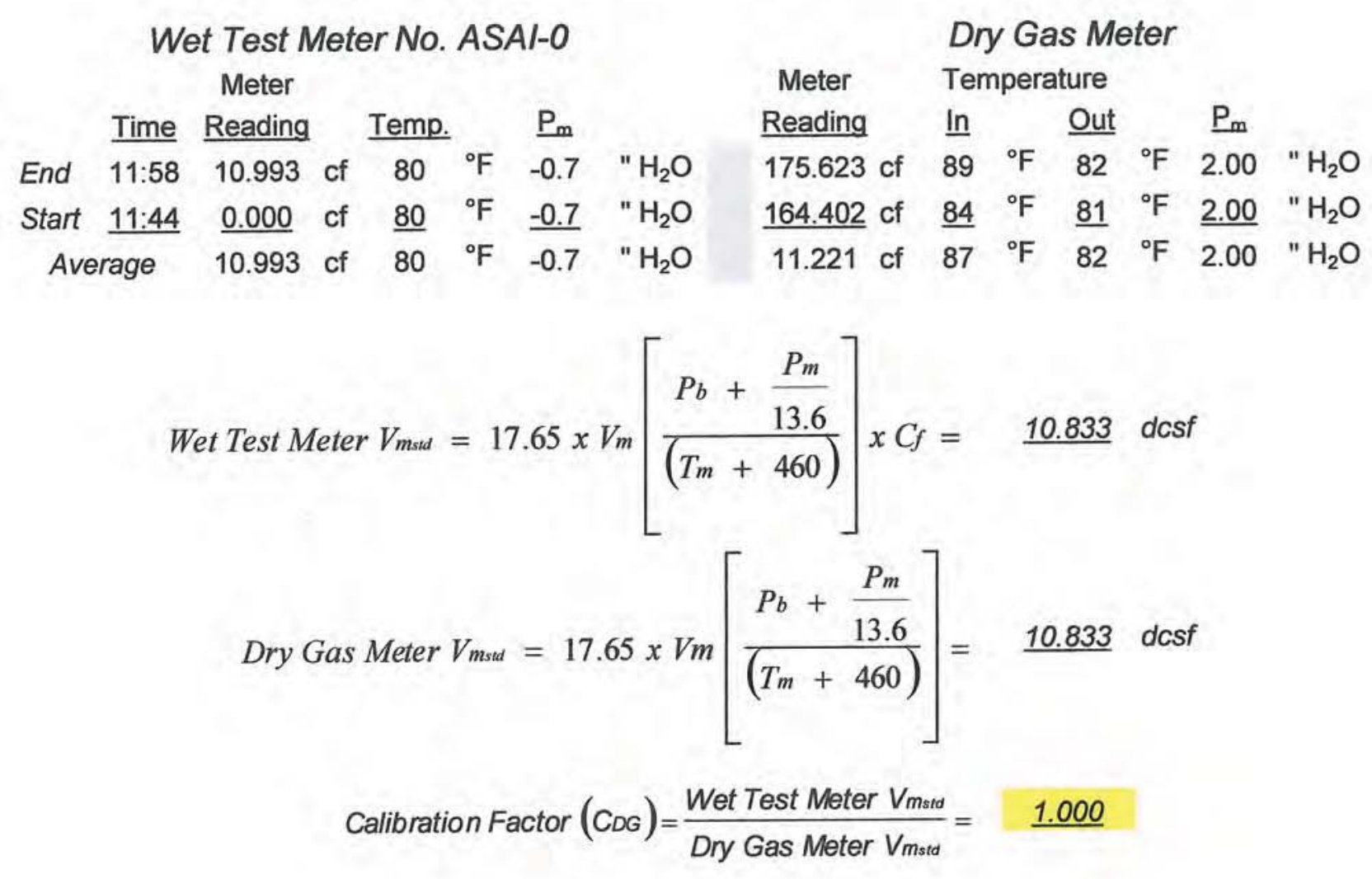


Calibration Data

Dry Gas Meter Calibration

Meter Console No.

Date Calibration Performed:
ASAI 1-1

04/03/07

$$
\begin{array}{rlrl}
\text { Run } \frac{1}{\mathrm{~Pb}} & =29.61 \\
\text { Wet Test Meter } \mathrm{C}_{\mathrm{f}}=1.020 \quad \mathrm{Hg} & \text { Console Pump Vacuum } & =-5.0 \mathrm{Hg}
\end{array}
$$

Wet Test Meter No. ASAl-O Meter

Dry Gas Meter Meter Temperature

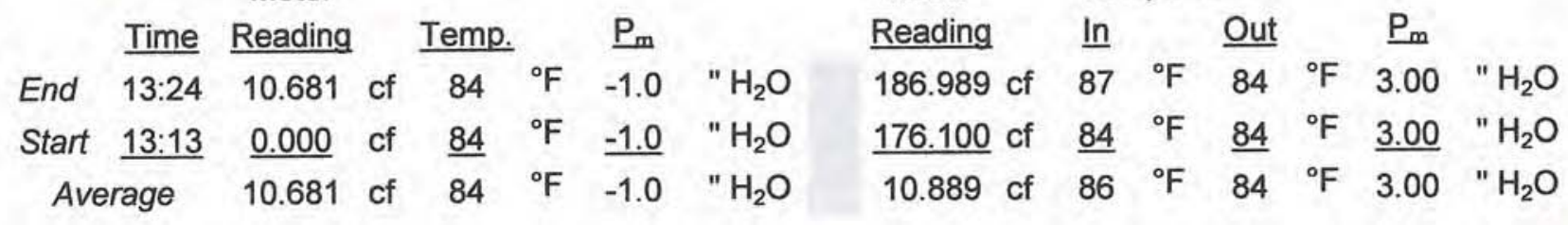

$$
\text { Calibration Factor }(C D G)=\frac{\text { Wet Test Meter } V_{\text {mstd }}}{\text { Dry Gas Meter } V_{m s t d}}=\underline{0.992}
$$




\title{
TIm
}

\author{
Calibration Data \\ Dry Gas Meter Calibration
}

\author{
Meter Console No. ASAI 1-1 \\ Date Calibration Performed: \\ 04/03/07
}

$\begin{aligned} \text { Run } \frac{1}{\mathrm{~Pb}} & =29.61 \\ \text { Wet Test Meter } \mathrm{C}_{\mathrm{f}}=1.020 \quad \mathrm{Hg} & \end{aligned}$

Wet Test Meter No. ASAI-0 Meter

Dry Gas Meter

\begin{tabular}{|c|c|c|c|c|c|c|c|c|c|c|c|c|c|}
\hline & & Meter & & & & & Meter & Ten & per & ure & & & \\
\hline & Time & Reading & Temp. & & $\underline{P_{m}}$ & & Reading & In & & Out & & $\underline{P_{m}}$ & \\
\hline End & $13: 39$ & $11.105 \mathrm{cf}$ & 84 & ${ }^{\circ} \mathrm{F}$ & -1.3 & $" \mathrm{H}_{2} \mathrm{O}$ & 199.982 cf & 91 & ${ }^{\circ} \mathrm{F}$ & 85 & ${ }^{\circ} \mathrm{F}$ & 4.00 & $" \mathrm{H}_{2} \mathrm{O}$ \\
\hline Start & $13: 29$ & $\underline{0.000} \mathrm{cf}$ & 84 & ${ }^{\circ} \mathrm{F}$ & -1.3 & " $\mathrm{H}_{2} \mathrm{O}$ & $\underline{188.648 \mathrm{cf}}$ & $\underline{87}$ & ${ }^{\circ} \mathrm{F}$ & 84 & ${ }^{\circ} \mathrm{F}$ & $\underline{4.00}$ & " $\mathrm{H}_{2} \mathrm{O}$ \\
\hline Ave & ge & 11.105 cf & 84 & ${ }^{\circ} \mathrm{F}$ & -1.3 & $" \mathrm{H}_{2} \mathrm{O}$ & 11.334 cf & 89 & ${ }^{\circ} \mathrm{F}$ & 85 & ${ }^{\circ} \mathrm{F}$ & 4.00 & $" \mathrm{H}_{2} \mathrm{O}$ \\
\hline
\end{tabular}

Wet Test Meter $V_{m s d}=17.65 \times V_{m}\left[\frac{P_{b}+\frac{P_{m}}{13.6}}{\left(T_{m}+460\right)}\right] x C_{f}=\quad \underline{10.847} \mathrm{dcsf}$

Dry Gas Meter $V_{m s d}=17.65 \times \mathrm{Vm}\left[\frac{P_{b}+\frac{P_{m}}{13.6}}{\left(T_{m}+460\right)}\right]=\underline{10.941} \mathrm{dcsf}$

Calibration Factor $\left(C_{D G}\right)=\frac{\text { Wet Test Meter } V_{m s t d}}{\text { Dry Gas Meter } V_{m s t d}}=\underline{0.991}$ 


Digital Temperature Indicator Calibration
$\begin{aligned} & \text { DTI Unit No. } \\ & \text { Date Calibration Performed: }\end{aligned}$

Meter Adjusted?

No

ASTM Reference Thermometer:

SN: 06000047

Range: $\quad-0.1^{\circ} \mathrm{C}$ to $1360.6^{\circ} \mathrm{C}$

Range:

Range:

Certified by:

Scot Jachson 04/03/07

Calibrator (Signature / Date)

Bill Heffey 04/03/07

QA Officer (Signature / Date) 


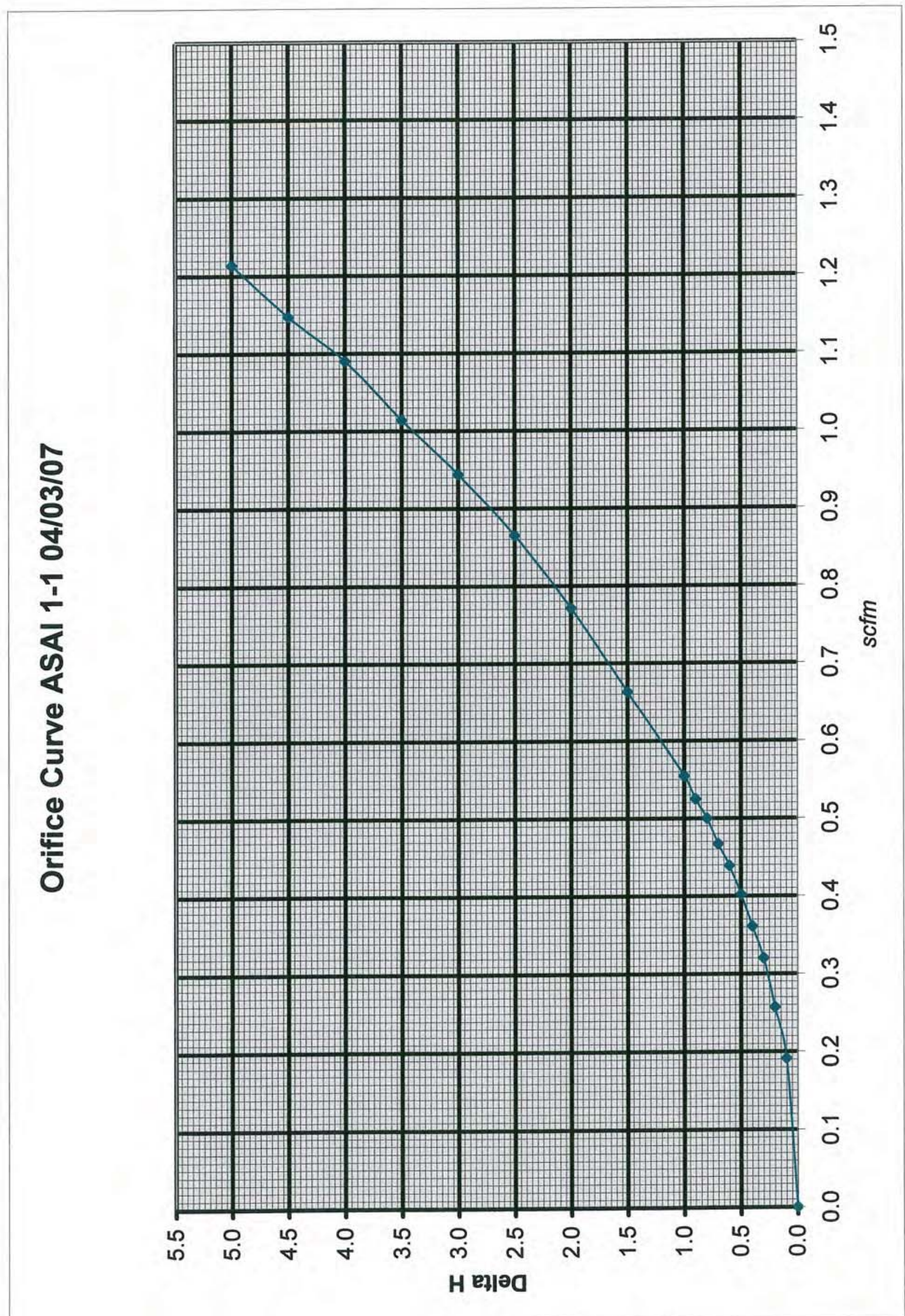

C-12 
Calibration Data

Dry Gas Meter Calibration

Meter Console No.

Date Calibration Performed:

\begin{tabular}{c} 
ASAI 1-2 \\
\hline $04 / 03 / 07$ \\
\hline
\end{tabular}

$\Delta \mathrm{H}$ Setting

\begin{tabular}{c}
$\left(\right.$ " $\left.\mathrm{H}_{2} \mathrm{O}\right)$ \\
\hline 0.50 \\
1.00 \\
1.50 \\
2.00 \\
3.00 \\
4.00 \\
Average
\end{tabular}

\begin{tabular}{c}
$\mathrm{C}_{\mathrm{DG}}$ \\
\hline 1.007 \\
\hline 1.008 \\
\hline 1.002 \\
\hline 1.001 \\
\hline 1.001 \\
\hline 1.002 \\
\hline 1.004 \\
\hline
\end{tabular}

Variation +:

Variation -:

$0.40 \%$ $-0.30 \%$

Certified by: Scot Jackson 4/03/07 Calibrator (Signature / Date) Bill Hefley 4/03/07 QA Officer (Signature / Date) 


\author{
Calibration Data \\ Dry Gas Meter Calibration
}

Meter Console No.

Date Calibration Performed:

ASAI 1-2

04/03/07

$$
\begin{aligned}
\text { Run } \frac{1}{\mathrm{~Pb}} & =29.56 \mathrm{Hg} \\
\text { Wet Test Meter } \mathrm{C}_{\mathrm{f}}=1.020 \quad \text { Console Pump Vacuum } & =-5.0 \mathrm{Hg}
\end{aligned}
$$

Wet Test Meter No. ASAI-O

\begin{tabular}{|c|c|c|c|c|c|c|c|}
\hline & Time & Reading & & Temp. & & $\underline{P_{m}}$ & \\
\hline End & $9: 02$ & 5.005 & $c f$ & 74 & ${ }^{\circ} \mathrm{F}$ & -0.2 & $" \mathrm{H}_{2} \mathrm{O}$ \\
\hline Start & $\underline{8: 50}$ & $\underline{0.000}$ & cf & $\underline{74}$ & ${ }^{\circ} \mathrm{F}$ & $\underline{-0.2}$ & $" \mathrm{H}_{2} \mathrm{O}$ \\
\hline & & 5.005 & cf & 74 & ${ }^{\circ} \mathrm{F}$ & -0.2 & $" \mathrm{H}_{2} \mathrm{O}$ \\
\hline
\end{tabular}
Meter

\section{Dry Gas Meter}

\begin{tabular}{|c|c|c|c|c|c|c|}
\hline eading & In & & Out & & $\underline{P_{m}}$ & \\
\hline $165.815 \mathrm{cf}$ & 81 & ${ }^{\circ} \mathrm{F}$ & 78 & ${ }^{\circ} \mathrm{F}$ & 0.50 & " $\mathrm{H}_{2} \mathrm{O}$ \\
\hline 30 & $\underline{8}$ & ${ }^{\circ} \mathrm{F}$ & 78 & $\mathrm{~F}$ & $\underline{0.50}$ & " $\mathrm{H}_{2} \mathrm{O}$ \\
\hline 113 & & ${ }^{\circ} \mathrm{F}$ & 78 & ${ }^{\circ} \mathrm{F}$ & 0.50 & $" \mathrm{H}_{2} \mathrm{O}$ \\
\hline
\end{tabular}

Meter Temperature

$$
\begin{gathered}
\text { Wet Test Meter } V_{m s d}=17.65 \times V_{m}\left[\frac{P_{b}+\frac{P_{m}}{13.6}}{\left(T_{m}+460\right)}\right] x C_{f}=\underline{4.985} d c s f \\
\text { Dry Gas Meter } V_{m s d}=17.65 \times \mathrm{Vm}\left[\frac{P_{b}+\frac{P_{m}}{13.6}}{\left(T_{m}+460\right)}\right]=\underline{4.951} \text { dcsf }
\end{gathered}
$$

$$
\text { Calibration Factor }\left(C_{D G}\right)=\frac{\text { Wet Test Meter } V_{\text {mstd }}}{\text { Dry Gas Meter } V_{\text {mstd }}}=1.007
$$




\author{
Calibration Data \\ Dry Gas Meter Calibration
}

Meter Console No.

ASAI 1-2

Date Calibration Performed:
Run 1 at 1.0" $\Delta \mathrm{H}$

Wet Test Meter $C_{f}=1.020$
$\mathrm{Pb}=29.56 " \mathrm{Hg}$ 


\author{
Calibration Data \\ Dry Gas Meter Calibration
}

Meter Console No.

ASAI 1-2

Date Calibration Performed:

04/03/07

$\begin{aligned} \text { Run } \frac{1}{\mathrm{~Pb}} & =29.57 \\ \text { Wet Test Meter } \mathrm{C}_{\mathrm{f}}=1.020 \quad \text { Console Pump Vacuum } & =-5.0 \mathrm{Hg}\end{aligned}$

Wet Test Meter No. ASAI-O Meter

\begin{tabular}{|c|c|c|c|c|c|c|}
\hline & Time & Reading & Temp. & & $\underline{P_{m}}$ & \\
\hline End & $9: 40$ & $10.523 \mathrm{cf}$ & 75 & ${ }^{\circ} \mathrm{F}$ & -0.6 & $" \mathrm{H}_{2} \mathrm{O}$ \\
\hline Start & $\underline{9: 25}$ & $\underline{0.000} \mathrm{cf}$ & $\underline{75}$ & ${ }^{\circ} \mathrm{F}$ & -0.6 & " $\mathrm{H}_{2} \mathrm{O}$ \\
\hline$A v$ & e & $10.523 \mathrm{cf}$ & 75 & ${ }^{\circ} \mathrm{F}$ & -0.6 & $" \mathrm{H}_{2} \mathrm{O}$ \\
\hline
\end{tabular}

Dry Gas Meter Meter Temperature

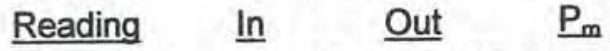
182.845 of $83 \quad{ }^{\circ} \mathrm{F} \quad 78 \quad{ }^{\circ} \mathrm{F} \quad 1.50 \quad " \mathrm{H}_{2} \mathrm{O}$ $\underline{172.100} \mathrm{cf} \quad \underline{78}{ }^{\circ} \mathrm{F} \quad \underline{78} \quad{ }^{\circ} \mathrm{F} \quad \underline{1.50} \quad " \mathrm{H}_{2} \mathrm{O}$ 10.745 of $81{ }^{\circ} \mathrm{F} \quad 78 \quad{ }^{\circ} \mathrm{F} \quad 1.50 \quad " \mathrm{H}_{2} \mathrm{O}$

$$
\begin{gathered}
\text { Wet Test Meter Vmsd }=17.65 \times V_{m}\left[\frac{P_{b}+\frac{P_{m}}{13.6}}{\left(T_{m}+460\right)}\right] \times C_{f}=\underline{10.455} \mathrm{dcsf} \\
\text { Dry Gas Meter } V_{m s d}=17.65 \times V_{m}\left[\frac{P_{b}+\frac{P_{m}}{13.6}}{\left(T_{m}+460\right)}\right]=\underline{10.438} \mathrm{dcsf} \\
\text { Calibration Factor }\left(C_{D G}\right)=\frac{\text { Wet Test Meter } V_{m s t d}}{\text { Dry Gas Meter } V_{m s t}}=\underline{1.002}
\end{gathered}
$$




\author{
Calibration Data \\ Dry Gas Meter Calibration
}

Meter Console No.

Date Calibration Performed:

ASAI 1-2

04/03/07

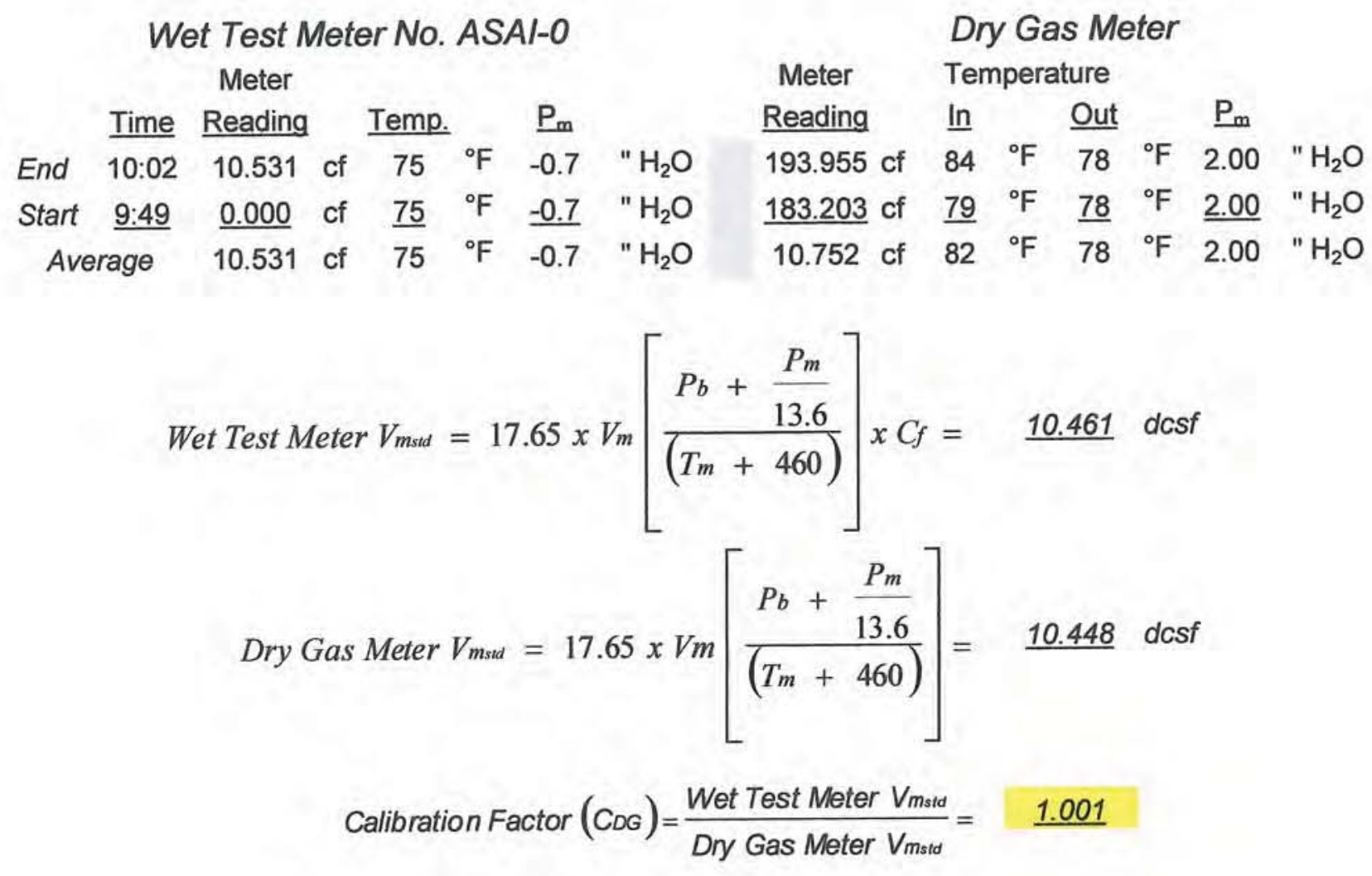




\author{
Calibration Data \\ Dry Gas Meter Calibration
}

Meter Console No.

Date Calibration Performed:

ASAI 1-2

04/03/07
Run 1 at 3.0" $\Delta \mathrm{H}$

Wet Test Meter $\mathrm{C}_{\mathrm{f}}=1.020$
$\mathrm{Pb}=29.57 " \mathrm{Hg}$

Console Pump Vacuum $=-5.0 \mathrm{Hg}$
Wet Test Meter No. ASAl-0 Meter

Time Reading Temp. $\quad \underline{\mathrm{P}_{\mathrm{m}}}$

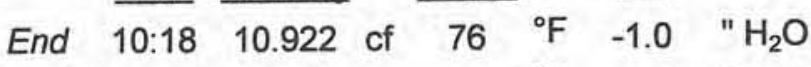

Start $\underline{10: 07} \underline{0.000}$ of $\underline{76} \quad{ }^{\circ} \mathrm{F} \quad \underline{-1.0} \quad " \mathrm{H}_{2} \mathrm{O}$

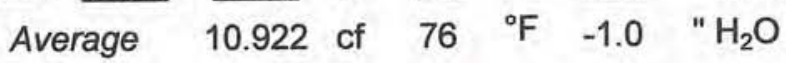

Dry Gas Meter

Meter Temperature

Reading In Out $\underline{P_{m}}$ 205.838 of $87 \quad{ }^{\circ} \mathrm{F} \quad 79 \quad{ }^{\circ} \mathrm{F} \quad 3.00 \quad " \mathrm{H}_{2} \mathrm{O}$ $\underline{194.706}$ of $\quad \underline{81}{ }^{\circ} \mathrm{F} \quad \underline{79}{ }^{\circ} \mathrm{F} \quad \underline{3.00} \quad " \mathrm{H}_{2} \mathrm{O}$ 11.132 of $84{ }^{\circ} \mathrm{F} \quad 79{ }^{\circ} \mathrm{F} \quad 3.00 \quad " \mathrm{H}_{2} \mathrm{O}$

$$
\begin{gathered}
\text { Wet Test Meter } V_{m s s}=17.65 \times V_{m}\left[\frac{P_{b}+\frac{P_{m}}{13.6}}{\left(T_{m}+460\right)}\right] x C_{f}=\underline{10.821} \mathrm{dcsf} \\
\text { Dry Gas Meter } V_{m s d}=17.65 \times V m\left[\frac{P_{b}+\frac{P_{m}}{13.6}}{\left(T_{m}+460\right)}\right]=\underline{10.809} \mathrm{dcsf} \\
\text { Calibration Factor }\left(C_{D G}\right)=\frac{\text { Wet Test Meter } V_{m s t d}}{D r y \text { Gas Meter } V_{m s t d}}=\underline{1.001}
\end{gathered}
$$




\author{
Calibration Data \\ Dry Gas Meter Calibration
}

Meter Console No.

Date Calibration Performed:
ASAI 1-2

04/03/07

$\begin{aligned} \text { Run } \frac{1}{\mathrm{~Pb}} & =29.58 \mathrm{Hg} \\ \text { Wet Test Meter } \mathrm{C}_{\mathrm{f}}=1.020 \quad \text { Console Pump Vacuum } & =-5.0 \mathrm{Hg}\end{aligned}$

Wet Test Meter No. ASAl-0 Meter

Dry Gas Meter

\begin{tabular}{|c|c|c|c|c|c|c|c|c|c|c|c|c|c|}
\hline & & Meter & & & & & Meter & Ten & ipera & ture & & & \\
\hline & Time & Reading & Temp. & & $\underline{P_{m}}$ & & $\underline{\text { Reading }}$ & $\underline{\ln }$ & & Out & & $\underline{P_{m}}$ & \\
\hline End & $10: 30$ & 10.229 cf & 76 & ${ }^{\circ} \mathrm{F}$ & -1.3 & $" \mathrm{H}_{2} \mathrm{O}$ & $216.832 \mathrm{cf}$ & 89 & ${ }^{\circ} \mathrm{F}$ & 80 & ${ }^{\circ} \mathrm{F}$ & 4.00 & $" \mathrm{H}_{2} \mathrm{O}$ \\
\hline Start & $10: 21$ & $\underline{0.000}$ cf & $\underline{76}$ & ${ }^{\circ} \mathrm{F}$ & -1.3 & $" \mathrm{H}_{2} \mathrm{O}$ & $\underline{206.408} \mathrm{cf}$ & $\underline{86}$ & ${ }^{\circ} \mathrm{F}$ & $\underline{79}$ & ${ }^{\circ} \mathrm{F}$ & $\underline{4.00}$ & $" \mathrm{H}_{2} \mathrm{O}$ \\
\hline Ave & & $10.229 \mathrm{cf}$ & 76 & ${ }^{\circ} \mathrm{F}$ & -1.3 & $" \mathrm{H}_{2} \mathrm{O}$ & 10.424 cf & 88 & ${ }^{\circ} \mathrm{F}$ & 80 & ${ }^{\circ} \mathrm{F}$ & 4.00 & " $\mathrm{H}_{2} \mathrm{O}$ \\
\hline
\end{tabular}

Wet Test Meter $V_{m s d}=17.65 \times V_{m}\left[\frac{P_{b}+\frac{P_{m}}{13.6}}{\left(T_{m}+460\right)}\right] \times C_{f}=\underline{10.130} \mathrm{dcsf}$
Dry Gas Meter $V_{m s d}=17.65 \times \mathrm{Vm}\left[\frac{P_{b}+\frac{P_{m}}{13.6}}{\left(T_{m}+460\right)}\right]=\underline{10.113} \mathrm{dcsf}$ Calibration Factor $(C D G)=\frac{\text { Wet Test Meter } V_{m s t d}}{\text { Dry Gas Meter } V_{m s t d}}=\underline{1.002}$ 


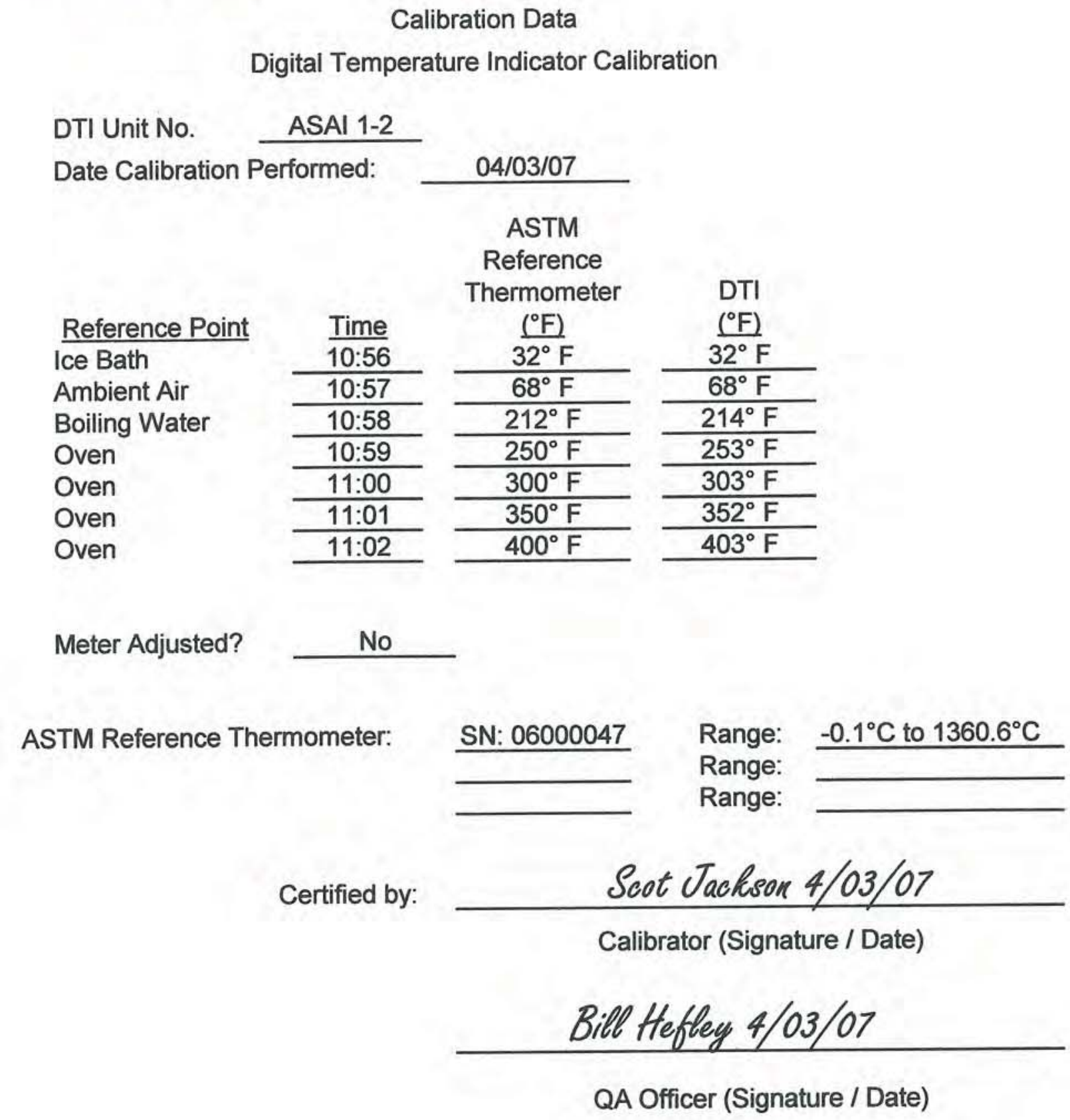




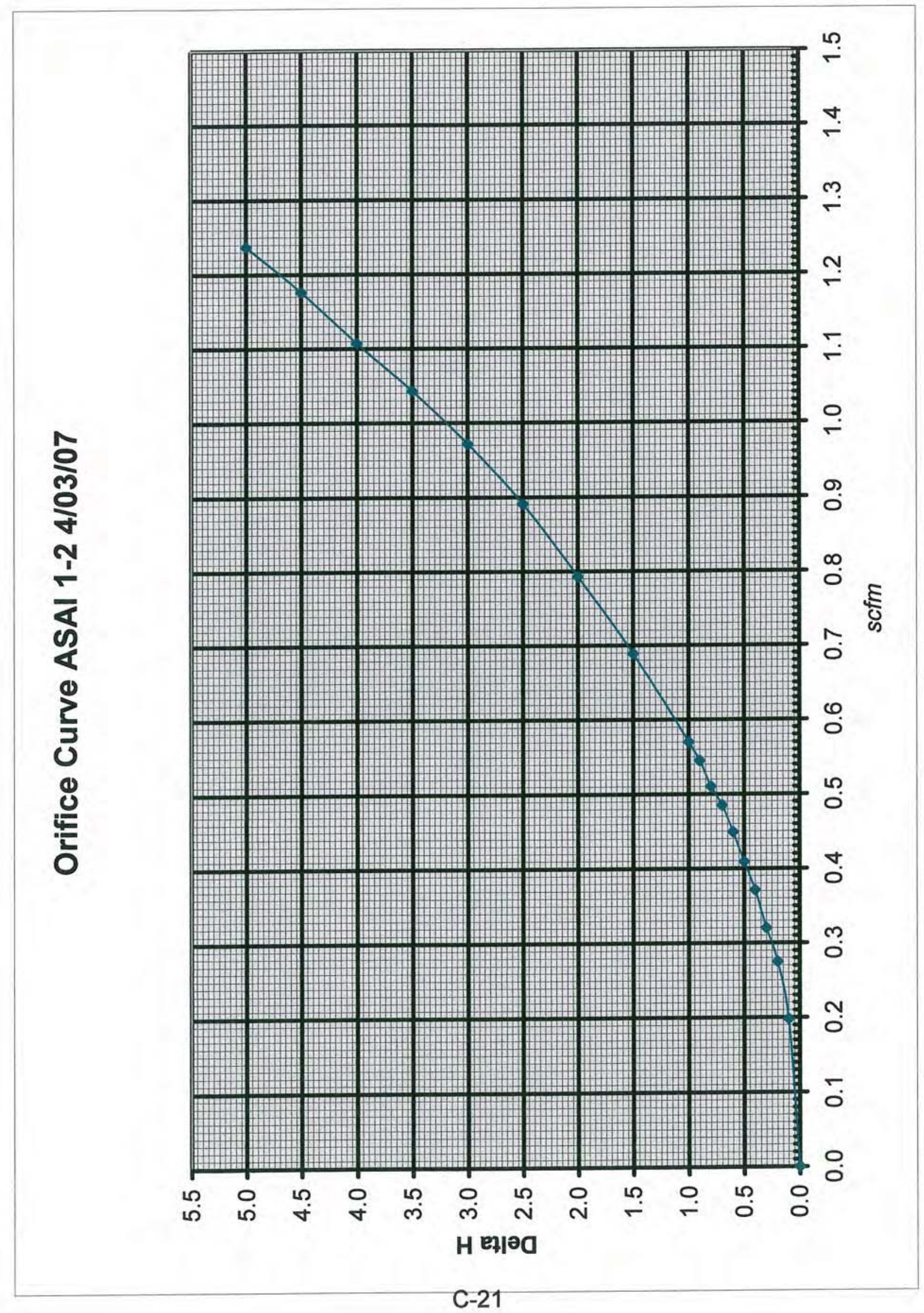




\section{AIRMPLNG ASSOCIATES, INC.}

\section{CALIBRATION DATA \\ PITOT TUBE CALIBRATION DATA}

$\begin{array}{lc}\text { Date: } & 03 / 29 / 07 \\ \text { I.D. \# } & \text { ASAI 2-1 } \\ \text { C }_{\text {pstd: }} & 0.990\end{array}$
Time: $11: 00$

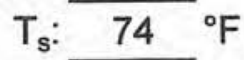

$\mathrm{Pb}: \overline{29.65} \mathrm{Hg}$

\begin{tabular}{|c|c|c|c|c|c|c|c|c|c|c|}
\hline \multirow{2}{*}{\begin{tabular}{c|} 
fps \\
Mark
\end{tabular}} & \multirow{2}{*}{$\begin{array}{c}\text { Desired } \\
\text { Calibration } \\
\text { Standard } \\
\end{array}$} & \multicolumn{2}{|c|}{$\begin{array}{l}\text { Calibration } \\
\text { Standard }\end{array}$} & \multirow{2}{*}{$\begin{array}{c}\sqrt{ } \text { Standard } \\
\text { Average }\end{array}$} & \multirow[b]{2}{*}{ High } & \multirow[b]{2}{*}{$\sqrt{ }$ High } & \multirow{2}{*}{$\begin{array}{l}\text { Cal. } \\
\text { Factor }\end{array}$} & \multirow[b]{2}{*}{ Low } & \multirow[b]{2}{*}{$\sqrt{ }$ Low } & \multirow{2}{*}{$\begin{array}{l}\text { Cal. } \\
\text { Factor }\end{array}$} \\
\hline & & Start & End & & & & & & & \\
\hline 20 & 0.09 & 0.09 & 0.09 & 0.300 & 0.13 & 0.361 & 0.824 & 0.13 & 0.361 & 0.824 \\
\hline 30 & 0.20 & 0.20 & 0.20 & 0.447 & 0.29 & 0.539 & 0.822 & 0.29 & 0.539 & 0.822 \\
\hline 40 & 0.36 & 0.36 & 0.36 & 0.600 & 0.52 & 0.721 & 0.824 & 0.52 & 0.721 & 0.824 \\
\hline 50 & 0.56 & 0.56 & 0.56 & 0.748 & 0.80 & 0.894 & 0.828 & 0.81 & 0.900 & 0.823 \\
\hline 60 & 0.81 & 0.81 & 0.81 & 0.900 & 1.15 & 1.072 & 0.831 & 1.15 & 1.072 & 0.831 \\
\hline 70 & 1.10 & 1.10 & 1.10 & 1.049 & 1.55 & 1.245 & 0.834 & 1.60 & 1.265 & 0.821 \\
\hline 80 & 1.43 & 1.45 & 1.45 & 1.204 & 2.10 & 1.449 & 0.823 & 2.10 & 1.449 & 0.823 \\
\hline \multirow[t]{2}{*}{90} & 1.81 & 1.80 & 1.80 & 1.342 & 2.60 & 1.612 & 0.824 & 2.60 & 1.612 & 0.824 \\
\hline & & & & & & & & & & \\
\hline 50 & & 056 & 0,56 & 0748 & 080 & 0894 & 0.828 & 080 & 0894 & 0828 \\
\hline 50 & 0.50 & 0.56 & 0.56 & 0.140 & 0.00 & \begin{tabular}{|l|}
0.894 \\
0.894
\end{tabular} & 0.828 & 0.80 & 0.894 & 0.828 \\
\hline & & & & & & & & & & \\
\hline & & & & & & & & & & \\
\hline & Average & & & & & & 0.826 & & & 0.824 \\
\hline
\end{tabular}

Summary of Results:

Normal high side calibraiton factor: $\quad 0.826$

variation $+0.97 \%$

variation -:

Normal low side calibration factor:

$-0.48 \%$

variation $+\frac{0.85 \%}{0.36 \%}$

variation -: $-0.36 \%$

\section{Certification:}

I certify that the Type S pitot tube, the standard type pitot tube, and the calibration setup meet or exceed all specifications, criteria and/or applicable design features and hereby assign a pitot tube calibration factor $\mathrm{C}_{\mathrm{p}}$ of: $\quad 0.826$

Certified by: $\quad \frac{\text { Scot Tackson 3/29/07 }}{\text { Calibrator (Signature/Date) }}$

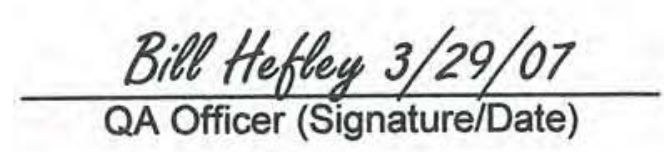




\section{AIR

Date:

I.D. \#

$\mathrm{C}_{\mathrm{pstd}}$
Time: $\quad 8: 30$

$\mathrm{T}_{\mathrm{s}}: 73^{\circ} \mathrm{F}$

$\mathrm{Pb}: 29.56$ " Hg

\begin{tabular}{|c|c|c|c|c|c|c|c|c|c|c|}
\hline fps & \begin{tabular}{|c|} 
Desired \\
Calibration
\end{tabular} & \multicolumn{2}{|c|}{$\begin{array}{l}\text { Calibration } \\
\text { Standard }\end{array}$} & \multirow{2}{*}{$\begin{array}{c}\sqrt{ } \text { Standard } \\
\text { Average }\end{array}$} & \multirow[b]{2}{*}{ High } & \multirow[b]{2}{*}{$\sqrt{H i g h}$} & \multirow{2}{*}{$\begin{array}{l}\text { Cal. } \\
\text { Factor }\end{array}$} & \multirow[b]{2}{*}{ Low } & \multirow[b]{2}{*}{$\sqrt{ }$ Low } & \multirow{2}{*}{$\begin{array}{l}\text { Cal. } \\
\text { Factor }\end{array}$} \\
\hline Mark & Standard & Start & End & & & & & & & \\
\hline 20 & 0.09 & 0.09 & 0.09 & 0.300 & 0.13 & 0.361 & 0.824 & 0.13 & 0.361 & 0.824 \\
\hline 30 & 0.20 & 0.20 & 0.20 & 0.447 & 0.29 & 0.539 & 0.822 & 0.29 & 0.539 & 0.822 \\
\hline 40 & 0.36 & 0.36 & 0.36 & 0.600 & 0.52 & 0.721 & 0.824 & 0.52 & 0.721 & 0.824 \\
\hline 50 & 0.56 & 0.56 & 0.56 & 0.748 & 0.81 & 0.900 & 0.823 & 0.81 & 0.900 & 0.823 \\
\hline 60 & 0.81 & 0.81 & 0.81 & 0.900 & 1.15 & 1.072 & 0.831 & 1.15 & 1.072 & 0.831 \\
\hline 70 & 1.10 & 1.10 & 1.10 & 1.049 & 1.60 & 1.265 & 0.821 & 1.60 & 1.265 & 0.821 \\
\hline 80 & 1.43 & 1.45 & 1.45 & 1.204 & 2.10 & 1.449 & 0.823 & 2.10 & 1.449 & 0.823 \\
\hline 90 & 1.81 & 1.80 & 1.80 & 1.342 & 2.60 & 1.612 & 0.824 & 2.60 & 1.612 & 0.824 \\
\hline & & & & & & & & & & \\
\hline & & & & & & & & & & \\
\hline 50 & 0.56 & 0.56 & 0.56 & 0.748 & 0.81 & 0.900 & 0.823 & 0.81 & 0.900 & 0.823 \\
\hline 50 & 0.56 & 0.56 & 0.56 & 0.748 & 0.81 & 0.900 & 0.823 & 0.81 & 0.900 & 0.823 \\
\hline & & & & & & & & & & \\
\hline & & & & & & & & & & \\
\hline & verage & & & & & & 0.824 & & & 0.824 \\
\hline
\end{tabular}

Summary of Results:

\begin{tabular}{rc} 
Normal high side calibraiton factor: & 0.824 \\
variation $+:$ & $0.85 \%$ \\
variation $-:$ & $-0.36 \%$ \\
\hline Normal low side calibration factor: & 0.824 \\
variation $+:$ & $0.85 \%$ \\
variation $-:-0.36 \%$
\end{tabular}

Certification:

I certify that the Type S pitot tube, the standard type pitot tube, and the calibration setup meet or exceed all specifications, criteria and/or applicable design features and hereby assign a pitot tube calibration factor $C_{p}$ of: $\quad 0.824$

Certified by:

Scot Jackson 4/3/07 Calibrator (Signature/Date)
Bill Hefley 4/3/07

QA Officer (Signature/Date) 


\section{SARMPUNG \\ ASSOCIATES, INC.}

CALIBRATION DATA

PITOT TUBE CALIBRATION DATA

\begin{tabular}{cc} 
Date: & $01 / 02 / 07$ \\
\hline I.D. \# $\frac{A S A I ~ 2-3}{0.990}$ \\
C $_{\text {pstd: }}$
\end{tabular}

Time: $15: 40$

$\mathrm{T}_{\mathrm{s}}: \quad 66^{\circ} \mathrm{F}$

$\mathrm{Pb}: 29.92$ " Hg

\begin{tabular}{|c|c|c|c|c|c|c|c|c|c|c|}
\hline \multirow{2}{*}{\begin{tabular}{|c|} 
fps \\
Mark \\
\end{tabular}} & \multirow{2}{*}{$\begin{array}{c}\text { Desired } \\
\text { Calibration } \\
\text { Standard }\end{array}$} & \multicolumn{2}{|c|}{$\begin{array}{l}\text { Calibration } \\
\text { Standard }\end{array}$} & \multirow{2}{*}{$\begin{array}{c}\sqrt{ } \text { Standard } \\
\text { Average }\end{array}$} & \multirow[b]{2}{*}{ High } & \multirow[b]{2}{*}{$\sqrt{ } \mathrm{High}$} & \multirow{2}{*}{$\begin{array}{l}\text { Cal. } \\
\text { Factor }\end{array}$} & \multirow[b]{2}{*}{ Low } & \multirow[b]{2}{*}{$\sqrt{ }$ Low } & \multirow{2}{*}{$\begin{array}{l}\text { Cal. } \\
\text { Factor }\end{array}$} \\
\hline & & Start & End & & & & & & & \\
\hline 20 & 0.09 & 0.09 & 0.09 & 0.300 & 0.13 & 0.361 & 0.824 & 0.13 & 0.361 & 0.824 \\
\hline 30 & 0.21 & 0.21 & 0.21 & 0.458 & 0.30 & 0.548 & 0.828 & 0.30 & 0.548 & 0.828 \\
\hline 40 & 0.37 & 0.37 & 0.37 & 0.608 & 0.54 & 0.735 & 0.819 & 0.53 & 0.728 & 0.827 \\
\hline 50 & 0.57 & 0.57 & 0.57 & 0.755 & 0.82 & 0.906 & 0.825 & 0.82 & 0.906 & 0.825 \\
\hline 60 & 0.83 & 0.83 & 0.83 & 0.911 & 1.20 & 1.095 & 0.823 & 1.20 & 1.095 & 0.823 \\
\hline 70 & 1.12 & 1.10 & 1.10 & 1.049 & 1.60 & 1.265 & 0.821 & 1.60 & 1.265 & 0.821 \\
\hline 80 & 1.47 & 1.50 & 1.50 & 1.225 & 2.15 & 1.466 & 0.827 & 2.15 & 1.466 & 0.827 \\
\hline \multirow[t]{2}{*}{90} & 1.86 & 1.85 & 1.85 & 1.360 & 2.65 & 1.628 & 0.827 & 2.65 & 1.628 & 0.827 \\
\hline & & & & & & & & & & \\
\hline 50 & 0.57 & 0.57 & 0.57 & 0.755 & 0.82 & 0.906 & 0.825 & 0.82 & 0.906 & 0.825 \\
\hline 50 & 0.57 & 0.57 & 0.57 & 0.755 & 0.82 & 0.906 & 0.825 & 0.82 & 0.906 & 0.825 \\
\hline & & & & & & & & & & \\
\hline & & & & & & & & & & \\
\hline$A$ & verage & & & & & & 0.824 & & & 0.825 \\
\hline
\end{tabular}

Summary of Results:

Normal high side calibraiton factor:

variation $+\frac{0.49 \%}{-0.61 \%}$

variation -:

Normal low side calibration factor:

$-0.61 \%$

variation $+\quad 0.36 \%$

variation $-:-0.48 \%$

Certification:

I certify that the Type S pitot tube, the standard type pitot tube, and the calibration setup meet or exceed all specifications, criteria and/or applicable design features and hereby assign a pitot tube calibration factor $C_{p}$ of: $\quad 0.824$

Certified by: $\quad \frac{\text { Patrick Selakovich 01/02/07 }}{\text { Calibrator (Signature/Date) }}$

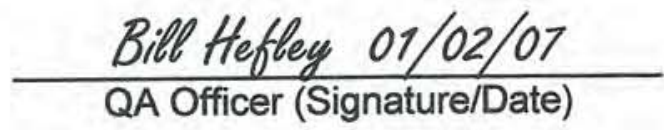


Date:

I.D. \# $\frac{\text { ASAI } 2-4}{0.990}$
Time: $14: 30$

$\mathrm{T}_{\mathrm{s}}: \quad 80^{\circ} \mathrm{F}$

$\mathrm{Pb}: \overline{29.62} \mathrm{Hg}$

\begin{tabular}{|c|c|c|c|c|c|c|c|c|c|c|}
\hline fps & $\begin{array}{c}\text { Desired } \\
\text { Calibration }\end{array}$ & \multicolumn{2}{|c|}{$\begin{array}{l}\text { Calibration } \\
\text { Standard }\end{array}$} & \multirow{2}{*}{$\begin{array}{c}\sqrt{ } \text { Standard } \\
\text { Average }\end{array}$} & \multirow[b]{2}{*}{ High } & \multirow[b]{2}{*}{$\sqrt{ }$ High } & \multirow{2}{*}{$\begin{array}{l}\text { Cal. } \\
\text { Factor }\end{array}$} & \multirow[b]{2}{*}{ Low } & \multirow[b]{2}{*}{$\sqrt{\text { Low }}$} & \multirow{2}{*}{$\begin{array}{l}\text { Cal. } \\
\text { Factor }\end{array}$} \\
\hline Mark & Standard & Start & End & & & & & & & \\
\hline 20 & 0.09 & 0.09 & 0.09 & 0.300 & 0.13 & 0.361 & 0.824 & 0.13 & 0.361 & 0.824 \\
\hline 30 & 0.20 & 0.20 & 0.20 & 0.447 & 0.29 & 0.539 & 0.822 & 0.29 & 0.539 & 0.822 \\
\hline 40 & 0.35 & 0.35 & 0.35 & 0.592 & 0.51 & 0.714 & 0.820 & 0.50 & 0.707 & 0.828 \\
\hline 50 & 0.55 & 0.55 & 0.55 & 0.742 & 0.81 & 0.900 & 0.816 & 0.80 & 0.894 & 0.821 \\
\hline 60 & 0.80 & 0.80 & 0.80 & 0.894 & 1.15 & 1.072 & 0.826 & 1.15 & 1.072 & 0.826 \\
\hline 70 & 1.08 & 1.10 & 1.10 & 1.049 & 1.60 & 1.265 & 0.821 & 1.60 & 1.265 & 0.821 \\
\hline 80 & 1.42 & 1.45 & 1.45 & 1.204 & 2.10 & 1.449 & 0.823 & 2.10 & 1.449 & 0.823 \\
\hline 90 & 1.79 & 1.80 & 1.80 & 1.342 & 2.60 & 1.612 & 0.824 & 2.60 & 1.612 & 0.824 \\
\hline & & & & & & & & & & \\
\hline & & & & & & & & & & \\
\hline 50 & 0.55 & 0.55 & 0.55 & 0.742 & 0.81 & 0.900 & 0.816 & 0.80 & 0.894 & 0.821 \\
\hline 50 & 0.55 & 0.55 & 0.55 & 0.742 & 0.81 & 0.900 & 0.816 & 0.80 & 0.894 & 0.821 \\
\hline & & & & & & & & & & \\
\hline & & & & & & & & & & 0824 \\
\hline & erage & & & & & & 0.822 & & & 0.824 \\
\hline
\end{tabular}

Summary of Results:

Normal high side calibraiton factor:

0.822

variation +:

$0.49 \%$

variation -:

$-0.73 \%$

Normal low side calibration factor:

0.824

variation +: $\quad 0.49 \%$

variation $-:-0.36 \%$

Certification:

I certify that the Type S pitot tube, the standard type pitot tube, and the calibration setup meet or exceed all specifications, criteria and/or applicable design features and hereby assign a pitot tube calibration factor $C_{p}$ of: $\quad 0.822$

Certified by:

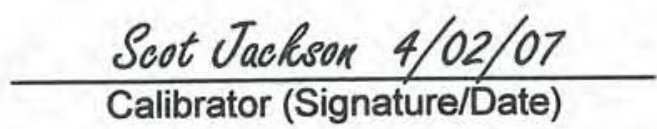




\section{AII

Calibration Data

Nozzle Calibration

\begin{aligned} & Nozzle Set No. ASAI - 5 \\ & \cline { 2 - 2 } Date Calibration Performed: $04 / 04 / 07 \\ &$\cline { 2 - 2 }\end{aligned}

$\begin{array}{llllllll}-1 & -2 & -3 & -4 & -5 & -6 & -7 & -8\end{array}$

\begin{tabular}{|c|c|c|c|c|c|c|c|c|}
\hline Measurement 1 & 0.154 & & 0.251 & 0.309 & 0.371 & 0.438 & 0.489 & 0.258 \\
\hline Measurement 2 & 0.154 & & 0.251 & 0.307 & 0.372 & 0.437 & 0.488 & 0.257 \\
\hline easurement 3 & 0.1 & & 0.252 & 0.309 & 0.372 & 0.439 & 0.489 & 0.258 \\
\hline leasurement 4 & 0.154 & & 0.252 & 07 & 0.372 & 0.438 & 0.489 & 0.259 \\
\hline leasurement 5 & 0.154 & & 0.251 & 0.308 & 0.372 & 0.437 & 0.490 & 0.258 \\
\hline leasurement 6 & 0.153 & & 0.252 & 0.309 & 0.371 & 0.437 & 0.489 & 0.258 \\
\hline easurement 7 & 0.154 & & 0.252 & 0.308 & 0.371 & 0.438 & 0.490 & 0.259 \\
\hline easurement 8 & 0.153 & & 0.253 & 0.307 & 0.371 & 0.437 & 0.488 & 0.258 \\
\hline Measurement 9 & 0.154 & & 0.252 & 0.308 & 0.372 & 0.438 & 0.490 & 0.258 \\
\hline leasurement 10 & 0.154 & & $\underline{0.252}$ & 0.307 & 0.372 & 0.439 & 0.489 & 0.258 \\
\hline Average & 0.154 & \#\#\#\# & 0.252 & 0.308 & 0.372 & 0.438 & 0.489 & 0.258 \\
\hline
\end{tabular}

Scot Jackson 4/04/07

Calibrator (Signature / Date)

Bill Hefley 4/04/07

QA Officer (Signature / Date) 


\section{AIR

Calibration Data

Nozzle Calibration

$\begin{aligned} \text { Nozzle Set No. } & \text { ASAI - } 6 \\ \text { Date Calibration Performed: } & 04 / 04 / 07 \\ & \end{aligned}$

$-1$

$0.114 \quad 0.192 \quad 0.253$

$-4$

$-5$

$-6$

$-7$ $-8$

Measurement 1

Measurement 2

Measurement 3

Measurement 4

Measurement 5

Measurement 6

Measurement 7

Measurement 8

Measurement 9

Measurement 10

Average

$\underline{0.307}$

$\begin{array}{lllll}0.371 & 0.425 & 0.485 & 0.259\end{array}$

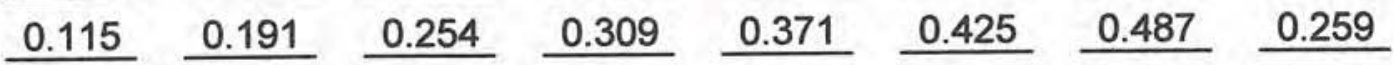

$\begin{array}{lllllllll}0.115 & 0.191 & 0.254 & 0.308 & 0.372 & 0.424 & 0.484 & 0.258\end{array}$

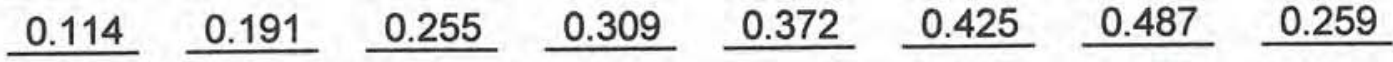

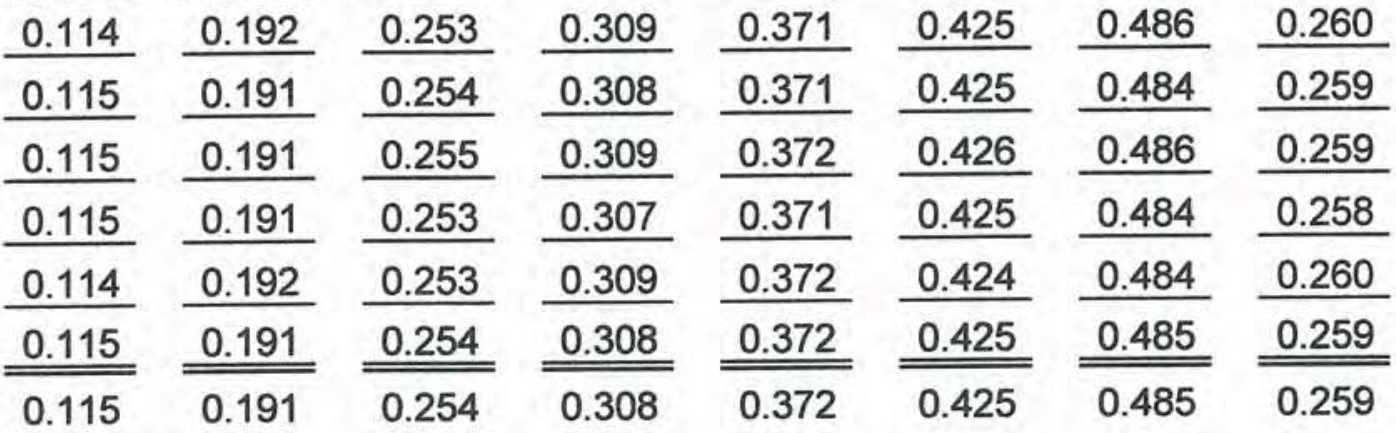

Scot Jacksen 4/04/07

Calibrator (Signature / Date)

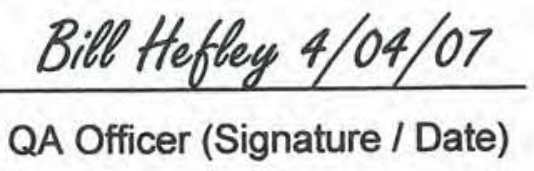




\section{AII AlR

\section{Calibration Data}

Nozzle Calibration

Nozzle Set No.
Date Calibration Performed:

$-1$
ASAI - 7

04/04/07

\begin{tabular}{|c|c|c|c|c|c|c|c|c|}
\hline & -1 & -2 & -3 & -4 & -5 & -6 & -7 & -8 \\
\hline Measurement 1 & 0.113 & 0.194 & 0.259 & 0.308 & 0.376 & 0.424 & 0.484 & 0.257 \\
\hline Measurement 2 & 0.114 & 0.194 & 0.258 & 0.308 & 0.376 & 0.425 & 0.487 & 0.258 \\
\hline Measurement 3 & 0.115 & 0.193 & 0.259 & 0.308 & 0.377 & 0.424 & 0.484 & 0.257 \\
\hline Measurement 4 & 0.115 & 0.193 & 0.258 & 0.309 & 0.378 & 0.425 & 0.486 & 0.258 \\
\hline Measurement 5 & 0.115 & 0.192 & 0.258 & 0.308 & 0.376 & 0.424 & 0.485 & 0.258 \\
\hline Measurement 6 & 0.114 & 0.192 & 0.259 & 0.309 & 0.376 & 0.426 & 0.485 & 0.258 \\
\hline Measurement 7 & 0.113 & 0.193 & 0.259 & 0.308 & 0.378 & 0.426 & 0.486 & 0.257 \\
\hline Measurement 8 & 0.114 & 0.193 & 0.258 & 0.308 & 0.377 & 0.424 & 0.486 & 0.258 \\
\hline Measurement 9 & 0.113 & 0.192 & 0.258 & 0.309 & 0.376 & 0.425 & 0.485 & 0.258 \\
\hline Measurement 10 & 0.114 & 0.192 & 0.259 & 0.308 & 0.377 & 0.426 & 0.484 & 0.257 \\
\hline Average & 0.114 & 0.193 & 0.259 & 0.308 & 0.377 & 0.425 & 0.485 & 0.258 \\
\hline & & & & & & \multicolumn{3}{|c|}{ Scot Tackson 4/04/07 } \\
\hline & & & & & & \multicolumn{3}{|c|}{ Calibrator (Signature / Date) } \\
\hline & & & & & & \multicolumn{3}{|c|}{ Bill Hefley 4/04/07 } \\
\hline & & & & & & \multicolumn{3}{|c|}{ QA Officer (Signature / Date) } \\
\hline
\end{tabular}


Post-test Calibration Data 


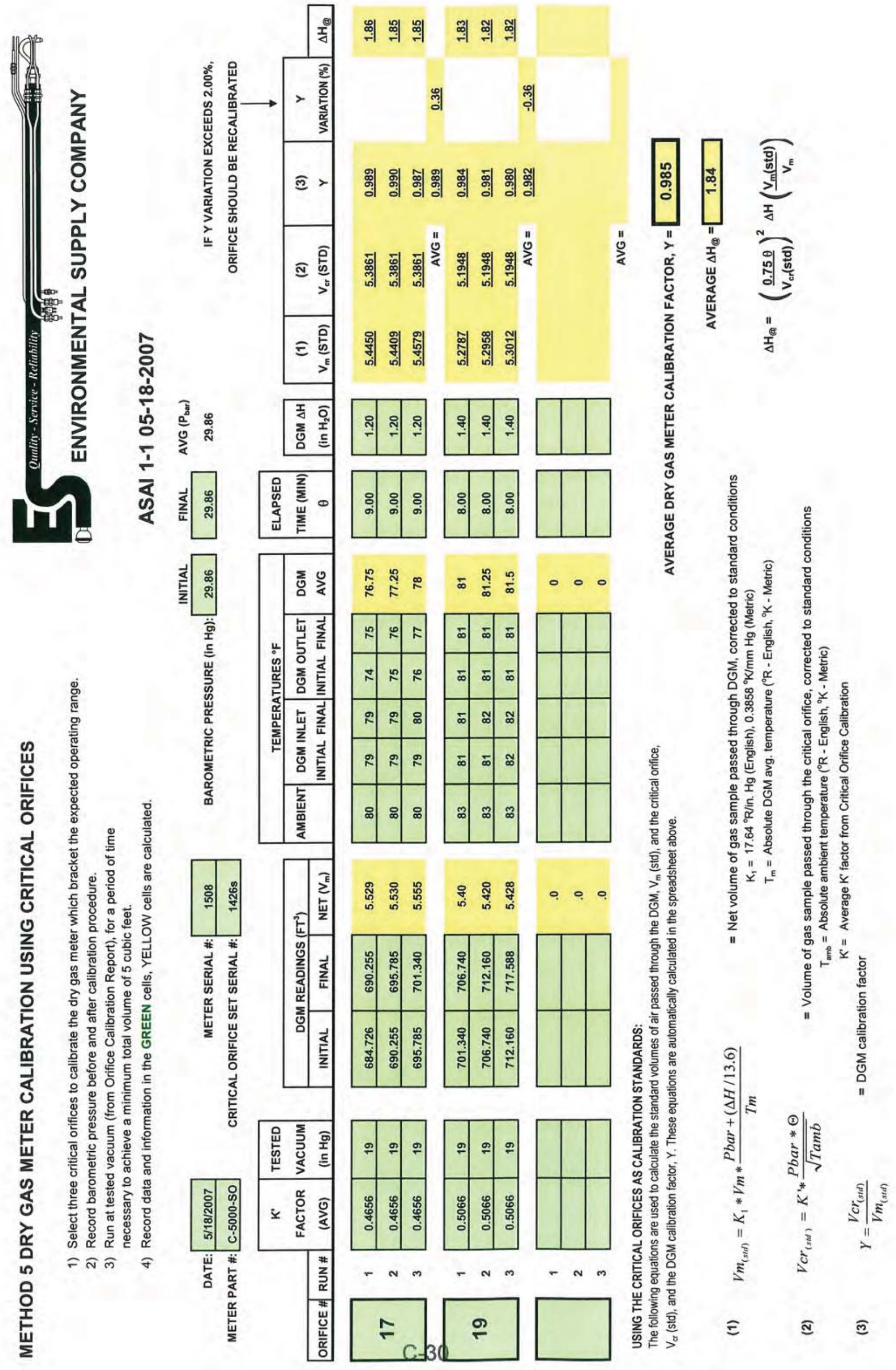


Calibration Data

Digital Temperature Indicator Calibration

DTI Unit No. ASAI 1-1

Date Calibration Performed:

\begin{tabular}{|c|c|}
\hline $05 / 18 / 07$ & \\
\hline $\begin{array}{c}\text { ASTM } \\
\text { Reference }\end{array}$ & \\
\hline Thermometer & DTI \\
\hline$\left({ }^{\circ} \mathrm{F}\right)$ & $\left({ }^{\circ} \mathrm{F}\right)$ \\
\hline $32^{\circ} \mathrm{F}$ & $31^{\circ} \mathrm{F}$ \\
\hline $80^{\circ} \mathrm{F}$ & $79^{\circ} \mathrm{F}$ \\
\hline $212^{\circ} \mathrm{F}$ & $214^{\circ} \mathrm{F}$ \\
\hline $250^{\circ} \mathrm{F}$ & $253^{\circ} \mathrm{F}$ \\
\hline $300^{\circ} \mathrm{F}$ & $303^{\circ} \mathrm{F}$ \\
\hline $350^{\circ} \mathrm{F}$ & $353^{\circ} \mathrm{F}$ \\
\hline $400^{\circ} \mathrm{F}$ & $404^{\circ} \mathrm{F}$ \\
\hline
\end{tabular}

Meter Adjusted?

No

Reference Point

Ice Bath

Ambient Air

Boiling Water

Oven

Oven

Oven

Oven

NIST Reference Calibrator:

\begin{tabular}{c}
$\frac{\text { Time }}{10: 45}$ \\
\hline $10: 46$ \\
\hline $10: 47$ \\
\hline $10: 48$ \\
\hline $10: 49$ \\
\hline $10: 50$ \\
\hline $10: 51$ \\
\hline
\end{tabular}

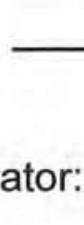

Certified by: $\quad$ Scot Jackson 5/18/07

Range: $\quad-0.2^{\circ} \mathrm{C}$ to $1,360.6^{c}$

Range:

Range:

Bill Hefley 5/18/07

QA Officer (Signature / Date) 


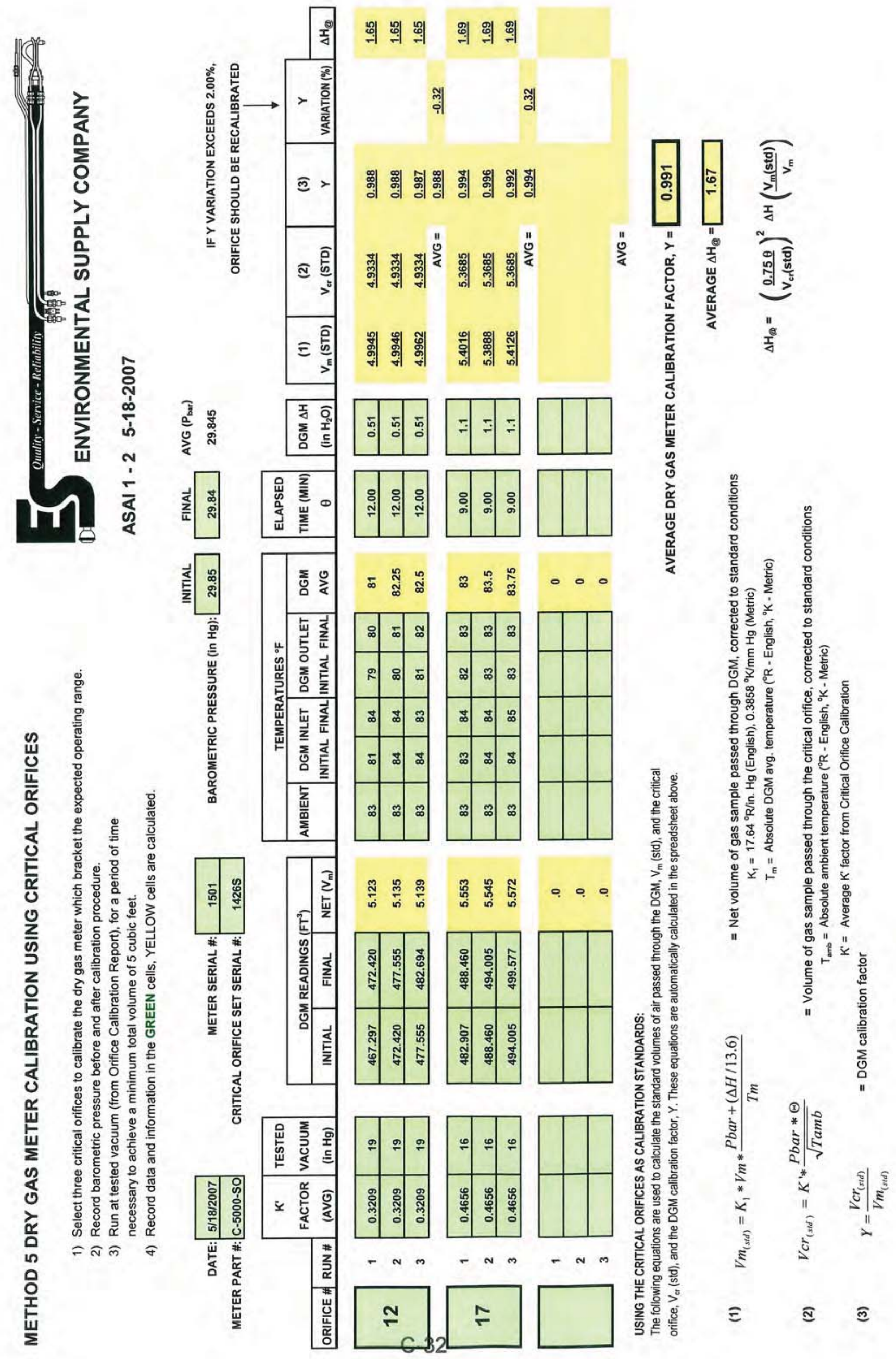


Calibration Data

Digital Temperature Indicator Calibration

DTI Unit No. ASAI 1-2

Date Calibration Performed:

\begin{tabular}{|c|c|}
\hline $05 / 18 / 07$ & \\
\hline $\begin{array}{c}\text { ASTM } \\
\text { Reference }\end{array}$ & \\
\hline Thermometer & DTI \\
\hline$\left({ }^{\circ} \mathrm{F}\right)$ & $\left({ }^{\circ} \mathrm{F}\right)$ \\
\hline $32^{\circ} \mathrm{F}$ & $32^{\circ} \mathrm{F}$ \\
\hline $83^{\circ} \mathrm{F}$ & $83^{\circ} \mathrm{F}$ \\
\hline $212^{\circ} \mathrm{F}$ & $213^{\circ} \mathrm{F}$ \\
\hline $250^{\circ} \mathrm{F}$ & $252^{\circ} \mathrm{F}$ \\
\hline $300^{\circ} \mathrm{F}$ & $302^{\circ} \mathrm{F}$ \\
\hline $350^{\circ} \mathrm{F}$ & $352^{\circ} \mathrm{F}$ \\
\hline $400^{\circ} \mathrm{F}$ & $401^{\circ} \mathrm{F}$ \\
\hline
\end{tabular}

Meter Adjusted?

No

Reference Point

Ice Bath

Ambient Air

Boiling Water

Oven

Oven

Oven

Oven

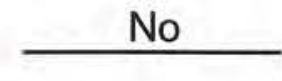

SN: 06000047

Certified by:
Range: $\quad-0.1^{\circ} \mathrm{C}$ to $1,360.6^{\mathrm{c}}$

Range:

Range:

Scot Jachson 5/18/07

Calibrator (Signature / Date)

Bill Hefley 5/18/07

QA Officer (Signature / Date) 


\section{II) ASSOCIATES, INC.}
CALIBRATION DATA
PITOT TUBE CALIBRATION DATA

\begin{tabular}{|c|c|}
\hline Date: & $05 / 21 / 07$ \\
\hline I.D. \# & ASAI 2-1 \\
\hline $\mathrm{C}_{\mathrm{pstd}}:$ & 0.990 \\
\hline
\end{tabular}

Time: $8: 30$

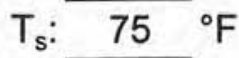

$\mathrm{Pb}: \overline{29.64} \mathrm{Hg}$

\begin{tabular}{|c|c|c|c|c|c|c|c|c|c|c|}
\hline $\begin{array}{c}\text { fps } \\
\text { Mark }\end{array}$ & $\begin{array}{c}\text { Desired } \\
\text { Calibration } \\
\text { Standard }\end{array}$ & \multicolumn{2}{c|}{$\begin{array}{c}\text { Calibration } \\
\text { Standard } \\
\text { Start }\end{array}$} & $\begin{array}{c}\text { E Standard } \\
\text { Average }\end{array}$ & High & v High & $\begin{array}{c}\text { Cal. } \\
\text { Factor }\end{array}$ & Low & v Low & $\begin{array}{c}\text { Cal. } \\
\text { Factor }\end{array}$ \\
\hline 20 & 0.09 & 0.09 & 0.09 & 0.300 & 0.13 & 0.361 & 0.824 & 0.13 & 0.361 & 0.824 \\
\hline 30 & 0.20 & 0.20 & 0.20 & 0.447 & 0.29 & 0.539 & 0.822 & 0.29 & 0.539 & 0.822 \\
\hline 40 & 0.36 & 0.36 & 0.36 & 0.600 & 0.52 & 0.721 & 0.824 & 0.52 & 0.721 & 0.824 \\
\hline 50 & 0.56 & 0.56 & 0.56 & 0.748 & 0.81 & 0.900 & 0.823 & 0.81 & 0.900 & 0.823 \\
\hline 60 & 0.80 & 0.81 & 0.81 & 0.900 & 1.20 & 1.095 & 0.813 & 1.20 & 1.095 & 0.813 \\
\hline 70 & 1.10 & 1.10 & 1.10 & 1.049 & 1.60 & 1.265 & 0.821 & 1.60 & 1.265 & 0.821 \\
\hline 80 & 1.43 & 1.45 & 1.45 & 1.204 & 2.10 & 1.449 & 0.823 & 2.10 & 1.449 & 0.823 \\
\hline 90 & 1.81 & 1.80 & 1.80 & 1.342 & 2.60 & 1.612 & 0.824 & 2.60 & 1.612 & 0.824 \\
\hline & & & & & & & & & & \\
\hline & & & & & & & & & & \\
\hline 50 & 0.56 & 0.56 & 0.56 & 0.748 & 0.80 & 0.894 & 0.828 & 0.81 & 0.900 & 0.823 \\
\hline 50 & 0.56 & 0.56 & 0.56 & 0.748 & 0.81 & 0.900 & 0.823 & 0.81 & 0.900 & 0.823 \\
\hline & & & & & & & & & & \\
\hline & & & & & & & & & & \\
\hline \multicolumn{2}{|l|}{ Average } & & & & & & 0.822 & & & 0.822 \\
\hline
\end{tabular}

Summary of Results:

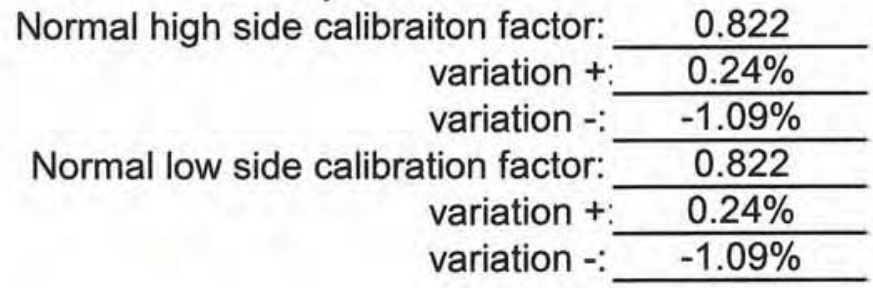

Certification:

I certify that the Type S pitot tube, the standard type pitot tube, and the calibration setup meet or exceed all specifications, criteria and/or applicable design features and hereby assign a pitot tube calibration factor $\mathrm{C}_{\mathrm{p}}$ of: $\quad 0.822$

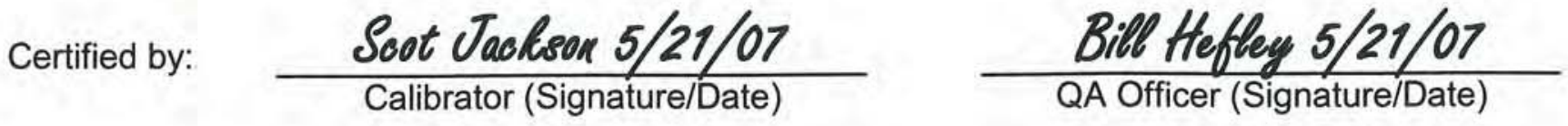




\title{
(1) SIRPRUNG ASSOCIATES, INC.
}

\author{
CALIBRATION DATA \\ PITOT TUBE CALIBRATION DATA
}

$\begin{array}{lc}\text { Date: } & 05 / 21 / 07 \\ \text { I.D. \# } \frac{\text { ASAl 2-2 }}{0.990} \\ \text { C }_{\text {pstd: }}\end{array}$

Time: $8: 30$

$\mathrm{T}_{\mathrm{s}}: \frac{8.30}{7{ }^{\circ} \mathrm{F}}$

$\mathrm{Pb}: \overline{29.64} " \mathrm{Hg}$

\begin{tabular}{|c|c|c|c|c|c|c|c|c|c|c|}
\hline \multirow{2}{*}{\begin{tabular}{|c|} 
fps \\
Mark
\end{tabular}} & \multirow{2}{*}{\begin{tabular}{|c|} 
Desired \\
Calibration \\
Standard
\end{tabular}} & \multicolumn{2}{|c|}{$\begin{array}{c}\text { Calibration } \\
\text { Standard }\end{array}$} & \multirow{2}{*}{$\begin{array}{c}\text { v Standard } \\
\text { Average }\end{array}$} & \multirow[b]{2}{*}{ High } & \multirow[b]{2}{*}{ v High } & \multirow{2}{*}{$\begin{array}{l}\text { Cal. } \\
\text { Factor }\end{array}$} & \multirow[b]{2}{*}{ Low } & \multirow[b]{2}{*}{ v Low } & \multirow{2}{*}{$\begin{array}{l}\text { Cal. } \\
\text { Factor }\end{array}$} \\
\hline & & Start & End & & & & & & & \\
\hline 20 & 0.09 & 0.09 & 0.09 & 0.300 & 0.13 & 0.361 & 0.824 & 0.13 & 0.361 & 0.824 \\
\hline 30 & 0.20 & 0.20 & 0.20 & 0.447 & 0.29 & 0.539 & 0.822 & 0.29 & 0.539 & 0.822 \\
\hline 40 & 0.36 & 0.36 & 0.36 & 0.600 & 0.52 & $\begin{array}{l}0.721 \\
\end{array}$ & 0.824 & 0.52 & 0.721 & 0.824 \\
\hline 50 & 0.56 & 0.56 & 0.56 & 0.748 & 0.81 & 0.900 & 0.823 & 0.81 & 0.900 & 0.823 \\
\hline 60 & 0.80 & 0.80 & 0.80 & 0.894 & 1.15 & 1.072 & 0.826 & 1.15 & 1.072 & 0.826 \\
\hline 70 & 1.10 & 1.10 & 1.10 & 1.049 & 1.60 & 1.265 & 0.821 & 1.60 & 1.265 & 0.821 \\
\hline 80 & 1.43 & 1.45 & 1.45 & 1.204 & 2.10 & \begin{tabular}{|l|l|}
1.449 \\
\end{tabular} & 0.823 & 2.10 & 1.449 & 0.823 \\
\hline \multirow[t]{2}{*}{90} & 1.81 & 1.80 & 1.80 & 1.342 & 2.60 & 1.612 & 0.824 & 2.60 & 1.612 & 0.824 \\
\hline & & & & & & & & & & \\
\hline & & & & & & & & & & \\
\hline 50 & 0.56 & 0.56 & 0.56 & 0.748 & 0.81 & 0.900 & 0.823 & 0.81 & 0.900 & 0.823 \\
\hline 50 & 0.56 & 0.56 & 0.56 & 0.748 & 0.81 & 0.900 & 0.823 & 0.81 & 0.900 & 0.823 \\
\hline & & & & & & & & & & \\
\hline & & & & & & & & & & \\
\hline & verage & & & & & & 0.823 & & & 0.823 \\
\hline
\end{tabular}

Summary of Results:

Normal high side calibraiton factor:

variation $+\quad 0.36 \%$

variation -: $-\frac{-0.24 \%}{0.823}$

Normal low side calibration factor: 0.823

variation $+\frac{0.36 \%}{-0.24 \%}$

variation $-: \frac{-0.24 \%}{-}$

Certification:

I certify that the Type S pitot tube, the standard type pitot tube, and the calibration setup meet or exceed all specifications, criteria and/or applicable design features and hereby assign a pitot tube calibration factor $\mathrm{C}_{\mathrm{p}}$ of: $\quad 0.823$
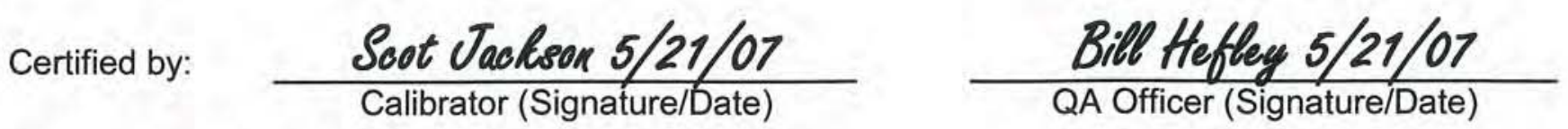


\section{II)}

\begin{abstract}
CALIBRATION DATA
PITOT TUBE CALIBRATION DATA
\end{abstract}

$\begin{array}{lc}\text { Date: } & \frac{05 / 21 / 07}{\text { I.D. \# }} \frac{\mathrm{ASAl} 2-3}{0.990} \\ \text { C }_{\text {pstd: }} & 0.9\end{array}$
Time: $15: 40$

$\mathrm{T}_{\mathrm{s}}: \frac{15: 40}{74}{ }^{\circ} \mathrm{F}$

$\mathrm{Pb}: \overline{29.64}$ " $\mathrm{Hg}$

\begin{tabular}{|c|c|c|c|c|c|c|c|c|c|c|}
\hline $\begin{array}{c}\text { fps } \\
\text { Mark }\end{array}$ & $\begin{array}{c}\text { Desired } \\
\text { Calibration } \\
\text { Standard }\end{array}$ & \multicolumn{2}{c|}{$\begin{array}{c}\text { Calibration } \\
\text { Standard } \\
\text { Start }\end{array}$} & $\begin{array}{c}\text { End } \\
\text { Vtandard } \\
\text { Average }\end{array}$ & High & v High & $\begin{array}{c}\text { Cal. } \\
\text { Factor }\end{array}$ & Low & v Low & $\begin{array}{c}\text { Cal. } \\
\text { Factor }\end{array}$ \\
\hline 20 & 0.09 & 0.09 & 0.09 & 0.300 & 0.13 & 0.361 & 0.824 & 0.13 & 0.361 & 0.824 \\
\hline 30 & 0.20 & 0.20 & 0.20 & 0.447 & 0.29 & 0.539 & 0.822 & 0.29 & 0.539 & 0.822 \\
\hline 40 & 0.36 & 0.36 & 0.36 & 0.600 & 0.52 & 0.721 & 0.824 & 0.52 & 0.721 & 0.824 \\
\hline 50 & 0.56 & 0.56 & 0.56 & 0.748 & 0.81 & 0.900 & 0.823 & 0.81 & 0.900 & 0.823 \\
\hline 60 & 0.81 & 0.80 & 0.80 & 0.894 & 1.15 & 1.072 & 0.826 & 1.15 & 1.072 & 0.826 \\
\hline 70 & 1.10 & 1.10 & 1.10 & 1.049 & 1.60 & 1.265 & 0.821 & 1.60 & 1.265 & 0.821 \\
\hline 80 & 1.43 & 1.45 & 1.45 & 1.204 & 2.10 & 1.449 & 0.823 & 2.10 & 1.449 & 0.823 \\
\hline 90 & 1.81 & 1.80 & 1.80 & 1.342 & 2.60 & 1.612 & 0.824 & 2.60 & 1.612 & 0.824 \\
\hline & & & & & & & & & & \\
\hline & & & & & & & & & & \\
\hline 50 & 0.56 & 0.56 & 0.56 & 0.748 & 0.81 & 0.900 & 0.823 & 0.81 & 0.900 & 0.823 \\
\hline 50 & 0.56 & 0.56 & 0.56 & 0.748 & 0.81 & 0.900 & 0.823 & 0.81 & 0.900 & 0.823 \\
\hline & & & & & & & & & & \\
\hline & & & & & & & & & & \\
\hline \multicolumn{2}{|r|}{ Average } & & & & & & 0.823 & & & 0.823 \\
\hline
\end{tabular}

Summary of Results:

\begin{tabular}{|c|c|}
\hline ibraiton factor. & 0893 \\
\hline variation + & $0.36 \%$ \\
\hline variation -: & $-0.24 \%$ \\
\hline Normal low side calibration factor: & 0.823 \\
\hline variation + & $0.36 \%$ \\
\hline variation & $-0.24 \%$ \\
\hline
\end{tabular}

Certification:

I certify that the Type S pitot tube, the standard type pitot tube, and the calibration setup meet or exceed all specifications, criteria and/or applicable design features and hereby assign a pitot tube calibration factor $C_{p}$ of: $\quad 0.823$

Certified by:

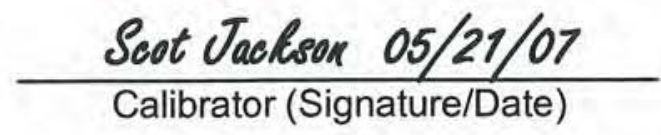

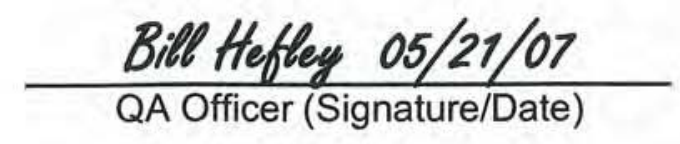




\section{AlR ASSOCIATES, INC.}

\section{CALIBRATION DATA \\ PITOT TUBE CALIBRATION DATA}

$\begin{array}{lc}\text { Date: } & \frac{05 / 21 / 07}{1 . D .} \# \frac{\mathrm{ASAl} 2-4}{0.990} \\ \text { C }_{\text {pstd }}: & 0.96\end{array}$

Time: $9: 45$

$\mathrm{T}_{\mathrm{s}}: 74^{\circ} \mathrm{F}$

$\mathrm{Pb}: \overline{29.63} \mathrm{Hg}$

\begin{tabular}{|c|c|c|c|c|c|c|c|c|c|c|}
\hline $\begin{array}{c}\text { fps } \\
\text { Mark }\end{array}$ & $\begin{array}{c}\text { Desired } \\
\text { Calibration } \\
\text { Standard }\end{array}$ & \multicolumn{2}{c|}{$\begin{array}{c}\text { Calibration } \\
\text { Standard } \\
\text { Start }\end{array}$} & $\begin{array}{c}\text { End } \\
\text { Etandard } \\
\text { Average }\end{array}$ & High & v High & $\begin{array}{c}\text { Cal. } \\
\text { Factor }\end{array}$ & Low & v Low & $\begin{array}{c}\text { Cal. } \\
\text { Factor }\end{array}$ \\
\hline 20 & 0.09 & 0.09 & 0.09 & 0.300 & 0.13 & 0.361 & 0.824 & 0.13 & 0.361 & 0.824 \\
\hline 30 & 0.20 & 0.20 & 0.20 & 0.447 & 0.29 & 0.539 & 0.822 & 0.29 & 0.539 & 0.822 \\
\hline 40 & 0.36 & 0.36 & 0.36 & 0.600 & 0.52 & 0.721 & 0.824 & 0.52 & 0.721 & 0.824 \\
\hline 50 & 0.56 & 0.56 & 0.56 & 0.748 & 0.80 & 0.894 & 0.828 & 0.80 & 0.894 & 0.828 \\
\hline 60 & 0.81 & 0.80 & 0.80 & 0.894 & 1.15 & 1.072 & 0.826 & 1.15 & 1.072 & 0.826 \\
\hline 70 & 1.10 & 1.10 & 1.10 & 1.049 & 1.60 & 1.265 & 0.821 & 1.60 & 1.265 & 0.821 \\
\hline 80 & 1.43 & 1.45 & 1.45 & 1.204 & 2.10 & 1.449 & 0.823 & 2.10 & 1.449 & 0.823 \\
\hline 90 & 1.81 & 1.80 & 1.80 & 1.342 & 2.60 & 1.612 & 0.824 & 2.60 & 1.612 & 0.824 \\
\hline & & & & & & & & & & \\
\hline & & & & & & & & & & \\
\hline 50 & 0.56 & 0.56 & 0.56 & 0.748 & 0.80 & 0.894 & 0.828 & 0.80 & 0.894 & 0.828 \\
\hline 50 & 0.56 & 0.56 & 0.56 & 0.748 & 0.80 & 0.894 & 0.828 & 0.80 & 0.894 & 0.828 \\
\hline & & & & & & & & & & \\
\hline & & & & & & & & & & \\
\hline \multicolumn{2}{l}{ Average } & & & & & & 0.824 & & & 0.824 \\
\hline
\end{tabular}

Summary of Results:

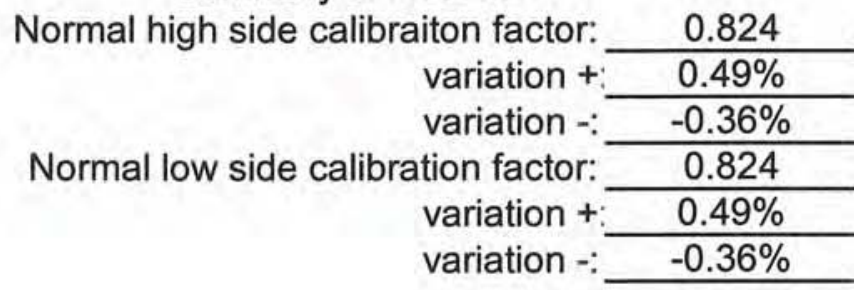

Certification:

I certify that the Type S pitot tube, the standard type pitot tube, and the calibration setup meet or exceed all specifications, criteria and/or applicable design features and hereby assign a pitot tube calibration factor $C_{p}$ of: $\quad 0.824$

Certified by:

Scot Jachson 5/21/07

Calibrator (Signature/Date)
Bill Hefley 5/21/07

QA Officer (Signature/Date) 


\section{III}

Calibration Data

Nozzle Calibration

\begin{aligned} Nozzle Set No. & ASAl - 5 \\ \cline { 2 - 2 } Date Calibration Performed: & $\frac{05 / 18 / 07}{}\end{aligned}$

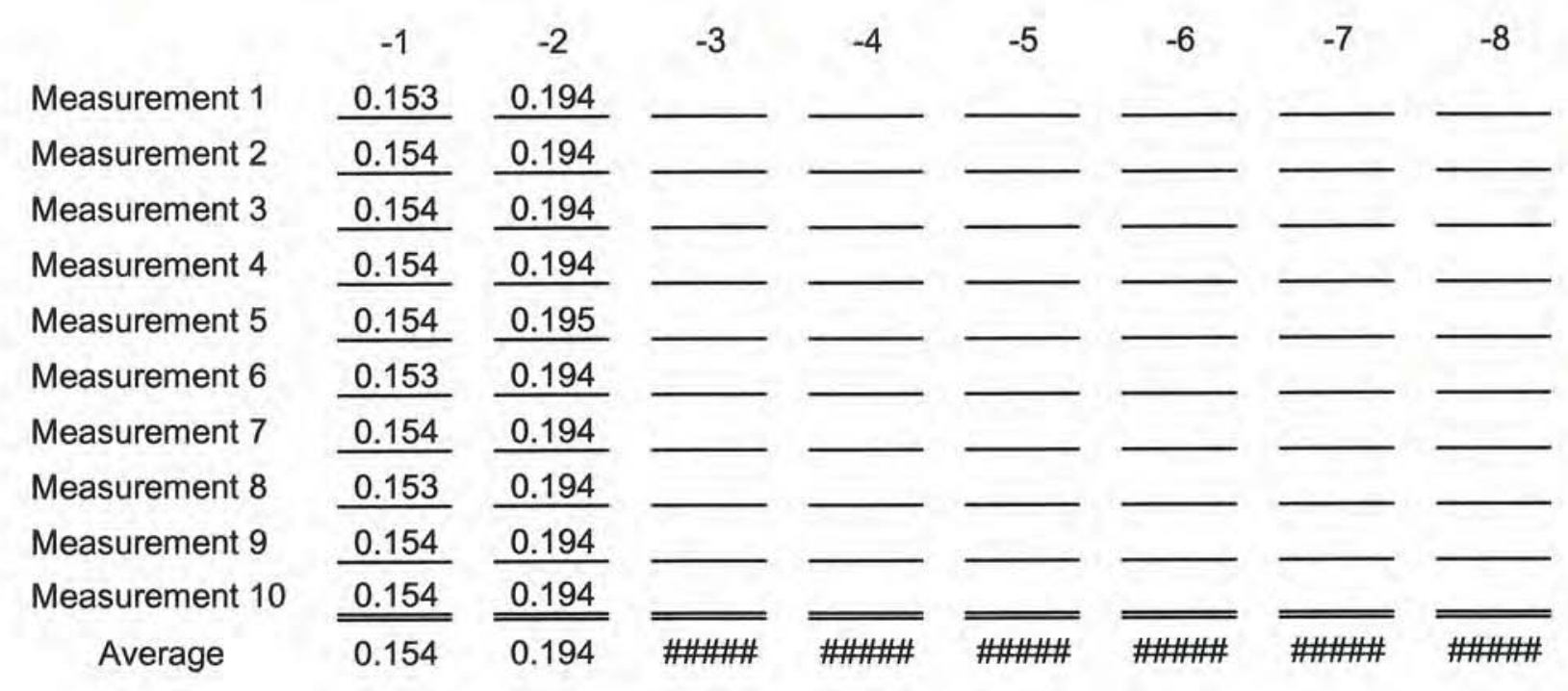

Soot Jackson 5/18/07

Calibrator (Signature / Date)

Bill Hefley 5/18/07

QA Officer (Signature / Date) 
Calibration Data

Nozzle Calibration

Nozzle Set No.

Date Calibration Performed:

ASAI - 6

$05 / 18 / 07$

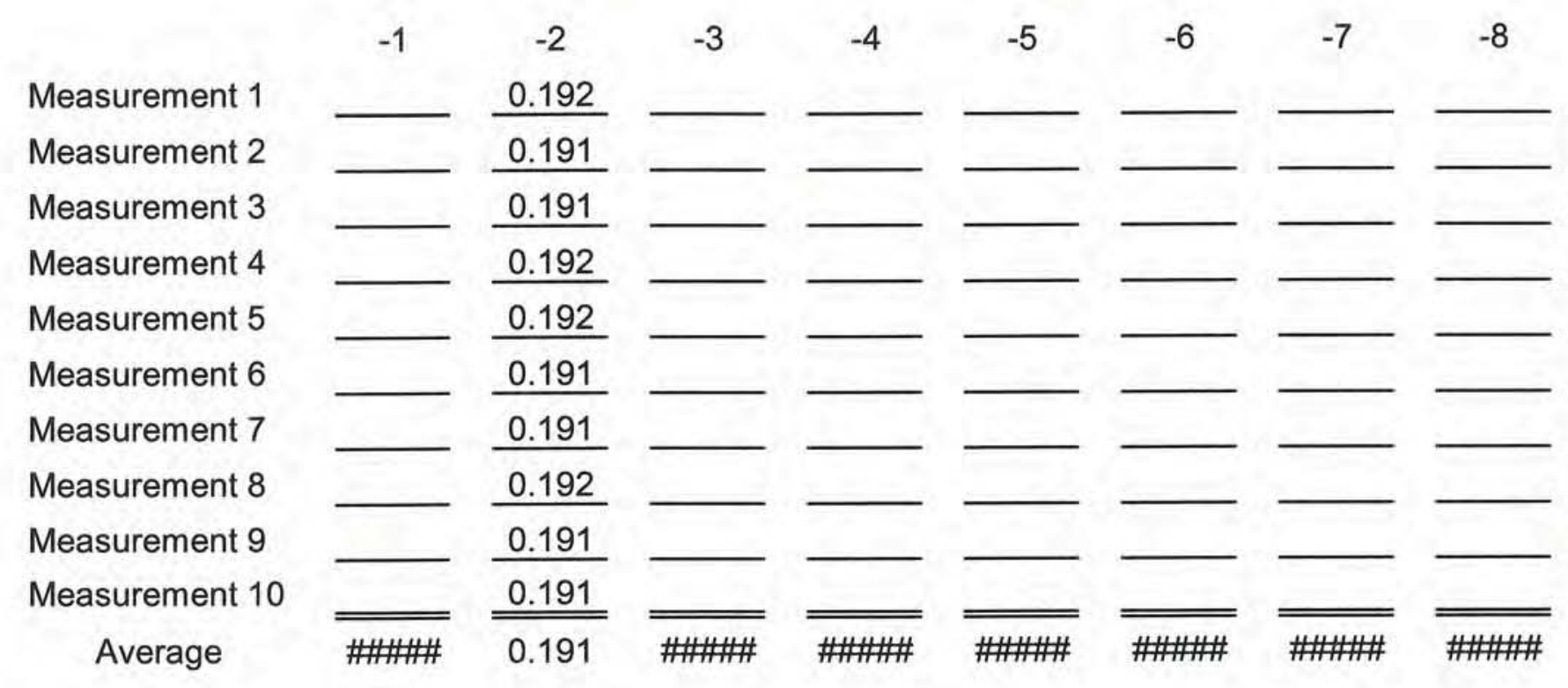

Soot Jachson 5/18/07

Calibrator (Signature / Date)

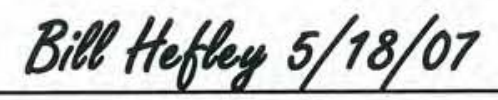

QA Officer (Signature / Date) 
Appendix D:

Field Data 


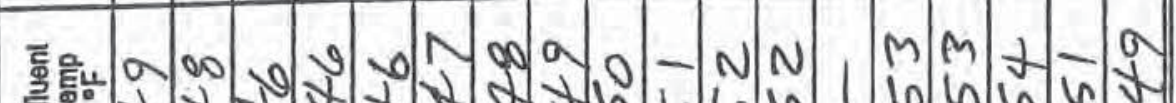

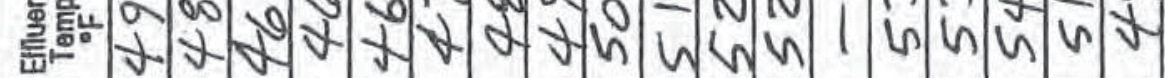

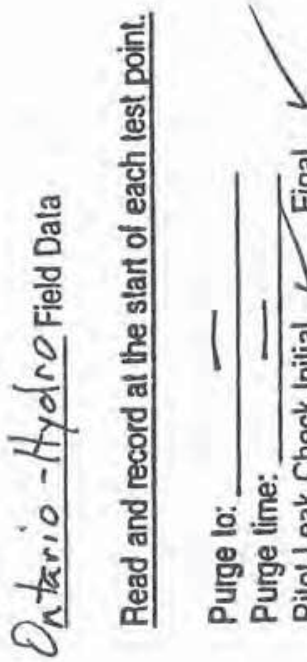

A

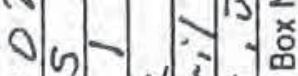

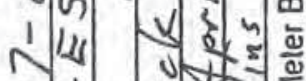

0.1 i

क म

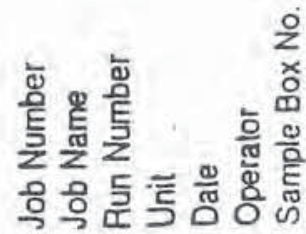

ठ․ㅠ.

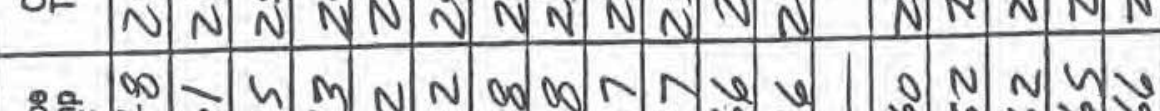

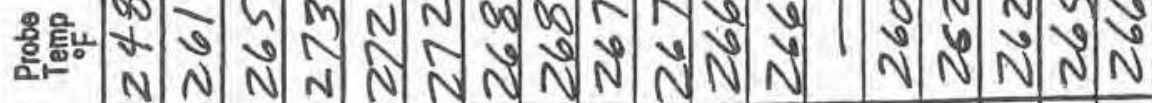

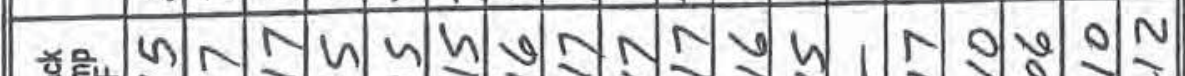

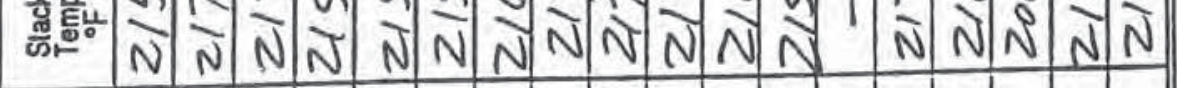

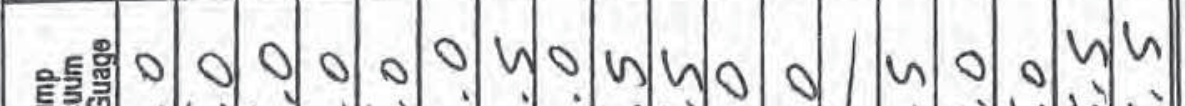

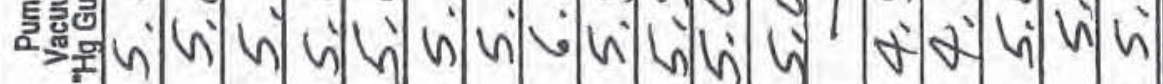

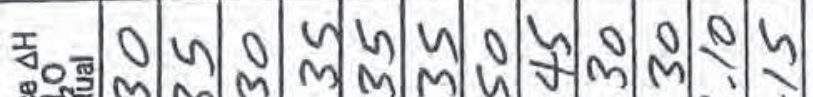

$a$ E

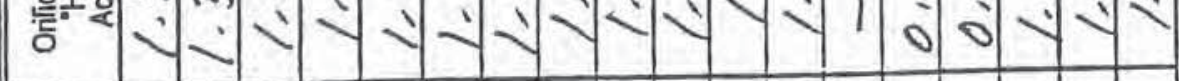

天о

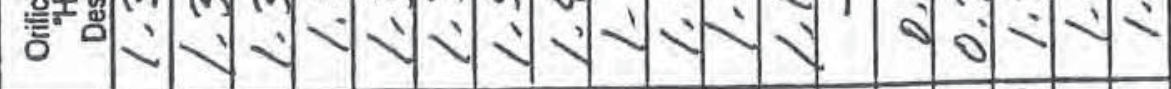

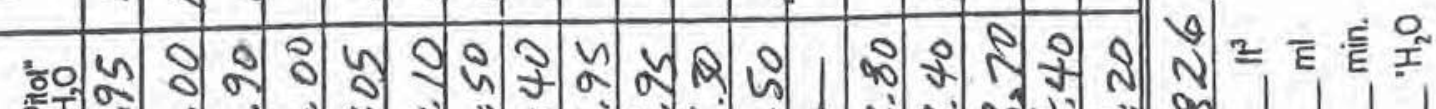

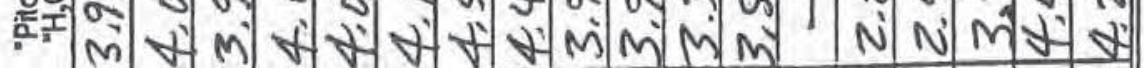

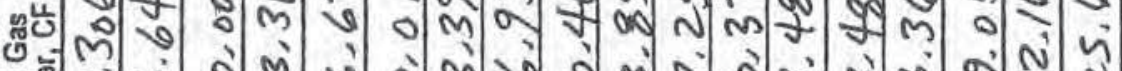

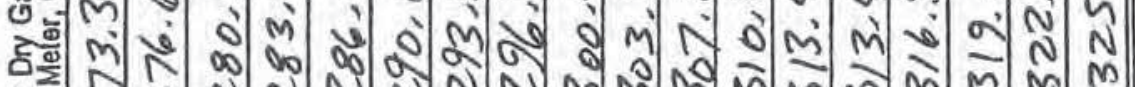
NNN N N N N MM M Mे M M M M M M

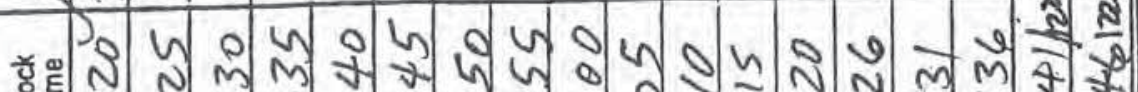

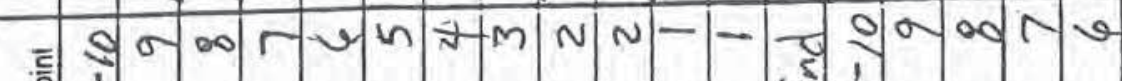
듬

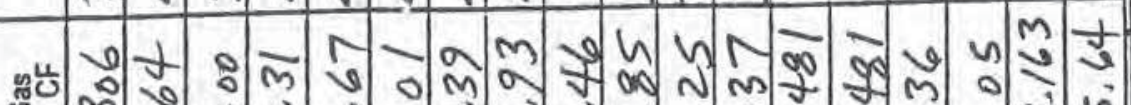

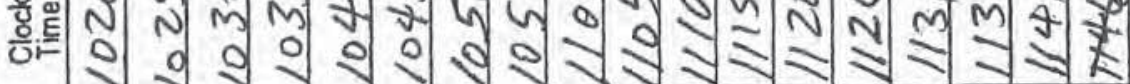

$\frac{1}{2} \frac{2}{2}$ $\overline{\bar{E}}$ i. के ॠँ

$x \times$ 敞 $\sqrt{0} \times$ 命

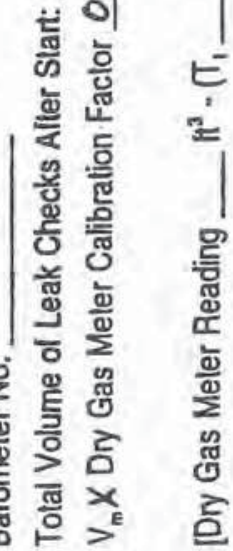
몰 ง

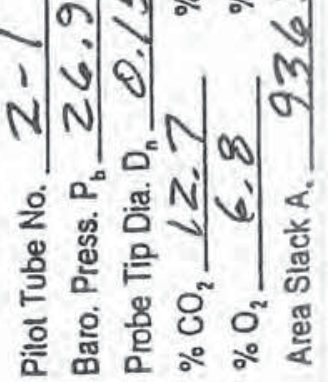

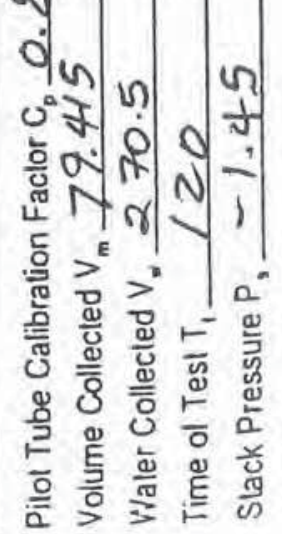


Impinger Box No. $M-3$

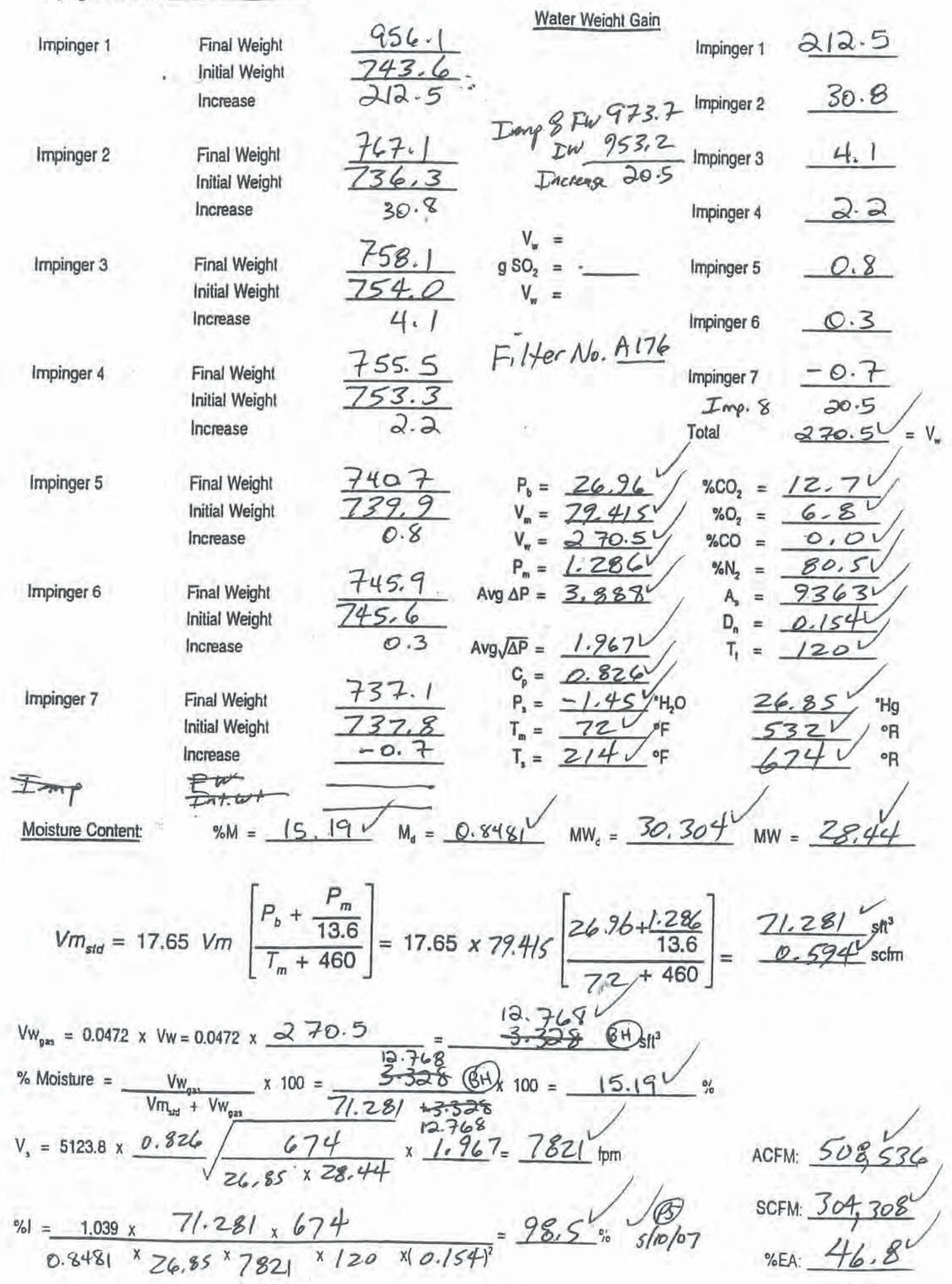




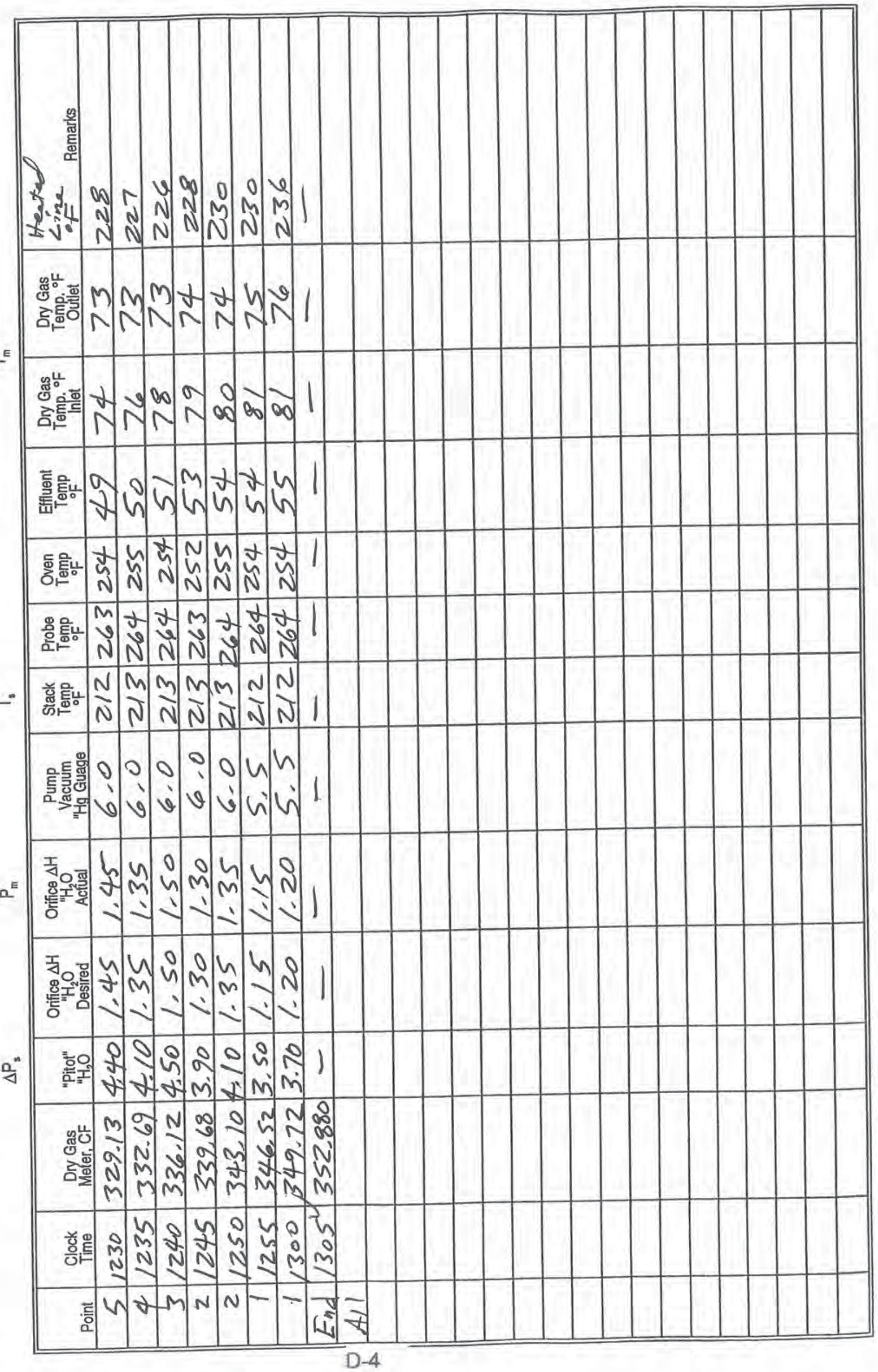



Impinger Box No.

$M-3$

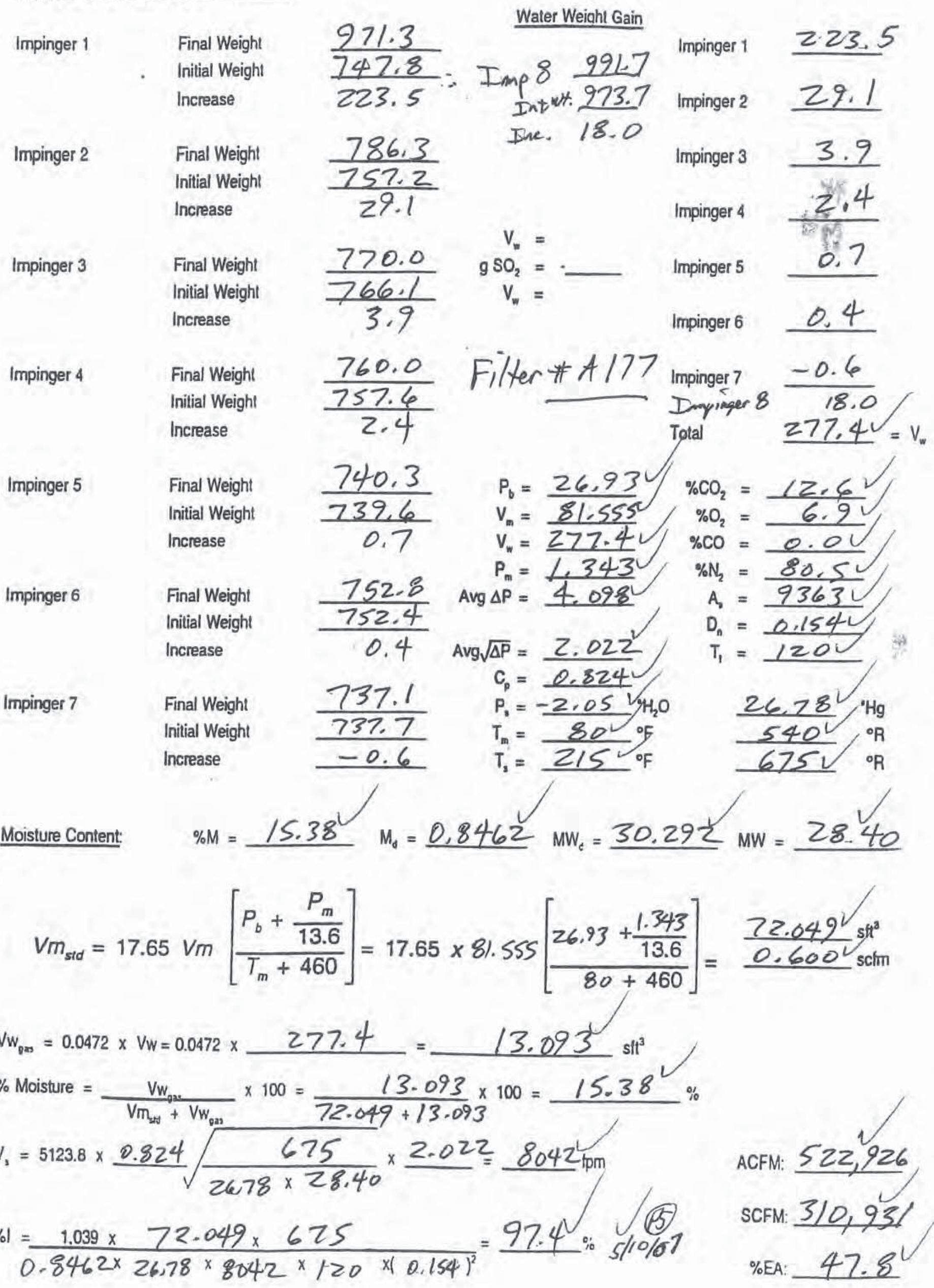




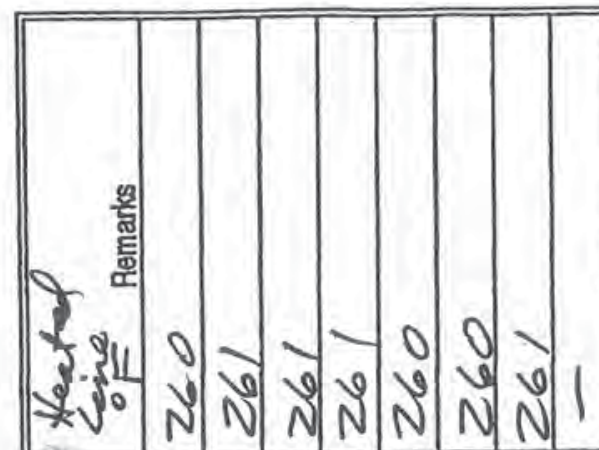

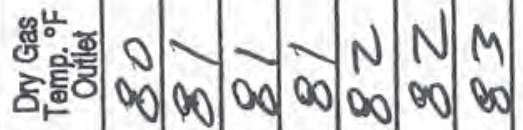

1

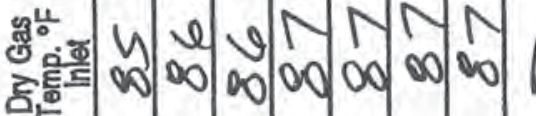

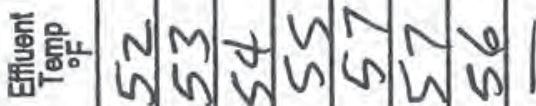

통

ơ

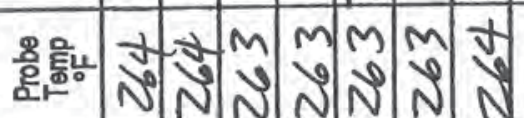

造

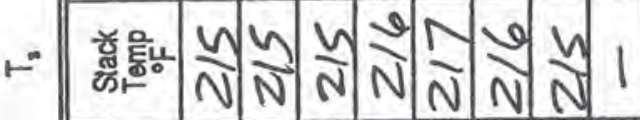

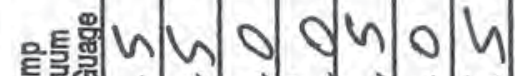

모웜

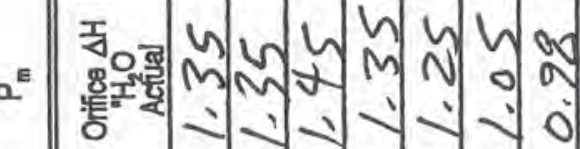

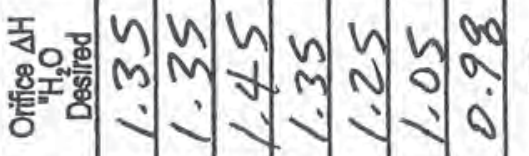

$n$
9
0

N.

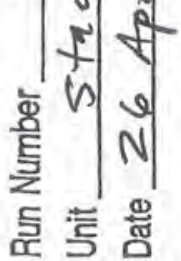

¿

ज小

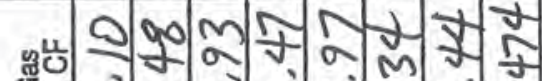

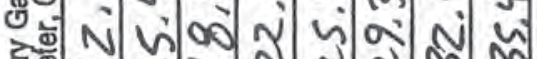

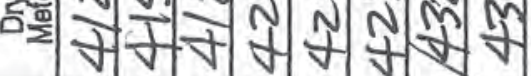

ชำ

아

$=\frac{1}{4}+m N \sim-7$ से 

Impinger Box No. $M-s$

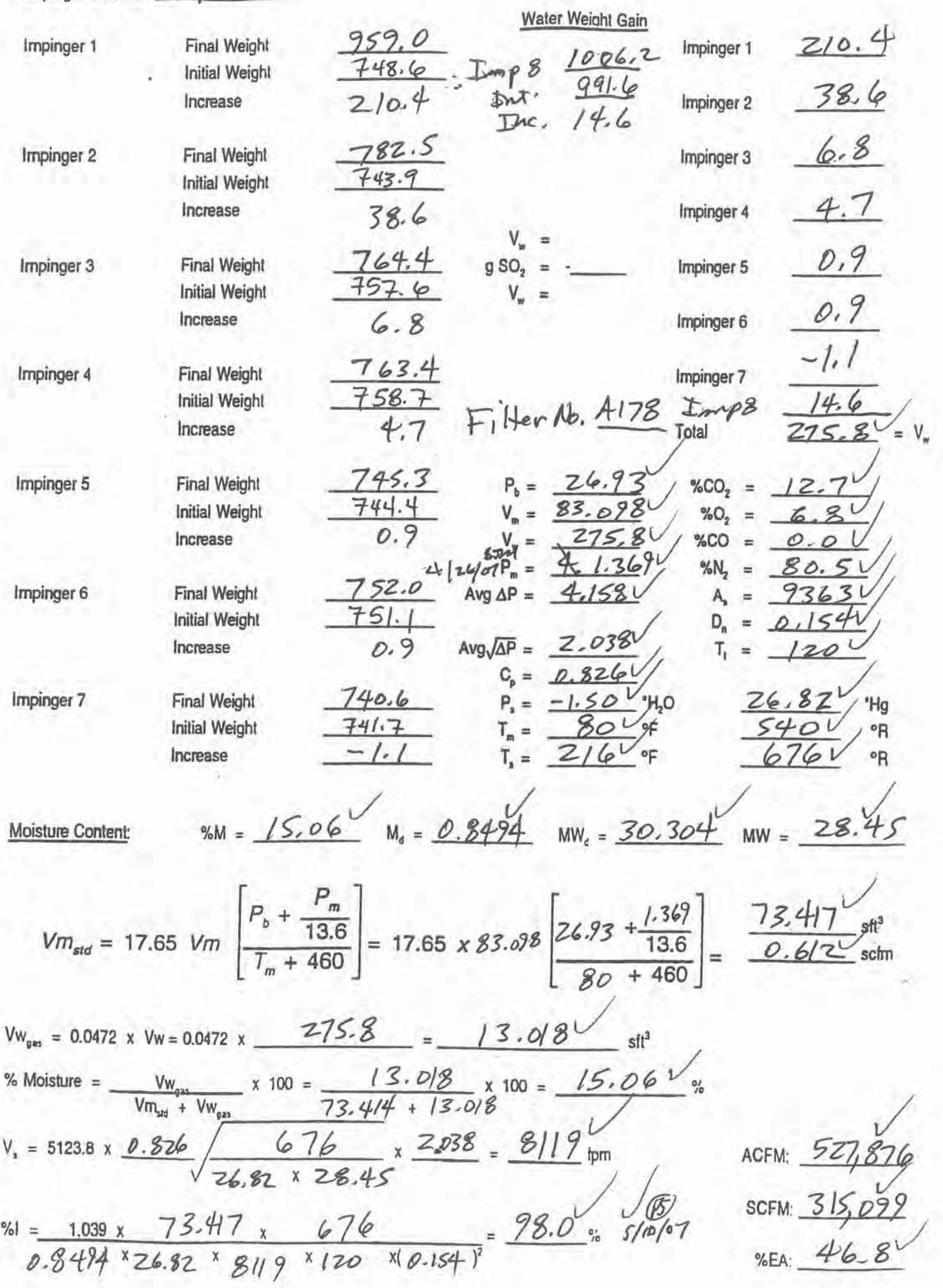




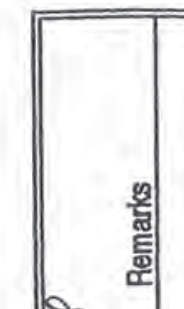

2.

हृ.

$\vdash^{E}$

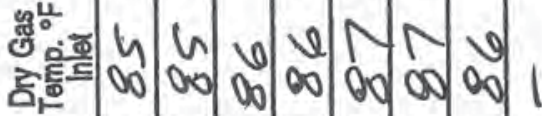

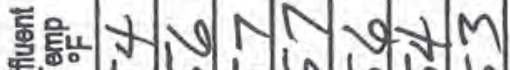

똟

告

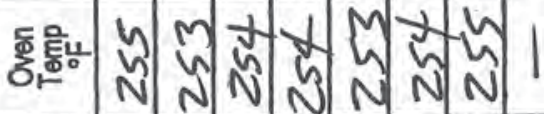

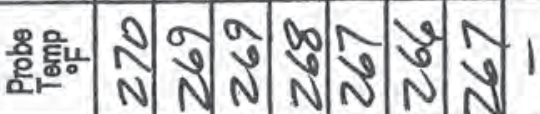

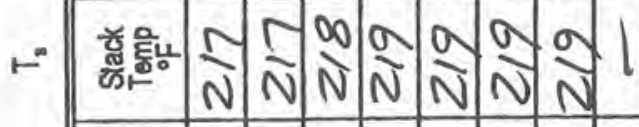

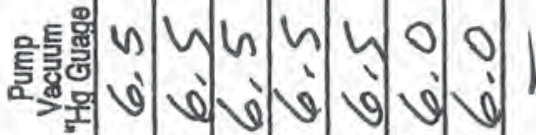

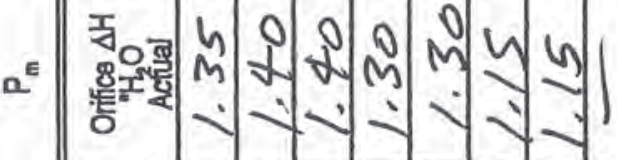

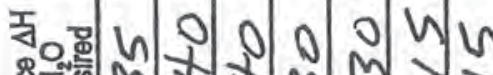

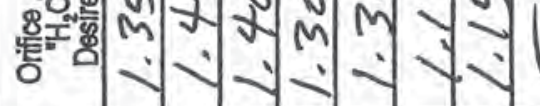

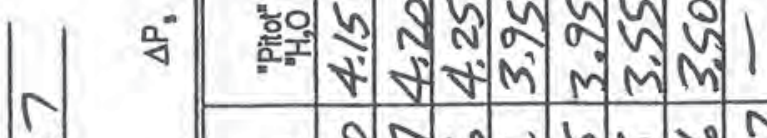

○)

N

MV

ff

$\$ 6$

ह $|N|$

宾专言

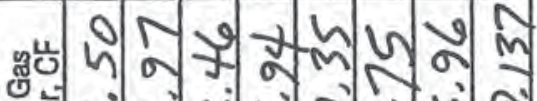

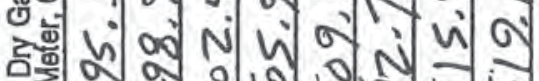

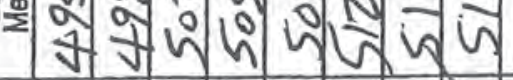

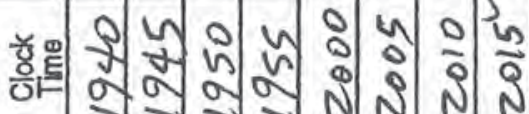

$$
\begin{aligned}
& \text { 言 } \begin{array}{l}
n \\
1 \\
N
\end{array}
\end{aligned}
$$




\section{ORSAT ANALYSIS DATA FORM}

Job \#: $\frac{O 7-024}{\text { Job Name: }} \frac{\frac{\text { ADA-ES }}{\text { Hardin, MT }}}{\text { Location: }}$
Date:
Operator:

Sample Location: Stack

Analytical Method:

3

Sample Type: Single Point Multi Point Grab Integrated

Leak Check: Time: 24 ms ( $\geq 4$ minutes) Rate: 0.0

Ambient Air Check:

\begin{tabular}{|l|c|}
\hline $\mathrm{CO}_{2}-\%$ Vol. & 0.0 \\
\hline $\mathrm{O}_{2}-\%$ Vol. & 20.9 \\
\hline $\mathrm{N}_{2}-\%$ Vol. & 79.1 \\
\hline
\end{tabular}

Run \#:

Run Time: $1020-1305$

\begin{tabular}{|l|c|c|c|c|}
\hline $\mathrm{CO}_{2}-\% \mathrm{Vol}$. & 12.7 & 12.8 & 12.7 & 12.7 \\
\hline $\mathrm{O}_{2}-\% \mathrm{Vol}$. & 6.8 & 6.7 & 6.8 & 6.8 \\
\hline $\mathrm{CO}-\% \mathrm{Vol}$. & 0.0 & 0.0 & 0.0 & 0.0 \\
\hline $\mathrm{N}_{2}-\% \mathrm{Vol}$. & 80.5 & 80.5 & 80.5 & 80.5 \\
\hline
\end{tabular}

Run \#: 2

Run Time: $1445-1650$

\begin{tabular}{|l|c|}
\hline $\mathrm{CO}_{2}-\%$ Vol. & $/ 2$. \\
\hline $\mathrm{O}_{2}-\%$ Vol. & 6.9 \\
\hline $\mathrm{CO}-\%$ Vol. & 0.0 \\
\hline $\mathrm{N}_{2}-\%$ Vol. & 80.5 \\
\hline
\end{tabular}

Analysis \#2

Analysis \#3

$12.6 \quad 12.6$

6.96 .9

$0.0 \quad 0.0$

80.5

80.5

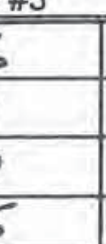

\begin{tabular}{|c|} 
Average $-\%$ Volume \\
\hline 12.6 \\
6.9 \\
0.0 \\
80.5 \\
\hline
\end{tabular}

Run \#: $\frac{3}{\text { Run Time: } 18 / 0-2015}$

$\mathrm{CO}_{2}-\%$ Vol.

Analysis \#1

Analysis \#2

Analysis \#3

Average - $\%$ Volume

$\mathrm{O}_{2}-\%$ Vol.

CO - \% Vol.

12. 7

$\mathrm{N}_{2}-\%$ Vol.

6. 8

0.0

80.5

\begin{tabular}{c|c}
12.7 & 12.7 \\
6.8 & 6.8 \\
0.0 & 0.0 \\
80.5 & 80.5
\end{tabular}

1.2. 7

6. 8

0.0 


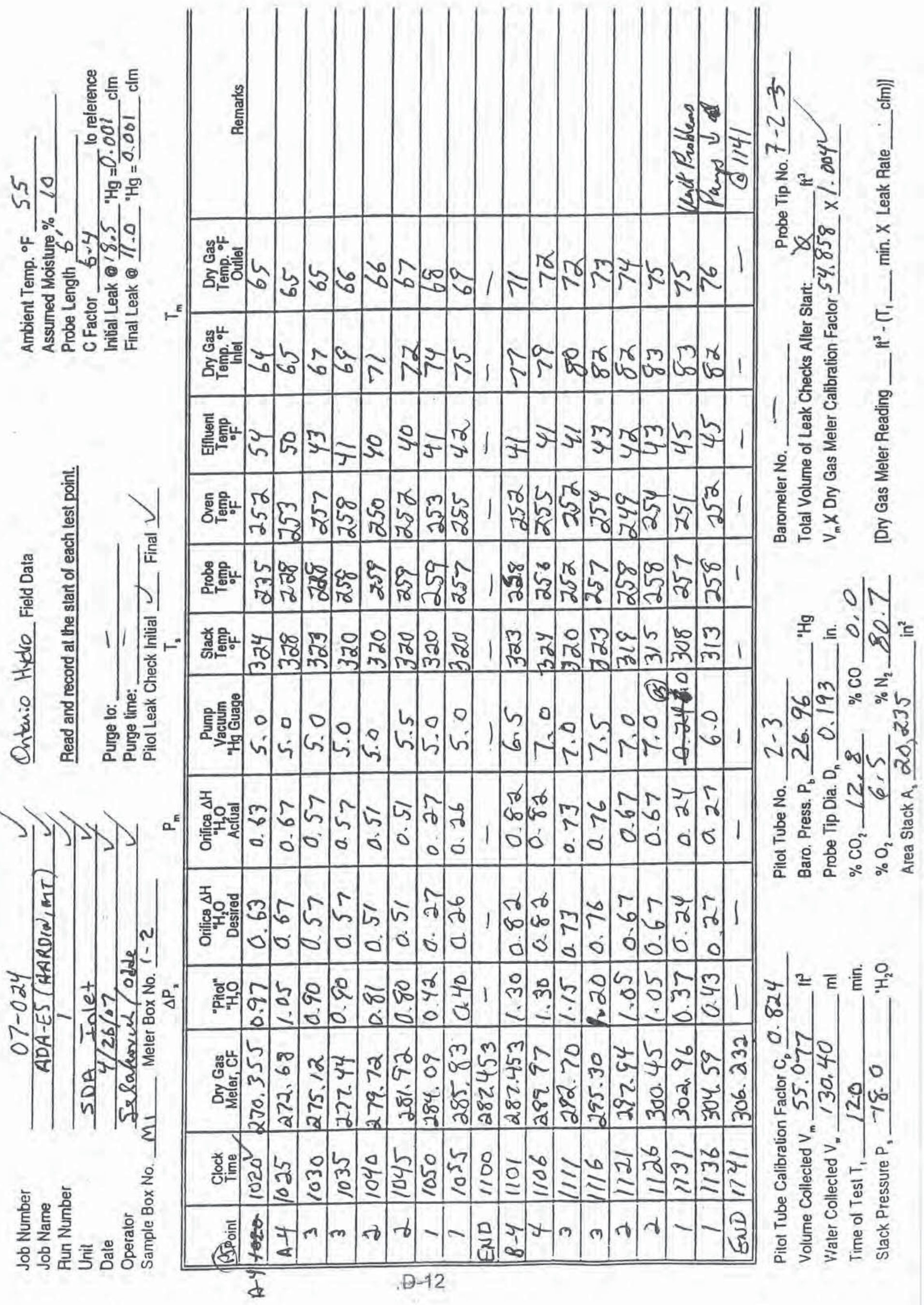


Impinger Box No.

M1

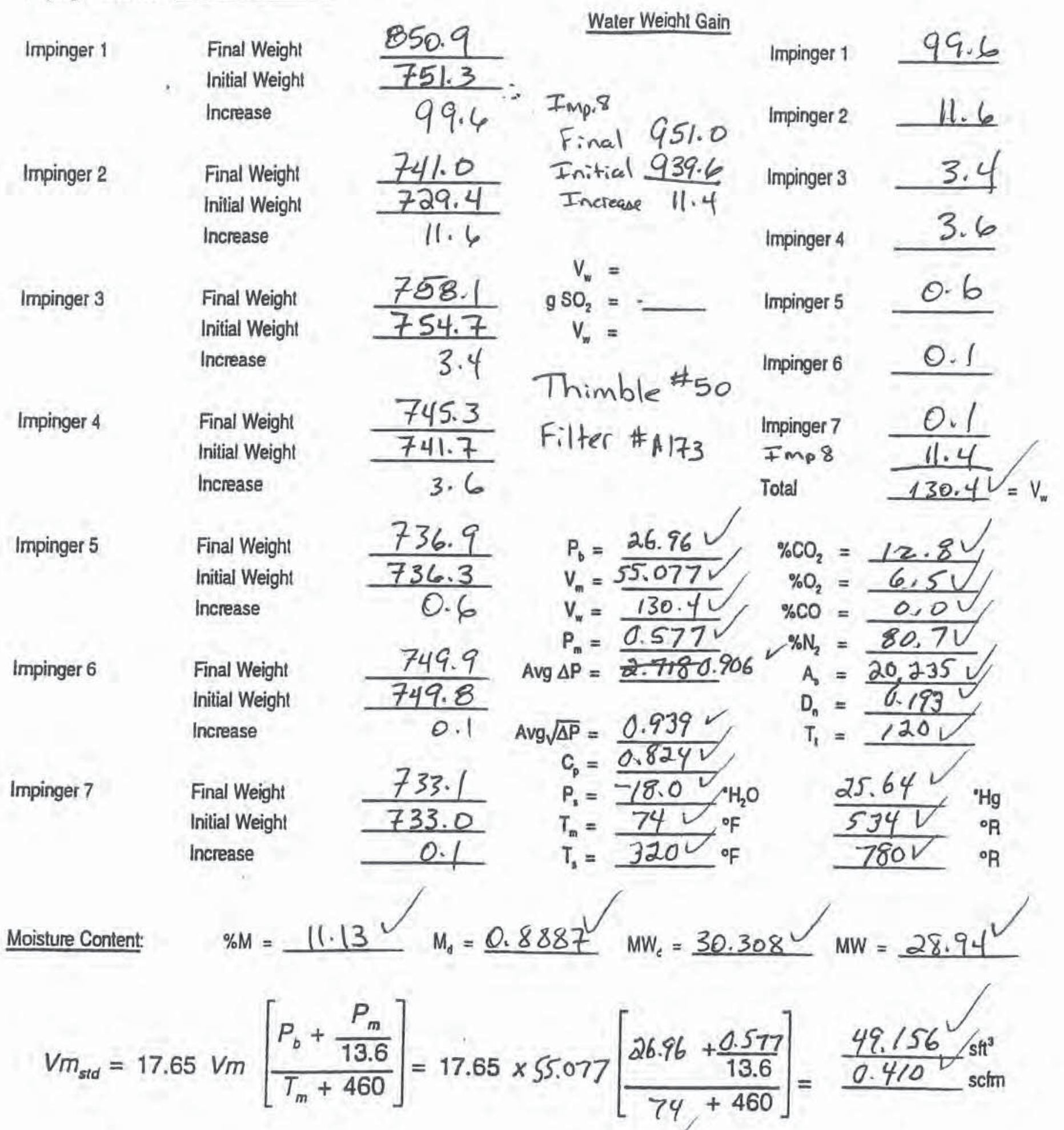

$V w_{p e s}=0.0472 \times V w=0.0472 \times$

130.4 $=6.155$ V $\mathrm{sIt}^{3}$

$\%$ Moisture = $\times 100=$ $6 \cdot 155 \times 100=$ $11.130 \%$

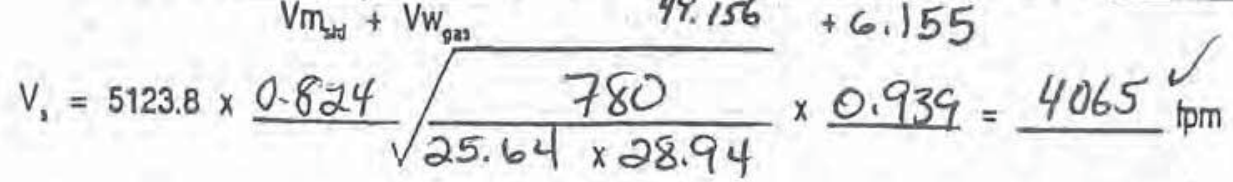
ACFM: $\frac{571,169^{\prime}}{295,561^{\prime}}$

$\%=\frac{1.039 \times 780 \times 49.156}{0.8887 \times 25.64 \times 4065 \times 120 \times(0.193)^{2}}=\frac{96.2 \%}{5 / 10 \% 7}$ 
善

(4) 5

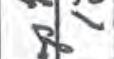

2

边.

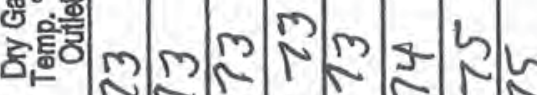

E nNina

造

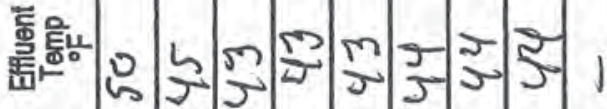

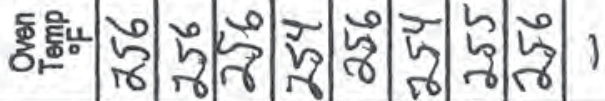

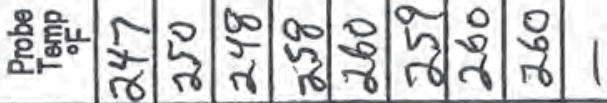

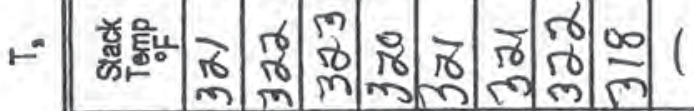

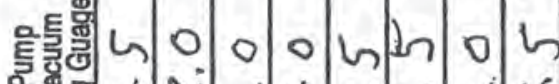

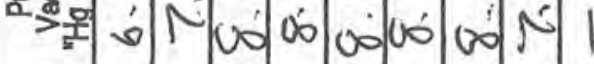

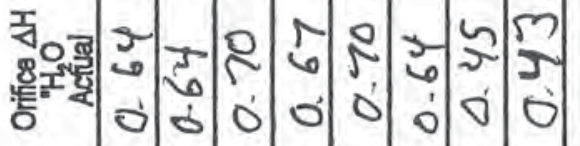

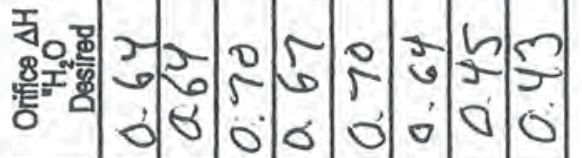

$a^{\circ}$

혼워 이의 0 . 015

- -0

巡

赔

nิm

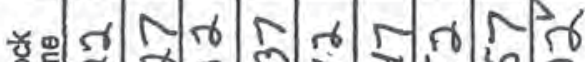

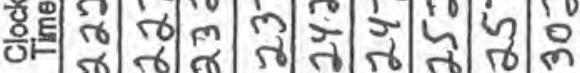

17

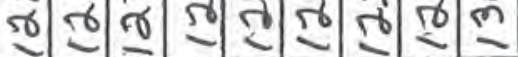

点部

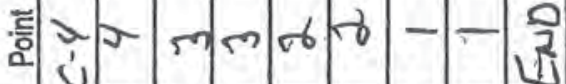




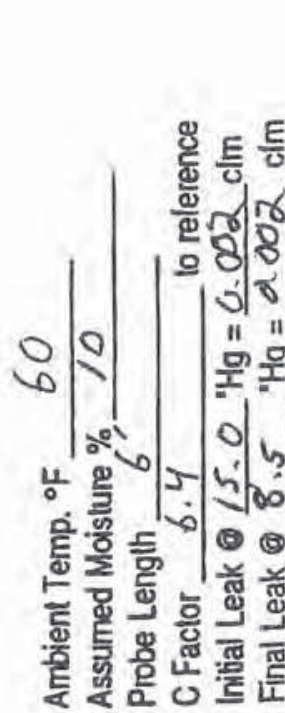

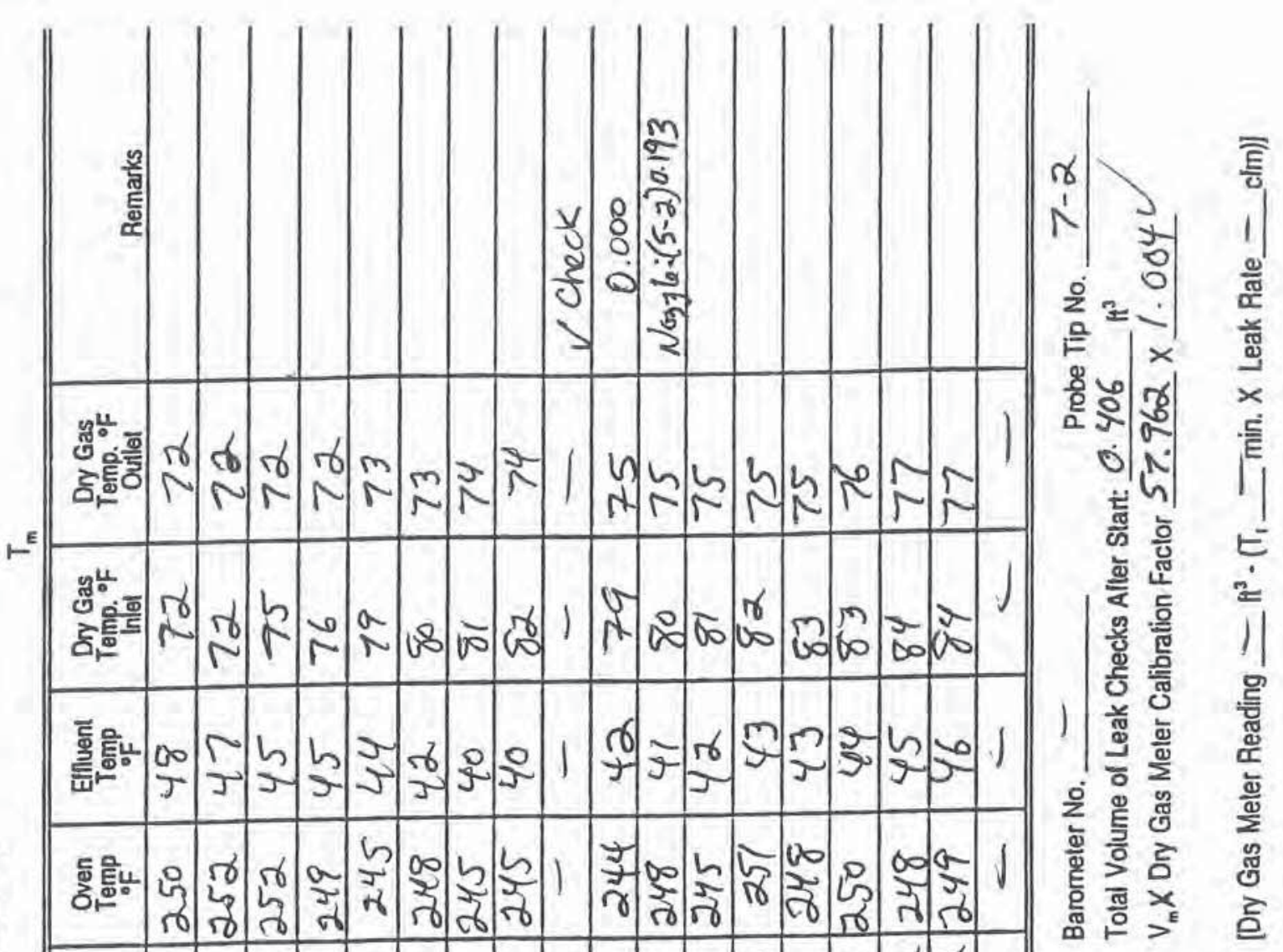

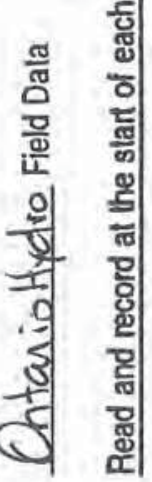

뜬

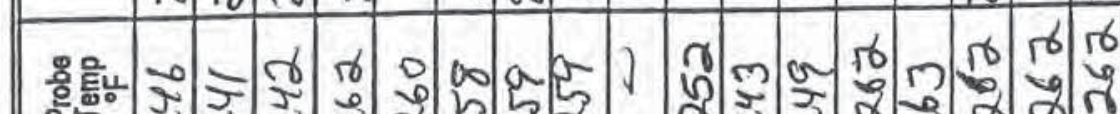

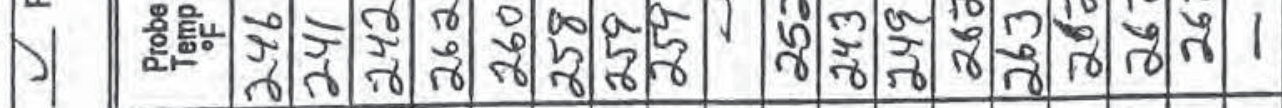

1 耨

० क०

बi⿺

으 홍

트윰

总罟흠

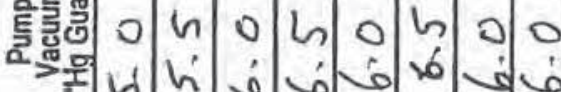

: 0.000 ln

MN N N

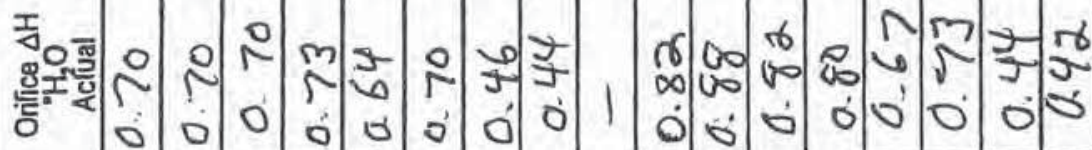

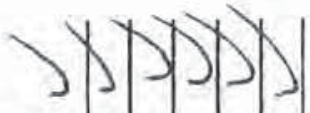

$a^{E}$

है

西

0.

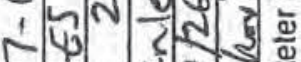

-

充

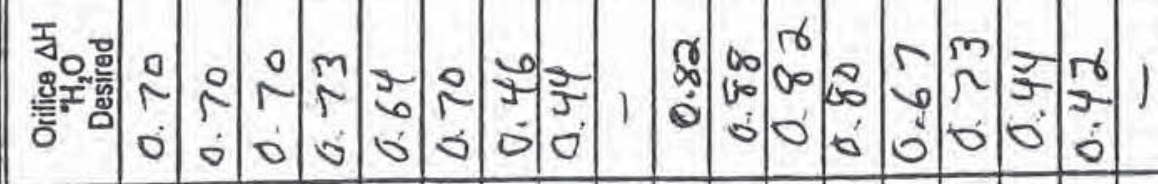

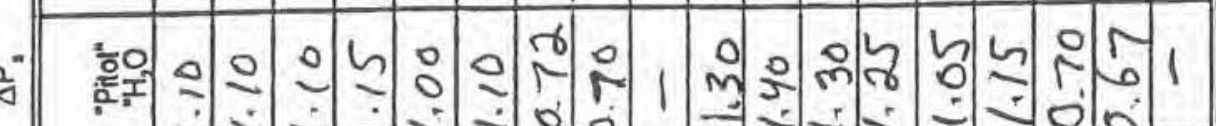

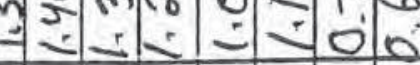

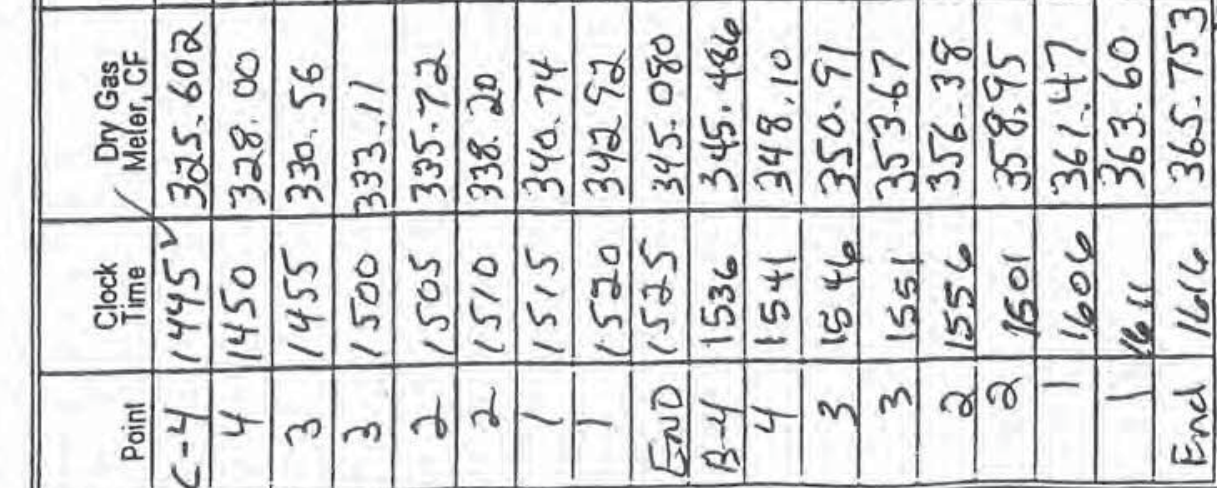

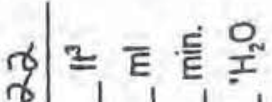

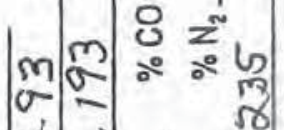

온

अช ชิ

$8 \int_{0}^{2}+2$

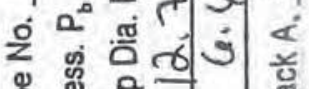

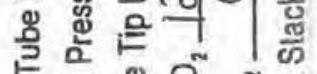

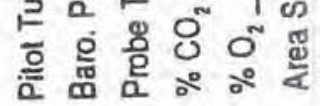

$\infty$

익

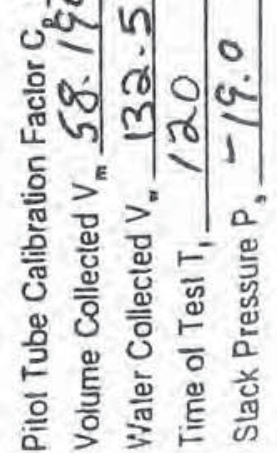


Impinger Box No. $M \alpha$

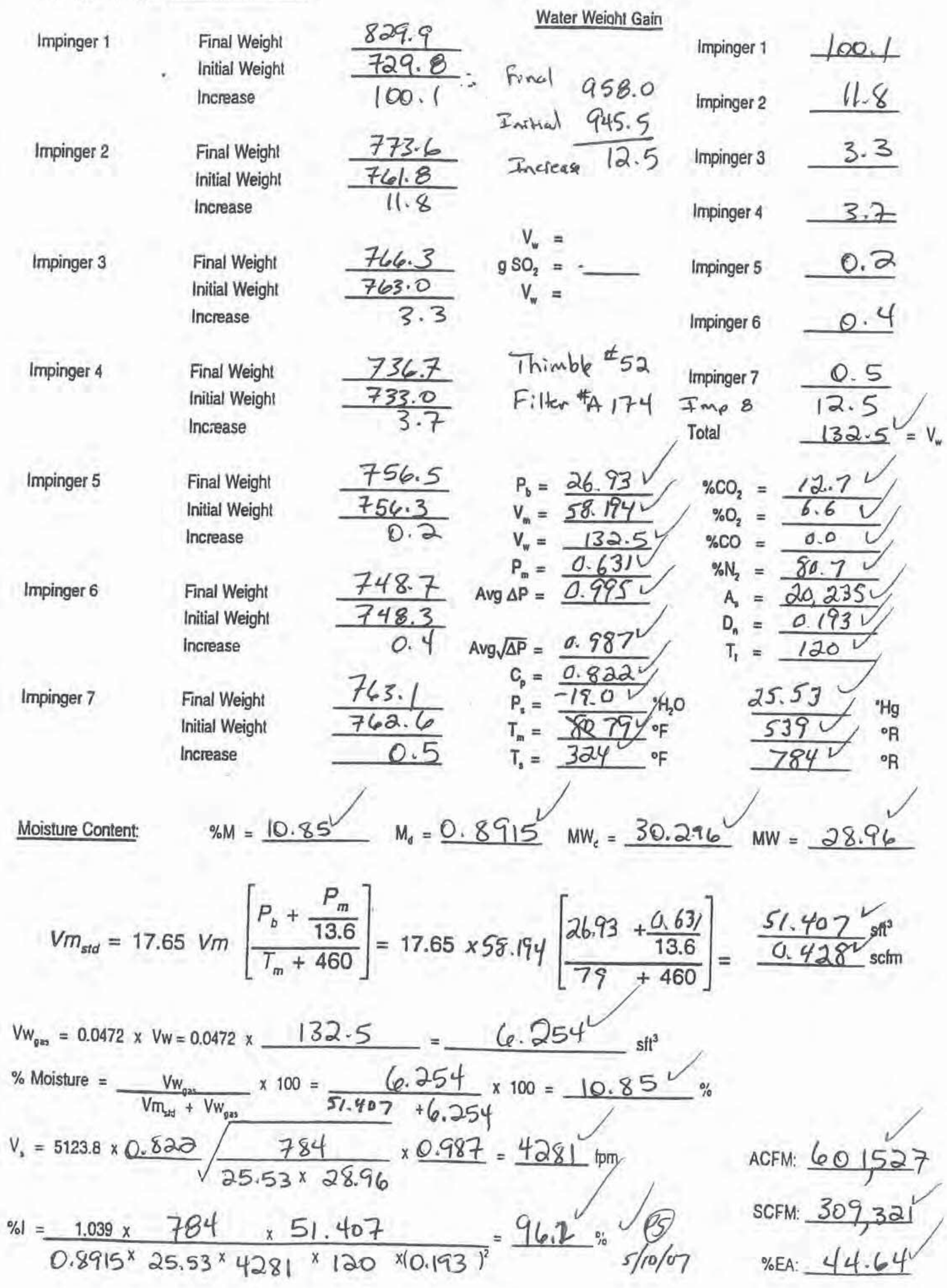




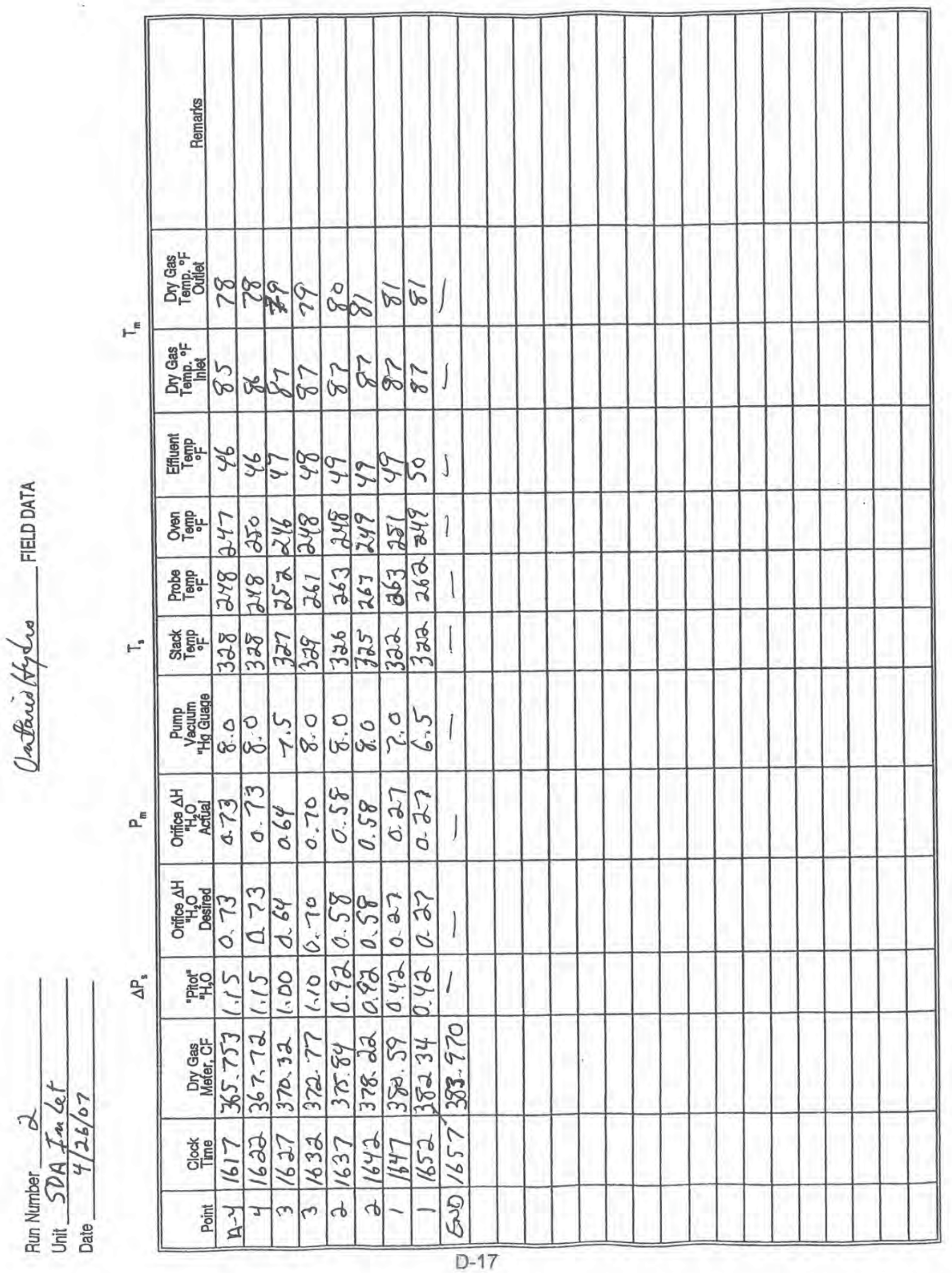


Impinger Box No.

$M I$

Impinger 1

Final Weight
$\begin{aligned} & \text { Initial Weight } \\ & \text { Increase }\end{aligned}$

Water Weiaht Gain

Impinger 196.8

Increase

Impinger 2

Final Weight

743.8

Final 960.4

Impinger $2 \quad 14.5$

Initial Weight

increase

\begin{tabular}{r}
$\frac{743.8}{729.3}$ \\
\hline 14.5 \\
$\frac{762.2}{760.0}$ \\
2.2
\end{tabular}

Initial 951.0

Increare 9.4

Impinger 3

22

Impinger 4

3.4

Impinger 3

Final Weight

$\mathrm{V}_{\mathrm{w}}=$

Initial Weight

Increase

$\mathrm{gSO}_{2}=$

Impinger

0.2

$\mathrm{V}_{\mathrm{w}}=$

Thimble $* 51$ Impinger $6 \quad 0.1$

Impinger 4

Final Weight

$\frac{750.7}{\frac{747.3}{3.4}}$

Filter ${ }_{A 175}$

Increase

Impinger 5

Final Weight

$\frac{732.4}{732.2}$

$P_{b}=\frac{26.93}{58.127}$
$V_{m}=\frac{126.5 \mathrm{~V}}{V_{w}}=\frac{1.626}{}$

$\frac{764.7}{764.6}$

$P_{m}=\frac{0.626}{0.986 v}$

Impinger 6

Final Weight

Initial Weight

Increase

Avg $\Delta \mathrm{P}=0.986 \mathrm{~V}$

$\operatorname{Avg} \sqrt{\Delta \mathrm{P}}=0.984 \mathrm{~V}$

$C_{p}=0.824 \mathrm{~L}$

$\operatorname{Total}_{\text {Impinger } 7} \frac{-0.1}{\frac{9.4}{126.5 \mathrm{~V}}}=\mathrm{V}_{w}$

Impinger 7

Final Weight

729.1

$P_{s}=-16.0 \mathrm{~V} / \mathrm{H}_{2} \mathrm{O}$

$T_{m}=87 \sim \mathrm{F}$

Increase

$-0.1$

$T_{1}=325 \mathrm{~V}{ }^{\circ} \mathrm{F}$

$\% \mathrm{CO}_{2}=12.7$

$\% \mathrm{O}_{2}=6.6$

$\% \mathrm{CO}=0.0 \mathrm{~V}$

$\% \mathrm{~N}_{2}=\frac{80.7 \mathrm{~V}}{20.235}$

$A_{3}=20,235$

$D_{n}=\frac{0.191 \mathrm{~V}}{170 \mathrm{~V}}$

$T_{1}=120$

$\frac{25.75 \mathrm{~V}}{\frac{547 \mathrm{~V}}{785 \mathrm{~V}}{ }^{\circ \mathrm{H}}}$

Moisture Content:

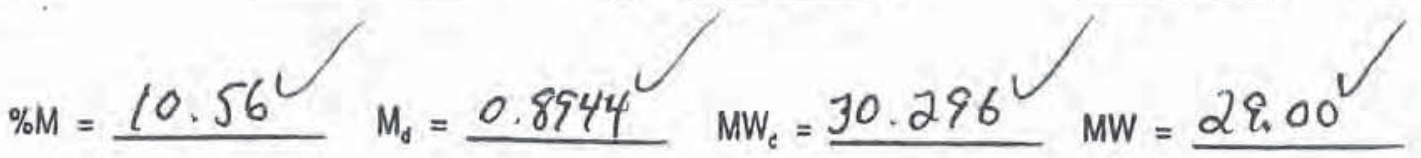

$$
V m_{s t d}=17.65 \mathrm{Vm}\left[\frac{P_{b}+\frac{P_{m}}{13.6}}{T_{m}+460}\right]=17.65 \times 58.127\left[\frac{26.93+\frac{0.626}{13.6}}{87+460}\right]=\frac{50.596}{0.422} \mathrm{sctm}
$$

$V_{W_{p s}}=0.0472 \times V_{W}=0.0472 \times$

126.5

5.971

$=$ $s t^{3}$

$\%$ Moisture = $\times 100=$

5.971 $\times 100=$ 10.56 $\%$

$v_{s}=5123.8 \times \frac{0.824}{\frac{785}{25.75 \times 29.00}} \times \frac{0.984}{4260} \mathrm{tpm}$

$\%=\frac{1.039 \times 785 \times 50.596}{0.8944 \times 25.75 \times 4260 \times 120 \times(0.191)^{2}}=96.1 \% \frac{\sqrt{(85)}}{510 \% 7}$

ACFM: 598,553

SCFM: $311,069 \mathrm{O}$

\%EA 44.6 
窑

势部

$\vdash$

高要

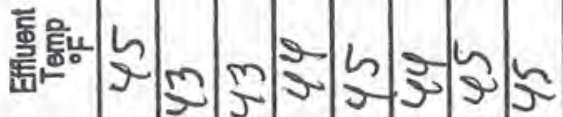

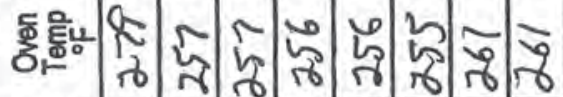

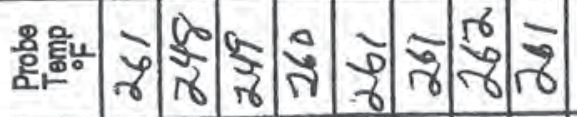

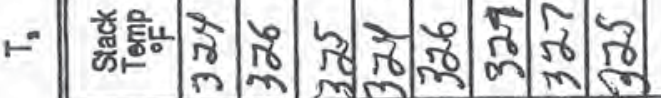

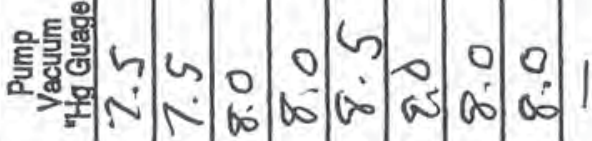

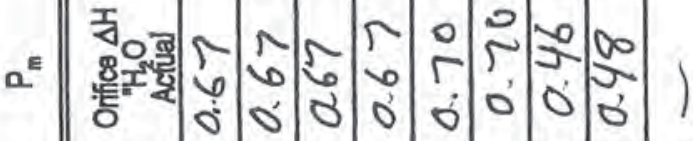

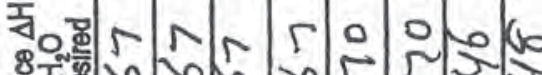

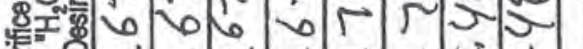

o a b́

ब.

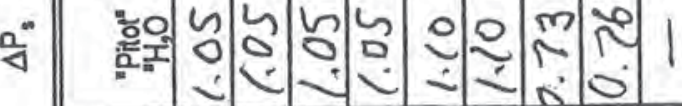

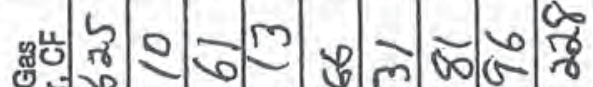

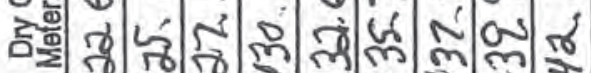

भी

7.

ol a m

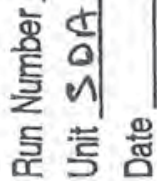

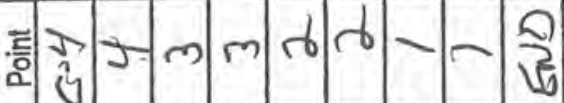




\section{ORSAT ANALYSIS DATA FORM}

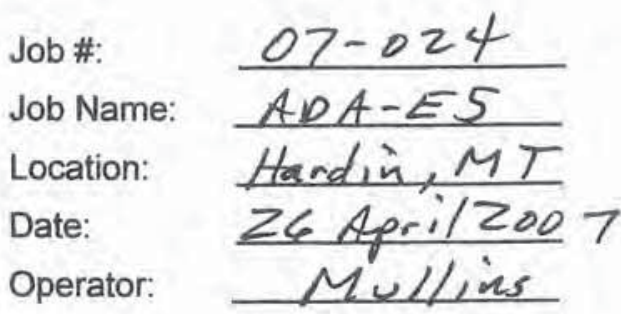

Sample Location: $\frac{S D A \text { In }}{3}$ Analytical Method:

Sample Type: Single Point/Multi Point Grab/4ntegrated

Leak Check: Time: 24 hus ( 24 minutes) Rate: 0.0

Ambient Air Check:

\begin{tabular}{|l|l|}
\hline $\mathrm{CO}_{2}-\%$ Vol. & 0.0 \\
\hline $\mathrm{O}_{2}-\%$ Vol. & 20.9 \\
\hline $\mathrm{N}_{2}-\%$ Vol. & 79.1 \\
\hline
\end{tabular}

Run \#:

Run Time:

\begin{tabular}{|l|r|r|r|c|}
\hline $\mathrm{CO}_{2}-\%$ Vol. & 12.8 & $12-8$ & 12.8 & 12.8 \\
\hline $\mathrm{O}_{2}-\%$ Vol. & 6.5 & 6.5 & 6.5 & 6.5 \\
\hline $\mathrm{CO}-\% \mathrm{Vol}$. & 0.0 & 0.0 & 0.0 & 0,0 \\
\hline $\mathrm{N}_{2}-\%$ Vol. & 80.7 & 80,7 & 80.7 & 80.7 \\
\hline
\end{tabular}

Run \#: $\quad$ Z

Run Time:

Analysis \#1

Analysis \#2

Analysis \#3

Average - $\%$ Volume

\begin{tabular}{|l|c|c|c|c|}
\hline $\mathrm{CO}_{2}-\%$ Vol. & 12.7 & 12.7 & 12.7 & 12.7 \\
\hline $\mathrm{O}_{2}-\%$ Vol. & 6.6 & 6.6 & 6.6 & 6.6 \\
\hline $\mathrm{CO}-\% \mathrm{Vol}$. & 0.0 & 0.0 & 0.0 & 0.0 \\
\hline $\mathrm{N}_{2}-\%$ Vol. & 80.7 & 80.7 & 80.7 & 80.7 \\
\hline
\end{tabular}

Run \#:

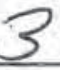

Run Time: Analysis \#1 Analysis \#2 Analysis \#3 Average - $\%$ Volume

\begin{tabular}{|l|r|r|r|c||}
\hline $\mathrm{CO}_{2}-\% \mathrm{Vol}$. & $12-7$ & 12.7 & 12.7 & 12.7 \\
\hline $\mathrm{O}_{2}-\% \mathrm{Vol}$. & $6-6$ & 6.6 & 6.6 & 6.6 \\
\hline $\mathrm{CO}-\% \mathrm{Vol}$. & 0.0 & 0.0 & 0.0 & 0.0 \\
\hline $\mathrm{N}_{2}-\% \mathrm{Vol}$. & 80.7 & 80.7 & 80.7 & 80.7 \\
\hline
\end{tabular}



Impinger Box No. $1-1$

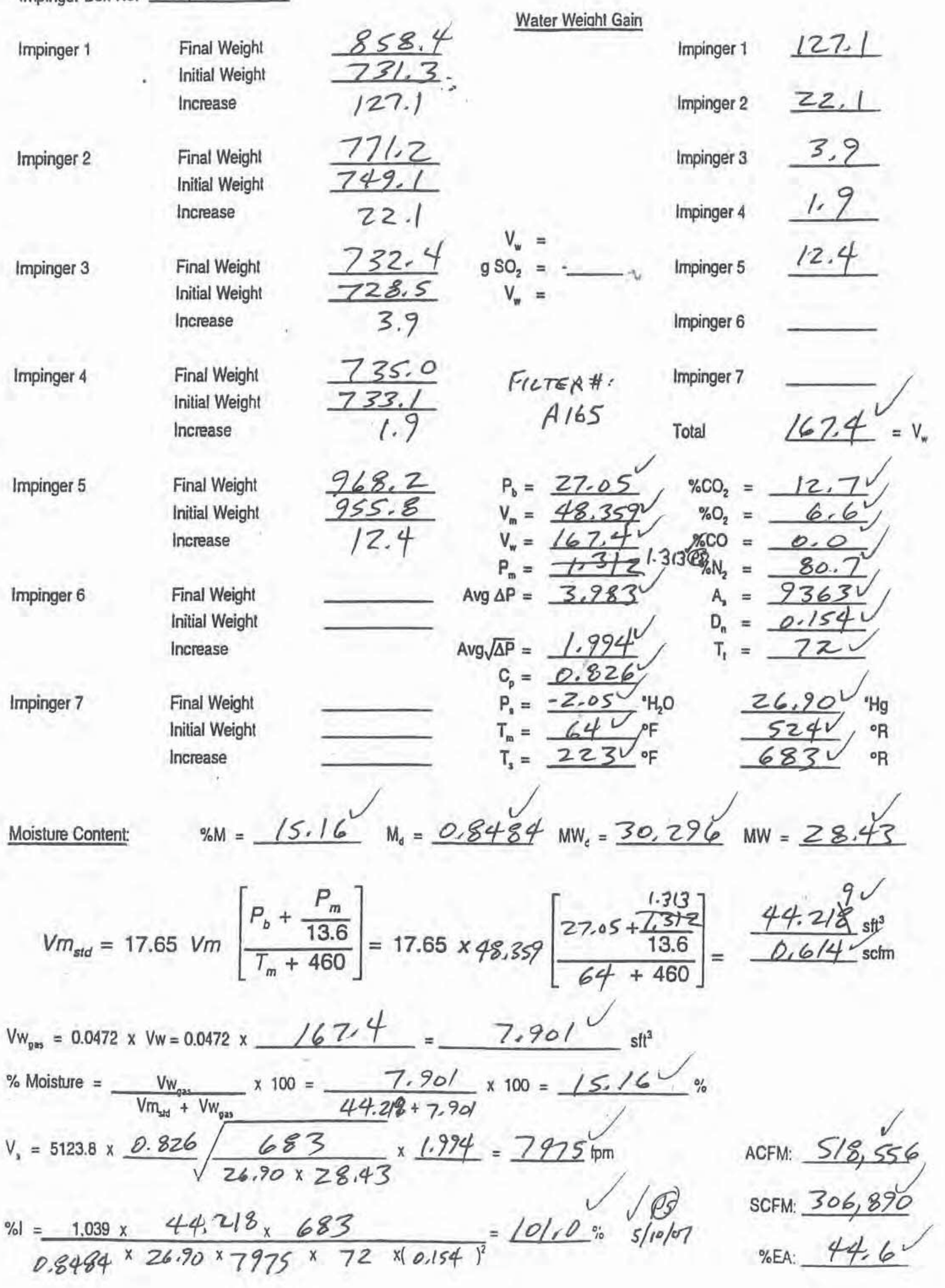




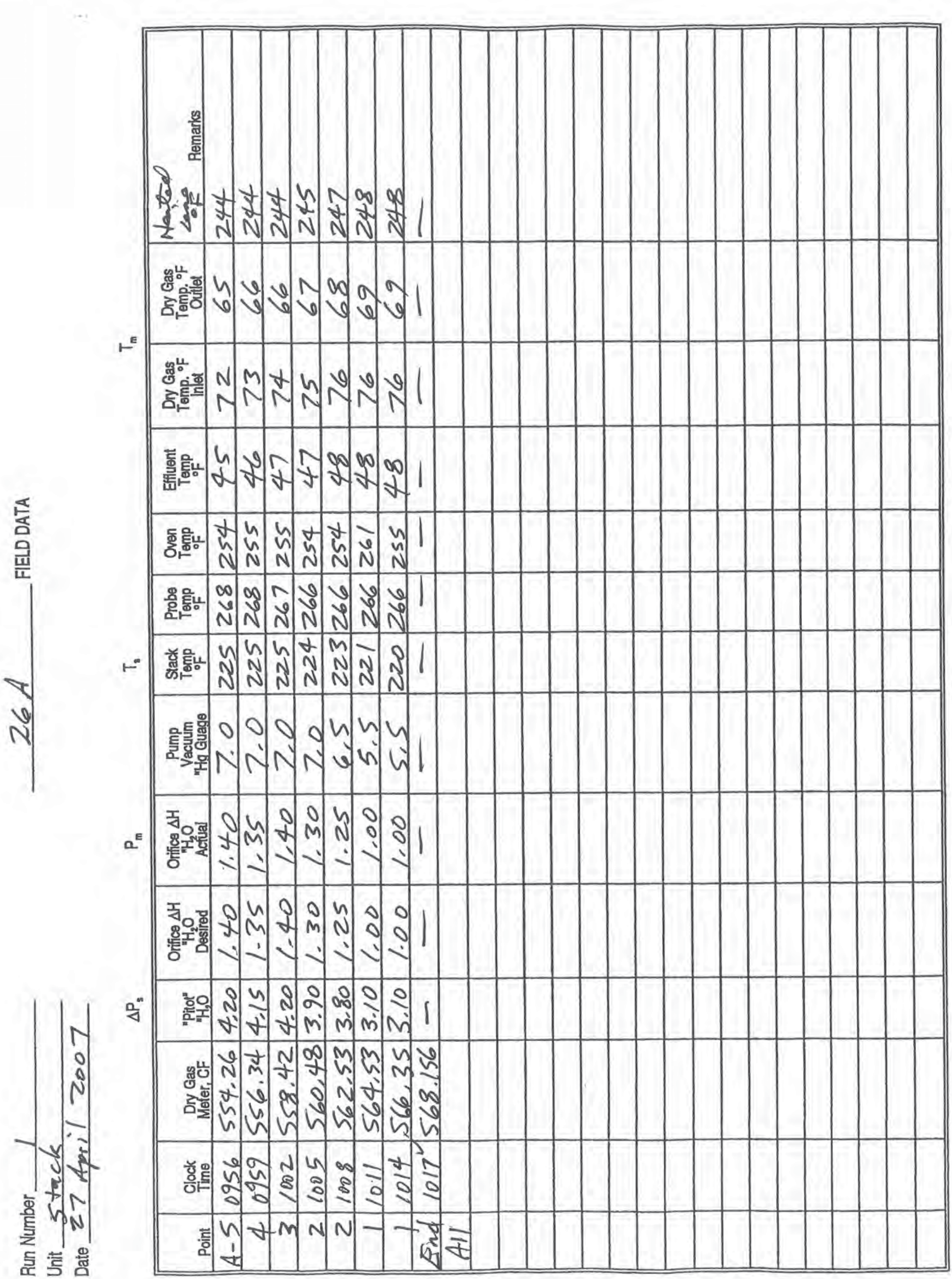



Impinger Box No. $1-2$

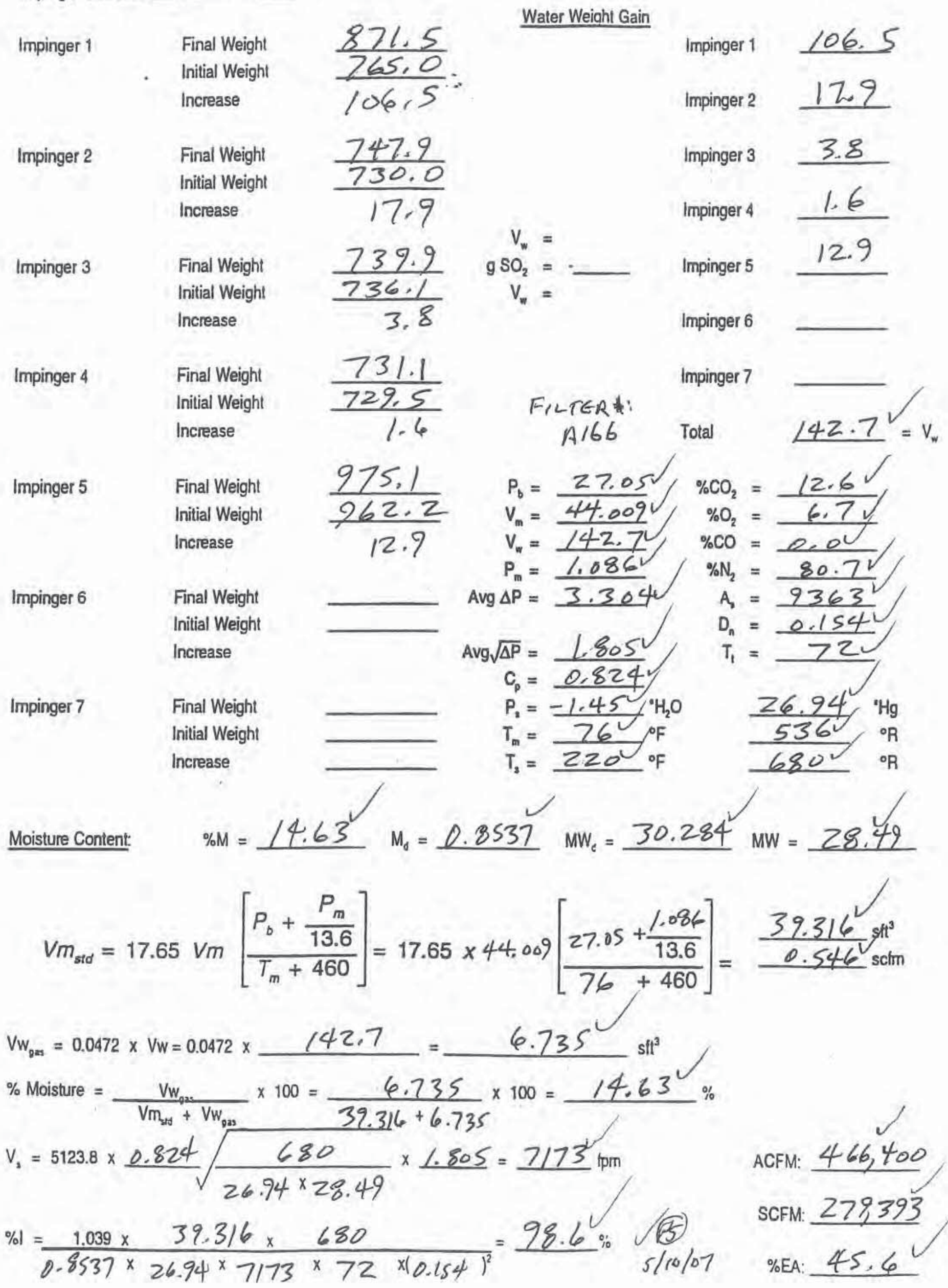


害

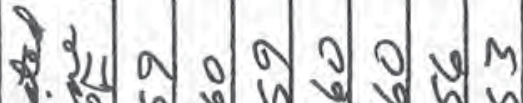

\&V N N N N N N

起

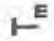

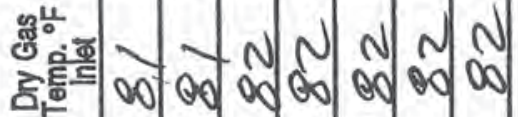

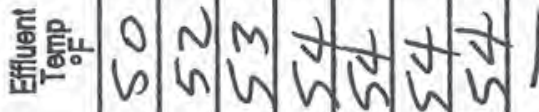

遥
亚

t

6

N

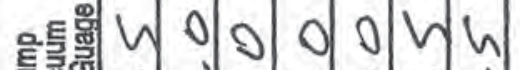

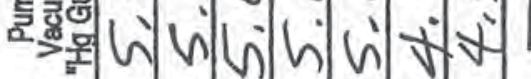

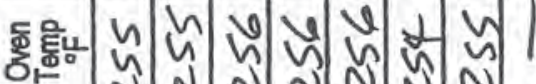

NNNNNNN

윻

呩N N N

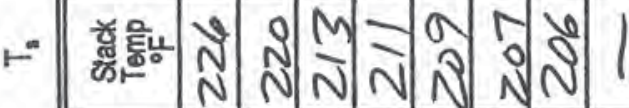

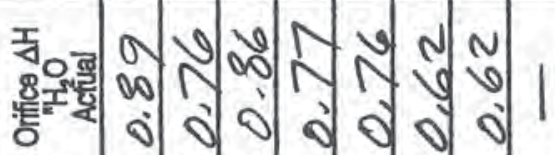

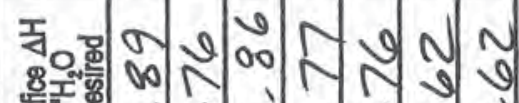

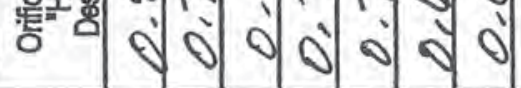

$\mid \begin{aligned} & n \\ & \text { ก } \\ & \text { | }\end{aligned}$

$\sqrt{1}=$

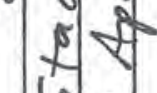

要 $N$

点

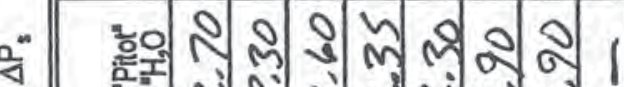

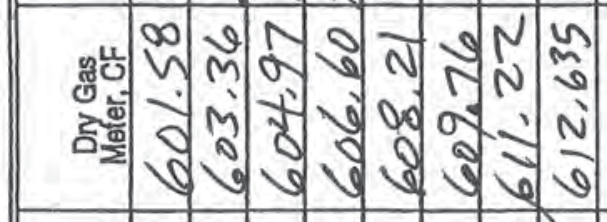

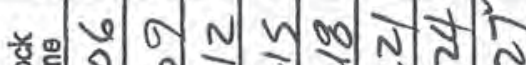

ठํㅠ N N N N N N N

둥 $\begin{aligned} & n \\ & \infty\end{aligned}$ 


\section{은 틍 틍}

(4)

.

$\left.0\right|_{1} \mid \begin{array}{lll}0 & 0 & 0 \\ & 11 & 0 \\ & 11 & 11\end{array}$

엔.

×

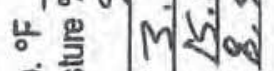

응

틀 亭

등혀 응 엉

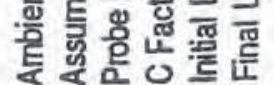

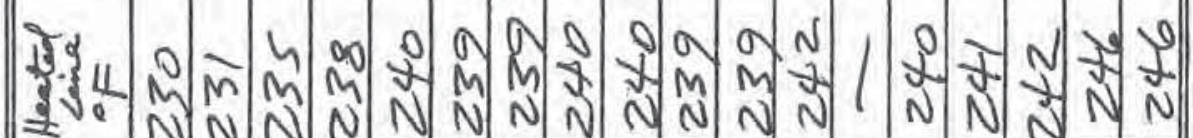

(

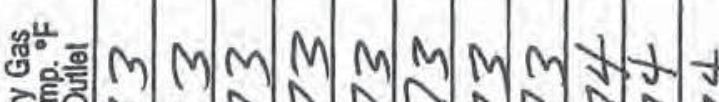

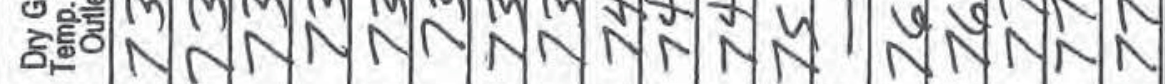

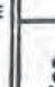

"3.

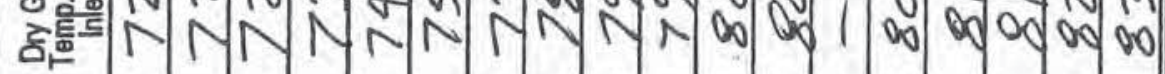

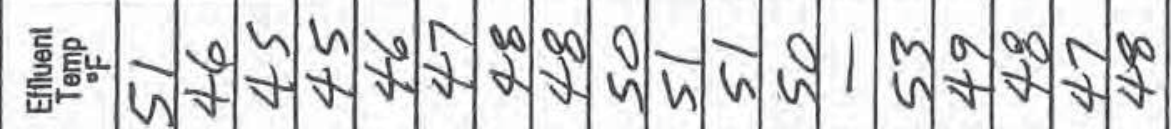

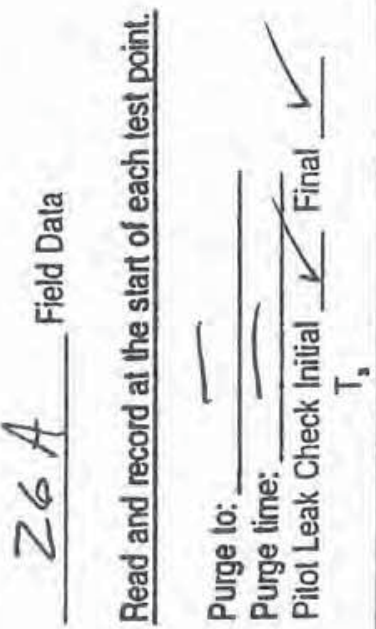

高高 。 N N NNN N NNNNNNN N NNN N

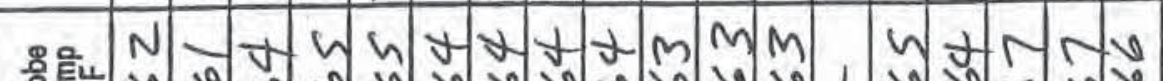

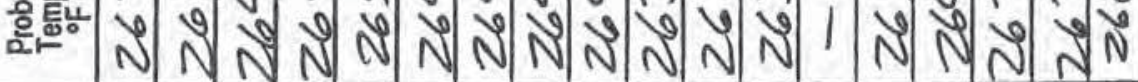
兽 N N Na N $\omega^{\circ} N N N N N N N N N N N N$ N N N N N N

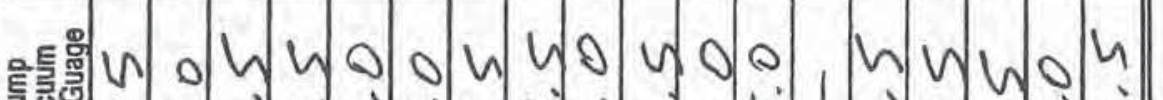
和

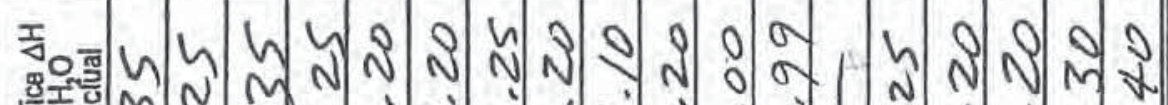

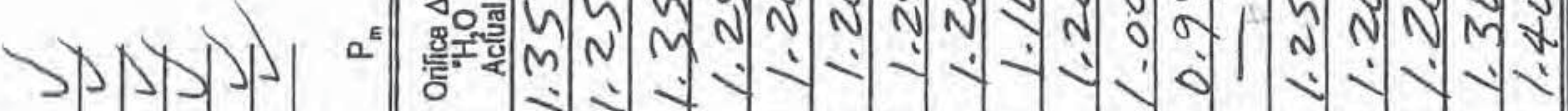

2.

$-5$

8.7

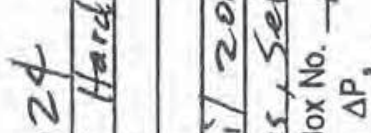

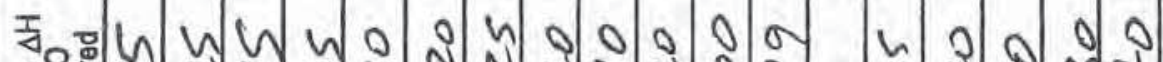

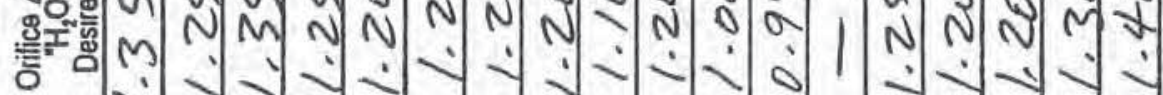

0 n m $=$.

这m

1. 1 से

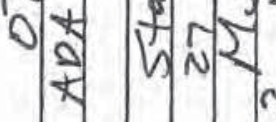

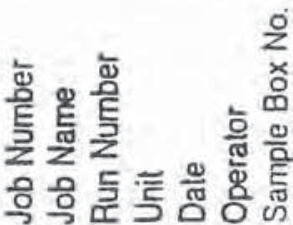

- 0 o 0 o 000 은

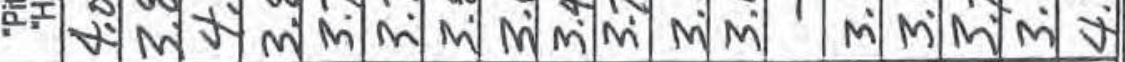
แ

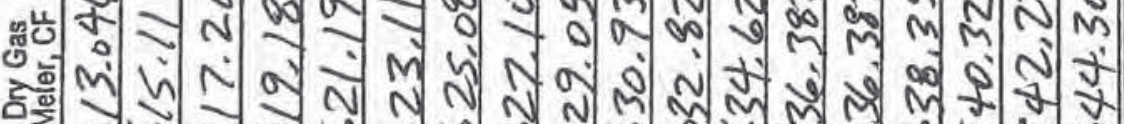
รง จे จ

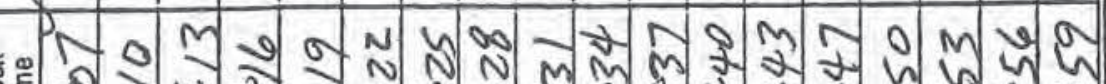
\%

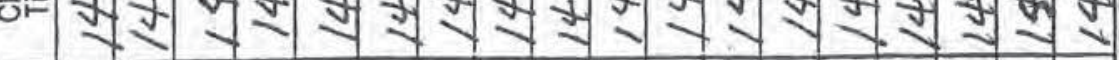

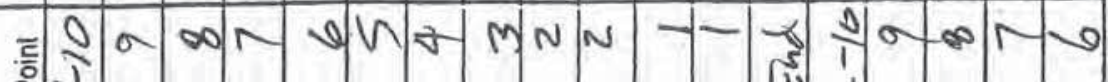
:

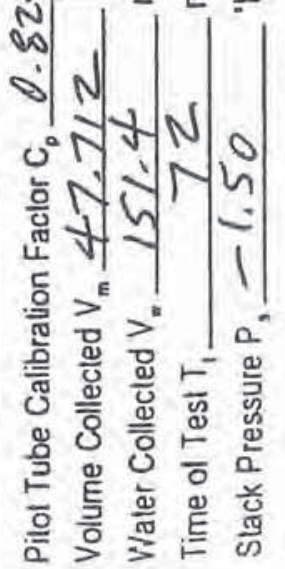


Impinger Box No.

$$
\begin{aligned}
& \text { Impinger 1 } \begin{array}{l}
\text { Final Weight } \\
\text { Initial Weight } \\
\text { Increase }
\end{array} \frac{862.7}{117.3 .4}= \\
& \text { Water Weiaht Gain } \\
& \text { Impinger } 2 \\
& \begin{array}{l}
\text { Final Weight } \\
\begin{array}{l}
\text { Initial Weight } \\
\text { Increase }
\end{array}
\end{array} \frac{753,6}{16,7} \\
& \text { Impinger } 3 \\
& \text { Final Weight } \\
& \text { Initial Weight } \\
& \text { Increase } \\
& \begin{array}{llll}
756.4 & \mathrm{v}_{w}= & \\
752.1 & \mathrm{gSO}_{2}= & \text { Impinger } 5 & 11.2 \\
\mathrm{v}_{w} & = &
\end{array} \\
& 4.3 \\
& \text { Impinger } 1 \\
& 117.3 \\
& \text { Impinger } 2 \\
& 16.7 \\
& \text { Impinger } 3 \quad 4.3 \\
& \text { Impinger } 4 \quad 1.9 \\
& \text { Impinger } 6 \\
& \text { Final Weight } \quad \frac{741.4}{759.5} \quad \text { Impinger } 7 \\
& \text { Initial Weight } \quad \frac{732.5}{19} \quad \text { FILTERH } \\
& \text { Increase } \quad 1,9 \\
& \begin{array}{ll}
\text { Impinger } 5 & \begin{array}{l}
\text { Final Weight } \\
\text { Initial Weight } \\
\text { Increase }
\end{array}
\end{array} \frac{930,3}{919,1} \\
& A / 67 \\
& P_{b}=26.99 \\
& v_{m}=47.712 \\
& v_{w}=151.4 \\
& P_{m}=\frac{1.2520}{3.810} \\
& \text { Avg } \Delta \mathrm{P}=3.810 \\
& \begin{aligned}
\operatorname{Avg} \sqrt{\Delta \mathrm{P}} & =1.949^{V} \\
C_{p} & =0.824
\end{aligned} \\
& \mathrm{P}_{2}=-1.50^{\mathrm{V}} \mathrm{H}_{2} \mathrm{O} \\
& \begin{array}{l}
T_{m}=78 \\
T_{1}=2280 \mathrm{~F}
\end{array} \\
& \text { Total } \\
& 151.4^{5}=v_{w}
\end{aligned}
$$

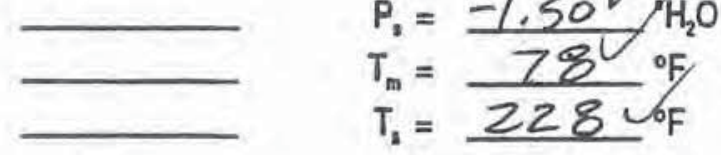




\section{ORSAT ANALYSIS DATA FORM}

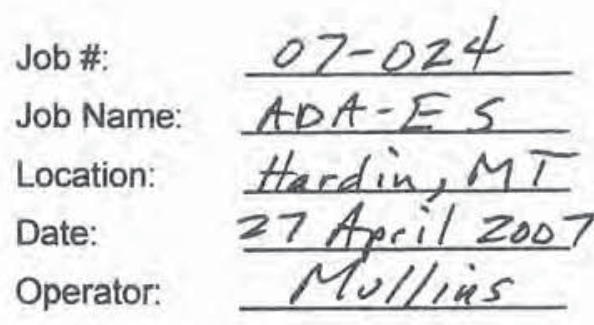

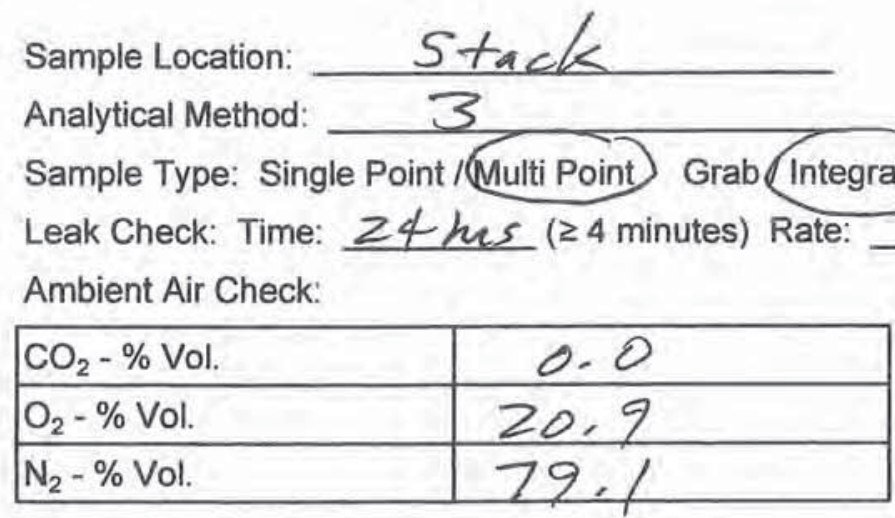

Run \#:

Run Time: $0900-1017$

\begin{tabular}{|l|r|r|c|c|}
\hline $\mathrm{CO}_{2}-\%$ Vol. & 12.6 & 12.8 & 12.8 & 12,7 \\
\hline $\mathrm{O}_{2}-\%$ Vol. & 6.6 & 6.7 & 6,5 & 6.6 \\
\hline $\mathrm{CO}-\% \mathrm{Vol}$. & 0.0 & 0.0 & 0.0 & 0,0 \\
\hline $\mathrm{N}_{2}-\% \mathrm{Vol}$. & 80.8 & 80.5 & 80.7 & 80.7 \\
\hline
\end{tabular}

Run \#: $z$

Run Time: $1110-1227$ Analysis \#1 Analysis \#2 Analysis \#3 Average - $\%$ Volume

\begin{tabular}{|l|c|c|c|c|}
\hline $\mathrm{CO}_{2}-\% \mathrm{Vol}$. & 12.6 & 12.6 & 12.6 & 12.6 \\
\hline $\mathrm{O}_{2}-\% \mathrm{Vol}$. & 6.7 & 6.7 & 6.7 & 6.7 \\
\hline $\mathrm{CO}-\% \mathrm{Vol}$. & 0.0 & 0.0 & 0.0 & 0.0 \\
\hline $\mathrm{N}_{2}-\% \mathrm{Vol}$. & 80.7 & 80.7 & 80.7 & 80.7 \\
\hline
\end{tabular}

Run \#: 3

Run Time: $1407-1523$ Analysis \#1 Analysis \#2 Analysis \#3 Average - $\%$ Volume

\begin{tabular}{|l|c|c|c|c|}
\hline $\mathrm{CO}_{2}-\% \mathrm{Vol}$. & 12.6 & 12.6 & 12.6 & 12.6 \\
\hline $\mathrm{O}_{2}-\% \mathrm{Vol}$. & 6.7 & 6.7 & 6.7 & 6.7 \\
\hline $\mathrm{CO}-\% \mathrm{Vol}$ & 0.0 & 0.0 & 0.0 & 0.0 \\
\hline $\mathrm{N}_{2}-\% \mathrm{Vol}$. & 80.7 & 80.7 & 80.7 & 80.7 \\
\hline
\end{tabular}




\section{Appendix E:}

EPA Test Methods 5 and 26A (Particulate Matter and Hydrogen Halides \& Halogens) Analytical Data - Stack 
Air Sampling Associates, Inc.

\section{Particulate Analysis Summary}

Project Number: $07-024$

Date Analysis Completed: $5 / 11 / 07$

Project Name: $A O A-E S$

Unit Tested: Stack (26A)

Project Location: Hardin, MT

Run Number:

Particulate Matter on Filter (mg):

Particulate Matter in Front Wash (mg):

Total Particulate Matter in "Front-Half" - MF (mg):

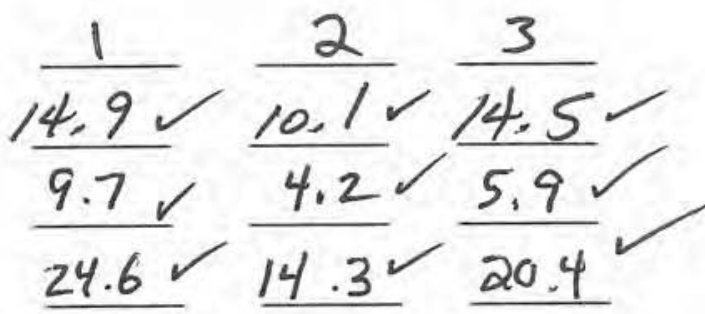

Particulate Matter in "Back-Half" (mg):

Total Particulate Matter in Sample - MT (mg):
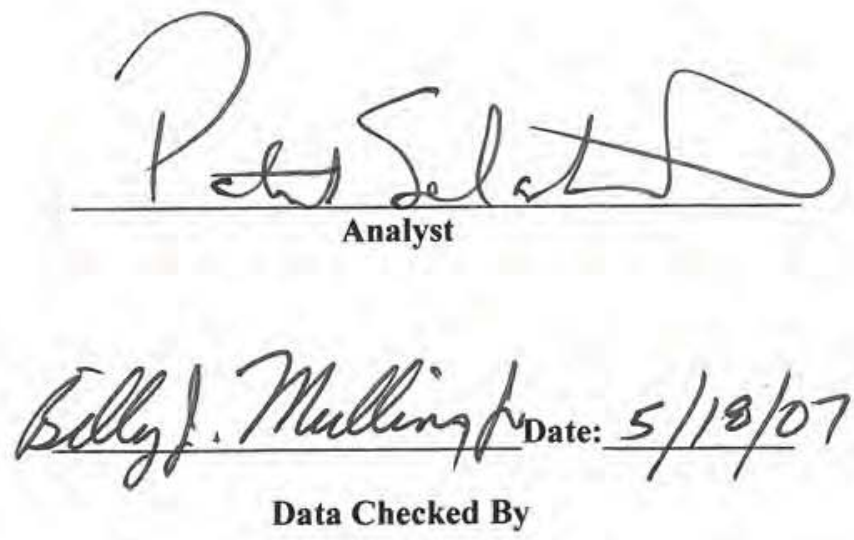

Version No. 1 5/30/06 


\section{Particulate Analysis EPA Method $26 \mathrm{H}$}

Stack Filters

Project No. $07-024$

Project Name ADA-ES
Location Unit Tested
Hardin, MT

\section{\begin{tabular}{l|l|l|l|l} 
Desiccator Time In & 0800 & $5 / 107$ & $08505 / 3107$ & 08
\end{tabular}}

Desiccator Time Out $0845 \mathrm{~S} / 3 / 0708405 / 4 / 107$

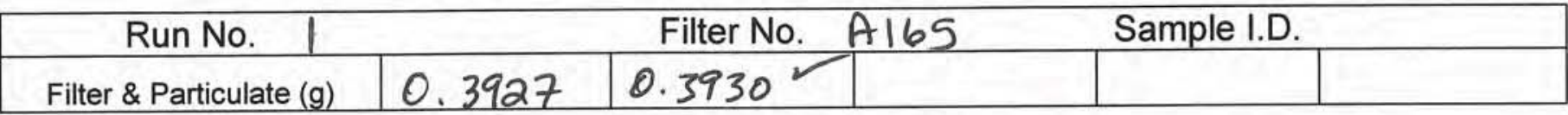

Filter \& Particulate Average (g) 0,3930 Initial Filter Weight $(\mathrm{g}) 0,3781$ Total Particulate $(\mathrm{mg})$ 14.92

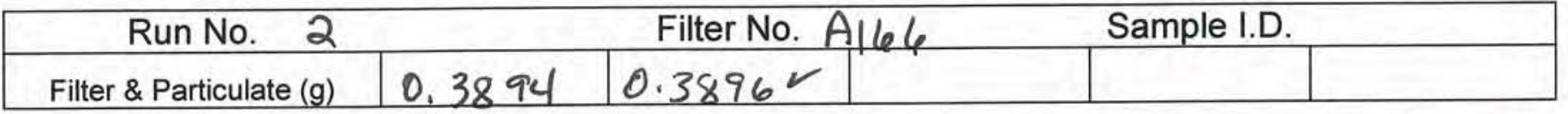

Filter \& Particulate Average (g) Initial Filter Weight (g) 0.3896 Total Particulate $(\mathrm{mg})$ 0.3795

$$
\text { Total Particulate (mg) }
$$
10.12

\begin{tabular}{|c|c|c|c|}
\hline Run No. 3 & & Filter No. A 167 & Sample I.D. \\
\hline Filter \& Particulate (g) & 0.3893 & $0.3896^{\circ}$ & \\
\hline
\end{tabular}

Filter \& Particulate Average (g) 0.3896 Initial Filter Weight $(\mathrm{g})$
Total Particulate $(\mathrm{mg})$
$\frac{0.3751}{14.5}$ 
Filter Weight Log

EPA Method 5

\begin{tabular}{|c|c|c|c|c|c|c|c|}
\hline \multirow[b]{3}{*}{ Filter No. } & Into Desicator & Weight & Weight & Weight & Weight & Weight & \multirow{3}{*}{$\begin{array}{c}\text { Weight } \\
\text { Used }\end{array}$} \\
\hline & Date & Date & Date & Date & Date & Date & \\
\hline & Time & Time & Time & Time & Time & Time & \\
\hline \multirow{3}{*}{$A 151$} & & 0.3827 & 0.3829 & & & & 0,3829 \\
\hline & 216107 & $2 / 7 / 07$ & $2 / 8 / 07$ & & & & \\
\hline & 1045 & 1051 & 1051 & & & & \\
\hline \multirow{3}{*}{$A 152$} & & 0.3698 & 0.3698 & & & & 0.3698 \\
\hline & & 2129107 & $3 / 09107$ & & & & \\
\hline & & 1648 & 0915 & & & & \\
\hline \multirow{3}{*}{ A153 } & & 0.3702 & 0.3704 & & & & 0.3704 \\
\hline & & 2128107 & 3101107 & & & & \\
\hline & & 1648 & 0915 & & & & \\
\hline \multirow{3}{*}{$A 154$} & & 0.3740 & 0.3741 & & & & 0.3741 \\
\hline & & $2 / 28 / 07$ & $3 / 1107$ & & & & \\
\hline & & 1648 & 0915 & & & & \\
\hline \multirow{3}{*}{$A 155$} & & 0.3424 & 0.3424 & & & & 0.3424 \\
\hline & & $2 / 28 / 07$ & 31,107 & & & & \\
\hline & & 1649 & 0916 & & & & \\
\hline \multirow{3}{*}{$A 156$} & & 0,3441 & 0.7443 & & & & 0.3443 \\
\hline & & $2 / 28 / 07$ & $3 / 1107$ & & & & \\
\hline & & 1649 & 0916 & & & & \\
\hline \multirow{3}{*}{ A 157} & & 0.3412 & 0.3414 & & & & 0.3414 \\
\hline & & $2 / 28 / 07$ & 311107 & & & & \\
\hline & & 1649 & 0417 & & & & \\
\hline \multirow{3}{*}{$A 158$} & & 0.3475 & 0.3479 & & & & 0.3479 \\
\hline & & $\partial / 28 / 07$ & 31,107 & & & & \\
\hline & & 1650 & 0917 & & & & \\
\hline \multirow{3}{*}{ A159 } & & 0.3447 & 0.3450 & & & & 0.3450 \\
\hline & & 2128107 & 31.107 & & & & \\
\hline & & 1650 & 0917 & & & & \\
\hline \multirow{2}{*}{$A 160$} & & 0.3668 & 0.3672 & & & & 0.3672 \\
\hline & & $7 / 28107$ & 311107 & & & & \\
\hline \multirow{3}{*}{$A / 61$} & & 1651 & 0918 & & & & \\
\hline & & 0,3657 & 0.3657 & & & & 0.3657 \\
\hline & & $\frac{2 / 28 / 07}{1651}$ & $\frac{311107}{0918}$ & & & & \\
\hline \multirow{3}{*}{ Al62 } & & 0.3633 & 0.3636 & & & & 0.3636 \\
\hline & & $2 / 28107$ & 31,107 & & & & \\
\hline & & 1651 & 0918 & & & & \\
\hline \multirow{3}{*}{$A 163$} & & 0.3732 & 0.3734 & & & & 0.7734 \\
\hline & & 2128107 & 31,102 & & & & \\
\hline & & 1652 & 0919 & & & & \\
\hline \multirow{3}{*}{$A 164$} & & 0.3748 & 0.3748 & & & & 0.3748 \\
\hline & & 2128107 & $31,1,2$ & & & & \\
\hline & & 1652 & 0919 & & & & \\
\hline \multirow{3}{*}{$A / 65$} & & 0.3783 & 0.3781 & & & $>$ & 0.7781 \\
\hline & & $212810 \mathrm{f}$ & 3101107 & & & & 2 \\
\hline & & 1652 & 10920 & & & & \\
\hline
\end{tabular}




\section{AIR \\ SAMPLING \\ ASSOCIATES, INC.}

Filter Weight Log

EPA Method 5

\begin{tabular}{|c|c|c|c|c|c|c|c|}
\hline \multirow[b]{3}{*}{ Filter No. } & Into Desicator & Weight & Weight & Weight & Weight & Weight & \multirow{3}{*}{$\begin{array}{l}\text { Weight } \\
\text { Used }\end{array}$} \\
\hline & Date & Date & Date & Date & Date & Date & \\
\hline & Time & Time & Time & Time & Time & Time & \\
\hline \multirow{3}{*}{$A 166$} & & 0,3795 & 0.3795 & & & & 0.3795 \\
\hline & $4 / 2 / 07$ & $4 / 12107$ & $4 / 17 / 07$ & & & & \\
\hline & 0800 & 0810 & 1423 & & & & \\
\hline \multirow{2}{*}{ A167 } & $\frac{4 / 2107}{200}$ & $\frac{0.3752}{411101}$ & $\frac{0.3751}{4 / 17107}$ & & & & 0.3751 \\
\hline & & 0815 & 423 & $\operatorname{som} 4(17) 07$ & & & \\
\hline \multirow{3}{*}{4168} & & 0.3880 & 1423 & 0.3879 & & & 0.3879 \\
\hline & $4 / 2 / 07$ & $4112 / 07$ & $4 / 12 / 07$ & & & & \\
\hline & 6800 & $\frac{6915}{0.2936}$ & $\frac{1422}{0.3835}$ & & & & 0.3835 \\
\hline \multirow{2}{*}{$416^{9}$} & $4 / 2 / 07$ & $4 i n 707$ & $4 / 17107$ & & & & \\
\hline & 0800 & $0 \% 15$ & $42 \geqslant$ & & & & \\
\hline \multirow{3}{*}{$A 170$} & & 0.3849 & 0.3848 & & & & 0.3848 \\
\hline & $\frac{4 / 2 / 07}{6 / 800}$ & $4 / 7 / 67$ & $4 / 0107$ & & & & \\
\hline & 8800 & $\frac{6815}{0.3819}$ & $\frac{1420}{0.3818}$ & & & & 0.3818 \\
\hline \multirow{2}{*}{$A 171$} & $4 / 2 / 67$ & $4 / 7 \%$ & $x \mid 1710$ & & & & \\
\hline & 6800 & $0 \$ 16$ & $1 / 420$ & & & & \\
\hline \multirow{5}{*}{$A 173$} & & 0.3762 & 0,3762 & & & & 0.3762 \\
\hline & $\begin{array}{l}4 / 2107 \\
0800\end{array}$ & $\begin{array}{l}4 / p / 07 \\
0016\end{array}$ & $\frac{4 / 107}{1420}$ & & & & \\
\hline & & 0.3536 & 0.3537 & $\nabla$ & & & \\
\hline & $42=107$ & 4120107 & $4 / 20107$ & & & & \\
\hline & 1500 & 1511 & 2230 & & & & \\
\hline \multirow{2}{*}{ Al74 } & 41407 & 0.3478 & 0.3479 & & & & \\
\hline & $\frac{41507}{1500}$ & $\frac{4120107}{1512}$ & $\frac{4120107}{2230}$ & & & & \\
\hline \multirow{3}{*}{ A 175} & & $\frac{1512}{0.3603}$ & 0.36031 & & & & \\
\hline & $4(1007$ & 4120107 & $4(20107$ & & & & \\
\hline & 1500 & 15,3 & 2230 & & & & \\
\hline \multirow{2}{*}{ A176 } & & 0.3667 & 0.3668 & 2 & & & \\
\hline & $\frac{4 / 23107}{1500}$ & $\frac{4120107}{15,4}$ & $\frac{4 / 26107}{2230}$ & & & & \\
\hline \multirow{3}{*}{1717} & & 0.3803 & 0.38060 & & & & \\
\hline & $41=107$ & 41520107 & $4(20107$ & & & & \\
\hline & 1500 & 1515 & 2230 & & & & \\
\hline \multirow{7}{*}{$A 178$} & पाzeी o- & $\frac{0.3820}{4120107}$ & $\frac{0.3820}{4(20 \% 7}$ & & & & \\
\hline & 1500 & 1516 & 2230 & & & & \\
\hline & & & & & & & \\
\hline & & & & & & & \\
\hline & & & & & & & \\
\hline & & & & & & & \\
\hline & & & & & & & \\
\hline
\end{tabular}




\section{AlR \\ ASSOCIATES, INC.}

Particulate Analysis EPA Method $26 \mathrm{~A}$

Page 1 of 1

Front Wash

Project No. $07-024$

Project Name $A D A-E S$

Location HARDIN, MT

Unit Tested STACK

Desiccator Time In $09405 / 8 / 07 \quad 10005 / 9 / 07 / 10275 / 10 / 07$

Desiccator Time Out $09505 / 9 / 07 / 1020 \quad 5 / 10 / 07 / 1005$ s/c1/07

\begin{tabular}{|c|c|c|c|c|}
\hline Run No. $I$ & & Volume $(\mathrm{ml})$ & 285 & Sample I.D. \\
\hline Final Weight $(\mathrm{g})$ & 112.0263 & 112.0270 & $112.0275^{5}$ & \\
\hline Initial Weight (g) & & & $112.0160^{\mathrm{V}}$ & \\
\hline Particulate Weight (g) & & & 0.0115 & \\
\hline
\end{tabular}

Particulate Average $(\mathrm{mg}) \quad 11.5$

Less Acetone Blank $(\mathrm{mg}) \quad 1.8$

Total Particulate $(\mathrm{mg})$

9.7

\begin{tabular}{|c|c|c|c|c|c|}
\hline Run No. 2 & & Volume $(\mathrm{ml})$ & 320 & Sample I.D. & \\
\hline Final Weight $(\mathrm{g})$ & 103.4507 & 103.4509 & $103.4513^{2}$ & & \\
\hline Initial Weight $(\mathrm{g})$ & & & 103.4451 & & \\
\hline Particulate Weight (g & & & 0.0062 & & \\
\hline
\end{tabular}

Particulate Average (mg) Less Acetone Blank (mg)

6.2

Total Particulate $(\mathrm{mg})$

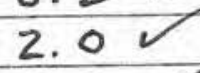

\begin{tabular}{|c|c|c|c|}
\hline \multicolumn{2}{|c|}{ Run No. 3 } & \multicolumn{3}{|c|}{ Volume $(\mathrm{ml})$} \\
\hline Final Weight $(\mathrm{g})$ & 103.5997 & 103.6000 & \\
\hline Initial Weight $(\mathrm{g})$ & & & \\
\hline Particulate Weight $(\mathrm{g})$ & & & \\
\hline
\end{tabular}

\begin{tabular}{|c|c|}
\hline 340 & Sample I.D. \\
\hline $103.6002^{\prime}$ & \\
\hline 103.5921 & \\
\hline 0.00817 & \\
\hline
\end{tabular}

Particulate Average (mg)

Less Acetone Blank (mg)

8.1

Total Particulate $(\mathrm{mg})$

2.2

5.9

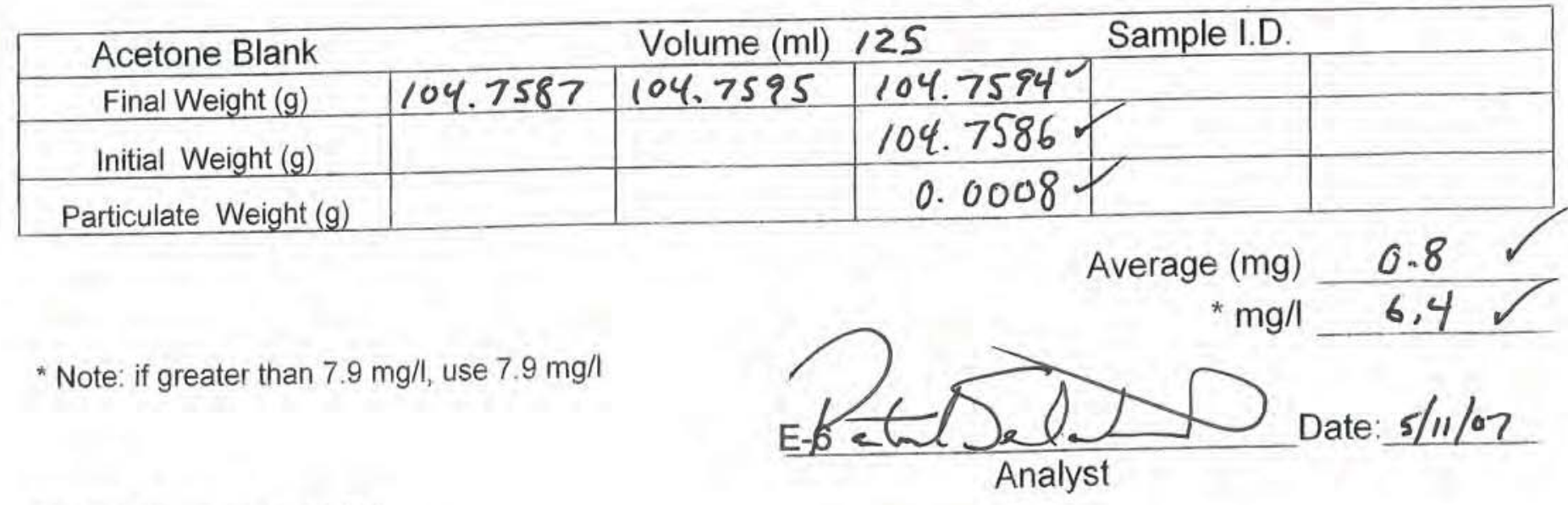


III AIR

SAMPLING

ASSOCIATES, INC.

Particulate Analysis EPA Method $26 \mathrm{~A}$

Front Half Tare Weights

Project No. $07-024$

Location Hardin, $M T$

Project Name AOA - ES Unit Tested Stack

Desiccator Time In $0800 \quad 5 / 107 \quad 08505 / 3107 \quad 08505 / 4 / 07$

Desiccator Time Out lo 845 5/3 $107 \quad 08455 / 4 / 070835$ 5/7/0

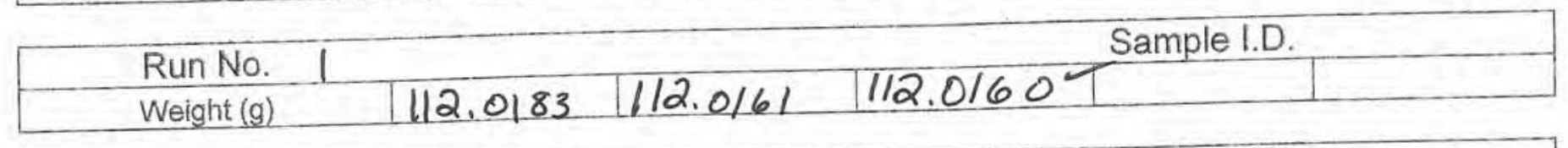

\begin{tabular}{|l|l|l|l|l|l|}
\hline Run No. 2 & \multicolumn{1}{|l|}{ Sample I.D. } \\
\hline Weight $(\mathrm{g})$ & & 103.4463 & 103.4447 & $103.445 /$ & \\
\hline
\end{tabular}

\begin{tabular}{|l|l|l|l|l|}
\hline Run No. 3 & Sample I.D. \\
\hline Weight $(\mathrm{g})$ & 103.5925 & 103.5921 & & \\
\hline Acetone Blank & 104.7597 & 104.7584 & $104.75864^{\text {Sample I.D. }}$ & \\
\hline Weight $(\mathrm{g})$ & 104 & \\
\hline
\end{tabular}

suit /Mys

E-7

Version No. $107 / 06 / 06$ 
H7E040102 Analytical Report

Sample Receipt Documentation ......................................... $\quad 26$

Wet Chemistry ............................................................... 29

Sample Summary .................................................. 30

QC Summary ……................................................ 40

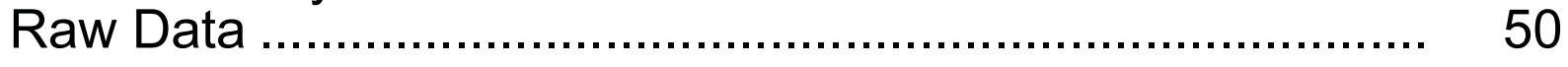

Sample Receipt Documentation ....................................... 152

Total Number of Pages ...................................................... 154 


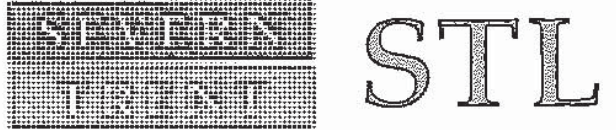

STL Knoxville

5815 Middlebrook Pike

Knoxville, TN 37921

Tel: 8652913000 Fax: 8655844315

www.stl-inc.com

\section{ANALYTICAL REPORT}

Hardin Montana - April 2007

Lot \#: H7B040102

Bill Hefley

Aix Sampling Associates, Inc.

407-2 B Holfords's Prairie Roa Lewisville, TX 75056

SEVERN TRENT LABORATORIES, INC.

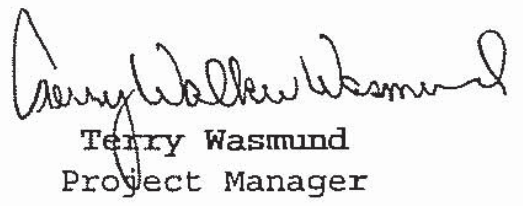

May 21, 2007 
ANALYTICAL METHODS SUMMARY

H7E040102

\begin{tabular}{|c|c|}
\hline PARAMETER & $\begin{array}{l}\text { ANALYTICAL } \\
\text { METHOD }\end{array}$ \\
\hline Bromine Emissions & KNOX $0050 / 26 \mathrm{~A}$ Mod \\
\hline Chlorine Emissions & KNOX $0050 / 26 \mathrm{~A} \operatorname{Mod}$ \\
\hline Hydrogen Bromide Emissions & KNOX $0050 / 26 \mathrm{~A}$ Mod \\
\hline Hydrogen Chloride Emissions & KNOX $0050 / 26 \mathrm{~A} \operatorname{Mod}$ \\
\hline Hydrogen Fluoride Emissions & KNOX $0050 / 26 \mathrm{~A}$ Mod \\
\hline \multicolumn{2}{|l|}{ References: } \\
\hline $\begin{array}{l}\text { Severn Trent Laboratories Knoxville, } \\
\text { Operating Procedure. }\end{array}$ & Facility Standard \\
\hline
\end{tabular}




\section{SAMPLE SUMMARY}

H7E040102

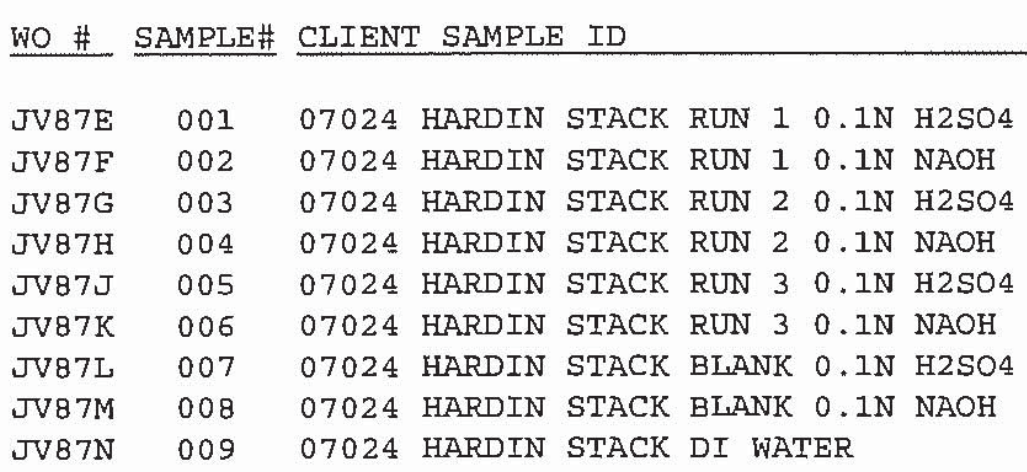

SAMPLED SAMP

DATE TIME

NOTE (S) :

- The analytical results of the samples listed above are presented on the following pages.

- All calculations are performed before rounding to avoid round-off errors in calculased results.

- Results noted as "ND" were not detected at or above the stated limit.

- This report must not be reproduced, except in full, wilhout the written approval of the haboratory.

- Results for the foilowing parameters are never reported on a dry weight basis: color, corrosivity, density, flasijpoint, ignilability, layers, odor,

paint fitter test, pH, porosity pressure, reactivity, redox potential, specific gravity, spot tests, solids, solubility, temperature, viscosity, and weight.

$04 / 27 / 07 \quad 11: 55$

$04 / 27 / 07 \quad 12: 00$

$04 / 27 / 07 \quad 13: 55$

$04 / 27 / 07 \quad 14: 00$

04/27/07 16:40

$04 / 27 / 07 \quad 16: 45$

04/27/07 14:50

04/27/07 14:00

$04 / 27 / 07 \quad 14: 55$ 
QC DATA ASSOCIATION SUMMARY

H7B040102

Sample Preparation and Analysis Control Numbers

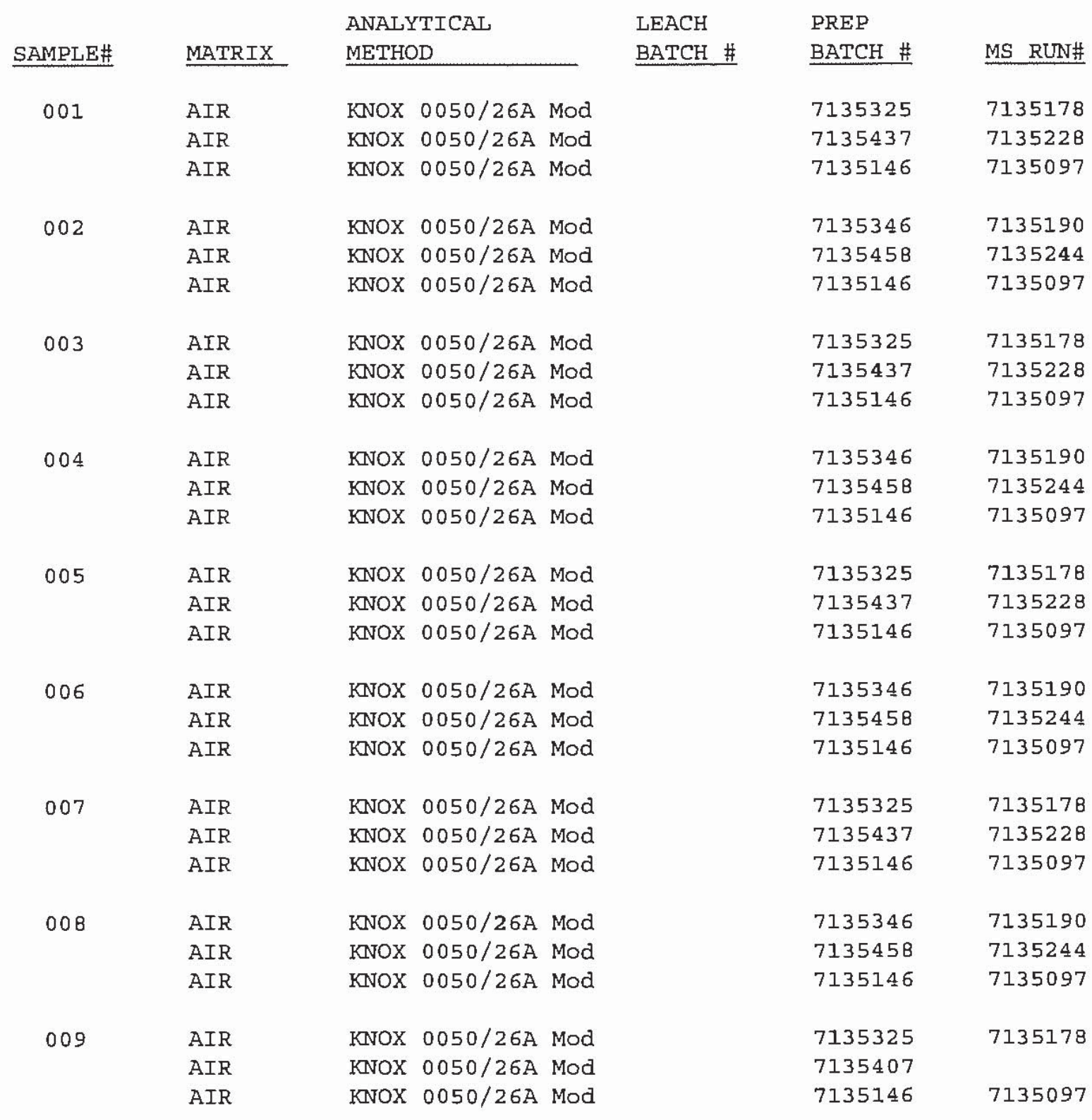




\section{PROJECT NARRATIVE H7E040102}

The results reported herein are applicable to the samples submitted for analysis only.

This report shall not be reproduced except in full, without the written approval of the laboratory.

The original chain of custody documentation is included with this report.

\section{Sample Receipt}

Custody seals were not present upon sample receipt at STL Knoxville.

\section{Quality Control and Data Interpretation}

Unless otherwise noted, all holding times and QC criteria were met and the test results shown in this report meet all applicable NELAC requirements.

Samples were analyzed for chloride $\left(\mathrm{Cl}^{-}\right)$, fluoride $\left(\mathrm{F}^{*}\right)$ and bromide $(\mathrm{Br})$ by ion chromatography using SOP number KNOX-WC-005 (based on EPA methods 9056, 9057 and $26 \mathrm{~A}$ ). Results for the $\mathrm{H}_{2} \mathrm{SO}_{4}$ impinger samples were reported as total $\mu \mathrm{g}$ hydrogen chloride $(\mathrm{HCl})$, total $\mu \mathrm{g}$ hydrogen fluoride $(\mathrm{HF})$ and total $\mu \mathrm{g}$ hydrogen bromide $(\mathrm{HBr})$. Results for the $\mathrm{NaOH}$ impinger samples were reported as total $\mu \mathrm{g}$ chlorine $\left(\mathrm{Cl}_{2}\right)$, total $\mu \mathrm{g}$ hydrogen fluoride (HF) and total $\mu \mathrm{g}$ hydrogen bromine $\left(\mathrm{Br}_{2}\right)$.

Please note that the halogen fluorine $(\mathrm{F})$ is not expected to be present in stack gas as diatomic fluorine $\left(\mathrm{F}_{2}\right)$. Fluorine reacts with water vapor in a favored reaction that forms $\mathrm{HF}: 2 \mathrm{~F}_{2}+2 \mathrm{H}_{2} \mathrm{O} \rightarrow 4 \mathrm{HF}+\mathrm{O}_{2}$. Therefore, the results from the $\mathrm{H}_{2} \mathrm{SO}_{4}$ impingers and the $\mathrm{NaOH}$ impingers are reported as $\mathrm{HF}$, and no $\mathrm{F}_{2}$ is expected in the samples.

Results were calculated using the following equations:

$\mathrm{HX}, \mathrm{ug}=\left(\mathrm{X}^{-}, \mathrm{ug} / \mathrm{mL}\right) *($ Sample Volume, $\mathrm{mL}) *\left(\frac{\text { Molecular Weight } \mathrm{HX}}{\text { Molecular Weight } \mathrm{X}^{-}}\right) *($ Bench Dilution $)$

STL Knoxville maintains the following certifications, approvals and accreditations: Arkansas DEQ Cert. \#05-043-0, California DHS ELAP Cert. \#2423, Colorado DPHE, Connecticut DPH Cert. \#PH-0223, Florida DOH Cert. \#E87177, Georgia DNR Cert. \#906, Hawaii DOH, Illinois EPA Cert. \#000687, Indiana DOH Cert. \#C-TN-02, lowa DNR Cert. \#375, Kansas DHE Cert. \#E-10349, Kentucky DEP Lab ID \#90101, Louisiana DEQ Cert. \#03079, Louisiana DOHH Cert. 㐖A030024, Maryland DHMH Cert. \#277, Massachusetts DEP Cert. \#M-TN009, Michigan DEQ Lab ID \#9933, New Jersey DEP Cert. \#TN001, New York DOH Lab DEHNR Cert. \#64, Ohio EPA VAP Cert. \#CL0059, Oklahoma DEQ ID \#9415, Pennsylvania DEP Cert. \#68-00576, South Carolina DHEC Lab ID \#84001001, Tennessee DOH Lab ID $\# 02014$, Utah DOH Cert. \# QUAN3, Virginia DGS Lab ID $\# 00165$, Washington DOE Lab \#C120, West Virginia DEP Cert. \#345, Wisconsin DNR Lab ID \#998044300, Naval Facilities Engineering Service Center and USDA Soil Permit \#S-46424. This list of approvals is subject to change and does not imply that laboratory certification is available for all parameters reported in this environmental sample data report. 


\title{
PROJECT NARRATIVE H7E040102
}

\author{
$\mathrm{Cl}_{2}, \mathrm{Br} 2 \mathrm{ug}=\left(\mathrm{X}^{-}, \mathrm{ug} / \mathrm{mL}\right) *($ Sample Volume, $\mathrm{mL}) *($ Bench Dilution $)$
}

$\mathrm{NaOH}$ impinger samples were treated with sodium thiosulfate $\left(\mathrm{Na}_{2} \mathrm{~S}_{2} \mathrm{O}_{3}\right)$ prior to the final analysis in order to convert residual hypochlorite $(\mathrm{OCl})$ to chloride ion. The presence of hypobromite is also assumed to be converted to bromide under these conditions.

Note: A sample volume of $100 \mathrm{~mL}$ was used to convert the results to total $\mu \mathrm{g}$ for the method blanks, laboratory control samples, and client reagent blanks.

For demonstration of analytical method performance on these samples, STL Knoxville analyzed matrix spikes (MS) and matrix spike duplicates (MSD). Acceptable recoveries of these spikes demonstrate that quantitation from this particular stack gas matrix is accurate and acceptable. Impinger samples containing $0.1 \mathrm{~N} \mathrm{H}_{2} \mathrm{SO} 4$ and $0.1 \mathrm{~N} \mathrm{NaOH}$ display matrix interference effects causing poor method performance and possibly giving unreliable data unless the interference is removed. Therefore, the samples were diluted in the lab to remove the interference for a more accurate chloride response. The samples may be analyzed at increasing dilutions along with matrix spikes until matrix spikes recover from the sample within laboratory control limits. The ion chromatograph calibration range used to quantitate the sample results permits a standard ten-fold sample dilution while supporting the reporting limit with the low calibration standard.

The dilution factor reported on the sample result form does not represent the bench dilution factor. It is actually the combination of factors required by the method to convert the anion reporting limit and method detection limit from $\mu \mathrm{g} / \mathrm{mL}$ to total $\mu \mathrm{g}$. It may appear to be elevated because it includes the total sample volume in $\mathrm{mL}$.

STL Knoxville maintains the following certifications, approvals and accreditations: Arkansas DEQ Cert. \#05-043-0, California DHS ELAP Cert. \#2423, Colorado DPHE, Connecticut DPH Cert. " Georgia DNR Cert. \#906, Hawaii DOH, Illinois EPA Cert. \#000687, Indiana DOH Ccrt. \#C-TN-02, Iowa DNR Cert. \#375, Kansas DHE Cert. \#E-10349, Kentucky DEP Lab ID \#90101, Louisiana DEQ Cert. \#03079, Louisiana DOHH Cert. ${ }_{\pi}$ LA030024, Maryland DHMH Cert. \#277, Massachusetts DEP Cert. \#M-TN009, Michigan DEQ Lab ID \#9933, New Jerscy DEP Cert. \#TN001, New York DOH Lab \#10781, North Carolina DPH Lab ID \#21705, North Carolina DEHNR Cert. \#64, Ohio EPA VAP Cert. \#CL0059, Oklahoma DEQ ID South Carolina DHEC Lab ID \#84001001, Tennessee DOH Lab ID \#02014, Utah DOH Cert. \# QUAN3, Virginia DGS Lab ID \#00165, Washington DOE Lab \#Cl20, West Virginia DEP Cert. \#345, Wisconsin DNR Lab ID Naval Facilities Engineering Service Center and USDA Soil Permit \#S-46424. This list of approvals is subject to change and does not imply that laboratory certification is available for all parameters reported in this environmental sample data report. 
Sample Data Summary 
Air Sampling Associates, Inc.

client Sample ID: 07024 HARDIN STACK RUN 1 0.1N H2SO4

General Chemistry

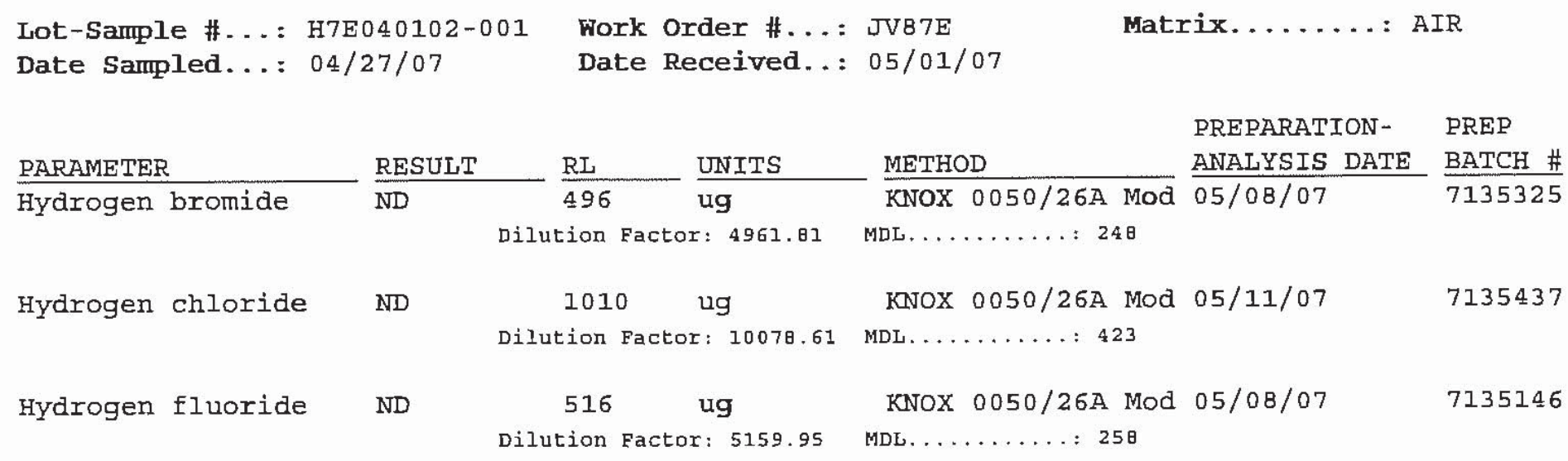


Air Sampling Associates, Inc.

client Sample ID: 07024 HARDIN STACK RUN $10.1 \mathrm{~N}$ NAOH

General Chenistry

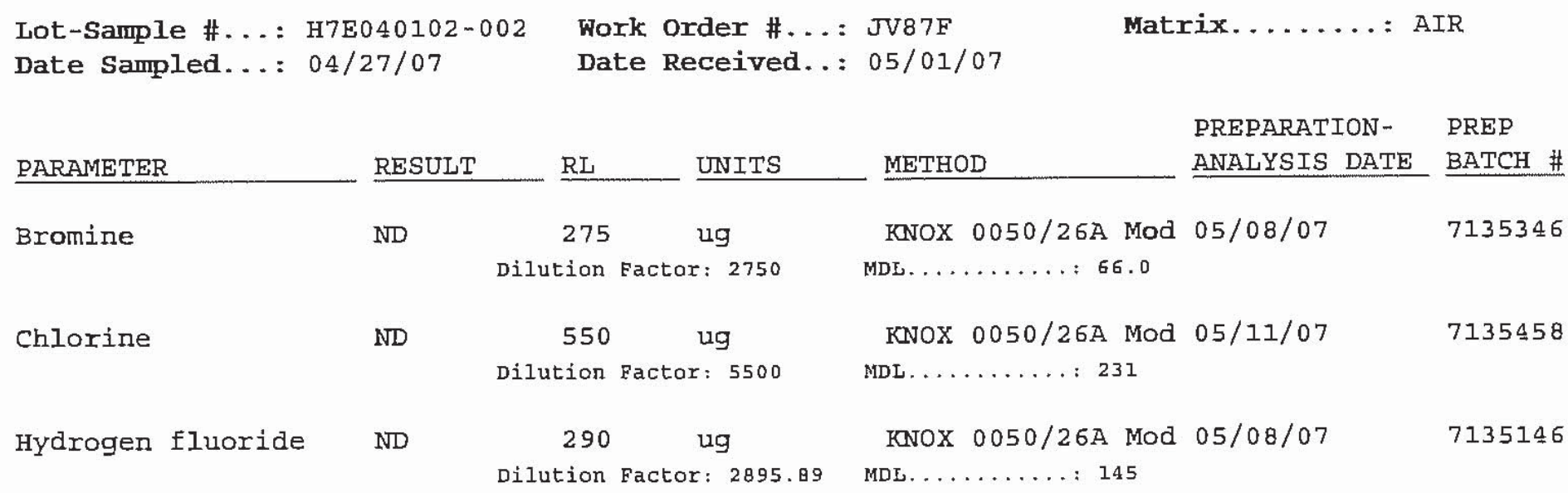


Air Sampling Associates, Inc.

client Sample ID: 07024 HARDIN STACK RUN 2 0.1N H2SO4

General Chemistry

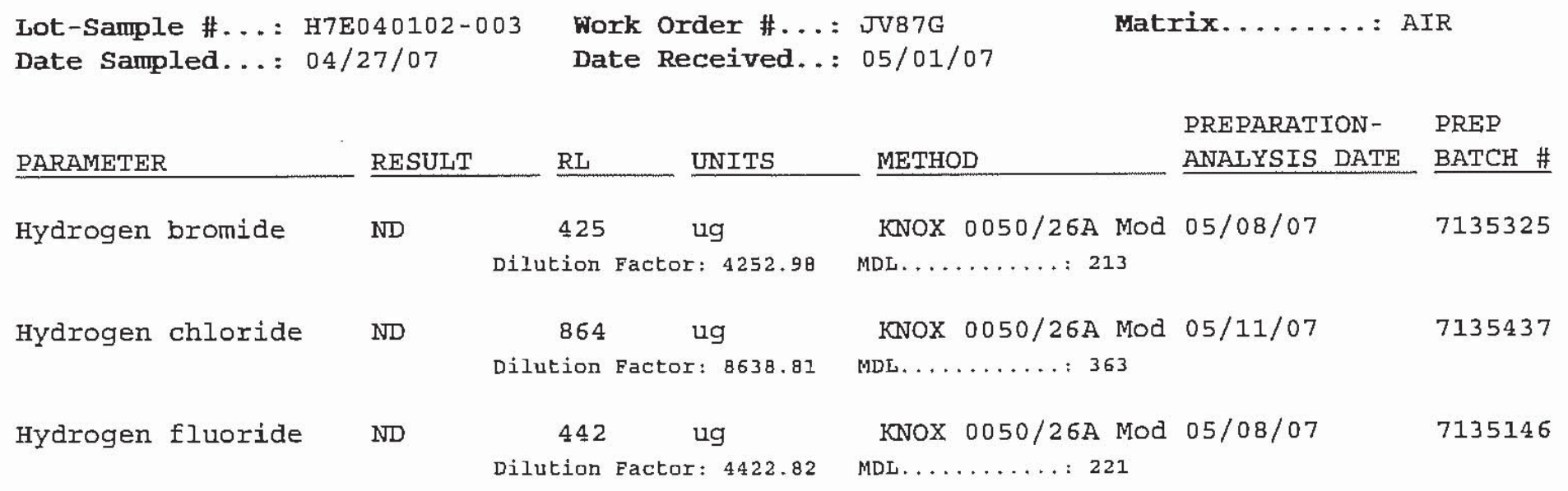


Air Sampling Associates, Inc.

client Sample ID: 07024 HARDIN STACK RUN 20. IN NAOH

General Chemistry

Lot-Sample \#...: H7E040102-004
Date Sampled...: $04 / 27 / 07$
Work Order \#...: JV87H

Date Received. . : 05/01/07
Matrix....... A AIR

PREPARATION - PREP ANALYSIS DATE BATCH \#

PARAMETER

RESULT

RL

UNITS

METHOD

ND 250 ug

Dilution Factor: 2500

KNOX 0050/26A Mod 05/08/07

MDL......... 60.0

Chlorine

ND

500

$\mathrm{ug}$

Dilution Factor: 5000

KNOX 0050/26A Mod 05/11/07 MDL.......... : 210

Hydrogen fluoride
ND $\mathrm{ug}$

KNOX 0050/26A Mod 05/08/07
7135346

7135458

7135146

Dilution Factor: 2632.63 
Air Sampling Associates, Inc.

client Sample ID: 07024 HARDIN STACK RUN 30. IN H2SO4

General Chemistry

Lot-Sample \#... : H7E040102-005

Date Sampled...: 04/27/07
Fork Order \#...: JV87J

Date Received. .: 05/01/07
Matrix.......: AIR

PREPARATION - PREP ANALYSIS DATE BATCH \#

PARAMETER

RESULT

RL

UNITS

METHOD

ND

481

ug Dilution Factor: 4809.92

KNOX 0050/26A Mod 05/08/07

7135325

Hydrogen bromide

$\mathrm{ND}$

$977 \mathrm{ug}$

Dilution Factor: 9770.08

KNOX $0050 / 26 \mathrm{~A} \operatorname{Mod} 05 / 11 / 07$

MDL......... : 410

Hydrogen fluoride
7135437

7135146
$500 \quad \mathrm{ug}$

Dilution Factor: 5002
KNOX 0050/26A Mod 05/08/07

MDL......... : 250 
Air Sampling Associates, Inc.

Client Sample ID: 07024 HARDIN STACK RUN $30.1 \mathrm{~N}$ NAOH

General Chemistry

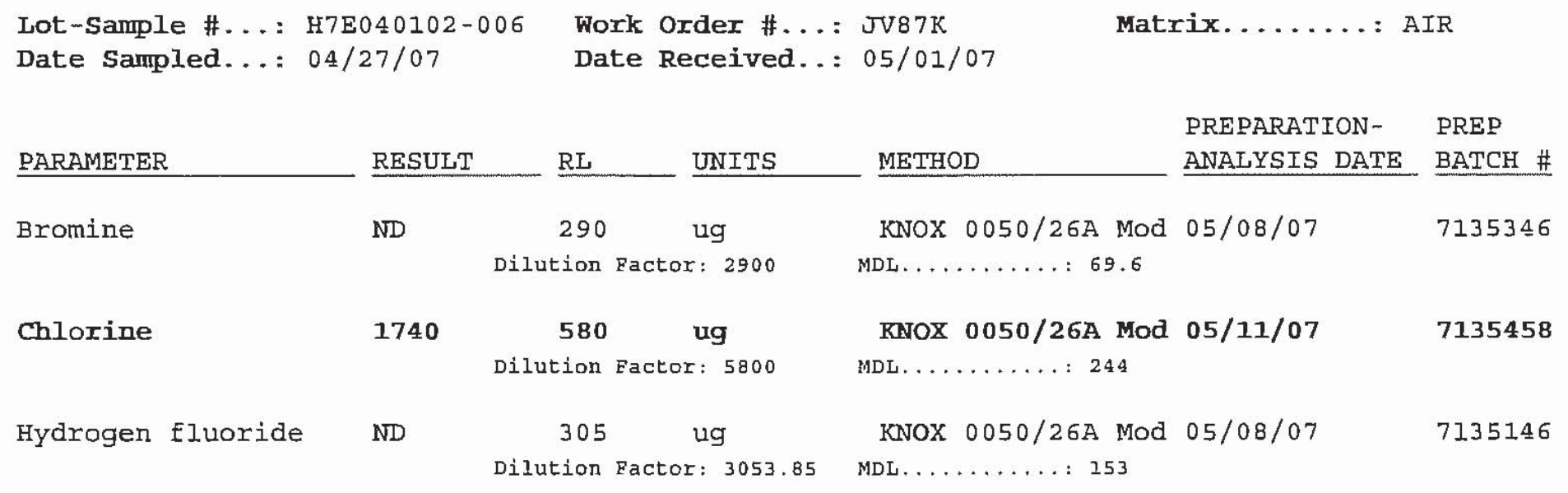


Air Sampling Associates, Inc.

Client Sarmle ID: 07024 HARDIN STACK BLANK 0.1N H2SO4

General Chemistry

Lot-Sample \#...: H7E040102-007 Work Order \#...: JV87L

Date Sampled...: 04/27/07

\begin{abstract}
Date Received... 05/01/07
\end{abstract}
Matrix....... AIR

PREPARATION - PREP METHOD ANALYSIS DATE BATCH \#
RESULT

ND

Hydrogen bromide

Hydrogen chloride

Hydrogen fluoride

$\mathrm{ND}$
RL

101

Dilution Factor: 1012.61

KNOX 0050/26A Mod 05/08/07

MDL. . . . . . . 50.6

KNOX 0050/26A Mod 05/11/07
Dilution Factor: 4113.72

$411 \quad \mathrm{ug}$

105 ug

Dilution Factor: 1053.05
KNOX 0050/26A Mod 05/08/07

MDL . . . . . . : 52.7
7135325

7135437

7135146 
Air Sampling Associates, Inc.

client Sample m: 07024 HARDIN STACK BLANK 0.IN NAOH

General Chemistry

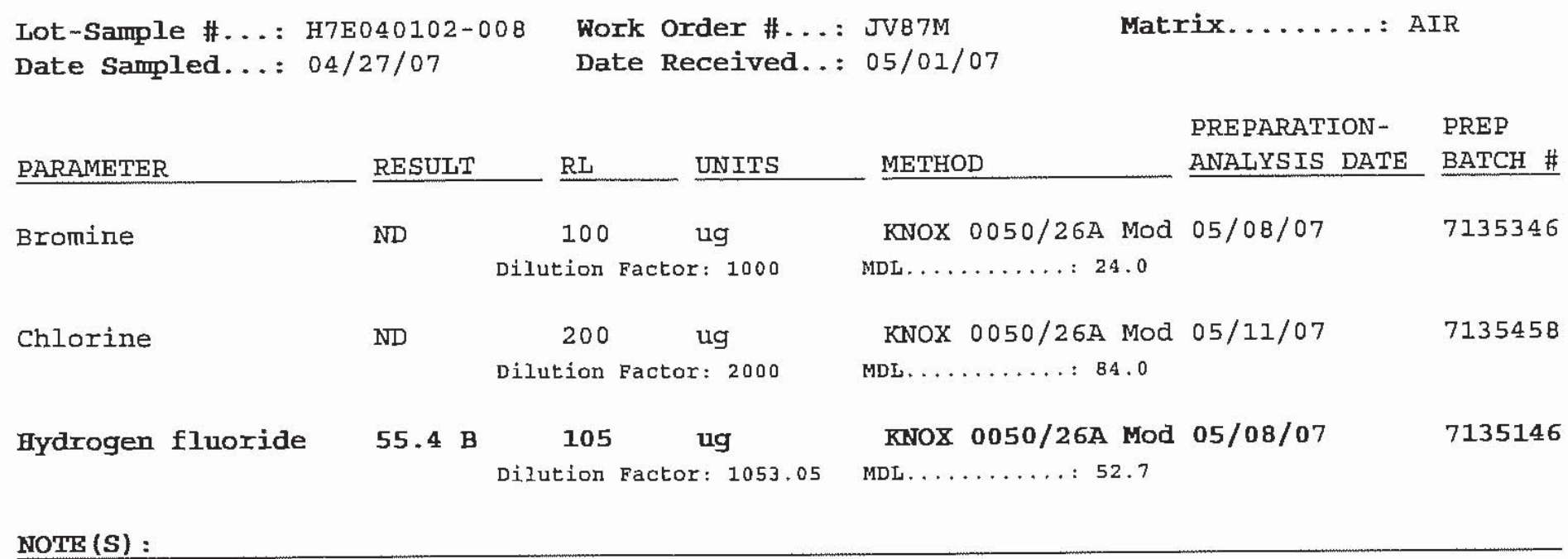

RL. Reporting Limit

B Estimated result. Result is tess than RL. 
Air Sampling Associates, Inc.

Client Sample ID: 07024 HARDIN STACK DI WATER

General Chemistry

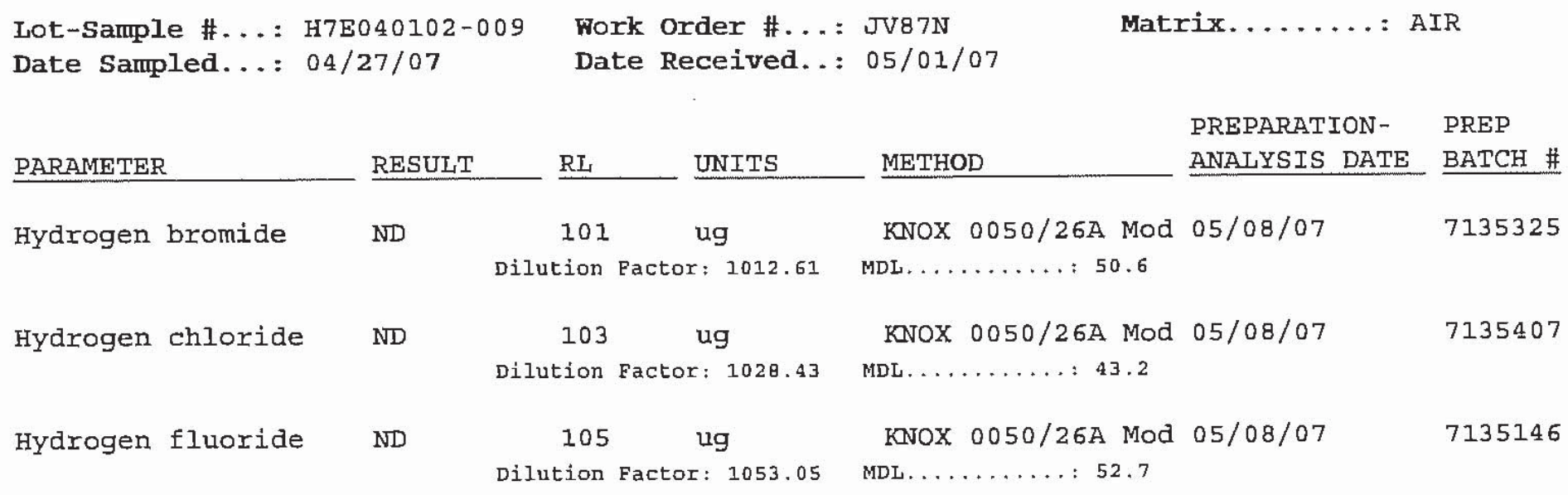


METHOD BLANK REPORT

General Chemistry

client Irot \#...: H7E040102

Matrix......: AIR

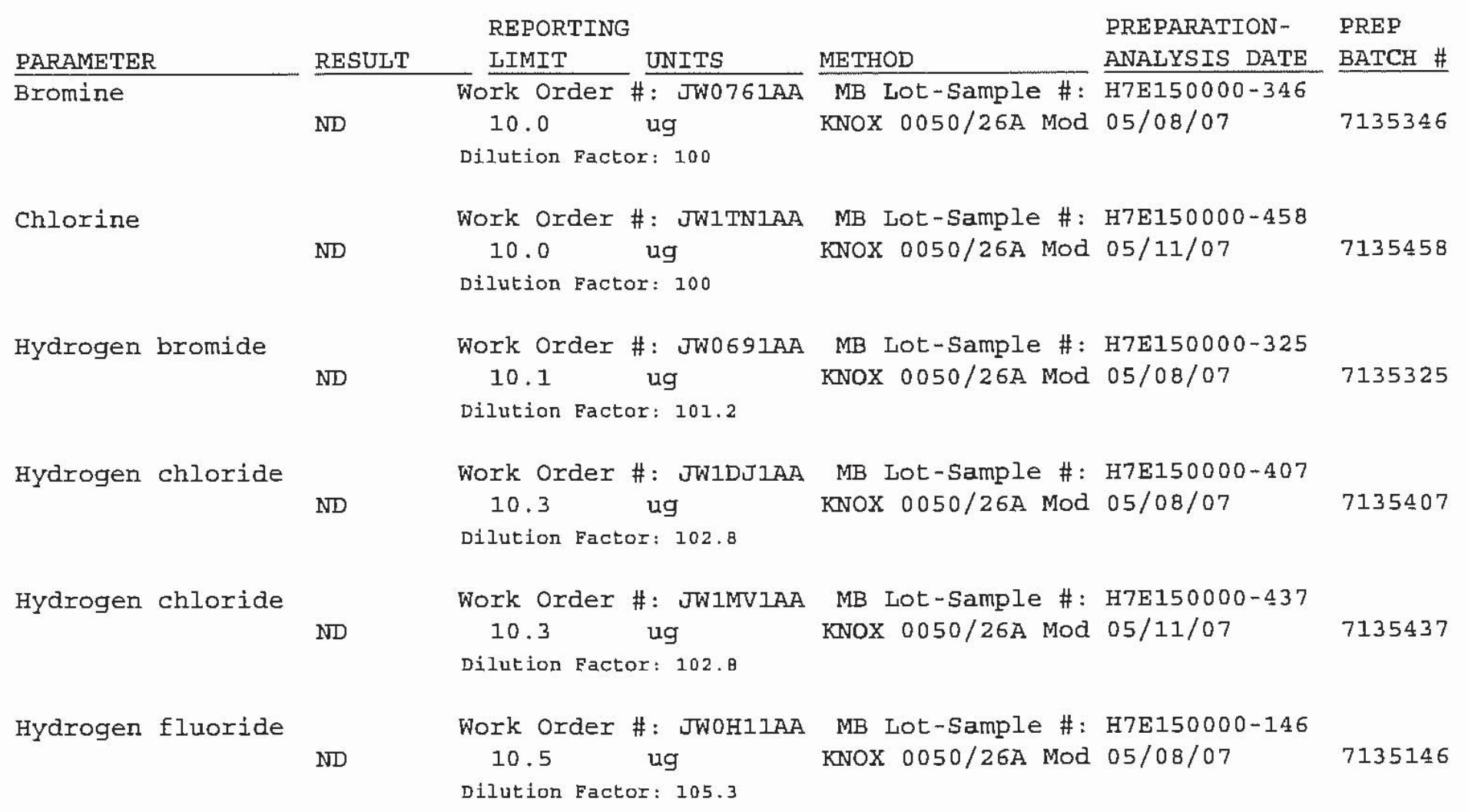

NOTE (S) :

Calculations are performed before rounding to avoid round-off errors in calculated results. 
LABORATORY CONTROL SAMPLE EVAWUATION REPORT

General Chemistry

Lot-Sample \#...: H7E040102

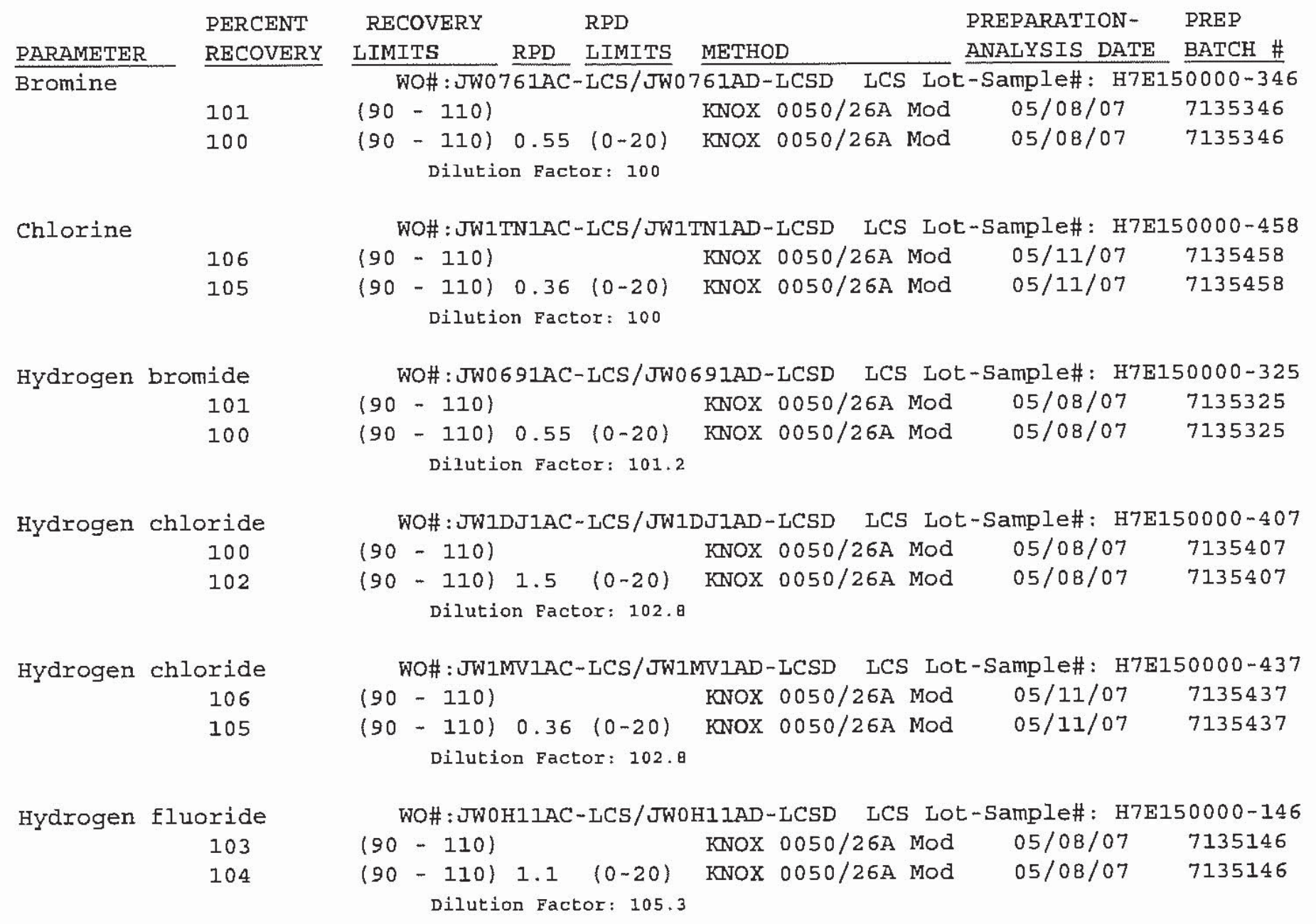

NOTB (S) :

Calculations are performed before rounding to avoid round-off errors in calculated results. 


\section{IAABORATORY CONTROL SAMPLE DATA REPORT}

General Chemistry

Lot-Sample \#...: H7E040102

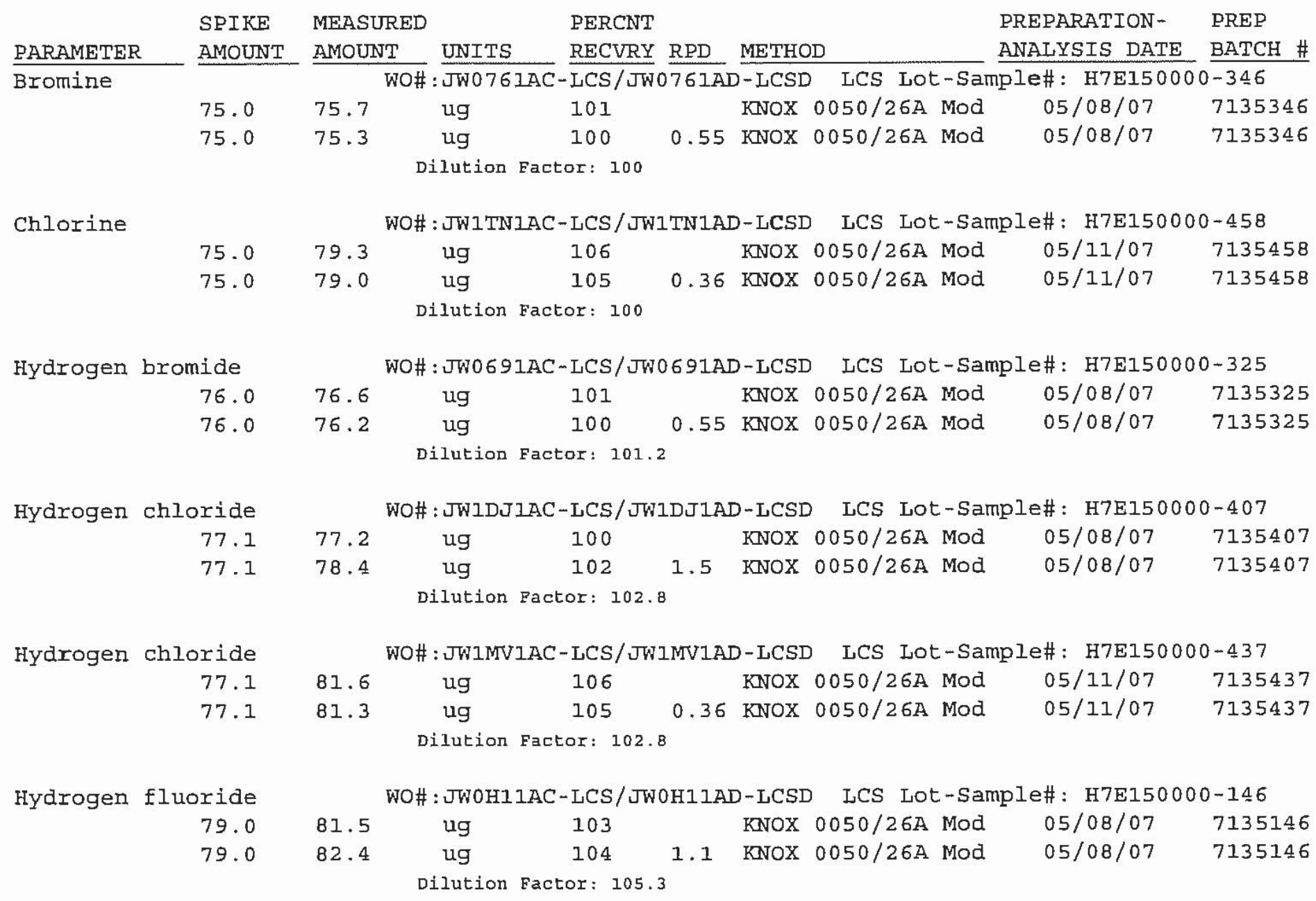


IABORATORY CONTROL SAMPLB EVALUATION REPORT

\section{General Chemistry}

Client Iot \#...: H7E040102

Matrix....... AIR

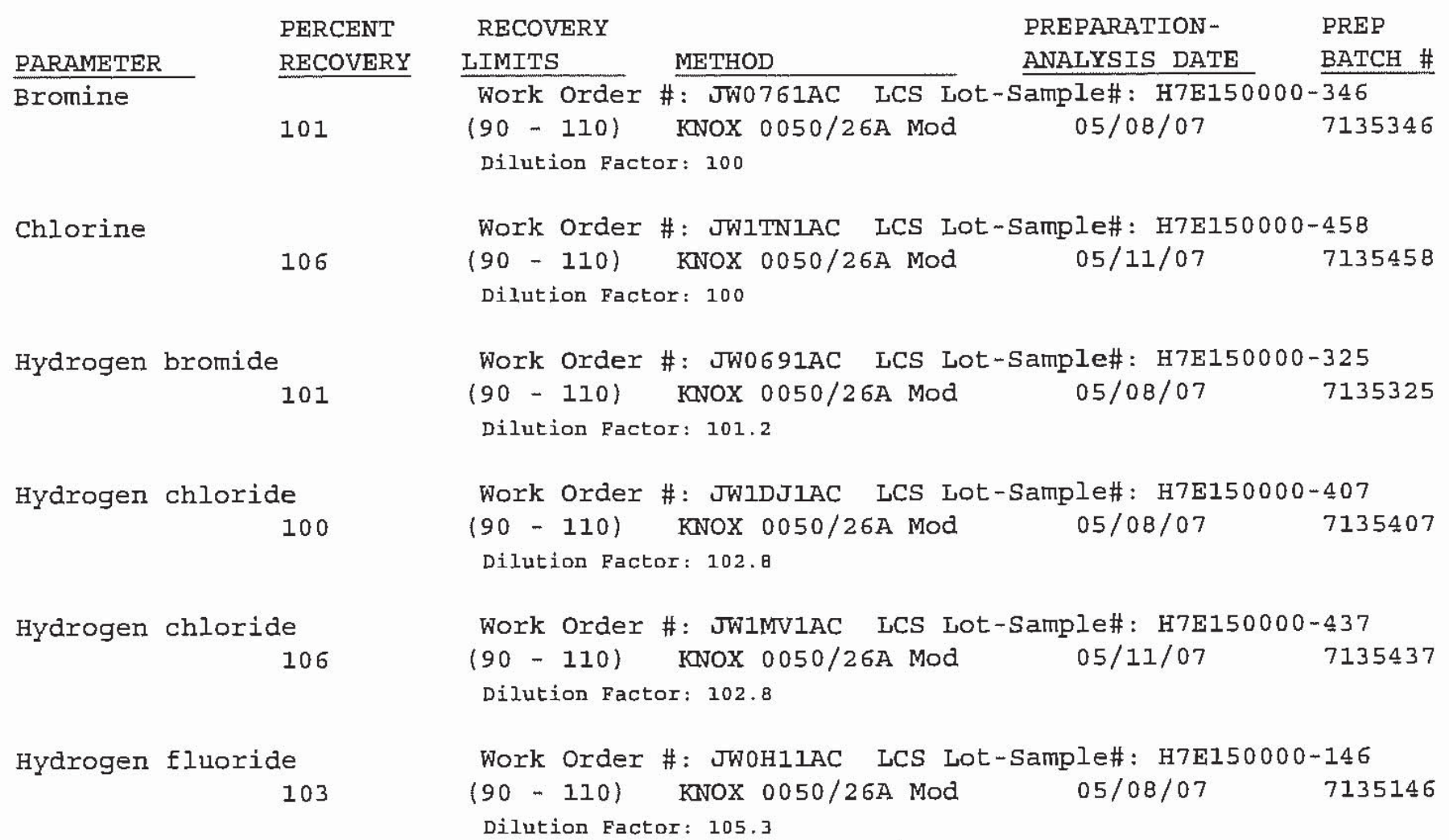

NOTE (S) :

Calculations are performed before rounding to avoid round-off errors in ealculated resuits. 
LABORATORY CONTROL SAMPIE DATA REPORT

General Chemistry

client Iot \#...: H7E040102

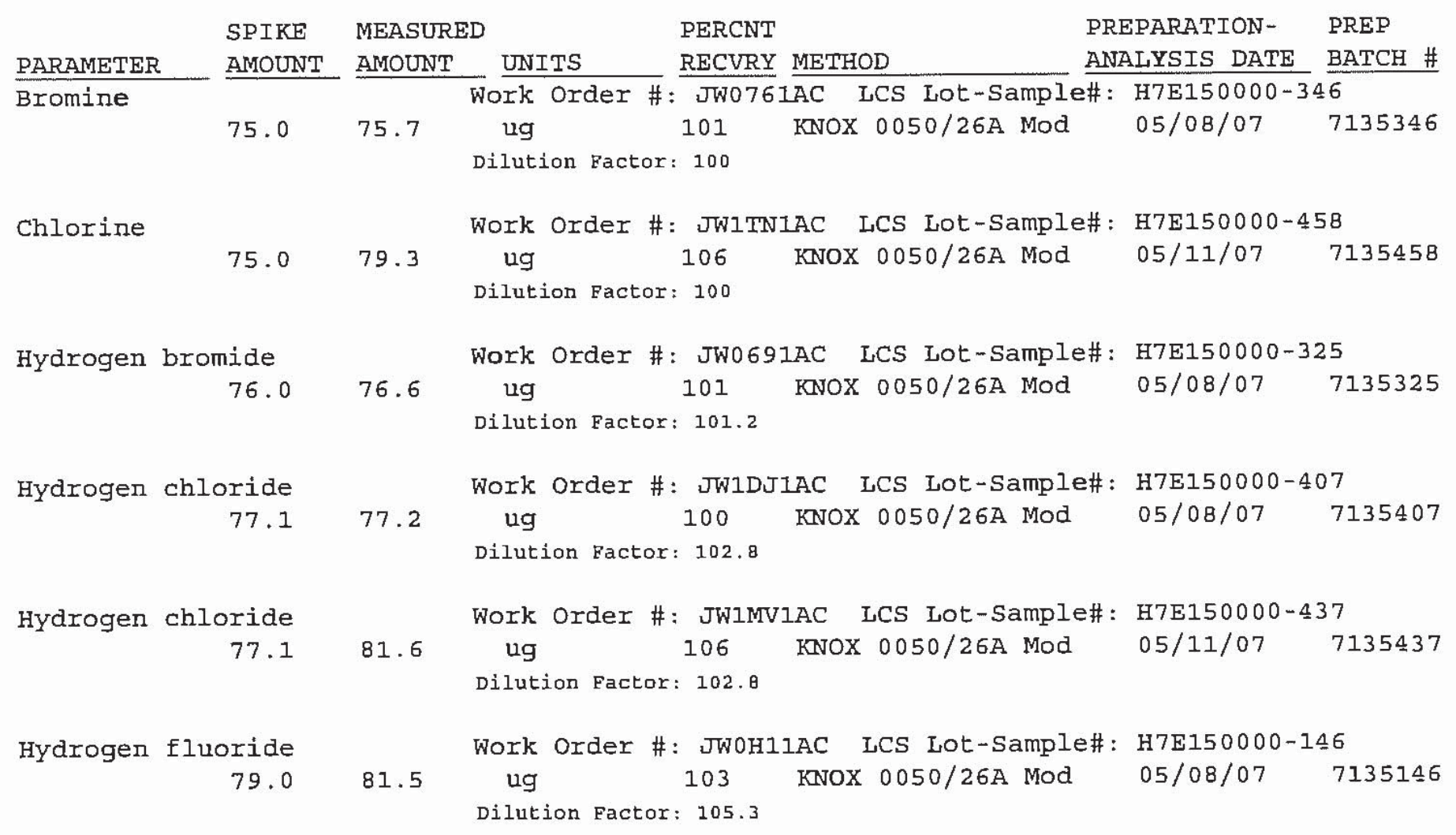

NOTE (S) :

Calculations are performed before rounding to avoid round-off etrors in calculated results. 
MATRIX SPIKK SAMPLE EVALUATION REPORT

General Chemistry

client Lot \#...: H7E040102

Date Sampled...: 04/27/07
Date Received. . : 05/01/07
Matrix....... AIR

PREPARATION- PREP ANALYSIS DATE BATCH \#
Chlorine

$$
95
$$

96

Hydrogen bromide

102

107

Hydrogen chloride

$$
89
$$

85

Hydrogen fluoride

104

102

Hydrogen fluoride

$$
114
$$

114

$$
\begin{array}{r}
(75-125 \\
(75-125
\end{array}
$$
KNOX 0050/26A Mod

KNOX 0050/26A Mod

WO\#: JV87GLAF-MS/JV87GIAG-MSD MS Lot-Sample \#: H7E040102-003

(75 - 125) KNOX 0050/26A Mod 05/08/07 7135325

$(75-125) \quad 4.6 \quad(0-20) \quad$ KNOX 0050/26A Mod 05/08/07 7135325

Dilution Factor: 4252 .

WO\#: JV87GLAC-MS/JV87G1AD-MSD MS Lot-Sample \#: H7E040102-003

(75 - 125) KNOX 0050/26A Mod 05/11/07 7135437

$\begin{array}{llll}(75-125) & 4.3 & (0-20) & \text { KNOX } 0050 / 26 \mathrm{~A} \operatorname{Mod} \quad 05 / 11 / 07 \quad 7135437\end{array}$

Dilution Factor: 8638 .

WO\#: JV87GIAJ-MS/JV87GIAK-MSD MS Lot-Sample \#: H7E040102-003

(75 - 125) KNOX 0050/26A Mod 05/08/07 7135146

$(75-125) \quad 1.5 \quad(0-20) \quad$ KNOX 0050/26A Mod 05/08/07 7135146

Dilution Factor: 4422 .

WO\#: JV87HLAJ-MS/JV87HIAK-MSD MS Lot-Sample \#: H7E040102-004

(75 - 125) KNOX 0050/26A Mod 05/08/07 7135146

$(75-125) 0.14(0-20)$ KNOX $0050 / 26 \mathrm{~A} \operatorname{Mod} 05 / 08 / 07 \quad 7135146$

Dilution Factor: 2632.

NOTB (S) :

Calculations are performed before rounding to avoid round-off crrors in calculated tesults. 
MATRIX SPIKE SAMPLE DATA REPORT

General Chemistry

Client Lot \#...: H7E040102

Date sampled...: 04/27/07
Matrix....... AIR

Date Received. .: 05/01/07

\begin{tabular}{|c|c|c|c|c|c|c|c|c|c|c|}
\hline PARAMETER & $\begin{array}{l}\text { SAMPLE } \\
\text { AMOUNT }\end{array}$ & $\begin{array}{l}\text { SPIKE } \\
\text { AMT }\end{array}$ & $\begin{array}{l}\text { MEASRD } \\
\text { AMOUNT }\end{array}$ & UNITS & $\begin{array}{l}\text { PERC } \\
\text { RECV }\end{array}$ & RPD & METHO & & $\begin{array}{l}\text { PREPARATION- } \\
\text { ANALYSIS DATE }\end{array}$ & $\begin{array}{l}\text { PREP } \\
\text { BATCH \# }\end{array}$ \\
\hline \multirow[t]{4}{*}{ Bromine } & & & WO\# : & JV87HIAI & $\sqrt{3887}$ & $\overline{A G-M S D}$ & MS & Lot-Sample & e \#: H7E040102 & $-\overline{004}$ \\
\hline & ND & 500 & 505 & ug & 101 & & KNOX & $0050 / 26 \mathrm{~A}$ & $05 / 08 / 07$ & 7135346 \\
\hline & $\mathrm{ND}$ & 500 & 560 & ug & 112 & 10 & KNOX & $0050 / 26 \mathrm{~A}$ & $05 / 08 / 07$ & 7135346 \\
\hline & & & Dilut & on Factor & & & & & & \\
\hline \multirow[t]{4}{*}{ Chlorine } & & & WO\# : & JV87HIAC & / JV87 & $\mathrm{D}$-MSD & MS & Lot-Sample & e \#: H7E040102 & -004 \\
\hline & ND & 1000 & 954 & ug & 95 & & KNOX & $0050 / 26 \mathrm{~A}$ & $05 / 11 / 07$ & 7135458 \\
\hline & ND & 1000 & 959 & ug & 96 & 0.50 & KNOX & $0050 / 26 \mathrm{~A}$ & $05 / 11 / 07$ & 7135458 \\
\hline & & & Dilut & on Factor & & & & & & \\
\hline \multirow[t]{4}{*}{ Hydrogen } & bromide & & WO\# : & JV87GIAI & $1 / 5087$ & AG-MSI & MS & Lot-Sample & e \#: H7E040102 & -003 \\
\hline & ND & 851 & 869 & ug & 102 & & KNOX & $0050 / 26 \mathrm{~A}$ & $05 / 08 / 07$ & 7135325 \\
\hline & ND & 851 & 910 & ug & 107 & 4.6 & KNOX & $0050 / 26 \mathrm{~A}$ & $05 / 08 / 07$ & 7135325 \\
\hline & & & Dilut & on Factor & & & & & & \\
\hline \multirow[t]{4}{*}{ Hydrogen } & chloride & & WO\# : & JV87GIAC & $1 / 3087$ & $A D-M S I$ & MS & Lot-Sample & e \#: H7E040102 & -003 \\
\hline & $\mathrm{ND}$ & 1730 & 1530 & ug & 89 & & KNOX & $0050 / 26 \mathrm{~A}$ & $05 / 11 / 07$ & 7135437 \\
\hline & $\mathrm{ND}$ & 1730 & 14070 & ug & 85 & 4.3 & KNOX & $0050 / 26 \mathrm{~A}$ & $05 / 11 / 07$ & 7135437 \\
\hline & & & Dilut & on Factor & & & & & & \\
\hline \multirow[t]{4}{*}{ Hydrogen } & fluoride & & WO\# : & JV87GIA & $/ 5087$ & AK-MSI & MS & Lot-Sample & e \#: H7E040102 & -003 \\
\hline & ND & 885 & 916 & ug & 104 & & KNOX & $0050 / 26 \mathrm{~A}$ & $05 / 08 / 07$ & 7135146 \\
\hline & ND & 885 & 902 & ug & 102 & 1.5 & KNOX & $0050 / 26 \mathrm{~A}$ & $05 / 08 / 07$ & 7135146 \\
\hline & & & Dilut & on Factor & & & & & & \\
\hline \multirow[t]{3}{*}{ Hydrogen } & fluoride & & wO\# : & JV87HIA & / Jv87 & $A K-M S I$ & MS & Lot-Sample & Le \#: H7E040102 & -004 \\
\hline & $\mathrm{ND}$ & 527 & 601 & ug & 114 & & KNOX & $0050 / 26 \mathrm{~A}$ & $05 / 08 / 07$ & 7135146 \\
\hline & $\mathrm{ND}$ & 527 & 602 & ug & 114 & 0.14 & KNOX & $0050 / 26 \mathrm{~A}$ & $05 / 08 / 07$ & 7135146 \\
\hline
\end{tabular}

NOTE (S) :

Calculations are performed before rounding to avoid round-off errors in calculated results. 
MATRIX SPIKR SAMPLE EVALUATION REPORT

General Chemistry

client Lot \#...: H7E040102 Date Sampled... : 04/27/07
Date Received..: 05/01/07

LIMITS

Work Order \#...: JVB7H1AF KNOX 0050/26A Mod

Dilution Factor: 2500

Work Order \#...: JVB7H1AC (75 - 125) KNOX 0050/26A Mod pilution Factor: 5000

Hydrogen bromide 102

Hydrogen chloride 89

Hydrogen fluoride 104

Hydrogen fluoride 114
Work Order \#...: JV87G1AF (75 - 125) KNOX 0050/26A Mod Dilution Factor: 4252 .

Work Order \#...: JV87G1AC (75 - 125) KNOX 0050/26A Mod Dilution Pactor: 8638.

Work order \#...: JV87G1AJ (75 - 125) KNOX 0050/26A Mod Dilution Pactor: 4422 .

Work Order \#...: JV87H1AJ (75 - 125) KNOX 0050/26A Mod Dilution Factor: 2632.
Matrix........ AIR

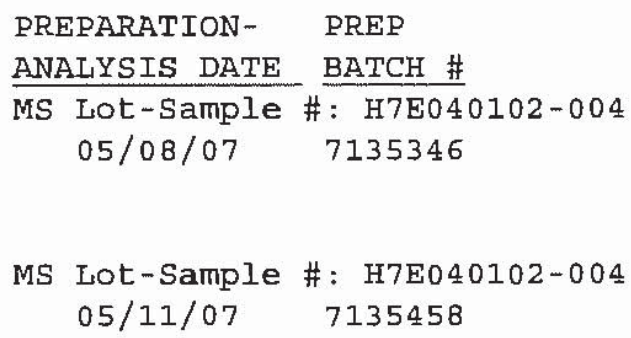

MS Lot-Sample \#: H7E040102-003 $05 / 08 / 07 \quad 7135146$

MS Lot-Sample \#: H7E040102-004 $05 / 08 / 07 \quad 7135146$

\section{$\operatorname{NOTB}(S)$ :}


MATRIX SPIKE SAMPLB DATA REPORT

General Chemistry

client Lot \#...: H7E040102

Date Sampled...: 0 0 $\$ 27 / 07$
Matrix....... A AIR

Date Received..: 05/01/07

\begin{tabular}{|c|c|c|c|c|c|c|c|c|c|}
\hline PARAMETER & $\begin{array}{l}\text { SAMPLE } \\
\text { AMOUNT }\end{array}$ & $\begin{array}{l}\text { SPIKE } \\
\text { AMT } \\
\end{array}$ & $\begin{array}{l}\text { MEASURED } \\
\text { AMOUNT }\end{array}$ & UNITS & $\begin{array}{l}\text { PERCENT } \\
\text { RECOVERY }\end{array}$ & $\underline{\text { METHO }}$ & & $\begin{array}{l}\text { PARATION- } \\
\text { ALYSIS DAT }\end{array}$ & $\begin{array}{l}\text { PREP } \\
\text { BATCH \# }\end{array}$ \\
\hline \multirow[t]{3}{*}{ Bromine } & & & \multicolumn{3}{|c|}{$\overline{\text { Work order \#...: JV87H1AF }}$} & \multirow{3}{*}{ KNOX } & \multicolumn{3}{|c|}{ MS Lot-Sample \#: H7E040102-004 } \\
\hline & \multirow[t]{2}{*}{$\mathrm{ND}$} & \multirow[t]{2}{*}{500} & 505 & ug & 101 & & \multirow{2}{*}{\multicolumn{2}{|c|}{$05 / 08 / 07$}} & \multirow[t]{2}{*}{7135346} \\
\hline & & & Dilution $\mathrm{F}$ & Pactor: 2500 & & & & & \\
\hline \multirow[t]{3}{*}{ Chlorine } & \multirow{3}{*}{ ND } & \multirow{3}{*}{1000} & Work ord & ler \#...: : & JVB7H1AC & \multirow{3}{*}{ KNOX } & \multicolumn{3}{|c|}{ MS Lot-Sample \#: H7E0 $\$ 0102-004$} \\
\hline & & & 954 & ug & 95 & & \multirow[t]{2}{*}{$0050 / 26 \mathrm{~A}$} & \multirow[t]{2}{*}{$05 / 11 / 07$} & \multirow{2}{*}{7135458} \\
\hline & & & Dilution & Pactor: 5000 & & & & & \\
\hline \multirow{3}{*}{ Hydrogen } & \multirow{3}{*}{$\begin{array}{c}\text { bromide } \\
\text { ND }\end{array}$} & \multirow{3}{*}{851} & Work ord & ler \#...: : & JVB7GIAF & \multirow{3}{*}{ KNOX } & \multicolumn{3}{|c|}{ MS Lot-Sample \#: H7E040102-003 } \\
\hline & & & 869 & ug & 102 & & \multirow[t]{2}{*}{$0050 / 26 \mathrm{~A}$} & \multirow[t]{2}{*}{$05 / 08 / 07$} & \multirow[t]{2}{*}{7135325} \\
\hline & & & Dilution & Factor: 4252 & & & & & \\
\hline \multirow[t]{3}{*}{ Hydrogen } & chloride & \multirow{3}{*}{1730} & Work ord & ler \#...: & JV87GIAC & \multirow{3}{*}{ KNOX } & \multicolumn{3}{|c|}{ MS Lot-Sample \#: H7E040102-003 } \\
\hline & \multirow[t]{2}{*}{$\mathrm{ND}$} & & 1530 & ug & 89 & & \multirow[t]{2}{*}{$0050 / 26 \mathrm{~A}$} & \multirow[t]{2}{*}{$05 / 11 / 07$} & \multirow[t]{2}{*}{713543} \\
\hline & & & Dilution & Eactor: 8638 & & & & & \\
\hline \multirow[t]{3}{*}{ Hydrogen } & fluoride & & Work ord & ler \#...: & $\pi 8870$ & & \multicolumn{3}{|c|}{ MS Lot-Sample \#: H7E040102-003 } \\
\hline & $\mathrm{ND}$ & 885 & 916 & ug & 104 & KNOX & $0050 / 26 \mathrm{~A}$ & $05 / 08 / 07$ & 7135146 \\
\hline & & & Dilution & Factor: 4422 & & & & & \\
\hline Hydrogen & fluoride & & Work Ord & ler \#...: & JV871 & & MS IC & H7EO & $0102-004$ \\
\hline & ND & 527 & 601 & ug & 114 & KNOX & $0050 / 26 \mathrm{~A}$ & $05 / 08 / 07$ & 7135146 \\
\hline & & & 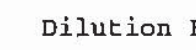 & ctor: 2632 & & & & & \\
\hline
\end{tabular}

\section{NOTE (S) :}




\section{Sample Receipt Documentation}



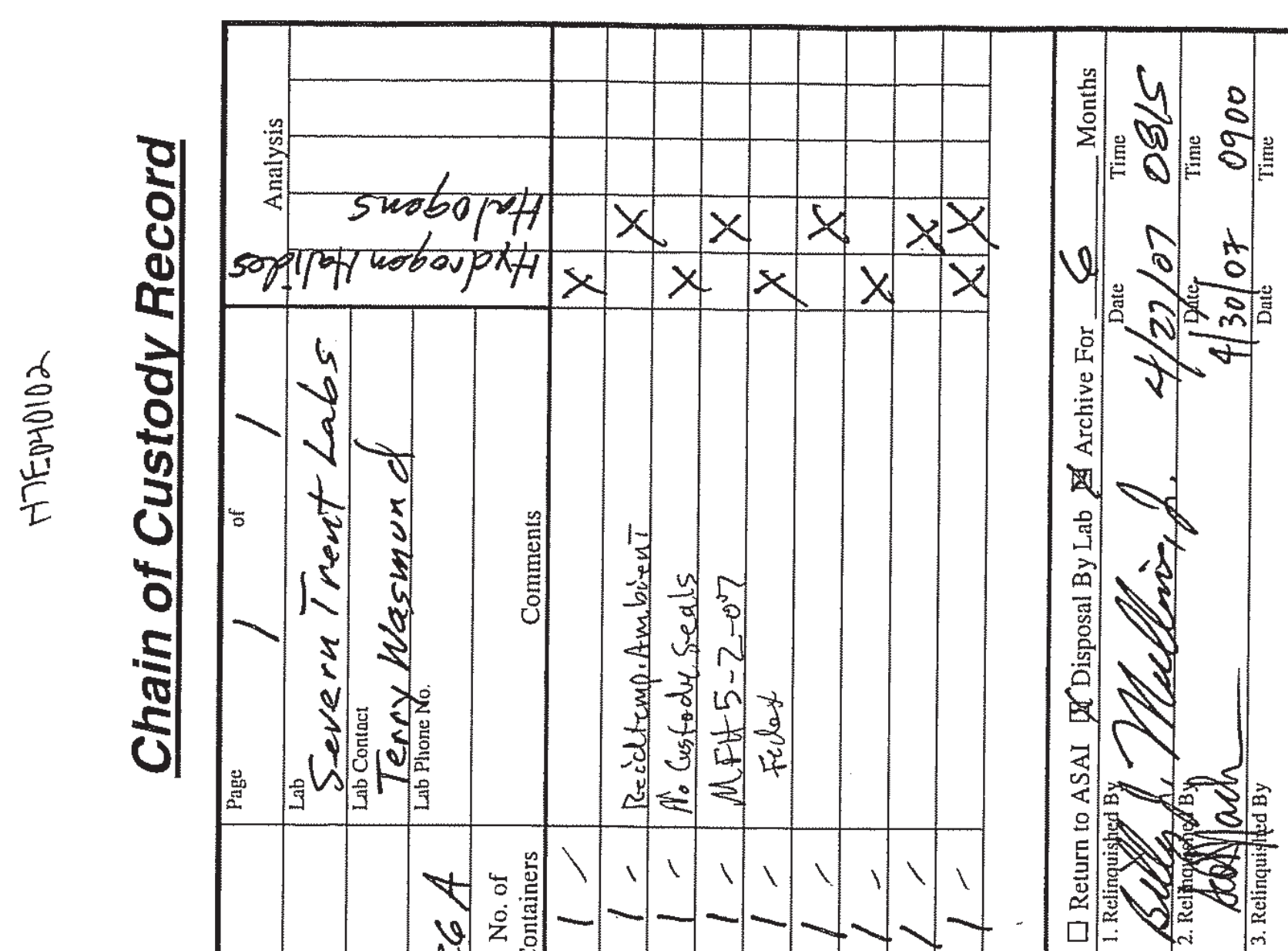

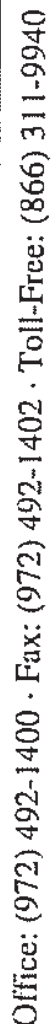

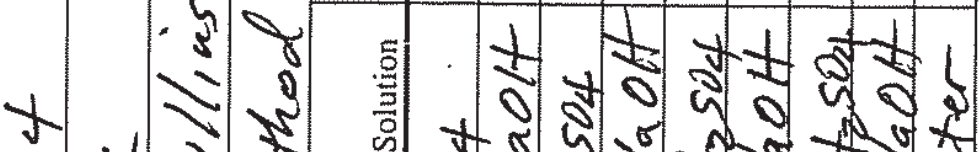

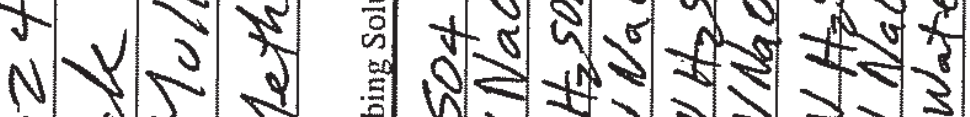
0 र 5 : 1.50 र

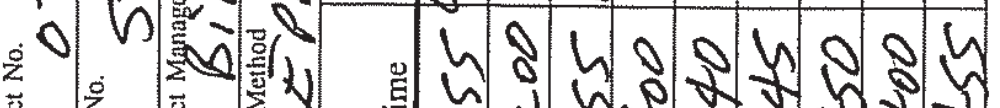
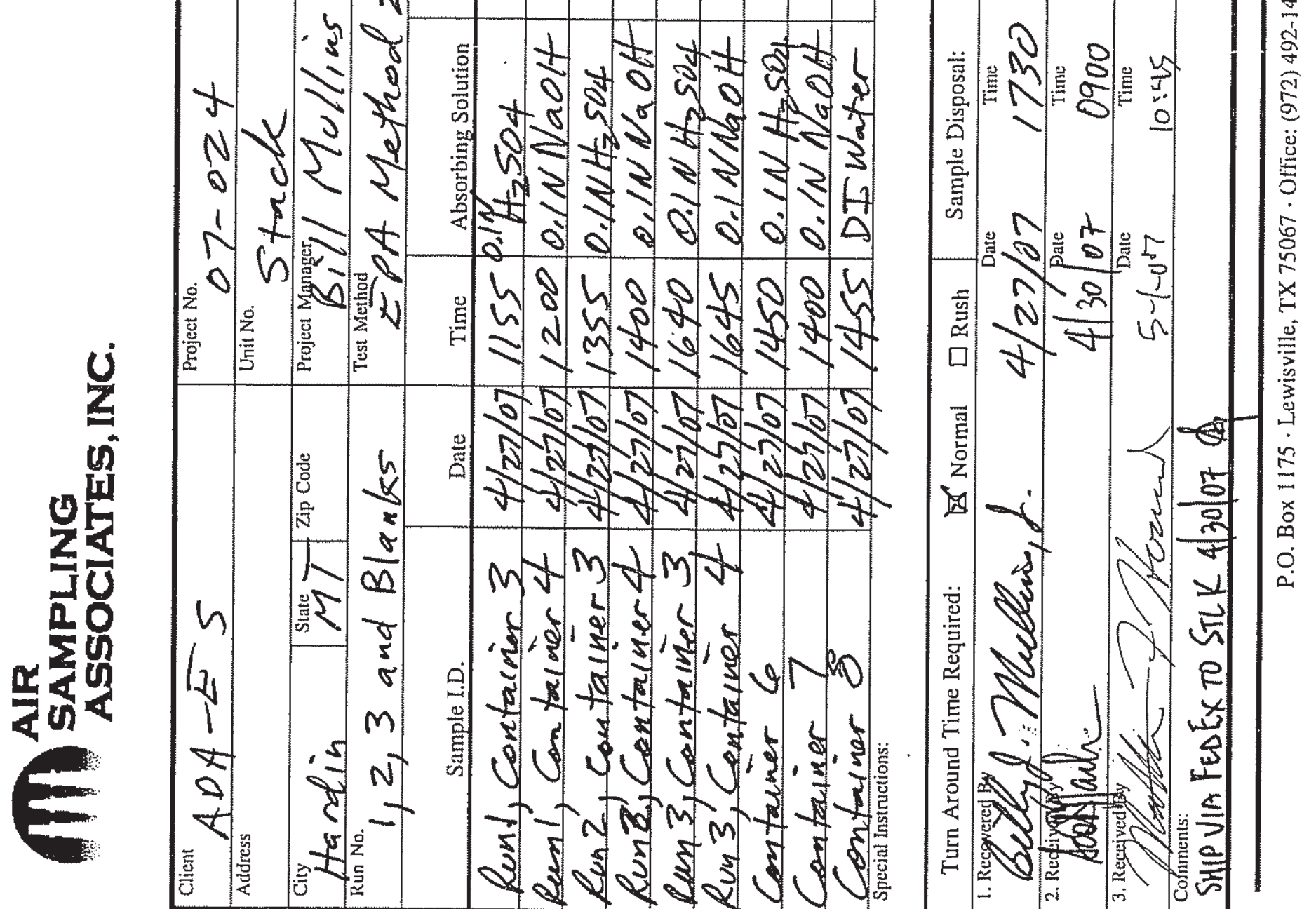


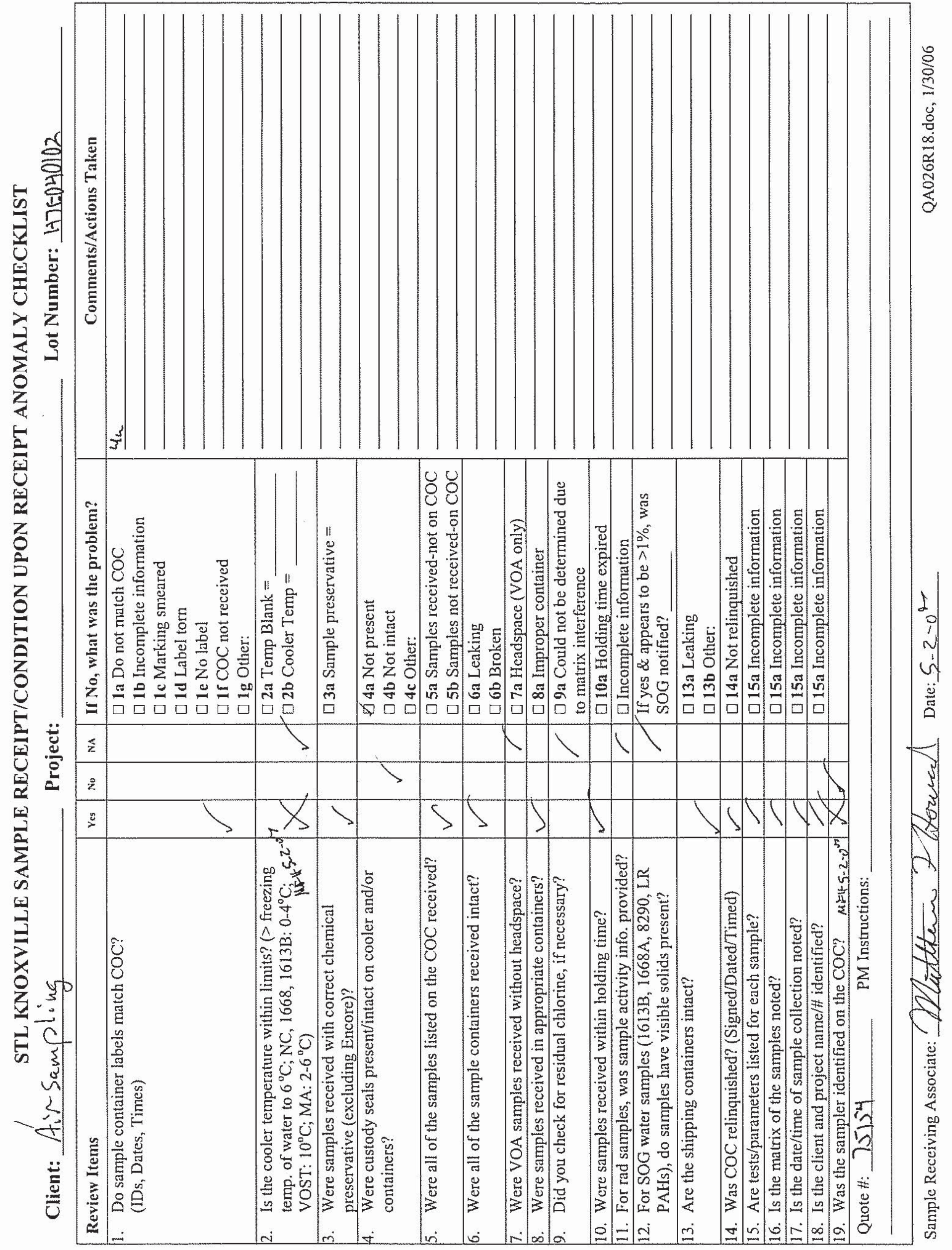


Wet Chemistry 
Sample Summary 
Air Sampling Associates, Inc.

Client Sample ID: 07024 HARDTN STACK RON 1 0.IN H2SO4

General Chemistry

Lot-Sanple \#.. : : H7E040102-001

Date Sampled...: 04/27/07
Work Order \#...: JV87E

Date Received..: 05/01/07
Matrix....... A AIR

PREPARATION- PREP ANALYSIS DATE BATCH \#

METHOD KNOX 0050/26A Mod 05/08/07 7135325 Dilution Factor: 4961.81 MDL.......... 248

Hydrogen chloride ND $1010 \mathrm{ug}$ Dilution Factor: 10078.61 KNOX 0050/26A Mod 05/11/07 MDL......... 423

Hydrogen fluoride ND $516 \quad \mathrm{ug}$ ig 5159.95 KNOX 0050/26A Mod 05/08/07 MDL.......... 258
7135437

7135146 
Air Sampling Associates, Inc.

\section{Client Sample ID: 07024 HARDIN STACK RON 1 0.IN NAOH}

\section{General Chemistry}

Lot-Sample \#... : H7E040102-002

Date Sampled...: 04/27/07
Work Order \#...: TVB $7 \mathrm{~F}$

Date Received..: 05/01/07
Matrix....... AIR

PREPARATION - PREP ANALYSIS DATE BATCH \#

PARAMETER

RESULT RL UNITS METHOD KNOX 0050/26A Mod 05/08/07 MDL......... 66.0 Dilution Factor: 2750 KNOX 0050/26A Mod 05/11/07 MDL......... 231

Hydrogen fluoride
ND

ND

ND
7135346

7135458

7135146
$550 \quad \mathrm{ug}$ Dilution Factor: 5500

$290 \quad u g$ Dilution Factor; 2895.89
KNOX 0050/26A Mod 05/08/07 MDL.......... 145 
Air Sampling Associates, Inc.

client Sample ID: 07024 HARDIN STACK RUN 2 o.1N H2SO4

General Chemistry

Lot-Sample \#...: H7E040102-003

Date Sampled...: 04/27/07
Work Order \#...: JV87G

Date Received. .: 05/01/07
Matrix....... A AIR

PREPARATION - PREP ANALYSIS DATE BATCH \#

PARAMETER

RESULT

RL

UNITS

METHOD

425 ug

Dilution Factor: 4252.98

KNOX 0050/26A Mod 05/08/07

MDL........ 213

Hydrogen chloride ND

864 ug

Dilution Factor: 8638.

KNOX 0050/26A Mod 05/11/07

MDL........ : 363

442 ug

Dilution Factor: 4422.82
KNOX 0050/26A Mod 05/08/07 MDL......... : 221
7135325

7135437

7135146
Hydrogen fluoride

ND 
Air Sampling Associates, Inc.

Client Sample ID: 07024 HARDIN STACK RUN 20. IN NAOH

\section{General Chemistry}

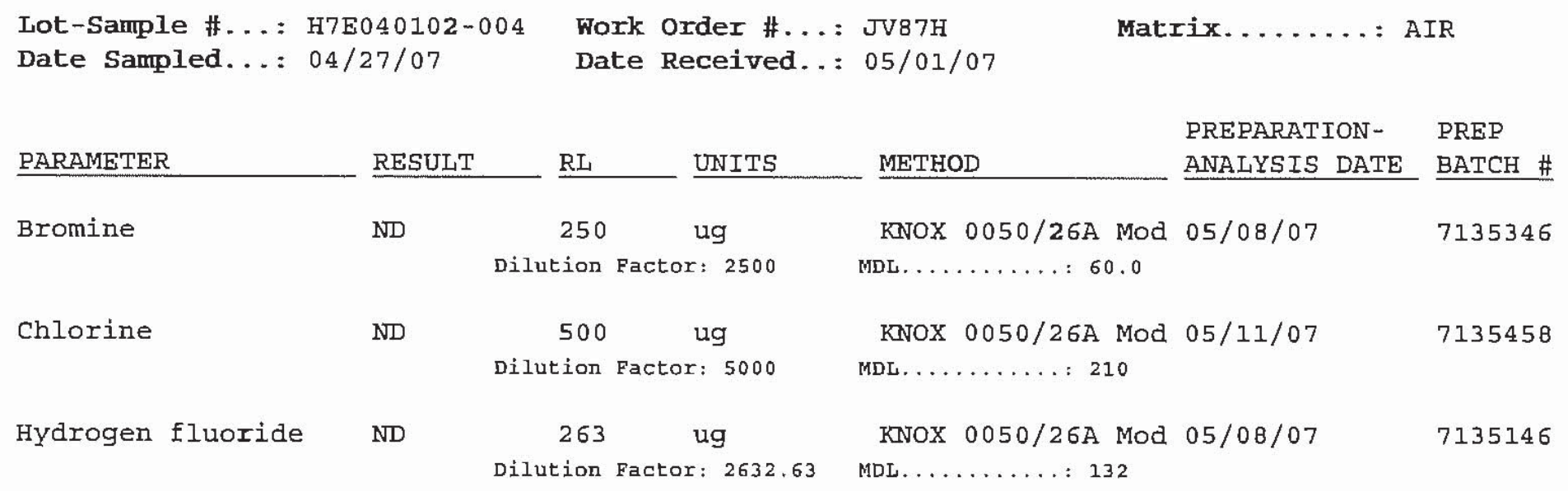


Air Sampling Associates, Inc.

Client Sample ID: 07024 HARDTN STACK RUN 30. IN H2SO4

\section{General Chemistry}

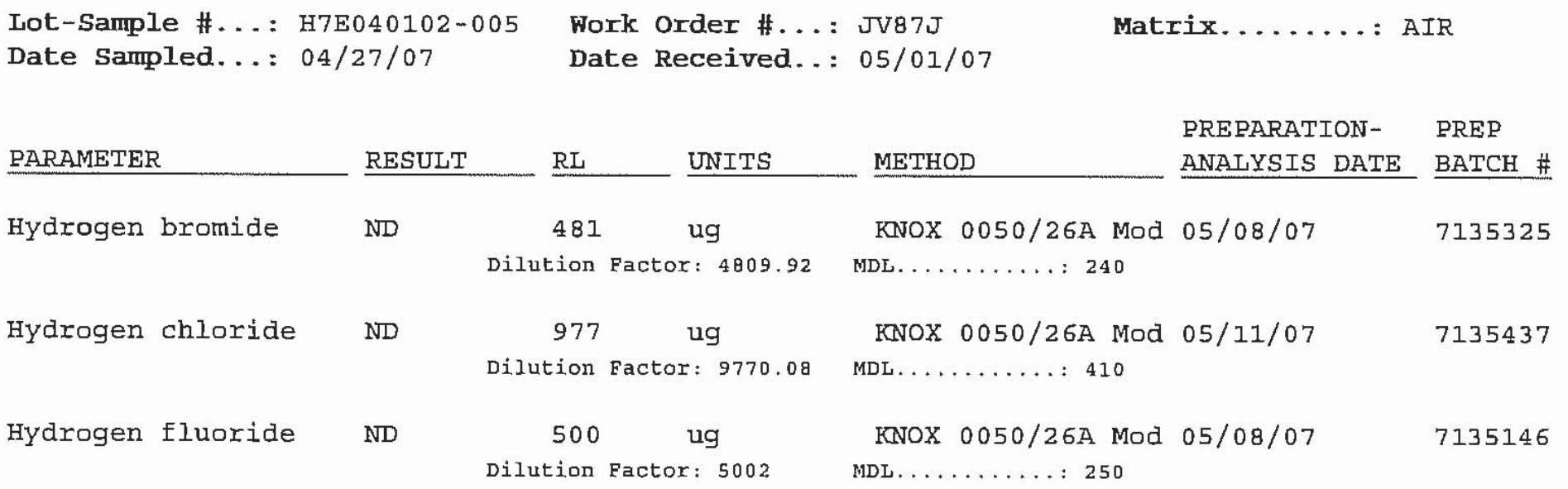


Air Sampling Associates, Inc.

client Sample ID: 07024 HARDTN STACK RUN 30 . IN NAOH

General Chemistry

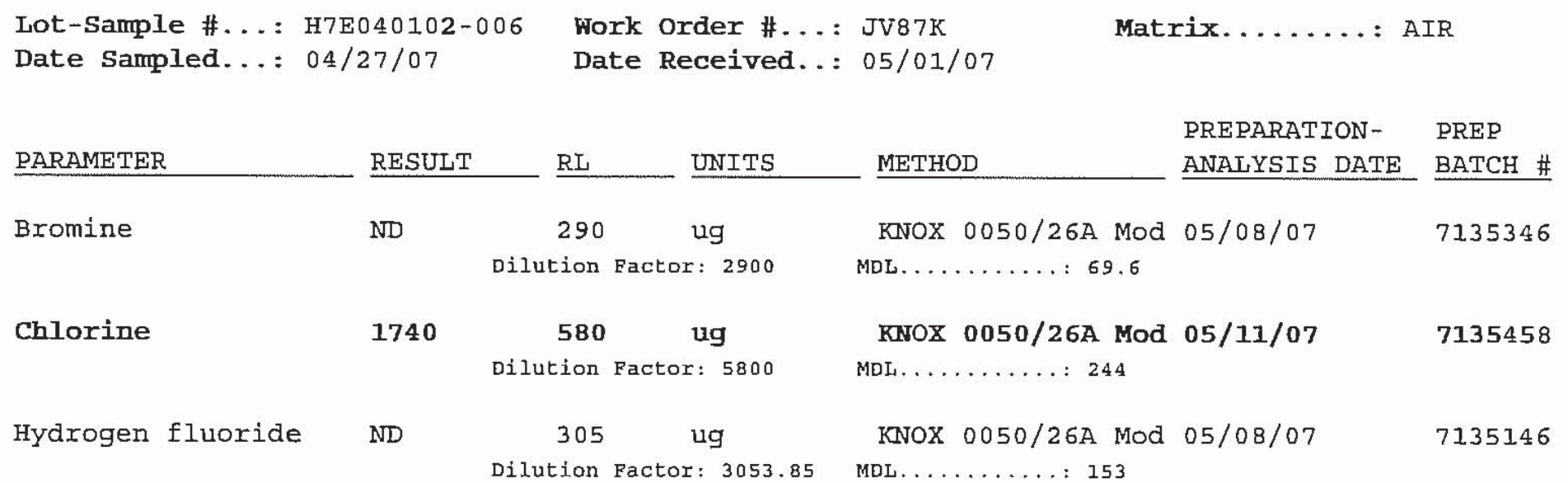


Air Sampling Associates, Inc.

client Sample ID: 07024 HARDIN STACK BLANK $0.1 \mathrm{~N}$ H2SO4

General Chemistry

Lot-Sanple \#...: H7E040102-007

Date Sampled...: 04/27/07
Work Order \#... : JV87L

Date Received..: 05/01/07
Matrix....... : AIR

PREPARATION- PREP ANALYSIS DATE BATCH \#

\section{PARAMETER}

RESULT

$\underline{\text { RI }}$

UNITS

101

ug

Dilution Factor: 1012.61

ND

411

ug Dilution Factor: 4113.72

ND
METHOD

KNOX 0050/26A Mod 05/08/07

MDL......... 50.6

KNOX 0050/26A Mod 05/11/07 MDL......... 173

KNOX 0050/26A Mod 05/08/07

MDL........ 52.7
7135325

7135437

7135146

\author{
Dilution Factor: 1053.05
}


Air Sampling Associates, Inc.

client Sample ID: 07024 HARDIN STACK BLANK 0. WN NAOH

General Chemistry

Lot-Sample \#...: H7E040102-008

Date Sampled...: 04/27/07
Work order \#...: JV87M

Date Received..: 05/01/07
Matrix......: AIR

PREPARATION-

PREP ANALYSIS DATE BATCH \#

PARAMETER

RESULT

$\underline{\mathrm{RL}}$

UNITS

METHOD

ND

100

ug

Dilution Factor: 1000

KNOX 0050/26A Mod 05/08/07

MDL......... 24.0

ND $200 \quad$ ug

Dilution Factor: 2000

55.4 B $105 \quad$ ug

Dilution Factor: 1053.05
KNOX 0050/26A Mod 05/11/07

MDL.......... 84.0

KNOX 0050/26A Mod 05/08/07

MDL .........: 52.7
7135346

7135458

7135146

\section{NOTE (S)}

RL Reporting Limit

B Estimated result. Result is less unan RL. 
Air Sampling Associates, Inc.

Client Sample ID: 07024 HARDIN STACK DI WATRR

General Chemistry

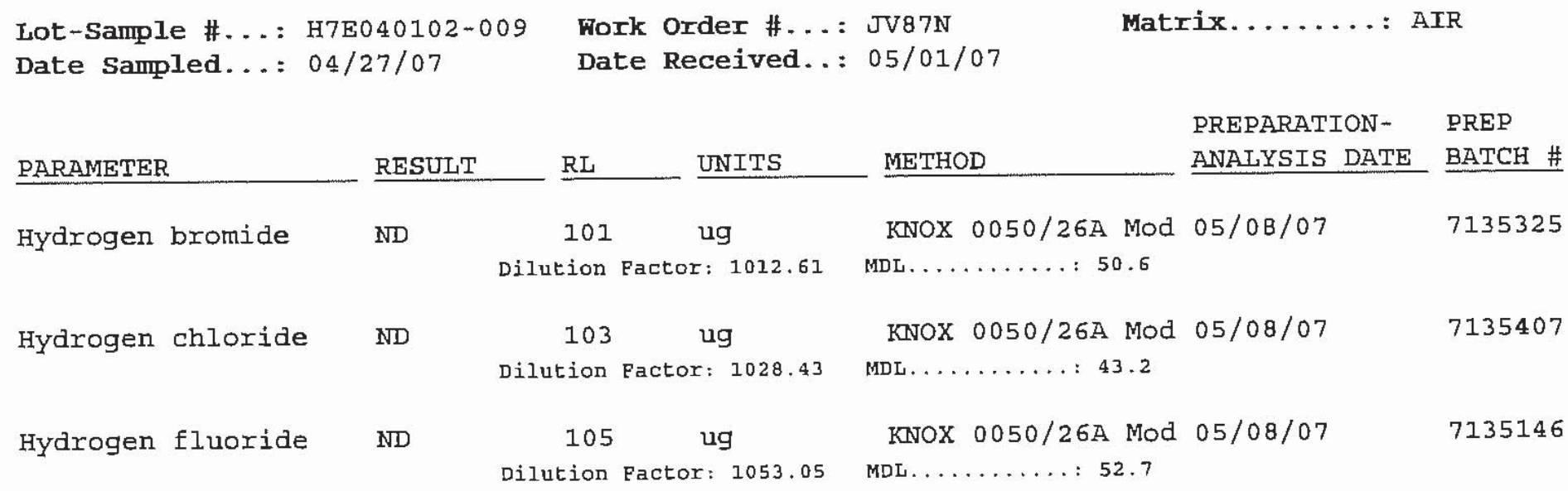


QC Summary 
MRTHOD BIANK REPORT

General Chemistry

Client Lot \#...: H7E040102

Matrix.......: AIR

\begin{tabular}{|c|c|c|c|c|c|}
\hline PARAMETER & RESULT & $\begin{array}{l}\text { REPORTING } \\
\text { LIMIT UNITS }\end{array}$ & METHOD & $\begin{array}{l}\text { PREPARATION- } \\
\text { ANALYSIS DATE }\end{array}$ & $\begin{array}{l}\text { PREP } \\
\text { BATCH \# }\end{array}$ \\
\hline Bromine & ND & $\begin{array}{l}\text { Work Order \#: JW0761AA } \\
10.0 \text { ug } \\
\text { Dilution Factor: } 100\end{array}$ & $\begin{array}{l}\text { MB Lot-Sample \#: } \\
\text { KNox } 0050 / 26 \mathrm{~A} \text { Mod }\end{array}$ & $\begin{array}{l}\text { H7E150000-346 } \\
05 / 08 / 07\end{array}$ & 7135346 \\
\hline Chlorine & ND & $\begin{array}{l}\text { Work Order \#: JWITNIAA } \\
10.0 \text { ug } \\
\text { Dilution Factor: } 100\end{array}$ & $\begin{array}{l}\text { MB Lot-Sample \#: } \\
\text { kNox } 0050 / 26 \text { A Mod }\end{array}$ & $\begin{array}{l}\text { H7E } 150000-458 \\
05 / 11 / 07\end{array}$ & 7135458 \\
\hline Hydrogen bromide & ND & 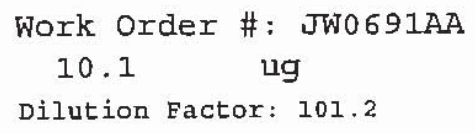 & $\begin{array}{l}\text { MB Lot-Sample \#: } \\
\text { KNOX 0050/26A Mod }\end{array}$ & $\begin{array}{l}\mathrm{H} 7 \mathrm{E} 150000-325 \\
05 / 08 / 07\end{array}$ & 7135325 \\
\hline Hydrogen chloride & ND & $\begin{array}{l}\text { Work Order \#: JWIDJIAA } \\
10.3 \text { ug } \\
\text { Dilution Factor: } 102.8\end{array}$ & $\begin{array}{l}\text { MB Lot-Sample \#: } \\
\text { KNOX } 0050 / 26 \mathrm{~A} \text { Mod }\end{array}$ & $\begin{array}{l}\mathrm{H} 7 \mathrm{E} 150000-407 \\
05 / 08 / 07\end{array}$ & 7135407 \\
\hline Hydrogen chloride & $\mathrm{ND}$ & $\begin{array}{l}\text { Work Order \#: JWIMVIAA } \\
10.3 \text { ug } \\
\text { Dilution Factor: } 102.8\end{array}$ & $\begin{array}{l}\text { MB Lot-Sample \#: } \\
\text { KNOX } 0050 / 26 \mathrm{~A} \text { Mod }\end{array}$ & $\begin{array}{l}\text { H7E150000-437 } \\
05 / 11 / 07\end{array}$ & 7135437 \\
\hline Hydrogen fluoride & ND & $\begin{array}{l}\text { Work order \#: JWOHIIAA } \\
10.5 \text { ug } \\
\text { Dilution Factor: } 105.3\end{array}$ & $\begin{array}{l}\text { MB Lot-Sample \#: } \\
\text { KNOX } 0050 / 26 \mathrm{~A} \text { Mod }\end{array}$ & $\begin{array}{l}\text { H7E } 150000-146 \\
05 / 08 / 07\end{array}$ & 7135146 \\
\hline
\end{tabular}

$\operatorname{NOTB}(\mathrm{S})$ :

Calculations are performed before rounding to avoid round-off errors in calculated results. 


\section{ILABORATORY CONTROL SAMPLE EVALUATION REPORT}

\section{General Chemistry}

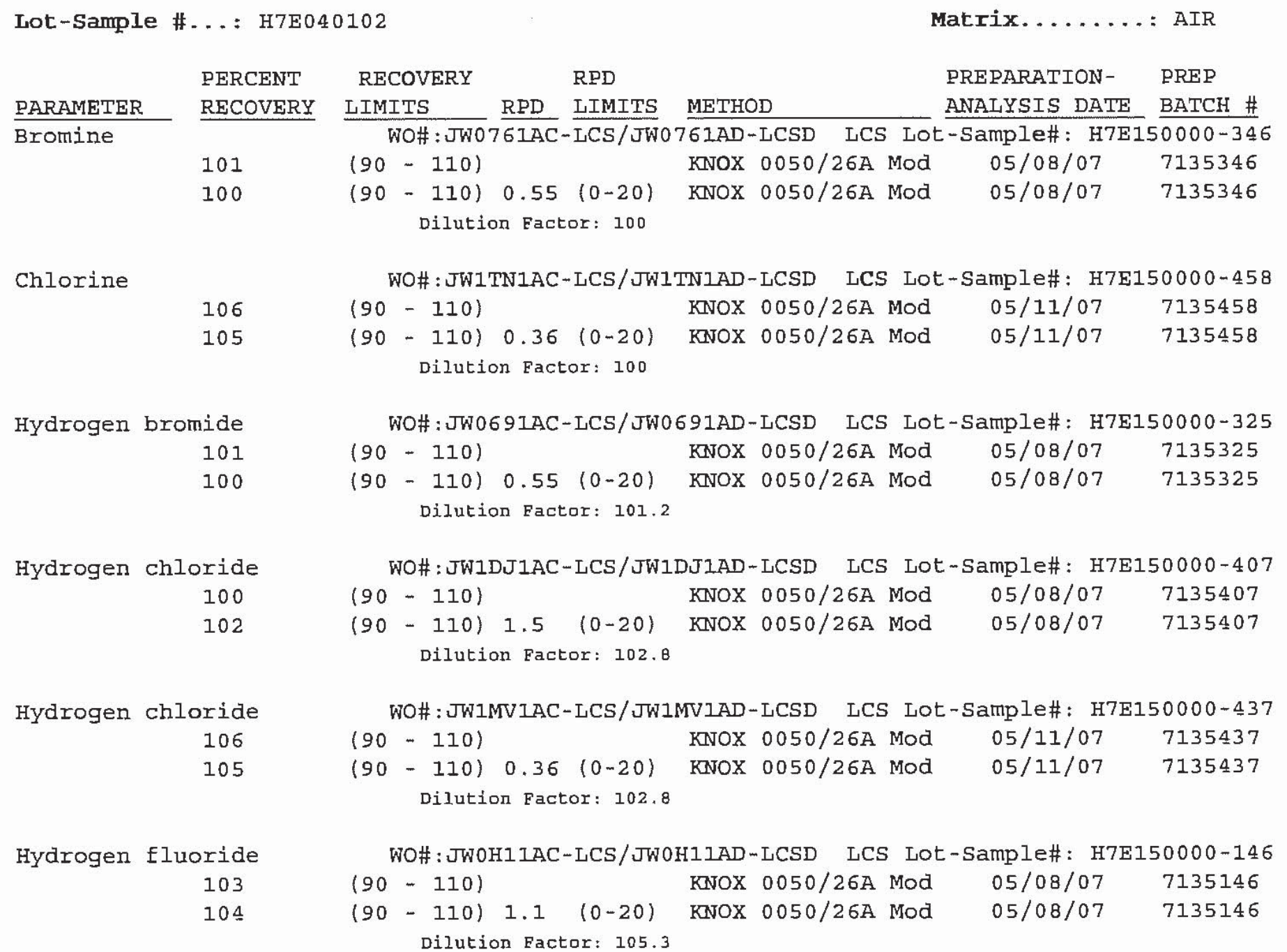

$\operatorname{NOTR}(5):$

Calculations are performed before rounding to avoid round-off errors in calculated results. 
LABORATORY CONTROL SAMPLE DATA REPORT

General Chemistry

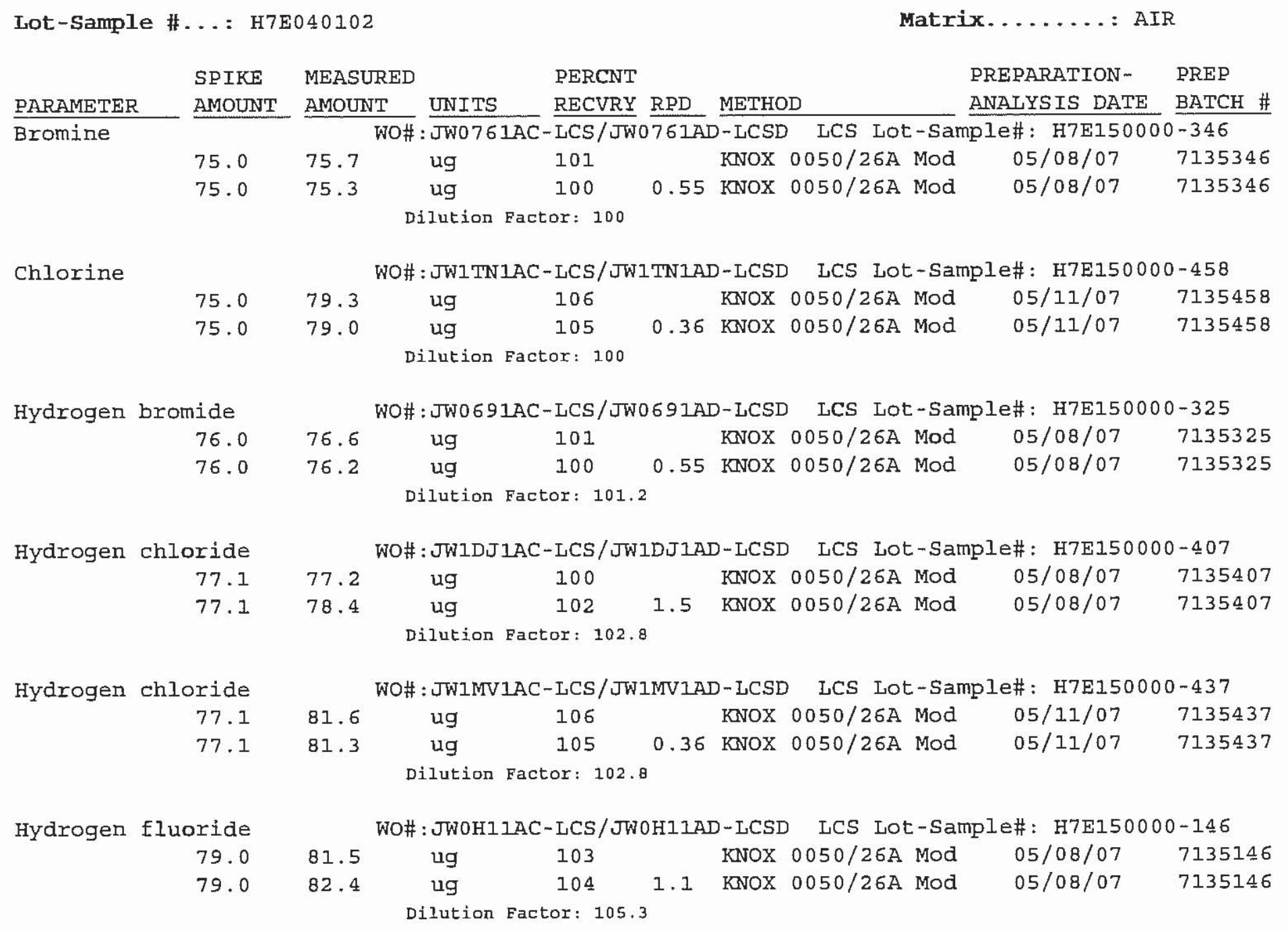

NOTE (S) :

Calculations are performed before rounding to avoid round-aff crrofs in calculated results. 
IABORATORY CONTROL SAMPLS EVALUATION REPORT

\section{General Chemistry}

client Lot \#...: H7E040102

Matrix....... AIR

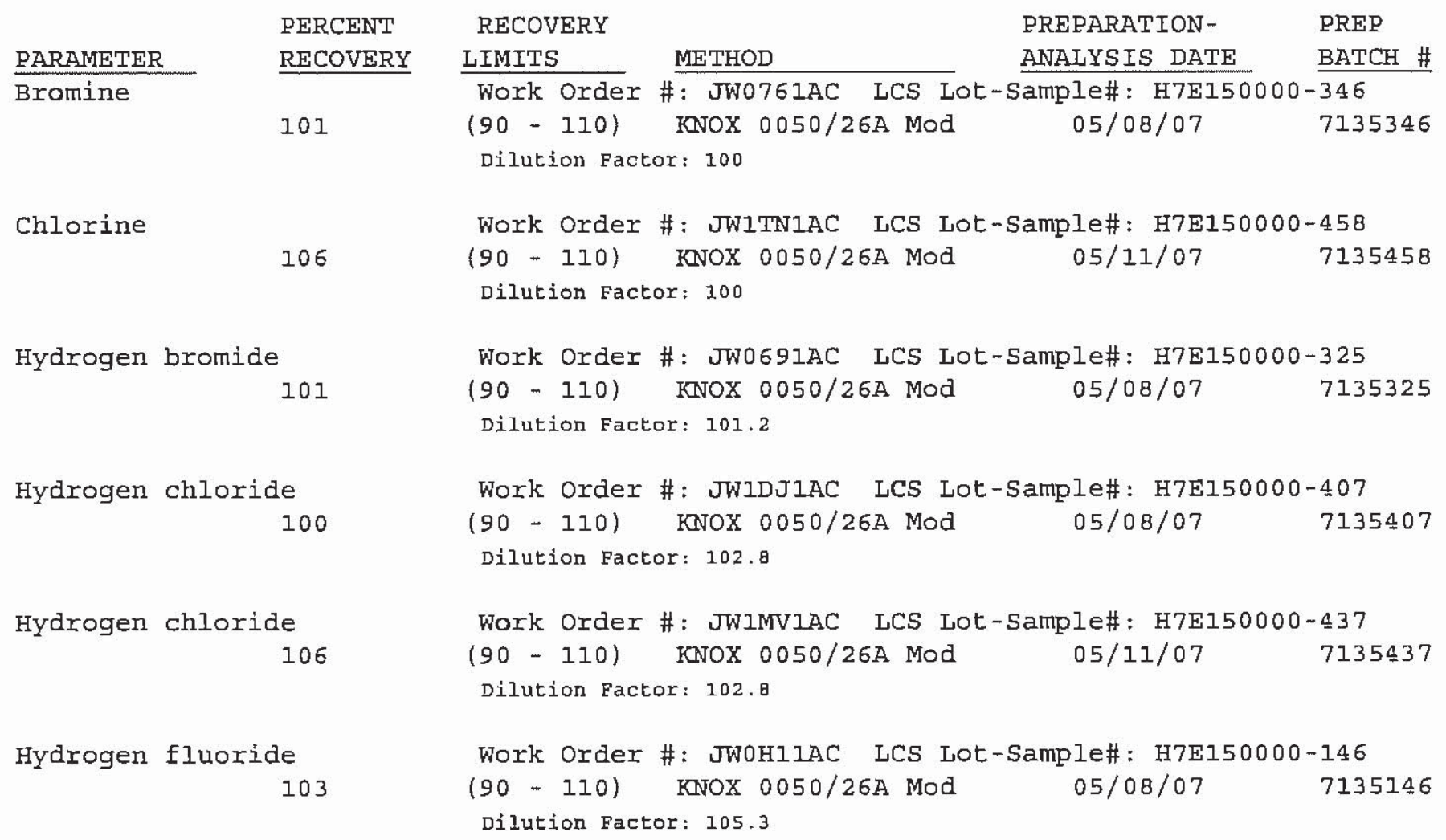

\section{NOTE (S) :}

Calculations are performed before rounding to avoid round-off errors in calculated results. 


\section{LABORATORY CONTROL SAMPLE DATA REPORT}

\section{General Chemistry}

Client Lot \#...: H7E040102

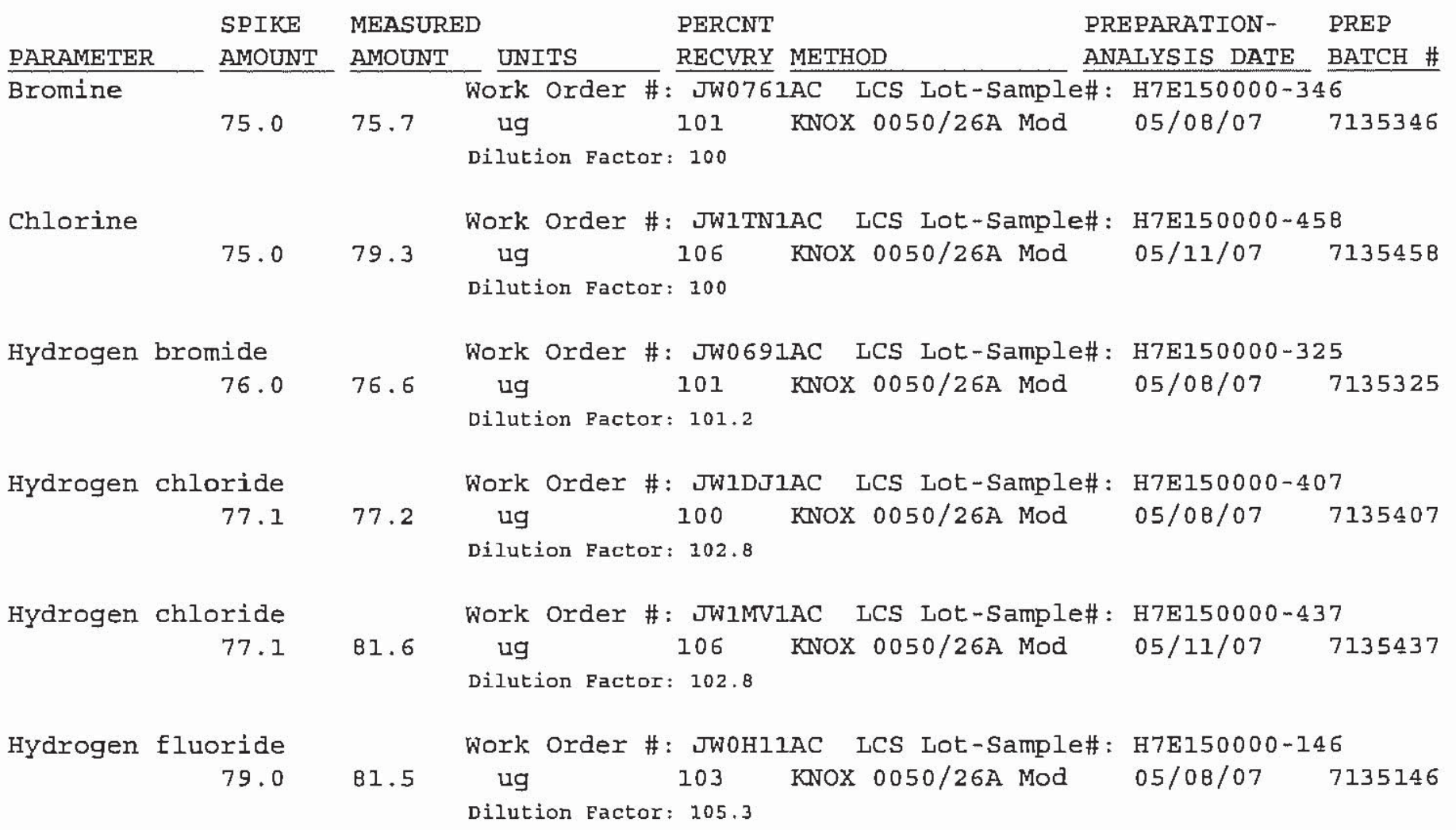

\section{NOTE $(S)$ :}

Calculations are performed before rounding to avoid round-off errors in calculated resuls. 
MATRIX SPIKR SAMPLF EVAUUATION REPORT

General Chemistry

Client Lot \#...: H7E040102

Date Sampled...: 04/27/07
Date Received..: 05/01/07
Matrix........ AIR

PREPARATION - PREP ANALYSIS DATE BATCH \#

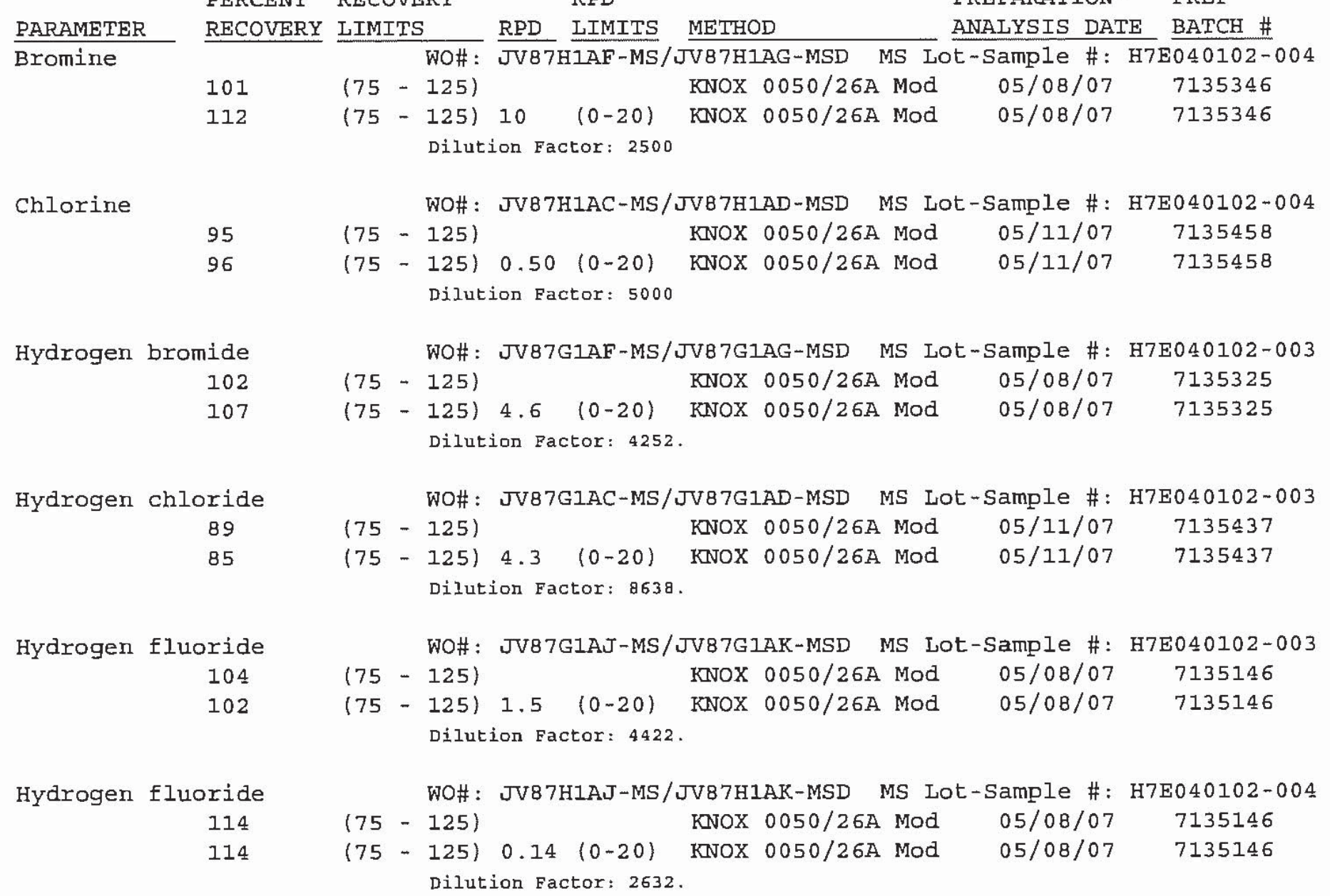

NOTE (S) :

Calculations are performed before rounding to avoid round-off errors in calculated results. 


\section{MATRIX SPIKF SAMPLE DATA REPORT}

\section{General Chemistry}

client Irot \#...: H7E040102 Date sampled...: 04/27/07
Matrix........ AIR

Date Received.. : 05/01/07

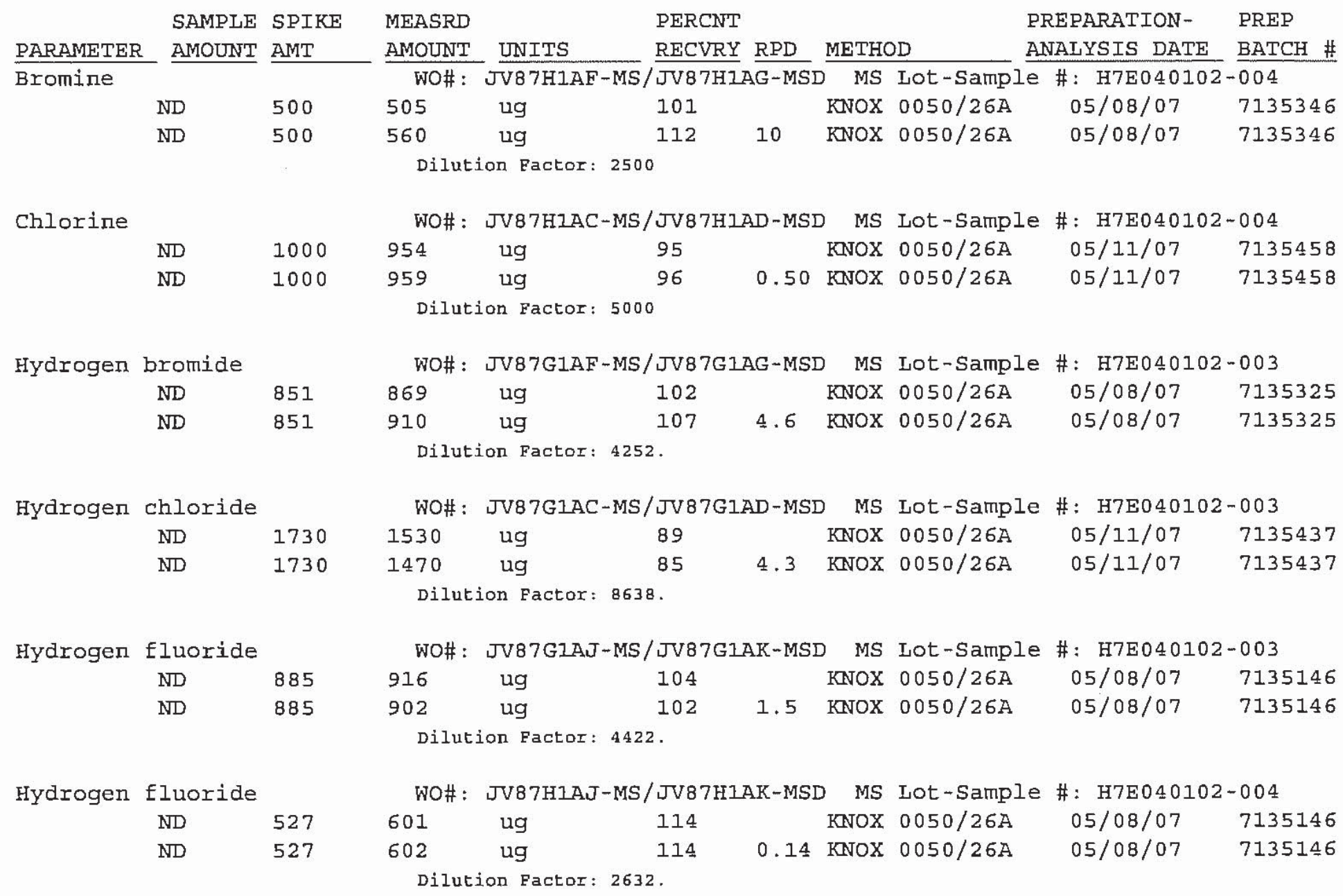

NOTE (S) :

Calculations are performed before rounding to avoid round-off errors in calculated results. 
MATRIX SPIKK SAMPIE EVALUATION REPORT

\section{General Chemistry}

Client Lot \#...: H7E040102

Date Sampled...: 04/27/07
Date Received..: 05/01/07
Matrix........ AIR

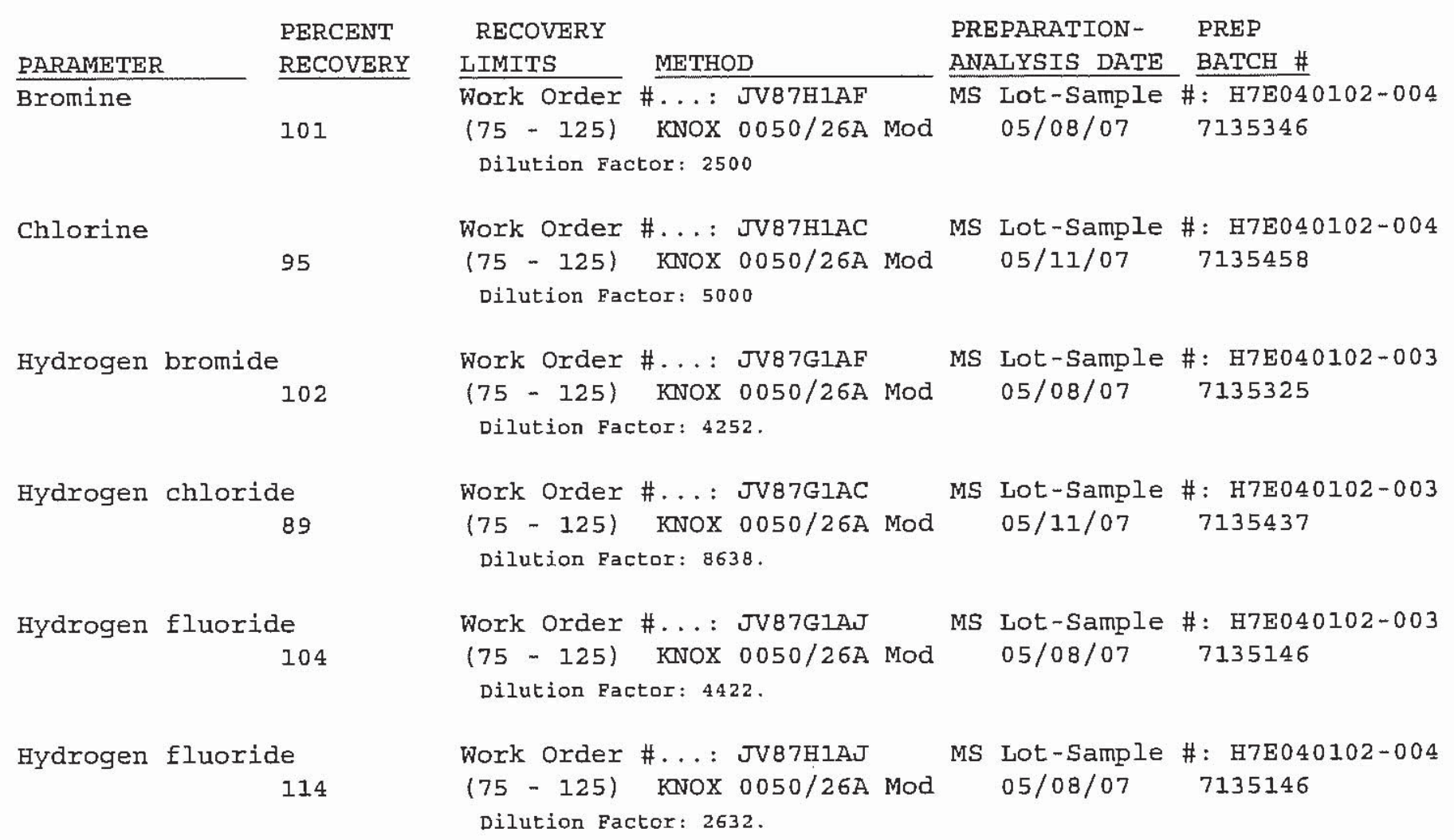




\section{MATRIX SPIKE SAMPLE DATA REPORT}

\section{General Chemistry}

client Lot \#...: H7E040102

Date Sampled...: 04/27/07
Date Received..: 05/01/07
Matrix.......: AIR

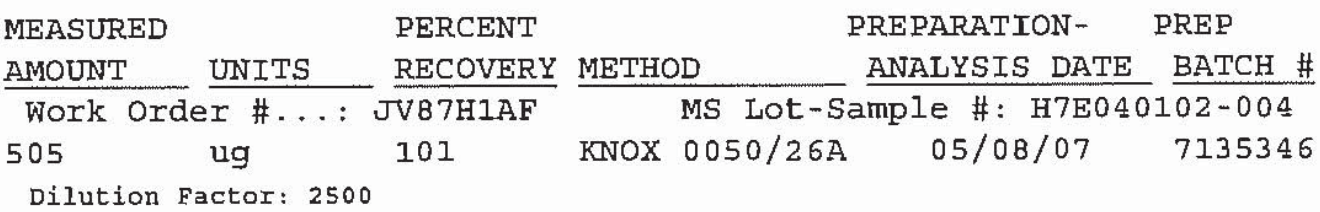

Work Order \#...: JVB7H1AC MS Lot-Sample \#: H7E040102-004

Chlorine

ND $\quad 1000$

954

ug

95

Dilution Factor: 5000

Hydrogen bromide

ND

851

$869 \quad$ ug 102

Dilution Factor: 4252 .

Hydrogen chloride

ND

173

Wor

r \#...: JV87G1AC

1530 ug 89

Dilution Factor: 8638 .

Hydrogen fluoride

ND

Work Order \#...: JVB7G1AJ

$\begin{array}{llll}885 & 916 & \text { ug }\end{array}$

Dilution Factor: 4422 .

Hydrogen fluoride

ND KNOX $0050 / 26 \mathrm{~A} \quad 05 / 11 / 07 \quad 7135458$

MS Lot-Sample \#: H7E040102-003 KNOX 0050/26A 05/08/07 7135325

MS Lot-Sample \#: H7E040102-003 KNOX $0050 / 26 \mathrm{~A} \quad 05 / 11 / 07 \quad 7135437$

MS Lot-Sample \#: H7E040102-003 KNOX $0050 / 26 \mathrm{~A} \quad 05 / 08 / 07 \quad 7135146$

MS Lot-Sample \#: H7E040102-004 KNOX 0050/26A 05/08/07 7135146
Work Order \#...: JV87H1AJ

601 ug 114

Dilution Factor: 2632.

\section{$\operatorname{NOTB}(S)$ :}




\section{Raw Data}




\section{Hydrogen Chloride / Chlorine}




\section{STL Knoxville IC Initial Calibration Data Review / Narrative Checklist \\ Methods: 300.0, 9056, 9057 and 26A, SOP: KNOX-WC-0005, Rev. 8 \\ Page 1 of 1 \\ (D)}

\section{Analvsis Date: $\quad 4 / 16 / 07$ \\ File ID: D16A- LOWANIONS - 041607-QUAD}

\begin{tabular}{|c|c|c|c|c|c|}
\hline Initial Calibration Review Items & N/A & Yes & No & If No, why is data reportable? & $\begin{array}{c}\text { 2nd } \\
V\end{array}$ \\
\hline 1. Were at least 5 levels of each analyte analyzed? & & $\checkmark$ & & & \\
\hline 2. Is low level standard concentration $\leq R L$ ? & & $\sqrt{ }$ & & & \\
\hline $\begin{array}{l}\text { 3. Are the correlation coefficients }(r) \geq 0.995 \text {; } \\
r^{2} \geq 0.990 \text { ? }\end{array}$ & & $\sqrt{ }$ & & & \\
\hline $\begin{array}{l}\text { 4. For method } 300.0 \text {, was the calibration curve } \\
\text { processed using linear reqression? }\end{array}$ & $\checkmark$ & & & & $N / A$ \\
\hline $\begin{array}{l}\text { 5. For manual integrated standards, are before/after } \\
\text { chromatogramss provided with initials/date/reason? }\end{array}$ & & $\sqrt{ }$ & $\cdots$ & $\begin{array}{l}\text { Reasons: } S=\text { Split peak, } U=\text { Undetected peak, I=Incorrect } \\
\text { peak integration, } B=B \text { aseline correction, } W=\text { Wrong peak } \\
\text { chosen by data system }\end{array}$ & \\
\hline
\end{tabular}

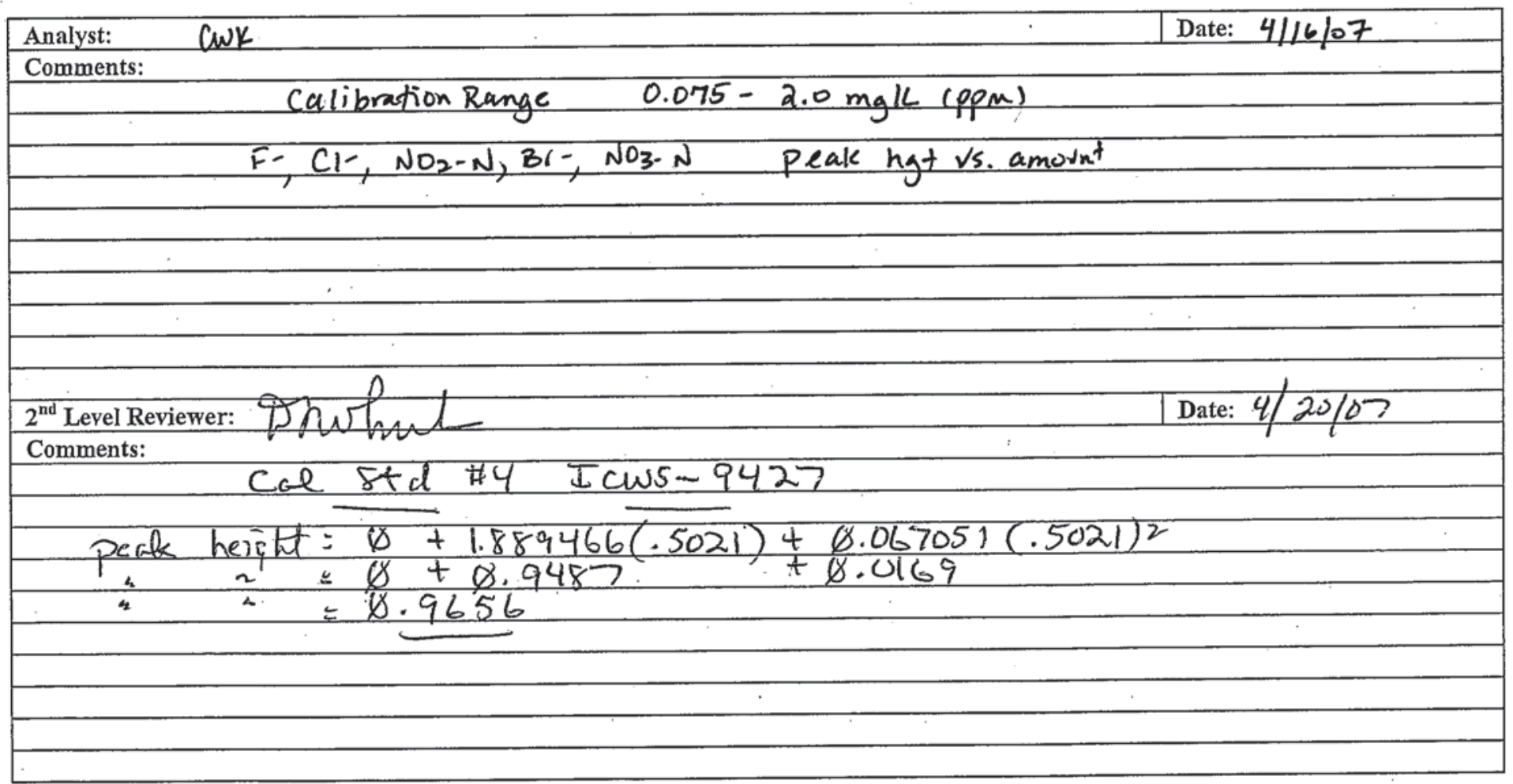




\section{CAL STD \#1 ICWS-9424}

\section{QUAD}

Sample Name: $\quad$ CAL STD \#1 ICWS-9424

Vial Number:

Sample Type:

Control Program:

Quantif. Method:

Recording Time:

Run Time ( $\mathrm{min})$ :
269

standard

ANIONS_AS14A

ANIONS

4/16/2007 12:21

14.75
Injection Volume:

Channel:

50.0

Wavelength:

Bandwidth:

Dilution Factor:

Sample Weight:

Sample Amount:
ECD_1

n.a.

n.a.

1.0000

1.0000

1.0000

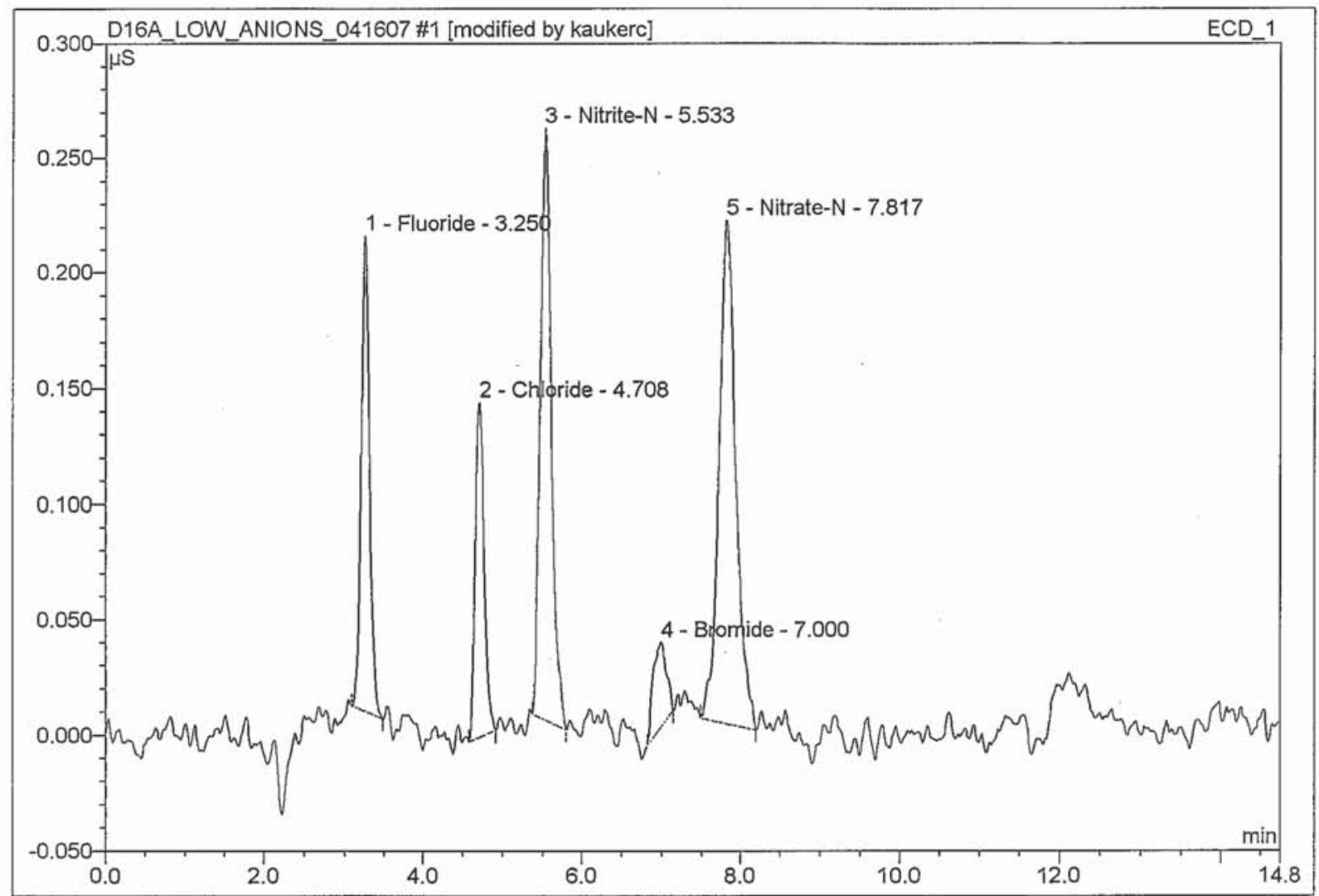

\begin{tabular}{|r|r|l|r|r|r|r|c|}
\hline No. & $\begin{array}{c}\text { Ret.Time } \\
\text { min }\end{array}$ & \multicolumn{1}{|c|}{ Peak Name } & $\begin{array}{c}\text { Height } \\
\mu \text { S }\end{array}$ & $\begin{array}{c}\text { Area } \\
\mu S^{*} \min \end{array}$ & $\begin{array}{c}\text { Rel.Area } \\
\%\end{array}$ & $\begin{array}{c}\text { Amount } \\
\text { mg/L }\end{array}$ & $\begin{array}{c}\text { Peak } \\
\text { Type }\end{array}$ \\
\hline 1 & 3.25 & Fluoride & 0.206 & 0.025 & 17.24 & 0.0747 & BMB \\
\cline { 2 - 7 } 2 & 4.71 & Chloride & 0.145 & 0.019 & 13.13 & 0.0764 & BMB \\
\hline 3 & 5.53 & Nitrite-N & 0.256 & 0.041 & 28.40 & 0.0749 & BMB \\
\hline 4 & 7.00 & Bromide & 0.037 & 0.007 & 4.96 & 0.0629 & BMB $^{*}$ \\
\hline 5 & 7.82 & Nitrate-N & 0.218 & 0.053 & 36.28 & 0.0764 & BMB \\
\hline Total: & & & 0.861 & 0.146 & 100.00 & 0.365 & \\
\hline
\end{tabular}

(10) cut 4116107 


\section{CAL STD \#1 ICWS-9424}

\section{QUAD}

Sample Name: $\quad$ CAL STD \#1 ICWS-9424

Vial Number: $\quad 269$

Sample Type: unknown

Control Program: ANIONS_AS14A

Quantif. Method: ANIONS

Recording Time:

Run Time (min):
4/16/2007 12:21

14.75
Injection Volume:

Channel:

Wavelength:

Bandwidth:

Dilution Factor:

Sample Weight:

Sample Amount:
50.0

ECD_1

n.a.

n.a.

1.0000

1.0000

1.0000

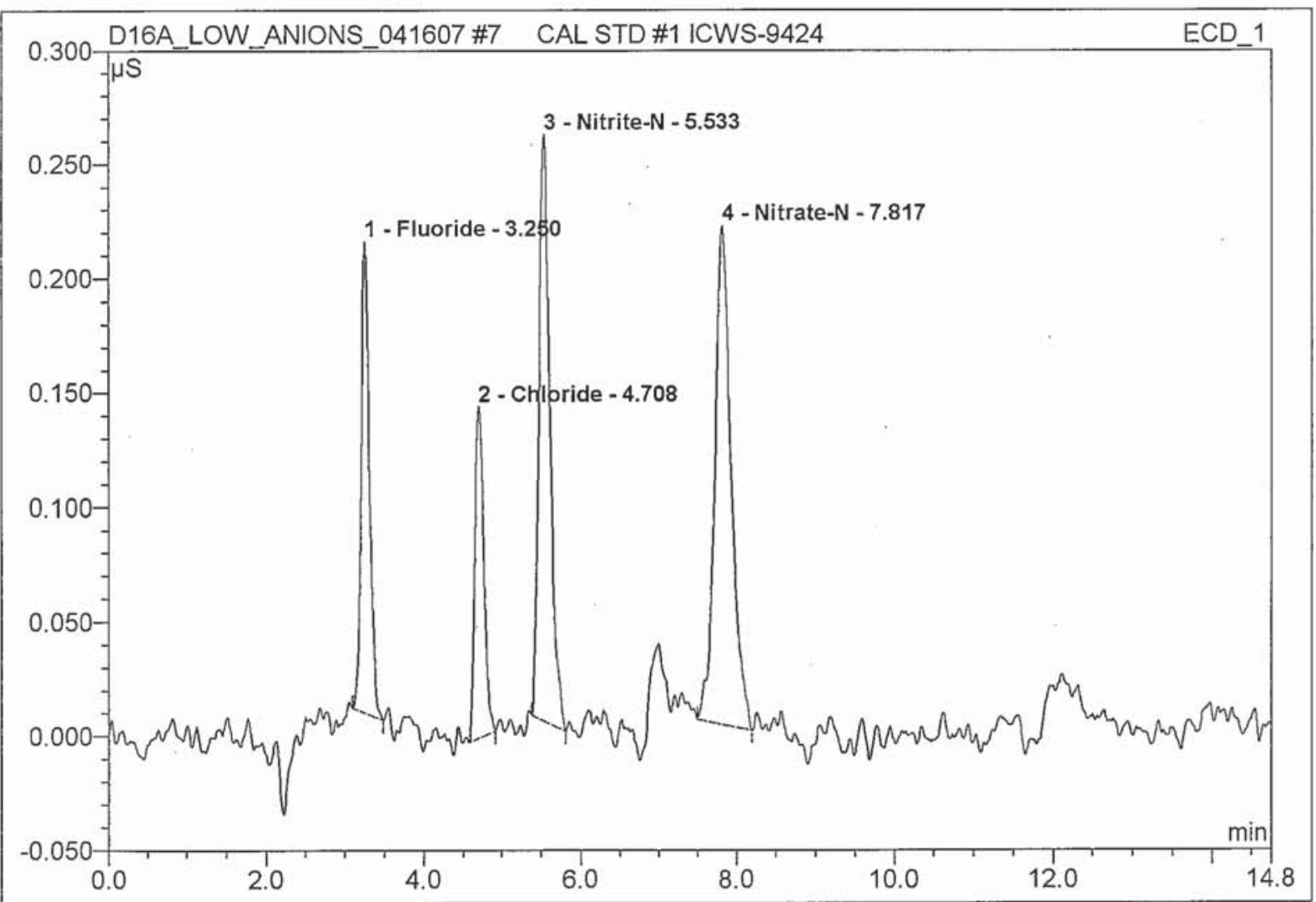

\begin{tabular}{|c|c|l|r|r|r|r|c|}
\hline No. & $\begin{array}{c}\text { Ret.Time } \\
\min \end{array}$ & \multicolumn{1}{|c|}{ Peak Name } & $\begin{array}{c}\text { Height } \\
\mu \text { S }\end{array}$ & $\begin{array}{c}\text { Area } \\
\mu S^{*} \text { min }\end{array}$ & $\begin{array}{c}\text { Rel.Area } \\
\%\end{array}$ & $\begin{array}{c}\text { Amount } \\
\mathrm{mg} / \mathrm{L}\end{array}$ & $\begin{array}{c}\text { Peak } \\
\text { Type }\end{array}$ \\
\hline 1 & 3.25 & Fluoride & 0.206 & 0.025 & 18.14 & 0.0860 & BMB \\
\hline 2 & 4.71 & Chloride & 0.145 & 0.019 & 13.81 & 0.0819 & BMB \\
\hline 3 & 5.53 & Nitrite-N & 0.256 & 0.041 & 29.88 & 0.0833 & BMB \\
\hline 4 & 7.82 & Nitrate-N & 0.218 & 0.053 & 38.17 & 0.0846 & BMB \\
\hline Total: & & & 0.825 & 0.139 & 100.00 & 0.336 & \\
\hline
\end{tabular}




\section{CAL STD \#2 ICWS-9425}

\section{QUAD}

$\begin{array}{ll}\text { Sample Name: } & \text { CAL STD \#2 ICWS-9425 } \\ \text { Vial Number: } & 270 \\ \text { Sample Type: } & \text { standard } \\ \text { Control Program: } & \text { ANIONS_AS14A } \\ \text { Quantif. Method: } & \text { ANIONS } \\ \text { Recording Time: } & 4 / 16 / 200712: 38 \\ \text { Run Time (min): } & 14.75\end{array}$

$\begin{array}{ll}\text { Injection Volume: } & 50.0 \\ \text { Channel: } & \text { ECD_1 } \\ \text { Wavelength: } & \text { n.a. } \\ \text { Bandwidth: } & \text { n.a. } \\ \text { Dilution Factor: } & 1.0000 \\ \text { Sample Weight: } & 1.0000 \\ \text { Sample Amount: } & 1.0000\end{array}$

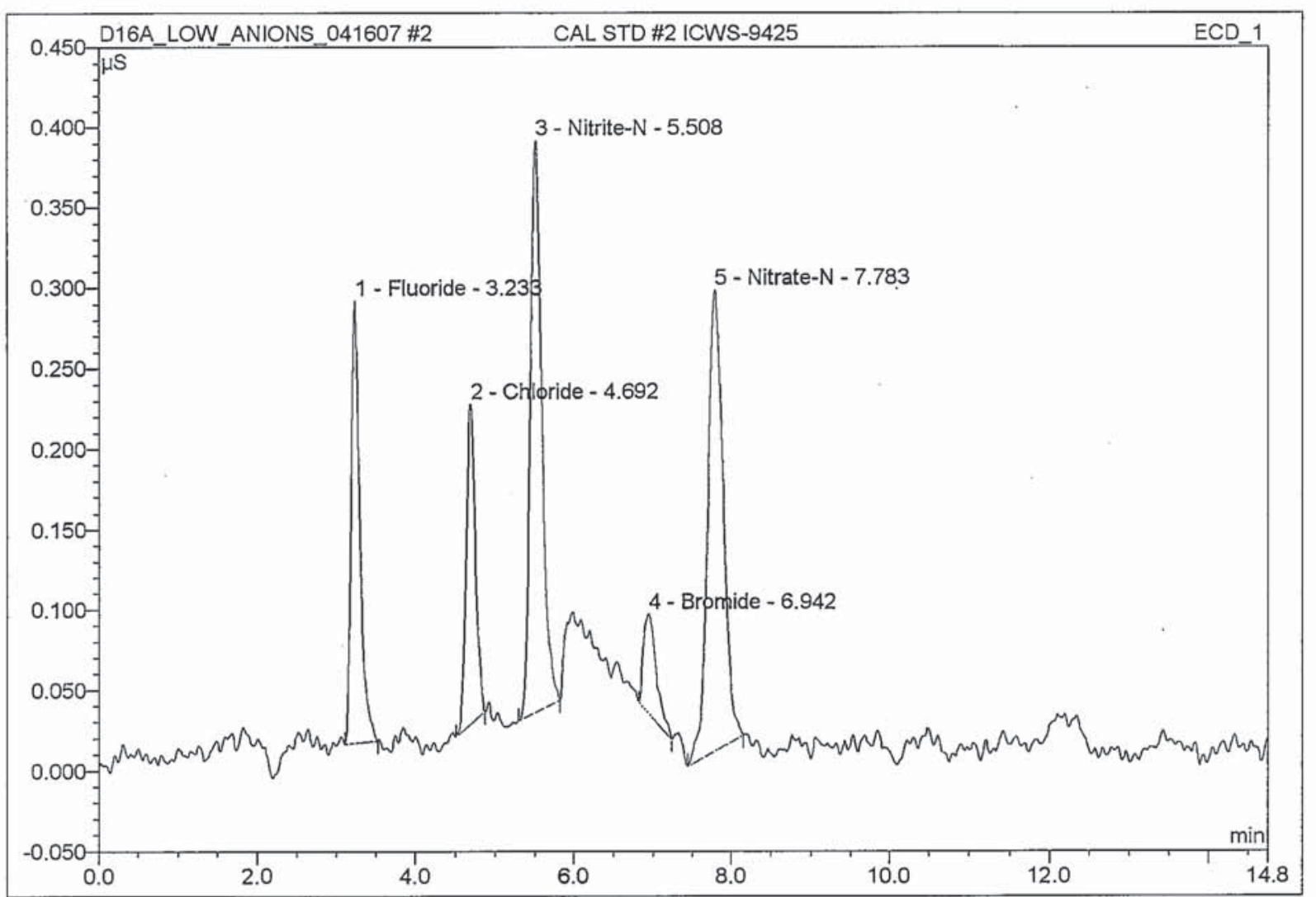

\begin{tabular}{|c|c|l|r|r|r|r|c|}
\hline No. & $\begin{array}{c}\text { Ret.Time } \\
\mathrm{min}\end{array}$ & \multicolumn{1}{|c|}{ Peak Name } & $\begin{array}{c}\text { Height } \\
\mu \mathrm{S}\end{array}$ & $\begin{array}{c}\text { Area } \\
\mu \mathbf{S}^{*} \min \end{array}$ & $\begin{array}{c}\text { Rel.Area } \\
\%\end{array}$ & $\begin{array}{c}\text { Amount } \\
\mathrm{mg} / \mathrm{L}\end{array}$ & $\begin{array}{c}\text { Peak } \\
\text { Type }\end{array}$ \\
\hline 1 & 3.23 & Fluoride & 0.275 & 0.035 & 17.75 & 0.0997 & BMB \\
\cline { 2 - 8 } 2 & 4.69 & Chloride & 0.199 & 0.027 & 13.66 & 0.1049 & BMB \\
\hline 3 & 5.51 & Nitrite-N & 0.356 & 0.059 & 29.75 & 0.1038 & BMB \\
\hline 4 & 6.94 & Bromide & 0.062 & 0.012 & 5.83 & 0.1058 & BMB \\
\hline 5 & 7.78 & Nitrate-N & 0.286 & 0.065 & 33.01 & 0.1003 & BMB \\
\hline Total: & & & 1.177 & 0.198 & 100.00 & 0.514 & \\
\hline
\end{tabular}




\section{CAL STD \#3 ICWS-9426}

\section{QUAD}

$\begin{array}{ll}\text { Sample Name: } & \text { CAL STD \#3 ICWS-9426 } \\ \text { Vial Number: } & 306 \\ \text { Sample Type: } & \text { standard } \\ \text { Control Program: } & \text { ANIONS_AS14A } \\ \text { Quantif. Method: } & \text { ANIONS } \\ \text { Recording Time: } & 4 / 16 / 200712: 55 \\ \text { Run Time (min): } & \mathbf{1 4 . 7 5}\end{array}$

Injection Volume: $\quad \mathbf{5 0 . 0}$ Channel: ECD_1

Wavelength:

n.a.

Bandwidth:

n.a.

Dilution Factor: $\quad 1.0000$

Sample Weight: $\quad 1.0000$

Run Time (min):

14.75

Sample Amount:

1.0000

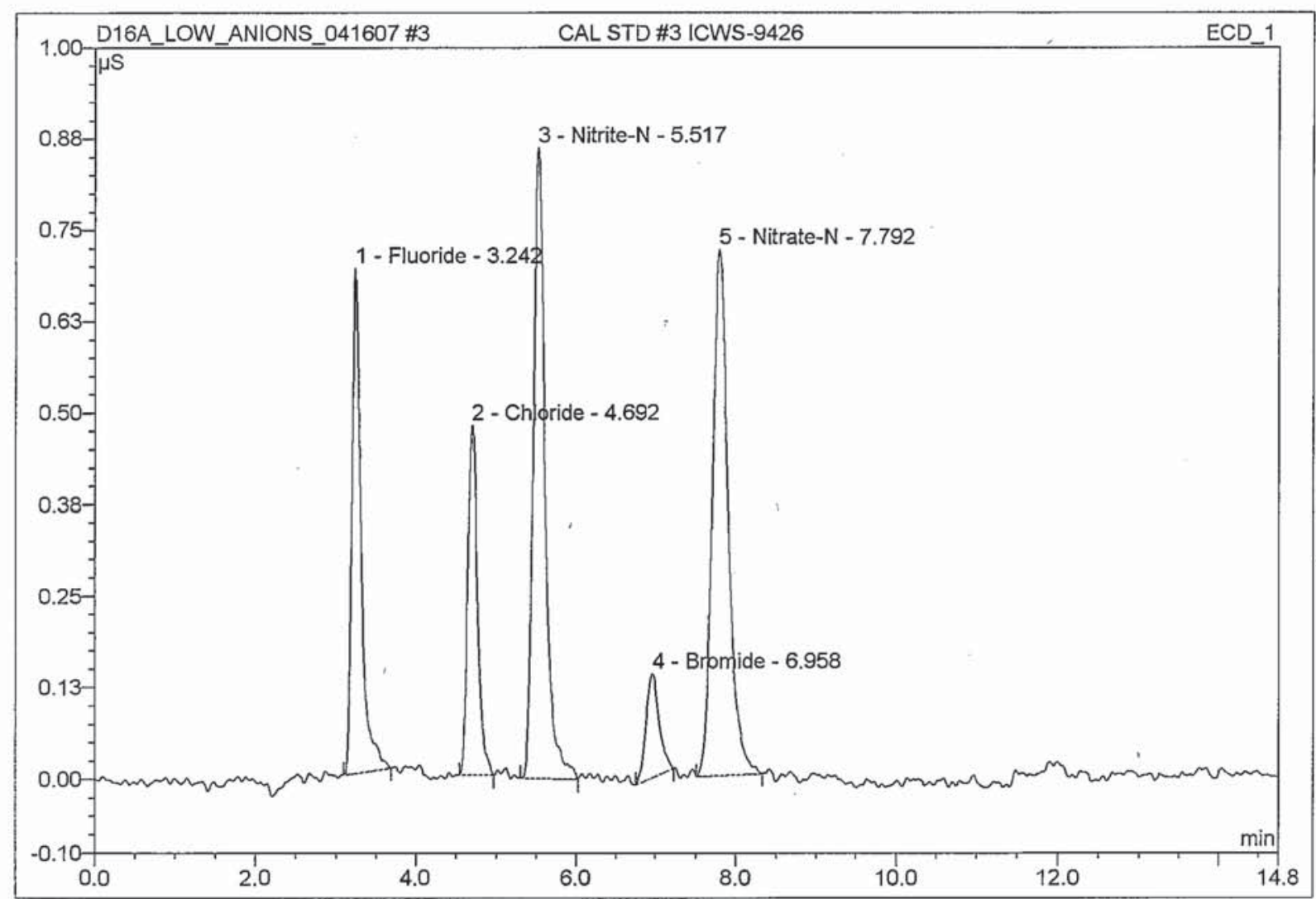

\begin{tabular}{|r|r|l|r|r|r|r|c|}
\hline No. & $\begin{array}{c}\text { Ret.Time } \\
\text { min }\end{array}$ & \multicolumn{1}{|c|}{ Peak Name } & $\begin{array}{c}\text { Height } \\
\mu \mathrm{S}\end{array}$ & $\begin{array}{c}\text { Area } \\
\mu \mathbf{S}^{*} \mathrm{~min}\end{array}$ & $\begin{array}{c}\text { Rel.Area } \\
\%\end{array}$ & $\begin{array}{c}\text { Amount } \\
\mathrm{mg} / \mathrm{L}\end{array}$ & $\begin{array}{c}\text { Peak } \\
\text { Type }\end{array}$ \\
\hline 1 & 3.24 & Fluoride & 0.690 & 0.093 & 18.64 & 0.2478 & BMB \\
\cline { 2 - 8 } & 4.69 & Chloride & 0.477 & 0.066 & 13.28 & 0.2505 & BMB \\
\hline 3 & 5.52 & Nitrite-N & 0.860 & 0.149 & 29.80 & 0.2492 & BMB \\
\hline 4 & 6.96 & Bromide & 0.141 & 0.027 & 5.37 & 0.2416 & BMB \\
\hline 5 & 7.79 & Nitrate-N & 0.717 & 0.165 & 32.92 & 0.2490 & BMB \\
\hline Total: & & & 2.886 & 0.500 & 100.00 & 1.238 & \\
\hline
\end{tabular}




\section{CAL STD \#4 ICWS-9427}

\section{QUAD}

Sample Name: $\quad$ CAL STD \#4 ICWS-9427

Vial Number: $\quad \mathbf{3 0 7}$

Sample Type: $\quad$ standard

Control Program: ANIONS_AS14A

Quantif. Method: ANIONS

Recording Time: $\quad$ 4/16/2007 13:12

Run Time (min): $\quad 14.75$
Injection Volume: $\quad \mathbf{5 0 . 0}$

Channel:

ECD_1

Wavelength:

n.a.

Bandwidth: n.a.

Dilution Factor: $\quad \mathbf{1 . 0 0 0 0}$

Sample Weight: $\quad \mathbf{1 . 0 0 0 0}$

Sample Amount: $\quad \mathbf{1 . 0 0 0 0}$

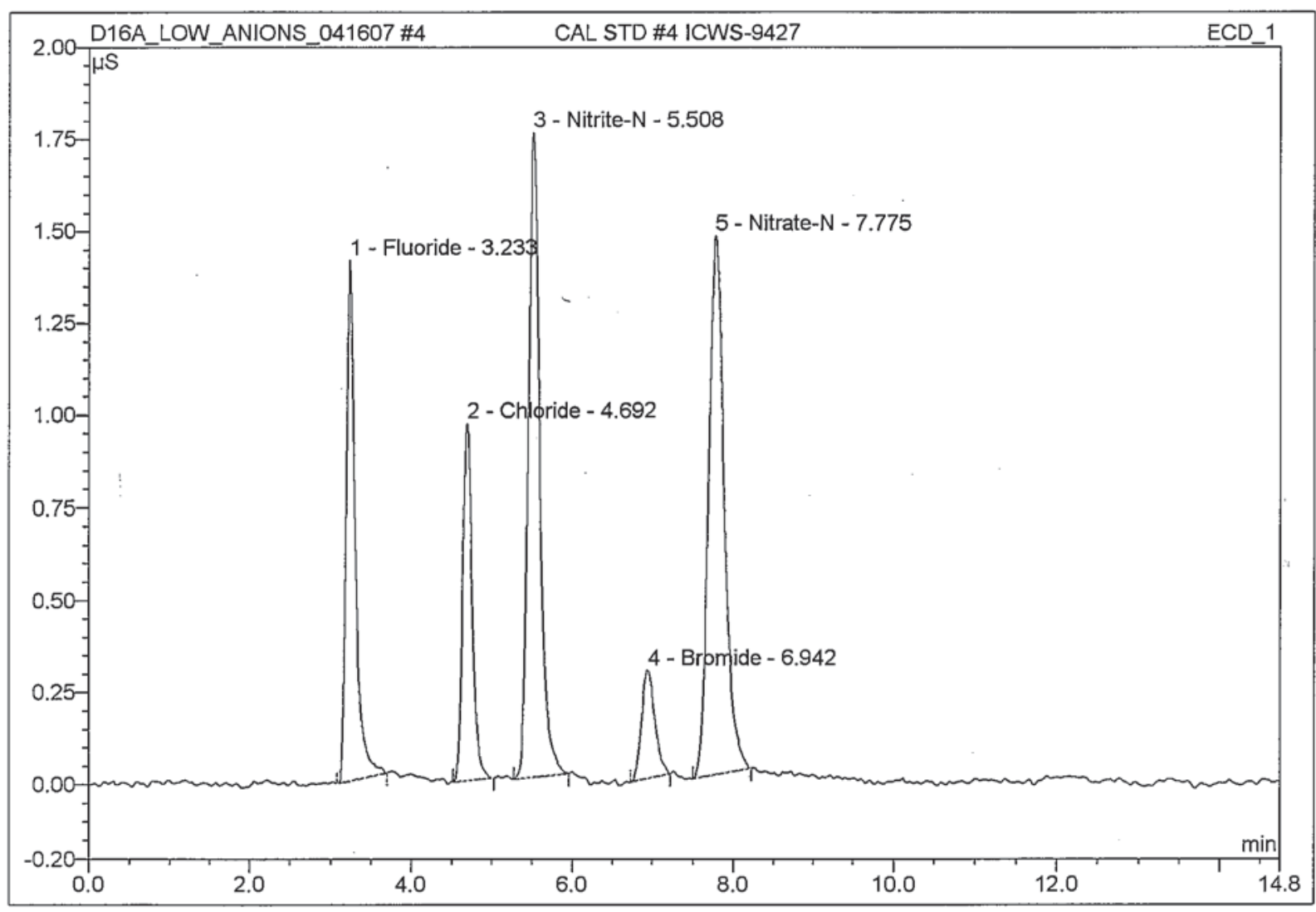

\begin{tabular}{|r|r|l|r|r|r|r|c|}
\hline No. & $\begin{array}{c}\text { Ret.Time } \\
\text { min }\end{array}$ & \multicolumn{1}{|c|}{ Peak Name } & $\begin{array}{c}\text { Height } \\
\mu \mathrm{S}\end{array}$ & $\begin{array}{c}\text { Area } \\
\boldsymbol{\mu} \mathbf{S}^{*} \min \end{array}$ & $\begin{array}{c}\text { Rel.Area } \\
\%\end{array}$ & $\begin{array}{c}\text { Amount } \\
\mathrm{mg} / \mathrm{L}\end{array}$ & $\begin{array}{c}\text { Peak } \\
\text { Type }\end{array}$ \\
\hline 1 & 3.23 & Fluoride & 1.411 & 0.187 & 18.69 & 0.4989 & BMB \\
\cline { 2 - 8 } 2 & 4.69 & Chloride & 0.966 & 0.133 & 13.37 & 0.5021 & BMB \\
\hline 3 & 5.51 & Nitrite-N & 1.748 & 0.292 & 29.29 & 0.5007 & BMB \\
\hline 4 & 6.94 & Bromide & 0.294 & 0.056 & 5.62 & 0.5020 & BMB \\
\hline 5 & 7.78 & Nitrate-N & 1.459 & 0.330 & 33.04 & 0.4994 & BMB \\
\hline Total: & & & 5.878 & 0.998 & 100.00 & 2.503 & \\
\hline
\end{tabular}




\section{CAL STD \#5 ICWS-9423}

QUAD

Sample Name: CAL STD \#5 ICWS-9423

Vial Number: $\quad \mathbf{3 0 8}$

Sample Type: $\quad$ standard

Control Program: ANIONS_AS14A

Quantif. Method: $\quad$ ANIONS

Recording Time: $\quad 4 / 16 / 2007$ 13:29

Run Time (min): $\quad 14.75$

Injection Volume: $\quad \mathbf{5 0 . 0}$

Channel:

Wavelength:

Bandwidth:

Dilution Factor:

Sample Weight:

Sample Amount:
ECD_1

n.a.

n.a.

1.0000

1.0000

1.0000

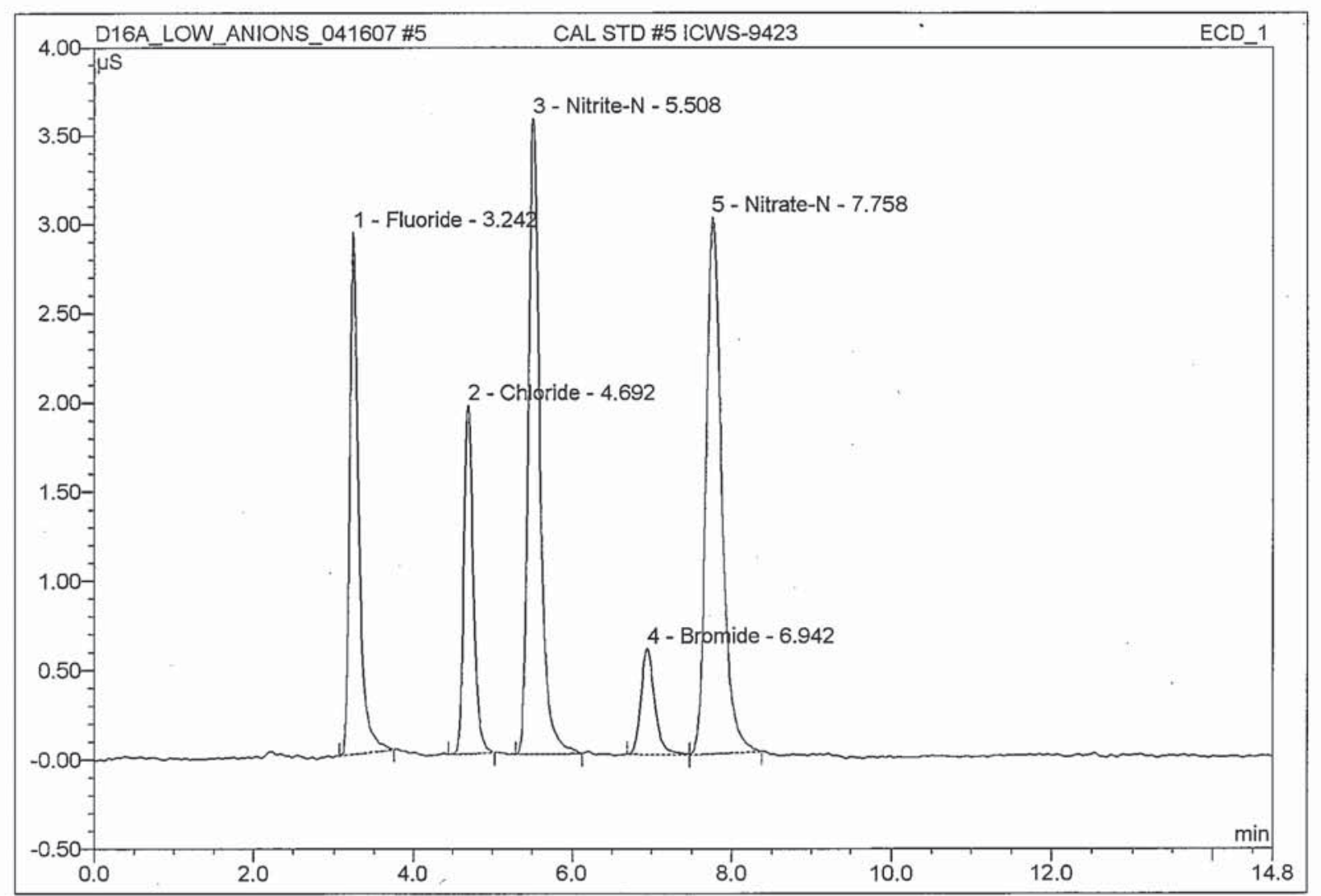

\begin{tabular}{|c|c|l|r|r|r|r|c|}
\hline No. & $\begin{array}{c}\text { Ret.Time } \\
\text { min }\end{array}$ & \multicolumn{1}{|c|}{ Peak Name } & $\begin{array}{c}\text { Height } \\
\mu \text { S }\end{array}$ & $\begin{array}{c}\text { Area } \\
\mu S^{*} \text { min }\end{array}$ & $\begin{array}{c}\text { Rel.Area } \\
\%\end{array}$ & $\begin{array}{c}\text { Amount } \\
\mathrm{mg} / \mathrm{L}\end{array}$ & $\begin{array}{c}\text { Peak } \\
\text { Type }\end{array}$ \\
\hline 1 & 3.24 & Fluoride & 2.922 & 0.382 & 18.55 & 1.0018 & $\mathrm{BMB}$ \\
\cline { 2 - 7 } 2 & 4.69 & Chloride & 1.951 & 0.269 & 13.07 & 0.9972 & $\mathrm{BMB}$ \\
\hline 3 & 5.51 & Nitrite-N & 3.567 & 0.606 & 29.40 & 0.9992 & $\mathrm{BMB}$ \\
\hline 4 & 6.94 & Bromide & 0.593 & 0.119 & 5.76 & 1.0027 & $\mathrm{BMb}$ \\
\hline 5 & 7.76 & Nitrate-N & 3.007 & 0.684 & 33.21 & 1.0006 & $\mathrm{bMB}$ \\
\hline Total: & & & 12.039 & 2.060 & 100.00 & 5.001 & \\
\hline
\end{tabular}




\section{CAL STD \#6 ICWS-9428}

\section{QUAD}

$\begin{array}{ll}\text { Sample Name: } & \text { CAL STD \#6 ICWS-9428 } \\ \text { Vial Number: } & 309 \\ \text { Sample Type: } & \text { standard } \\ \text { Control Program: } & \text { ANIONS_AS14A } \\ \text { Quantif. Method: } & \text { ANIONS } \\ \text { Recording Time: } & 4 / 16 / 200713: 46 \\ \text { Run Time (min): } & 14.75\end{array}$

Injection Volume: $\quad \mathbf{5 0 . 0}$ Channel:

Wavelength:

Bandwidth:

Dilution Factor:

Sample Weight:

Sample Amount:
ECD_1

n.a.

n.a.

1.0000

1.0000

1.0000

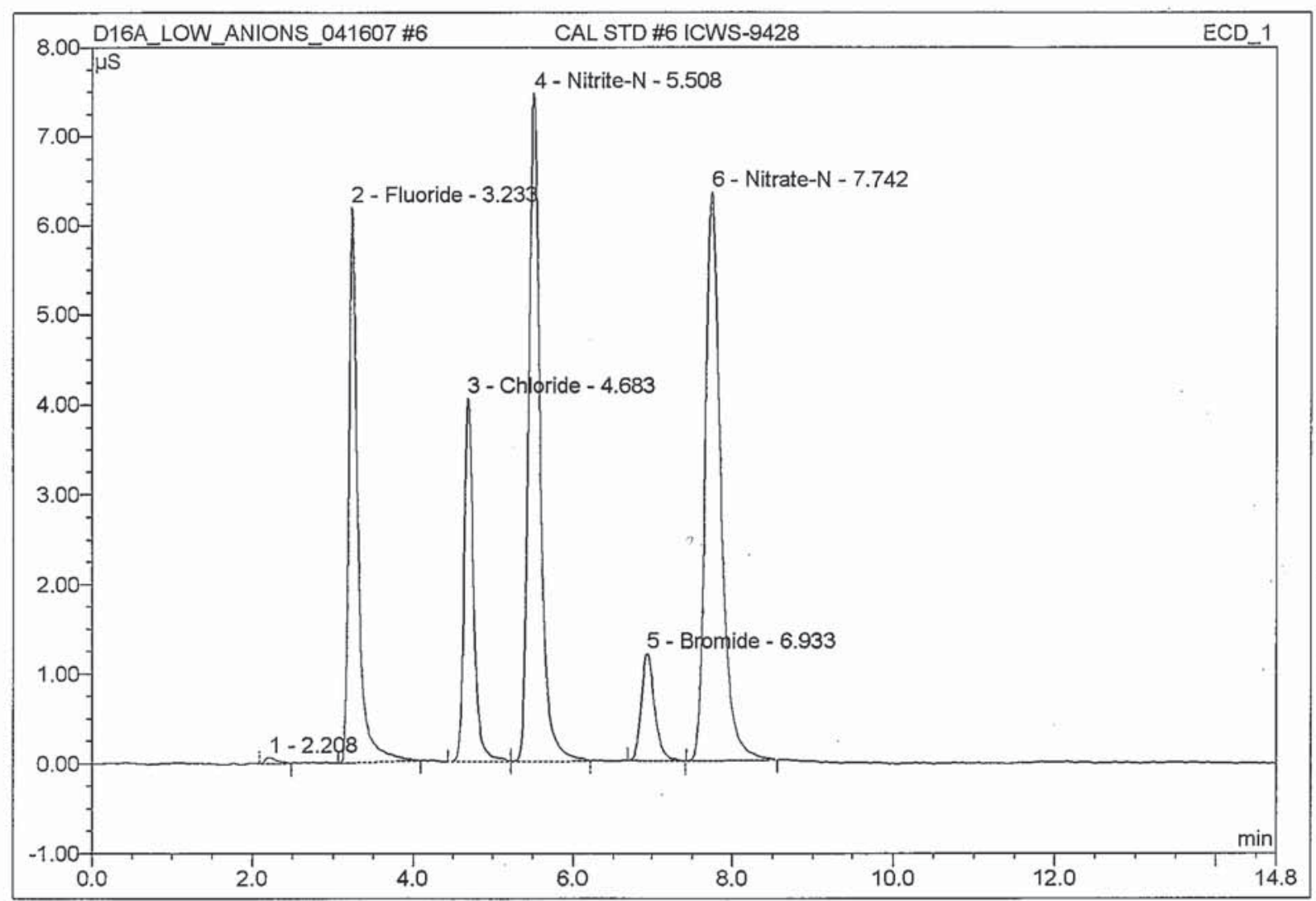

\begin{tabular}{|r|r|l|r|r|r|r|c|}
\hline No. & $\begin{array}{c}\text { Ret.Time } \\
\text { min }\end{array}$ & \multicolumn{1}{|c|}{ Peak Name } & $\begin{array}{c}\text { Height } \\
\mu \text { S }\end{array}$ & \multicolumn{1}{c|}{$\begin{array}{c}\text { Area } \\
\mu \mathbf{S}^{*} \min \end{array}$} & $\begin{array}{c}\text { Rel.Area } \\
\%\end{array}$ & $\begin{array}{c}\text { Amount } \\
\text { mg/L }\end{array}$ & $\begin{array}{c}\text { Peak } \\
\text { Type }\end{array}$ \\
\hline 1 & 2.21 & n.a. & 0.068 & 0.011 & 0.26 & n.a. & BMB \\
\hline 2 & 3.23 & Fluoride & 6.188 & 0.821 & 18.97 & 1.9997 & BMB \\
\hline 3 & 4.68 & Chloride & 4.048 & 0.574 & 13.25 & 2.0005 & BM \\
\hline 4 & 5.51 & Nitrite-N & 7.457 & 1.253 & 28.94 & 2.0002 & MB \\
\hline 5 & 6.93 & Bromide & 1.200 & 0.236 & 5.44 & 1.9993 & BMB \\
\hline 6 & 7.74 & Nitrate-N & 6.339 & 1.435 & 33.13 & 1.9999 & BMB \\
\hline Total: & & & 25.301 & 4.330 & 100.00 & 10.000 & \\
\hline
\end{tabular}




\begin{tabular}{|llll|}
\hline \multicolumn{1}{|l|}{ Q EALSTQ\#4HEWS-9427 } & & \\
QUAD & & & \\
\hline Sample Name: & CAL STD \#4 ICWS-9427 & & \\
Vial Number: & 307 & Injection Volume: & 50.0 \\
Sample Type: & standard & Channel: & ECD_1 \\
Control Program: & ANIONS_AS14A & Wavelength: & n.a. \\
Quantif. Method: & ANIONS & Bandwidth: & n.a. \\
Recording Time: & $4 / 16 / 200713: 12$ & Dilution Factor: & 1.0000 \\
Run Time (min): & 14.75 & Sample Weight: & 1.0000 \\
\hline
\end{tabular}

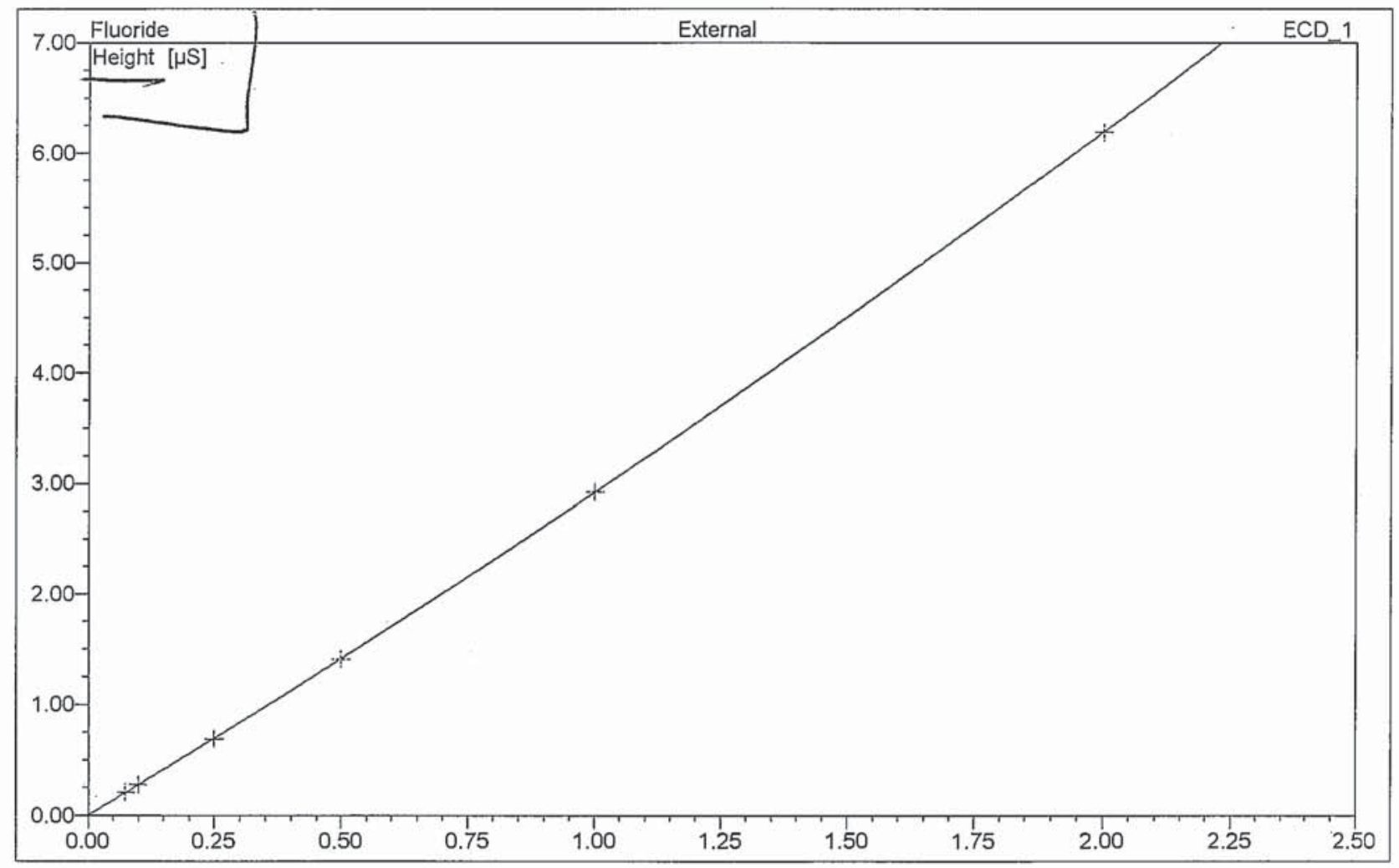

\begin{tabular}{|c|c|c|c|c|c|c|c|c|}
\hline No. & $\begin{array}{c}\text { Ret.Time } \\
\text { min }\end{array}$ & Peak Name & Cal.Type & Points & \begin{tabular}{|c|} 
Corr.Coeff. \\
(R)
\end{tabular} & Offset & Slope & Curve \\
\hline 1 & 3.23 & Fluoride & Quad & 6 & 0.9996 & 0.0000 & 2.739293 & 0.177604 \\
\hline 2 & 4.69 & Chloride & Quad & 6 & 0.9998 & 0.0000 & 1.889466 & 0.067051 \\
\hline 3 & 5.51 & Nitrite-N & Quad & 6 & 0.9997 & 0.0000 & 3.411458 & 0.158481 \\
\hline 4 & 6.94 & Bromide & Quad & 6 & 1.0000 & 0.0000 & 0.581641 & 0.009270 \\
\hline 5 & 7.78 & Nitrate-N & Quad & 6 & 0.9996 & 0.0000 & 2.839897 & 0.164894 \\
\hline Average: & & & & & 0.9997 & 0.0000 & 2.2924 & 0.1155 \\
\hline
\end{tabular}




\begin{tabular}{|llll|}
\hline QUAD & & & \\
Sample Name: & CAL STD \#4 ICWS-9427 & & \\
Vial Number: & 307 & Injection Volume: & 50.0 \\
Sample Type: & standard & Channel: & ECD_1 \\
Control Program: & ANIONS_AS14A & Wavelength: & n.a. \\
Quantif. Method: & ANIONS & Bandwidth: & n.a. \\
Recording Time: & $4 / 16 / 2007$ 13:12 & Dilution Factor: & 1.0000 \\
Run Time (min): & 14.75 & Sample Weight: & 1.0000 \\
\end{tabular}

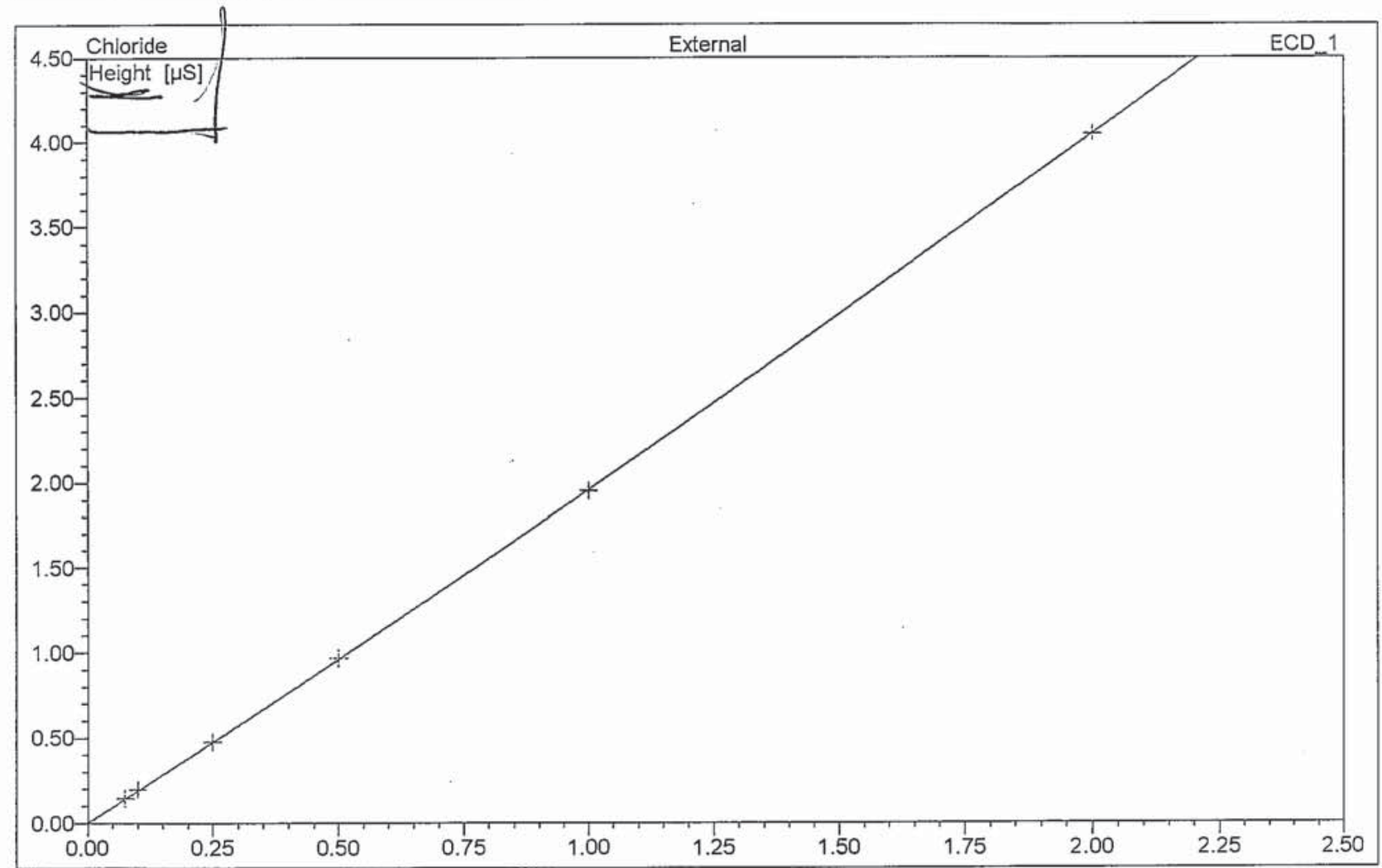

\begin{tabular}{|c|c|c|c|c|c|c|c|c|}
\hline No. & \begin{tabular}{|c|}
$\min ^{R}$ \\
Ret.Time
\end{tabular} & Peak Name & Cal.Type & Points & $\begin{array}{c}\text { Corr.Coeff. } \\
\text { (R) }\end{array}$ & Offset & Slope & Curve \\
\hline 1 & 3.23 & Fluoride & Quad & 6 & 0.9996 & 0.0000 & 2.739293 & 0.177604 \\
\hline 2 & 4.69 & Chloride & Quad & 6 & 0.9998 & 0.0000 & 1.889466 & 0.067051 \\
\hline 3 & 5.51 & Nitrite-N & Quad & 6 & 0.9997 & 0.0000 & 3.411458 & 0.158481 \\
\hline 4 & 6.94 & Bromide & Quad & 6 & 1.0000 & 0.0000 & 0.581641 & 0.009270 \\
\hline 5 & 7.78 & Nitrate-N & Quad & 6 & 0.9996 & 0.0000 & 2.839897 & 0.164894 \\
\hline Average: & & - & & & 0.9997 & 0.0000 & 2.2924 & 0.1155 \\
\hline
\end{tabular}




\begin{tabular}{|llll|}
\hline \multicolumn{1}{|c|}{ CALSTD\#4HCWS-9427-(3) } & & \\
QUAD & & & \\
\hline Sample Name: & CAL STD \#4 ICWS-9427 & Injection Volume: & 50.0 \\
Vial Number: & 307 & Channel: & ECD_1 \\
Sample Type: & standard & Wavelength: & n.a. \\
Control Program: & ANIONS_AS14A & Bandwidth: & n.a. \\
Quantif. Method: & ANIONS & Dilution Factor: & 1.0000 \\
Recording Time: & $4 / 16 / 2007$ 13:12 & Sample Weight: & 1.0000 \\
Run Time (min): & $\mathbf{1 4 . 7 5}$ & Sample Amount: & 1.0000 \\
\hline
\end{tabular}

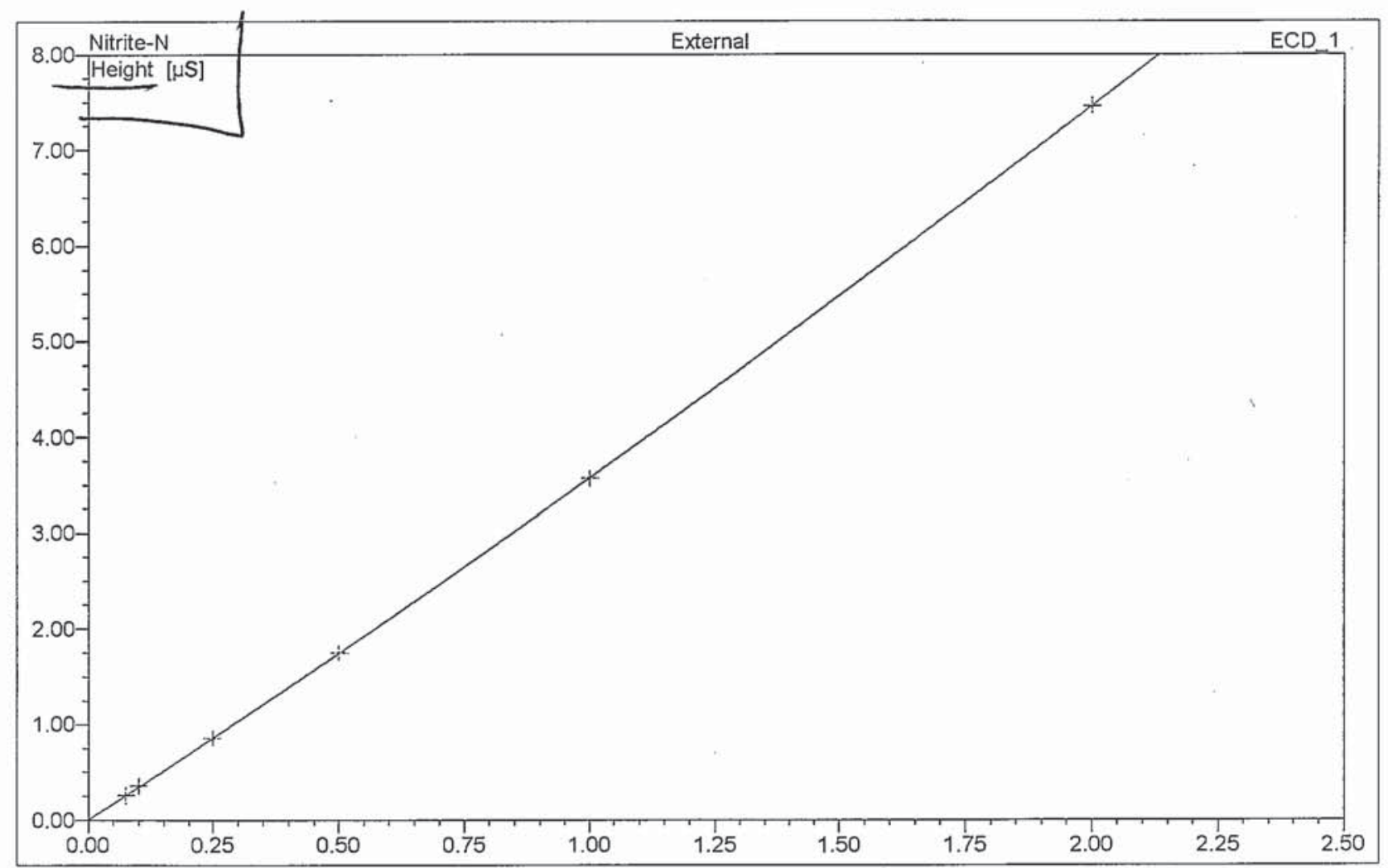

\begin{tabular}{|c|c|c|c|c|c|c|c|c|}
\hline No. & \begin{tabular}{|} 
Ret.Time \\
$\min$
\end{tabular} & Peak Name & Cal.Type & Points & $\begin{array}{c}\text { Corr.Coeff. } \\
\text { (R) }\end{array}$ & Offset & Slope & Curve \\
\hline 1 & 3.23 & Fluoride & Quad & 6 & 0.9996 & 0.0000 & 2.739293 & 0.177604 \\
\hline 2 & 4.69 & Chloride & Quad & 6 & 0.9998 & 0.0000 & 1.889466 & 0.067051 \\
\hline 3 & 5.51 & Nitrite-N & Quad & 6 & 0.9997 & 0.0000 & 3.411458 & 0.158481 \\
\hline 4 & 6.94 & Bromide & Quad & 6 & 1.0000 & 0.0000 & 0.581641 & 0.009270 \\
\hline 5 & 7.78 & Nitrate-N & Quad & 6 & 0.9996 & 0.0000 & 2.839897 & 0.164894 \\
\hline Average: & & & & & 0.9997 & 0.0000 & 2.2924 & 0.1155 \\
\hline
\end{tabular}




\begin{tabular}{|llll|}
\hline 4 GALSTO\#4TCWS-9427 ( & & \\
QUAD & & & \\
\hline Sample Name: & CAL STD \#4 ICWS-9427 & & \\
Vial Number: & 307 & Injection Volume: & 50.0 \\
Sample Type: & standard & Channel: & ECD_1 \\
Control Program: & ANIONS_AS14A & Wavelength: & n.a. \\
Quantif. Method: & ANIONS & Bandwidth: & n.a. \\
Recording Time: & $4 / 16 / 2007$ 13:12 & Dilution Factor: & 1.0000 \\
Run Time (min): & 14.75 & Sample Weight: & 1.0000 \\
\end{tabular}

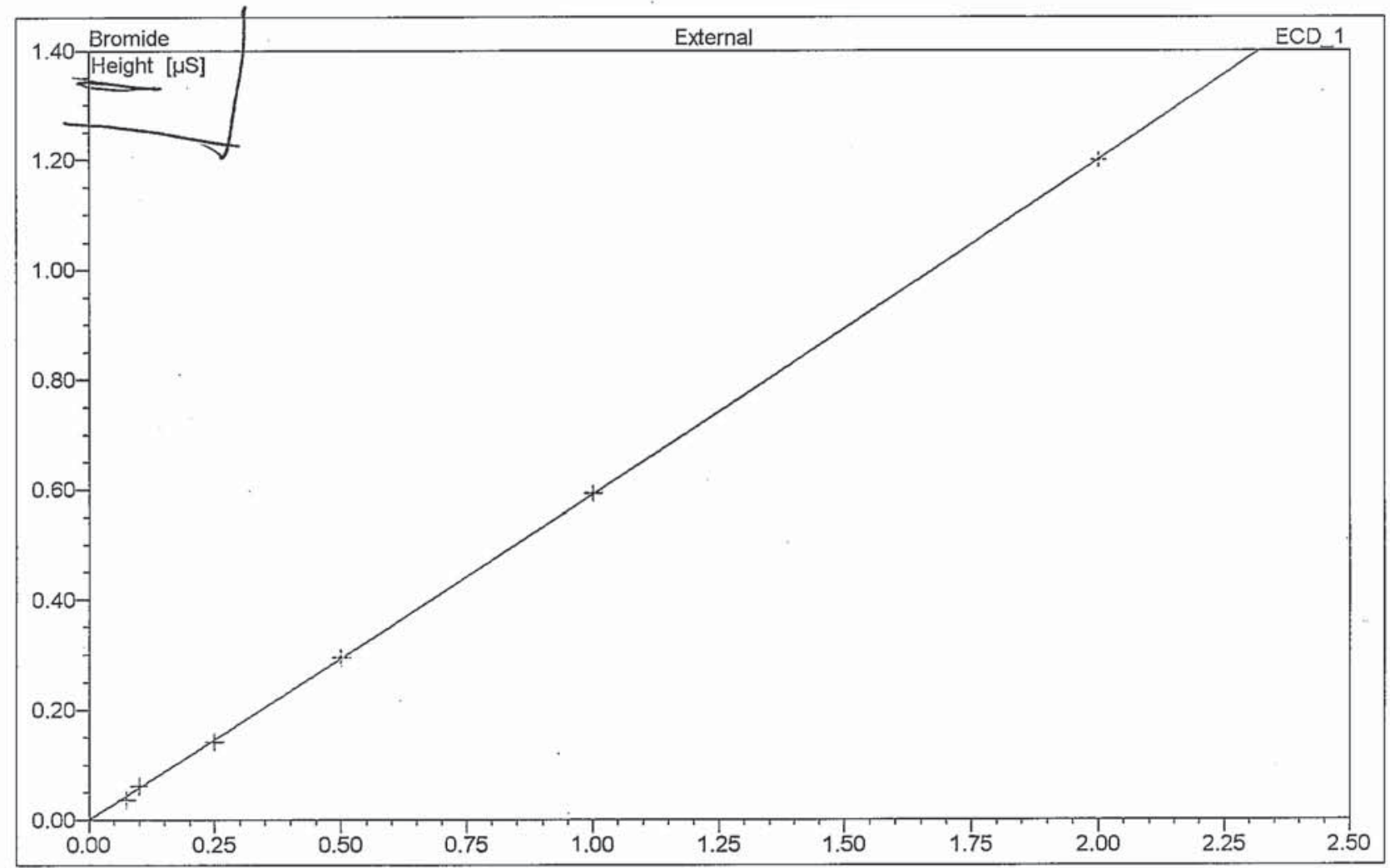

\begin{tabular}{|r|c|l|l|l|c|c|c|c|}
\hline No. & $\begin{array}{c}\text { Ret.Time } \\
\text { min }\end{array}$ & Peak Name & Cal.Type & Points & $\begin{array}{c}\text { Corr.Coeff. } \\
(\mathbf{R})\end{array}$ & Offset & Slope & Curve \\
\hline 1 & 3.23 & Fluoride & Quad & 6 & 0.9996 & 0.0000 & 2.739293 & 0.177604 \\
\hline 2 & 4.69 & Chloride & Quad & 6 & 0.9998 & 0.0000 & 1.889466 & 0.067051 \\
\hline 3 & 5.51 & Nitrite-N & Quad & 6 & 0.9997 & 0.0000 & 3.411458 & 0.158481 \\
\hline 4 & 6.94 & Bromide & Quad & 6 & $\sqrt{1.0000}$ & 0.0000 & 0.581641 & 0.009270 \\
\hline 5 & 7.78 & Nitrate-N & Quad & 6 & 0.9996 & 0.0000 & 2.839897 & 0.164894 \\
\hline Average: & \multicolumn{9}{|l}{} & & 0.9997 & 0.0000 & 2.2924 & 0.1155 \\
\hline
\end{tabular}




\begin{tabular}{|c|c|c|c|}
\hline \multicolumn{2}{|c|}{$\begin{array}{l}4 \text { CALSSTD \#4-1ENS-9427 } \\
\text { QUAD }\end{array}$} & & \\
\hline Sample Name: & CAL STD \#4 ICWS-9427 & Injection Volume: & 50.0 \\
\hline Vial Number: & 307 & Channel: & ECD_1 \\
\hline Sample Type: & standard & Wavelength: & n.a. \\
\hline Control Program: & ANIONS_AS14A & Bandwidth: & n.a. \\
\hline Quantif. Method: & ANIONS & Dilution Factor: & 1.0000 \\
\hline Recording Time: & $4 / 16 / 2007$ 13:12 & Sample Weight: & 1.0000 \\
\hline Run Time (min): & 14.75 & Sample Amount: & 1.0000 \\
\hline
\end{tabular}

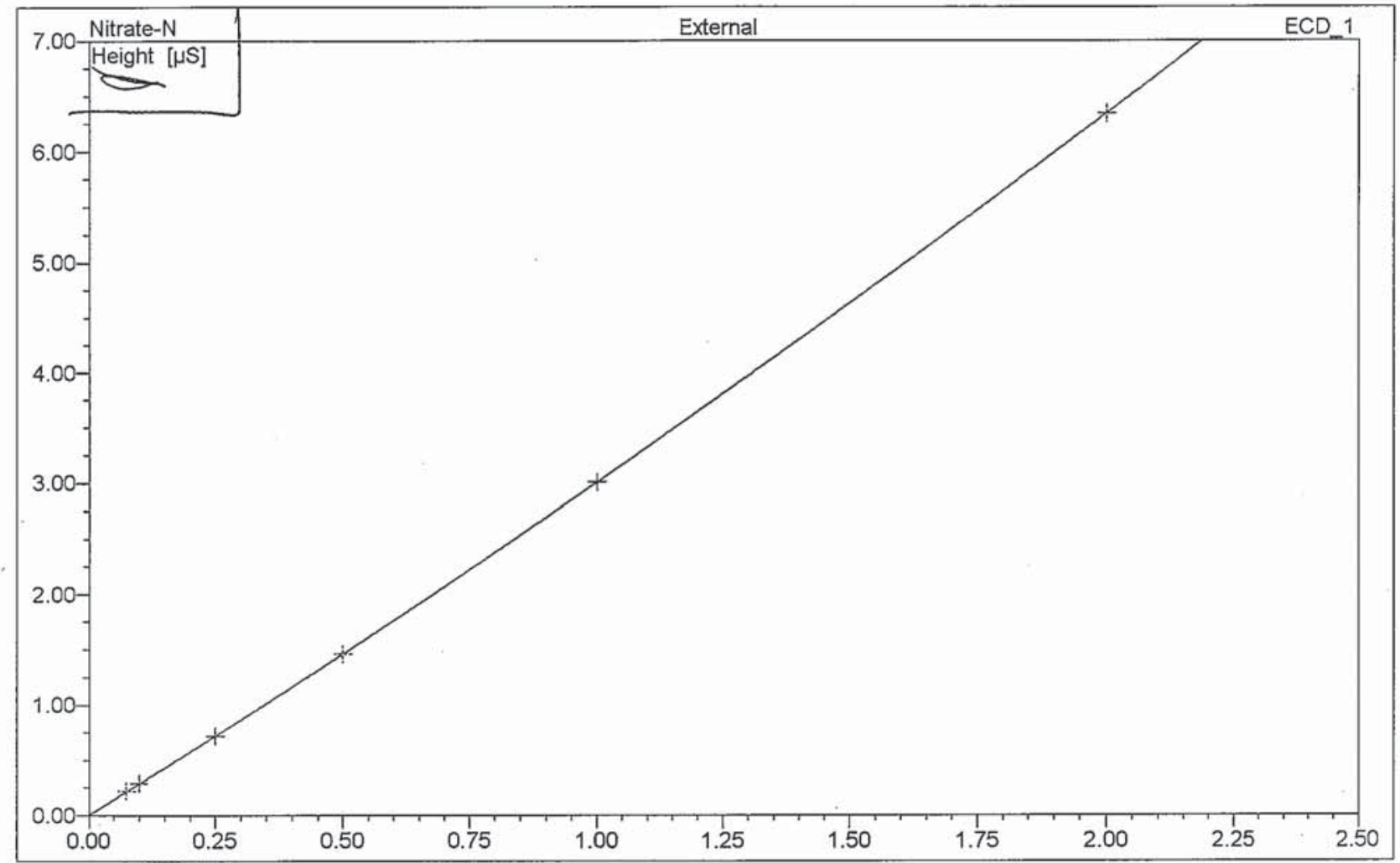

\begin{tabular}{|c|c|c|c|c|c|c|c|c|}
\hline No. & \begin{tabular}{|c|} 
Ret.Time \\
$\min$
\end{tabular} & Peak Name & Cal.Type & Points & $\begin{array}{c}\text { Corr.Coeff. } \\
\text { (R) }\end{array}$ & Offset & Slope & Curve \\
\hline 1 & 3.23 & Fluoride & Quad & 6 & 0.9996 & 0.0000 & 2.739293 & 0.177604 \\
\hline 2 & 4.69 & Chloride & Quad & 6 & 0.9998 & 0.0000 & 1.889466 & 0.067051 \\
\hline 3 & 5.51 & Nitrite-N & Quad & 6 & 0.9997 & 0.0000 & 3.411458 & 0.158481 \\
\hline 4 & 6.94 & Bromide & Quad & 6 & 1.0000 & 0.0000 & 0.581641 & 0.009270 \\
\hline 5 & 7.78 & Nitrate-N & Quad & 6 & 0.9996 & 0.0000 & 2.839897 & 0.164894 \\
\hline Average: & & & & & 0.9997 & 0.0000 & 2.2924 & 0.1155 \\
\hline
\end{tabular}




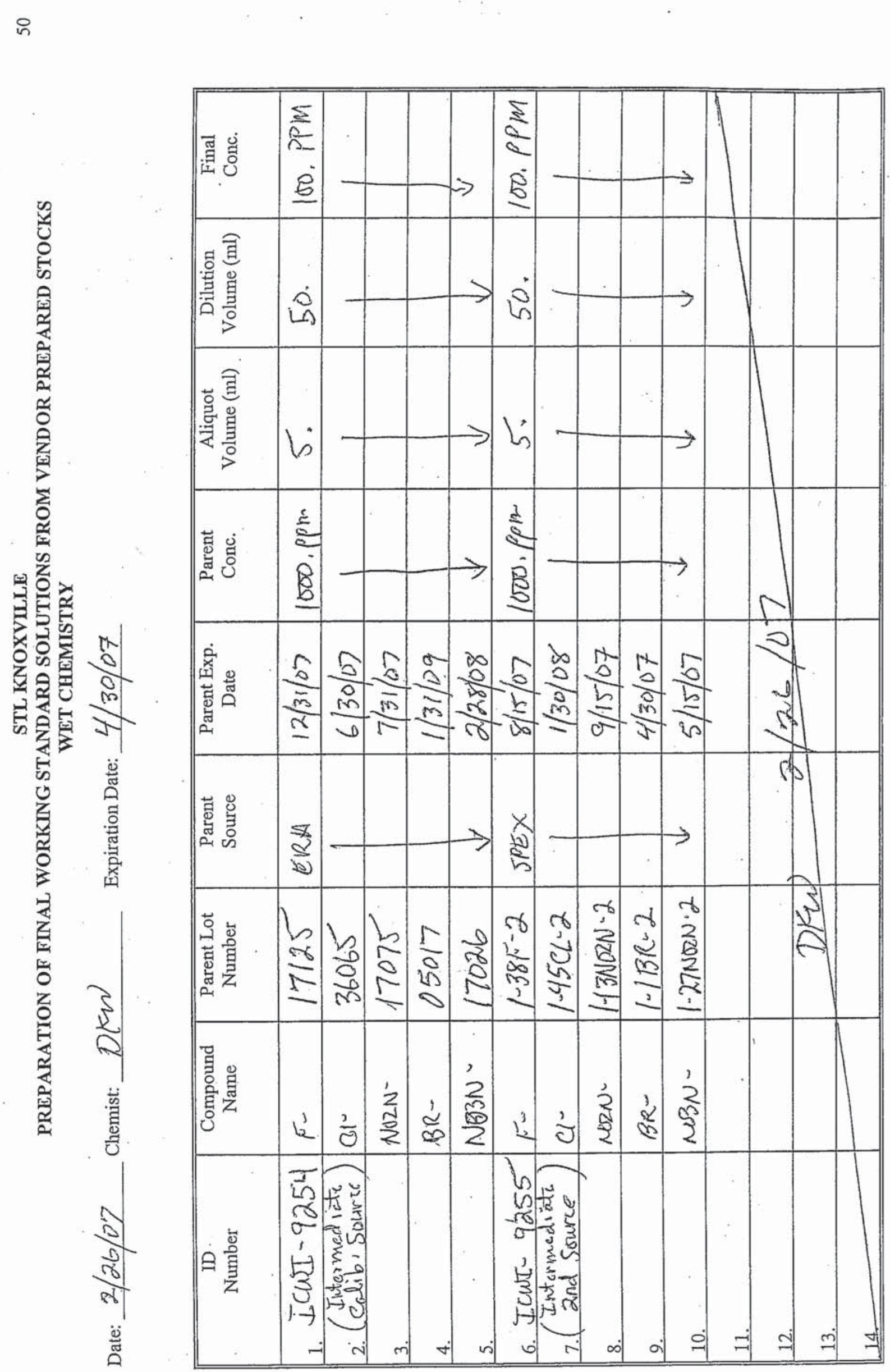


]

으

¿̊ః
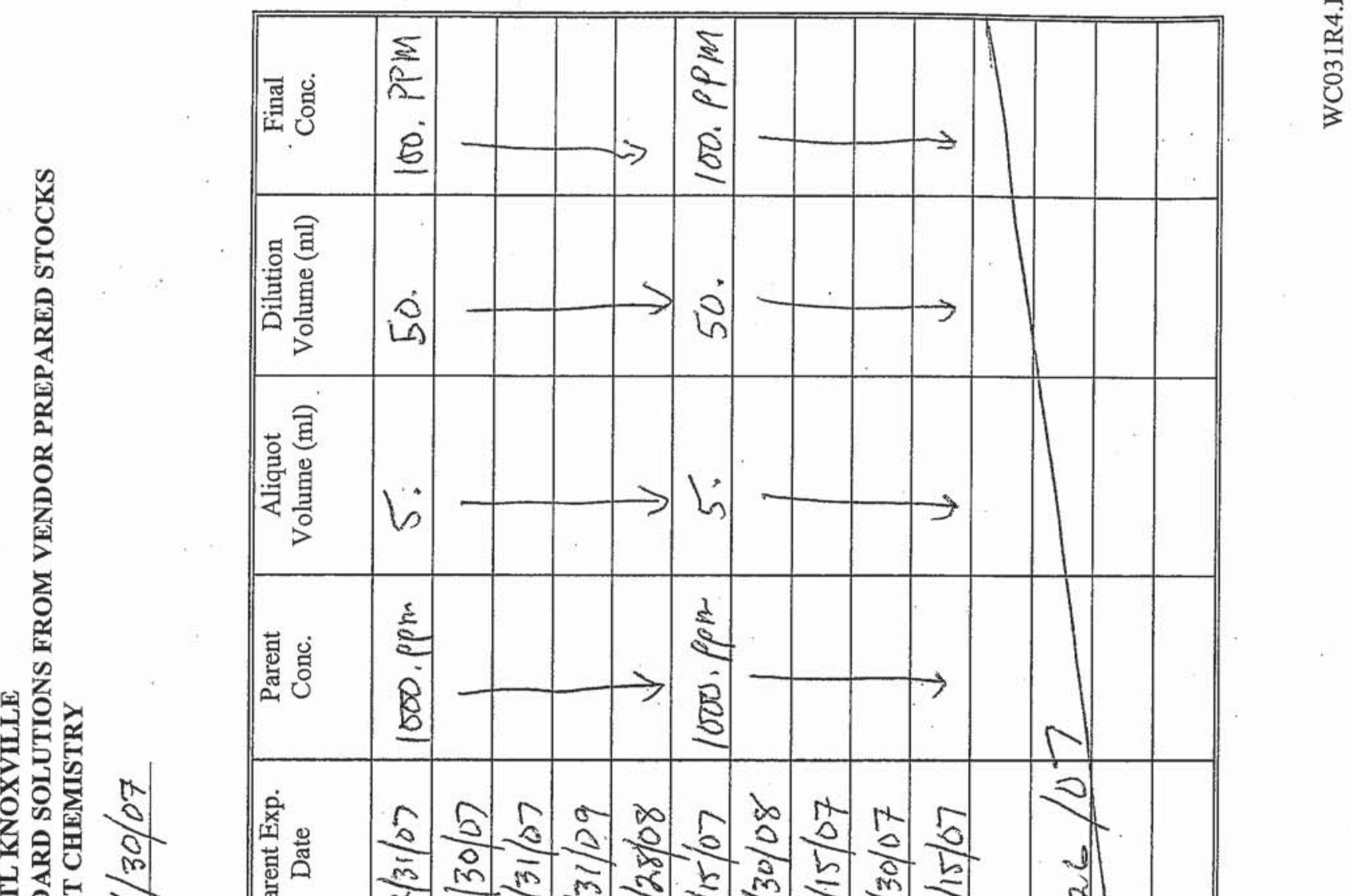

]

䜌言

高亳言

is

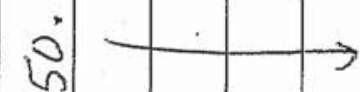

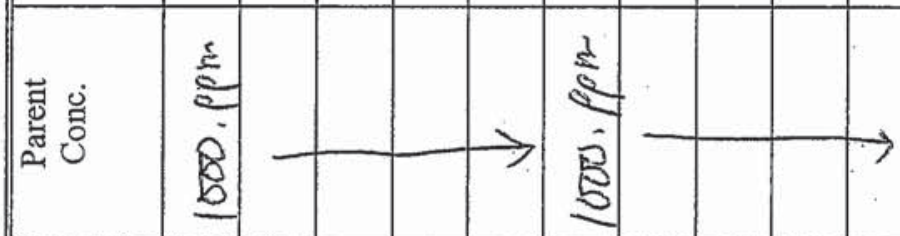

7

离

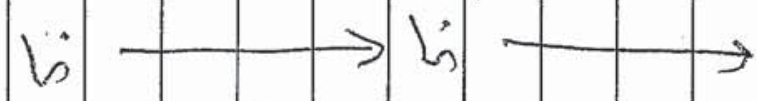

7

7

?

so

$=$

$\rightarrow$ t)

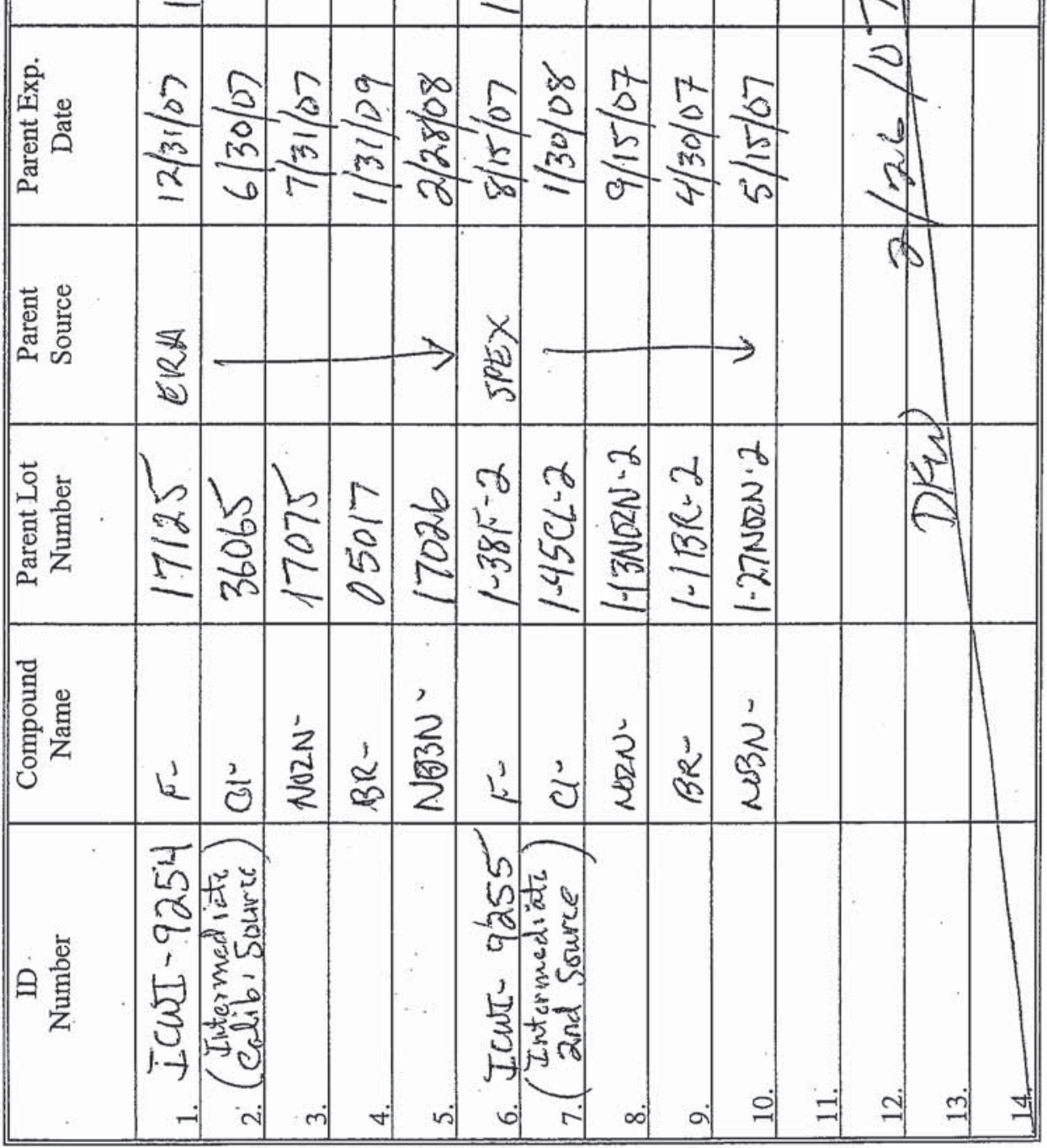



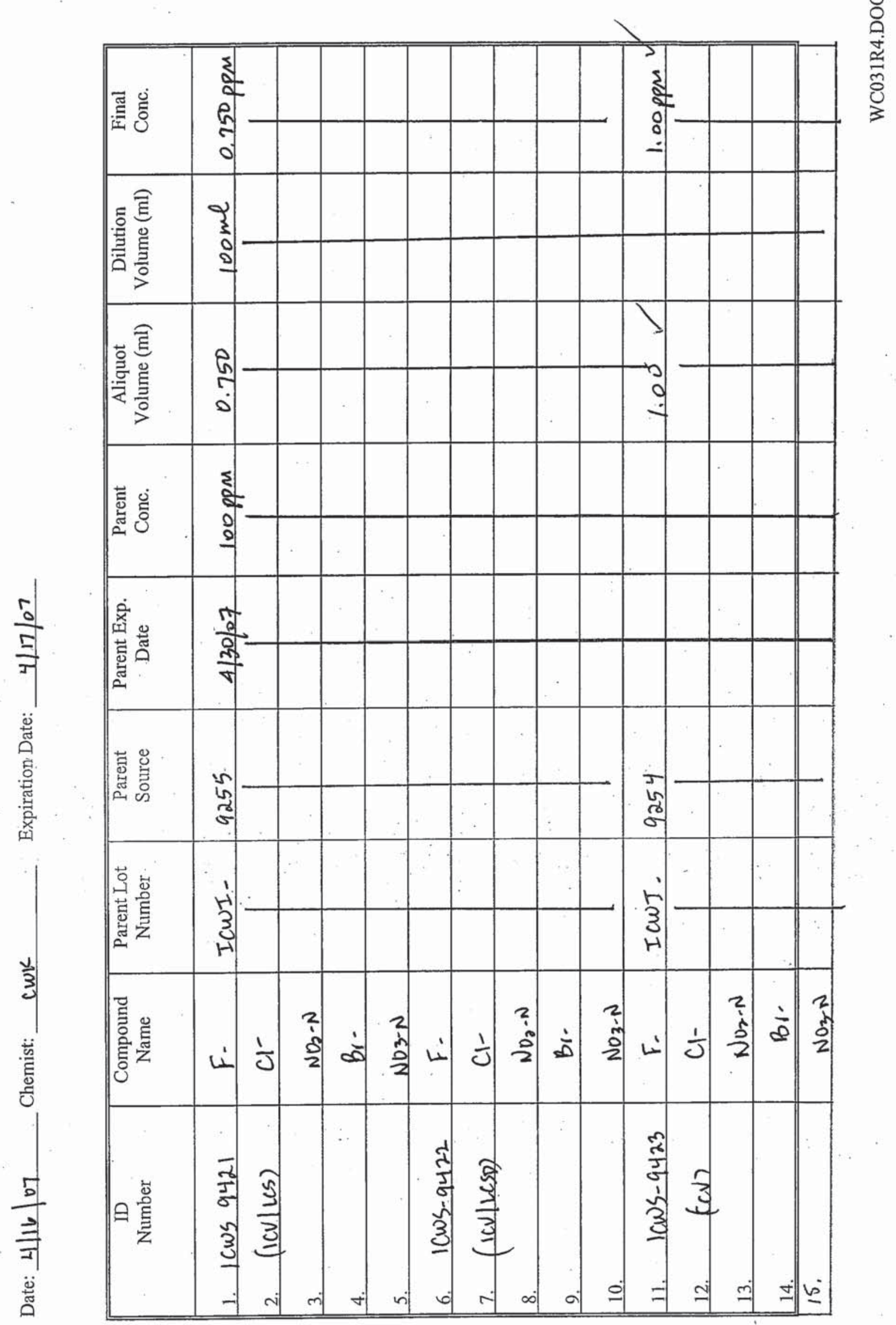

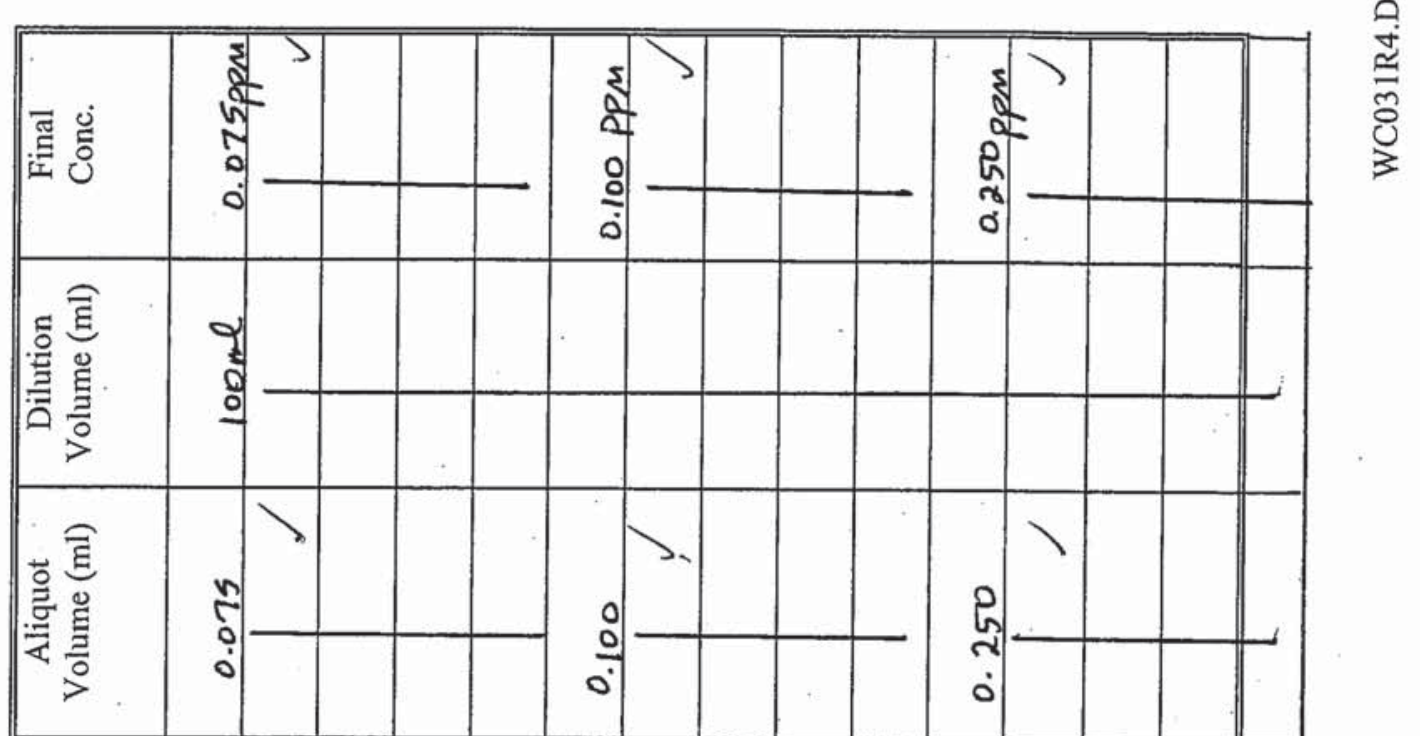

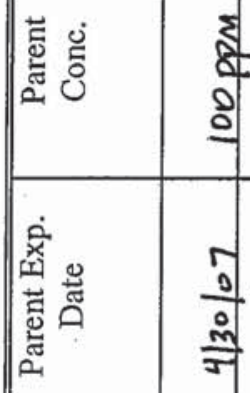

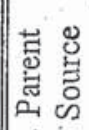

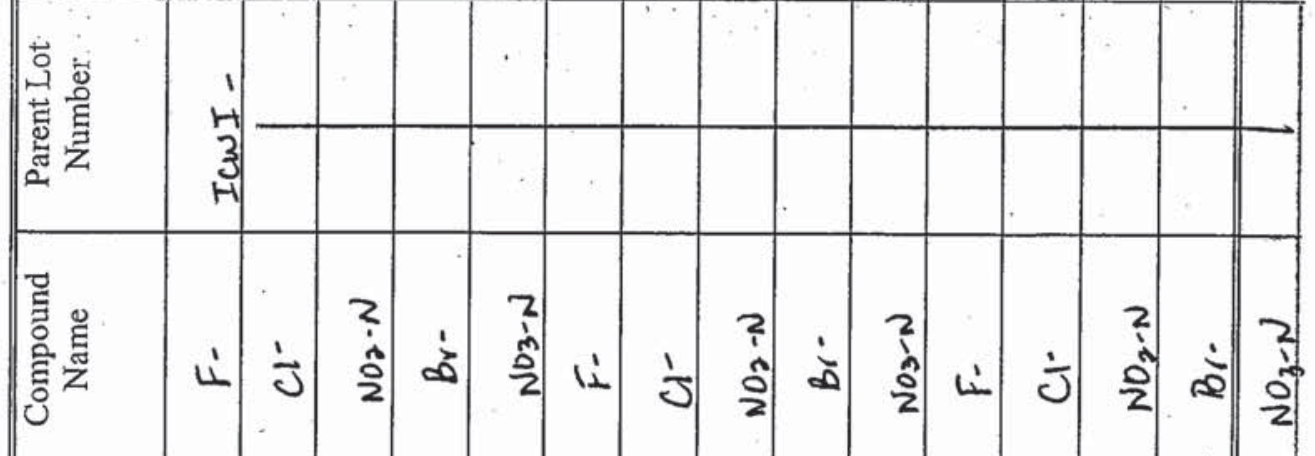

$\frac{\frac{1}{2}}{\frac{3}{7}}$

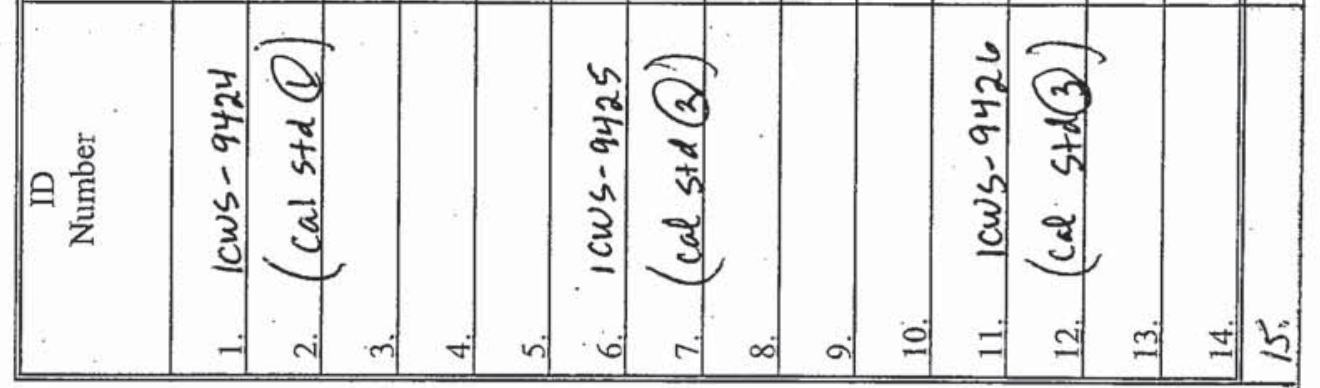




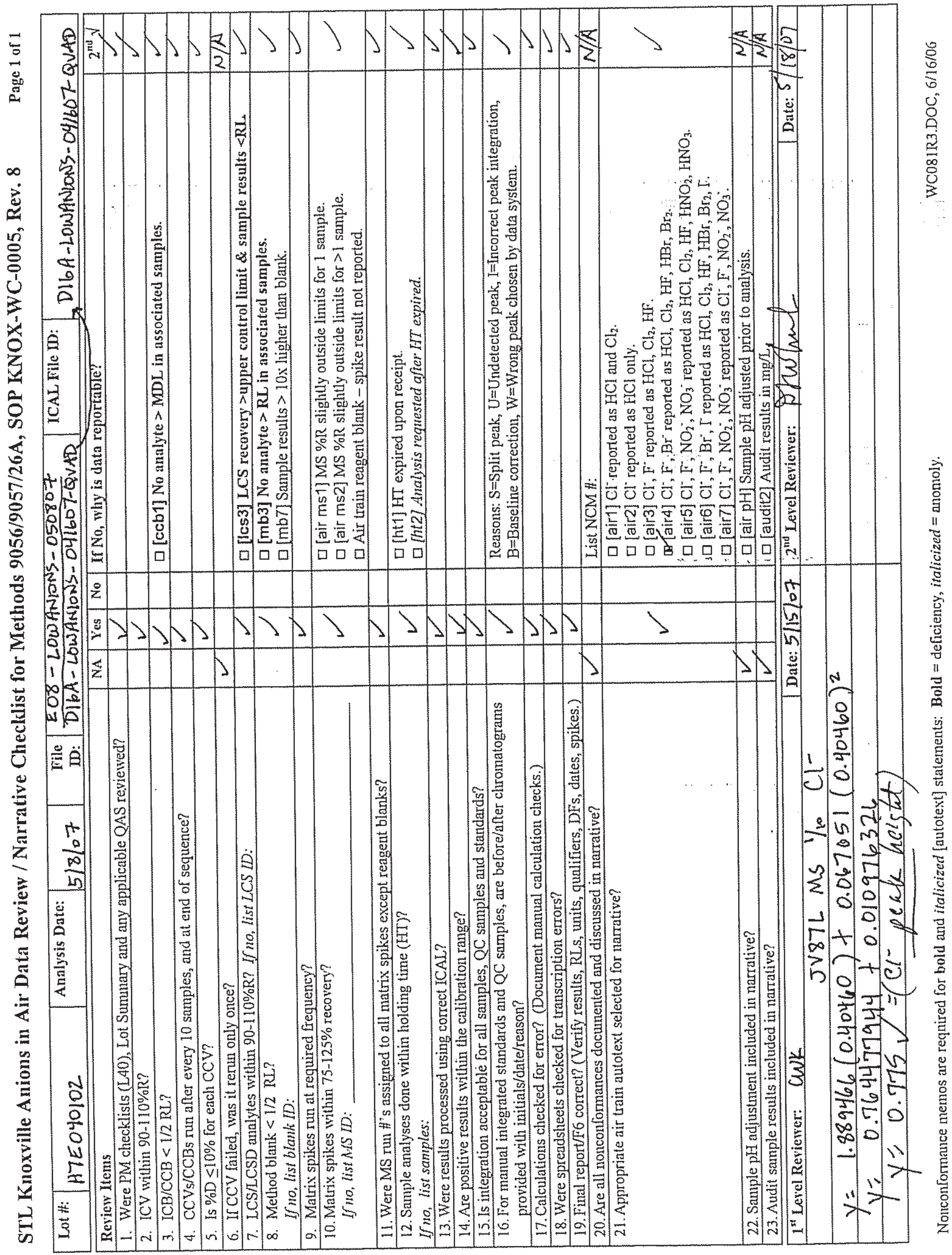




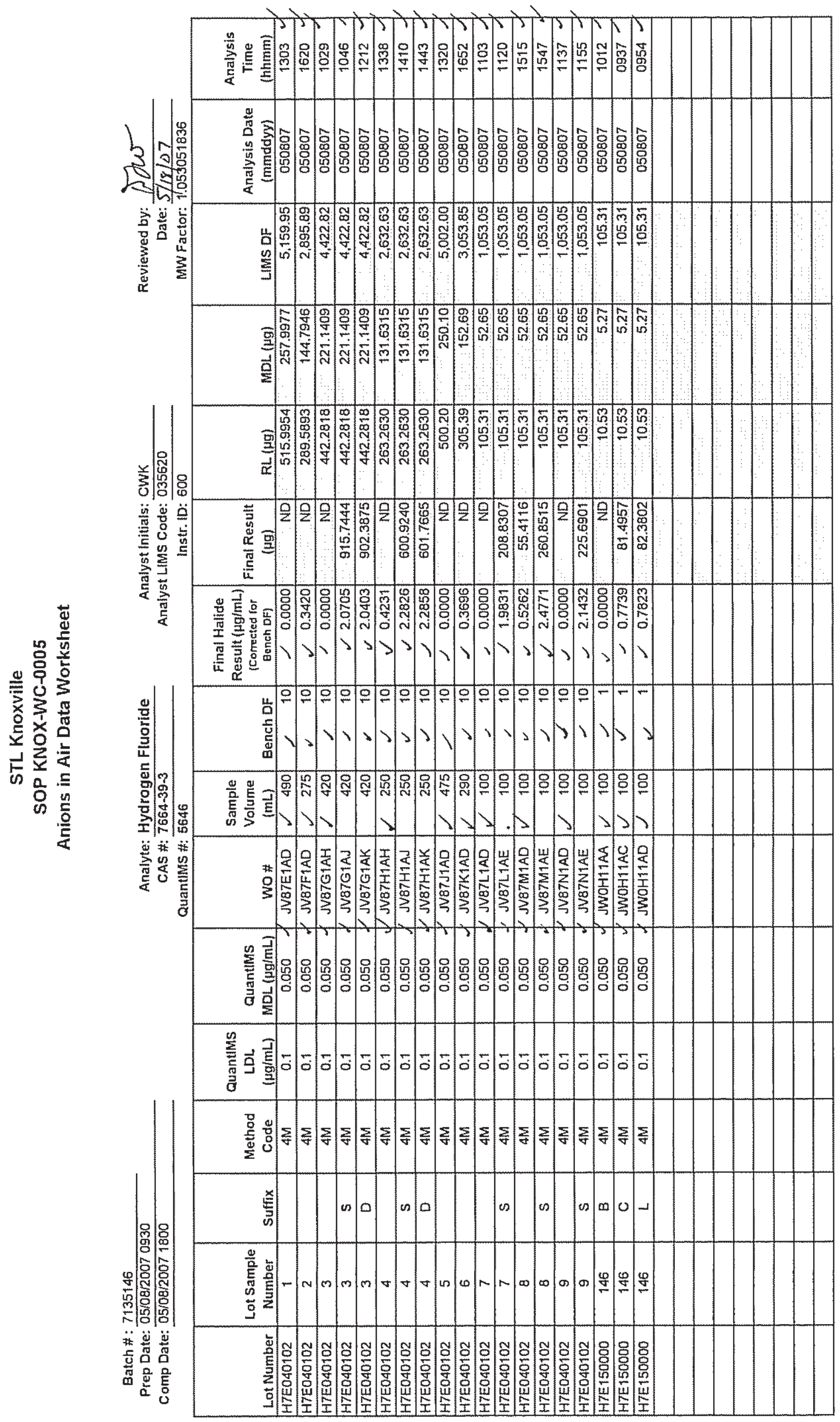



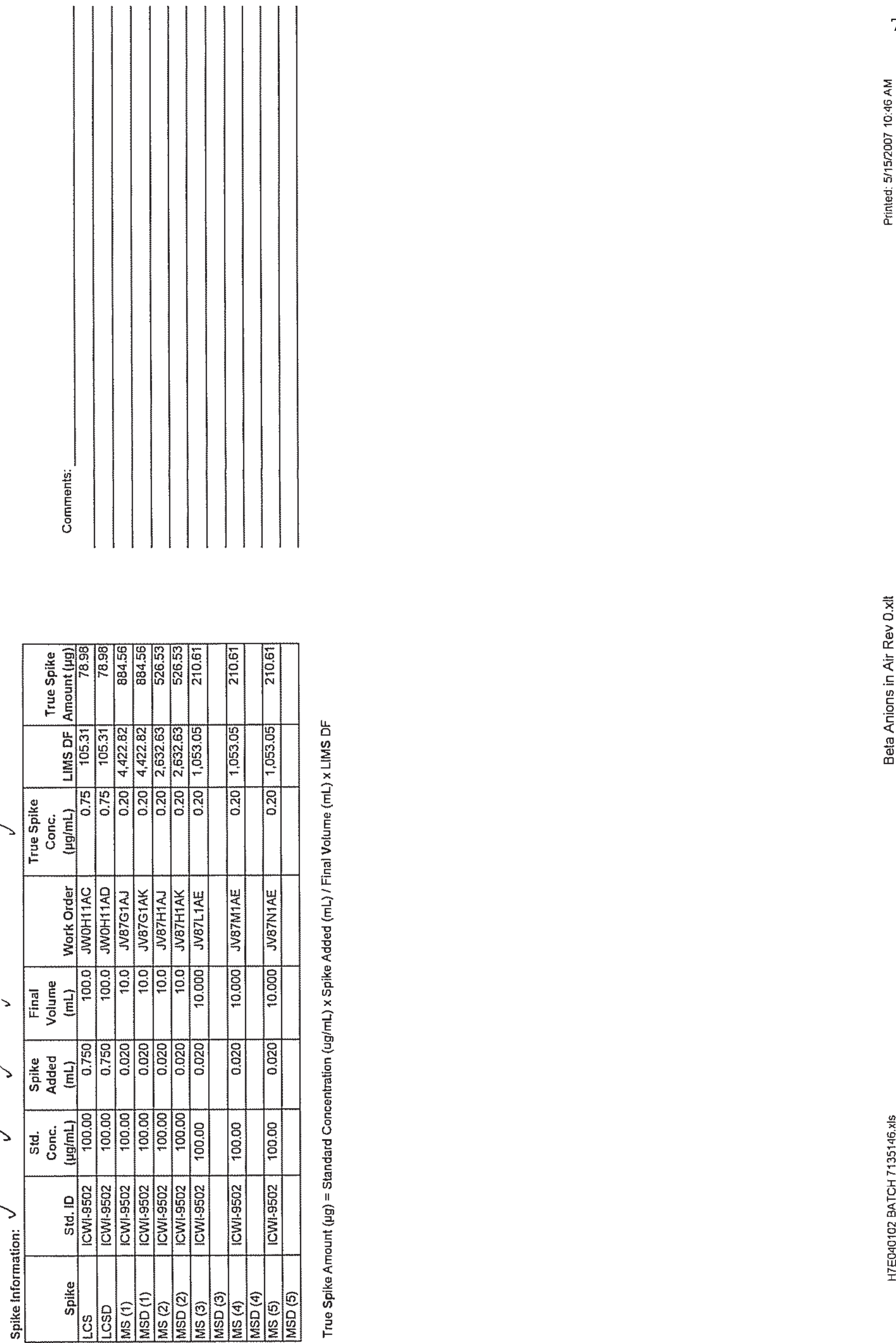


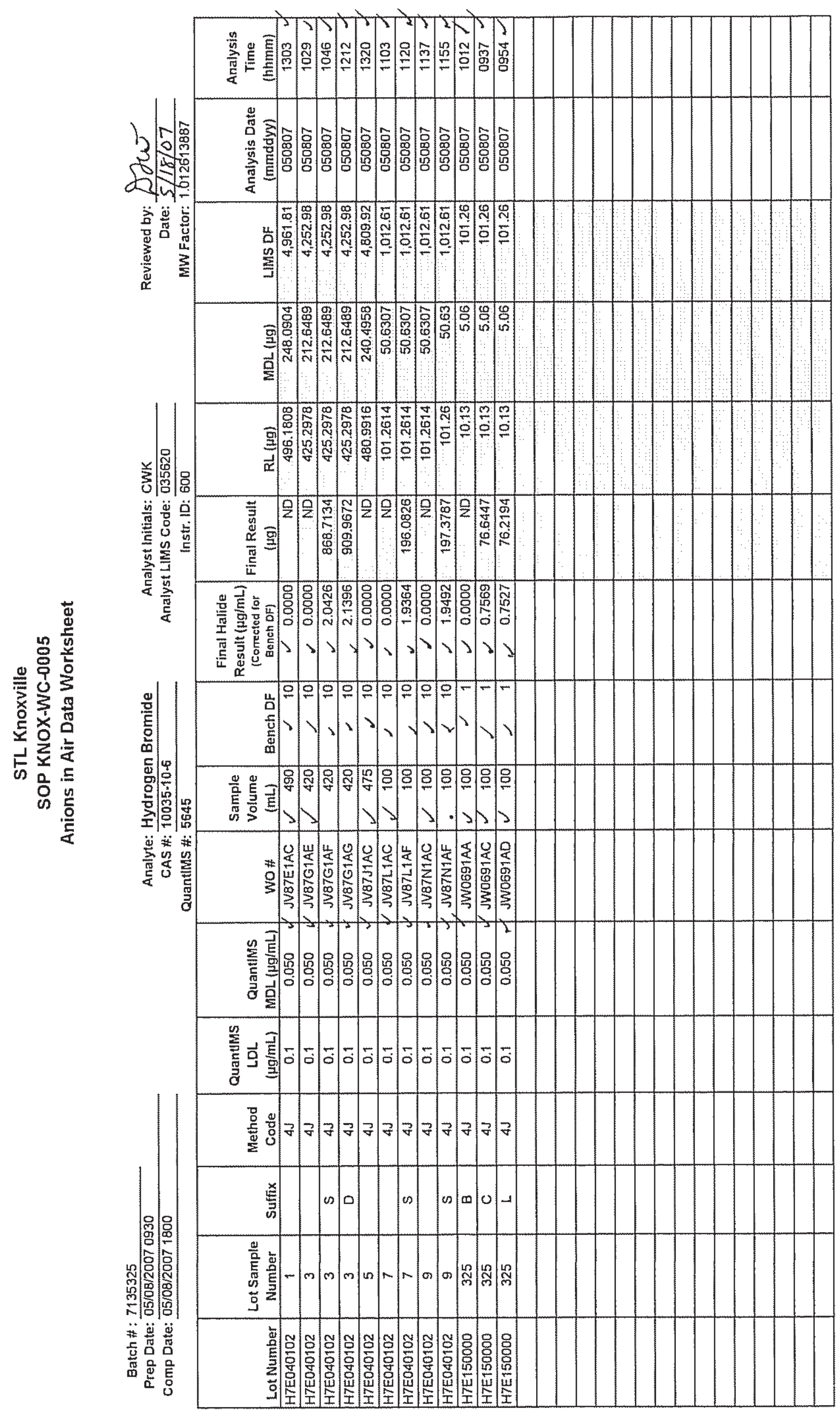




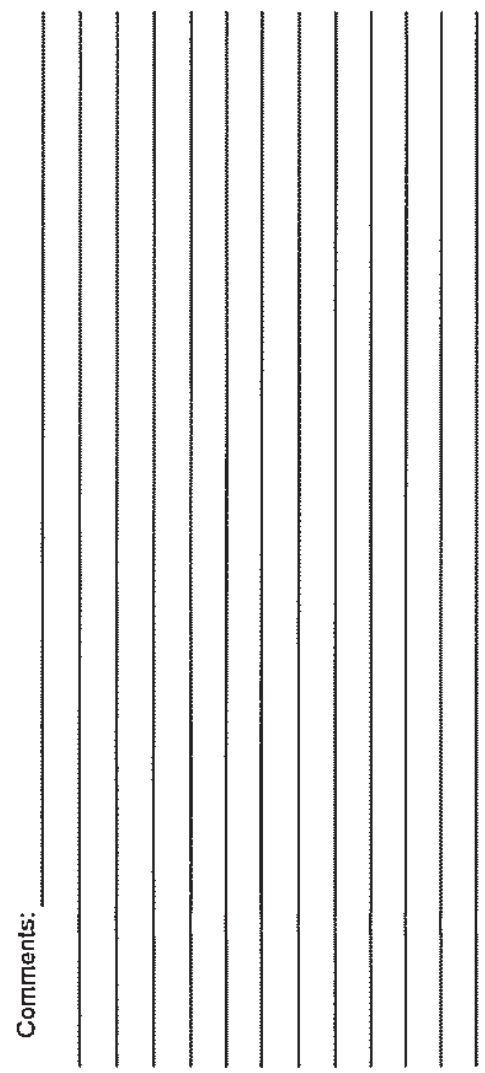

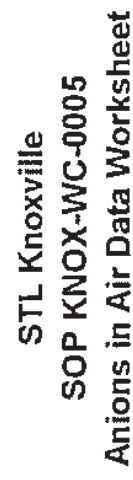

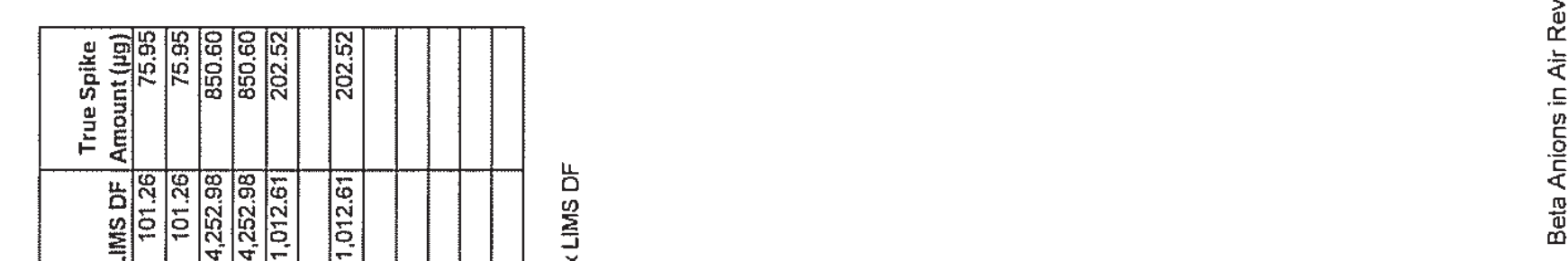

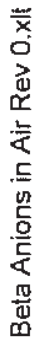




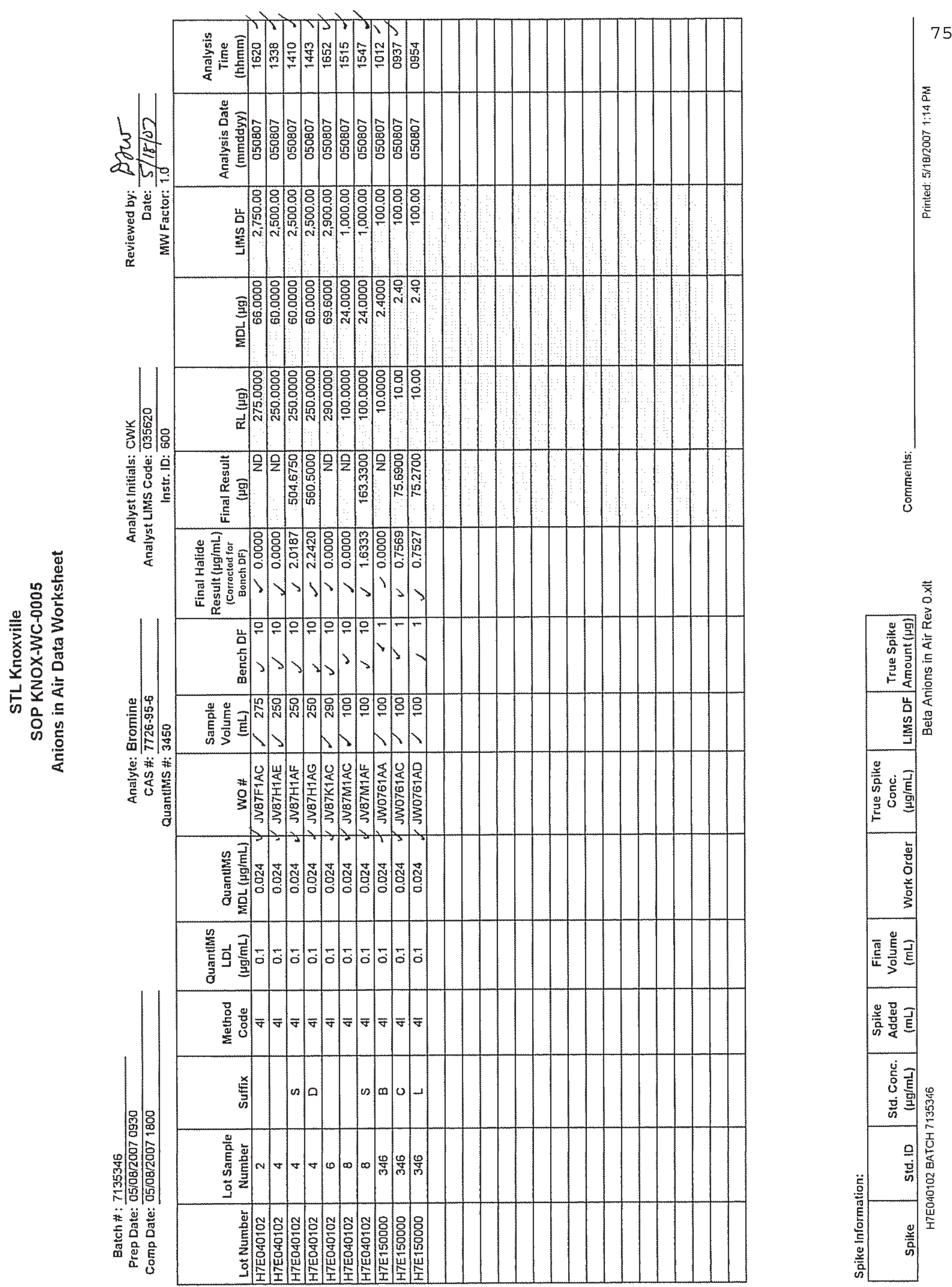



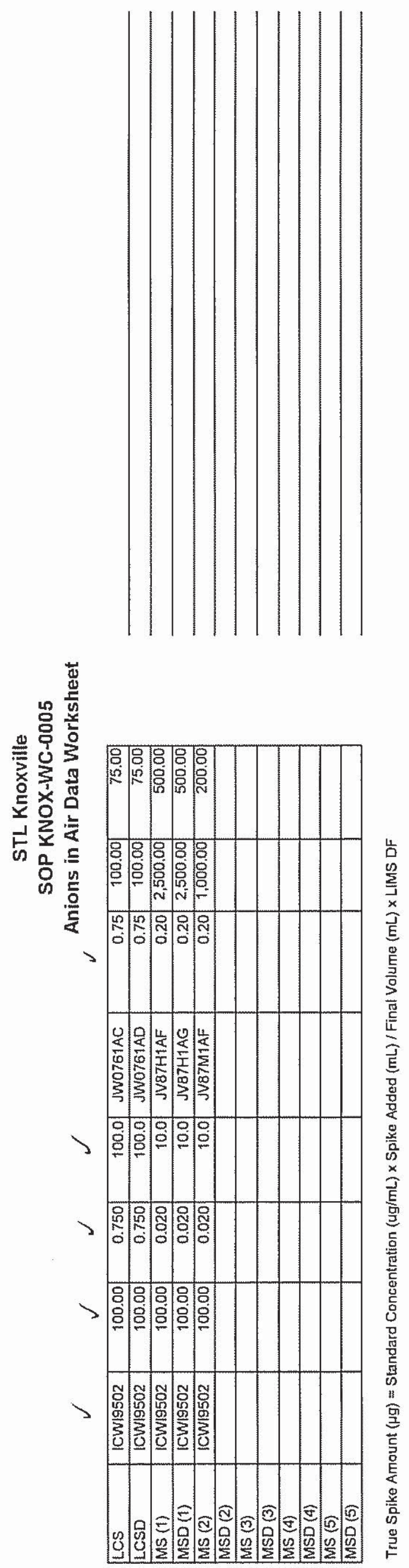

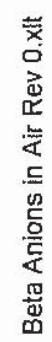




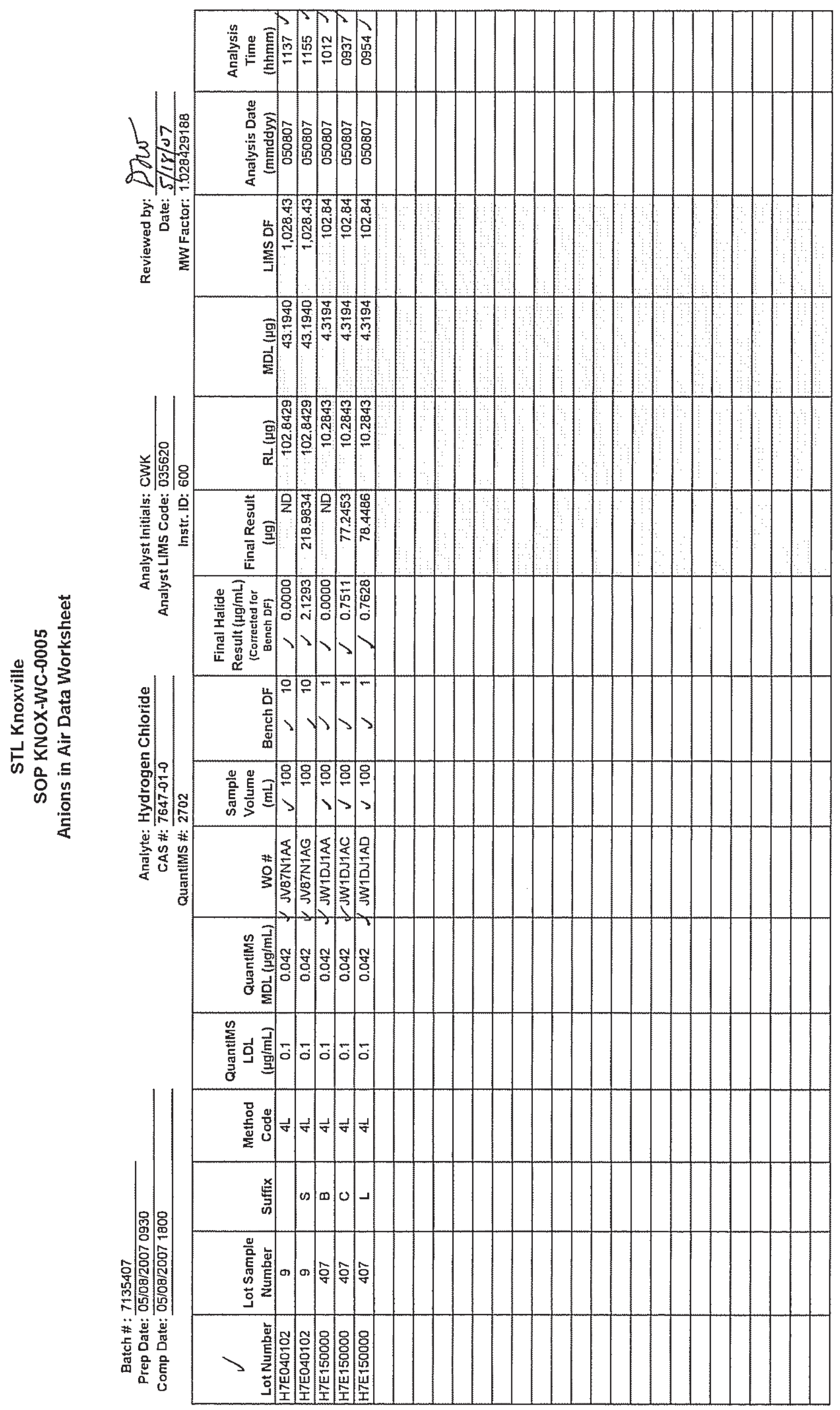




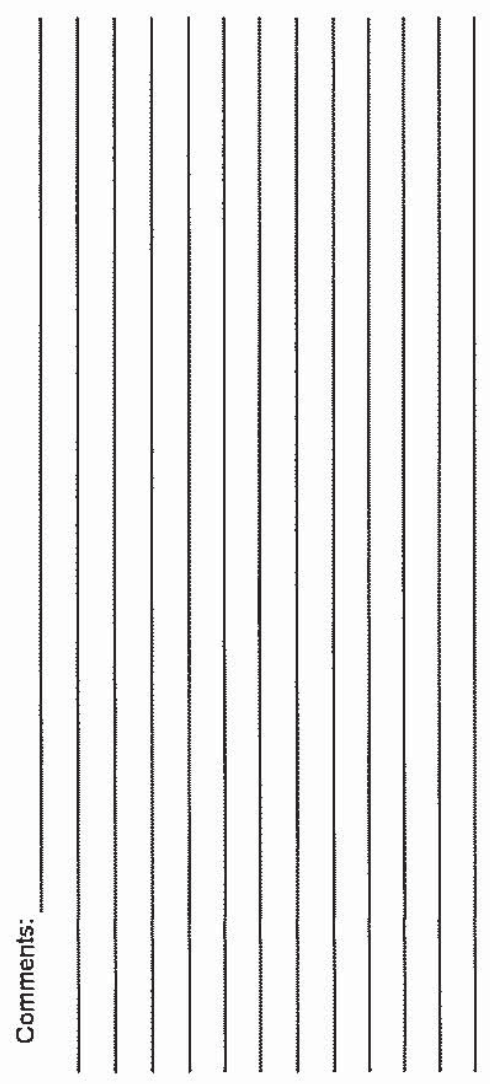

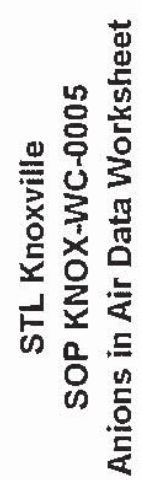

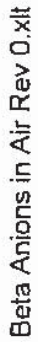

$>$ 蹗
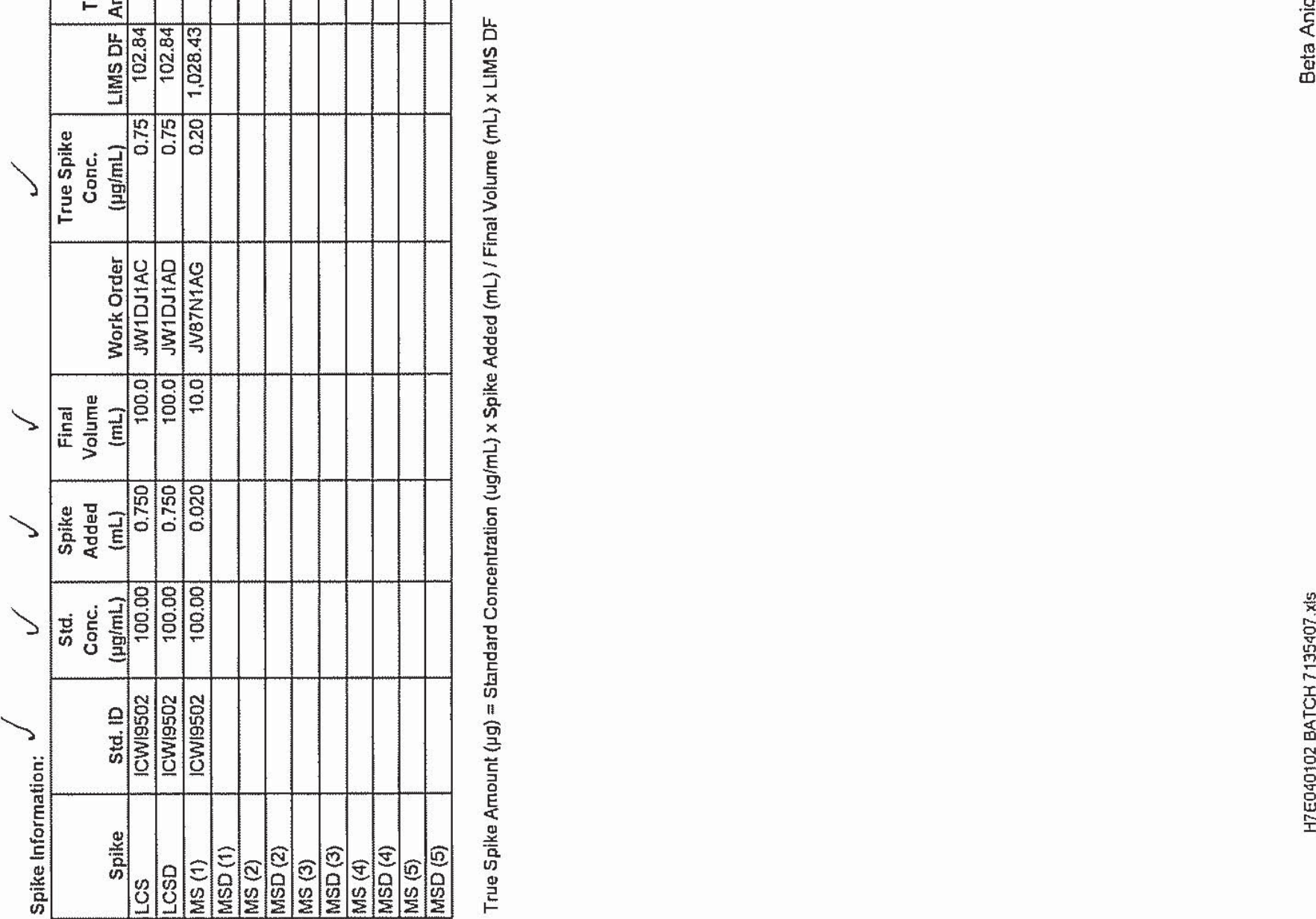
STL Knoxville

Dionex IC Runlog Cover Page

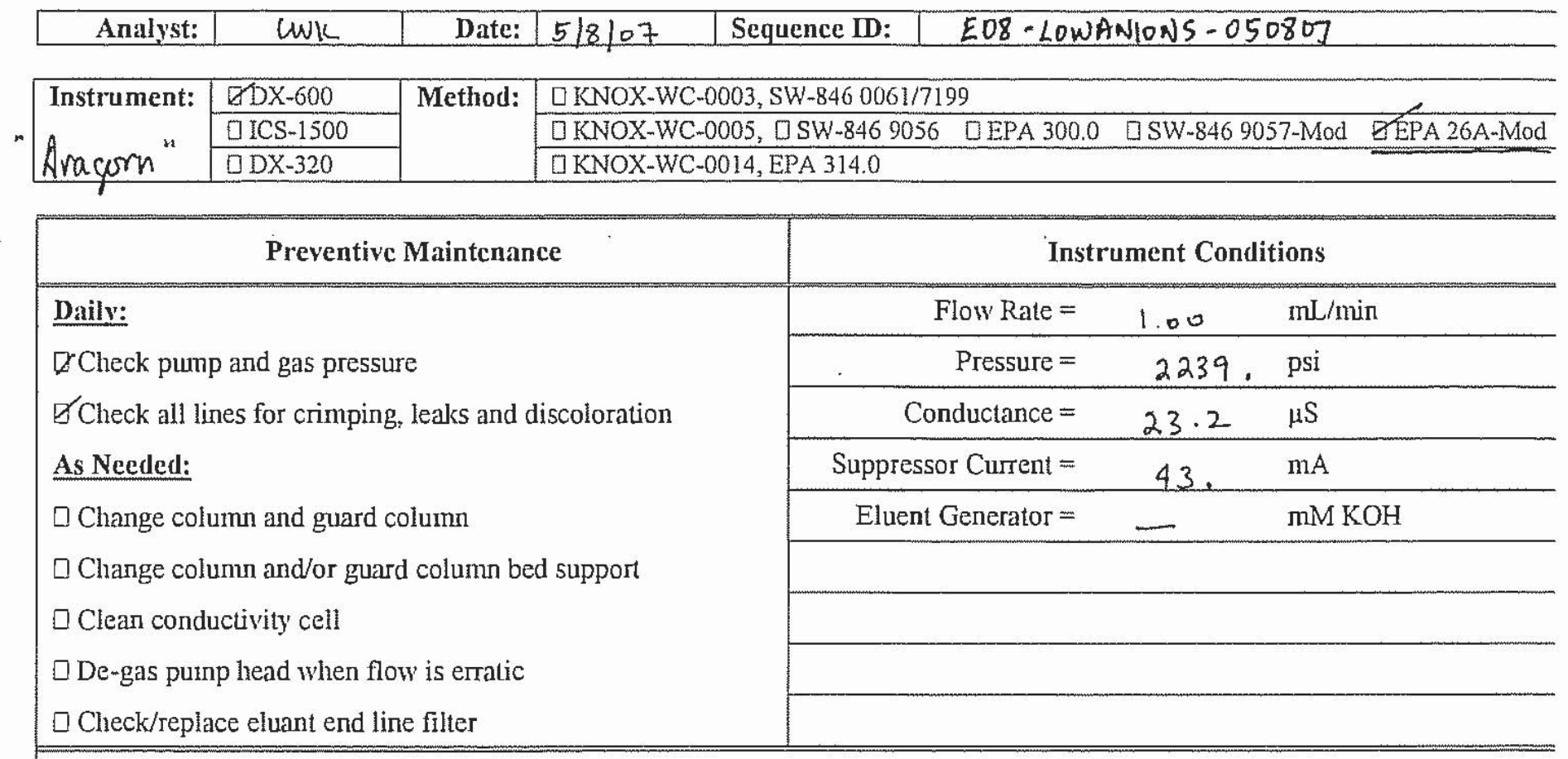

\begin{tabular}{|c|c|c|c|c|c|c|}
\hline \multicolumn{7}{|c|}{ MS/MSD Spike Information } \\
\hline WO \# & Compound & Spike ID & Parent Conc. & Spikc Added $(\mathrm{mL})$ & Final Volume (mL) & Final Conc. \\
\hline$J \sqrt{ } 87 \mathrm{~N}$ & $F$ & FowI-9502 & $100 P P M$ & $0.020 \mathrm{ml}$ & lome & $0.20 \mathrm{ppm}$ \\
\hline & $\mathrm{Cl}^{-}$ & & 1 & 1 & 1 & 11 \\
\hline & $B r^{\prime}$ & & & & & \\
\hline JV87L & F- & & & & & \\
\hline 1 & $\mathrm{Cl}_{-}$ & & & & & \\
\hline 1 & $B r-$ & & & & & \\
\hline JV87M & $F-$ & & & 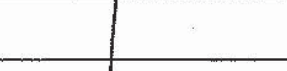 & & \\
\hline 1 & $\mathrm{Cl}_{-}$ & & & & & \\
\hline 1 & $\mathrm{Br}-$ & & & & & \\
\hline 10876 & $\mathrm{~F}$ & & & & & \\
\hline 1 & $\mathrm{Cl}^{-}$ & & & & & \\
\hline 1 & $\mathrm{Br}-$ & & & & & \\
\hline $3 \sqrt{37} 7 \mathrm{H}$ & $F$ & & & & & \\
\hline , & $\mathrm{Cl}^{-}$ & & & & & \\
\hline Commiquts: & Br- & & & $T$ & & 1 \\
\hline
\end{tabular}

\footnotetext{
Batch: $7135146\left(F^{-}\right)$Batch: 7135407 (HCl for DI H20 Blank)

Batch: 7135325 ( $\left.\mathrm{HBr}^{\circ}\right)$

Batch: $7135346\left(B_{r_{2}}\right)$

$D$ Sodium Thiosulfate added to $\mathrm{NaOH}$ impinger samples.
} 
Sequence: $\quad$ E08_LOW_ANIONS_050807

Page 1 of 3

Operator: kaukerc

Printed: 5/9/2007 8:05:35 AM

Title:

Datasource: Aragorn_net

Location: IC ARAGORN

Timebase: IC_Aragorn

Created:

5/8/2007 9:30:07 AM by kaukerc

\#Samples:

30

Last Updale:

5/8/2007 3:07:18 PM by kaukerc

\begin{tabular}{|r|l|l|r|r|r|r|}
\hline No. & Name & Comment & Inj. Vol. & Inj. Date/Time & Dil. Factor & *Mulkiplier \\
[Liters
\end{tabular}


Title:

Datasource:

Location:

Timebase:

Aragorn_nel

\#Samples:

\begin{tabular}{|c|c|c|c|c|c|}
\hline No. & Name & Weight & Type & Status & Program \\
\hline 1 & (1) CAL STD \#1 ICWS-9424 & 1.0000 & Standard & Finished & ANIONS_AS14A \\
\hline 2 & 0 CAL STD \#2 ICWS-9425 & 1.0000 & Standard & Finished & ANIONS_AS14A \\
\hline 3 & (1) CAL STD \#3ICWS-9426 & 1.0000 & Standard & Finished & ANIONS_AS14A \\
\hline 4 & (1) CAL STD \#4 ICWS-9427 & 1.0000 & Standard & Finished & ANIONS AS14A \\
\hline 5 & (1) CAL STD \#5 ICWS-9423 & 1.0000 & Standard & Finished & ANIONS_AS14A \\
\hline 6 & (1) CAL STD \#6 ICWS-9428 & 1.0000 & Standard & Finished & ANIONS AS14A \\
\hline 7 & [2] ICV/LCS ICWS-9527 & 1.0000 & Unknown & Finished & ANIONS_AS14A \\
\hline 8 & 包 ICVILCSD ICWS-9528 & 1.0000 & Unknown & Finished & ANIONS_AS14A \\
\hline 9 & (?) ICB/METHOD BLK & 1.0000 & Unknown & Finished & ANIONS_AS14A \\
\hline 10 & ? H7E040102 JV87G 1/10 & 1.0000 & Unknown & Finished & ANIONS_AS14A \\
\hline 11 & [2] H7E040102 JVB7G MS 1/10 0.2 PPM F, CL, BR & 1.0000 & Unknown & Finished & ANIONS_AS14A \\
\hline 12 & ? H7E040102 JVB7L $1 / 10$ & 1.0000 & Unknown & Finished & ANIONS_AS14A \\
\hline 13 & ? H7E040102 JVB7L MS 1/10 0.2 PPM F, BR. CL & 1.0000 & Unknown & Finished & ANIONS_AS14A \\
\hline 14 & (?) H7E040102 JV87N $1 / 10$ & 1,0000 & Unknown & Finished & ANIONS_AS14A \\
\hline 15 & (3) H7E040102 JV87N MS 1/10 0.2 PPM BR, F, CL & 1.0000 & Unknown & Finished & ANIONS_AS14A \\
\hline 16 & 高 H7E040102 JVB7G MSD 1/10 0.2 PPM CL, BR, F & 1.0000 & Unknown & Finished & ANIONS_AS14A \\
\hline 17 & ?] CCV ICWS-9529 & 1.0000 & Unknown & Finished & ANIONS_AS14A \\
\hline 18 & [ $\mathrm{CCB}$ & 1.0000 & Unknown & Finished & ANIONS_AS14A \\
\hline 19 & [?] H7E040102 JVB7E 1/10 & 1.0000 & Unknown & Finished & ANIONS_AS14A \\
\hline 20 & [. H7E040102 JVB7J 1/10 & 1.0000 & Unknown & Finished & ANIONS_AS14A \\
\hline 21 & [? H7E040102 JVB7H 1/10 & 1.0000 & Unknown & Finished & ANIONS_AS14A_CI2 \\
\hline 22 & [? H7E040102 JV87H MS 1/10 0.2 PPM CL, BR, F & 1.0000 & Unknown & Finished & ANIONS_AS14A_Cl2 \\
\hline 23 & ?] H7E040102 JV87H MSD 1/10 0.2 PPM CL, BR, F & 1.0000 & Unknown & Finished & ANIONS_AS14A_Cl2 \\
\hline 24 & ? H7E040102 JV87M 1/10 & 1.0000 & Unknown & Finished & ANIONS_AS14A_Cl2 \\
\hline 25 & [? H7E040102 JVB7M MS 1/10 0.2 PPM CL, F, BR & 1.0000 & Unknown & Finished & ANIONS_AS14A_Cl2 \\
\hline 26 & [?] H7E040102 JV87F 1/10 & 1.0000 & Unknown & Finished & ANIONS_AS14A_Cl2 \\
\hline 27 & [] H7E040102 JV87K 1/10 & 1.0000 & Unknown & Finished & ANIONS_AS14A_Cl2 \\
\hline 28 & ] CCV ICWS-9529 & 1.0000 & Unknown & Finished & ANIONS_AS14A \\
\hline 29 & CCB & 1.0000 & Unknown & Finished & ANIONS_AS14A \\
\hline 30 & [?] SHUTDOWN & 1.0000 & Unknown & Finished & SHUTDOWN \\
\hline
\end{tabular}


Title:

Dalasource:

Location:

Timebase:

Aragorn_net

\#Samples:

IC_ARAGORN

IC Aragorn

Created:

5/8/2007 9:30:07 AM by kaukerc 30

Last Update: 5/8/2007 3:07:18 PM by kaukerc

\begin{tabular}{|c|c|c|}
\hline No. & Name & Method \\
\hline 1 & (1) CAL STD \#1 ICWS-9424 & ANIONS \\
\hline 2 & (1) CAL STD \#2 ICWS-9425 & ANIONS \\
\hline 3 & (1) CAL STD \#3 ICWS-9426 & ANIONS \\
\hline 4 & (1) CAL STD \#4 ICWS-9427 & ANIONS \\
\hline 5 & (9) CAL STD \#5 ICWS-9423 & ANIONS \\
\hline 6 & (9) CAL STD \#6 ICWS-9428 & ANIONS \\
\hline 7 & (2) ICV/LCS ICWS-9527 & ANIONS \\
\hline 8 & (2) ICV/LCSD ICWS-9528 & ANIONS \\
\hline 9 & [2] ICB/METHOD BLK & ANIONS \\
\hline 10 & (2) H7E040102 JV87G 1/10 & ANIONS \\
\hline 11 & 2. H7E040102 JV87G MS $1 / 100.2$ PPM F, CL, BR & ANIONS \\
\hline 12 & [? H7E040102 JV87L 1/10 & ANIONS \\
\hline 13 & [7] H7E040102 JV87L MS 1/10 0.2 PPM F, BR. CL & ANIONS \\
\hline 14 & [? H7E040102 JV87N $1 / 10$ & ANIONS \\
\hline 15 & H7E040102 JV87N MS $1 / 100.2$ PPM BR, F, CL & ANIONS \\
\hline 16 & ? H7E040102 JV87G MSD 1/10 0.2 PPM CL, BR, F & ANIONS \\
\hline 17 & 竞 CCVICWS-9529 & ANIONS \\
\hline 18 & (2) $\mathrm{CCB}$ & ANIONS \\
\hline 19 & (2) H7E040102 JV87E 1/10 & ANIONS \\
\hline 20 & H7E 040102 JV87J $1 / 10$ & ANIONS \\
\hline 21 & (2) H7E040102 JV87H $1 / 10$ & ANIONS \\
\hline 22 & H7E040102 JV87H MS $1 / 100.2$ PPM CL, BR, F & ANIONS \\
\hline 23 & H7) H7E040102 JVB7H MSD 1/10 0.2 PPM CL, BR, F & ANIONS \\
\hline 24 & [3] H7E040102 JV87M 1/10 & ANIONS \\
\hline 25 & H7E040102 JV87M MS $1 / 100.2$ PPM CL, F, BR & ANIONS \\
\hline 26 & H7E040102 JV87F $1 / 10$ & ANIONS \\
\hline 27 & (2) H7E040102 JV87K 1/10 & ANIONS \\
\hline 28 & (2) CCVICWS-9529 & ANIONS \\
\hline 29 & 这 $\mathrm{CCB}$ & ANIONS \\
\hline 30 & [?] SHUTDOWN & ANIONS \\
\hline
\end{tabular}




\section{ICVILCS ICWS-9527}

\section{QUAD}

\section{Sample Name: ICV/LCS ICWS-9527}

Vial Number:

Sample Type:

Control Program:

Quantif, Method:

Recording Time:

Run Time (min):
310

unknown

ANIONS_AS14A

ANIONS

5/8/2007 9:37

14.75

$\begin{array}{ll}\text { Injection Volume: } & \mathbf{5 0 . 0} \\ \text { Channel: } & \text { ECD_1 } \\ \text { Wavelength: } & \text { n.a. } \\ \text { Bandwidth: } & \text { n.a. } \\ \text { Dilution Factor: } & \mathbf{1 . 0 0 0 0} \\ \text { Sample Weight: } & \mathbf{1 . 0 0 0 0} \\ \text { Sample Amount: } & \mathbf{1 . 0 0 0 0}\end{array}$

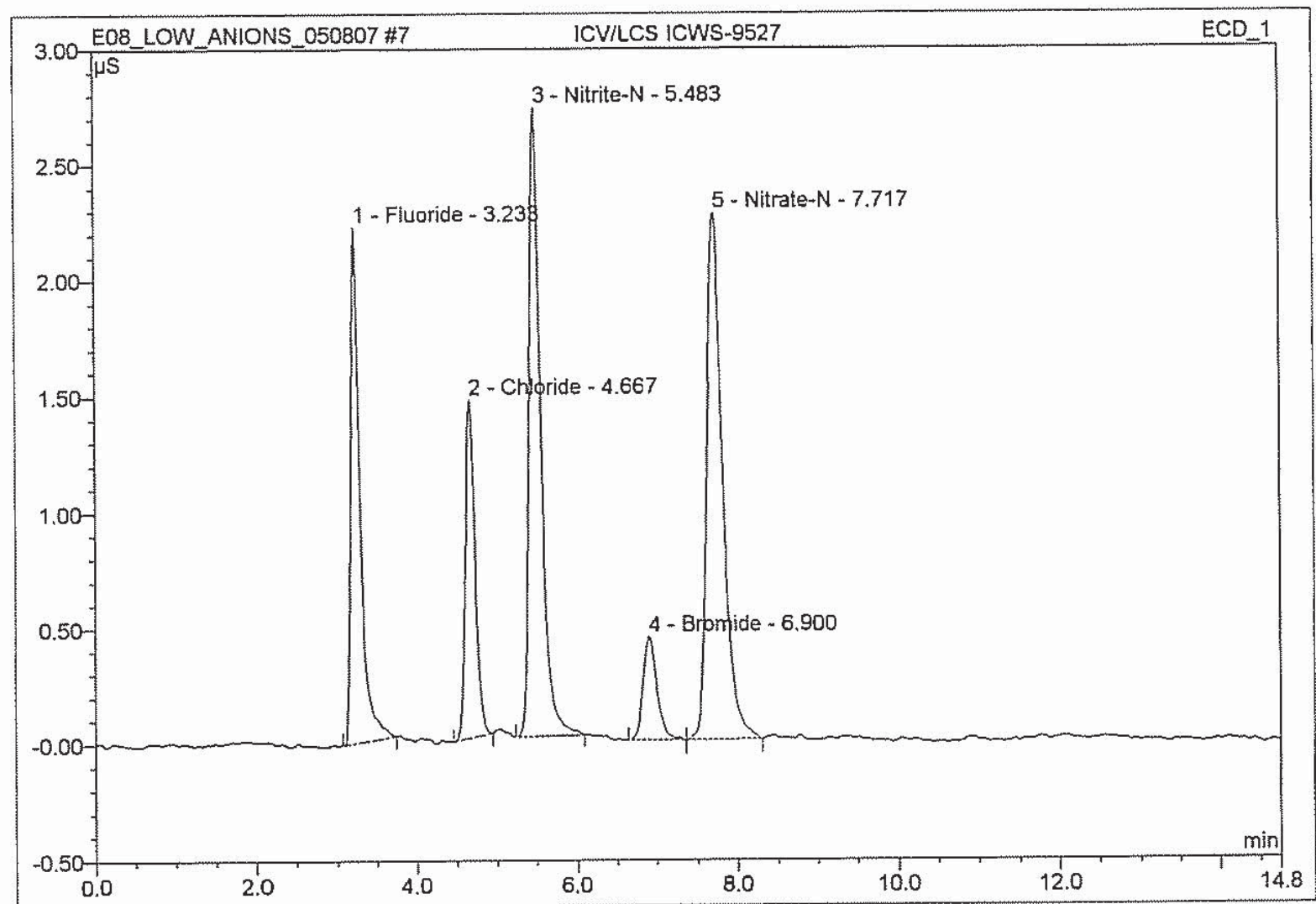

\begin{tabular}{|r|r|l|r|r|r|r|r|}
\hline No. & $\begin{array}{c}\text { Ret.Time } \\
\text { min }\end{array}$ & \multicolumn{1}{|c|}{ Peak Name } & $\begin{array}{c}\text { Height } \\
\mu S\end{array}$ & $\begin{array}{c}\text { Area } \\
\mu S^{*} \min \end{array}$ & $\begin{array}{c}\text { Rel.Area } \\
\%\end{array}$ & $\begin{array}{c}\text { Amount } \\
\text { mg/L }\end{array}$ & $\begin{array}{c}\text { Peak } \\
\text { Type }\end{array}$ \\
\hline 1 & 3.23 & Fluoride & 2.226 & 0.303 & 19.23 & 0.7739 & BMB \\
\cline { 2 - 8 } 2 & 4.67 & Chloride & 1.457 & 0.203 & 12.87 & 0.7511 & BMB \\
\hline 3 & 5.48 & Nitrite-N & 2.713 & 0.461 & 29.20 & 0.7680 & BMB \\
\hline 4 & 6.90 & Bromide & 0.446 & 0.089 & 5.63 & 0.7569 & BM \\
\hline 5 & 7.72 & Nitrate-N & 2.272 & 0.522 & 33.07 & 0.7659 & $\mathrm{MB}$ \\
\hline Total: & & & 9.114 & 1.577 & 100.00 & 3.816 & \\
\hline
\end{tabular}

JWOHIIAC HF-

JWO691AC ABC

JWD761AC

default_letter/Integration

JWIDJIAC

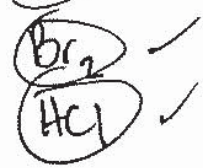




\section{ICVILCSD ICWS-9528}

\section{QUAD}

Sample Name: $\quad$ ICV/LCSD ICWS-9528

Vial Number: $\quad 311$

Sample Type: $\quad$ unknown

Control Program: ANIONS_AS14A

Quantif. Method: ANIONS

Recording Time: $\quad$ 5/8/2007 9:54

Run Time (min): $\quad 14.75$

$\begin{array}{ll}\text { Injection Volume: } & \mathbf{5 0 . 0} \\ \text { Channel: } & \text { ECD_1 } \\ \text { Wavelength: } & \text { n.a. } \\ \text { Bandwidth: } & \text { n.a. } \\ \text { Dilution Factor: } & 1.0000 \\ \text { Sample Weight: } & 1.0000 \\ \text { Sample Amount: } & 1.0000\end{array}$

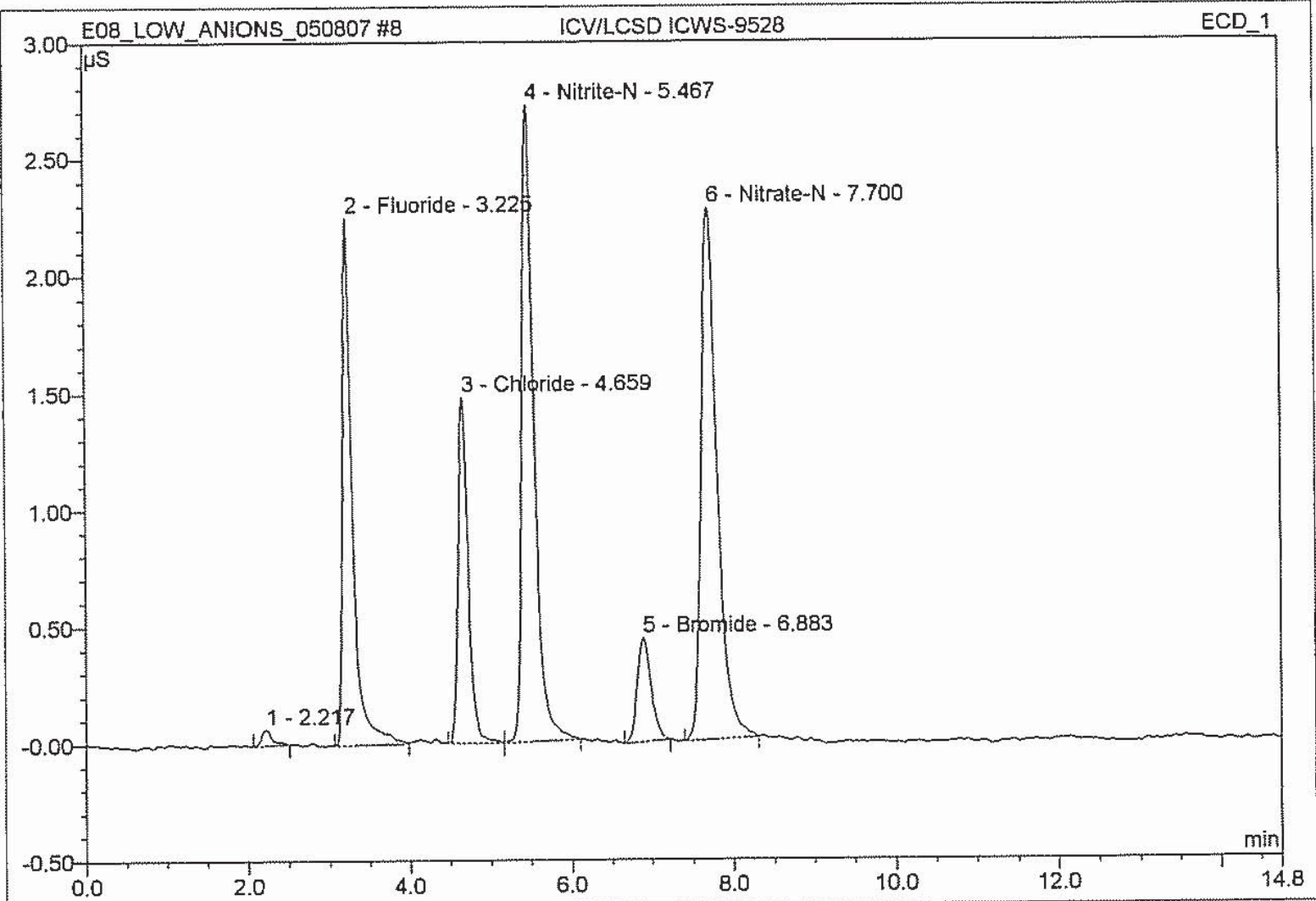

\begin{tabular}{|r|r|l|r|r|r|r|r|}
\hline No. & $\begin{array}{c}\text { Ret.Time } \\
\text { min }\end{array}$ & \multicolumn{1}{|c|}{ Peak Name } & $\begin{array}{c}\text { Height } \\
\mu S\end{array}$ & $\begin{array}{c}\text { Area } \\
\mu S^{*} \min \end{array}$ & $\begin{array}{c}\text { Rel.Area } \\
\%\end{array}$ & $\begin{array}{c}\text { Amount } \\
\text { mg/L }\end{array}$ & $\begin{array}{c}\text { Peak } \\
\text { Type }\end{array}$ \\
\hline 1 & 2.22 & n.a. & 0.068 & 0.011 & 0.65 & n.a. & BMB \\
\hline 2 & 3.23 & Fluoride & 2.252 & 0.322 & 19.77 & 0.7823 & BMB \\
\hline 3 & 4.66 & Chloride & 1.480 & 0.213 & 13.08 & 0.7628 & BMb \\
\hline 4 & 5.47 & Nitrite-N & 2.720 & 0.471 & 28.95 & 0.7697 & bMB \\
\hline 5 & 6.88 & Bromide & 0.443 & 0.088 & 5.39 & 0.7527 & BMB \\
\hline 6 & 7.70 & Nitrate-N & 2.273 & 0.523 & 32.16 & 0.7664 & BMB \\
\hline Total: & & & 9.236 & 1.628 & 100.00 & 3.834 & \\
\hline
\end{tabular}

default_letter/Integration
Chromeleon (c) Dionex 1996-2001 Version 6.50 SP10a Build 1065 


\section{ICB/METHOD BLK}

\section{QUAD}

Sample Name: ICB/METHOD BLK

Vial Number: $\quad 312$

Sample Type: $\quad$ unknown

Control Program: ANIONS_AS14A

Quantif. Method: ANIONS

Recording Time: $\quad$ 5/8/2007 10:12

Run Time ( $\mathrm{min})$ : $\quad \mathbf{1 4 . 7 5}$
Injection Volume:

Channel:

Wavelength:

Bandwidth:

Dilution Factor:

Sample Weight:

Sample Amount:
50.0

ECD_1

n.a.

n.a.

1.0000

1.0000

1.0000

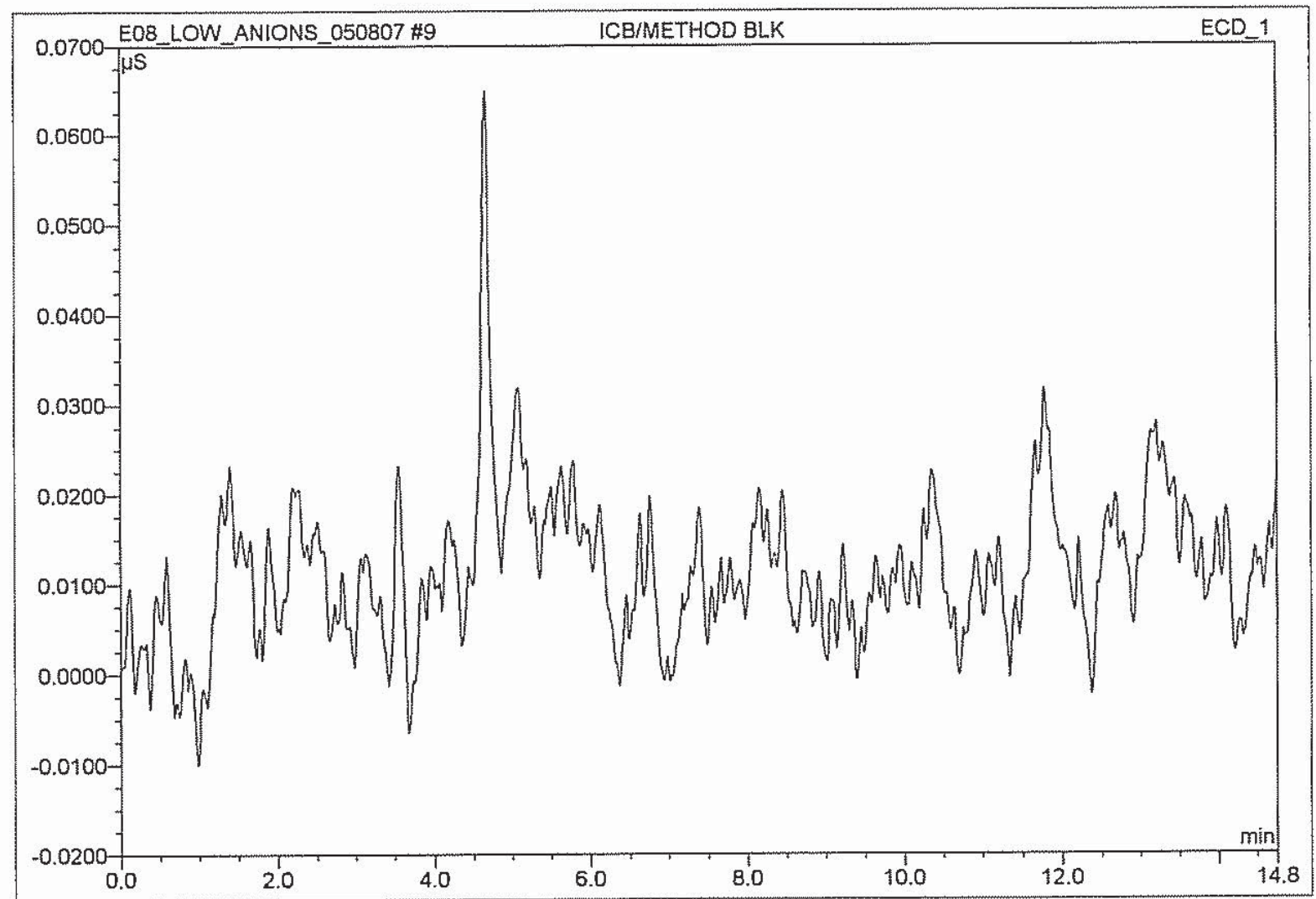

\begin{tabular}{|c|c|c|c|c|c|c|c|}
\hline No. & $\begin{array}{c}\text { Ret.Time } \\
\mathrm{min}\end{array}$ & Peak Name & $\begin{array}{c}\text { Height } \\
\mu \mathrm{S}\end{array}$ & $\begin{array}{c}\text { Area } \\
\mu \mathrm{S}^{*} \mathrm{~min}\end{array}$ & $\begin{array}{c}\text { Rel.Area } \\
\%\end{array}$ & $\begin{array}{c}\text { Amount } \\
\mathrm{mg} / \mathrm{L}\end{array}$ & $\begin{array}{c}\text { Peak } \\
\text { Type }\end{array}$ \\
\hline Total: & & & 0.000 & 0.000 & 0.00 & 0.000 & \\
\hline
\end{tabular}




\section{H7E040102 JV87G 1/10}

\section{QUAD}

Sample Name: H7E040102 JV87G 1/10

Vial Number: $\quad \mathbf{3 1 3}$

Sample Type: unknown

Control Program: ANIONS_AS14A

Quantif. Method: ANIONS

Recording Time: $\quad$ 5/8/2007 10:29

Run Time (min): $\quad 14.75$

$\begin{array}{ll}\text { Injection Volume: } & \mathbf{5 0 . 0} \\ \text { Channel: } & \text { ECD_1 } \\ \text { Wavelength: } & \text { n.a. } \\ \text { Bandwidth: } & \text { n.a. } \\ \text { Dilution Factor: } & \mathbf{1 0 . 0 0 0 0} \\ \text { Sample Weight: } & \mathbf{1 . 0 0 0 0} \\ \text { Sample Amount: } & \mathbf{1 . 0 0 0 0}\end{array}$

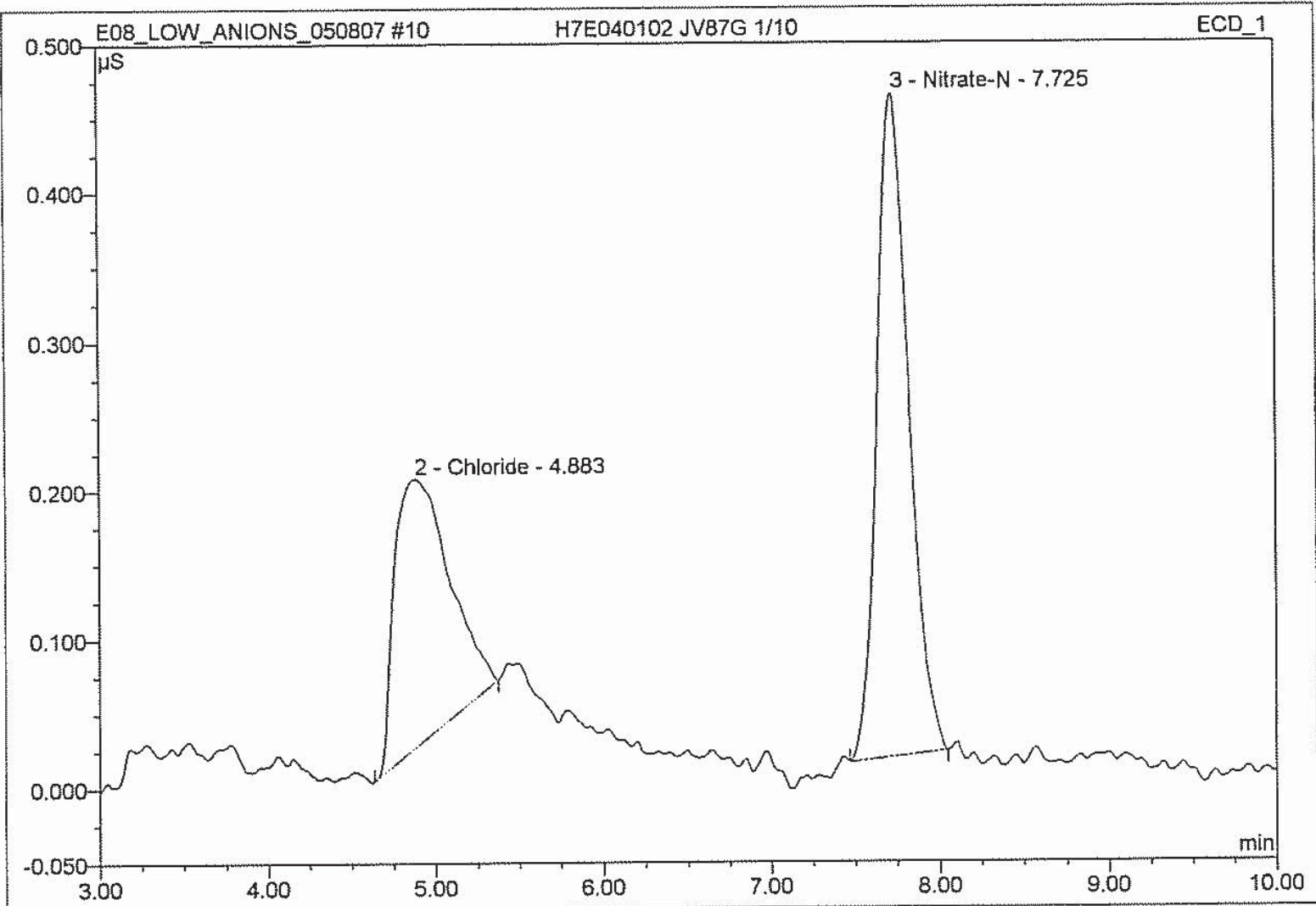

\begin{tabular}{|r|c|l|r|r|r|r|r|}
\hline No. & $\begin{array}{c}\text { Ret.Time } \\
\text { min }\end{array}$ & \multicolumn{1}{|c|}{ Peak Name } & $\begin{array}{c}\text { Height } \\
\mu S\end{array}$ & $\begin{array}{c}\text { Area } \\
\mu S^{*} \text { min }\end{array}$ & $\begin{array}{c}\text { Rel.Area } \\
\%\end{array}$ & $\begin{array}{c}\text { Amount } \\
\text { mg/L }\end{array}$ & $\begin{array}{r}\text { Peak } \\
\text { Type }\end{array}$ \\
\hline 1 & 2.05 & n.a. & 0.140 & 0.092 & 0.14 & n.a. & BMB \\
\hline 2 & 4.88 & Chloride & 0.180 & 0.068 & 0.10 & 0.9509 & BMB \\
\hline 3 & 7.73 & Nitrate-N & 0.445 & 0.098 & 0.15 & 1.5525 & BMB \\
\hline 4 & 11.61 & n.a. & 196.046 & 67.179 & 99.62 & n.a. & BMB \\
\hline Total: & & & 196.812 & 67.438 & 100.00 & 2.503 & \\
\hline
\end{tabular}

$F \cdot \quad N D$

Br. ND

default_letter/Integration

Chromeleon (c) Dionex 1996-2001

Version 6.50 SP10a Build 1065 


\section{H7E040102 JV87G MS 1/10 0.2 PPM F, CL, BR}

\section{QUAD}

Sample Name:

Vial Number:

Sample Type:

Control Program:

Quantif. Method:

Recording Time:

Run Time (min):
H7E040102 JV87G MS 1/10 0.2 PPM F, CL, Injection Volume: 314 unknown

ANIONS_AS14A

ANIONS

$5 / 8 / 200710: 46$

14.75
Channel:

Wavelength:

Bandwidth:

Dilution Factor:

Sample Weight:

Sample Amount:
50.0

ECD_1

n.a.

n.a.

10.0000

1.0000

1.0000

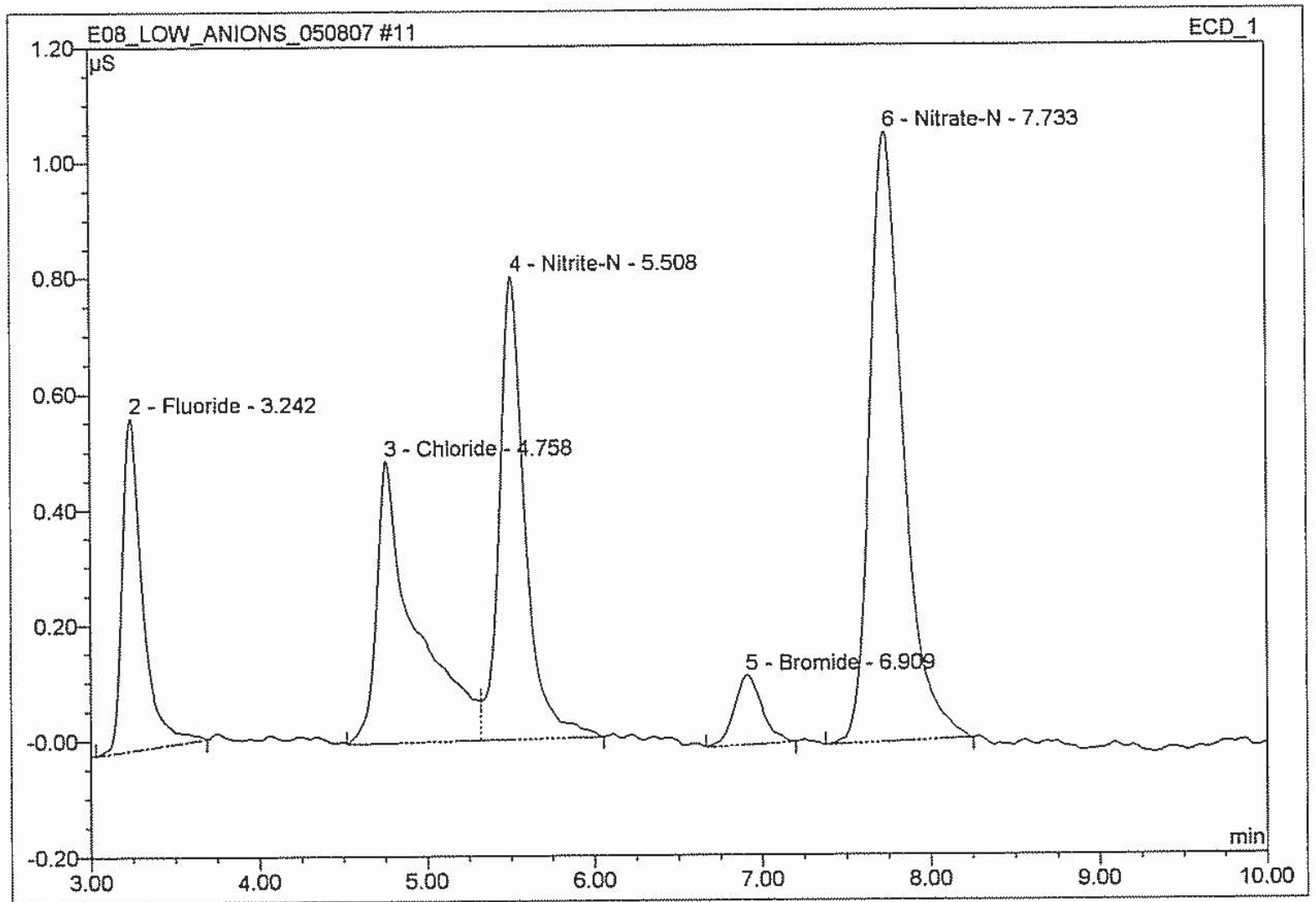

\begin{tabular}{|c|c|l|r|r|r|r|c|}
\hline No. & $\begin{array}{c}\text { Ret.Time } \\
\min \end{array}$ & \multicolumn{1}{|c|}{ Peak Name } & $\begin{array}{c}\text { Height } \\
\mu S\end{array}$ & $\begin{array}{c}\text { Area } \\
\mu S^{*} \text { min }\end{array}$ & $\begin{array}{c}\text { Rel.Area } \\
\%\end{array}$ & $\begin{array}{c}\text { Amount } \\
\text { mg/L }\end{array}$ & $\begin{array}{r}\text { Peak } \\
\text { Type }\end{array}$ \\
\hline 1 & 2.07 & n.a. & 0.134 & 0.057 & 0.08 & n.a. & BMB \\
\cline { 2 - 7 } 2 & 3.24 & Fluoride & 0.575 & 0.081 & 0.12 & 2.0705 & BMB \\
\hline 3 & 4.76 & Chloride & 0.488 & 0.132 & 0.19 & 2.5608 & BM \\
\hline 4 & 5.51 & Nitrite-N & 0.800 & 0.142 & 0.21 & 2.3205 & MB \\
\hline 5 & 6.91 & Bromide & 0.119 & 0.023 & 0.03 & 2.0426 & BMB \\
\hline 6 & 7.73 & Nitrate-N & 1.055 & 0.242 & 0.36 & 3.6371 & BMB \\
\hline 7 & 11.63 & n.a. & 195.771 & 67.048 & 99.00 & n.a. & BMB \\
\hline Total: & & & 198.942 & 67.725 & 100.00 & 12.631 & \\
\hline
\end{tabular}




\section{H7E040102 JV87L 1/10}

\section{QUAD}

Sample Name: H7E040102 JV87L 1/10

Vial Number: $\quad \mathbf{3 1 5}$

Sample Type: unknown

Control Program: ANIONS_AS14A

Quantif. Method: ANIONS

Recording Time: $\quad 5 / 8 / 200711: 03$

Run Time (min):

$\begin{array}{ll}\text { Injection Volume: } & \mathbf{5 0 . 0} \\ \text { Channel: } & \text { ECD_1 } \\ \text { Wavelength: } & \text { n.a. } \\ \text { Bandwidth: } & \text { n.a. } \\ \text { Dilution Factor: } & 10.0000 \\ \text { Sample Weight: } & 1.0000 \\ \text { Sample Amount: } & 1.0000\end{array}$

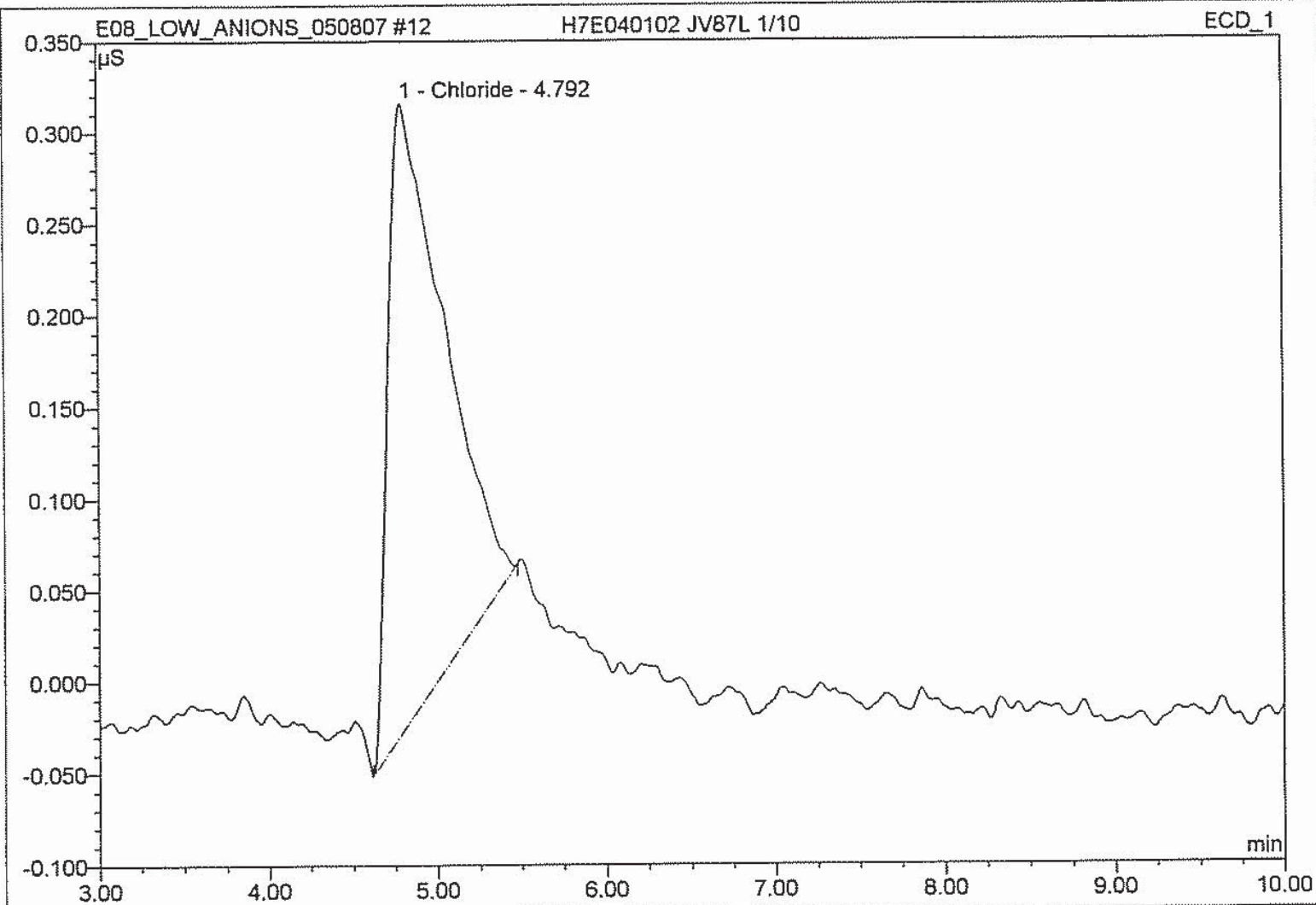

\begin{tabular}{|c|c|l|r|r|r|r|r|}
\hline No. & $\begin{array}{c}\text { Ret.Time } \\
\mathrm{min}\end{array}$ & \multicolumn{1}{|c|}{ Peak Name } & $\begin{array}{c}\text { Height } \\
\mu \mathrm{S}\end{array}$ & $\begin{array}{c}\text { Area } \\
\mu \mathrm{S}^{\star} \mathrm{min}\end{array}$ & $\begin{array}{c}\text { Rel.Area } \\
\%\end{array}$ & $\begin{array}{c}\text { Amount } \\
\mathrm{mg} / \mathrm{L}\end{array}$ & $\begin{array}{r}\text { Peak } \\
\text { Type }\end{array}$ \\
\hline 1 & 4.79 & Chloride & 0.344 & 0.132 & 0.09 & 1.8095 & BMB \\
\hline 2 & 11.47 & n.a. & 379.279 & 152.718 & 99.91 & n.a. & BMB \\
\hline Total: & & & 379.623 & 152.851 & 100.00 & 1.810 & \\
\hline
\end{tabular}

F. ND $\mathcal{L}$

Br- ND

default_letter/Integration

Chromeleon (c) Dionex 1996-2001

Version 6.50 SP10a Build 1065 


\section{H7E040102 JV87L MS 1/10 0.2 PPM F, BR. CL}

\section{QUAD}

\begin{tabular}{llll}
\hline Sample Name: & H7E040102 JV87L MS 1/10 0.2 PPM F, BR. Injection Volume: & $\mathbf{5 0 . 0}$ \\
Vial Number: & 316 & Channel: & ECD_1 \\
Sample Type: & unknown & Wavelength: & n.a. \\
Control Program: & ANIONS_AS14A & Bandwidth: & n.a. \\
Quantif. Method: & ANIONS & Dilution Factor: & 10.0000 \\
Recording Time: & $5 / 8 / 200711: 20$ & Sample Weight: & 1.0000 \\
Run Time (min): & $\mathbf{1 4 . 7 5}$ & Sample Amount: & 1.0000 \\
\hline
\end{tabular}

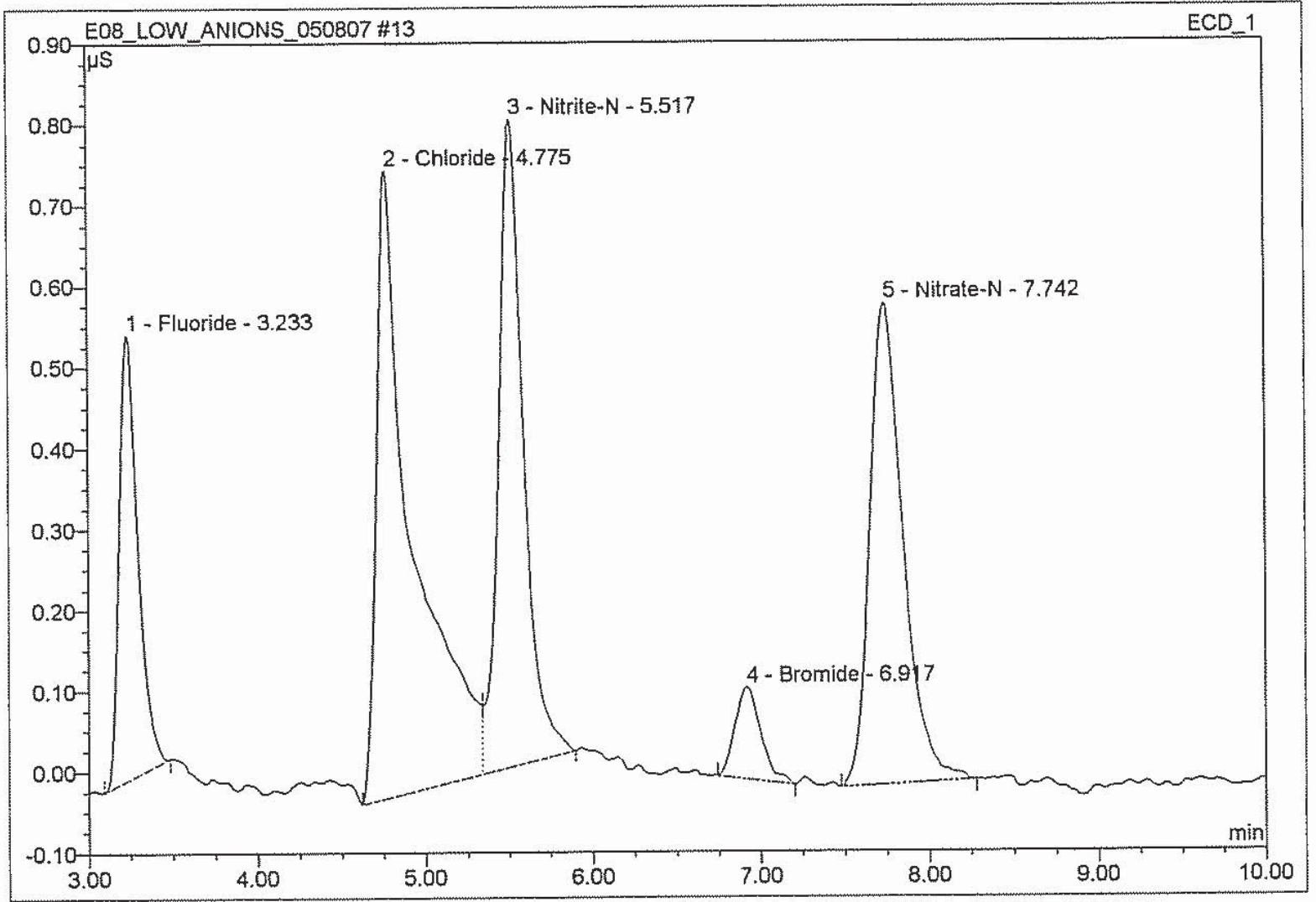

\begin{tabular}{|c|c|l|r|r|r|r|c|}
\hline No. & $\begin{array}{c}\text { Ret.Time } \\
\text { min }\end{array}$ & \multicolumn{1}{|c|}{ Peak Name } & $\begin{array}{c}\text { Height } \\
\mu S\end{array}$ & $\begin{array}{c}\text { Area } \\
\mu S^{*} \min \end{array}$ & $\begin{array}{c}\text { Rel.Area } \\
\%\end{array}$ & $\begin{array}{c}\text { Amount } \\
\mathrm{mg} / \mathrm{L}\end{array}$ & $\begin{array}{c}\text { Peak } \\
\text { Type }\end{array}$ \\
\hline 1 & 3.23 & Fluoride & 0.550 & 0.071 & 0.05 & 1.9831 & BMB \\
\hline 2 & 4.78 & Chloride & 0.775 & 0.195 & 0.13 & 4.0460 & $\mathrm{BM}$ \\
\hline 3 & 5.52 & Nitrite- $N$ & 0.800 & 0.139 & 0.09 & 2.3189 & MB \\
\hline 4 & 6.92 & Bromide & 0.113 & 0.020 & 0.01 & 1.9364 & BMB \\
\hline 5 & 7.74 & Nitrate- $\mathrm{N}$ & 0.594 & 0.137 & 0.09 & 2.0668 & BMB \\
\hline 6 & 11.47 & n.a. & 375.971 & 150.940 & 99.63 & n.a. & BMB \\
\hline Total: & & & 378.803 & 151.501 & 100.00 & 12.351 & \\
\hline
\end{tabular}




\section{H7E040102 JV87N 1/10}

\section{QUAD}

Sample Name: H7E040102 JV87N 1/10

Vial Number: $\quad 317$

Sample Type: unknown

Control Program: ANIONS_AS14A

Quantif. Method: ANIONS

Recording Time: $\quad$ 5/8/2007 11:37

Run Time (min):

\subsection{5}

$\begin{array}{ll}\text { Injection Volume: } & \mathbf{5 0 . 0} \\ \text { Channel: } & \text { ECD_1 } \\ \text { Wavelength: } & \text { n.a. } \\ \text { Bandwidth: } & \text { n.a. } \\ \text { Dilution Factor: } & 10.0000 \\ \text { Sample Weight: } & 1.0000 \\ \text { Sample Amount: } & \mathbf{1 . 0 0 0 0}\end{array}$

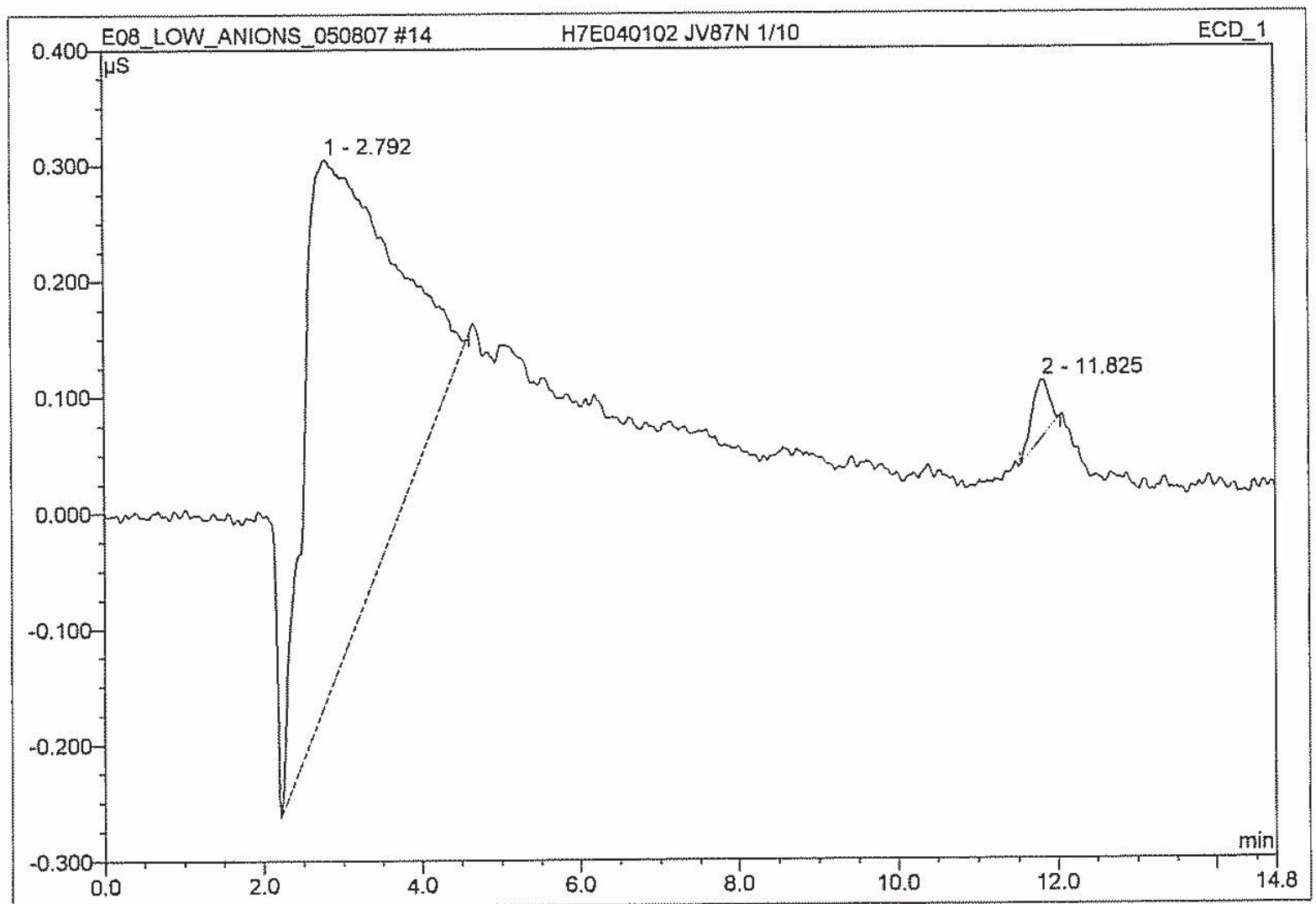

\begin{tabular}{|r|c|l|r|r|r|r|r|}
\hline No. & $\begin{array}{c}\text { Ret.Time } \\
\text { min }\end{array}$ & \multicolumn{1}{|c|}{ Peak Name } & $\begin{array}{c}\text { Height } \\
\mu S\end{array}$ & $\begin{array}{c}\text { Area } \\
\mu S^{*} \min \end{array}$ & $\begin{array}{c}\text { Rel.Area } \\
\%\end{array}$ & $\begin{array}{c}\text { Amount } \\
\text { mg/L }\end{array}$ & $\begin{array}{r}\text { Peak } \\
\text { Type }\end{array}$ \\
\hline 1 & 2.79 & n.a. & 0.467 & 0.570 & 97.84 & n.a. & BMB \\
\hline 2 & 11.83 & n.a. & 0.049 & 0.013 & 2.16 & n.a. & BMB \\
\hline Total: & & & 0.516 & 0.582 & 100.00 & 0.000 & \\
\hline
\end{tabular}

$$
\begin{aligned}
& F-N D \\
& C l-N D \\
& B r-N D
\end{aligned}
$$

default_letter/Integration 


\section{H7E040102 JV87N MS 1/10 0.2 PPM BR, F, CL}

\section{QUAD}

\begin{tabular}{llll}
\hline Sample Name: & H7E040102 JV87N MS 1/10 0.2 PPM BR, F, Injection Volume: & $\mathbf{5 0 . 0}$ \\
Vial Number: & $\mathbf{3 1 8}$ & Channel: & ECD_1 \\
Sample Type: & unknown & Wavelength: & n.a. \\
Control Program: & ANIONS_AS14A & Bandwidth: & n.a. \\
Quantif. Method: & ANIONS & Dilution Factor: & 10.0000 \\
Recording Time: & $\mathbf{5 / 8 / 2 0 0 7 ~ 1 1 : 5 5}$ & Sample Weight: & 1.0000 \\
Run Time (min): & $\mathbf{1 4 . 7 5}$ & Sample Amount: & $\mathbf{1 . 0 0 0 0}$
\end{tabular}

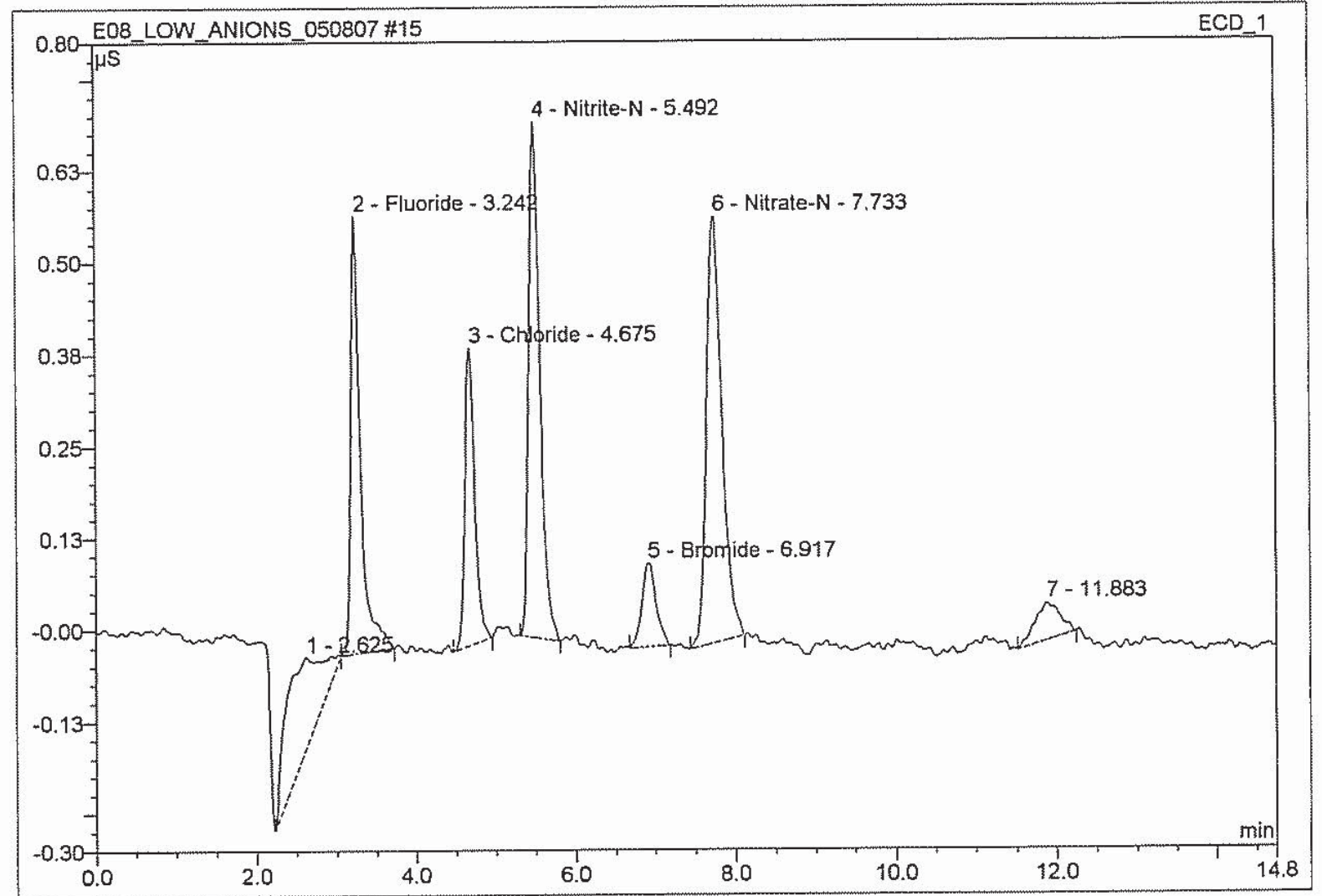

\begin{tabular}{|r|r|l|r|r|r|r|r|}
\hline No. & $\begin{array}{c}\text { Ret.Time } \\
\text { min }\end{array}$ & \multicolumn{1}{|c|}{ Peak Name } & $\begin{array}{c}\text { Height } \\
\mu S\end{array}$ & $\begin{array}{c}\text { Area } \\
\mu S^{*} \text { min }\end{array}$ & $\begin{array}{c}\text { Rel.Area } \\
\%\end{array}$ & $\begin{array}{c}\text { Amount } \\
\text { mg/L }\end{array}$ & $\begin{array}{r}\text { Peak } \\
\text { Type }\end{array}$ \\
\hline 1 & 2.63 & n.a. & 0.122 & 0.070 & 14.07 & n.a. & BMB \\
\cline { 2 - 7 } 2 & 3.24 & Fluoride & 0.595 & 0.085 & 16.97 & 2.1432 & bMB \\
\hline 3 & 4.68 & Chloride & 0.405 & 0.058 & 11.52 & 2.1293 & BMB \\
\hline 4 & 5.49 & Nitrite-N & 0.702 & 0.115 & 22.95 & 2.0393 & BMB \\
\hline 5 & 6.92 & Bromide & 0.114 & 0.023 & 4.68 & 1.9492 & BMB \\
\hline 6 & 7.73 & Nitrate-N & 0.581 & 0.131 & 26.18 & 2.0216 & BMB \\
\hline 7 & 11.88 & n.a. & 0.051 & 0.018 & 3.62 & n.a. & BMB \\
\hline Total: & & & 2.570 & 0.501 & 100.00 & 10.283 & \\
\hline
\end{tabular}




\begin{tabular}{|llll|}
\hline 16 H7E040102 JV87G MSD 1/10 0.2 PPM CL, BR, F & \\
QUAD & & \\
\hline Sample Name: & H7E040102 JV87G MSD 1/10 0.2 PPM CL, E Injection Volume: & 50.0 \\
Vial Number: & $\mathbf{3 1 8}$ & Channel: & ECD_1 \\
Sample Type: & unknown & Wavelength: & n.a. \\
Control Program: & ANIONS_AS14A & Bandwidth: & n.a. \\
Quantif. Method: & ANIONS & Dilution Factor: & 10.0000 \\
Recording Time: & $\mathbf{5 / 8 / 2 0 0 7} 12: 12$ & Sample Weight: & 1.0000 \\
Run Time (min): & $\mathbf{1 4 . 7 5}$ & Sample Amount: & 1.0000 \\
\hline
\end{tabular}

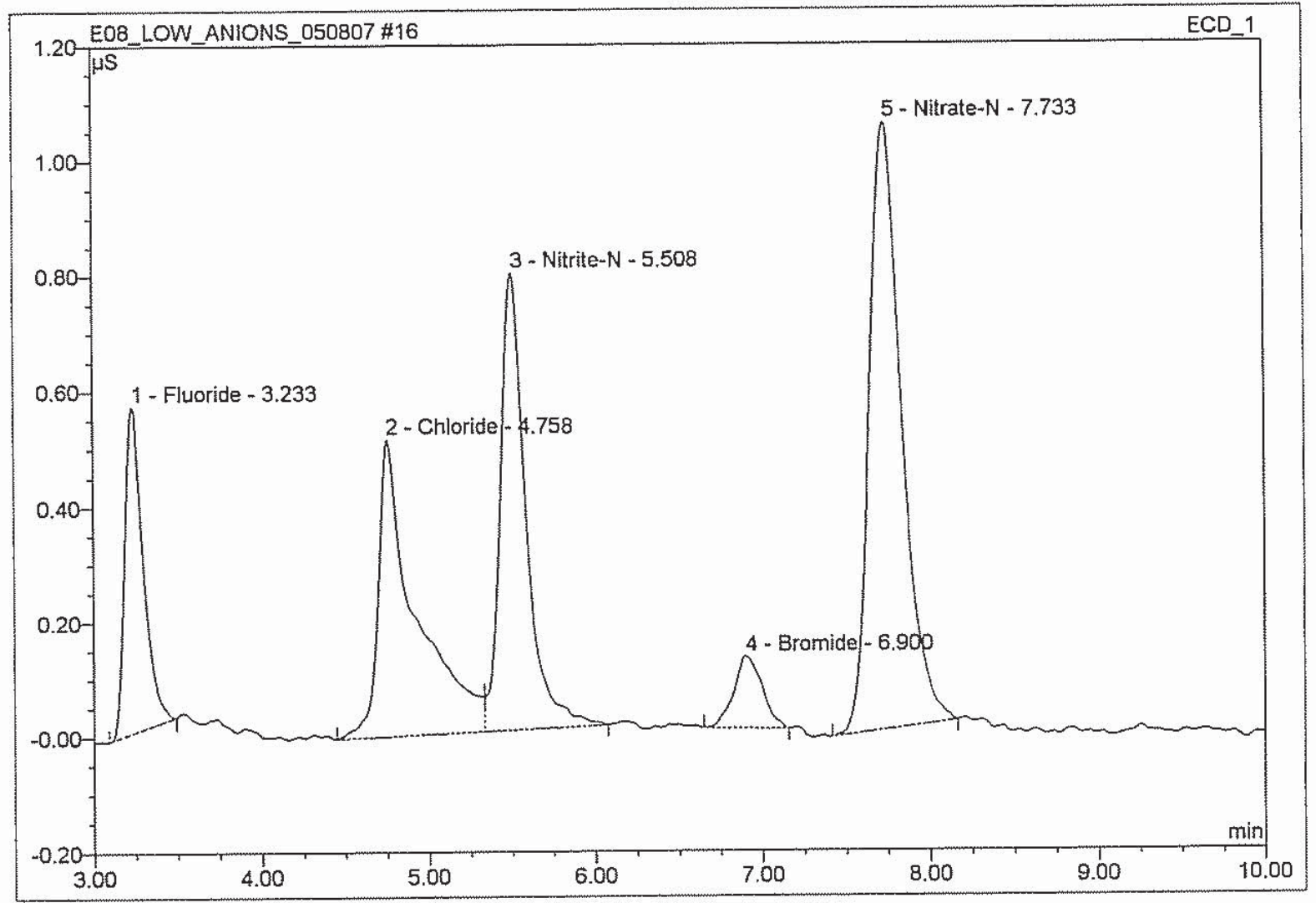

\begin{tabular}{|c|c|l|r|r|r|r|c|}
\hline No. & $\begin{array}{c}\text { Ret.Time } \\
\text { min }\end{array}$ & \multicolumn{1}{|c|}{ Peak Name } & $\begin{array}{c}\text { Height } \\
\mu S\end{array}$ & $\begin{array}{c}\text { Area } \\
\mu S^{*} \text { min }\end{array}$ & $\begin{array}{c}\text { Rel.Area } \\
\%\end{array}$ & $\begin{array}{c}\text { Amount } \\
\text { mg/L }\end{array}$ & $\begin{array}{c}\text { Peak } \\
\text { Type }\end{array}$ \\
\hline 1 & 3.23 & Fluoride & 0.566 & 0.072 & 0.11 & 2.0403 & BMB \\
\hline 2 & 4.76 & Chloride & 0.515 & 0.134 & 0.20 & 2.6993 & BM \\
\hline 3 & 5.51 & Nitrite-N & 0.792 & 0.141 & 0.21 & 2.2976 & MB \\
\hline 4 & 6.90 & Bromide & 0.125 & 0.025 & 0.04 & 2.1396 & BMB \\
\hline 5 & 7.73 & Nitrate-N & 1.054 & 0.236 & 0.35 & 3.6333 & BMB \\
\hline 6 & 11.62 & n.a. & 196.506 & 67.389 & 99.10 & n.a. & BMB \\
\hline Total: & & & 199.558 & 67.998 & 100.00 & 12.810 & \\
\hline
\end{tabular}




\section{CCV ICWS-9529}

\section{QUAD}

Sample Name: $\quad$ CCV ICWS-9529

Vial Number: $\quad \mathbf{3 2 0}$

Sample Type: $\quad$ unknown

Control Program: ANIONS_AS14A

Quantif. Method: ANIONS

Recording Time: $\quad$ 5/8/2007 12:29

Run Time (min): $\quad 14.75$

$\begin{array}{ll}\text { Injection Volume: } & \mathbf{5 0 . 0} \\ \text { Channel: } & \text { ECD_1 } \\ \text { Wavelength: } & \text { n.a. } \\ \text { Bandwidth: } & \text { n.a. } \\ \text { Dilution Factor: } & 1.0000 \\ \text { Sample Weight: } & 1.0000 \\ \text { Sample Amount: } & 1.0000\end{array}$

$\begin{array}{ll}\text { Injection Volume: } & 50.0 \\ \text { Channel: } & \text { ECD_1 }\end{array}$

Wavelength: $\quad$ n.a.

Dilution Factor: $\quad \mathbf{1 . 0 0 0 0}$

Sample Weight: $\quad 1.0000$

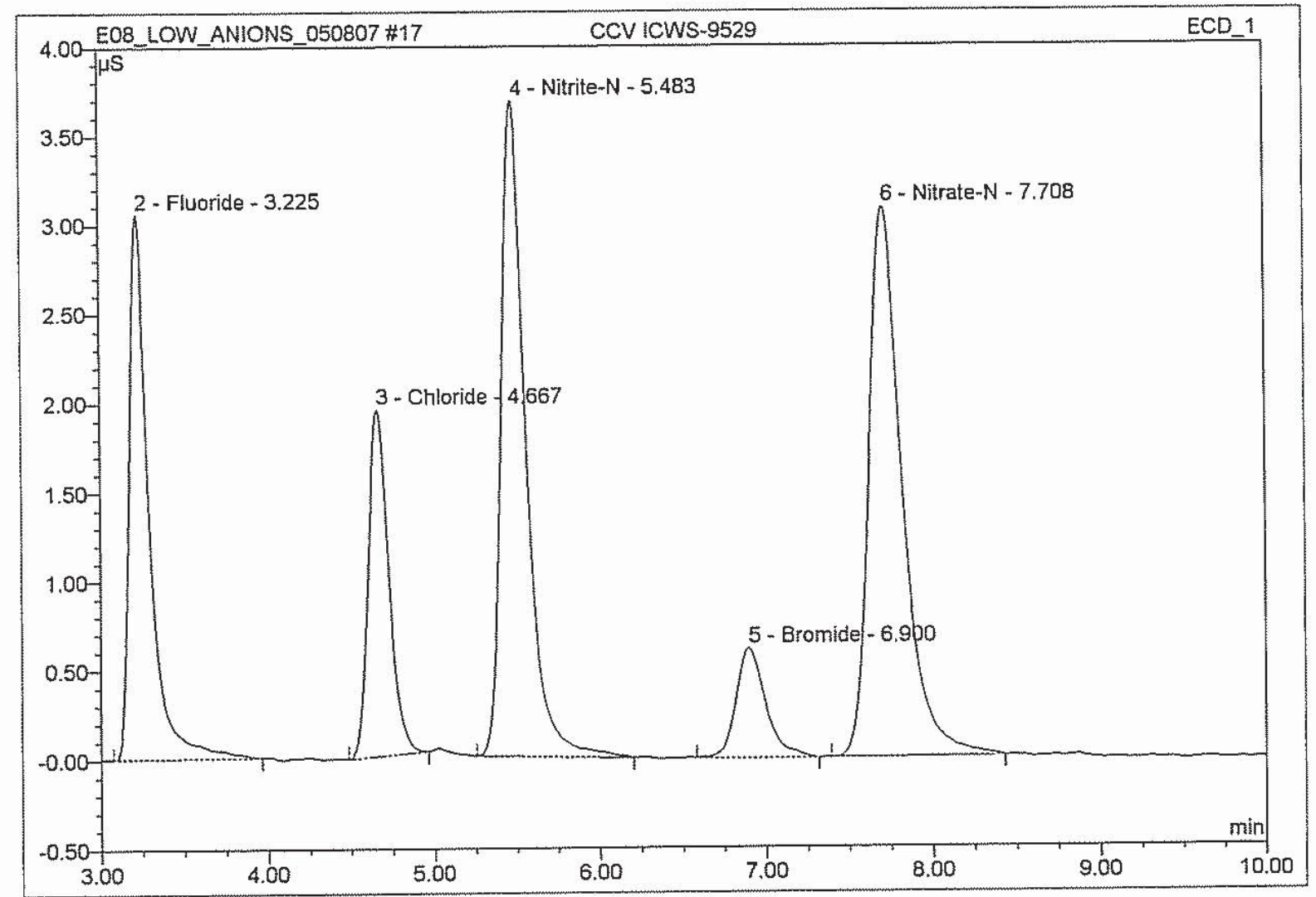

\begin{tabular}{|c|c|l|r|r|r|r|c|}
\hline No. & $\begin{array}{c}\text { Ret.Time } \\
\text { min }\end{array}$ & \multicolumn{1}{|c|}{ Peak Name } & $\begin{array}{c}\text { Height } \\
\mu S\end{array}$ & $\begin{array}{c}\text { Area } \\
\mu S^{\star} \min \end{array}$ & $\begin{array}{c}\text { Rel.Area } \\
\%\end{array}$ & $\begin{array}{c}\text { Amount } \\
\text { mg/L }\end{array}$ & $\begin{array}{c}\text { Peak } \\
\text { Type }\end{array}$ \\
\hline 1 & 2.21 & n.a. & 0.076 & 0.011 & 0.49 & n.a. & BMB \\
\hline 2 & 3.23 & Fluoride & 3.057 & 0.420 & 19.28 & 1.0452 & BMB \\
\hline 3 & 4.67 & Chloride & 1.946 & 0.276 & 12.69 & 0.9948 & BMB \\
\hline 4 & 5.48 & Nitrite-N & 3.680 & 0.631 & 28.98 & 1.0296 & BMB \\
\hline 5 & 6.90 & Bromide & 0.612 & 0.126 & 5.79 & 1.0345 & BMB \\
\hline 6 & 7.71 & Nitrate-N & 3.084 & 0.714 & 32.78 & 1.0250 & BMB \\
\hline Total: & & & 12.456 & 2.178 & 100.00 & 5.129 & \\
\hline
\end{tabular}




\section{$18 \mathrm{CCB}$}

\section{QUAD}

Sample Name: CCB

Vial Number: $\quad 321$

Sample Type: $\quad$ unknown

Control Program: ANIONS_AS14A

Quantif. Method: ANIONS

Recording Time: $\quad 5 / 8 / 2007$ 12:46

Run Time (min): $\quad 14.75$

$\begin{array}{ll}\text { Injection Volume: } & \mathbf{5 0 . 0} \\ \text { Channel: } & \text { ECD_1 } \\ \text { Wavelength: } & \text { n.a. } \\ \text { Bandwidth: } & \text { n.a. } \\ \text { Dilution Factor: } & 1.0000 \\ \text { Sample Weight: } & 1.0000 \\ \text { Sample Amount: } & 1.0000\end{array}$

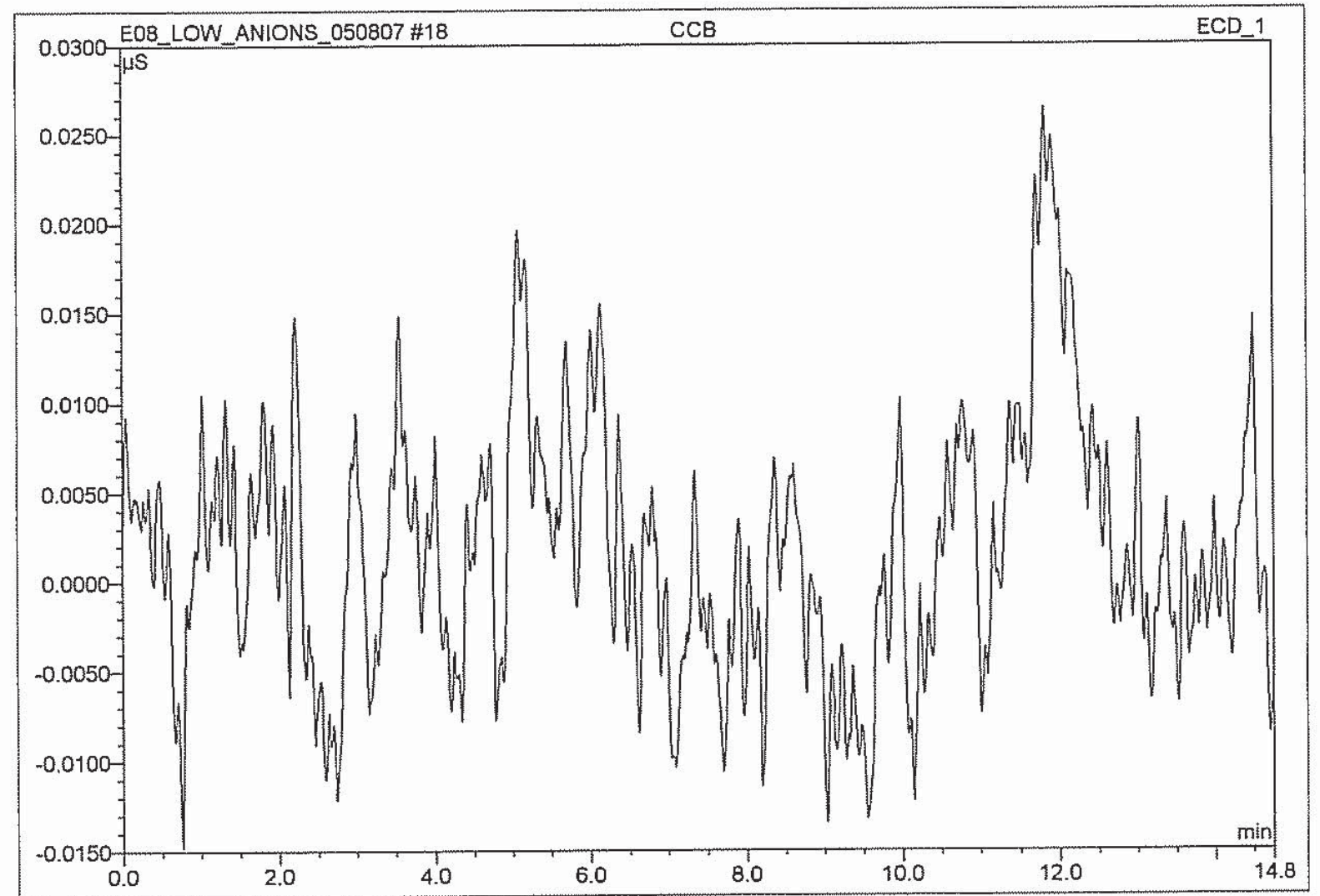

\begin{tabular}{|c|c|c|c|c|c|c|c|}
\hline No. & $\begin{array}{c}\text { Ret.Time } \\
\mathrm{min}\end{array}$ & Peak Name & $\begin{array}{c}\text { Height } \\
\mu \mathrm{S}\end{array}$ & $\begin{array}{c}\text { Area } \\
\mu \mathrm{S}^{\star} \mathrm{min}\end{array}$ & $\begin{array}{c}\text { Rel.Area } \\
\%\end{array}$ & $\begin{array}{c}\text { Amount } \\
\mathrm{mg} / \mathrm{L}\end{array}$ & $\begin{array}{c}\text { Peak } \\
\text { Type }\end{array}$ \\
\hline Total: & & & 0.000 & 0.000 & 0.00 & 0.000 & \\
\hline
\end{tabular}

(ND)

default_letter/Integration

Chromeleon (c) Dionex 1996-2001

Version 6.50 SP10a Build 1065 


\section{H7E040102 JV87E 1/10}

\section{QUAD}

Sample Name: H7E040102 JV87E 1/10

Vial Number: $\quad 358$

Sample Type:

Control Program:

Quantif. Method:

Recording Time:

Run Time (min): unknown

ANIONS_AS14A

ANIONS

5/8/2007 13:03

14.74

$\begin{array}{ll}\text { Injection Volume: } & \mathbf{5 0 . 0} \\ \text { Channel: } & \text { ECD_1 } \\ \text { Wavelength: } & \text { n.a. } \\ \text { Bandwidth: } & \text { n.a. } \\ \text { Dilution Factor: } & \mathbf{1 0 . 0 0 0 0} \\ \text { Sample Weight: } & \mathbf{1 . 0 0 0 0} \\ \text { Sample Amount: } & \mathbf{1 . 0 0 0 0}\end{array}$

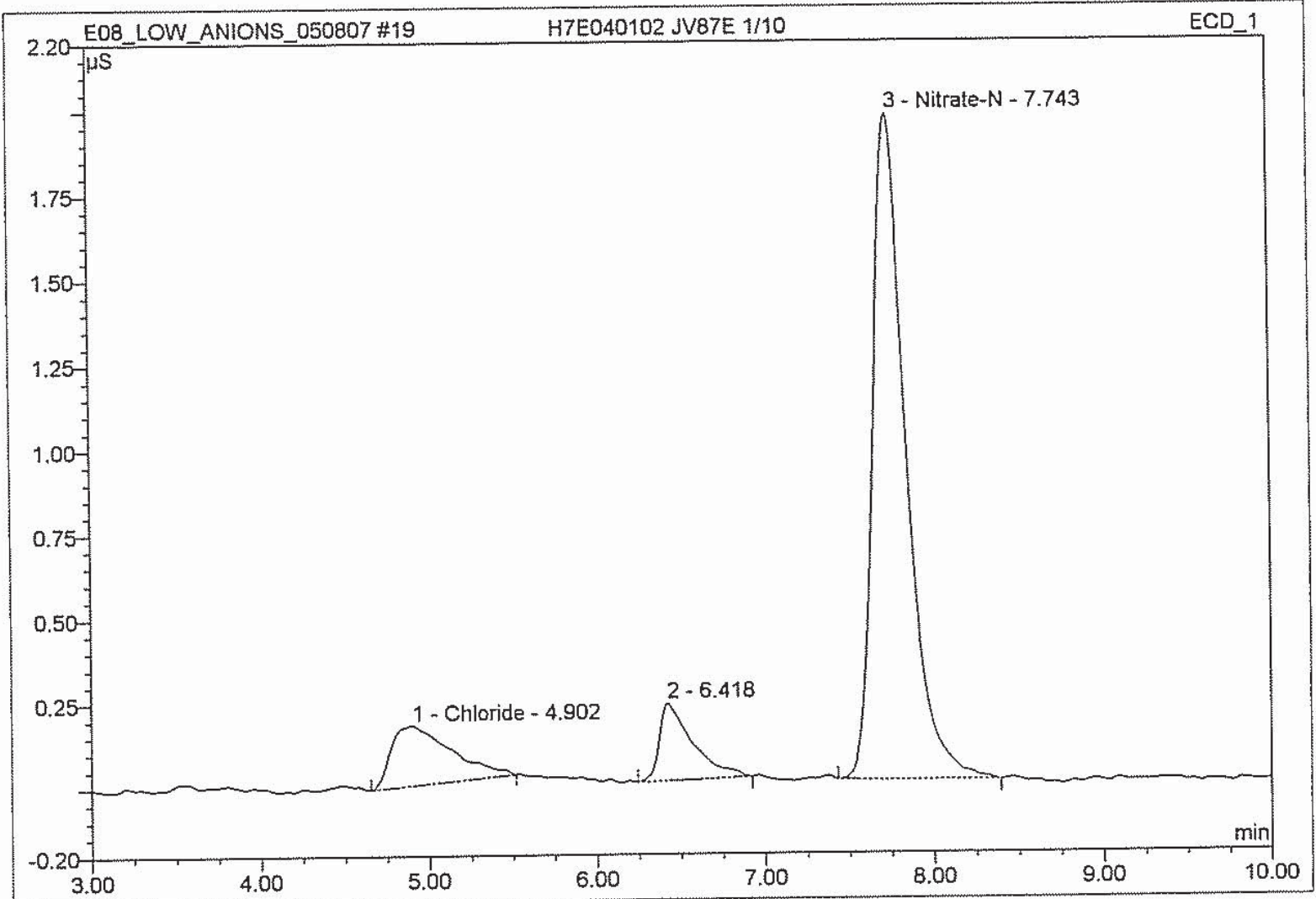

\begin{tabular}{|c|c|l|r|r|r|r|r|}
\hline No. & $\begin{array}{c}\text { Ret.Time } \\
\mathrm{min}\end{array}$ & \multicolumn{1}{|c|}{ Peak Name } & $\begin{array}{c}\text { Height } \\
\mu \mathrm{S}\end{array}$ & $\begin{array}{c}\text { Area } \\
\mu \mathrm{S}^{\star} \mathrm{min}\end{array}$ & $\begin{array}{c}\text { Rel.Area } \\
\%\end{array}$ & $\begin{array}{c}\text { Amount } \\
\mathrm{mg} / \mathrm{L}\end{array}$ & $\begin{array}{r}\text { Peak } \\
\text { Type }\end{array}$ \\
\hline 1 & 4.90 & Chloride & 0.177 & 0.073 & 0.12 & 0.9358 & BMB \\
\hline 2 & 6.42 & n.a. & 0.228 & 0.052 & 0.09 & n.a. & BMB \\
\hline 3 & 7.74 & Nitrate-N & 1.963 & 0.447 & 0.76 & 6.6551 & BMB \\
\hline 4 & 11.65 & n.a. & 173.723 & 58.567 & 99.03 & n.a. & BMB \\
\hline Total: & & & 176.091 & 59.140 & 100.00 & 7.591 & \\
\hline
\end{tabular}

$F-$ NQ

Br. ND 1

Chromeleon (c) Dionex 1996-2001

default_letter/Integration

Version 6.50 SP10a Build 1065 


\section{H7E040102 JV87J 1/10}

\section{QUAD}

Sample Name: H7E040102 JV87J 1/10

Vial Number: $\quad \mathbf{3 5 9}$

Sample Type:

Control Program:

Quantif. Method:

Recording Time:

Run Time (min): unknown

ANIONS_AS14A

ANIONS

5/8/2007 13:20

14.75
Injection Volume:

Channel:

Wavelength:

Bandwidth:

Dilution Factor:

Sample Weight:

Sample Amount:
50.0

ECD_1

n.a.

n.a.

10.0000

1.0000

1.0000

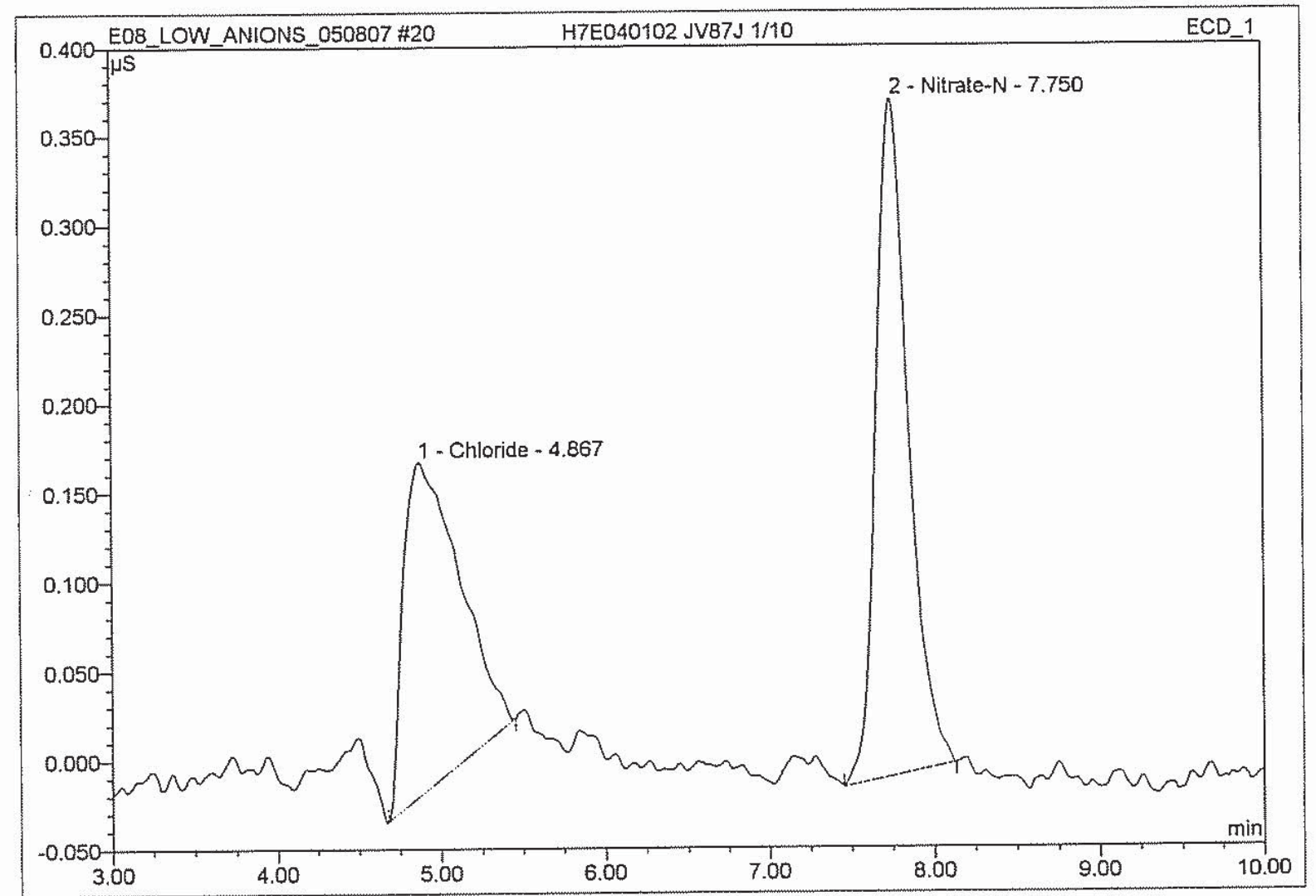

\begin{tabular}{|r|c|l|r|r|r|r|r|}
\hline No. & $\begin{array}{c}\text { Ret.Time } \\
\mathrm{min}\end{array}$ & \multicolumn{1}{|c|}{ Peak Name } & $\begin{array}{c}\text { Height } \\
\mu \mathrm{S}\end{array}$ & $\begin{array}{c}\text { Area } \\
\mu \mathrm{S}^{\star} \mathrm{min}\end{array}$ & $\begin{array}{c}\text { Rel.Area } \\
\%\end{array}$ & $\begin{array}{c}\text { Amount } \\
\mathrm{mg} / \mathrm{L}\end{array}$ & $\begin{array}{c}\text { Peak } \\
\text { Type }\end{array}$ \\
\hline 1 & 4.87 & Chloride & 0.187 & 0.074 & 0.12 & 0.9858 & BMB \\
\cline { 2 - 8 } 2 & 7.75 & Nitrate-N & 0.379 & 0.087 & 0.14 & 1.3249 & BMB \\
\hline 3 & 11.63 & n.a. & 182.308 & 61.865 & 99.74 & n.a. & BMB \\
\hline Total: & & & 182.874 & 62.026 & 100.00 & 2.311 & \\
\hline
\end{tabular}

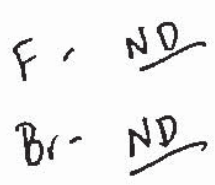

default_letter/lntegration 


\section{H7E040102 JV87H 1/10}

\section{QUAD}

Sample Name: H7E040102 JV87H 1/10

Vial Number: $\quad \mathbf{3 6 0}$

Sample Type:

Control Program:

Quantif. Method:

Recording Time:

Run Time ( $\mathrm{min}$ ): unknown

ANIONS_AS14A_CI2

ANIONS

5/8/2007 13:38

30.00

$\begin{array}{ll}\text { Injection Volume: } & \mathbf{5 0 . 0} \\ \text { Channel: } & \text { ECD_1 } \\ \text { Wavelength: } & \text { n.a. } \\ \text { Bandwidth: } & \text { n.a. } \\ \text { Dilution Factor: } & \mathbf{1 0 . 0 0 0 0} \\ \text { Sample Weight: } & \mathbf{1 . 0 0 0 0} \\ \text { Sample Amount: } & \mathbf{1 . 0 0 0 0}\end{array}$

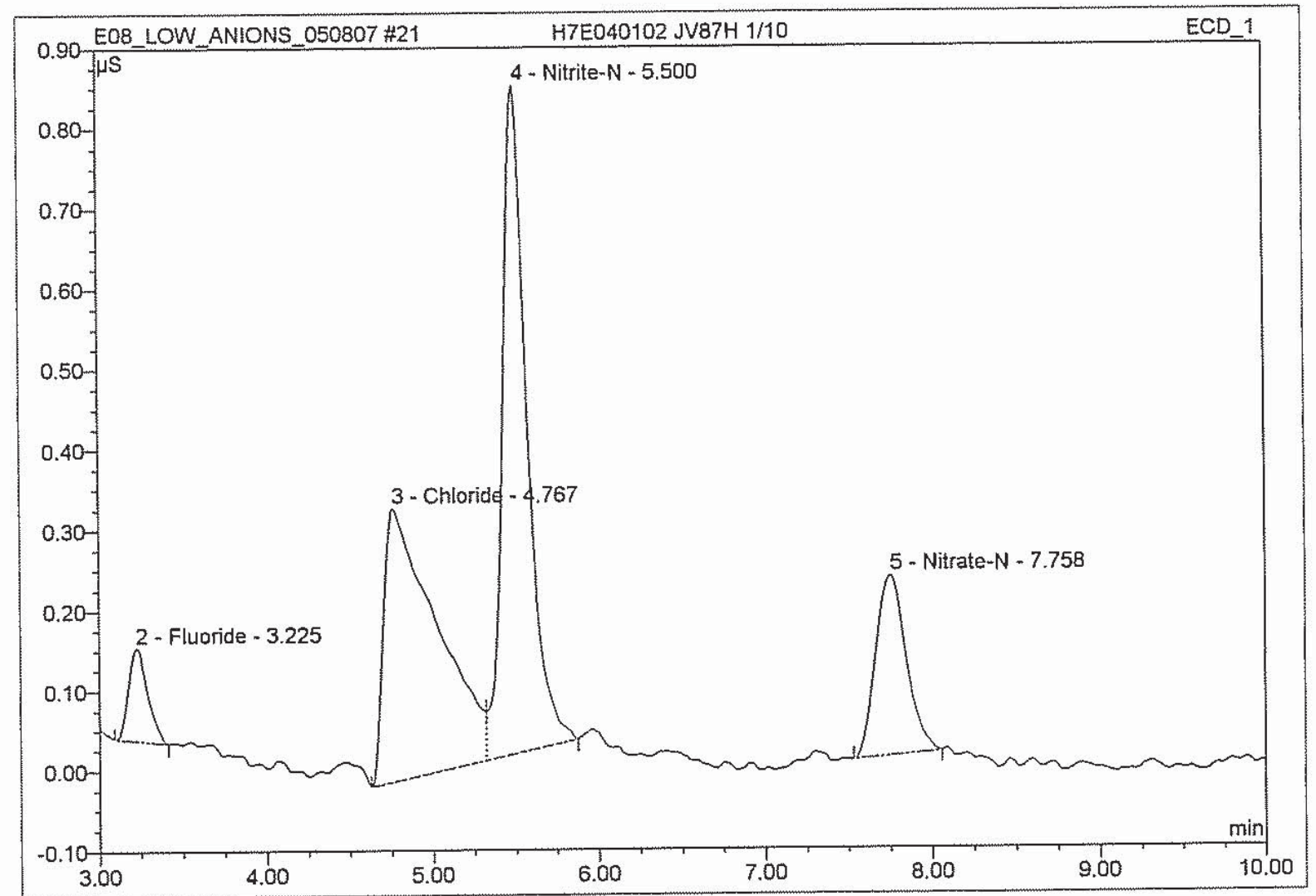

\begin{tabular}{|r|r|l|r|r|r|r|c|}
\hline No. & $\begin{array}{c}\text { Ret.Time } \\
\min \end{array}$ & \multicolumn{1}{|c|}{ Peak Name } & $\begin{array}{c}\text { Height } \\
\mu S\end{array}$ & $\begin{array}{c}\text { Area } \\
\mu S^{*} \text { min }\end{array}$ & $\begin{array}{c}\text { Rel.Area } \\
\%\end{array}$ & $\begin{array}{c}\text { Amount } \\
\text { mg/L }\end{array}$ & $\begin{array}{r}\text { Peak } \\
\text { Type }\end{array}$ \\
\hline 1 & 2.22 & n.a. & 1.792 & 0.286 & 0.45 & n.a. & BMB \\
\hline 2 & 3.23 & Fluoride & 0.116 & 0.015 & 0.02 & 0.4231 & BMB \\
\hline 3 & 4.77 & Chloride & 0.341 & 0.126 & 0.20 & 1.7947 & BM \\
\hline 4 & 5.50 & Nitrite-N & 0.832 & 0.143 & 0.23 & 2.4108 & MB \\
\hline 5 & 7.76 & Nitrate-N & 0.225 & 0.048 & 0.08 & 0.7876 & BMB \\
\hline 6 & 11.74 & n.a. & 33.057 & 10.445 & 16.50 & n.a. & BMB \\
\hline 7 & 21.48 & n.a. & 59.159 & 52.237 & 82.52 & n.a. & BMB \\
\hline Total: & & & 95.522 & 63.300 & 100.00 & 5.416 & \\
\hline
\end{tabular}




\begin{tabular}{|llll|}
\hline 22 H7E040102 JV87H MS 1/10 0.2 PPM CL, BR, F & \\
QUAD & & \\
\hline Sample Name: & H7E040102 JV87H MS 1/10 0.2 PPM CL, BF Injection Volume: & 50.0 \\
Vial Number: & 361 & Channel: & ECD_1 \\
Sample Type: & unknown & Wavelength: & n.a. \\
Control Program: & ANIONS_AS14A_Cl2 & Bandwidth: & n.a. \\
Quantif. Method: & ANIONS & Dilution Factor: & 10.0000 \\
Recording Time: & $5 / 8 / 200714: 10$ & Sample Weight: & 1.0000 \\
Run Time (min): & $\mathbf{3 0 . 0 0}$ & Sample Amount: & $\mathbf{1 . 0 0 0 0}$ \\
\hline
\end{tabular}

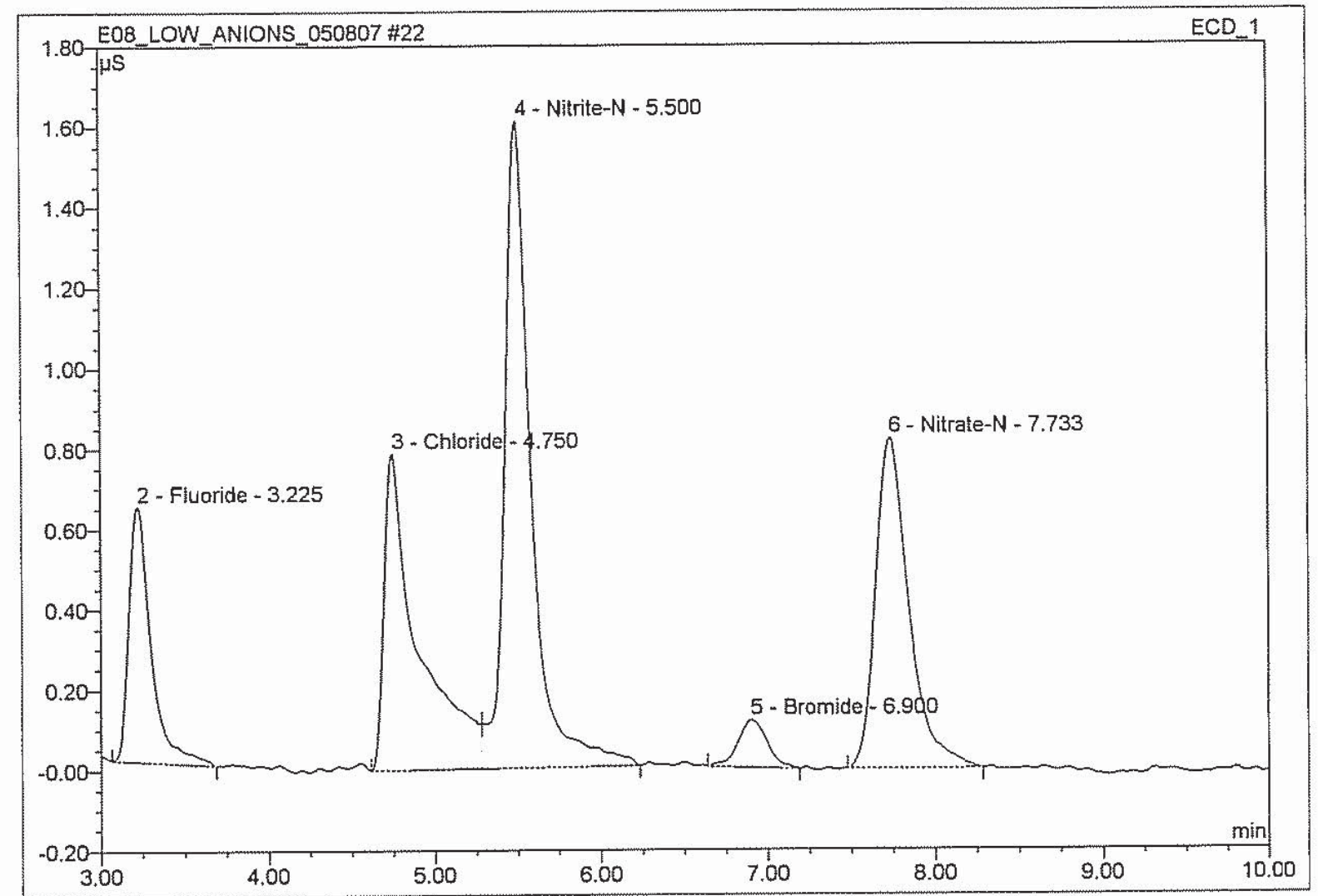

\begin{tabular}{|r|r|l|r|r|r|r|c|}
\hline No. & $\begin{array}{c}\text { Ret.Time } \\
\text { min }\end{array}$ & \multicolumn{1}{|c|}{ Peak Name } & $\begin{array}{c}\text { Height } \\
\mu \mathrm{S}\end{array}$ & $\begin{array}{c}\text { Area } \\
\mu \mathrm{S}^{*} \min \end{array}$ & $\begin{array}{c}\text { Rel.Area } \\
\%\end{array}$ & $\begin{array}{c}\text { Amount } \\
\mathrm{mg} / \mathrm{L}\end{array}$ & $\begin{array}{r}\text { Peak } \\
\text { Type }\end{array}$ \\
\hline 1 & 2.22 & n.a. & 1.790 & 0.281 & 0.44 & n.a. & BMB \\
\hline 2 & 3.23 & Fluoride & 0.635 & 0.097 & 0.15 & 2.2826 & BMB \\
\hline 3 & 4.75 & Chloride & 0.787 & 0.187 & 0.29 & 4.1073 & BM \\
\hline 4 & 5.50 & Nitrite-N & 1.607 & 0.298 & 0.47 & 4.6122 & MB \\
\hline 5 & 6.90 & Bromide & 0.118 & 0.024 & 0.04 & 2.0187 & BMB \\
\hline 6 & 7.73 & Nitrate-N & 0.821 & 0.190 & 0.30 & 2.8439 & BMB \\
\hline 7 & 11.74 & n.a. & 32.943 & 10.389 & 16.29 & n.a. & BMB \\
\hline 8 & 21.48 & n.a. & 59.244 & 52.317 & 82.02 & n.a. & BMB \\
\hline Total: & & & 97.945 & 63.783 & 100.00 & 15.865 & \\
\hline
\end{tabular}




\section{H7E040102 JV87H MSD 1/10 0.2 PPM CL, BR, F}

\section{QUAD}

\begin{tabular}{llcl}
\hline Sample Name: & H7E040102 JV87H MSD 1/10 0.2 PPM CL, Elnjection Volume: & $\mathbf{5 0 . 0}$ \\
Vial Number: & 362 & Channel: & ECD_1 \\
Sample Type: & unknown & Wavelength: & n.a. \\
Control Program: & ANIONS_AS14A_Cl2 & Bandwidth: & n.a. \\
Quantif. Method: & ANIONS & Dilution Factor: & 10.0000 \\
Recording Time: & $5 / 8 / 200714: 43$ & Sample Weight: & 1.0000 \\
Run Time (min): & $\mathbf{3 0 . 0 0}$ & Sample Amount: & $\mathbf{1 . 0 0 0 0}$ \\
\hline
\end{tabular}

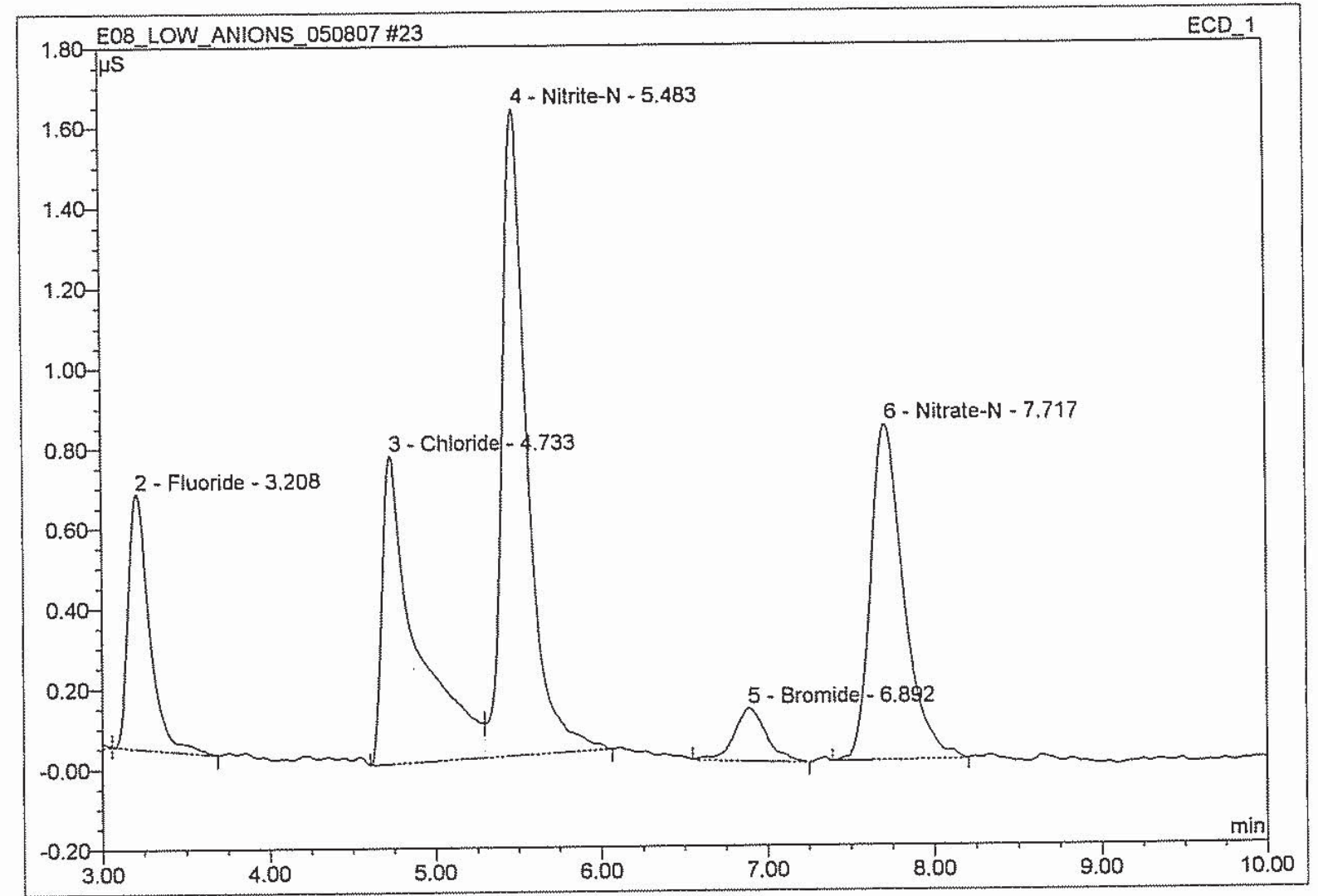

\begin{tabular}{|r|r|l|r|r|r|r|c|}
\hline No. & $\begin{array}{c}\text { Ret.Time } \\
\text { min }\end{array}$ & \multicolumn{1}{|c|}{ Peak Name } & $\begin{array}{c}\text { Height } \\
\mu S\end{array}$ & $\begin{array}{c}\text { Area } \\
\mu S^{\star} \text { min }\end{array}$ & $\begin{array}{c}\text { Rel.Area } \\
\%\end{array}$ & $\begin{array}{c}\text { Amount } \\
\text { mg/L }\end{array}$ & $\begin{array}{c}\text { Peak } \\
\text { Type }\end{array}$ \\
\hline 1 & 2.21 & n.a. & 1.797 & 0.298 & 0.47 & n.a. & BMB \\
\cline { 2 - 8 } 2 & 3.21 & Fluoride & 0.635 & 0.094 & 0.15 & 2.2858 & bMB \\
\hline 3 & 4.73 & Chloride & 0.769 & 0.185 & 0.29 & 4.0119 & BM \\
\hline 4 & 5.48 & Nitrite-N & 1.612 & 0.283 & 0.44 & 4.6263 & MB \\
\hline 5 & 6.89 & Bromide & 0.131 & 0.029 & 0.05 & 2.2420 & BMB \\
\hline 6 & 7.72 & Nitrate-N & 0.837 & 0.193 & 0.30 & 2.8969 & BMB \\
\hline 7 & 11.72 & n.a. & 32.958 & 10.408 & 16.25 & n.a. & BMB \\
\hline 8 & 21.43 & n.a. & 59.421 & 52.511 & 82.01 & n.a. & BMB \\
\hline 9 & 29.03 & n.a. & 0.133 & 0.028 & 0.04 & n.a. & BMB \\
\hline Total: & & & 98.293 & 64.029 & 100.00 & 16.063 & \\
\hline
\end{tabular}




\section{H7E040102 JV87M 1/10}

\section{QUAD}

\begin{tabular}{llll|}
\hline Sample Name: & H7E040102 JV87M 1/10 & Injection Volume: & $\mathbf{5 0 . 0}$ \\
Vial Number: & $\mathbf{3 6 3}$ & Channel: & ECD_1 \\
Sample Type: & unknown & Wavelength: & n.a. \\
Control Program: & ANIONS_AS14A_Cl2 & Bandwidth: & n.a. \\
Quantif. Method: & ANIONS & Dilution Factor: & $\mathbf{1 0 . 0 0 0 0}$ \\
Recording Time: & $\mathbf{5 / 8 / 2 0 0 7 ~ 1 5 : 1 5}$ & Sample Weight: & $\mathbf{1 . 0 0 0 0}$ \\
Run Time (min): & $\mathbf{3 0 . 0 0}$ & Sample Amount: & $\mathbf{1 . 0 0 0 0}$ \\
\hline
\end{tabular}

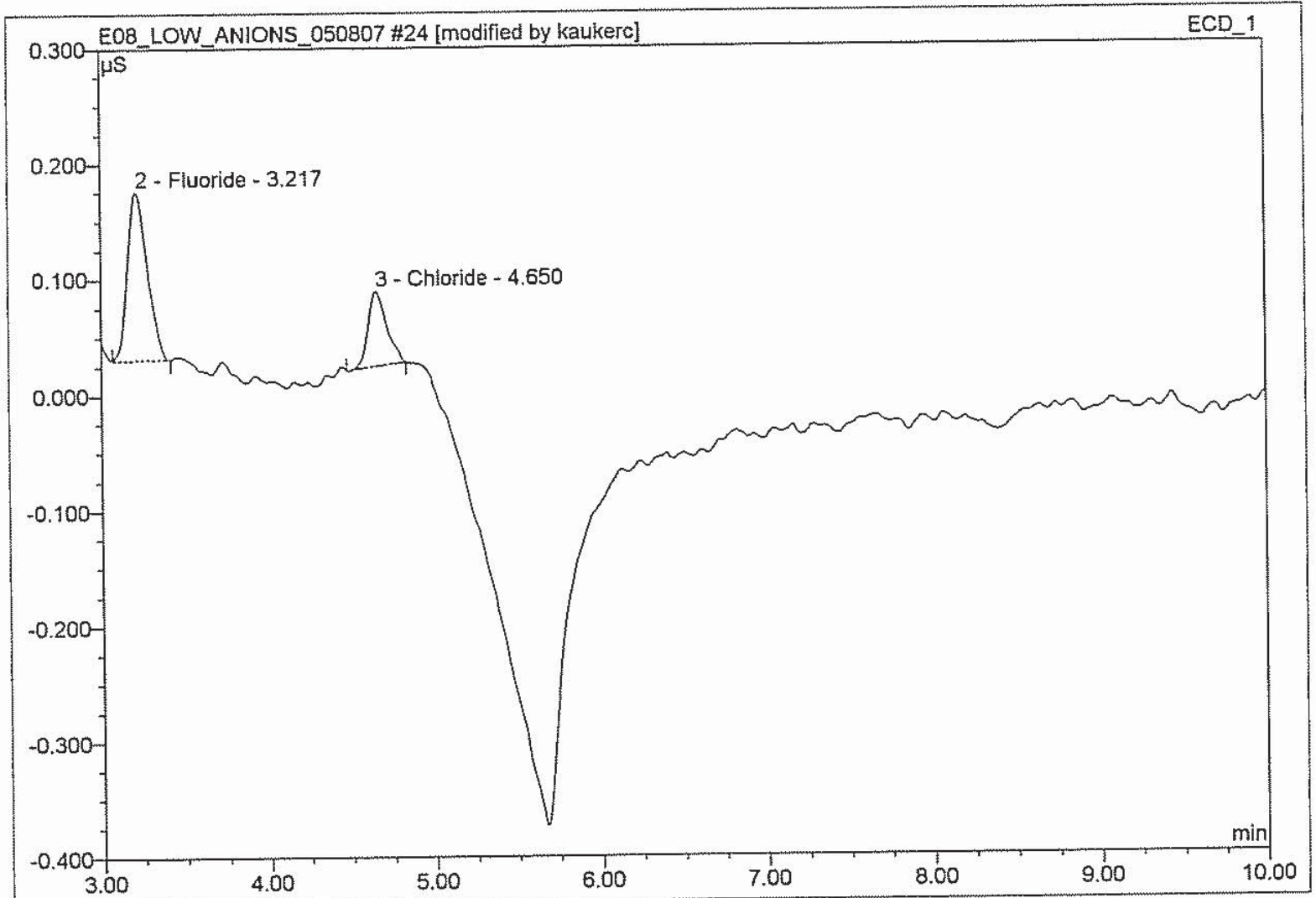

\begin{tabular}{|r|c|l|r|r|r|r|r|}
\hline No. & $\begin{array}{c}\text { Ret.Time } \\
\text { min }\end{array}$ & \multicolumn{1}{|c|}{ Peak Name } & $\begin{array}{c}\text { Height } \\
\mu S\end{array}$ & $\begin{array}{c}\text { Area } \\
\mu S^{*} \min \end{array}$ & $\begin{array}{c}\text { Rel.Area } \\
\%\end{array}$ & $\begin{array}{c}\text { Amount } \\
\text { mg/L }\end{array}$ & $\begin{array}{r}\text { Peak } \\
\text { Type }\end{array}$ \\
\hline 1 & 2.22 & n.a. & 1.845 & 0.309 & 0.59 & n.a. & BMB \\
\cline { 2 - 7 } & 3.22 & Fluoride & 0.145 & 0.020 & 0.04 & 0.5262 & BMB \\
\hline 3 & 4.65 & Chloride & 0.065 & 0.008 & 0.02 & 0.3411 & BMB \\
\hline 4 & 11.74 & n.a. & 0.373 & 0.124 & 0.24 & n.a. & BMB \\
\hline 5 & 21.48 & n.a. & 58.847 & 52.039 & 99.12 & n.a. & BMB \\
\hline Total: & & & 61.274 & 52.500 & 100.00 & 0.867 & \\
\hline
\end{tabular}

(B) Cuk 519107

default_letter/Integration

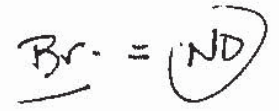

Chromeleon (c) Dionex 1996-2001 Version 6.50 SP10a Build 1065 


\section{H7E040102 JV87M 1/10}

\section{QUAD}

Sample Name: H7E040102 JV87M 1/10

Vial Number:

Sample Type:

Control Program:

Quantif. Method:

Recording Time:

Run Time ( $\mathrm{min}$ ):
363

unknown

ANIONS_AS14A_Cl2

ANIONS

5/8/2007 15:15

30.00

$\begin{array}{ll}\text { Injection Volume: } & \mathbf{5 0 . 0} \\ \text { Channel: } & \text { ECD_1 } \\ \text { Wavelength: } & \text { n.a. } \\ \text { Bandwidth: } & \text { n.a. } \\ \text { Dilution Factor: } & 10.0000 \\ \text { Sample Weight: } & 1.0000 \\ \text { Sample Amount: } & 1.0000\end{array}$

$E C D_{-1}$

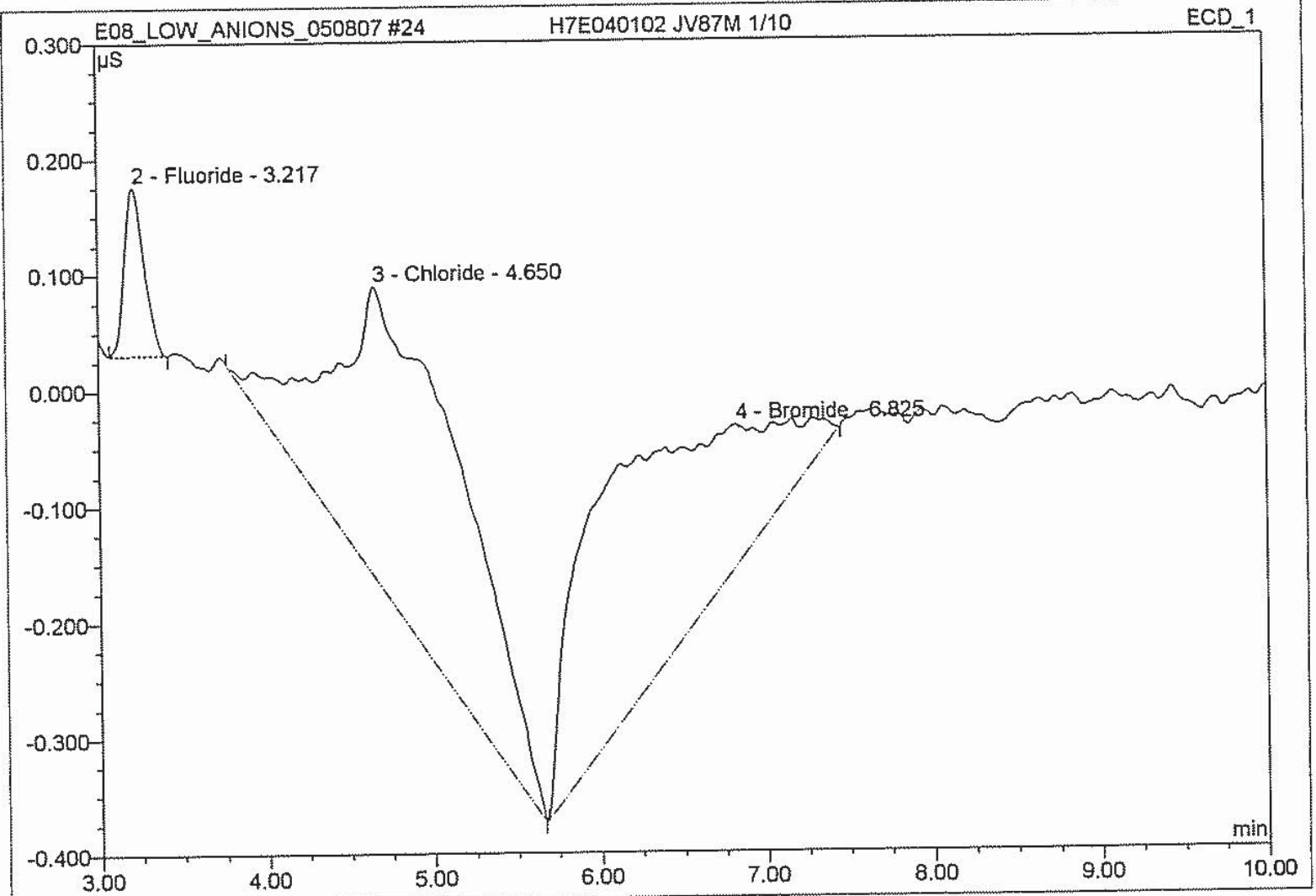

\begin{tabular}{|c|c|l|r|r|r|r|r|}
\hline No. & $\begin{array}{c}\text { Ret.Time } \\
\text { min }\end{array}$ & \multicolumn{1}{|c|}{ Peak Name } & $\begin{array}{c}\text { Height } \\
\mu S\end{array}$ & $\begin{array}{c}\text { Area } \\
\mu S^{*} \min \end{array}$ & $\begin{array}{c}\text { Rel.Area } \\
\%\end{array}$ & $\begin{array}{c}\text { Amount } \\
\text { mg/L }\end{array}$ & $\begin{array}{r}\text { Peak } \\
\text { Type }\end{array}$ \\
\hline 1 & 2.22 & n.a. & 1.845 & 0.309 & 0.58 & n.a. & BMB \\
\hline 2 & 3.22 & Fluoride & 0.145 & 0.020 & 0.04 & 0.5262 & BMB \\
\hline 3 & 4.65 & Chloride & 0.251 & 0.252 & 0.48 & 1.3202 & BMB \\
\hline 4 & 6.83 & Bromide & 0.121 & 0.234 & 0.44 & 2.0738 & BMB \\
\hline 5 & 11.74 & n.a. & 0.373 & 0.124 & 0.23 & n.a. & BMB \\
\hline 6 & 21.48 & n.a. & 58.847 & 52.039 & 98.23 & n.a. & BMB \\
\hline Total: & & & 61.581 & 52.978 & 100.00 & 3.920 & \\
\hline
\end{tabular}

original 


\begin{tabular}{|llll|}
\hline 25 H7E040102 JV87M MS 1/10 0.2 PPM CL, F, BR & \\
QUAD & & \\
\hline Sample Name: & H7E040102 JV87M MS 1/10 0.2 PPM CL, F, Injection Volume: & $\mathbf{5 0 . 0}$ \\
Vial Number: & 364 & Channel: & ECD_1 \\
Sample Type: & unknown & Wavelength: & n.a. \\
Control Program: & ANIONS_AS14A_Cl2 & Bandwidth: & n.a. \\
Quantif. Method: & ANIONS & Dilution Factor: & 10.0000 \\
Recording Time: & $\mathbf{5 / 8 / 2 0 0 7 ~ 1 5 : 4 7}$ & Sample Weight: & $\mathbf{1 . 0 0 0 0}$ \\
Run Time (min): & $\mathbf{3 0 . 0 0}$ & Sample Amount: & $\mathbf{1 . 0 0 0 0}$ \\
\hline
\end{tabular}

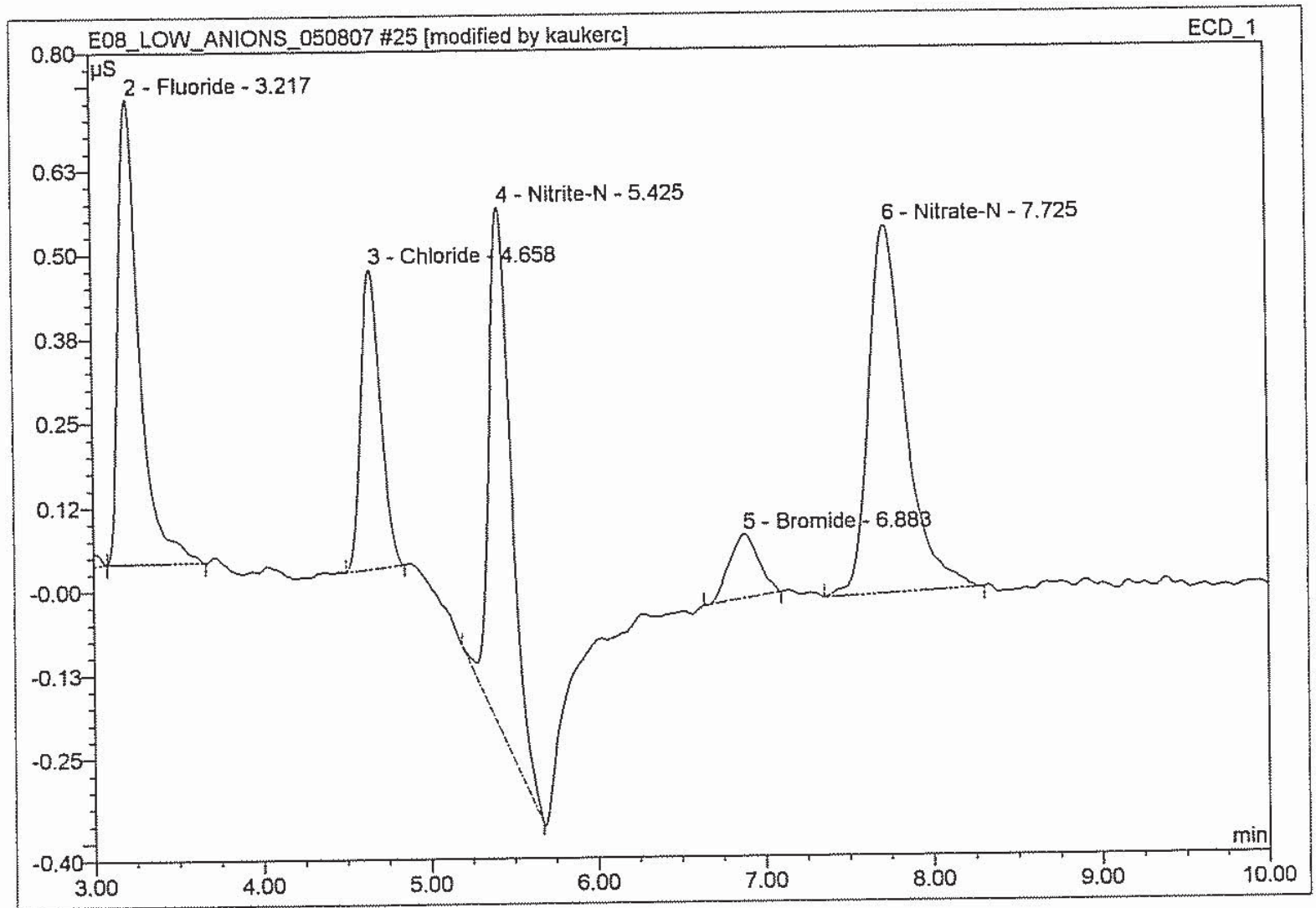

\begin{tabular}{|c|c|c|c|c|c|c|c|}
\hline No. & $\begin{array}{c}\text { Ret.Time } \\
\text { min }\end{array}$ & Peak Name & $\begin{array}{c}\text { Height } \\
\mu S\end{array}$ & $\begin{array}{c}\text { Area } \\
\mu S^{*} \min \end{array}$ & $\begin{array}{c}\text { Rel.Area } \\
\% \\
\end{array}$ & $\begin{array}{c}\text { Amount } \\
\mathrm{mg} / \mathrm{L}\end{array}$ & $\begin{array}{l}\text { Peak } \\
\text { Type }\end{array}$ \\
\hline 1 & 2.22 & n.a. & 1.854 & 0.341 & 0.64 & n.a. & $\mathrm{BMb}^{*}$ \\
\hline 2 & 3.22 & Fluoride & 0.689 & 0.100 & 0.19 & 2.4771 & $\mathrm{bMB}$ \\
\hline 3 & 4.66 & Chloride & 0.444 & 0.060 & 0.11 & 2.3314 & $\mathrm{BMB}$ \\
\hline 4 & 5.43 & Nitrite-N & 0.778 & 0.117 & 0.22 & 2.2570 & BMB \\
\hline 5 & 6.88 & Bromide & 0.095 & 0.019 & 0.04 & 1.6333 & $\mathrm{BMB}^{*}$ \\
\hline 6 & 7.73 & Nitrate-N & 0.545 & 0.139 & 0.26 & 1.8987 & $\mathrm{BMB}^{*}$ \\
\hline 7 & 11.74 & n.a. & 0.372 & 0.130 & 0.24 & n.a. & $\mathrm{BMB}$ \\
\hline 8 & 21.48 & n.a. & 58.878 & 52.186 & 98.29 & n.a. & $\mathrm{BMB}$ \\
\hline Total: & & & 63.656 & 53.093 & 100.00 & 10.598 & \\
\hline
\end{tabular}




\section{H7E040102 JV87M MS 1/10 0.2 PPM CL, F, BR}

\section{QUAD}

\begin{tabular}{llll}
\hline Sample Name: & H7E040102 JV87M MS 1/10 0.2 PPM CL, F, Injection Volume: & $\mathbf{5 0 . 0}$ \\
Vial Number: & $\mathbf{3 6 4}$ & Channel: & ECD_1 \\
Sample Type: & unknown & Wavelength: & n.a. \\
Control Program: & ANIONS_AS14A_CI2 & Bandwidth: & n.a. \\
Quantif. Method: & ANIONS & Dilution Factor: & 10.0000 \\
Recording Time: & $\mathbf{5 / 8 / 2 0 0 7 ~ 1 5 : 4 7}$ & Sample Weight: & 1.0000 \\
Run Time (min): & $\mathbf{3 0 . 0 0}$ & Sample Amount: & $\mathbf{1 . 0 0 0 0}$ \\
\hline
\end{tabular}

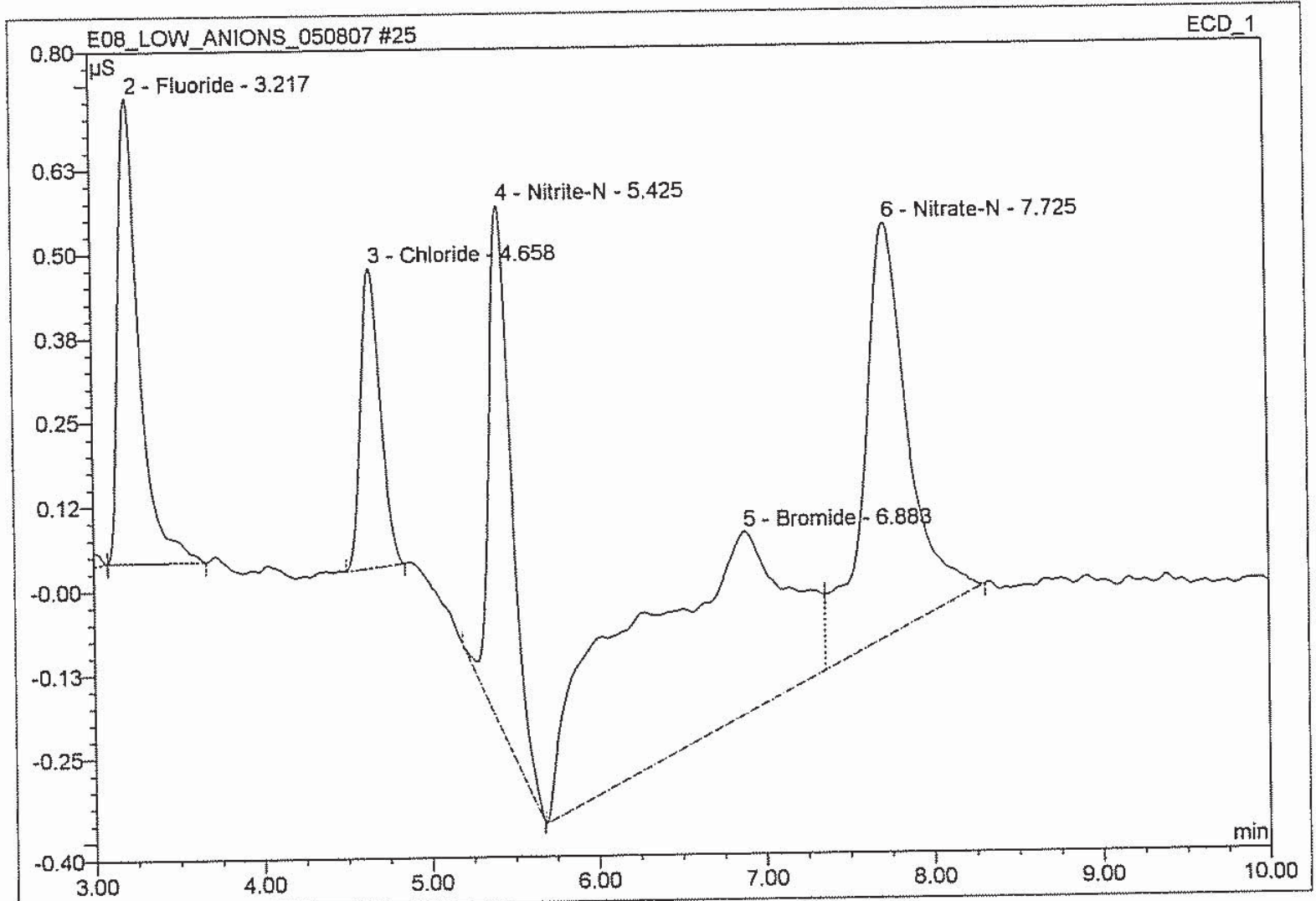

\begin{tabular}{|c|c|l|r|r|r|r|c|}
\hline No. & $\begin{array}{c}\text { Ret.Time } \\
\text { min }\end{array}$ & \multicolumn{1}{|c|}{ Peak Name } & $\begin{array}{c}\text { Height } \\
\mu S\end{array}$ & $\begin{array}{c}\text { Area } \\
\mu S^{\star} \text { min }\end{array}$ & $\begin{array}{r}\text { Rel.Area } \\
\%\end{array}$ & $\begin{array}{c}\text { Amount } \\
\text { mg/L }\end{array}$ & $\begin{array}{r}\text { Peak } \\
\text { Type }\end{array}$ \\
\hline 1 & 2.22 & n.a. & 1.854 & 0.341 & 0.64 & n.a. & BMB \\
\hline 2 & 3.22 & Fluoride & 0.689 & 0.100 & 0.19 & 2.4771 & bMB \\
\hline 3 & 4.66 & Chloride & 0.444 & 0.060 & 0.11 & 2.3314 & BMB \\
\hline 4 & 5.43 & Nitrite-N & 0.778 & 0.117 & 0.22 & 2.2570 & BMB \\
\hline 5 & 6.88 & Bromide & 0.270 & 0.326 & 0.61 & 4.6164 & BM \\
\hline 6 & 7.73 & Nitrate-N & 0.614 & 0.192 & 0.36 & 2.1361 & MB \\
\hline 7 & 11.74 & n.a. & 0.372 & 0.130 & 0.24 & n.a. & BMB \\
\hline 8 & 21.48 & n.a. & 58.878 & 52.186 & 97.63 & n.a. & BMB \\
\hline Total: & & & 63.900 & 53.453 & 100.00 & 13.818 & \\
\hline
\end{tabular}




\section{H7E040102 JV87F 1/10}

\section{QUAD}

\begin{tabular}{|llll|}
\hline Sample Name: & H7E040102 JV87F 1/10 & Injection Volume: & $\mathbf{5 0 . 0}$ \\
Vial Number: & 365 & Channel: & ECD_1 \\
Sample Type: & unknown & Wavelength: & n.a. \\
Control Program: & ANIONS_AS14A_Cl2 & Bandwidth: & n.a. \\
Quantif. Method: & ANIONS & Dilution Factor: & $\mathbf{1 0 . 0 0 0 0}$ \\
Recording Time: & $\mathbf{5 / 8 / 2 0 0 7 ~ 1 6 : 2 0}$ & Sample Weight: & 1.0000 \\
Run Time (min): & $\mathbf{3 0 . 0 0}$ & Sample Amount: & $\mathbf{1 . 0 0 0 0}$ \\
\hline
\end{tabular}

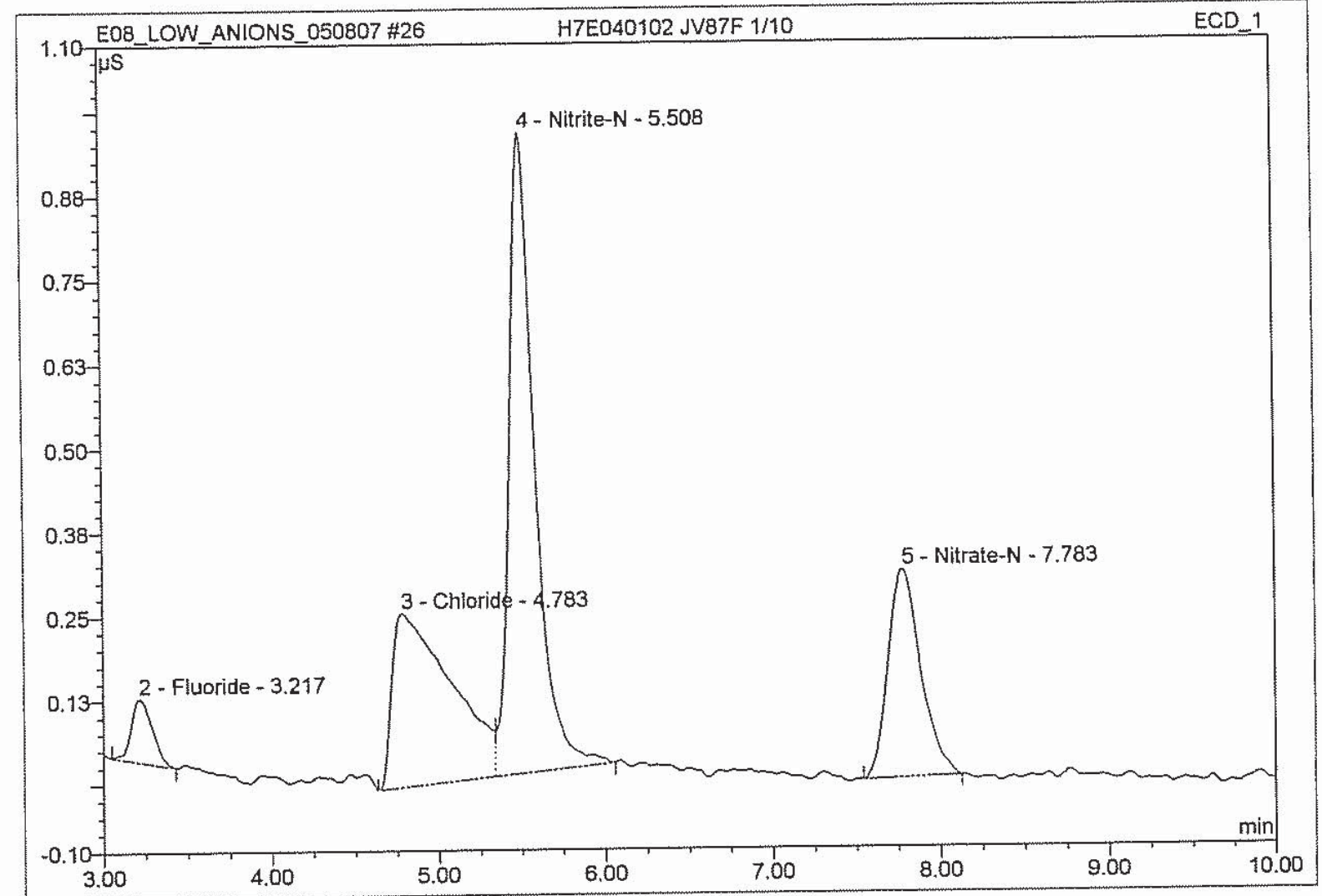

\begin{tabular}{|r|r|l|r|r|r|r|c|}
\hline No. & $\begin{array}{c}\text { Ret.Time } \\
\min \end{array}$ & \multicolumn{1}{|c|}{ Peak Name } & $\begin{array}{c}\text { Height } \\
\mu S\end{array}$ & $\begin{array}{c}\text { Area } \\
\mu S^{*} \min \end{array}$ & $\begin{array}{r}\text { Rel.Area } \\
\%\end{array}$ & $\begin{array}{c}\text { Amount } \\
\text { mg/L }\end{array}$ & $\begin{array}{r}\text { Peak } \\
\text { Type }\end{array}$ \\
\hline 1 & 2.22 & n.a. & 1.620 & 0.253 & 0.37 & n.a. & BMB \\
\hline 2 & 3.22 & Fluoride & 0.094 & 0.014 & 0.02 & 0.3420 & BMB \\
\hline 3 & 4.78 & Chloride & 0.258 & 0.106 & 0.16 & 1.3612 & BM \\
\hline 4 & 5.51 & Nitrite-N & 0.952 & 0.169 & 0.25 & 2.7554 & MB \\
\hline 5 & 7.78 & Nitrate-N & 0.309 & 0.070 & 0.10 & 1.0815 & BMB \\
\hline 6 & 11.72 & n.a. & 46.693 & 14.705 & 21.66 & n.a. & BMB \\
\hline 7 & 14.23 & n.a. & 0.153 & 0.029 & 0.04 & n.a. & BMB \\
\hline 8 & 21.49 & n.a. & 59.176 & 52.535 & 77.39 & n.a. & BMB \\
\hline Total: & & & 109.256 & 67.881 & 100.00 & 5.540 & \\
\hline
\end{tabular}




\section{H7E040102 JV87K 1/10}

QUAD

\begin{tabular}{|llll|}
\hline Sample Name: & H7E040102 JV87K 1/10 & Injection Volume: & $\mathbf{5 0 . 0}$ \\
Vial Number: & 366 & Channel: & ECD_1 \\
Sample Type: & unknown & Wavelength: & n.a. \\
Control Program: & ANIONS_AS14A_CI2 & Bandwidth: & n.a. \\
Quantif. Method: & ANIONS & Dilution Factor: & $\mathbf{1 0 . 0 0 0 0}$ \\
Recording Time: & $\mathbf{5 / 8 / 2 0 0 7 ~ 1 6 : 5 2}$ & Sample Weight: & $\mathbf{1 . 0 0 0 0}$ \\
Run Time (min): & $\mathbf{3 0 . 0 0}$ & Sample Amount: & $\mathbf{1 . 0 0 0 0}$ \\
\hline
\end{tabular}

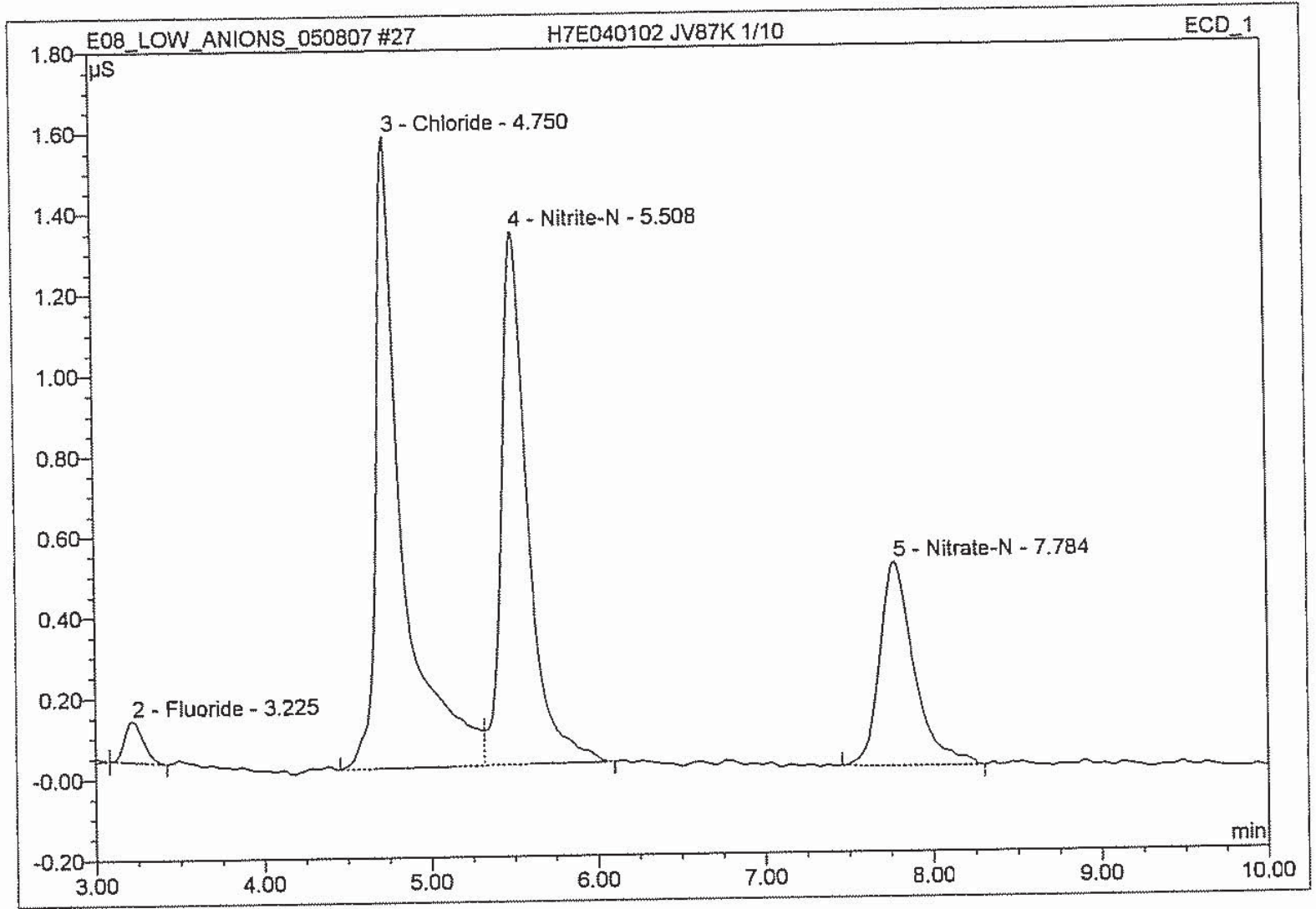

\begin{tabular}{|r|r|l|r|r|r|r|r|}
\hline No. & $\begin{array}{c}\text { Ret.Time } \\
\text { min }\end{array}$ & \multicolumn{1}{|c|}{ Peak Name } & $\begin{array}{c}\text { Height } \\
\mu S\end{array}$ & $\begin{array}{c}\text { Area } \\
\mu S^{*} \text { min }\end{array}$ & $\begin{array}{r}\text { Rel.Area } \\
\%\end{array}$ & $\begin{array}{c}\text { Amount } \\
\text { mg/L }\end{array}$ & $\begin{array}{r}\text { Peak } \\
\text { Type }\end{array}$ \\
\hline 1 & 2.22 & n.a. & 1.568 & 0.262 & 0.37 & n.a. & BMB \\
\cline { 2 - 8 } & 3.23 & Fluoride & 0.101 & 0.013 & 0.02 & 0.3696 & bMB \\
\hline 3 & 4.75 & Chloride & 1.565 & 0.285 & 0.41 & 8.0532 & BM \\
\hline 4 & 5.51 & Nitrite-N & 1.320 & 0.240 & 0.34 & 3.8034 & MB \\
\hline 5 & 7.78 & Nitrate-N & 0.503 & 0.119 & 0.17 & 1.7531 & BMB \\
\hline 6 & 11.71 & n.a. & 51.792 & 16.380 & 23.42 & n.a. & BMB \\
\hline 7 & 21.49 & n.a. & 59.311 & 52.645 & 75.27 & n.a. & BMB \\
\hline Total: & & & 116.161 & 69.944 & 100.00 & 13.979 & \\
\hline
\end{tabular}<smiles>[Mg]C1CCCC1[Te]</smiles> 


\section{CCV ICWS-9529}

\section{QUAD}

Sample Name: $\quad$ CCV ICWS-9529

Vial Number: $\quad 368$

Sample Type: unknown

Control Program: ANIONS_AS14A

Quantif. Method: ANIONS

Recording Time: $\quad$ 5/8/2007 17:25

Run Time (min): $\quad \mathbf{1 4 . 7 5}$

$\begin{array}{ll}\text { Injection Volume: } & \mathbf{5 0 . 0} \\ \text { Channel: } & \text { ECD_1 } \\ \text { Wavelength: } & \text { n.a. } \\ \text { Bandwidth: } & \text { n.a. } \\ \text { Dilution Factor: } & 1.0000 \\ \text { Sample Weight: } & 1.0000 \\ \text { Sample Amount: } & \mathbf{1 . 0 0 0 0}\end{array}$

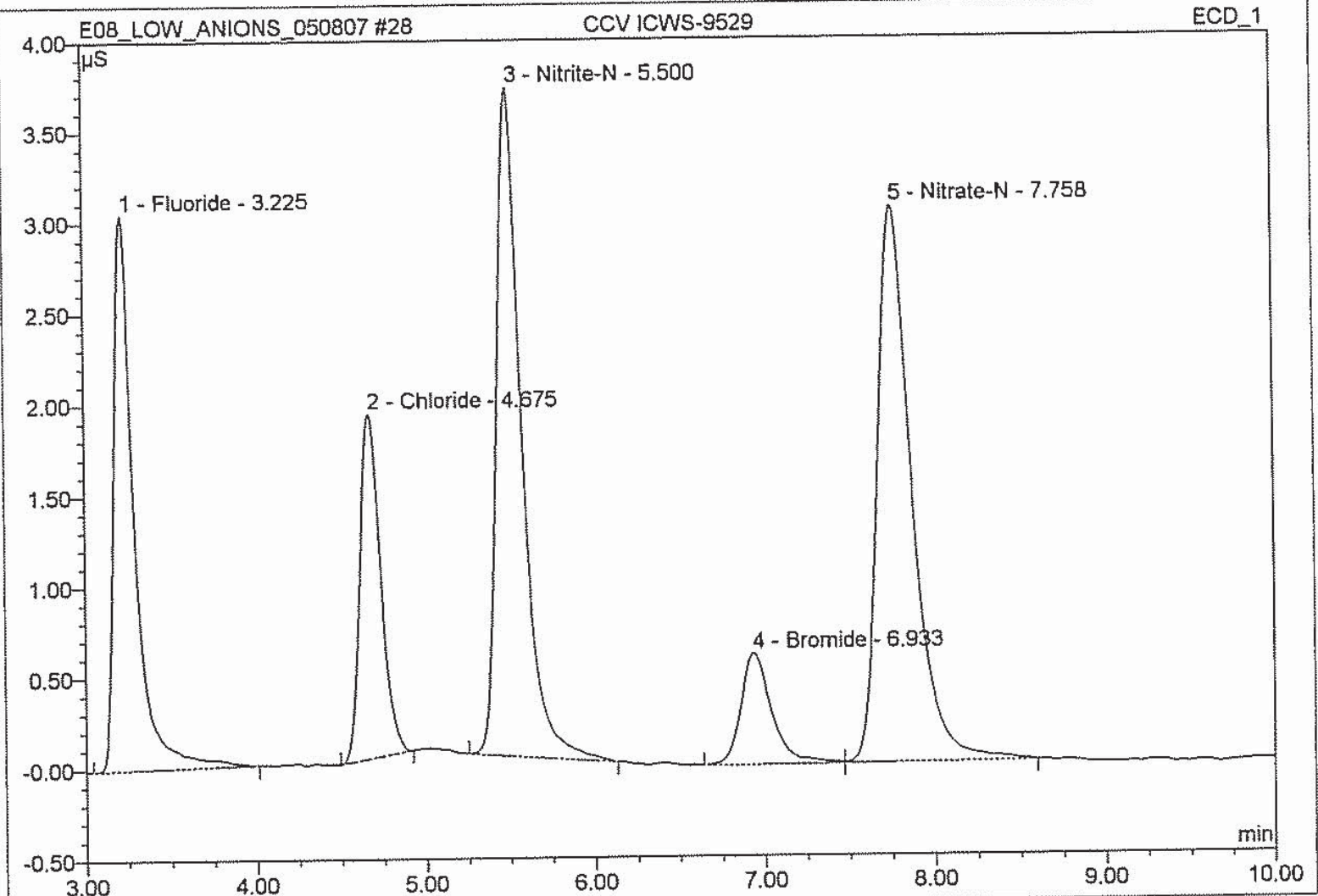

\begin{tabular}{|r|c|l|r|r|r|r|c|}
\hline No. & $\begin{array}{c}\text { Ret.Time } \\
\text { min }\end{array}$ & \multicolumn{1}{|c|}{ Peak Name } & $\begin{array}{c}\text { Height } \\
\mu S\end{array}$ & $\begin{array}{c}\text { Area } \\
\mu S^{\star} \min \end{array}$ & $\begin{array}{c}\text { Rel.Area } \\
\%\end{array}$ & $\begin{array}{c}\text { Amount } \\
\text { mg/L }\end{array}$ & $\begin{array}{c}\text { Peak } \\
\text { Type }\end{array}$ \\
\hline 1 & 3.23 & Fluoride & 3.054 & 0.423 & 19.61 & 1.0441 & $\mathrm{BMB}$ \\
\cline { 2 - 8 } & 4.68 & Chloride & 1.893 & 0.265 & 12.31 & 0.9684 & $\mathrm{BMB}$ \\
\hline 3 & 5.50 & Nitrite-N & 3.676 & 0.629 & 29.14 & 1.0283 & $\mathrm{BMB}$ \\
\hline 4 & 6.93 & Bromide & 0.609 & 0.128 & 5.91 & 1.0298 & $\mathrm{BM}$ \\
\hline 5 & 7.76 & Nitrate-N & 3.058 & 0.713 & 33.03 & 1.0169 & $\mathrm{MB}$ \\
\hline Total: & & & 12.289 & 2.157 & 100.00 & 5.087 & \\
\hline
\end{tabular}




\begin{tabular}{|llll|}
\hline 29 CCB & & & \\
QUAD & & & \\
\hline Sample Name: & CCB & Injection Volume: & $\mathbf{5 0 . 0}$ \\
Vial Number: & 369 & Channel: & ECD_1 \\
Sample Type: & unknown & Wavelength: & n.a. \\
Control Program: & ANIONS_AS14A & Bandwidth: & n.a. \\
Quantif. Method: & ANIONS & Dilution Factor: & $\mathbf{1 . 0 0 0 0}$ \\
Recording Time: & $\mathbf{5 / 8 / 2 0 0 7 1 7 : 4 2}$ & Sample Weight: & $\mathbf{1 . 0 0 0 0}$ \\
Run Time (min): & $\mathbf{1 4 . 7 5}$ & Sample Amount: & $\mathbf{1 . 0 0 0 0}$ \\
\hline
\end{tabular}

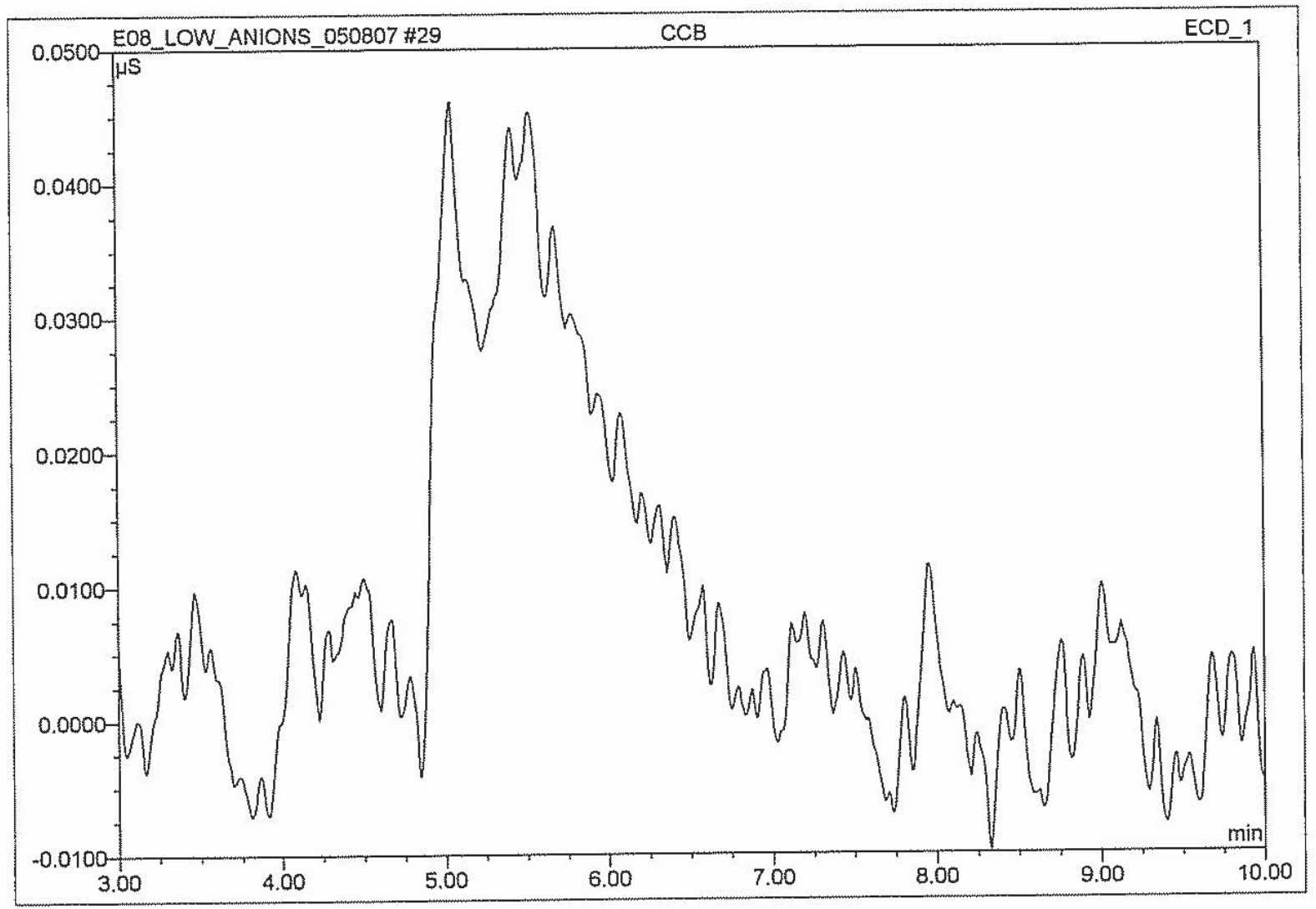

\begin{tabular}{|c|c|c|c|c|c|c|c|}
\hline No. & $\begin{array}{c}\text { Ret.Time } \\
\mathrm{min}\end{array}$ & Peak Name & $\begin{array}{c}\text { Height } \\
\mu \mathrm{S}\end{array}$ & $\begin{array}{c}\text { Area } \\
\mu \mathrm{S}^{\star} \mathrm{min}\end{array}$ & $\begin{array}{c}\text { Rel.Area } \\
\%\end{array}$ & $\begin{array}{c}\text { Amount } \\
\mathrm{mg} / \mathrm{L}\end{array}$ & $\begin{array}{c}\text { Peak } \\
\text { Type }\end{array}$ \\
\hline Total: & & & 0.000 & 0.000 & 0.00 & 0.000 & \\
\hline
\end{tabular}




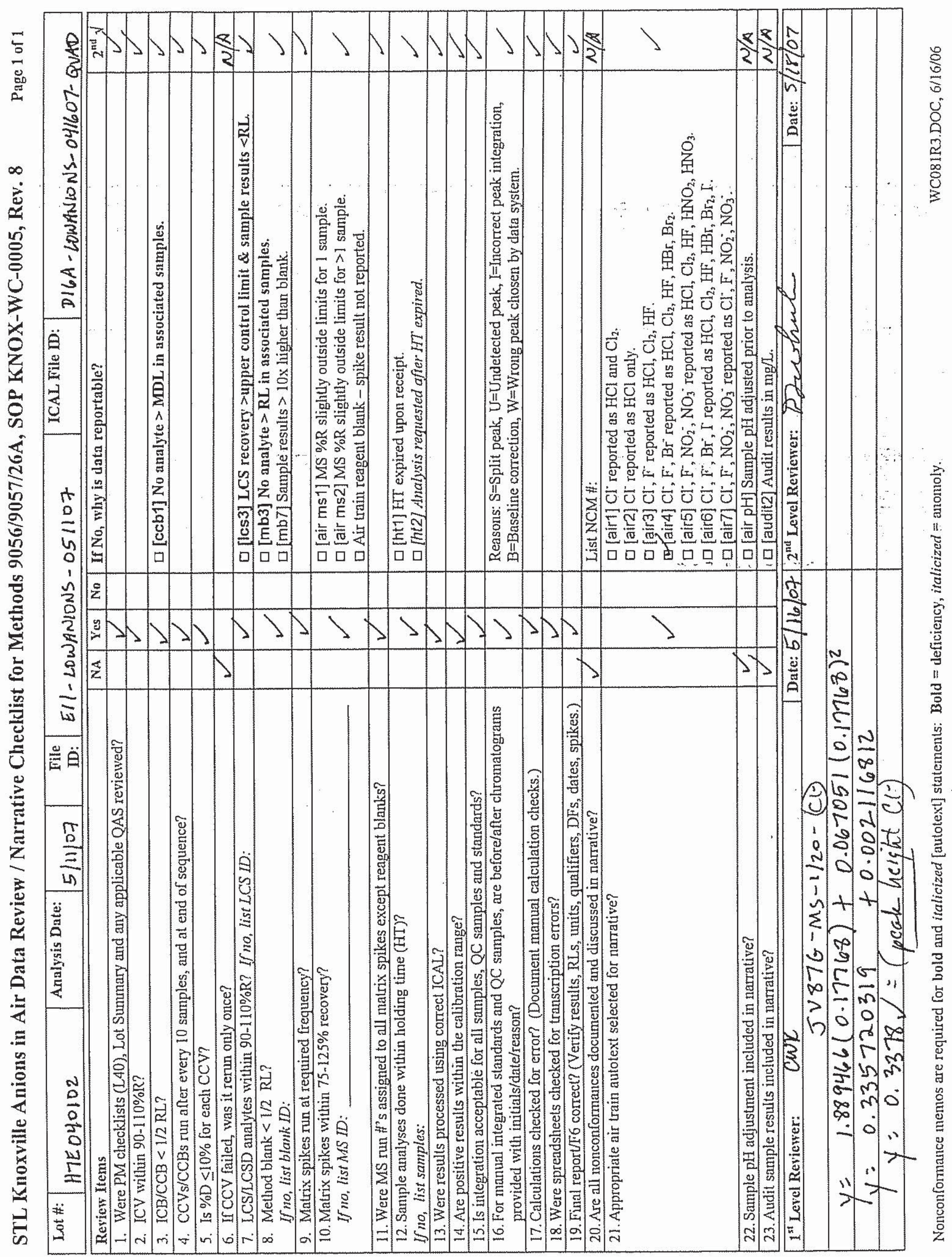




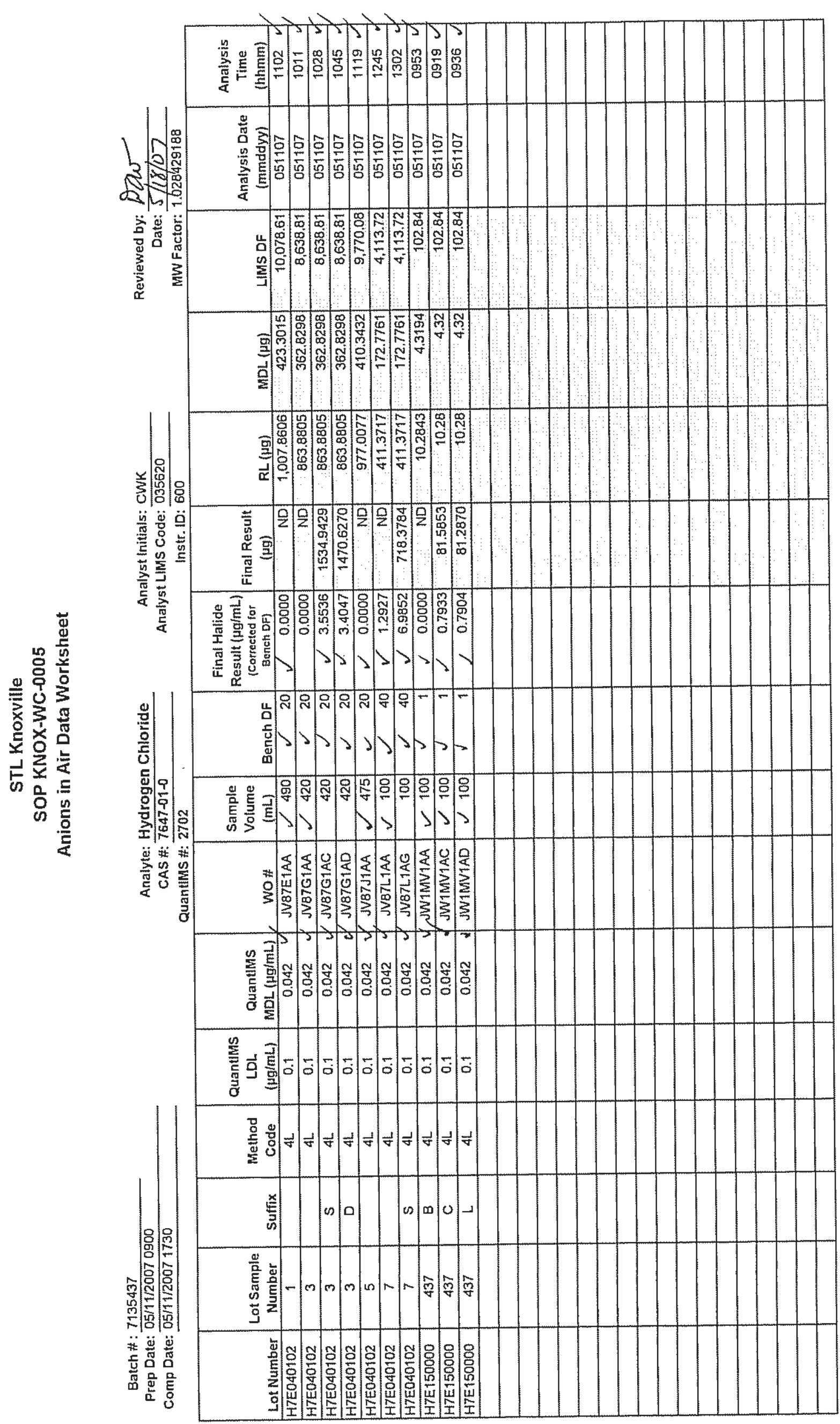



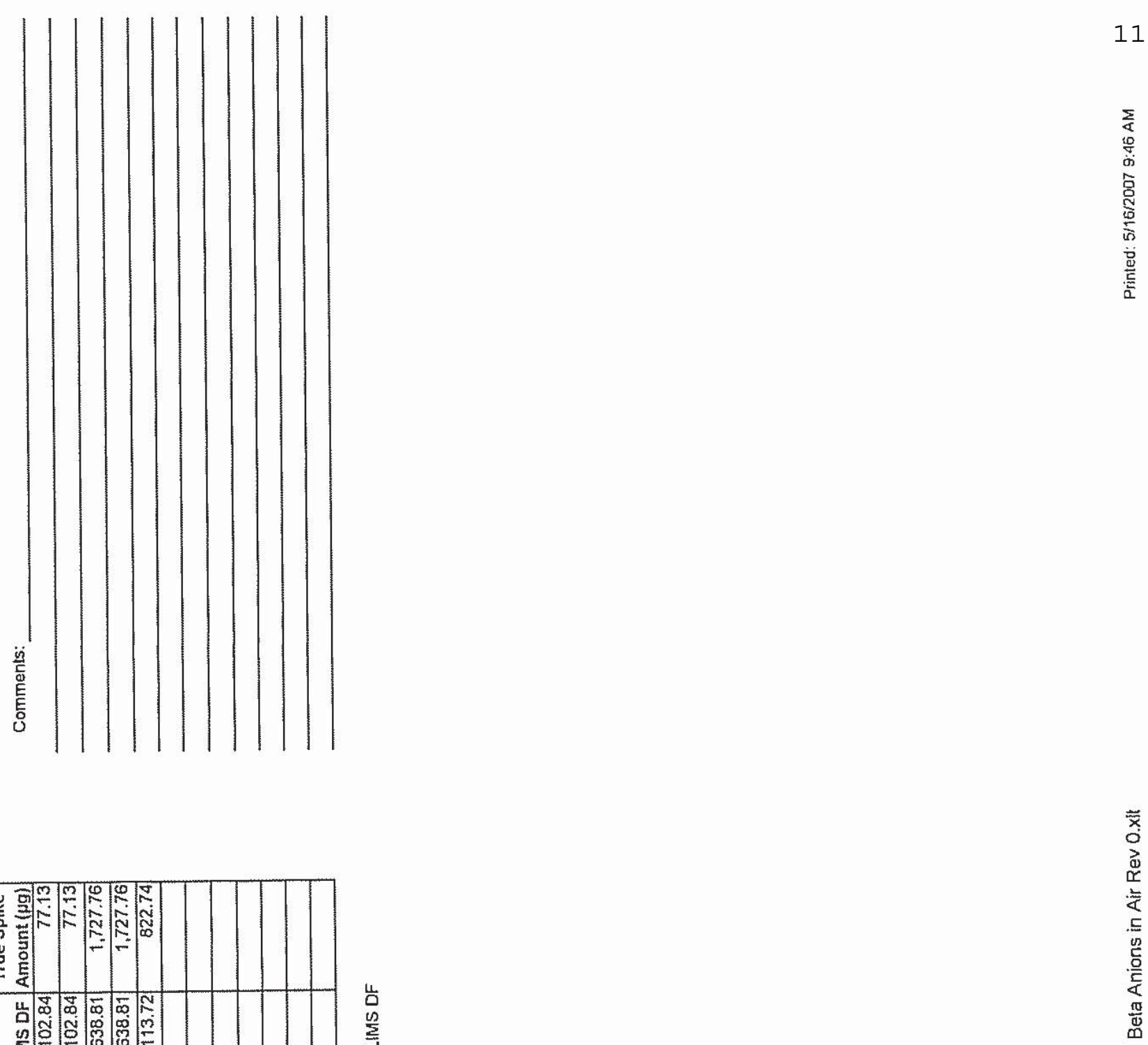

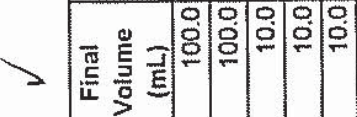

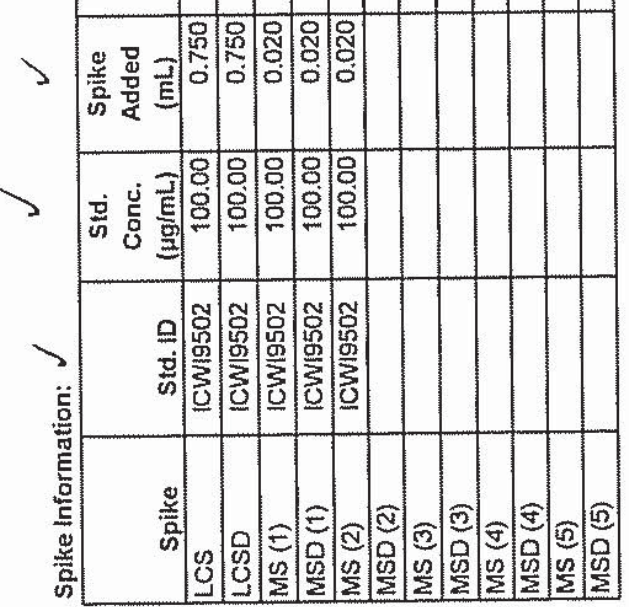




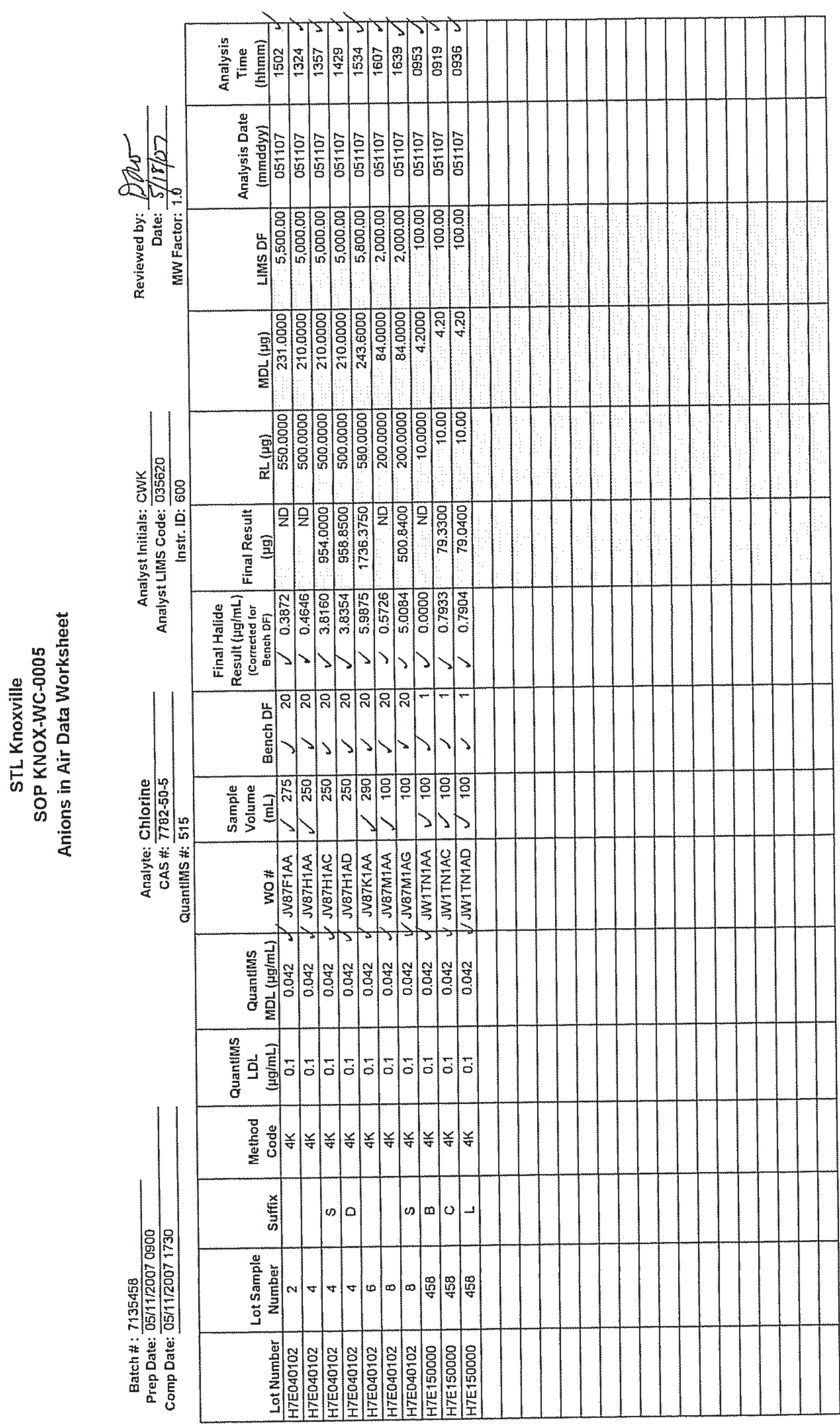




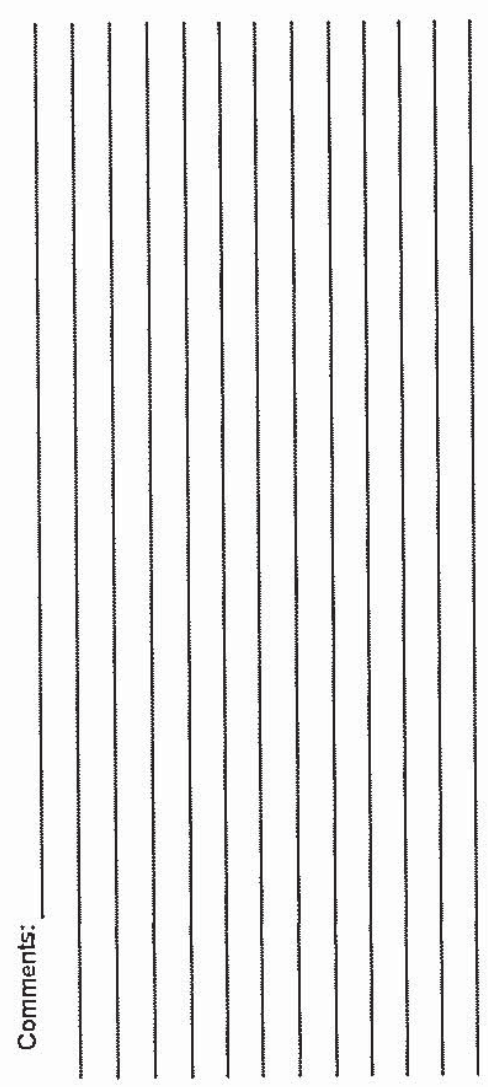

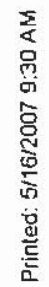

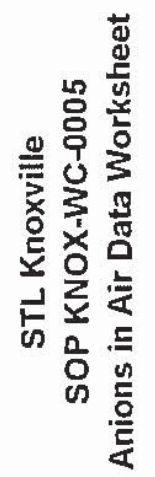

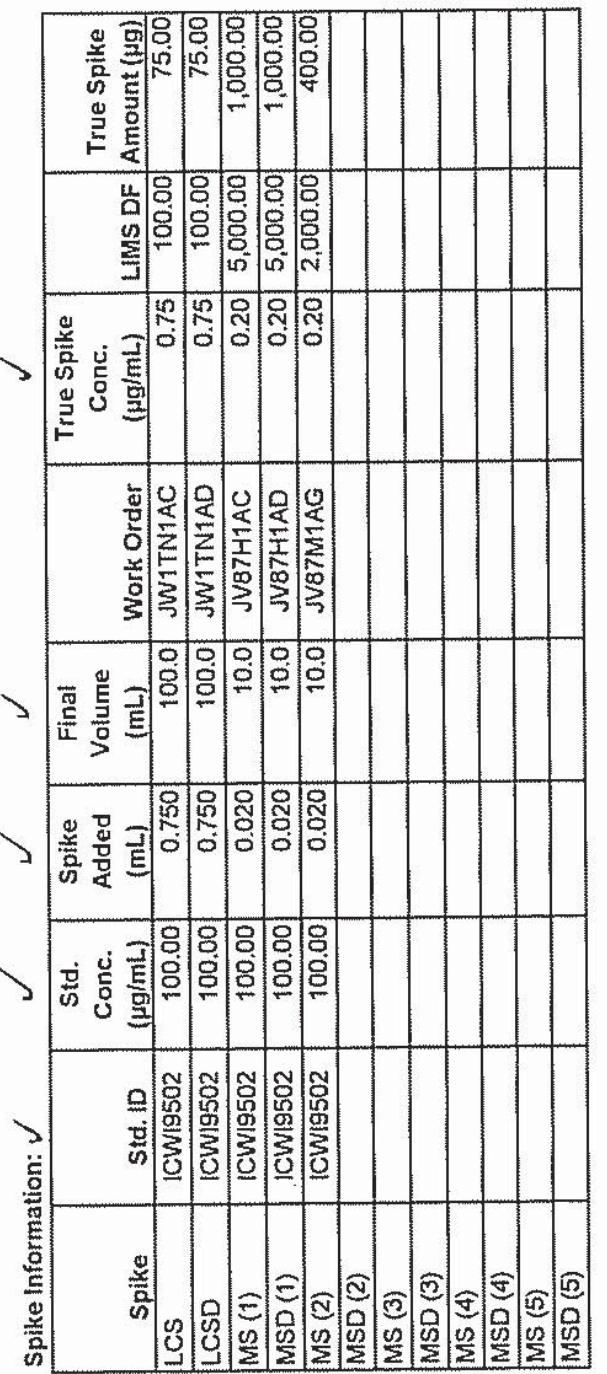

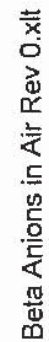


STL Knoxville

Dionex IC Runlog Cover Page

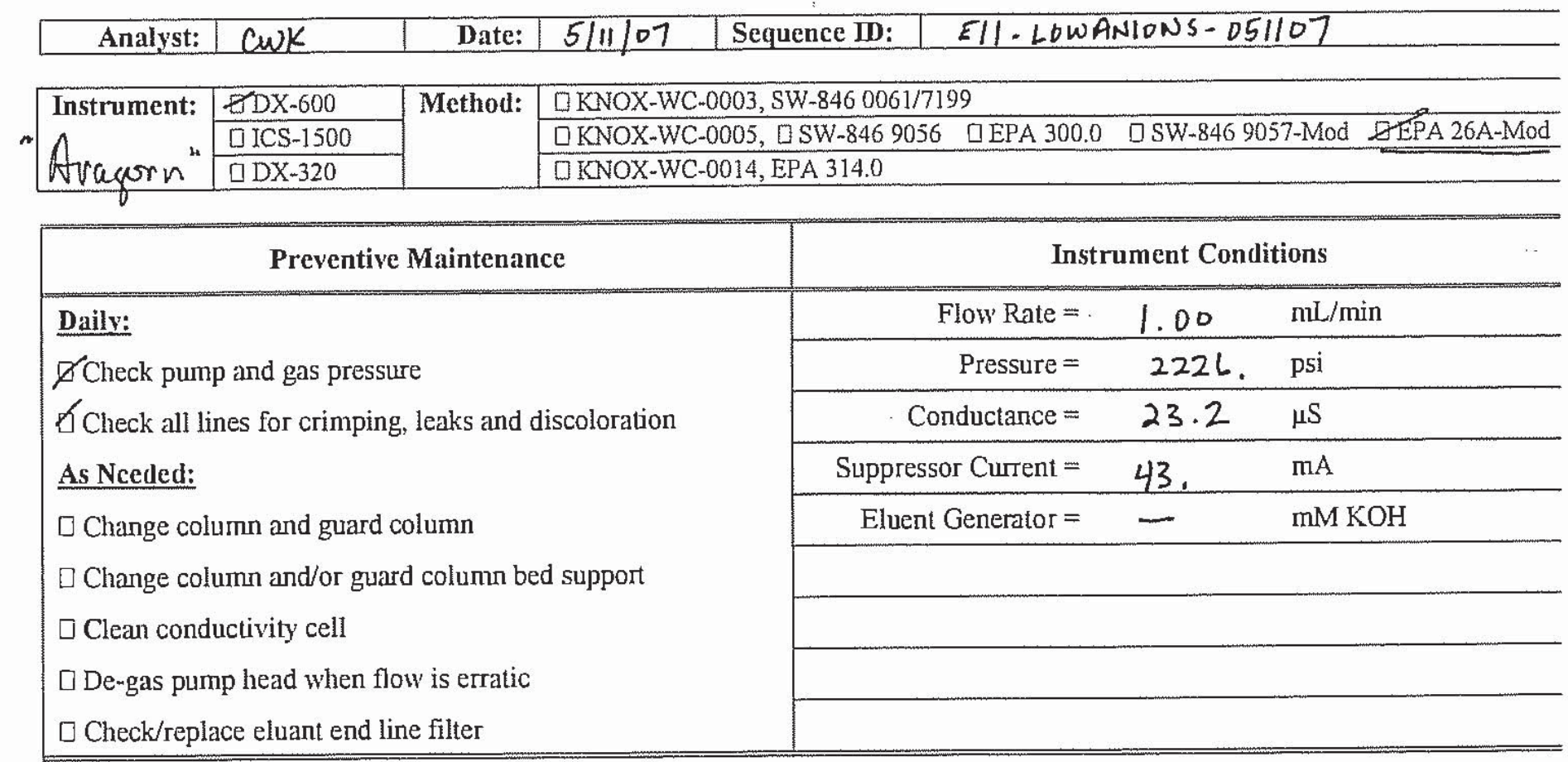

\begin{tabular}{|c|c|c|c|c|c|c|}
\hline \multicolumn{7}{|c|}{ MS/MSD Spike Information } \\
\hline WO\# & Compound & Spike ID & Parent Conc. & Spike Added (mL) & Final Volume (mL) & Final Conc. \\
\hline JV87G & $\mathrm{Cl}^{-}$ & IowI 9502 & $100 \mathrm{ppm}$ & $0.020 \mathrm{ml}$ & $10 \mathrm{ml}$ & $0.20 \mathrm{ppM}$ \\
\hline JV87L & $\mathrm{Cl}_{-}$ & ICWI 9502 & $100 \mathrm{pg} M$ & $0.020 \mathrm{me}$ & $10 \mathrm{ml}$ & $0.20 \mathrm{pPM}$ \\
\hline $\mathrm{JV} 87 \mathrm{H}$ & $\mathrm{Cl}^{-}$ & ICWI-9502 & $100 \mathrm{ppm}$ & $0.020 \mathrm{ml}$ & $10 \mathrm{ml}$ & $0.20 P P M$ \\
\hline $\mathrm{JV} 87 \mathrm{M}$ & $\mathrm{Cl}^{-}$ & IOWI-9502 & 100 PPM & $0.020 \mathrm{ml}$ & $10 \mathrm{ml}$ & $0.20 \mathrm{ppm}$ \\
\hline & & & & & & \\
\hline & & & & & & \\
\hline & & & & & & \\
\hline & & & & & & \\
\hline & & & & & & \\
\hline & & & & & & \\
\hline & & & & & & \\
\hline & & & & & & \\
\hline
\end{tabular}

Comments:

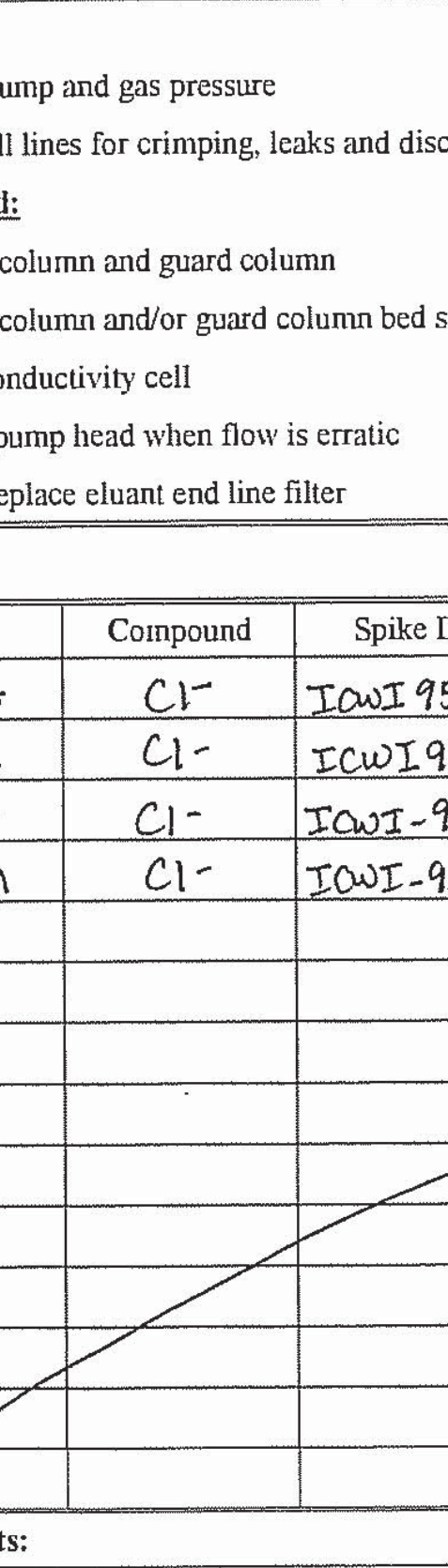

Batch: $7135437(\mathrm{HCl})$

Batch: $7135458\left(\mathrm{Cl}_{2}\right)$

Sodium Thiosulfate added to $\mathrm{NaOH}$ impinger sanples. 
Sequence: Operator:

E11_LOW_ANIONS_051107 kaukerc

Tille:

Datasource: Aragorn_net

Location: IC_ARAGORN

Timebase: IC_Aragorn

\#Samples: $\quad 30$
Page 1 of 2 Printed: 5/14/2007 8:05:09 AM

Created: Last Update:
5/11/2007 9:18:07 AM by kaukerc 5/11/2007 2:10:16 PM by kaukerc

\begin{tabular}{|c|c|c|c|c|c|c|c|c|}
\hline No. & Nan & & Comment & Inj. Vol. & Inj. Date/Time & Dil. Factor & $\begin{array}{r}{ }^{*} \text { Multiplier } \\
\text { [Liters] }\end{array}$ & Weight \\
\hline 1 & 要 & CAL STD \#1 ICWS-9424 & QUAD & 50.0 & 4/16/2007 12:21:03 PM & 1.0000 & & 1.0000 \\
\hline 2 & 0 & CAL STD \#2 ICWS-9425 & QUAD & 50.0 & 4/16/2007 12:38:14 PM & 1.0000 & & 1.0000 \\
\hline 3 & (7) & CAL STD \#3 ICWS-9426 & QUAD & 50.0 & 4/16/2007 12:55:24 PM & 1.0000 & & 1.0000 \\
\hline 4 & (1) & CAL STD \#4 ICWS-9427 & QUAD & 50.0 & 4/16/2007 1:12:34 PM & 1.0000 & & 1.0000 \\
\hline 5 & D & CAL STD \#5 ICWS-9423 & QUAD & 50.0 & 4/16/2007 1:29:45 PM & 1.0000 & & 1.0000 \\
\hline 6 & (1) & CAL STD \#6 ICWS-9428 & QUAD & 50.0 & $4 / 16 / 20071: 46: 55 \mathrm{PM}$ & 1.0000 & & 1.0000 \\
\hline 7 & 圆 & ICVILCS ICWS-9562 & QUAD & 50.0 & 5/11/2007 9:19:37 AM & 1.0000 & & 1.0000 \\
\hline 8 & ? & ICVILCSD ICWS-9563 & QUAD & 50.0 & $5 / 11 / 20079: 36: 47$ AM & 1.0000 & & 1.0000 \\
\hline 9 & 通 & ICB/METHOD BLK & QUAD & 50.0 & $5 / 11 / 20079: 53: 57 \mathrm{AM}$ & 1.0000 & & 1.0000 \\
\hline 10 & ใี? & H7E040102 JV87G $1 / 20$ & QUAD & 50.0 & $5 / 11 / 2007$ 10:11:07 AM & 20.0000 & & 1.0000 \\
\hline 11 & ? & H7E040102 JV87G MS 1/20 0.2 PPM CL & QUAD & 50.0 & $5 / 11 / 2007$ 10:28:18 AM & 20.0000 & & 1.0000 \\
\hline 12 & ? & H7E040102 JV87G MSD 1/20 0.2 PPM CL & QUAD & 50.0 & $5 / 11 / 200710: 45: 28 \mathrm{AM}$ & 20.0000 & & 1.0000 \\
\hline 13 & ?] & H7E040102 JV87E 1/20 & QUAD & 50.0 & $5 / 11 / 200711: 02: 39 \mathrm{AM}$ & 20.0000 & & 1.0000 \\
\hline 14 & ? & H7E040102 JV87J $1 / 20$ & QUAD & 50.0 & 5/11/2007 11:19:49 AM & 20.0000 & & 1.0000 \\
\hline 15 & ? & H7E040102 JV87L 1/20 & QUAD & 50.0 & $5 / 11 / 200711: 36: 59 \mathrm{AM}$ & 20.0000 & & 1.0000 \\
\hline 16 & ? & H7E040102 JV87L MS 1/20 0.2 PPM CL & QUAD & 50.0 & $5 / 11 / 200711: 54: 10 \mathrm{AM}$ & 20.0000 & & 1.0000 \\
\hline 17 & ? & CCVICWS-9564 & QUAD & 50.0 & $5 / 11 / 2007$ 12:11:20 PM & 1.0000 & & 1.0000 \\
\hline 18 & ? & $\mathrm{CCB}$ & QUAD & 50.0 & $5 / 11 / 2007$ 12:28:32 PM & 1.0000 & & 1.0000 \\
\hline 19 & 匇 & H7E040102 JV87L 1/40 & QUAD & 50.0 & $5 / 11 / 2007$ 12:45:42 PM & 40.0000 & & 1.0000 \\
\hline 20 & 党 & H7E040102 JV87L MS 1/40 0.2 PPM CL & QUAD & 50.0 & $5 / 11 / 2007$ 1:02:53 PM & 40.0000 & & 1.0000 \\
\hline 21 & 通 & H7E040102 JV87H 1/20 & QUAD & 50.0 & $5 / 11 / 2007$ 1:24:54 PM & 20.0000 & & 1.0000 \\
\hline 22 & ? & H7E040102 JV87H MS 1/20 0.2 PPM CL & QUAD & 50.0 & $5 / 11 / 20071: 57: 19 \mathrm{PM}$ & 20.0000 & & 1.0000 \\
\hline 23 & ? & H7E040102 JV87H MSD 1/20 0.2 PPM CL & QUAD & 50.0 & $5 / 11 / 20072: 29: 45 \mathrm{PM}$ & 20.0000 & & 1.0000 \\
\hline 24 & ? & H7E040102 JV87F 1/20 & QUAD & 50,0 & 5/11/2007 3:02:10 PM & 20.0000 & & 1.0000 \\
\hline 25 & ใด & H7E040102 JV87K 1/20 & QUAD & 50.0 & $5 / 11 / 2007$ 3:34:36 PM & 20.0000 & & 1.0000 \\
\hline 26 & 挣 & H7E040102 JV87M 1/20 & QUAD & 50.0 & 5/11/2007 4:07:01 PM & 20.0000 & & 1.0000 \\
\hline 27 & ? & H7E040102 JV87M MS 1/20 0.2 PPM CL & QUAD & 50.0 & 5/11/2007 4:39:27 PM & 20.0000 & & 1.0000 \\
\hline 28 & ? & CCV ICWS-9564 & QUAD & 50.0 & $5 / 11 / 2007$ 5:11:52 PM & 1,0000 & & 1.0000 \\
\hline 29 & 爷 & $\mathrm{CCB}$ & QUAD & 50.0 & 5/11/2007 5:29:03 PM & 1.0000 & & 1.0000 \\
\hline 30 & ? & SHUTDOWN & QUAD & 50.0 & $5 / 11 / 2007$ 5:46:13 PM & 1.0000 & & 1.0000 \\
\hline
\end{tabular}


Sequence:

Title:

Datasource: Aragorn_net

Location: IC_ARAGORN

Timebase: $\quad$ IC_Aragorn

Created:

\begin{tabular}{|c|c|c|c|c|c|}
\hline No: & Name & Type & Status & Program & Method \\
\hline 1 & (1) CAL STD \#1 ICWS-9424 & Standard & Finished & ANIONS_AS14A & ANIONS \\
\hline 2 & 7 CAL STD \#2 ICWS-9425 & Standard & Finished & ANIONS_AS14A & ANIONS \\
\hline 3 & 17 CAL STD \#3 ICWS-9426 & Standard & Finished & ANIONS_AS14A & ANIONS \\
\hline 4 & (]) CAL STD \#4 ICWS-9427 & Standard & Finished & ANIONS_AS14A & ANIONS \\
\hline 5 & (1) CAL STD \#5 ICWS-9423 & Standard & Finished & ANIONS_AS14A & ANIONS \\
\hline 6 & 8 CAL STD \#6 ICWS-9428 & Standard & Finished & ANIONS_AS14A & ANIONS \\
\hline 7 & [? ICVILCS ICWS-9562 & Unknown & Finished & ANIONS_AS14A & ANIONS \\
\hline 8 & [?] ICV/LCSD ICWS-9563 & Unknown & Finished & ANIONS_AS14A & ANIONS \\
\hline 9 & [3] ICBMETHOD BLK & Unknown & Finished & ANIONS_AS14A & ANIONS \\
\hline 10 & (3) H7E040102 JV87G 1/20 & Unknown & Finished & ANIONS_AS14A & ANIONS \\
\hline 11 & ?] H7E040102 JV87G MS 1/200.2 PPM CL & Unknown & Finished & ANIONS_AS14A & ANIONS \\
\hline 12 & H7 HE040102 JV87G MSD 1/20 0.2 PPM CL & Unknown & Finished & ANIONS_AS14A & ANIONS \\
\hline 13 & H7E040102 JV87E 1/20 & Unknown & Finished & ANIONS_AS14A & ANIONS \\
\hline 14 & 商 H7E040102 JV87J 1/20 & Unknown & Finished & ANIONS_AS14A & ANIONS \\
\hline 15 & ? H7E040102 JV87L 1/20 & Unknown & Finished & ANIONS_AS14A & ANIONS \\
\hline 16 & [? H7E040102 JV87L MS 1/20 0.2 PPM CL & Unknown & Finished & ANIONS_AS14A & ANIONS \\
\hline 17 & ? CCVICWS-9564 & Unknown & Finished & ANIONS_AS14A & ANIONS \\
\hline 18 & ? & Unknown & Finished & ANIONS_AS14A & ANIONS \\
\hline 19 & (?) H7E040102 JV87L $1 / 40$ & Unknown & Finished & ANIONS_AS14A & ANIONS \\
\hline 20 & (2) H7E040102 JV87L MS $1 / 400.2 \mathrm{PPM} \mathrm{CL}$ & Unknown & Finished & ANIONS_AS14A & ANIONS \\
\hline 21 & (2) H7E040102 JV87H 1/20 & Unknown & Finished & ANIONS_AS14A_Cl2 & ANIONS \\
\hline 22 & ? H7E040102 JV87H MS 1/20 0.2 PPM CL & Unknown & Finished & ANIONS_AS14A_Cl2 & ANIONS \\
\hline 23 & [? H7E040102 JV87H MSD 1/20 0.2 PPM CL & Unknown & Finished & ANIONS_AS14A_Cl2 & ANIONS \\
\hline 24 & H7E040102 JV87F 1/20 & Unknown & Finished & ANIONS_AS14A_CI2 & ANIONS \\
\hline 25 & ? H7E040102 JV87K $1 / 20$ & Unknown & Finished & ANIONS_AS14A_CI2 & ANIONS \\
\hline 26 & [? H7E040102 JV87M 1/20 & Unknown & Finished & ANIONS_AS14A_Cl2 & ANIONS \\
\hline 27 & (7) H7E040102 JV87M MS 1/200.2 PPM CL & Unknown & Finished & ANIONS_AS14A_CI2 & ANIONS \\
\hline 28 & 角 CCVICWS-9564 & Unknown & Finished & ANIONS_AS14A & ANIONS \\
\hline 29 & ? $\mathrm{CCB}$ & Unknown & Finished & ANIONS_AS14A & ANIONS \\
\hline 30 & [?] SHUTDOWN & Unknown & Finished & SHUTDOWN & ANIONS \\
\hline
\end{tabular}




\section{ICV/LCS ICWS-9562}

\section{QUAD}

Sample Name: ICV/LCS ICWS-9562

Vial Number: $\quad \mathbf{3 1 0}$

Sample Type: unknown

Control Program: ANIONS_AS14A

Quantif. Method: ANIONS

Recording Time: $\quad$ 5/11/2007 9:19

Run Time (min): $\quad 14.75$
Injection Volume: $\quad \mathbf{5 0 . 0}$

Channel:

ECD_1

Wavelength: n.a.

Bandwidth: $\quad$ n.a.

Dilution Factor: $\quad \mathbf{1 . 0 0 0 0}$

Sample Weight: $\quad \mathbf{1 . 0 0 0 0}$

Sample Amount: $\quad \mathbf{1 . 0 0 0 0}$

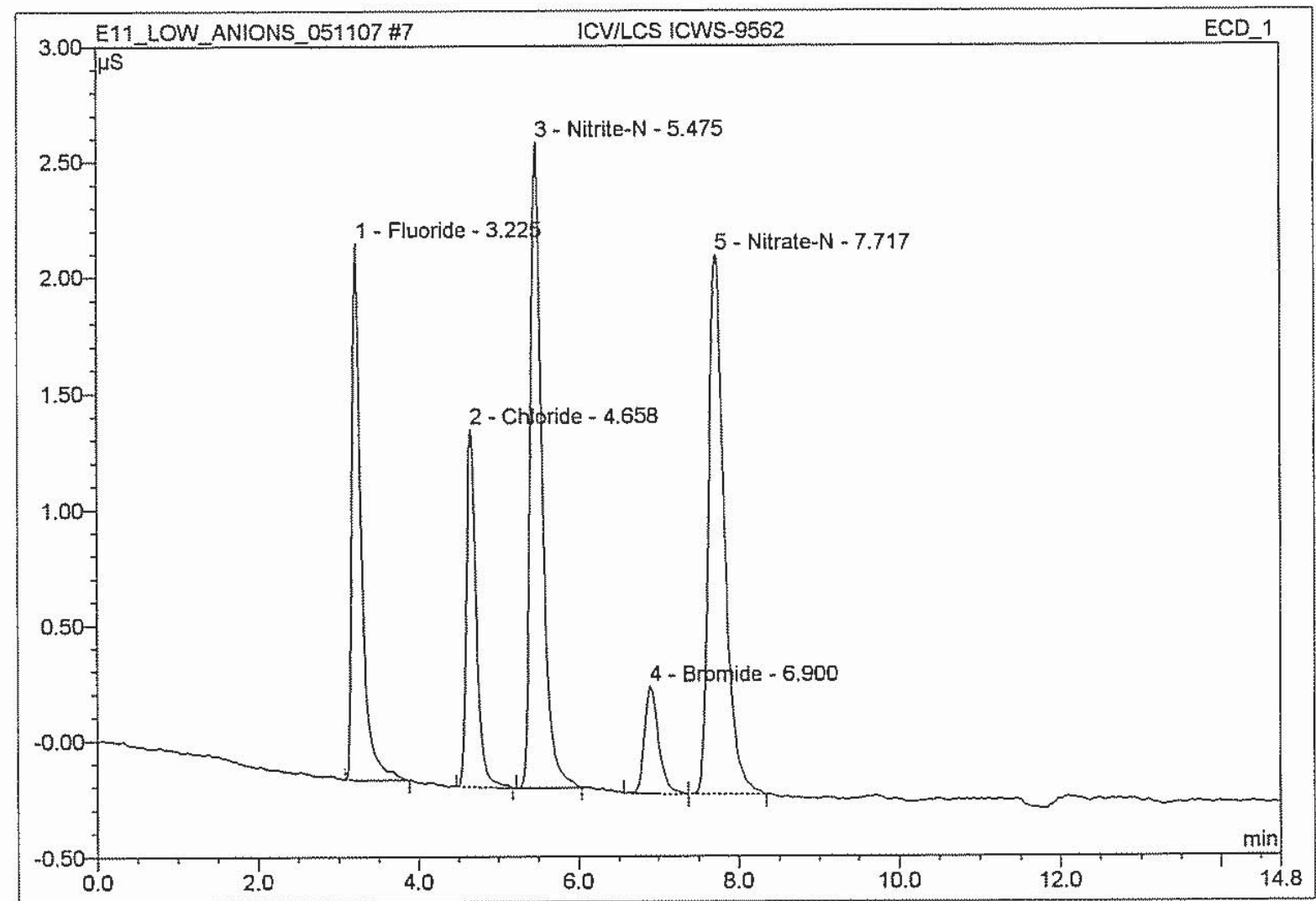

\begin{tabular}{|c|c|l|r|r|r|r|c|}
\hline No. & $\begin{array}{c}\text { Ret.Time } \\
\text { min }\end{array}$ & \multicolumn{1}{|c|}{ Peak Name } & $\begin{array}{c}\text { Height } \\
\mu S\end{array}$ & $\begin{array}{c}\text { Area } \\
\mu S^{*} \min \end{array}$ & $\begin{array}{c}\text { Rel.Area } \\
\%\end{array}$ & $\begin{array}{c}\text { Amount } \\
\text { mg/L }\end{array}$ & $\begin{array}{c}\text { Peak } \\
\text { Type }\end{array}$ \\
\hline 1 & 3.23 & Fluoride & 2.315 & 0.316 & 19.33 & 0.8034 & BMB \\
\cline { 2 - 7 } 2 & 4.66 & Chloride & 1.541 & 0.220 & 13.43 & 0.7933 & BMB \\
\hline 3 & 5.48 & Nitrite-N & 2.786 & 0.473 & 28.95 & 0.7879 & BMB \\
\hline 4 & 6.90 & Bromide & 0.460 & 0.094 & 5.78 & 0.7811 & BMb \\
\hline 5 & 7.72 & Nitrate-N & 2.327 & 0.532 & 32.51 & 0.7837 & $\mathrm{bMB}$ \\
\hline Total: & & & 9.430 & 1.635 & 100.00 & 3.949 & \\
\hline
\end{tabular}

JWITNIAC (C13)

JWIMVIAC (H4)

default_letter/Integration

Chromeleon (c) Dionex 1996-2001

Version 6.50 SP10a Build 1065 


\section{ICV/LCSD ICWS-9563}

\section{QUAD}

Sample Name: ICV/LCSD ICWS-9563

Vial Number:

Sample Type:

Control Program:

Quantif. Method:

Recording Time:

Run Time ( $\mathrm{min}$ ):
311

unknown

ANIONS_AS14A

ANIONS

5/11/2007 9:36

14.75

$\begin{array}{ll}\text { Injection Volume: } & \mathbf{5 0 . 0} \\ \text { Channel: } & \text { ECD_1 } \\ \text { Wavelength: } & \text { n.a. } \\ \text { Bandwidth: } & \text { n.a. } \\ \text { Dilution Factor: } & \mathbf{1 . 0 0 0 0} \\ \text { Sample Weight: } & 1.0000 \\ \text { Sample Amount: } & \mathbf{1 . 0 0 0 0}\end{array}$

$\begin{array}{ll}\text { Injection Volume: } & \mathbf{5 0 . 0} \\ \text { Channel: } & \text { ECD_1 }\end{array}$

Wavelength: n.a.

Bandwidth:

n.a.

1.0000

Sample Amount: $\quad \mathbf{1 . 0 0 0 0}$

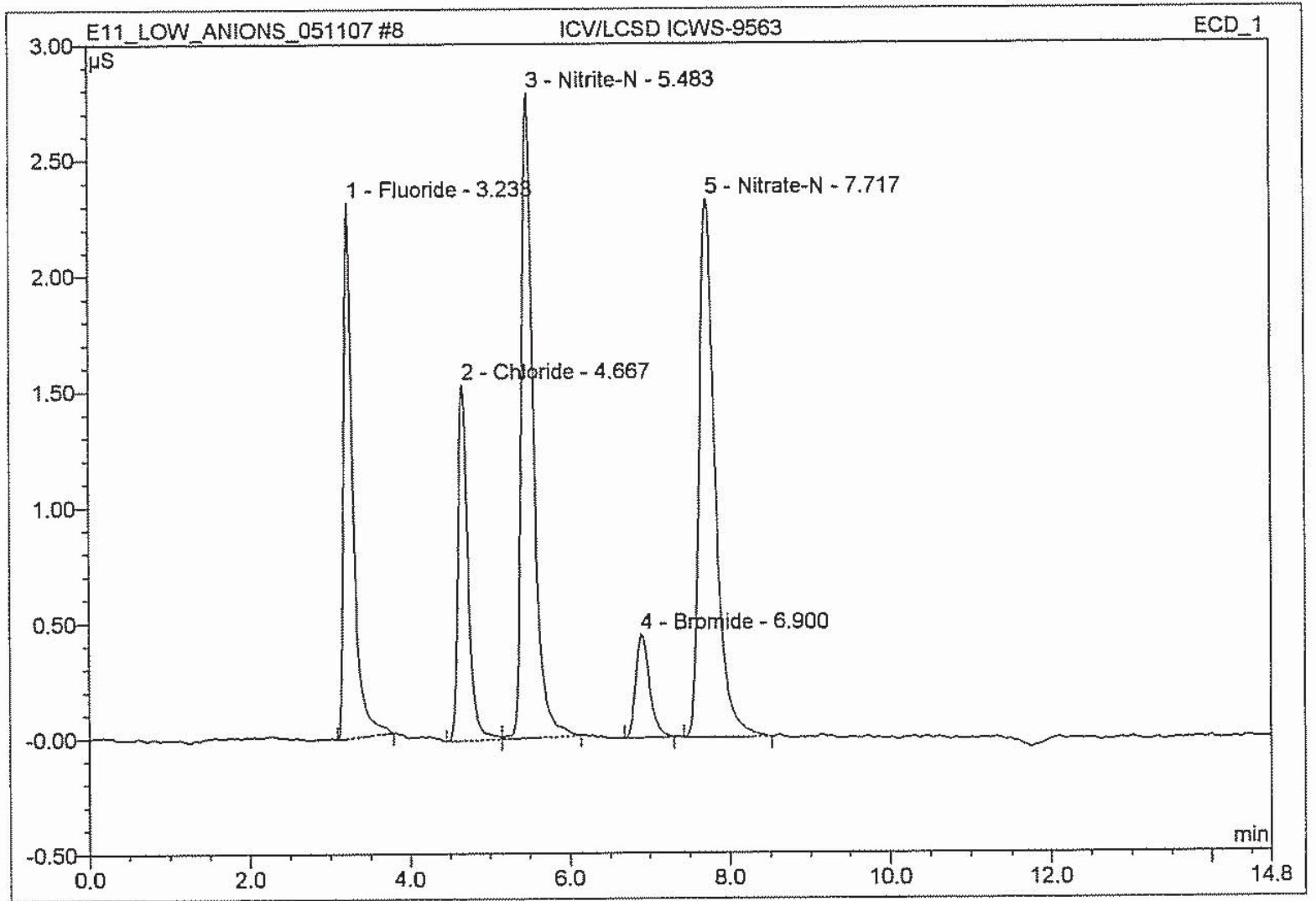

\begin{tabular}{|c|c|l|r|r|r|r|c|}
\hline No. & $\begin{array}{c}\text { Ret.Time } \\
\min \end{array}$ & \multicolumn{1}{|c|}{ Peak Name } & $\begin{array}{c}\text { Height } \\
\mu \mathrm{S}\end{array}$ & $\begin{array}{c}\text { Area } \\
\mu \mathrm{S}^{*} \mathrm{~min}\end{array}$ & $\begin{array}{c}\text { Rel.Area } \\
\%\end{array}$ & $\begin{array}{c}\text { Amount } \\
\mathrm{mg} / \mathrm{L}\end{array}$ & $\begin{array}{c}\text { Peak } \\
\text { Type }\end{array}$ \\
\hline 1 & 3.23 & Fluoride & 2.314 & 0.313 & 19.03 & 0.8029 & $\mathrm{BMB}$ \\
\cline { 2 - 8 } 2 & 4.67 & Chloride & 1.535 & 0.221 & 13.44 & 0.7904 & $\mathrm{BM}$ \\
\hline 3 & 5.48 & Nitrite-N & 2.784 & 0.483 & 29.34 & 0.7873 & $\mathrm{MB}$ \\
\hline 4 & 6.90 & Bromide & 0.448 & 0.090 & 5.45 & 0.7606 & $\mathrm{BMB}$ \\
\hline 5 & 7.72 & Nitrate-N & 2.325 & 0.538 & 32.74 & 0.7830 & $\mathrm{BMB}$ \\
\hline Total: & & & 9.406 & 1.645 & 100.00 & 3.924 & \\
\hline
\end{tabular}

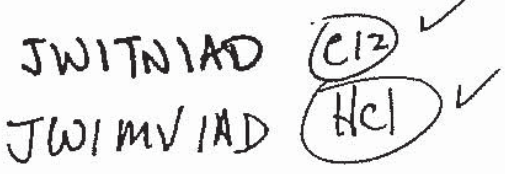

default_letter/Integration

Chromeleon (c) Dionex 1996-2001 Version 6.50 SP10a Build 1065 


\section{ICB/METHOD BLK}

\section{QUAD}

Sample Name: ICB/METHOD BLK

Vial Number: $\quad 312$

Sample Type: $\quad$ unknown

Control Program: ANIONS_AS14A

Quantif. Method: ANIONS

Recording Time: $\quad$ 5/11/2007 9:53

Run Time (min):

14.75

$\begin{array}{ll}\text { Injection Volume: } & \mathbf{5 0 . 0} \\ \text { Channel: } & \text { ECD_1 } \\ \text { Wavelength: } & \text { n.a. } \\ \text { Bandwidth: } & \text { n.a. } \\ \text { Dilution Factor: } & 1.0000 \\ \text { Sample Weight: } & 1.0000 \\ \text { Sample Amount: } & 1.0000\end{array}$

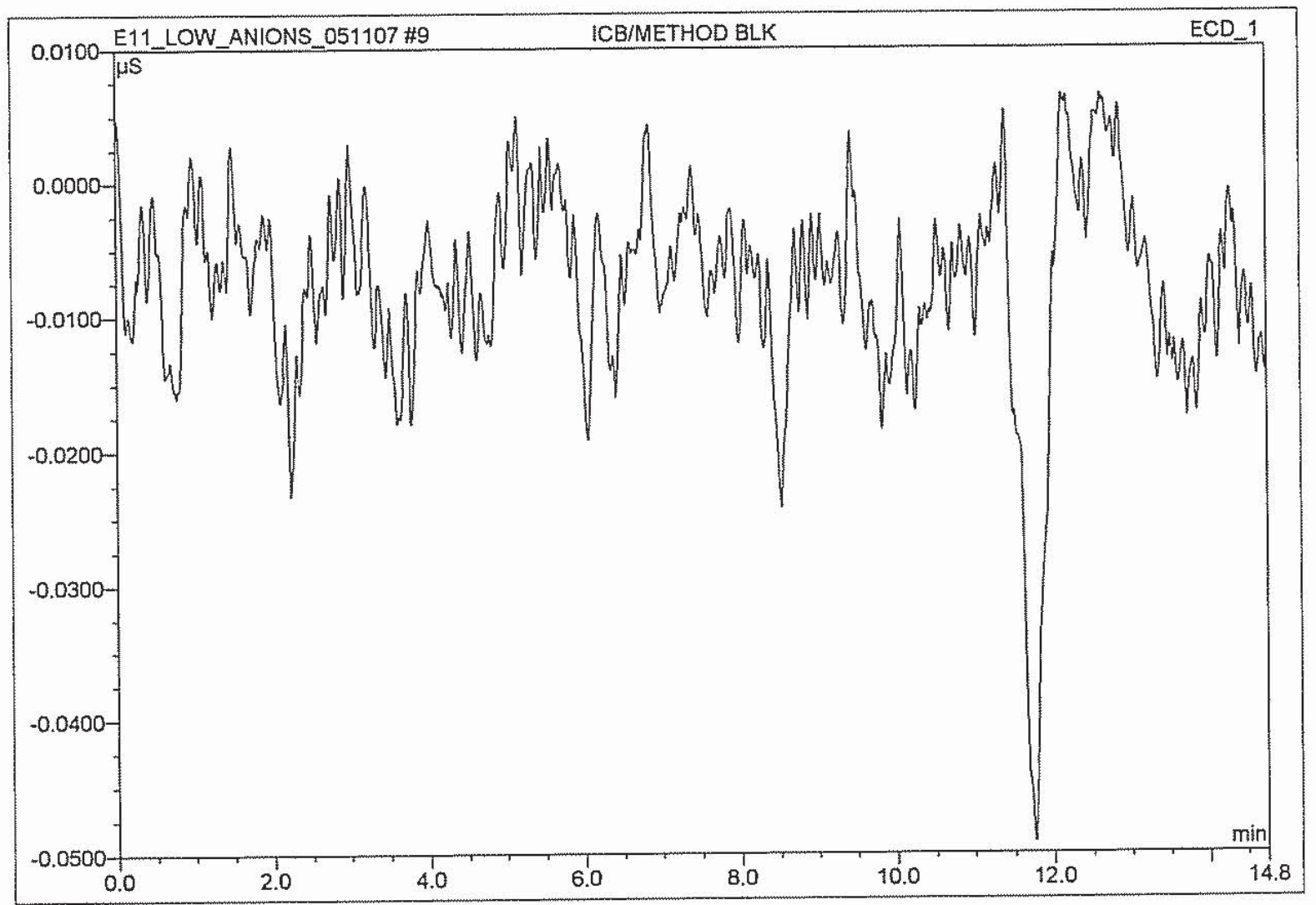

\begin{tabular}{|c|c|c|c|c|c|c|c|}
\hline No. & $\begin{array}{c}\text { Ret.Time } \\
\mathrm{min}\end{array}$ & Peak Name & $\begin{array}{c}\text { Height } \\
\mu \mathrm{S}\end{array}$ & $\begin{array}{c}\text { Area } \\
\mu \mathrm{S}^{\star} \mathrm{min}\end{array}$ & $\begin{array}{c}\text { Rel.Area } \\
\%\end{array}$ & $\begin{array}{c}\text { Amount } \\
\mathrm{mg} / \mathrm{L}\end{array}$ & $\begin{array}{c}\text { Peak } \\
\text { Type }\end{array}$ \\
\hline Total: & & & 0.000 & 0.000 & 0.00 & 0.000 & \\
\hline
\end{tabular}

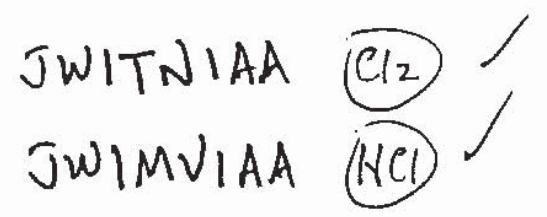




\section{H7E040102 JV87G 1/20}

\section{QUAD}

Sample Name: $\quad$ H7E040102 JV87G 1/20

Vial Number: $\quad \mathbf{3 1 3}$

Sample Type: unknown

Control Program: ANIONS_AS14A

Quantif. Method: ANIONS

Recording Time: $\quad 5 / 11 / 2007$ 10:11
Run Time (min): $\quad \mathbf{1 4 . 7 5}$

$\begin{array}{ll}\text { Injection Volume: } & \mathbf{5 0 . 0} \\ \text { Channel: } & \text { ECD_1 } \\ \text { Wavelength: } & \text { n.a. } \\ \text { Bandwidth: } & \text { n.a. } \\ \text { Dilution Factor: } & \mathbf{2 0 . 0 0 0 0} \\ \text { Sample Weight: } & \mathbf{1 . 0 0 0 0} \\ \text { Sample Amount: } & \mathbf{1 . 0 0 0 0}\end{array}$

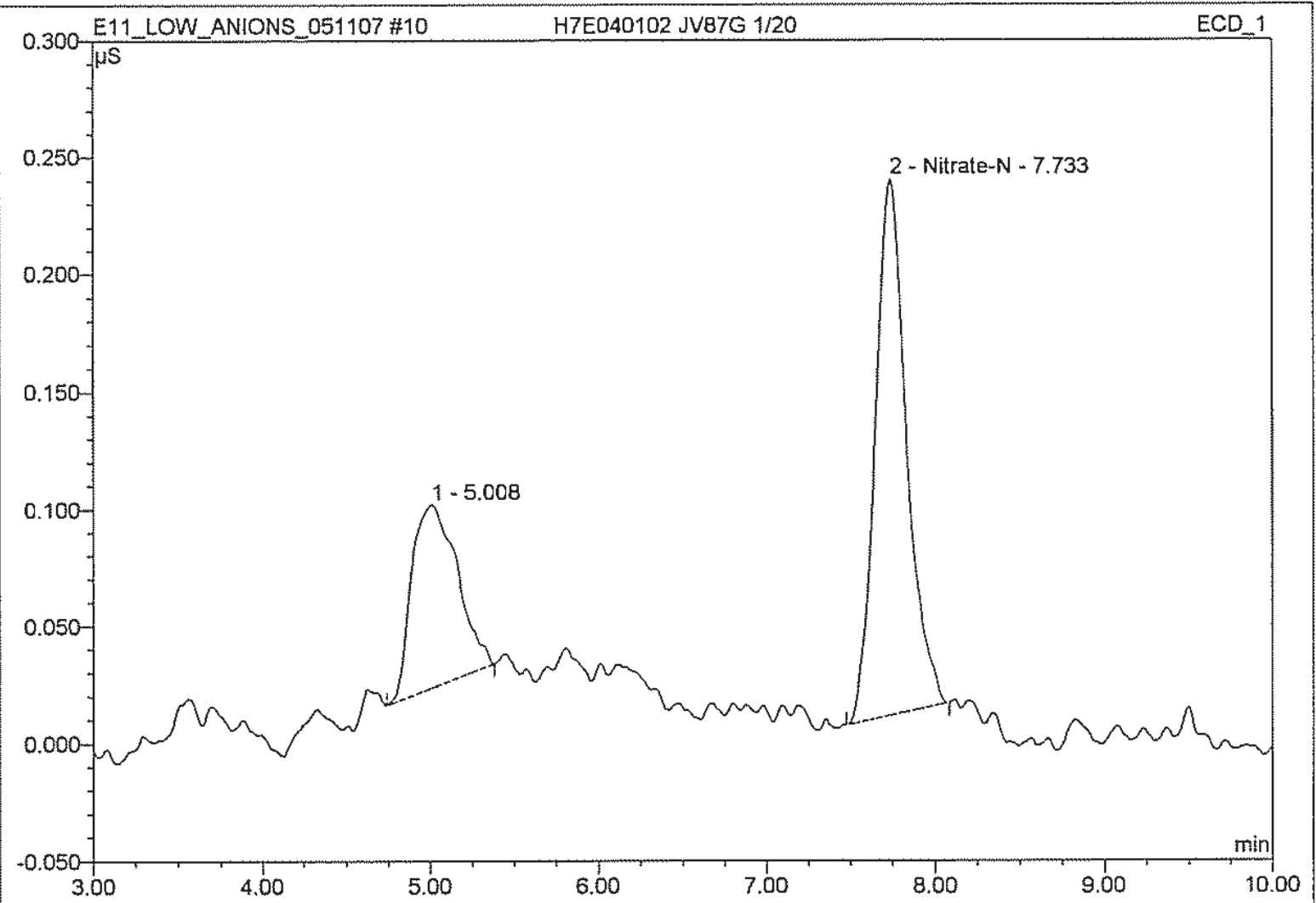

\begin{tabular}{|r|r|l|r|r|r|r|r|}
\hline No. & $\begin{array}{c}\text { Ret.Time } \\
\mathrm{min}\end{array}$ & \multicolumn{1}{|c|}{ Peak Name } & $\begin{array}{c}\text { Height } \\
\mu \mathrm{S}\end{array}$ & $\begin{array}{c}\text { Area } \\
\mu \mathrm{S}^{\star} \mathrm{min}\end{array}$ & $\begin{array}{c}\text { Rel.Area } \\
\%\end{array}$ & $\begin{array}{c}\text { Amount } \\
\mathrm{mg} / \mathrm{L}\end{array}$ & $\begin{array}{r}\text { Peak } \\
\text { Type }\end{array}$ \\
\hline 1 & 5.01 & n.a. & 0.078 & 0.025 & 0.08 & n.a. & BMB \\
\cline { 2 - 7 } 2 & 7.73 & Nitrate-N & 0.229 & 0.049 & 0.15 & 1.6023 & BMB \\
\hline 3 & 11.68 & n.a. & 100.826 & 32.375 & 99.77 & n.a. & BMB \\
\hline Total: & & & 101.133 & 32.449 & 100.00 & 1.602 & \\
\hline
\end{tabular}

$\left(\begin{array}{c}\text { sample soreming run (c) } 1 / 10 \\ \text { w/ ms unacceptable results }\end{array}\right)$ default_letter/Integration

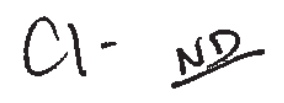

etw

Chromeleon (c) Dionex 1996-2001 Version 6.50 SP10a Build 1065 


\begin{tabular}{|llll|}
\hline 11 H7E040102 JV87G MS 1/20 0.2 PPM CL & & \\
QUAD & & & \\
\hline Sample Name: & H7E040102 JV87G MS 1/20 0.2 PPM CL & Injection Volume: & 50.0 \\
Vial Number: & 314 & Channel: & ECD_1 \\
Sample Type: & unknown & Wavelength: & n.a. \\
Control Program: & ANIONS_AS14A & Bandwidth: & n.a. \\
Quantif. Method: & ANIONS & Dilution Factor: & $\mathbf{2 0 . 0 0 0 0}$ \\
Recording Time: & $\mathbf{5 / 1 1 / 2 0 0 7 ~ 1 0 : 2 8}$ & Sample Weight: & $\mathbf{1 . 0 0 0 0}$ \\
Run Time (min): & $\mathbf{1 4 . 7 5}$ & Sample Amount: & $\mathbf{1 . 0 0 0 0}$ \\
\hline
\end{tabular}

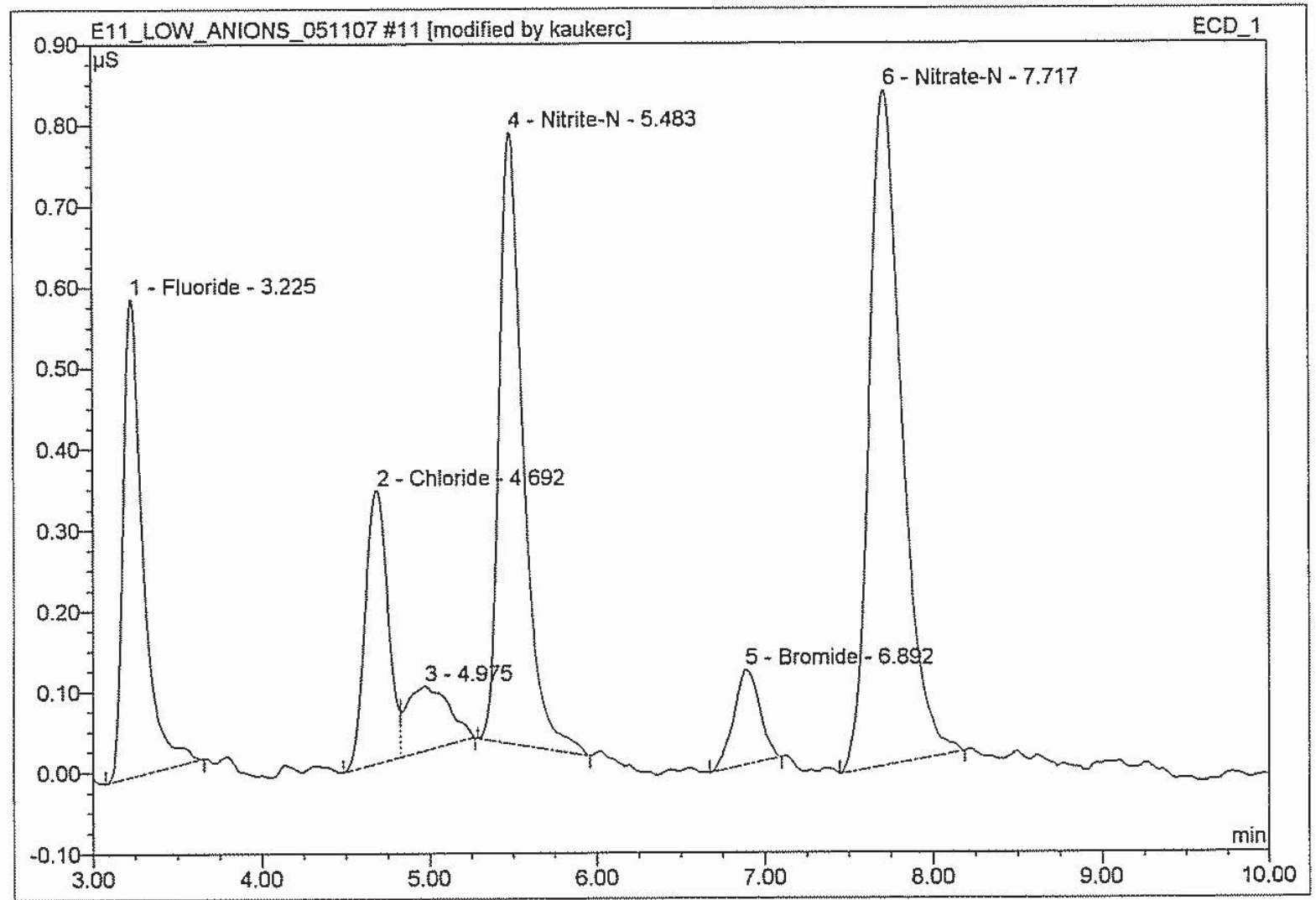

\begin{tabular}{|c|c|c|c|c|c|c|c|}
\hline No. & $\begin{array}{l}\text { Ret.Time } \\
\text { min }\end{array}$ & Peak Name & $\begin{array}{c}\text { Height } \\
\mu \mathrm{S}\end{array}$ & $\begin{array}{c}\text { Area } \\
\mu S^{*} \min \end{array}$ & $\begin{array}{c}\text { Rel.Area } \\
\% \\
\end{array}$ & $\begin{array}{c}\text { Amount } \\
\text { mg/L }\end{array}$ & $\begin{array}{l}\text { Peak } \\
\text { Type }\end{array}$ \\
\hline 1 & 3.23 & Fluoride & 0.591 & 0.081 & 0.25 & 4.2554 & $\mathrm{BMB}$ \\
\hline 2 & 4.69 & Chloride & 0.338 & 0.053 & 0.16 & 3.5536 & $\mathrm{BM}^{*} \mathrm{~V}$ \\
\hline 3 & 4.98 & n.a. & 0.080 & 0.024 & 0.07 & n.a. & $\mathrm{MB}^{*}$ \\
\hline 4 & 5.48 & Nitrite-N & 0.755 & 0.125 & 0.38 & 4.3834 & BMB \\
\hline 5 & 6.89 & Bromide & 0.117 & 0.021 & 0.06 & 4.0243 & BMB \\
\hline 6 & 7.72 & Nitrate-N & 0.834 & 0.188 & 0.57 & 5.7775 & BMB \\
\hline 7 & 11.67 & n.a. & 100.794 & 32.400 & 98.51 & ก.a. & $\mathrm{BMB}$ \\
\hline Total: & & & 103.509 & 32.892 & 100.00 & 21.994 & \\
\hline
\end{tabular}

(B) cuk 5/11/07 


\section{H7E040102 JV87G MS 1/20 0.2 PPM CL}

\section{QUAD}

\begin{tabular}{llll}
\hline Sample Name: & H7E040102 JV87G MS 1/20 0.2 PPM CL & Injection Volume: & $\mathbf{5 0 . 0}$ \\
Vial Number: & $\mathbf{3 1 4}$ & Channel: & ECD_1 \\
Sample Type: & unknown & Wavelength: & n.a. \\
Control Program: & ANIONS_AS14A & Bandwidth: & n.a. \\
Quantif. Method: & ANIONS & Dilution Factor: & $\mathbf{2 0 . 0 0 0 0}$ \\
Recording Time: & $\mathbf{5 / 1 1 / 2 0 0 7 ~ 1 0 : 2 8}$ & Sample Weight: & $\mathbf{1 . 0 0 0 0}$ \\
Run Time (min): & $\mathbf{1 4 . 7 5}$ & Sample Amount: & $\mathbf{1 . 0 0 0 0}$ \\
\hline
\end{tabular}

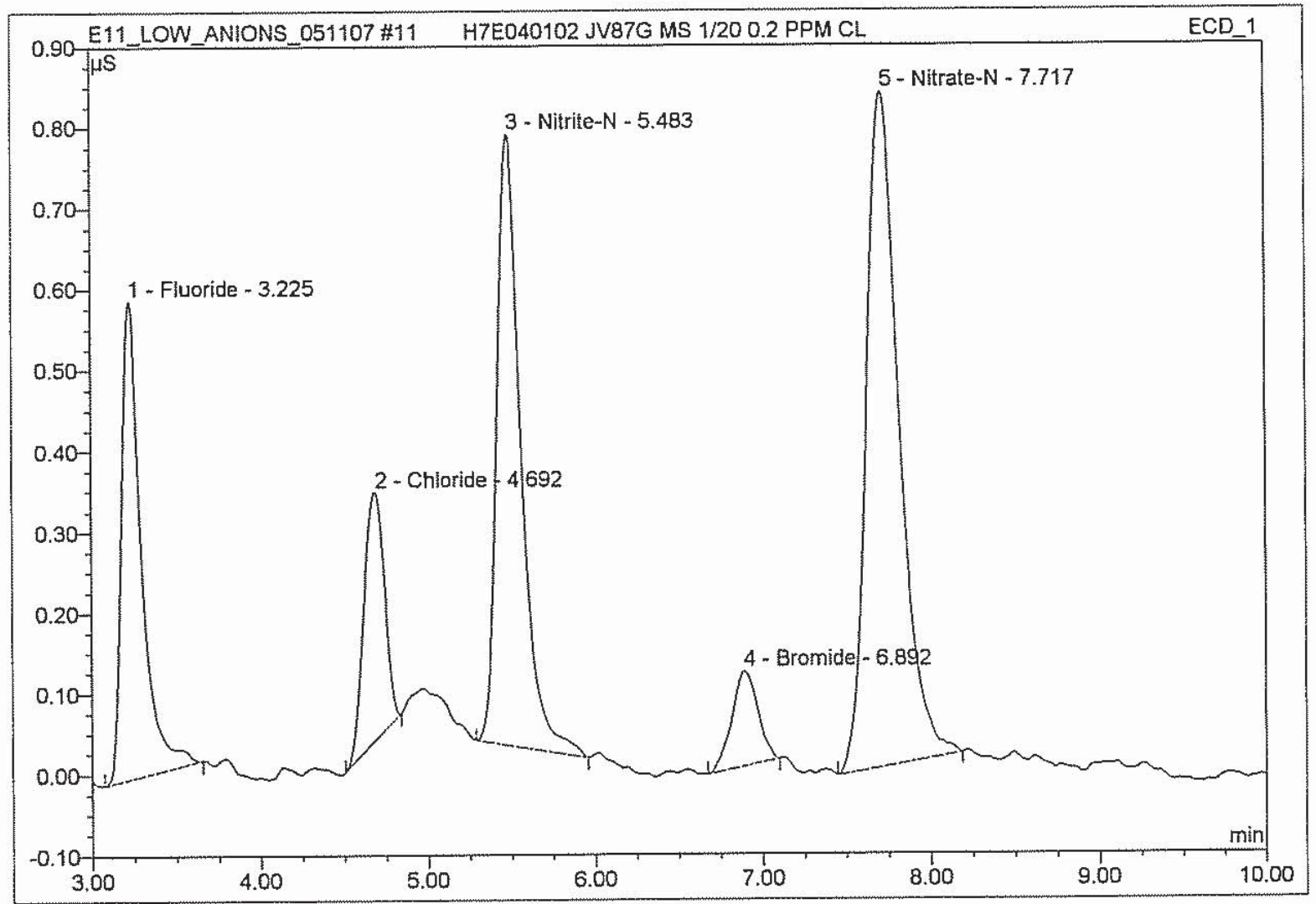

\begin{tabular}{|c|c|l|r|r|r|r|c|}
\hline No. & $\begin{array}{c}\text { Ret.Time } \\
\min \end{array}$ & \multicolumn{1}{|c|}{ Peak Name } & $\begin{array}{c}\text { Height } \\
\mu S\end{array}$ & $\begin{array}{c}\text { Area } \\
\mu S^{*} \min \end{array}$ & $\begin{array}{c}\text { Rel.Area } \\
\%\end{array}$ & $\begin{array}{c}\text { Amount } \\
\mathrm{mg} / \mathrm{L} .\end{array}$ & $\begin{array}{c}\text { Peak } \\
\text { Type }\end{array}$ \\
\hline 1 & 3.23 & Fluoride & 0.591 & 0.081 & 0.25 & 4.2554 & BMB \\
\hline 2 & 4.69 & Chloride & 0.306 & 0.044 & 0.13 & 3.2202 & BMB \\
\hline 3 & 5.48 & Nitrite-N & 0.755 & 0.125 & 0.38 & 4.3834 & BMB \\
\hline 4 & 6.89 & Bromide & 0.117 & 0.021 & 0.06 & 4.0243 & BMB \\
\hline 5 & 7.72 & Nitrate-N & 0.834 & 0.188 & 0.57 & 5.7775 & BMB \\
\hline 6 & 11.67 & n.a. & 100.794 & 32.400 & 98.60 & n.a. & BMB \\
\hline Total: & & & 103.398 & 32.859 & 100.00 & 21.661 & \\
\hline
\end{tabular}

origind 


\section{H7E040102 JV87G MSD 1/20 0.2 PPM CL}

\section{QUAD}

\begin{tabular}{llll|}
\hline Sample Name: & H7E040102 JV87G MSD 1/20 0.2 PPM CL & Injection Volume: & 50.0 \\
Vial Number: & $\mathbf{3 1 5}$ & Channel: & ECD_1 \\
Sample Type: & unknown & Wavelength: & n.a. \\
Control Program: & ANIONS_AS14A & Bandwidth: & n.a. \\
Quantif. Method: & ANIONS & Dilution Factor: & $\mathbf{2 0 . 0 0 0 0}$ \\
Recording Time: & $\mathbf{5 / 1 1 / 2 0 0 7 ~ 1 0 : 4 5}$ & Sample Weight: & 1.0000 \\
Run Time (min): & $\mathbf{1 4 . 7 5}$ & Sample Amount: & $\mathbf{1 . 0 0 0 0}$ \\
\hline
\end{tabular}

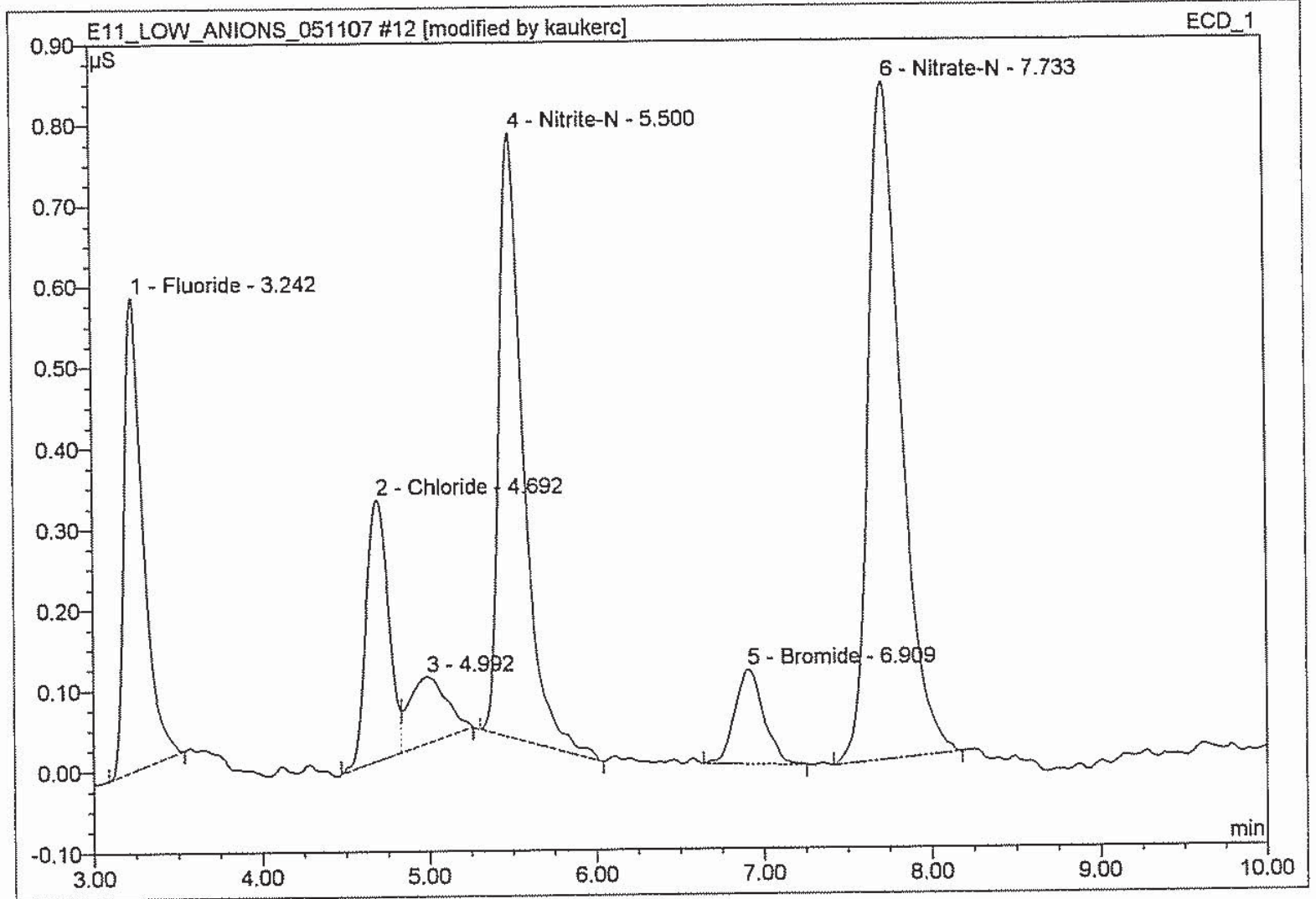

\begin{tabular}{|r|r|l|r|r|r|r|c|}
\hline No. & $\begin{array}{c}\text { Ret.Time } \\
\mathrm{min}\end{array}$ & \multicolumn{1}{|c|}{ Peak Name } & $\begin{array}{c}\text { Height } \\
\mu \mathrm{S}\end{array}$ & $\begin{array}{c}\text { Area } \\
\mu \mathrm{S}^{*} \mathrm{~min}\end{array}$ & $\begin{array}{c}\text { Rel.Area } \\
\%\end{array}$ & $\begin{array}{c}\text { Amount } \\
\mathrm{mg} / \mathrm{L}\end{array}$ & $\begin{array}{r}\text { Peak } \\
\text { Type }\end{array}$ \\
\hline 1 & 3.24 & Fluoride & 0.585 & 0.077 & 0.23 & 4.2106 & $\mathrm{BMB}$ \\
\cline { 2 - 8 } & 4.69 & Chloride & 0.324 & 0.050 & 0.15 & 3.4047 & $\mathrm{BM}^{*}$ \\
\hline 3 & 4.99 & n.a. & 0.082 & 0.022 & 0.07 & n.a. & $\mathrm{MB}^{*}$ \\
\hline 4 & 5.50 & Nitrite-N & 0.748 & 0.125 & 0.38 & 4.3422 & $\mathrm{BMB}^{*}$ \\
\hline 5 & 6.91 & Bromide & 0.117 & 0.023 & 0.07 & 3.9942 & $\mathrm{BMB}$ \\
\hline 6 & 7.73 & Nitrate-N & 0.838 & 0.189 & 0.57 & 5.8035 & $\mathrm{BMB}$ \\
\hline 7 & 11.68 & n.a. & 101.163 & 32.484 & 98.53 & n.a. & BMB \\
\hline Total: & & & 103.857 & 32.969 & 100.00 & 21.755 & \\
\hline
\end{tabular}

(B) ank $5 / 1107$ 


\section{H7E040102 JV87G MSD 1/20 0.2 PPM CL}

\section{QUAD}

Sample Name: H7E040102 JV87G MSD 1/20 0.2 PPM CL

Vial Number:

Sample Type:

Control Program:

Quantif. Method:

Recording Time:

Run Time (min):
315

unknown

ANIONS_AS14A

ANIONS

$5 / 11 / 2007$ 10:45

14.75

$\begin{array}{ll}\text { Injection Volume: } & \mathbf{5 0 . 0} \\ \text { Channel: } & \text { ECD_1 } \\ \text { Wavelength: } & \text { n.a. } \\ \text { Bandwidth: } & \text { n.a. } \\ \text { Dilution Factor: } & \mathbf{2 0 . 0 0 0 0} \\ \text { Sample Weight: } & \mathbf{1 . 0 0 0 0} \\ \text { Sample Amount: } & \mathbf{1 . 0 0 0 0}\end{array}$

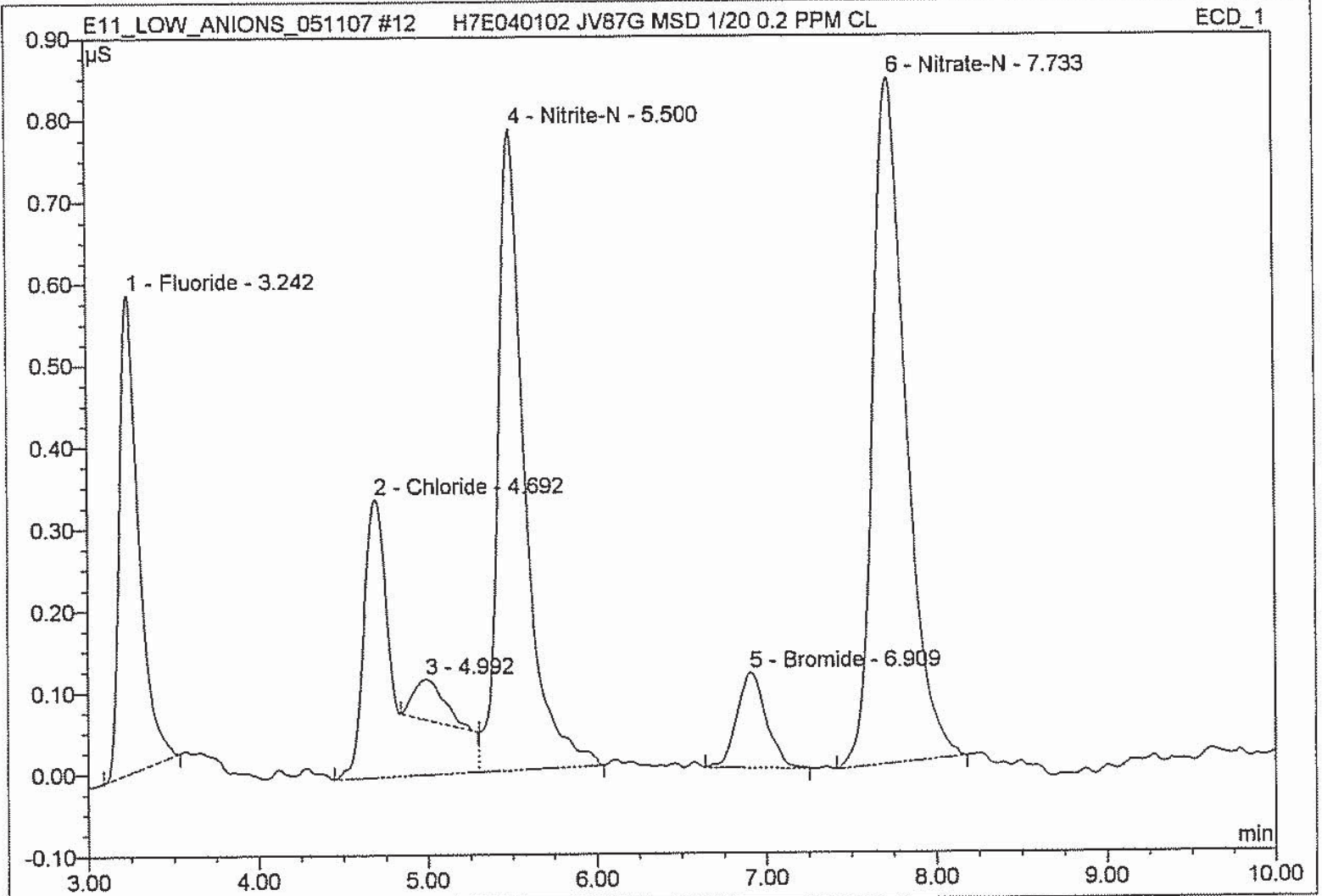

\begin{tabular}{|c|c|l|r|r|r|r|c|}
\hline No. & $\begin{array}{c}\text { Ret.Time } \\
\text { min }\end{array}$ & \multicolumn{1}{|c|}{ Peak Name } & $\begin{array}{c}\text { Height } \\
\mu S\end{array}$ & $\begin{array}{c}\text { Area } \\
\mu S^{*} \text { min }\end{array}$ & $\begin{array}{c}\text { Rel.Area } \\
\%\end{array}$ & $\begin{array}{c}\text { Amount } \\
\text { mg/L }\end{array}$ & $\begin{array}{r}\text { Peak } \\
\text { Type }\end{array}$ \\
\hline 1 & 3.24 & Fluoride & 0.585 & 0.077 & 0.23 & 4.2106 & BMB \\
\cline { 2 - 7 } 2 & 4.69 & Chloride & 0.340 & 0.084 & 0.25 & 3.5747 & BM \\
\hline 3 & 4.99 & n.a. & 0.050 & 0.011 & 0.03 & n.a. & Rd \\
\hline 4 & 5.50 & Nitrite-N & 0.784 & 0.142 & 0.43 & 4.5472 & MB \\
\hline 5 & 6.91 & Bromide & 0.117 & 0.023 & 0.07 & 3.9942 & BMB \\
\hline 6 & 7.73 & Nitrate-N & 0.838 & 0.189 & 0.57 & 5.8035 & BMB \\
\hline 7 & 11.68 & n.a. & 101.163 & 32.484 & 98.41 & n.a. & BMB \\
\hline Total: & & & 103.876 & 33.010 & 100.00 & 22.130 & \\
\hline
\end{tabular}

original

default_letter/Integration

Chromeleon (c) Dionex 1996-2001

Version 6.50 SP10a Build 1065 


\section{H7E040102 JV87E 1/20}

\section{QUAD}

Sample Name: H7E040102 JV87E 1/20

Vial Number: $\quad 316$

Sample Type:

Control Program:

Quantif. Method:

Recording Time:

Run Time (min): unknown

ANIONS_AS14A

ANIONS

5/11/2007 11:02

14.75
Injection Volume:

Channel:

Wavelength:

Bandwidth:

Dilution Factor:

Sample Weight:

Sample Amount:
50.0

ECD_1

n.a.

n.a.

20.0000

1.0000

1.0000

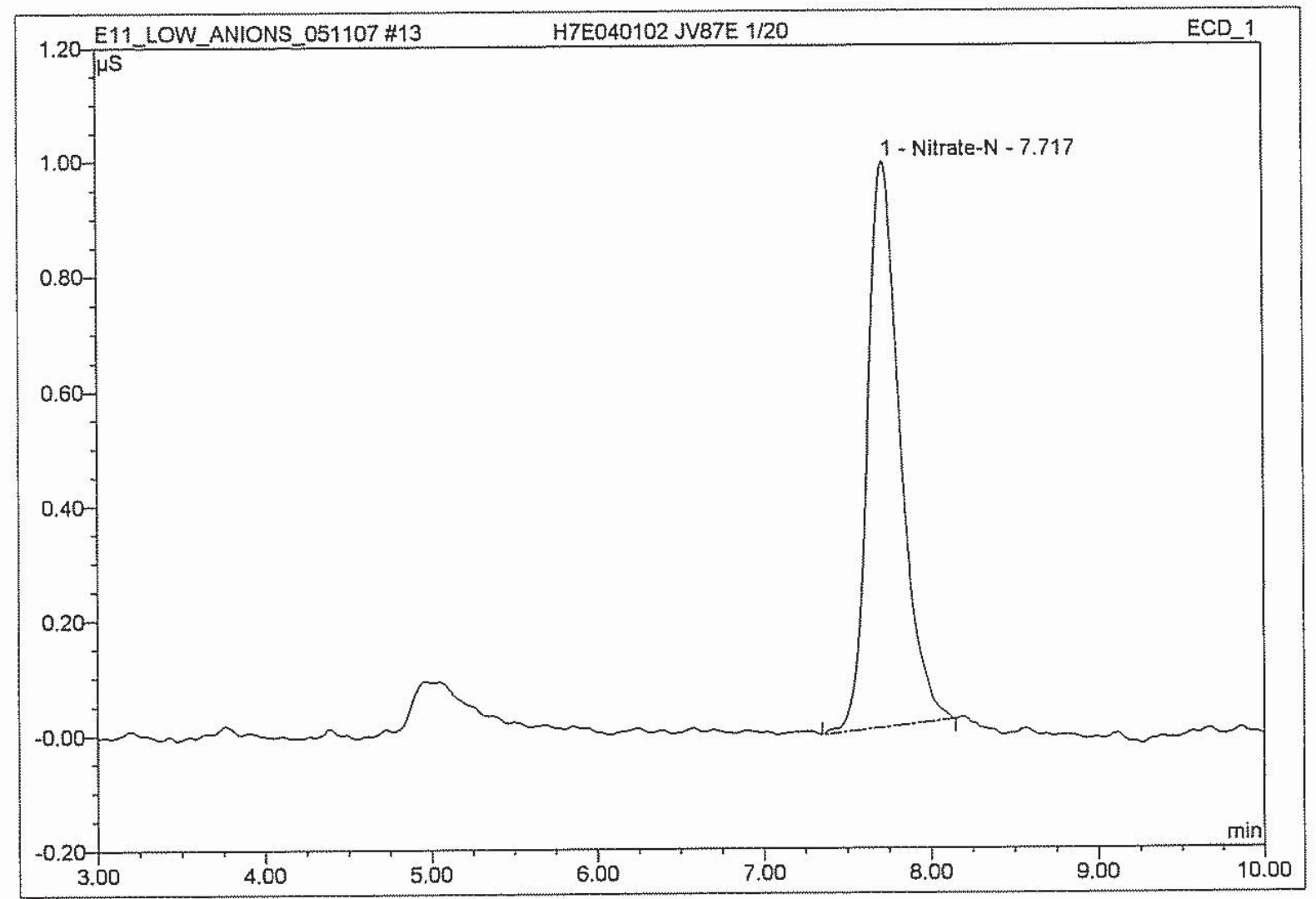

\begin{tabular}{|r|c|l|r|r|r|r|r|}
\hline No. & $\begin{array}{c}\text { Ret.Time } \\
\mathrm{min}\end{array}$ & \multicolumn{1}{|c|}{ Peak Name } & $\begin{array}{c}\text { Height } \\
\mu \mathrm{S}\end{array}$ & $\begin{array}{c}\text { Area } \\
\mu \mathrm{S}^{\star} \mathrm{min}\end{array}$ & $\begin{array}{c}\text { Rel.Area } \\
\%\end{array}$ & $\begin{array}{c}\text { Amount } \\
\mathrm{mg} / \mathrm{L}\end{array}$ & $\begin{array}{r}\text { Peak } \\
\text { Type }\end{array}$ \\
\hline 1 & 7.72 & Nitrate-N & 0.986 & 0.223 & 0.80 & 6.8085 & BMB \\
\hline 2 & 11.68 & n.a. & 86.175 & 27.478 & 99.20 & n.a. & BMB \\
\hline Total: & & & 87.161 & 27.701 & 100.00 & 6.808 & \\
\hline
\end{tabular}

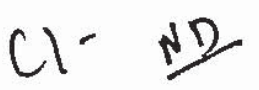

default_letter/Integration 


\section{H7E040102 JV87J 1/20}

\section{QUAD}

Sample Name: H7E040102 JV87J 1/20

Vial Number: $\quad 317$

Sample Type:

Control Program:

Quantif. Method:

Recording Time:

Run Time ( $\mathrm{min}$ ): unknown

ANIONS_AS14A

ANIONS

5/11/2007 11:19

14.75

$\begin{array}{ll}\text { Injection Volume: } & \mathbf{5 0 . 0} \\ \text { Channel: } & \text { ECD_1 } \\ \text { Wavelength: } & \text { n.a. } \\ \text { Bandwidth: } & \text { n.a. } \\ \text { Dilution Factor: } & 20.0000 \\ \text { Sample Weight: } & 1.0000 \\ \text { Sample Amount: } & 1.0000\end{array}$

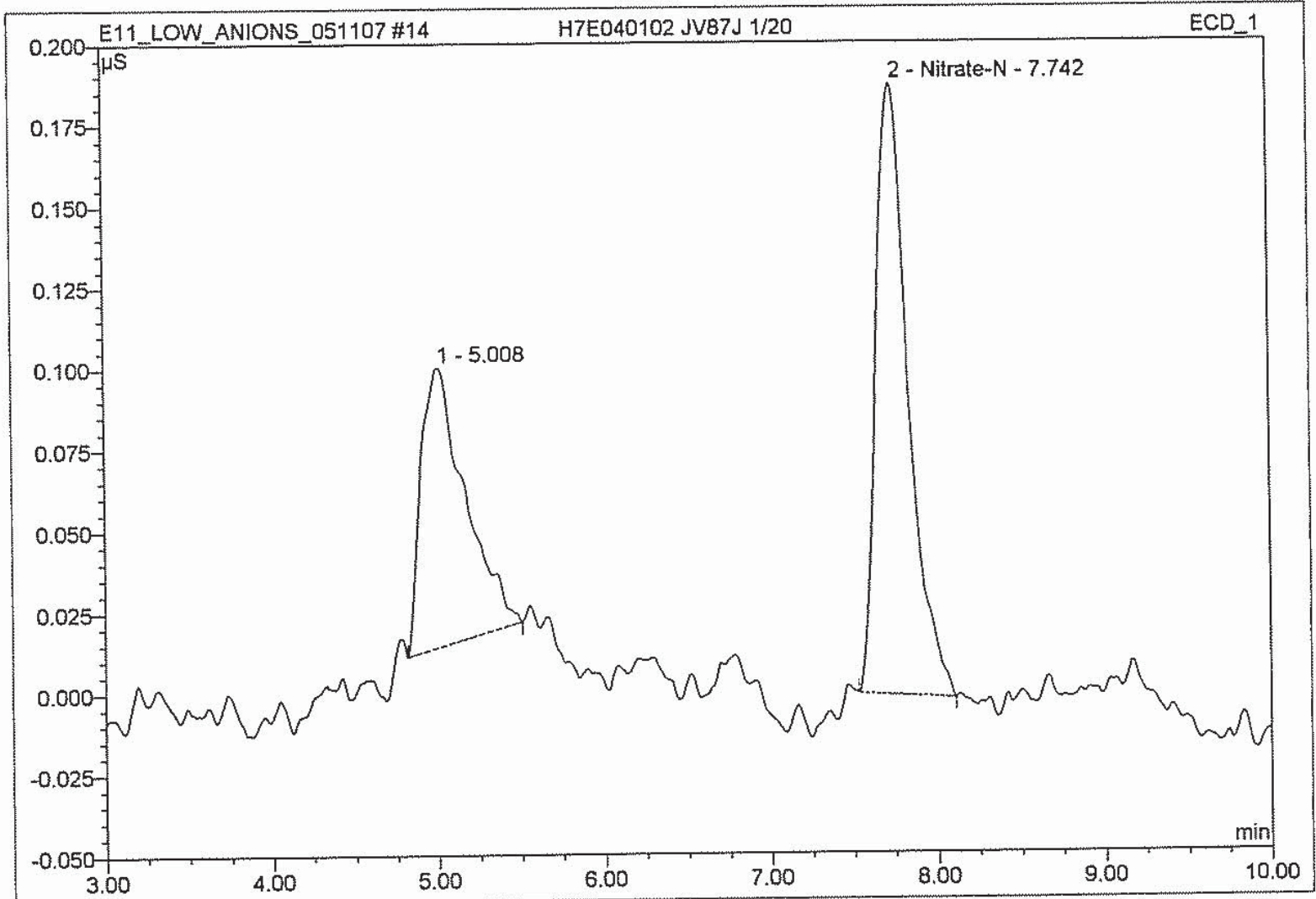

\begin{tabular}{|c|c|l|r|r|r|r|r|}
\hline No. & $\begin{array}{c}\text { Ret.Time } \\
\mathrm{min}\end{array}$ & \multicolumn{1}{|c|}{ Peak Name } & $\begin{array}{c}\text { Height } \\
\mu \mathrm{S}\end{array}$ & $\begin{array}{c}\text { Area } \\
\mu \mathrm{S}^{\star} \mathrm{min}\end{array}$ & $\begin{array}{c}\text { Rel.Area } \\
\%\end{array}$ & $\begin{array}{c}\text { Amount } \\
\mathrm{mg} / \mathrm{L}\end{array}$ & $\begin{array}{r}\text { Peak } \\
\text { Type }\end{array}$ \\
\hline 1 & 5.01 & n.a. & 0.086 & 0.027 & 0.09 & n.a. & BMB \\
\cline { 2 - 7 } & 7.74 & Nitrate-N & 0.188 & 0.042 & 0.14 & 1.3207 & BMB \\
\hline 3 & 11.68 & n.a. & 92.387 & 29.526 & 99.77 & n.a. & BMB \\
\hline Total: & & & 92.661 & 29.595 & 100.00 & 1.321 & \\
\hline
\end{tabular}




\section{H7E040102 JV87L 1/20}

\section{QUAD}

Sample Name: H7E040102 JV87L 1/20

Vial Number: $\quad 318$

Sample Type: unknown

Control Program: ANIONS_AS14A

Quantif. Method: ANIONS

Recording Time: $\quad$ 5/11/2007 11:36

Run Time (min): $\quad 14.75$

$\begin{array}{ll}\text { Injection Volume: } & \mathbf{5 0 . 0} \\ \text { Channel: } & \text { ECD_1 } \\ \text { Wavelength: } & \text { n.a. } \\ \text { Bandwidth: } & \text { n.a. } \\ \text { Dilution Factor: } & 20.0000 \\ \text { Sample Weight: } & 1.0000 \\ \text { Sample Amount: } & 1.0000\end{array}$

Injection Volume: $\quad \mathbf{5 0 . 0}$

Wavelength: $\quad$ n.a.

Bandwidth: n.a.

Dilution Factor: $\quad 20.0000$

Sample Amount: $\quad \mathbf{1 . 0 0 0 0}$

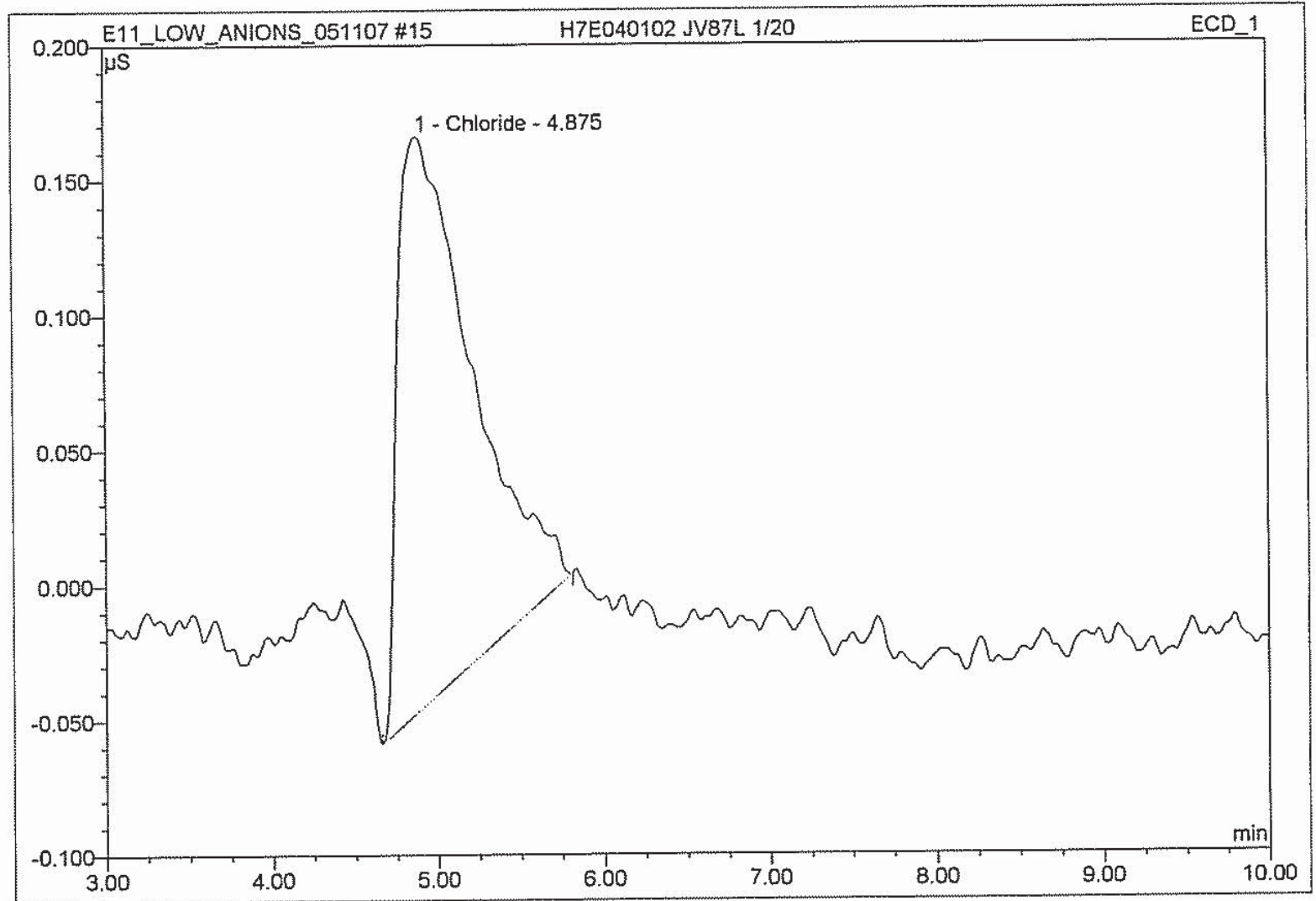

\begin{tabular}{|r|c|l|r|r|r|r|r|}
\hline No. & $\begin{array}{c}\text { Ret. Time } \\
\text { min }\end{array}$ & \multicolumn{1}{|c|}{ Peak Name } & $\begin{array}{c}\text { Height } \\
\mu S\end{array}$ & $\begin{array}{c}\text { Area } \\
\mu S^{*} \text { min }\end{array}$ & $\begin{array}{c}\text { Rel.Area } \\
\%\end{array}$ & $\begin{array}{c}\text { Amount } \\
\mathrm{mg} / \mathrm{L}\end{array}$ & $\begin{array}{r}\text { Peak } \\
\text { Type }\end{array}$ \\
\hline 1 & 4.88 & Chloride & 0.213 & 0.111 & 0.15 & 2.2455 & BMB \\
\hline 2 & 11.58 & n.a. & 216.215 & 75.199 & 99.85 & n.a. & BMB \\
\hline Total: & & & 216.428 & 75.310 & 100.00 & 2.246 & \\
\hline
\end{tabular}

\section{Peak Shape and RT do not match Chlonide Rewen at higher dilution}

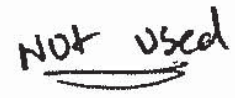




\section{H7E040102 JV87L MS 1/20 0.2 PPM CL}

\section{QUAD}

Sample Name: H7E040102 JV87L MS 1/20 0.2 PPM CL

Vial Number: $\quad \mathbf{3 1 8}$

Sample Type: unknown

Control Program: ANIONS_AS14A

Quantif. Method: ANIONS

Recording Time: $\quad$ 5/11/2007 11:54

Run Time (min): $\quad 14.75$

$\begin{array}{ll}\text { Injection Volume: } & \mathbf{5 0 . 0} \\ \text { Channel: } & \text { ECD_1 } \\ \text { Wavelength: } & \text { n.a. } \\ \text { Bandwidth: } & \text { n.a. } \\ \text { Dilution Factor: } & \mathbf{2 0 . 0 0 0 0} \\ \text { Sample Weight: } & 1.0000 \\ \text { Sample Amount: } & 1.0000\end{array}$

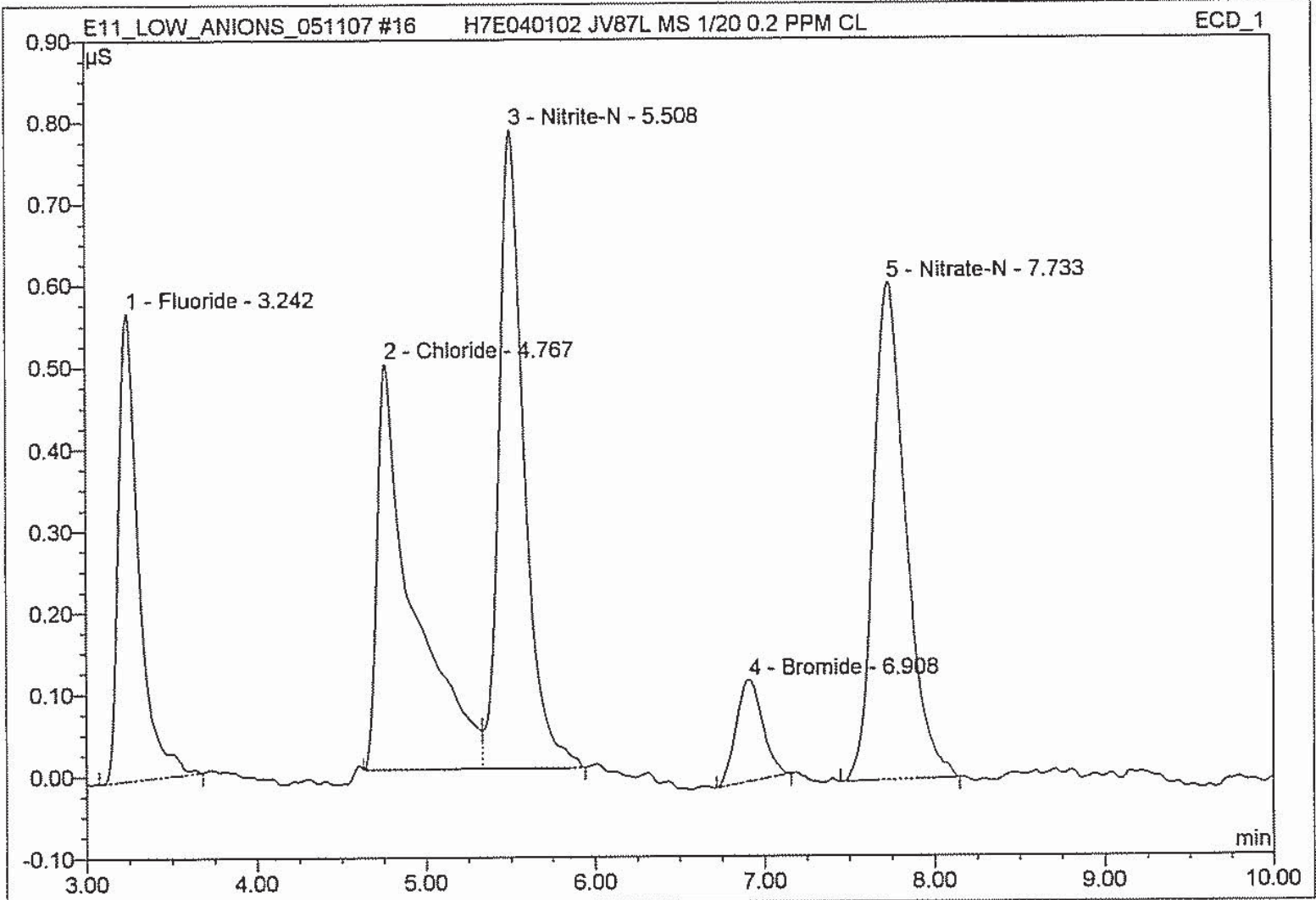

\begin{tabular}{|c|c|l|r|r|r|r|c|}
\hline No. & $\begin{array}{c}\text { Ret.Time } \\
\text { min }\end{array}$ & \multicolumn{1}{|c|}{ Peak Name } & $\begin{array}{c}\text { Height } \\
\mu S\end{array}$ & $\begin{array}{c}\text { Area } \\
\mu S^{*} \min \end{array}$ & $\begin{array}{c}\text { Rel.Area } \\
\%\end{array}$ & $\begin{array}{c}\text { Amount } \\
\text { mg/L }\end{array}$ & $\begin{array}{c}\text { Peak } \\
\text { Type }\end{array}$ \\
\hline 1 & 3.24 & Fluoride & 0.571 & 0.079 & 0.10 & 4.1138 & BMB \\
\hline 2 & 4.77 & Chloride & 0.496 & 0.122 & 0.16 & 5.2018 & BM \\
\hline 3 & 5.51 & Nitrite-N & 0.781 & 0.134 & 0.18 & 4.5297 & MB \\
\hline 4 & 6.91 & Bromide & 0.124 & 0.023 & 0.03 & 4.2477 & BMB \\
\hline 5 & 7.73 & Nitrate-N & 0.607 & 0.134 & 0.18 & 4.2255 & BMB \\
\hline 6 & 11.59 & n.a. & 216.202 & 75.125 & 99.35 & n.a. & BMB \\
\hline Total: & & & 218.781 & 75.616 & 100.00 & 22.319 & \\
\hline
\end{tabular}

default_letter/Integration

$N / 30 \% R$ - NOT HSED

Chromeleon (c) Dionex 1996-2001 Version 6.50 SP10a Build 1065 


\section{CCV ICWS-9564}

\section{QUAD}

Sample Name: $\quad$ CCV ICWS-9564

Vial Number: $\quad \mathbf{3 2 0}$

Sample Type: unknown

Control Program: ANIONS_AS14A

Quantif. Method: ANIONS

Recording Time: $\quad 5 / 11 / 2007$ 12:11

Run Time (min): $\quad 14.75$

$\begin{array}{ll}\text { Injection Volume: } & \mathbf{5 0 . 0} \\ \text { Channel: } & \text { ECD_1 } \\ \text { Wavelength: } & \text { n.a. } \\ \text { Bandwidth: } & \text { n.a. } \\ \text { Dilution Factor: } & \mathbf{1 . 0 0 0 0} \\ \text { Sample Weight: } & \mathbf{1 . 0 0 0 0} \\ \text { Sample Amount: } & \mathbf{1 . 0 0 0 0}\end{array}$

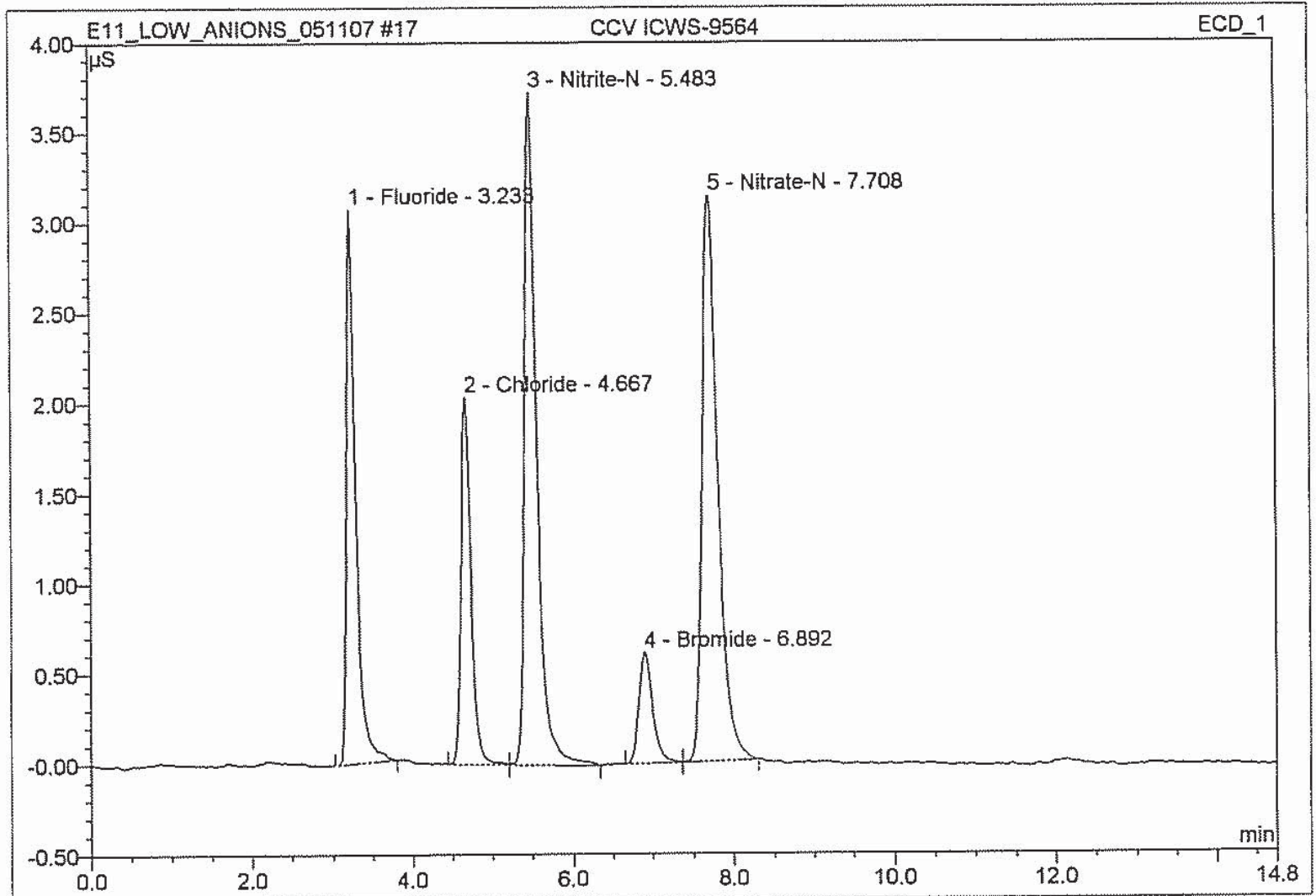

\begin{tabular}{|c|c|l|r|r|r|r|c|}
\hline No. & $\begin{array}{c}\text { Ret.Time } \\
\text { min }\end{array}$ & \multicolumn{1}{|c|}{ Peak Name } & $\begin{array}{c}\text { Height } \\
\mu S\end{array}$ & $\begin{array}{c}\text { Area } \\
\mu S^{*} \min \end{array}$ & $\begin{array}{c}\text { Rel.Area } \\
\%\end{array}$ & $\begin{array}{c}\text { Amount } \\
\text { mg/L }\end{array}$ & $\begin{array}{c}\text { Peak } \\
\text { Type }\end{array}$ \\
\hline 1 & 3.23 & Fluoride & 3.076 & 0.411 & 18.80 & 1.0512 & BMB \\
\cline { 2 - 7 } 2 & 4.67 & Chloride & 2.033 & 0.289 & 13.24 & 1.0378 & $\mathrm{BM}$ \\
\hline 3 & 5.48 & Nitrite-N & 3.728 & 0.650 & 29.75 & 1.0423 & $\mathrm{MB}$ \\
\hline 4 & 6.89 & Bromide & 0.616 & 0.122 & 5.58 & 1.0412 & $\mathrm{BMb}$ \\
\hline 5 & 7.71 & Nitrate-N & 3.139 & 0.713 & 32.64 & 1.0423 & $\mathrm{bMB}$ \\
\hline Total: & & & 12.592 & 2.186 & 100.00 & 5.215 & \\
\hline
\end{tabular}




\section{$18 \mathrm{CCB}$}

\section{QUAD}

Sample Name: $\quad$ CCB

Vial Number: $\quad 321$

Sample Type: $\quad$ unknown

Control Program: ANIONS_AS14A

Quantif. Method: ANIONS

Recording Time: $\quad 5 / 11 / 2007$ 12:28

Run Time (min): $\quad 14.75$

$\begin{array}{ll}\text { Injection Volume: } & 50.0 \\ \text { Channel: } & \text { ECD_1 } \\ \text { Wavelength: } & \text { n.a. } \\ \text { Bandwidth: } & \text { n.a. } \\ \text { Dilution Factor: } & 1.0000 \\ \text { Sample Weight: } & 1.0000 \\ \text { Sample Amount: } & 1.0000\end{array}$

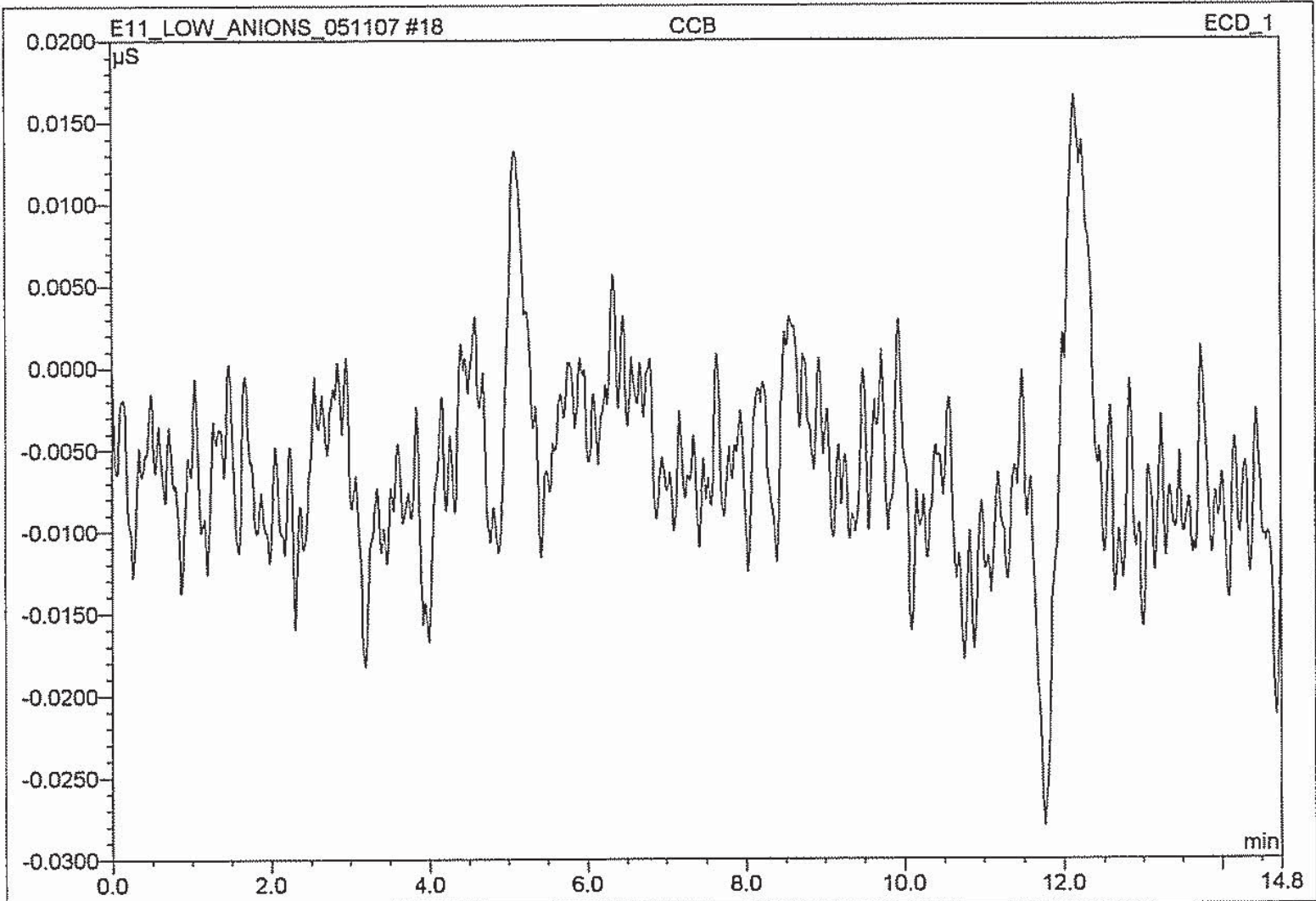

\begin{tabular}{|c|c|c|c|c|c|c|c|}
\hline No. & $\begin{array}{c}\text { Ret.Time } \\
\mathrm{min}\end{array}$ & Peak Name & $\begin{array}{c}\text { Height } \\
\mu \mathrm{S}\end{array}$ & $\begin{array}{c}\text { Area } \\
\mu \mathrm{S}^{*} \mathrm{~min}\end{array}$ & $\begin{array}{c}\text { Rel.Area } \\
\%\end{array}$ & $\begin{array}{c}\text { Amount } \\
\mathrm{mg} / \mathrm{L}\end{array}$ & $\begin{array}{c}\text { Peak } \\
\text { Type }\end{array}$ \\
\hline Total: & & & 0.000 & 0.000 & 0.00 & 0.000 & \\
\hline
\end{tabular}




\section{H7E040102 JV87L $1 / 40$}

\section{QUAD}

Sample Name: H7E040102 JV87L 1/40

Vial Number:

Sample Type:

Control Program:

Quantif. Method:

Recording Time:

Run Time (min):
358

unknown

ANIONS_AS14A

ANIONS

5/11/2007 12:45
14.75
Injection Volume:

Channel:

Wavelength:

Bandwidth:

Dilution Factor:

Sample Weight:

Sample Amount:
50.0

ECD_1

n.a.

n.a.

40.0000

1.0000

1.0000

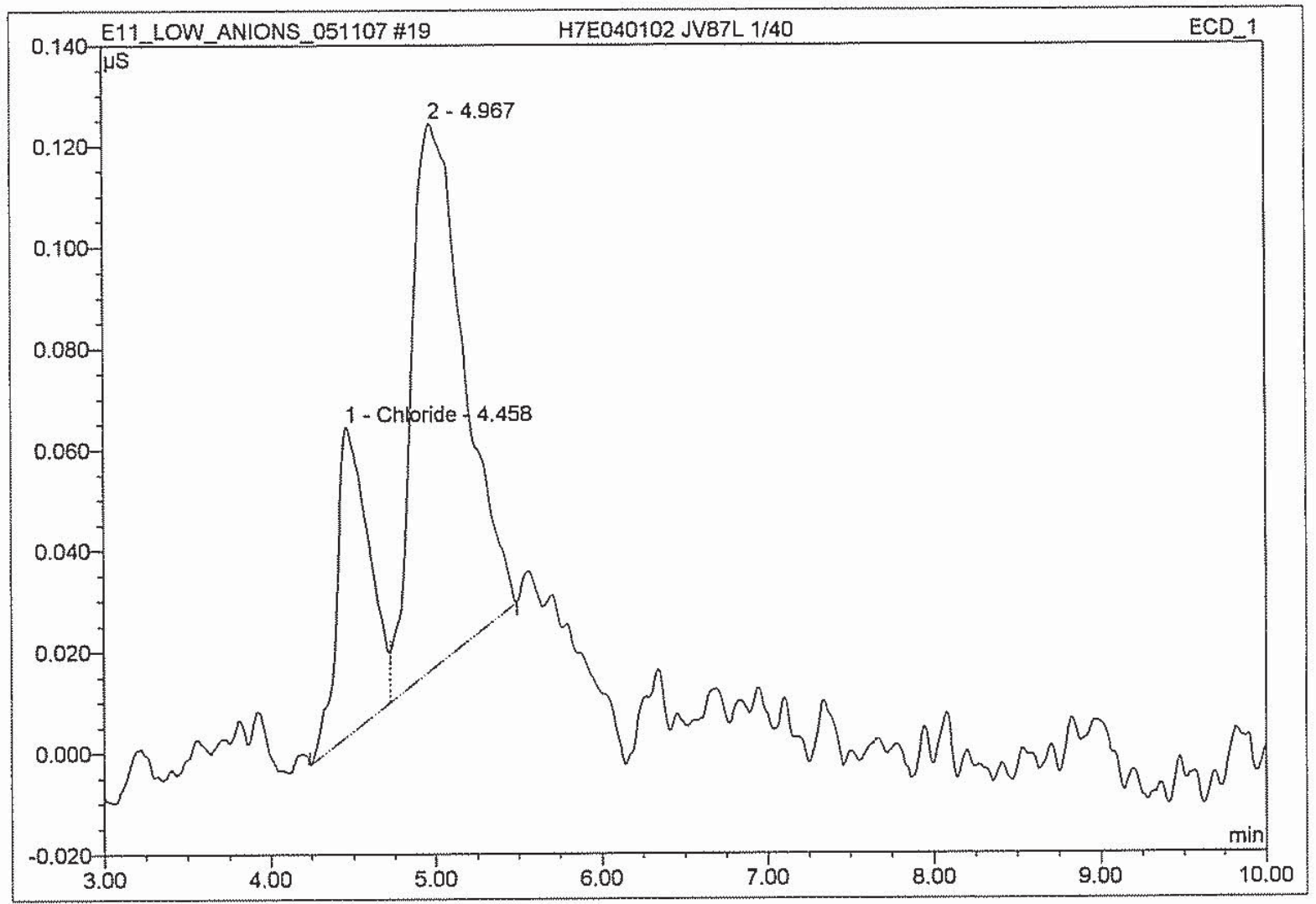

\begin{tabular}{|r|c|l|r|r|r|r|c|}
\hline No. & $\begin{array}{c}\text { Ret.Time } \\
\min \end{array}$ & \multicolumn{1}{|c|}{ Peak Name } & $\begin{array}{c}\text { Height } \\
\mu S\end{array}$ & $\begin{array}{c}\text { Area } \\
\mu S^{\star} \min \end{array}$ & $\begin{array}{c}\text { Rel.Area } \\
\%\end{array}$ & $\begin{array}{c}\text { Amount } \\
\text { mg/L }\end{array}$ & $\begin{array}{c}\text { Peak } \\
\text { Type }\end{array}$ \\
\hline 1 & 4.46 & Chloride & 0.061 & 0.014 & 0.04 & 1.2927 & BM \\
\cline { 2 - 8 } 2 & 4.97 & n.a. & 0.108 & 0.040 & 0.11 & n.a. & MB \\
\hline 3 & 11.68 & n.a. & 107.880 & 34.874 & 99.85 & n.a. & BMB \\
\hline Total: & & & 108.050 & 34.927 & 100.00 & 1.293 & \\
\hline
\end{tabular}




\section{H7E040102 JV87L MS 1/40 0.2 PPM CL}

\section{QUAD}

\begin{tabular}{|llll|}
\hline Sample Name: & H7E040102 JV87L MS 1/40 0.2 PPM CL & Injection Volume: & 50.0 \\
Vial Number: & 359 & Channel: & ECD_1 \\
Sample Type: & unknown & Wavelength: & n.a. \\
Control Program: & ANIONS_AS14A & Bandwidth: & n.a. \\
Quantif. Method: & ANIONS & Dilution Factor: & 40.0000 \\
Recording Time: & $\mathbf{5 / 1 1 / 2 0 0 7 ~ 1 3 : 0 2}$ & Sample Weight: & 1.0000 \\
Run Time (min): & $\mathbf{1 4 . 7 5}$ & Sample Amount: & $\mathbf{1 . 0 0 0 0}$ \\
\hline
\end{tabular}

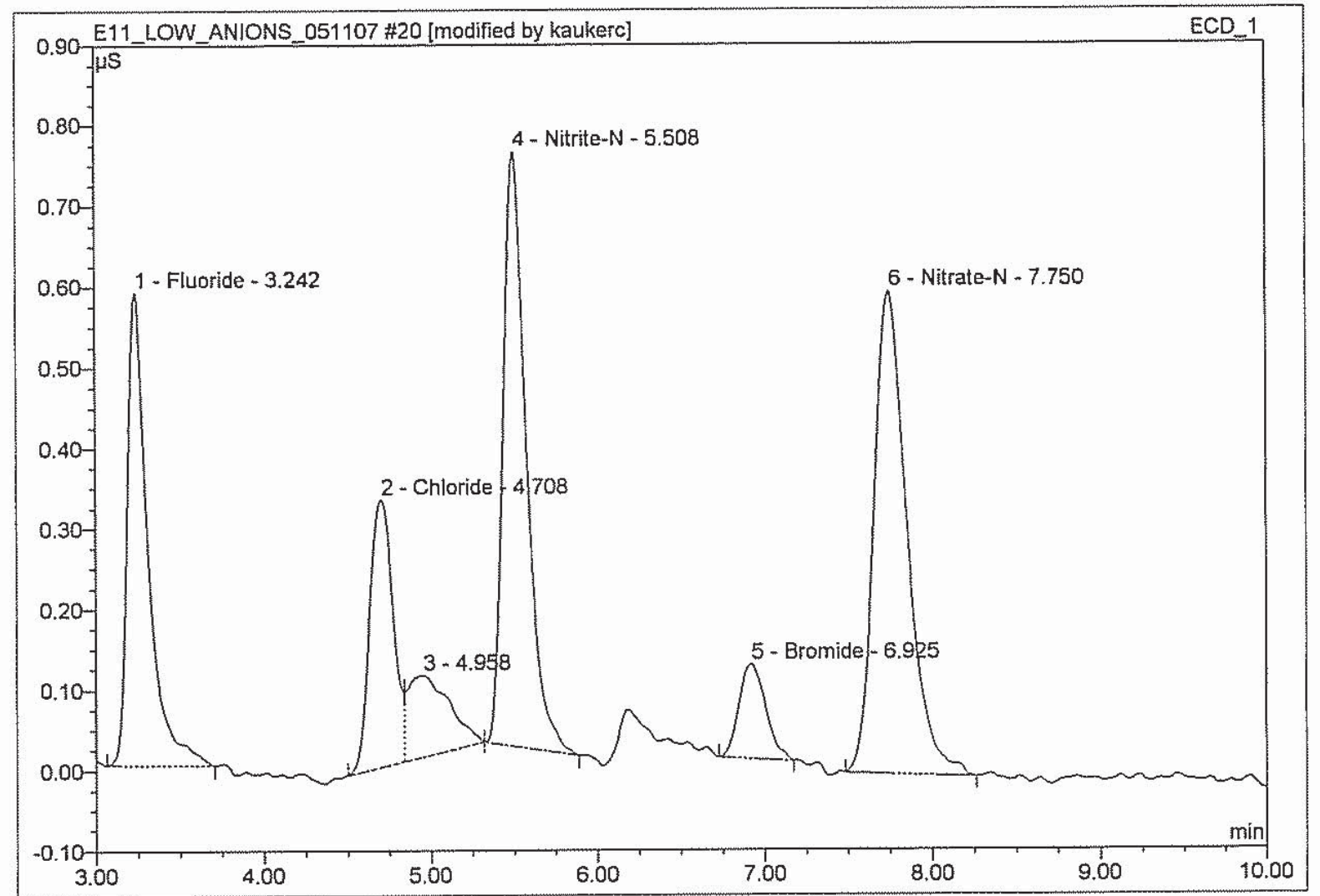

\begin{tabular}{|r|r|l|r|r|r|r|c|}
\hline No. & $\begin{array}{c}\text { Ret.Time } \\
\mathrm{min}\end{array}$ & \multicolumn{1}{|c|}{ Peak Name } & $\begin{array}{c}\text { Height } \\
\mu \mathrm{S}\end{array}$ & $\begin{array}{c}\text { Area } \\
\mu \mathrm{S}^{*} \min \end{array}$ & $\begin{array}{c}\text { Rel.Area } \\
\%\end{array}$ & $\begin{array}{c}\text { Amount } \\
\mathrm{mg} / \mathrm{L}\end{array}$ & $\begin{array}{c}\text { Peak } \\
\text { Type }\end{array}$ \\
\hline 1 & 3.24 & Fluoride & 0.586 & 0.084 & 0.24 & 8.4451 & $\mathrm{BMB}$ \\
\hline & 4.71 & Chloride & 0.332 & 0.054 & 0.16 & 6.9852 & $\mathrm{BM}^{*}$ \\
\hline 3 & 4.96 & n.a. & 0.101 & 0.029 & 0.08 & n.a. & $\mathrm{Mb}^{*}$ \\
\hline 4 & 5.51 & Nitrite-N & 0.737 & 0.118 & 0.34 & 8.5601 & bMB $^{*}$ \\
\hline 5 & 6.93 & Bromide & 0.117 & 0.022 & 0.06 & 8.0237 & BMB \\
\hline 6 & 7.75 & Nitrate-N & 0.597 & 0.136 & 0.39 & 8.3143 & BMB \\
\hline 7 & 11.68 & n.a. & 106.245 & 34.294 & 98.72 & n.a. & BMB \\
\hline Total: & & & 108.716 & 34.738 & 100.00 & 40.328 & \\
\hline
\end{tabular}

(B) avk $5 / 1107$

default_letter/Integration 


\section{H7E040102 JV87L MS 1/40 0.2 PPM CL}

\section{QUAD}

Sample Name: $\quad$ H7E040102 JV87L MS 1/40 0.2 PPM CL

Vial Number:

Sample Type:

Control Program:

Quantif. Method:

Recording Time:

Run Time (min):
359

unknown

ANIONS_AS14A

ANIONS

5/11/2007 13:02

14.75
Injection Volume:

Channel:

Wavelength:

Bandwidth:

Dilution Factor:

Sample Weight:

Sample Amount:
50.0

ECD_1

n.a.

n.a.

40.0000

1.0000

1.0000

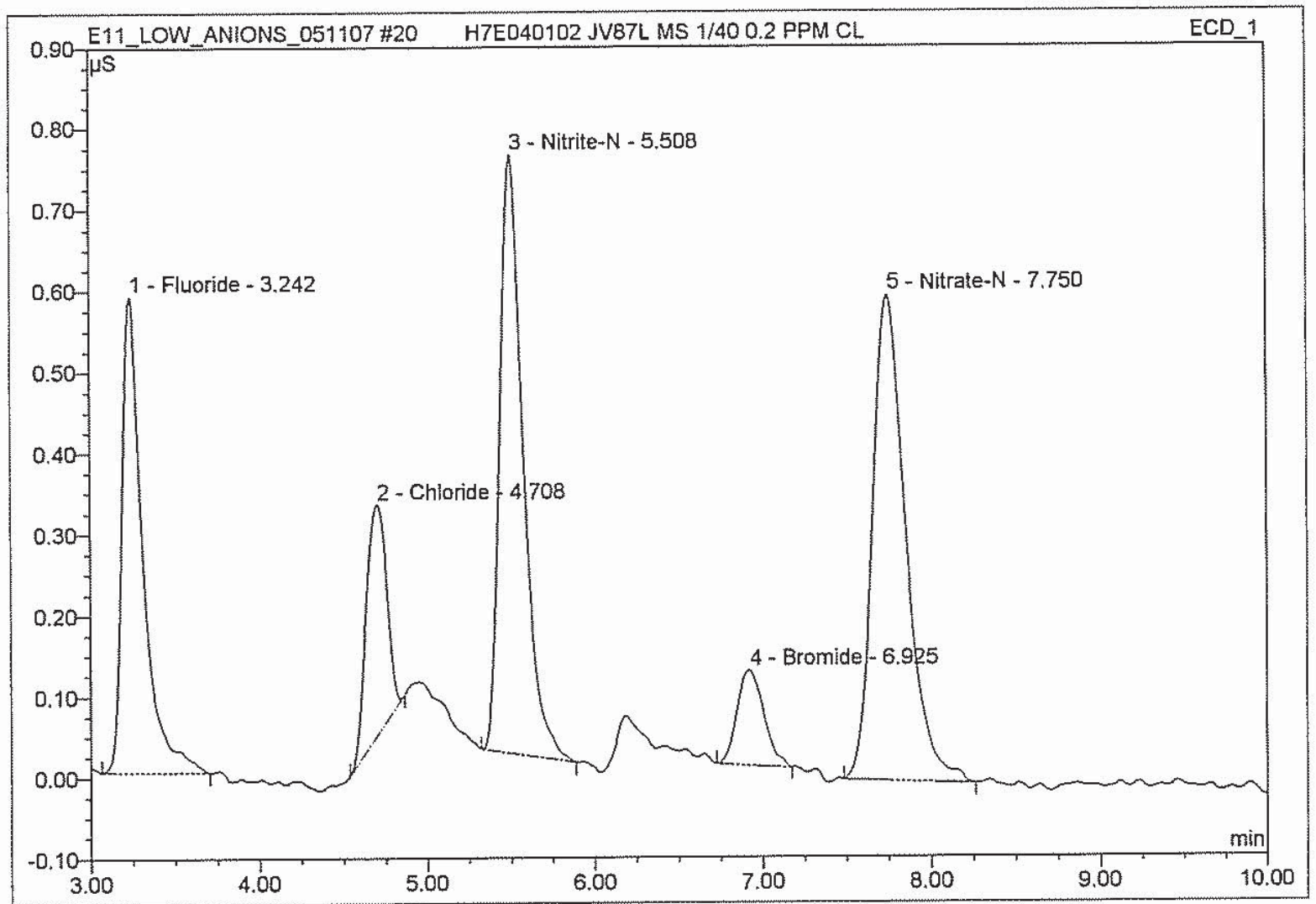

\begin{tabular}{|c|c|l|r|r|r|r|c|}
\hline No. & $\begin{array}{c}\text { Ret.Time } \\
\text { min }\end{array}$ & \multicolumn{1}{|c|}{ Peak Name } & $\begin{array}{c}\text { Height } \\
\mu S\end{array}$ & $\begin{array}{c}\text { Area } \\
\mu S^{*} \min \end{array}$ & $\begin{array}{c}\text { Rel.Area } \\
\%\end{array}$ & $\begin{array}{c}\text { Amount } \\
\text { mg/L }\end{array}$ & $\begin{array}{c}\text { Peak } \\
\text { Type }\end{array}$ \\
\hline 1 & 3.24 & Fluoride & 0.586 & 0.084 & 0.24 & 8.4451 & BMB \\
\hline 2 & 4.71 & Chloride & 0.283 & 0.040 & 0.12 & 5.9506 & BMB \\
\hline 3 & 5.51 & Nitrite-N & 0.737 & 0.118 & 0.34 & 8.5601 & BMB \\
\hline 4 & 6.93 & Bromide & 0.117 & 0.022 & 0.06 & 8.0237 & BMB \\
\hline 5 & 7.75 & Nitrate-N & 0.597 & 0.136 & 0.39 & 8.3143 & BMB \\
\hline 6 & 11.68 & n.a. & 106.245 & 34.294 & 98.85 & n.a. & BMB \\
\hline Total: & & & 108.565 & 34.695 & 100.00 & 39.294 & \\
\hline
\end{tabular}

onginal 


\section{H7E040102 JV87H 1/20}

\section{QUAD}

\begin{tabular}{llll}
\hline Sample Name: & H7E040102 JV87H 1/20 & Injection Volume: & 50.0 \\
Vial Number: & 360 & Channel: & ECD_1 \\
Sample Type: & unknown & Wavelength: & n.a. \\
Control Program: & ANIONS_AS14A_CI2 & Bandwidth: & n.a. \\
Quantif. Method: & ANIONS & Dilution Factor: & 20.0000 \\
Recording Time: & $5 / 11 / 200713: 24$ & Sample Weight: & 1.0000 \\
Run Time (min): & 30.00 & Sample Amount: & 1.0000 \\
\hline
\end{tabular}

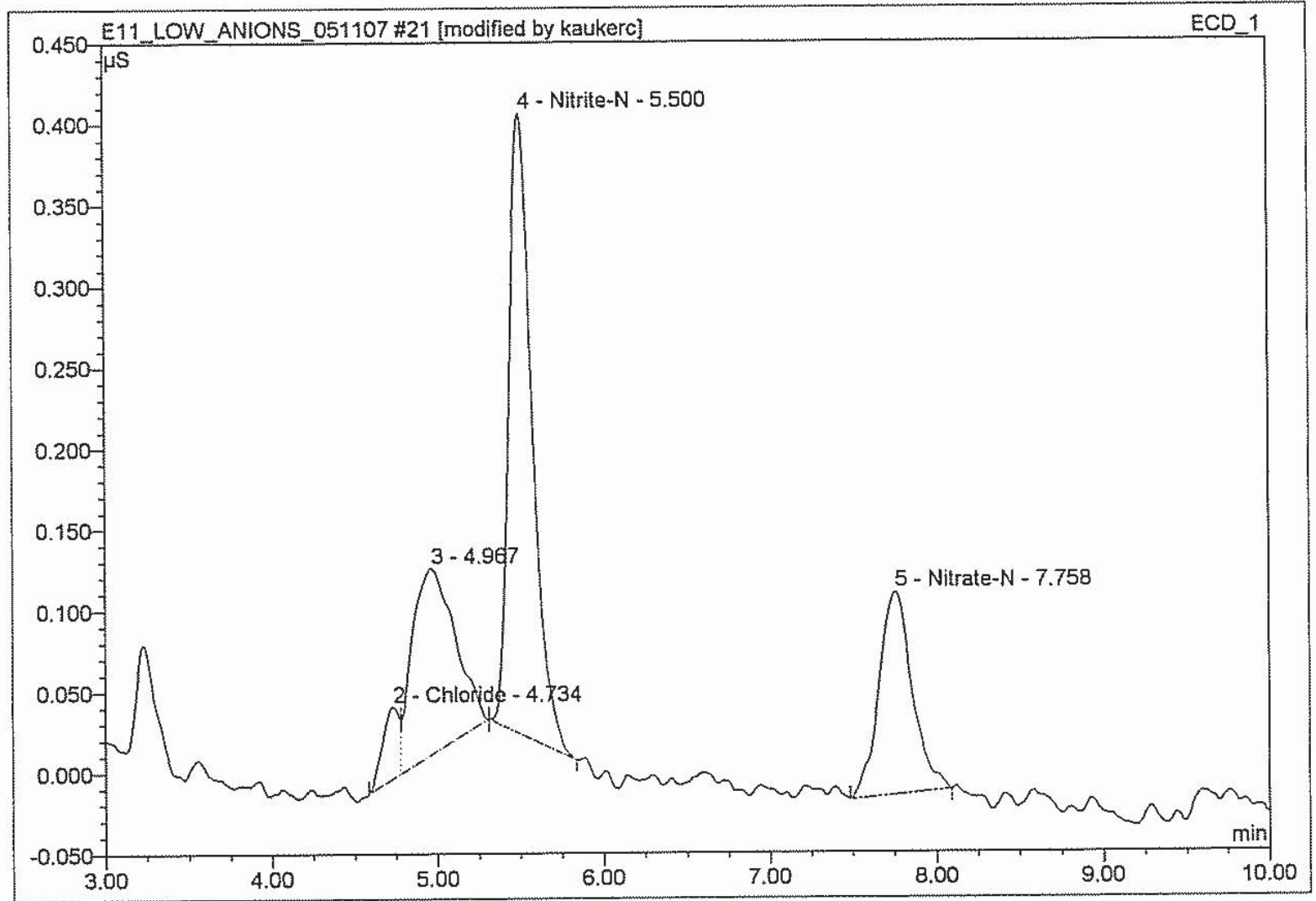

\begin{tabular}{|c|c|c|c|c|c|c|c|}
\hline No. & $\begin{array}{c}\text { Ret.Time } \\
\text { min }\end{array}$ & Peak Name & $\begin{array}{c}\text { Height } \\
\mu \mathrm{S}\end{array}$ & $\begin{array}{c}\text { Area } \\
\mu S^{\star} \min \end{array}$ & $\begin{array}{c}\text { Rel.Area } \\
\%\end{array}$ & $\begin{array}{c}\text { Amount } \\
\mathrm{mg} / \mathrm{L}\end{array}$ & $\begin{array}{l}\text { Peak } \\
\text { Type }\end{array}$ \\
\hline 1 & 2.23 & n.a. & 1.272 & 0.198 & 0.34 & n.a. & $\mathrm{BMB}$ \\
\hline 2 & 4.73 & Chloride & 0.044 & 0.005 & 0.01 & 0.4646 & $\mathrm{BM}^{*}$ \\
\hline 3 & 4.97 & n.a. & 0.114 & 0.035 & 0.06 & n.a. & $\mathrm{Mb}^{*}$ \\
\hline 4 & 5.50 & Nitrite-N & 0.383 & 0.062 & 0.11 & 2.2318 & $\mathrm{bMB}^{*}$ \\
\hline 5 & 7.76 & Nitrate-N & 0.124 & 0.029 & 0.05 & 0.8746 & $\mathrm{BMB}$ \\
\hline 6 & 11.74 & n.a. & 15.648 & 5.013 & 8.58 & n.a. & $\mathrm{BMB}$ \\
\hline 7 & 21.43 & n.a. & 60.010 & 53.084 & 90.86 & n.a. & BMB \\
\hline Total: & & & 77.596 & 58.426 & 100.00 & 3.571 & \\
\hline
\end{tabular}

(No scpardion at
$1 / 1$ s screeniug rum )
(B) $\dot{q}($ a

cwe $5(11 / 0)$
Chromeleon (c) Dionex 1996-2001

Version 6.50 SP10a Build 1065 


\section{H7E040102 JV87H 1/20}

\section{QUAD}

Sample Name: H7E040102 JV87H 1/20

Vial Number: $\quad \mathbf{3 6 0}$

Sample Type: unknown

Control Program: ANIONS_AS14A_Cl2

Quantif. Method: ANIONS

Recording Time: $\quad 5 / 11 / 2007$ 13:24

Run Time (min): $\quad \mathbf{3 0 . 0 0}$

$\begin{array}{ll}\text { Injection Volume: } & \mathbf{5 0 . 0} \\ \text { Channel: } & \text { ECD_1 } \\ \text { Wavelength: } & \text { n.a. } \\ \text { Bandwidth: } & \text { n.a. } \\ \text { Dilution Factor: } & \mathbf{2 0 . 0 0 0 0} \\ \text { Sample Weight: } & \mathbf{1 . 0 0 0 0} \\ \text { Sample Amount: } & \mathbf{1 . 0 0 0 0}\end{array}$

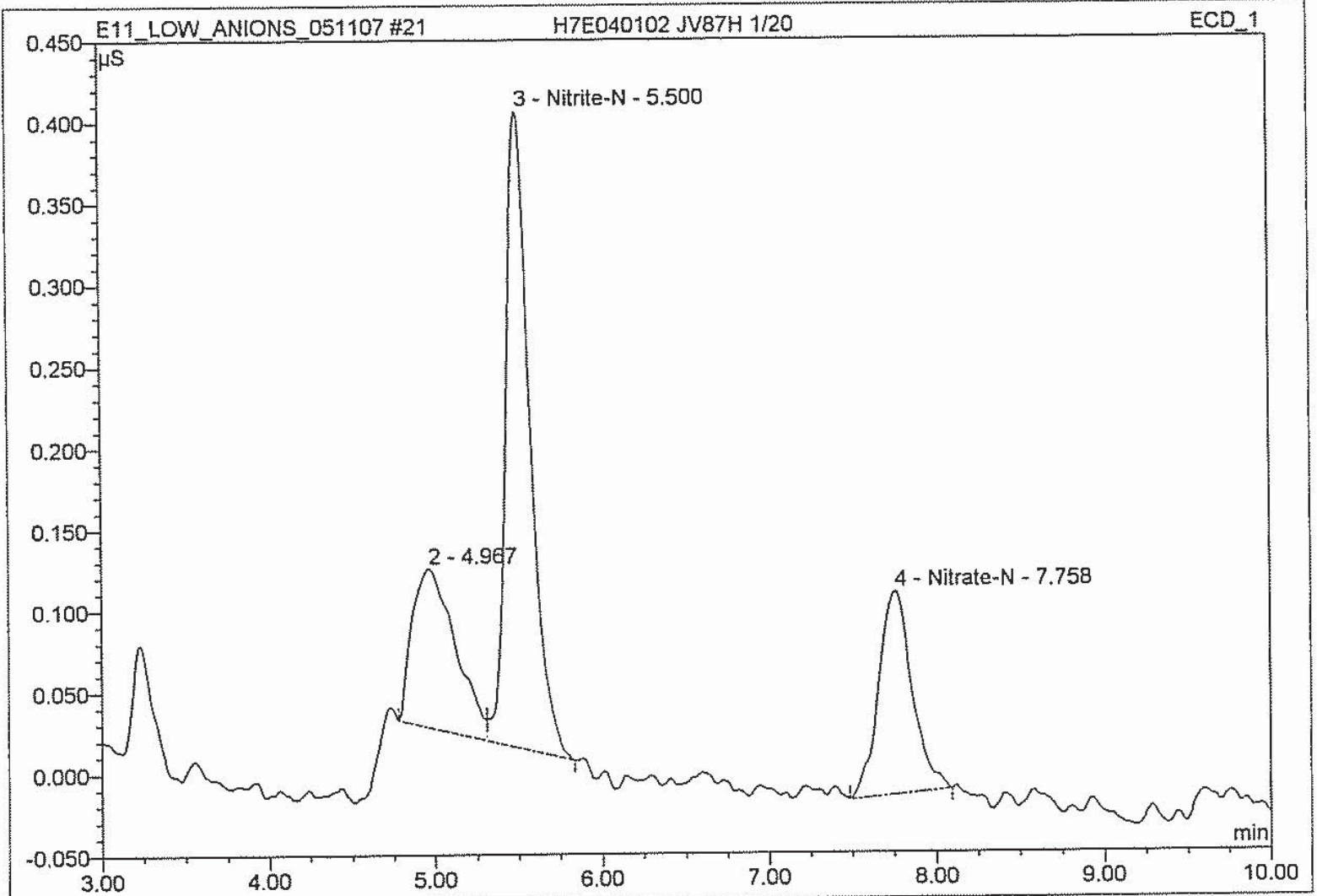

\begin{tabular}{|r|r|l|r|r|r|r|c|}
\hline No. & $\begin{array}{c}\text { Ret.Time } \\
\text { min }\end{array}$ & \multicolumn{1}{|c|}{ Peak Name } & $\begin{array}{c}\text { Height } \\
\mu S\end{array}$ & $\begin{array}{c}\text { Area } \\
\mu S^{*} \min \end{array}$ & $\begin{array}{c}\text { Rel.Area } \\
\%\end{array}$ & $\begin{array}{c}\text { Amount } \\
\text { mg/L }\end{array}$ & $\begin{array}{r}\text { Peak } \\
\text { Type }\end{array}$ \\
\hline 1 & 2.23 & n.a. & 1.272 & 0.198 & 0.34 & n.a. & BMB \\
\hline 2 & 4.97 & n.a. & 0.097 & 0.029 & 0.05 & n.a. & BM \\
\hline 3 & 5.50 & Nitrite-N & 0.391 & 0.065 & 0.11 & 2.2795 & MB \\
\hline 4 & 7.76 & Nitrate-N & 0.124 & 0.029 & 0.05 & 0.8746 & BMB \\
\hline 5 & 11.74 & n.a. & 15.648 & 5.013 & 8.58 & n.a. & BMB \\
\hline 6 & 21.43 & n.a. & 60.010 & 53.084 & 90.87 & n.a. & BMB \\
\hline Total: & & & 77.543 & 58.418 & 100.00 & 3.154 & \\
\hline
\end{tabular}

onginal

default_letter/Integration

Chromeleon (c) Dionex 1996-2001

Version 6.50 SP10a Build 1065 


\section{H7E040102 JV87H MS 1/20 0.2 PPM CL}

\section{QUAD}

Sample Name: $\quad$ H7E040102 JV87H MS 1/20 0.2 PPM CL

Vial Number:

Sample Type:

Control Program:

Quantif. Method:

Recording Time:

Run Time (min):
361

unknown

ANIONS_AS14A_Cl2

ANIONS

$5 / 11 / 2007$ 13:57

30.00
Injection Volume:

Channel:

Wavelength:

Bandwidth:

Dilution Factor:

Sample Weight:

Sample Amount:
50.0

ECD_1

n.a.

n.a.

20.0000

1.0000

1.0000

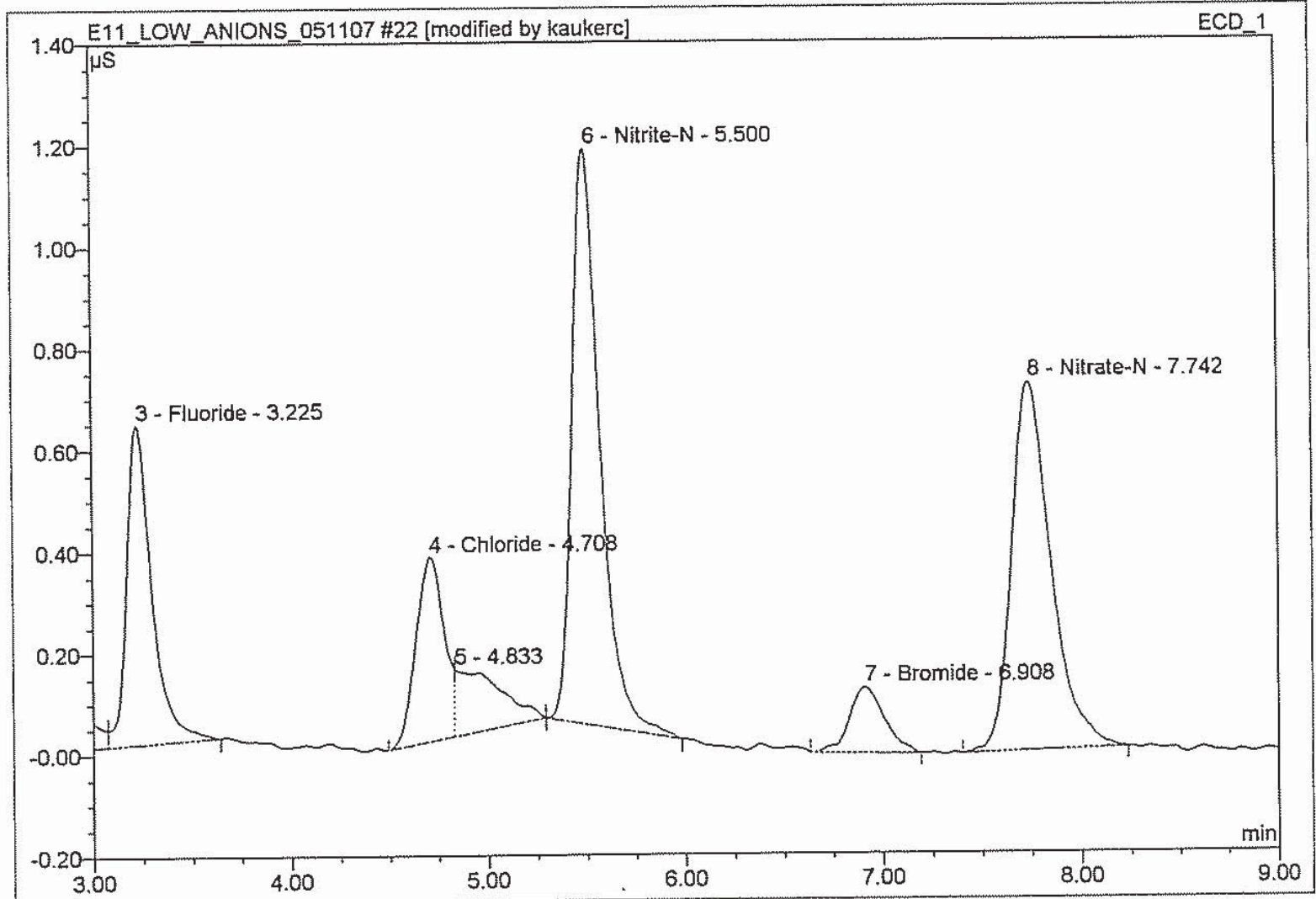

\begin{tabular}{|r|r|l|r|r|r|r|c|}
\hline No. & $\begin{array}{c}\text { Ret.Time } \\
\text { min }\end{array}$ & \multicolumn{1}{|c|}{ Peak Name } & $\begin{array}{c}\text { Height } \\
\mu S\end{array}$ & $\begin{array}{c}\text { Area } \\
\mu S^{\star} \text { min }\end{array}$ & $\begin{array}{r}\text { Rel.Area } \\
\%\end{array}$ & $\begin{array}{c}\text { Amount } \\
\text { mg/L }\end{array}$ & $\begin{array}{r}\text { Peak } \\
\text { Type }\end{array}$ \\
\hline 1 & 2.22 & n.a. & 1.275 & 0.213 & 0.36 & n.a. & BM \\
\cline { 2 - 7 } 2 & 2.81 & n.a. & 0.119 & 0.022 & 0.04 & n.a. & M \\
\cline { 2 - 7 } 3 & 3.23 & Fluoride & 0.628 & 0.093 & 0.16 & 4.5220 & MB \\
\hline 4 & 4.71 & Chloride & 0.363 & 0.061 & 0.10 & 3.8160 & $\mathrm{BM}^{\star}$ \\
\hline 5 & 4.83 & n.a. & 0.132 & 0.033 & 0.06 & n.a. & $\mathrm{Mb}^{\star}$ \\
\hline 6 & 5.50 & Nitrite-N & 1.133 & 0.187 & 0.32 & 6.5446 & $\mathrm{bMB}$ \\
\hline 7 & 6.91 & Bromide & 0.130 & 0.027 & 0.05 & 4.4445 & $\mathrm{BMB}$ \\
\hline 8 & 7.74 & Nitrate-N & 0.725 & 0.166 & 0.28 & 5.0308 & $\mathrm{BMB}$ \\
\hline 9 & 11.74 & n.a. & 15.711 & 5.048 & 8.57 & n.a. & BMB \\
\hline 10 & 21.43 & n.a. & 59.925 & 53.035 & 90.07 & n.a. & BMB \\
\hline Total: & & & 80.142 & 58.885 & 100.00 & 24.358 & \\
\hline
\end{tabular}

default_letter/Integration

(5) awk 5-11-07

Chromeleon (c) Dionex 1996-2001 Version 6.50 SP10a Build 1065 


\section{H7E040102 JV87H MS 1/20 0.2 PPM CL}

\section{QUAD}

Sample Name: $\quad$ H7E040102 JV87H MS 1/20 0.2 PPM CL

Vial Number:

Sample Type:

Control Program:

Quantif. Method:

Recording Time:

Run Time (min):
361

unknown

ANIONS_AS14A_CI2

ANIONS

5/11/2007 13:57

30.00

$\begin{array}{ll}\text { Injection Volume: } & \mathbf{5 0 . 0} \\ \text { Channel: } & \text { ECD_1 } \\ \text { Wavelength: } & \text { n.a. } \\ \text { Bandwidth: } & \text { n.a. } \\ \text { Dilution Factor: } & \mathbf{2 0 . 0 0 0 0} \\ \text { Sample Weight: } & \mathbf{1 . 0 0 0 0} \\ \text { Sample Amount: } & \mathbf{1 . 0 0 0 0}\end{array}$

$\begin{array}{ll}\text { Injection Volume: } & \mathbf{5 0 . 0} \\ \text { Channel: } & \mathrm{ECD}\end{array}$

Wavelength:

20.0000

1.0000

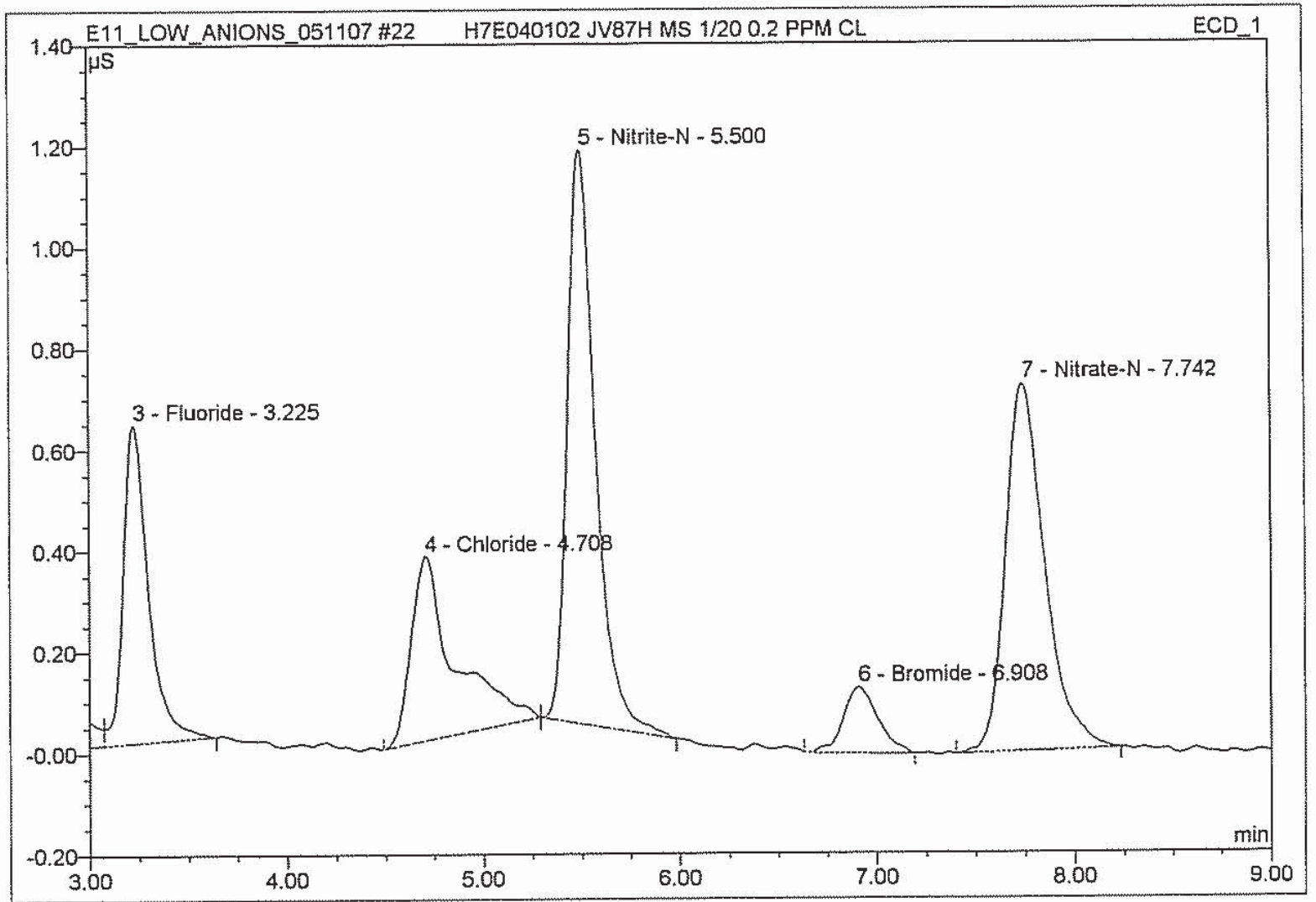

\begin{tabular}{|r|r|l|r|r|r|r|r|}
\hline No. & $\begin{array}{c}\text { Ret.Time } \\
\text { min }\end{array}$ & \multicolumn{1}{|c|}{ Peak Name } & $\begin{array}{c}\text { Height } \\
\mu S\end{array}$ & $\begin{array}{c}\text { Area } \\
\mu S^{*} \min \end{array}$ & $\begin{array}{c}\text { Rel.Area } \\
\%\end{array}$ & $\begin{array}{c}\text { Amount } \\
\text { mg/L }\end{array}$ & $\begin{array}{r}\text { Peak } \\
\text { Type }\end{array}$ \\
\hline 1 & 2.22 & n.a. & 1.275 & 0.213 & 0.36 & n.a. & BM \\
\cline { 2 - 8 } & 2.81 & n.a. & 0.119 & 0.022 & 0.04 & n.a. & M \\
\hline 3 & 3.23 & Fluoride & 0.628 & 0.093 & 0.16 & 4.5220 & MB \\
\hline 4 & 4.71 & Chloride & 0.363 & 0.094 & 0.16 & 3.8160 & BMB \\
\hline 5 & 5.50 & Nitrite-N & 1.133 & 0.187 & 0.32 & 6.5446 & bMB \\
\hline 6 & 6.91 & Bromide & 0.130 & 0.027 & 0.05 & 4.4445 & BMB \\
\hline 7 & 7.74 & Nitrate-N & 0.725 & 0.166 & 0.28 & 5.0308 & BMB \\
\hline 8 & 11.74 & n.a. & 15.711 & 5.048 & 8.57 & n.a. & BMB \\
\hline 9 & 21.43 & n.a. & 59.925 & 53.035 & 90.07 & n.a. & BMB \\
\hline Total: & & & 80.009 & 58.885 & 100.00 & 24.358 & \\
\hline
\end{tabular}

default_letter/Integration

original

Chromeleon (c) Dionex 1996-2001

Version 6.50 SP10a Build 1065 


\section{H7E040102 JV87H MSD 1/20 0.2 PPM CL}

\section{QUAD}

Sample Name: H7E040102 JV87H MSD 1/20 0.2 PPM CL

Vial Number: $\quad 362$

Sample Type: unknown

Control Program: ANIONS_AS14A_CI2

Quantif. Method: ANIONS

Recording Time: $\quad$ 5/11/2007 14:29

Run Time ( $\mathrm{min}): \quad 30.00$

$\begin{array}{ll}\text { Injection Volume: } & \mathbf{5 0 . 0} \\ \text { Channel: } & \text { ECD_1 } \\ \text { Wavelength: } & \text { n.a. } \\ \text { Bandwidth: } & \text { n.a. } \\ \text { Dilution Factor: } & 20.0000 \\ \text { Sample Weight: } & 1.0000 \\ \text { Sample Amount: } & \mathbf{1 . 0 0 0 0}\end{array}$

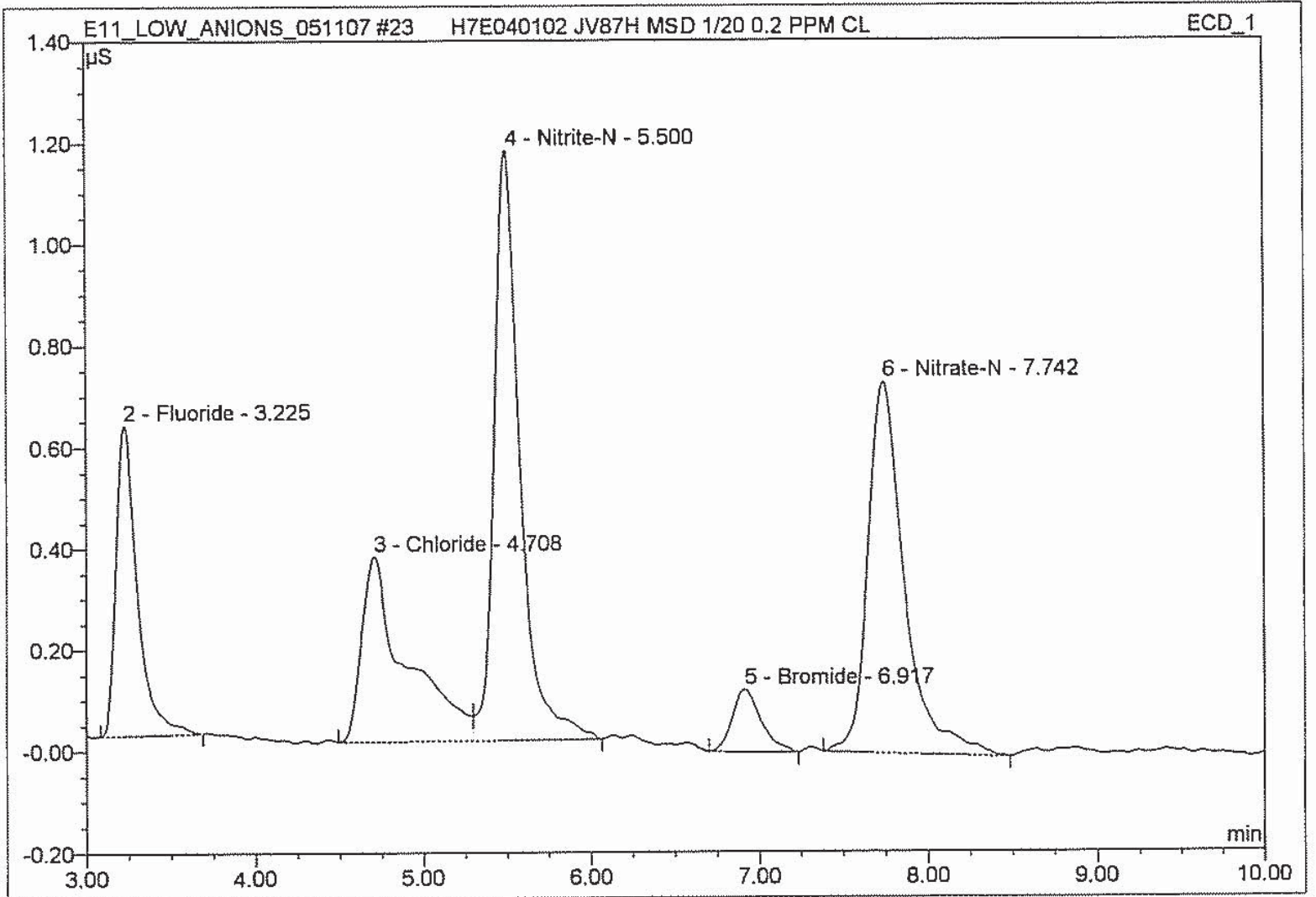

\begin{tabular}{|c|c|l|r|r|r|r|c|}
\hline No. & $\begin{array}{c}\text { Ret.Time } \\
\text { min }\end{array}$ & \multicolumn{1}{|c|}{ Peak Name } & $\begin{array}{c}\text { Height } \\
\mu S\end{array}$ & $\begin{array}{c}\text { Area } \\
\mu S^{*} \text { min }\end{array}$ & $\begin{array}{c}\text { Rel.Area } \\
\%\end{array}$ & $\begin{array}{c}\text { Amount } \\
\text { mg/L }\end{array}$ & $\begin{array}{r}\text { Peak } \\
\text { Type }\end{array}$ \\
\hline 1 & 2.22 & n.a. & 1.258 & 0.209 & 0.36 & n.a. & BMB \\
\hline 2 & 3.23 & Fluoride & 0.611 & 0.089 & 0.15 & 4.3972 & BMB \\
\hline 3 & 4.71 & Chloride & 0.365 & 0.112 & 0.19 & 3.8354 & BM \\
\hline 4 & 5.50 & Nitrite-N & 1.162 & 0.206 & 0.35 & 6.7095 & MB \\
\hline 5 & 6.92 & Bromide & 0.122 & 0.025 & 0.04 & 4.1855 & BMB \\
\hline 6 & 7.74 & Nitrate-N & 0.732 & 0.183 & 0.31 & 5.0818 & BMB \\
\hline 7 & 11.74 & n.a. & 15.427 & 4.978 & 8.44 & n.a. & BMB \\
\hline 8 & 21.43 & n.a. & 60.019 & 53.194 & 90.17 & n.a. & BMB \\
\hline Total: & & & 79.697 & 58.995 & 100.00 & 24.209 & \\
\hline
\end{tabular}




\section{H7E040102 JV87F 1/20}

\section{QUAD}

\begin{tabular}{llll}
\hline Sample Name: & H7E040102 JV87F 1/20 & Injection Volume: & $\mathbf{5 0 . 0}$ \\
Vial Number: & $\mathbf{3 6 3}$ & Channel: & ECD_1 \\
Sample Type: & unknown & Wavelength: & n.a. \\
Control Program: & ANIONS_AS14A_CI2 & Bandwidth: & n.a. \\
Quantif. Method: & ANIONS & Dilution Factor: & $\mathbf{2 0 . 0 0 0 0}$ \\
Recording Time: & $\mathbf{5 / 1 1 / 2 0 0 7 1 5 : 0 2}$ & Sample Weight: & $\mathbf{1 . 0 0 0 0}$ \\
Run Time (min): & $\mathbf{3 0 . 0 0}$ & Sample Amount: & $\mathbf{1 . 0 0 0 0}$ \\
\hline
\end{tabular}

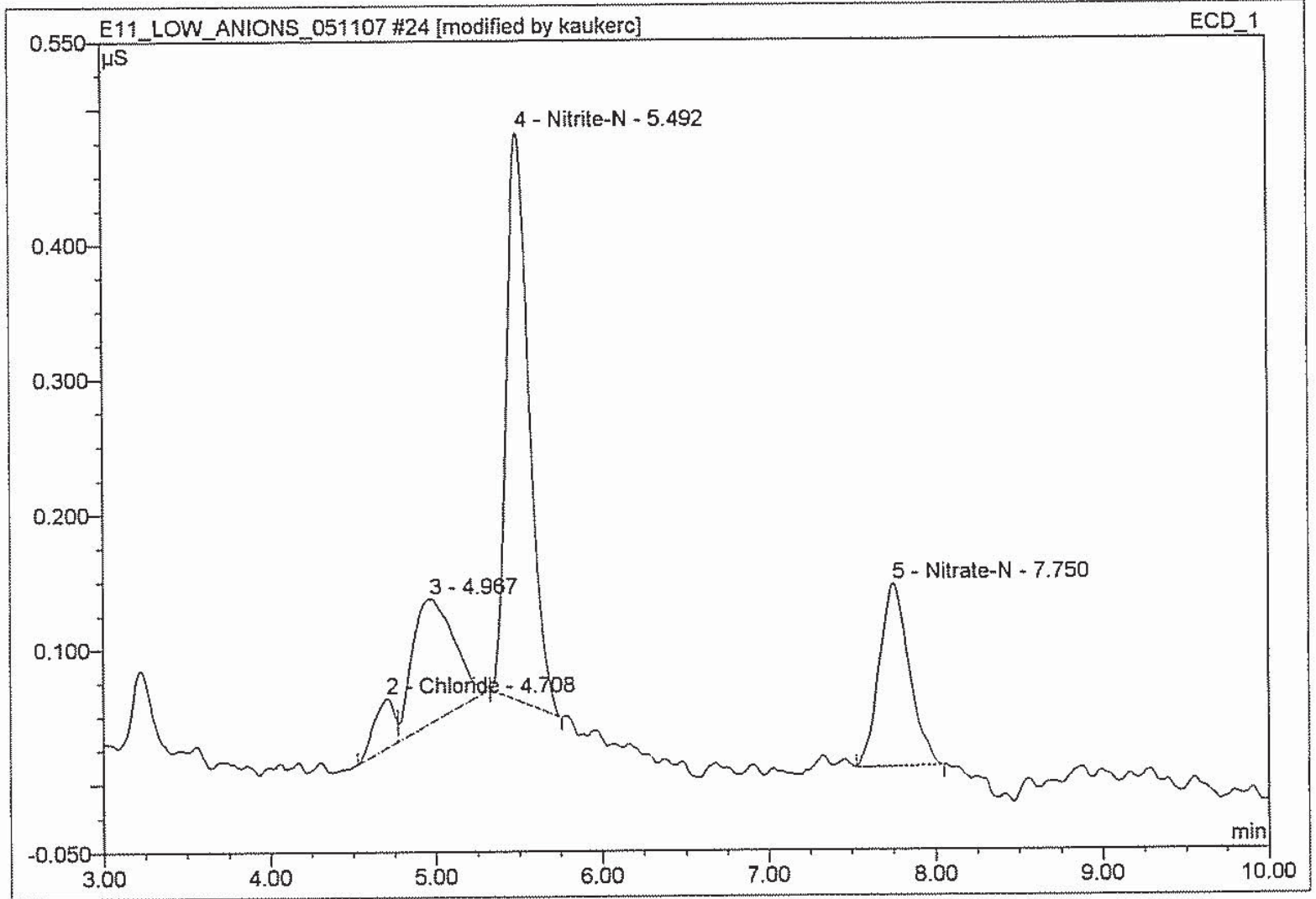

\begin{tabular}{|c|c|c|c|c|c|c|c|}
\hline No. & $\begin{array}{l}\text { Ret.Time } \\
\text { min }\end{array}$ & Peak Name & $\begin{array}{c}\text { Height } \\
\mu S\end{array}$ & $\begin{array}{c}\text { Area } \\
\mu S^{\star} \min \end{array}$ & \begin{tabular}{|c|} 
Rel.Area \\
$\%$
\end{tabular} & $\begin{array}{c}\begin{array}{c}\text { Amount } \\
\mathrm{mg} / \mathrm{L}\end{array} \\
\end{array}$ & $\begin{array}{l}\text { Peak } \\
\text { Type }\end{array}$ \\
\hline 1 & 2.22 & n.a. & 1.188 & 0.196 & 0.32 & n.a. & BMB \\
\hline 2 & 4.71 & Chloride & 0.037 & 0.005 & 0.01 & 0.3872 & $\mathrm{BM}^{\star}$ \\
\hline 3 & 4.97 & n.a. & 0.092 & 0.027 & 0.05 & n.a. & $\mathrm{Mb}^{\star}$ \\
\hline 4 & 5.49 & Nitrite-N & 0.420 & 0.066 & 0.11 & 2.4496 & $\mathrm{bMB}^{*}$ \\
\hline 5 & 7.75 & Nitrate-N & 0.135 & 0.028 & 0.05 & 0.9500 & $\mathrm{BMB}$ \\
\hline 6 & 11.73 & n.a. & 21.534 & 6.857 & 11.36 & n.a. & $\mathrm{BMB}$ \\
\hline 7 & 21.43 & n.a. & 60.032 & 53.175 & 88.10 & n.a. & $\mathrm{BMB}$ \\
\hline Total: & & & 83.439 & 60.355 & 100.00 & 3.787 & \\
\hline
\end{tabular}

$\left(\begin{array}{l}\text { No SEPARATION at } \\ 1 / 10 \text { sereening run }\end{array}\right)$ default_letter/Integration

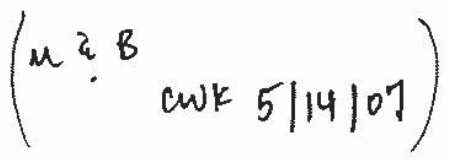

Chromeleon (c) Dionex 1996-2001 Version 6.50 SP10a Build 1065 


\section{H7E040102 JV87F 1/20}

\section{QUAD}

Sample Name: H7E040102 JV87F 1/20

Vial Number:

Sample Type:

Control Program:

Quantif. Method:

Recording Time:

Run Time (min):
363

unknown

ANIONS_AS14A_CI2

ANIONS

5/11/2007 15:02

30.00
Injection Volume:

Channel:

Wavelength:

Bandwidth:

Dilution Factor:

Sample Weight:

Sample Amount:
50.0

ECD_1

n.a.

n.a.

20.0000

1.0000

1.0000

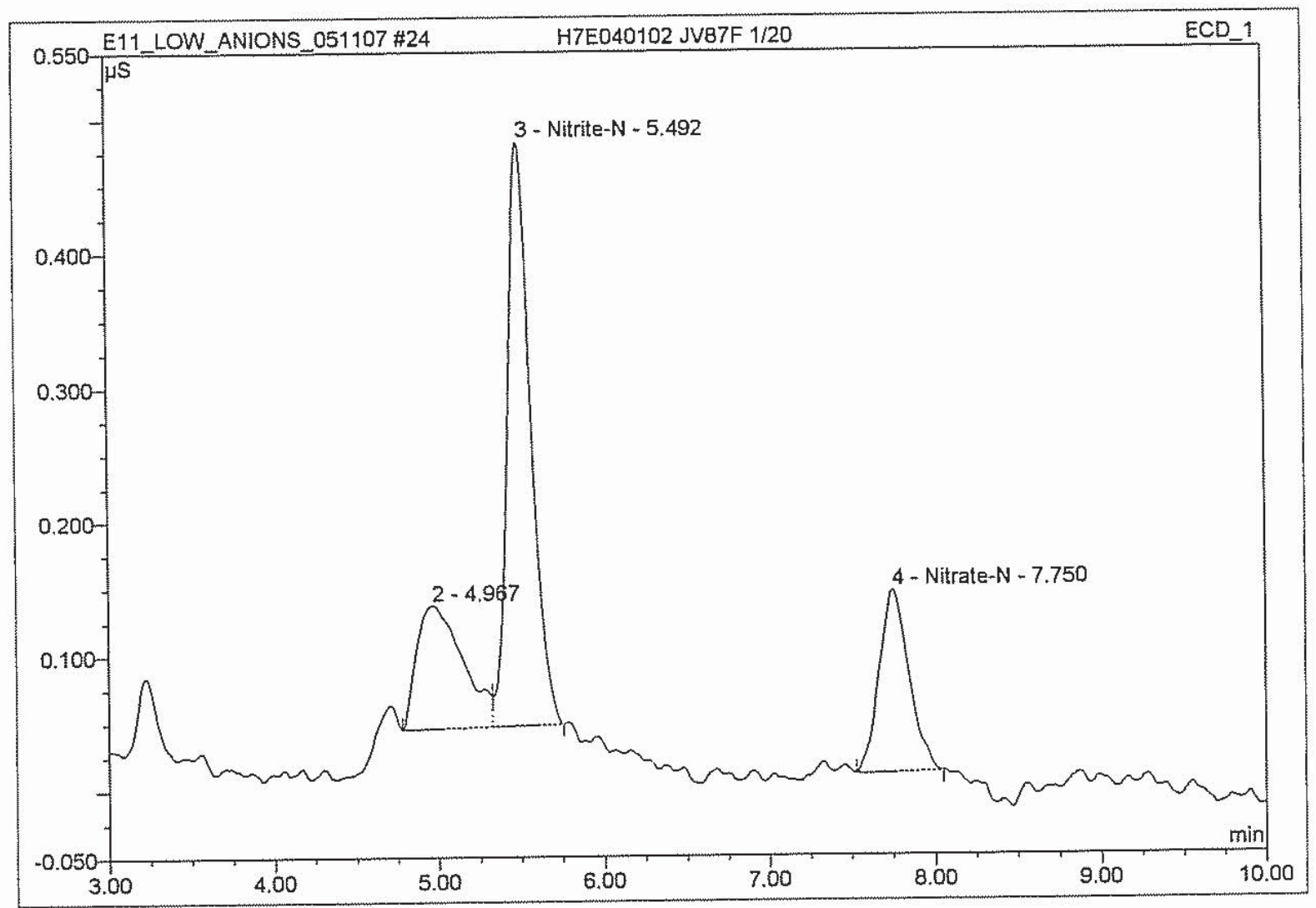

\begin{tabular}{|c|c|l|r|r|r|r|c|}
\hline No. & $\begin{array}{c}\text { Ret.Time } \\
\text { min }\end{array}$ & \multicolumn{1}{|c|}{ Peak Name } & $\begin{array}{c}\text { Height } \\
\mu S\end{array}$ & $\begin{array}{c}\text { Area } \\
\mu S^{*} \min \end{array}$ & $\begin{array}{c}\text { Rel.Area } \\
\%\end{array}$ & $\begin{array}{c}\text { Amount } \\
\text { mg/L }\end{array}$ & $\begin{array}{r}\text { Peak } \\
\text { Type }\end{array}$ \\
\hline 1 & 2.22 & n.a. & 1.188 & 0.196 & 0.32 & n.a. & BMB \\
\hline 2 & 4.97 & n.a. & 0.091 & 0.029 & 0.05 & n.a. & BM \\
\hline 3 & 5.49 & Nitrite-N & 0.434 & 0.071 & 0.12 & 2.5296 & MB \\
\hline 4 & 7.75 & Nitrate-N & 0.135 & 0.028 & 0.05 & 0.9500 & BMB \\
\hline 5 & 11.73 & n.a. & 21.534 & 6.857 & 11.36 & n.a. & BMB \\
\hline 6 & 21.43 & n.a. & 60.032 & 53.175 & 88.10 & n.a. & BMB \\
\hline Total: & & & 83.415 & 60.356 & 100.00 & 3.480 & \\
\hline
\end{tabular}

original

default_letter/Integration 


\section{H7E040102 JV87K 1/20 \\ QUAD}

Sample Name: H7E040102 JV87K 1/20

Vial Number:

Sample Type:

Control Program:

Quantif. Method:

Recording Time:

Run Time (min):
364

unknown

ANIONS_AS14A_Cl2

ANIONS

5/11/2007 15:34

30.00
Injection Volume: $\quad \mathbf{5 0 . 0}$

Channel:

Wavelength:

Bandwidth:

Dilution Factor:

Sample Weight:

Sample Amount:
ECD_1

n.a.

n.a.

20.0000

1.0000

1.0000

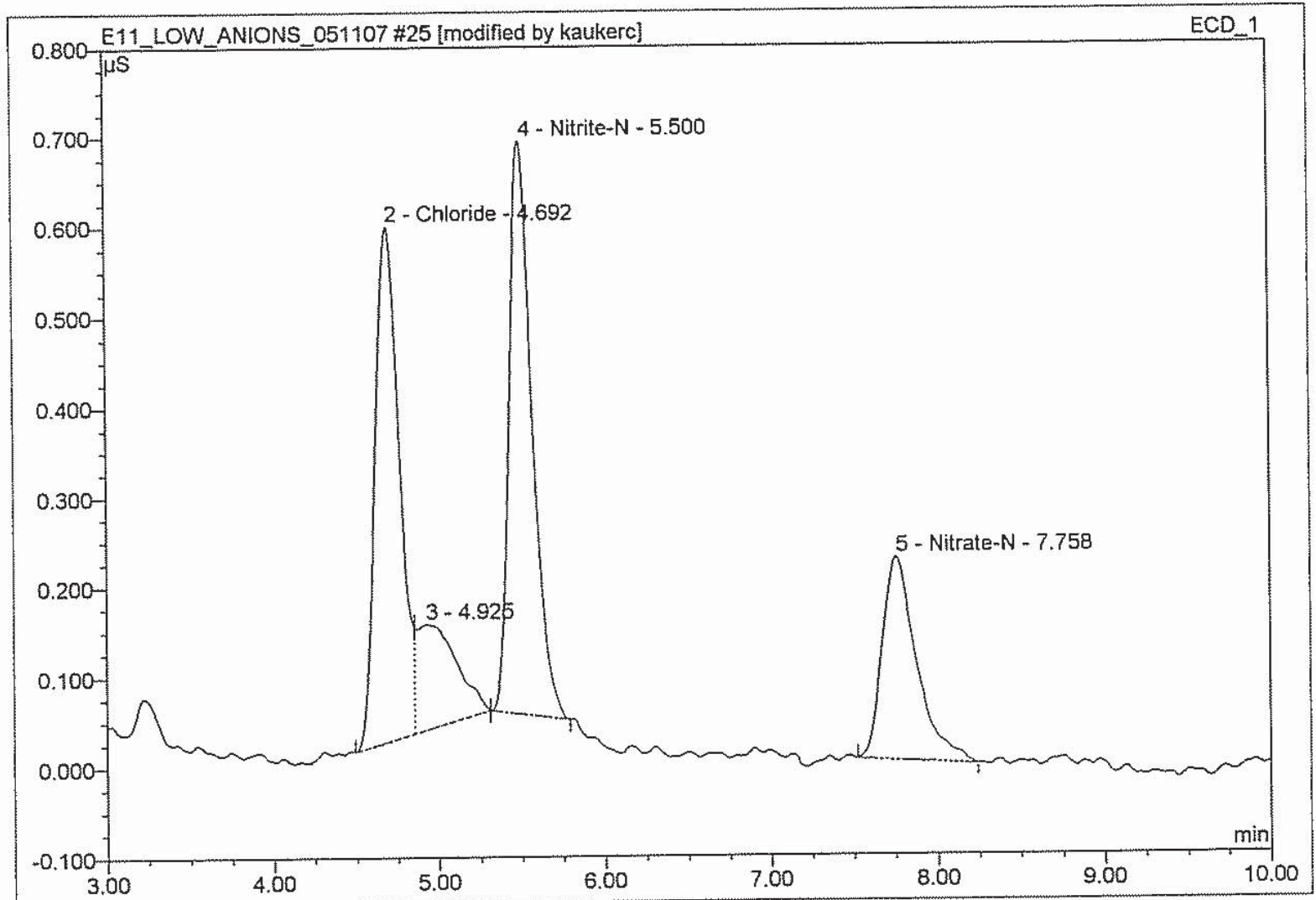

\begin{tabular}{|r|c|l|r|r|r|r|r|}
\hline No. & $\begin{array}{c}\text { Ret.Time } \\
\text { min }\end{array}$ & \multicolumn{1}{|c|}{ Peak Name } & $\begin{array}{c}\text { Height } \\
\mu S\end{array}$ & $\begin{array}{c}\text { Area } \\
\mu S^{*} \text { min }\end{array}$ & $\begin{array}{c}\text { Rel.Area } \\
\%\end{array}$ & $\begin{array}{c}\text { Amount } \\
\text { mg/L }\end{array}$ & $\begin{array}{r}\text { Peak } \\
\text { Type }\end{array}$ \\
\hline 1 & 2.22 & n.a. & 1.150 & 0.177 & 0.29 & n.a. & BMB \\
\cline { 2 - 8 } & 4.69 & Chloride & 0.572 & 0.097 & 0.16 & 5.9875 & BM $^{*}$ \\
\hline 3 & 4.93 & n.a. & 0.116 & 0.032 & 0.05 & n.a. & Mb $^{*}$ \\
\hline 4 & 5.50 & Nitrite-N & 0.636 & 0.101 & 0.17 & 3.6954 & bMB \\
\hline 5 & 7.76 & Nitrate-N & 0.224 & 0.053 & 0.09 & 1.5728 & BMB \\
\hline 6 & 11.73 & n.a. & 24.190 & 7.716 & 12.62 & n.a. & BMB \\
\hline 7 & 21.44 & n.a. & 59.923 & 52.974 & 86.63 & n.a. & BMB \\
\hline Total: & & & 86.811 & 61.151 & 100.00 & 11.256 & \\
\hline
\end{tabular}

default_letter/Integration

(S) Cuk 5/14/07

Chromeleon (c) Dionex 1996-2001

Version 6,50 SP10a Build 1065 


\section{H7E040102 JV87K 1/20}

\section{QUAD}

\begin{tabular}{llll|}
\hline Sample Name: & H7E040102 JV87K 1/20 & Injection Volume: & $\mathbf{5 0 . 0}$ \\
Vial Number: & $\mathbf{3 6 4}$ & Channel: & ECD_1 \\
Sample Type: & unknown & Wavelength: & n.a. \\
Control Program: & ANIONS_AS14A_CI2 & Bandwidth: & n.a. \\
Quantif. Method: & ANIONS & Dilution Factor: & $\mathbf{2 0 . 0 0 0 0}$ \\
Recording Time: & $\mathbf{5 / 1 1 / 2 0 0 7 ~ 1 5 : 3 4}$ & Sample Weight: & $\mathbf{1 . 0 0 0 0}$ \\
Run Time (min): & $\mathbf{3 0 . 0 0}$ & Sample Amount: & $\mathbf{1 . 0 0 0 0}$ \\
\hline
\end{tabular}

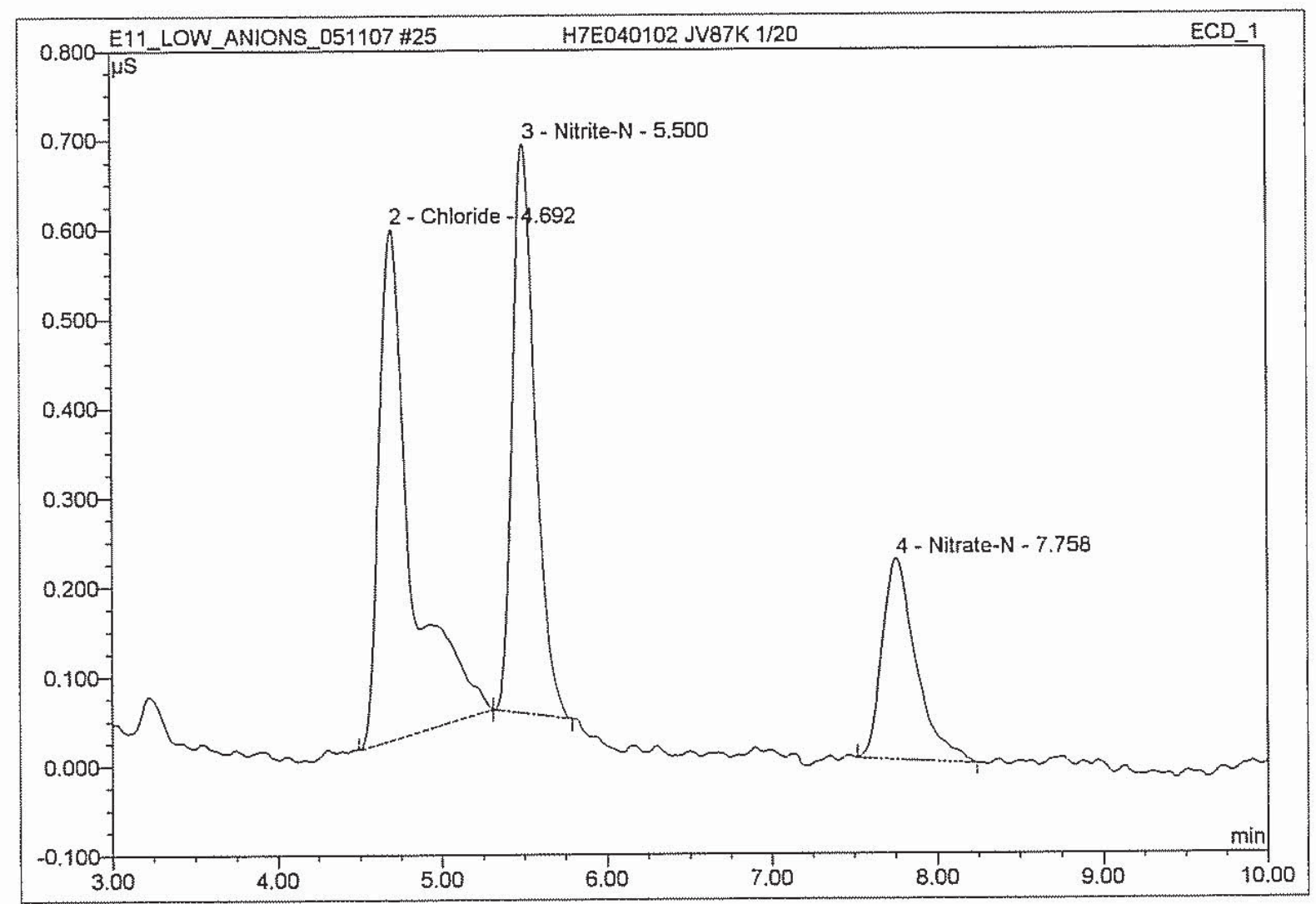

\begin{tabular}{|c|c|l|r|r|r|r|c|}
\hline No. & $\begin{array}{c}\text { Ret.Time } \\
\min \end{array}$ & \multicolumn{1}{|c|}{ Peak Name } & $\begin{array}{c}\text { Height } \\
\mu S\end{array}$ & $\begin{array}{c}\text { Area } \\
\mu S^{*} \min \end{array}$ & $\begin{array}{c}\text { Rel.Area } \\
\%\end{array}$ & $\begin{array}{c}\text { Amount } \\
\text { mg/L }\end{array}$ & $\begin{array}{c}\text { Peak } \\
\text { Type }\end{array}$ \\
\hline 1 & 2.22 & n.a. & 1.150 & 0.177 & 0.29 & n.a. & BMB \\
\hline 2 & 4.69 & Chloride & 0.572 & 0.129 & 0.21 & 5.9875 & BMB \\
\hline 3 & 5.50 & Nitrite-N & 0.636 & 0.101 & 0.17 & 3.6954 & bMB \\
\hline 4 & 7.76 & Nitrate-N & 0.224 & 0.053 & 0.09 & 1.5728 & BMB \\
\hline 5 & 11.73 & n.a. & 24.190 & 7.716 & 12.62 & n.a. & BMB \\
\hline 6 & 21.44 & n.a. & 59.923 & 52.974 & 86.63 & n.a. & BMB \\
\hline Total: & & & 86.695 & 61.151 & 100.00 & 11.256 & \\
\hline
\end{tabular}

original 


\section{H7E040102 JV87M 1/20}

\section{QUAD}

Sample Name: H7E040102 JV87M 1/20

Vial Number: $\quad 365$

Sample Type: $\quad$ unknown

Control Program: ANIONS_AS14A_CI2

Quantif. Method: ANIONS

Recording Time:

Run Time (min):
5/11/2007 16:07

30.00

$\begin{array}{ll}\text { Injection Volume: } & 50.0 \\ \text { Channel: } & \text { ECD_1 } \\ \text { Wavelength: } & \text { n.a. } \\ \text { Bandwidth: } & \text { n.a. } \\ \text { Dilution Factor: } & 20.0000 \\ \text { Sample Weight: } & 1.0000 \\ \text { Sample Amount: } & 1.0000\end{array}$

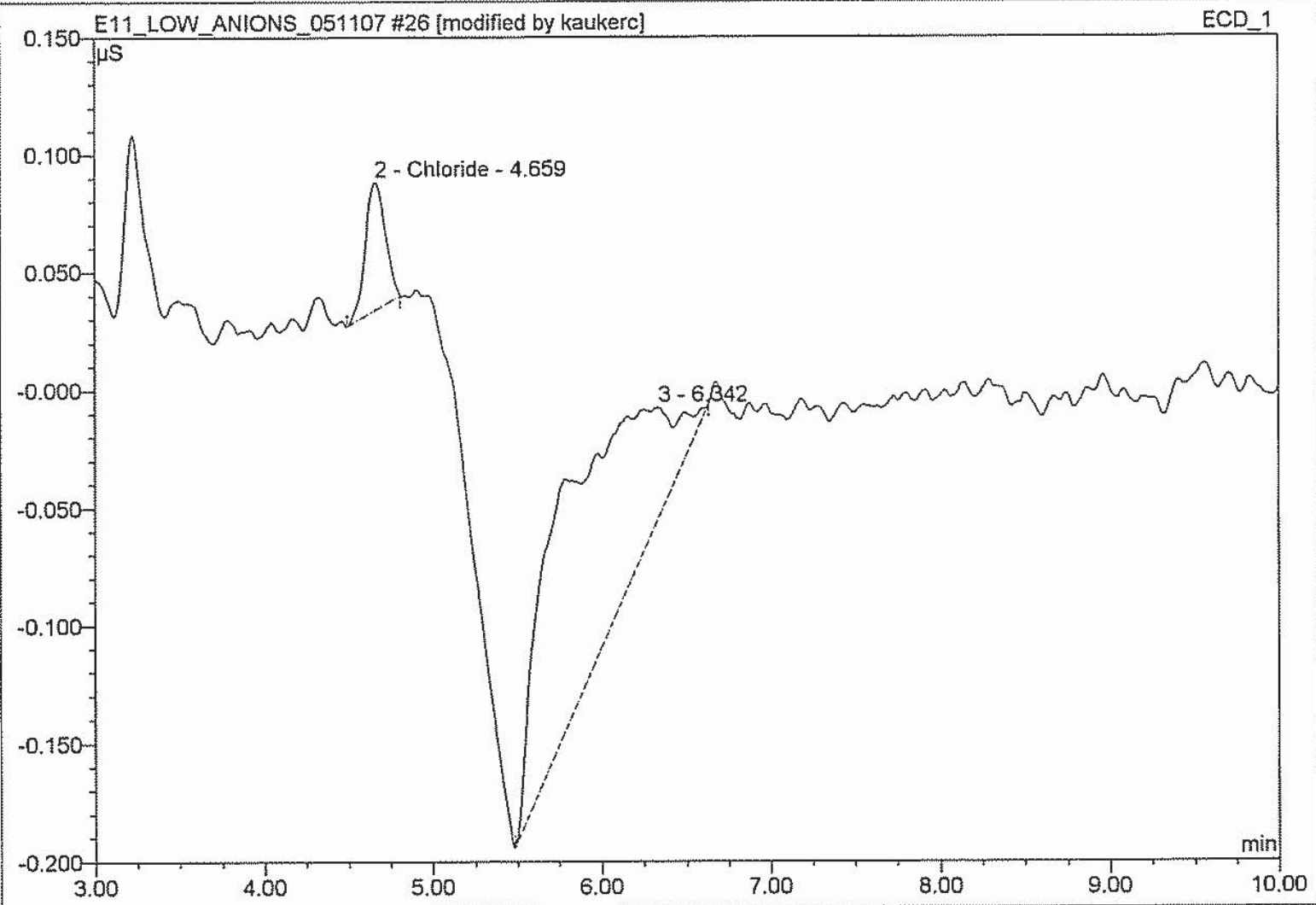

\begin{tabular}{|r|r|l|r|r|r|r|r|}
\hline No. & $\begin{array}{c}\text { Ret.Time } \\
\min \end{array}$ & \multicolumn{1}{|c|}{ Peak Name } & $\begin{array}{c}\text { Height } \\
\mu S\end{array}$ & $\begin{array}{c}\text { Area } \\
\mu S^{*} \min \end{array}$ & $\begin{array}{r}\text { Rel.Area } \\
\%\end{array}$ & $\begin{array}{c}\text { Amount } \\
\mathrm{mg} / \mathrm{L}\end{array}$ & $\begin{array}{r}\text { Peak } \\
\text { Type }\end{array}$ \\
\hline 1 & 2.23 & n.a. & 1.279 & 0.221 & 0.41 & n.a. & BMB \\
\cline { 2 - 7 } 2 & 4.66 & Chloride & 0.054 & 0.007 & 0.01 & 0.5726 & BMB $^{*}$ \\
\hline 3 & 6.34 & n.a. & 0.046 & 0.070 & 0.13 & n.a. & BMB \\
\hline 4 & 11.73 & n.a. & 0.315 & 0.104 & 0.19 & n.a. & BMB \\
\hline 5 & 21.44 & n.a. & 59.746 & 52.963 & 99.25 & n.a. & BMB \\
\hline Total: & & & 61.441 & 53.366 & 100.00 & 0.573 & \\
\hline
\end{tabular}

(i) oulk 5/14107 


\section{H7E040102 JV87M 1/20}

\section{QUAD}

Sample Name: H7E040102 JV87M 1/20

Vial Number:

Sample Type:

Control Program:

Quantif. Method:

Recording Time:

Run Time (min):
365

unknown

ANIONS_AS14A_CI2

ANIONS

5/11/2007 16:07

30.00

$\begin{array}{ll}\text { Injection Volume: } & \mathbf{5 0 . 0} \\ \text { Channel: } & \text { ECD_1 } \\ \text { Wavelength: } & \text { n.a. } \\ \text { Bandwidth: } & \text { n.a. } \\ \text { Dilution Factor: } & \mathbf{2 0 . 0 0 0 0} \\ \text { Sample Weight: } & \mathbf{1 . 0 0 0 0} \\ \text { Sample Amount: } & \mathbf{1 . 0 0 0 0}\end{array}$

$\begin{array}{ll}\text { Injection Volume: } & \mathbf{5 0 . 0} \\ \text { Channel: } & \text { ECD_1 }\end{array}$

Wavelength: n.a.

Bandwidth: n.a.

Dilution Factor: $\quad \mathbf{2 0 . 0 0 0 0}$

Sample Amount: $\quad \mathbf{1 . 0 0 0 0}$

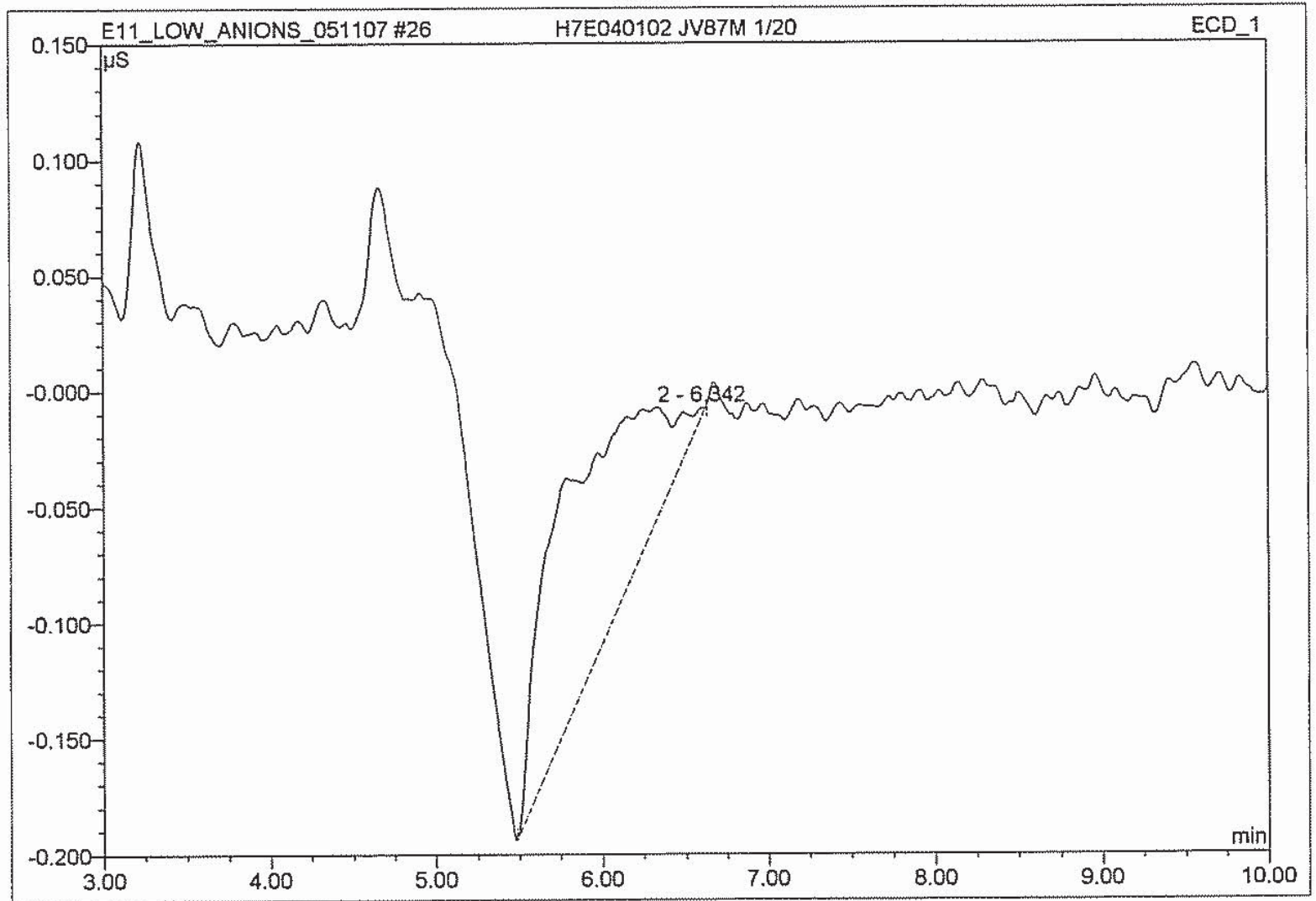

\begin{tabular}{|r|r|l|r|r|r|r|r|}
\hline No. & $\begin{array}{c}\text { Ret.Time } \\
\text { min }\end{array}$ & \multicolumn{1}{|c|}{ Peak Name } & $\begin{array}{c}\text { Height } \\
\mu \text { S }\end{array}$ & $\begin{array}{c}\text { Area } \\
\mu S^{*} \text { min }\end{array}$ & $\begin{array}{c}\text { Rel.Area } \\
\%\end{array}$ & $\begin{array}{c}\text { Amount } \\
\text { mg/L }\end{array}$ & $\begin{array}{r}\text { Peak } \\
\text { Type }\end{array}$ \\
\hline 1 & 2.23 & n.a. & 1.279 & 0.221 & 0.41 & n.a. & BMB \\
\hline 2 & 6.34 & n.a. & 0.046 & 0.070 & 0.13 & n.a. & BMB \\
\hline 3 & 11.73 & n.a. & 0.315 & 0.104 & 0.19 & n.a. & BMB \\
\hline 4 & 21.44 & n.a. & 59.746 & 52.963 & 99.26 & n.a. & BMB \\
\hline Total: & & & 61.387 & 53.358 & 100.00 & 0.000 & \\
\hline
\end{tabular}

original 


\section{H7E040102 JV87M MS 1/20 0.2 PPM CL}

\section{QUAD}

\begin{tabular}{llll|}
\hline Sample Name: & H7E040102 JV87M MS 1/20 0.2 PPM CL & Injection Volume: & $\mathbf{5 0 . 0}$ \\
Vial Number: & 366 & Channel: & ECD_1 \\
Sample Type: & unknown & Wavelength: & n.a. \\
Control Program: & ANIONS_AS14A_CI2 & Bandwidth: & n.a. \\
Quantif. Method: & ANIONS & Dilution Factor: & 20.0000 \\
Recording Time: & $5 / 11 / 200716: 39$ & Sample Weight: & 1.0000 \\
Run Time (min): & $\mathbf{3 0 . 0 0}$ & Sample Amount: & 1.0000 \\
\hline
\end{tabular}

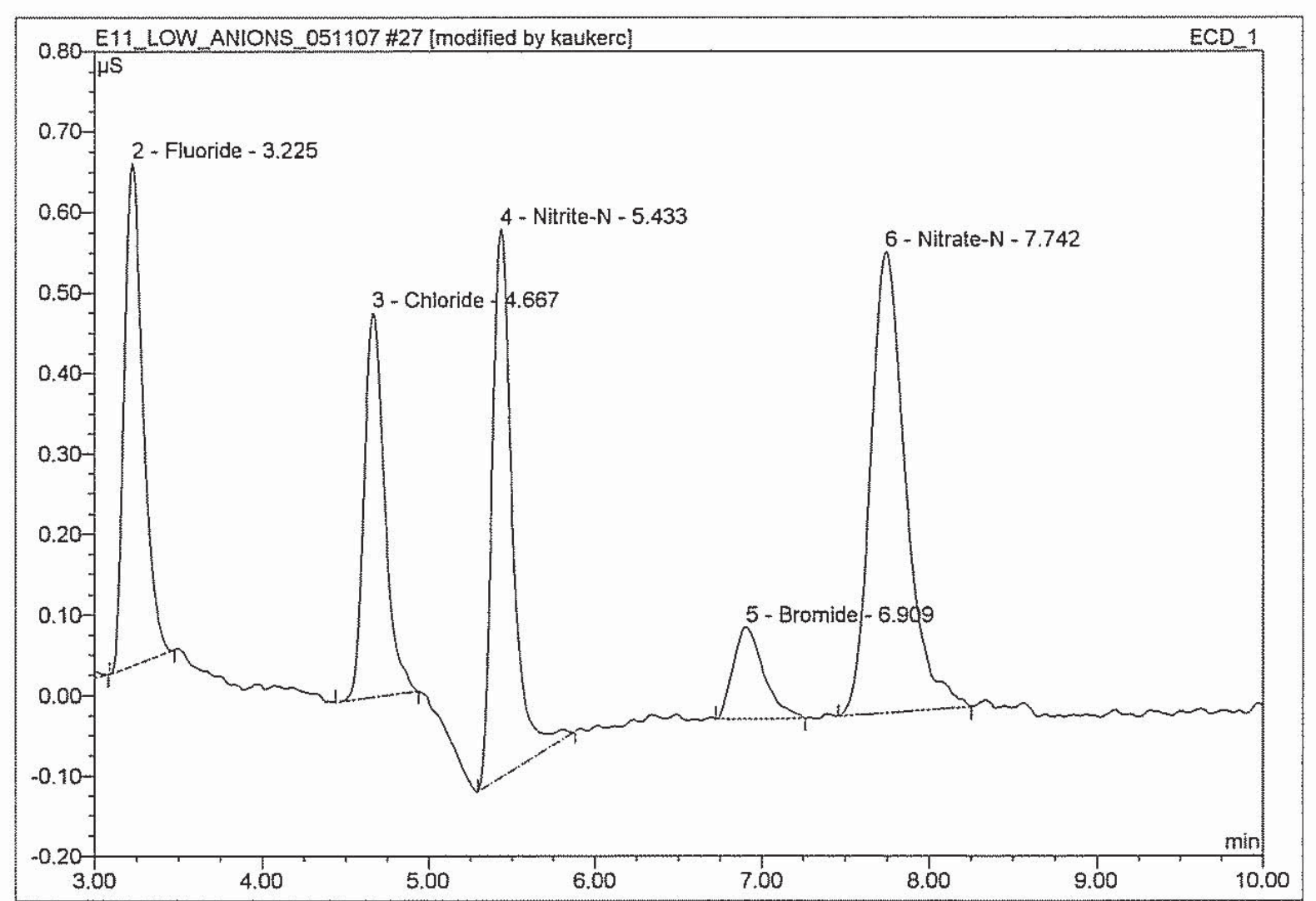

\begin{tabular}{|r|r|l|r|r|r|r|c|}
\hline No. & $\begin{array}{c}\text { Ret.Time } \\
\mathrm{min}\end{array}$ & \multicolumn{1}{|c|}{ Peak Name } & $\begin{array}{c}\text { Height } \\
\mu \mathrm{S}\end{array}$ & $\begin{array}{c}\text { Area } \\
\mu \mathrm{S}^{*} \mathrm{~min}\end{array}$ & $\begin{array}{c}\text { Rel.Area } \\
\%\end{array}$ & $\begin{array}{c}\text { Amount } \\
\mathrm{mg} / \mathrm{L}\end{array}$ & $\begin{array}{r}\text { Peak } \\
\text { Type }\end{array}$ \\
\hline 1 & 2.23 & n.a. & 1.294 & 0.235 & 0.43 & n.a. & BMB \\
\hline 2 & 3.23 & Fluoride & 0.624 & 0.081 & 0.15 & 4.4915 & $\mathrm{BMB}$ \\
\hline 3 & 4.67 & Chloride & 0.477 & 0.068 & 0.13 & 5.0084 & $\mathrm{BMB}^{*}$ \\
\hline 4 & 5.43 & Nitrite-N & 0.681 & 0.096 & 0.18 & 3.9589 & $\mathrm{BMB}$ \\
\hline 5 & 6.91 & Bromide & 0.114 & 0.024 & 0.05 & 3.8980 & $\mathrm{BMB}$ \\
\hline 6 & 7.74 & Nitrate-N & 0.573 & 0.139 & 0.26 & 3.9898 & $\mathrm{BMB}$ \\
\hline 7 & 11.73 & n.a. & 0.333 & 0.112 & 0.21 & n.a. & BMB \\
\hline 8 & 21.44 & n.a. & 59.890 & 53.174 & 98.60 & n.a. & BMB \\
\hline Total: & & & 63.987 & 53.929 & 100.00 & 21.347 & \\
\hline
\end{tabular}




\section{H7E040102 JV87M MS 1/20 0.2 PPM CL}

\section{QUAD}

\begin{tabular}{llll|}
\hline Sample Name: & H7E040102 JV87M MS 1/20 0.2 PPM CL & Injection Volume: & $\mathbf{5 0 . 0}$ \\
Vial Number: & $\mathbf{3 6 6}$ & Channel: & ECD_1 \\
Sample Type: & unknown & Wavelength: & n.a. \\
Control Program: & ANIONS_AS14A_Cl2 & Bandwidth: & n.a. \\
Quantif. Method: & ANIONS & Dilution Factor: & $\mathbf{2 0 . 0 0 0 0}$ \\
Recording Time: & $\mathbf{5 / 1 1 / 2 0 0 7 ~ 1 6 : 3 9}$ & Sample Weight: & $\mathbf{1 . 0 0 0 0}$ \\
Run Time (min): & $\mathbf{3 0 . 0 0}$ & Sample Amount: & $\mathbf{1 . 0 0 0 0}$ \\
\hline
\end{tabular}

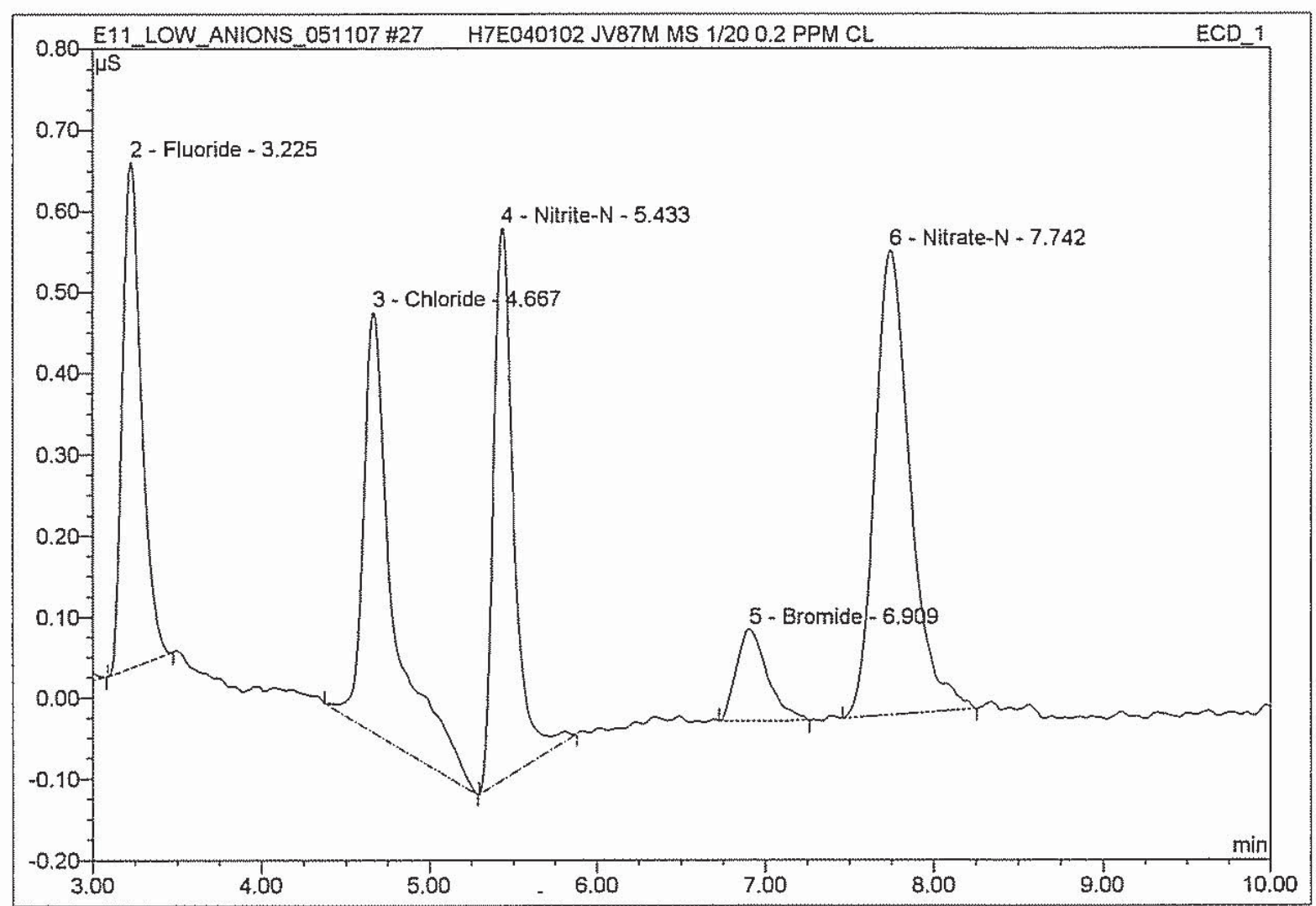

\begin{tabular}{|r|r|l|r|r|r|r|r|}
\hline No. & $\begin{array}{c}\text { Ret.Time } \\
\min \end{array}$ & \multicolumn{1}{|c|}{ Peak Name } & $\begin{array}{c}\text { Height } \\
\mu S\end{array}$ & $\begin{array}{c}\text { Area } \\
\mu S^{*} \min \end{array}$ & $\begin{array}{c}\text { Rel.Area } \\
\%\end{array}$ & $\begin{array}{c}\text { Amount } \\
\text { mg/L }\end{array}$ & $\begin{array}{r}\text { Peak } \\
\text { Type }\end{array}$ \\
\hline 1 & 2.23 & n.a. & 1.294 & 0.235 & 0.43 & n.a. & BMB \\
\hline 2 & 3.23 & Fluoride & 0.624 & 0.081 & 0.15 & 4.4915 & BMB \\
\hline 3 & 4.67 & Chloride & 0.519 & 0.107 & 0.20 & 5.4359 & BMB \\
\hline 4 & 5.43 & Nitrite-N & 0.681 & 0.096 & 0.18 & 3.9589 & BMB \\
\hline 5 & 6.91 & Bromide & 0.114 & 0.024 & 0.05 & 3.8980 & BMB \\
\hline 6 & 7.74 & Nitrate-N & 0.573 & 0.139 & 0.26 & 3.9898 & BMB \\
\hline 7 & 11.73 & n.a. & 0.333 & 0.112 & 0.21 & n.a. & BMB \\
\hline 8 & 21.44 & n.a. & 59.890 & 53.174 & 98.53 & n.a. & BMB \\
\hline Total: & & & 64.028 & 53.968 & 100.00 & 21.774 & \\
\hline
\end{tabular}




\section{CCV ICWS-9564}

\section{QUAD}

Sample Name: $\quad$ CCV ICWS-9564

Vial Number: $\quad 368$

Sample Type: $\quad$ unknown

Control Program: ANIONS_AS14A

Quantif. Method: ANIONS

Recording Time: $\quad 5 / 11 / 2007$ 17:11

Run Time (min): $\quad 14.75$

$\begin{array}{ll}\text { Injection Volume: } & \mathbf{5 0 . 0} \\ \text { Channel: } & \text { ECD_1 } \\ \text { Wavelength: } & \text { n.a. } \\ \text { Bandwidth: } & \text { n.a. } \\ \text { Dilution Factor: } & \mathbf{1 . 0 0 0 0} \\ \text { Sample Weight: } & 1.0000 \\ \text { Sample Amount: } & \mathbf{1 . 0 0 0 0}\end{array}$

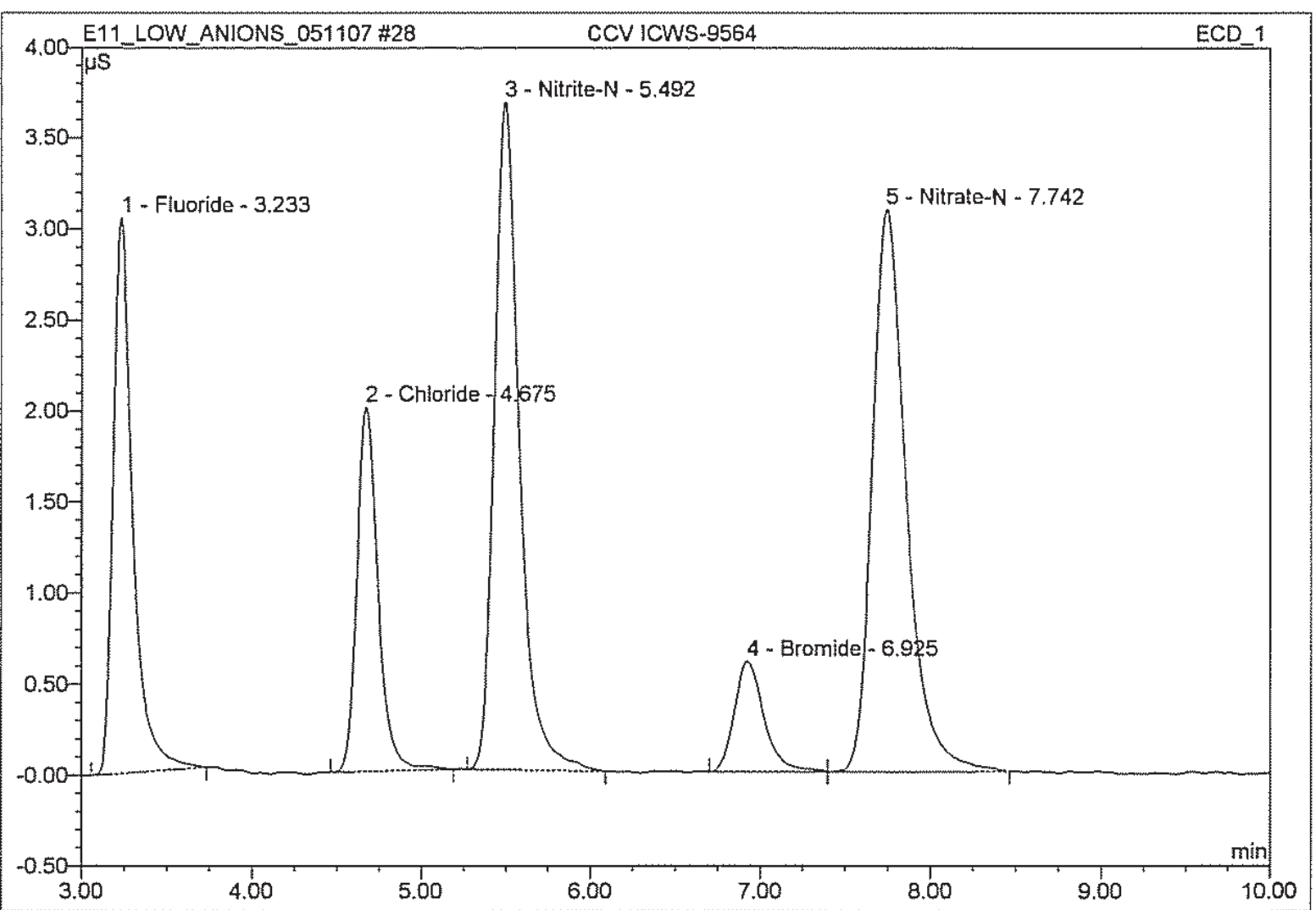

\begin{tabular}{|c|c|l|r|r|r|r|c|}
\hline No. & $\begin{array}{c}\text { Ret.Time } \\
\text { min }\end{array}$ & \multicolumn{1}{|c|}{ Peak Name } & $\begin{array}{c}\text { Height } \\
\mu S\end{array}$ & $\begin{array}{c}\text { Area } \\
\mu S^{*} \min \end{array}$ & $\begin{array}{c}\text { Rel.Area } \\
\%\end{array}$ & $\begin{array}{c}\text { Amount } \\
\mathrm{mg} / \mathrm{L}\end{array}$ & $\begin{array}{c}\text { Peak } \\
\text { Type }\end{array}$ \\
\hline 1 & 3.23 & Fluoride & 3.053 & 0.404 & 18.68 & 1.0440 & BMB \\
\cline { 2 - 7 } 2 & 4.68 & Chloride & 2.002 & 0.290 & 13.39 & 1.0223 & BMB \\
\hline 3 & 5.49 & Nitrite-N & 3.668 & 0.631 & 29.14 & 1.0263 & BMB \\
\hline 4 & 6.93 & Bromide & 0.607 & 0.124 & 5.71 & 1.0263 & BMb \\
\hline 5 & 7.74 & Nitrate-N & 3.090 & 0.716 & 33.08 & 1.0269 & $\mathrm{bMB}$ \\
\hline Total: & & & 12.420 & 2.165 & 100.00 & 5.146 & \\
\hline
\end{tabular}




\section{$29 \mathrm{CCB}$}

\section{QUAD}

\begin{tabular}{llll|}
\hline Sample Name: & CCB & Injection Volume: & $\mathbf{5 0 . 0}$ \\
Vial Number: & $\mathbf{3 6 9}$ & Channel: & ECD_1 \\
Sample Type: & unknown & Wavelength: & n.a. \\
Control Program: & ANIONS_AS14A & Bandwidth: & n.a. \\
Quantif. Method: & ANIONS & Dilution Factor: & $\mathbf{1 . 0 0 0 0}$ \\
Recording Time: & $\mathbf{5 / 1 1 / 2 0 0 7 ~ 1 7 : 2 9}$ & Sample Weight: & $\mathbf{1 . 0 0 0 0}$ \\
Run Time (min): & $\mathbf{1 4 . 7 5}$ & Sample Amount: & $\mathbf{1 . 0 0 0 0}$ \\
\hline
\end{tabular}

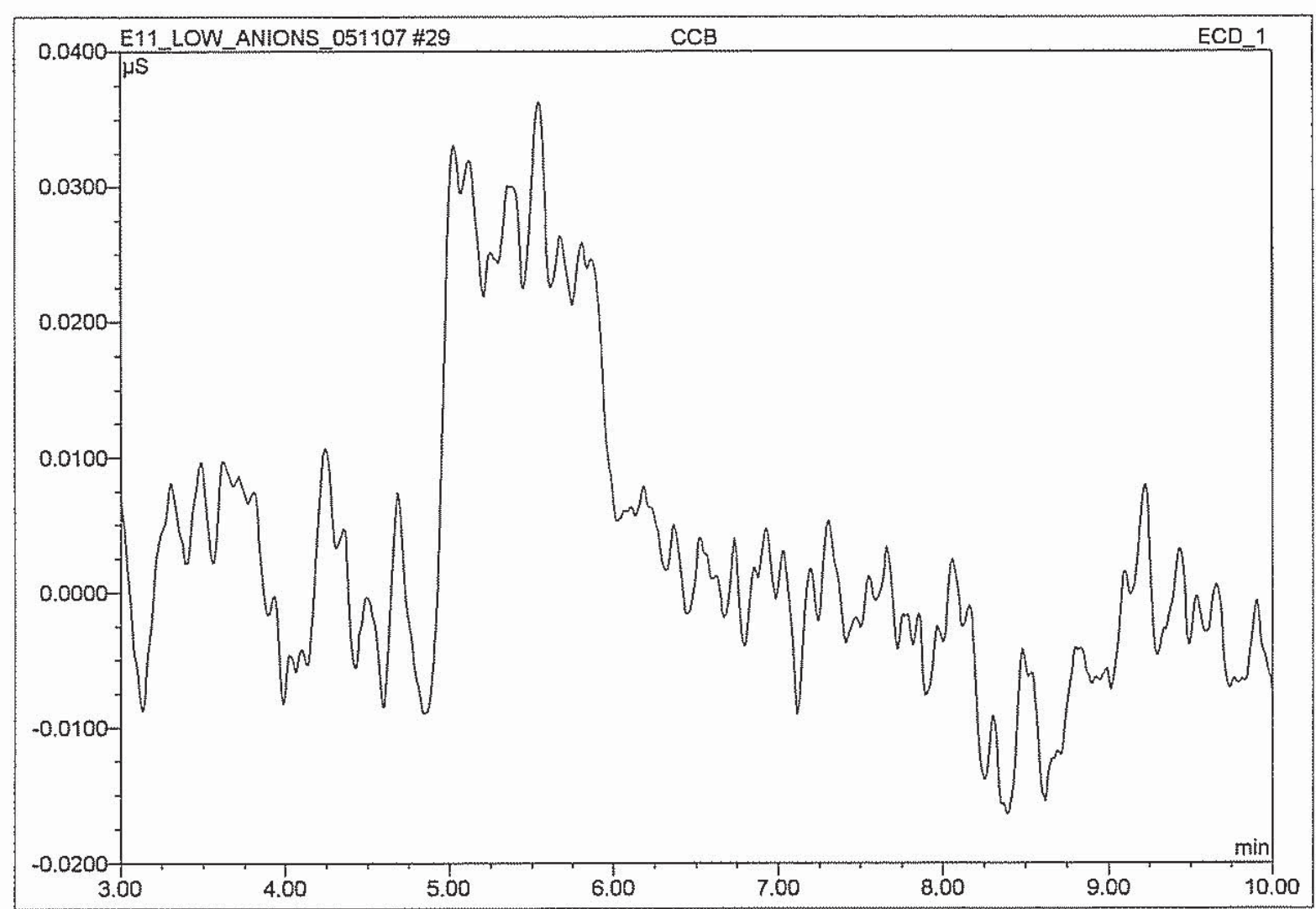

\begin{tabular}{|c|c|c|c|c|c|c|c|}
\hline No. & $\begin{array}{c}\text { Ret.Time } \\
\mathrm{min}\end{array}$ & Peak Name & $\begin{array}{c}\text { Height } \\
\mu \mathrm{S}\end{array}$ & $\begin{array}{c}\text { Area } \\
\mu \mathrm{S}^{*} \mathrm{~min}\end{array}$ & $\begin{array}{c}\text { Rel.Area } \\
\%\end{array}$ & $\begin{array}{c}\text { Amount } \\
\mathrm{mg} / \mathrm{L}\end{array}$ & $\begin{array}{c}\text { Peak } \\
\text { Type }\end{array}$ \\
\hline Total: & & & 0.000 & 0.000 & 0.00 & 0.000 & \\
\hline
\end{tabular}


古

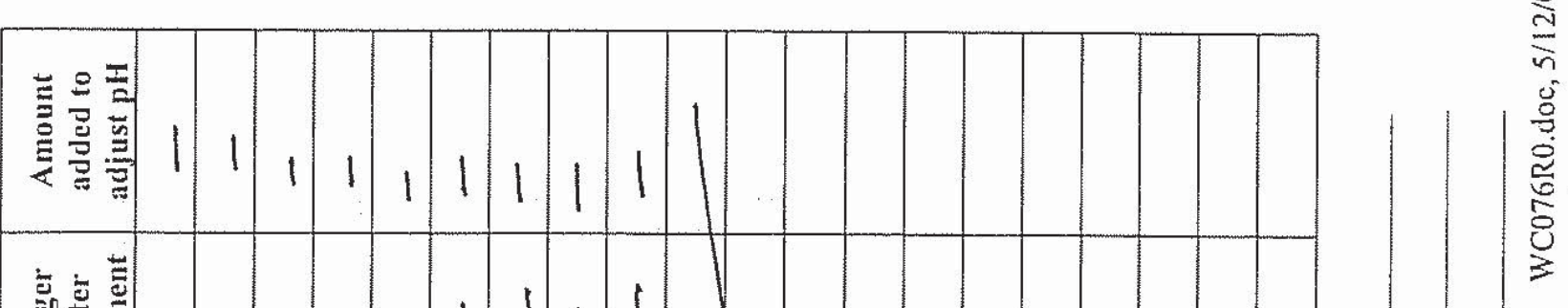

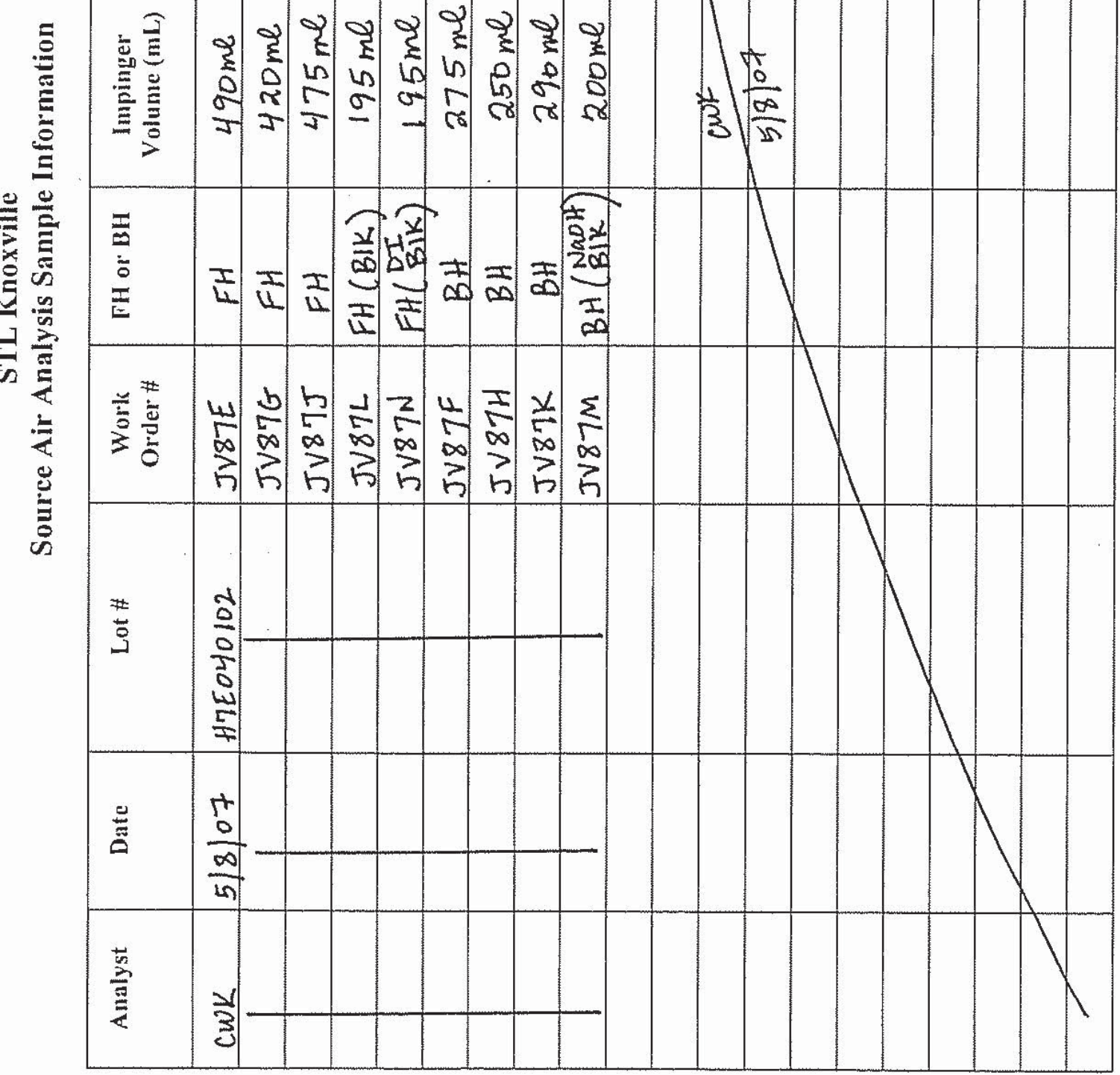



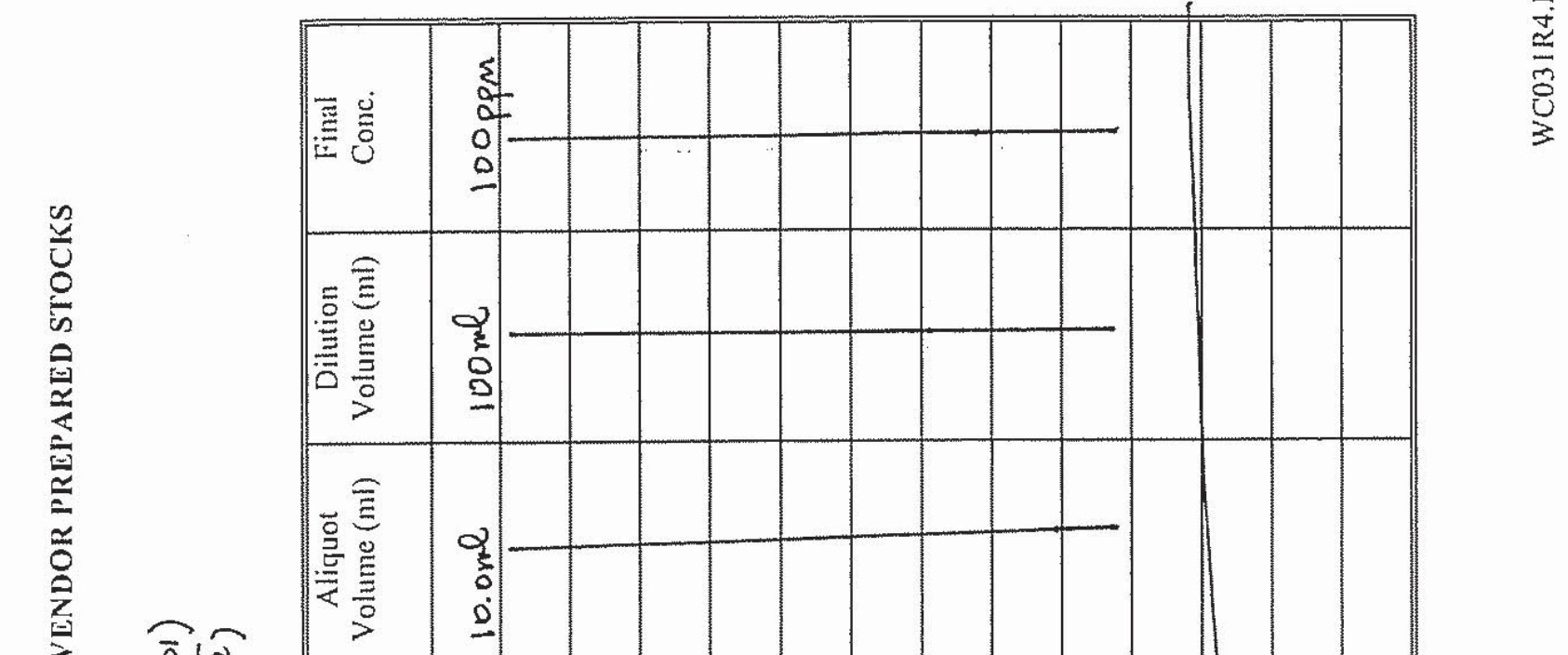

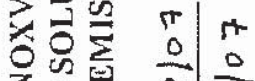
背望

的它包

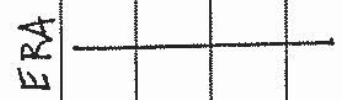

㐫

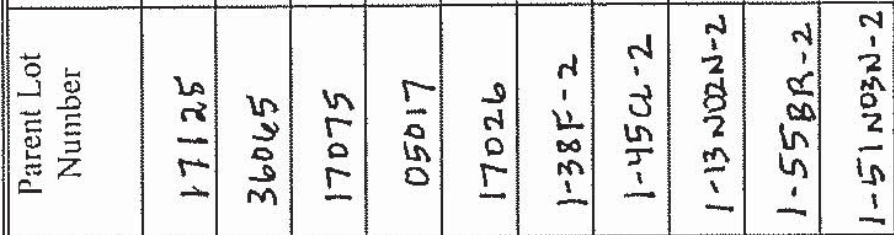




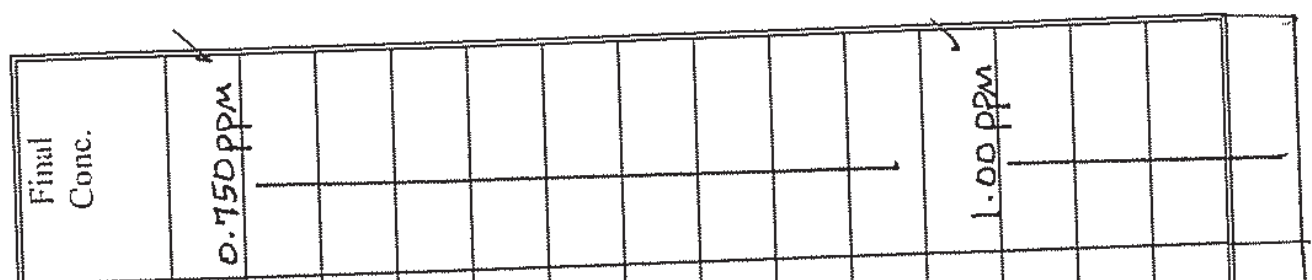

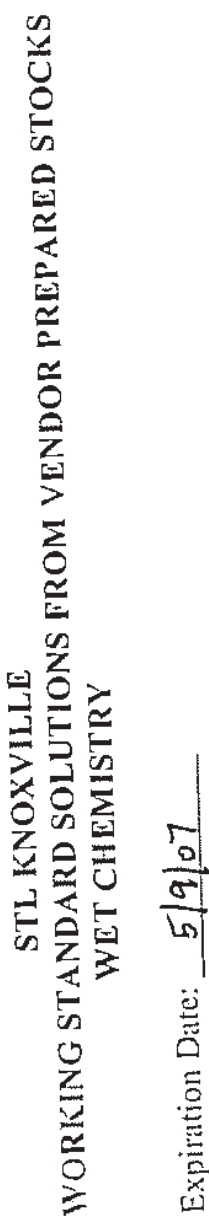
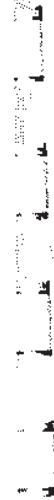

.

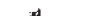

-

$\cdots$

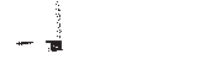

․․․

-

- $\frac{1}{2}$

$-\infty$

L。

$=$

$1 . \quad \equiv$

Is

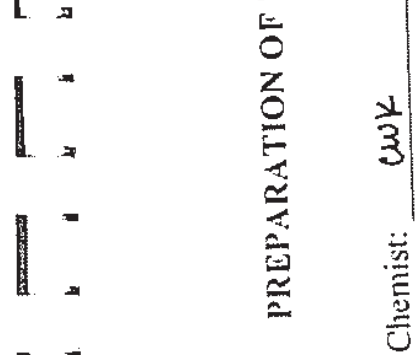

$1:$

望

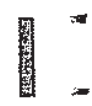

点

(

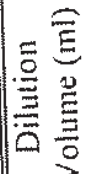

总

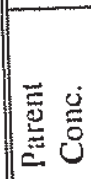

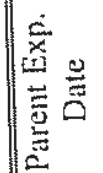

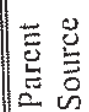

譬意

雍

2
0
9
0

譬量

3

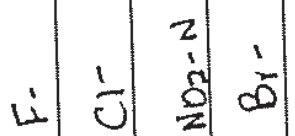

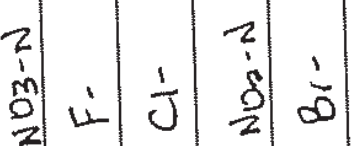

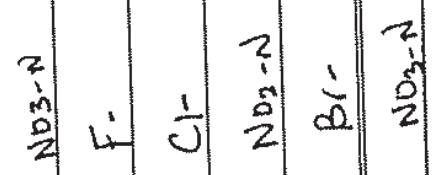
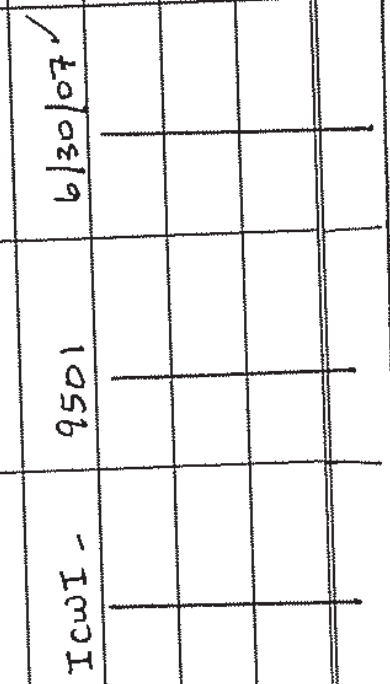

告 告

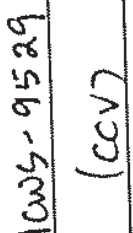

$\exists$

$\therefore \circ= \pm \therefore$ 


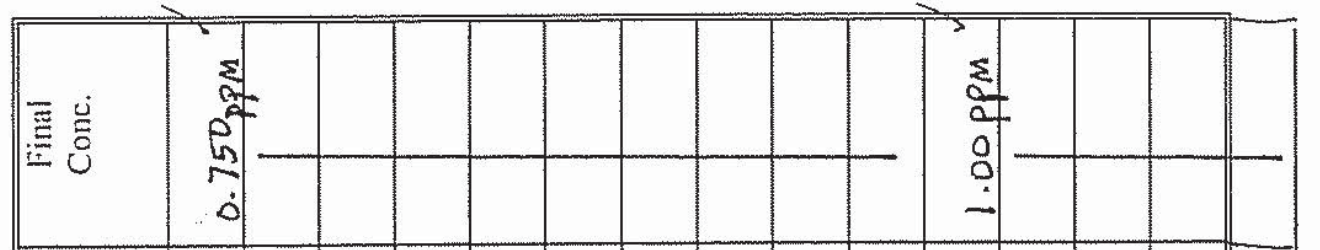

$=\frac{\widehat{a}}{\stackrel{\Xi}{\Xi}}$

염

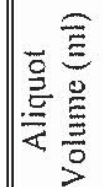

है

$-\frac{2}{0}$

를

势谣

\section{I}

高

$\sum$

\begin{tabular}{|l|l|l|l|l|l|l|l|l|l|l}
\hline & & & & & & & & & & \\
\hline
\end{tabular}

药 竞

บ

总 总

离。

焉焉

$\frac{0}{\frac{0}{0}}$

蒿总

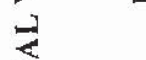

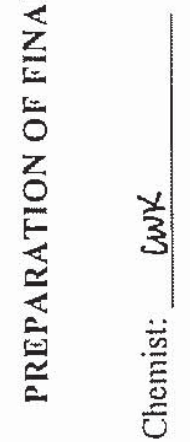

苍总

$>$

ถิ)

|

㟔
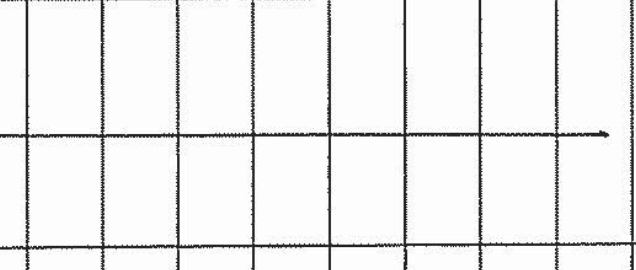

2
है
0
$\dot{0}$

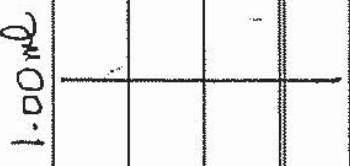

$\frac{5}{20}$

\begin{tabular}{|c|c|c|c|c|c|c|c|c|c|c|c|c|c|c|c|}
\hline 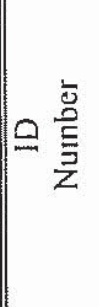 & 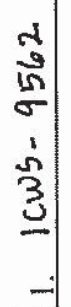 & $\frac{3}{3}$ & $m$ & $\rightarrow$ & जी & $\begin{array}{l}m \\
\vec{n} \\
5 \\
5 \\
3 \\
0 \\
0\end{array}$ & $\begin{array}{l}0 \\
0 \\
3 \\
3\end{array}$ & $\infty$ & बi & 의 & $\begin{array}{l}5 \\
5 \\
5 \\
\vdots \\
3 \\
3 \\
=\end{array}$ & $\begin{array}{l}3 \\
y \\
y\end{array}$ & 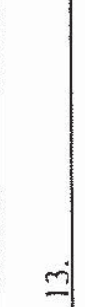 & \pm & $\underline{v}$ \\
\hline
\end{tabular}


Sample Receipt Documentation 

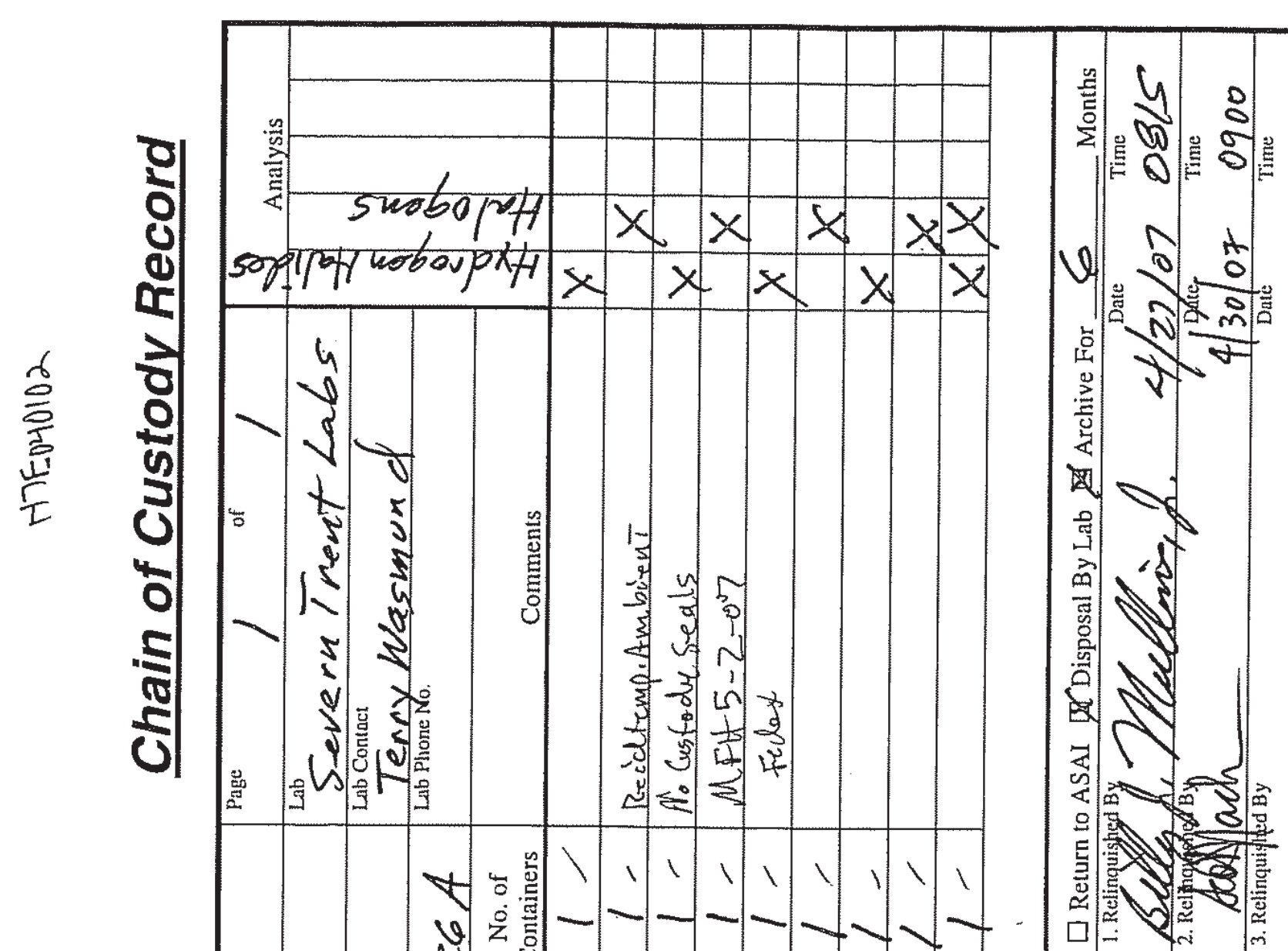

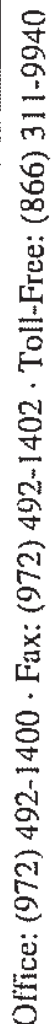

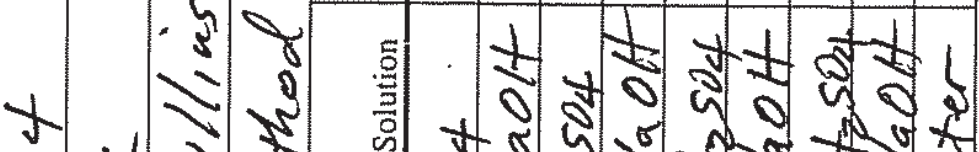

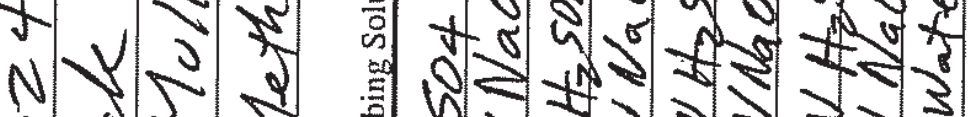
0 रर 5 : 1.50 र

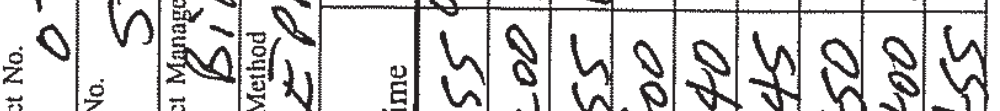
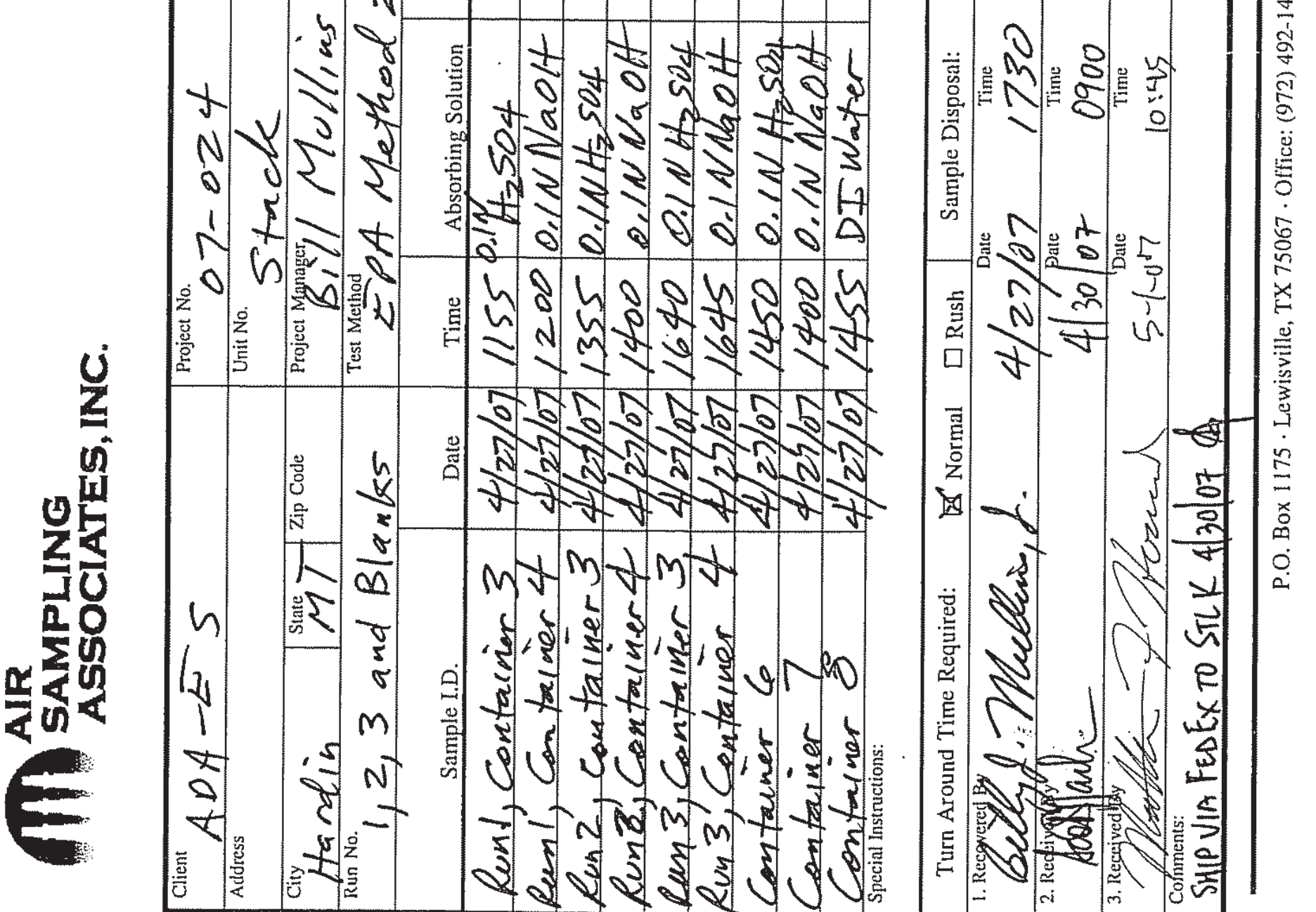


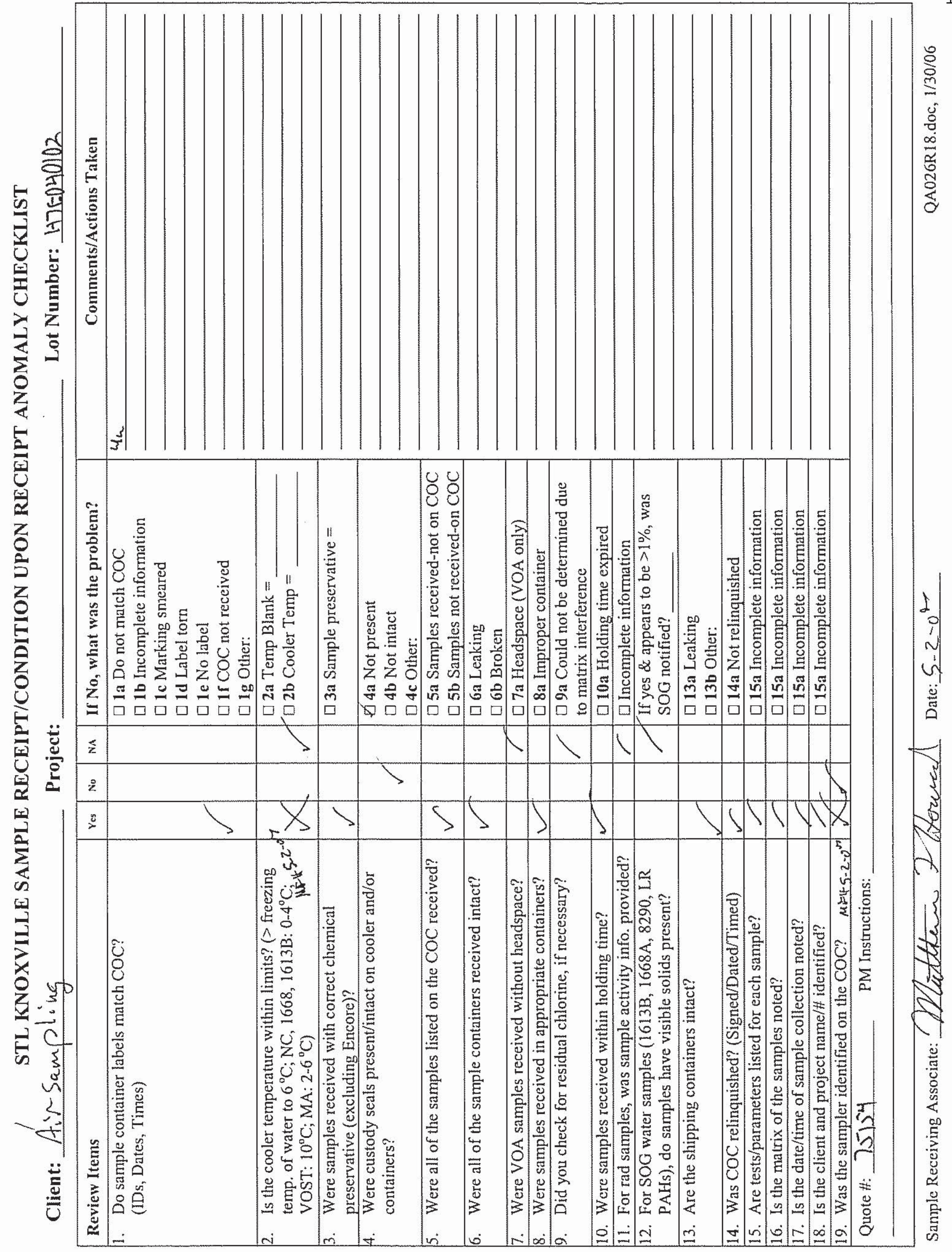


Appendix F:

EPA Test Methods 5, 17 (Particulate Matter), and ASTM D6784-02 (Ontario Hydro) Mercury Speciation Test Method Analytical Data 
TIT AIR

SAMPLING

ASSOCIATES, INC.

Air Sampling Associates, Inc.

Particulate Analysis Summary

Project Number: $07-024$

Project Name: $A D A-E S$
Date Analysis Completed: $5 / 11 / 07$

Unit Tested: SDA Inlet Duct

Project Location: Ha rd in, MT

Run Number:

Particulate Matter on Filter (mg):

$$
\text { " "Thistles: }
$$

Particulate Matter in Front Wash (mg):

Total Particulate Matter in "Front-Half" - MF (mg):

Particulate Matter in "Back-Half” (mg):

Total Particulate Matter in Sample - MT (mg):
Ontario -Hydro Train

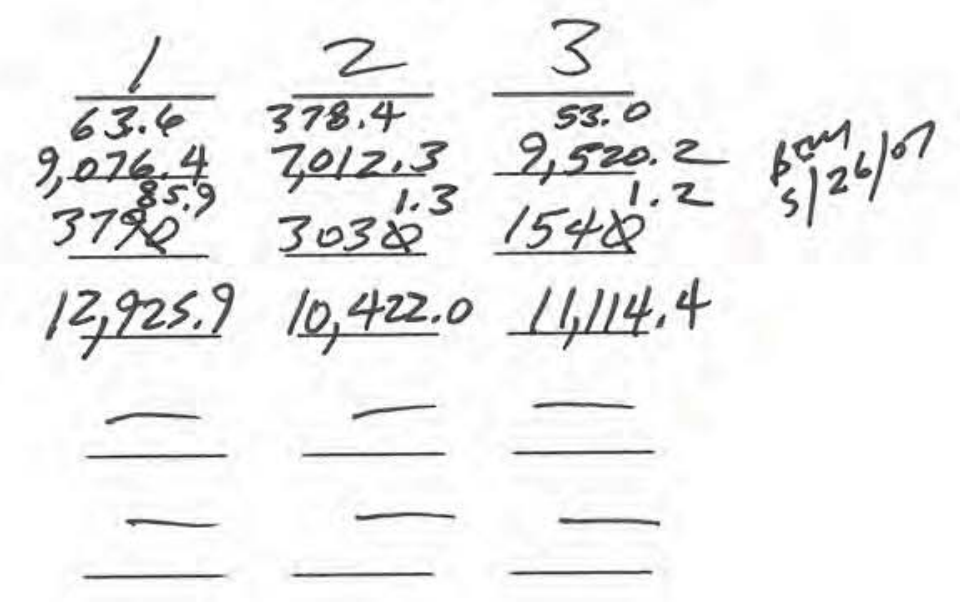

$\frac{\text { Severn Trent Laboratories }}{\text { Analyst }}$

${ }_{\text {Data Checked By }}$

Version No. 1 5/30/06

F-2 
Particulate Analysis EPA Method Ontais - Hydro

Thimber

Stack Filters

Project No. $07-024$

Project Name ADA-ES Location Hardin, MT

\begin{tabular}{|r|l|l|l|l|l|}
\hline Desiccator Time In & & & & & \\
\hline Desiccator Time Out & & & & & \\
\hline
\end{tabular}

\begin{tabular}{|c|c|c|c|c|c|}
\hline Run No. & Filter No. 50 & & \\
\hline Filter \& Particulate (g) & & & & & \\
\hline
\end{tabular}

Filter \& Particulate Average (g) 11.7251 Initial Filter Weight $(\mathrm{g}) 2.6487$ Total Particulate $(\mathrm{mg}) 9,076.4$

\begin{tabular}{|c|c|c|c|c|c|}
\hline Run No. & Filter No. 52 & & \\
\hline Filter \& Particulate (g) & & & & & \\
\hline
\end{tabular}

Filter \& Particulate Average (g) 9.8597 Initial Filter Weight $(\mathrm{g}) \geq .8474$

Total Particulate $(\mathrm{mg}) \& 7,012.3$

\begin{tabular}{|c|l|l|l|l|l|}
\hline Run No. 3 & Filter No. 5/ & & \\
\hline Filter \& Particulate $(\mathrm{g})$ & & & & & \multicolumn{3}{c|}{ Sample I.D. } \\
\hline
\end{tabular}

Filter \& Particulate Average (g) 12.3807 Initial Filter Weight $(\mathrm{g}) 2.8605$ Total Particulate $(\mathrm{mg}) \frac{2,520.2}{9}$

$\frac{\text { Severn Trent Labosatories }}{\text { Analyst }}$ 
Particulate Analysis EPA Method Ontario-Hydro

Stack Filters

Project No. $07-024$

Project Name $A D A-E S$
Location Unit Tested

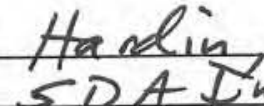

Tre a 7

\begin{tabular}{|r|l|l|l|l|l|}
\hline Desiccator Time In & & & & \\
\hline Desiccator Time Out & & & & & \\
\hline
\end{tabular}

\begin{tabular}{|c|c|c|c|c|c|}
\hline Run No. & Filter No. A-173 & \multicolumn{3}{c|}{ Sample I.D. } \\
\hline Filter \& Particulate (g) & & & & & \\
\hline
\end{tabular}

Filter \& Particulate Average (g) 0.4173

Initial Filter Weight $(\mathrm{g}) 0.3537$

Total Particulate (mg) 63,6

\begin{tabular}{|c|c|c|c|c|}
\hline Run No. & Filter No. $A-174$ & \multicolumn{1}{c|}{ Sample I.D. } \\
\hline Filter \& Particulate $(\mathrm{g})$ & & & & \\
\hline
\end{tabular}

Filter \& Particulate Average (g) 0.7263 Initial Filter Weight $(\mathrm{g}) 0.3479$ Total Particulate $(\mathrm{mg})$ 378.4

\begin{tabular}{|c|l|l|l|l|}
\hline Run No. 3 & Filter No. $A-175 \quad$ Sample I.D. \\
\hline Filter \& Particulate $(\mathrm{g})$ & & & & \\
\hline
\end{tabular}

Filter \& Particulate Average $(\mathrm{g}) 0.4133$ Initial Filter Weight (g) 0,3603 Total Particulate $(\mathrm{mg})$ 


\section{AIR \\ SAMPLING \\ ASSOCIATES, INC.}

Filter Weight Log

EPA Method 5

\begin{tabular}{|c|c|c|c|c|c|c|c|}
\hline \multirow[b]{3}{*}{ Filter No. } & Into Desicator & Weight & Weight & Weight & Weight & Weight & \multirow{3}{*}{$\begin{array}{l}\text { Weight } \\
\text { Used }\end{array}$} \\
\hline & Date & Date & Date & Date & Date & Date & \\
\hline & Time & Time & Time & Time & Time & Time & \\
\hline \multirow{2}{*}{$A 166$} & & 0,3795 & 0.3795 & & & & 0.3795 \\
\hline & $4 / 2 / 07$ & $4 / 13$ or & $4 / 17 / 07$ & & & & \\
\hline \multirow{3}{*}{ A167 } & $\frac{0800}{4 / 2107}$ & $\frac{6840}{0.3752}$ & $\frac{1423}{0.3751}$ & & & & 0,375 \\
\hline & 000 & सा17न & $4 / 12107$ & & & & \\
\hline & & $\frac{0815}{0.2380}$ & 1423 & $\operatorname{sm} 4(1) 101$ & & & \\
\hline \multirow{3}{*}{4168} & $4 / 2107$ & & 1423 & 0.3879 & & & 0.3879 \\
\hline & 6800 & $\frac{41.7 / 07}{69}$ & $\frac{4 / 12 / 9}{1422}$ & & & & \\
\hline & $101=7$ & 0.3936 & 0,3235 & & & $=$ & 0.3835 \\
\hline \multirow{2}{*}{4169} & $4 / 2 / 07$ & $\frac{41.7107}{0.315}$ & 410707 & & & & \\
\hline & & $\frac{0825}{0.3849}$ & $\frac{142,2}{0.3848}$ & & - & & 0.3848 \\
\hline \multirow[t]{2}{*}{4170} & $\frac{4 / 2 / 07}{4800}$ & $4 / 7 / 67$ & $4 / 17 / 07$ & & & & \\
\hline & 0800 & $\frac{6.315}{0.3819}$ & $\frac{1420}{0.3818}$ & & & & \\
\hline \multirow{2}{*}{$A 171$} & $4 / 207$ & 41967 & xin/0 & & & & 0.3818 \\
\hline & 6890 & $\therefore 816$ & 1420 & & & & \\
\hline \multirow{3}{*}{$A 172$} & 12107 & $\frac{0.3762}{4 / 10107}$ & $\frac{0,3762}{410107}$ & & & & 0.3762 \\
\hline & $7 / 2500$ & $\frac{78}{6816}$ & $\frac{4 / 1707}{1420}$ & & & & \\
\hline & & 0.3536 & 0.3537 & $\nu$ & & & 0,3537 \\
\hline \multirow{2}{*}{ A173 } & 4 1.d & 4120107 & $4 / 20107$ & & & & \\
\hline & 1500 & $\frac{1511}{0.3478}$ & $\frac{2230}{0.3479}$ & & & & \\
\hline \multirow{3}{*}{$A(74$} & 41507 & $\frac{0.54+8}{4120107}$ & $\frac{0.347^{9}}{4120107}$ & & & & 0.3479 \\
\hline & 1500 & 1512 & $\frac{4120107}{2230}$ & & & & \\
\hline & & 0.3603 & 0.3603 & & & & 0.3603 \\
\hline \multirow[t]{2}{*}{$A 175$} & 4120107 & 4126107 & 4120107 & & & & \\
\hline & 1500 & 15,3 & 2230 & & & & \\
\hline \multirow{3}{*}{$A 176$} & 41907 & $\frac{0.3667}{4.20107}$ & $\frac{0.3668}{4(2607}$ & 2 & & & 0.3668 \\
\hline & 1500 & 1514 & 2230 & & & & \\
\hline & & 0.3803 & 0.3806 & & & & 0,3806 \\
\hline \multirow{2}{*}{$A 177$} & $41=3107$ & 4 trecos & $4(20) \cdot 7)$ & & & & \\
\hline & 1500 & $\frac{15,5}{3020}$ & $\frac{2230}{0.3220}$ & & & & \\
\hline \multirow{2}{*}{$A 178$} & $4 \mid \frac{10}{00}$ & 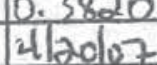 & $\begin{array}{l}\frac{0.3820}{4(20107} \\
4(20)\end{array}$ & & & & 0.3820 \\
\hline & 1500 & 1516 & 2230 & & & & \\
\hline & & & & & & & \\
\hline & & & & & & & \\
\hline & & & & & & & \\
\hline & & & & & & & \\
\hline & & & & & & & \\
\hline
\end{tabular}




\section{SARMPLING \\ ASSOCIATES, INC.}

Thimble Tare Weight Log

EPA Method 17

\begin{tabular}{|c|c|c|c|c|c|c|c|}
\hline \multirow[b]{3}{*}{ Filter No. } & Into Desicator & Weight & Weight & Weight & Weight & Weight & \multirow{3}{*}{$\begin{array}{l}\text { Weight } \\
\text { Used }\end{array}$} \\
\hline & Date & Date & Date & Date & Date & Date & \\
\hline & Time & Time & Time & Time & Time & Time & \\
\hline \multirow{3}{*}{45} & & 24.941 & 2.7944 & & & & $2.79+4$ \\
\hline & 9129106 & $10 \mathrm{k} / \mathrm{os}_{6}$ & $10 / 3106$ & & & & \\
\hline & 1200 & 1635 & 1100 & & & & \\
\hline \multirow{3}{*}{46} & 1 & 27987 & 2.7990 & & & & 2.7990 \\
\hline & & $10 / 2106$ & 1013106 & & & & \\
\hline & & 1636 & 1101 & & & & \\
\hline \multirow{3}{*}{47} & & 2.5514 & 2.55147 & & & & 2.5514 \\
\hline & & $10 / 2 / 06$ & $10 / 3106$ & & & & \\
\hline & & 1637 & 1162 & & & & \\
\hline \multirow{3}{*}{48} & & 2.7781 & 2.7785 & & & & 2.7785 \\
\hline & & $10 / 2106$ & 1013106 & & & & \\
\hline & & 1638 & 1103 & & $=$ & & \\
\hline \multirow{3}{*}{49} & & 2.7795 & 2.77997 & & & & 2.7799 \\
\hline & & $10 / 2 / 06$ & $10 / 3106$ & & & & \\
\hline & & 1639 & 1104 & & & & \\
\hline \multirow{3}{*}{50} & & 2.6485 & 2.6487 & $\bar{z}$ & & & 2.6487 \\
\hline & & $10 / 2 / 06$ & 1013106 & & & & \\
\hline & & 1640 & 1105 & & & & \\
\hline \multirow{3}{*}{51} & & 2.8600 & 2.8605 & & & & 2.8605 \\
\hline & & $10 / 2106$ & 1013106 & & & & 는 \\
\hline & & 1641 & 100 & & & & \\
\hline \multirow{3}{*}{52} & & 2.8470 & 2.8474 & & & & 2.8474 \\
\hline & & $10 / 2 / 06$ & 1013106 & & & & \\
\hline & & 1642 & 1107 & & & & \\
\hline \multirow{3}{*}{53} & & 2.1349 & 2.7352 & $E$ & & & 2.7352 \\
\hline & $\frac{1}{2}$ & $10 / 2106$ & 1013106 & & & & \\
\hline & & 1623 & no 8 & & & & \\
\hline \multirow{3}{*}{54} & & 3.5794 & 3,5795 & $\angle$ & & & 3.5795 \\
\hline & $11 / 28106$ & $1 / 21 / 06$ & $11 / 22 / 06$ & & & & \\
\hline & 1360 & 1350 & 6745 & & & & \\
\hline \multirow{3}{*}{55} & & 2.8785 & 28788 & $\angle$ & & & 2.8788 \\
\hline & & $11 / 21 / 06$ & $14 / 22 / 06$ & & & & \\
\hline & & 1351 & 00.66 & & & & \\
\hline \multirow{3}{*}{56} & & 2.4725 & 2.4724 & $\overline{7}$ & & & 2.4724 \\
\hline & & $11 / 21 / 06$ & $11 / 22 / 06$ & & & & \\
\hline & & 1352 & 6747 & & & & \\
\hline \multirow{3}{*}{57} & & 2.9916 & 2.99114 & E & & & 2.9911 \\
\hline & & $11 / 2106$ & $11 / 22 / 06$ & & & & \\
\hline & & 1353 & 2748 & & & & \\
\hline \multirow{3}{*}{58} & & 3.4660 & 3.4656 & $=$ & & & 3,4656 \\
\hline & & $11 / 21 / 06$ & $11 / 22 / 06$ & & & & \\
\hline & & 254 & 0749 & & & & \\
\hline \multirow{3}{*}{59} & & 3,1165 & 3.1160 & 2 & & & 3.1160 \\
\hline & & 112106 & 11/22/06 & & & & \\
\hline & 文 & 1355 & 6750 & & & & \\
\hline
\end{tabular}


AIR

SAMPLING

ASSOCIATES, INC.

Air Sampling Associates, Inc.

Particulate Analysis Summary

Project Number: $07-024$

Project Name: $A D A-E 5$

Project Location: Hardin, $M T$
Date Analysis Completed: $5 / 11 / 07$

Unit Tested: Stack
Run Number:

Particulate Matter on Filter (mg):

Particulate Matter in Front Wash (mg):

Total Particulate Matter in "Front-Half" - MF (mg):

Particulate Matter in "Back-Half" (mg):

Total Particulate Matter in Sample - MT (mg):
Ontario -Hydro Train

$\begin{array}{llll}\frac{1}{18.8} & \frac{2}{19.5} & \frac{3}{18.9} \\ \frac{24.8}{43.6} & \frac{13.6}{33.1} & \frac{54.3}{73.2}\end{array}$

$\frac{\text { Severn Trent Laboratories }}{\text { Analyst }}$

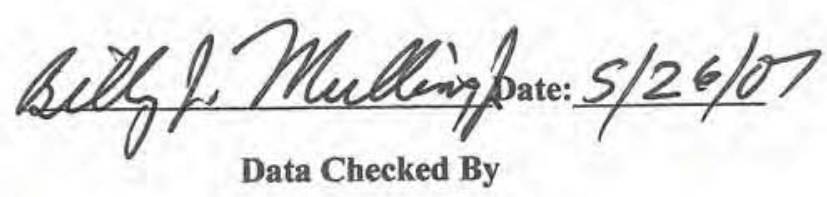

Version No. 1 5/30/06

F-7 

of

Particulate Analysis EPA Method Onfario-1 Hydro

Stack Filters

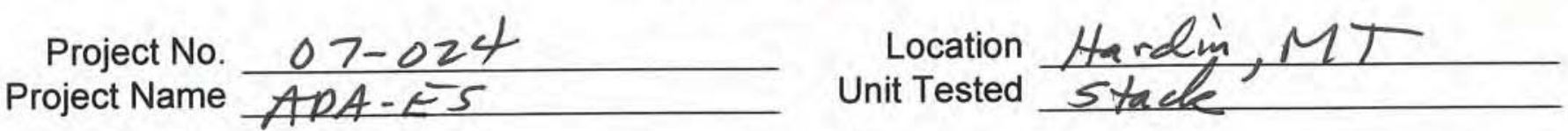

\begin{tabular}{|r|l|l|l|l|l|}
\hline Desiccator Time In & & & & \\
\hline Desiccator Time Out & & & & & \\
\hline
\end{tabular}

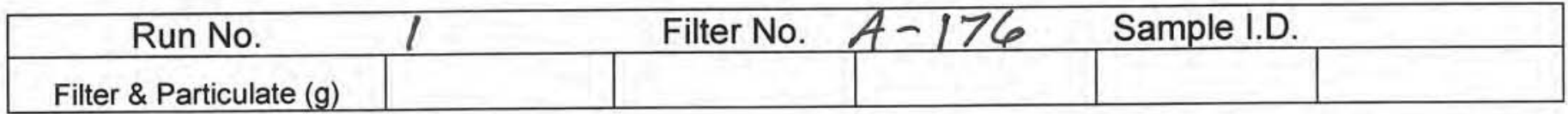

Filter \& Particulate Average (g) 0,3856 Initial Filter Weight $(\mathrm{g}) 0.3668$ Total Particulate (mg) 18,8

\begin{tabular}{|c|c|c|c|c|}
\hline Run No. Filter No. $A-177$ & \multicolumn{3}{|c|}{ Sample I.D. } \\
\hline Filter \& Particulate (g) & & & & \\
\hline
\end{tabular}

Filter \& Particulate Average (g) 0,4001 Initial Filter Weight $(\mathrm{g}) 0,3806$ Total Particulate $(\mathrm{mg})$ 19,5

\begin{tabular}{|c|l|l|l|l|}
\hline Run No. 3 & Filter No. $A-178$ Sample I.D. \\
\hline Filter \& Particulate $(\mathrm{g})$ & & & & \\
\hline
\end{tabular}

$\begin{array}{rc}\text { Filter \& Particulate Average }(\mathrm{g}) & 0.4009 \\ \begin{array}{r}\text { Initial Filter Weight }(\mathrm{g}) \\ \text { Total Particulate }(\mathrm{mg})\end{array} & \frac{0.3820}{18,9}\end{array}$

$\frac{\text { Seren Trent Laboratories }}{\text { Analyst }}$ 
H7E040218 Analytical Report

Sample Receipt Documentation

Metals ................................................................................ 80

Sample Results ................................................ 81

QC Summary ...................................................... 112

Quality Control Results Mercury ...................................... 125

Raw Data Mercury ….............................................. 143

Miscellaneous Data.............................................. 174

Wet Chemistry ............................................................... 186

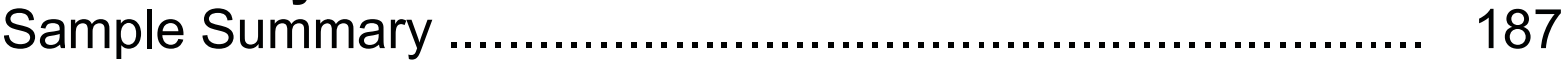

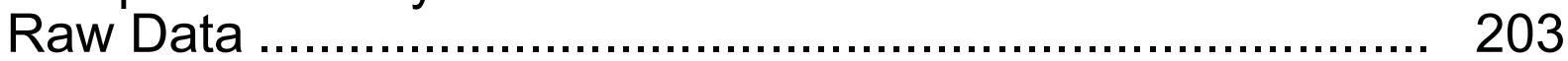

Sample Receipt Documentation ........................................ 212

Total Number of Pages ..................................................... 220 


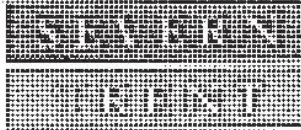

STL Knoxville

5815 Middiebrook Pike

Knoxvilie, TN 37921

Tel: 8652913000 Fax: 8655844315

www.stl-inc.corn

\section{ANALYTICAL REPORT}

PROJECT NO. 07-024

Hardin Montana - April 2007

Lot \#: H7E040218

\section{Bìll Hefley}

Air Sampling Associates, Inc. 407-2 B Holfords's Prairie Roa Lewisville, TX 75056

SEVERN TRENT LABORATORIES, INC.

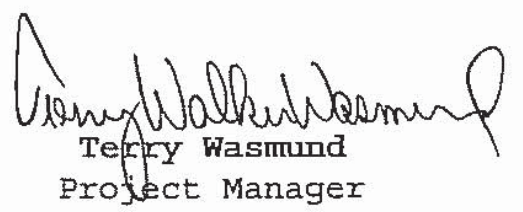

May 21, 2007 
ANALYTICAL METHODS SUMMARY

H7E040218

$\begin{array}{ll}\text { PARAMETER } & \begin{array}{l}\text { ANALYTICAL } \\ \text { METHOD }\end{array} \\ \begin{array}{l}\text { Mercury (Ontario Hydro) } \\ \text { Particulate Emissions }\end{array} & \begin{array}{l}\text { ASTM D6784-02 } \\ \text { CFR60A 5 }\end{array} \\ \text { References: } & \\ \text { ASTM Annual Book Of ASTM Standards. } & \\ \text { CFR60A "Test Methods", 40 CFR, Part 60, Appendix A, July 1, } 1993 .\end{array}$




\section{SAMPLE SUMMARY}

H7B040218

\begin{tabular}{ll} 
WO \# & SAMPLE \\
\cline { 2 - 2 } JWAEW & 001 \\
JWAFF & 002 \\
JWAFK & 003 \\
JWAFR & 004 \\
JWAFW & 005 \\
JWAFX & 006 \\
JWAF5 & 007 \\
JWAGJ & 008 \\
JWAGK & 009 \\
JWAGN & 010 \\
JWAGP & 011 \\
JWAGR & 012 \\
JWAG8 & 013 \\
JWAHC & 014 \\
JWAHE & 015 \\
JWAHH & 016 \\
JWAHL & 017 \\
JWAHM & 018 \\
JWAH3 & 019 \\
JWAH5 & 020 \\
JWAH6 & 021 \\
JWAH7 & 022 \\
JWAJA & 023 \\
JWAJH & 024 \\
JWAJR & 025 \\
JWAJV & 026 \\
JWAJ5 & 027 \\
JWAJ9 & 028 \\
JWAKF & 029 \\
JWAKG & 030 \\
JWAKK & 031 \\
JWAK0 & 032 \\
JWAK9 & 033 \\
JWALL & 034 \\
JWALV & 035 \\
JWAL3 & 036 \\
&
\end{tabular}

\begin{abstract}
CLIENT SAMPLE ID
\end{abstract}
HARDIN STACK RUN 1 FILTER Al76

HARDIN STACK RUN I ACETONE

HARDIN STACK RUN $1 \mathrm{FH}$

HARDIN STACK RUN $1 \mathrm{KCL}$

HARDIN STACK RUN 1 HNO3/H2O2

HARDIN STACK RUN I KMNO4/H2SO4

HARDIN STACK RUN 2 FILTER A177

HARDIN STACK RUN 2 ACETONE

HARDIN STACK RUN 2 FH

HARDIN STACK RUN 2 KCL

HARDIN STACK RUN 2 HNO3/H2O2

HARDIN STACK RUN 2 KMNO4/H2SO4

HARDIN STACK RUN 3 FILTER AI78

HARDIN STACK RUN 3 ACETONE

HARDIN STACK RUN $3 \mathrm{FH}$

HARDIN STACK RUN 3 KCL

HARDIN STACK RUN 3 HNO3/H2O2

HARDIN STACK RUN 3 KMNO4/H2SO4

HARDIN SDA INLET DUCT RUN 1 FILTER (THIMBLE 50)

HARDIN SDA INLET DUCT RUN I FILTER (A173)

HARDIN SDA INLET DUCT RUN I ACETONE

HARDIN SDA INUET DUCT RUN 1 FH

HARDIN SDA INLET DUCT RUN $1 \mathrm{KCL}$

HARDIN SDA INLET DUCT RUN 1 HNO3/H2O2

HARDIN SDA INLET DUCT RUN 1 KMNO4,H2SO4

HARDIN SDA INLET DUCT RUN 2 FILTER (THIMBLE 52)

HARDIN SDA INLET DUCT RUN 2 FILTER (A174)

HARDIN SDA INLET DUCT RUN 2 ACETONE

HARDIN SDA INLET DUCT RUN $2 \mathrm{FH}$

HARDIN SDA INLET DUCT RUN 2 KCL

HARDIN SDA INLET DUCT RUN 2 HNO3/H2O2

HARDIN SDA INLET DUCT RUN 2 KMNO4/H2SO4

HARDIN SDA INLET DUCT RUN 3 FILTER (THIMBLE 51)

HARDIN SDA INLET DUCT RUN 3 FILTER (A175)

HARDIN SDA INLET DUCT RUN 3 ACETONE

HARDIN SDA INLET DUCT RUN $3 \mathrm{FH}$
SAMPLED SAMP DATE TIME

$04 / 26 / 07 \quad 14: 05$ 04/26/07 16:01 04/26/07 16:01 $04 / 26 / 07 \quad 13: 50$ $04 / 26 / 07 \quad 13: 32$ $04 / 26 / 07 \quad 13: 43$ $04 / 26 / 07 \quad 19: 49$ $04 / 26 / 07 \quad 19: 57$ $04 / 26 / 07 \quad 19: 59$ $04 / 26 / 07 \quad 19: 51$ $04 / 26 / 07 \quad 17: 23$ $04 / 26 / 07 \quad 17: 32$ 04/26/07 22:11 $04 / 26 / 07 \quad 22: 13$ $04 / 26 / 07 \quad 22: 12$ 04/26/07 22:13 04/26/07 21:51 04/26/07 21:59 $04 / 26 / 07 \quad 15: 25$ $04 / 26 / 07 \quad 15: 24$ $04 / 26 / 07 \quad 15: 52$ $04 / 26 / 07 \quad 15: 54$ $04 / 26 / 07 \quad 14: 48$ $04 / 26 / 07 \quad 14: 32$ $04 / 26 / 07 \quad 14: 41$ $04 / 26 / 07 \quad 19: 36$ $04 / 26 / 07$ 19:18 $04 / 26 / 07 \quad 19: 44$ $04 / 26 / 07 \quad 19: 46$ 04/26/07 19:08 $04 / 26 / 07 \quad 18: 43$ $04 / 26 / 07$ 18:29 $04 / 26 / 0721: 33$ $04 / 26 / 07 \quad 21: 29$ $04 / 26 / 07 \quad 21: 47$ $04 / 26 / 07 \quad 21: 50$ 


\section{SAMPLE SUMIMARY}

H7B 040218

\begin{tabular}{ll}
\hline & \\
JWAMG & 037 \\
JWAMK & 038 \\
JWAMP & 039 \\
JWAM2 & 040 \\
JWAM8 & 041 \\
JWAND & 042 \\
JWANG & 043 \\
JWAPF & 044 \\
JWAPP & 045
\end{tabular}

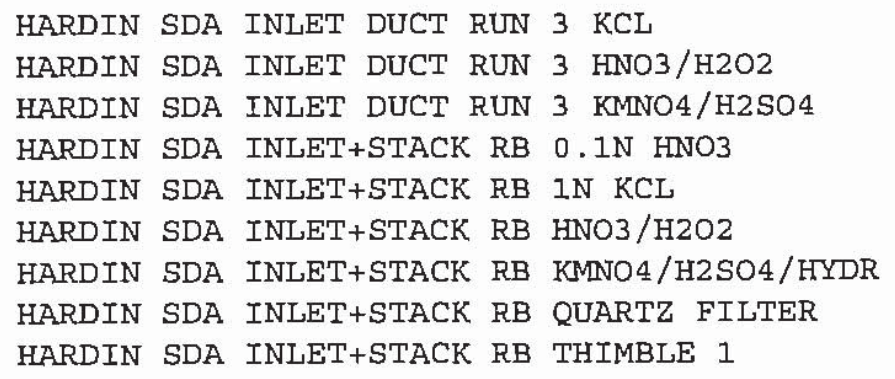

HARDIN SDA INLET DUCT RUN 3 KCL

HARDIN SDA INLET DUCT RUN 3 HNO3/H2O2

HARDIN SDA INLET DUCT RUN 3 KMNO4/H2SO4

HARDIN SDA INLET+STACK RB $0.1 \mathrm{~N}$ HNO3

HARDIN SDA INLET+STACK RB IN KCL

HARDIN SDA INLET+STACK RB HNO3/H2O2

HARDIN SDA INLET+STACK RB KMNO4/H2SO4/HYDR

HARDIN SDA INLET+STACK RB QUARTZ FILTER

HARDIN SDA INLET+STACK RB THIMBLE 1

SAMPLED SAMP

DATE TIME

04/26/07 21:22

$04 / 26 / 07 \quad 21: 06$

04/26/07 21:11

$04 / 26 / 07 \quad 22: 17$

$04 / 26 / 07 \quad 17: 43$

$04 / 26 / 07 \quad 17: 46$

$04 / 26 / 07 \quad 17: 48$

$04 / 26 / 0720: 16$

$04 / 26 / 0720: 16$

\section{NOTE (S) :}

- The analytical results of the saniples listed above are presented on the following pages.

- All caleulations are performed before rourding to avoid round-off errors in calculated results.

- Results noted as "ND" were not detected at or above the stated limis.

- This report must not be reproduced, except in full, without the written approval of the laboratory.

- Results for the following parameters are never repored on a dry weight basis: color, corrosivity, density, Mashpoint, ignitability, layers, odor, paint fitter test, ph, porosity pressure, teactivity, redox potential, specifie gravity, spot tests, solids, solubility, temperature, viscosity, and weight. 


\section{QC DATA ASSOCIATION SUMMARY}

H7E040218

Sample Preparation and Analysis Control Numbers

\begin{tabular}{|c|c|c|c|c|c|}
\hline SAMPLE\# & MATRIX & $\begin{array}{l}\text { ANALYTICAL } \\
\text { METHOD }\end{array}$ & $\begin{array}{l}\text { LEACH } \\
\text { BATCH \# }\end{array}$ & $\begin{array}{l}\text { PREP } \\
\text { BATCH \# }\end{array}$ & MS RUN\#\# \\
\hline 001 & AIR & CFR60A 5 & & 7130097 & \\
\hline 002 & AIR & CFR60A 5 & & 7130097 & \\
\hline 003 & AIR & ASTM D6784-02 & & 7134095 & \\
\hline 004 & AIR & ASTM D6784-02 & & 7127427 & 7127247 \\
\hline 005 & AIR & ASTM D6784-02 & & 7127421 & 7127246 \\
\hline 006 & AIR & A.STM D6784-02 & & 7127428 & 7127248 \\
\hline 007 & AIR & CFR60A 5 & & 7130097 & \\
\hline 008 & AIR & CFR60A 5 & & 7130097 & \\
\hline 009 & AIR & ASTM D6784-02 & & 7134095 & \\
\hline 010 & AIR & A.STM D6784-02 & & 7127427 & \\
\hline 011 & AIR & A.STM D6784-02 & & 7127421 & \\
\hline 012 & AIR & ASTM D6784-02 & & 7127428 & \\
\hline 013 & AIR & CFR60A 5 & & 7130097 & \\
\hline 014 & AIR & CFR60A 5 & & 7130097 & \\
\hline 015 & AIR & ASTM D6784-02 & & 7134095 & \\
\hline 016 & AIR & ASTM D $6784-02$ & & 7127427 & \\
\hline 017 & AIR & ASTM D6784-02 & & 7127421 & \\
\hline 018 & AIR & ASTM D6784-02 & & 7127428 & \\
\hline 019 & AIR & CFR60A 5 & & 7130097 & \\
\hline 020 & AIR & CFR60A 5 & & 7130097 & \\
\hline 021 & AIR & CFR60A 5 & & 7130097 & \\
\hline
\end{tabular}

(Continued on next page) 


\section{QC DATA ASSOCIATION SUMMARY}

H7E040218

Sample Preparation and Analysis Control Numbers

\begin{tabular}{|c|c|c|c|c|c|}
\hline SAMPLE\# & MATRIX & $\begin{array}{l}\text { ANALYTICAL } \\
\text { METHOD }\end{array}$ & $\begin{array}{l}\text { LEACH } \\
\text { BATCH \# }\end{array}$ & $\begin{array}{l}\text { PREP } \\
\text { BATCH \# }\end{array}$ & MS RUN\# \\
\hline 022 & AIR & ASTM D6784-02 & & 7134095 & \\
\hline 023 & AIR & ASTM D6784-02 & & 7127427 & \\
\hline 024 & AIR & ASTM D6784-02 & & 7127421 & \\
\hline 025 & AIR & ASTM D6784-02 & & 7127428 & \\
\hline 026 & AIR & CFR60A 5 & & 7130097 & \\
\hline 027 & AIR & CFR60A 5 & & 7130097 & \\
\hline 028 & AIR & CFR60A 5 & & 7130097 & \\
\hline 029 & AIR & ASTM D6784-02 & & 7134095 & \\
\hline 030 & AIR & ASTM D67B4-02 & & 7127427 & \\
\hline 031 & AIR & ASTM D6784-02 & & 7127421 & \\
\hline 032 & AIR & ASTM D6784-02 & & 7127428 & \\
\hline 033 & AIR & CFR60A 5 & & 7130097 & \\
\hline 034 & AIR & CFR60A 5 & & 7130097 & \\
\hline 035 & AIR & CFR60A 5 & & 7130097 & \\
\hline 036 & AIR & ASTM D6784-02 & & 7134095 & \\
\hline 037 & AIR & ASTM D6784-02 & & 7127427 & \\
\hline 038 & AIR & ASTM D6784-02 & & 7127421 & \\
\hline 039 & $\mathrm{AIR}$ & ASTM D6784-02 & & 7127428 & \\
\hline 040 & AIR & ASTM D6784-02 & & 7134095 & \\
\hline 041 & AIR & ASTM D6784-02 & & 7127427 & \\
\hline 042 & AIR & ASTM D6784-02 & & 7127421 & \\
\hline
\end{tabular}

(Continued on next page) 


\section{QC DATA ASSOCIATION SUMMARY}

H7E040218

Sample Preparation and Analysis Control Numbers

$\begin{array}{clllll}\text { SAMPLE\# } & \text { MATRIX } & \begin{array}{l}\text { ANALYTICAL } \\ \text { METHOD }\end{array} & \begin{array}{l}\text { LEACH } \\ \text { BATCH \# }\end{array} & \begin{array}{l}\text { PREP } \\ \text { BATCH \# }\end{array} & \text { MS RUN\# } \\ 043 & \text { AIR } & \text { ASTM D6784-02 } & & 7127428 \\ 044 & \text { AIR } & \text { ASTM D6784-02 } & & 7134095 \\ 045 & \text { AIR } & \text { ASTM D6784-02 } & & 7134095\end{array}$




\section{PROJECT NARRATIVE H7E040218}

The results reported herein are applicable to the samples submitted for analysis only.

This report shall not be reproduced except in full, without the written approval of the laboratory.

The original chain of custody documentation is included with this report.

\section{Sample Receipt}

Custody seals were not present upon sample receipt at STL Knoxville.

\section{Quality Control and Data Interpretation}

Unless otherwise noted, all holding times and QC criteria were met and the test results shown in this report meet all applicable NELAC requirements.

\section{Metals}

These stack gas samples were prepared and analyzed using STL Knoxville standard operating procedure KNOX-IP-0006 which is based on ASTM Method D6784-02, "Standard Test Method for Elemental, Oxidized, Particle-Bound and Total Mercury in Flue Gas Generated from Coal-Fired Stationary Sources (Ontario Hydro Method)" with modifications from EPA Method 29. SW-846 Method 7470A, as incorporated in STL Knoxville standard operating procedure KNOX-MT-0009, was used to perform the final instrument analysis.

These front half samples were digested using a modified procedure of the Ontario Hydro SOP (KNOX-IP-0006). Samples (JWAH7) HARDIN SDA INLET DUCT RUN 1 FH, (JWAKF) HARDIN SDA INLET DUCT RUN 2 FH and (JWAL3) HARDIN INLET DUCT RUN 3 FH were received with a thimble, filter, $0.1 \mathrm{~N}$ nitric acid probe rinse and an acetone rinse. Samples (JWAFK) HARDIN STACK RUN 1 FH, (JWAGK) HARDIN STACK RUN 2 FH and (JWAHE) HARDIN STACK RUN $3 \mathrm{FH}$ were received with a filter, $0.1 \mathrm{~N}$ nitric acid probe rinse and an acetone rinse.(JWAM2) HARDIN SDA INLET+STACK RB 0.1N HN03 is a reagent blank. (JWAPF) HARDIN SDA INLET+STACK RB QUARTZ FILTER and (JWAPP) HARDIN SDA INLET+STACK RB THIMBLE 1 are reagent blanks. A particulate

STL Knoxville maintains the following certifications, approvals and accreditations: Arkansas DEQ Cert. H05-043-0, California DHS ELAP Cert. \#2423, Colorado DPHE, Connecticut DPH Cert. \#PH-0223, Florida DOH Cert. \#E87177, Georgia DNR Cert. \#906, Hawaii DOH, Illinois EPA Cert. \#000687, Indiana DOH Cert. \#C-TN-02, Iowa DNR Cert.

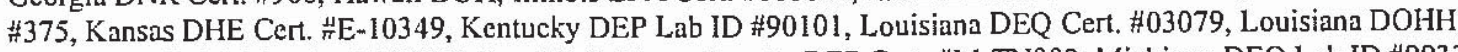
Cert. \#LA030024, Maryland DHMH Cert. \#277, Massachusetts DEP Cert. \#M-TN009, Michigan DEQ Lab ID \#9933, New Jersey DEP Cert. \#TN001, New York DOH Lab " 10781 , North Carolina DPH Lab ID \#21705, North Carolina DEHNR Cert. \#64, Ohio EPA VAP Cert. \#CL0059, Oklahoma DEQ ID South Carolina DHEC Lab ID \#84001001, Tennessee DOH Lab ID \#02014, Utah DOH Cert. \# QUAN3, Virginia DGS Lab ID \#00165, Washington DOE Lab \#C120, West Virginia DEP Cert. \#345, Wisconsin DNR Lab ID \#998044300, Naval Facilities Engineering Service Center and USDA Soil Permit \#S-46424. This list of approvals is subject to change and does not imply that laboratory certification is available for all parameters reported in this environmental sample data report. 


\section{PROJECT NARRATIVE H7E040218}

analysis was performed prior to the metals preparation. Some of the samples had large quantities of particulate matter. Instead of taking portions of the particulate and probe rinses to digest the entire front half of each sample was digested. This was done using acid rinsed glass beakers labeled with the corresponding sample ID. For samples that had a thimble and a filter, the thimble and the filter were torn into pieces and placed in the beaker along with the ash, acetone rinse, and the nitric probe rinse. For samples that had a filter and no thimble, the filter was torn into pieces and placed in the beaker along with the acetone rinse and the nitric probe rinse. For the reagent blank samples; the thimble was torn into pieces and placed into a beaker, the filter was torn into pieces and placed into a beaker, and the $0.1 \mathrm{~N}$ nitric rinse was poured into a beaker. Added to samples JWAFK, JWAGK, JWAHE, JWAH7, JWAKF, and JWAL3 was $6 \mathrm{~mL}$ concentrated HN03 and $3 \mathrm{~mL}$ conc. HCl. For sample JWAM2, $3 \mathrm{~mL}$ conc. HN03 added, and for JWAPF and JWAPP $3 \mathrm{~mL}$ conc. $\mathrm{HN} 03$ and $3 \mathrm{~mL}$ conc. $\mathrm{HCl}$ was added. The method blank, LCS and LCSD were also digested in beakers in which $20 \mathrm{~mL} 0.1 \mathrm{~N} \mathrm{HN03}$ was added to each beaker along with $6 \mathrm{~mL}$ conc. HN03 and $3 \mathrm{~mL}$ conc. HCl. The LCS/LCSD were spiked with $50 \mathrm{uL}$ of a $\mathrm{Hg} 10 \mathrm{ppm}$ standard. To samples JWAPF and JWAPP an additional $100 \mathrm{~mL}$ of $0.1 \mathrm{~N} \mathrm{HN} 03$ was added to prevent the samples from going dry. All of the beakers were placed onto a hotplate at 90-95 degrees celsius. After a reduction in sample volume to 50 $\mathrm{mL}$, the samples were removed from the hotplate and allowed to cool and then they were filtered thru Whatman 41 filter paper, if needed, and brought to a final volume of $100 \mathrm{~mL}$ with DI water. A portion of this digestate $(50 \mathrm{~mL})$ was taken for mercury prep and analysis.

Results were calculated using the following equation:

$H g, u g=(H g, u g / L) *($ Digestate Volume, $L) *\left(\frac{\text { Final Volume Hg Digestate }(m L)}{\text { Volume Digestate Used }(m L)}\right) *$ Bench Dilution

Please note that the dilution factor reported on the sample result form for the front half samples is actually a combination of preparation factors and bench dilution factors.

For the $5 \% \mathrm{HNO}_{3} / 10 \% \mathrm{H}_{2} \mathrm{O}_{2}$ impinger samples, a 10 milliliter portion of the sample as received was processed for mercury. The $\mathrm{KCl}$ and $4 \% \mathrm{KMnO}_{4} / 10 \% \mathrm{H}_{2} \mathrm{SO}_{4}$ impinger samples were treated with hydroxylamine hydrochloride, followed by removal of a $25 \mathrm{~mL}$ portion of sample for mercury processing. Results were calculated using the equation listed below. For

STL Knoxville maintains the following certifications, approvals and accreditations: Arkansas DEQ Cert. $\frac{.1}{\pi} 05-043-0$, California DHS ELAP Cert. \$2423, Colorado DPHE, Connecticut DPH Cert. \#PH-0223, Florida DOH Cert. \#E87177, Georgia DNR Cert. \#906, Hawaii DOH, Illinois EPA Cert. \#000687, Indiana DOH Cert. \#C-TN-02, lowa DNR Cert. \#375, Kansas DHE Cert. \#E-10349, Kentucky DEP Lab ID \#90101, Louisiana DEQ Cert. \#03079, Louisiana DOHH Cert. \#LA030024, Maryland DHMH Cert. \#277, Massachusetts DEP Cert. \#M-TN009, Michigan DEQ Lab ID \#9933, New Jerscy DEP Cert. \#TN001, New York DOH Lab \#10781, North Carolina DPH Lab ID \#21705, North Carolina

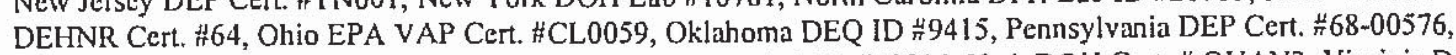
South Carolina DHEC Lab ID \#84001001, Tennessee DOH Lab ID \#02014, Utah DOH Cert. \# QUAN3, Virginia DGS Lab ID \#00165, Washington DOE Lab \#C120, West Virginia DEP Cert. \#345, Wisconsin DNR Lab ID \#998044300, Naval Facilities Engineering Service Center and USDA Soil Pernit \#S-46424. This list of approvals is subject to change and does not imply that laboratory certification is available for all parameters reported in this environmental sample data report. 


\section{PROJECT NARRATIVE H7E040218}

the $\mathrm{KCl}$ and $4 \% \mathrm{KMnO}_{4} / 10 \% \mathrm{H}_{2} \mathrm{SO}_{4}$ impinger samples, the sample volume includes the volume of hydroxylamine hydrochloride added to the sample.

$H g, u g=(H g, u g / L) *($ Sample Volume, $L) *\left(\frac{\text { FinalVolume Hg Digestate }(m L)}{\text { Volume Sample Digested }(m L)}\right) *$ Bench Dilution

Please note that the dilution factor reported on the sample result form for the back half impingers is actually the combination of preparation factors (not just a dilution factor) required by the method to convert the $\mathrm{Hg}$ reporting limits and method detection limits in concentration units from ug/L to a total ug unit:

DilutionFactor $=($ Volume, $L) *\left(\frac{\text { FinalVolume Hg Digestate }(m L)}{\text { Volume Sample Digested }(m L)}\right) *$ Bench Dilution

The matrix spike/matrix spike duplicate recoveries for sample HARDIN STACK RUN 1 KMNO4/H2S04 were outside control limits for mercury because the native analyte concentration in the sample was at least four times greater than the spike level. The laboratory control sample showed acceptable results indicating that the analysis was in control.

\section{Wet Chemistry}

The measurement of the mass of particulate matter trapped by the particulate filter and probe rinse derived from an M-5 sampling train was performed using SOP number KNOX-WC0006 (based on EPA Methods 0050 and 5). Microfiber filters, Petri dishes, and $150 \mathrm{~mL}$ beakers are carefully inspected and tare weighed to constant weight. After sample collection, the filters are dried, and then carefully weighed to constant weight to determine the mass of particulate matter trapped on the filters. The acetone probe rinse solution is evaporated to dryness, and then weighed to constant weight to determine the total particulate mass collected in the rinse. The total particulate mass collected by an M-5 train is the sum of the particulate filter and the acetone probe rinse residue weights.

Filter thimbles and disks that were not prepared by STL Knoxville were submitted for analysis. The reported weight is the gross weight, which includes the total weight of the filter and the entrained particulate material. Unfortunately, the laboratory information system will

STL Knoxville maintains the following certifications, approvals and accreditations: Arkansas DEQ Cert. $\# 05-043-0$, California DHS ELAP Cert. \#2423, Colorado DPHE, Connecticut DPH Cert. \#PH-0223, Florida DOH Cert. \#E87177, Georgia DNR Cert. \#906, Hawaii DOH, Illinois EPA Cert. \#000687, Indiana DOH Cert. \#C-TN-02, Iowa DNR Cert. $\# 375$, Kansas DHE Cert. 華E 10349, Kentucky DEP Lab ID $\# 90101$, Louisiana DEQ Cert. \#03079, Louisiana DOHH Cert. \#LA030024, Maryland DHMH Cert. \#277, Massachusetts DEP Cert. \#M-TN009, Michigan DEQ Lab ID \#9933, New Jersey DEP Cert. HTN001, New York DOH Lab H10781, North Carolina DPH Lab ID H21705, North Carolina DEHNR Cert. \#64, Ohio EPA VAP Cert. \#CL0059, Oklahoma DEQ ID \#9415, Pennsylvania DEP Cert. \#68-00576, South Carolina DHEC Lab ID $\# 84001001$, Tennessee DOH Lab ID $\# 02014$, Utah DOH Cert. \# QUAN3, Virginia DGS Lab ID H00165, Washington DOE Lab 금 120 , West Virginia DEP Cert. \#345, Wisconsin DNR Lab ID \#998044300, Naval Facilities Engineering Service Center and USDA Soil Permit \#S-46424. This list of approvals is subject to change and does not imply that laboratory certification is available for all parameters reported in this environmental sample data report. 


\section{PROJECT NARRATIVE H7E040218}

not report sufficient digits to obtain a precise net particulate weight when the tare weight is subtracted from the gross weight. The actual raw data is tabulated below for the user's convenience.

\begin{tabular}{|c|c|l|c|}
\hline $\begin{array}{c}\text { Lot-Sample } \\
\text { Number }\end{array}$ & $\begin{array}{c}\text { Work Order } \\
\text { Number }\end{array}$ & \multicolumn{1}{|c|}{ Client ID } & $\begin{array}{c}\text { Gross } \\
\text { Weight } \\
\text { Average } \\
\text { (mg) }\end{array}$ \\
\hline H7E040218-001 & $\begin{array}{c}\text { JWAEW1A } \\
\text { A }\end{array}$ & HARDIN STACK RUN 1 FILTER A176 & 385.6 \\
\hline H7E040218-007 & JWAF51AA & HARDIN STACK RUN 2 FILTER A177 & 400.1 \\
\hline H7E040218-013 & JWAG81AA & HARDIN STACK RUN 3 FILTER A178 & 400.9 \\
\hline H7E040218-019 & JWAH31AA & $\begin{array}{l}\text { HARDIN SDA INLET DUCT RUN 1 FILTER } \\
\text { (THIMBLE 50) }\end{array}$ & 11725.1 \\
\hline H7E040218-020 & JWAH51AA & HARDIN SDA INLET DUCT RUN 1 FILTER (A173) & 417.3 \\
\hline H7E040218-026 & JWAJV1AA & $\begin{array}{l}\text { HARDIN SDA INLET DUCT RUN 2 FILTER } \\
\text { (THIMBLE 52) }\end{array}$ & 9859.7 \\
\hline H7E0402 I8-027 & JWAJ51AA & HARDIN SDA INLET DUCT RUN 2 FILTER (A174) & 726.3 \\
\hline H7E040218-033 & JWAK91AA & $\begin{array}{l}\text { HARDIN SDA INLET DUCT RUN 3 FILTER } \\
\text { (THIMBLE 51) }\end{array}$ & 12380.7 \\
\hline H7E040218-034 & JWALL1AA & HARDIN SDA INLET DUCT RUN 3 FILTER (A175) & 413.3 \\
\hline
\end{tabular}

The acetone probe rinse samples were extremely hygroscopic and gained weight in the dessicator. To obtain the most representative result, the average of the first two weights was reported. The inconsistency indicates that there may be a slight positive bias to the results.

STL Knoxville maintains the following certifications, approvals and accreditations: Arkansas DEQ Cert. H05-043-0, California DHS ELAP Cert. \#2423, Colorado DPHE, Connecticut DPH Cert. \#PH-0223, Florida DOH Cert. \#E87177, - Georgia DNR Cert. \#906, Hawaii DOH, Illinois EPA Cert. \#000687, Indiana DOH Cert. \#C-TN-02, lowa DNR Cert. \#375, Kansas DHE Cert. \#E-10349, Kentucky DEP Lab ID \#90101, Louisiana DEQ Cert. \#03079, Louisiana DOHH Cert. \#LA.030024, Maryland DHMH Cert. \#277, Massachusetts DEP Cert. \#M-TN009, Michigan DEQ Lab ID \#9933, New Jcrsey DEP Cert. \#TN001, New York DOH Lab \#10781, North Carolina DPH Lab ID \#21705, North Carolina DEHNR Cert. \#64, Ohio EPA VAP Cert. \#CL0059, Oklahoma DEQ ID \#9415, Pennsylvania DEP Cert. \#68-00576,

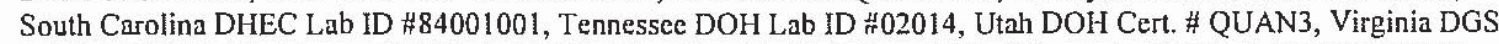
Lab ID \#00165, Washington DOE Lab \#C120, West Virginia DEP Cert. \#345, Wisconsin DNR Lab ID \#998044300, Naval Facilities Engineering Service Center and USDA Soil Permit \#S-46424. This list of approvals is subject to change and does not imply that laboratory certification is available for all parameters reported in this environmental sample data report. 
Sample Data Summary 
Air Sampling Associates, Inc.

Client Sample ID: HARDIN STACK RON 1 FH

TOTAL Metals

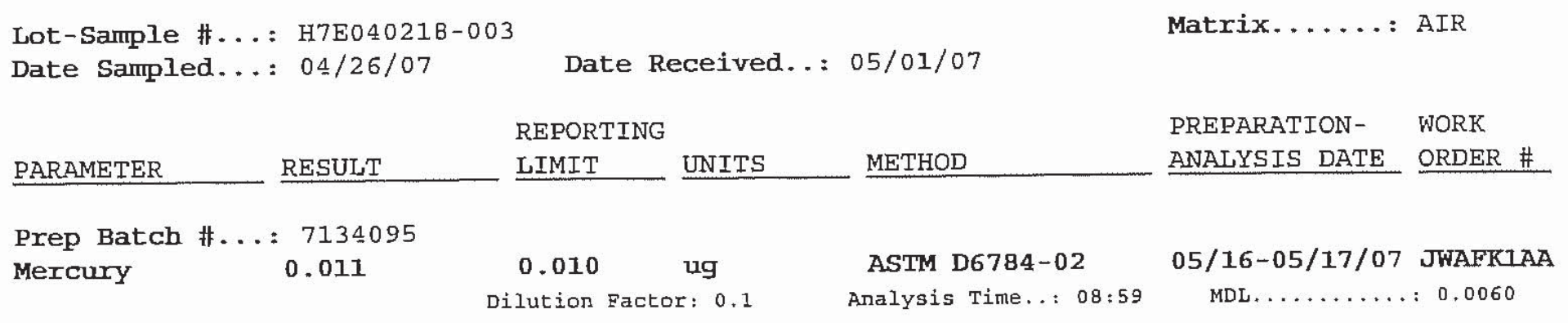


Air Sampling Associates, Inc.

client Sample ID: HARDIN STACK RUN 1 KCL

TOTAL Metals

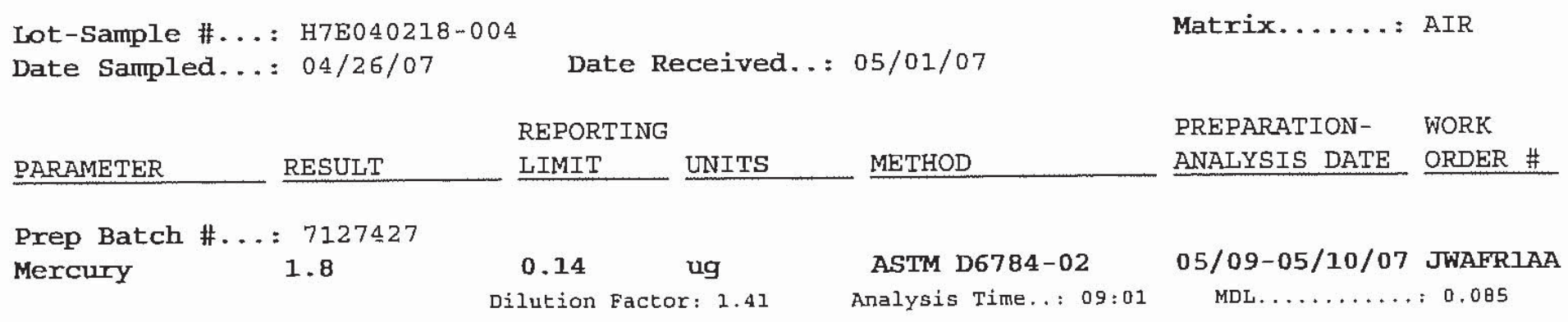


Air Sampling Associates, Inc.

Client Sample ID: HARDIN STACK RUN 1 HNO3/H2O2

TOTAL Metals

Lot-Sample \#...: H7E040218-005

Date Sampled...: 04/26/07

Date Received..: 05/01/07

REPORTING

PARAMETER

RESULT

Prep Batch \#. . : 7127421

Mercury

0.15 ug

Dilution Factor: 0.7
Matrix......: AIR

PREPARATION- WORK ANALYSIS DATE ORDER \#
ASTM D6784-02

Analysis Time..: 08:36
05/09-05/10/07 JWAFW1AA MDL $\ldots \ldots \ldots \ldots: 0.042$ 
Air Sampling Associates, Inc.

Client Sample ID: HARDTN STACK RUN 1 KMNO4/H2SO4

TOTAL Metals

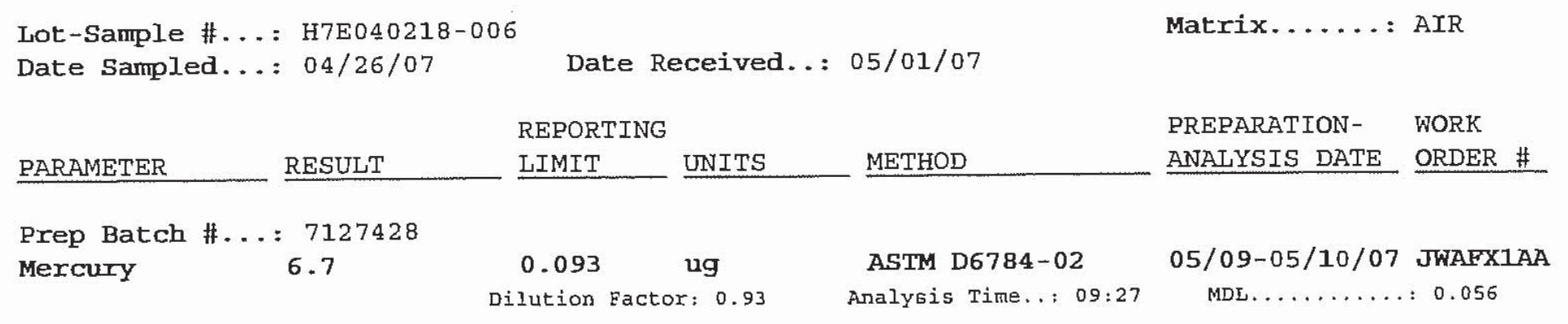


Air Sampling Associates, Inc.

client Sample ID: HARDIN STACK RUN 2 FH

TOTAL Metals

Lot-Sample \#...: H7E040218-009

Date Sampled...: 0 $\leqslant / 26 / 07$

Date Received..: 05/01/07

REPORTING

PARAMETER

RESULT

Prep Batch 柆...: 7134095

Mercury
0.058 ug

Dilution Factor: 0.1
Matrix...... AIR

PREPARATION - WORK ANALYSIS DATE ORDER \#

ASTM D6784-02

Analysis Time..: 08:53
05/16-05/17/07 JWAGK1AA MDL........ 0.0060 
Air Sampling Associates, Inc.

Client Sample ID: HARDIN STACK RUN 2 KCL

TOTAL Metals

Lot-Sample \#. . : H7E040218-010

Date Sampled...: 04/26/07

PARAMETER

RESULT

Prep Batch \#...: 7127427

Mercury

\begin{abstract}
1.8
\end{abstract}
Date Received..: 05/01/07

REPORTING

LIMIT

UNITS

METHOD

$\begin{array}{ccc}0.14 & \text { ug } & \text { ASTM D6784-02 } \\ \text { Dilution Factor: 1.41 AnalYsis Time..: 09:11 }\end{array}$

Matrix...... AIR

PREPARATION- WORK ANALYSIS DATE ORDER \#

05/09-05/10/07 JWAGNDAA 
Air Sampling Associates, Inc.

client Sample ID: HARDIN STACK RUN 2 HNO3/H2O2

TOTAL Metals

Lot-Sample \#...: H7E040218-011

Date Sampled...: 04/26/07

Date Received..: 05/01/07

REPORTING

PARAMETER

RESULT

Prep Batch \#...: 7127421

Mercury

0.19

ug

Dilution Factor: 0.73
Matrix......: AIR

PREPARATION- WORK ANALYSIS DATE ORDER \#
ASTM D6784-02

Analysis Time..: $08: 46$
05/09-05/10/07 JWAGPLAA MDL......... 0.044 
Air Sampling Associates, Inc.

Client Sample ID: HARDIN STACK RUN 2 KMNO4/H2SO4

TOTAL Metals

Lot-Sample \#...: H7E040218-012

Date Sampled...: $0 \leqslant / 26 / 07$

Date Received..: 05/01/07

REPORTING

PARAMETER

RESULT

Prep Batch \#...: 7127428

Mercury
7.3 ug

Dilution Factor: 1.02 METHOD

ASTM D6784-02

Analysis Time..: 09:37
Matrix....... AIR

PREPARATION - WORK ANALYSIS DATE ORDER \#

05/09-05/10/07 JWAGRIAA MDL......... 0.061 
Air Sampling Associates, Inc.

\section{Client Sample ID: HARDIN STACK RUN 3 FH}

TOTAL Metals

Lot-Sample \#...: H7E040218-015

Date Sampled...: 04/26/07

Date Received..: 05/01/07

REPORTING LIMIT UNITS METHOD

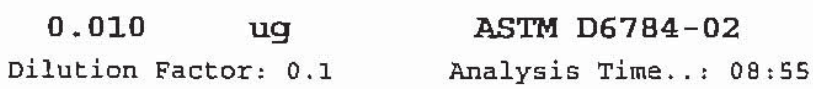

Matrix.....: AIR

PREPARATION- WORK ANALYSIS DATE ORDER \#

05/16-05/17/07 JWAHE1AA MDL.......... 0.0060 
Air Sampling Associates, Inc.

client Sample ID: HARDIN STACK RUN 3 KCL

TOTAL Metals

Lot-Sample \#... : H7E040218-016

Date Sampled...: 04/26/07

Date Received..: 05/01/07

REPORTING

LIMIT

UNITS

0.14 ug

Dilution Factor: 1.41
METHOD

Matrix....... AIR

PREPARATION- WORK ANALYSIS DATE ORDER \#

ASTM D6784-02 05/09-05/10/07 JWAHH1AA

Analysis Time..: 09:12 
Air Sampling Associates, Inc.

Client Sample ID: HARDIN STACK RUN 3 HNO3/H2O2

TOTAL Metals

Lot -Sample \#...: H7E040218-017

Date Sampled...: 04/26/07

PARAMETER

RESULT

Prep Batch \#...: 7127421

Mercury
0.11
Date Received. . : 05/01/07

REPORTING

LIMIT

UNITS

0.068 ug
Dilution Factor: 0.68
Matrix......: AIR

PREPARATION- WORK ANALYSIS DATE ORDER \#
05/09-05/10/07 JWAHL1AA MDL.........: 0.041 
Air Sampling Associates, Inc.

client Sample ID: HARDIN STACK RUN 3 KMNO4/H2SO4

TOTAL Metals

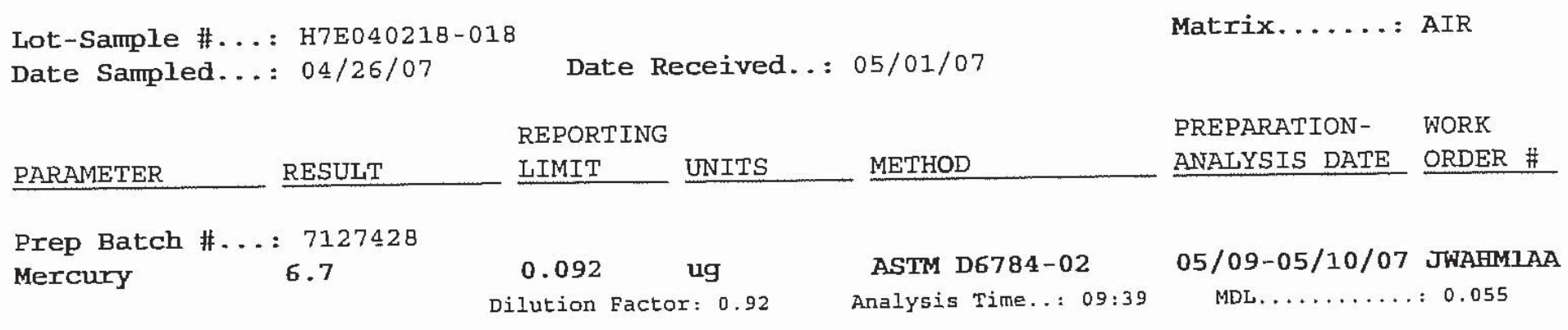


Aix Sampling Associates, Inc.

client Sample ID: HARDIN SDA INLFT DUCT RUN 1 FH

TOTAL Metals

Lot-Sample 唗. : H7E040218-022

Date Sampled...: 04/26/07

PARAMETER

Prep Batch \#...: 7134095

Mexcury
1. 3
Date Received. : : 05/01/07

REPORTING

IIMIT

UNITS

0.050 ug

Dilution Factor: 0.5
Matrix...... AIR

PREPARATION - WORK ANALYSIS DATE ORDER \#

05/16-05/17/07 JWAH71AA MDL......... 0.030 
Air Sampling Associates, Inc.

Client Sample ID: HARDIN SDA INLET DUCT RUN I KCL

TOTAL Metals

Lot-Sample \#...: H7E040218-023

Date Sampled...: 04/26/07

PARAMETER

Prep Batch \#...: 7127427

Mercury
Date Received. .: 05/01/07

REPORTING

LIMIT

0.11

ug

Dilution Factor: 1.06 METHOD
Matrix...... AIR

PREPARATION- WORK ANALYSIS DATE ORDER \#

ASTM D6784-02

Analysis Time..: 09:15
05/09-05/10/07 JWAJAIAA MDL......... 0.064 
Air Sampling Associates, Inc.

Client Sample ID: HARDIN SDA INLET DUCT RUN 1 HNO3/H2O2

TOTAL Metals

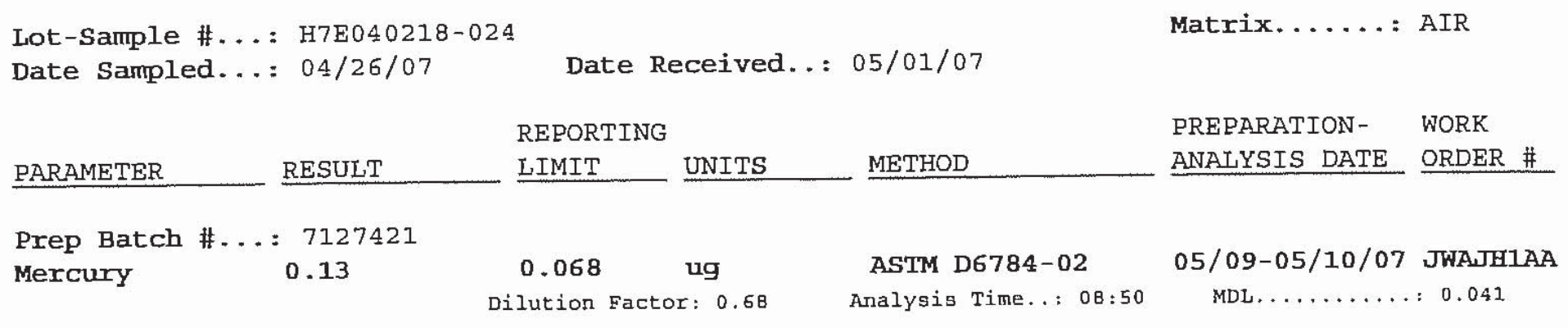


Air Sampling Associates, Inc.

Client Sample ID: HARDIN SDA INLET DUCT RUN 1 KMNO4,H2SO4

TOTAL Metals

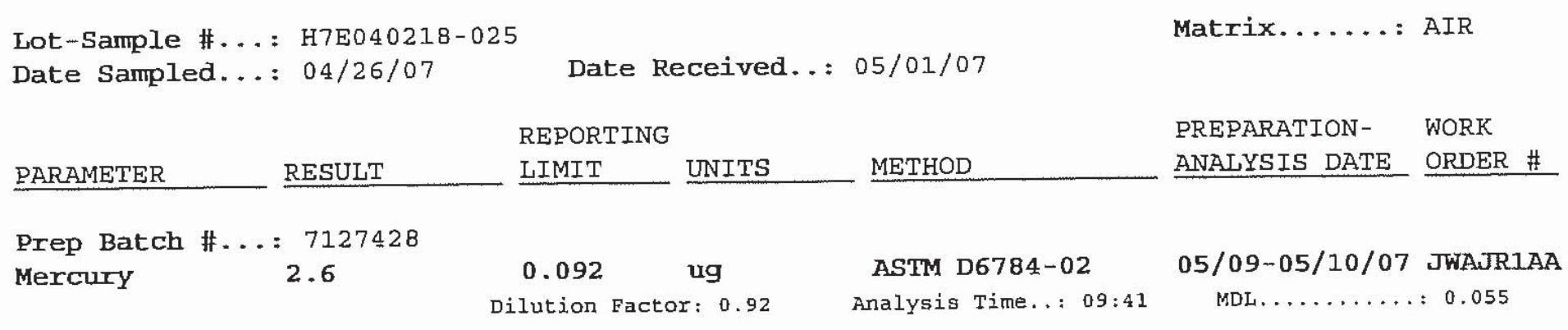


Aix Sampling Associates, Inc.

client Sample ID: HARDIN SDA INLET DUCT RUN 2 FH

TOTAL Metals

Lot-Sample \#...: H7E040218-029

Date Sampled...: 04/26/07

PARAMETER

Prep Batch \#...: 7134095

Mercury
0.97

Date Received..: 05/01/07

REPORTING LIMIT

UNITS
METHOD

ASTM D6784-02

Analysis Time..: 09:55

Matrix...... AIR

PREPARATION- WORK ANALYSIS DATE ORDER \#

05/16-05/17/07 JWAKPIAA MDL......... 0.030 
Air Sampling Associates, Inc.

Client Sample ID: HARDIN SDA INLET DOCT RUN 2 KCL

TOTAL Metals

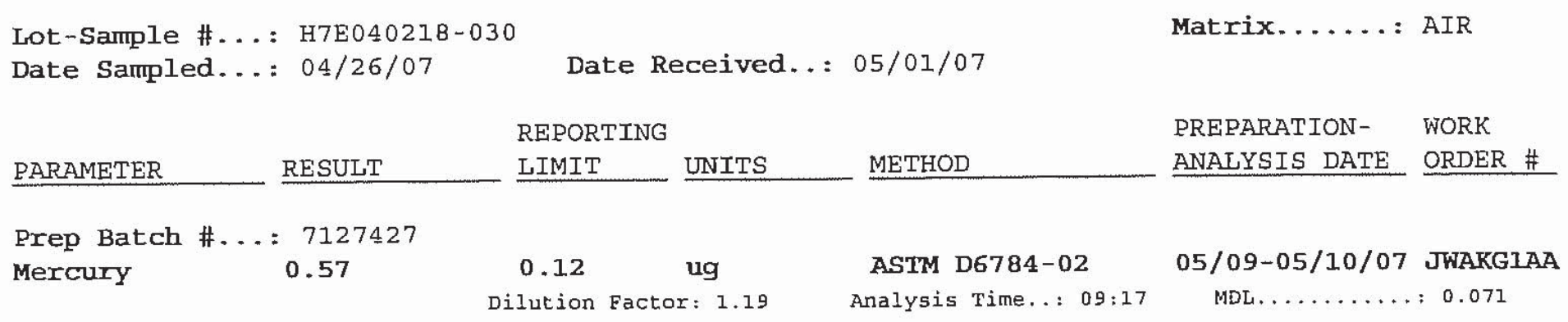


Air Sampling Associates, Inc.

Client Sample ID: HARDIN SDA INLET DUCT RUN 2 HNO3/H2O2

TOTAL Metals

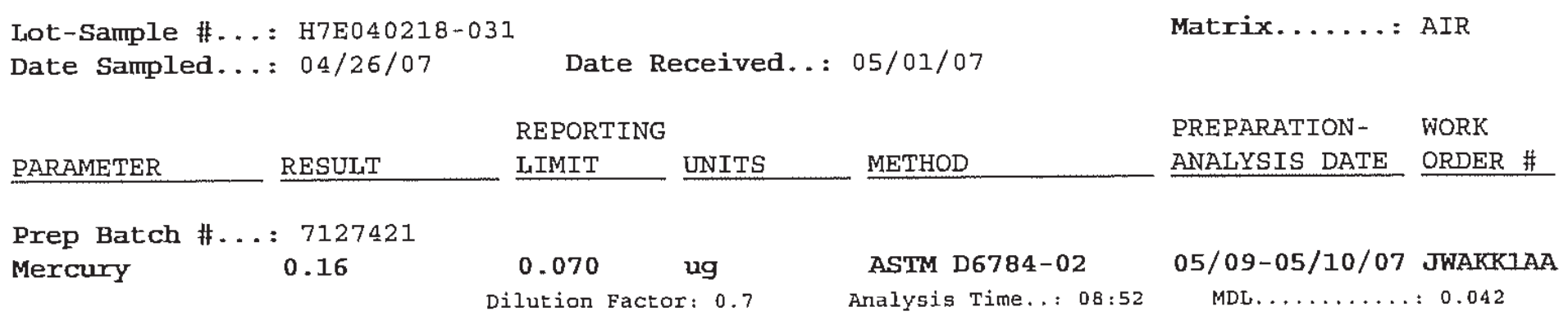


Air Sampling Associates, Inc.

client Sample ID: HARDIN SDA INLET DUCT RUN 2 KMNO4/H2SO4

TOTAL Metals

Lot-Sample \#...: H7E040218-032

Date Sampled...: 04/26/07

Date Received..: 05/01/07

REPORTING

PARAMETER

RESULT

Prep Batch \#. . : 7127428

Mercury
3.1
LIMIT

0.092

ug

Dilution Factor: 0.92
Matrix...... AIR

PREPARATION- WORK ANALYSIS DATE ORDER \#

ASTM D6784-02

Analygis Time..: 09:43
05/09-05/10/07 JWAK01AA MDL......... 0.055 
Air Sampling Associates, Inc.

Client Sample ID: HARDIN SDA INLET DUCT RUN 3 FH

TOTAL Metals

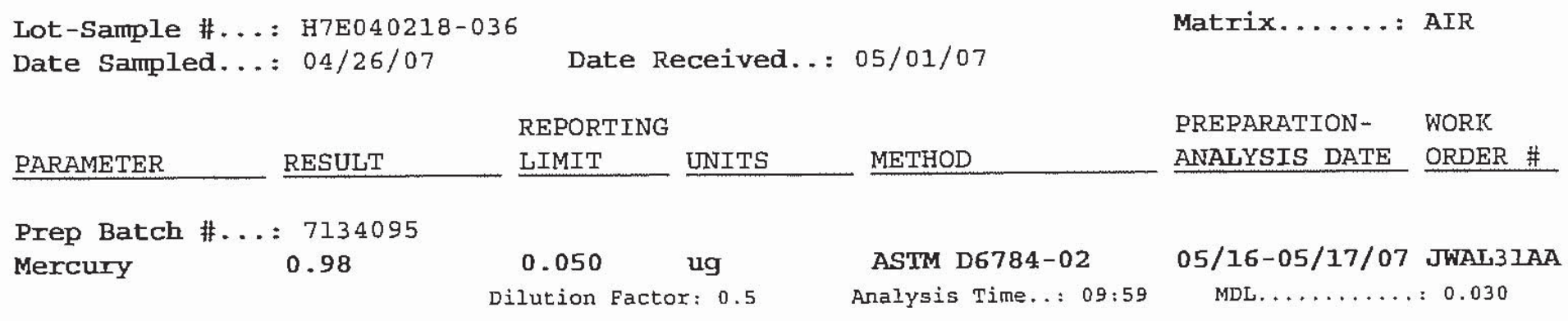


Air Sampling Associates, Inc.

client Sample ID: HARDIN SDA INLET DUCT RUN 3 KCL

TOTAL Metals

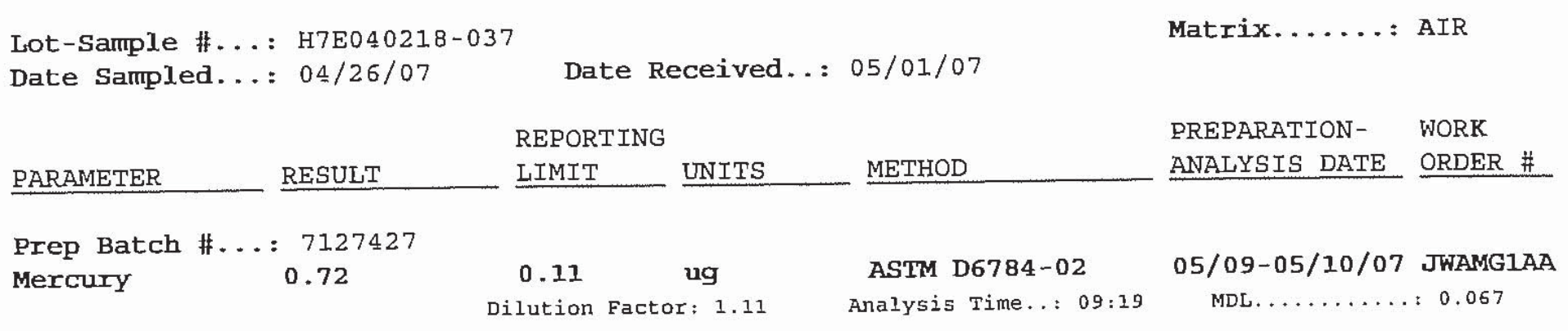


Air Sampling Associates, Inc.

Client Sample ID: HARDIN SDA INLET DUCT RUN 3 HNO3/H2O2

TOTAL Metals

Lot-Sample \#...: H7E040218-038

Date Sampled...: 04/26/07

PARAMETER

Prep Batch \#...: 7127421

Mercury
0.17

0.073 ug

Dilution Factor: 0.73
Date Received..: 05/01/07

REPORTING

UNITS

METHOD

ASTM D6784-02

Analysis Time..: 08:54
Matrix......: AIR

PREPARATION- WORK ANALYSIS DATE ORDER \#

05/09-05/10/07 JWAMKבAA MDL......... 0.044 
Air Sampling Associates, Inc.

client Sample ID: HARDIN SDA INLFT DUCT RUN 3 KMNO4/H2SO4

TOTAL Metals

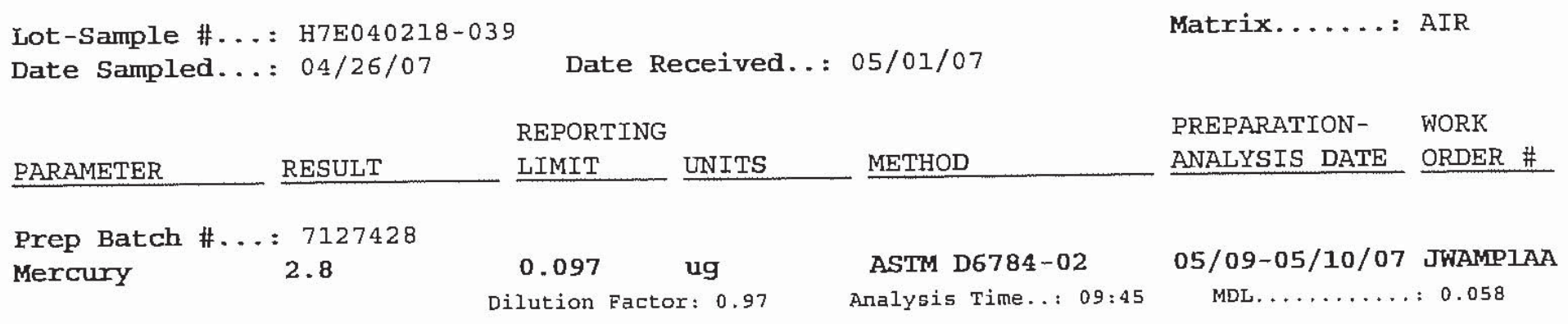


Air Sampling Associates, Inc.

Client Sample ID: HARDIN SDA INLET+STACK RB 0. IN HNO3

TOTAL Metals

Lot-Sample \#... : H7E040218-040

Date Sampled...: 04/26/07

PARAMETER

Prep Batch \#...: 7134095

Mercury
ND

0.010 ug
Dilution Factor: 0.1

Date Received..: 05/01/07

REPORTING

UNITS

METHOD

ASTM D6784-02

Analysis Time..: 09:03
Matrix......: AIR

PREPARATION- WORK ANALYSIS DATE ORDER \#

05/16-05/17/07 JWAM21AA MDL......... 0.0060 
Air Sarmpling Associates, Inc.

Client Sample ID: HARDIN SDA INLFT+STACK RB IN KCL

TOTAL Metals

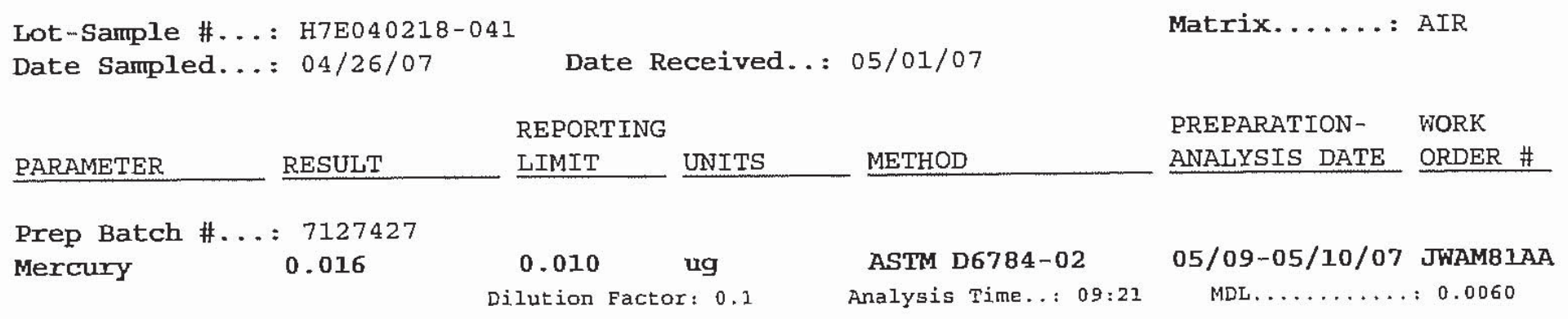


Air Sampling Associates, Inc.

Client Sample ID: HARDIN SDA INL.BT+STACK RB HNO3/H2O2

TOTAL Metals

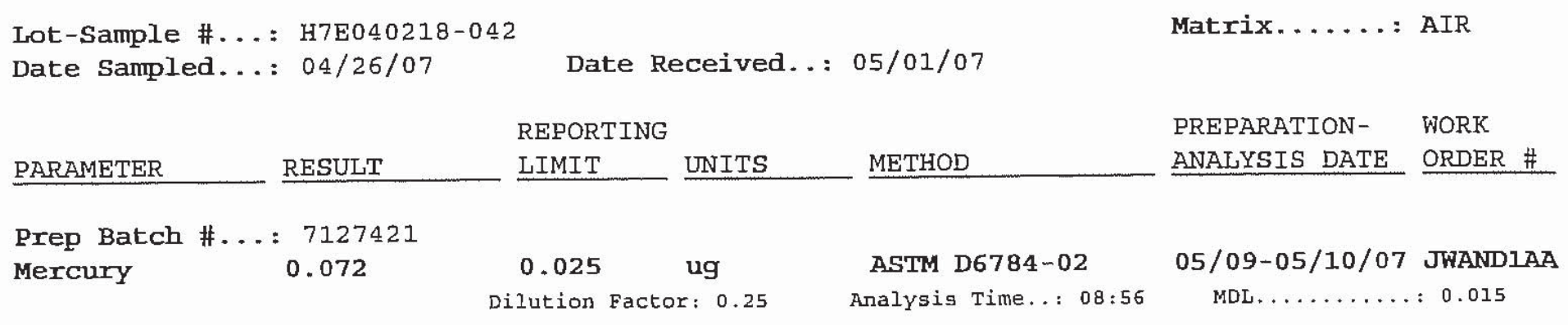


Air Sampling Associates, Inc.

client Sample ID: HARDIN SDA INLET+STACK RB KMNO4/H2SO4/HYDR

TOTAL Metals

Lot-Sample \#...: H7E040218-043

Date Sampled...: 04/26/07

PARAMETER

Prep Batch \#...: 7127428

Mercury
ND
Date Received..: 05/01/07

REPORTING LIMIT UNITS

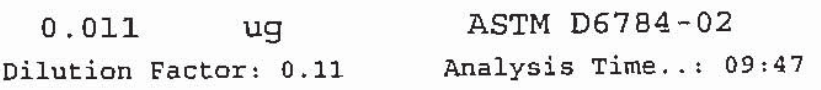

Matrix...... AIR

PREPARATION- WORK ANALYSIS DATE ORDER \#

05/09-05/10/07 JWANGIAA MDL......... 0.0066 
Air Sampling Associates, Inc.

Client Sample ID: HARDIN SDA INLET+STACK RB QUARTZ FILTER.

TOTAL Metals

Lot-Sample \#...: H7E040218-044

Date Sampled...: 04/26/07

Date Received..: 05/01/07

REPORTING

PARAMETER

RESULT

Prep Batch \#...: 7134095

Mercury

ND
$0.010 \mathrm{ug}$

Dilution Factor: 0.1
Matrix......: AIR

PREPARATION- WORK ANALYSIS DATE ORDER \#

05/16-05/17/07 JWAPF1AA MDL.........; 0.0060 


\section{Air Sampling Associates, Inc.}

Client Sample ID: HARDIN SDA INLET+STACK RB THIMBLE 1

TOTAL Metals

Lot-Sample \#. . . : H7E040218-045

Date Sampled... : 04/26/07

$\begin{array}{lll}\text { PARAMETER } & \text { RESULT } \\ \text { Prep Batch \#...: } & 7134095 \\ \text { Mercury } & 0.032\end{array}$

Date Received..: 05/01/07

REPORTING

LIMIT

UNITS

0.010 ug

Dilution Factor: 0.1

METHOD

Matrix...... AIR

PREPARATION- WORK ANALYSIS DATE ORDER \#

ASTM D6784-02 05/16-05/17/07 JWAPP1AA

Analysis Time... 09:07 
METHOD BLANK RBPORT

TOTAL Metals

Client Lot \#...: H7E040218

REPORTING

PARAMETER

RESULT

LIMIT

UNITS

METHOD

MB Lot-Sample \#: H7E070000-421 Prep Batch \#.. : 7127421

Mercury

ND

0.025

ug

ASTM D6784-02

05/09-05/10/07 JWFFG1AA

Dilution Factor: 0.25

Analysis Time..: 08:30

MB Lot-Sample \#: H7E070000-427 Prep Batch \#. . : 7127427

Mercury

ND

0.010

ug

ASTM D6784-02

05/09-05/10/07 JWFFK1AA

Dilution Factor: 0.1

Analysis Time..; 08:58

MB Lot-Sample \#: H7E070000-428 Prep Batch \#.. : 7127428

Mercury

ND

0.010

ug

ASTM D6784-02

05/09-05/10/07 JWFFN1AA

Dilution Factor: 0.1

Analysis Time..: $09: 23$

MB Lot-Sample \#: H7E140000-095 Prep Batch \#...: 7134095

Mercury

$\mathrm{ND}$

0.010

ug

ASTM D6784-02

05/16-05/17/07 JWWQH1AA

Dilution Factor: 0.1

Analysis Time..: 08:40

NOTE $(S):$

Calculations are performed before rounding to avoid round-off errors in calculated results. 


\section{LABORATORY CONTROI, SAMPLE EVALUATION REPORT}

TOTAL Metals

\begin{tabular}{|c|c|c|c|c|}
\hline \multirow[b]{2}{*}{ PARAMETER } & \multirow{2}{*}{$\begin{array}{l}\text { PERCENT } \\
\text { RECOVERY } \\
\end{array}$} & RECOVERY & \multicolumn{2}{|c|}{ RPD } \\
\hline & & I.IMITS & $\mathrm{RPD}$ & LIMITS \\
\hline \multirow[t]{2}{*}{ Mercury } & 104 & $(80-120)$ & & \\
\hline & 102 & $(80-120)$ & 1.6 & $(0-20)$ \\
\hline
\end{tabular}

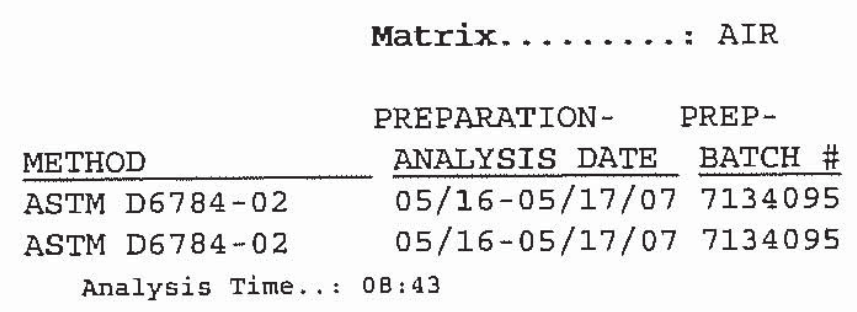

NOTE (S) :

Calculations are performed beforc rounding to avoid round-off errors in calcufated results. 


\section{LABORATORY CONTROL SAMPLE DATA RBPORT}

\section{TOTAL Metals}

Lot-Sample \#. . : : H7E040218

Matrix....... A AIR

$$
\text { SPIKE MEASURED }
$$

PERCNT

PREPARATION- PREP

PARAMETER AMOUNT AMOUNT

UNITS

Mercury

$0.500 \quad 0.519$ ug

RECVRY RPD METHOD

0.500

0.511

ug 104

ASTM D6784-02

ANALYSIS DATE BATCH \# 05/16-05/17/07 7134095

Dilution Factor: 0.1

1.6 ASTM D6784-02

$05 / 16-05 / 17 / 07 \quad 7134095$

Analysis Time..: 08:43

NOTE (S) :

Calculations are performed before rounding to avoid round-off errors in calculated resulis. 
LABORATORY CONTROL SAMPLE EVALUATION RBPORT

TOTAL Metals

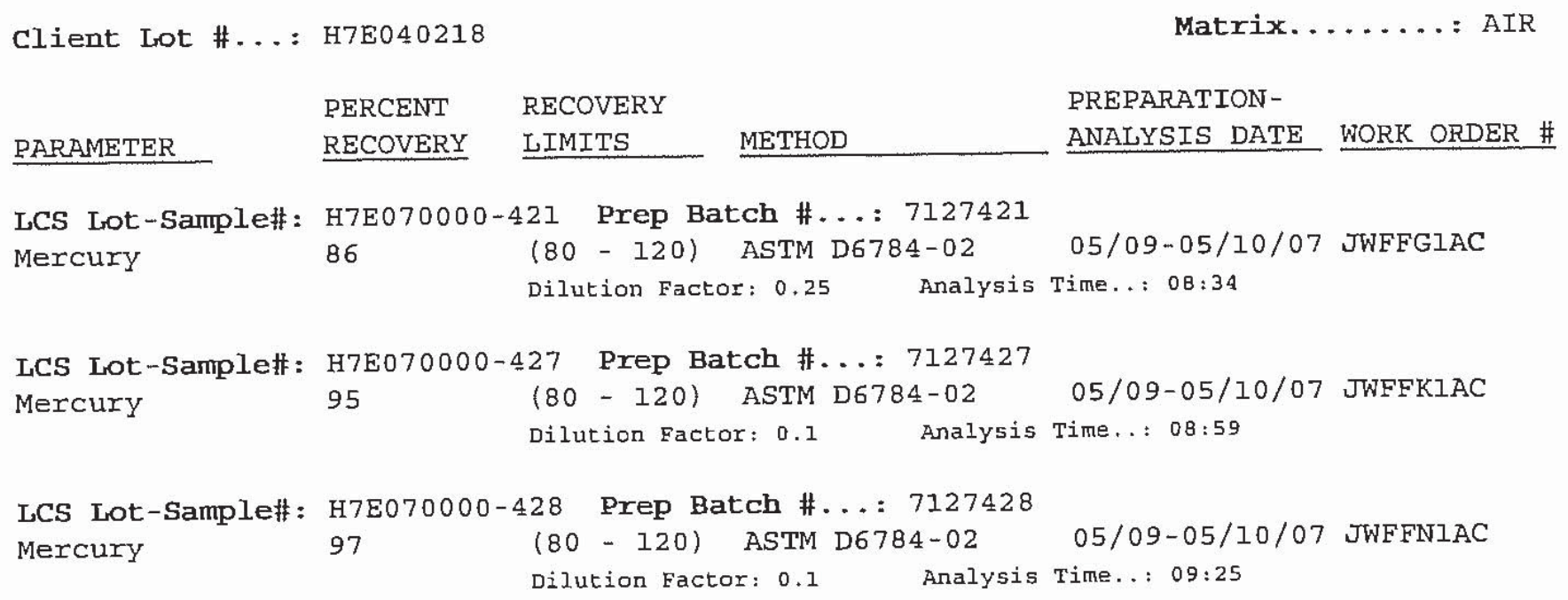

NOTB (S) :

Calculations are performed before rounding to avoid round-off errors in calculated resulls. 


\section{LABORATORY CONTROL SAMPLE DATA REPORT}

TOTAL Metals

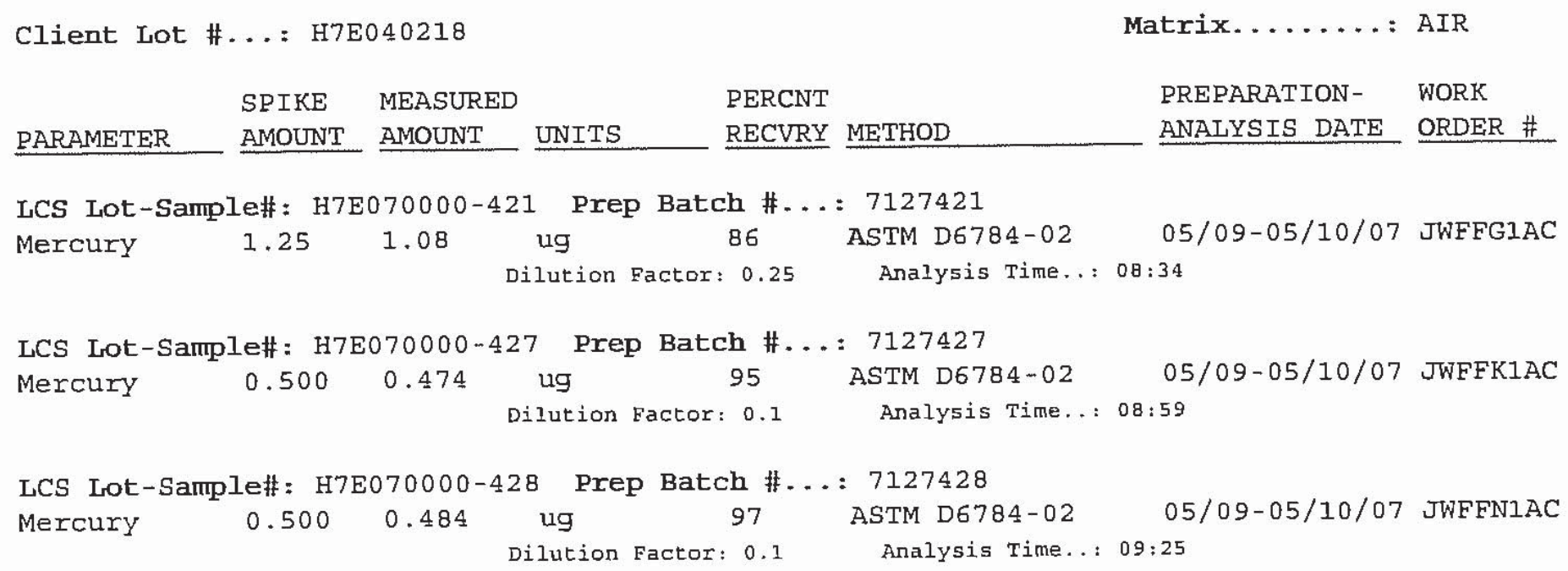

NOTE $(S)$ :

Calculations are performed before rounding to avoid round-off errots in calculated results. 
MATRIX SPIKF SAMPLE EVALUATION RBPORT

TOTAL Metals

Client Lot \#...: H7E040218

Date Sampled...: 04/26/07
Matrix........ AIR

Date Received. .: 05/01/07

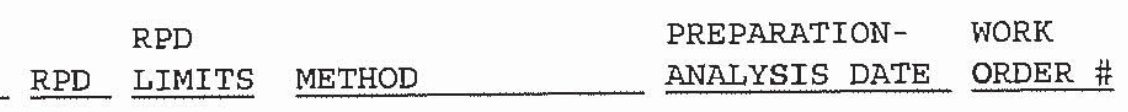

05/09-05/10/07 JWAFW1AC

05/09-05/10/07 JWAFWIAD

ASTM D678 -02

MS Lot-Sample \#: H7E040218-005 Prep Batch \#...: 7127421

$\begin{array}{ll}\text { Mercury } & 98 \\ & 101\end{array}$

$(80-120)$

$(80$ - 120) $2.5 \quad(0-20)$ ASTM D6784-02

Dilution Factor; 0.7

Analysis Time..: $08: 38$

NOTE (S) :

Calculations are performed before rounding to avoid round-off errors in calculated results. 
MATRIX SPIKG SAMPLB DATA REPORT

TOTAL Metals

Client Lot \#...: H7E040218

Date Sampled...: 04/26/07
Date Received..: 05/01/07

PERCNT

RECVRY RPD METHOD
Matrix....... AIR

PREPARATION- WORK ANALYSIS DATE ORDER \#

MS Lot-Sample \#: H7E040218-005 Prep Batch \#...: 7127421

Mercury

$$
\begin{aligned}
& 0.15 \quad 0.700 \quad 0.840 \text { ug } \quad 98 \text { ASTM D6784-02 05/09-05/10/07 JWAFWIAC } \\
& 0.150 .700 \quad 0.861 \text { ug } 101 \quad 2.5 \text { ASTM D6784-02 05/09-05/10/07 JWAFWIAD }
\end{aligned}
$$

NOTE (S) :

Calculations ate performed before rounding to avoid round-off errors in calculated results. 
MATRIX SPIKB SAMPLE EVALUATION REPORT

TOTAL Metals

Client Lot \#...: H7E040218

Date Sampled...: 04/26/07
Date Received..: 05/01/07

$\begin{array}{lllll}\text { PERCENT } & \text { RECOVERY } & \text { RPD } & \text { PREPARATION } & \text { WORK } \\ \text { RECOVERY } & \text { LIMITS }\end{array}$

MS Lot-Sample \#: H7E040218-004 Prep Batch \#...: 7127427

$\begin{array}{cccccc}\text { Mercury } & 102 & (80-120) & \text { ASTM D6784-02 } \\ & 95 & (80-120) & 3.1 & (0-20) & \text { ASTM D6784-02 } \\ & \text { Dilution Factor: } 1.41 \\ & \text { Analysis Time..: } 09: 03\end{array}$

Matrix........ AIR

05/09-05/10/07 JWAFRIAC 05/09-05/10/07 JWAFR1AD

$\operatorname{NOTB}(S)$ :

Calculations are performed before rounding to avoid round-off errors in calculated results. 
MATRIX SPIKF SAMPLE DATA RBPORT

TOTAL Metals

Client Lot \#...: H7E04021B

Date Sampled...: 04/26/07
Matrix...........AIR

Date Received..: 05/01/07

PERCNT

PREPARATION-

WORK

AMOUNT UNITS

Prep Batch \#.. : 7127427

MS Lot-San
Mercury

$$
1.8
$$

1.41

1.41
3.26

3.16 ug

ug

Dilution Factor: 1.41

Analysis Time..: 09:03
102 ASTM D6784-02 05/09-05/10/07 JWAFRIAC

953.1 ASTM D67B4-02 05/09-05/10/07 JWAFRIAD

NOTE (S) :

Calculations are performed before rounding to avoid round-off errors in calculated resulis. 


\section{MATRIX SPIKE SAMPLE EVALUATION RBPORT}

TOTAL Metals

Client Lot \#...: H7E04021B

Date Sampled...: 04/26/07
Matrix.......: AIR

Date Received..: 05/01/07

\begin{tabular}{|c|c|c|c|c|c|c|}
\hline PERCENT & RECOVERY & & RPD & & PREPARATION - & WORK \\
\hline RECOVERY & LIMITS & RPD & LIMITS & METHOD & ANALYSIS DATE & ORDER \# \\
\hline
\end{tabular}

MS Lot-Sample \#: H7E040218-006 Prep Batch \#...: 7127428

Mercury
ASTM D67B4-02

ASTM D6784-02

NC, MSB $\quad(80-120)$

NC, MSB $\quad(B 0-120)$$$
\text { Dilution Factor: } 0.93
$$

Analysis Time..; 09:34
05/09-05/10/07 JWAFXIAC

05/09-05/10/07 JWAFXIAD

$\operatorname{NOTE}(S)$ :

Calculations are performed before rounding to avoid round-off errors in calculated results.

NC The recovery and/or RPD were not calculated.

MSB The recovery and RPD were not calculated beenuse the sample anount was greater dian four times the spike amount. 
MATRIX SPIKE SAMPLE DATA REPORT

TOTAL Metals

Client Lot \#...: H7E040218

Date Sampled...: 04/26/07
Matrix....... AIR

Date Received..: 05/01/07

PERCNT

RECVRY RPD METHOD

PREPARATION ANALYSIS DATE

WORK AMOUNT UNITS

MS Lot-Sample \#: H7E040218-006 Prep Batch \#...: 7127428 Mercury

$\begin{array}{cccc}6.7 & 0.930 & 7.54 & \text { ug } \\ 6.7 & 0.930 & 8.01 \text { ugalifiers : NC, MSB } \\ & & \text { Qualifiers : NC, MSB } \\ & \text { Dilution Factor: } 0.93 \\ & \text { Analysis Time..: 09:34 }\end{array}$

ASTM D6784-02 05/09-05/10/07 JWAFXIAC

ASTM D6784-02 05/09-05/10/07 JWAFX1AD

\section{$\operatorname{NOTB}(\mathrm{S}):$}

Calculations are performed before rounding to avoid round-off errors in calcutated results.

NC The recovery and/or RPD were not calculated.

MSB The recovery and RPD were not calculated because the sample amount was greater than four times the spike amount. 


\section{STL Knoxville}

\section{Mercury Data Reporting Form}

Post Digestion Spike
Units: $u g / L(p p b)$

Instrument ID: Leeman HydraAA Hg

Data File Name: M051707. PRN

\begin{tabular}{|c|c|c|c|c|}
\hline Element & $\begin{array}{c}\text { PDS } \\
\text { JWAGKA }\end{array}$ & $\begin{array}{c}\text { Original Sample } \\
\text { JWAGK }\end{array}$ & Spike Added & $\begin{array}{c}\text { Percent } \\
\text { Recovery }\end{array}$ \\
\hline $\mathrm{Hg}$ & 1.56 & 0.52 & 1.0 & 104 \\
\hline
\end{tabular}


Sample Data Summary 
Air Sampling Associates, Inc.

Client Sample ID: HARDIN STACK RON 1 FILTKR A176

General Chemistry

Lot-Sample \#...: : H7E040218-001

Date sampled...: 04/26/07
Work Order \#...: JWAEW

Date Received. .: 05/01/07
Matrix....... A AIR

PREPARATION- PREP

ANALYSIS DATE BATCH \#
PARAMETER

Particulates (total) 386
RESULT

RL

0.50

Dilution Factor: 1
METHOD

CFR6 OA 5

MDL . . . . . . 0.50 
Air Sampling Associates, Inc.

client sample ID: HARDIN STACK RUN 1 ACETONE

General Chemistry

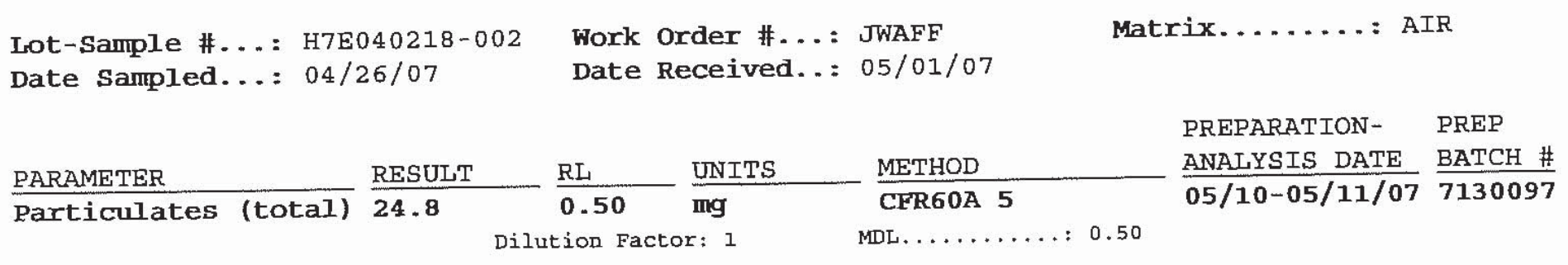


Air Sampling Associates, Inc.

client Sample ID: HARDIN STACK RUN 2 FILTER A177

General Chemistry

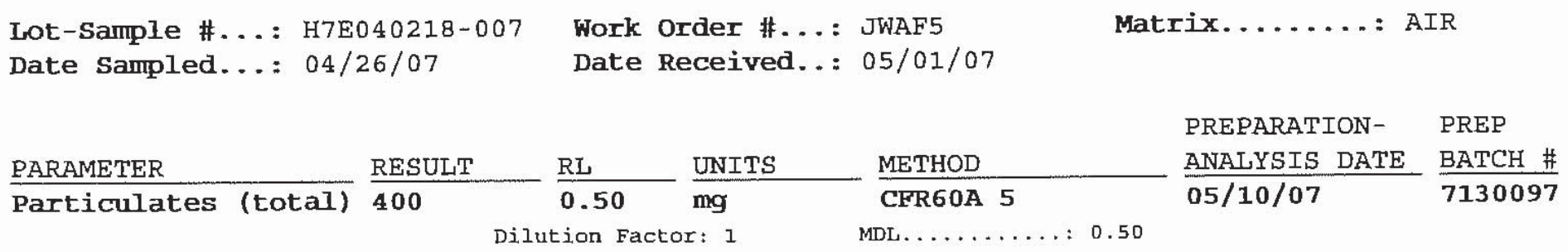


Air Sampling Associates, Inc.

client Sample ID: HARDIN STACK RUN 2 ACFTONE

General Chemistry

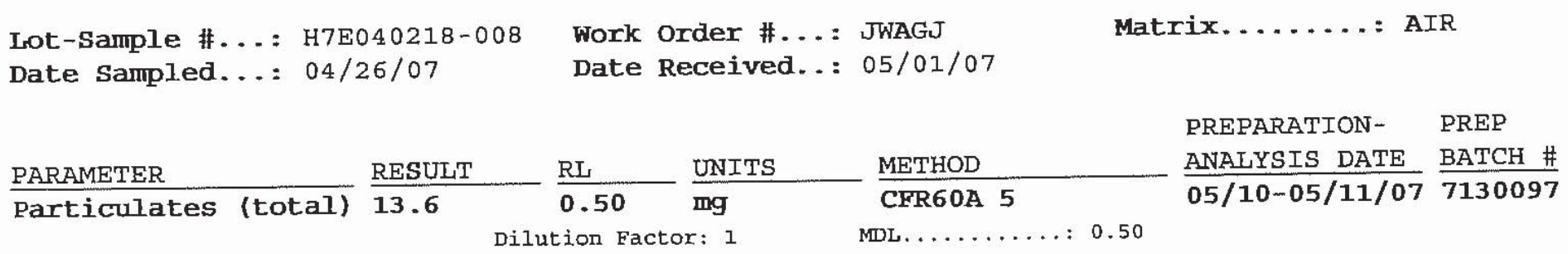


Air Sampling Associates, Inc.

Client Sample ID: HARDIN STACK RUN 3 FILTKR A178

General Chemistry

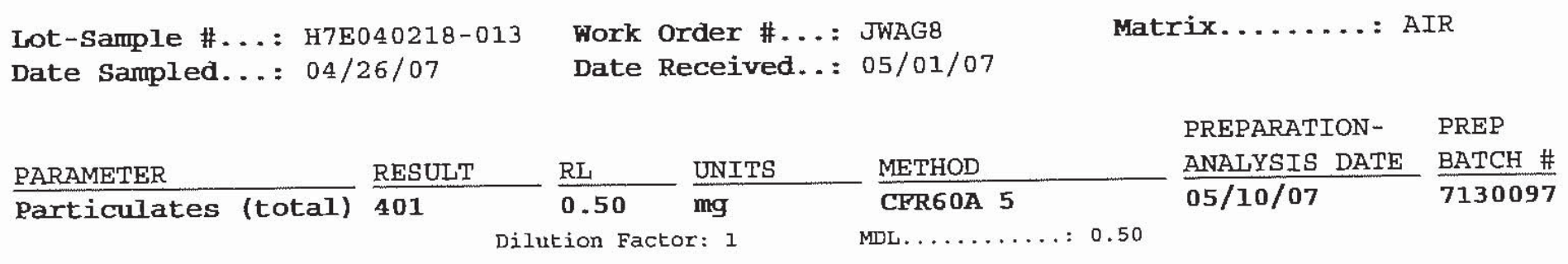


Air Sampling Associates, Inc.

client sample ID: HARDIN STACK RUN 3 ACETONE

General Chemistry

Iot-Sample \#...: H7E040218-014

Date Sampled...: 04/26/07
Hork Order \#...: JWAHC

Date Received..: 05/01/07
Matrix........

PREPARATION- PREP ANALYSIS DATE BATCH \# 05/10-05/11/07 7130097
PARAMETER

RESULT

Particulates (total) 54.3
RL

0.50

UNITS

ing Dilution Factor: 1
METHOD

CFR60A 5


Air Sampling Associates, Inc.

client sample ID: HARDIN SDA INLET DUCT RUN 1 FILTBR (THTMBLE 50)

General Chemistry

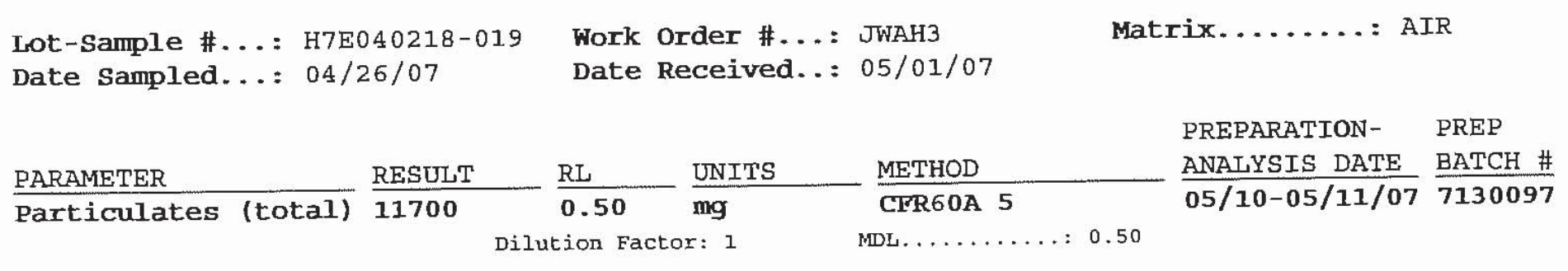


Air Sampling Associates, Inc.

Client Sample ID: HARDIN SDA INLET DUCT RUN 1 FILTKR (A173)

General Chemistry

Iot-Sample \#...: H7E040218-020

Date Sampled...: 04/26/07 work Order \#... : JWAH5

Date Received..: 05/01/07
Matrix....... A AIR

PREPARATION- PREP

ANALYSIS DATE BATCH \#
PARAMETER

Particulates (total) 417
RESULT

$\underline{\mathrm{RL}}$

UNITS

mg

Dilution Factor: 1
METHOD

CFR60A 5

MDL......... 0.50 
Air Sampling Associates, Inc.

Client Sample W: HARDTN SDA INLET DUCT RUN 1 ACETONE

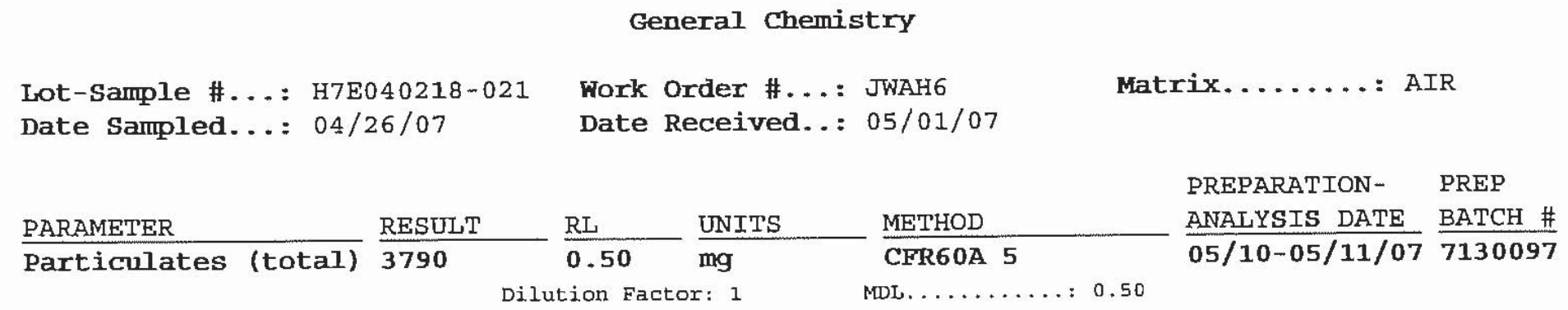


Air Sampling Associates, Inc.

client Sample ID: HARDIN SDA INLET DUCT RUN 2 FILTER (THIMBLE 52)

General Chemistry

Iot-Sample \#...: H7E040218-026

Date Sampled...: 04/26/07
Work Order \#...: JWAJV

Date Received... 05/01/07
Matrix....... AIR

PREPARATION - PREP ANALYSIS DATE BATCH \# 05/10-05/11/07 7130097
PARAMETER

Particulates (total)
RESULT 9860
RI

Dilution Factor: 1
METHOD CFR60A 5 MDL........ 0.50 
Air Sampling Associates, Inc.

client sample ID: HARDIN SDA INLET DUCT RUN 2 FILTBR (A174)

General Chemistry

Lot-Sample \#...: H7E040218-027

Date Sampled...: 04/26/07
Work Order \#...: JWAJ5

Date Received..: 05/01/07
Matrix....... AIR

PREPARATION - PREP

ANALYSIS DATE BATCH \#
PARAMETER

Particulates (total)
RESULT

726
RL

0.50

UNIT

Dilution Factor: 1
METHOD

CFR60A 5

MDL......... 0.50 
Air Sampling Associates, Inc.

Client Sample ID: HARDIN SDA INIFT DUCT RUN 2 ACBTONE

General Chemistry

Lot-Sample \#... : H7E040218-028

Date Sampled...: $04 / 26 / 07$
Hork Order \#...: JWAJ9

Date Received... 05/01/07
Matrix....... AIR

PREPARATION- PREP ANALYSIS DATE BATCH \# 05/10-05/11/07 $\overline{7130097}$
PARAMETER

RESULT

particulates (total) $\mathbf{3 0 3 0}$
RL 0.50 m!g
METHOD CFR60A 5 MDL. . . . . . . 0.50 
Air Sampling Associates, Inc.

client sample ID: HARDIN SDA INLET DUCT RUN 3 FILTER (THTMBLE 51)

General Chemistry

Lot-Sample \#...: H7E040218-033

Date Sampled...: 04/26/07
Work Order \#...: JWAK9

Date Received..: 05/01/07
Matrix........ AIR

PREPARATION - PREP ANALYSIS DATE BATCH \#
PARAMETER

RESULT

Particulates (total) 12400
$\mathrm{RL}$

0.50 mg

Dilution Factor: 1
METHOD

CFR60A 5 
Air Sampling Associates, Inc.

Client Sample ID: HARDIN SDA INLET DUCT RUN 3 FILTBR (A175)

General Chemistry

Lot-Sample \#...: H7E040218-034

Date sampled...: 04/26/07
Work Order \#...: JWALI

Date Received..: 05/01/07
Matrix.......: AIR

PREPARATION - PREP

ANALYSIS DATE BATCH \#
PARAMETER

RESULT

Particulates (total) 413

$-\mathrm{RI}$

Dilution Factor: 1
UNITS
METHOD

CFR60A 5 
Air Sampling Associates, Inc.

Client Sample ID: HARDIN SDA INLET DUCT RUN 3 ACETONE

General Chemistry

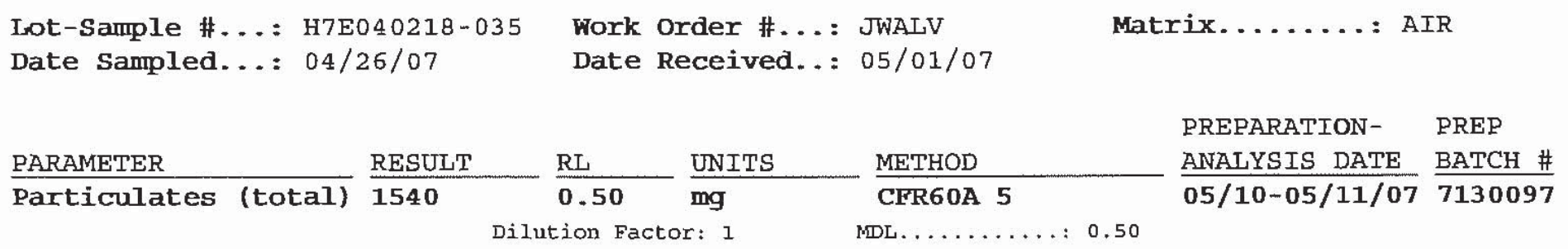


Sample Receipt Documentation 


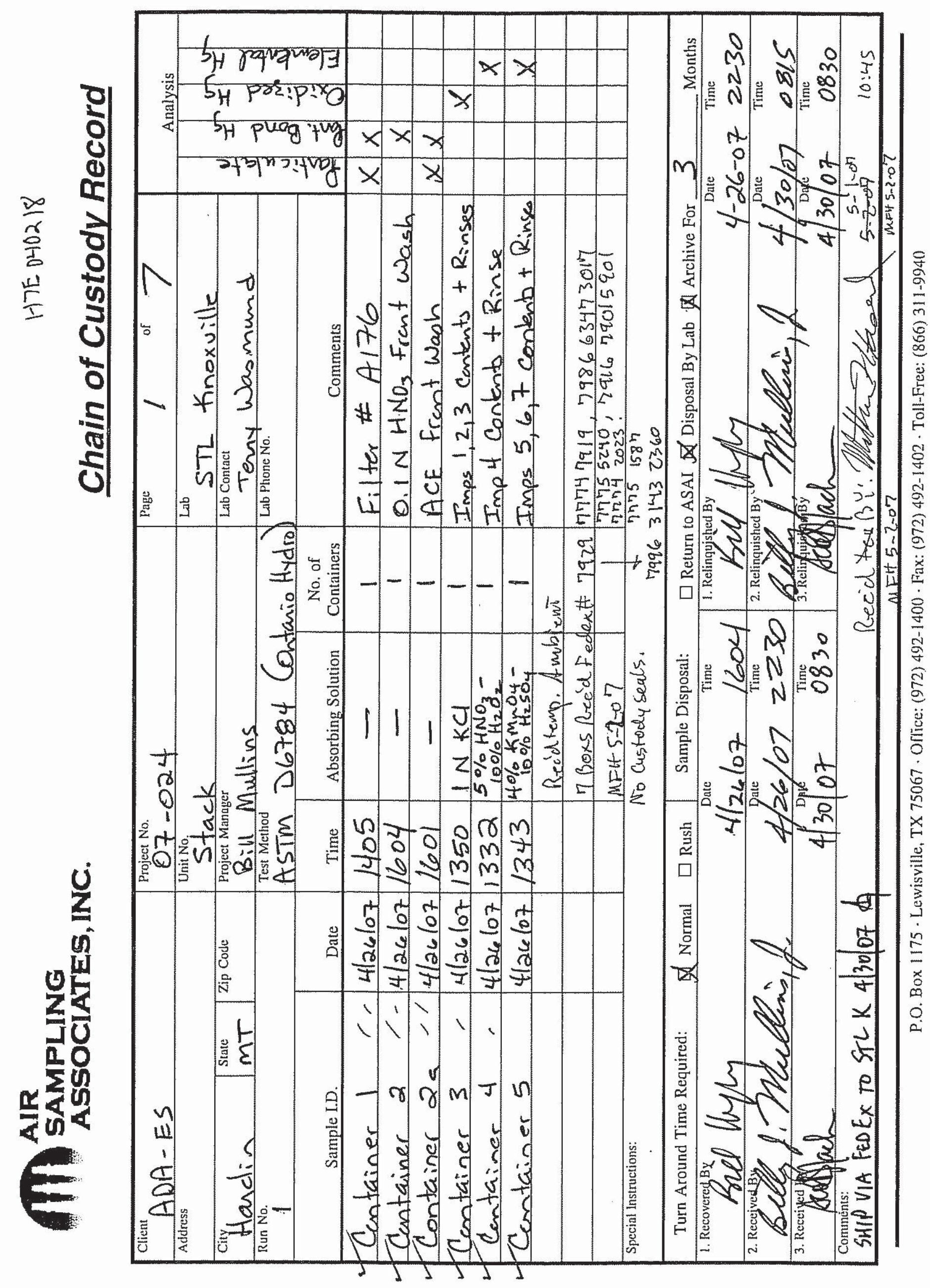




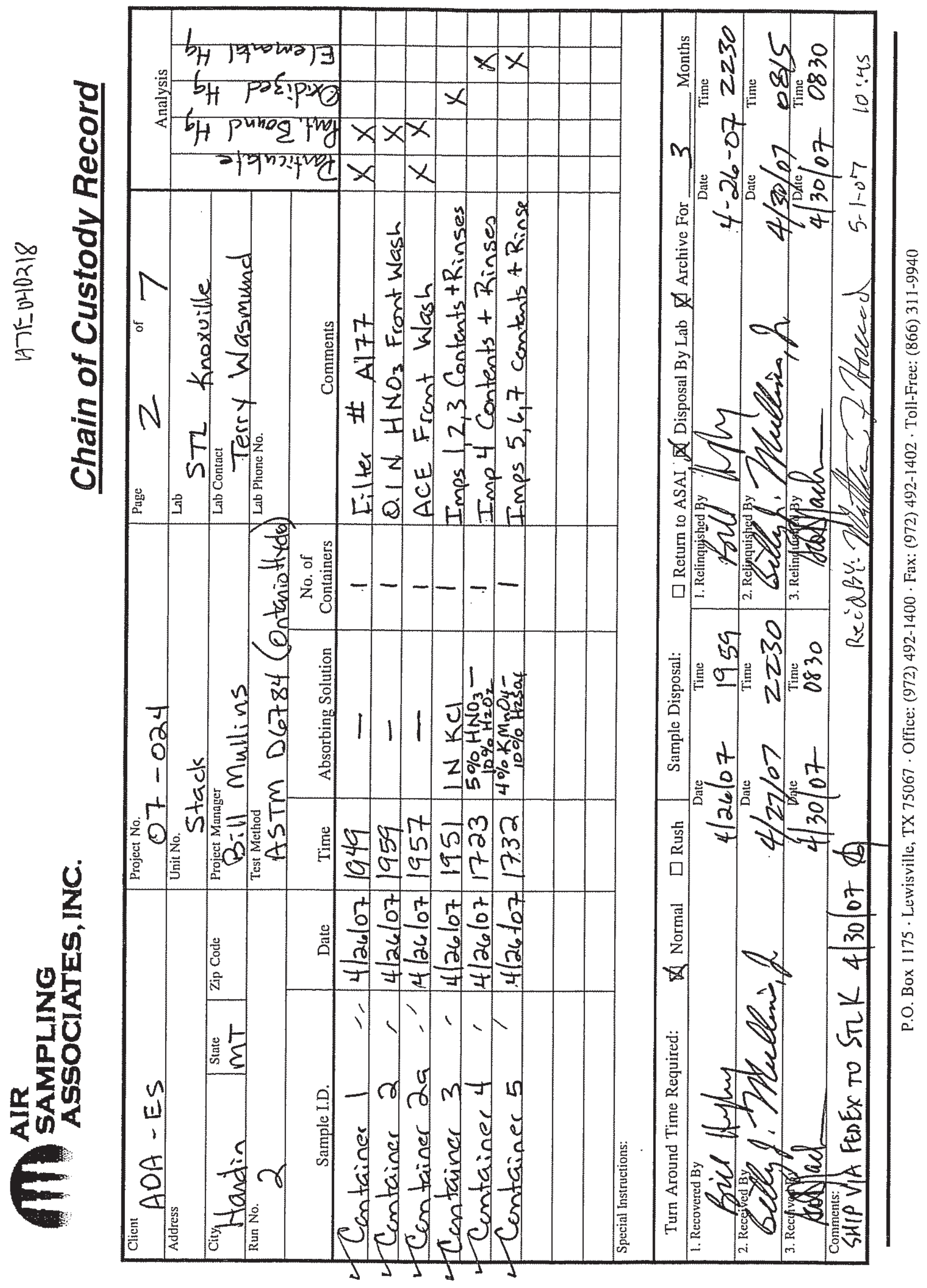




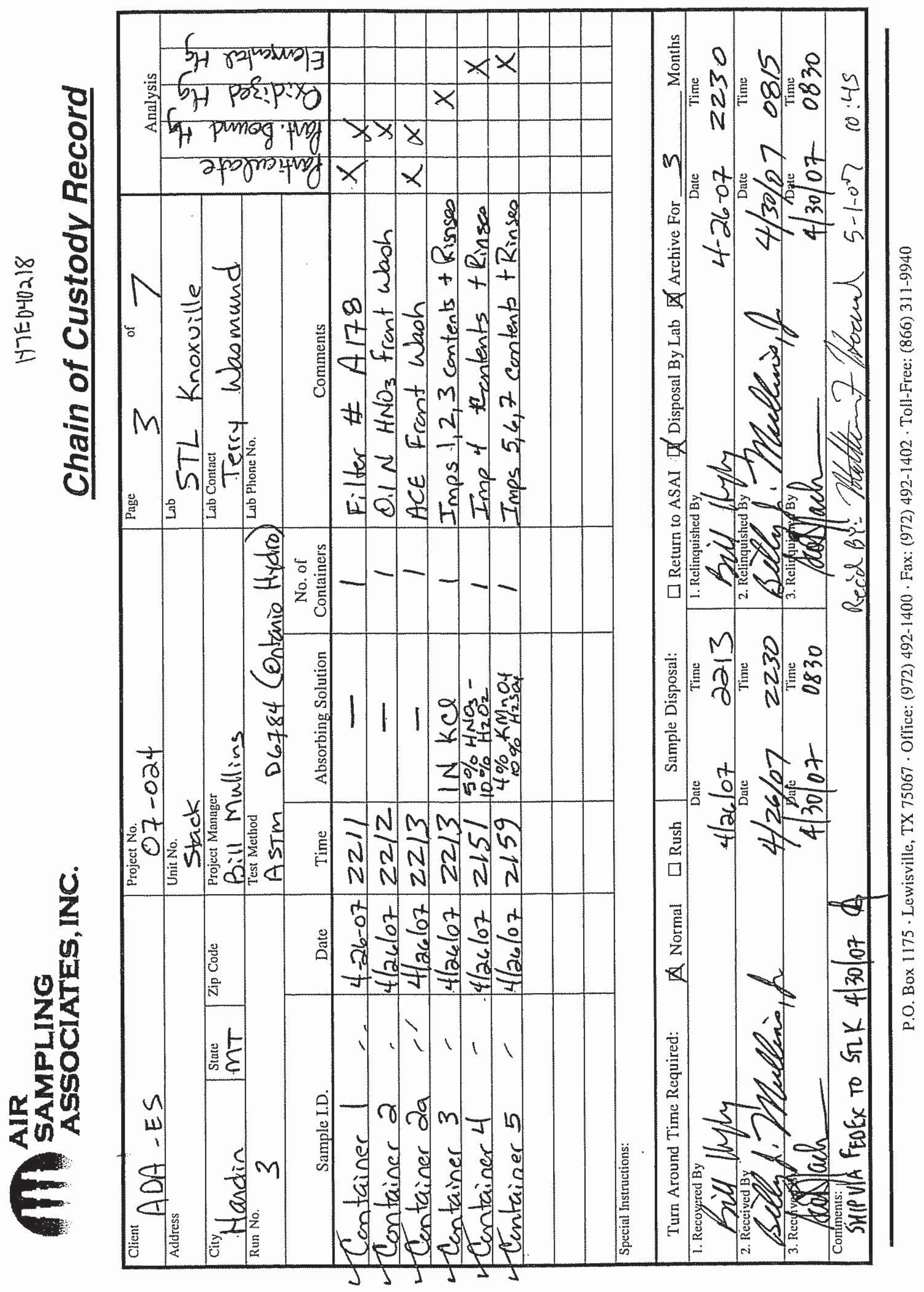




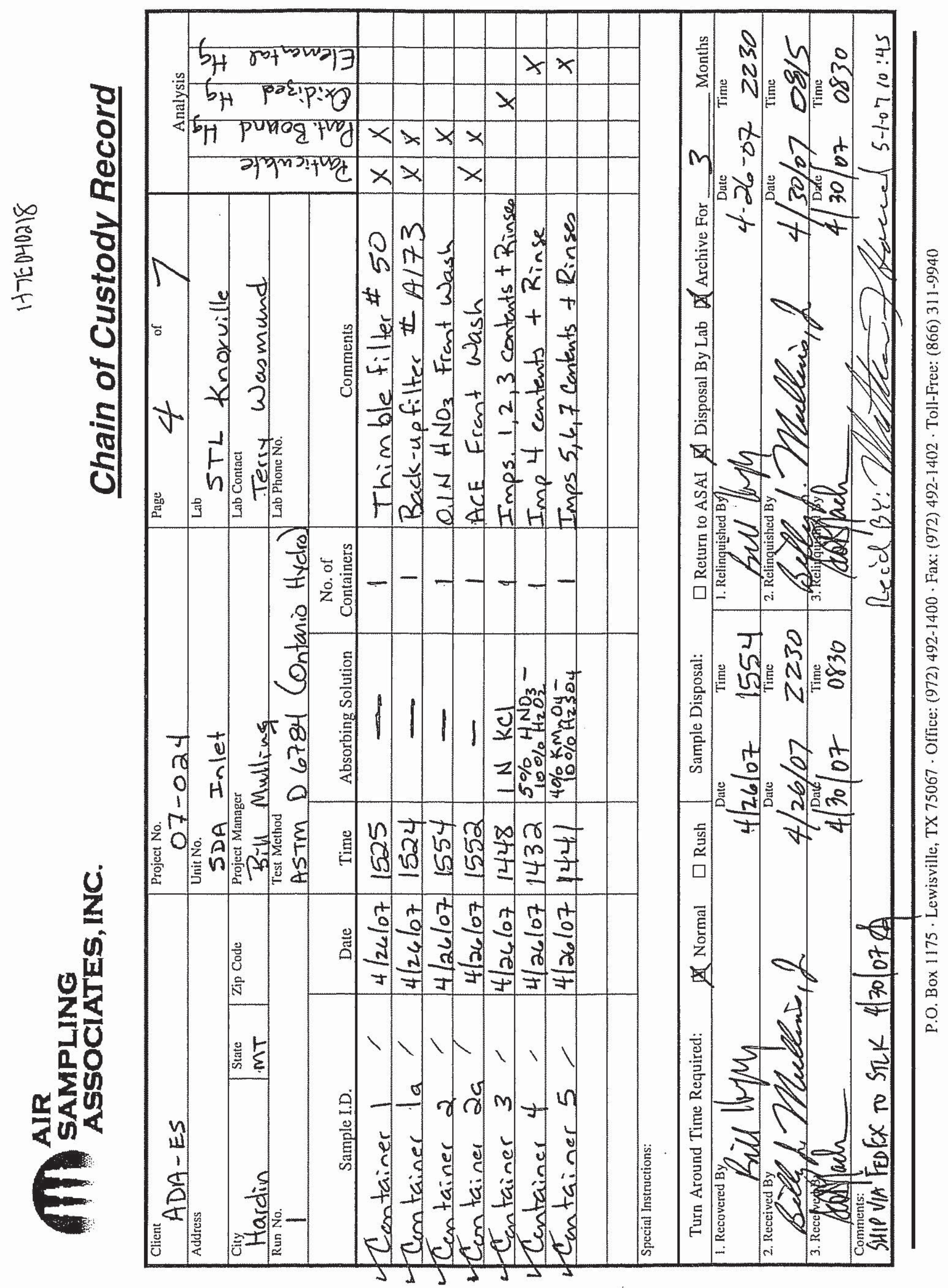




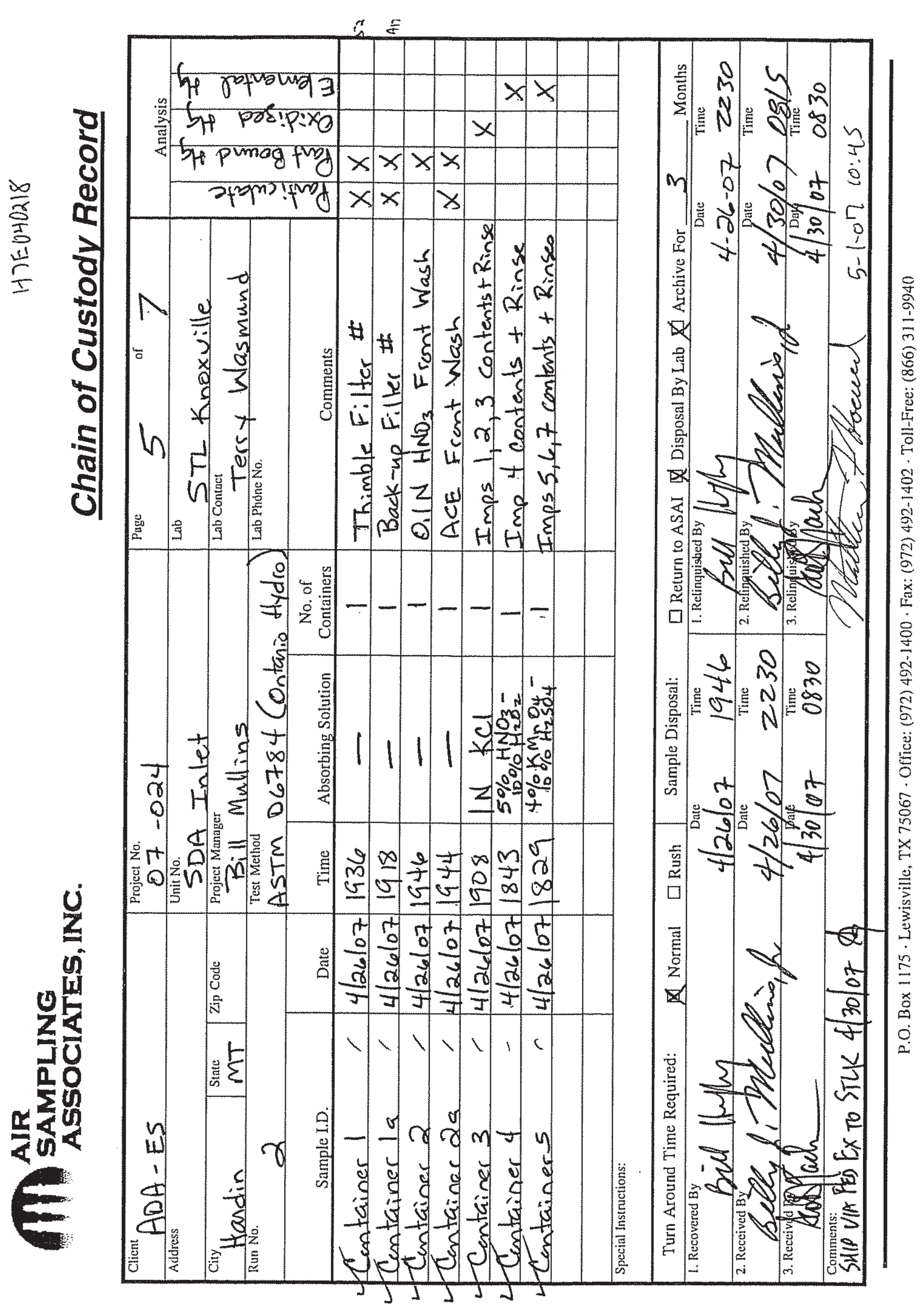




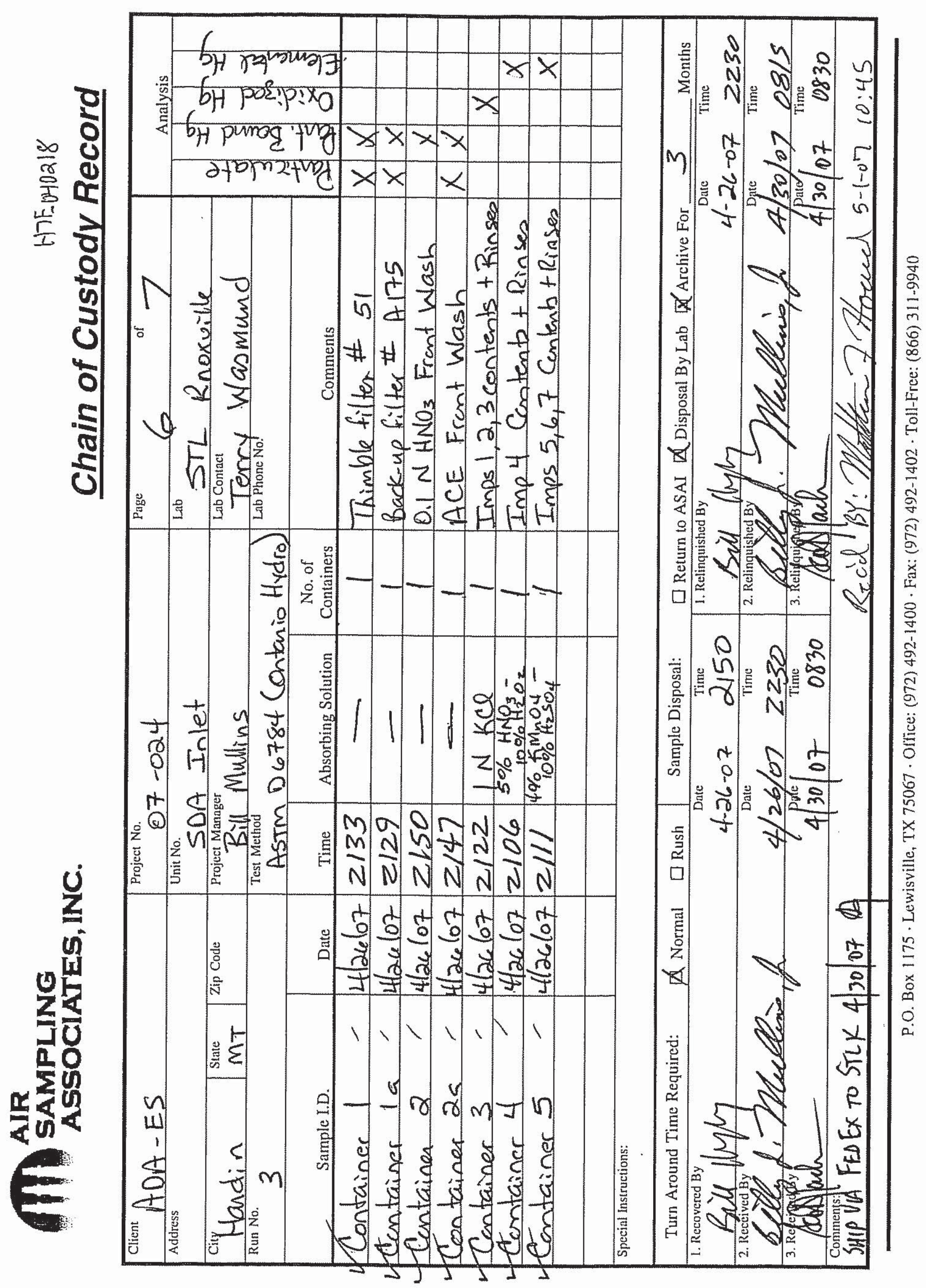




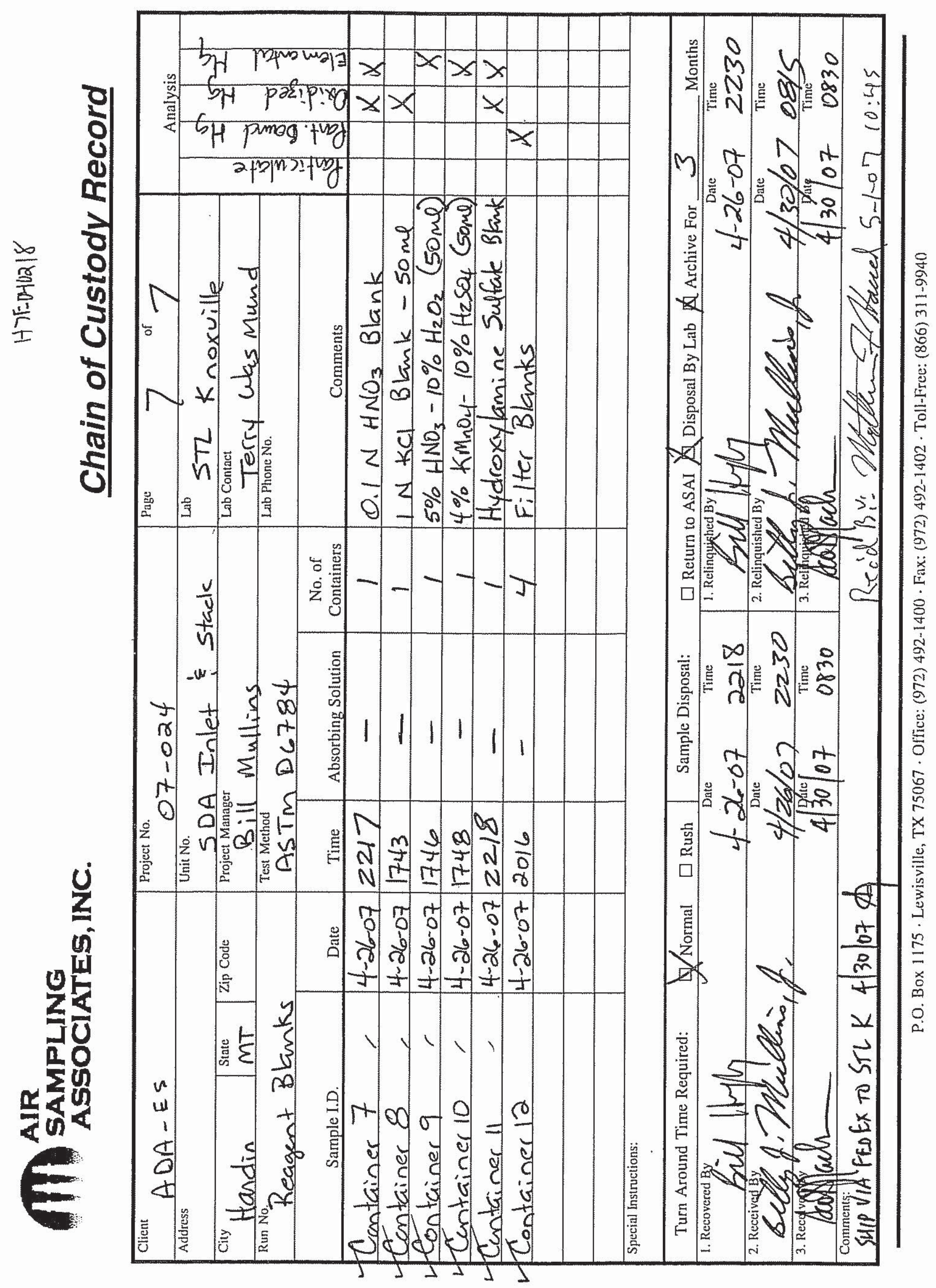




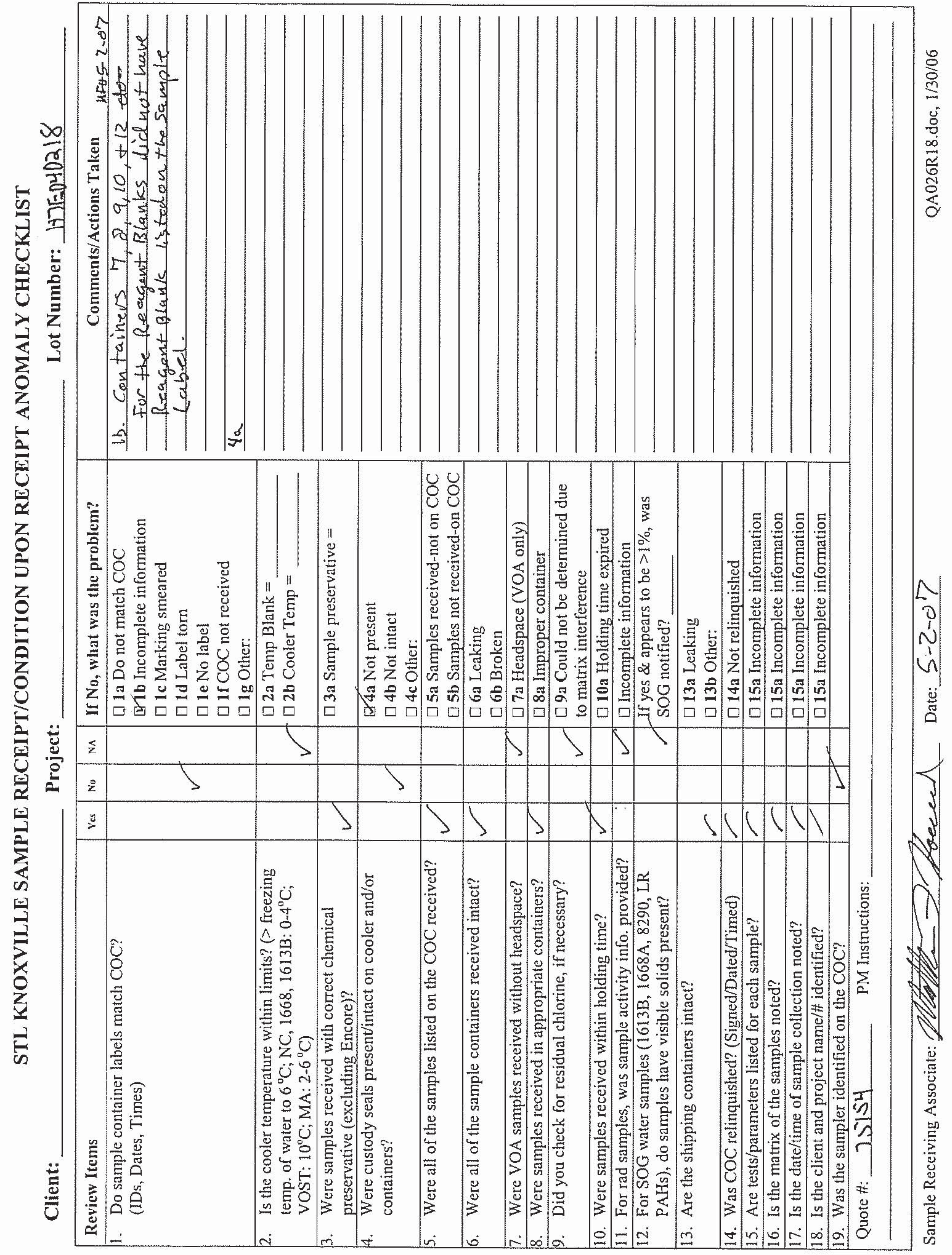


Metals 
Sample Results 
Air Sampling Associates, Inc.

Client Sample ID: HARDIN STACK RUN 1 FH

TOTAL Metals

Lot-Sample \#...: H7E0. $0218-003$

Date Sampled...: 04/26/07

Date Received..: 05/01/07

REPORTING

LIMIT

UNITS

Prep Batch \#...: 7134095

Mercury

0.010 ug

Dilution Factor: 0.1
METHOD

Matrix......: AIR

PREPARATION - WORK ANALYSIS DATE ORDER \#

ASTM D6784-02

05/16-05/17/07 JWAFK1AA

MDL......... 0.0060 
Air Sampling Associates, Inc.

Client Sample ID: HARDIN STACK RUN I KCL

TOTAL Metals

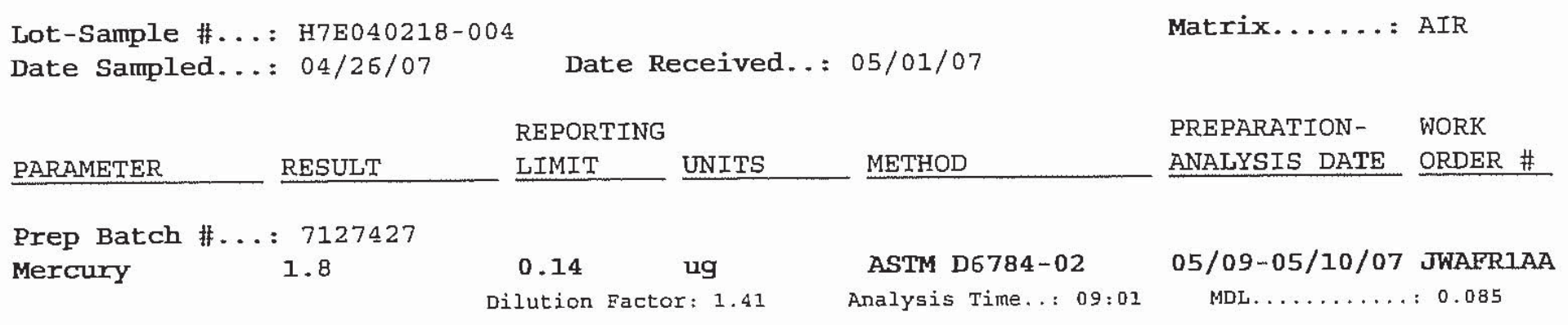


Air Sampling Associates, Inc.

client Sample ID: HARDIN STACK RUN I HNO3/H2O2

TOTAY Metals

Lot-Sample \#. . : H7E04021B-005

Date Sampled...: 04/26/07

PARAMETER

Prep Batch \#...: 7127421

Mercury

0.15
Date Received..: 05/01/07

REPORTING

LIMIT

UNITS

METHOD

0.070 ug
ASTM D6784-02
Analysion Factor: $0.7 \quad$ Time. : 00:36

Matrix....... AIR

PREPARATION- WORK ANALYSIS DATE ORDER \#

05/09-05/10/07 JWAFWבAA MDL.........: 0.042 
Air Sampling Associates, Inc.

\section{Client Sample ID: HARDIN STACK RUN I KMNO4/H2SO4}

TOTAL Metals

Lot-Sample \#. . : H7E040218-006

Date Sampled...: 04/26/07

Date Received. .: 05/01/07

REPORTING LIMIT UNITS METHOD

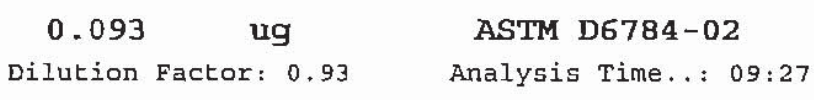

Matrix......: AIR

PREPARATION - WORK ANALYSIS DATE ORDER \#

05/09-05/10/07 JWAFX1AA MDL. ........ ; 0.056 
Air Sampling Associates, Inc.

Client Sample ID: HARDIN STACK RUN 2 FH

\section{TOTAL Metals}

Lot-Sarmple \#...: H7E040218-009

Date Sampled...: 04/26/07

$\begin{array}{ll}\text { PARAMETER } & \text { RESULT } \\ \text { Prep Batch \#...: } & 7134095 \\ \text { Mercury } & 0.058\end{array}$

0.058
Date Received..: 05/01/07

REPORTING

LIMIT

0.010 ug
Dilution Eactor: 0.1

ASTM D6784-02

Analysis Time..: 08:53
Matrix...... AIR

PREPARATION- WORK ANALYSIS DATE ORDER \#

05/16-05/17/07 JWAGKבAA 
Air Sampling Associates, Inc.

Client Sample ID: HARDIN STACK RUN 2 KCL

TOTAL Metals

Lot-Sample \#...: H7E040218-010

Date Sampled...: 04/26/07

PARAMETER

RESULT

Prep Batch \#...: 7127427

Mercury
1.8
0.14

Dilution Factor: 1.41
Date Received..: 05/01/07

REPORTING

UNITS

METHOD

ASTM D6784-02

Analysis Time..: 09:11
Matrix....... AIR

PREPARATION- WORK ANALYSIS DATE ORDER \#

05/09-05/10/07 JWAGNIAA 
Air Sampling Associates, Inc.

client Sample ID: HARDIN STACK RUN 2 HNO3/H2O2

TOTAL Metals

Irot-Sample \#...: H7E040218-011

Date Sampled...: 04/26/07

PARAMETER

Prep Batch \#...: 7127421

Mercury
0.19
Date Received. .: 05/01/07

REPORTING

LIMIT

UNITS
METHOD
Matrix.....: AIR

PREPARATION- WORK ANALYSIS DATE ORDER \#

$\begin{array}{lccc}0.073 & \text { ug } & \text { ASTM D6784-02 } & 05 / 09-05 / 10 / 07 \text { JWAGPIAA } \\ \text { Dilution Factor: } 0.73 & \text { Analysis Time..: 08:46 } & \text { MDL........ } 0.044\end{array}$


Air Sampling Associates, Inc.

client Sample ID: HARDIN STACK RUN 2 KMNO4/H2SO4

TOTAL Metals

Lot-Sample

Date Sarmpled...: 04/26/07

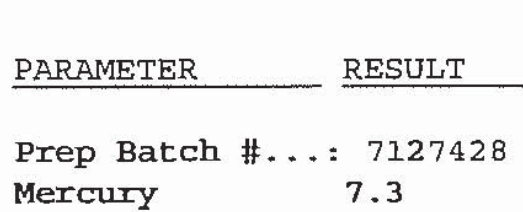

Date Received..: 05/01/07

REPORTING LIMIT UNITS

0.10 ug
ASTM D6784-02
Analysis Time..: 09:37

Matrix.....: AIR

PREPARATION- WORK ANALYSIS DATE ORDER \#

05/09-05/10/07 JWAGRIAA MDL .......... 0.061 
Air Sampling Associates, Inc.

\section{Client Sample ID: HARDIN STACK RUN 3 FH}

TOTAL Metals

Lot-Sample \#. . : H7E040218-015

Date Sampled...: 04/26/07

Date Received. .: 05/01/07

REPORTING

PARAMETER

RESULT

Prep Batch \#...: 7134095

Mercury

0.029
$0.010 \mathrm{ug}$

Dilution Eactor: 0.1
Matrix......: AIR

PREPARATION - WORK METHOD ANALYSIS DATE ORDER \#
ASTM D6784-02 05/16-05/17/07 JWAHELAA 
Air Sampling Associates, Inc.

Client Sample ID: HARDIN STACK RUN 3 KCL

TOTAL Metals

Lot-Sample \#...: H7E04021B-016

Date Sampled...: 04/26/07

Date Received..: 05/01/07

REPORTING

LIMIT

UNITS

Prep Batch \#...: 7127427

Mercury

1.7

0.14

ug

Dilution Factor: 1.41
METHOD

Matrix....... AIR

PREPARATION- WORK ANALYSIS DATE ORDER \#

ASTM D6784-02

05/09-05/10/07 JWAHН1AA

MDL.......... 0.085 
Air Sampling Associates, Inc.

Client Sample ID: HARDIN STACK RUN 3 HNO3/H2O2

TOTAL Metals

Lot-Sample \#... : H7E040218-017

Date Sampled...: 04/26/07

PARAMETER

Prep Batch \#...: 7127421

Mercury

0.11
Date Received. .: 05/01/07

REPORTING

LIMIT

UNITS

METHOD

0.068 ug
Dilution Factor: $0.68 \quad$ Analysis Time..: 08:48

Matrix...... AIR

PREPARATION - WORK ANALYSIS DATE ORDER \#

05/09-05/10/07 JWAHWIAA MOL ..........: 0.041 
Air Sampling Associates, Inc.

client Sample ID: HARDIN STACK RUN 3 KMNO4/H2SO4

TOTAL Metals

Lot-Sample \#...: H7E040218-018

Date Sampled...: 04/26/07

PARAMETER

Prep Batch \#...: 7127428

Mercury
6.7
Date Received..: 05/01/07

REPORTING LIMIT UNITS

ug

Dilution Factor: 0.92
Matrix...... : AIR

PREPARATION- WORK ANALYSIS DATE ORDER \#
05/09-05/10/07 JWAHMבAA MDL $\ldots \ldots \ldots \ldots: 0.055$ 
Air Sampling Associates, Inc.

Client Sample ID: HARDIN SDA INLET DUCT RUN 1 FH TOTAL Metals

Lot-Sample \#...: H7E040218-022

Date Sampled...: 04/26/07

PARAMETER

Prep Batch \#...: 7134095

Mercury
1.3
Date Received. : 05/01/07

REPORTING LIMIT UNITS METHOD

ASTM D6784-02
Analysis Time..: 09:57

Matrix.....: AIR

PREPARATION - WORK ANALYSIS DATE ORDER \#

05/16-05/17/07 JWAB7LAA MDL......... 0.030 
Air Sampling Associates, Inc.

Client sample ID: HARDIN SDA INLET DUCT RUN 1 KCL

TOTAL Metals

Lot-Sample \#...: H7E040218-023

Date Sampled...: 04/26/07

PARAMETER

Prep Batch \#...: 7127427

Mercury

$$
0.65
$$

Date Received..: 05/01/07

REPORTING

LIMIT

UNITS

ug

Dilution Factor: 1.06 METHOD
Matrix...... AIR

PREPARATION - WORK ANALYSIS DATE ORDER \#

ASTM D6784-02

Aralysis Time...: 09:15
05/09-05/10/07 JWAJAIAA MDL.......... 0.064 
Air Sampling Associates, Inc.

client Sample ID: HARDIN SDA INLET DUCT RUN 1 HNO3/H2O2

TOTAL Metals

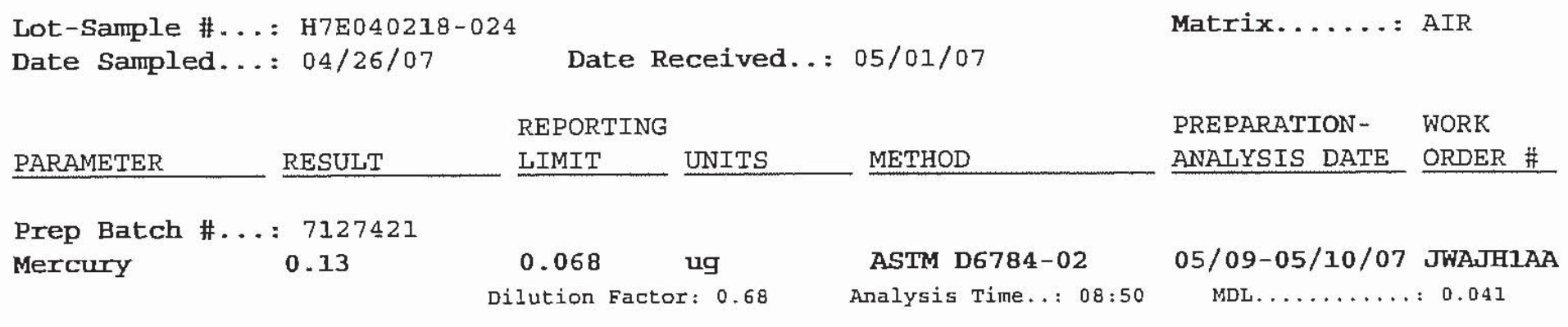


Air Sampling Associates, Inc.

Client Sample ID: HARDIN SDA INLET DUCT RUN 1 KMNO4,H2SO4

TOTAL Metals

Lot-Sample \#. . : H7E040218-025

Date Sampled...: 04/26/07
RESULT

Prep Batch \#...: 7127428

Mercury
2.6
Date Received..: 05/01/07

REPORTING

LIMIT UNITS

0.092 ug

Dilution Factor: 0.92
Matrix....... A AIR

PREPARATION- WORK ANALYSIS DATE ORDER \#

$$
\text { ASTM D6784-02 05/09-05/10/07 JWAJRIAA }
$$

Analysis Time..: 09:41 
Air Sampling Associates, Inc.

Client Sample ID: HARDIN SDA INLET DUCT RUN 2 FH

TOTAL Metals

Lot-Sample \#...: H7E040218-029

Date Sampled...: 04/26/07

Date Received. .: 05/01/07

REPORTING

PARAMETER

RESULT

Prep Batch \#...: 7134095

Mercury

0.97
0.050 ug

Dilution Factor: 0.5
ASTM 06784-02

Analysis Time..: 09:55
Matrix...... AIR

PREPARATION - WORK ANALYSIS DATE ORDER \#

05/16-05/17/07 JWAKFLAA MDL......... : 0.030 
Air Sampling Associates, Inc.

Client Sample ID: HARDIN SDA INEFT DUCT RUN 2 KCL

TOTAL Metals

Lot-Sample \#. . . : H7E040218-030

Date Sampled...: 04/26/07

PARAMETER

Prep Batch \#...: 7127427

Mercury

$$
0.57
$$

Date Received. .: 05/01/07

REPORTING LIMIT

UNITS METHOD

ASTM D6784-02

Analysis Time..: 09:17

Matrix......: AIR

PREPARATION - WORK ANALYSIS DATE ORDER \#

05/09-05/10/07 JWAKGIAA MDL......... 0.071 
Aix Sampling Associates, Inc.

Client Sample ID: HARDIN SDA INLET DUCT RUN 2 HNO3/H2O2

TOTAL Metals

Lot-Sample \#...: H7E040218-031

Date Sampled...: 04/26/07

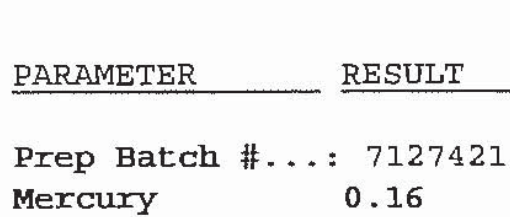

Date Received..: 05/01/07

REPORTING LIMIT UNITS

ug

0.070 ug
ilution Eactor: 0.7

ASTM D6784-02

Analysis Time..: 08:52
Matrix...... AIR

PREPARATION - WORK ANALYSIS DATE ORDER \#

05/09-05/10/07 JWAKKIAA 
Air Sampling Associates, Inc.

client Sample ID: HARDIN SDA INLET DUCT RUN 2 KMNO4/H2SO4

TOTAL Metals

Lot-Sample \#. . . : H7E040218-032

Date Sampled...: 04/26/07

PARAMETER

Prep Batch 荈: 7127428

Mercury
3.1
Date Received. .: 05/01/07

REPORTING

LIMIT UNITS

0.092 ug

Dilution Factor: 0.92
Matrix...... AIR

PREPARATION- WORK ANALYSIS DATE ORDER \#

\section{ASTM D6784-02}

Analysis Time..: 09:43
05/09-05/10/07 JWAK01AA MDL. ......... 0.055 
Air Sampling Associates, Inc.

Client Sample ID: HARDIN SDA INLET DUCT RUN 3 FH

TOTAL Metals

Lot-Sample \#...: H7E040218-036

Date Sampled...: 04/26/07

PARAMETER

Erep Batch \#...: 7134095

Mercury

0.98
Date Received. .: 05/01/07

REPORTING LIMIT UNITS METHOD

ASTM D6784-02

Analysis Time..: 09:59
Matrix.....: AIR

PREPARATION- WORK ANALYSIS DATE ORDER \#

05/16-05/17/07 JWAL31AA MDL ........ 0.030 
Air Sampling Associates, Inc.

Client Sample ID: HARDIN SDA INLFT DUCT RUN 3 KCL

TOTAL Metals

Lot-Sample \#...: H7E040218-037

Date Sampled...: 04/26/07

PARAMETER

Prep Batch \#...: 7127427

Mercury

0.72
Date Received..: 05/01/07

REPORTING

LIMIT

UNITS

ug

Dilution Factor: 1.11 METHOD
Matrix...... AIR

PREPARATION- WORK ANALYSIS DATE ORDER \#

ASTM D6784-02 05/09-05/10/07 JWAMGLAA Analysis Time..: 09:19 
Air Sampling Associates, Inc.

client Sample ID: HARDIN SDA INHCT DUCT RUN 3 HNO3/H2O2

TOTAL Metals

Lot-Sample \#...: H7E040218-038

Date Sampled...: 04/26/07

PARAMETER

Prep Batch \#...: 7127421

Mercury

0.17
Date Received..: 05/01/07

REPORTING

LIMIT

UNITS

ug

Dilution Factor: 0.73
Matrix......: AIR

PREPARATION- WORK ANALYSIS DATE ORDER \#

ASTM D6784-02 05/09-05/10/07 JWAMKבAA

Analysis Time... 08:54 
Air Sampling Associates, Inc.

Client Sample ID: HARDIN SDA INLET DUCT RUN 3 KMNO4/H2SO4

TOTAL Metals

Lot-Sample \#...: H7E040218-039

Date Sampled...: 04/26/07

PARAMETER

Prep Batch \#...: 7127428

Mercury
2.8
Date Received. .: 05/01/07

REPORTING LIMIT UNITS 0.097 ug
ilution Factor: 0.97 METHOD
Matrix...... AIR

PREPARATION - WORK ANALYSIS DATE ORDER \# ASTM D6784-02 05/09-05/10/07 JWAMPLAA Analysis Time... 09:45 
Air Sampling Associates, Inc.

client Sample ID: HARDIN SDA INLET+STACK RB 0. IN HNO3

TOTAL Metals

Lot-Sample \#...: H7E040218-040

Date Sampled...: 04/26/07

Date Received..: 05/01/07

REPORTING

PARAMETER

RESULT

Prep Batch \#...: 7134095

Mercury

ND
LIMIT

0.010

ug

Dilution Factor: 0.1
Matrix......: AIR

PREPARATION- WORK ANALYSIS DATE ORDER \#

METHOD

ASTM D6784-02

Analysis Time..: 09:03
05/16-05/17/07 JWAM21AA MDL......... 0.0060 
Air Sampling Associates, Inc.

client Sample ID: HARDIN SDA INLUT+STACK RB IN KCL

TOTAL Metals

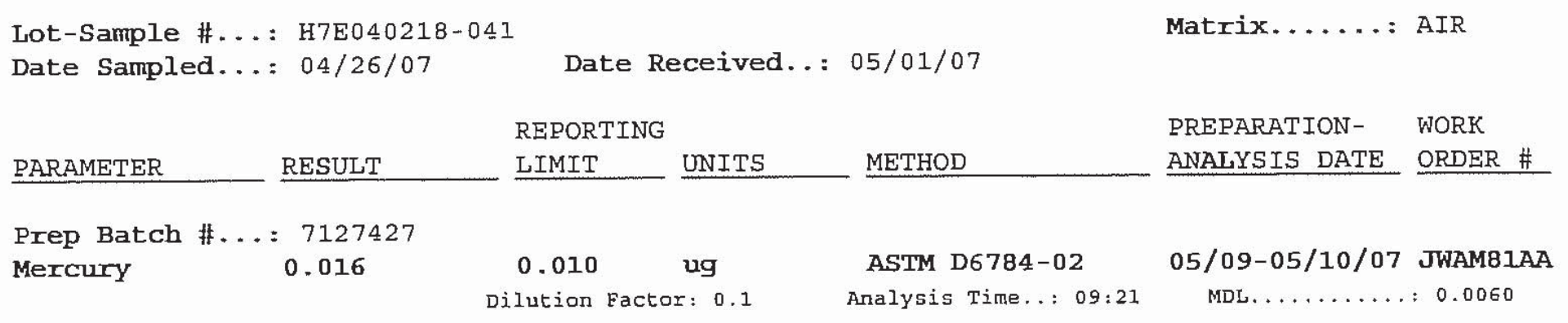


Air Sampling Associates, Inc.

client Sample ID: HARDIN SDA INLET+STACK RB HNO3/H2O2

TOTAL Metals

Lot-Sample \#...: H7E040218-042

Date Sampled...: 04/26/07

PARAMETER R_ RESULT

Prep Batch \#...: 7127421

Mercury

$0.025 \quad$ ug
Dilution Factor: 0.25

Date Received..: 05/01/07

REPORTING

LIMIT UNITS METHOD

ASTM D6784-02

Analysis Time.,: 08:56
Matrix...... AIR

PREPARATION - WORK ANALYSIS DATE ORDER \#

05/09-05/10/07 JWANDIAA MDL......... 0.015 
Air Sampling Associates, Inc.

client Sample ID: HARDIN SDA INLET+STACK RB KMNO4/H2SO4/HYDR

TOTAL Metals

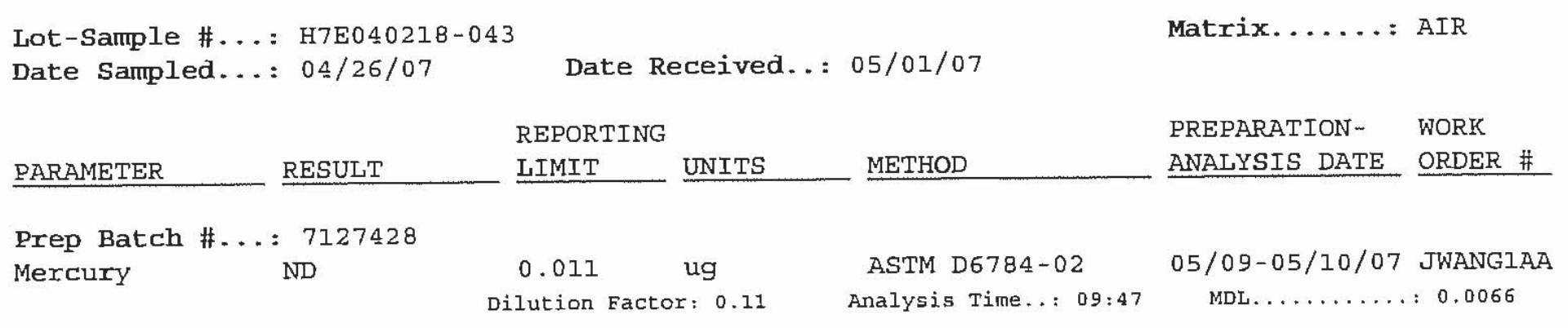


Air Sampling Associates, Inc.

Client Sample ID: HARDIN SDA INLET+STACK RB QUARTZ FILTER

TOTAL Metals

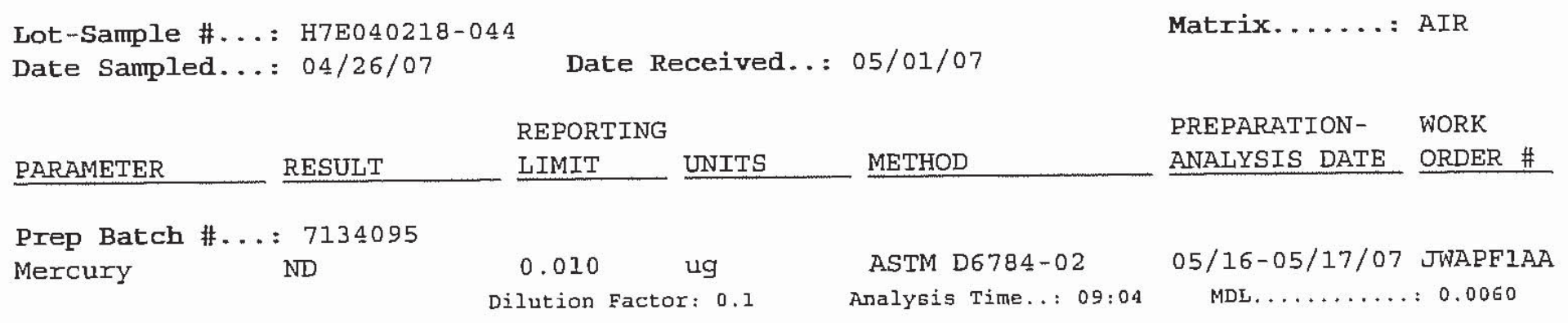


Air Sampling Associates, Inc.

client Sample ID: HARDIN SDA INLET+STACK RB THIMBLE 1

TOTAL Metals

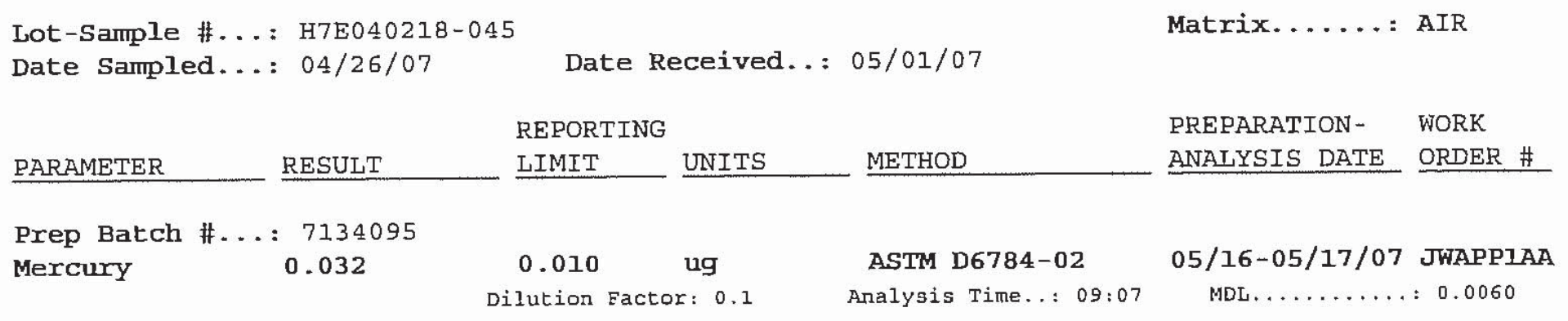


QC Summary 


\section{METHOD BLANK REPORT}

TOTAL Metals

Client Lot \#...: H7E040218

Matrix....... A AIR

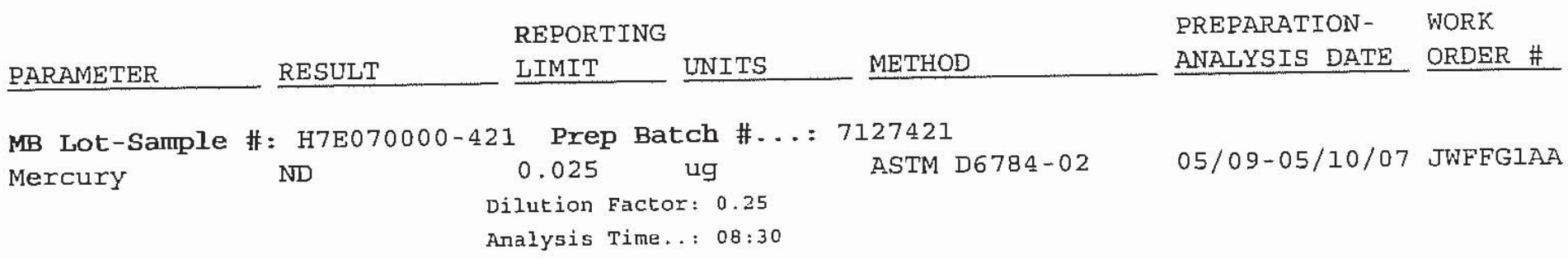

MB Lot-Sample \#: H7E070000-427 Prep Batch \#...: 7127427

$\begin{array}{ccc}\text { Mercury ND } & 0.010 \text { ug } \\ \text { Dilution Factor: } 0.1\end{array}$

ASTM D6784-02 05/09-05/10/07 JWFFKIAA

Analysis Time..: 08:58

MB Lot-Sample \#: H7E070000-428 Prep Batch \#..: 7127428

Mercury

ND

0.010

ug

A.STM D6784-02

05/09-05/10/07 JWFFNIAA Dilution Factor: 0.1

Analysis Time.. : 09:23

MB Lot-Sample \#: H7E140000-095 Prep Batch \#...: 7134095

$\begin{array}{ccc}\text { Mercury ND } & 0.010 \text { ug } \\ \text { Dilution Factor: } 0.1\end{array}$

ASTM D678:-02

05/16-05/17/07 JWWQH1AA

NOTE (S) :

Calculations are performed before sounding to avoid round-off errors in calculated resulis. 
LABORATORY CONTROL SAMPLE EVALUATION RBPORT

TOTAL Metals

Lot-Sample \#...: H7E040218

Matrix....... A AIR

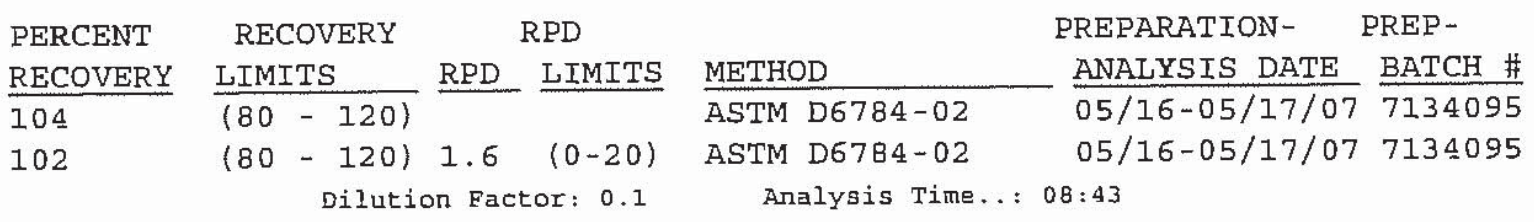

NOTE (S) :

Calculations are performed before rounding to avoid round-off errors in calculated results. 


\section{LAABORATORY CONTROL SAMPLE DATA REPORT}

\section{TOTAL Metals}

Lot-Sample \#...: H7E040218

Matrix....... AIR

\begin{tabular}{|c|c|c|c|c|c|c|c|c|}
\hline PARAMETER & $\begin{array}{l}\text { SPIKE } \\
\text { AMOUNT }\end{array}$ & $\begin{array}{l}\text { MEASURED } \\
\text { AMOUNT }\end{array}$ & UNITS & $\begin{array}{c}\text { PERCNT } \\
\text { RECVRY }\end{array}$ & $\underline{R P D}$ & METHOD & $\begin{array}{l}\text { PREPARATION- } \\
\text { ANALYSIS DATE }\end{array}$ & $\begin{array}{l}\text { PREP } \\
\text { BATCH \# }\end{array}$ \\
\hline \multirow[t]{2}{*}{ Mercury } & 0.500 & 0.519 & $\mathrm{ug}$ & 104 & & ASTM D6784-02 & $05 / 16-05 / 17 / 07$ & 7134095 \\
\hline & 0.500 & 0.511 & ug & 102 & 1.6 & ASTM D6784-02 & $05 / 16-05 / 17 / 07$ & 7134095 \\
\hline
\end{tabular}

$\operatorname{NOTE}(S):$

Calculations are perfotmed before rounding to avoid round-off errors in calculated results. 
IAABORATORY CONTROL SAMPLE EVALUATION REPORT

\section{TOTAL Metals}

Client Lot 非...: H7E040218

Matrix........ AIR

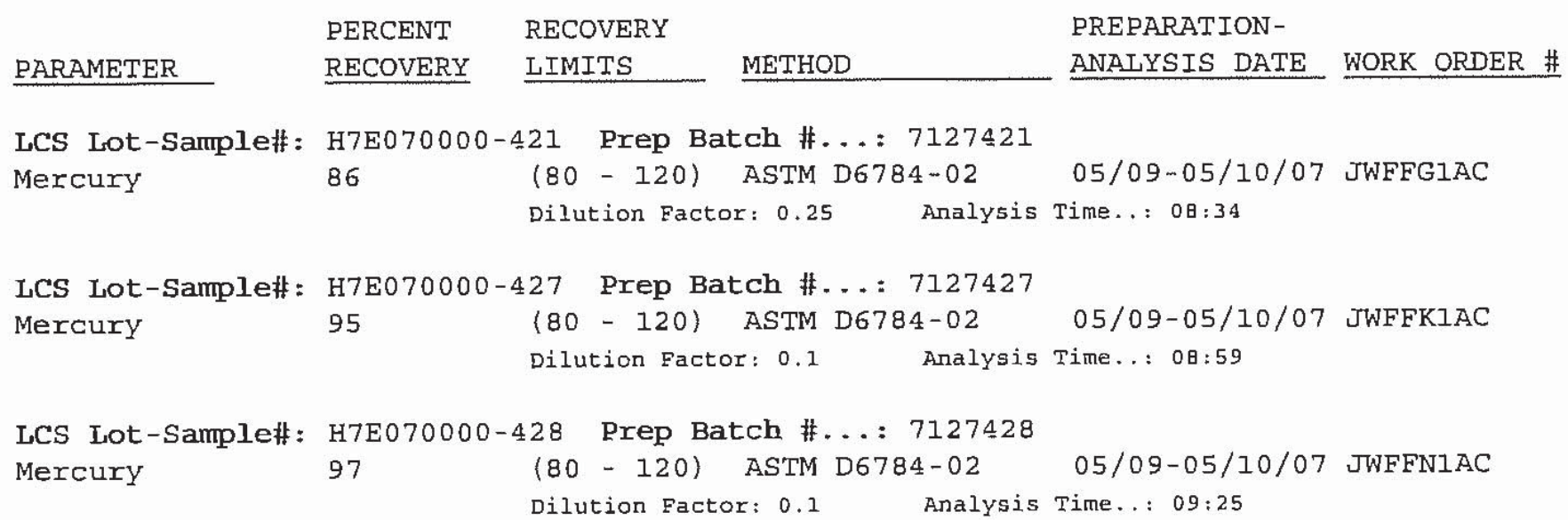

NOTE (S) :

Calculations are performed before rounding to avoid round-off errots in calculated resulis. 


\section{I.ABORATORY CONTROL SAMPLE DATA RFPORT}

TOTAL Metals

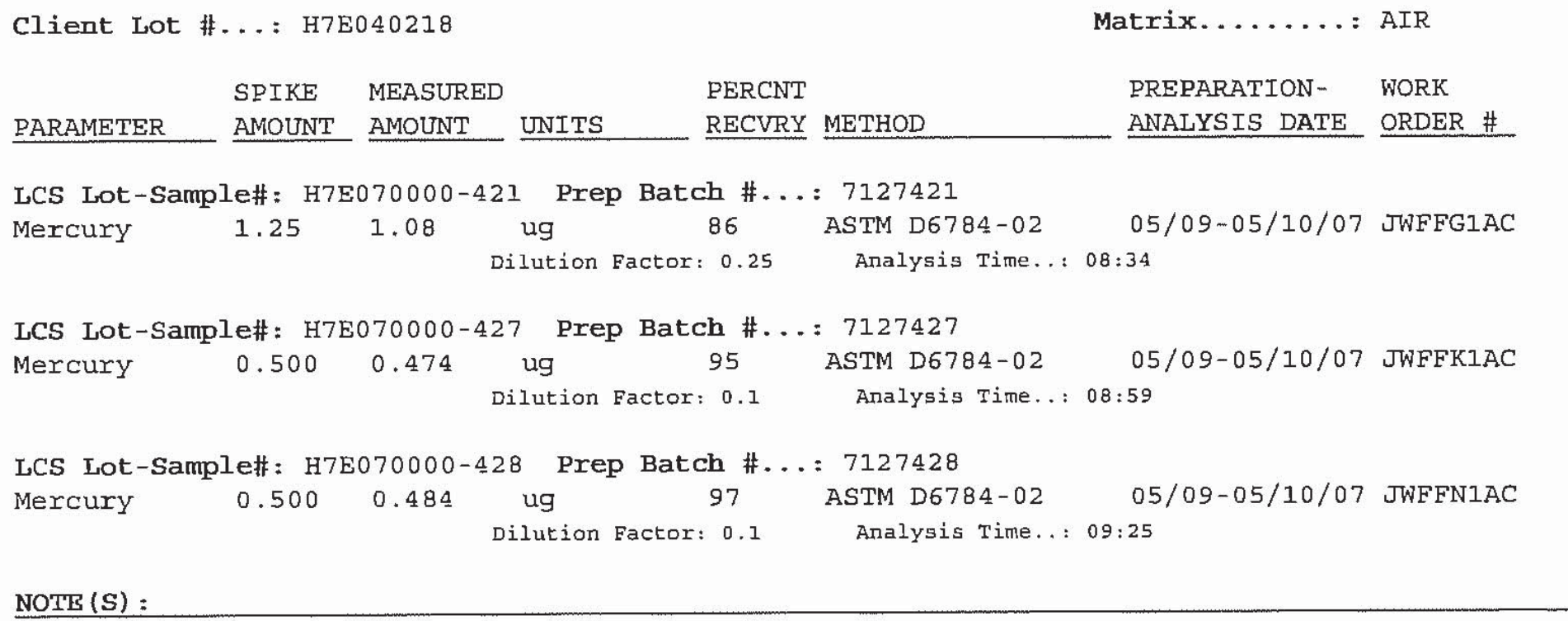

Calculations ate performed before rounding to avoid round-off errors in calculated results. 
MATRIX SPIKE SAMPLE GVALUATION REPORT

TOTAL Metals

Client Irot \#...: H7E040218

Date Sampled...: 04/26/07
Date Received. .: 05/01/07

\begin{tabular}{|c|c|c|c|c|}
\hline PERCENT & RECOVERY & & RPD & \\
\hline RECOVERY & LIMITS & $\mathrm{RPD}$ & LIMITS & METHOD \\
\hline
\end{tabular}

MS Lot-Sample \#: H7E040218-005 Prep Batch \#. . : 7127421

Mercury

98

101

\author{
$(80-120)$ \\ $(80$ - 120) $2.5(0-20)$ ASTM D6784-02 \\ Dilution Factor: 0.7 \\ Analysis Time..: 08:38
}

Matrix.......: AIR

PREPARATION - WORK ANALYSIS DATE ORDER \#

05/09-05/10/07 JWAFW1AC 05/09-05/10/07 JWAFWIAD

\section{NOTE $(S)$ :}

Calculations are performed before rounding to avoid round-off errors in calculated results. 
MATRIX SPIKE SAMPLE DATA REPORT

TOTAL Metals

Client Lot \#...: H7E040218

Date Sampled...: 04/26/07
Date Received. : : 05/01/07 RECVRY RPD METHOD
Matrix......: AIR

PREPARATION- WORK ANALYSIS DATE ORDER \#

PARAMETER AMOUNT AMT

AMOUNT UNITS

\#...: 7127421

MS Lot-S
Mercury

$$
0.15
$$

0.700

0.840

Prep Batch \#...

0.15

0.700

0.861

ug

$\begin{array}{lllll}98 & & \text { ASTM D6784-02 } & 05 / 09-05 / 10 / 07 & \text { JWAFW1AC } \\ 101 & 2.5 & \text { ASTM D6784-02 } & 05 / 09-05 / 10 / 07 & \text { JWAFWIAD }\end{array}$

$\begin{array}{llllll}98 & & \text { ASTM } & \text { D6784-02 } & 05 / 09-05 / 10 / 07 & \text { JWAFW1AC } \\ 101 & 2.5 & \text { ASTM D6784-02 } & 05 / 09-05 / 10 / 07 & \text { JWAFW1AD }\end{array}$

Dilution Factor: 0.7

Analysis Time..: 08:38

NOTE $(S):$

Calculations are performed before rounding to avoid round-off errors in calculated results. 
MATRIX SPIKE SAMPLE EVALUATION REPORT

TOTAL Metals

Client Lot \#...: H7E040218

Date Sampled...: 04/26/07
Matrix........ AIR

Date Received. .: 05/01/07

RPD

RPD LIMITS METHOD

$\begin{array}{llll}\text { PERCENT } & \text { RECOVERY } \\ \text { RECOVERY } & \text { LIMITS }\end{array}$ RPD LIMITS METHOD

MS Lot-Sample \#: H7E040218-004 Prep Batch \#...: 7127427

$\begin{array}{llll}\text { Mercury } & 102 & (80-120) & \text { ASTM D6784-02 }\end{array}$

$95(80-120) 3.1(0-20)$ ASTM D6784-02

Dilution Factor: 1.41

Analysis Time. : 09:03
PREPARATION - WORK ANALYSIS DATE ORDER \#

05/09-05/10/07 JWAFR1AC 05/09-05/10/07 JWAFRIAD

NOTE (S) :

Calculations are performed before rounding to avoid round-off errors in calculated results. 


\section{MATRIX SPIKE SAMPLE DATA REPORT}

TOTAL Metals

Client Lot \#...: H7E040218

Date Sampled...: 04/26/07
Matrix........ AIR

Date Received..: 05/01/07

PERCNT

RECVRY RPD METHOD
PREPARATION- WORK ANALYSIS DATE ORDER \#

PARAMETER AMOUNT AMT AMOUNT UNITS

Prep Batch \#...: 7127427 MS Lot-S
Mercury

$\begin{array}{rrrrr}1.8 & 1.41 & 3.26 & \text { ug } & 102 \\ 1.8 & 1.41 & 3.16 \text { ug } & 95 \\ & & \text { Dilution Factor: } 1.41 \\ & & \text { Analysis Time. : } & 09: 03\end{array}$

102

Analysis Time... 09:03 3.1 ASTM D6784-02 05/09-05/10/07 JWAFRIAD

NOTE $(S)$ :

Calculations are performed before rounding to avoid round-off errors in calculated resulls. 
MATRIX SPIKE SAMPLE EVALUATION REPORT

TOTAL Metals

Client Lot \#...: H7E040218

Date Sampled...: 04/26/07
Date Received..: 05/01/07

$\begin{array}{llll}\text { PERCENT } & \text { RECOVERY } & \text { RPD } & \\ \text { RECOVERY } & \text { LIMITS }\end{array}$

Matrix........ AIR

PREPARATION- WORK ANALYSIS DATE ORDER \#

05/09-05/10/07 JWAFX1AC 05/09-05/10/07 JWAFX1AD

MS Lot-Sample \#: H7E040218-006 Prep Batch \#...: 7127428

Mercury

NC, MSB $(80-120)$

ASTM D6784-02

ASTM D6784-02

05/09-05/10/07 JWAFX1AD

Analysis Time..: 09:34

NOTE (S) :

Calculations are performed before rounding to avoid round-off errors in calculated results.

NC The recovery and/or RPD were not calculated.

MSB The recovery and RPD were not calculated because the sample amount was greater than four times the spike amount. 
MATRIX SPIKE SAMPLE DATA REPORT

TOTAL Metals

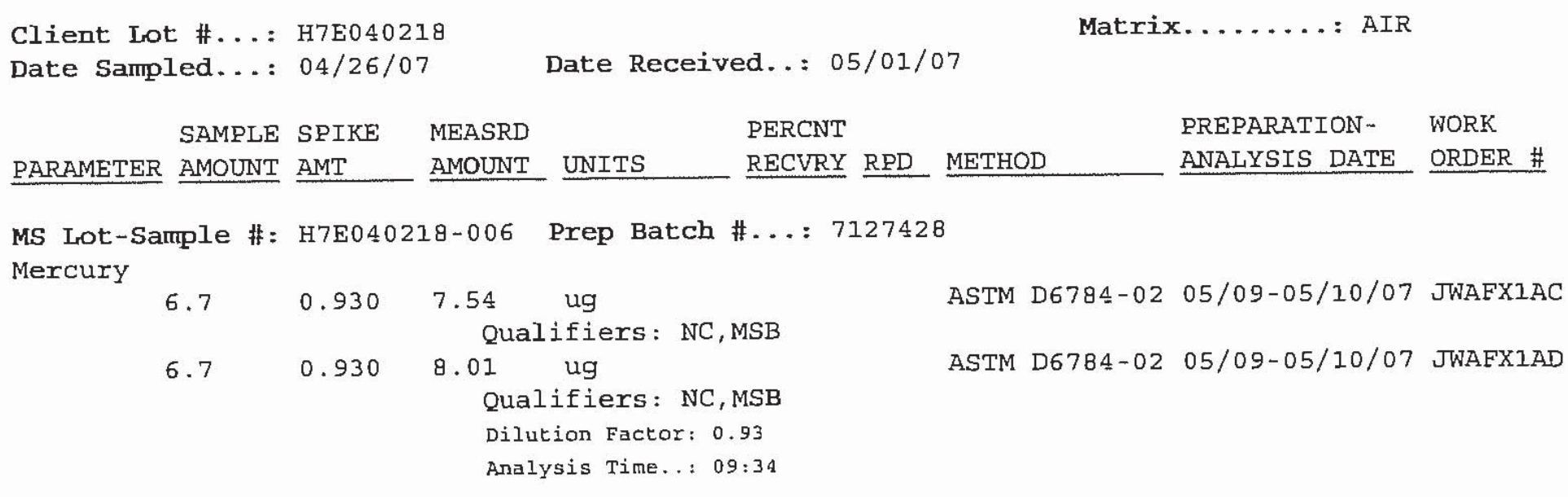

\section{NOTE (S) :}

Calculations are performed before rounding to avoid round-off errors in calculated results.

NC The recovery and/or RPD were not calculated.

MSB The tecovery and RPD were not calcuated because the sample amount was greater dian four times ule spike amoumt. 


\section{STL Knoxville}

\section{Mercury Data Reporting Form}

Post Digestion Spike

Units: ug/L (ppb)

Instrument ID: Leeman HydraAA. Hg

Data File Name: M051707. PRN

\begin{tabular}{|c|c|c|c|c|}
\hline Element & $\begin{array}{c}\text { PDS } \\
\text { JWAGKA }\end{array}$ & $\begin{array}{c}\text { Original Sample } \\
\text { JWAGK }\end{array}$ & Spike Added & $\begin{array}{c}\text { Percent } \\
\text { Recovery }\end{array}$ \\
\hline $\mathrm{Hg}$ & 1.56 & 0.52 & 1.0 & 104 \\
\hline
\end{tabular}




\section{Quality Control Results}

Mercury 


\section{STL Knoxville}

Mercury Data Reporting Form

Initial Calibration Verification

Units: ug/L (ppb)

Instrument ID: Leeman HydraAA Hg An

Data File Name: M051007.PRN

\begin{tabular}{|c|c|c|c|c|c|c|c|c|c|c|c|c|c|}
\hline \multirow[b]{2}{*}{ Elem } & \multirow[b]{2}{*}{$\begin{array}{l}\text { True } \\
\text { Conc }\end{array}$} & \multicolumn{2}{|c|}{$\begin{array}{c}\text { Ck2icv } \\
\text { 5/10/2007 } \\
\text { 8:10 AM }\end{array}$} & & \multirow[b]{2}{*}{$\begin{array}{l}\% \\
\operatorname{Rec}\end{array}$} & \multirow{2}{*}{\multicolumn{2}{|c|}{\begin{tabular}{c|}
$\%$ \\
$\operatorname{Rec}$ \\
\end{tabular}}} & \multirow[b]{2}{*}{ Found } & \multirow[b]{2}{*}{$\begin{array}{l}\% \\
\mathrm{Rec}\end{array}$} & \multirow[b]{2}{*}{ Found } & \multirow[b]{2}{*}{$\begin{array}{l}\% \\
\operatorname{Rec}\end{array}$} & \multirow[b]{2}{*}{ Found } & \multirow[b]{2}{*}{$\begin{array}{l}\% \\
\text { Rec }\end{array}$} \\
\hline & & Found & $\begin{array}{c}\% \\
\operatorname{Rec} \\
\end{array}$ & Found & & & & & & & & & \\
\hline $\mathrm{Hg}$ & 2.5 & 2.53 & 101.2 & & & & & & & & & & \\
\hline
\end{tabular}




\section{STL Knoxville}

\section{Mercury Data Reporting Form}

Continuing Calibration Verification

Units: $u g / L(p p b)$

Instrument ID: Leeman HydraAA Hg An

Data File Name: M051007.PRN

\begin{tabular}{|c|c|c|c|c|c|c|c|c|c|c|c|c|c|}
\hline \multirow[b]{2}{*}{ Elem } & \multirow[b]{2}{*}{$\begin{array}{l}\text { True } \\
\text { Conc }\end{array}$} & \multicolumn{2}{|c|}{$\begin{array}{c}\text { Ck3ccv } \\
5 / 10 / 2007 \\
8: 16 \mathrm{AM} \\
\end{array}$} & \multicolumn{2}{|c|}{$\begin{array}{c}\text { Ck3ccv } \\
5 / 10 / 2007 \\
8: 42 \mathrm{AM}\end{array}$} & \multicolumn{2}{|c|}{$\begin{array}{c}\mathrm{Ck} 3 \mathrm{ccv} \\
5 / 10 / 2007 \\
9: 05 \mathrm{AM} \\
\end{array}$} & \multicolumn{2}{|c|}{$\begin{array}{c}\text { Ck3ccv } \\
5 / 10 / 2007 \\
9: 30 \mathrm{AM}\end{array}$} & \multicolumn{2}{|c|}{$\begin{array}{c}\text { Ck3ccv } \\
5 / 10 / 2007 \\
9: 52 \text { AM } \\
\end{array}$} & \multicolumn{2}{|c|}{$\begin{array}{c}\text { Ck3ccv } \\
5 / 10 / 2007 \\
10: 18 \mathrm{AM}\end{array}$} \\
\hline & & Found & $\begin{array}{l}\% \\
\operatorname{Rec}\end{array}$ & Found & $\begin{array}{l}\% \\
\operatorname{Rec}\end{array}$ & Found & $\begin{array}{c}\% \\
\operatorname{Rec}\end{array}$ & Found & $\begin{array}{c}\% \\
\operatorname{Rec}\end{array}$ & Found & $\begin{array}{c}\% \\
\operatorname{Rec} \\
\end{array}$ & Found & $\begin{array}{c}\% \\
\operatorname{Rec}\end{array}$ \\
\hline $\mathrm{Hg}$ & 5.0 & 5.32 & 106.4 & 5.37 & 107.4 & 5.30 & 106.0 & 5.23 & 104.6 & 5.25 & 105.0 & 5.32 & 106.4 \\
\hline
\end{tabular}




\section{STL Knoxville}

\section{Mercury Data Reporting Form}

Continuing Calibration Verification 


\section{STL Knoxville}

\section{Mercury Data Reporting Form}

Contract Required Detection Limit Standard(s)

Units: $\mathrm{ug} / \mathrm{L}(\mathrm{ppb})$

Instrument ID: Leeman HydraAA Hg An

Data File Name: M051007.PRN

\begin{tabular}{|c|c|c|c|c|c|c|c|c|c|c|c|c|c|}
\hline \multirow[b]{2}{*}{ Elem } & \multirow[b]{2}{*}{$\begin{array}{l}\text { True } \\
\text { Conc }\end{array}$} & \multicolumn{2}{|c|}{$\begin{array}{c}\text { CRA } \\
5 / 10 / 2007 \\
8: 15 \mathrm{AM} \\
\end{array}$} & \multirow[b]{2}{*}{ Found } & \multirow[b]{2}{*}{$\begin{array}{c}\% \\
\operatorname{Rec}\end{array}$} & \multirow[b]{2}{*}{ Found } & \multirow[b]{2}{*}{$\begin{array}{c}\% \\
\operatorname{Rec}\end{array}$} & \multirow[b]{2}{*}{ Found } & \multirow[b]{2}{*}{$\begin{array}{l}\% \\
\operatorname{Rec}\end{array}$} & \multirow[b]{2}{*}{ Found } & \multirow[b]{2}{*}{$\begin{array}{l}\% \\
\operatorname{Rec}\end{array}$} & \multirow[b]{2}{*}{ Found } & \multirow[b]{2}{*}{$\begin{array}{l}\% \\
\operatorname{Rec} \\
\end{array}$} \\
\hline & & Found & $\begin{array}{c}\% \\
\mathrm{Rec} \\
\end{array}$ & & & & & & & & & & \\
\hline $\mathrm{Hg}$ & 0.2 & 0.21 & 105.5 & & & & & & & & & & \\
\hline
\end{tabular}




\section{STL Knoxville}

\section{Mercury Data Reporting Form}

Initial Calibration Blank(s)
Units: $u g / L(p p b)$

Instrument ID: Leeman HydraAA $\mathrm{Hg}$ An

Data File Name: M051007.PRN

\begin{tabular}{|c|c|c|c|c|c|c|c|c|c|c|c|c|c|}
\hline \multirow[b]{2}{*}{ Elem } & \multirow[b]{2}{*}{$\begin{array}{c}\text { Reporting } \\
\text { Limit }\end{array}$} & \multicolumn{2}{|c|}{$\begin{array}{c}\text { ICB } \\
5 / 10 / 2007 \\
8: 13 \mathrm{AM}\end{array}$} & \multirow[b]{2}{*}{ Found } & \multirow[b]{2}{*}{ Flag } & \multirow[b]{2}{*}{ Found } & \multirow[b]{2}{*}{ Flag } & \multirow[b]{2}{*}{ Found } & \multirow[b]{2}{*}{ Flag } & \multirow[b]{2}{*}{ Found } & \multirow[b]{2}{*}{ Flag } & \multirow[b]{2}{*}{ Found } & \multirow[b]{2}{*}{ Flas } \\
\hline & & Found & Flag & & & & & & & & & & \\
\hline$\overline{\mathrm{Hg}}$ & 0.1 & 0.06 & $\mathrm{U}$ & & & & & & & & & & \\
\hline
\end{tabular}




\section{STL Knoxville}

\section{Mercury Data Reporting Form}

Continuing Calibration Blank(s)
Units: ug/L (ppb)

Instrument ID: Leeman HydraAA Hg Ana

Data File Name: M051007.PRN

\begin{tabular}{|c|c|c|c|c|c|c|c|c|c|c|c|c|c|}
\hline \multirow[b]{2}{*}{ Elcm } & \multirow[b]{2}{*}{$\begin{array}{c}\text { Reporting } \\
\text { Limil }\end{array}$} & \multicolumn{2}{|c|}{$\begin{array}{l}\text { Cklccb } \\
5 / 11 / 2(1) 7 \\
5: 18 \text { AM }\end{array}$} & \multicolumn{2}{|c|}{$\begin{array}{c}\text { Cklccb } \\
5 / 10 / 20107 \\
8:++\mathrm{AM}\end{array}$} & \multicolumn{2}{|c|}{$\begin{array}{c}\text { Ckiccb } \\
5 / 1(1 / 20)(1) \\
9: 07 \mathrm{AM}\end{array}$} & \multicolumn{2}{|c|}{$\begin{array}{c}\text { Cklccb } \\
5 / 10 / 2007 \\
1): 32 \mathrm{AM}\end{array}$} & \multicolumn{2}{|c|}{$\begin{array}{c}\mathrm{Ck} 1 \mathrm{ccb} \\
5 / 10 / 20(07 \\
9: 5+\mathrm{AM}\end{array}$} & \multicolumn{2}{|c|}{$\begin{array}{c}\text { Cklccb } \\
5 / 10 / 2(1) 7 \\
10: 21 \mathrm{AM}\end{array}$} \\
\hline & & Found & Fling & Found & Flag & Found & Flag & Found & Flag & Found & Flag & Found & Flag \\
\hline $\mathrm{Hg}$ & 0.1 & 0,166 & $\mathrm{U}$ & 0.06 & $\mathrm{U}$ & 0.06 & $\mathrm{U}$ & 0.06 & U & 0.06 & $\mathrm{U}$ & 0.06 & $\mathrm{U}$ \\
\hline
\end{tabular}




\section{STL Knoxville}

\section{Mercury Data Reporting Form}

\section{Continuing Calibration Blank(s)}

Units: $u g / L(p p b)$

Instrument ID: Leeman HydraAA Hg An

Data File Name: M051007.PRN

\begin{tabular}{|c|c|c|c|c|c|c|c|c|c|c|c|c|c|}
\hline \multirow[b]{2}{*}{ Elem } & \multirow{2}{*}{$\begin{array}{l}\text { Reporting } \\
\text { Limit }\end{array}$} & \multicolumn{2}{|c|}{$\begin{array}{c}\text { Cklccb } \\
5 / 10 / 2007 \\
10: 45 \mathrm{AM}\end{array}$} & \multicolumn{2}{|c|}{$\begin{array}{c}\text { Ck1ccb } \\
5 / 10 / 2007 \\
11: 12 \mathrm{AM}\end{array}$} & \multicolumn{2}{|c|}{$\begin{array}{c}\text { Cklccb } \\
5 / 10 / 2007 \\
11: 22 \mathrm{AM}\end{array}$} & \multirow[b]{2}{*}{ Found } & \multirow[b]{2}{*}{ Flag } & \multirow[b]{2}{*}{ Found } & \multirow[b]{2}{*}{ Flag } & \multirow[b]{2}{*}{ Found } & \multirow[b]{2}{*}{ Flag } \\
\hline & & Found & Flag & Found & Flag & Found & Flag & & & & & & \\
\hline $\mathrm{Hg}$ & 0.1 & 0.06 & $\mathrm{U}$ & 0.06 & $\bar{U}$ & 0.06 & $\bar{U}$ & & & & & & \\
\hline
\end{tabular}




\section{STL Knoxville}

\section{Mercury Data Reporting Form}

Instrument Detection Limits

IDL Completion Date:12/14/2006
Units: ug/L (ppb)

Instrument ID: Leeman HydraAA Hg An

Data File Name: M051007.PRN

\begin{tabular}{|c|c|c|c|}
\hline Element & $\begin{array}{c}\text { Wavelength } \\
(\mathbf{n m})\end{array}$ & $\begin{array}{c}\text { Reporting } \\
\text { Limit }\end{array}$ & IDL \\
\hline $\mathrm{Hg}$ & 253.70 & 0.1 & 0.06 \\
\hline
\end{tabular}




\section{STL Knoxville}

\section{Sample ID Nomenclature}

The sample ID consists of 5 alpha-numeric characters followed by a suffix in the 6 th position that designates the sample type:

\begin{tabular}{cll} 
Suffix & & \multicolumn{1}{c}{ Sample Type: } \\
\cline { 1 - 1 } B & & Method Blank \\
C & & Laboratory Control Sample \\
L & & Laboratory Control Sample Duplicate \\
S & & Matrix Spike \\
D & & Matrix Spike Duplicate \\
X & & Sample Duplicate \\
P & & Serial Dilution \\
A & Post Digestion Spike \\
Z\# & Dilution; \# = Dilution Factor
\end{tabular}




\section{STL Knoxville}

\section{Mercury Data Reporting Form}

Initial Calibration Verification
Units: $u g / L(p p b)$

Instrument ID: Leeman HydraAA Hg An

Data File Name: M051707.PRN

\begin{tabular}{|c|c|c|c|c|c|c|c|c|c|c|c|c|c|}
\hline \multirow[b]{2}{*}{ Elem } & \multirow[b]{2}{*}{$\begin{array}{l}\text { True } \\
\text { Conc }\end{array}$} & \multicolumn{2}{|c|}{$\begin{array}{c}\text { Ck2icv } \\
\text { 5/17/2007 } \\
\text { 8:19 AM }\end{array}$} & & & & & & & \multirow[b]{2}{*}{ Found } & \multirow[b]{2}{*}{$\begin{array}{c}\% \\
\operatorname{Rec}\end{array}$} & \multirow[b]{2}{*}{ Found } & \multirow[b]{2}{*}{$\begin{array}{l}\% \\
\text { Rec }\end{array}$} \\
\hline & & Found & $\begin{array}{l}\% \\
\text { Rec }\end{array}$ & Found & $\begin{array}{l}\% \\
\operatorname{Rec}\end{array}$ & Found & $\begin{array}{l}\% \\
\operatorname{Rec}\end{array}$ & Found & $\begin{array}{l}\% \\
\operatorname{Rec}\end{array}$ & & & & \\
\hline $\mathrm{Hg}$ & 2.5 & 2.54 & 101.6 & & & & & & & & & & \\
\hline
\end{tabular}




\section{STL Knoxville}

\section{Mercury Data Reporting Form}

Continuing Calibration Verification
Units: ug/L (ppb)

Instrument ID: Leeman HydraAA Hg An

Data File Name: M051707.PRN

\begin{tabular}{|c|c|c|c|c|c|c|c|c|c|c|c|c|c|}
\hline \multirow[b]{2}{*}{ Elem } & \multirow[b]{2}{*}{$\begin{array}{l}\text { True } \\
\text { Conc }\end{array}$} & \multicolumn{2}{|c|}{$\begin{array}{c}\text { Ck3ccv } \\
5 / 17 / 2007 \\
8: 25 \mathrm{AM}\end{array}$} & \multicolumn{2}{|c|}{$\begin{array}{c}\mathrm{Ck} 3 \mathrm{ccv} \\
5 / 17 / 2007 \\
8: 49 \mathrm{AM} \\
\end{array}$} & \multicolumn{2}{|c|}{$\begin{array}{c}\text { Ck3ccv } \\
5 / 17 / 2007 \\
9: 13 \mathrm{AM}\end{array}$} & \multicolumn{2}{|c|}{$\begin{array}{c}\text { Ck3ccv } \\
5 / 17 / 2007 \\
9: 39 \mathrm{AM} \\
\end{array}$} & \multicolumn{2}{|c|}{$\begin{array}{c}\text { Ck3ccv } \\
5 / 17 / 2007 \\
10: 07 \mathrm{AM} \\
\end{array}$} & & \\
\hline & & Found & $\begin{array}{c}\% \\
\operatorname{Rec}\end{array}$ & Found & $\begin{array}{c}\% \\
\operatorname{Rec}\end{array}$ & Found & $\begin{array}{c}\% \\
\operatorname{Rec}\end{array}$ & Found & $\begin{array}{c}\% \\
\text { Rec }\end{array}$ & Found & $\begin{array}{c}\% \\
\text { Rec }\end{array}$ & Found & $\begin{array}{c}\% \\
\text { Rec }\end{array}$ \\
\hline $\mathrm{Hg}$ & 5.0 & 5.06 & 101.2 & 5.24 & 104.8 & 5.16 & 103.2 & 5.26 & 105.2 & 5.23 & 104.6 & & \\
\hline
\end{tabular}


STL Knoxville

Mercury Data Reporting Form

Contract Required Detection Limit Standard(s)

Units: $u g / L(p p b)$

Instrument ID: Leeman HydraAA Hg An

Data File Name: M051707.PRN

\begin{tabular}{|c|c|c|c|c|c|c|c|c|c|c|c|c|c|}
\hline \multirow[b]{2}{*}{ Elem } & \multirow[b]{2}{*}{$\begin{array}{l}\text { True } \\
\text { Conc }\end{array}$} & \multicolumn{2}{|c|}{$\begin{array}{c}\text { CRA } \\
5 / 17 / 2007 \\
8: 23 \mathrm{AM} \\
\end{array}$} & \multirow[b]{2}{*}{ Found } & \multirow[b]{2}{*}{$\begin{array}{c}\% \\
\operatorname{Rec}\end{array}$} & \multirow[b]{2}{*}{ Found } & \multirow[b]{2}{*}{$\begin{array}{l}\% \\
\operatorname{Rec}\end{array}$} & \multirow[b]{2}{*}{ Found } & \multirow[b]{2}{*}{$\begin{array}{l}\% \\
\operatorname{Rec}\end{array}$} & \multirow[b]{2}{*}{ Found } & \multirow[b]{2}{*}{$\begin{array}{c}\% \\
\operatorname{Rec} \\
\end{array}$} & \multirow[b]{2}{*}{ Found } & \multirow[b]{2}{*}{$\begin{array}{l}\% \\
\operatorname{Re}\end{array}$} \\
\hline & & Found & $\begin{array}{c}\% \\
\operatorname{Rec}\end{array}$ & & & & & & & & & & \\
\hline $\mathrm{Hg}$ & 0.2 & 0.20 & 100.0 & & & & & & & & & & \\
\hline
\end{tabular}




\section{STL Knoxville}

\section{Mercury Data Reporting Form}

Initial Calibration Blank(s)

Units: $u g / L(p p b)$

Instrument ID: Leeman HydraAA Hg An

Data File Name: M051707.PRN

\begin{tabular}{|c|c|c|c|c|c|c|c|c|c|c|c|c|c|}
\hline \multirow[b]{2}{*}{ Elem } & \multirow[b]{2}{*}{$\begin{array}{c}\text { Reporting } \\
\text { Limit }\end{array}$} & \multicolumn{2}{|c|}{$\begin{array}{c}\text { ICB } \\
5 / 17 / 2007 \\
8: 21 \mathrm{AM} \\
\end{array}$} & \multirow[b]{2}{*}{ Found } & \multirow[b]{2}{*}{ Flag } & \multirow[b]{2}{*}{ Found } & \multirow[b]{2}{*}{ Flag } & \multirow[b]{2}{*}{ Found } & \multirow[b]{2}{*}{ Flag } & \multirow[b]{2}{*}{ Found } & \multirow[b]{2}{*}{ Flag } & \multirow[b]{2}{*}{ Found } & \multirow[b]{2}{*}{ Flag } \\
\hline & & Found & Flag & & & & & & & & & & \\
\hline $\mathrm{Hg}$ & 0.1 & 0.06 & $U$ & & & & & & & & & & \\
\hline
\end{tabular}




\section{STL Knoxville}

\section{Mercury Data Reporting Form}

Continuing Calibration Blank(s)
Units: $u g / L(p p b)$

Instrument ID: Leeman HydraAA Hg Ana

Data File Name: M051707.PRN

\begin{tabular}{|c|c|c|c|c|c|c|c|c|c|c|c|c|c|}
\hline \multirow[b]{2}{*}{ Elcm } & \multirow{2}{*}{$\begin{array}{l}\text { Reporling } \\
\text { Limil } \\
\end{array}$} & \multicolumn{2}{|c|}{$\begin{array}{l}\text { Cklccb } \\
5 / 17 / 2007 \\
8: 27 \mathrm{AM}\end{array}$} & \multicolumn{2}{|c|}{$\begin{array}{c}\text { Cklccb } \\
5 / 17 / 2(107 \\
8: 51 \mathrm{AM}\end{array}$} & \multicolumn{2}{|c|}{$\begin{array}{c}\text { Cklccb } \\
5 / 17 / 2007 \\
9: 15 \mathrm{AM}\end{array}$} & \multicolumn{2}{|c|}{$\begin{array}{c}\text { Cklccb } \\
5 / 17 / 2007 \\
9:+1 \mathrm{AM}\end{array}$} & \multicolumn{2}{|c|}{$\begin{array}{c}\text { Cklccb } \\
5 / 17 / 2007 \\
10:(09) \mathrm{AM}\end{array}$} & \multirow[b]{2}{*}{ Found } & \multirow[b]{2}{*}{ Flag } \\
\hline & & Found & Flag & Found & Flag & Found & Flag & Found & Fliag & Found & Flag & & \\
\hline $\mathrm{Hg}$ & 0.1 & 0.06 & $\mathrm{U}$ & 0.06 & $\mathrm{U}$ & 0.06 & $\mathrm{U}$ & 0.06 & U & 0.06 & $\mathrm{U}$ & & \\
\hline
\end{tabular}




\section{STL Knoxville}

\section{Mercury Data Reporting Form}

Post Digestion Spike

Units: $u g / L(p p b)$

Instrument ID: Leeman HydraAA $\mathrm{Hg}$

Data File Name: M051707. PRN

\begin{tabular}{|c|c|c|c|c|}
\hline Element & $\begin{array}{c}\text { PDS } \\
\text { JWAGKA }\end{array}$ & $\begin{array}{c}\text { Original Sample } \\
\text { JWAGK }\end{array}$ & Spike Added & $\begin{array}{c}\text { Percent } \\
\text { Recovery }\end{array}$ \\
\hline \hline $\mathrm{Hg}$ & 1.56 & 0.52 & 1.0 & 104 \\
\hline
\end{tabular}




\section{STL Knoxville}

\section{Mercury Data Reporting Form}

Instrument Detection Limits

IDL Completion Date:12/14/2006
Units: $u g / L(p p b)$

Instrument ID: Leeman HydraAA Hg An

Data File Name: M051707.PRN

\begin{tabular}{|c|c|c|c|}
\hline Element & $\begin{array}{c}\text { Wavelength } \\
(\mathbf{n m})\end{array}$ & $\begin{array}{c}\text { Reporting } \\
\text { Limit }\end{array}$ & IDL \\
\hline $\mathrm{Hg}$ & 253.70 & 0.1 & 0.06 \\
\hline
\end{tabular}




\section{STL Knoxville}

\section{Sample ID Nomenclature}

The sample ID consists of 5 alpha-numeric characters followed by a suffix in the 6th position that designates the sample type:

\begin{tabular}{cll} 
Suffix & & \multicolumn{1}{c}{ Sample Type: } \\
\cline { 1 - 1 } B & & Method Blank \\
C & & Laboratory Control Sample \\
L & & Laboratory Control Sample Duplicate \\
S & & Matrix Spike \\
D & & Matrix Spike Duplicate \\
X & & Sample Duplicate \\
P & Serial Dilution \\
A & Post Digestion Spike \\
Z\# & Dilution; \#= Dilution Factor
\end{tabular}

Mercury Report Generator Version 2.0 
Raw Data Mercury 


\section{STL Knoxville \\ Mercury Analysis Cover Sheet}

Scanned

\begin{tabular}{|r|c|c|l|}
\hline Analyst & Dtw & Instrument & Leeman Hydra AA Mercury Analyzer \\
\hline Run Date & $5 / 10 / 07$ & Chart Name & M05 1007 \\
\hline
\end{tabular}

\begin{tabular}{||l|l|c|l||}
\hline \multicolumn{2}{|l|}{ Daily Maintenance } & \multicolumn{2}{c|}{ As Needed Maintenance } \\
\hline$\square$ & Check tubing. & $\square$ & Adjust/change Hg lamp. \\
\hline \multirow{2}{*}{$\begin{array}{l}\text { Check maintenance schedule } \\
\text { flags. }\end{array}$} & $\square$ & Clean or replace optical cell. \\
\cline { 3 - 4 } & & $\square$ & Lubricate pump and autosampler arm. \\
\cline { 3 - 4 } & $\square$ & Change the drying tube. \\
\hline
\end{tabular}


Instrument Upload

Started Fri May 11 09:10:41 2007 by WILBURND

Run Log - Page 1 :

Data File: UPL\$KNX_DATA_ROOT: $<$ LHG $>$ M051007.PRN; 1

\begin{tabular}{|c|c|c|c|c|c|c|c|}
\hline$\#$ & WorkOrder & Dilution & Date & Time & Batch & Lot & Instrument \\
\hline- & 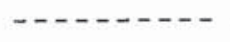 & $\ldots-$ & $-\ldots-$ & $\cdots$ & -- & - - & \\
\hline 1 & CK2ICV & 1.00 & $10-M A Y-2007$ & $08: 10: 56$ & & & HG \\
\hline 2 & $I C B$ & 1.00 & $10-M A Y-2007$ & $08: 13: 07$ & & & HG \\
\hline 3 & CRA. & 1.00 & $10-M A Y-2007$ & $08: 15: 03$ & & & HG \\
\hline 4 & $\mathrm{CK} 3 \mathrm{CCV}$ & 1.00 & $10-M A Y-2007$ & $08: 16: 49$ & & & HG \\
\hline 5 & CKICCB & 1.00 & $10-M A Y-2007$ & $08: 18: 39$ & & & HG \\
\hline 6 & JWE4MBT & 1.00 & $10-M A Y-2007$ & $08: 20: 37$ & 7128346 & $\mathrm{H} 7 \mathrm{E} 070000$ & HG \\
\hline 7 & JWHCHCT & 1.00 & $10-M A Y-2007$ & $08: 22: 24$ & 7128346 & $\mathrm{H} 7 \mathrm{E} 080000$ & HG \\
\hline 8 & JV36RT & 1.00 & $10-M A Y-2007$ & $08: 24: 13$ & 7128346 & H7E020140 & HG \\
\hline 9 & JV36RST & 1.00 & $10-M A Y-2007$ & $08: 26: 00$ & 7128346 & H7E020140 & HG \\
\hline 10 & JV36RDT & 1.00 & $10-M A Y-2007$ & $08: 27: 59$ & 7128346 & H7E020140 & HG \\
\hline 11 & JWFFGB & 0.25 & $10-M A Y-2007$ & $08: 30: 38$ & 7127421 & $\mathrm{H} 7 \mathrm{E} 070000$ & HG \\
\hline 12 & JWFFGC & 0.25 & $10-M A Y-2007$ & $08: 34: 38$ & 7127421 & $\mathrm{H} 7 \mathrm{E} 070000$ & HG \\
\hline 13 & JWAFW & 0.70 & $10-M A Y-2007$ & $08: 36: 59$ & 7127421 & $\mathrm{H} 7 \mathrm{E} 040218$ & HG \\
\hline 14 & JWAFWS & 0.70 & $10-M A Y-2007$ & $08: 38: 46$ & 7127421 & $\mathrm{H} 7 \mathrm{E} 040218$ & HG \\
\hline 15 & JWAFWD & 0.70 & $10-M A Y-2007$ & $08: 40: 56$ & 7127421 & $\mathrm{H} 7 \mathrm{E} 040218$ & HG \\
\hline 16 & $\mathrm{CK} 3 \mathrm{CCV}$ & 1.00 & $10-M A Y-2007$ & $08: 42: 43$ & & & HG \\
\hline 17 & CKICCB & 1.00 & $10-M A Y-2007$ & $08: 44: 33$ & & & HG \\
\hline 18 & JWAGP & 0.73 & $10-M A Y-2007$ & $08: 46: 33$ & 7127421 & $\mathrm{H} 7 \mathrm{E} 040218$ & HG \\
\hline 19 & JWAHL & 0.68 & $10-M A Y-2007$ & $08: 48: 29$ & 7127421 & H7E040218 & $\mathrm{HG}$ \\
\hline 20 & JWAJH & 0.68 & $10-M A Y-2007$ & $08: 50: 17$ & 7127421 & $\mathrm{H} 7 \mathrm{E} 040218$ & HG \\
\hline 21 & JWAKK & 0.70 & $10-M A Y-2007$ & $08: 52: 26$ & 7127421 & $\mathrm{H} 7 \mathrm{E} 040218$ & HG \\
\hline 22 & JWAMK & 0.73 & $10-M A Y-2007$ & $08: 54: 13$ & 7127421 & $\mathrm{H} 7 \mathrm{E} 040218$ & HG \\
\hline 23 & JWAND & 0.25 & $10-M A Y-2007$ & $08: 56: 14$ & 7127421 & $\mathrm{H} 7 \mathrm{E} 040218$ & HG \\
\hline 24 & JWFFKB & 0.10 & $10-M A Y-2007$ & $08: 58: 03$ & 7127427 & $\mathrm{H} 7 \mathrm{E} 070000$ & HG \\
\hline 25 & JWFFKC & 0.10 & $10-M A Y-2007$ & $08: 59: 51$ & 7127427 & $\mathrm{H} 7 \mathrm{E} 070000$ & HG \\
\hline 26 & JWAFR & 1.41 & $10-M A Y-2007$ & $09: 01: 42$ & 7127427 & $\mathrm{H} 7 \mathrm{E} 040218$ & HG \\
\hline 27 & JWAFRS & 1.41 & $10-M A Y-2007$ & $09: 03: 31$ & 7127427 & $\mathrm{H} 7 \mathrm{E} 040218$ & HG \\
\hline 28 & CK3CCV & 1.00 & $10-M A Y-2007$ & $09: 05: 20$ & & & HG \\
\hline 29 & CK1CCB & 1.00 & $10-M A Y-2007$ & $09: 07: 16$ & & & HG \\
\hline 30 & JWAFRD & 1.41 & $10-M A Y-2007$ & $09: 09: 23$ & 7127427 & $\mathrm{H} 7 \mathrm{E} 040218$ & HG \\
\hline 31 & JWAGN & 1.41 & $10-M A Y-2007$ & $09: 11: 10$ & 7127427 & H7E0 40218 & HG \\
\hline 32 & JWAHH & 1.41 & $10-\mathrm{MAY}-2007$ & $09: 12: 57$ & 7127427 & H7E0 040218 & HG \\
\hline 33 & JWAJA & 1.06 & $10-\mathrm{MAY}-2007$ & $09: 15: 15$ & 7127427 & H7E0 40218 & HG \\
\hline 34 & JWAKG & 1.19 & $10-M A Y-2007$ & $09: 17: 04$ & 7127427 & H7E0 40218 & HG \\
\hline 35 & JWAMG & 1.11 & $10-M A Y-2007$ & $09: 19: 22$ & 7127427 & H7E0 40218 & HG \\
\hline 36 & JWAM8 & 0.10 & $10-\mathrm{MAY}-2007$ & $09: 21: 29$ & 7127427 & $\mathrm{H} 7 \mathrm{E} 040218$ & HG \\
\hline 37 & JWFFNB & 0.10 & $10-\mathrm{MAY}-2007$ & $09: 23: 17$ & 7127428 & $\mathrm{H} 7 \mathrm{E} 070000$ & HG \\
\hline 38 & JWEFNC & 0.10 & $10-\mathrm{MAY}-2007$ & $09: 25: 30$ & 7127428 & $\mathrm{H} 7 \mathrm{E} 070000$ & HG \\
\hline 39 & JWAFX & 0.93 & $10-M A Y-2007$ & $09: 27: 42$ & 7127428 & $\mathrm{H} 7 \mathrm{E} 040218$ & HG \\
\hline 40 & CK3CCV & 1.00 & 10-MAY-2007 & $09: 30: 13$ & & & HG \\
\hline 41 & CKICCB & 1.00 & 10-MAY-2007 & $09: 32: 13$ & & & HG \\
\hline 42 & JWAFXS & 0.93 & $10-M A Y-2007$ & $09: 34: 10$ & 7127428 & $\mathrm{H} 7 \mathrm{E0} 40218$ & HG \\
\hline 43 & JWAFXD & $0: 93$ & 10-MAY-2007 & $09: 35: 57$ & 7127428 & H7E0 040218 & HG \\
\hline 44 & JWAGR & 1.02 & 10-MAY-2007 & $09: 37: 47$ & 7127428 & H7E 040218 & HG \\
\hline
\end{tabular}


Instrument Upload

Started Fri May 11 09:10:41 2007 by WILBURND

Data File: UPL\$KNX_DATA_ROOT:<LHG $>$ M0 51007.PRN; 1

Run Log - Page 2 :

\begin{tabular}{|c|c|c|c|c|c|c|c|}
\hline \# & WorkOrder & Dilution & Date & Time & Batch & Lot & Instrume \\
\hline-- & 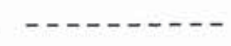 & 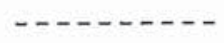 & $--1-1-1$ & $-\ldots-$ & $-\cdots-$ & $\cdots-\cdots$ & \\
\hline 45 & JWAHM & 0.92 & 10-MAY-2007 & $09: 39: 37$ & 7127428 & $\mathrm{H} 7 \mathrm{E} 040218$ & HG \\
\hline 46 & JWAJR & 0.92 & 10-MAY-2007 & $09: 41: 25$ & 7127428 & $\mathrm{H} 7 \mathrm{E} 040218$ & HG \\
\hline 47 & JWAKO & 0.92 & 10-MAY-2007 & $09: 43: 24$ & 7127428 & $\mathrm{H} 7 \mathrm{E} 040218$ & HG \\
\hline 48 & JWAMP & 0.97 & 10-MAY-2007 & $09: 45: 13$ & 7127428 & $\mathrm{H} 7 \mathrm{E} 040218$ & HG \\
\hline 49 & JWANG & 0.11 & 10-MAY-2007 & $09: 47: 21$ & 7127428 & $\mathrm{H} 7 \mathrm{E} 040218$ & HG \\
\hline 50 & JWJG6B & 1.00 & $10-M A Y-2007$ & $09: 49: 08$ & 7129089 & $\mathrm{H} 7 \mathrm{E} 090000$ & HG \\
\hline 51 & JWJG6C & 1.00 & $10-M A Y-2007$ & $09: 51: 00$ & 7129089 & $\mathrm{H} 7 \mathrm{E} 090000$ & HG \\
\hline 52 & $\mathrm{CK} 3 \mathrm{CCV}$ & 1.00 & $10-M A Y-2007$ & $09: 52: 51$ & & & HG \\
\hline 53 & $\mathrm{CK} \perp \mathrm{CCB}$ & 1.00 & $10-M A Y-2007$ & $09: 54: 38$ & & & HG \\
\hline 54 & JV7R5 & 2.10 & $10-M A Y-2007$ & $09: 56: 46$ & 7129089 & $\mathrm{H} 7 \mathrm{E} 030258$ & HG \\
\hline 55 & JV7VF & 2.20 & $10-M A Y-2007$ & $09: 59: 27$ & 7129089 & H7E030258 & HG \\
\hline 56 & JV7VFS & 2.20 & $10-M A Y-2007$ & $10: 01: 28$ & 7129089 & $\mathrm{H} 7 \mathrm{E} 030258$ & HG \\
\hline 57 & JV7VFD & 2.20 & $10-M A Y-2007$ & $10: 03: 47$ & 7129089 & $\mathrm{H} 7 \mathrm{E} 030258$ & HG \\
\hline 58 & JV7VL & 2.10 & $10-M A Y-2007$ & $10: 05: 35$ & 7129089 & $\mathrm{H} 7 \mathrm{E} 030258$ & HG \\
\hline 59 & JV7WG & 2.10 & $10-M A Y-2007$ & $10: 08: 22$ & 7129089 & $\mathrm{H} 7 \mathrm{E} 030258$ & HG \\
\hline 60 & JWFGW & 2.20 & 10-MAY-2007 & $10: 10: 10$ & 7129089 & H7E070168 & HG \\
\hline 61 & JWFGWS & 2.20 & 10-MAY-2007 & $10: 12: 30$ & 7129089 & H7E070168 & HG \\
\hline 62 & JWFGWD & 2.20 & 10-MAY-2007 & $10: 15: 19$ & 7129089 & H7E0 070168 & HG \\
\hline 63 & JWFJD & 2.10 & $10-M A Y-2007$ & $10: 17: 07$ & 7129089 & H7E0 070168 & HG \\
\hline 64 & $\mathrm{CK} 3 \mathrm{CCV}$ & 1.00 & 10-MAY-2007 & $10: 18: 57$ & & & HG \\
\hline 65 & $\mathrm{CK} \perp \mathrm{CCB}$ & 1.00 & $10-M A Y-2007$ & $10: 21: 14$ & & & HG \\
\hline 66 & JWFJ2 & 2.10 & $10-M A Y-2007$ & $10: 23: 01$ & 7129089 & H7E070168 & HG \\
\hline 67 & JWFKG & 2.20 & 10-MAY-2007 & $10: 25: 50$ & 7129089 & H7E070168 & HG \\
\hline 68 & JWJG8B & 0.10 & 10-MAY-2007 & $10: 27: 51$ & 7129090 & $\mathrm{H} 7 \mathrm{E} 090000$ & HG \\
\hline 69 & JWJG8C & 0.10 & 10-MAY-2007 & $10: 29: 42$ & 7129090 & $\mathrm{H} 7 \mathrm{E} 090000$ & HG \\
\hline 70 & JV7TF & 0.74 & 10-MAY-2007 & $10: 31: 38$ & 7129090 & $\mathrm{H} 7 \mathrm{E} 030258$ & HG \\
\hline 71 & JV7VG & 0.77 & 10-MAY-2007 & $10: 33: 49$ & 7129090 & $\mathrm{H} 7 \mathrm{E} 030258$ & HG \\
\hline 72 & JV7VGS & 0.77 & 10-MAY-2007 & $10: 35: 37$ & 7129090 & $\mathrm{H} 7 \mathrm{E} 030258$ & HG \\
\hline 73 & JV7VGD & 0.77 & $10-M A Y-2007$ & $10: 37: 29$ & 7129090 & $\mathrm{H} 7 \mathrm{E} 030258$ & HG \\
\hline 74 & JV7VQ & 0.77 & 10-MAY-2007 & $10: 39: 31$ & 7129090 & $\mathrm{H} 7 \mathrm{E} 030258$ & HG \\
\hline 75 & JV7WH & 0.76 & 10-MAY-2007 & $10: 41: 21$ & 7129090 & $\mathrm{H} 7 \mathrm{E} 030258$ & HG \\
\hline 76 & $\mathrm{CK} 3 \mathrm{CCV}$ & 1.00 & 10-MAY-2007 & $10: 43: 21$ & & & HG \\
\hline 77 & CKICCB & 1.00 & 10-MAY-2007 & $10: 45: 07$ & & & HG \\
\hline 78 & JV880 & 0.27 & 10-MAY -2007 & $10: 46: 54$ & 7129090 & $\mathrm{H} 7 \mathrm{E} 030258$ & HG \\
\hline 79 & JWFG1 & 0.80 & $10-M A Y-2007$ & $10: 48: 46$ & 7129090 & H7E070168 & HG \\
\hline 80 & JWFGIS & 0.80 & 10-MAY-2007 & $10: 50: 45$ & 7129090 & H7E070168 & HG \\
\hline 81 & JWFGID & 0.80 & $10-M A Y-2007$ & $10: 52: 48$ & 7129090 & H7E070168 & HG \\
\hline 82 & JWFHN & 0.20 & 10-MAY-2007 & $10: 54: 39$ & 7129090 & H7E070168 & HG \\
\hline 83 & JWFJE & 0.77 & 10-MAY-2007 & $10: 56: 27$ & 7129090 & H7E070168 & HG \\
\hline 84 & JWFJ4 & 0.77 & 10-MAY-2007 & $10: 58: 28$ & 7129090 & H7E0 70168 & HG \\
\hline 85 & JWFKJ & 0.80 & $10-\mathrm{MAY}-2007$ & $11: 00: 42$ & 7129090 & H7E070168 & HG \\
\hline 86 & JWE4MBT & 1.00 & $10-M A Y-2007$ & $11: 05: 38$ & 7128346 & $\mathrm{H} 7 \mathrm{E} 070000$ & HG \\
\hline 87 & JWFHN & 0.20 & $10-M A Y-2007$ & $11: 07: 30$ & 7129090 & H7E070168 & HG \\
\hline 88 & $\mathrm{CK} 3 \mathrm{CCV}$ & 1.00 & 10-MAY-2007 & $11: 09: 49$ & & & HG \\
\hline
\end{tabular}




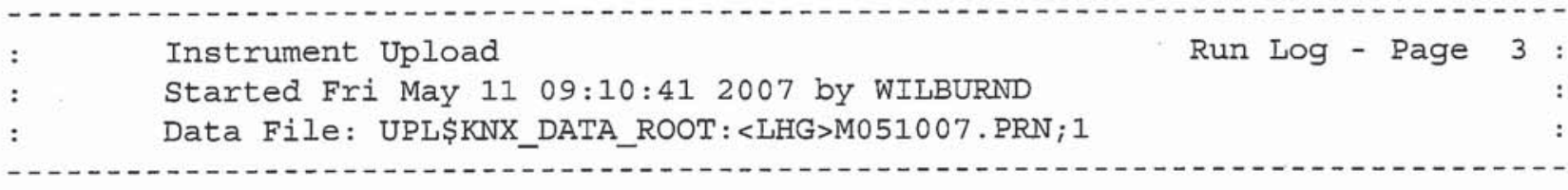

\begin{tabular}{lllllll} 
\# WorkOrder & Dilution & Date & Time & Batch & Lot & Instrument \\
\hline 89 CK1CCB & 1.00 & 10 MAY-2007 & $11: 12: 26$ & & & HG \\
90 JWFG1Z10 & 8.00 & 10 MAY-2007 & $11: 14: 33$ & 7129090 & H7E070168 & HG \\
91 JWFG1SZ10 & 8.00 & 10 MAY-2007 & $11: 16: 21$ & 7129090 & H7E070168 & HG \\
92 JWFG1DZ10 & 8.00 & 10 MAY-2007 & $11: 18: 51$ & 7129090 & H7E070168 & HG \\
93 CK3CCV & 1.00 & 10 MAY-2007 $11: 20: 39$ & & & HG \\
94 CK1CCB & 1.00 & 10 MAY-2007 $11: 22: 37$ & & & HG
\end{tabular}


STL Knoxville

Method Information

Method Name: Ontario-Hydro Method

\begin{tabular}{|c|c|}
\hline Instrument Calibrated Every ? Hours: & 24 \\
\hline Correlation Coefficient $>/=$ : & 0.995 \\
\hline CRA Required?: & Yes \\
\hline Duplicate RPD: & 0 \\
\hline
\end{tabular}

Element: $\mathrm{Hg}$

Chart Number: m051007.prn

$\begin{array}{rr}\text { CRA Recovery Range: } & 30.5 \\ \text { ICV Recovery Range: } & 10.5 \\ \text { CCV Recovery Range: } & 20.5 \\ \text { LCS Recovery Range: } & 20.5 \\ \text { MS/MSD Recovery Range: } & 20.5\end{array}$

Letter to Signify Blanks: B

Letter to Signify LCS's: C

Letter to Signify LCSD's: L

Letter to Signify Dilutions: $\quad Z$

Letter to Signify Duplicates: $\mathrm{X}$

Letter to Signify MS's: S

Letter to Signify MSD's: D

Letters to Signify MSA's: MSA+

Number of Additions: $\quad 3$
Number of Calibration Standards: 6

Run ICV Immediately After Calibration?: Yes

Number of Samples Between CCVs: $\quad 10$

Calculate Duplicates by CLP or SW-846?: SW-846 Protocol

\section{Calibration Standard Concentrations (ug/L)}

$\begin{array}{lllll}\text { Standard } 1 \text { Conc: } 0.1 & \text { Standard } 2 \text { Conc: } 0.2 & \text { Standard } 3 \text { Conc: } 0.5 & \text { Standard } 4 \text { Conc: } 1 \\ \text { Standard } 5 \text { Conc: } 5 & \text { Standard } 6 \text { Conc: } 10 & \text { Standard } 7 \text { Conc: } 0 & \text { Standard } 8 \text { Conc: } 0\end{array}$

ICB/CCB Check Level (ug/L): $0.099 \quad$ Prep Blank Check Level (ug/L): $0.099 \quad$ Reporting Limit (ug/L): 0.1
IDL (ug/L): 0.06
Date of IDL: $12 / 14 / 2006$ 
STL Knoxville Mercury Data Review Checklist

Method: Ontario-Hydro Method

\begin{tabular}{|c|c|c|c|c|c|c|c|}
\hline Analyst: & \multicolumn{2}{|c|}{ DKW } & Instrument: & \multicolumn{3}{c|}{ Leeman Hydra AA Mercury Analyzer } \\
\hline Run Date: & May-10-07 & Calibration Number: & 1 & Chart Name: & m051007.prn & Element: & Hg \\
\hline
\end{tabular}

\begin{tabular}{|c|c|c|c|l|}
\hline A. Calibration/Instrument Run QC & N/A & Yes & No & Comments \\
\hline 1. Instrument calibrated every 24 hours? & & $\mathrm{X}$ & & Calibration was 3 hours and 26 minutes long \\
\hline $\begin{array}{l}\text { 2. Instrument calibrated using a minimum of a blank and 5 } \\
\text { standards? }\end{array}$ & & $\mathrm{X}$ & & \\
\hline $\begin{array}{l}\text { 3. Do standard absorbances increase as standard } \\
\text { concentrations increase? }\end{array}$ & & $\mathrm{X}$ & & \\
\hline 4. Instrument calibrated with standards at the SOP levels? & & $\mathrm{X}$ & & \\
\hline 5. Correlation coefficient greater than or equal to 0.995? & & $\mathrm{X}$ & Correlation coefficient was 0.9999 \\
\hline 6. ICV analyzed directly after calibration? & & $\mathrm{X}$ & & \\
\hline 7. ICV within specified control limits? & & $\mathrm{X}$ & & \\
\hline 8. ICB analyzed immediately after ICV? & & $\mathrm{X}$ & & \\
\hline 9. ICB within acceptable range? & & $\mathrm{X}$ & & \\
\hline 10. CRA analyzed at the beginning of the run? & & \\
\hline 11. CRA results within acceptable range? & & $\mathrm{X}$ & & \\
\hline 12. All CCV's within specified control limits? & & \\
\hline 13. All CCB's within acceptable range? & & $\mathrm{X}$ & & \\
\hline 14. All CCB's analyzed immediately after CCV's? & & $\mathrm{X}$ & & \\
\hline
\end{tabular}

B. Client Sample and QC Sample Results
1.Were all sample results within the linear range of the instrument?
2. Were there any samples with results more negative than Report Limit?
3.Were samples analyzed within the holding time?

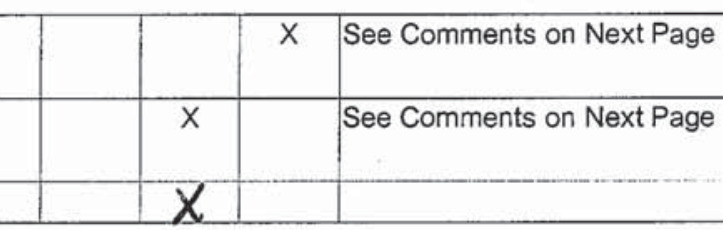

\section{Preparation/Matrix QC}

\begin{tabular}{|l|c|c|c|c|}
\hline 1. Was one method blank prepared for each batch? & & $X$ & & \\
\hline 2. Were all method blanks less than the specified level? & & & $\mathrm{X}$ & See Comments on Next Page \\
\hline 3. Was an LCS prepared for each batch? & & $X$ & & \\
\hline 4. Were all LCS recoveries within QC limits? & & $\mathrm{X}$ & & \\
\hline 5. Were matrix spikes, sample duplicates and/or post- & & $X$ & & \\
digestion spikes run at required frequency? & & & $\mathrm{X}$ & See Comments on Next Page \\
\hline 6. Were all matrix spike recoveries within QC limits? & & & \\
\hline 7. Were post-digestion spike recoveries within QC limits? & $\mathrm{X}$ & & \\
\hline 8. Were duplicate RPDs within QC limits? & & $\mathrm{X}$ & & \\
\hline
\end{tabular}

\section{Other}

1. Current IDL data on file?

2. Current MDL data on file?

3. Are all nonconformances documented appropriately?

4.Were all project specific instructions followed?

Question

Number: Problem(s) Found:

B1 Sample JWFG1S at 5/10/2007 10:50:45 AM exceeded linear range and an acceptable dilution was NOT found.

B1 Sample JWFG1D at 5/10/2007 10:52:48 AM exceeded linear range and an acceptable dilution was NOT found.

B2 Sample JWFHN at 5/10/2007 10:54:39 AM: Original sample is more negative than report limit. Dilute sample and reanalyze. Preveloks sample abave Calbration limit. Reanalyzed

C2 Method Blank JWE4MBT at 5/10/2007 8:20:37 AM had 0.92 ppb contamination. ?

C2 Method Blank JWE4MBT at 5/10/2007 11:05:38 AM had 0.95 ppb contamination. TSBE RE-DIGESTED 
STL Knoxville Mercury Data Review Checklist

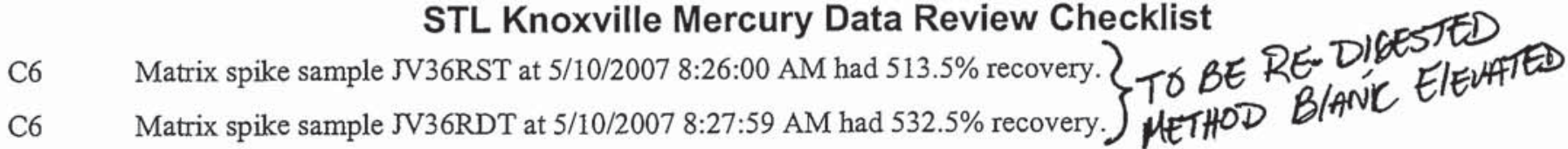

C6 Matrix spike sample JWAFXD at 5/10/2007 9:35:57 AM had 144\% recovery. MSB, NC

C6 Matrix spike sample JWFG1S at 5/10/2007 10:50:45 AM had 69.99\% recovery.

C6 Matrix spike sample JWFG1D at 5/10/2007 10:52:48 AM had 19.99\% recovery.

C6 Matrix spike sample JWFG1SZ10 at 5/10/2007 11:16:21 AM had 5.000\% recovery.

C6 Matrix spike sample JWFG1DZ10 at 5/10/2007 11:18:51 AM had 8.000\% recovery. Analyst: D(te)

Date: $5 / 10 / 07$ 


\section{STL KNOXVILLE}

\section{INTERMEDLATE STANDARD - CALIBRATION}

Stock Std Name: PLHG-2Y

Element: $\mathbf{H g}$

Stock Source: Spex

Stock Std Lot \#: $12 \cdot 168 \mathrm{HG}$

Stock Std Expiration Date: $4 / 30 / 2008$

$\mathrm{HNO}_{3}$ Lot B 44032
Stock Vol (ml)/100ml: $1 \mathrm{ml}$

Date Prepared: $5-1-0$.

Expiration Date: $6-1-0$ n

QC Check: 105020 ?

Initials: D

Matrix: $2 \% \mathrm{HNO}_{3}$

\section{WORKING STANDARD - CALIBRATION}

One ml Intermediate Standard $/ 100 \mathrm{ml} \mathbf{0 . 1 5} \%$ Concentrated Nitric Acid

\begin{tabular}{|c|c|c|c|c|}
\hline 1. I-CAL $3425-10-1$ & Initials DKW & Date: 050107 & Exp. Date: 050207 & HN03 Lot: B44032. \\
\hline 2. I-CAL $3425-10-2$ & Initials DKW & Date: 050707 & Exp. Date: 050807 & HN03 Lot: 844032 \\
\hline 3. I-CAL $3425-10-3$ & Initials $\mu N D$ & Date: $5 / 8107$ & Exp. Date: $5 / 9 / 07$ & HN03 Lot 344032 \\
\hline 4. I-CAL $3425-10-4$ & Initials DKW & Date: 050907 & Exp. Date: $05 / 10 / 0 n$ & HNO3 Lot: 844032 \\
\hline 5. .I-CAL & Initials & Date: & Exp. Date: 1 & HNO3 Lot: \\
\hline 6. .I-CAL & Initials & Date: & Exp. Date: & HNO3 Lot: \\
\hline 7. .I-CAL & Initials & Date: & Exp. Date: & HN03 Lot: \\
\hline 8. .I-CAL_ & Initials & Date: & Exp. Date: & HNO3 Lot: \\
\hline 9. .I-CAL & Initials & Date: & Exp. Date: & HNO3 Lot: \\
\hline 10..I-CAL & Initials & Date: & Exp. Date: & HN03 Lot: \\
\hline 11..I-CAL & Initials & Date: & Exp. Date: & HN03 Lot: \\
\hline 12..I-CAL & Initials & Date: & Exp: Date: & HNO3 Lot: \\
\hline 13. I-CAL & Initials & Date: & Exp. Date: & HNO3 Lot: \\
\hline 14. I-CAL & Initials & Date: & Exp. Date: & HN03 Lot: \\
\hline 15. .I-CAL & Initials & Date: & Exp. Date: & HNO3 Lot: \\
\hline 16. .I-CAL & Initials & Date: & Exp. Date: & HN03 Lot: \\
\hline 17. .I-CAL_ & Initials & Date: & Exp. Date: & HEN03 Lot: \\
\hline 18. .I-CAL & Initials & Date: & Exp. Date: & HNO3 Lot: \\
\hline 19. I-CAL & Initials & Date: & Exp. Date: & HN03 Lot: \\
\hline 20. .I-CAL & Initials & Date: & Exp. Date: & HN03 Lot: \\
\hline . .I-CAL & Initials & Date: & Exp. Date: & HNO3 Lot: \\
\hline 22. .I-CAL & Initials & Date: & Exp. Date: & HN03 Lot: \\
\hline . I-CAL & Initials & Date: & Exp. Date: & HNO3 Lot: \\
\hline .I-CAL & Initials & Date: & Exp. Date: & HN03 Lot: \\
\hline . I-CAL & Initials & Date: & Exp. Date: & HNO3 Lot: \\
\hline
\end{tabular}




\section{STL KNOXVILLE}

\section{INTERMEDIATE STANDARD - VERIFICATION}

Stock Std Name: ICP-080

Element: $\mathrm{Hg}$

Stock Source: Ultra

Stock Std Expiration Date: $28-31-08$

Stock Conc.: $1000 \mathrm{ppm}$

Acid Lot: B44032
Stock Vol (ml)/100ml: $1.0 \mathrm{ml}$

Date:Prepared: $5-1-07$

Expiration Date: $6-1-07$

QC Check: M050207

Initials: DFW

Matrix: $2 \% \mathrm{HNO}_{3}$

\section{WORKING STANDARD - VERIFICATION}

$1 \mathrm{ml}$ Intermediate Standard $/ 100 \mathrm{ml} 0.15 \%$ Concentrated Nitric Acid

1. I-VER $3426-11-1$

2. I-VER $3426-11-2$

3. I-VER $3426-11-3$

4. I-VER

5. I-VER

6. I-VER

7. I-VER

8. I-VER

9. I-VER

10. I-VER

11. I-VER

12. I-VER

13. I-VER

14. I-VER

15. I-VER

16. I-VER

17. I-VER

18. I-VER

19. I-VER

20. I-VER

21. I-VER

22. I-VER

23. I-VER

24. I-VER

25. I-VER
Initials_DKed Initials Torri) Initials DRN Initials Initials Initials Initials Initials Initials Initials Initials Initials Initials Initials Initials Initials Initials Initials Initials Initials Initials Initials Initials Initials Initials
Date: 050107 Exp. Date:054207 HN03 Lot: 344032 Date:050807 Exp. Date:05080 7 HN03 Lot: B44032 Date:050907 Exp. Date:050 07 HN03 Lot 344032 Date: ___Exp. Date: Date: Exp. Date: Date: __ Exp. Date: Date: ___ Exp. Date: Date: ___ Exp. Date: Date:_Exp. Date: Date:__Exp. Date: Date: ___ Exp. Date: Date: ___Exp. Date: HNO3 Lot: HN03 Lot: HN03 Lot: HN03 Lot: HNO3 Lot: HNO3 Lot: HN03 Lot: HN03 Lot: Date:___ Exp. Date: Date: ___ Exp. Date: HN03 Lot: HN03 Lot: Date: __ Exp Date: Date: ___ Exp. Date: Date: ___ Exp. Date: Date: _Exp. Date: Date:__ Exp. Date: Date: ___ Exp. Date: Date: ___Exp. Date: HNO3 Lot: HNO3-Lot: HN03 Lot: HNO3 Lot: HN03 Lot: HN03 Lot: HNO3 Lot: HN03 Lot: Date: __ Exp. Date: Date: Exp. Date: HNO3 Lot: Date: ___Exp. Date: Date: ___Exp. Date: HN03 Lot: HNO3 Lot: HNO3 Lot: 
Protocol: 7ptcurve

Linear
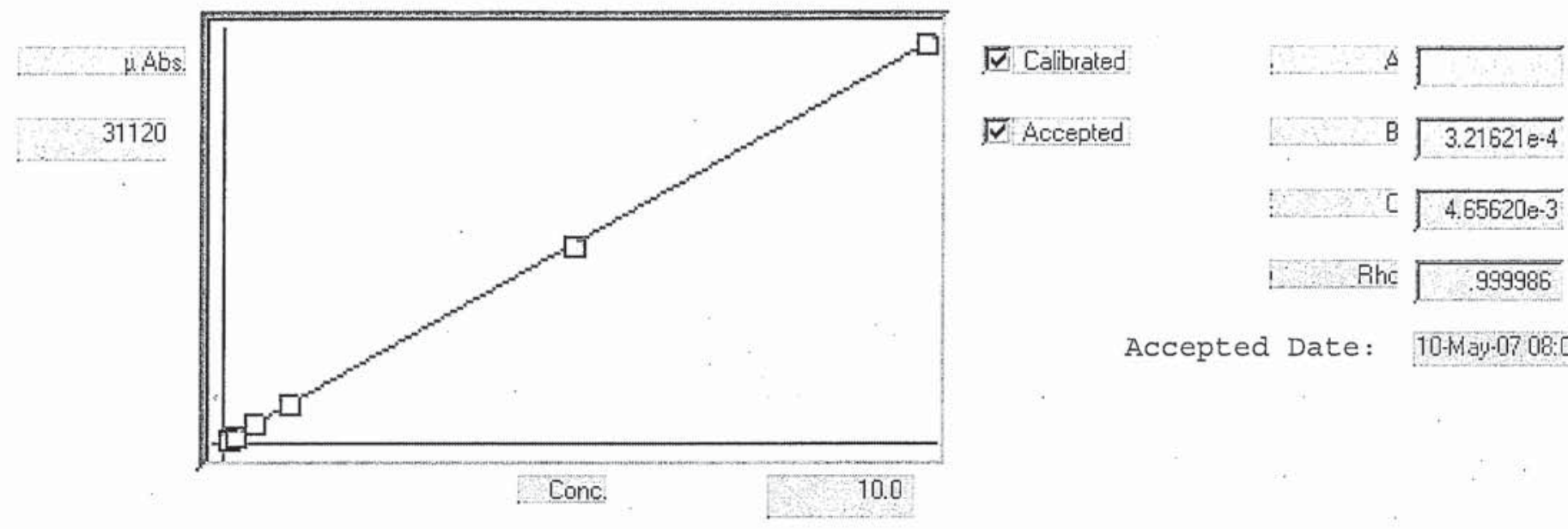

Accepted Date: 10-May-0708:08

S

Mean SD or \%RSD

$\operatorname{Rep} 1 \operatorname{Rep} 2 \operatorname{Rep} 3 \mid \operatorname{Rep} 4 \operatorname{Rep} 5$

Conc.
.00000
.10000
.20000
.50000
1.0000
5.0000
10.000

\begin{tabular}{r|r|r|r} 
Calc. & Dev. & Mean & SD or \%RSD \\
\hline .026 & -.026 & -95 & 1 \\
.105 & .005 & 313 & $0 \%$ \\
.210 & .010 & 639 & $0 \%$ \\
.521 & .021 & 1604 & $0 \%$ \\
1.01 & .008 & 3121 & $0 \%$ \\
4.97 & -.032 & 15433 & $0 \%$ \\
10.0 & .014 & 31120 & $0 \%$ \\
& & &
\end{tabular}

\begin{tabular}{r|r|r|}
-95 & & \\
313 & & \\
638 & & \\
1604 & & \\
3121 & & \\
15433 & & \\
31120 & & \\
& &
\end{tabular}


STL - Knoxville

$07: 56: 08$ 10 May 2007
Folder: M051007

Protocol: 7ptcurve

Line Conc. Units

SD/RSD

1

2

3

4 5

*** Standard: 1 Rep: 1

Seq : 0

$07: 56: 08 \quad 10$ May 07 HG

$\begin{array}{llll}\mathrm{Hg} & .000 \quad \mathrm{ppb} & -95\end{array}$

*** Standard: 2 Rep: 1

Seq : 1

$07: 58: 27 \quad 10$ May 07 HG

Hg $\quad .100 \quad \mathrm{ppb} \quad 313$

*** Standard: 3 Rep: 1

Seq : 2

08:00:17 10 May 07 HG

$\begin{array}{llll}\mathrm{Hg} & .200 \quad \mathrm{ppb} & 638\end{array}$

*** Standard: 4 Rep: 1

Seq : 3

08:02:06 10 May 07 HG

Hg $.500 \quad 1604$

*** Standard: 5 Rep: 1

Seq: $4 \quad 08: 04: 2610$ May 07 HG

Hg $\quad 1.00 \quad \mathrm{ppb} \quad 3121$

*** Standard: 6 Rep: 1

Seq: $508: 06: 2610$ May 07 HG

Hg $\quad 5.00 \quad$ ppb $\quad 15433$

*** Standard: 7 Rep: 1

Seq: $608: 08: 1810$ May 07 HG

Hg $\quad 10.0 \quad \mathrm{ppb} \quad 31120$

*** Check Standard: $2 \mathrm{Ck} 2 \mathrm{icv} \quad \mathrm{Seq}: 7 \quad 08: 10: 5610$ May 07 HG Line Flag \%RCV. Found True Units SD/RSD

Hg $\quad 201.2 .53 \quad 2.50 \quad \mathrm{ppb} \quad .000 \quad \%$

*** Sample ID: ICB Seq: $8 \quad$ 08:13:07 10 May 07 HG Hg $\quad .049 \quad \mathrm{ppb} \quad .000^{\mathrm{ICB}} \% .049$

*** Sample ID: CRA Seq: $9 \quad 08: 15: 03$ 10 May 07 HG Hg $\quad .211 .000 \% \quad .211$

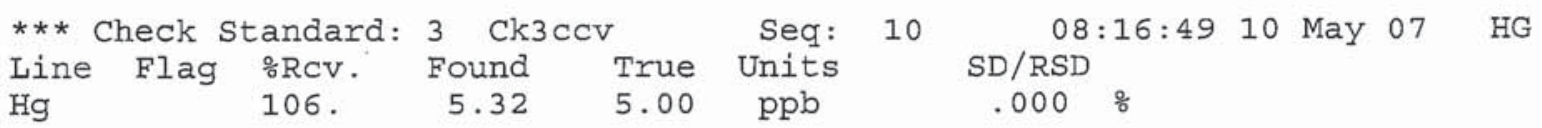

*** Check Standard: 1 Cklccb Seq: 11 08:18:39 10 May 07 HG Line Flag Found Range $(+/-)$ Units SD/RSD

$\begin{array}{lllll}\mathrm{Hg} & -.008 & .059 & \mathrm{ppb} & .000 \quad \%\end{array}$

*** Sample ID: JWE4MBT Seq: $12 \quad$ 08:20:37 10 May 07 HG REANAlyzEHg $.929 \quad \mathrm{ppb} \quad .000^{7128346} .929$

*** Sample ID: JWHCHCT Seq: $13 \quad 08: 22: 2410$ May 07 HG $\mathrm{Hg} \quad 5.55 \quad \mathrm{ppb} \quad .000 \quad \% \quad 5.55$

*** Sample ID: JV36RT Seq: $14 \quad$ 08:24:13 10 May 07 HG $\mathrm{Hg} \quad .005 \quad \mathrm{ppb} \quad .000 \quad \% \quad .005$

*** Sample ID: JV36RST Seq: $15 \quad$ 08:26:00 10 May 07 HG

$\mathrm{Hg} \quad 5.14 \quad \mathrm{ppb} \quad .000 \% 5.14$ 
STL - Knoxville

$08: 27: 5910$ May 2007
Folder: M051007

Protocol: 7ptcurve
Line Conc. Units

*** Sample ID: JV36RDT

SD/RSD

1

Seq : 16

$08: 27: 5910$ May 07 HG

$\begin{array}{lllll}\mathrm{Hg} & 5.33 & \mathrm{ppb} & .000 \quad \frac{\circ}{8} \quad 5.33\end{array}$

*** Sample ID: JWFFGB

Seq : $\quad 17$

$08: 30: 38 \quad 10$ May $07 \quad$ HG

$\begin{array}{lllll}\mathrm{Hg} & .022 & \mathrm{ppb} & .000 \% & 022\end{array}$

*** Sample ID: JWFFGC

Seq: $1808: 34: 3810$ May 07 HG

$\begin{array}{lllll}\mathrm{Hg} & 4.30 \quad \mathrm{ppb} & .000 \quad \% & 4.30\end{array}$

*** Sample ID: JWAFW

Seq: $1908: 36: 5910$ May 07 HG

$\begin{array}{lllll}\mathrm{Hg} & .216 & \mathrm{ppb} & .000 & \%\end{array}$

*** Sample ID: JWAFWS

Seq : $20 \quad 08: 38: 4610$ May 07 HG

$\begin{array}{llll}\mathrm{Hg} & 1.20 \quad \mathrm{ppb} & .000 \% & \% 20\end{array}$

*** Sample ID: JWAFWD

Seq: $21 \quad 08: 40: 5610$ May 07 HG

Hg $\quad 1.23 \quad \mathrm{ppb} \quad .000 \% 1.23$

$\begin{array}{lllllll}* * * \text { Check Standard: } & 3 \mathrm{Ck} 3 \mathrm{CCV} & \text { Seq: } 22 & 08: 42: 43 & 10 \text { May } 07 \text { HG } \\ \text { Line Flag \%RCV. } & \text { Found True Units } & \text { SD/RSD } & & & \end{array}$ Hg $\quad 107 . \quad 5.37 \quad 5.00 \quad \mathrm{ppb} \quad 000 \quad \frac{8}{8}$

*** Check Standard: 1 Cklccb Line Flag Found Range $(+/-)$ Units

$\mathrm{Hg} \quad-.046 \quad .059 \quad \mathrm{ppb}$

Seq: $23 \quad 08: 44: 3310$ May 07 HG $\mathrm{SD} / \mathrm{RSD}$ $.000 \%$

Seq: $24 \quad 08: 46: 3310$ May 07 HG

*** Sample ID: JWAGP

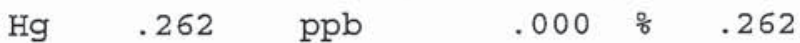

*** Sample ID: JWAHL Seq: $25 \quad 08: 48: 2910$ May 07 HG

$\mathrm{Hg} \quad .169 \quad \mathrm{ppb} \quad .000 \quad \% \quad .169$

*** Sample ID: JWAJH

Seq: $26 \quad 08: 50: 17$ 10 May 07 HG

$\mathrm{Hg} \quad .198 \quad \mathrm{ppb} \quad .000 \quad \% \quad .198$

*** Sample ID: JWAKK S Seq: $27 \quad 08: 52: 2610$ MaY 07 HG Hg $\quad .224 \quad$ ppb $\quad .000 \quad \% \quad .224$

*** Sample ID: JWAMK $\quad$ Seq: $28 \quad 08: 54: 13 \quad 10$ May 07 HG

Hg $\quad .235 \quad 0 \quad \mathrm{ppb} \quad .000 \quad \% \quad .235$

*** Sample ID: JWAND Seq: $29 \quad 08: 56: 1410$ May 07 HG

$\mathrm{Hg} \quad .288 \quad \mathrm{ppb} \quad .000 \quad \% \quad .288$

*** Sample ID: JWFFKB Seq: $30 \quad$ 08:58:03 10 May 07 HG

$\mathrm{Hg} \quad .005 \quad \mathrm{ppb} \quad .000^{7127427} \quad .005$

*** Sample ID: JWFFKC Seq: $31 \quad 08: 59: 51$ 10 May 07 HG 
STL - Knoxville

09:01:42 10 May 2007

Line Conc. Units

*** Sample ID: JWAFR

$\mathrm{Hg} \quad 1.29 \quad \mathrm{ppb}$ .000

*** Sample ID: JWAFRS

$\mathrm{SD} / \mathrm{RSD}$

Folder: M051007

Protocol: 7ptcurve

$\begin{array}{lllll}\mathrm{Hg} & 2.31 & \mathrm{ppb} & .000 \quad \% & 2.31\end{array}$

*** Check Standard: $3 \mathrm{Ck} 3 \mathrm{ccV}$

Line Flag \%RCv. Found True

$\mathrm{Hg}$ 106 .

$5.30 \quad 5.0$

1

2

3

Seq: 32

09:01:42 10 May 07

HG

*** Check Standard: $1 \mathrm{Cklccb}$

Line Flag Found Range $(+/-)$ Units

$\mathrm{Hg}$

$-.008 \quad .059 \quad \mathrm{ppb}$

*** Sample ID: JWAFRD
Seq : 34
Units
$\mathrm{ppb}$

Seq: 33

09:03:31 10 May 07

HG

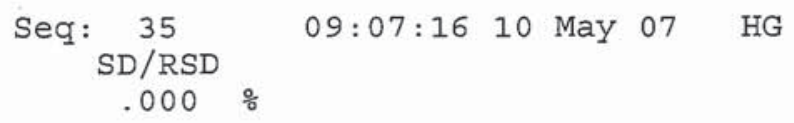

Seq: 36 09:09:23 10 May 07 HG

Hg $\quad 2.24 \quad \mathrm{ppb} \quad .000 \quad \% \quad 2.24$

*** Sample ID: JWAGN Seq: $37 \quad 09: 11: 10.10$ May 07 HG

Hg $\quad 1.29 \quad \mathrm{ppb} \quad .000 \quad \% \quad 1.29$

*** Sample ID: JWAHH Seq: 38 09:12:57 10 May 07 HG

$\begin{array}{lllll}\mathrm{Hg} & 1.18 \quad \mathrm{ppb} & .000 \quad \% & 1.18\end{array}$

*** Sample ID: JWAJA

Seq: $3909: 15: 1510$ May 07 HG

$\begin{array}{llllll} & \mathrm{Hg} & .615 & \mathrm{ppb} & .000 & \circ\end{array}$

*** Sample ID: JWAKG

Seq: $40 \quad 09: 17: 04 \quad 10$ May 07 HG

$\begin{array}{llllll}\mathrm{Hg} & .483 & \mathrm{ppb} & .000 & \% & .483\end{array}$

*** Sample ID: JWAMG

Seq: $4109: 19: 2210$ May 07 HG

$\begin{array}{llllll}\mathrm{Hg} & .650 & \mathrm{ppb} & .000 & \% & .650\end{array}$

$* * *$ Sample ID: JWAM8

Seq: 42 09:21:29 10 May 07 HG

$\begin{array}{llllll}\mathrm{Hg} & .157 & \mathrm{ppb} & .000 & \% & .157\end{array}$

*** Sample ID: JWFFNB

Seq: $43 \quad 09: 23: 17 \quad 10$ May 07 HG

$\mathrm{Hg} \quad-.042 \quad \mathrm{ppb}$ 7127428

Seq: $.000 \quad-.042$

*** Sample ID: JWFFNC

Seq: $44 \quad 09: 25: 30 \quad 10$ May 07 HG

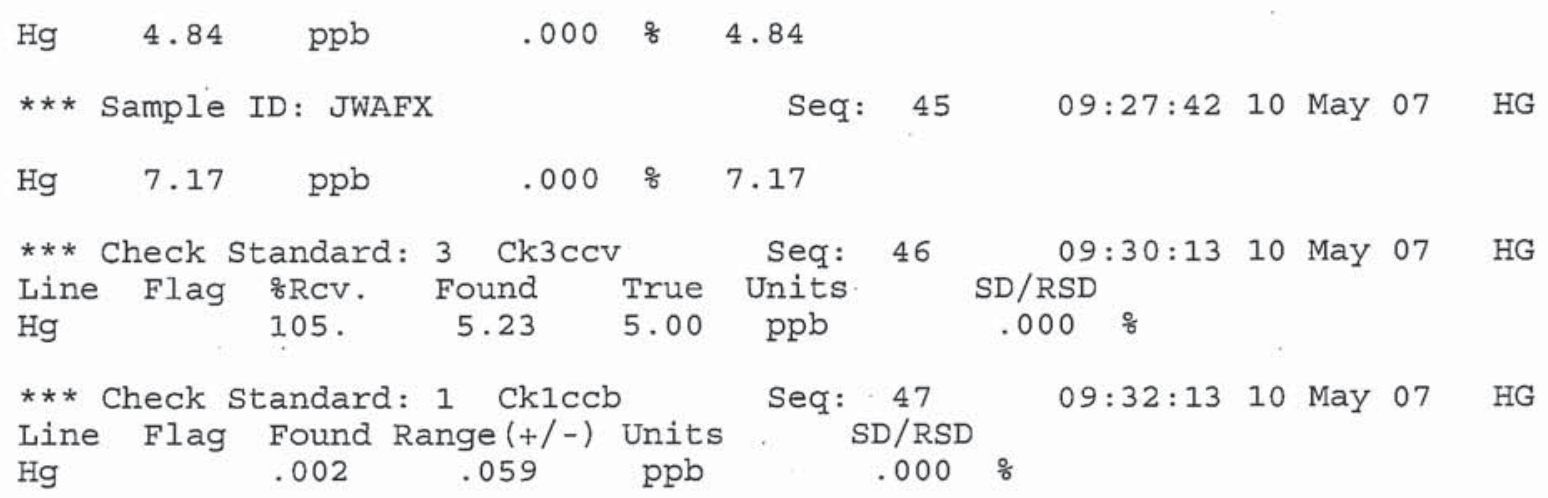


STL - Knoxville

09:34:10 10 May 2007

Line Conc. Units

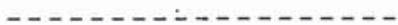

*** Sample ID: JWAFXS

Hg $8.11 \quad \mathrm{ppb}$

*** Sample ID: JWAFXD

$\mathrm{Hg} \quad 8.61 \quad \mathrm{ppb}$

*** Sample ID: JWAGR

Hg $\quad 7.12 \quad \mathrm{ppb}$

*** Sample ID: JWAHM

Hg $\quad 7.24 \quad \mathrm{ppb}$

*** Sample ID: JWAJR

Hg 2.88 $\quad \mathrm{ppb}$

*** Sample ID: JWAKO

$\mathrm{Hg} \quad 3.35 \quad \mathrm{ppb}$

*** Sample ID: JWAMP

Hg $\quad 2.89 \quad \mathrm{ppb}$

*** Sample ID: JWANG

$\mathrm{Hg} \quad .009 \quad \mathrm{ppb}$

*** Sample ID: JWJG6B

$\mathrm{Hg} \quad-.003 \quad \mathrm{ppb}$

*** Sample ID: JWJG6C

Hg $\quad 4.86 \quad \mathrm{ppb}$

*** Check Standard: $3 \mathrm{Ck} 3 \mathrm{ccv}$

Line Flag \%RCV. Found True

$\mathrm{Hg}$

*** Check Standard: 1 Cklccb

Line Flag Found Range(+/-) Units

$\begin{array}{llll}\mathrm{Hg} & -.018 & .059 & \mathrm{ppb}\end{array}$

*** Sample ID: JV7R5

$\mathrm{Hg} \quad .006 \quad \mathrm{ppb}$

*** Sample ID: JV7VF

$\mathrm{Hg} \quad .011 . \mathrm{ppb}$

*** Sample ID: JV7VFS

$\mathrm{Hg} \quad 1.00 \quad \mathrm{ppb}$

*** Sample ID: JV7VFD

Hg $\quad 1.01 \quad \mathrm{ppb}$ 7129089
Folder: M051007

Protocol: 7ptcurve

1

3

4

5

Seq: 48 09:34:10 10 May 07 HG

8.11

Seq: 49 09:35:57 10 May 07 HG $.000 \div 8.61$

Seq: $50 \quad 09: 37: 47 \quad 10$ May 07 HG

$.000 \% 7.12$

Seq: 51 09:39:37 10 May 07 HG $.000 \quad 7.24$

Seq: 52 09:41:25 10 May 07 HG $.000 \% 2.88$

Seq: $53 \quad 09: 43: 24$ 10 May 07 HG $.000 \% 3.35$

Seq : $54 \quad 09: 45: 13$ 10 May 07 HG $.000 \quad 2.89$

Seq: 55 09:47:21 10 May 07 HG $.000 \quad 8 \quad .009$

Seq: 56 09:49:08 10 May 07 HG

Seq: 57 09:51:00 10 May 07 HG 4.86

Seq: $58 \quad 09: 52: 51 \quad 10$ May 07 HG Units SD/RSD

$\mathrm{ppb} \quad .000 \%$

Seq: 59 09:54:38 10 May 07 HG $\mathrm{SD} / \mathrm{RSD}$ $.000 \%$

Seq: $60 \quad 09: 56: 4610$ May 07 HG $.000 \% \quad .006$

Seq: 61 09:59:27 10 May 07 HG $.000 \% \quad .011$

Seq: 62 10:01:28 10 May 07 HG $.000 \% 1.00$

Seq: $63 \quad 10: 03: 47 \quad 10$ May 07 HG 
STL - Knoxville

$10: 05: 3510$ May 2007
Folder: M051007

Protocol: 7ptcurve

Line Conc. Units SD/RSD $\quad 1 \quad 3 \quad 2 \quad 3 \quad 4 \quad 5$

*** Sample ID: JV7VL

Seq : 64

$10: 05: 3510$ May 07 HG

$\begin{array}{llllll}\mathrm{Hg} & -.031 & \mathrm{ppb} & .000 \quad \% & -.031\end{array}$

*** Sample ID: JV7WG

Seq: $65 \quad 10: 08: 22 \quad 10$ May 07 HG

$\begin{array}{llllll}\mathrm{Hg} & .020 & \mathrm{ppb} & .000 \quad \% & .020^{\circ}\end{array}$

*** Sample ID: JWFGW

Seq: $66 \quad 10: 10: 10 \quad 10$ May 07 HG

$\begin{array}{llllll}\mathrm{Hg} & .005 & \mathrm{ppb} & .000 \quad \% & .005\end{array}$

*** Sample ID: JWFGWS Seq: $67 \quad$ 10:12:30 10 May 07 HG

Hg $\quad 1.08 \quad \mathrm{ppb} \quad .000 \quad \% \quad 1.08$

*** Sample ID: JWFGWD Seq: $68 \quad$ 10:15:19 10 May 07 HG

$\mathrm{Hg} \quad 1.09 \quad \mathrm{ppb} \quad .000 \% 1.09$

*** Sample ID: JWFJD Seq: $69 \quad$ 10:17:07 10 May 07 HG

$\mathrm{Hg} \quad-.019 \quad \mathrm{ppb} \quad .000 \quad \% \quad-.019$

*** Check standard: $3 \mathrm{Ck3CCV}$ Seq: $70 \quad 10: 18: 57 \quad 10$ May 07 HG

Line Flag \%RCv. Found True Units SD/RSD

$\mathrm{Hg} \quad 106 . \quad 5.32 \quad 5.00 \quad \mathrm{ppb} \quad .000 \quad \%$

*** Check Standard: 1 Cklccb Seq: 71 10:21:14 10 May 07 HG

Line Flag Found Range $(+/-)$ Units

$\mathrm{Hg}-.034 \quad .059 \quad \mathrm{ppb}$

$\mathrm{SD} / \mathrm{RSD}$ .000 웅

*** Sample ID: JWFJ2

Seq: $72 \quad 10: 23: 01$ 10 May 07 HG

$\begin{array}{lllll}\mathrm{Hg} & .029 & \mathrm{ppb} & .000 \quad 029\end{array}$

*** Sample ID: JWFKG

Seq: $73 \quad 10: 25: 5010$ May 07 HG

$\begin{array}{llllll}\mathrm{Hg} & .049 & \mathrm{ppb} & .000 \quad \% & .049\end{array}$

*** Sample ID: JWJG8B

Seq: $74 \quad 10: 27: 51 \quad 10$ May 07 HG

$\mathrm{Hg} \quad .012 \quad \mathrm{ppb}$ 7129090

*** Sample ID: JWJG8C $.000 \div .012$

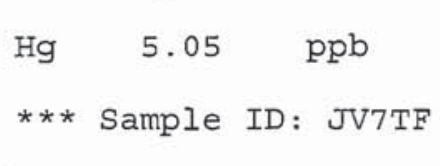

Seq: $75 \quad 10: 29: 42 \quad 10$ May 07 HG

$\begin{array}{lllll}\mathrm{Hg} & .012 & \mathrm{ppb} & .000 \quad \% & .012\end{array}$

*** Sample ID: JV7VG Seq: $77 \quad$ 10:33:49 10 May 07 HG

$\mathrm{Hg} \quad .016 \quad \mathrm{ppb} \quad .000 \quad \% \quad .016$

*** Sample ID: JV7VGS Seq: $78 \quad 10: 35: 37 \quad 10$ May 07 HG

Hg $\quad 1.11 \quad \mathrm{ppb} \quad .000 \quad \% \quad 1.11$

*** Sample ID: JV7VGD Seq: $79 \quad$ 10:37:29 10 May 07 HG

$\begin{array}{llllll} & \mathrm{Hg} & .994 \quad \mathrm{ppb} & .000 & \% & .994\end{array}$ 
STL - Knoxville

10:39:31 10 May 2007

Line Conc. Units

-

*** Sample ID: JV7VQ

$\mathrm{Hg} \quad .003 \quad \mathrm{ppb}$

*** Sample ID: JV7WH

$.000 \% \quad .003$
Folder: M051007

Protocol: 7ptcurve

$\mathrm{Hg} \quad .004 \quad \mathrm{ppb} \quad .000 \quad \% \quad .004$

$\begin{array}{llllllll}* * \text { Check Standard: } & 3 \text { Ck3ccv } & \text { Seq: } 82 & 10: 43: 21 & 10 \text { May } 07 & \text { HG } \\ \text { Line Flag } \% \text { Rcv. } & \text { Found } & \text { True Units } & \text { SD/RSD } & & & \\ \text { Hg } & 105 . & 5.27 & 5.00 & \text { ppb } & .000 \% & \end{array}$

$\begin{array}{llllll}\mathrm{Hg} & 105 & 5.27 & 5.00 & \mathrm{ppb} & .000 \%\end{array}$

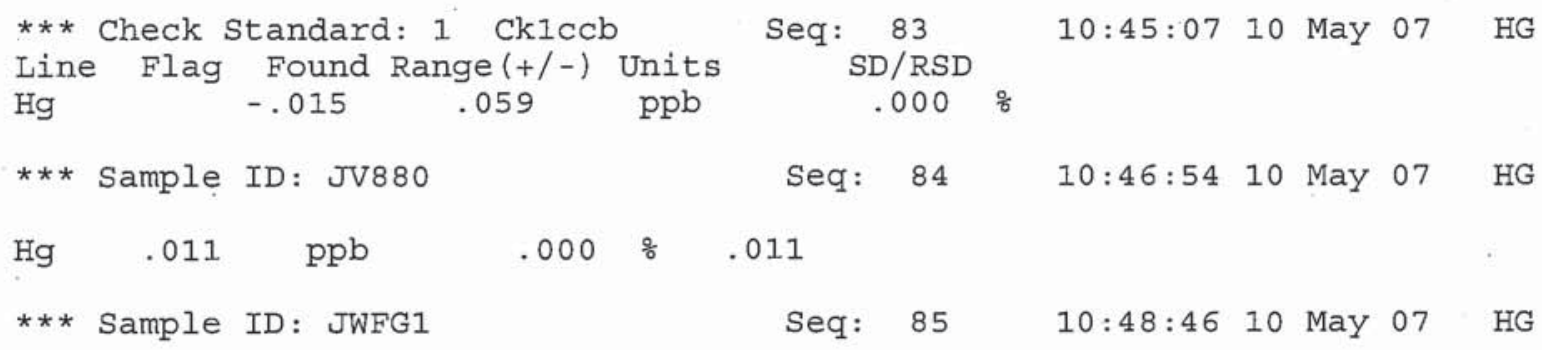

DILUTE Hg $21.5 \quad \mathrm{ppb} \quad .000$ \% 21.5

f *** Sample ID: JWFG1S Seq: $86 \quad 10: 50: 4510$ May 07 HG

$\begin{array}{llllll}\mathrm{Hg} & 22.2 & \mathrm{ppb} & .000 \quad \% & 22.2\end{array}$

*** Sample ID: JWFG1D Seq: $87 \quad 10: 52: 48 \quad 10$ May 07 HG

L $\begin{array}{lllll}\mathrm{Hg} & 21.7 & \mathrm{ppb} & .000 & \%\end{array}$

*** Sample ID: JWFHN Seq: $88 \quad 10: 54: 3910$ May 07 HG

RE-ANALYZE REVEIOUS SAMPLE HIGH

*** Sample ID: JWFJE Seq: $89 \quad$ 10:56:27 10 May 07 HG

Hg $\quad 3.45 \quad \mathrm{ppb} \quad .000 \% 3.45$

*** Sample ID: JWFJ4 Seq: $90 \quad 10: 58: 2810$ May 07 HG

$\begin{array}{llllll}\mathrm{Hg} & .026 \quad \mathrm{ppb} & .000 \quad \% \quad .026\end{array}$

*** Sample ID: JWFKJ Seq: 91 11:00:42 10 May 07 HG

Hg $\quad 6.60 \quad \mathrm{ppb} \quad .000 \% 6.60$

*** Sample ID: JWE4MBT Seq: 92 11:05:38 10 May 07 HG

Hg $\quad .957 \quad \mathrm{ppb} \quad .000 \% \quad .957$

*** Sample ID: JWFHN Seq: 93 11:07:30 10 May 07 HG

$\mathrm{Hg} \quad-.027 \mathrm{ppb} \quad .000 \frac{\mathrm{q}}{6}-.027$

*** Check Standard: $3 \mathrm{Ck} 3 \mathrm{ccv}$ Seq

Line Flag :Rcv. Found True Units

Hg 105. $5.24 \quad 5.00 \quad \mathrm{ppb}$
$94 \quad 11: 09: 49 \quad 10$ May 07 HG $S D / R S D$ $.000 \%$
*** Check Standard: $1 \mathrm{Cklccb}$

Line Flag Found Range $(+/-)$ Units

$\mathrm{Hg}$
Seq : 95

$\mathrm{SD} / \mathrm{RSD}$ $.000 \div$ 
STL - Knoxville

Folder: M051007

Protocol: 7ptcurve

Page 7160

$11: 14: 3310$ May 2007

SD/RSD

1

2

3

Seq : 96

$11: 14: 33 \quad 10$ May 07 HG

*** Sample ID: JWFG1Z10

$.000 \div 2.15$

$\mathrm{Hg}$

$\begin{array}{llll}2.15 & \mathrm{ppb} & .000 \% & 2.15\end{array}$

*** Sample ID: JWFG1SZ10

Seq: $97 \quad 11: 16: 21$ 10 May 07 HG

$\mathrm{Hg} \quad 2.20 \quad \mathrm{ppb} \quad .000 \quad \% \quad 2.20$

*** Sample ID: JWFGIDZ10

Seq: 98 11:18:51 10 May 07 HG

$\begin{array}{llll}\mathrm{Hg} & 2.23 & \mathrm{ppb} & .000\end{array}$

*** Check Standard: $3 \mathrm{Ck} 3 \mathrm{ccv}$ Seq:

Line Flag $\%$ RCV. Found True Units

Hg $\quad$ 106. $5.32 \quad 5.00 \quad \mathrm{ppb}$

99 11:20:39 10. May 07 HG

*** Check Standard: $1 \mathrm{Ck} 1 \mathrm{ccb}$

Line Flag Found Range(+/-) Units

$\mathrm{Hg}$

.038

.059

$\mathrm{ppb}$

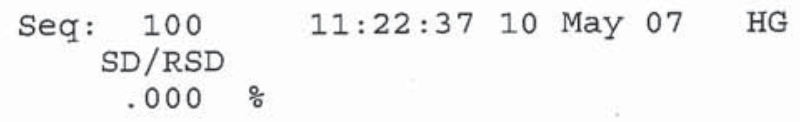




\section{STL Knoxville Mercury Analysis Cover Sheet}

\begin{tabular}{|r|l|l|l|}
\hline Analyst & DKW & Instrument & Leeman Hydra AA Mercury Analyzer \\
\hline Run Date & $05 / 17 / 07$ & Chart Name & M05/707 \\
\hline
\end{tabular}

\begin{tabular}{||c|l|c|l||}
\hline \multicolumn{2}{|l|}{ Daily Maintenance } & \multicolumn{2}{c|}{ As Needed Maintenance } \\
\hline \multirow{2}{*}{\begin{tabular}{l} 
Check tubing. \\
\cline { 3 - 4 }
\end{tabular}} & $\begin{array}{l}\text { Check maintenance schedule } \\
\text { flags. }\end{array}$ & $\square$ & Clean or replace optical cell. \\
\cline { 3 - 4 } & $\square$ & Lubricate pump and autosampler arm. \\
\cline { 3 - 4 } & $\square$ & Change the drying tube. \\
\hline
\end{tabular}


Instrument Upload

Started Thu May 17 08:55:28 2007 by WILBURND

: Data File: UPL $\$$ KNX_DATA_ROOT : $<$ LHG $>$ M051707.PRN; 1

Run Log - Page 1 :

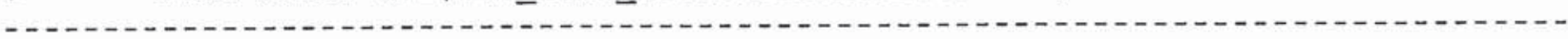

\begin{tabular}{|c|c|c|c|c|c|c|c|}
\hline \# & WorkOrder & Dilution & Date & Time & Batch & Lot & Instrumen \\
\hline & & $\ldots$ & - & $-\cdots-\cdots$ & $\cdots$ & - & \\
\hline 1 & CK2ICV & 1.00 & 17-MAY-2007 & $08: 19: 54$ & & & HG \\
\hline 2 & $I C B$ & 1.00 & 17-MAY-2007 & $08: 21: 41$ & & & HG \\
\hline 3 & CRA & 1.00 & $17-M A Y-2007$ & $08: 23: 27$ & & & HG \\
\hline 4 & $\mathrm{CK} 3 \mathrm{CCV}$ & 1.00 & $17-M A Y-2007$ & $08: 25: 13$ & & & HG \\
\hline 5 & $\mathrm{CK} \perp \mathrm{CCB}$ & 1.00 & 17-MAY-2007 & $08: 27: 29$ & & & HG \\
\hline 6 & JWXH9BT & 1.00 & $17-\mathrm{MAY}-2007$ & $08: 29: 25$ & 7135462 & $\mathrm{H} 7 \mathrm{E} 140000$ & HG \\
\hline 7 & JW2DACT & 1.00 & 17-MAY-2007 & $08: 31: 26$ & 7135462 & $\mathrm{H} 7 \mathrm{E} 150000$ & HG \\
\hline 8 & JWQNGT & 1.00 & 17-MAY-2007 & $08: 33: 14$ & 7135462 & H7E110126 & HG \\
\hline 9 & JWPEDT & 1.00 & 17-MAY-2007 & $08: 35: 02$ & 7135462 & $\mathrm{H} 7 \mathrm{E} 100314$ & HG \\
\hline 10 & JWPEDST & 1.00 & 17-MAY - 2007 & $08: 37: 20$ & 7135462 & H7E100314 & HG \\
\hline 11 & JWPEDDT & 1.00 & 17-MAY-2007 & $08: 39: 09$ & 7135462 & H7E100314 & HG \\
\hline 12 & JWWQHB & 0.10 & $17-M A Y-2007$ & $08: 40: 57$ & 7134095 & $\mathrm{H} 7 \mathrm{E} 140000$ & HG \\
\hline 13 & JWWQHC & 0.10 & 17-MAY -2007 & $08: 43: 16$ & 7134095 & $\mathrm{H} 7 \mathrm{E} 140000$ & HG \\
\hline 14 & JWWQHL & 0.10 & 17-MAY-2007 & $08: 45: 44$ & 7134095 & $\mathrm{H} 7 \mathrm{E} 140000$ & HG \\
\hline 15 & JWAKF & 0.50 & $17-M A Y-2007$ & $08: 47: 32$ & 7134095 & H7E 040218 & HG \\
\hline 16 & $\mathrm{CK} 3 \mathrm{CCV}$ & 1.00 & 17-MAY-2007 & $08: 49: 30$ & & & HG \\
\hline 17 & CKICCB & 1.00 & 17-MAY - 2007 & $08: 51: 46$ & & & HG \\
\hline 18 & JWAGK & 0.10 & 17-MAY -2007 & $08: 53: 36$ & 7134095 & $\mathrm{H} 7 \mathrm{E} 040218$ & HG \\
\hline 19 & JWAHE & 0.10 & $17-\mathrm{MAY}-2007$ & $08: 55: 22$ & 7134095 & $\mathrm{H} 7 \mathrm{E} 040218$ & HG \\
\hline 20 & JWAH7 & 0.50 & 17-MAY -2007 & $08: 57: 31$ & 7134095 & H7E0 40218 & HG \\
\hline 21 & JWA FK & 8.10 & 17-MAY -2007 & $08: 59: 18$ & 7134095 & $\mathrm{H} 7 \mathrm{E} 040218$ & HG \\
\hline 22 & JWAL 3 & 0.50 & 17-MAY-2007 & $09: 01: 05$ & 7134095 & $\mathrm{H} 7 \mathrm{E} 040218$ & HG \\
\hline 23 & JWAM2 & 0.10 & 17-MAY - 2007 & $09: 03: 03$ & 7134095 & $\mathrm{H} 7 \mathrm{E} 040218$ & HG \\
\hline 24 & JWAPF & 0.10 & 17-MAY -2007 & $09: 04: 56$ & 7134095 & $\mathrm{H} 7 \mathrm{E} 040218$ & HG \\
\hline 25 & JWAPP & 0.10 & 17-MAY-2007 & $09: 07: 43$ & 7134095 & $\mathrm{H} 7 \mathrm{E} 040218$ & HG \\
\hline 26 & JW2DPB & 1.00 & 17-MAY - 2007 & $09: 09: 41$ & 7136046 & $\mathrm{H} 7 \mathrm{E} 160000$ & HG \\
\hline 27 & JW2DPC & 1.00 & 17-MAY -2007 & $09: 11: 28$ & 7136046 & $\mathrm{H} 7 \mathrm{E} 160000$ & HG \\
\hline 28 & $\mathrm{CK} 3 \mathrm{CCV}$ & 1.00 & 17-MAY -2007 & $09: 13: 16$ & & & HG \\
\hline 29 & $\mathrm{CK} \perp \mathrm{CCB}$ & 1.00 & 17-MAY-2007 & $09: 15: 24$ & & & HG \\
\hline 30 & JW2DPL & 1.00 & 17-MAY-2007 & $09: 17: 22$ & 7136046 & $\mathrm{H} 7 \mathrm{E} 160000$ & HG \\
\hline 31 & JWFGE & 1.00 & 17-MAY-2007 & $09: 19: 33$ & 7136046 & H7E070168 & HG \\
\hline 32 & JWFG8 & 1.00 & $17-\mathrm{MAY}-2007$ & $09: 21: 19$ & 7136046 & H7E070168 & HG \\
\hline 33 & JWFH4 & 1.00 & 17-MAY-2007 & $09: 23: 31$ & 7136046 & H7E070168 & HG \\
\hline 34 & JWFJN & 1.00 & $17-\mathrm{MAY}-2007$ & $09: 27: 16$ & 7136046 & H7E0 70168 & HG \\
\hline 35 & JWFKE & 1.00 & $17-M A Y-2007$ & $09: 29: 04$ & 7136046 & H7E0 70168 & HG \\
\hline 36 & JWFMK & 1.00 & $17-\mathrm{MAY}-2007$ & $09: 30: 55$ & 7136046 & $\mathrm{H} 7 \mathrm{E} 070168$ & HG \\
\hline 37 & JWFGEA & 1.00 & $17-\mathrm{MAY}-2007$ & $09: 34: 05$ & 7136046 & $\mathrm{H} 7 \mathrm{E} 070168$ & HG \\
\hline 38 & JWAFKA & 1.00 & $17-M A Y-2007$ & $09: 36: 07$ & 7134095 & $\mathrm{H} 7 \mathrm{E} 040218$ & HG \\
\hline 39 & JWM3FBT & 1.00 & $17-\mathrm{MAY}-2007$ & $09: 37: 59$ & 7136191 & $\mathrm{H} 7 \mathrm{E} 100000$ & HG \\
\hline 40 & $\mathrm{CK} 3 \mathrm{CCV}$ & 1.00 & $17-\mathrm{MAY}-2007$ & $09: 39: 52$ & & & HG \\
\hline 41 & CKICCB & 1.00 & $17-\mathrm{MAY}-2007$ & $09: 41: 40$ & & & HG \\
\hline 42 & JW2WCCT & 1.00 & 17-MAY-2007 & $09: 43: 29$ & 7136191 & $\mathrm{H} 7 \mathrm{E} 160000$ & HG \\
\hline 43 & JWMXKT & 1.00 & $17-M A Y-2007$ & $09: 45: 16$ & 7136191 & H7E100161 & HG \\
\hline 44 & JWMXKST & 1.00 & $17-M A Y-2007$ & $09: 47: 35$ & 7136191 & H7E100161 & HG \\
\hline
\end{tabular}


Instrument Upload

Run Log - Page 2 :

Started Thu May 17 08:55:28 2007 by WILBURND

Data File: UPL\$KNX_DATA_ROOT:<LHG >M051707.PRN; 1

\begin{tabular}{|c|c|c|c|c|c|c|c|}
\hline \# & WorkOrder & Dilution & Date & Time & Batch & Lot & Instrument \\
\hline & 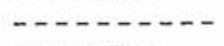 & $---1-1-1$ & 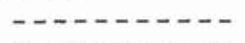 & 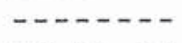 & 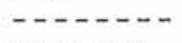 & $\cdots-$ & \\
\hline 45 & JWMXKDT & 1.00 & 17-MAY-2007 & $09: 50: 34$ & 7136191 & H7E100161 & HG \\
\hline 46 & TEST & 1.00 & 17-MAY-2007 & $09: 53: 05$ & & & HG \\
\hline 47 & JWAKFZ5 & 1.00 & 17-MAY-2007 & $09: 55: 07$ & 7134095 & H7E040218 & HG \\
\hline 48 & JWAH7Z5 & 1.00 & 17-MAY-2007 & $09: 57: 05$ & 7134095 & H7E040218 & HG \\
\hline 49 & JWAL $3 Z 5$ & 1.00 & 17-MAY-2007 & $09: 59: 12$ & 7134095 & $\mathrm{H} 7 \mathrm{E} 040218$ & HG \\
\hline 50 & JWAGKA & 1.00 & 17-MAY-2007 & $10: 05: 48$ & 7134095 & $\mathrm{H} 7 \mathrm{E} 040218$ & HG \\
\hline 51 & $\mathrm{CK} 3 \mathrm{CCV}$ & 1.00 & $17-\mathrm{MAY}-2007$ & $10: 07: 37$ & & & HG \\
\hline 52 & CKICCB & 1.00 & 17-MAY-2007 & $10: 09: 23$ & & & HG \\
\hline
\end{tabular}




\section{STL KNOXVILLE}

\section{INTERMEDIATE STANDARD -- CALIBRATION}

Stock Std Name: PLHG-2Y

Element: $\mathrm{Hg}$

Stock Source: Spex

Stock Std Lot \#: $12 \cdot 168 \mathrm{HG}$

Stock Std Expiration Date: $4 / 30 / 2008$

Stock Conc.; $1000 \mathrm{ppm}$

$\mathrm{HNO}_{3}$ Lot $\mathrm{B} 44032$
Stock Vol $(\mathrm{ml}) / 100 \mathrm{ml}: 1 \mathrm{ml}$

Date Prepared: 5-1-0n

Expiration Date: $6-1-07$

QC Check: 1050207

Initials: Drw

Matrix: $2 \% \mathrm{HNO}_{3}$

\section{WORKING STANDARD - CALIBRATION}

One ml Intermediate Standard $/ 100 \mathrm{ml} 0.15 \%$ Concentrated Nitric Acid

1. I-CAL $3425-10-1$ Initials DKW

2. I-CAL $3425-10-2$ Initials DKW

3. I-CAL $3425-10-3$ Initials $\mu w D$

4. I-CAL $3425-10-4$ Initials DKW

5. I-CAL $3425-10-5$

6. I-CAL $34230-10-6$

7. I-CAL $3425-10-7$

8. .I-CAL

9. .I-CAL

10..I-CAL

11..I-CAL

12..I-CAL

13. I-CAL

14. I-CAL

15. I-CAL

16. I-CAL

17. I-CAL

18. I-CAL

19. .I-CAL

20. I-CAL

21. I-CAL

22. I-CAL

23. I-CAL

24. I-CAL

25. I-CAL Initials DKW Initials DKeW Initials Dow Initials Initials Initials Initials Initials Initials Initials Initials Initials Initials Initials Initials Initials Initials Initials Initials Initials Initials
Date: 050107

Exp. Date: 050207 HN03 Lot: 344032 Date: 050707 Exp. Date: 050807 HN03 Lot: 844032 Date: $5 / 8 / 07$ Exp. Date: $5 / 9 / 07$ HN03 Lot: Date: 050907 Exp. Date: $05 / 10 / 07$ HN03 Lot: Date: 051007 Exp. Date: $05 / 11 / 01$ HN03 Lot: Date: 051407 Exp. Date: $05 / 507$ HN03 Lot: Date:051607 Exp. Date: 057707 HN03 Lot: Date:_Exp. Date: Date:_Exp. Date: Date: Exp. Date: Date: Exp. Date: Date:___ Exp. Date: Date: Exp. Date: Date:_Exp. Date: Date: Exp. Date: Date: Exp. Date: Date: Exp. Date:

Date: Exp. Date:

Date: Exp. Date:

Date: Exp. Date:

Date: Exp. Date: Date: Exp. Date: Date: Exp. Date: Date: Exp. Date: Date: Exp. Date: HNO3 Lot: B.44032 844032 Bu403á B4403: HNO3 Lot: HN03 Lot: HN03 Lot: HN03 Lot: HN03 Lot: HN03 Lot: HN03 Lot: HN03 Lot: HNO3 Lot: HN03 Lot: HN03 Lot: HN03 Lot: HN03 Lot: HNO3 Lot: HNO3 Lot: HN03 Lot: HN03 Lot:

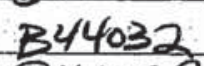




\section{STL KNOXVILLE}

\section{INTERMEDIATE STANDARD - VERIFICATION}

Stock Std Name: ICP-080

Element: $\mathrm{Hg}$

Stock Source: Ultra.

Stock Std Lot \#: FOO 424

Stock Std Expiration Date: $\quad 08-3 /-08$

Stock Conc.: $1000 \mathrm{ppm}$

Acid Lot: B44032
Stock Vol $(\mathrm{ml}) / 100 \mathrm{ml}: 1.0 \mathrm{ml}$

Date Prepared: $5-1-07$

Expiration Date: $\frac{6-1-07}{6}$

QC Check: $M 050207$

Initials: DEW

Matrix: $2 \% \mathrm{HNO}_{3}$

\section{WORKING STANDARD - VERIFICATION}

$1 \mathrm{ml}$ Intermediate Standard/100 $\mathrm{ml} 0.15 \%$ Concentrated Nitric Acid

1. I-VER $3426-11-1$

2. I-VER $3426-11-2$

3. I-VER $3426-11-3$

4. I-VER $3426-11-4$

5. I-VER $3426-11-5$

6. I-VER $3426-11-6$

7. I-VER

8. I-VER

9. I-VER

10. I-VER

11. I-VER

12. I-VER

13. I-VER

14. I-VER

15. I-VER

16. I-VER

17. I-VER

18. I-VER

19. I-VER

20. I-VER

21. I-VER

22. I-VER

23. I-VER

24. I-VER

25. I-VER
Initials Deee Initials ID Cu: Initials DRH Initials DXE Initials DKW Initials I/CW Initials Initials Initials Initials Initials Initials Initials Initials Initials. Initials Initials Initials Initials Initials Initials Initials Initials Initials Initials
Date: 050107 Exp. Date: 054207 FN03 Lot: 344032 Date:050807 Exp. Date:05080 7 HN03 Lot:B44082 Date:050907 Exp. Date:056307 HN03 Lot: 344032 Date:05/007Exp, Date:05/107 HN03 Lot: 344032 Date:05/407Exp. Date:05/507HN03 Lot: 344032 Date05/607 Exp. Date:05170\%17N03 Lot:B44032 Date:___Exp. Date: HN03 Lot: Date: ___ Exp. Date: HN03 Lot: Date:_Exp. Date:__ HN03 Lot: Date:___ Exp. Date: ___ HN03 Lot: Date: Exp. Date:__ HN03 Lot: Date:_Exp. Date:___ HN03 Lot: Date: Exp. Date:___ HN03 Lot: Date: ___ Exp. Date:___ HN03 Lot: Date: ___._. Exp. Date: ____ HN03 Lot: Date: ___ Exp. Date: ___ HN03 Lot: Date: ___ Exp. Date:___ HN03 Lot: Date:___ Exp. Date:__ HN03 Lot: Date: ___ Exp. Date: __ HN03 Lot: Date: ___ Exp. Date: ___ HN03 Lot: Date: ___Exp. Date:___ HN03 Lot: Date: ___ Exp. Date: ___ HN03 Lot: Date: ___ Exp. Date: ___ HN03 Lot: Date: __ Exp. Date:__ HN03 Lot: Date:___ Exp. Date:__ HNO3 Lot: 


\section{STL Knoxville}

\section{Method Information}

Method Name: Ontario-Hydro Method

Instrument Calibrated Every ? Hours: 24

Correlation Coefficient $>/=: \quad 0.995$

CRA Required?: Yes

Duplicate RPD: $\quad 20$
Element: $\mathrm{Hg}$ Chart Number: m051707.prn

Number of Calibration Standards: 6

Run ICV Immediately After Calibration?: Yes

Number of Samples Between CCVs: 10

Calculate Duplicates by CLP or SW-846?: SW-846 Protocol

$\begin{array}{rr}\text { CRA Recovery Range: } & 30.5 \\ \text { ICV Recovery Range: } & 10.5 \\ \text { CCV Recovery Range: } & 20.5 \\ \text { LCS Recovery Range: } & 20.5 \\ \text { MS/MSD Recovery Range: } & 20.5\end{array}$

Letter to Signify Blanks: B

Letter to Signify LCS's: C

Letter to Signify LCSD's: L

Letter to Signify Dilutions: $\quad Z$

Letter to Signify Duplicates: $\mathrm{X}$

Letter to Signify MS's: $\quad$ S

Letter to Signify MSD's: D

Letters to Signify MSA's: MSA+

Number of Additions: $\quad 3$
CRA Conc. (ug/L): $\quad 0.2$

ICV Conc. (ug/L): $\quad 2.5$

CCV Conc. (ug/L): 5

LCS Spike Level (ug/L): $\quad 5$

MS Spike Level (ug/L): $\quad 1$

\section{Calibration Standard Concentrations (ug/L)}

Standard 1 Conc: 0.1

Standard 5 Conc: 5
Standard 2 Conc: 0.2

Standard 6 Conc: 10
Standard 3 Conc: 0.5

Standard 7 Conc: 0
Standard 4 Conc: 1

Standard 8 Conc: 0

ICB/CCB Check Level (ug/L): $0.099 \quad$ Prep Blank Check Level (ug/L): $0.099 \quad$ Reporting Limit (ug/L): 0.1

IDL (ug/L): 0.06

Date of IDL: $12 / 14 / 2006$ 
STL Knoxville Mercury Data Review Checklist

\section{Method: Ontario-Hydro Method}

\begin{tabular}{|c|c|c|c|c|c|c|c|}
\hline Analyst: & \multicolumn{3}{|c|}{ DKW } & Instrument: & \multicolumn{2}{c|}{ Leeman Hydra AA Mercury Analyzer } \\
\hline Run Date: & May-17-07 & Calibration Number: & 1 & Chart Name: & m051707.prn & Element: & Hg \\
\hline
\end{tabular}

\section{A. Calibration/Instrument Run QC}

1. Instrument calibrated every 24 hours?

2. Instrument calibrated using a minimum of a blank and 5 standards?

3. Do standard absorbances increase as standard concentrations increase?

4. Instrument calibrated with standards at the SOP levels?

5. Correlation coefficient greater than or equal to 0.995 ?

6. ICV analyzed directly after calibration?

7. ICV within specified control limits?

8. ICB analyzed immediately after ICV?

9. ICB within acceptable range?

10. CRA analyzed at the beginning of the run?

11. CRA results within acceptable range?

12. All CCV's within specified control limits?

13. All CCB's within acceptable range?

14. All CCB's analyzed immediately after CCV's?

\section{B. Client Sample and QC Sample Results}
1.Were all sample results within the linear range of the instrument?
2. Were there any samples with results more negative
than Report Limit?
3.Were samples analyzed within the holding time?

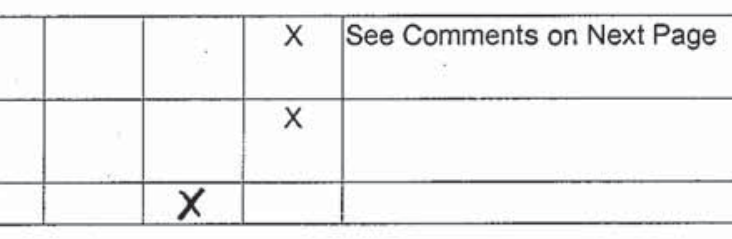

\section{Preparation/Matrix QC}

1.Was one method blank prepared for each batch?

2. Were all method blanks less than the specified level?

3.Was an LCS prepared for each batch?

4. Were all LCS recoveries within QC limits?

5. Were matrix spikes, sample duplicates and/or post-

digestion spikes run at required frequency?

6.Were all matrix spike recoveries within $\mathrm{QC}$ limits?

7.Were post-digestion spike recoveries within $\mathrm{QC}$ limits?

8. Were duplicate RPDs within QC limits?

\begin{tabular}{|l|c|c|l|}
\hline N/A & Yes & No & Comments \\
\hline & $X$ & & Calibration was 2 hours and 3 minutes long \\
\hline & $X$ & & \\
& $X$ & & \\
& $X$ & & \\
\hline & $X$ & & Correlation coefficient was 0.9999 \\
\hline & $X$ & & \\
\hline & $X$ & & \\
\hline & $X$ & & \\
\hline & $X$ & & \\
\hline & $X$ & & \\
\hline & $X$ & & \\
\hline & $X$ & & \\
\hline & $X$ & & \\
\hline
\end{tabular}

\section{Other}

1. Current IDL data on file?

2. Current MDL data on file?

3. Are all nonconformances documented appropriately?

4.Were all project specific instructions followed?

\begin{tabular}{|l|l|l|}
\hline$x$ & & \\
\hline$x$ & & \\
\hline$x$ & & \\
\hline$x$ & & \\
\hline$x$ & & \\
\hline & $\mathrm{x}$ & See Comments on Next Page \\
\hline$x$ & & \\
\hline $\mathrm{X}$ & & \\
\hline
\end{tabular}

Question

Number: Problem(s) Found:

B1 Sample JWAFKA at 5/17/2007 9:36:07 AM exceeded linear range and an acceptable dilution was NOT found.

C6 Matrix spike sample JWPEDST at 5/17/2007 8:37:20 AM had 5\% recovery.

C6 Matrix spike sample JWPEDDT at 5/17/2007 8:39:09 AM had $\$ 2 \%$ recovery.

C6 Matrix spike sample JWMXKST at 5/17/2007 9:47:35 AM had 577.7\% recovery.

C6 Matrix spike sample JWMXKDT at 5/17/2007 9:50:34 AM had $50.7 \%$ recovery. 
Analyst: DTL SThoxville Mercury Data Review Checklist $10 / 24$

Analyst Comments: 
Protocol: 7ptcurve

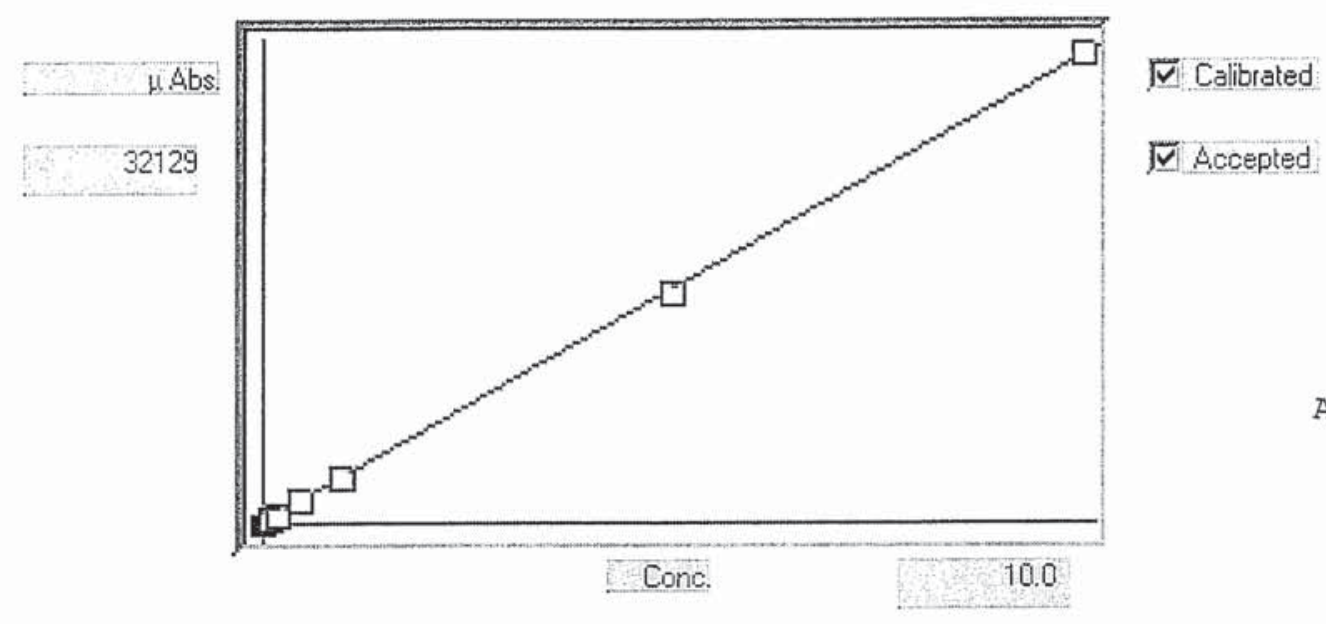

\section{Linear}

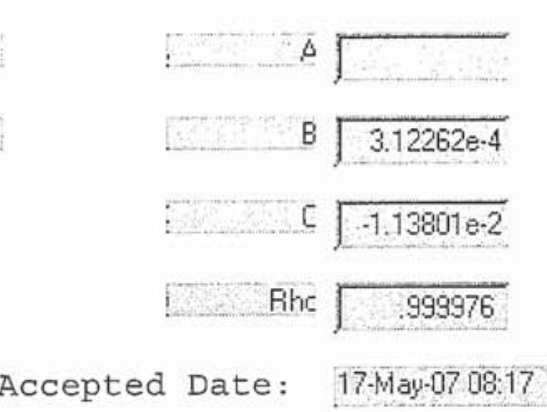

Calc.

Dev. Mean SD or \%RSD

318

318
730

1690

3299

15891

32129
Rep $1 \operatorname{Rep} 2 \operatorname{Rep} 3 \operatorname{Rep} 4 \operatorname{Rep} 5$

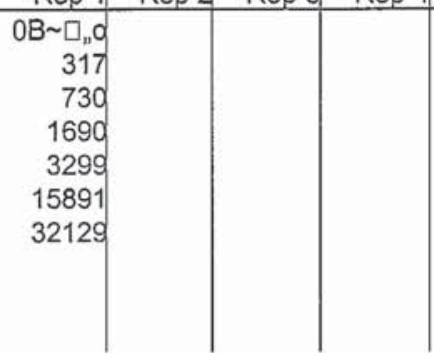




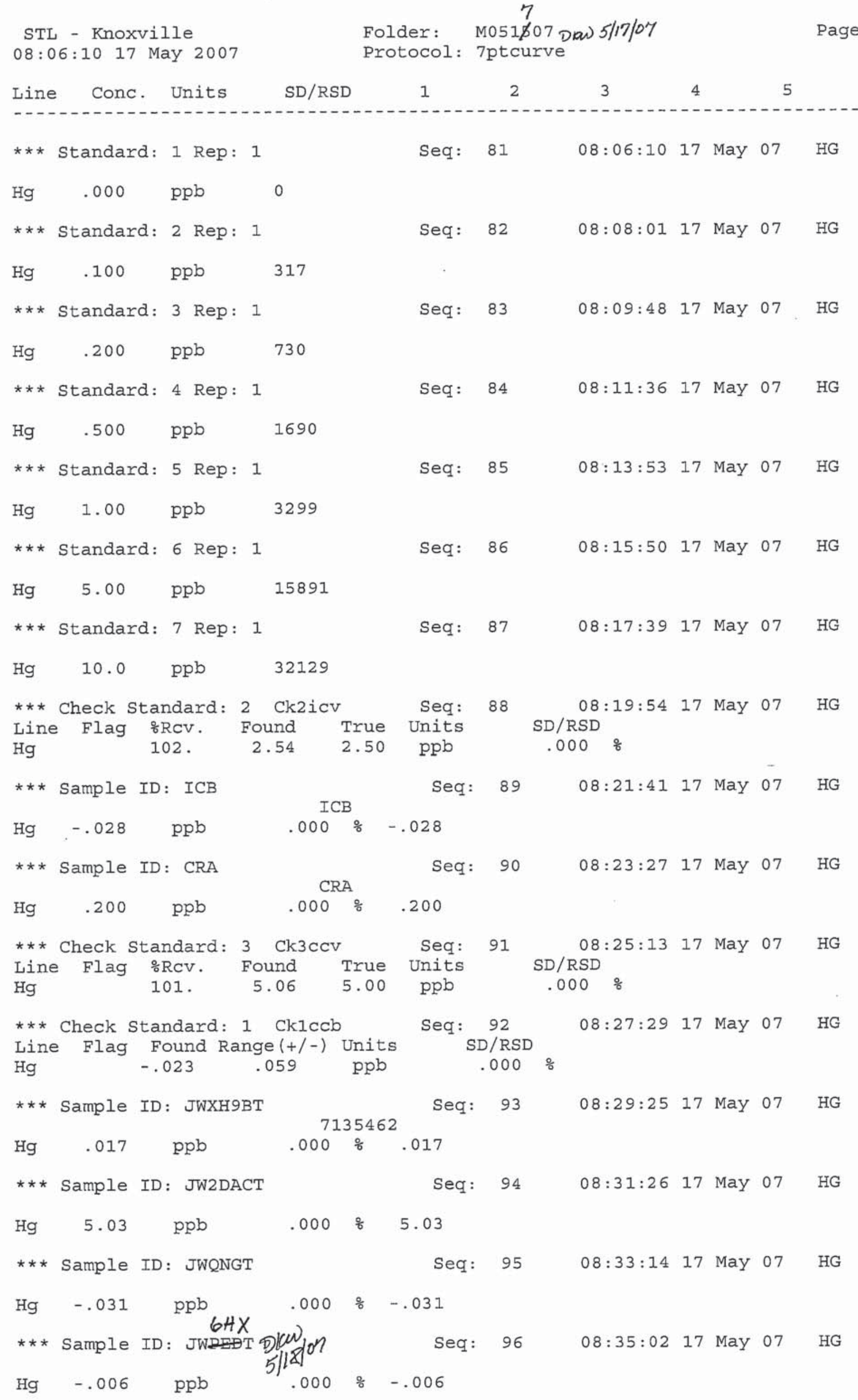


STL - Knoxville

08:37:20 17 May 2007 $\begin{array}{ll} & 7 \\ \text { Folder: } & \text { M051\%07 D/aw } 5 / 12 / 07 \\ \text { Protocol: } & 7 \text { ptcurve }\end{array}$
Line Conc. Units SD/RSD

*** Sample ID: JWHXST D/pulo?

Seq : $\quad 97$

3

$.000 \% 5.06$

Hg $\quad 5.06 \quad \mathrm{ppb}$

$64 X$
$D K W$
$5 / 18 / 07$

Seq : 98

08:39:09 17 May 07 HG

$\begin{array}{lllll}\mathrm{Hg} & 5.12 & \mathrm{ppb} & .000 & \circ\end{array}$

*** Sample ID: JWWQHB

Hg $\quad-.003 \quad \mathrm{ppb}$

$7134095 \mathrm{OH} F \mathrm{FH}$

Seq: $9908: 40: 5717$ May 07 HG $.000 \div-.003$

*** Sample ID: JWWQHC

Seq: 100 08:43:16 17 May 07 HG

$\begin{array}{llll}\mathrm{Hg} & 5.19 \quad \mathrm{ppb} & .000 \% & 5.19\end{array}$

*** Sample ID: JWWQHL

Seq: $101 \quad 08: 45: 4417$ May $07 \quad$ HG

$\mathrm{Hg} \quad 5.11 \quad \mathrm{ppb} \quad .000 \quad \% \quad 5.11$

*** Sample ID: JWA $\begin{array}{lllll}\mathrm{Hg} & 10.2 \quad \mathrm{ppb} & .000 \quad 10.2\end{array}$

*** Check Standard: 3 Ck3ccv 4 Seq: 103 08:49:30 17 May 07 HG
Line Flag \&RCV. Found True Units
SD/RSD $\mathrm{Hg} \quad 105 . \quad 5.24 \quad 5.00 \quad \mathrm{ppb} \quad .000 \quad \%$

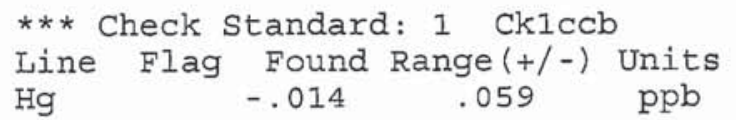

Seq: $104 \quad 08: 51: 4617$ May 07 HG

*** Sample ID: JWAGK

Seq: 105 08:53:36 17 May 07 HG

$\begin{array}{llllll} & \mathrm{Hg} & .582 & \mathrm{ppb} & .000 & \%\end{array}$

*** Sample ID: JWAHE

Seq: 106 08:55:22 17 May 07 HG

$\begin{array}{llllll} & \mathrm{Hg} & .286 & \mathrm{ppb} & .000 \quad \% & .286\end{array}$

Sample ID: JWAH7

Seq: 107 08:57:31 17 May 07 HG

$\begin{array}{lllll}\mathrm{Hg} & 14.0 & \mathrm{ppb} & .000 \% & \%\end{array}$

*** Sample ID: JWA FK $\begin{array}{llllll}H g & .113 & \mathrm{ppb} & .000 & \% & .113\end{array}$

*** Sample ID: JWAL3

Seq: 109 09:01:05 17 May 07 HG

$\begin{array}{lllll}\mathrm{Hg} & 10.2 & \mathrm{ppb} & .000 & \%\end{array}$

*** Sample ID: JWAM2

Seq: 110 09:03:03 17 May 07 HG

$\begin{array}{llllll}\mathrm{Hg} & -.026 & \mathrm{ppb} & .000 \quad \% & -.026\end{array}$

*** Sample ID: JWAPF

Seq: 111 09:04:56 17 May 07 HG

$\begin{array}{llllll}\mathrm{Hg} & .035 & \mathrm{ppb} & .000 \quad \% & .035\end{array}$

Sample ID: JWAPP

Seq: 112 09:07:43 17 May 07 HG

$\begin{array}{llllll} & \mathrm{Hg} & .322 & \mathrm{ppb} & .000 & \%\end{array}$ 
STL - Knoxville

09:09:41 17 May 2007
Folder: M051\$007 Daw 5/17/07

Protocol: 7ptcurve

Iine Conc. Units

SD/RSD

1

2

3

4

5

*** Sample ID: JW2DPB

Seq : 113

09:09:41 17 May 07 HG

$\mathrm{Hg} \quad .005 \quad \mathrm{ppb} \quad .000 \frac{7136046}{8} .005$

*** Sample ID: JW2DPC Seq: $114 \quad 09: 11: 2817$ May 07 HG

Hg $\quad 5.20 \quad \mathrm{ppb} \quad .000 \quad \% \quad 5.20$

$\begin{array}{llllllll}* * \text { Check Standard: } & 3 \mathrm{Ck} 3 \mathrm{ccv} & \text { Seq: } & 115 & 09: 13: 16 & 17 \text { May } 07 & \text { HG } \\ \text { Iine Flag \%Rcv. } & \text { Found True Units } & & \text { SD/RSD }\end{array}$

Hg 103. $\quad 5.16 \quad 5.00 \quad \mathrm{ppb} \quad .000 \quad \%$

*** Check Standard: 1 Cklccb Seq: $116 \quad 09: 15: 24 \quad 17$ May 07 HG

Line Flag Found Range $(+/-)$ Units SD/RSD

Hg $\quad-.018 \quad .059 \quad \mathrm{ppb} \quad .000 \%$

*** Sample ID: JW2DPL $\quad$ Seq: $117 \quad 09: 17: 2217$ May 07 HG

$\mathrm{Hg} \quad 5.08 \quad \mathrm{ppb} \quad .000 \quad 5.08$

*** Sample ID: JWFGE Seq: $118 \quad 09: 19: 3317$ May 07 HG

Hg $\quad .066 \quad$ ppb $\quad .000 \quad \% \quad .066$

*** Sample ID: JWFG8 Seq: $119 \quad 09: 21: 1917$ May 07 HG

$\mathrm{Hg} \quad-.006 \quad \mathrm{ppb} \quad .000 \quad \% \quad-.006$

*** Sample ID: JWFH4 Seq: $120 \quad 09: 23: 3117$ May 07 HG

$\mathrm{Hg} \quad .104 \quad \mathrm{ppb} \quad .000 \quad \% \quad .104$

*** Sample ID: JWFJN $\quad$ Seq: $121 \quad 09: 27: 1617$ May 07 HG

Hg $\quad .022 \quad \mathrm{ppb} \quad .000 \quad \% \quad .022$

*** Sample ID: JWFKE Seq: $122 \quad$ 09:29:04 17 May 07 HG

$\mathrm{Hg} \quad .085 \quad \mathrm{ppb} \quad .000 \quad \frac{\circ}{8} \quad .085$

*** Sample ID: JWFMK Seq: $123 \quad 09: 30: 5517$ May 07 HG

$\mathrm{Hg} \quad-.001 \quad \mathrm{ppb} \quad .000 \% \quad-.001$

*** Sample ID: JWFGEA Seq: $124 \quad$ 09:34:05 17 May 07 HG

$\mathrm{Hg} \quad 1.14 \quad \mathrm{ppb} \quad .000^{2}$ \% 1.14

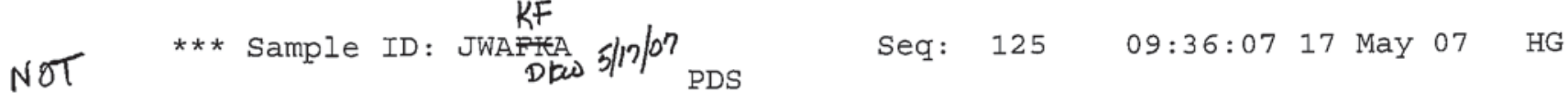
DILUTED Hg $\quad 10.3 \quad \mathrm{ppb} \quad .000 \quad \% \quad 10.3$

USED ANJTHER PDS

$\mathrm{Hg} \quad-.024 \quad \mathrm{ppb} \quad .000 \frac{\circ}{8}-.024$

*** Check Standard: $3 \mathrm{Ck} 3 \mathrm{ccv} \quad$ Seq: $127 \quad 09: 39: 52 \quad 17$ May 07 HG

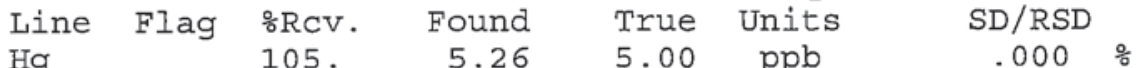

*** Check Standard: 1 Cklccb Seq: 128 09:41:40 17 May 07 HG

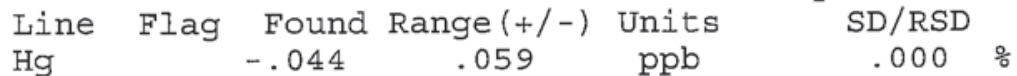


STL - Knoxville

09:43:29 17 May 2007

Line Conc. Units SD/RSD

1

2

3

4

5

*** Sample ID: JW2WCCT

Seq: 129 09:43:29 17 May 07 HG

$\mathrm{Hg}$

$5.42 \mathrm{ppb}$ $.000 \% 5.42$

*** Sample ID: JWMXKT

Seq: 130 09:45:16 17 May 07 HG

$\begin{array}{llllll}\mathrm{Hg} & .023 & \mathrm{ppb} & .000 \quad \% & .023\end{array}$

*** Sample ID: JWMXKST

Seq: 131 09:47:35 17 May 07 HG

$\begin{array}{lllll}\mathrm{Hg} & 5.80 \quad \mathrm{ppb} & .000 \quad \% \quad 5.80\end{array}$

*** Sample ID: JWMXKDT

Seq: 132 09:50:34 17 May 07 HG

$\begin{array}{lllll}\mathrm{Hg} & 5.53 \quad \mathrm{ppb} & .000 \quad \% \quad 5.53\end{array}$

*** Sample ID: TEST BLANK

Seq: 133 09:53:05 17 May 07 HG

$\mathrm{Hg} \quad .011 \quad \mathrm{ppb} \not K F \quad .000^{\text {TCLP }} \% .011$

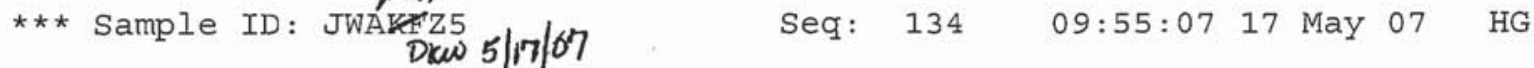

$\begin{array}{lllll}\mathrm{Hg} & 1.94 \quad \mathrm{ppb} & .000 & \% & 1.94\end{array}$

*** Sample ID: JWAH7z5 Seq: $135 \quad$ 09:57:05 17 May 07 HG

Hg $\quad 2.66 \quad$ ppb $\quad .000 \quad \% \quad 2.66$

*** Sample ID: JWAL3Z5 Seq: $136 \quad$ 09:59:12 17 May 07 HG

Hg $\quad 1.96 \quad$ ppb $\quad .000 \quad \frac{8}{7} \quad 1.96$

*** Sample ID: JWAGKA

Seq: $137 \quad 10: 05: 48 \quad 17$ May $07 \quad$ HG

Hg $\quad 1.56 \quad \mathrm{ppb} \quad .000^{\mathrm{PDS}} \% 1.56$

*** Check Standard: $3 \mathrm{Ck} 3 \mathrm{ccv} \quad$ Seq: $138 \quad$ 10:07:37 17 May 07 HG

Line Flag \%Rcv. Found True Units SD/RSD

Hg $\quad 105 . \quad 5.23 \quad 5.00 \quad \mathrm{ppb} \quad .000 \quad \%$

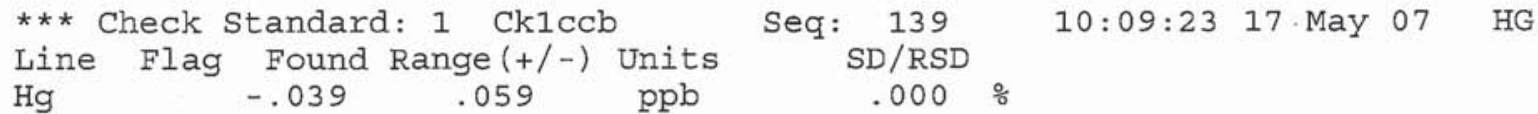

